\title{
External Dose-Rate Conversion Factors for Calculation of Dose to the Public
}

July 1988

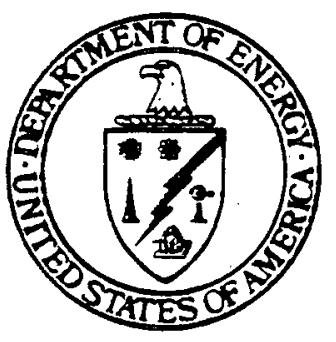

U.S. Department of Energy

Assistant Secretary for Environment, Safety and Health

Office of Environmental Guidance and Compliance

Washington, D.C. 20545
This document is

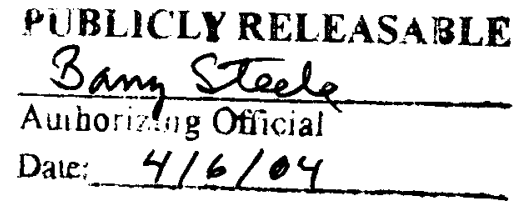




\section{DISCLAIMER}

This report was prepared as an account of work sponsored by an agency of the United States Government. Neither the United States Government nor any agency Thereof, nor any of their employees, makes any warranty, express or implied, or assumes any legal liability or responsibility for the accuracy, completeness, or usefulness of any information, apparatus, product, or process disclosed, or represents that its use would not infringe privately owned rights. Reference herein to any specific commercial product, process, or service by trade name, trademark, manufacturer, or otherwise does not necessarily constitute or imply its endorsement, recommendation, or favoring by the United States Government or any agency thereof. The views and opinions of authors expressed herein do not necessarily state or reflect those of the United States Government or any agency thereof. 


\section{DISCLAIMER}

Portions of this document may be illegible in electronic image products. Images are produced from the best available original document. 

LIST OF TABLES . . . . . . . . . . . . . . . . . . . . . . . . v v

1. INTRODUCTION ......................... . . . . 1

1.1 Description of External Dose-Rate Conversion Factors. . . . 1

1.2 Adjustments to Previously Documented Methodology. . . . . . 3

1.3 Outline of Report... . . . . . . . . . . . . . . . 5

2. CALCULATION OF EXTERNAL DOSE-RATE FACTORS FOR PHOTONS AND

ELECTRONS. . . . . . . . . . . . . . . . . . . . . . . . . . . . 7

2.1 Fhoton Dose-Rate Factors for Body Organs. . . . . . . . . . . . . 7

2.1.1 Immersion in contaminated air. . . . . . . . . . . . 8

2.1.2 Immersion in contaminated water. . . . . . . . . . . 12

2.1.3 Exposure to a contaminated ground surface. . . . . . 13

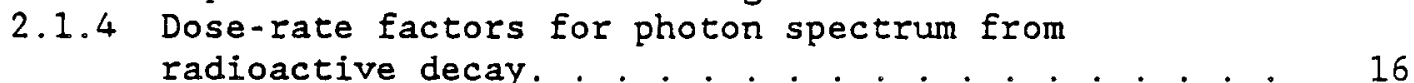

2.2 Electron Dose-Rate Factors for Skin . . . . . . . . . . . . . . . 17

2.2.1 Immersion in contaminated water. . . . . . . . . . . 18

2.2.2 Immersion in contaminated air. . . . . . . . . . . . 19

2.2.3 Exposure to a contaminated ground surface. . . . . . 21

2.2 .4 Dose-rate factors for electron spectrum from
radioactive decay. . . . . . . . . . . . . . . . . . 24

2.3 Effective Dose-Rate Factors for Photons. . . . . . . . . . . 27

3. IMPLEMENTATION OF EXTERNAL DOSE-RATE FACTORS FOR PHOTONS AND ELECTRONS . . . . . . . . . . . . . . . . . . . . . . . . . . . 29

3.1 Radiations from Spontaneous Fission and Bremsstrahlung. . . 30

3.1.1 Spontaneous fission. . . . . . . . . . . . . . . . . 30

3.1.2 Bremsstrahlung. . . . . . . . . . . . . . . . . . . 30

3.2 Corrections to the External Dose-Rate Factors... . . . . 31

3.2.1 Effects of indoor residence. . . . . . . . . . . . . . . . . . 32

3.2.2 Effects of ground roughness. . . . . . . . . . . . 35

3.2.3 Exposure during boating activities... . . . . . . . 36

3.2 .4 Exposure to contaminated shorelines. . . . . . . . . 37

3.2.5 Guidance on application of dose-reduction factors. . 38

3.3 External Dose-Rate Factors for Sources in Soil. . . . . . . 39

3.3.1 Photon dose-rate factors... . . . . . . . . . . . 39

3.3.2 Electron dose-rate factors . . . . . . . . . . . . . 40

3.4 External Dose-Rate Factors for Radioactive Decay Chains . . 41

REFERENCES . . . . . . . . . . . . . . . . . . . 45 
$\underline{\text { Page }}$

APPENDIX A. TABLES OF DOSE-RATE CONVERSION FACTORS FOR EXTERNAL EXPOSURE TO PHOTONS AND ELECTRONS ............ . 49

A.1 Dose-Rate Factors for Immersion in Contaminated Air . . . . 51

A.2 Dose-Rate Factors for Immersion in Contaminated Water . . . 91

A.3 Dose-Rate Factors for Exposure to a Contaminated Ground Surface. . . . . . . . . . . . . . . . . . . . 131

APPENDIX B. DIAGRAMS OF RADIOACTIVE DECAY CHAINS. . . . . . . . . . 171

APPENDIX C. IISTING OF THE DOSFACTER COMPUTER CODE. . . . . . . . . 191 
LIST OF FIGURES

Figure

$\underline{\text { Page }}$

B.I Diagrams of Radioactive Decay Chains. . . . . . . . . . . . . 171

LIST OF TABLES

Table

$\underline{\text { Page }}$

1 Organ-specific weighting factors for calculation of

effective dose equivalents. . . . . . . . . . . . . . . . . 28

A.1 Dose-rate factors for body organs and effective dose-rate

factor for immersion in contaminated air. . . . . . . . . . . 52

A. 2 Dose-rate factors for body organs and effective dose-rate

factor for immersion in contaminated water. . . . . . . . . . . 92

A.3 Dose-rate factors for body organs and effective dose-rate

factor for exposure $1 \mathrm{~m}$ above contaminated ground surface. 
This report presents a tabulation of dose-rate conversion factors for external exposure to photons and electrons emitted by radionuclides in the environment. ${ }^{*}$ This report was prepared in conjunction with criteria for limiting dose equivalents to members of the public from operations of the U.S. Department of Energy (DOE), as given in DOE Order 5400.3, "Radiation Protection of thr. Public and the Environment." The dose-rate conversion factors are provided for use by the DOE and its contractors in performing calculations of external dose equivalents to members of the public.

The dose-rate conversion factors for external exposure to photons and electrons presented in this report are based on a methodology developed at Oak Ridge National Laboratory. $(1,2)$ However, as described in Section 1.2, some adjustments of the previously documented methodology have been made in obtaining the dose-rate conversion factors in this report.

\subsection{Description of External Dose-Rate Conversion Factors}

Dose-rate conversion factors for external exposure, which also are referred to as dose-rate factors, represent the external dose-equivalent rate per unit concentration of a radionuclide in the environment for a particular mode of exposure. The exposure modes considered for members of the public include the following:

- immersion in a contaminated atmospheric cloud;

- immersion in contaminated water; and

- exposure to a contaminated ground surface.

These exposure modes also are referred to as air immersion, water immersion, and ground-surface exposure.

$*$ This report was prepared at Oak Ridge National Laboratory by D. C. Kocher, with the assistance of K. F. Eckerman. 
The estimation of external dose from arbitrary distributions of radionuclides in the environment is a difficult problem. Therefore, simplified and idealized exposure conditions often are assumed; namely, that the distribution of sources at the location of an exposed individual is effectively infinite or semi-infinite in extent and that the radionuclide concentrations are uniform throughout the source region. In particular, a semi-infinite source region is assumed for immersion in contaminated air, and an infinite source region is assumed for immersion in contaminated water and exposure to a contaminated ground surface.

If we assume that an infinite or semi-infinite source region has a uniform radionuclide concentration $C$ at time $t$, then the instantaneous external dose-equivalent rate to an exposed individual at that time, $\mathrm{dH}(t) / \mathrm{dt}$, can be written in the general form

$$
\mathrm{dH}(t) / \mathrm{d} t-C(t) \times \mathrm{DRF},
$$

where DRF denotes the dose-rate factor for external exposure and is independent of time. The dose-rate factor thus is defined as the external dose-equivalent rate per unit radionuclide concentration.

The external dose-rate factor is determined uniquely for a given radionuclide by the radiation type (i.e., photons or electrons), the mode of exposure (e.g., immersion in contaminated air), and the body organ or combination of organs for which the external dose-equivalent rate is desired. The dose-rate factor thus embodies all aspects of the calculation of external dose equivalents except for the radionuclide concentration, which is estimated either by direct measurement or by use of models for environmental transport of radionuclides. The determination of radionuclide concentrations in the environment, including their dependence on time after a release, is beyond the scope of this report.

In comparing calculated radiation doses with applicable limits for members of the public, the quantity of interest is the annual dose equivalent, $H$. For external exposure, $H$ is the integral of the instantaneous dose-equivalent rate over a time period of one year and, from eq. (1), can be written in the general form

$$
H=D R F \times \int C(t) d t .
$$




\subsection{Adjustments to Previously Documented Methodology}

Some adjustments of the previously documented methodology $(1,2)$ have been made in calculating the external dose-rate factors presented in this report. The adjustments to the DOSFACTER computer code used to perform the calculations are described as follows.

- Calculations of photon and electron dose-rate factors for the body surface have been eliminated, since the body surface does not represent a tissue at risk from radiation exposure.

- Calculations of photon dose-rate factors for "total body," which represent the total energy absorbed in the body divided by the body mass, have been replaced by effective dose-rate factors for photons as described below. The effective dose-rate factor provides the more appropriate quantity for use in estimating risk from both external and internal exposures.

- Effective dose-rate factors for external exposure to photons have been calculated using the weighting factors for specific body organs recommended by the International Commission on Radiological Protection (ICRP). (3) The effective dose-rate factors presented in this report were obtained from photon dose-rate factors for specific organs calculated according to the methodology documented previously. (1) Although dose-rate factors for skin are calculated in this report and a weighting factor for skin has been recommended by the ICRP for use in assessing the detriment to population groups (i.e., the collective dose), (4) neither the photon nor the electron dose-rate factor for skin is included in the effective dose-rate factors tabulated in this report. This approach is consistent with the ICRP recommendation that the dose to skin be excluded in calculating effective dose equivalents to maximally exposed individuals in critical groups of the population. (4) 
- The previous calculations of electron dose-rate factors for skin at a depth below the body surface of $70 \mu \mathrm{m}(1)$ have been revised slightly by assuming a depth of $7 \mathrm{mg} / \mathrm{cm}^{2}$, as recommended by the ICRP; ${ }^{(3)}$ this depth represents an average value over the body surface which is appropriate for use in environmental dose assessments. Since the density of epidermal tissue is assumed to be $1.12 \mathrm{~g} / \mathrm{cm}^{3}$, (5) the adjustment from $70 \mu \mathrm{m}$ to $7 \mathrm{mg} / \mathrm{cm}^{2}$ represents a $12 \%$ decrease in the assumed depth of radiosensitive tissues of the skin. However, in most cases, the resulting increases in electron dose-rate factors for skin are small (e.g., about 38 for immersion in an atmospheric cloud of $85 \mathrm{Kr}$ ). In addition, the previous calculations of electron doserate factors for skin obtained as an average over the entire thickness of the dermis between 50 and $1250 \mu \mathrm{m}(1)$ have been eliminated, since the basal cell layer of the epidermis is regarded by the ICRP as the principal skin tissue at risk from radiation exposure. (3) Finally, the DOSFACTER code now includes calculations of electron dose-rate factors for skin at depths below the body surface of 4,8 , and $40 \mathrm{mg} / \mathrm{cm}^{2}$, as recommended by whitton; (6) these depths represent appropriate values for different parts of the body surface. However, electron dose-rate factors for these depths of the skin are not tabulated in this report.

- In the previous version of the DOSFACTER code, ${ }^{(1)}$ electron dose-rate factors for skin were calculated incorrectly for the particular case of ground-surface exposure to radionuclides that emit high-energy electrons. Specifically, for emitted electron energies above about $4.5 \mathrm{MeV}$, the subroutine BGRND that performs numerical integrations of eq. (25) in Section 2.2.3 of this report underestimated the electron dose-rate factor at a depth of $70 \mu \mathrm{m}$ by an amount that increased with increasing energy. The largest error was about $50 \%$ for emitted electron energies near $10 \mathrm{MeV}$, which is the highest electron energy occurring in the decay of radionuclides considered in this report. (7) This error has been corrected in the present calculations. However, the electron dose-rate factors for skin for ground-surface exposure are increased for only a few radionuclides, since the electrons emitted by most of the radionuclides considered in this report have 
energies less than $4.5 \mathrm{MeV}$ (7) Furthermore, for those few radionuclides that are affected by the correction, the resulting increase in the dose-rate factor is only a few percent or less, because most of the electrons emitted by these radionuclides also have energies less than $4.5 \mathrm{MeV}$ ( (7)

- The output of the DOSFACTER code has been reformatted so that all dose-rate factors for a given exposure mode are presented in a single table. This table gives ( 1 ) the photon dose-rate factors for all organs except skin, (2) the effective dose-rate factor for photons based on the dose-rate factors for all organs except skin, and (3) the photon, electron, and photon plus electron (i.e., total) dose-rate factors for skin.

\subsection{Outline of Report}

The remainder of this report is organized as follows. Section 2 presents the equations used to calculate the external dose-rate factors for photons and electrons, including photon dose-rate factors for all body organs, electron dose-rate factors for skin, and effective dose-rate factors for photons. This section also describes the data bases in the DOSFACTER computer code and the other assumptions used in calculating the dose-rate factors. Section 3 is concerned with implementation of the methodology presented in Section 2 and includes discussions of (1) potentially important radiations from the decay of radionuclides in the environment that are not taken into account in calculating the doserate factors, (2) corrections that can be applied to the dose-rate factors tabulated in this report to take into account the effects of indoor residence, ground roughness, exposure during boating activities, and exposure to contaminated shorelines, (3) methods for calculating external dose-rate factors for exposure to sources distributed with depth in soil, and (4) application of the dose-rate factors to the important cases of radionuclides that decay to radioactive daughter products. 
Appendix A presents the tables of dose-rate conversion factors for external exposure to photons and electrons for immersion in contaminated air, immersion in contaminated water, and exposure to a contaminated ground surface. Appendix B presents diagrams of the parent-daughter relationships for all decay chains involving two or more of the radionuclides considered in this report. These diagrams show the halflives, modes of decay, and branching fractions for each decay chain. Finally, Appendix $C$ presents a complete listing of the version of the DOSFACTER computer code used in the calculations for this report. 
2. CALCULATION OF EXTERNAL DOSE-RATE FACTORS

FOR PHOTONS AND ELECTRONS

Photons and electrons usually are the only important radiations emitted by radionuclides in the environment that can penetrate the body surface and give a dose to radiosensitive tissues. This section presents the equations used to calculate the dose-rate factors for external exposure to photons and electrons for immersion in contaminated air, immersion in contaminated water, and exposure to a contaminated ground surface. The data bases in the DOSFACTER computer code and other assumptions used to obtain the results in Appendix $A$ also are described.

For each radiation type and exposure mode, we first present the dose-rate factor equations for specific body organs from monoenergetic sources. These equations then are generalized to the spectrum of photons or electrons arising from the most common modes of radioactive decay, i.e., beta decay, alpha decay, and isomeric transitions. (7) Finally, calculation of the effective dose-rate factor for external exposure to photons is described.

In unusual circumstances, spontaneous fission and bremsstrahlung also can produce penetrating radiations of potential importance to estimation of external dose to members of the public. These processes are not taken into account in calculating the dose-rate factors presented in this report but are discussed briefly in Section 3.1.

As discussed in Section 1, the methodology for calculating the external dose-rate factors for photons and electrons has been documented previously, $(1,2)$ except for the adjustments described in Section 1.2. The primary purpose of presenting a complete description of the methodology in this report is to permit an understanding of the calculations without the need for access to other documents.

\subsection{Photon Dose-Rate Factors for Body Organs}

The photon dose-rate factors for body organs presented in this report are based on Monte Carlo simulations of organ doses for a reference individual immersed in a contaminated atmospheric cloud. The Monte Carlo 
simulations of organ doses for immersion in contaminated air also provide the basis for the photon dose-rate factors for body organs for immersion in contaminated water and exposure to a contaminated ground surface.

In all calculations of photon dose-rate factors, the reference individual is assumed to be an adult hermaphrodite of mass $70 \mathrm{~kg}$ and height $174 \mathrm{~cm}$ in which the shape, location, and composition of most of the body organs are described by snyder et al. ( 8 ) The organs for which the model of Snyder et al. was modified for the calculations in this report include ovaries, uterus, breast, and red bone marrow. The dose-rate factors for ovaries and uterus are based on a body mass $(58 \mathrm{~kg}$ ) and height $(163 \mathrm{~cm})$ that are more appropriate for an adult female, as described by $O^{\prime}$ Brien and Sanna. $(9,10)$ Breast was not considered by Snyder et al., and we have assumed that the photon dose-rate factor for breast is the average of the values for skin and lungs at all energies. The description of red bone marrow by Snyder et al. assumes that the skeleton is a homogeneous mixture of bone and bone marrow. This model has been modified, as described by Kerr, (11) to take into account that red marrow is located in the trabecular bone cavities of the skeleton, with the result that bone provides a significant amount of shielding of the red marrow for photon energies below about $0.2 \mathrm{MeV}$ [see also section 2.2 .1 of ref. (1)]

\subsubsection{Immersion in contaminated air}

For immersion in contaminated air, an exposed individual is assumed to be standing at the boundary of a semi-infinite (i.e., hemispherical) atmospheric cloud with uniform source concentration. For a unit concentration of sources of monoenergetic photons $(\gamma)$ of emitted energy $E_{\gamma}$, the photon dose-rate factor for body organ $k$, denoted by $D R F_{\gamma}^{k}$, is given by

$$
\operatorname{DRF}_{\gamma}^{\mathrm{k}}\left(E_{\gamma}\right)=(1 / 2)\left(\mathrm{KE}_{\gamma} / \rho_{a}\right) \mathrm{G}_{\gamma}^{\mathrm{k}}\left(\mathrm{E}_{\gamma}\right)
$$

where $K$ is a constant described below, $\rho_{a}$ is the density of air, and $G_{\gamma}^{k}$ is the ratio of dose-equivalent rate in organ $k$ to absorbed dose rate in air for photons of energy $E_{\gamma}$ and is obtained as described below. 
The factor $\mathrm{KE}_{\boldsymbol{\gamma}} / \rho_{\mathrm{a}}$ in eq. (3) is the absorbed dose rate in air per unit concentration of monoenergetic sources of energy $E_{\gamma}$ in an infinite, uniformly contaminated atmospheric cloud of density $\rho_{a}$. This factor is obtained from the requirement of conservation of energy that the energy emitted per unit volume must equal the energy absorbed per unit volume throughout the source region and from the definition of absorbed dose as the energy absorbed per unit mass of material in the source medium.

The factor of $1 / 2$ in eq. (3) was recommended by Dillman (12) to express the assumption that the source region is semi-infinite rather than infinite in extent for all emitted photon energies. However, calculations by Ryman et al. (13) of the effects of the air-ground interface on the absorbed dose rate in air at $1 \mathrm{~m}$ above ground, which represents the average height of body organs for an individual standing on the ground, have shown that assuming the factor of $1 / 2$ for all photon energies

(1) increasingly underestimates the dose as the energy decreases below $30 \mathrm{keV}$, e.g., by about 708 at $10 \mathrm{keV}$ and by a factor of 2 below $6 \mathrm{keV}$, (2) overestimates the dose at energies between $30 \mathrm{keV}$ and $1 \mathrm{MeV}$, but only by about $20 \%$ near $70 \mathrm{keV}$ and less at other energies, and

(3) underestimates the dose at energies above $1 \mathrm{MeV}$, but only by a few percent. An energy-dependent correction factor for air-ground interface effects has not been incorporated in the calculations in this report, because the correction factor (1) is less than $20 \%$ at the photon energies of primary importance to external dose, (2) has been calculated only at the single height above ground of $1 \mathrm{~m}$ rather than as an average over the height of the exposed individual, and (3) does not take into account additional perturbations of the radiation field in air above ground caused by the presence of the exposed individual.

The value of the constant $\mathrm{K}$ in eq. (3) depends on the units in which the dose-rate factor is expressed. In this report, the dose-rate factors for air immersion are given in units of mrem/y per $\mu \mathrm{Ci} / \mathrm{m}^{3}$; i.e., the unit concentration of sources is assumed to be $1 \mu \mathrm{Ci} / \mathrm{m}^{3}$ and the dose-equivalent rates in body organs are expressed in mrem/y. Then, if the density of air is expressed in $\mathrm{g} / \mathrm{m}^{3}$ and the energy of the emitted photon in $\mathrm{MeV}, \mathrm{K}$ is obtained as follows from the relationships that $1 \mu \mathrm{Ci}$ of activity is $3.7 \times 10^{4}$ disintegrations (dis) per second, an absorbed dose of 1 rad is $100 \mathrm{erg}$ of absorbed energy per gram of material in the source medium, and 
$1 \mathrm{MeV}$ of energy is $1.6 \times 10^{-6} \mathrm{erg}$ :

$$
\begin{aligned}
K= & \left(3.7 \times 10^{4} \mathrm{dis} / \mu \mathrm{Ci}-\mathrm{s}\right)\left(10^{-2} \mathrm{~g} \text {-rad } / \mathrm{erg}\right)\left(1.6 \times 10^{-6} \mathrm{erg} / \mathrm{MeV}\right) \\
& \times\left(10^{3} \mathrm{mrad} / \mathrm{rad}\right)\left(3.1536 \times 10^{7} \mathrm{~s} / \mathrm{y}\right) \\
= & 1.87 \times 10^{7} \mathrm{~g}-\mathrm{mrad} / \mathrm{MeV}-\mu \mathrm{Ci}-\mathrm{y} .
\end{aligned}
$$

Thus, the units on the right-hand side of eq. (3) are $\mathrm{mrad} / \mathrm{y}$ per $\mu \mathrm{Ci} / \mathrm{m}^{3}$, and the dose-rate factor is obtained in units of mrem $/ \mathrm{y}$ per $\mu \mathrm{Ci} / \mathrm{m}^{3}$ by assuming a quality factor of one for converting absorbed dose in rad to dose equivalent in rem.

The dose-rate factors for air immersion presented in this report are based on the calculations of Eckerman et al. (14) for an infinite, uniformly contaminated cloud source. Briefly, Eckerman et al. obtained dose-rate factors for different body organs from (1) organ doses from monoenergetic sources at the body surface calculated using Monte Carlo methods by $O^{\prime} B r i e n$ and Sanna $(9,10)$ and (2) the energy spectra of scattered photons in air from monoenergetic sources in an infinite, uniformly contaminated atmospheric cloud calculated by Dillman. (12) The organ dose-rate factors from monoenergetic sources in an atmospheric cloud were obtained by integrating the organ doses per photon per unit area at the body surface given by $O^{\prime} B r i e n$ and Sanna over the spectrum of scattered photons in air given by Dillman for each source energy considered. The results of these calculations in units of $\mathrm{Sv} / \mathrm{y}$ per $\mathrm{Bq} / \mathrm{cm}^{3}$ for an assumed air density of $1.29 \times 10^{3} \mathrm{~g} / \mathrm{m}^{3}$ at $0^{\circ} \mathrm{C}$ and $760 \mathrm{~mm}$ pressure are given in Table 2 of ref. (2).

Jacob et al. (15) recently noted that the calculations of O'Brien and Sanna $(9,10)$ described above contain an error which results in a systematic underestimation of the organ dose-rate factors for air immersion obtained by Eckerman et al. (14) and used in this report. The dose-rate factors are underestimated by about 10-308 depending on the organ, and the same systematic underestimation of photon dose-rate factors occurs in the results for water immersion and ground-surface exposure described in Sections 2.1.2 and 2.1.3. The photon dose-rate factors in this report have not been corrected for this error, because revised results in the form needed for the DOSFACTER code were not available. (Oak Ridge National Laboratory is undertaking an effort to produce an appropriate set 
of corrected photon dose-rate factors using Monte Carlo methods.) It also is important to bear in mind that the error in the present calculations usually will be much less than any error in measurements or calculations of radionuclide concentrations in the environment. Thus, the error in the dose-rate factors generally is not of concern with regard to the accuracy of estimates of external dose.

The dose-rate factor for immersion in contaminated air in eq. (3) is expressed in terms of the ratio of organ dose-equivalent rate to absorbed dose rate in air, $G_{\gamma}^{k}$, rather than directly in terms of the dose-rate factors for air immersion calculated by Eckerman et al. (14) and documented in ref. (2), because this ratio also is needed in calculating organ doserate factors for immersion in contaminated water and exposure to a contaminated ground surface (see Sections 2.1.2 and 2.1.3). Again, as defined in eq. (3), the values of $G_{y}^{k}$ for different body organs as a function of photon energy are obtained from the organ dose-rate factors for an infinite cloud source calculated by Eckerman et al. divided by the dose-rate factor in air, $\mathrm{KE}_{\gamma} / \rho_{\mathrm{a}}$, for the same unit source concentration. The values of $G_{\gamma}^{k}$ used in the present calculations are listed in the function subprogram FAC in Appendix $C$. Results are given for 23 body organs at 15 photon energies between 0.01 and $10 \mathrm{MeV}$.

The density of air assumed for use in eq. (3) is $1.189 \times 10^{3} \mathrm{~g} / \mathrm{m}^{3}$. This value is appropriate for dry air at $20^{\circ} \mathrm{C}$ and $750 \mathrm{~mm}$ pressure, and is about $8 \%$ less than the density at $0^{\circ} \mathrm{C}$ and $760 \mathrm{~mm}$ pressure assumed by Eckerman et al. (14) Thus, the organ dose-rate factors for air immersion given in Appendix A.1 are about 88 greater than those that would be obtained directly from Table 2 of ref. (2). The lower air density assumed in the present calculations also results in similar increases in the organ dose-rate factors for exposure to a contaminated ground surface presented in Section 2.1.3.

The dose-rate factors for air immersion in Appendix A.1 assume continuous exposure outdoors for 100 of the time during a year. Correction factors which account for shielding of photons by building structures during indoor residence are discussed in section 3.2.1. 


\subsubsection{Immersion in contaminated water}

For immersion in contaminated water, an exposed individual is assumed to be submersed in an infinite medium with uniform source concentration. Thus, as with the dose-rate factor for air immersion in eq. (3), the dose-rate factor for immersion in contaminated water can be obtained from the principle of conservation of energy. However, since calculations of organ dose-rate factors for water immersion, similar to those of Eckerman et al. (14) for air immersion described in section 2.1.1, are not available in the literature, we further assume that the ratio of dose-equivalent rate for organ $k$ to dose-equivalent rate in tissue at the body surface of the exposed individual is the same for the two exposure modes. Thus, for immersion in contaminated water, the dose-rate factor for organ $k$ is given in terms of the ratio of organ dose-equivalent rate to absorbed dose rate in air for air immersion, $G_{\gamma}^{k}$, in eq. (3) as

$$
\mathrm{DRF}_{\gamma}^{\mathrm{k}}\left(\mathrm{E}_{\boldsymbol{\gamma}}\right)=\left(\mathrm{KE}_{\boldsymbol{\gamma}} / \rho_{\mathrm{w}}\right)\left(\mathrm{R}_{\gamma}^{\mathrm{w}} / \mathrm{R}_{\gamma}^{a}\right) G_{\gamma}^{\mathrm{k}}\left(E_{\gamma}\right)
$$

where $K$ is the constant calculated in eq. (4), $\rho_{\mathrm{w}}$ is the density of water and is assumed to be $0.99823 \times 10^{6} \mathrm{~g} / \mathrm{m}^{3}$ for a temperature of $20^{\circ} \mathrm{C}$, and $\mathrm{R}_{\gamma}^{\mathrm{W}}$ and $R_{\gamma}^{a}$ are the ratio of energy absorption in tissue to energy absorption in water ( $w$ ) and air (a) for emitted energy $E_{\gamma}$, respectively, obtained as described below. The dose-rate factors are in units of mrem $/ \mathrm{y}$ per $\mu \mathrm{Ci} / \mathrm{m}^{3}$. The energy-absorption ratios $R_{\gamma}^{w}$ and $R_{\gamma}^{a}$ are obtained from the photon mass energy-absorption coefficients, $\mu_{\mathrm{en}} / \rho$, in tissue ( $t$ ), water, and air as follows:

$$
\begin{aligned}
& R_{\gamma}^{\mathrm{w}}=\left(\mu_{\mathrm{en}} / \rho\right)_{t} /\left(\mu_{\mathrm{en}} / \rho\right)_{\mathrm{w}}, \\
& \mathrm{R}_{\gamma}^{\mathrm{a}}=\left(\mu_{\mathrm{en}} / \rho\right)_{t} /\left(\mu_{\mathrm{en}} / \rho\right)_{\mathrm{a}} .
\end{aligned}
$$

For both air immersion and water immersion, the emission of monoenergetic photons uniformly throughout the source region results in a continuous energy spectrum of photons between zero energy and the emitted energy at every point in the source region; and it is this continuous spectrum of photons that is incident upon the body surface. In the present calculations, the energy-absorption ratios $\mathrm{R}_{\gamma}^{\mathrm{a}}$ for air immersion in eq. (7) were obtained from the calculations of Dillman, ${ }^{(12)}$ in which the ratios of 
mass energy-absorption coefficients in tissue and air are averaged over the spectrum of photons in an infinite, uniformly contaminated atmospheric cloud. The values of $R_{\gamma}^{a}$ at 25 photon energies between 0.01 and $10 \mathrm{MeV}$ are listed in the subroutine CALC in Appendix $C$.

The mass energy-absorption coefficients in tissue and water are nearly equal at all photon energies. Therefore, it is an excellent approximation to evaluate the energy-absorption ratio $R_{\gamma}^{W}$ for water immersion in eq. (6) at the emitted energy $E_{\boldsymbol{\gamma}}$, instead of averaging over the spectrum of photons in an infinite, uniformly contaminated water medium; this approximation has been used in the present calculations. The mass energy-absorption coefficients in tissue and water at 25 photon energies between 0.01 and $10 \mathrm{MeV}$ were obtained from refs. (16-18) and are listed in the subroutine CALC in Appendix $C$.

The dose-rate factors for water immersion in Appendix A.2 assume continuous exposure for $100 \%$ of the time during a year. While this assumption may be reasonable for air immersion and ground-surface exposure, a more realistic exposure time is needed for water immersion. An exposure time of 18 is recommended in the absence of site-specific information; ${ }^{(19)}$ i.e., the dose-rate factors in Appendix A.2 should be reduced by a factor of 100 in estimating external dose equivalents to merbers of the public. An additional correction factor which accounts for photon exposure during boating activities is discussed in Section 3.2.3.

\subsubsection{Exposure to a contaminated ground surface}

For exposure to a contaminated ground surface, an individual is assumed to be standing on a smooth, infinite plane with uniform source concentration. Since calculations of organ dose-rate factors for groundsurface exposure, similar to those of Eckerman et al. (14) for air immersion described in Section 2.1.1, were not available at the time this methodology was developed, we have assumed that the ratio of organ doseequivalent rate to absorbed dose rate in air at any height above ground is the same as the ratio $G_{\gamma}^{k}$ for air immersion defined with eq. (3). Therefore, for exposure to a contaminated ground surface, the dose-rate factor for organ $k$ is calculated from the product of absorbed dose rate in 
air above ground and the ratio $G_{\gamma}^{k}$ as

$$
\operatorname{DRF}_{\gamma}^{\mathrm{k}}\left(z, E_{\gamma}\right)=\mathrm{KE}_{\gamma} \int_{\sigma} \Phi_{\gamma}^{\mathrm{a}}\left(r, E_{\gamma}\right) \mathrm{d} \sigma \times \mathrm{G}_{\gamma}^{\mathrm{k}}\left(\mathrm{E}_{\gamma}\right)
$$

where $\mathrm{K}$ is the constant calculated in eq. (4), $z$ is the height of the receptor location above the ground surface denoted by $\sigma, \Phi_{\gamma}^{\mathrm{a}}$ is the pointisotropic specific absorbed fraction $(16,20)$ for photons in air described below, and $r$ is the distance from any point on the ground surface to the receptor location.

The specific absorbed fraction in air, $\Phi_{\gamma}^{a}$, is defined as the fraction of the emitted energy $E_{\gamma}$ that is absorbed per unit mass of air at a distance $r$ from an isotropic point source. In this report, the dose-rate factors for exposure to a contaminated ground surface are given in units of $\mathrm{mrem} / \mathrm{y}$ per $\mu \mathrm{Ci} / \mathrm{m}^{2}$. Therefore, from the units for the constant $\mathrm{K}$ in eq. (4), the specific absorbed fraction is given in units of $\mathrm{g}^{-1}$.

The specific absorbed fraction for photons in air is given by ${ }^{(16)}$

$$
\Phi_{\gamma}^{a}\left(r, E_{\gamma}\right)=\left(1 / 4 \pi r^{2}\right)\left(\mu_{e n} / \rho\right)_{a} B_{e n}^{a}\left(\mu_{a} r\right) \exp \left(-\mu_{a} r\right)
$$

where $\left(\mu_{\mathrm{en}} / \rho\right)_{\mathrm{a}}$ and $\mu_{\mathrm{a}}$ are the mass energy-absorption and linear attenuation coefficients in air, respectively, $B_{e n}^{a}$ is the energyabsorption buildup factor in air described below, and each of these parameters is a function of the photon energy $E_{\boldsymbol{\gamma}}$. In order to obtain the specific absorbed fraction in units of $\mathrm{g}^{-1}$, the mass energy-absorption coefficient is given in units of $\mathrm{cm}^{2} / \mathrm{g}$, the linear attenuation coefficient in $\mathrm{cm}^{-1}$, and the distance $r$ in $\mathrm{cm}$. The buildup factor is dimensionless.

We use the Berger form of the buildup factor given by ${ }^{(21)}$

$$
B_{e n}^{a}\left(\mu_{a} r\right)=1+\left(C_{a} \mu_{a} r\right) \exp \left(D_{a} \mu_{a} r\right)
$$

where the coefficients $C_{a}$ and $D_{a}$ are functions of the photon energy $E_{\gamma}$. These coefficients are obtained from linear least-squares fits of eq. (10) to published buildup factors in air. $(22,23)$

By integration of eq. (8) over an infinite ground surface using eqs. (9) and (10) and the relation

$$
\mathrm{d} \sigma=2 \pi \mathrm{r} d r
$$

we obtain the dose-rate factor for organ $k$ for exposure at a height $z$ 
above a contaminated ground surface in the form

$$
\begin{aligned}
\operatorname{DRF}_{\gamma}^{k}\left(z, E_{\gamma}\right)=(1 / 2) \mathrm{KE}_{\gamma}\left(\mu_{\mathrm{en}} / \rho\right) a & \left\{E_{1}\left(\mu_{\mathrm{a}} z\right)\right. \\
& \left.-\left[\mathrm{C}_{\mathrm{a}} /\left(\mathrm{D}_{\mathrm{a}}-1\right)\right] \exp \left[\left(\mathrm{D}_{\mathrm{a}}-1\right) \mu_{\mathrm{a}} z\right]\right\} \mathrm{G}_{\gamma}^{\mathrm{k}}\left(\mathrm{E}_{\boldsymbol{\gamma}}\right),
\end{aligned}
$$

where $E_{1}$ is the first-order exponential integral given by

$$
E_{1}\left(\mu_{\mathrm{a}} z\right)=\int_{z}^{\infty}(1 / r) \exp \left(-\mu_{\mathrm{a}} r\right) d r .
$$

The dose-rate factors in this report are calculated for the single height $z=100 \mathrm{~cm}(1 \mathrm{~m})$ above ground.

In implementing eqs. (12) and (13), mass energy-absorption and attenuation coefficients in air for 25 photon energies between 0.01 and $10 \mathrm{MeV}$ were obtained from refs. (17) and (18) and are listed in the subroutine CALC in Appendix $C$. Values of linear attenuation coefficients in $\mathrm{cm}^{-1}$ are obtained from the mass attenuation coefficients in $\mathrm{cm}^{2} / \mathrm{g}$ by multiplying by the density of air $\left(1.189 \times 10^{-3} \mathrm{~g} / \mathrm{cm}^{3}\right)$. Values of the coefficients $C_{a}$ and $D_{a}$ in the Berger form of the buildup factor in eq. (10) also are listed in the subroutine CALC in Appendix $C$. The exporrential integral in eq. (13) is calculated using polynomial and rational approximations given by eqs. 5.1 .53 and 5.1 .56 of ref. (24).

As discussed in Section 2.2.3 of ref. (1), the use in eq. (8) of the ratio $G_{\gamma}^{k}$ for air immersion often results in underestimates of photon dose-rate factors for ground-surface exposure, because the radiation field at a height of $1 \mathrm{~m}$ above ground (1) has an angular distribution that is strongly peaked in the direction almost parallel to the ground surface (i.e., in the direction almost normal to the body surface), rather than an isotropic angular distribution as in the case of air immersion, and (2) has an energy distribution that is weighted more toward higher energies for a given emitted energy than the energy distribution for air immersion. Recent calculations by Jacob et a1. (15) for plane sources on the ground, which also simulate the effects of ground roughness by locating the sources at a depth of $3 \mathrm{~mm}$ in soil, indicate that the photon dose-rate factors for ground-surface exposure in this report may be underestimated by as much as $40 \%$ for emitted photon energies above $200 \mathrm{keV}$ but may be overestimated for energies below $50 \mathrm{keV}$. These results have 
not been incorporated in the calculations in this report, partly for the reasons discussed in Section 2.1 .1 and partly because the dose-rate factor in eq. (12) does not incorporate the effects of ground roughness which were included in the calculations of Jacob et al.

The dose-rate factors for ground-surface exposure in Appendix A.3 assume continuous exposure outdoors for $100 \%$ of the time during a year. Correction factors to the photon dose-rate factors for this exposure mode which account for shielding by building structures during indoor residence, ground roughness, and exposure to contaminated shorelines are discussed in Sections 3.2.1, 3.2.2, and 3.2.4, respectively. Methods for calculating external dose-rate factors for exposure to photon sources distributed with depth in soil are discussed in Section 3.3.1.

\subsubsection{Dose-rate factors for photon spectrum from radioactive decay}

The spectrum of photons emitted by radionuclides is assumed to consist entirely of discrete $\gamma$ - and $x$-rays from beta decay, alpha decay, or isomeric transitions [e.g., see ref. (7)]. Therefore, for a particular radionuclide and exposure mode, the photon dose-rate factor for organ $k$ is given in terms of the dose-rate factors for monoenergetic sources by

$$
D R F_{\gamma}^{k}=\sum_{i} f_{\gamma_{i}} D R F_{\gamma}^{k}\left(E_{\gamma_{i}}\right)
$$

where $f_{\gamma_{i}}$ is the intensity of the $i$ th photon of energy $E_{\gamma_{i}}$ in number per decay and the summation includes all discrete photons in the decay spectrum of the radionuclide. Contributions to the dose-rate factors from the continuous spectra of photons from spontaneous fission or bremstrahlung are not taken into account in the results tabulated in this report but are discussed in Section 3.1 . 


\subsection{Electron Dose-Rate Factors for Skin}

This section presents the equations for the electron dose-rate factors as a function of depth in tissue for air immersion, water immersion, and ground-surface exposure. Skin is the only organ for which electron dose-rate factors are calculated in this report. Calculations were not performed for the lens of the eye, because the dose-rate factors always are less than those for skin due to the greater depth below the body surface of the radiosensitive tissues for the lens of the eye. (3) Thus, limitation of external electron dose to the skin to ensure prevention of non-stochastic radiation effects also will prevent such effects in the lens of the eye. Electron dose-rate factors need not be considered for any other organs, due to the relatively short range in tissue of electrons from radioactive decay.

The DOSFACTER code listed in Appendix $C$ calculates electron dose-rate factors for four different depths of the skin below the body surface: 4 , 8 , and $40 \mathrm{mg} / \mathrm{cm}^{2}$ to represent exposures of specific parts of the body, as recommended by Whitton, ${ }^{(6)}$ and $7 \mathrm{mg} / \mathrm{cm}^{2}$, as recommended by the ICRP. (3) Results are presented in Appendix A of this report only for the depth of $7 \mathrm{mg} / \mathrm{cm}^{2}$, since this depth is an average value that is appropriate for environmental exposures which result in relatively uniform irradiations of the entire body surface.

In the electron dose-rate factor equations for monoenergetic sources in Sections 2.2.1-2.2.3, the symbol $\epsilon$ denotes electrons in general. Then, in Section 2.2.4, the symbols $e$ and $\beta$ specifically denote discrete electrons from atomic de-excitation processes and continuous electrons from beta decay, respectively. The dose-rate factor as a function of depth in tissue is presented first for immersion in contaminated water, because the equation for this exposure mode contains terms that are used in the equation for immersion in contaminated air. 


\subsubsection{Immersion in contaminated water}

For immersion in an infinite, uniformly contaminated water medium, the electron dose-rate factor at depth $x$ in tissue ( $t$ ) from a unit concentration of sources of monoenergetic electrons $(\epsilon)$ of emitted energy $E_{\epsilon}$ is given by $(25)$

$$
\mathrm{DRF}_{\epsilon}^{\tau}\left(\mathrm{x}, \mathrm{E}_{\epsilon}\right)=\left(\mathrm{KE}_{\epsilon} / \rho_{\mathrm{W}}\right) \mathrm{R}_{\epsilon}^{\mathrm{W}} \mathrm{q}\left(\mathrm{x}, \mathrm{E}_{\epsilon}\right) \mathrm{G}_{\epsilon}^{\tau}\left(\mathrm{x} / \mathrm{r}_{0}^{\mathrm{W}}, \mathrm{E}_{\epsilon}\right)
$$

where $\mathrm{K}$ is the constant calculated in eq. (4), $\rho_{\mathrm{w}}$ is the density of water and is assumed to be $0.99823 \times 10^{6} \mathrm{~g} / \mathrm{m}^{3}, R_{\epsilon}^{\mathrm{w}}$ is the ratio of energy absorption in tissue to energy absorption in water for energy $E_{\epsilon}, q$ is a leakage correction factor, $G_{\epsilon}^{t}$ is a geometrical reduction factor defined as the ratio of dose-equivalent rate at depth $\mathrm{x}$ in a semi-infinite tissue medium to absorbed dose rate in the contaminated water, and $r_{0}^{W}$ is the mean electron range in water for energy $E_{\epsilon}$. The quantities $R_{\epsilon}^{w}, G_{\epsilon}^{t}$; and $q$ are described below. The dose-rate factor is in units of mrem/y per $\mu \mathrm{Ci} / \mathrm{m}^{3}$.

The energy-absorption ratio $\mathrm{R}_{\epsilon}^{\mathrm{W}}$ is obtained from the electron mass stopping powers, $\mathrm{dE} / \rho \mathrm{dx}$, in tissue $(t)$ and water $(w)$ as

$$
R_{\epsilon}^{W}=(d E / \rho d x)_{t} /(d E / \rho d x)_{w} .
$$

Since the stopping powers in tissue and water are nearly equal at all electron energies, the ratio in eq. (16) is evaluated at the emitted energy $E_{\epsilon}$, rather than as an average over the spectrum of electrons in an infinite, uniformly contaminated water medium. The mass stopping powers in tissue and water at 25 electron energies between 0.01 and $10 \mathrm{MeV}$ were obtained from ref. (26) and are listed in the subroutine CALC in Appendix $C$.

The geometrical reduction factor $G_{\epsilon}^{t}$ is analogous to the ratio of dose-equivalent rate for organ $k$ to absorbed dose rate in air, $G_{\gamma}^{k}$, in the photon dose-rate factor equations in section 2.1. This factor is obtained from electron specific absorbed fractions in water calculated using Monte Carlo methods, as described by Berger. (25) Water is assumed to be a tissue-equivalent material, and the geometrical reduction factor assumes that a semi-infinite tissue medium is in contact with a semi-infinite, uniformly contaminated water medium. The contaminated water is treated as semi-infinite in extent because electrons cannot penetrate through the 
body. Thus, any point on the body surface is irradiated by only half of the source region, and $G_{\epsilon}^{t}$ has the value one-half at the body surface and decreases with increasing depth in tissue. The geometrical reduction factors used in the present calculations were obtained from ref. (27), and the values at 25 electron energies between 0.01 and $10 \mathrm{MeV}$ and at 21 values of the ratio $\mathrm{x} / \mathrm{r}_{0}^{\mathrm{w}}$ (i.e., the depth below the body surface relative to the electron range in water) are listed in the function subprogram $G$ in Appendix $C$. Values of the electron range in water at 25 energies between 0.01 and $10 \mathrm{MeV}$ were obtained from ref. (26) and are listed in the subroutine CALC in Appendix $C$.

The leakage correction factor $q$ accounts for the finite lateral extent of the body surface and is given by the empirical formula ${ }^{(25)}$

$$
q\left(x, E_{\epsilon}\right)-1-q_{1}\left(E_{\epsilon}\right) q_{2}(x / a)
$$

where the parameter a depends only on the emitted energy $E_{\epsilon}$. Values of $q_{1}$ and a for 17 electron energies between 0.02 and $4 \mathrm{MeV}$ and values of $q_{2}$ for 15 values of the ratio $x / a$ between 0 and 1 were obtained from ref. (25) and are listed in the function subprogram $G$ in Appendix $C$.

As noted in Section 2.1.2, the dose-rate factors for water immersion in Appendix A.2 assume exposure for 1008 of the time, but a more realistic exposure time (e.g., 18) ${ }^{(19)}$ should be applied in estimating dose equivalents to members of the public. Electron dose-rate factors for skin from exposure during boating activities are discussed in section 3.2.3.

\subsubsection{Immersion in contaminated air}

For an exposed individual standing at the boundary of a semiinfinite, uniformly contaminated atmospheric cloud, we assume that the electron range in air for emitted energy $E_{\epsilon}$ is less than the average height of the body surface above ground, which is taken to be $1 \mathrm{~m}$. Therefore, the source region is assumed to be effectively infinite in extent, and the electron dose-rate factor at depth $\mathrm{x}$ in tissue for immersion in contaminated air is given in terms of the geometrical reduction factor $G_{\epsilon}^{t}$ for immersion in contaminated water as ${ }^{(25)}$ 


$$
\operatorname{DRF}_{\epsilon}^{t}\left(x, E_{\epsilon}\right)=\left(\mathrm{KE}_{\epsilon} / \rho_{\mathrm{a}}\right)\left(\mathrm{R}_{\epsilon}^{\mathrm{a}} / \alpha\right) \mathrm{q}\left(\mathrm{x}, \mathrm{E}_{\epsilon}\right) \mathrm{G}_{\epsilon}^{t}\left(\mathrm{x} / \mathrm{r}_{0}^{\mathrm{w}}, \mathrm{E}_{\epsilon}\right)
$$

where $\mathrm{K}$ is the constant calculated in eq. (4), $\rho_{a}$ is the density of air and is assumed to be $1.189 \times 10^{3} \mathrm{~g} / \mathrm{m}^{3}, \mathrm{R}_{\epsilon}^{a} / \alpha$ is the ratio of energy absorption in tissue to energy absorption in air for emitted energy $E_{\epsilon}$ described below, and the leakage correction factor $q$ and the geometrical reduction factor $G_{\epsilon}^{t}$ are described in Section 2.2.1. The dose-rate factor is in units of mrem/y per $\mu \mathrm{Ci} / \mathrm{m}^{3}$.

The energy-absorption ratio $\mathrm{R}_{\epsilon}^{\mathrm{a}} / \alpha$ consists of two factors. The first factor is the ratio of mass stopping powers in tissue and air,

$$
R_{\epsilon}^{a}=(d E / \rho d x)_{t} /(d E / \rho d x)_{a},
$$

which is evaluated at the emitted energy $E_{\epsilon}$. The second factor, $\alpha$, also depends on the emitted energy and accounts for the variations in mass stopping powers in tissue and air over the continuous energy spectrum of electrons incident on the body surface for the monoenergetic source of energy $E_{\epsilon}$ in an infinite, uniformly contaminated atmospheric cloud. The mass stopping powers in tissue and air at 25 electron energies between 0.01 and $10 \mathrm{MeV}$ and values of $\alpha$ at 8 electron energies between 0.01 and $2 \mathrm{MeV}$ were obtained from refs. (26) and (25), respectively, and are listed in the subroutine CAIC in Appendix $C$.

The assumption that the source region is effectively infinite in extent for immersion in contaminated air results in overestimates of electron dose-rate factors in tissue whenever the electron energy is sufficiently high that the range is greater than $1 \mathrm{~m}$ in air plus the assumed depth of tissue below the body surface. As described in Section 2.2.3 below, electron dose-rate factors for skin at a depth of $7 \mathrm{mg} / \mathrm{cm}^{2}$ in tissue are overestimated for emitted electron energies above $0.36 \mathrm{MeV}$. The extent of overestimation increases with increasing electron energy above this value and approaches a factor of two at the highest electron energies occurring in radioactive decay. (7)

As noted in Section 2.1.1, the dose-rate factors for air immersion in Appendix A.1 assume exposure outdoors for $100 \%$ of the time. The effects of indoor residence on electron dose-rate factors for skin for air immersion are discussed in Section 3.2.1. 


\subsubsection{Exposure to a contaminated ground surface}

The geometrical reduction factor $G_{\epsilon}^{t}$ used in calculating electron dose-rate factors as a function of depth in tissue for air immersion and water immersion cannot be used in calculating dose-rate factors for exposure to a contaminated ground surface, because the geometrical reduction factor applies only to the case of a source volume that is effectively semi-infinite in extent and is in contact with the body surface of an exposed individual. Thus, the dose-rate factor for groundsurface exposure must be obtained by explicit consideration of the specific absorbed fraction for electrons.

In principle, the electron dose-rate factor at any depth in tissue for exposure to a contaminated ground surface should be calculated by considering transport of electrons in air between any source point on the ground and the assumed height of the body surface above ground followed by transport through tissue to the assumed depth of the skin. However, electron specific absorbed fractions for transport through two different media have not been considered in the literature, so the problem must be reduced to a consideration of electron transport through a single medium.

The calculations in this report assume that exposures of the skin occur at a single height of $100 \mathrm{~cm}$ ( $1 \mathrm{~m}$ ) above ground. Consideration of electron transport through air followed by tissue is reduced to a consideration of transport through air alone by replacing the assumed thickness of tissue by an equivalent thickness of air which is added to the assumed height of the body surface above ground. (This assumption produces more accurate results than the alternative of considering transport through tissue alone by replacing the assumed height of the body surface above ground by an equivalent thickness of tissue, because most of the energy loss of electrons in transport between the ground surface and the depth of skin below the body surface occurs during transport through 1 m of air.) Thus, for height $z$ of the body surface above ground and depth $x$ in tissue, the effective height $z^{\prime}$ in air above ground at which the electron dose-rate factor in tissue is calculated is given by

$$
z^{\prime}=z+1.14\left(\rho_{t} / \rho_{a}\right) x
$$

where $\rho_{t}$ and $\rho_{\mathrm{a}}$ are the densities of tissue and air, respectively, and the 
factor 1.14 approximates the ratio of mass stopping powers in tissue and air for any electron energy. (25) (Since the calculations in the DOSFACTER code are performed by expressing the distances $x, z$, and $z$ ' in units of $\mathrm{g} / \mathrm{cm}^{2}$, rather than $\mathrm{cm}$, the density of tissue is not used explicitly in calculating the effective height $z^{\prime}$.)

The electron dose-rate factor in tissue at the height $z^{\prime}$ in air above ground defined by eq. (20) then is given by

$$
\operatorname{DRF}_{\epsilon}^{t}\left(z^{\prime}, E_{\epsilon}\right)=(1 / 2) \mathrm{KE}_{\epsilon} \mathrm{R}_{\epsilon}^{\mathrm{a}} \int_{\sigma} \Phi_{\epsilon}^{\mathrm{a}}\left(r, \mathrm{E}_{\epsilon}\right) \mathrm{d} \sigma
$$

where $\mathrm{K}$ is the constant calculated in eq. (4), $\mathrm{R}_{\epsilon}^{\mathrm{a}}$ is the ratio of mass stopping powers in tissue and air evaluated at the emitted energy $E_{\epsilon}$ [see eq. (19) ], $\sigma$ denotes the ground surface, $\Phi_{\epsilon}^{a}$ is the specific absorbed fraction for electrons in air, and $r$ is the distance from any point on the ground surface to the receptor location at height $z^{\prime}$ in air above ground. The factor of $1 / 2$ accounts for the impenetrability of the body to electrons, so that any point on the body surface is irradiated by only half of the source region. The dose-rate factor is in units of mrem/y per $\mu \mathrm{Ci} / \mathrm{m}^{2}$.

An analytical equation for the electron specific absorbed fraction, analogous to eq. (9) for photons, has not been developed. Thus, eq. (21) must be evaluated numerically using tabulations of electron specific absorbed fractions. In order to facilitate numerical integrations, we use the electron scaled point kernel, $F_{\epsilon}$, developed by Berger, $(25,27)$ which is defined in terms of the specific absorbed fraction by the equation

$$
F_{\epsilon}\left(r / r_{0}, E_{\epsilon}\right) d\left(r / r_{0}\right)=4 \pi \rho \Phi_{\epsilon}\left(r, E_{\epsilon}\right) r^{2} d r \text {, }
$$

where $r_{0}$ is the electron range for energy $E_{\epsilon}$ in the medium of density $\rho$. Thus, scaling of the specific absorbed fraction is accomplished by expressing distances in units of the electron range.

Tables of scaled point kernels in water, $F_{\epsilon}^{W}$, are given by Berger, and the results for 25 electron energies between 0.01 and $10 \mathrm{MeV}$ and 25 values of the scaled distance in water, $r / r_{0}^{W}$, between 0 and 1.20 are listed in the subroutine BGRND in Appendix $C$. The dose-rate factor in eq. (21) depends on the electron scaled point kernel in air, $F_{\epsilon}^{a}$, which is obtained from the scaled point kernel in water according to the 
equation ${ }^{(25)}$

$$
F_{\epsilon}^{a}\left(r / r_{0}^{a}, E_{\epsilon}\right)=\alpha^{\prime} F_{\epsilon}^{w}\left(\alpha^{\prime} r / r_{0}^{a}, E_{\epsilon}\right)
$$

where $\alpha^{\prime}$ is a scaling parameter that depends on the energy $E_{\epsilon}$. Values of $\alpha^{\prime}$ for 8 electron energies between 0.01 and $2 \mathrm{MeV}$ were obtained from ref. (25) and are listed in the subroutine BGRND in Appendix $C$.

By expressing eq. (21) in terms of the scaled point kernel in air defined in eqs. (22) and (23) and using the element of surface area do given by eq. (11), we obtain the electron dose-rate factor in tissue above ground in the form

$$
\operatorname{DRF}_{\epsilon}^{t}\left(z^{\prime}, E_{\epsilon}\right)=(1 / 4)\left(K E_{\epsilon} / P_{a}\right)\left(1 / r_{0}^{a}\right) R_{\epsilon}^{a} \Omega\left(z^{\prime}, E_{\epsilon}\right)
$$

where

$$
\Omega\left(z^{\prime}, E_{\varepsilon}\right)=\int_{z^{\prime} / r_{0}^{a}}^{\infty}(1 / u) F_{\epsilon}^{a}\left(u, E_{\epsilon}\right) d u
$$

and $u$ is the scaled distance $r / r_{0}^{a}$. Again, $z^{\prime}$ is given by eq. (20) and embodies the assumed height of the body surface above ground $(100 \mathrm{~cm})$ and the assumed depth of the skin below the body surface $\left(7 \mathrm{mg} / \mathrm{cm}^{2}\right.$ for the calculations presented in this report).

The integral over scaled distance $u$ in eq. (25) is evaluated numerically using Simpson's rule. For values of u greater than about 1.20, the electron scaled point kernel and, thus, the integral in eq. (25) are zero. For values of $u$ less than 0.05 , the integration is facilitated by use of the excellent approximation that the scaled point kernel for any energy varies linearly with $u$ for values between 0 and 0.05 . (27) [For $u<0.05$, the previous version of the DOSFACTER code ${ }^{(1)}$ incorrectly calculated the integral in eq. (25), as described in Section 1.2. For a height of the body surface above ground $0: 100 \mathrm{~cm}$ and a depth in tissue of $7 \mathrm{mg} / \mathrm{cm}^{2}$, scaled distances less than 0.05 correspond to emitted electron energies greater than about $4.5 \mathrm{MeV} .{ }^{(26)}$ ]

As shown in Fig. 9 of ref. (1), calculation of electron dose-rate factors for skin for ground-surface exposure at a single height of $1 \mathrm{~m}$ above ground provides a reasonable approximation to the dose-rate factor averaged over the height of an exposed individual for emitted electron 
energies above about $1 \mathrm{MeV}$. As the energy decreases below $1 \mathrm{MeV}$, however, the dose-rate factor at $1 \mathrm{~m}$ increasingly underestimates the value averaged over the height of the body. Indeed, for energies below $0.36 \mathrm{MeV}$, the dose-rate factor at $1 \mathrm{~m}$ is zero for a depth of skin of $7 \mathrm{mg} / \mathrm{cm}^{2}$, based on eq. (20) and known electron ranges in air, ${ }^{(26)}$ which is a particularly inappropriate approximation to the dose-rate factor for portions of the skin closer to the ground surface. However, in cases where the external dose to skin from exposure to electron sources on the ground surface is underestimated, the external dose to skin generally will be unimportant compared with the dose to other organs from internal and external exposures.

As noted in Section 2.1.3, the dose-rate factors for ground-surface exposure in Appendix A.3 assume exposure outdoors for 100 of the time. The effects of indoor residence, ground roughness, and exposure to contaminated shorelines on electron dose-rate factors for skin for ground-surface exposure are discussed in Sections 3.2.1, 3.2.2, and 3.2.4, respectively. External dose-rate factors for exposure to electron sources distributed with depth in soil are discussed in Section 3.3.2.

\subsubsection{Dose-rate factors for electron spectrum from radioactive decay}

The spectrum of electrons emitted by radionuclides is assumed to consist of (1) discrete electrons (e) from Auger and internal conversion processes following beta decay, alpha decay, or isomeric transitions and $(2)$ continuous electrons ( $\beta$ ) from beta decay [e.g., see ref. (7)]. Therefore, for a particular radionuclide and exposure mode, the electron dose-rate factor for skin is given in terms of the dose-rate factors for monoenergetic sources by

$$
D R F_{\epsilon}^{t}=\sum_{i} f_{e_{i}} D R F_{\epsilon}^{t}\left(E_{e_{i}}\right)+\sum_{j} f_{\beta_{j}} \int_{0}^{E_{\beta_{j}}^{m}} N_{\beta_{j}}(E) D R F_{\epsilon}^{t}(E) d E
$$

where $f_{e_{i}}$ is the intensity of the $i$ th discrete electron of energy $E_{e_{i}}$ in number per decay, $f_{\beta_{j}}$ is the intensity of the $j$ th continuous beta transition with endpoint energy $E_{\beta_{j}}^{m}$ in number per decay, $N_{\beta_{j}}$ (E) is the 
probability per unit energy for emission of an electron with energy between $E$ and $E+d E$ for the $j$ th continuous beta transition, obtained from the Fermi theory of beta decay ${ }^{(28)}$ as described below, and the summations include all discrete and continuous electrons in the decay spectrum of the radionuclide. Contributions to the dose-rate factors from the continuous spectrum of electrons from spontaneous fission are not taken into account in the results tabulated in this report but are discussed in Section 3.1 .

Evaluation of the second term on the right-hand side of eq. (26) requires knowledge of the energy distribution function for beta decay, $\mathrm{N}_{\beta}(E)$. A rigorous calculation of this function is a formidable task requiring extensive computer calculations $(29,30)$ which are not feasible for the large number of radionuclides considered in this report. Rather, we have used an approximation, developed by Evans, ${ }^{(31)}$ which simplifies the calculation yet also yields adequate numerical accuracy (i.e., often within 18 of the results from rigorous calculations).

The energy distribution function for beta decay, properly normalized so that the probability per decay that an electron is emitted with energy between zero and the endpoint is unity, is given by (28)

$$
N_{\beta}(E)-\frac{f_{0}(E) f_{1}(E) f_{C}(Z, E)}{\int_{0}^{E_{\beta}^{m}} f_{0}(E) f_{1}(E) f_{C}(Z, E) d E},
$$

where $f_{0}(E)$ is the statistical factor for an allowed transition, $f_{1}(E)$ is the correction factor for a first-forbidden unique transition, $f_{C}(z, E)$ is the correction factor for the Coulomb (electrostatic) interaction, $Z$ is the atomic number of the daughter nucleus, and $E_{\beta}^{\mathfrak{m}}$ is the endpoint energy. The factors $f_{0}, f_{1}$, and $f_{C}$ are dimensionless. Because of the small rest mass of the electron, relativistic relationships must be used for these factors.

The statistical factor for an allowed transition is given by

$$
f_{0}=\eta w\left(w^{m}-w\right)^{2} \text {, }
$$

where $W$ is the total energy of the electron, $\eta$ is the electron momentum, and $w^{m}$ is the total energy for the endpoint of the spectrum. In terms of the electron kinetic energy $E$ in $\mathrm{MeV}$ and the rest-mass energy of $0.511 \mathrm{MeV}$, the total energy and momentum are given by 


$$
\begin{aligned}
& W=E / 0.511+1, \\
& \eta=\left(W^{2}-1\right)^{1 / 2} .
\end{aligned}
$$

The correction factor for a first-forbidden unique transition (i.e., a transition between initial and final nuclear states of opposite parity and spins differing by two units of angular momentum) is given by

$$
f_{1}=\eta^{2}+\left(w^{m}-w\right)^{2}
$$

For allowed transitions or higher forbidden transitions, the factor $f_{1}$ is assumed to be unity.

The simplifying approximation to the energy distribution function for beta decay developed by Evans involves the relativistic correction factor for the Coulomb interaction, which is written as $(31)$

$$
f_{C}=\eta^{2 s} \exp (\pi y)|\Gamma(1+s+i y)|^{2} \text {. }
$$

where

$$
\begin{aligned}
& s=\left[1-(z / 137)^{2}\right]^{1 / 2}-1 \\
& y= \pm z W / 137 \eta .
\end{aligned}
$$

In eq. (32), $\Gamma$ is the complex gamma function which, in the present calculations, is evaluated for argument $z=1+s+i y$ using the power-series approximation

$$
1 / \Gamma(z)=\sum_{k} c_{k} z^{k}
$$

where the coefficients $c_{k}$ are given with eq. 6.1 .34 of ref. (32). The parameter $y$ in eq. (34) is positive for $\beta^{-}$decay and negative for $\beta^{+}$ decay.

The integral over energy in the denominator of eq. (27) is evaluated numerically using Simpson's rule. As input to the numerical procedure, the integrand is calculated at $10 \mathrm{keV}$ intervals between zero energy and the endpoint energy of each beta spectrum. 
The ICRP has recommended that limits on dose equivalent to whole body or specific body organs be replaced by a limit on the effective dose equivalent from external and internal exposures. (3) A limit on effective dose equivalent is intended to provide a limit on risk from stochastic radiation effects for any uniform or nonuniform irradiations of the body. The DOE has adopted the ICRP recommendation in its current radiation protection standards for members of the public.

As defined by the ICRP, $(3,4)$ the effective dose-rate factor for any of the three external exposure modes considered in this report, denoted by $D R F_{E}$, is a weighted sum of the dose-rate factors for several body organs:

$$
D R F_{E}=\sum_{k} w_{k} D R F^{k} \text {, }
$$

where $k$ is an index denoting each organ, $w_{k}$ is a weighting factor giving the proportion of the stochastic risk resulting from the kth organ under conditions of uniform whole-body irradiation, and $D R F^{k}$ is the external dose-rate factor for the kth organ for the particular exposure mode.

The organ-specific weighting factors $w_{k}$ assigned by the ICRP ${ }^{(3)}$ are given in Table 1 . In the present calculations, "gonads" is assumed to be the testes, since the external dose-rate factors for this organ always are greater than those for the ovaries, and "bone surfaces" is assumed to be the skeleton. The organ labeled "remainder" refers to the five organs other than those listed in the table which have the highest external dose-rate factors, and a weighting factor $w_{k}=0.06$ is assigned to each of these organs. For external exposure, the organs included in the "remainder" category vary with photon energy. For example, at $0.05 \mathrm{MeV}$, this category includes bladder, kidneys, liver, marrow, and thymus; but at $2 \mathrm{MeV}$, bladder and liver are replaced by brain and upper large intestine.

It is important to note that the dose-rate factor for skin is not included in the effective dose-rate factor calculated in this report, because the ICRP has recommended that the dose to skin be excluded in calculating effective dose equivalents to maximally exposed individuals in critical population groups. (4) Thus, for external exposure, the effective dose-rate factor results from photon irradiations only, and the dose-rate 
Table 1. Organ-specific weighting factors for calculation of effective dose equivalent ${ }^{a}$

\begin{tabular}{ll}
\hline \multicolumn{1}{c}{ Organ } & $\mathrm{w}_{\mathrm{k}}$ \\
\hline & 0.25 \\
Gonads & 0.15 \\
Breast & 0.12 \\
Red marrow & 0.12 \\
Lungs & 0.03 \\
Thyroid & 0.03 \\
Rone surfaces & 0.30 \\
\hline
\end{tabular}

Qvalues from ref. (3).

factor for skin is used principally to evaluate compliance with dose limits for individual organs which ensure prevention of non-stochastic radiation effects. However, the ICRP also has recommended that the dose to skin may be included in the effective dose equivalent with a weighting factor of 0.01 in assessing the collective detriment from exposures of population groups. (4) The listings in Appendix A of external dose-rate factors for skin for photons plus electrons permit inclusion of the dose to skin in the effective dose equivalent with its appropriate weighting factor, if so desired. 
3. IMPLEMENTATION OF EXTERNAL DOSE-RATE FACTORS FOR PHOTONS AND ELECTRONS

The dose-rate conversion factors for external exposure to photons and electrons that result from implementation of the methodology presented in Section 2 are listed in Appendix A. Dose-rate factors have been calculated for approximately 500 radionuclides of potential importance in environmental dose assessments. The energies and intensities of the photons and electrons used to calculate the dose-rate factors for each radionuclide were obtained from a compilation of evaluated radioactive decay data. (7) All other data required for the calculations are contained in the DOSFACTER code itself; these data have been described in Sections 2.1-2.3 and are given in the code listing in Appendix $C$.

The dose-rate factors for immersion in contaminated air and immersion in contaminated water are given in units of mrem/y per $\mu \mathrm{Ci} / \mathrm{m}^{3}$, and the values for exposure to a contaminated ground surface are given in units of $\mathrm{mrem} / \mathrm{y}$ per $\mu \mathrm{Ci} / \mathrm{m}^{2}$. If dose-rate factors in units of $\mathrm{Sv} / \mathrm{y}$ per $\mathrm{Bq} / \mathrm{cm}^{3}$ for air immersion and water immersion and in units of $\mathrm{Sv} / \mathrm{y}$ per $\mathrm{Bq} / \mathrm{cm}^{2}$ for ground-surface exposure are desired, as given in refs. (1) and (2), then the values for air immersion and water immersion in this report should be reduced by a factor of $3.7 \times 10^{3}$ and the values for ground-surface exposure should be reduced by a factor of $3.7 \times 10^{5}$.

The remainder of this section discusses some potentially important considerations in applying the dose-rate factors in Appendix A to obtain realistic estimates of external dose equivalents to members of the public. These considerations include (1) penetrating radiations from radioactive decay that are not taken into account in the calculations, (2) corrections that can be applied to the dose-rate factors to take into account the effects of indoor residence, ground roughness, exposure during boating activities, and exposure to contaminated shorelines, (3) methods for calculating external dose-rate factors for exposure to sources distributed with depth in soil, and (4) application of the dose-rate factors to the important cases of radionuclides that decay to radioactive daughter products. 


\subsection{Radiations from Spontaneous Fission and Bremsstrahlung}

The external dose-rate factors in Appendix A take into account only photons and electrons from beta decay, alpha decay, and isomeric transitions. In unusual circumstances, penetrating radiations from spontaneous fission decay or bremsstrahlung also could be important in estimating external dose equivalents to members of the public.

\subsubsection{Spontaneous fission}

Spontaneous fission occurs in the decay of several radionuclides in the actinide series, and results in the emission of photons, electrons, and neutrons as well as fission fragments. However, spontaneous fission is an important decay mode for only a few radionuclides $\left(e . g .,{ }^{248} \mathrm{~cm}\right.$, ${ }^{252} \mathrm{Cf},{ }^{254} \mathrm{Cf}$, and $\left.{ }^{256} \mathrm{Fm}\right)$; in these cases, the dose-rate factors in this report considerably underestimate external dose equivalents.

Branching fractions for spontaneous fission for the radionuclides considered in this report are given in Appendix 5 of ref. (7). Empirical relationships for the continuous energy spectra of photons, electrons, and neutrons from spontaneous fission that could be applied to the estimation of external dose equivalents are given by Dillman and Jones. (33) However, external dose equivalents from spontaneous fission usually will be unimportant in dose assessments for members of the public, either because radionuclides with significant branching fractions for spontaneous fission will occur in relatively small concentrations in the environment or because dose equivalents from internal exposure will be much more important for these radionuclides.

\subsubsection{Bremsstrahlung}

Bremsstrahlung is the continuous spectrum of photons produced primarily by the slowing down of electrons emitted in radioactive decay by interaction with the material surrounding the radiating atom (i.e., air, water, or soil for radionuclides in the environment). Bremsstrahlung also 
is produced by interaction of an ejected electron with the decaying nucleus itself, but this contribution is negligible. (30) Bremsstrahlung usually will be important in estimating external dose equivalents only for radionuclides that are pure beta emitters with relatively high decay energies (e.g., ${ }^{90} \mathrm{Sr}$ and its daughter ${ }^{90} \mathrm{Y}$ ).

Methods for calculating the spectrum of bremsstrahlung in a number of materials have been implemented by Dillman. (30) Simplified methods, based on an approximation given by Evans, (31) have been implemented by O'Donnel1 et al. (34) However, contributions to external dose equivalents from bremsstrahlung usually will be unimportant in dose assessments for members of the public, either because of the presence of significant concentrations of photon-emitting radionuclides in the environment or because, for radionuclides that are pure beta emitters, dose equivalents from internal exposure will be much more important.

\subsection{Corrections to the External Dose-Rate Factors}

This section discusses corrections that can be applied to the external dose-rate factors for photons and electrons in Appendix $A$ to take into account the effects of indoor residence, ground roughness, exposure during boating activities, and exposure to contaminated shorelines. These corrections, which are referred to as dose-reduction factors, are multiplicative factors that are less than or equal to unity and, thus, serve to reduce estimates of external dose equivalents. These factors may be applied to the external dose-rate factors for particular exposure modes to obtain more realistic estimates of dose equivalents to members of the public.

Two separate factors must be taken into account in estimating the effects of dose-reduction factors on external dose equivalents to members of the public: (1) the reduction in dose during a particular exposure situation (e.g., during indoor residence) and (2) the fraction of the time during a year that the reduction in dose occurs. Thus, in estimating annual dose equivalents, the dose-reduction factor for a particular exposure situation must be weighted by the fraction of the year during which the reduction in dose is assumed to occur. Both of these factors 
are discussed in Sections 3.2.1-3.2.4.

None of the dose-reduction factors for external exposure discussed in this section were applied to the photon and electron dose-rate factors tabulated in Appendix A. Guidance on use of the dose-reduction factors in estimating external dose equivalents to members of the public is presented in Section 3.2.5.

\subsubsection{Effects of indoor residence}

The dose-rate factors for immersion in contaminated air in Appendix A.1 and exposure to a contaminated ground surface in Appendix A. 3 assume that exposed individuals spend all of the time outdoors. A more realistic dose assessment for these exposure modes would take into account the effects of shielding by building structures in reducing external dose equivalents during indoor residence.

Fnoton exposures. For routine releases from nuclear reactors, the U.S. Nuclear Regulatory Commission (NRC) recommends that the following dose-reduction factors be applied in calculating external dose equivalents from photons to take into account both the shielding provided by building structures during indoor residence and the fraction of the time individuals spend indoors: (35)

Photon dose-reduction factors for air immersion and ground-surface exposure to account for indoor residence -

- 0.7 for maximally exposed individuals;

- 0.5 for average individuals in population groups.

In both cases, the recommended shielding factor during indoor residence is 0.5. The difference in the two dose-reduction factors then reflects the assumed fraction of the time spent indoors, i.e., about $50 \%$ for maximally exposed individuals but nearly $100 \%$ for average individuals in population groups. (35) These dose-reduction factors were based on an analysis by Burson and Profio. (36) Similar values, based on a compilation by Oakley, (37) have been recommended by the National Council on Radiation Protection and Measurements. (38) 
The dose-reduction factors listed above may also be applied to routine releases from sources other than nuclear reactors and to accidental releases. In general, however, the values are most appropriate for emitted photon energies above a few hundred keV. For photons of lower energy, use of these dose-reduction factors may considerably overestimate external dose equivalents during indoor residence. (

The photon dose-reduction factors for indoor residence recommended by the NRC are based on a shielding factor that is representative of living areas in single-family homes. In some cases, however, it may be more appropriate to assume that exposed individuals are residing in basements of homes or in schools, apartment houses, or office buildings. These structures generally provide significantly greater shielding than living areas in single-family homes. For immersion in contaminated air, representative shielding factors range from 0.6 for the basement of a wood frame house to 0.2 or less for a large office or industrial-type building; and, for ground-surface exposure, representative shielding factors range from 0.1 for the basement of a home to 0.005 for the basement of large, multi-story structures. (36)

The photon dose-reduction factors for indoor residence for groundsurface exposure recommended by Burson and Profio ${ }^{(36)}$ for a variety of structures and adopted by the $\mathrm{NRC}^{(35)}$ for single-family homes take into account activity deposited on the outside walls and roof of the structure, as well as activity deposited on the ground surface. A simple model for estimating dose-reduction factors for activity deposited on the outside walls and roof of a building is given in ref. (39).

Electron exposures. The NRC has not considered the effects of indoor residence on external dose equivalents from electrons. (35) However, since electrons from radioactive decay cannot penetrate building structures, external dose equivalents from electrons during indoor residence can be estimated on the basis of concentrations of radionuclides in indoor air and on the floor of the building [e.g., see section II of ref. (39)].

The following discussion of dose-reduction factors for electrons during indoor residence applies primarily to routine (i.e., chronic) releases of radionuclides to the atmosphere, in which case steady-state concentrations of airborne radionuclides inside and outside buildings may be assumed. For acute or accidental releases, however, the relationship 
between indoor and outdoor airborne concentrations of radionuclides will vary with time during and after a release, and will depend on the timing and extent of building ventilation. Therefore, for such releases, it may be prudent to assume no reduction in external dose equivalents from electrons during indoor residence.

For noble-gas radionuclides, air immersion is the only external exposure mode of concern. The effects of indoor residence on electron dose equivalents to skin should be negligible during chronic releases (i.e., the dose-reduction factor should be essentially unity), unless the range of the emitted electrons in air is somewhat greater than the interior dimensions of building rooms, because the indoor and outdoor air concentrations for noble gases will be nearly the same. For ${ }^{85} \mathrm{Kr}$, for example, the electron energies from beta decay are less than $0.687 \mathrm{MeV}^{(7)}$ and the corresponding electron ranges in air are less than $2.6 \mathrm{~m}$, (26) which is a representative radius of rooms in single-family homes. Thus, a dose-reduction factor of unity during indoor exposures to electrons from ${ }^{85} \mathrm{Kr}$ decay is a reasonable assumption. However, if the electron range in air is somewhat greater than the dimensions of building rooms (i.e., if the emitted electron energy is about $1 \mathrm{MeV}$ or greater), then the assumption of a dose-reduction factor of unity will overestimate electron dose equivalents to skin from exposure to noble gases during indoor residence. The extent of overestimation as it depends on the electron range in air relative to the dimensions of building rooms has not been treated in the literature.

For depositing radionuclides, the indoor air concentration can be considerably less than the outdoor air concentration during chronic releases. (39) Thus, if the indoor and outdoor deposition velocities are comparable, then the dose-reduction factors for external exposure to electrons during indoor residence can be substantial for both air immersion and ground-surface exposure. Similar to the case of noble gases discussed above, the dose-reduction factor for electrons for air immersion or ground-surface exposure can be assumed equal to the ratio of indoor and outdoor concentrations of airborne or deposited radionuclides, respectively, provided the electron range in air is comparable to or less than the dimensions of building rooms. For greater electron ranges in air, this assumption again overestimates electron dose equivalents to skin 
during indoor residence, but by unknown amounts.

Exposure times. As stated previously, indoor residence times recommended by the $\mathrm{NRC}^{(35)}$ for maximally exposed and average individuals are 508 and nearly 1008 , respectively. These values apply to residence in homes or apartments. However, substantially lower residence times are more appropriate for other locations; e.g., residence times of about $15 \%$ for schools and 25 for office buildings would be reasonable.

\subsubsection{Effects of ground roughness}

The dose-rate factors for exposure to a contaminated ground surface in Appendix A.3 assume that the source region is a smooth plane. A more realistic dose assessment for this exposure mode would take into account the effects of ground roughness in reducing external dose equivalents, i.e., the shielding provided by terrain irregularities and surface vegetation for sources on the ground surface. A dose-reduction factor for ground roughness would be applied in addition to the dose-reduction factor for indoor residence discussed in section 3.2.1. Thus, no additional consideration of exposure times is needed in estimating the effects of ground roughness on external dose equivalents.

Photon exposures. The NRC has not considered photon dose-reduction factors for ground-surface exposure that take into account ground roughness. (35) However, dose-reduction factors for a photon spectrum representative of fallout following releases from nuclear reactor accidents are given by Burson and Profio. (36) The recommended values range from essentially 1.0 for paved areas to about 0.5 for a deeply plowed field, and a representative average value is about 0.7 . As in the case of building shielding during indoor residence discussed in Section 3.2.1, the average dose-reduction factor for ground roughness should overestimate external dose equivalents from ground-surface exposure if the radionuclides emit mostly low-energy photons. (39)

Electron exposures. Electron dose-reduction factors for groundsurface exposure that take into account ground roughness have not been treated in the literature. However, some reduction in dose due to terrain irregularities and surface vegetation undoubtedly occurs. Furthermore, 
the electron dose is likely to be reduced by a greater amount due to ground roughness than the photon dose. Therefore, in estimating electron dose-rate factors for ground-surface exposure, it would be reasonable to use the appropriate photon dose-reduction factors for ground roughness, since the resulting electron dose probably would not be underestimated.

\subsubsection{Exposure during boating activities}

The dose-rate factors for immersion in contaminated water in Appendix A.2 assume immersion in an infinite source region and, thus, are appropriate for exposure while swimming. External exposure to contaminated water also may occur during boating activities, but dosereduction factors in this case have not been considered by the NRC.

Photon exposures. For photons, a dose-reduction factor of 0.5 during boating activities is a reasonable value that is unlikely to underestimate external dose equivalents. This dose-reduction factor (1) takes into account that the source region is effectively semi-infinite in extent for an exposed individual located at the boundary of the air-water interface and (2) assumes that the shielding of photons provided by the typically thin hull of a boat is negligible. The latter assumption will result in overestimates of external dose equivalents during boating activities for radionuclides that emit mostly low-energy photons.

Electron exposures. For electrons, it is reasonable to assume a dose-reduction factor of zero during boating activities, i.e., that the electron dose-equivalent rate is zero. This recommendation is based on the assumption that the hull of a boat provides complete shielding from electron sources in the water.

Exposure times. The exposure time for boating activities should be estimated on a site-specific basis. For individuals who reside on a boat for all or part of a year, an exposure time of $50 \%$ or more would be reasonable. For individuals who engage only in recreational boating activities, an exposure time as high as $5 \%$ would be reasonable. 


\subsubsection{Exposure to contaminated shorelines}

The dose-rate factors for exposure to a contaminated ground surface in Appendix A.3 assume that the source region is infinite in extent and, thus, are appropriate for large areas that are contaminated by deposition of radionuclides from the atmosphere or by irrigation with contaminated water. External exposure to a contaminated ground surface also may occur during fishing or hiking along shorelines of finite width that are contaminated by deposition of radionuclides from water. (40)

Photon exposures. For exposure to photons from contaminated shorelines of finite width, the NRC has recommended the following dosereduction factors for different types of shorelines: (35)

Photon dose-reduction factors for exposure to contaminated shorelines -

- 0.1 for discharge canal bank;

- 0.2 for river shoreline;

- 0.3 for lake shore;

-0.5 for ocean shore;

- 1.0 for tidal basin.

These values would be applied in addition to the dose-reduction factor for ground roughness discussed in Section 3.2.2, and they do not include consideration of exposure times.

Electron exposures. Dose-reduction factors for exposure to electrons from contaminated shorelines have not been considered in the literature. Because of the relatively short range of electrons in air, the dose-rate factor from exposure to shorelines of finite width should be about the same as the dose-rate factor for exposure to an infinite ground surface; i.e., the dose-reduction factor for exposure to contaminated shorelines should be essentially unity. However, as discussed in Section 3.2.2, an electron dose-reduction factor for ground roughness, based on an appropriate value for photons, could be taken into account in estimating electron dose-rate factors for exposure to contaminated shorelines. 
Exposure times. In estimating external dose equivalents from shoreline exposure, the NRC has recommended exposure times for use in lieu of site-specific data. (35) The values for an adult are 12 hours per year for maximally exposed individuals and 8 hours per year for average individuals in population groups. However, if the dose assessment takes into account other age groups, then the recommended exposure times are considerably higher; i.e., for teenagers, the recommended values are 67 hours per year for maximally exposed individuals and 47 hours per year for average individuals in population groups. (35)

\subsubsection{Guidance on application of dose-reduction factors}

Dose-reduction factors for indoor residence, ground roughness, exposure during boating activities, and exposure to contaminated shorelines have not been used in calculating the dose-rate factors for external exposure to photons and electrons in Appendix A. However, dosereduction factors may be applied to these results at the discretion of each DOE site in estimating external dose equivalents to members of the public. Any. use of dose-reduction factors should be fully documented; i.e., specific information should be given regarding (1) the reduction in external dose equivalents from photons or electrons that is assumed for a particular exposure situation and (2) the fraction of the time during which such dose reductions are assumed to occur.

Use of the dose-reduction factors discussed in this report is particularly warranted whenever annual dose equivalents estimated without use of these factors approach or exceed applicable limits. Otherwise, it may be unimportant that not considering the dose-reduction factors is likely to result in overestimates of external dose equivalents for members of the public, because the various dose-reduction factors often will be considerably less than the magnitude of uncertainties in measured or calculated concentrations of radionuclides in the environment.

If dose-reduction factors are applied to a particular exposure situation, then it should be borne in mind that the values for photons and electrons generally are different. However, this difference is important only in estimating external dose equivalents to the skin, because skin is 
the only organ for which the total dose may result from photon and electron irradiations. A separate accounting of photon and electron dose-reduction factors for skin is facilitated by the listings of photon and electron dose-rate factors for skin in Appendix A. In estimating external dose equivalents to any organ except skin or in estimating effective dose equivalents from external exposure to photons, only the dose-reduction factors for photons would be considered.

\subsection{External Dose-Rate Factors for Sources in Soil}

The photon dose-reduction factor for ground roughness discussed in Section 3.2.2 does not describe the shielding provided by soil as radionuclides penetrate into the ground over time, e.g., by natural infiltration or plowing of soils. Consideration of external exposure to sources in soil may be important in estimating dose equivalents to maximally exposed individuals, particularly during long-term releases to the environment or if the soil is plowed regularly, or in estimating collective dose equivalents during the environmental residence time of the released radionuclides. In these cases, an assumption that deposited radionuclides remain on the ground surface usually would result in considerable overestimates of external dose equivalents.

\subsubsection{Photon dose-rate factors}

Photon dose-rate factors in air above ground from monoenergetic sources that are distributed uniformly in slab sources in soil at various depths below the ground surface have been calculated by Kocher and sjoreen. (41) The dose-rate factors for particular radionuclides then may be obtained from the known photon spectra ${ }^{(7)}$ according to eq. (14). The assumption of uniformly contaminated slab sources is appropriate for frequently plowed soils or for use with linear compartment models that describe downward migration of radionuclides in soil $[\mathrm{e} . \mathrm{g}$. , see ref. (42)]. Only sources within about $1 \mathrm{~m}$ of the ground surface need to be considered, because depths of soil greater than $1 \mathrm{~m}$ provide essentially 
complete shielding of photons from radioactive decay for an exposed individual standing on the ground surface.

Kocher and Sjoreen ${ }^{(41)}$ also have calculated photon dose-rate factors in air above ground for monoenergetic sources that are distributed exponentially with depth in soil. Such a source distribution may be appropriate for the case of natural infiltration of radionuclides into soil during a chronic deposition onto the ground surface.

The photon dose-rate factors in air above ground for particular radionuclides distributed in soil, calculated as described above, can be converted to effective dose-rate factors for an exposed individual standing on the ground by using (1) the ratios of photon dose-equivalent rates in body organs to absorbed dose rate in air as a function of emitted photon energy listed in the function subprogram FAC in Appendix $C$ [i.e., the quantity $G_{\gamma}^{k}$ defined with eq. (3)], and (2) the organ-specific weighting factors for the effective dose equivalent given in Table 1 (see Section 2.3). However, for all radionuclides that emit significant intensities of photons with energies greater than a few hundred keV, which are of greatest concern in estimating external dose equivalents from exposure to contaminated soil, it is an excellent approximation to assume that the ratio of effective dose-rate factor to the dose-rate factor in air is 0.7 .

\subsubsection{Electron dose-rate factors}

External exposure to electrons from radionuclides distributed with depth in soil generally can be neglected, because a few $\mathrm{cm}$ (and often only a few $\mathrm{mm}$ ) of soil provides complete shielding of electrons from radioactive decay for a receptor location $1 \mathrm{~m}$ above ground. (26) Thus, calculations of electron dose equivalents from sources deposited on the ground need to consider only those sources that remain on or very near the surface. 


\subsection{External Dose-Rate Factors for Radioactive Decay Chains}

In applying the dose-rate factors for external exposure in Appendix A to estimates of dose equivalents to members of the public, it is important to understand that the values for each radionuclide do not include any possible contributions from radioactive daughter products. Rather, the tabulations contain separate entries for all such daughter products. For example, the photon dose-rate factors listed for ${ }^{137} \mathrm{Cs}$ are zero, because all photons result from the decay of the $137 \mathrm{~m}_{\mathrm{Ba}}$ daughter, (7) and a separate listing of the photon dose-rate factors for the daughter is provided. The parent-daughter relationships, including half-lives, modes of decay, and decay branching fractions, for all radionuclides considered in this report are shown in Appendix $B$.

In the particular case of ${ }^{137} \mathrm{Cs}$ decay to $137 \mathrm{~m}_{\mathrm{Ba}}$ noted above, it generally is reasonable to assume that the activity of $137 \mathrm{~m}_{\mathrm{Ba}}$ is in secular equilibrium with the activity of ${ }^{137} \mathrm{Cs}$ at any location in the environment and at any time, due to the short half-life of the daughter and the similarity in environmental behavior of the parent and daughter. Thus, the dose-rate factors for $137 \mathrm{~m}$ Ba can be multiplied by the known branching fraction ${ }^{(7)}$ of 0.946 for decay of ${ }^{137} \mathrm{Cs}$ to $137 \mathrm{~m}_{\mathrm{Ba}}$ and added to the corresponding dose-rate factors for ${ }^{137} \mathrm{Cs}$. A similar assumption is reasonable for other decay chains involving short-lived daughter products and parent and daughter radionuclides that exhibit similar environmental behavior (e.g., ${ }^{106} \mathrm{Ru}$ decay to ${ }^{106} \mathrm{Rh}$ ).

In many potentially important cases, however, it may not be reasonable to assume secular equilibrium in the environment between a radionuclide and its radioactive daughter products. Examples include the complex decay chains involving radionuclides in the actinide series and their radioactive daughter products, in which parent and daughter radionuclides may exhibit significant differences in environmental behavior, decay chains involving noble gases and radionuclides that can deposit on the ground surface (e.g., ${ }^{88} \mathrm{Kr}$ decay to ${ }^{88} \mathrm{Rb}$ ), and decay chains in which the parent and daughter radionuclides are reasonably long-lived and have similar half-lives (e.g., ${ }^{95} \mathrm{Zr}$ decay to ${ }^{95} \mathrm{Nb}$ ). In these cases, the dose-rate factors for a parent and its daughters can be combined only after proper consideration of the equations describing production and 
decay of daughter radionuclides over time ${ }^{(31)}$ and differences in environmental behavior of the parent and daughters.

In estimating external dose equivalents to members of the public, contributions from radioactive daughters produced following a release of a parent radionuclide to the environment generally must be considered when the half-lives of the daughters are comparable to or less than (1) the half-life of the parent and (2) the time period of concern for the dose assessment (e.g., any one-year period during the operating lifetime of a facility). Specific examples of decay chains in which contributions from radioactive daughters produced in the environment generally must be considered in estimating external dose equivalents are given in the previous two paragraphs. It should be emphasized that contributions from such daughters that occur with the parent in releases to the environment also must be taken into account.

However, in estimating external dose equivalents to members of the public during the operating lifetime of a facility (e.g., over a period of a few decades), contributions from radioactive daughters produced following a release of a parent radionuclide to the environment generally can be neglected if the half-life of the daughter (1) is much longer than the half-life of the parent (i.e., by about three orders of magnitude or more, depending on the half-life of the parent and the relative magnitudes of the external dose-rate factors for the parent and daughter) or (2) is much longer than the time period of concern for the dose assessment, regardless of the half-life of the parent. An example of the first situation would be a release of ${ }^{99}$ Mo with a half-life of 66.02 hours

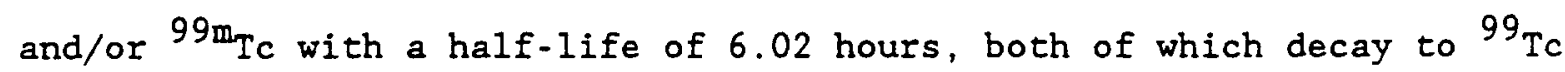
with a half-life of $2.13 \times 10^{5}$ years. An example of the second situation would be a release of $234 \mathrm{U}$ with a half-life of $2.445 \times 10^{5}$ years, which decays to ${ }^{230}$ Th with a half-life of $7.7 \times 10^{4}$ years. In both examples, the activity of the long-lived daughters produced in the environment will be insignificant during the time period of concern for the dose assessment, compared with the activity of the parents released to the environment, and the contributions to external dose equivalents from the daughters can be ignored. (These examples are illustrative only, because the external dose from ${ }^{99} \mathrm{Tc}, 234 \mathrm{~J}$, and ${ }^{230} \mathrm{Th}$ always will be insignificant compared with the dose from internal exposures.) It again should be 
emphasized, however, that long-lived daughter products also may occur with the parent in releases to the environment, in which case the daughters must be considered in estimating external dose equivalents during the operating lifetime of a facility.

For purposes other than estimating external dose equivalents to members of the public during the operating lifetime of a facility, the contributions from radioactive daughters produced following a release of a parent radionuclide to the environment may need to be taken into account, regardless of the half-lives of the daughters relative to either the half-life of the parent or the duration of releases. For example, if the collective dose equivalent in the exposed population is to be calculated over the residence time in the environment of the released radionuclides and their daughters, e.g., as part of an analysis to determine if releases are ALARA (As Low As Reasonably Achievable), then in principle the contributions from all daughters should be taken into account until radioactive decay is complete. The contributions from the daughters may be more important than those from the parents in determining collective dose equivalents over the environmental residence time of all radionuclides, even when maximum annual dose equivalents to individuals, which occur during the release period, are determined primarily by exposure to the parents. In practice, however, the collective dose equivalent may be calculated only over the operating lifetime of a facility; or calculations beyond the operating lifetime may be truncated by specifying either a time cutoff (e.g., 10,000 years) or a minimum annual dose equivalent to individuals (e.g., $1 \mathrm{mrem}$ ) for inclusion in the collective dose. With such procedures, contributions to external dose equivalents from long-lived daughters produced in the environment then could be neglected in many cases.

The discussions in the previous two paragraphs illustrate the difficulty in formulating firm guidelines regarding situations where contributions from long-lived daughters produced in the environment either must be taken into account or always can be neglected in calculating external dose equivalents to members of the public. Practical experience in calculating annual dose equivalents to maximally exposed individuals and collective dose equivalents in exposed populations for expected releases of radionuclides from operating facilities will be the most 
useful means of developing appropriate guidelines. However, the guidelines that may be developed from practical experience also will depend on general procedures that are established for performing dose assessments. An important example discussed above is whether collective dose equivalents are to be calculated only during the operating lifetime of a facility or, in addition, for an extended period of time thereafter. 


\section{REFERENCES}

1. D. C. Kocher, Dose-Rate Conversion Factors for External Exposure to Photons and Electrons, NUREG/CR-1918, ORNL/NUREG-79, Oak Ridge National Laboratory (1981).

2. D. C. Kocher, "Dose-Rate Conversion Factors for External Exposure to Photons and Electrons," Health Phys. 45, 665 (1983).

3. International Commission on Radiological Protection, "Recommendations of the International Commission on Radiological Protection, " ICRP Publication 26, Ann. ICRP 1, No. 3 (1977).

4. International Commission on Radiological Protection, "A Compilation of the Major Concepts and Quantities in Use by ICRP," ICRP Publication 42, Ann. ICRP 14, No. 4 (1984).

5. International Commission on Radiological Protection, Report of the Task Group on Reference Man, ICRP Publication 23, Pergamon Press, Oxford, U.K. (1975).

6. J. T. Whitton, "New Values for Epidermal Thickness and Their Importance," Health Phys. 24, 1 (1973).

7. D. C. Kocher, Radioactive Decay Data Tables, DOE/TIC-11026, U.S. Department of Energy (1981).

8. W. S. Snyder, M. R. Ford, G. G. Warner, and H. L. Fisher, Jr., "Estimates of Absorbed Fractions for Monoenergetic Photon Sources Uniformly Distributed in Various Organs of a Heterogeneous Phantom," MIRD Pamphlet No. 5, J. Nuc1. Med. Suppl. 3 (1969).

9. K. O'Brien and R. Sanna, "The Effect of the Male-Female Body-Size Difference on Absorbed Dose-Rate Distributions in Humans from Natural Gamma Rays," Health Phys. 34, 107 (1978).

10. K. O'Brien, "Human Dose from Radiation of Terrestrial Origin," p. 1163 in Natural Radiation Environment III, Vo1. 2, CONF-780422, U.S. Department of Energy (1980).

11. G. D. Kerr, "A Review of Organ Doses from Isotropic Fields of $\gamma$ Rays," Health Phys. 39, 3 (1980).

12. L. T. Dillman, "Absorbed Gamma Dose Rate for Immersion in a SemiInfinite Radioactive Cloud," Health Phys. 27, 571 (1974).

13. J. C. Ryman, R. E. Faw, and.K. Shultis, "Air-Ground Interface Effects on $y$-Ray Submersion Dose," Health Phys. 41, 759 (1981).

14. K. F. Eckerman, G. D. Kerr, and R. Raridon, "Organ Doses from Isotropic Cloud Sources of Gamma Rays," Health Phys. 39, 1054 (1980). 
15. P. Jacob, H. G. Paretzke, H. Rosenbaum, and M. Zank1, "Effective Dose Equivalents for Photon Exposures from Plane Sources on the Ground," Radiat. Protect. Dosim. 14, 299 (1986).

16. M. J. Berger, "Energy Deposition in Water by Photons from Point Isotropic Sources," J. Nucl. Med. Suppl. 1, 15 (1968).

17. J. H. Hubbell, Photon Cross Sections, Attenuation Coefficients, and Energy Absorption Coefficients from $10 \mathrm{keV}$ to $100 \mathrm{GeV}$, NSRDS-NBS 29, National Bureau of Standards (1969).

18. U.S. Department of Health, Education, and Welfare, Radiological Health Handbook (1970).

19. G. G. Killough and L. R. McKay, A Methodology for Calculating Radiation Doses from Radioactivity Released to the Environment, ORNL-4992, Oak Ridge National Laboratory (1976).

20. R. Loevinger and M. Berman, "A Schema for Absorbed-Dose Calculations for Biologically-Distributed Radionuclides," J. Nucl. Med. Suppl. 1, 7 (1968).

21. D. K. Trubey, A Survey of Empirical Functions Used to Fit Gamma-Ray Buildup Factors, ORNL-RSIC-10, Oak Ridge National Laboratory (1966).

22. A. B. Chilton, "Broad Beam Attenuation," p. 202 in Engineering Compendium on Radiation Shielding, Vol. I, edited by R. G. Jaeger, Springer-Verlag, New York (1968).

23. F. H. Clark, "Gamma-Ray Buildup Factors for Sand, Air and Wood (Cellulose)," Nucl. Appl. 6, 588 (1969).

24. W. Gautschi and W. F. Cahill, "Exponential Integral and Related Functions," p. 227 in Handbook of Mathematical Functions, edited by M. Abramowitz and I. A. Stegun, Dover Press, New York (1965).

25. M. J. Berger, "Beta-Ray Dose in Tissue-Equivalent Material Immersed in a Radioactive Cloud," Health Phys. 26, 1 (1974).

26. National Academy of Sciences-National Research Council, Studies in Penetration of Charged Particles in Matter, NAS-NRC Publication 1133 (1964).

27. M. J. Berger, Improved Point Kernels for Electron and Beta-Ray Dosimetry, NBSIR 73-107, National Bureau of Standards (1973).

28. C. S. Wu and S. A. Moskowski, Beta Decay, Interscience, New York (1966).

29. N. B. Gove and M. J. Martin, "Log-f Tables for Beta Decay," Nucl. Data Tables 10, 205 (1971). 
30. I. T. Dillman, EDISTR - A Computer Program to Obtain a Nuclear Decay Data Base for Radiation Dosimetry, ORNL/TM-6689, Oak Ridge National Laboratory (1980).

31. R. D. Evans, The Atomic Nucleus, Addison-Wesley, Reading, MA (1952).

32. P. J. Davis, "Gamma Function and Related Functions," p. 253 in Handbook of Mathematical Functions, edited by M. Abramowitz and I. A. Stegun, Dover Press, New York (1965).

33. L. T. Dillman and T. D. Jones, "Internal Dosimetry of Spontaneously Fissioning Nuclides," Health Phys. 29, 111 (1975).

34. F. R. O'Donnel1, D. C. Kocher, O. W. Burke, and F. H. Clark, CONDOS II - A Tool for Estimating Radiation Doses from RadionuclideContaining Consumer Products, NUREG/CR-2068, ORNL/NUREG/TM-454, Oak Ridge National Laboratory (1981).

35. U.S. Nuclear Regulatory Commission, Regulatory Guide 1.109. Calculation of Annual Doses to Man from Routine Releases of Reactor Effluents for the Purpose of Evaluating Compliance with 10 CFR Part 50, Appendix I (1977).

36. 2. G. Burson and A. E. Profio, "Structure Shielding in Reactor Accidents," Health Phys. 33, 287 (1977).

37. D. T. Oakley, Natural Radiation Exposure in the United States, ORP/SID 72-1, U.S. Environmental Protection Agency (1972).

38. National Council on Radiation Protection and Measurements, Natural Background Radiation in the United States, NCRP Report No. 45 (1975).

39. D. C. Kocher, "Effects of Indoor Residence on Radiation Doses from Routine Releases of Radionuclides to the Atmosphere," Nucl. Technol. 48,171 (1980).

40. J. K. Soldat, N. M. Robinson, and D. A. Baker, Models and Computer Codes for Evaluating Environmental Radiation Doses, BNWL-1754, Pacific Northwest Laboratory (1974).

41. D. C. Kocher and A. L. Sjoreen, "Dose-Rate Conversion Factors for External Exposure to Photon Emitters in Soil," Health Phys. 48, 193 (1985).

42. A. L. Sjoreen, D. C. Kocher, G. G. Killough, and C. W. Miller, MLSOIL and DFSOIL - Computer Codes to Estimate Effective Ground Surface Concentrations for Dose Computations, ORNL-5974, Oak Ridge National Laboratory (1984). 

APPENDIX A

TABLES OF DOSE-RATE CONVERSION FACTORS FOR

EXTERNAL EXPOSURE TO PHOTONS AND ELECTRONS

This appendix presents the tables of dose-rate conversion factors for external exposure to photons and electrons. Appendix A.I presents the results for immersion in contaminated air, Appendix A.2 the results for immersion in contaminated water, and Appendix A.3 the results for exposure to a contaminated ground surface.

The tables of dose-rate factors for each of the three exposure modes are described briefly as follows.

- Each radionuclide is identified by its name and half-life; the notation " $M$ " with the radionuclide name denotes a metastable excited state of the isotope. The units for the half-lives are denoted as follows: $\mathrm{S}$ - seconds; $M$ - minutes; $H$ - hours; $D$ - days; and $\mathrm{Y}=$ years.

- For all body organs except skin, the dose-rate factor applies to photons only.

- The entry labeled "EFFECTIVE" is the effective dose-rate factor for external exposure to photons, which is a weighted sum of photon dose-rate factors for 11 specific body organs, excluding skin. The weighting factors applied to each organ are listed in Table 1 (see Section 2.3).

- For skin, separate dose-rate factors are given for photons and electrons, and the entry labeled "TOTAL" is the sum of the two. The dose-rate factor for electrons applies to a depth in tissue below the body surface of $7 \mathrm{mg} / \mathrm{cm}^{2}$. Again, the photon and electron dose-rate factors for skin are not included in the effective dose-rate factor. 
- The dose-rate factors for all three exposure modes assume exposure for 1008 of the time. However, as discussed in Section 2.1.2, the values for immersion in contaminated water in Appendix A.2 should be reduced by a substantial amount to reflect a more realistic exposure time for members of the public. Reduction of the dose-rate factors for this exposure mode by a factor of 100 is recommended in lieu of site-specific information.

- The dose-rate factors for each radionuclide do not include possible contributions from radioactive daughter products. The treatment of dose-rate factors for daughter products in estimating external dose equivalents to members of the public is discussed in Section 3.4, and the parent-daughter relationships for the radionuclides considered in this report are shown in Appendix $B$.

- The dose-rate factors for the three exposure modes do not take into account the effects of indoor residence, ground roughness, exposure during boating activities, and exposure to contaminated shorelines. Dose-reduction factors for these situations are discussed in Section 3.2. These dose-reduction factors may be applied to specific external exposure situations, at the discretion of the user, to provide more realistic estimates of external dose equivalents to members of the public.

Dose-rate factors for external exposure above ground to radionuclides that are distributed with depth in soil are not presented in this appendix. However, methods for their calculation are described in Section 3.3.

The tables of external dose-rate factors listed in this appendix are available in computer-readable form from the Engineering Physics Information Center, Oak Ridge National Laboratory, P.O. Box X, Oak Ridge, TN 37831. Requests for these data should refer to the code DOSFACTER-DOE and to this report by title and number. 


\section{A.1 Dose-Rate Factors for Immersion}

in Contaminated Air

The following tables give the dose-rate conversion factors for external exposure to photons and electrons for immersion in a semiinfinite, uniformly contaminated atmospheric cloud. Dose-reduction factors which can be applied to these results to take into account external exposure to a contaminated atmospheric cloud during indoor residence are discussed in section 3.2.1. 


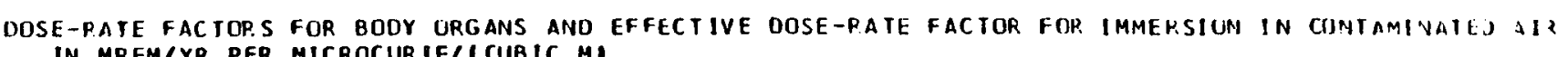

\begin{tabular}{|c|c|c|c|c|c|c|c|c|c|c|}
\hline NUCL IDE & HALF-LIFE & ADRENALS & BLADOER & BRAIN & BREAST & HEAR T & $\begin{array}{l}\text { SMALL } \\
\text { INTESTINE }\end{array}$ & $\begin{array}{c}\text { UPPEP } \\
\text { LARGE } \\
\text { INTESTIVE }\end{array}$ & $\begin{array}{c}\text { LOHER } \\
\text { LARGE } \\
\text { INTESTIINE }\end{array}$ & Q IONEYS \\
\hline $\begin{array}{l}H-3 \\
B E-7 \\
B E-10 \\
C-11 \\
C-14\end{array}$ & $\begin{array}{r}12.28 \mathrm{Y} \\
53.44 \mathrm{D} \\
1.6 E 6 \mathrm{Y} \\
20.48 \mathrm{H} \\
5.73 E 3 \mathrm{Y}\end{array}$ & $\begin{array}{l}0.0 \\
2.14 E+02 \\
0.0 \\
4.39 E+03 \\
0.0\end{array}$ & $\begin{array}{l}0.0 \\
2.02 E \cdot 02 \\
0.0 \\
4.17 E \cdot 03 \\
0.0\end{array}$ & $\begin{array}{l}0.0 \\
2.25 E \cdot 02 \\
0.0 \\
4.65 E \cdot 03 \\
0.0\end{array}$ & $\begin{array}{l}0.0 \\
2.71 E+02 \\
0.0 \\
5.54 E+03 \\
0.0\end{array}$ & $\begin{array}{l}0.0 \\
1.98 E \cdot 02 \\
0.0 \\
4.08 E \cdot 03 \\
0.0\end{array}$ & $\begin{array}{l}0.0 \\
1.78 E+02 \\
0.0 \\
3.67 E+03 \\
0.0\end{array}$ & $\begin{array}{l}0.0 \\
2.10 E+02 \\
0.0 \\
4.33 E+03 \\
0.0\end{array}$ & $\begin{array}{l}0.0 \\
1.94 E \cdot 02 \\
0.0 \\
4.00 E+03 \\
0.0\end{array}$ & $\begin{array}{l}0.0 \\
2.10 E+02 \\
0.0 \\
4.33 E+03 \\
0.0\end{array}$ \\
\hline $\begin{array}{l}N-13 \\
N-16 \\
0-15 \\
F-18 \\
N A-22\end{array}$ & $\begin{array}{r}9.97 \mathrm{M} \\
7.13 \mathrm{~S} \\
122.24 \mathrm{~S} \\
109.74 \mathrm{M} \\
2.602 \mathrm{Y}\end{array}$ & $\begin{array}{l}4.40 E+03 \\
2.44 E+04 \\
4.40 E+03 \\
4.26 E+03 \\
9.52 E+03\end{array}$ & $\begin{array}{l}4.17 E+03 \\
2.47 E+04 \\
4.18 E+03 \\
4.05 E+03 \\
9.29 E \cdot 03\end{array}$ & $\begin{array}{l}4.65 E+03 \\
2.62 E+04 \\
4.66 E+03 \\
4.51 E+03 \\
1.04 E+04\end{array}$ & $\begin{array}{l}5.54 E+03 \\
3.15 E+04 \\
5.55 E+03 \\
5.37 E+03 \\
1.19 E \cdot 04\end{array}$ & $\begin{array}{l}4.08 E+03 \\
2.43 E+04 \\
4.08 E+03 \\
3.95 E+03 \\
9.15 E+03\end{array}$ & $\begin{array}{l}3.67 E+03 \\
2.33 E+04 \\
3.67 E+03 \\
3.56 E+03 \\
8.35 E+03\end{array}$ & $\begin{array}{l}4.33 E+03 \\
2.00 E+04 \\
4.33 E+03 \\
4.20 E+03 \\
9.73 E+03\end{array}$ & $\begin{array}{l}4.00 E+03 \\
2.44 E \cdot 04 \\
4.00 E+03 \\
3.87 E+03 \\
8.97 E+03\end{array}$ & $\begin{array}{l}4.33 E+03 \\
2.76 E+04 \\
4.33 E+03 \\
4.19 E+03 \\
9.72 E+03\end{array}$ \\
\hline $\begin{array}{l}N A-24 \\
M G-27 \\
M G-2 B \\
A L-26 \\
A L-28\end{array}$ & $\begin{array}{l}15.00 \mathrm{H} \\
9.458 \mathrm{M} \\
20.91 \mathrm{H} \\
7.2 \mathrm{H} \\
2.240 \mathrm{M}\end{array}$ & $\begin{array}{l}1.97 E+04 \\
4.02 E+03 \\
5.95 E+03 \\
1.21 E+04 \\
B .21 E+03\end{array}$ & $\begin{array}{l}2.02 E+04 \\
3.90 E+03 \\
5.87 E+03 \\
1.22 E+04 \\
8.41 E+03\end{array}$ & $\begin{array}{l}2.25 E+04 \\
4.39 E+03 \\
6.59 E+03 \\
1.36 E+04 \\
9.44 E+03\end{array}$ & $\begin{array}{l}2.54 E+04 \\
4.91 E+03 \\
7.46 E+03 \\
1.54 E+04 \\
1.05 E \cdot 04\end{array}$ & $\begin{array}{l}2.02 E+04 \\
3.85 E+03 \\
5.80 E+03 \\
1.21 E+04 \\
8.38 E+03\end{array}$ & $\begin{array}{l}1.89 E+04 \\
3.49 E+03 \\
5.32 E+03 \\
1.11 E+04 \\
7.79 E+03\end{array}$ & $\begin{array}{l}2.15 E+04 \\
4.10 E+03 \\
6.17 E+03 \\
1.28 E+04 \\
8.91 E+03\end{array}$ & $\begin{array}{l}1.99 E+04 \\
3.77 E+03 \\
5.69 E * 03 \\
1.18 E+04 \\
8.22 E+03\end{array}$ & $\begin{array}{l}2.18 E+04 \\
4.08 E+03 \\
6.18 E+03 \\
1.29 E+04 \\
8.94 E+03\end{array}$ \\
\hline $\begin{array}{l}S 1-31 \\
s 1-32 \\
p-32 \\
p-33 \\
5-35\end{array}$ & 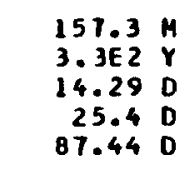 & $\begin{array}{l}3.87 E+00 \\
0.0 \\
0.0 \\
0.0 \\
0.0\end{array}$ & $\begin{array}{l}3.85 E \cdot 00 \\
0.0 \\
0.0 \\
0.0 \\
0.0\end{array}$ & $\begin{array}{l}4.34 E \cdot 00 \\
0.0 \\
0.0 \\
0.0 \\
0.0\end{array}$ & $\begin{array}{l}4.80 E \cdot 00 \\
0.0 \\
0.0 \\
0.0 \\
0.0\end{array}$ & $\begin{array}{l}3.81 E+00 \\
0.0 \\
0.0 \\
0.0 \\
0.0\end{array}$ & $\begin{array}{l}3.51 E+00 \\
0.0 \\
0.0 \\
0.0 \\
0.0\end{array}$ & $\begin{array}{l}4.06 E+03 \\
0.0 \\
0.0 \\
0.0 \\
0.0\end{array}$ & $\begin{array}{l}3.74 E+00 \\
0.0 \\
0.0 \\
0.0 \\
0.0\end{array}$ & $\begin{array}{l}4.05 E+00 \\
0.0 \\
0.0 \\
0.0 \\
0.0\end{array}$ \\
\hline $\begin{array}{l}C L-36 \\
C L-38 \\
A R-37 \\
A R-39 \\
A R-41\end{array}$ & 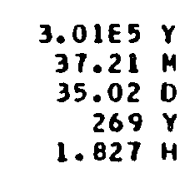 & $\begin{array}{l}3.05 E-06 \\
1.08 E+03 \\
4.86 E-04 \\
0.0 \\
5.60 E+03\end{array}$ & $\begin{array}{l}5.50 E-13 \\
7.28 E+03 \\
8.76 E-11 \\
0.0 \\
5.58 E+03\end{array}$ & $\begin{array}{l}2.43 E-07 \\
8.15 E+03 \\
3.87 E-05 \\
0.0 \\
6.29 E+03\end{array}$ & $\begin{array}{l}2.39 E-04 \\
9.08 E \cdot 03 \\
3.81 E-02 \\
0.0 \\
6.95 E+03\end{array}$ & $\begin{array}{l}3.97 E-07 \\
7.27 E+03 \\
6.33 E-05 \\
0.0 \\
5.52 E+03\end{array}$ & $\begin{array}{l}5.12 E-09 \\
E .18 E+03 \\
8.16 E-07 \\
0.0 \\
5.09 E+03\end{array}$ & $\begin{array}{l}7.54 E-07 \\
7.73 E+03 \\
1.20 E-06 \\
0.0 \\
5.88 E+03\end{array}$ & $\begin{array}{l}1.25 E-06 \\
7.14 E \cdot 03 \\
1.99 E-04 \\
0.0 \\
5.42 E+03\end{array}$ & $\begin{array}{l}3.65 E-09 \\
7.78 E+03 \\
5.82 E-07 \\
0.0 \\
5.87 E \cdot 03\end{array}$ \\
\hline $\begin{array}{l}K-40 \\
K-42 \\
K-43 \\
C A-41 \\
C A-45\end{array}$ & $\begin{array}{r}1.277 E 9 Y \\
12.36 \mathrm{H} \\
22.6 \mathrm{H} \\
1.03 E 5 \mathrm{Y} \\
162.7 \mathrm{D}\end{array}$ & $\begin{array}{l}6.00 E+02 \\
1.21 E+03 \\
4.23 E+03 \\
1.13 E-03 \\
8.76 E-09\end{array}$ & $\begin{array}{l}6.04 E+02 \\
1.22 E+03 \\
4.00 E+03 \\
2.03 E-10 \\
8.07 E-13\end{array}$ & $\begin{array}{l}7.71 E+02 \\
1.38 E+03 \\
4.45 E+03 \\
9.00 E-05 \\
9.39 E-10\end{array}$ & $\begin{array}{l}8.51 E+02 \\
1.52 E+03 \\
5.35 E+03 \\
8.84 E-02 \\
4.19 E-07\end{array}$ & $\begin{array}{l}6.78 E+02 \\
1.21 E+03 \\
3.92 E+03 \\
1.47 E-04 \\
1.43 E-09\end{array}$ & $\begin{array}{l}6.29 E+02 \\
1.13 E+03 \\
3.53 E+03 \\
1.89 E-06 \\
7.56 E-11\end{array}$ & $\begin{array}{l}7.22 E \cdot 02 \\
1.29 E+03 \\
4.16 E+03 \\
2.79 E-06 \\
1.15 E-11\end{array}$ & $\begin{array}{l}t .66 E+02 \\
1.19 E+03 \\
3.85 E+03 \\
4.63 E-04 \\
4.36 E-09\end{array}$ & $\begin{array}{l}7.22 E \cdot 02 \\
1.29 E+03 \\
4.16 E+03 \\
1.35 E-06 \\
1.09 E-10\end{array}$ \\
\hline $\begin{array}{l}\text { CA-4Y } \\
\text { CA-49 } \\
\text { SC-44 } \\
\text { SC- }-46 \\
S C-46 M\end{array}$ & 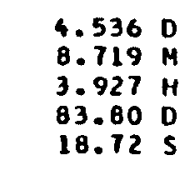 & $\begin{array}{l}4.64 E+03 \\
1.59 E+04 \\
9.34 E+03 \\
8.99 E+03 \\
3.19 E+02\end{array}$ & $\begin{array}{l}4.61 E+03 \\
1.64 E+04 \\
9.06 E+03 \\
8.79 E+03 \\
3.49 E+02\end{array}$ & $\begin{array}{l}5.20 E+03 \\
1.00 E+04 \\
1.02 E+04 \\
9.90 E+03 \\
3.74 E+02\end{array}$ & $\begin{array}{l}5.76 E+03 \\
2.07 E+04 \\
1.16 E+04 \\
1.10 E+04 \\
5.9 B E+02\end{array}$ & $\begin{array}{l}4.56 E+03 \\
1.64 E+04 \\
8.92 E+03 \\
8.69 E+03 \\
3.44 E+02\end{array}$ & $\begin{array}{l}4.20 E+03 \\
1.55 E+04 \\
8.11 E+03 \\
7.92 E+03 \\
3.00 E+02\end{array}$ & $\begin{array}{l}4.86 E+03 \\
1.74 E+04 \\
9.48 E+03 \\
9.25 E+03 \\
3.68 E+02\end{array}$ & $\begin{array}{l}4.47 E+03 \\
1.62 E+04 \\
8.74 E+03 \\
8.50 E+03 \\
3.37 E+02\end{array}$ & $\begin{array}{l}4.85 E+03 \\
1.79 E+04 \\
9.47 E+03 \\
9.22 E+03 \\
3.55 E+02\end{array}$ \\
\hline $\begin{array}{l}S C-47 \\
S C-48 \\
S C-49 \\
T 1-44 \\
T I-45\end{array}$ & $\begin{array}{r}3.422 \mathrm{O} \\
43.67 \mathrm{H} \\
57.4 \mathrm{M} \\
47.3 \mathrm{Y} \\
3.08 \mathrm{H}\end{array}$ & $\begin{array}{l}4.64 E+02 \\
1.49 E+04 \\
4.78 E+00 \\
4.46 E \cdot 02 \\
3.75 E \cdot 03\end{array}$ & $\begin{array}{l}4.25 E+02 \\
1.47 E+04 \\
4.88 E+00 \\
4.76 E+02 \\
3.56 E+03\end{array}$ & $\begin{array}{l}4.55 E+02 \\
1.65 E+04 \\
5.48 E+00 \\
4.72 E+02 \\
3.97 E+03\end{array}$ & $\begin{array}{l}7.09 E+02 \\
1.03 E+04 \\
6.07 E+00 \\
9.51 E+02 \\
4.73 E+03\end{array}$ & $\begin{array}{l}1.45 E+04 \\
4.86 E+00 \\
4.23 E+02 \\
3.48 E+03\end{array}$ & $\begin{array}{l}3.67 E+02 \\
1.33 E+04 \\
4.52 E+00 \\
3.78 E+02 \\
3.13 E+03\end{array}$ & $\begin{array}{l}4.45 E+02 \\
1.55 E+04 \\
5.17 E+00 \\
4.53 E+02 \\
3.70 E+03\end{array}$ & $\begin{array}{l}4.12 E+02 \\
1.42 E+04 \\
4.77 E+00 \\
4.03 E+02 \\
3.41 E+03\end{array}$ & $\begin{array}{l}\text { 4. } 33 E+02 \\
1.54 E+04 \\
5.18 E+00 \\
4.92 E+02 \\
3.70 E+03\end{array}$ \\
\hline
\end{tabular}




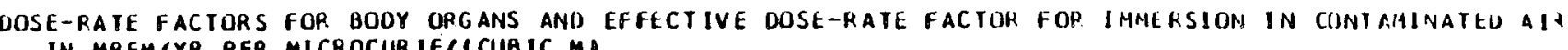

\begin{tabular}{|c|c|c|c|c|c|c|c|c|c|}
\hline HALF & LIVER & LUNGS & MARROW & MAI & OVARIES & PANCREAS & KEELETON & SP LEEN & S TOMA \\
\hline $\begin{array}{r}12.28 Y \\
53.44 \\
1.6 E 6 \\
20.48 \\
Y \\
5.73 E 3 Y\end{array}$ & $\begin{array}{l}0.0 \\
2.01 E+02 \\
0.0 \\
4.15 E+03 \\
0.0\end{array}$ & $\begin{array}{l}0.0 \\
2.16 E+02 \\
0.0 \\
4.65 E+03 \\
0.0\end{array}$ & $\begin{array}{l}0.0 \\
2.52 E+02 \\
0.0 \\
5.13 E \cdot 03 \\
0.0\end{array}$ & $\begin{array}{l}0.0 \\
2.22 E+02 \\
0.0 \\
4.56 E+03 \\
0.0\end{array}$ & $\begin{array}{l}0.0 \\
1.92 E+02 \\
0.0 \\
3.98 E+03 \\
0.0\end{array}$ & $\begin{array}{l}0.0 \\
1.73 E+02 \\
0.0 \\
3.57 E \cdot 03 \\
0.0\end{array}$ & $\begin{array}{l}0.0 \\
2.56 E+02 \\
0.0 \\
5.21 E+03 \\
0.0\end{array}$ & $\begin{array}{l}0.0 \\
1.97 E+02 \\
0.0 \\
4.05 E+03 \\
0.0\end{array}$ & $\begin{array}{l}0.0 \\
1.99 E+02 \\
0.0 \\
4.09 E+03 \\
0.0\end{array}$ \\
\hline $\begin{array}{r}9.97 \\
7.13 \\
122.24 \\
109.74 \\
2.602 \\
2.602\end{array}$ & $\begin{array}{l}4.15 E+03 \\
2.46 E+04 \\
4.15 E+03 \\
4.02 E+03 \\
9.26 E+03\end{array}$ & $\begin{array}{l}4.45 E+03 \\
2.53 E+04 \\
4.46 E+03 \\
4.32 E+03 \\
9.86 E+03\end{array}$ & $\begin{array}{l}5.13 E+03 \\
2.65 E+04 \\
5.13 E \bullet 03 \\
4.91 E \bullet 03 \\
1.08 E \bullet 04\end{array}$ & $\begin{array}{l}4.56 E+03 \\
2.74 E+04 \\
4.56 E+03 \\
4.42 E+03 \\
1.00 E+04\end{array}$ & $\begin{array}{l}3.99 E \cdot 03 \\
2.22 E+04 \\
3.99 E+03 \\
3.86 E+03 \\
8.85 E+03\end{array}$ & $\begin{array}{l}3.57 E \cdot 03 \\
2.27 E * 04 \\
3.57 E+03 \\
3.46 E+03 \\
8.00 E+03\end{array}$ & & & $\begin{array}{l}4.09 E+03 \\
2.51 E+04 \\
4.10 E+03 \\
3.97 E+03 \\
9.09 E+03\end{array}$ \\
\hline $\begin{array}{l}15.00 \mathrm{H} \\
9.450 \mathrm{H} \\
20.91 \mathrm{H} \\
7.2 \mathrm{H} \\
2.240 \mathrm{H}\end{array}$ & $\begin{array}{l}2.03 E+04 \\
3.88 E * 03 \\
5.85 E+03 \\
1.22 E \cdot 04 \\
8.44 E+03\end{array}$ & $\begin{array}{l}2.13 E+04 \\
4.13 E * 03 \\
6.21 E+03 \\
1.29 E+04 \\
8.80 E \cdot 03\end{array}$ & $\begin{array}{l}2.19 E+04 \\
4.46 E+03 \\
6.66 E * 03 \\
1.37 E+04 \\
9.15 E \cdot 03\end{array}$ & $\begin{array}{l}2.17 E+04 \\
4.19 E+03 \\
6.28 E+03 \\
1.31 E+04 \\
0.97 E+03\end{array}$ & $\begin{array}{l}1.83 E+04 \\
3.76 E+03 \\
5.57 E+03 \\
1.13 E+04 \\
7.76 E+03\end{array}$ & $\begin{array}{l}1.78 E+04 \\
3.35 E+03 \\
5.06 E+03 \\
1.06 E+04 \\
1.34 E+03\end{array}$ & $\begin{array}{l}2.22 E+04 \\
4.53 E+03 \\
6.77 E+03 \\
1.40 E+04 \\
9.31 E+03\end{array}$ & $\begin{array}{l}1.95 E \bullet 04 \\
3.16 E+03 \\
5.66 E * 03 \\
1.18 E+04 \\
8.10 E+03\end{array}$ & $\begin{array}{l}2.00 E+04 \\
3.83 E+03 \\
5.74 E+03 \\
1.19 E+04 \\
8.21 E+03\end{array}$ \\
\hline $\begin{array}{r}157.3 \mathrm{M} \\
3.362 \mathrm{Y} \\
14.29 \mathrm{D} \\
25.4 \mathrm{D} \\
87.44 \mathrm{O}\end{array}$ & $\begin{array}{l}3.84 E+00 \\
0.0 \\
0.0 \\
0.0 \\
0.0\end{array}$ & $\begin{array}{l}4.07 E+00 \\
0.0 \\
0.0 \\
0.0 \\
0.0\end{array}$ & $\begin{array}{l}4.28 E+00 \\
0.0 \\
0.0 \\
0.0 \\
0.0\end{array}$ & $\begin{array}{l}4.10 E+00 \\
0.0 \\
0.0 \\
0.0 \\
0.0\end{array}$ & $\begin{array}{l}3.66 E+00 \\
0.0 \\
0.0 \\
0.0\end{array}$ & $\begin{array}{l}3.33 E+00 \\
0.0 \\
0.0 \\
0.0 \\
0.0\end{array}$ & $\begin{array}{l}4.35 E+00 \\
0.0 \\
0.0 \\
0.0 \\
0.0\end{array}$ & $\begin{array}{l}0.71 E^{2}+1 \\
0.0 \\
0.0 \\
0.0 \\
0.0\end{array}$ & $\begin{array}{l}.76 \mathrm{E}+ \\
.0 \\
.0 \\
.0 \\
.0\end{array}$ \\
\hline 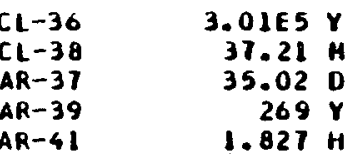 & $\begin{array}{l}2.96 E-08 \\
7.32 E \cdot 03 \\
4.72 E-06 \\
0.0 \\
5.57 E+03\end{array}$ & $\begin{array}{l}2.41 E-06 \\
7.69 E \cdot 03 \\
3.84 E-04 \\
0.0 \\
5.89 E+03\end{array}$ & $\begin{array}{l}1.45 E-06 \\
7.89 E+03 \\
2.31 E-04 \\
0.0 \\
6.19 E+03\end{array}$ & $\begin{array}{l}7.91 E-01 \\
7.79 E \cdot 03 \\
1.26 E-04 \\
0.0 \\
5.94 E+03\end{array}$ & $\begin{array}{l}1.19 E-06 \\
6.65 E+03 \\
1.90 E-04 \\
0.0 \\
5.29 E+03\end{array}$ & $\begin{array}{l}1.78 E-06 \\
6.38 E+03 \\
2.84 E-04 \\
0.0 \\
4.82 E+03\end{array}$ & $\begin{array}{l}3.91 E-06 \\
8.03 E+03 \\
6.24 E-04 \\
0.0 \\
6.29 E \cdot 03\end{array}$ & $\begin{array}{l}6.08 E-07 \\
7.01 E+03 \\
1.10 E-04 \\
0.0 \\
5.38 E+03\end{array}$ & $\begin{array}{l}6.21 E-07 \\
7.15 E+03 \\
9.90 E-05 \\
0.0 \\
5.44 E+03\end{array}$ \\
\hline $\begin{array}{r}1.277 E 9 Y \\
12.36 \mathrm{H} \\
22.6 \mathrm{H} \\
1.03 E 5 \mathrm{Y} \\
162.7 \mathrm{D}\end{array}$ & $\begin{array}{l}6.84 E+02 \\
1.22 E * 03 \\
3.98 E+03 \\
1.10 E-05 \\
3.00 E-10\end{array}$ & & $\begin{array}{l}7.54 E+02 \\
1.35 E+03 \\
4.94 E+03 \\
5.35 E-04 \\
6.84 E-09\end{array}$ & & & & & & $\begin{array}{l}3.66 E+02 \\
1.19 E+03 \\
3.93 E+03 \\
2.30 E-04 \\
2.71 E-09\end{array}$ \\
\hline $\begin{array}{l}4.536 \\
8.719 \mathrm{M} \\
3.927 \mathrm{H} \\
83.80 \mathrm{D} \\
18.72 \mathrm{~S}\end{array}$ & $\begin{array}{l}4.60 E * 03 \\
1.65 E * 04 \\
9.02 E+03 \\
0.74 E * 03 \\
3.49 E+02\end{array}$ & $\begin{array}{l}4.87 E+03 \\
1.71 E+04 \\
9.62 E+03 \\
9.30 E+03 \\
3.84 E+02\end{array}$ & $\begin{array}{l}5.14 E+03 \\
1.75 E+04 \\
1.06 E+04 \\
9.93 E+03 \\
5.84 E+02\end{array}$ & $\begin{array}{l}4.92 E+03 \\
1.77 E+04 \\
9.77 E+03 \\
9.41 E+03 \\
3.52 E+02\end{array}$ & & & & & $3.45 E+0$ \\
\hline $\begin{array}{r}3.422 \mathrm{O} \\
43.67 \mathrm{H} \\
57.4 \mathrm{M} \\
47.3 \mathrm{Y} \\
3.08 \mathrm{H}\end{array}$ & $\begin{array}{l}4.24 E+02 \\
1.46 E+04 \\
4.90 E+00 \\
4.71 E+02 \\
3.54 E+03\end{array}$ & $\begin{array}{l}1.55 E+04 \\
5.15 E+00 \\
5.20 E+02 \\
3.00 E+03\end{array}$ & $\begin{array}{l}6.07 E+02 \\
1.65 E+04 \\
5.32 E * 00 \\
9.11 E+02 \\
4.38 E * 03\end{array}$ & $\begin{array}{l}1.57 E+04 \\
5.20 E+00 \\
3.31 E+02 \\
3.09 E+03\end{array}$ & $\begin{array}{l}3.68 E+02 \\
1.40 E+04 \\
4.51 E * 00 \\
3.88 E+02 \\
3.40 E+03\end{array}$ & $\begin{array}{l}3.56 E+02 \\
1.26 E+04 \\
4.25 E \bullet 00 \\
3.20 E+02 \\
3.05 E+03\end{array}$ & $\begin{array}{l}1.68 E+04 \\
5.41 E+00 \\
9.36 E+02 \\
4.45 E+03\end{array}$ & $\begin{array}{l}1.41 E * 04 \\
4.70 E \cdot 00 \\
4.33 E+02 \\
3.46 E * 03\end{array}$ & $\begin{array}{l}1.44 \mathrm{E}+04 \\
4.76 \mathrm{E} \cdot 00 \\
4.42 \mathrm{E}+02 \\
3.49 \mathrm{E}+03\end{array}$ \\
\hline
\end{tabular}


DOSE-RATE FACTORS FOR BODY ORGANS AND EFFECTIVE DOSE-RATE FACTOR FOR IMMERSION IN CUNTAMINATEO AIU

\begin{tabular}{|c|c|c|c|c|c|c|c|c|c|}
\hline NUCL IDE & LF-LIFE & TESTES & THYMUS & THYROID & UTERUS & FECTIVE & $\begin{array}{c}\text { SKIN } \\
\text { (PHOTONI }\end{array}$ & $\begin{array}{l}\text { SKIN } \\
\text { IELECTRON) }\end{array}$ & $\begin{array}{l}\text { SKIN } \\
\text { ITOTAL, }\end{array}$ \\
\hline $\begin{array}{l}H-3 \\
B E-7 \\
B E-10 \\
C-11 \\
C-14\end{array}$ & $\begin{array}{r}12.28 Y \\
53.44 \mathrm{~V} \\
1.6 \mathrm{E} 6 \mathrm{Y} \\
20.48 \mathrm{M} \\
5.13 E 3 \mathrm{Y}\end{array}$ & $\begin{array}{l}0.0 \\
2.91 E+02 \\
0.0 \\
5.98 E+03 \\
0.0\end{array}$ & $\begin{array}{l}0 \\
19 E+02 \\
0 \\
52 E+03 \\
0\end{array}$ & $\begin{array}{l}0 \\
69 E+02 \\
0 \\
53 E+03 \\
0\end{array}$ & $\begin{array}{l}.0 \\
.76 E+02 \\
0 \\
.62 E+03 \\
0\end{array}$ & $\begin{array}{l}0.0 \\
2.49 E+02 \\
0.0 \\
5.11 E+03 \\
0.0\end{array}$ & $\begin{array}{l}0.0 \\
3.31 E+02 \\
0.0 \\
6.83 E+03 \\
0.0\end{array}$ & $\begin{array}{l}0.0 \\
0.0 \\
1.10 E+03 \\
2.65 E+03 \\
2.91 E+01\end{array}$ & $\begin{array}{l}0.0 \\
3.31 E+02 \\
1.10 E+03 \\
9.48 E+03 \\
2.91 E+01\end{array}$ \\
\hline $\begin{array}{l}N-13 \\
N-16 \\
D-15 \\
F-18 \\
N A-22\end{array}$ & $\begin{array}{r}9.97 \mathrm{M} \\
7.13 \mathrm{~S} \\
122.24 \mathrm{~S} \\
109.74 \mathrm{M} \\
2.602 \mathrm{Y}\end{array}$ & $\begin{array}{l}5.98 E+03 \\
3.38 E \cdot 04 \\
5.98 E \cdot 03 \\
5.79 E \cdot 03 \\
1.31 E \cdot 04\end{array}$ & $\begin{array}{l}.52 E+03 \\
.75 E+04 \\
.53 E \cdot 03 \\
.39 E+03 \\
.02 E+04\end{array}$ & $\begin{array}{l}.53 E+03 \\
.06 E+04 \\
.54 E \cdot 03 \\
.36 E \cdot 03 \\
.23 E+04\end{array}$ & $\begin{array}{l}.62 E+03 \\
618 E+04 \\
63 E+03 \\
.51 E+03 \\
.16 E+03\end{array}$ & $\begin{array}{l}11 E+03 \\
.93 E+04 \\
.12 E+03 \\
.95 E+03 \\
.12 E+04\end{array}$ & $\begin{array}{l}6.83 E+03 \\
3.55 E+04 \\
6.84 E+03 \\
6.62 E+03 \\
1.50 E+04\end{array}$ & & $\begin{array}{l}1.04 E+04 \\
5.00 E+04 \\
1.24 E+04 \\
8.08 E+03 \\
1.61 E+04\end{array}$ \\
\hline $\begin{array}{l}N A-24 \\
M G-27 \\
M G-28 \\
A L-26 \\
A L-28\end{array}$ & $\begin{array}{l}15.00 \mathrm{H} \\
9.458 \mathrm{M} \\
20.91 \mathrm{H} \\
7.2 \mathrm{H} \mathrm{Y} \\
2.240 \mathrm{M}\end{array}$ & $\begin{array}{l}2.77 E+04 \\
5.47 E+03 \\
8.21 E+03 \\
1.69 E+04 \\
1.15 E+04\end{array}$ & $\begin{array}{l}2.19 E \bullet 04 \\
4.36 E \bullet 03 \\
6.51 E \bullet 03 \\
1.32 E \bullet 04 \\
9.12 E \bullet 03\end{array}$ & $\begin{array}{l}2.54 E+04 \\
5.21 E+03 \\
7.76 E+03 \\
1.57 E+04 \\
1.07 E+04\end{array}$ & $\begin{array}{l}1.82 E+04 \\
3.38 E+03 \\
5.17 E+03 \\
1.09 E+04 \\
1.62 E+03\end{array}$ & $\begin{array}{l}2.39 E+04 \\
4.68 E+03 \\
7.03 E+03 \\
1.45 E+04 \\
9.92 E+03\end{array}$ & $\begin{array}{l}3.10 E+04 \\
6.32 E \cdot 03 \\
9.46 E+03 \\
1.92 E+04 \\
1.31 E+04\end{array}$ & $\begin{array}{l}4.05 E+03 \\
5.29 E+03 \\
7.06 E+02 \\
2.88 E+03 \\
9.87 E+03\end{array}$ & $\begin{array}{l}3.50 E+04 \\
1.16 E+04 \\
1.02 E+04 \\
2.21 E+04 \\
2.30 E+04\end{array}$ \\
\hline $\begin{array}{l}S 1-31 \\
51-32 \\
p-32 \\
p-33 \\
s-35\end{array}$ & $\begin{array}{rl}157.3 & M \\
3.3 E 2 & Y \\
14.29 & D \\
25.4 & 0 \\
87.44 & D\end{array}$ & $\begin{array}{l}5.34 E \cdot 00 \\
0.0 \\
0.0 \\
0.0\end{array}$ & $\begin{array}{l}4.28 E \cdot 00 \\
0.0 \\
0.0 \\
0.0\end{array}$ & $\begin{array}{l}\text { TE・00 } \\
b \\
0\end{array}$ & $\begin{array}{l}40 E+00 \\
0 \\
0 \\
0 \\
0\end{array}$ & $\begin{array}{l}4.58 E+00 \\
0.0 \\
0.0 \\
0.0 \\
0.0\end{array}$ & $\begin{array}{l}E .14 E+00 \\
0.0 \\
0.0 \\
0.0 \\
0.0\end{array}$ & $\begin{array}{l}4.39 E+03 \\
9.72 E+01 \\
5.23 E+03 \\
1.63 E+02 \\
3.59 E+01\end{array}$ & $\begin{array}{l}4.40 E+03 \\
9.72 E+01 \\
5.23 E+03 \\
1.63 E+02 \\
3.59 E+01\end{array}$ \\
\hline $\begin{array}{l}C L-36 \\
C L-38 \\
A R-37 \\
A R-39 \\
A R-41\end{array}$ & 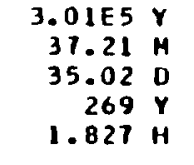 & $\begin{array}{l}2.21 E-05 \\
9.95 E+03 \\
3.53 E-03 \\
0.0 \\
7.74 E+03\end{array}$ & $\begin{array}{l}5.09 E-10 \\
1.86 E \cdot 03 \\
8.12 E-08 \\
0.0 \\
6.19 E \cdot 03\end{array}$ & $\begin{array}{l}1.52 E-06 \\
9.18 E \cdot 03 \\
2.43 E-04 \\
0.0 \\
7.34 E+03\end{array}$ & $\begin{array}{l}2.22 E-10 \\
6.62 E+03 \\
3.54 E-08 \\
0.0 \\
4.94 E+03\end{array}$ & $\begin{array}{l}.59 E+03 \\
.76 E-03 \\
.0 \\
.63 E+03\end{array}$ & $\begin{array}{l}5.09 E-04 \\
1.13 E+04 \\
8.12 E-02 \\
0.0 \\
8.89 E+03\end{array}$ & $\begin{array}{l}1.50 E+03 \\
1.24 E+04 \\
0.0 \\
1.26 E+03 \\
3.30 E+03\end{array}$ & $\begin{array}{l}1.50 E+03 \\
2.37 E+04 \\
8.12 E-02 \\
1.26 E+03 \\
1.22 E+04\end{array}$ \\
\hline $\begin{array}{l}K-40 \\
K-42 \\
K-43 \\
C A-41 \\
C A-45\end{array}$ & $\begin{array}{r}1.277 E 9 \mathrm{Y} \\
12.36 \mathrm{H} \\
22.6 \mathrm{H} \\
1.03 E 5 \mathrm{Y} \\
162.7 \mathrm{D}\end{array}$ & $\begin{array}{l}9.45 E+02 \\
1.69 E+03 \\
5.73 E+03 \\
8.19 E-03 \\
6.11 E-08\end{array}$ & $\begin{array}{l}7.54 E \cdot 02 \\
1.34 E \cdot 03 \\
4.38 E \cdot 03 \\
1.88 E-07 \\
2.77 E-11\end{array}$ & $\begin{array}{l}1.58 E+03 \\
5.36 E+03 \\
5.64 E-04 \\
7.69 E-09\end{array}$ & $\begin{array}{l}6.11 E \bullet 02 \\
1.10 E \bullet 03 \\
3.48 E \bullet 03 \\
0.23 E-0 B \\
1.03 E-12\end{array}$ & $\begin{array}{l}4.91 E+03 \\
1.57 E-02 \\
8.17 E-00\end{array}$ & $\begin{array}{l}1.08 E+03 \\
1.93 E+03 \\
6.54 E+03 \\
1.80 E-01 \\
7.05 E-07\end{array}$ & $\begin{array}{l}3.28 E+03 \\
1.15 E+04 \\
1.97 E+03 \\
0.0 \\
1.70 E+02\end{array}$ & $\begin{array}{l}4.36 E+03 \\
1.35 E+04 \\
0.51 E+03 \\
1.80 E-01 \\
1.70 E+02\end{array}$ \\
\hline $\begin{array}{l}C A-47 \\
C A-49 \\
\text { SC-44 } \\
\text { SC- } 46 \\
S C-46 M\end{array}$ & 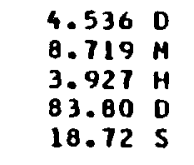 & $\begin{array}{l}6.40 E+03 \\
2.24 E+04 \\
1.28 E \cdot 04 \\
1.23 E+04 \\
5.35 E+02\end{array}$ & $\begin{array}{l}5.11 E+03 \\
1.76 E+04 \\
9.98 E \bullet 03 \\
9.82 E \bullet 03 \\
4.12 E+02\end{array}$ & $\begin{array}{l}1.11 E+04 \\
5.28 E+02\end{array}$ & $\begin{array}{l}4.07 E+03 \\
1.47 E+04 \\
1.91 E * 03 \\
1.65 E+03 \\
3.07 E+02\end{array}$ & $\begin{array}{l}1.09 E+04 \\
1.05 E+04 \\
4.73 E+02\end{array}$ & $\begin{array}{l}7.36 E+03 \\
2.45 E+04 \\
1.47 E+04 \\
1.42 E+04 \\
5.73 E+02\end{array}$ & $\begin{array}{l}4.47 E+03 \\
4.04 E+02 \\
1.73 E+02\end{array}$ & $\begin{array}{l}9.66 E+03 \\
3.12 E+04 \\
1.91 E+04 \\
1.46 E+04 \\
1.45 E+02\end{array}$ \\
\hline $\begin{array}{l}S C-47 \\
S C-48 \\
S C-49 \\
11-44 \\
I I-45\end{array}$ & $\begin{array}{rr}3.422 & \mathrm{O} \\
43.67 & \mathrm{H} \\
57.4 & \mathrm{H} \\
47.3 \mathrm{Y} \\
3.08 \mathrm{H}\end{array}$ & $\begin{array}{l}6.43 E+02 \\
2.04 E+04 \\
6.69 E+00 \\
7.94 E+02 \\
5.10 E+03\end{array}$ & $\begin{array}{l}4.95 E+02 \\
1.64 E \cdot 04 \\
5.31 E \bullet 00 \\
6.18 E \cdot 02 \\
3.86 E \cdot 03\end{array}$ & $\begin{array}{l}6.31 E+02 \\
1.95 E+04 \\
6.23 E+00 \\
7.0 B E 002 \\
4.12 E+03\end{array}$ & $\begin{array}{l}3.74 E+02 \\
1.28 E+04 \\
4.41 E+00 \\
3.79 E+02 \\
3.09 E+03\end{array}$ & $\begin{array}{l}1.75 \mathrm{E}+04 \\
5.76 \mathrm{E}+00 \\
6.73 \mathrm{E}+02 \\
4.36 \mathrm{E}+03\end{array}$ & $\begin{array}{l}6.94 E+02 \\
2.36 E+04 \\
7.62 E+00 \\
8.60 E+02 \\
5.83 E+03\end{array}$ & $\begin{array}{l}7.98 E+02 \\
1.26 E+03 \\
6.31 E+03 \\
1.34 E-01 \\
2.64 E+03\end{array}$ & $\begin{array}{l}2.48 E+04 \\
6.32 E+03 \\
8.60 E+02 \\
8.47 E+03\end{array}$ \\
\hline
\end{tabular}


DOSE-PATE FACTORS FOR BOOY ORGANS AND EFFECTIVE DOSE-PATE FACTOR FOR IMMERSION IN CONTAMINATES MIR
IN MREM/YR PER MICROCUR IE/ICUOIC MI

\begin{tabular}{|c|c|c|c|c|c|c|c|c|c|c|}
\hline CLIDE & LF-LIFE & DRENALS S & BLADDER & BRAIN & BREAST & HE AR T & $\begin{array}{l}\text { SMALL } \\
\text { INIESIINE }\end{array}$ & $\begin{array}{l}\text { UPPER } \\
\text { LARGE } \\
\text { NIESTIVE }\end{array}$ & $\begin{array}{c}\text { LUWER } \\
\text { LARGE } \\
\text { INIESTINEE }\end{array}$ & CIONEY Y \\
\hline $\begin{array}{l}1-51 \\
1-48 \\
1-49 \\
1-52 \\
1-49\end{array}$ & 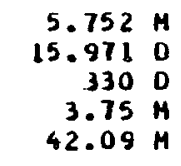 & $\begin{array}{l}1.60 E+03 \\
1.29 E+04 \\
3.39 E-03 \\
6.29 E+03 \\
4.46 E+03\end{array}$ & $\begin{array}{l}.49 E+03 \\
.26 E+04 \\
.10 E-10 \\
.33 E+03 \\
.24 E+03\end{array}$ & $\begin{array}{l}.64 E+03 \\
.42 E+C 4 \\
70 E-04 \\
13 E+03 \\
70 E+03\end{array}$ & $\begin{array}{l}.09 E+03 \\
.59 E+04 \\
.65 E-01 \\
.87 E+03 \\
.82 E+03\end{array}$ & $\begin{array}{l}46 E+03 \\
25 E+04 \\
41 E-04 \\
27 E+03 \\
24 E+03\end{array}$ & $\begin{array}{l}31 E+03 \\
14 E+04 \\
69 E-06 \\
01 E+03 \\
71 E+03\end{array}$ & $\begin{array}{l}.55 E+03 \\
.33 E+04 \\
.37 E-06 \\
.67 E+03 \\
.39 E+03\end{array}$ & $\begin{array}{l}1.74 E \cdot 03 \\
1.22 E+04 \\
1.39 E-03 \\
6.15 E+03 \\
4.05 E+03\end{array}$ & $\begin{array}{l}1.55 E+03 \\
1.33 E+04 \\
4.06 E-00 \\
6.67 E+03 \\
4.39 E+03\end{array}$ \\
\hline $\begin{array}{l}-51 \\
1-52 \\
1-524 \\
-53\end{array}$ & $\begin{array}{r}27.704 \\
5.591 \\
21.4 \\
3.7 E 6 \\
312.7 \\
30\end{array}$ & $\begin{array}{l}1.36 E+02 \\
1.53 E \cdot 04 \\
1.04 E \cdot 04 \\
6.27 E-03 \\
3.76 E+03\end{array}$ & $\begin{array}{l}1.25 E+02 \\
1.50 E+04 \\
1.03 E \cdot 04 \\
1.13 E-09 \\
3.63 E+03\end{array}$ & $\begin{array}{l}1.37 E * 02 \\
1.69 E * 04 \\
1.15 E * 04 \\
5.00 E-04 \\
4.09 E+03\end{array}$ & $\begin{array}{l}1.00 E+0 Z \\
1.89 E+04 \\
1.31 E * 04 \\
4.91 E-01 \\
4.59 E+03\end{array}$ & $\begin{array}{l}.22 E+02 \\
048 E+04 \\
01 E+04 \\
17 E-04 \\
.58 E+03\end{array}$ & $\begin{array}{l}.10 E \cdot 02 \\
.35 E \cdot 04 \\
.26 E+03 \\
.05 E-05 \\
.25 E+03\end{array}$ & $\begin{array}{l}1.29 E+02 \\
1.58 E * 04 \\
1.08 E+04 \\
1.55 E-05 \\
3.81 E+03\end{array}$ & $\begin{array}{l}1.21 E * 02 \\
1.45 E+04 \\
9.92 E+03 \\
2.51 E-03 \\
3.51 E+03\end{array}$ & $\begin{array}{l}1.29 E+02 \\
1.57 E+04 \\
1.07 E+04 \\
1.52 E-06 \\
3.80 E+03\end{array}$ \\
\hline $\begin{array}{l}-56 \\
-58 \\
-57 \\
-52\end{array}$ & $\begin{array}{r}2.5785 \mathrm{H} \\
1.47 \mathrm{H} \\
8.275 \mathrm{H} \\
2.7 \mathrm{Y} \\
44.63 \mathrm{O}\end{array}$ & $\begin{array}{l}7.84 E+03 \\
3.12 E+02 \\
3.17 E+03 \\
8.25 E-03 \\
5.24 E+03\end{array}$ & $\begin{array}{l}7.83 E+03 \\
3.00 E+02 \\
2.98 E+03 \\
1.49 E-09 \\
5.18 E+03\end{array}$ & $\begin{array}{l}0.78 E+03 \\
3.33 E+02 \\
3.29 E+03\end{array}$ & $\begin{array}{l}9.81 E+03 \\
4.16 E+02 \\
4.16 E+03 \\
6.46 E-01 \\
6.47 E \cdot 03\end{array}$ & $\begin{array}{l}.78 E+03 \\
95 E+02 \\
.92 E+03 \\
07 E-03 \\
13 E+03\end{array}$ & & & & $\begin{array}{l}\text { 8. } 29 E+03 \\
3.12 E+02 \\
3.08 E+03 \\
9.89 E-06 \\
5.44 E+03\end{array}$ \\
\hline $\begin{array}{l}0-56 \\
0-57 \\
0-58 \\
0-584 \\
0-60\end{array}$ & $\begin{array}{rl}270.9 & 0 \\
70.800 & 0 \\
9.15 & \mathrm{H} \\
5.271 & 8\end{array}$ & $\begin{array}{l}1.67 E \cdot 04 \\
4.97 E+02 \\
4.35 E+03 \\
1.46 E-02 \\
1.10 E+04\end{array}$ & $\begin{array}{l}1.67 E+04 \\
4.64 E+02 \\
4.19 E+03 \\
3.02 E-03 \\
1.09 E+04\end{array}$ & $1.23 E \cdot 04$ & $\begin{array}{l}2.10 E+04 \\
9.24 E+02 \\
5.35 E+03 \\
9.47 E-01 \\
1.36 E+04\end{array}$ & & & & & $\begin{array}{l}4.38 E+03 \\
5.84 E-03 \\
1.15 E+04\end{array}$ \\
\hline $\begin{array}{l}-604 \\
-61 \\
-56 \\
-56 \\
-57 \\
-59\end{array}$ & $\begin{array}{l}1.650 \mathrm{H} \\
6.10 \mathrm{O} \\
36.08 \mathrm{H} \\
7.5 \mathrm{Y} \mathrm{Y}\end{array}$ & $\begin{array}{l}1.73 E+01 \\
3.19 E+02 \\
7.62 E+03 \\
8.47 E+03 \\
1.39 E-02\end{array}$ & $\begin{array}{l}1.77 E+01 \\
3.32 E+02 \\
7.32 E+03 \\
8.44 E+03 \\
2.50 E-09\end{array}$ & $\begin{array}{l}1.92 E+01 \\
3.44 E+02 \\
8.17 E+03 \\
9.48 E+03 \\
1.11 E-03\end{array}$ & $\begin{array}{l}2.63 E+01 \\
5.68 E \cdot 02 \\
9.65 E+03 \\
1.07 E+04 \\
1.09 E+00\end{array}$ & & & 12. & & $\begin{array}{l}3.47 E+02 \\
7.64 E+03 \\
8.88 E+03 \\
1.66 E-05\end{array}$ \\
\hline $\begin{array}{l}-63 \\
-65 \\
1-61 \\
1-62 \\
-64\end{array}$ & $\begin{array}{r}100.1 \mathrm{Y} \\
2.520 \mathrm{H} \\
3.400 \mathrm{H} \\
9.74 \mathrm{H} \\
12.701 \mathrm{H}\end{array}$ & $\begin{array}{l}2.41 E+03 \\
3.56 E \cdot 03 \\
4.34 E+03 \\
8.16 E+02\end{array}$ & $\begin{array}{l}4.12 E+03 \\
1.76 E+02\end{array}$ & $\begin{array}{l}2.71 E+03 \\
3.78 E \cdot 03 \\
4.59 E+03 \\
8.65 E+02\end{array}$ & $\begin{array}{l}3.01 E+03 \\
4.49 E+03 \\
5.47 E+03 \\
1.03 E+03\end{array}$ & $\begin{array}{l}2.38 E+03 \\
3.32 E+03 \\
4.03 E+03 \\
7.59 E+02\end{array}$ & & $\begin{array}{l}0.0 \\
2.53 E+03 \\
3.52 E+03 \\
4.2 \theta E+03 \\
8.05 E+02\end{array}$ & $\begin{array}{l}2.34 E+03 \\
3.25 E+03 \\
3.95 E+03 \\
7.44 E+02\end{array}$ & $\begin{array}{l}3.52 E \bullet 0 \\
4.28 E \bullet 0 \\
8.05 E \bullet 0\end{array}$ \\
\hline $\begin{array}{l}y-65 \\
y-69 \\
-694\end{array}$ & $\begin{array}{r}9.26 \mathrm{H} \\
244.4 \mathrm{O} \\
5556 \mathrm{H} \\
13.76 \mathrm{H}\end{array}$ & $\begin{array}{l}2.58 E+03 \\
2.58 E-02 \\
1.79 E+03\end{array}$ & $\begin{array}{l}1.84 E+03 \\
2.53 E+03 \\
2.42 E-02 \\
1.68 E+03\end{array}$ & $\begin{array}{l}2.04 \mathrm{E} \cdot 03 \\
2.86 \mathrm{E}+03 \\
2.67 \mathrm{E}-02 \\
1.86 \mathrm{E}+03\end{array}$ & $\begin{array}{l}2.46 E+03 \\
3.17 E+03 \\
3.32 E-02 \\
2.29 E+03\end{array}$ & $\begin{array}{l}4.32 E \cdot 02 \\
1.79 E \cdot 03 \\
2.51 E \cdot 03 \\
2.36 E-02 \\
1.64 E+03\end{array}$ & $\begin{array}{l}2.29 E+03 \\
2.12 E-02 \\
1.4 B E+03\end{array}$ & $\begin{array}{l}2.60 E-03 \\
2.50 E-02 \\
1.74 E+03\end{array}$ & & $\begin{array}{l}4.55 E \bullet 0 \\
1.91 \mathrm{E} \bullet 0 \\
2.66 E * 0 \\
2.50 \mathrm{E}-0 \\
1.74 \mathrm{E} * 0\end{array}$ \\
\hline$E-68$ & $\begin{array}{rl}9.40 & H \\
3.261 & 0 \\
68.0 & H \\
14.1 & H \\
288 & 0\end{array}$ & $\begin{array}{l}1.27 E \cdot 04 \\
3.28 E-02\end{array}$ & $\begin{array}{l}3.90 E+03 \\
1.27 E \cdot 04 \\
7.42 E-09\end{array}$ & $\begin{array}{l}1.42 E+04 \\
2.63 E-03\end{array}$ & $\begin{array}{l}8.99 E+02 \\
5.86 E+03 \\
1.60 E+04 \\
2.54 E+00\end{array}$ & $\begin{array}{l}5.46 E+02 \\
3.01 E+03 \\
1.26 E+04 \\
4.29 E-03\end{array}$ & $\begin{array}{l}1.16 E+04 \\
5.73 E-05\end{array}$ & $\begin{array}{l}4.05 E * 03 \\
1.34 E+04 \\
7.74 E-05\end{array}$ & $\begin{array}{l}1.15 E+04 \\
5.37 E * 02 \\
3.74 E * 03 \\
1.24 E+04 \\
1.35 E-02\end{array}$ & $\begin{array}{l}5.78 E+0 \\
4.04 E * 0 \\
1.35 E+0 \\
4.16 E-0\end{array}$ \\
\hline
\end{tabular}


DOSE-RATE FACTORS FOR BODY ORGANS ANO EFFECTIVE DOSE-RATE FACTOR FOR IMMERSION IN CONTAMINATED AL
IN MREM/YR PER MICROCURIE/ICUBIC MI

\begin{tabular}{|c|c|c|c|c|c|c|c|c|c|c|}
\hline LIOE & LF-LIFE & LIVER & LUNGS & ARROH & $\begin{array}{c}\text { RED } \\
\text { MARROH }\end{array}$ & VARIES & ANCREAS & KELETON & SP LEEN & YA \\
\hline $\begin{array}{l}1-51 \\
-48 \\
-49 \\
-52 \\
8-49\end{array}$ & $\begin{array}{r}5.752 \\
15.971 \\
330 \\
30 \\
3.75 \mathrm{M} \\
42.09 \mathrm{M}\end{array}$ & $\begin{array}{l}.49 E+03 \\
26 E+04 \\
29 E-05 \\
32 E+03 \\
.22 E+03\end{array}$ & $\begin{array}{l}.60 E \cdot 03 \\
.34 E \cdot 04 \\
.68 E-03 \\
.68 E+03 \\
.54 E+03\end{array}$ & $\begin{array}{l}95 E+03 \\
44 E+04 \\
61 E-03 \\
98 E+03 \\
42 E+03\end{array}$ & $\begin{array}{l}65 E+03 \\
36 E+04 \\
79 E-04 \\
73 E+03 \\
55 E+03\end{array}$ & $\begin{array}{l}E+03 \\
E+04 \\
E-03 \\
E+03 \\
E+03 \\
E+03\end{array}$ & $\begin{array}{l}28 E+03 \\
09 E+04 \\
98 E-03 \\
49 E+03 \\
60 E+03\end{array}$ & $\begin{array}{l}98 E+03 \\
46 E+04 \\
35 E-03 \\
09 E+03 \\
51 E+03\end{array}$ & $\begin{array}{l}6 E+03 \\
22 E+04 \\
34 E-04 \\
0 E+03 \\
2 E+03\end{array}$ & $\begin{array}{l}1.41 E+03 \\
1.24 E+04 \\
6.90 E-04 \\
6.16 E+03 \\
4.16 E+03\end{array}$ \\
\hline $\begin{array}{l}R-51 \\
N-52 \\
N-52 M \\
N-53 \\
N-54 \\
N-54\end{array}$ & 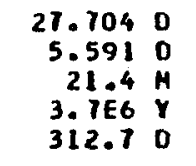 & $\begin{array}{l}1.0 \\
6.1 \\
3.6\end{array}$ & & & & & & & & $\begin{array}{l}1.23 E+0 \\
1.47 E+0 \\
1.00 E+0 \\
1.28 E-0 \\
3.57 E+0\end{array}$ \\
\hline $\begin{array}{l}\text { FE-5 } \\
\text { FE-5 } \\
\text { FE-5 }\end{array}$ & $\begin{array}{r}2.5785 \mathrm{H} \\
1.47 \mathrm{H} \\
8.275 \mathrm{H} \\
2.7 \mathrm{Y} \\
44.63 \mathrm{O}\end{array}$ & $\begin{array}{l}.83 E+03 \\
.99 E+02 \\
.997 E+03 \\
.02 E-05\end{array}$ & $\begin{array}{l}29 E+03 \\
.21 E+02 \\
.20 E+03 \\
.52 E-03 \\
.48 E+03\end{array}$ & $9.72 E+03$ & & & & & & $\begin{array}{l}1.68 \mathrm{E}- \\
5.06 \mathrm{EF}\end{array}$ \\
\hline $\begin{array}{l}c 0-56 \\
00-57 \\
00-58 \\
c 0-584 \\
c 0-60\end{array}$ & $\begin{array}{rl}78.76 & 0 \\
270.9 & 0 \\
70.80 & 0 \\
9.15 & H \\
5.271 & Y\end{array}$ & $\begin{array}{l}1.67 E+04 \\
4.63 E+02 \\
4.1 T E+03 \\
2.13 E-03 \\
1.09 E+04\end{array}$ & $\begin{array}{l}.76 E+04 \\
.12 E+02 \\
.45 E+03 \\
.29 E-02 \\
.15 E+04\end{array}$ & $\begin{array}{l}1.05 E+04 \\
.04 E+02 \\
6 B E+02\end{array}$ & & & & & & $\begin{array}{l}4.58 E+ \\
4.12 E+ \\
4.76 E- \\
1.06 E+\end{array}$ \\
\hline $\begin{array}{l}C O-61 \\
N I-56 \\
N 1-57 \\
N I-59\end{array}$ & $\begin{array}{r}10.41 \mathrm{M} \\
1.650 \mathrm{H} \\
6.10 \mathrm{O} \\
36.08 \mathrm{H} \\
T .5 \mathrm{E}\end{array}$ & $\begin{array}{l}1.16 E \cdot 01 \\
3.29 E \cdot 02 \\
7.29 E \cdot 03 \\
8.43 E+03 \\
1.35 E-04\end{array}$ & $\begin{array}{l}1.89 E \\
3.57 E \\
7.80 E \\
8.93 E\end{array}$ & $\begin{array}{l}5.27 E+02 \\
8.85 E+03 \\
9.54 E+03 \\
6.58 E-03\end{array}$ & $\begin{array}{l}72 E+01 \\
77 E+02 \\
38 E+03 \\
03 E+03 \\
30 E-03\end{array}$ & $\begin{array}{l}1.62 E+01 \\
2.90 E+02 \\
6.94 E+03 \\
7.93 E+03 \\
5.43 E-03\end{array}$ & & & & $\begin{array}{l}3.13 E+ \\
7.19 E+ \\
8.24 E E \\
2.83 E-\end{array}$ \\
\hline $\begin{array}{l}1-63 \\
I-65 \\
1-61\end{array}$ & $\begin{array}{r}100.1 \mathrm{Y} \\
2.520 \mathrm{H} \\
3.408 \mathrm{H} \\
9.74 \mathrm{M} \\
12.701 \mathrm{H}\end{array}$ & $\begin{array}{l}2.40 E+03 \\
3.37 E+03 \\
4.10 E+03 \\
7.72 E+02\end{array}$ & $\begin{array}{l}.0 \\
.54 E * \\
.61 E: \\
.40 E: \\
.28 E+\end{array}$ & $9.50 E+02$ & $\begin{array}{l}.57 E+03 \\
.69 E+03 \\
.50 E+03 \\
.47 E+02\end{array}$ & $\begin{array}{l}2.27 E+03 \\
3.23 E+03 \\
3.94 E+03 \\
7.41 E+02\end{array}$ & & & & $\begin{array}{l}2.35 \mathrm{EA} \\
3.32 \mathrm{Et} \\
4.04 \mathrm{Et} \\
7.61 \mathrm{E*}\end{array}$ \\
\hline $\begin{array}{l}2 N-62 \\
2 N-65 \\
2 N-69 \\
2 N-694\end{array}$ & 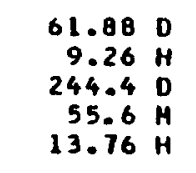 & $\begin{array}{l}4.43 E+02 \\
1.03 E+03 \\
2.52 E+03 \\
2.41 E-02 \\
1.67 E+03\end{array}$ & $\begin{array}{l}4.85 E+02 \\
1.96 E+03 \\
2.68 E+03 \\
2.59 E-02 \\
1.80 E+03\end{array}$ & $\begin{array}{l}2.85 E+03 \\
3.09 E-02 \\
2.13 E+03\end{array}$ & & & & & & $\begin{array}{l}1.80 E+ \\
2.48 E+ \\
2.37 \mathrm{E}- \\
1.65 \mathrm{EA}\end{array}$ \\
\hline $\begin{array}{l}G A-66 \\
G A-67 \\
G A-68 \\
G A-72 \\
G E-68\end{array}$ & $\begin{array}{r}9.40 \mathrm{H} \\
3.261 \mathrm{D} \\
68.0 \mathrm{H} \\
14.1 \mathrm{H} \\
288 \mathrm{O}\end{array}$ & $\begin{array}{l}1.18 E+04 \\
5.60 E+02 \\
3.88 E+03 \\
1.27 E+04 \\
3.28 E-04\end{array}$ & $\begin{array}{l}6.11 E+02 \\
4.16 E+03 \\
1.34 E+04 \\
2.60 E-02\end{array}$ & $\begin{array}{l}8.54 E+02 \\
4.77 E+03 \\
1.41 E+04 \\
1.58 E-02\end{array}$ & $\begin{array}{l}5.81 E+02 \\
4.25 E+03 \\
1.36 E+04 \\
0.54 E-03\end{array}$ & & & $\begin{array}{l}1.33 E+04 \\
8.74 E+02 \\
4.84 E+03 \\
1.44 E+04 \\
4.22 E-02\end{array}$ & $\begin{array}{l}1.14 E 044 \\
5.52 E+02 \\
3.78 E 003 \\
1.22 E 0.04 \\
7.45 E-03\end{array}$ & 6.75E- \\
\hline
\end{tabular}


DOSE-PATE FACTORS FUR BODY ORGANS ANO EFFECTIVE DOSE-RATE FACTCR fOK IMMERSION IN CONTAMINATES als

\begin{tabular}{|c|c|c|c|c|c|c|c|c|c|}
\hline NUCL IOE & LLF-LIFE & TESTES & THYMUS & THYROIO & UTERUS & EFFECTIVE & $\begin{array}{c}\text { SKIN } \\
\text { (PHOTONI }\end{array}$ & $\begin{array}{l}\text { SKIN } \\
\text { IELECTRONI }\end{array}$ & $\begin{array}{l}\text { SKIN } \\
\text { ITOTALI }\end{array}$ \\
\hline $\begin{array}{l}V I-51 \\
V-48 \\
V-49 \\
V-52 \\
C R-49\end{array}$ & $\begin{array}{r}5.752 \mathrm{M} \\
15.971 \mathrm{D} \\
330 \mathrm{D} \\
3.75 \mathrm{M} \\
42.09 \mathrm{H}\end{array}$ & $\begin{array}{l}2.16 E+03 \\
1.77 E+04 \\
2.46 E-02 \\
8.74 E+03 \\
6.13 E+03\end{array}$ & $\begin{array}{l}1.65 E+03 \\
1.40 E+04 \\
5.66 E-07 \\
6.98 E+03 \\
4.65 E+03\end{array}$ & $\begin{array}{l}2.03 E+03 \\
1.67 E \cdot 04 \\
1.69 E-03 \\
8.25 E+03 \\
5.72 E+03\end{array}$ & $\begin{array}{l}1.31 E+03 \\
1.11 E+04 \\
2.41 E-01 \\
5.64 E+03 \\
3.68 E+03\end{array}$ & $\begin{array}{l}1.87 E+03 \\
1.51 E+04 \\
4.71 E-02 \\
7.50 E+03 \\
5.25 E+03\end{array}$ & $\begin{array}{l}2.43 E+03 \\
2.03 E+04 \\
5.66 E-01 \\
1.00 E+04 \\
6.97 E+03\end{array}$ & $\begin{array}{l}6.67 E+03 \\
9.32 E+02 \\
0.0 \\
8.40 E+03 \\
4.45 E+03\end{array}$ & $\begin{array}{l}9.11 E \div 03 \\
2.13 E+04 \\
5.66 E-01 \\
1.84 E+04 \\
1.14 E+04\end{array}$ \\
\hline $\begin{array}{l}C R-51 \\
M N-52 \\
M N-52 M \\
M N-53 \\
M N-54\end{array}$ & $\begin{array}{rr}27.704 & 0 \\
5.591 & 0 \\
21.4 & N \\
3.7166 & Y \\
312.7 & 0\end{array}$ & $\begin{array}{l}1.82 E+02 \\
2.10 E+04 \\
1.44 E+04 \\
4.55 E-02 \\
5.11 E+03\end{array}$ & $\begin{array}{l}1.37 E+02 \\
1.66 E+04 \\
1.12 E+04 \\
1.05 E-06 \\
4.05 E+03\end{array}$ & $\begin{array}{l}1.71 E+02 \\
1.98 E \cdot 04 \\
1.35 E \cdot 04 \\
3.14 E-03 \\
4.86 E+03\end{array}$ & $\begin{array}{l}1.10 E+02 \\
1.32 E+04 \\
9.06 E+03 \\
4.58 E-07 \\
3.14 E+03\end{array}$ & $\begin{array}{l}1.58 E+02 \\
1.79 E+04 \\
1.23 E+04 \\
8.72 E-02 \\
4.37 E+03\end{array}$ & $\begin{array}{l}2.05 E \cdot 02 \\
2.41 E+04 \\
1.65 E+04 \\
1.05 E+00 \\
5.91 E+03\end{array}$ & $\begin{array}{l}0.0 \\
4.22 E \cdot 02 \\
0.96 E+03 \\
0.0 \\
0.0\end{array}$ & $\begin{array}{l}2.05 E+02 \\
2.45 E+04 \\
2.54 E+04 \\
1.05 E+00 \\
5.91 E+03\end{array}$ \\
\hline $\begin{array}{l}M N-56 \\
M N-57 \\
F E-52 \\
F E-55 \\
F E-59\end{array}$ & $\begin{array}{r}2.5785 \mathrm{H} \\
1.47 \mathrm{M} \\
8.275 \mathrm{H} \\
2.7 \mathrm{Y} \\
44.630\end{array}$ & $\begin{array}{l}1.08 E+04 \\
4.31 E+02 \\
4.32 E+03 \\
5.99 E-02 \\
7.20 E \cdot 03\end{array}$ & $\begin{array}{l}8.59 E+03 \\
3.36 E+02 \\
3.28 E+03 \\
1.38 E-06 \\
5.77 E+03\end{array}$ & $\begin{array}{l}1.02 E+04 \\
4.10 E+02 \\
4.05 E+03 \\
4.13 E-03 \\
6.86 E * 03\end{array}$ & $\begin{array}{l}6.96 E+03 \\
2.62 E+02 \\
2.60 E+03 \\
6.02 E-07 \\
4.55 E+03\end{array}$ & $\begin{array}{l}9.31 E+03 \\
3.71 E+02 \\
3.72 E+03 \\
1.15 E-01 \\
6.17 E+03\end{array}$ & $\begin{array}{l}1.24 E+04 \\
4.92 E+02 \\
4.8 B E+03 \\
1.38 E+00 \\
8.30 E+03\end{array}$ & $\begin{array}{l}6.39 E+03 \\
8.68 E+03 \\
1.30 E+03 \\
0.0 \\
4.65 E+02\end{array}$ & $\begin{array}{l}1.88 E+04 \\
9.17 E+03 \\
6.17 E+03 \\
1.38 E+00 \\
8.76 E+03\end{array}$ \\
\hline $\begin{array}{l}C 0-56 \\
C 0-57 \\
C 0-58 \\
C 0-584 \\
C 0-60\end{array}$ & $\begin{array}{rr}78.76 & 0 \\
270.99 & 0 \\
70.80 & 0 \\
9.15 & H \\
5.271 & Y\end{array}$ & $\begin{array}{l}2.31 E+04 \\
7.22 E+02 \\
5.91 E+03 \\
9.93 E-02 \\
1.51 E+04\end{array}$ & $\begin{array}{l}1.83 E+04 \\
5.57 E+02 \\
4.65 E+03 \\
2.79 E-03 \\
1.21 E+04\end{array}$ & $\begin{array}{l}2.15 E * 04 \\
7.16 E+02 \\
5.60 E+03 \\
1.34 E-02 \\
1.44 E+04\end{array}$ & $\begin{array}{l}1.48 E * 04 \\
4.03 E+02 \\
3.63 E+03 \\
5.29 E-04 \\
9.63 E+03\end{array}$ & & & $\begin{array}{l}8.93 E+02 \\
1.12 E+01 \\
1.63 E+02 \\
0.0 \\
2.93 E+02\end{array}$ & $\begin{array}{l}2.71 E+04 \\
7.86 E+02 \\
6.99 E+03 \\
2.00 E+00 \\
1.77 E+04\end{array}$ \\
\hline $\begin{array}{l}\mathrm{CO}-60 \mathrm{H} \\
\mathrm{CO}-61 \\
\mathrm{NI}-56 \\
N 1-57 \\
N I-59\end{array}$ & $\begin{array}{r}10.47 \mathrm{M} \\
1.650 \mathrm{H} \\
6.10 \mathrm{O} \\
36.08 \mathrm{H} \\
7.5 \mathrm{H}\end{array}$ & $\begin{array}{l}2.60 E \cdot 01 \\
5.22 E \cdot 02 \\
1.04 E+04 \\
1.17 E+04 \\
1.01 E-01\end{array}$ & $\begin{array}{l}2.04 E \cdot 01 \\
4.08 E \cdot 02 \\
0.14 E+03 \\
9.25 E+03 \\
2.32 E-06\end{array}$ & $\begin{array}{l}2.46 E * 01 \\
5.08 E \cdot 02 \\
9.85 E * 03 \\
1.10 E * 04 \\
6.93 E-03\end{array}$ & $\begin{array}{l}1.51 E \cdot 01 \\
2.70 E \cdot 02 \\
6.38 E * 03 \\
7.51 E * 03 \\
1.01 E-06\end{array}$ & $\begin{array}{l}2.21 E+01 \\
4.41 E+02 \\
0.92 E+03 \\
1.01 E+04 \\
1.93 E-01\end{array}$ & $\begin{array}{l}3.17 E+01 \\
5.84 E+02 \\
1.19 E+04 \\
1.34 E+04 \\
2.32 E+00\end{array}$ & $\begin{array}{l}1.05 E+01 \\
3.28 E+03 \\
8.73 E+00 \\
9.34 E+02 \\
0.0\end{array}$ & $\begin{array}{l}4.22 E+01 \\
3.86 E+03 \\
1.19 E+04 \\
1.43 E+04 \\
2.32 E+00\end{array}$ \\
\hline $\begin{array}{l}N 1-63 \\
N I-65 \\
C U-61 \\
C U-62 \\
C U-64\end{array}$ & $\begin{array}{r}100.1 \mathrm{Y} \\
2.520 \mathrm{H} \\
3.408 \mathrm{H} \\
9.74 \mathrm{H} \\
12.701 \mathrm{H}\end{array}$ & $\begin{array}{l}0.0 \\
3.33 E+03 \\
4.84 E+03 \\
5.90 E+03 \\
1.11 E+03\end{array}$ & $\begin{array}{l}0.0 \\
2.66 E+03 \\
3.69 E+03 \\
4.47 E+03 \\
8.42 E+02\end{array}$ & $\begin{array}{l}3.15 E+03 \\
4.51 E+03 \\
5.47 E+03 \\
1.03 E+03\end{array}$ & $\begin{array}{l}0.0 \\
2.13 E * 03 \\
2.95 E * 03 \\
3.58 E * 03 \\
6.74 E * 02\end{array}$ & $\begin{array}{l}0.0 \\
2.86 E+03 \\
4.15 E+03 \\
5.05 E+03 \\
9.49 E+02\end{array}$ & $\begin{array}{l}3.82 E+03 \\
5.54 E+03 \\
6.75 E+03 \\
1.27 E+03\end{array}$ & $\begin{array}{l}0.0 \\
4.72 E+03 \\
2.23 E+03 \\
1.03 E+04 \\
6.93 E+02\end{array}$ & $\begin{array}{l}0.0 \\
8.54 E+03 \\
1.77 E+03 \\
1.70 E+04 \\
1.96 E+03\end{array}$ \\
\hline $\begin{array}{l}C U-67 \\
2 N-62 \\
2 N-65 \\
2 N-69 \\
2 N-694\end{array}$ & $\begin{array}{r}61.880 \\
9.26 \mathrm{H} \\
244.4 \mathrm{D} \\
55.8 \mathrm{H} \\
13.76 \mathrm{H}\end{array}$ & $\begin{array}{l}0.74 E * 02 \\
2.64 E \bullet 03 \\
3.53 E * 03 \\
3.49 E-02 \\
2.42 E * 03\end{array}$ & $\begin{array}{l}5.17 E+02 \\
2.01 E+03 \\
2.83 E+03 \\
2.63 E-02 \\
1.83 E+03\end{array}$ & $\begin{array}{l}6.57 E+02 \\
2.46 E+03 \\
3.37 E+03 \\
3.24 E-02 \\
2.25 E+03\end{array}$ & $\begin{array}{l}3.8 \theta E+02 \\
1.59 E+03 \\
2.22 E+03 \\
2.11 E-02 \\
1.47 E+03\end{array}$ & & $\begin{array}{l}7.33 E+02 \\
3.04 E+03 \\
4.08 E+03 \\
3.96 E-02 \\
2.75 E+03\end{array}$ & & $\begin{array}{l}1.38 E+03 \\
3.16 E+03 \\
4.08 E+03 \\
2.10 E+03 \\
2.91 E+03\end{array}$ \\
\hline $\begin{array}{l}G A-66 \\
G A-67 \\
G A-68 \\
G A-72 \\
G E-68\end{array}$ & 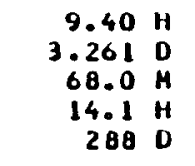 & $\begin{array}{l}1.63 E+04 \\
8.46 E+02 \\
5.58 E+03 \\
1.76 E+04 \\
2.38 E-01\end{array}$ & $\begin{array}{l}1.28 E+04 \\
6.46 E+02 \\
4.23 E+03 \\
1.39 E+04 \\
5.95 E-06\end{array}$ & $\begin{array}{l}1.50 E+04 \\
8.14 E+02 \\
5.17 E+03 \\
1.64 E+04 \\
1.66 E-02\end{array}$ & $\begin{array}{l}1.05 E+04 \\
4.89 E+02 \\
3.39 E+03 \\
1.13 E+04 \\
3.36 E-06\end{array}$ & $\begin{array}{l}1.41 E+04 \\
7.37 E+02 \\
4.77 E+03 \\
1.51 E+04 \\
4.52 E-01\end{array}$ & $\begin{array}{l}1.83 E+04 \\
9.36 E+02 \\
6.30 E+03 \\
2.00 E+04 \\
5.41 E+00\end{array}$ & $\begin{array}{l}7.80 E * 03 \\
1.39 E * 01 \\
5.67 E * 03 \\
3.62 E+03 \\
0.0\end{array}$ & $\begin{array}{l}2.61 E * 04 \\
9.50 E * 02 \\
1.21 E * 04 \\
2.36 E * 04 \\
5.41 E+00\end{array}$ \\
\hline
\end{tabular}


DOSE-PATE FACTCRS HOR BOOY OPGANS AND EFFECTIVE OUSE-PATE FACTOP FOR. IMMERSION IN COMIAMIVATES MIR
IN MREMAYR PER MICROCURIE/ICUBIC MI

\begin{tabular}{|c|c|c|c|c|c|c|c|c|c|c|}
\hline NUCL IDE & HALF-LIFE & DRENALS & BLADOER & BP.AIN & BPEAST & HE AR.T & $\begin{array}{c}\text { SMALL } \\
\text { INTESTINE }\end{array}$ & $\begin{array}{c}\text { UPPER } \\
\text { LARGE } \\
\text { INTESTINE }\end{array}$ & $\begin{array}{c}\text { LOWER } \\
\text { LARGE } \\
\text { INTESTINE }\end{array}$ & Y IDNEYS \\
\hline $\begin{array}{l}G E-71 \\
G E-77 \\
A S-72 \\
A S-73 \\
A S-74\end{array}$ & $\begin{array}{r}11.80 \\
11.30 \mathrm{H} \\
26.0 \mathrm{H} \\
80.300 \\
17.77 \mathrm{D}\end{array}$ & $\begin{array}{l}32 E-02 \\
67 E+03 \\
89 E+03 \\
23 E+01 \\
33 E+03\end{array}$ & $\begin{array}{l}51 E-09 \\
47 E+03 \\
60 E+03 \\
45 E+01 \\
18 E+03\end{array}$ & $\begin{array}{l}66 E-03 \\
95 E+03 \\
50 E+03 \\
25 E+01 \\
55 E+03\end{array}$ & $\begin{array}{l}57 E+00 \\
02 E+03 \\
03 E+03 \\
05 E+01 \\
16 E+03\end{array}$ & $\begin{array}{l}4.34 E-03 \\
4.39 E+03 \\
7.47 E+03 \\
1.10 E+01 \\
3.11 E+03\end{array}$ & $\begin{array}{l}79 E-05 \\
.98 E+03 \\
.76 E+03 \\
.05 E+01 \\
.80 E+03\end{array}$ & $\begin{array}{l}7.83 E-05 \\
4.66 E+03 \\
7.94 E+03 \\
1.25 E+01 \\
3.30 E+03\end{array}$ & $\begin{array}{l}1.37 E-02 \\
4.32 E+03 \\
7.32 E+03 \\
1.10 E+01 \\
3.05 E+03\end{array}$ & $\begin{array}{l}4.21 E-05 \\
4.65 E+03 \\
7.93 E+03 \\
1.62 E+01 \\
3.30 E+03\end{array}$ \\
\hline $\begin{array}{l}A S-76 \\
A S-77 \\
S E-73 \\
S E-75 \\
S E-79\end{array}$ & $\begin{array}{r}26.32 \mathrm{H} \\
38.8 \mathrm{H} \\
7.15 \mathrm{H} \\
119.78 \mathrm{D} \\
6.5 E 4 \mathrm{~V}\end{array}$ & & & & & & & & $\begin{array}{l}1.76 E+03 \\
3.31 E+01 \\
4.20 E+03 \\
1.47 E+03 \\
0.0\end{array}$ & $\begin{array}{l}1.91 E+03 \\
3.55 E+01 \\
4.56 E+03 \\
1.56 E+03 \\
0.0\end{array}$ \\
\hline $\begin{array}{l}8 R-77 \\
8 R-80 \\
8 R-804 \\
8 R-82 \\
B R-83\end{array}$ & $\begin{array}{r}57.04 \mathrm{H} \\
17.4 \mathrm{M} \\
4.42 \mathrm{H} \\
35.30 \mathrm{H} \\
2.39 \mathrm{H}\end{array}$ & & & & $\begin{array}{l}.71 E+03 \\
.07 E+02 \\
.32 E+01 \\
.45 E+04 \\
.05 E+01\end{array}$ & & & & & $\begin{array}{l}1.33 E+03 \\
3.24 E+02 \\
3.02 E+01 \\
1.19 E+04 \\
3.18 E+01\end{array}$ \\
\hline $\begin{array}{l}2-84 \\
2-85 \\
2-79\end{array}$ & 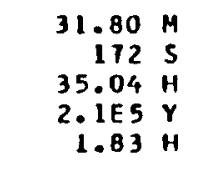 & & & & & & & & & $\begin{array}{l}.26 E+03 \\
.06 E+02 \\
.07 E+03 \\
.05 E+01 \\
.54 E-02\end{array}$ \\
\hline $\begin{array}{l}K R-88 \\
K R-89\end{array}$ & $\begin{array}{r}10.72 \mathrm{Y} \\
4.48 \mathrm{H} \\
76.3 \mathrm{M} \\
2.84 \mathrm{H} \\
3.16 \mathrm{M}\end{array}$ & & & & & & & & & \\
\hline $\begin{array}{l}K R-90 \\
R B-81 \\
R B-82 \\
R B-83 \\
R B-84\end{array}$ & 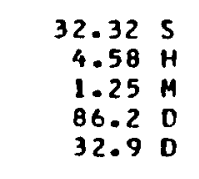 & $\begin{array}{l}5.67 E+03 \\
2.61 E \bullet 03 \\
4.74 E \cdot 03 \\
2.16 E+03 \\
3.99 E \cdot 03\end{array}$ & & & & & & & & \\
\hline $\begin{array}{l}R B-86 \\
R B-87 \\
R B-88 \\
R B-89 \\
R B-90\end{array}$ & 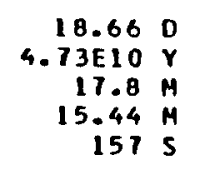 & $\begin{array}{l}4.22 E \cdot 02 \\
0.0 \\
2.98 E+03 \\
9.55 E+03 \\
1.07 E \cdot 04\end{array}$ & $\begin{array}{l}4.14 E \cdot 02 \\
0.0 \\
3.03 E+03 \\
9.58 E+03 \\
1.08 E \div 04\end{array}$ & $\begin{array}{l}3.0 \\
3.39 \mathrm{E} \\
1.07 \mathrm{E} \\
1.19 \mathrm{E}\end{array}$ & $\begin{array}{l}5.17 E+02 \\
0.0 \\
3.78 E+03 \\
1.20 E+04 \\
1.38 E \cdot 04\end{array}$ & $\begin{array}{l}4.10 E+02 \\
0.0 \\
3.02 E \cdot 03 \\
9.52 E \cdot 03 \\
1.00 E+04\end{array}$ & $\begin{array}{l}3.75 E+02 \\
0.0 \\
2.80 E \cdot 03 \\
8.79 E \cdot 03 \\
1.02 E \cdot 04\end{array}$ & $\begin{array}{l}4.37 E+02 \\
0.0 \\
3.21 E+03 \\
1.01 E+04 \\
1.15 E+04\end{array}$ & & $\begin{array}{l}4.35 E+02 \\
0.0 \\
3.23 E * 03 \\
1.02 E * 04 \\
1.19 E * 04\end{array}$ \\
\hline $\begin{array}{l}R B-904 \\
S R-82 \\
S R-85 \\
S R-854 \\
S R-874\end{array}$ & $\begin{array}{r}258 \mathrm{~S} \\
25.0 \mathrm{O} \\
64.84 \mathrm{D} \\
67.66 \mathrm{M} \\
2.805 \mathrm{H}\end{array}$ & $\begin{array}{l}1.55 E \cdot 04 \\
2.19 E-01 \\
2.20 E+03 \\
9.26 E+02 \\
1.37 E+03\end{array}$ & $\begin{array}{l}3.92 E-04 \\
2.09 E+03 \\
8.45 E+02 \\
1.28 E+03\end{array}$ & $\begin{array}{l}2.33 E+03 \\
9.08 E+02 \\
1.41 E+03\end{array}$ & $\begin{array}{l}1.24 E+00 \\
2.78 E+03 \\
1.31 E+03 \\
1.78 E+03\end{array}$ & $\begin{array}{l}1.56 E+04 \\
4.08 E-02 \\
2.04 E+03 \\
8.25 E+02 \\
1.25 E+03\end{array}$ & $\begin{array}{l}4.33 E-03 \\
1.84 E \cdot 03 \\
7.36 E \cdot 02 \\
1.13 E \cdot 03\end{array}$ & $\begin{array}{l}1.66 E+04 \\
8.29 E-04 \\
2.17 E+03 \\
8.73 E+02 \\
1.33 E+03\end{array}$ & $\begin{array}{l}1.54 E+04 \\
1.18 E-01 \\
2.00 E+03 \\
8.20 E+02 \\
1.23 E 003\end{array}$ & $\begin{array}{l}1.69 \mathrm{E}+04 \\
9.39 \mathrm{E}-03 \\
2.17 \mathrm{E}+03 \\
8.69 \mathrm{E}+02 \\
1.33 \mathrm{E}+03\end{array}$ \\
\hline
\end{tabular}




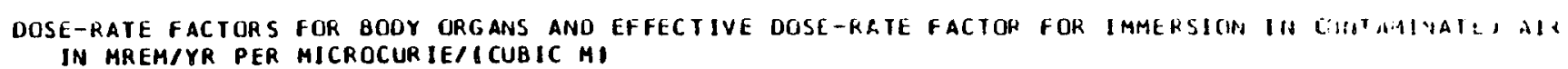

\begin{tabular}{|c|c|c|c|c|c|c|c|c|c|c|}
\hline NUCL IDE & HALF-LIFE & LIVER & LUNGS & MARROH & $\begin{array}{c}\text { REO } \\
\text { MARROW }\end{array}$ & OVARIES & PANCREAS & SKELETON & SPLEEN & STOM \\
\hline $\begin{array}{l}G E-71 \\
G E-77 \\
A S-72 \\
A S-73 \\
A S-74\end{array}$ & 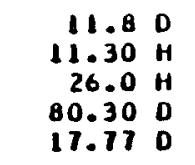 & $\begin{array}{l}3.32 \mathrm{E}-04 \\
4.45 \mathrm{E} \cdot 03 \\
1.56 \mathrm{E} \cdot 03 \\
1.39 \mathrm{E}+01 \\
3.16 \mathrm{E}+03\end{array}$ & $\begin{array}{l}2.63 E-02 \\
4.76 E \cdot 03 \\
8.08 E \cdot 03 \\
1.55 E \cdot 01 \\
3.38 E \cdot 03\end{array}$ & $\begin{array}{l}1.59 E-02 \\
5.53 E \cdot 03 \\
8.99 E \cdot 03 \\
2.80 E+01 \\
3.84 E \cdot 03\end{array}$ & $\begin{array}{l}0.64 E-03 \\
4.85 E \cdot 03 \\
8.24 E \cdot 03 \\
7.76 E * 00 \\
3.46 E * 03\end{array}$ & $\begin{array}{l}1.30 E-02 \\
4.18 E+03 \\
7.26 E+03 \\
1.13 E+01 \\
3.05 E+03\end{array}$ & $\begin{array}{l}1.94 E-02 \\
3.84 E+03 \\
6.52 E+03 \\
8.35 E+00 \\
2.72 E+03\end{array}$ & $\begin{array}{l}4.27 E-02 \\
5.63 E+03 \\
9.14 E+03 \\
2.8 \theta E * 01 \\
3.90 E \cdot 03\end{array}$ & $\begin{array}{l}7.54 E-03 \\
4.35 E+03 \\
7.35 E+03 \\
1.19 E+01 \\
3.08 E+03\end{array}$ & $\begin{array}{l}6.83 E-03 \\
4.38 E+03 \\
7.46 E+03 \\
1.25 E+01 \\
3.12 E+03\end{array}$ \\
\hline $\begin{array}{l}A S-76 \\
A S-77 \\
S E-73 \\
S E-75 \\
S E-19\end{array}$ & $\begin{array}{r}26.32 \mathrm{H} \\
38.8 \mathrm{H} \\
7.15 \mathrm{H} \\
119.78 \mathrm{O} \\
6.5 E 4 \mathrm{Y}\end{array}$ & $\begin{array}{l}1.02 E+03 \\
3.42 E+01 \\
4.38 E+03 \\
1.52 E \cdot 03 \\
0.0\end{array}$ & $\begin{array}{l}1.94 E \cdot 03 \\
3.70 E \bullet 01 \\
4.71 E \bullet 03 \\
1.65 E \bullet 03 \\
0.0\end{array}$ & $\begin{array}{l}2.15 E * 03 \\
4.71 E+01 \\
5.64 E+03 \\
2.24 E * 03 \\
0.0\end{array}$ & $\begin{array}{l}1.98 E+03 \\
3.75 E+01 \\
4.75 E+03 \\
1.63 E \bullet 03 \\
0.0\end{array}$ & $\begin{array}{l}1.75 E+03 \\
3.14 E+01 \\
4.14 E+03 \\
1.36 E+03 \\
0.0\end{array}$ & $\begin{array}{l}1.5 T E+03 \\
2.94 E+01 \\
3.74 E+03 \\
1.29 E+03 \\
0.0\end{array}$ & $\begin{array}{l}2.19 E+03 \\
4.80 E+01 \\
5.73 E+03 \\
2.29 E+03 \\
0.0\end{array}$ & $\begin{array}{l}1.77 E+03 \\
3.37 E+01 \\
4.27 E+03 \\
1.50 E+03 \\
0.0\end{array}$ & $\begin{array}{l}1.79 \mathrm{E} \\
3.38 \mathrm{E} \\
4.31 \mathrm{E} \\
1.50 \mathrm{E} \\
0.0\end{array}$ \\
\hline $\begin{array}{l}B R-77 \\
B R-80 \\
8 R-80 M \\
B R-82 \\
8 R-83\end{array}$ & $\begin{array}{r}57.04 \mathrm{H} \\
17.4 \mathrm{H} \\
4.42 \mathrm{H} \\
35.30 \mathrm{H} \\
2.39 \mathrm{H}\end{array}$ & $\begin{array}{l}1.28 E \cdot 03 \\
3.10 E \bullet 02 \\
1.72 E+01 \\
1.13 E \cdot 04 \\
3.04 E \cdot 01\end{array}$ & $\begin{array}{l}1.30 E \cdot 03 \\
3.32 E \cdot 02 \\
1.97 E \cdot 01 \\
1.21 E \bullet 04 \\
3.2 T E \cdot 01\end{array}$ & $\begin{array}{l}1.64 E+03 \\
3.75 E+02 \\
3.45 E * 01 \\
1.31 E+04 \\
3.74 E * 01\end{array}$ & $\begin{array}{l}1.40 E * 03 \\
3.39 E * 02 \\
7.90 E * 00 \\
1.22 E \bullet 04 \\
3.34 E * 01\end{array}$ & $\begin{array}{l}1.21 E+03 \\
2.99 E+02 \\
1.43 E+01 \\
1.09 E+04 \\
2.93 E+01\end{array}$ & $\begin{array}{l}1.10 E \cdot 03 \\
2.67 E+02 \\
9.48 E \cdot 00 \\
9.77 E+03 \\
2.62 E+01\end{array}$ & $\begin{array}{l}1.67 E+03 \\
3.81 E+02 \\
3.53 E+01 \\
1.34 E+04 \\
3.80 E+01\end{array}$ & $\begin{array}{l}1.25 E+03 \\
3.02 E+02 \\
1.35 E+01 \\
1.10 E+04 \\
2.97 E+01\end{array}$ & $\begin{array}{l}1.26 \\
3.06 \\
1.50 \\
1.11 \\
3.00\end{array}$ \\
\hline $\begin{array}{l}8 R-84 \\
8 R-85 \\
K R-79 \\
K R-81 \\
K R-834\end{array}$ & $\begin{array}{r}31.80 \\
172 \\
35 \\
35.04 \\
2.165 \\
1.83 \\
1.83\end{array}$ & $\begin{array}{l}8.64 E \bullet 03 \\
2.90 E+02 \\
1.03 E \bullet 03 \\
3.91 E \bullet 01 \\
1.37 E-02\end{array}$ & $\begin{array}{l}9.07 E \bullet 03 \\
3.09 E \bullet 02 \\
1.10 E \bullet 03 \\
4.24 E \bullet 01 \\
5.88 E-02\end{array}$ & $\begin{array}{l}9.44 E+03 \\
3.32 E+02 \\
1.30 E+03 \\
5.48 E+01 \\
6.44 E-02\end{array}$ & $\begin{array}{l}9.29 E+03 \\
3.13 E+02 \\
1.13 E+03 \\
4.36 E+01 \\
2.03 E-02\end{array}$ & & $\begin{array}{l}7.57 E+03 \\
2.50 E+02 \\
8.83 E+02 \\
3.38 E+01 \\
3.62 E-02\end{array}$ & & $\begin{array}{l}8.32 E+03 \\
2.81 E+02 \\
1.00 E+03 \\
3.88 E+01 \\
2.52 E-02\end{array}$ & $\begin{array}{l}8.55 \mathrm{E} \\
2.86 \mathrm{E} \\
1.01 \mathrm{E} \\
3.87 \mathrm{E} \\
2.63 \mathrm{E}\end{array}$ \\
\hline $\begin{array}{l}K R-85 \\
K R-85 M \\
K R-87 \\
K R-88 \\
K R-89\end{array}$ & $\begin{array}{r}10.72 \mathrm{Y} \\
4.48 \mathrm{H} \\
76.3 \mathrm{M} \\
2.84 \mathrm{H} \\
3.16 \mathrm{M}\end{array}$ & $\begin{array}{l}9.08 E \cdot 00 \\
6.16 E \cdot 02 \\
3.75 E+03 \\
9.61 E+03 \\
8.55 E+03\end{array}$ & $\begin{array}{l}9.75 E \cdot 00 \\
6.73 E \bullet 02 \\
3.95 E \bullet 03 \\
1.01 E \bullet 04 \\
9.01 E \bullet 03\end{array}$ & $\begin{array}{l}1.12 E+01 \\
9.66 E+02 \\
4.22 E+03 \\
1.05 E+04 \\
9.54 E+03\end{array}$ & $\begin{array}{l}9.97 E+00 \\
6.46 E+02 \\
4.04 E+03 \\
1.03 E+04 \\
9.19 E+03\end{array}$ & $\begin{array}{l}0.73 E+00 \\
5.42 E+02 \\
3.42 E+03 \\
8.72 E+03 \\
7.87 E \cdot 03\end{array}$ & $\begin{array}{l}7.81 E+00 \\
5.19 E+02 \\
3.27 E+03 \\
8.38 E+03 \\
7.46 E+03\end{array}$ & & $\begin{array}{l}8.87 E+00 \\
6.14 E+02 \\
3.61 E+03 \\
9.22 E+03 \\
8.25 E+03\end{array}$ & $\begin{array}{l}9.43 \mathrm{E} \\
8.42 \mathrm{E}\end{array}$ \\
\hline $\begin{array}{l}K R-90 \\
R B-81 \\
R B-82 \\
R B-83 \\
R B-84\end{array}$ & 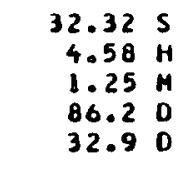 & $\begin{array}{l}5.60 E+03 \\
2.44 E+03 \\
4.48 E+03 \\
2.04 E+03 \\
3.02 E+03\end{array}$ & $\begin{array}{l}5.94 E+03 \\
2.63 E+03 \\
4.81 E+03 \\
2.19 E+03 \\
4.09 E+03\end{array}$ & $\begin{array}{l}6.45 E+03 \\
3.17 E+03 \\
5.50 E+03 \\
2.50 E+03 \\
4.49 E+03\end{array}$ & $\begin{array}{l}5.99 E+03 \\
2.67 E+03 \\
4.91 E+03 \\
2.24 E+03 \\
4.15 E+03\end{array}$ & $\begin{array}{l}5.26 E+03 \\
2.30 E+03 \\
4.31 E+03 \\
1.96 E+03 \\
3.70 E \cdot 03\end{array}$ & $\begin{array}{l}4.84 E+03 \\
2.10 E+03 \\
3.86 E+03 \\
1.75 E+03 \\
3.30 E+03\end{array}$ & $\begin{array}{l}6.56 E+03 \\
3.22 E+03 \\
5.59 E+03 \\
2.54 E+03 \\
4.5 I E+03\end{array}$ & $\begin{array}{l}5.42 E \cdot 03 \\
2.40 E+03 \\
4.37 E+03 \\
1.99 E * 03 \\
3.71 E * 03\end{array}$ & $\begin{array}{l}2.01 E 0 \\
3.78 E\end{array}$ \\
\hline $\begin{array}{l}R B-86 \\
R B-87 \\
R B-8 B \\
R B-89 \\
R B-90\end{array}$ & $\begin{array}{r}18.66 \mathrm{D} \\
4.73 E 10 \mathrm{~V} \\
17.8 \mathrm{M} \\
15.44 \mathrm{M} \\
157 \mathrm{~S}\end{array}$ & $\begin{array}{l}4.12 E+02 \\
0.0 \\
3.04 E \cdot 03 \\
9.58 E \cdot 03 \\
1.09 E \cdot 04\end{array}$ & $\begin{array}{l}4.38 E \cdot 02 \\
0.0 \\
3.20 E \cdot 03 \\
1.01 E \cdot 04 \\
1.13 E \cdot 04\end{array}$ & $\begin{array}{l}4.66 E+02 \\
0.0 \\
3.32 E+03 \\
1.06 E+04 \\
1.17 E+04\end{array}$ & $\begin{array}{l}4.43 E+02 \\
0.0 \\
3.24 E+03 \\
1.03 E+04 \\
1.18 E+04\end{array}$ & $\begin{array}{l}3.98 E+02 \\
0.0 \\
2.80 E+03 \\
8.95 E+03 \\
9.71 E+03\end{array}$ & $\begin{array}{l}3.57 E \cdot 02 \\
0.0 \\
2.64 E+03 \\
8.33 E+03 \\
9.69 E+03\end{array}$ & $\begin{array}{l}4.74 E * 02 \\
0.0 \\
3.37 E+03 \\
1.08 E * 04 \\
1.19 E * 04\end{array}$ & $\begin{array}{l}3.99 E+02 \\
0.0 \\
2.92 E+03 \\
9.23 E+03 \\
1.05 E+04\end{array}$ & $\begin{array}{l}2.97 \mathrm{E} \\
9.42 \mathrm{E} \\
1.09 \mathrm{E}\end{array}$ \\
\hline $\begin{array}{l}R B-904 \\
S R-82 \\
S R-85 \\
S R-85 M \\
S R-874\end{array}$ & $\begin{array}{rr}250 & 5 \\
25.0 & 0 \\
64.84 & 0 \\
67.66 & M \\
2.805 & H\end{array}$ & $\begin{array}{l}1.57 E+04 \\
1.38 E-02 \\
2.08 E+03 \\
8.42 E+02 \\
1.28 E+03\end{array}$ & $\begin{array}{l}1.65 E \cdot 04 \\
2.09 E-01 \\
2.23 E \cdot 03 \\
9.15 E \cdot 02 \\
1.37 E \cdot 03\end{array}$ & $\begin{array}{l}1.72 E+04 \\
2.00 E-01 \\
2.57 E \cdot 03 \\
1.24 E \cdot 03 \\
1.66 E \cdot 03\end{array}$ & $\begin{array}{l}1.69 E+04 \\
7.04 E-02 \\
2.28 E+03 \\
9.23 E+02 \\
1.42 E+03\end{array}$ & $\begin{array}{l}1.44 E+04 \\
1.12 E-01 \\
2.00 E+03 \\
1.47 E+02 \\
1.20 E+03\end{array}$ & $\begin{array}{l}1.38 E+04 \\
1.33 E-01 \\
1.79 E \cdot 03 \\
1.22 E+02 \\
1.10 E+03\end{array}$ & $\begin{array}{l}1.75 E+04 \\
3.42 E-01 \\
2.61 E+03 \\
1.27 E+03 \\
1.69 E+03\end{array}$ & $\begin{array}{l}1.51 E+04 \\
7.98 E-02 \\
2.03 E+03 \\
8.38 E+02 \\
1.26 E+03\end{array}$ & $\begin{array}{l}1.30 E 0 \\
8.16 \mathrm{E} \\
2.05 \mathrm{E} \\
8.32 \mathrm{E} \\
1.26 \mathrm{E}\end{array}$ \\
\hline
\end{tabular}


DOSE-P.ATE FACTORS FOR BODY OPGANS AND EFFECTIVE DUSE-RATE FACTUR FOR. IMMERSION IN CONTAMINATEI) AIR

\begin{tabular}{|c|c|c|c|c|c|c|c|c|c|}
\hline IUCLIDE & HALF-LIFE & TESTES & THYMUS & THYROID & UTERUS & FECTIVE & $\begin{array}{c}\text { SKIN } \\
\text { I PHOTONI }\end{array}$ & $\begin{array}{l}\text { SKIN } \\
\text { IELECTRONI }\end{array}$ & $\begin{array}{l}\text { SKIN } \\
\text { ITOTALI }\end{array}$ \\
\hline $\begin{array}{l}\text { GE-71 } \\
G E-77 \\
A S-72 \\
A S-73 \\
A S-74\end{array}$ & 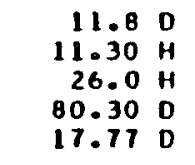 & $\begin{array}{l}2.40 E-01 \\
6.36 E+03 \\
1.08 E+04 \\
2.75 E+01 \\
4.53 E+03\end{array}$ & $\begin{array}{l}02 E-06 \\
94 E+03 \\
33 E \cdot 03 \\
94 E+01 \\
47 E+03\end{array}$ & $\begin{array}{l}68 E-02 \\
01 E+03 \\
01 E+04 \\
53 E+01 \\
22 E+03\end{array}$ & $\begin{array}{l}39 E-06 \\
92 E+03 \\
61 E+03 \\
07 E+00 \\
75 E+03\end{array}$ & $\begin{array}{l}4.57 E-01 \\
5.48 E+03 \\
9.20 E+03 \\
2.29 E+01 \\
3.87 E+03\end{array}$ & $\begin{array}{l}5.47 E+00 \\
1.23 E+03 \\
1.23 E+04 \\
4.5 B E+01 \\
5.20 E+03\end{array}$ & $\begin{array}{l}0.0 \\
4.87 E+03 \\
8.19 E+03 \\
0.0 \\
1.88 E+03\end{array}$ & $\begin{array}{l}5.47 E * 00 \\
1.21 E+04 \\
2.05 E+04 \\
4.5 B E+01 \\
7.08 E * 03\end{array}$ \\
\hline $\begin{array}{l}A S-76 \\
A S-77 \\
S E-73 \\
S E-75 \\
S E-79\end{array}$ & $\begin{array}{r}26.32 \mathrm{H} \\
38.8 \mathrm{H} \\
7.15 \mathrm{H} \\
119.78 \mathrm{O} \\
6.5 \mathrm{~F}\end{array}$ & $\begin{array}{l}2.58 E+03 \\
5.02 E+01 \\
6.37 E+03 \\
2.26 E+03 \\
0.0\end{array}$ & $\begin{array}{l}2.00 E+03 \\
3.81 E+01 \\
4.82 E+03 \\
.12 E+03 \\
0.0\end{array}$ & $\begin{array}{l}2.42 \mathrm{E} * 03 \\
4.75 \mathrm{E} \cdot 01 \\
5.93 \mathrm{E} \cdot 03 \\
2.16 \mathrm{E} \cdot 03 \\
0.0\end{array}$ & & & $\begin{array}{l}2.96 E+03 \\
5.62 E+01 \\
7.24 E+03 \\
2.50 E+03 \\
0.0\end{array}$ & $\begin{array}{l}8.43 E \bullet 03 \\
1.34 E * 03 \\
2.76 E+03 \\
1.70 E * 01 \\
3.93 E \bullet 01\end{array}$ & $\begin{array}{l}1.14 E * 04 \\
1.40 E * 03 \\
1.00 E+04 \\
2.52 E+03 \\
3.93 E * 01\end{array}$ \\
\hline $\begin{array}{l}B R-17 \\
B R-80 \\
B R-80 M \\
8 R-82 \\
B R-83\end{array}$ & $\begin{array}{r}57.04 \mathrm{H} \\
17.4 \mathrm{M} \\
4.42 \mathrm{H} \\
35.30 \mathrm{H} \\
2.39 \mathrm{H}\end{array}$ & $\begin{array}{l}1.85 E+03 \\
4.44 E+02 \\
4.82 E+01 \\
1.60 E * 04 \\
4.38 E \cdot 01\end{array}$ & $\begin{array}{l}1.41 E+03 \\
3.41 E+02 \\
2.77 E+01 \\
1.26 E \bullet 04 \\
3.33 E+01\end{array}$ & $\begin{array}{l}1.74 E+03 \\
4.15 E+02 \\
3.79 E+01 \\
1.51 E \cdot 04 \\
4.06 E \cdot 01\end{array}$ & & & & $\begin{array}{l}1.30 E+01 \\
5.54 E+03 \\
0.0 \\
5.97 E+02 \\
2.10 E+03\end{array}$ & $\begin{array}{l}2.13 E+03 \\
6.05 E+03 \\
8.29 E+01 \\
1.90 E+04 \\
2.15 E+03\end{array}$ \\
\hline $\begin{array}{l}8 R-84 \\
8 R-85 \\
K R-79 \\
K R-81 \\
K R-83 M\end{array}$ & 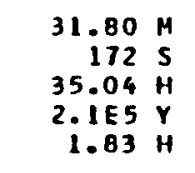 & $\begin{array}{l}1.19 E \cdot 04 \\
4.07 E+02 \\
1.4 B E+03 \\
5.82 E+01 \\
3.78 E-01\end{array}$ & & & & & & $\begin{array}{l}1.00 E+04 \\
7.87 E+03 \\
1.16 E+02 \\
0.0 \\
0.0\end{array}$ & $\begin{array}{l}2.34 E+04 \\
0.34 E+03 \\
1.81 E+03 \\
1.43 E+01 \\
4.28 E * 00\end{array}$ \\
\hline $\begin{array}{l}R-85 \\
R-85 M \\
R-87 \\
R-88 \\
R-89\end{array}$ & $\begin{array}{r}10.72 \mathrm{Y} \\
4.48 \mathrm{H} \\
76.3 \mathrm{M} \\
2.84 \mathrm{H} \\
3.16 \mathrm{M}\end{array}$ & $\begin{array}{l}1.31 E+01 \\
9.28 E+02 \\
5.18 E+03 \\
1.31 E+04 \\
1.18 E+04\end{array}$ & & & & & & & $\begin{array}{l}1.58 E * 03 \\
2.62 E * 03 \\
1.65 E * 04 \\
1.73 E * 04 \\
2.43 E * 04\end{array}$ \\
\hline $\begin{array}{l}K R-90 \\
R B-81 \\
R B-82 \\
R B-83 \\
R B-84\end{array}$ & 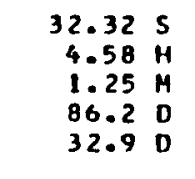 & $\begin{array}{l}7.83 E+03 \\
3.55 E+03 \\
6.44 E+03 \\
2.93 E+03 \\
5.43 E+03\end{array}$ & $\begin{array}{l}6.19 E+03 \\
2.69 E+03 \\
4.90 E+03 \\
2.23 E+03 \\
4.26 E+03\end{array}$ & $\begin{array}{l}7.37 E+03 \\
3.32 E \bullet 03 \\
5.98 E+03 \\
2.72 E+03 \\
5.13 E+03\end{array}$ & $\begin{array}{l}4.96 E \bullet 03 \\
2.14 E \bullet 03 \\
3.91 E \bullet 03 \\
1.78 E \bullet 03 \\
3.34 E \bullet 03\end{array}$ & $\begin{array}{l}6.73 E * 03 \\
3.05 E+03 \\
5.51 E+03 \\
2.51 E+03 \\
4.64 E+03\end{array}$ & $\begin{array}{l}8.94 E+03 \\
4.03 E \cdot 03 \\
7.37 E \cdot 03 \\
3.37 E \cdot 03 \\
6.26 E .03\end{array}$ & $\begin{array}{l}1.05 E * 04 \\
1.32 E * 03 \\
1.13 E * 04 \\
0.0 \\
1.19 E * 03\end{array}$ & $\begin{array}{l}1.94 E \cdot 04 \\
5.35 E \bullet 03 \\
1.87 E+04 \\
3.37 E \cdot 03 \\
T .45 E \cdot 03\end{array}$ \\
\hline $\begin{array}{l}R B-86 \\
R B-87 \\
R B-88 \\
R B-89 \\
R B-90\end{array}$ & 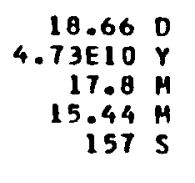 & $\begin{array}{l}5.78 E+02 \\
0.0 \\
4.16 E+03 \\
1.32 E+04 \\
1.49 E+04\end{array}$ & $\begin{array}{l}4.64 E+02 \\
0.0 \\
3.30 E+03 \\
1.05 E \cdot 04 \\
1.18 E+04\end{array}$ & $\begin{array}{l}5.52 E+02 \\
0.0 \\
3.87 E+03 \\
1.24 E+04 \\
1.35 E+04\end{array}$ & $\begin{array}{l}3.62 E+02 \\
0.0 \\
2.12 E * 03 \\
0.52 E+03 \\
9.63 E+03\end{array}$ & $\begin{array}{l}4.95 E+02 \\
0.0 \\
3.58 E+03 \\
1.14 E+04 \\
1.29 E+04\end{array}$ & $\begin{array}{l}6.67 E+02 \\
0.0 \\
4.72 E+03 \\
1.51 E+04 \\
1.63 E+04\end{array}$ & $\begin{array}{l}5.09 E+03 \\
1.06 E+02 \\
1.72 E+04 \\
8.03 E+03 \\
1.62 E+04\end{array}$ & $\begin{array}{l}5.16 E+03 \\
1.86 E+02 \\
2.19 E+04 \\
2.31 E+04 \\
3.25 E+04\end{array}$ \\
\hline $\begin{array}{l}R B-904 \\
S R-82 \\
S R-85 \\
S R-85 M \\
S R-874\end{array}$ & $\begin{array}{rr}258 & 5 \\
25.0 & 0 \\
64.84 & 0 \\
67.66 & M \\
2.805 & H\end{array}$ & $\begin{array}{l}2.16 E+04 \\
1.49 E+00 \\
2.99 E+03 \\
1.25 E+03 \\
1.85 E+03\end{array}$ & $\begin{array}{l}1.71 E+04 \\
2.76 E-03 \\
2.27 E+03 \\
9.52 E+02 \\
1.40 E \bullet 03\end{array}$ & $\begin{array}{l}1.99 E+04 \\
2.45 E-01 \\
2.77 E+03 \\
1.20 E+03 \\
1.12 E+03\end{array}$ & $\begin{array}{l}1.40 E+04 \\
7.76 E-05 \\
1.81 E \cdot 03 \\
7.45 E+02 \\
1.12 E \bullet 03\end{array}$ & $\begin{array}{l}1.86 E+04 \\
1.70 E+00 \\
2.56 E+03 \\
1.10 E+03 \\
1.60 E+03\end{array}$ & $\begin{array}{l}2.42 E+04 \\
1.46 E+01 \\
3.44 E+03 \\
1.38 E+03 \\
2.10 E+03\end{array}$ & $\begin{array}{l}1.12 E+04 \\
0.9 \\
2.25 E+01 \\
3.08 E+01 \\
4.44 E+02\end{array}$ & $\begin{array}{l}3.55 E+04 \\
1.46 E+01 \\
3.46 E+03 \\
1.41 E+03 \\
2.54 E+03\end{array}$ \\
\hline
\end{tabular}




\begin{tabular}{|c|c|c|c|c|c|c|c|c|c|c|}
\hline ICLIOE & HALF-LIFE & AORENALS & BLADOER & QRAIN & BREAST & HE AR T & $\begin{array}{l}\text { SMALL } \\
\text { INTESTINE }\end{array}$ & $\begin{array}{l}\text { UPPER } \\
\text { LARGE } \\
\text { INTESTINE }\end{array}$ & $\begin{array}{l}\text { LOWER } \\
\text { LARGE } \\
\text { INIESTINE }\end{array}$ & CIONEYS \\
\hline $\begin{array}{l}5 R-89 \\
S R-90 \\
S R-91 \\
S R-92 \\
S R-93\end{array}$ & $\begin{array}{rr}50.55 & 0 \\
28.6 & Y \\
9.5 & H \\
2.71 & H \\
7.3 & M\end{array}$ & $\begin{array}{l}0.17 E-01 \\
0.0 \\
3.08 E+03 \\
5.84 E+03 \\
1.01 E+04\end{array}$ & $\begin{array}{l}5.98 E-01 \\
0.0 \\
2.99 E \cdot 03 \\
5.83 E \cdot 03 \\
9.93 E+03\end{array}$ & $\begin{array}{l}6.73 E-01 \\
0.0 \\
3.36 E+03 \\
6.57 E+03 \\
1.11 E+04\end{array}$ & $\begin{array}{l}7.52 E-01 \\
0.0 \\
3.11 E+03 \\
7.28 E+03 \\
1.26 E+04\end{array}$ & $\begin{array}{l}5.91 E-01 \\
0.0 \\
2.95 E+03 \\
5.77 E+03 \\
9.82 E+03\end{array}$ & $\begin{array}{l}5.36 E-01 \\
0.0 \\
2.68 E+03 \\
5.34 E+03 \\
8.99 E+03\end{array}$ & $\begin{array}{l}0.29 E-01 \\
0.0 \\
3.14 E+03 \\
6.15 E+03 \\
1.04 E+04\end{array}$ & $\begin{array}{l}5.78 E-01 \\
0.0 \\
2.89 E+03 \\
5.67 E+03 \\
9.63 E+03\end{array}$ & $\begin{array}{l}6.21 E-01 \\
0.0 \\
3.13 E+05 \\
6.14 E+03 \\
1.05 E+04\end{array}$ \\
\hline $\begin{array}{l}y-86 \\
y-87 \\
y-88 \\
y-90 \\
y-90 H\end{array}$ & $\begin{array}{r}14.74 \mathrm{H} \\
80.3 \mathrm{H} \\
106.60 \mathrm{D} \\
64.1 \mathrm{H} \\
3.19 \mathrm{H}\end{array}$ & $\begin{array}{l}1.62 E+04 \\
1.96 E+03 \\
1.24 E+04 \\
0.0 \\
2.72 E+03\end{array}$ & $\begin{array}{l}1.59 E+04 \\
1.86 E+03 \\
1.25 E+04 \\
0.0 \\
2.55 E \cdot 03\end{array}$ & $\begin{array}{l}1.79 E+04 \\
2.07 \mathrm{E}+03 \\
1.41 \mathrm{E}+04 \\
0.0 \\
2.00 \mathrm{E}+03\end{array}$ & $\begin{array}{l}2.02 E+04 \\
2.50 E+03 \\
1.56 E+04 \\
0.0 \\
3.00 E+03\end{array}$ & $\begin{array}{l}1.58 E+04 \\
1.82 E+03 \\
1.25 E+04 \\
0.0 \\
2.49 E+03\end{array}$ & $\begin{array}{l}1.44 E \cdot 04 \\
1.63 E+03 \\
1.15 E \cdot 04 \\
0.0 \\
2.23 E+03\end{array}$ & $\begin{array}{l}1.68 E+04 \\
1.93 E+03 \\
1.33 E+04 \\
0.0 \\
2.04 E+03\end{array}$ & $\begin{array}{l}1.55 E+04 \\
1.18 E+03 \\
1.22 E+04 \\
0.0 \\
2.45 E+03\end{array}$ & $\begin{array}{l}1.68 E+04 \\
1.93 E \cdot 03 \\
1.33 E+04 \\
0.0 \\
2.63 E+03\end{array}$ \\
\hline $\begin{array}{l}Y-91 \\
Y-91 M \\
Y-92 \\
Y-93 \\
Z R-86\end{array}$ & $\begin{array}{r}58.51 \\
49.71 \\
3.54 \\
10.11 \mathrm{H} \\
16.5 \mathrm{H}\end{array}$ & $\begin{array}{l}1.59 E+01 \\
2.31 E+03 \\
1.12 E+03 \\
4.05 E+02 \\
1.17 E+03\end{array}$ & $\begin{array}{l}1.57 E+01 \\
2.20 E+03 \\
1.10 E+03 \\
4.01 E+02 \\
1.08 E+03\end{array}$ & $\begin{array}{l}1.77 E+01 \\
2.45 E+03 \\
1.24 E+03 \\
4.47 E+02 \\
1.16 E+03\end{array}$ & $\begin{array}{l}1.96 E+01 \\
2.88 E+03 \\
1.38 E+03 \\
5.18 E+02 \\
1.64 E+03\end{array}$ & $\begin{array}{l}1.56 E+01 \\
2.15 E+03 \\
1.09 E+03 \\
3.97 E+02 \\
1.05 E+03\end{array}$ & $\begin{array}{l}1.43 E+01 \\
1.93 E+03 \\
9.92 E \bullet 02 \\
3.65 E+02 \\
9.40 E+02\end{array}$ & $\begin{array}{l}1.66 \mathrm{E}+01 \\
2.28 \mathrm{E} * 03 \\
1.16 \mathrm{E} * 03 \\
4.22 \mathrm{E} \cdot 02 \\
1.11 \mathrm{E}+03\end{array}$ & $\begin{array}{l}1.53 E * 01 \\
2.10 E * 03 \\
1.06 E \cdot 03 \\
3.90 E \cdot 02 \\
1.04 E+03\end{array}$ & $\begin{array}{l}1.65 E+01 \\
2.28 E+03 \\
1.15 E+03 \\
4.23 E+02 \\
1.11 E+03\end{array}$ \\
\hline $\begin{array}{l}2 R-88 \\
2 R-89 \\
2 R-93 \\
2 R-95 \\
2 R-97\end{array}$ & $\begin{array}{r}83.4 \mathrm{D} \\
78.43 \mathrm{H} \\
1.53 E 6 \mathrm{~V} \\
64.02 \mathrm{D} \\
16.90 \mathrm{H}\end{array}$ & $\begin{array}{l}1.65 E+03 \\
5.16 E+03 \\
0.0 \\
3.29 E+03 \\
7.99 E+02\end{array}$ & $\begin{array}{l}1.54 E \cdot 03 \\
4.99 E \cdot 03 \\
0.0 \\
3.16 E+03 \\
7.82 E * 02\end{array}$ & $\begin{array}{l}1.69 E+03 \\
5.61 E+03 \\
0.0 \\
3.55 E+03 \\
0.77 E+02\end{array}$ & $\begin{array}{l}2.14 E+03 \\
6.35 E+03 \\
0.0 \\
4.03 E+03 \\
1.00 E+03\end{array}$ & $\begin{array}{l}1.50 E+03 \\
4.92 E+03 \\
0.0 \\
3.11 E+03 \\
7.72 E+02\end{array}$ & $\begin{array}{l}1.35 E+03 \\
4.46 E+03 \\
0.0 \\
2.01 E \cdot 03 \\
7.05 E+02\end{array}$ & $\begin{array}{l}1.59 E+03 \\
5.24 E+03 \\
0.0 \\
3.31 E+03 \\
8.21 E+02\end{array}$ & $\begin{array}{l}1.48 E+03 \\
4.82 E+03 \\
0.0 \\
3.04 E+03 \\
7.57 E+02\end{array}$ & $\begin{array}{l}1.59 E+03 \\
5.22 E+03 \\
0.0 \\
3.30 E \cdot 03 \\
8.20 E+02\end{array}$ \\
\hline $\begin{array}{l}N B-90 \\
N B-91 \\
N B-91 M \\
N B-92 \\
N B-924\end{array}$ & 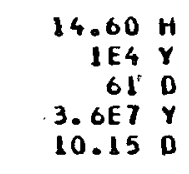 & $\begin{array}{l}1.95 E+04 \\
7.17 E+00 \\
1.88 E+02 \\
6.66 E+03 \\
4.34 E+03\end{array}$ & $\begin{array}{l}1.97 E * 04 \\
6.87 E * 00 \\
1.86 E+02 \\
6.42 E+03 \\
4.22 E+03\end{array}$ & $\begin{array}{l}2.20 E * 04 \\
7.74 E \bullet 00 \\
2.09 E * 02 \\
7.21 E \bullet 03 \\
4.75 E \bullet 03\end{array}$ & & & & $\begin{array}{l}2.09 E+04 \\
7.13 E+00 \\
1.90 E+02 \\
6.73 E+03 \\
4.44 E+03\end{array}$ & & $\begin{array}{l}2.10 E \cdot 04 \\
7.23 E+00 \\
1.95 E+02 \\
6.71 E+03 \\
4.42 E \cdot 03\end{array}$ \\
\hline $\begin{array}{l}N B-934 \\
N B-94 \\
N B-94 M \\
N B-95 \\
N B-954\end{array}$ & $\begin{array}{r}14.6 \mathrm{Y} \\
2.03 E 4 \mathrm{Y} \\
6.26 \mathrm{M} \\
35.06 \mathrm{D} \\
80.6 \mathrm{H}\end{array}$ & $\begin{array}{l}1.24 E-01 \\
7.06 E+03 \\
1.91 E+01 \\
3.43 E+03 \\
2.56 E+02\end{array}$ & $\begin{array}{l}7.54 E-03 \\
6.81 E+03 \\
1.81 E+01 \\
3.30 E+03 \\
2.33 E+02\end{array}$ & $\begin{array}{l}2.40 E-02 \\
7.65 E * 03 \\
2.04 E \bullet 01 \\
3.70 E * 03 \\
2.50 E * 02\end{array}$ & & & & & & $\begin{array}{l}4.07 \mathrm{E}-02 \\
3.12 \mathrm{E}+03 \\
1.91 \mathrm{E}+01 \\
3.45 \mathrm{E}+03 \\
2.40 \mathrm{E}+02\end{array}$ \\
\hline $\begin{array}{l}N B-96 \\
N B-97 \\
N B-97 M \\
M O-91 \\
M O-93\end{array}$ & $\begin{array}{r}23.35 \mathrm{H} \\
72.1 \mathrm{H} \\
60 \mathrm{~S} \\
15.49 \mathrm{M} \\
3.5 E 3 \mathrm{Y}\end{array}$ & $\begin{array}{l}1.10 E+04 \\
2.94 E+03 \\
3.26 E+03 \\
4.22 E+03 \\
6.92 E-01\end{array}$ & $\begin{array}{l}1.06 E * 04 \\
2.82 E * 03 \\
3.13 E \bullet 03 \\
4.01 E \bullet 03 \\
4.22 E-02\end{array}$ & $\begin{array}{l}1.19 E+04 \\
3.16 E+03 \\
3.52 E+03 \\
4.47 E+03 \\
1.34 E-01\end{array}$ & $\begin{array}{l}1.35 E+04 \\
3.63 E+03 \\
3.99 E+03 \\
5.32 E+03 \\
1.55 E+01\end{array}$ & $\begin{array}{l}1.05 E+04 \\
2.77 E+03 \\
3.08 E+03 \\
3.92 E+03 \\
1.77 E-01\end{array}$ & $\begin{array}{l}9.50 E+03 \\
2.50 E \bullet 03 \\
2.78 E+03 \\
3.53 E+03 \\
5.95 E-02\end{array}$ & $\begin{array}{l}1.11 E+04 \\
2.94 E+03 \\
3.2 \forall E+03 \\
4.16 E+03 \\
5.60 E-02\end{array}$ & $\begin{array}{l}1.32 E+04 \\
2.71 E+03 \\
3.01 E+03 \\
3.85 E+03 \\
4.36 E-01\end{array}$ & $\begin{array}{l}1.11 E+04 \\
2.94 E+03 \\
3.21 E+03 \\
4.16 E+03 \\
2.2 B E-01\end{array}$ \\
\hline $\begin{array}{l}M 0-99 \\
M O-101 \\
\text { IC-95 } \\
\text { IC-954 } \\
\text { IC-96 }\end{array}$ & $\begin{array}{r}66.02 \mathrm{H} \\
14.61 \mathrm{M} \\
20.0 \mathrm{H} \\
61 \\
4.28\end{array}$ & $\begin{array}{l}0.85 E+02 \\
6.71 E * 03 \\
3.52 E+03 \\
2.93 E+03 \\
1.12 E \cdot 04\end{array}$ & $\begin{array}{l}6.55 E+02 \\
6.63 E+03 \\
3.39 E+03 \\
2.79 E+03 \\
1.08 E+04\end{array}$ & $\begin{array}{l}7.32 E+02 \\
7.43 E+03 \\
3.01 E+03 \\
3.11 E+03 \\
1.22 E \bullet 04\end{array}$ & $\begin{array}{l}8.64 E+02 \\
8.44 E+03 \\
4.32 E+03 \\
3.74 E+03 \\
1.37 E+04\end{array}$ & $\begin{array}{l}6.45 E+02 \\
6.56 E+03 \\
3.34 E+03 \\
2.75 E+03 \\
1.07 E+04\end{array}$ & $\begin{array}{l}5.81 E+02 \\
6.01 E+03 \\
3.02 E+03 \\
2.48 E * 03 \\
9.67 E+03\end{array}$ & $\begin{array}{l}6.86 E+02 \\
6.98 E+03 \\
3.55 E+03 \\
2.92 E+03 \\
1.14 E+04\end{array}$ & $\begin{array}{l}0.31 E+02 \\
6.43 E+03 \\
3.27 E+03 \\
2.70 E+03 \\
1.04 E+04\end{array}$ & $\begin{array}{l}6.83 E+02 \\
6.98 E+03 \\
3.54 E+03 \\
2.91 E+03 \\
1.13 E+04\end{array}$ \\
\hline
\end{tabular}




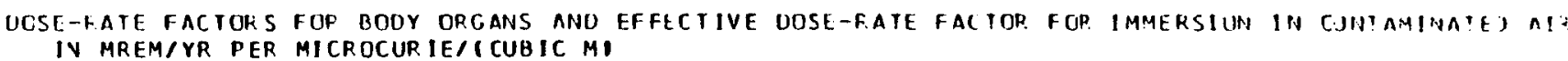

\begin{tabular}{|c|c|c|c|c|c|c|c|c|c|c|}
\hline NUCL IOE & HALF-LIFE & LIVER & LUNGS & MARROH & $\begin{array}{c}\text { PEED } \\
\text { MARROW }\end{array}$ & OVARIES & ANCPEAS & SKELETON & SPLEEN & STOMACH \\
\hline $\begin{array}{l}5 R-89 \\
S R-90 \\
5 R-91 \\
S R-92 \\
S R-93\end{array}$ & 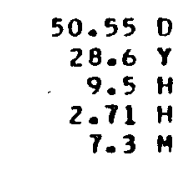 & $\begin{array}{l}.95 E-01 \\
.0 \\
.97 E+03 \\
082 E 03 \\
.91 E+03\end{array}$ & $\begin{array}{l}.34 E-01 \\
.0 \\
11 E+003 \\
.16 E E 03 \\
05 E+04\end{array}$ & $\begin{array}{l}.82 E-01 \\
.0 \\
.42 E+03 \\
.47 E+03 \\
.14 E+04\end{array}$ & $\begin{array}{l}.43 E-01 \\
.0 \\
.21 E+03 \\
.22 E+03 \\
.07 E+04\end{array}$ & $\begin{array}{l}5.77 E-01 \\
0.0 \\
2.87 E+03 \\
5.51 E+03 \\
9.37 E \cdot 03\end{array}$ & $\begin{array}{l}14 E-01 \\
0 \\
.57 E+03 \\
.05 E+03 \\
56 E+03\end{array}$ & $\begin{array}{l}6.94 E-01 \\
0.0 \\
3.48 E+03 \\
6.58 E+03 \\
1.15 E+04\end{array}$ & $\begin{array}{l}5.16 E-01 \\
0.0 \\
2.08 E+03 \\
5.63 E+03 \\
9.59 E+03\end{array}$ & $\begin{array}{l}5.88 E-01 \\
0.0 \\
2.93 E+03 \\
5.68 E+03 \\
9.75 E+03\end{array}$ \\
\hline $\begin{array}{l}\gamma-86 \\
\gamma-87 \\
\gamma-88 \\
\gamma-90 \\
\gamma-90 M\end{array}$ & $\begin{array}{r}14.74 \mathrm{H} \\
80.3 \mathrm{H} \\
106.60 \mathrm{O} \\
64.11 \mathrm{H} \\
3.19 \mathrm{H}\end{array}$ & $\begin{array}{l}1.59 E+04 \\
1.85 E \cdot 03 \\
1.25 E \cdot 04 \\
0.0 \\
2.53 E \cdot 03\end{array}$ & $\begin{array}{l}1.69 E+04 \\
1.99 E+03 \\
1.32 E+04 \\
0.0 \\
2.73 E+03\end{array}$ & $\begin{array}{l}1.01 E+04 \\
2.31 E * 03 \\
1.38 E \cdot 04 \\
0.0 \\
3.36 E+03\end{array}$ & $\begin{array}{l}1.71 E+04 \\
2.04 E+03 \\
1.34 E+04 \\
0.0 \\
2.78 E+03\end{array}$ & $\begin{array}{l}1.51 E * 04 \\
1.77 E+03 \\
1.17 E+04 \\
0.0 \\
2.36 E+03\end{array}$ & $\begin{array}{l}1.30 E+04 \\
1.59 E+03 \\
1.09 E+04 \\
0.0 \\
2.18 E \cdot 03\end{array}$ & $\begin{array}{l}1.84 E * 04 \\
2.34 E * 03 \\
1.41 E * 04 \\
0.0 \\
3.42 E * 03\end{array}$ & $\begin{array}{l}1.54 E * 04 \\
1.81 E * 03 \\
1.21 E * 04 \\
0.0 \\
2.49 E * 03\end{array}$ & $\begin{array}{l}1.56 E+04 \\
1.82 E+03 \\
1.23 E+04 \\
0.0 \\
2.50 E+03\end{array}$ \\
\hline $\begin{array}{l}Y-91 \\
Y-91 M \\
Y-92 \\
Y-93 \\
Z R-86\end{array}$ & $\begin{array}{r}58.51 \mathrm{D} \\
49.71 \\
3.54 \\
10.1 \mathrm{H} \\
16.5 \mathrm{H}\end{array}$ & $\begin{array}{l}1.57 E+01 \\
2.18 E+03 \\
1.09 E+03 \\
4.01 E+02 \\
1.07 E+03\end{array}$ & & $\begin{array}{l}1.75 E+01 \\
2.66 E+03 \\
1.25 E+03 \\
4.65 E \cdot 02 \\
1.51 E+03\end{array}$ & & $\begin{array}{l}1.50 E+01 \\
2.10 E+03 \\
1.05 E+03 \\
3.12 E+02 \\
9.69 E+02\end{array}$ & $\begin{array}{l}1.36 E \cdot 01 \\
1.8 B E \cdot 03 \\
9.47 E \cdot 02 \\
3.4 B E+02 \\
9.23 E+02\end{array}$ & $\begin{array}{l}1.78 E+01 \\
2.10 E+03 \\
1.27 E+03 \\
4.73 E+02 \\
1.54 E+03\end{array}$ & $\begin{array}{l}1.52 E+01 \\
2.13 E+03 \\
1.06 E+03 \\
3.88 E+02 \\
1.06 E+03\end{array}$ & $\begin{array}{l}1.54 E+01 \\
2.15 E+03 \\
1.08 E+03 \\
3.93 E+02 \\
1.06 E+03\end{array}$ \\
\hline $\begin{array}{l}2 R-88 \\
2 R-89 \\
2 R-93 \\
2 R-95 \\
2 R-97\end{array}$ & $\begin{array}{r}83.4 \mathrm{O} \\
78.43 \mathrm{H} \\
1.53 \mathrm{E} 6 \mathrm{~V} \\
64.02 \mathrm{O} \\
16.90 \mathrm{H}\end{array}$ & $\begin{array}{l}1.53 E+03 \\
4.97 E+03 \\
0.0 \\
3.14 E+03 \\
7.80 E+02\end{array}$ & $\begin{array}{l}1.65 E \cdot 03 \\
5.30 E+03 \\
0.0 \\
3.35 E+03 \\
8.30 E+02\end{array}$ & $\begin{array}{l}1.98 E \cdot 03 \\
5.77 E+03 \\
0.0 \\
3.68 E+03 \\
9.04 E+02\end{array}$ & $\begin{array}{l}1.70 E \cdot 03 \\
5.38 E \cdot 03 \\
0.0 \\
3.41 F \cdot 03\end{array}$ & $\begin{array}{l}1.44 E+03 \\
4.81 E+03 \\
0.0 \\
3.05 E+03 \\
1.41 E+02\end{array}$ & $\begin{array}{l}2 E+03 \\
8 E+03 \\
1 E+03 \\
5 E+02\end{array}$ & $\begin{array}{l}2.02 E+03 \\
5.87 E+03 \\
0.0 \\
3.74 E+03 \\
9.19 E+02\end{array}$ & $\begin{array}{l}1.50 E+03 \\
4.82 E+03 \\
0.0 \\
3.05 E+03 \\
7.56 E+02\end{array}$ & $\begin{array}{l}1.51 E+03 \\
4.91 E+03 \\
0.0 \\
3.11 E+03 \\
7.66 E+02\end{array}$ \\
\hline $\begin{array}{l}N B-90 \\
N B-91 \\
N B-91 M \\
N B-92 \\
N B-92 M\end{array}$ & 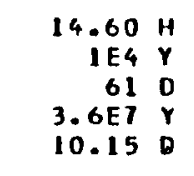 & $\begin{array}{l}1.98 E+04 \\
6.90 E+00 \\
1.85 E * 02 \\
6.39 E+03 \\
4.19 E+03\end{array}$ & $\begin{array}{l}2.08 E \cdot 04 \\
7.89 E \bullet 00 \\
1.97 E+02 \\
6.82 E \bullet 03 \\
4.47 E \bullet 03\end{array}$ & $\begin{array}{l}2.20 E \cdot 04 \\
9.10 E+00 \\
2.09 E+02 \\
7.4 \theta E+03 \\
4.79 E+03\end{array}$ & & & & & & $\begin{array}{l}1.94 E+04 \\
6.99 E+00 \\
1.82 E+02 \\
6.31 E+03 \\
4.14 E+03\end{array}$ \\
\hline $\begin{array}{l}N B-934 \\
N B-94 \\
N B-94 M \\
N B-95 \\
N B-95 M\end{array}$ & $\begin{array}{r}14.6 r \\
2.03 E 4 \gamma \\
6.26 \mathrm{H} \\
35.06 \mathrm{~V} \\
86.6 \mathrm{H}\end{array}$ & $\begin{array}{l}2.33 E-02 \\
6.16 E+03 \\
1.80 E+01 \\
3.28 E+03 \\
2.32 E+02\end{array}$ & $\begin{array}{l}1.33 E \\
7.22 E \\
1.97 E \\
3.50 E \\
2.52 E\end{array}$ & $\begin{array}{l}1.65 \\
7.88 \\
2.14 \\
3.83 \\
3.38\end{array}$ & $\begin{array}{l}4.4 \\
7.3 \\
1.9 \\
3.5 \\
2.5\end{array}$ & $\begin{array}{l}7.85 \\
6.57 \\
1.17 \\
3.19 \\
2.07\end{array}$ & $\begin{array}{l}7.5 \\
5.8 \\
1.5 \\
2.8 \\
2.0\end{array}$ & & $\begin{array}{l}-02 \\
03 \\
03 \\
01 \\
01 \\
03 \\
02\end{array}$ & $\begin{array}{l}6.56 E-02 \\
6.69 E+03 \\
1.80 E+01 \\
3.24 E+03 \\
2.29 E \cdot 02\end{array}$ \\
\hline $\begin{array}{l}N B-96 \\
N B-97 \\
N B-97 M \\
M O-91 \\
M O-93\end{array}$ & 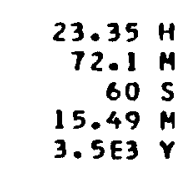 & $\begin{array}{l}1.06 E * 04 \\
2.80 E * 03 \\
3.11 E \cdot 03 \\
3.99 E \cdot 03 \\
1.31 E-01\end{array}$ & $\begin{array}{l}1.13 E+04 \\
3.00 E+03 \\
3.32 E+03 \\
4.2 \theta E+03 \\
7.43 E-01\end{array}$ & $\begin{array}{l}1.23 E+04 \\
3.33 E \cdot 03 \\
3.65 E \cdot 03 \\
4.92 E \cdot 03 \\
9.26 E-01\end{array}$ & $\begin{array}{l}1.14 E * 04 \\
3.05 E * 03 \\
3.38 E * 03 \\
4.38 E * 03 \\
2.51 E-01\end{array}$ & $\begin{array}{l}1.02 E+04 \\
2.72 E+03 \\
3.02 E+03 \\
3.83 E+03 \\
4.40 E-01\end{array}$ & $\begin{array}{l}9.12 E+03 \\
2.41 E+03 \\
2.68 E+03 \\
3.44 E+03 \\
4.24 E-01\end{array}$ & $\begin{array}{l}1.25 E+04 \\
3.3 B E+03 \\
3.71 E+03 \\
5.00 E+03 \\
1.22 E+00\end{array}$ & $\begin{array}{l}1.03 E+04 \\
2.12 E+03 \\
3.02 E+03 \\
3.89 E+03 \\
3.36 E-01\end{array}$ & $\begin{array}{l}1.04 E \cdot 04 \\
2.17 E+03 \\
3.08 E+03 \\
3.94 E+03 \\
3.67 E-01\end{array}$ \\
\hline $\begin{array}{l}\text { MO-99 } \\
M O-101 \\
\text { TC-95 } \\
\text { TC-95M } \\
\text { TC }-96\end{array}$ & $\begin{array}{r}66.02 \mathrm{H} \\
14.61 \mathrm{H} \\
20.0 \mathrm{H} \\
611 \mathrm{O} \\
4.28 \mathrm{O}\end{array}$ & $\begin{array}{l}6.51 E * 02 \\
6.62 E \cdot 03 \\
3.37 E \cdot 03 \\
2.78 E+03 \\
1.08 E+04\end{array}$ & $\begin{array}{l}6.98 E+02 \\
7.03 E+03 \\
3.60 E+03 \\
2.98 E+03 \\
1.15 E+04\end{array}$ & $\begin{array}{l}7.94 E+02 \\
7.57 E+03 \\
3.93 E+03 \\
3.43 E \bullet 03 \\
1.25 E+04\end{array}$ & $\begin{array}{l}7.06 E+02 \\
1.11 E+03 \\
3.66 E+03 \\
3.02 E+03 \\
1.17 E+04\end{array}$ & $\begin{array}{l}6.26 E * 02 \\
6.24 E * 03 \\
3.27 E * 03 \\
2.65 E+03 \\
1.05 E * 04\end{array}$ & $\begin{array}{l}5.60 E+02 \\
5.73 E+03 \\
2.90 E+03 \\
2.39 E+03 \\
9.29 E+03\end{array}$ & $\begin{array}{l}8.08 E+02 \\
7.70 E+03 \\
3.99 E+03 \\
3.49 E+03 \\
1.27 E * 04\end{array}$ & $\begin{array}{l}6.34 E+02 \\
6.40 E+03 \\
3.27 E+03 \\
2.71 E+03 \\
1.04 E+04\end{array}$ & $\begin{array}{l}6.49 E+03 \\
3.33 E+03 \\
2.75 E+03 \\
1.06 E+04\end{array}$ \\
\hline
\end{tabular}




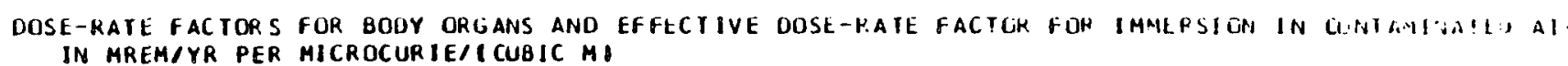

\begin{tabular}{|c|c|c|c|c|c|c|c|c|c|}
\hline NUCL IDE & HALF-LIFE & TESTES & THYMUS & JHYROID & UTERUS & EFFECTIVE & $\begin{array}{c}\text { SKIN } \\
\text { I PHOTONI }\end{array}$ & $\begin{array}{l}\text { SKIN } \\
\text { IELECTRONI }\end{array}$ & $\begin{array}{l}\text { SKIN } \\
\text { GTOTAL, }\end{array}$ \\
\hline $\begin{array}{l}S R-89 \\
S R-90 \\
S R-91 \\
5 R-92 \\
S R-93\end{array}$ & $\begin{array}{rr}50.55 & 0 \\
28.6 & Y \\
9.5 & H \\
2.71 & H \\
7.3 & M\end{array}$ & $\begin{array}{l}8.38 E-01 \\
0.0 \\
4.19 E+03 \\
0.08 E+03 \\
1.39 E+04\end{array}$ & $\begin{array}{l}0.69 E-01 \\
0.0 \\
3.33 E+03 \\
6.45 E+03 \\
1.09 E+04\end{array}$ & $\begin{array}{l}.01 E-01 \\
.0 \\
.99 E+03 \\
.64 E+03 \\
.30 E+04\end{array}$ & $\begin{array}{l}5.18 E-01 \\
0.0 \\
2.60 E+03 \\
5.18 E+03 \\
6.75 E+03\end{array}$ & $\begin{array}{l}7.17 E-01 \\
0.0 \\
3.59 E+03 \\
6.93 E+03 \\
1.19 E+04\end{array}$ & $\begin{array}{l}9.70 E-01 \\
0.0 \\
4.84 E+03 \\
9.27 E+03 \\
1.59 E+04\end{array}$ & $\begin{array}{l}4.38 E+03 \\
1.11 E+03 \\
4.94 E+03 \\
1.11 E+03 \\
7.02 E+03\end{array}$ & $\begin{array}{l}4.38 E+03 \\
1.11 E+03 \\
9.78 E+03 \\
1.04 E+04 \\
2.29 E+04\end{array}$ \\
\hline $\begin{array}{l}y-86 \\
y-87 \\
y-88 \\
y-90 \\
Y-90 H\end{array}$ & $\begin{array}{r}14.74 \mathrm{H} \\
80.3 \mathrm{H} \\
106.60 \mathrm{O} \\
64.1 \mathrm{H} \\
3.19 \mathrm{H}\end{array}$ & $\begin{array}{l}2.22 E \cdot 04 \\
2.67 E \bullet 03 \\
1.73 E \bullet 04 \\
0.0 \\
3.70 E \bullet 03\end{array}$ & $\begin{array}{l}1.75 E * 04 \\
2.01 E \bullet 03 \\
1.37 E \bullet 04 \\
0.0 \\
2.80 E+03\end{array}$ & $\begin{array}{l}2.09 E+04 \\
2.47 E+03 \\
1.61 E+04 \\
0.0 \\
3.46 E+03\end{array}$ & $\begin{array}{l}1.41 E+04 \\
1.62 E * 03 \\
1.12 E+04 \\
0.0 \\
2.22 E+03\end{array}$ & $\begin{array}{l}1.91 E+04 \\
2.29 E+03 \\
1.4 B E+04 \\
0.0 \\
3.19 E+03\end{array}$ & $\begin{array}{l}2.55 E+04 \\
3.06 E+03 \\
1.97 E+04 \\
0.0 \\
4.17 E+03\end{array}$ & $\begin{array}{l}1.69 E+03 \\
9.58 E \cdot 00 \\
5.31 E+00 \\
7.38 E+03 \\
3.16 E+02\end{array}$ & $\begin{array}{l}2.72 E+04 \\
3.07 E+03 \\
1.97 E+04 \\
7.38 E+03 \\
4.49 E+03\end{array}$ \\
\hline $\begin{array}{l}\gamma-91 \\
\gamma-91 M \\
\gamma-92 \\
\gamma-93 \\
2 R-86\end{array}$ & $\begin{array}{rr}58.51 & 0 \\
49.71 & M \\
3.54 & H \\
10.1 & H \\
16.5 & H\end{array}$ & $\begin{array}{l}2.18 E \cdot 01 \\
3.13 E+03 \\
1.54 E \bullet 03 \\
5.59 E \cdot 02 \\
1.59 E \cdot 03\end{array}$ & $\begin{array}{l}1.75 E+01 \\
2.39 E \bullet 03 \\
1.22 E \cdot 03 \\
4.40 E \cdot 02 \\
1.20 E+03\end{array}$ & $\begin{array}{l}2.08 E+01 \\
2.92 E+03 \\
1.46 E+03 \\
5.25 E+02 \\
1.51 E+03\end{array}$ & $\begin{array}{l}1.38 E+01 \\
1.90 E+03 \\
9.61 E+02 \\
3.57 E+02 \\
9.45 E+02\end{array}$ & $\begin{array}{l}1.87 E+01 \\
2.68 E+03 \\
1.32 E+03 \\
4.82 E+02 \\
1.39 E+03\end{array}$ & $\begin{array}{l}2.52 E+01 \\
3.59 E+03 \\
1.77 E+03 \\
0.36 E+02 \\
1.81 E \bullet 03\end{array}$ & $\begin{array}{l}4.55 E+03 \\
1.95 E+02 \\
1.18 E+04 \\
9.42 E+03 \\
5.33 E+01\end{array}$ & $\begin{array}{l}4.57 E+03 \\
3.79 E \cdot 03 \\
1.35 E+04 \\
1.01 E+04 \\
1.86 E+03\end{array}$ \\
\hline $\begin{array}{l}2 R-88 \\
2 R-89 \\
2 R-93 \\
2 R-95 \\
2 R-97\end{array}$ & $\begin{array}{r}03.4 \mathrm{D} \\
78.43 \mathrm{H} \\
1.53 E 6 \mathrm{Y} \\
64.02 \mathrm{D} \\
16.90 \mathrm{H}\end{array}$ & $\begin{array}{l}2.22 E \cdot 03 \\
7.03 E \cdot 03 \\
0.0 \\
4.46 E \cdot 03 \\
1.10 E \cdot 03\end{array}$ & $\begin{array}{l}1.67 E \cdot 03 \\
5.55 E * 03 \\
0.0 \\
3.50 E * 03 \\
8.62 E \cdot 02\end{array}$ & $\begin{array}{l}2.06 E+03 \\
6.67 E+03 \\
0.0 \\
4.22 E+03 \\
1.03 E+03\end{array}$ & $\begin{array}{l}1.34 E+03 \\
4.33 E+03 \\
0.0 \\
2.73 E+03 \\
6.88 E+02\end{array}$ & $\begin{array}{l}1.92 E+03 \\
6.01 E+03 \\
0.0 \\
3.81 E+03 \\
9.41 E+02\end{array}$ & $\begin{array}{l}2.53 E+03 \\
8.12 E+03 \\
0.0 \\
5.15 E+03 \\
1.26 E+03\end{array}$ & $\begin{array}{l}6.91 E+01 \\
6.83 E+02 \\
0.0 \\
4.46 E+02 \\
5.26 E+03\end{array}$ & $\begin{array}{l}2.60 E+03 \\
8.81 E+03 \\
0.0 \\
5.60 E+03 \\
6.52 E+03\end{array}$ \\
\hline $\begin{array}{l}N \theta-90 \\
N \theta-91 \\
N \theta-914 \\
N B-92 \\
N B-924\end{array}$ & 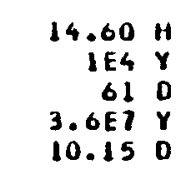 & $\begin{array}{l}2.72 E \bullet 04 \\
1.33 E \bullet 01 \\
2.62 E \bullet 02 \\
9.06 E * 03 \\
5.90 E * 03\end{array}$ & $\begin{array}{l}2.14 E+04 \\
7.47 E+00 \\
2.07 E+02 \\
1.12 E+03 \\
4.72 E+03\end{array}$ & $\begin{array}{l}2.52 E+04 \\
9.95 E+00 \\
2.47 E+02 \\
8.57 E+03 \\
5.64 E+03\end{array}$ & $\begin{array}{l}1.77 E+04 \\
5.96 E * 00 \\
1.63 E+02 \\
5.57 E+03 \\
3.66 E+03\end{array}$ & & $\begin{array}{l}3.08 E+04 \\
3.32 E+01 \\
3.17 E+02 \\
1.05 E+04 \\
6.85 E+03\end{array}$ & $\begin{array}{l}2.75 E+03 \\
4.98 E-01 \\
6.87 E+01 \\
1.04 E+01 \\
0.0\end{array}$ & $\begin{array}{l}3.36 E+04 \\
3.37 E+01 \\
3.86 E+02 \\
1.05 E+04 \\
6.85 E+03\end{array}$ \\
\hline $\begin{array}{l}N B-93 M \\
N B-94 \\
N B-94 M \\
N \theta-95 \\
N B-95\end{array}$ & $\begin{array}{r}14.6 Y \\
2.03 E 4 Y \\
6.26 \mathrm{M} \\
35.06 \mathrm{D} \\
86.6 \mathrm{H}\end{array}$ & $\begin{array}{l}7.84 E-01 \\
9.59 E \cdot 03 \\
2.84 E \cdot 01 \\
4.65 E+03 \\
3.46 E \bullet 02\end{array}$ & $\begin{array}{l}1.31 E-02 \\
7.57 E \cdot 03 \\
2.03 E \cdot 01 \\
3.66 E \cdot 03 \\
2.61 E \cdot 02\end{array}$ & $\begin{array}{l}2.13 E-01 \\
9.10 E * 03 \\
2.50 E+01 \\
4.41 E * 03 \\
3.29 E * 02\end{array}$ & $\begin{array}{l}6.80 E-04 \\
5.88 E \cdot 03 \\
1.56 E+01 \\
2.85 E+03 \\
2.05 E+02\end{array}$ & $\begin{array}{l}0.20 E+03 \\
2.42 E+01 \\
3.97 E+03 \\
3.04 E+02\end{array}$ & $\begin{array}{l}4.29 E \bullet 00 \\
1.11 E+04 \\
4.57 E+01 \\
5.37 E \bullet 03 \\
3.96 E \bullet 02\end{array}$ & $\begin{array}{l}0.0 \\
6.79 E+02 \\
1.4 B E+01 \\
3.14 E+01 \\
1.02 E+03\end{array}$ & $\begin{array}{l}4.29 E+00 \\
1.1 B E+04 \\
6.05 E+01 \\
5.40 E+03 \\
1.41 E+03\end{array}$ \\
\hline $\begin{array}{l}N B-96 \\
N B-97 \\
N B-97 M \\
M C-91 \\
M O-93\end{array}$ & 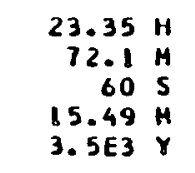 & $\begin{array}{l}1.49 E \bullet 04 \\
3.99 E+03 \\
4.42 E+03 \\
5.14 E+03 \\
4.39 E+00\end{array}$ & $\begin{array}{l}1.18 E+04 \\
3.10 E \bullet 03 \\
3.47 E \bullet 03 \\
4.35 E \cdot 03 \\
7.32 E-02\end{array}$ & $\begin{array}{l}1.42 E+04 \\
3.75 E+03 \\
4.18 E+03 \\
5.32 E+03 \\
1.19 E+00\end{array}$ & $\begin{array}{l}9.23 E+03 \\
3.44 E+03 \\
2.70 E+03 \\
3.49 E \div 03 \\
3.81 E-03\end{array}$ & $\begin{array}{l}3.41 E+03 \\
3.78 E+03 \\
4.91 E+03 \\
3.78 E+00\end{array}$ & $\begin{array}{l}1.72 E+04 \\
4.60 E * 03 \\
5.10 E+03 \\
6.56 E * 03 \\
2.40 E \cdot 01\end{array}$ & $\begin{array}{l}1.53 E+03 \\
3.33 E+03 \\
1.08 E+02 \\
1.11 E+04 \\
0.0\end{array}$ & $\begin{array}{l}1.8 B E * 04 \\
1.93 E * 03 \\
5.21 E+03 \\
1.83 E+04 \\
2.40 E * 01\end{array}$ \\
\hline $\begin{array}{l}M O-99 \\
M O-101 \\
\text { IC-95 } \\
\text { IC }-954 \\
\text { IC }-96\end{array}$ & 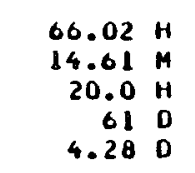 & $\begin{array}{l}9.32 E+02 \\
9.25 E+03 \\
4.78 E \cdot 03 \\
3.99 E \cdot 03 \\
1.52 E \cdot 04\end{array}$ & $\begin{array}{l}7.30 E+02 \\
7.30 E+03 \\
3.77 E+03 \\
3.11 E \cdot 03 \\
1.21 E+04\end{array}$ & $\begin{array}{l}8.85 E+02 \\
8.70 E+03 \\
4.53 E+03 \\
3.78 E+03 \\
1.45 E+04\end{array}$ & $\begin{array}{l}5.67 E+02 \\
5.86 E * 03 \\
2.93 E * 03 \\
2.43 E * 03 \\
9.37 E * 03\end{array}$ & $\begin{array}{l}7.94 \mathrm{E}+03 \\
4.08 \mathrm{E}+03 \\
3.43 \mathrm{E}+03 \\
1.30 \mathrm{E} \cdot 04\end{array}$ & $\begin{array}{l}1.07 E+03 \\
1.06 E+04 \\
5.54 E+03 \\
4.58 E+03 \\
1.76 E+04\end{array}$ & $\begin{array}{l}2.72 E+03 \\
3.79 E+03 \\
6.80 E+00 \\
3.90 E+01 \\
7.13 E+00\end{array}$ & $\begin{array}{l}3.79 E+03 \\
1.44 E+04 \\
5.55 E+03 \\
4.62 E+03 \\
1.76 E+04\end{array}$ \\
\hline
\end{tabular}


DOSE-RATE FACTORS FOR BODY ORGANS AND EFFECTIVE DCSE-PATE FACTOP. FOR IMMERSION IN CONIAMIVATEL AIS
IN MREMAYR PER MICROCUR IE/ICUBIC MI

\begin{tabular}{|c|c|c|c|c|c|c|c|c|c|c|}
\hline CLIDE & HALF-LIFE & RENALS $S$ & BLADDER & BP.AIN & REAST & HE AR. T & $\begin{array}{l}\text { SMALL } \\
\text { INTESTINE }\end{array}$ & $\begin{array}{c}\text { UPPER } \\
\text { LARSE } \\
\text { INTESTIVE }\end{array}$ & $\begin{array}{c}\text { LOWER } \\
\text { LARGE } \\
\text { INTESTINE }\end{array}$ & RlOMEYS \\
\hline $\begin{array}{l}\text { TC-964 } \\
\text { TC-97 } \\
\text { TC-97H } \\
\text { TC-98 } \\
\text { TC-99 }\end{array}$ & $\begin{array}{r}51.5 \mathrm{M} \\
2.6 E 6 \mathrm{Y} \\
89 \mathrm{D} \\
4.2 E 6 \mathrm{Y} \\
2.13 E 5 \mathrm{Y}\end{array}$ & 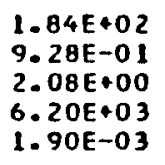 & & 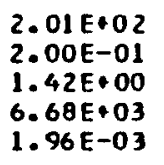 & & $.76 \mathrm{E}$ & $\begin{array}{l}60 E \cdot 02 \\
09 E-01 \\
09 E+00 \\
2 B E+03\end{array}$ & $\begin{array}{l}1.88 E \bullet 02 \\
1.61 E-01 \\
1.47 E+00 \\
6.22 E+03 \\
1.92 E-03\end{array}$ & $\begin{array}{l}.73 E+02 \\
.04 E-01 \\
.66 E+00 \\
.72 E+03 \\
.71 E-03\end{array}$ & $\begin{array}{l}1.87 E+02 \\
4.63 E-01 \\
1.80 E+00 \\
6.21 E+03 \\
1.93 E-03\end{array}$ \\
\hline $\begin{array}{l}T C-994 \\
T C-101 \\
R U-97 \\
R U-103 \\
R U-105\end{array}$ & $\begin{array}{r}6.02 \mathrm{H} \\
14.2 \mathrm{M} \\
2.9 \mathrm{D} \\
39.35 \mathrm{D} \\
4.44 \mathrm{H}\end{array}$ & $\begin{array}{l}1.48 \\
9.78 \\
2.08\end{array}$ & $\begin{array}{l}E+02 \\
E+03 \\
E+02 \\
E+03\end{array}$ & $\begin{array}{l}24 E+02 \\
50 E+03 \\
62 E+02 \\
20 E+03 \\
70 E+03\end{array}$ & & $\begin{array}{l}4.82 E+02 \\
1.34 E+03 \\
8.12 E+02 \\
1.93 E+03\end{array}$ & & $\begin{array}{l}5.15 E+02 \\
1.42 E+03 \\
9.23 E+02 \\
2.05 E+03 \\
3.45 E+03\end{array}$ & $\begin{array}{l}4.72 E+02 \\
1.33 E+03 \\
8.68 E+02 \\
1.89 E+03 \\
3.18 E+03\end{array}$ & $\begin{array}{l}4.96 E+02 \\
1.42 E+03 \\
9.22 E+02 \\
2.05 E+03 \\
3.44 E+03\end{array}$ \\
\hline $\begin{array}{l}U-106 \\
H-103 M \\
H-105 \\
H-105 M \\
H-106\end{array}$ & 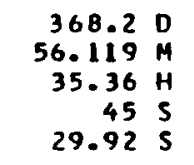 & $\begin{array}{l}0.0 \\
2.56 E-01 \\
3.34 E+02 \\
1.12 E \cdot 02 \\
9.05 E \cdot 02\end{array}$ & $\begin{array}{l}0.0 \\
1.49 E-01 \\
3.09 E+02 \\
1.03 E+02 \\
8.66 E \cdot 02\end{array}$ & $\begin{array}{l}8 E-02 \\
7 E+02 \\
0 E+02\end{array}$ & $\begin{array}{l}6 E+00 \\
5 E+02 \\
T E \cdot 02 \\
3 E+03\end{array}$ & $\begin{array}{l}0.0 \\
1.02 E-01 \\
3.01 E+02 \\
1.01 E+02 \\
8.50 E+02\end{array}$ & $\begin{array}{l}5 E-02 \\
0 E+02 \\
9 E+01 \\
T E+02\end{array}$ & $\begin{array}{l}0.0 \\
2.04 E-01 \\
3.18 E+02 \\
1.09 E+02 \\
9.03 E+02\end{array}$ & & $\begin{array}{l}0.0 \\
3.33 E-01 \\
3.18 E+02 \\
1.05 E+02 \\
9.02 E+02\end{array}$ \\
\hline $\begin{array}{l}P D-103 \\
P D-107 \\
P D-109 \\
A G-106 M \\
A G-108\end{array}$ & $\begin{array}{rl}16.961 & 0 \\
6.5 E 6 & \mathrm{~V} \\
13.453 \mathrm{H} \\
8.46 \mathrm{O} \\
2.37 \mathrm{M}\end{array}$ & $\begin{array}{l}2.4 B E+00 \\
0.0 \\
2.97 E+00 \\
1.23 E+04 \\
7.64 E+01\end{array}$ & $\begin{array}{l}1.34 E \cdot 00 \\
0.0 \\
2.82 E+00 \\
1.20 E+04 \\
7.28 E+01\end{array}$ & $\begin{array}{l}.07 E+00 \\
0 \\
14 E+00 \\
34 E+04 \\
13 E+01\end{array}$ & $\begin{array}{l}.87 E \\
.0 \\
.74 E \\
.54 E \\
.57 E\end{array}$ & $\begin{array}{l}1.12 E+00 \\
0.0 \\
2.75 E+00 \\
1.18 E+04 \\
1.13 E+01\end{array}$ & 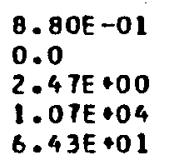 & $\begin{array}{l}1.96 E+03 \\
0.0 \\
2.92 E+03 \\
1.25 E+04 \\
7.58 E+01\end{array}$ & $\begin{array}{l}00 \\
00 \\
04 \\
01\end{array}$ & $\begin{array}{l}2.8 B E \bullet 00 \\
0.0 \\
2.92 E+00 \\
1.25 E+04 \\
7.57 E * 01\end{array}$ \\
\hline $\begin{array}{l}A G-108 M \\
A G-109 M \\
A G-110 \\
A G-110 M \\
A G-111\end{array}$ & $\begin{array}{rl}127 & Y \\
39.6 & S \\
24.57 & S \\
249.85 & 0 \\
7.46 & 0\end{array}$ & $\begin{array}{l}1.06 E+03 \\
1.35 E+01 \\
1.35 E+02 \\
1.22 E+04 \\
1.13 E+02\end{array}$ & $\begin{array}{l}0.73 E+03 \\
1.31 E+01 \\
1.29 E+02 \\
1.18 E+04 \\
1.05 E+02\end{array}$ & $\begin{array}{l}1.52 E+03 \\
1.29 E+01 \\
1.45 E+02 \\
1.33 E+04 \\
1.15 E+02\end{array}$ & $\begin{array}{l}8.02 E \div 03 \\
4.06 E \cdot 01 \\
1.67 E+02 \\
1.50 E+04 \\
1.50 E * 02\end{array}$ & & & & & $\begin{array}{l}7.01 E+03 \\
1.48 E+01 \\
1.35 E+02 \\
1.24 E+04 \\
1.08 E * 02\end{array}$ \\
\hline $\begin{array}{l}C D-109 \\
C D-111 M \\
C D-113 \\
C D-1134 \\
C D-115\end{array}$ & $\begin{array}{r}464 \mathrm{O} \\
48.7 \mathrm{M} \\
9.3 \mathrm{15} \mathrm{Y} \\
13.7 \mathrm{Y} \\
53.46 \mathrm{H}\end{array}$ & $\begin{array}{l}2.94 E+00 \\
1.19 E+03 \\
0.0 \\
0.0 \\
8.18 E+02\end{array}$ & $\begin{array}{l}2.22 E+00 \\
1.09 E \times 03 \\
0.0 \\
0.0 \\
8.33 E+02\end{array}$ & $\begin{array}{l}1.02 E+00 \\
1.17 E+03 \\
0.0 \\
0.0 \\
9.28 E \bullet 02\end{array}$ & $\begin{array}{l}3.34 E+01 \\
1.71 E * 03 \\
0.0 \\
0.0 \\
1.11 E+03\end{array}$ & $\begin{array}{l}1.16 E \cdot 00 \\
1.07 E \cdot 03 \\
0.0 \\
0.0 \\
8.14 E \cdot 02\end{array}$ & $\begin{array}{l}9.50 E+02 \\
0.0 \\
0.0 \\
7.33 E+02\end{array}$ & $\begin{array}{l}1.13 E+03 \\
0.0 \\
0.0 \\
8.64 E \bullet 02\end{array}$ & $\begin{array}{l}1.06 \mathrm{E}+03 \\
0.0 \\
0.0 \\
1.99 \mathrm{E} * 02\end{array}$ & $\begin{array}{l}4.89 E \cdot 00 \\
1.12 E+03 \\
0.0 \\
0.0 \\
8.65 E \cdot 02\end{array}$ \\
\hline $\begin{array}{l}C D-1 \\
C D M M \\
C D-117 \\
C D-117 M \\
1 N-111 \\
I N-113 M\end{array}$ & $\begin{array}{r}44.6 \mathrm{D} \\
2.49 \mathrm{H} \\
3.36 \mathrm{H} \\
2.83 \mathrm{D} \\
1.658 \mathrm{H}\end{array}$ & & & & & & & & & \\
\hline $\begin{array}{l}I N-116 \\
\text { IN-114M } \\
I N-115 \\
\text { IN-115M } \\
\text { IN-116M }\end{array}$ & $\begin{array}{r}71.9 \mathrm{~S} \\
49.51 \mathrm{D} \\
4.6 \mathrm{E} 15 \mathrm{Y} \\
4.36 \mathrm{H} \\
54.15 \mathrm{M}\end{array}$ & $\begin{array}{l}3.87 E+02 \\
0.0 \\
6.80 E+02 \\
1.10 E+04\end{array}$ & $\begin{array}{l}.64 E+02 \\
.0\end{array}$ & $0 E+02$ & $1.37 E+04$ & $\begin{array}{l}3.56 E+02 \\
0.0 \\
6.13 E+02 \\
1.08 E+04\end{array}$ & $E \cdot 02$ & $1.15 E+04$ & $\begin{array}{l}3.51 E+02 \\
0.0 \\
6.07 E+02 \\
1.06 E+04\end{array}$ & $\begin{array}{l}\text { 3. } 80 E \cdot 02 \\
0.0 \\
6.53 E+02 \\
1.15 E+04\end{array}$ \\
\hline
\end{tabular}




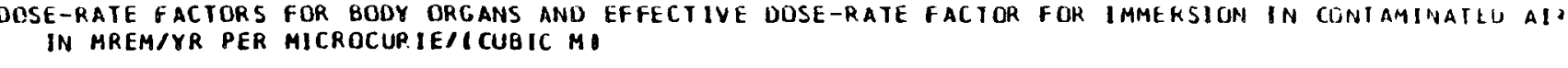

\begin{tabular}{|c|c|c|c|c|c|c|c|c|c|c|}
\hline ICL IOE & LF-LIFE & LIVER & LUNGS & MARROW & $\begin{array}{c}\text { REO } \\
\text { MARROH }\end{array}$ & OVARIES & ANCREAS & KELETON & SPLEEN & S TOMACH \\
\hline $\begin{array}{l}\text { IC-96M } \\
\text { IC-97 } \\
\text { IC }-97 M \\
\text { IC-98 } \\
\text { IC-99 }\end{array}$ & $\begin{array}{r}51.5 \mathrm{H} \\
2.6 E 6 \mathrm{Y} \\
89 \mathrm{O} \\
4.2 E 6 \mathrm{Y} \\
2.13 E 5 \mathrm{Y}\end{array}$ & $\begin{array}{l}1.78 E+02 \\
2.22 E-01 \\
1.41 E+00 \\
5.91 E+03 \\
1.89 E-03\end{array}$ & $\begin{array}{l}1.90 E+02 \\
1.02 E+00 \\
2.28 E+00 \\
6.32 E+03 \\
2.09 E-03\end{array}$ & $\begin{array}{l}.05 E+02 \\
.33 E+00 \\
.48 E+00 \\
0.99 E+03 \\
.55 E-03\end{array}$ & $\begin{array}{l}1.92 E+02 \\
3.45 E-01 \\
1.33 E+00 \\
6.44 E+03 \\
.53 E-03\end{array}$ & $\begin{array}{l}2 E+02 \\
0 E-01 \\
0 E+0 Q \\
6+03 \\
0 E-03\end{array}$ & $\begin{array}{l}4 E+02 \\
9 E-01 \\
3 E+00 \\
0 E+03 \\
0 E-03\end{array}$ & $\begin{array}{l}9 E+02 \\
B E+00 \\
35 E+00 \\
0 E+03 \\
0 E E-03\end{array}$ & $\begin{array}{l}.72 E+02 \\
.80 E-01 \\
.61 E+00 \\
.75 E+03 \\
.81 E-03\end{array}$ & $\begin{array}{l}.75 E+02 \\
.31 E-01 \\
.67 E+00 \\
.85 E+03 \\
.82 E-03\end{array}$ \\
\hline $\begin{array}{l}\text { TC-994 } \\
\text { TC-101 } \\
R U-97 \\
R U-103 \\
R U-105\end{array}$ & $\begin{array}{r}0.02 \mathrm{H} \\
14.2 \\
2.9 \\
39.35 \\
4.44 \\
6.40\end{array}$ & $\begin{array}{l}4.88 E+02 \\
1.37 E+03 \\
8.91 E+02 \\
1.96 E+03 \\
3.29 E+03\end{array}$ & $\begin{array}{l}5.38 E+02 \\
1.47 E+03 \\
9.68 E+02 \\
2.11 E+03 \\
3.52 E+03\end{array}$ & $\begin{array}{l}.21 E+02 \\
.83 E+03 \\
.30 E+03 \\
.43 E+03 \\
.97 E+03\end{array}$ & $\begin{array}{l}.91 E+02 \\
51 E+03 \\
70 E+02 \\
.16 E+03 \\
.59 E+03\end{array}$ & $\begin{array}{l}22 E+02 \\
26 E+03 \\
95 E+02 \\
89 E+03 \\
17 E+03\end{array}$ & $\begin{array}{l}.05 E+02 \\
.18 E+03 \\
.66 E+02 \\
.69 E+03 \\
.83 E+03\end{array}$ & & $\begin{array}{l}.88 E+02 \\
.35 E+03 \\
.85 E+02 \\
.92 E+03 \\
.20 E+03\end{array}$ & $\begin{array}{l}\text { 4. } 83 \mathrm{E}+02 \\
1.35 \mathrm{E}+03 \\
8.81 \mathrm{E}+02 \\
1.94 \mathrm{E}+03 \\
3.25 \mathrm{E}+03\end{array}$ \\
\hline $\begin{array}{l}R U-106 \\
R H-103 M \\
R H-105 \\
R H-105 H \\
R H-106\end{array}$ & 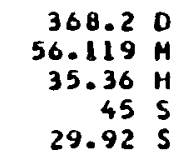 & $\begin{array}{l}0.0 \\
1.34 E-01 \\
3.07 E+02 \\
1.03 E+02 \\
0.61 E+02\end{array}$ & & $\begin{array}{l}0.0 \\
4.53 E-01 \\
4.17 E+02 \\
1.78 E+02 \\
1.04 E+03\end{array}$ & $\begin{array}{l}.0 \\
.07 E-01 \\
.43 E+02 \\
.01 E+02 \\
.41 E+02\end{array}$ & & & $\begin{array}{l}.0 \\
13 E-01 \\
.24 E+02 \\
83 E+02 \\
05 E+03\end{array}$ & $\begin{array}{l}.0 \\
.06 E-01 \\
.04 E+02 \\
.03 E+02 \\
.39 E+02\end{array}$ & $\begin{array}{l}0.0 \\
1.88 E-01 \\
3.03 E+02 \\
1.02 E+02 \\
8.50 E+02\end{array}$ \\
\hline $\begin{array}{l}P D-103 \\
P D-107 \\
P D-109 \\
A G-1064 \\
A G-108\end{array}$ & 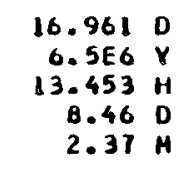 & $\begin{array}{l}1.29 E+00 \\
0.0 \\
2.80 E+00 \\
1.19 E+04 \\
1.23 E+01\end{array}$ & $\begin{array}{l}2.82 E+00 \\
0.0 \\
3.00 E+00 \\
1.27 E+04\end{array}$ & $\begin{array}{l}3.96 E+00 \\
0.0 \\
3.46 E+00 \\
1.39 E+04 \\
0.76 E+01\end{array}$ & $\begin{array}{l}1.29 E \cdot 00 \\
0.0 \\
3.07 E+00 \\
1.29 E+04 \\
.92 E \cdot 01\end{array}$ & $\begin{array}{l}.93 E * 00 \\
.0 \\
69 E+00 \\
14 E+04 \\
.99 E+01\end{array}$ & $\left\{\begin{array}{l}1 E+00 \\
1 E+00 \\
3 E+04\end{array}\right.$ & $\begin{array}{l}.49 E+00 \\
.0 \\
.52 E+00 \\
.42 E+04 \\
.91 E+01\end{array}$ & & $\begin{array}{l}1.82 E+00 \\
0.0 \\
2.76 E+00 \\
1.17 E+04 \\
7.15 E+01\end{array}$ \\
\hline $\begin{array}{l}A G-110 \\
A G-110 M \\
A G-111\end{array}$ & $\begin{array}{rr}127 & y \\
39.6 & 5 \\
24.57 & 5 \\
249.85 & 0 \\
7.46 & D\end{array}$ & $\begin{array}{l}.69 E+03 \\
.28 E+01 \\
.29 E+02\end{array}$ & & & & & & & & \\
\hline $\begin{array}{l}\text { CD-109 } \\
C D-111 M \\
C D-113 \\
C D-113 M \\
C D-115\end{array}$ & $\begin{array}{r}464 \mathrm{D} \\
48.7 \mathrm{H} \\
9.3 \mathrm{1} 15 \mathrm{Y} \\
13.7 \mathrm{Y} \\
53.46 \mathrm{H}\end{array}$ & $\begin{array}{l}1.05 E+00 \\
1.09 E+03 \\
0.0 \\
0.0 \\
8.28 E+02\end{array}$ & $\begin{array}{l}.57 E+00 \\
.18 E+03 \\
.0 \\
.0 \\
.89 E+02\end{array}$ & $\begin{array}{l}.40 E \cdot 00 \\
.60 E+03 \\
.0 \\
.0 \\
.03 E+03\end{array}$ & $\begin{array}{l}B E+00 \\
E+03 \\
B E+02\end{array}$ & $\begin{array}{l}E+00 \\
E+02 \\
E+02\end{array}$ & $\begin{array}{l}P E \div 00 \\
2 E+02 \\
3 E \div 02\end{array}$ & $\begin{array}{l}9 E+00 \\
4 E+03 \\
4 E+03\end{array}$ & $\begin{array}{l}1.96 E+00 \\
1.08 E+03 \\
0.0 \\
0.0 \\
8.09 E+02\end{array}$ & $\begin{array}{l}\text { 2. } 24 E+00 \\
1.08 E+03 \\
0.0 \\
0.0 \\
8.17 E+02\end{array}$ \\
\hline $\begin{array}{l}C D-115 M \\
C O-117 \\
C D-117 M \\
I N-111 \\
I N-113 M\end{array}$ & $\begin{array}{r}44.60 \\
2.49 \mathrm{H} \\
3.36 \mathrm{H} \\
2.830 \\
1.658 \mathrm{H}\end{array}$ & $\begin{array}{l}9.52 E+01 \\
4.72 E+03 \\
9.50 E+03 \\
1.51 E+03 \\
1.02 E+03\end{array}$ & $\begin{array}{l}1.01 E+02 \\
5.02 E+03 \\
1.00 E+04 \\
1.65 E+03 \\
1.10 E+03\end{array}$ & $\begin{array}{l}2.43 E+04 \\
1.05 E+04 \\
2.27 E+03 \\
1.32 E+03\end{array}$ & & & & & & \\
\hline $\begin{array}{l}1 N-114 \\
1 N-114 M \\
I N-115 \\
I N-115 M \\
I N-116 M\end{array}$ & $\begin{array}{r}71.9 \mathrm{~S} \\
49.51 \mathrm{O} \\
4.6 E 15 \mathrm{Y} \\
4.36 \mathrm{H} \\
54.15 \mathrm{M}\end{array}$ & $\begin{array}{l}1.32 E+02 \\
3.61 E+02 \\
0.0 \\
6.26 E+02 \\
1.09 E * 04\end{array}$ & $\begin{array}{l}1.41 E * 02 \\
3.91 E+02 \\
0.0 \\
6.77 E \cdot 02 \\
1.15 E \cdot 04\end{array}$ & $\begin{array}{l}4.75 E+02 \\
0.0 \\
8.40 E+02 \\
1.22 E+04\end{array}$ & $\begin{array}{l}1.44 E+02 \\
3.92 E+02 \\
0.0 \\
6.97 E+02 \\
1.17 E+04\end{array}$ & $\begin{array}{l}1.27 E+02 \\
3.40 E+02 \\
0.0 \\
5.79 E+02 \\
1.03 E+04\end{array}$ & $\begin{array}{l}3.10 E+02 \\
0.0 \\
5.41 E+02 \\
9.45 E+03\end{array}$ & $\begin{array}{l}1.61 E+02 \\
4.84 E \cdot 02 \\
0.0 \\
8.56 E+02 \\
1.24 E+04\end{array}$ & $\begin{array}{l}1.28 E+02 \\
3.55 E+02 \\
0.0 \\
6.18 E+02 \\
1.05 E+04\end{array}$ & $\begin{array}{l}1.30 E+02 \\
3.58 E+02 \\
0.0 \\
6.19 E+02 \\
1.07 E+04\end{array}$ \\
\hline
\end{tabular}



DOSE-RATE FACTURS FOR BODY URGAMS ANO EFFECTIVE DOSE-PATE FACTOR. FOR IMMEPSION IN COAITAMINATLU ALT

\begin{tabular}{|c|c|c|c|c|c|c|c|c|c|}
\hline JCL IOE & HALF-LIFE & TESTES & THYMUS & HY ROID & ITERUS & FECTIVE & $\begin{array}{l}\text { SKIN } \\
\text { HOTONI }\end{array}$ & $\begin{array}{l}\text { SKIN } \\
\text { LECTRONI }\end{array}$ & $\begin{array}{l}\text { SKIN } \\
\text { ITOTALI }\end{array}$ \\
\hline $\begin{array}{l}\text { TC-964 } \\
\mathrm{TC}-97 \\
\mathrm{TC}-97 \mathrm{M} \\
\mathrm{TC}-9 \mathrm{~B} \\
\mathrm{TC}-99\end{array}$ & $\begin{array}{r}51.5 \\
2.6 E 6 \\
B 9 \\
89 \\
4.2 E 6 Y \\
2.13 E 5 Y\end{array}$ & $\begin{array}{l}2.54 E+02 \\
5.16 E+00 \\
7.24 E+00 \\
8.42 E+03 \\
3.07 E-03\end{array}$ & $\begin{array}{l}99 E+02 \\
50 E-01 \\
64 E \cdot 00 \\
58 E+03\end{array}$ & $\begin{array}{l}39 E+02 \\
74 E+00 \\
65 E+00 \\
94 E+03\end{array}$ & $\begin{array}{l}55 E+02 \\
02 E-02 \\
90 E-01 \\
14 E+03 \\
55 E-03\end{array}$ & $\begin{array}{l}17 E+02 \\
70 E+00 \\
T B E+00\end{array}$ & $\begin{array}{l}.05 E+02 \\
.76 E+01 \\
.48 E+01 \\
.71 E+03 \\
.27 E-03\end{array}$ & $\begin{array}{l}0.0 \\
0.0 \\
3.51 E+01 \\
4.78 E+02 \\
2.24 E+02\end{array}$ & $\begin{array}{l}.05 E+02 \\
.76 E+01 \\
.99 E+01 \\
.02 E+04 \\
.24 E+02\end{array}$ \\
\hline $\begin{array}{l}\mathrm{TC}-99 \mathrm{M} \\
\mathrm{TC}-101 \\
\mathrm{RU}-97 \\
\mathrm{RU}-103 \\
\mathrm{RU}-105\end{array}$ & $\begin{array}{r}6.02 \mathrm{H} \\
14.2 \mathrm{M} \\
2.9 \mathrm{D} \\
39.35 \mathrm{D} \\
4.44 \mathrm{H}\end{array}$ & $\begin{array}{l}7.50 E+02 \\
1.99 E+03 \\
1.33 E+03 \\
2.83 E+03 \\
4.70 E+03\end{array}$ & $\begin{array}{l}5.78 E+02 \\
1.51 E+03 \\
1.00 E 003 \\
2.14 E+03\end{array}$ & $\begin{array}{l}.41 E+02 \\
1.87 E+03 \\
1.26 E+03 \\
2.62 E+03 \\
.43 E+03\end{array}$ & $\begin{array}{l}4.29 E+02 \\
1.20 E+03 \\
7.8 B E+02 \\
1.12 E+03 \\
2.87 E+03\end{array}$ & $\begin{array}{l}6.63 E+02 \\
1.73 E+03 \\
1.16 E+03 \\
2.42 E+03 \\
4.02 E+03\end{array}$ & $\begin{array}{l}8.05 E \cdot 02 \\
2.24 E+03 \\
1.49 E+03 \\
3.24 E+03 \\
5.40 E+03\end{array}$ & $\begin{array}{l}3.42 E+01 \\
3.37 E+03 \\
3.53 E+01 \\
1.56 E+02 \\
2.79 E+03\end{array}$ & $\begin{array}{l}8.40 E \cdot 02 \\
5.61 E \cdot 03 \\
1.53 E+03 \\
3.39 E+03 \\
8.18 E+03\end{array}$ \\
\hline $\begin{array}{l}\text { RU-1 106 } \\
\text { RH-1 03M } \\
\text { RH-1 05 } \\
\text { RH-105M } \\
\text { RH-1 } 06\end{array}$ & 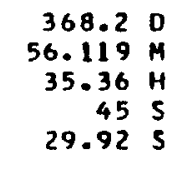 & $\begin{array}{l}0.0 \\
1.36 E+00 \\
4.49 E+02 \\
1.65 E+02 \\
1.23 E+03\end{array}$ & $\begin{array}{l}0.0 \\
1.60 E-01 \\
3.39 E+02 \\
1.23 E \cdot 02 \\
9.48 E \cdot 02\end{array}$ & $\begin{array}{l}.0 \\
.89 E-01 \\
0.21 E+02 \\
1.60 E+02 \\
.15 E+03\end{array}$ & $\begin{array}{l}0.0 \\
3.70 E-02 \\
2.71 E+02 \\
8.96 E+01 \\
1.53 E+02\end{array}$ & $\begin{array}{l}0.0 \\
9.99 E-01 \\
3.90 E+02 \\
1.45 E+02 \\
1.05 E+03\end{array}$ & $\begin{array}{l}0.0 \\
4.46 E+00 \\
5.04 E+02 \\
1.92 E+02 \\
1.41 E+03\end{array}$ & $\begin{array}{l}0.0 \\
0.0 \\
1.45 E+02 \\
2.18 E+02 \\
1.14 E+04\end{array}$ & $\begin{array}{l}0.0 \\
4.46 E * 00 \\
1.25 E \cdot 03 \\
4.11 E+02 \\
1.28 E+04\end{array}$ \\
\hline $\begin{array}{l}P D-103 \\
P D-107 \\
P D-109 \\
A G-106 M \\
A G-108\end{array}$ & 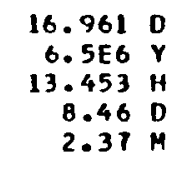 & $\begin{array}{l}1.20 E+01 \\
0.0 \\
4.03 E+00 \\
1.69 E+04 \\
1.04 E+02\end{array}$ & $\begin{array}{l}1.36 E+00 \\
0.0 \\
3.05 E+00 \\
1.32 E+04 \\
7.97 E+01\end{array}$ & $\begin{array}{l}5.16 E+00 \\
0.0 \\
3.73 E+00 \\
1.59 E+04 \\
9.70 E+01\end{array}$ & $\begin{array}{l}4.91 E-01 \\
0.0 \\
2.45 E+00 \\
1.05 E+04 \\
6.30 E+01\end{array}$ & $\begin{array}{l}8.89 E \bullet 00 \\
0.0 \\
3.45 E+00 \\
1.44 E+04 \\
8.88 E+01\end{array}$ & $\begin{array}{l}3.94 E+01 \\
0.0 \\
4.61 E \cdot 00 \\
1.94 E+04 \\
1.20 E+02\end{array}$ & $\begin{array}{l}0.0 \\
0.0 \\
2.44 E+03 \\
2.08 E+01 \\
4.54 E+03\end{array}$ & $\begin{array}{l}3.94 E+01 \\
0.0 \\
2.44 E+03 \\
1.94 E+04 \\
4.66 E+03\end{array}$ \\
\hline $\begin{array}{l}A G-108 M \\
A G-109 M \\
A G-110 \\
A G-110 M \\
A G-111\end{array}$ & 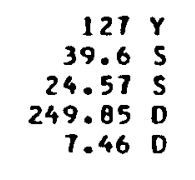 & $\begin{array}{l}1.83 E+02 \\
1.66 E+04 \\
1.53 E+02\end{array}$ & $\begin{array}{l}1.39 E+03 \\
1.62 E+01 \\
1.43 E+02 \\
1.31 E+04 \\
1.15 E+02\end{array}$ & $\begin{array}{l}8.99 E * 03 \\
2.32 E+01 \\
1.72 E+02 \\
1.57 E+04 \\
1.43 E+02\end{array}$ & & & & & $\begin{array}{l}1.11 E+04 \\
6.27 E+01 \\
9.60 E+03 \\
1.94 E+04 \\
2.52 E+03\end{array}$ \\
\hline $\begin{array}{l}C D-109 \\
C D-111 M \\
C D-113 \\
C D-113 M \\
C D-115\end{array}$ & $\begin{array}{r}464 \mathrm{O} \\
48.7 \mathrm{M} \\
9.3 \mathrm{E15} \mathrm{Y} \\
13.7 \mathrm{Y} \\
53.46 \mathrm{H}\end{array}$ & $\begin{array}{l}1.56 E+01 \\
1.63 E+03 \\
0.0 \\
0.0 \\
1.19 E+03\end{array}$ & $\begin{array}{l}2.03 E+00 \\
1.23 E+03 \\
0.0 \\
0.0 \\
9.05 E+02\end{array}$ & $\begin{array}{l}7.26 E+00 \\
1.55 E \cdot 03 \\
0.0 \\
0.0 \\
1.11 E+03\end{array}$ & $\begin{array}{l}3.23 E-01 \\
9.61 E+02 \\
0.0 \\
0.0 \\
7.24 E+02\end{array}$ & $\begin{array}{l}1.42 E+03 \\
0.0 \\
0.0 \\
1.02 E+03\end{array}$ & $\begin{array}{l}4.3 \text { TE+01 } \\
1.81 E+03 \\
0.0 \\
0.0 \\
1.37 E+03\end{array}$ & & $\begin{array}{l}4.37 E+01 \\
2.17 E+03 \\
2.82 E+02 \\
9.89 E+02 \\
3.41 E+03\end{array}$ \\
\hline $\begin{array}{l}C D-115 M \\
C D-117 \\
C D-117 M \\
\text { IN-111 } \\
\text { IN-113M }\end{array}$ & $\begin{array}{r}44.6 \mathrm{D} \\
2.49 \mathrm{H} \\
3.36 \mathrm{H} \\
2.83 \mathrm{D} \\
1.658 \mathrm{H}\end{array}$ & $\begin{array}{l}1.34 E+02 \\
6.62 E+03 \\
1.31 E+04 \\
2.27 E+03 \\
1.48 E+03\end{array}$ & $\begin{array}{l}1.07 E+02 \\
5.23 E \bullet 03 \\
1.04 E+04 \\
1.72 E+03 \\
1.11 E+03\end{array}$ & & & & & $\begin{array}{l}4.50 E+03 \\
3.03 E+03 \\
1.15 E+03 \\
1.27 E+02 \\
8.76 E+02\end{array}$ & $\begin{array}{l}4.65 E+03 \\
1.06 E+04 \\
1.61 E+04 \\
2.66 E+03 \\
2.56 E+03\end{array}$ \\
\hline $\begin{array}{l}N-114 \\
N-114 M \\
N-115 \\
N-115 M \\
N-116 M\end{array}$ & 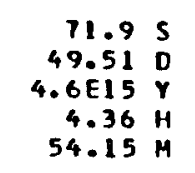 & $\begin{array}{l}1.89 E+02 \\
5.34 E+02 \\
0.0 \\
9.22 E+02 \\
1.51 E+04\end{array}$ & $\begin{array}{l}4.05 E+02 \\
0.0 \\
6.89 E+02 \\
1.21 E+04\end{array}$ & $\begin{array}{l}5.02 E+02 \\
0.0 \\
8.58 E+02 \\
1.43 E+04\end{array}$ & $\begin{array}{l}3.15 E+02 \\
0.0 \\
5.51 E+02 \\
9.67 E+03\end{array}$ & $\begin{array}{l}1.61 E+02 \\
4.59 E * 02 \\
0.0 \\
7.97 E * 02 \\
1.30 E+04\end{array}$ & $\begin{array}{l}2.17 E \cdot 02 \\
6.20 E \cdot 02 \\
0.0 \\
1.05 E+03 \\
1.74 E \cdot 04\end{array}$ & $\begin{array}{l}5.89 E * 03 \\
6.35 E * 02 \\
1.23 E * 02 \\
1.07 E * 03 \\
1.95 E * 03\end{array}$ & $\begin{array}{l}6.11 E \bullet 03 \\
1.26 E \bullet 03 \\
1.23 E \cdot 02 \\
2.12 E \cdot 03 \\
1.93 E \cdot 04\end{array}$ \\
\hline
\end{tabular}




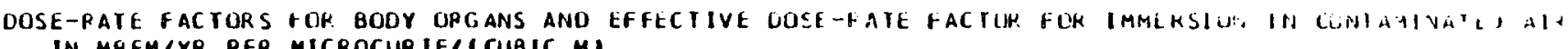

\begin{tabular}{|c|c|c|c|c|c|c|c|c|c|c|}
\hline LIOE & HALF-LIFE & ADRENALS & IODER & BRAIN & BREA & HE ART & $\begin{array}{l}\text { SMAL } \\
\text { INTESTINE }\end{array}$ & $\begin{array}{c}\text { UPPER } \\
\text { LARE } \\
\text { INTESTIIVE }\end{array}$ & $\begin{array}{l}\text { LOLW } \\
\text { LARGE } \\
\text { INESTINE }\end{array}$ & \\
\hline iit & $\begin{array}{r}43.8 \\
116.5 \\
115.10 \\
13.0\end{array}$ & 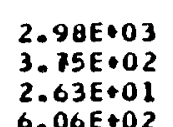 & 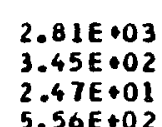 & 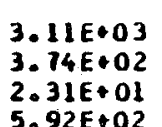 & 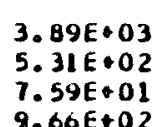 & 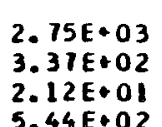 & 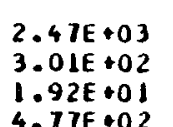 & 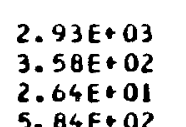 & 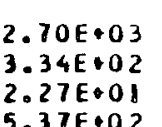 & \\
\hline & & 00 & $\begin{array}{l}5.55 E+02 \\
3.55 \times+00\end{array}$ & 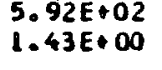 & $\begin{array}{l}9.96 \\
2.093 \\
2.03\end{array}$ & & & & & \\
\hline 12? & $\begin{array}{l}129.2 \\
9.64 \\
9.64\end{array}$ & 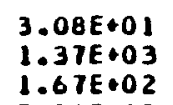 & 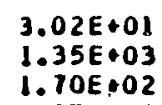 & 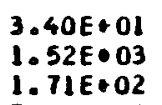 & 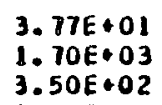 & 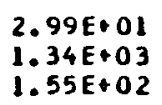 & 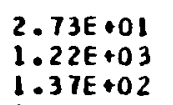 & 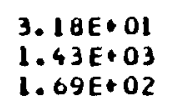 & & \\
\hline & $\begin{array}{l}2.80 \\
2.70 \\
2.70\end{array}$ & 02 & 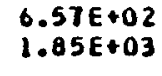 & $\left\{\begin{array}{l}\{=02 \\
E \in+03\end{array}\right.$ & $\begin{array}{l}1.10 \\
2.41 \\
2.41\end{array}$ & & 3 & i. & & \\
\hline & & $\begin{array}{l}8.39 E+03 \\
1.83 E=03 \\
1.82 E=04\end{array}$ & 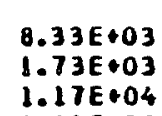 & 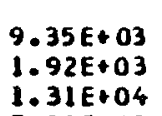 & 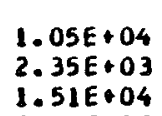 & & 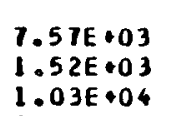 & 03 & & \\
\hline 123. & 18 & $\begin{array}{l}6.93 E E=03 \\
2.91 E+03\end{array}$ & 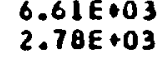 & $\begin{array}{l}7.39 \in E+03 \\
\text { 3. } 10 E+03\end{array}$ & & $\begin{array}{l}0.03 \\
03\end{array}$ & $\begin{array}{l}5.85 \\
2.46 \\
2.46\end{array}$ & $6.0^{x}$ & & \\
\hline 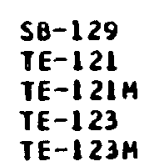 & 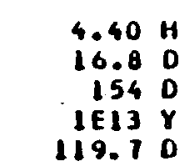 & 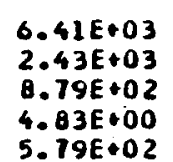 & 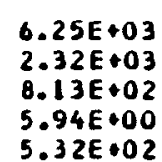 & 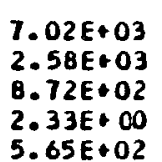 & 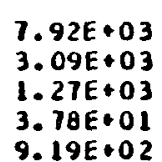 & & & & & \\
\hline . & 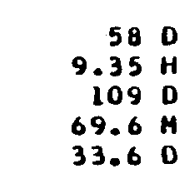 & 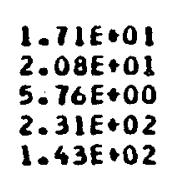 & 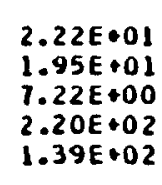 & 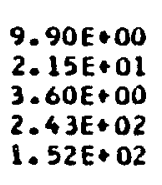 & 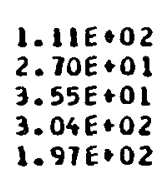 & 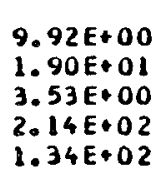 & 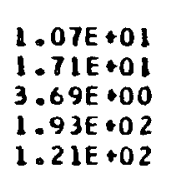 & $\begin{array}{l}2.06 \\
2.01 \\
2.04 \\
2.28 \\
1.245 \\
1.45\end{array}$ & & \\
\hline & & 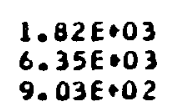 & 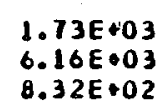 & $\begin{array}{l}0^{3} \\
0_{02}\end{array}$ & & & & & & \\
\hline & & $\begin{array}{l}4.0106=03 \\
9.966+03\end{array}$ & $\begin{array}{l}3.966 \\
9.706\end{array}$ & & & & & & & \\
\hline & & 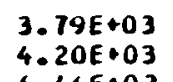 & 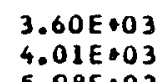 & $\begin{array}{l}3.99 \\
4.46 \\
4.46\end{array}$ & & & & & & \\
\hline & 00 & $1.89 E+01$ & 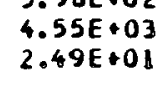 & $1.02 E+01$ & & & & & & \\
\hline & & & $\begin{array}{l}1.90 \\
3.05\end{array}$ & & & & & & & \\
\hline & & & & & & & & & & \\
\hline
\end{tabular}



DOSE-RATE FACTORS FOR BOOY ORGANS ANO EFFECTIVE DOSE-PATE FACTOR FOP. IMMEPSIOH IN CONTAMINATEI) AIR
IN MREMAYR PER MICROCUR IEIICUBIC MI

\begin{tabular}{|c|c|c|c|c|c|c|c|c|c|c|}
\hline LIDE & IFE & LIVER & LUNGS & ARROH & $\begin{array}{c}\text { RED } \\
\text { MARROW }\end{array}$ & VARIES & PANCREAS & KELETON & PLEEN & 5 TOMACH \\
\hline $\begin{array}{l}1 N-117 \\
1 N-117 M \\
S N-113 \\
S N-117 M \\
S N-119 M\end{array}$ & 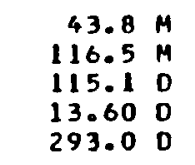 & $\begin{array}{l}80 E+03 \\
44 E+02 \\
29 E+01 \\
53 E+02 \\
43 E+00\end{array}$ & $\begin{array}{l}01 E+03 \\
13 E+02 \\
12 E+01 \\
09 E+02 \\
12 E+00\end{array}$ & $\begin{array}{l}E+03 \\
E+02 \\
E+01\end{array}$ & $\begin{array}{l}03 E+03 \\
75 E+02 \\
37 E+01 \\
72 E+02 \\
46 E+00\end{array}$ & & & & $\begin{array}{l}.74 E+03 \\
.41 E+02 \\
.26 E+01 \\
.53 E+02 \\
.41 E+00\end{array}$ & $\begin{array}{l}2.7 E E+03 \\
3.40 E+02 \\
2.33 E+01 \\
5.48 E+02 \\
2.76 E+00\end{array}$ \\
\hline $\begin{array}{l}S N-123 \\
S N-125 \\
S N-126 \\
S B-117\end{array}$ & $\begin{array}{r}129.20 \\
9.64 \\
1.085 \mathrm{Y} \\
2.80 \mathrm{H} \\
2.70 \mathrm{D}\end{array}$ & $\begin{array}{l}.01 E+01 \\
35 E \cdot 03 \\
.68 E+02 \\
53 E+02 \\
83 E+03\end{array}$ & $\begin{array}{l}19 E+01 \\
43 E \cdot 03 \\
.77 E \cdot 02 \\
16 E+02 \\
97 E+03\end{array}$ & $\begin{array}{l}39 E+01 \\
52 E+03 \\
20 E+02\end{array}$ & $\begin{array}{l}23 E+01 \\
45 E+03 \\
30 E+02 \\
91 E+02 \\
01 E+03\end{array}$ & & & & $\begin{array}{l}.91 E+01 \\
.30 E+03 \\
.59 E+02 \\
.49 E+02 \\
.79 E+03\end{array}$ & $\begin{array}{l}2.96 E+01 \\
1.33 E+03 \\
1.61 E+02 \\
6.46 E+02 \\
1.81 E+03\end{array}$ \\
\hline $\begin{array}{l}S B-126 \\
S B-126 M \\
S B-127\end{array}$ & $\begin{array}{rl}60.20 & 0 \\
2.77 & Y \\
12.4 & 0 \\
19.0 & \mathrm{M} \\
3.85 & 0\end{array}$ & $\begin{array}{l}2 E+03 \\
2 E+03 \\
16 E+04\end{array}$ & & & & & & & & $\begin{array}{l}\text { 8. } 15 E+03 \\
1.10 E+03 \\
1.15 E+04 \\
6.50 E+03 \\
2.73 E+03\end{array}$ \\
\hline & 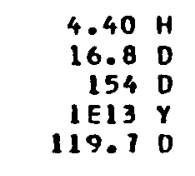 & $\begin{array}{l}6 \\
2 \\
8 \\
4 \\
5\end{array}$ & & & & & & & & $\begin{array}{l}13 E+03 \\
27 E+03 \\
97 E+02 \\
27 E+00 \\
24 E+02\end{array}$ \\
\hline $\begin{array}{l}T E-125 M \\
T E-127 \\
T E-12 T M \\
T E-129 \\
T E-129 M\end{array}$ & $\begin{array}{rr}58 & 0 \\
9.35 & H \\
109 & 0 \\
69.6 & M \\
33.6 & 0\end{array}$ & $\begin{array}{l}1.61 E+01 \\
1.94 E+01 \\
5.39 E+00 \\
2.18 E+02 \\
1.37 E+02\end{array}$ & $\begin{array}{l}19 E+01 \\
09 E+01 \\
029 E+00 \\
34 E+02\end{array}$ & $\begin{array}{l}3.64 E+01 \\
2.52 E+01 \\
1.20 E+01 \\
2.72 E+02\end{array}$ & & & & & & $\begin{array}{l}01 \\
01 \\
01 \\
00 \\
02 \\
02 \\
02\end{array}$ \\
\hline $\begin{array}{l}\text { TE-1 } 31 \\
T E-131 \mathrm{M} \\
T E-132 \\
T E-133 \\
T E-133 \mathrm{M}\end{array}$ & $\begin{array}{r}25.0 \mathrm{M} \\
30 \mathrm{H} \\
18.2 \mathrm{H} \\
12.45 \mathrm{M} \\
55.4 \mathrm{M}\end{array}$ & $\begin{array}{l}1.73 \mathrm{E}+ \\
6.14 \mathrm{E}+ \\
0.24 \mathrm{E}+ \\
3.95 \mathrm{E}+ \\
9.66 \mathrm{E}+\end{array}$ & & & & & & & & \\
\hline $\begin{array}{l}T E-134 \\
1-122 \\
1-123 \\
1-124 \\
1-125\end{array}$ & $\begin{array}{r}41.8 \mathrm{M} \\
3.62 \mathrm{M} \\
13.13 \mathrm{H} \\
4.18 \mathrm{D} \\
60.14 \mathrm{D}\end{array}$ & $\begin{array}{l}3.58 E+03 \\
3.98 E+03 \\
5.92 E+02 \\
4.53 E+03 \\
1.76 E+01\end{array}$ & $\begin{array}{l}3 \\
3 \\
2 \\
3 \\
1\end{array}$ & $\begin{array}{l}5.29 \mathrm{E} \\
4.06 \mathrm{E}\end{array}$ & & $\begin{array}{l}3.40 E+03 \\
3.82 E+03 \\
5.22 E+02 \\
4.31 E+03 \\
1.74 E+01\end{array}$ & & & & $\begin{array}{l}3.53 E+03 \\
3.93 E+03 \\
5.86 E+02 \\
4.46 E+03 \\
1.73 E+01\end{array}$ \\
\hline $\begin{array}{l}1-126 \\
1-128 \\
1-129 \\
1-130 \\
1-131\end{array}$ & $\begin{array}{r}12.93 \mathrm{D} \\
24.99 \mathrm{M} \\
1.57 E 7 \mathrm{Y} \\
12.36 \mathrm{H} \\
8.040 \mathrm{D}\end{array}$ & $\begin{array}{l}1.89 E+03 \\
3.03 E+02 \\
1.65 E+01 \\
8.97 E \cdot 03 \\
1.52 E \cdot 03\end{array}$ & $\begin{array}{l}3.26 E+02 \\
2.06 E+01 \\
9.60 E+03 \\
1.64 E \cdot 03\end{array}$ & $\begin{array}{l}2.30 E+03 \\
3.81 E+02 \\
3.51 E+01 \\
1.07 E \cdot 04 \\
1.99 E \cdot 03\end{array}$ & $\begin{array}{l}2.07 E+03 \\
3.34 E+02 \\
7.82 E+00 \\
9.19 E+03 \\
1.69 E+03\end{array}$ & $\begin{array}{l}1.82 E+03 \\
2.89 E+02 \\
1.4 B E+01 \\
8.67 E+03 \\
1.43 E+03\end{array}$ & $\begin{array}{l}9.7 \mathrm{TE}+00 \\
9.73 E+03 \\
1.32 E+03\end{array}$ & $\begin{array}{l}2.34 E+03 \\
3.87 E * 02 \\
3.62 E * 01 \\
1.09 E * 04 \\
2.02 E+03\end{array}$ & $\begin{array}{l}1.84 E+03 \\
2.97 E+02 \\
1.34 E+01 \\
8.73 E+03 \\
1.50 E+03\end{array}$ & $\begin{array}{l}1.99 E .02 \\
1.51 E .01 \\
8.86 E+03 \\
1.51 E+03\end{array}$ \\
\hline
\end{tabular}




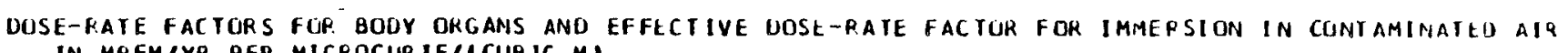

\begin{tabular}{|c|c|c|c|c|c|c|c|c|c|}
\hline NUCL SOE & HALF-LIFE & TESTES & THYMUS & THY RDID & UTERUS & EFFECIIVE & $\begin{array}{c}\text { SKIN } \\
\text { (PHOTONI }\end{array}$ & $\begin{array}{c}\text { SKIN } \\
\text { (ELECTRON I }\end{array}$ & $\begin{array}{l}\text { SKIN } \\
\text { ITOTALS }\end{array}$ \\
\hline $\begin{array}{l}I N-117 \\
1 N-117 M \\
S N-113 \\
S N-117 M \\
S N-119 M\end{array}$ & $\begin{array}{r}43.8 \mathrm{~N} \\
116.5 \mathrm{~N} \\
115.1 \\
13.60 \\
293.0 \mathrm{D}\end{array}$ & $\begin{array}{l}4.06 E+03 \\
5.13 E+02 \\
5.29 E+01 \\
8.59 E+02 \\
1.56 E+01\end{array}$ & $\begin{array}{l}3.10 E+03 \\
3.86 E+02 \\
2.66 E+01 \\
6.47 E+02 \\
3.43 E+00\end{array}$ & $\begin{array}{l}3.82 E \cdot 03 \\
4.85 E \cdot 02 \\
4.01 E \cdot 01 \\
8.32 E \cdot 02 \\
8.56 E \cdot 00\end{array}$ & $\begin{array}{l}2.44 E+03 \\
3.03 E+02 \\
1.81 E+01 \\
4.86 E+02 \\
7.22 E-01\end{array}$ & $\begin{array}{l}3.50 E+03 \\
4.47 E+02 \\
4.19 E+01 \\
7.54 E+02 \\
1.09 E+01\end{array}$ & $\begin{array}{l}4.61 E+03 \\
5.78 E \bullet 02 \\
8.97 E+01 \\
9.55 E \bullet 02 \\
3.62 E+01\end{array}$ & $\begin{array}{l}1.55 E+03 \\
3.12 E+03 \\
0.0 \\
4.82 E+02 \\
0.0\end{array}$ & $\begin{array}{l}6.16 E+03 \\
3.69 E+03 \\
8.97 E+01 \\
1.44 E+03 \\
3.62 E+01\end{array}$ \\
\hline $\begin{array}{l}S N-123 \\
S N-125 \\
S N-126 \\
S B-117 \\
S B-122\end{array}$ & 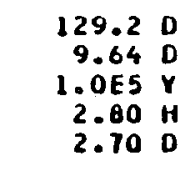 & $\begin{array}{l}4.21 E+01 \\
1.88 E+03 \\
2.87 E+02 \\
1.00 E+03 \\
2.63 E+03\end{array}$ & $\begin{array}{l}3.38 E+01 \\
1.50 E+03 \\
2.15 E+02 \\
7.56 E+02 \\
2.02 E+03\end{array}$ & $\begin{array}{l}4.02 E+01 \\
1.78 E+03 \\
2.80 E+02 \\
9.65 E+02 \\
2.45 E+03\end{array}$ & $\begin{array}{l}2.64 E+01 \\
1.19 E+03 \\
1.37 E+02 \\
5.72 E+02 \\
1.60 E+03\end{array}$ & $\begin{array}{l}3.60 E+01 \\
1.61 E+03 \\
2.44 E+02 \\
8.76 E+02 \\
2.25 E+03\end{array}$ & $\begin{array}{l}4.86 E+01 \\
2.17 E+03 \\
3.25 E+02 \\
1.13 E+03 \\
3.02 E+03\end{array}$ & $\begin{array}{l}3.91 E+03 \\
6.36 E+03 \\
1.41 E+02 \\
8.22 E+01 \\
4.19 E+03\end{array}$ & $\begin{array}{l}3.96 E+03 \\
8.53 E+03 \\
4.65 E+02 \\
1.21 E+03 \\
1.20 E+03\end{array}$ \\
\hline $\begin{array}{l}S B-124 \\
S B-125 \\
S B-126 \\
S B-126 M \\
S B-127\end{array}$ & 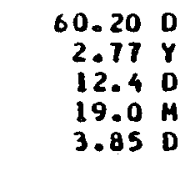 & $\begin{array}{l}1.16 E+04 \\
2.50 E+03 \\
1.65 E+04 \\
9.40 E+03 \\
3.95 E+03\end{array}$ & $\begin{array}{l}9.10 E+03 \\
1.89 E+03 \\
1.29 E+04 \\
7.27 E+03 \\
3.06 E+03\end{array}$ & $\begin{array}{l}1.08 E+04 \\
2.32 E \cdot 03 \\
1.56 E \cdot 04 \\
8.83 E+03 \\
3.71 E \bullet 03\end{array}$ & $\begin{array}{l}7.39 E+03 \\
1.50 E+03 \\
1.01 E+04 \\
5.73 E+03 \\
2.41 E+03\end{array}$ & $\begin{array}{l}9.95 E+03 \\
2.13 E+03 \\
1.41 E+04 \\
8.04 E+03 \\
3.38 E+03\end{array}$ & $\begin{array}{l}1.33 E+04 \\
2.87 E+03 \\
1.90 E+04 \\
1.08 E+04 \\
4.54 E+03\end{array}$ & $\begin{array}{l}2.63 E+03 \\
3.36 E+02 \\
1.98 E+03 \\
4.43 E+03 \\
2.05 E+03\end{array}$ & $\begin{array}{l}1.59 E+04 \\
3.21 E * 03 \\
2.10 E+04 \\
1.52 E+04 \\
6.58 E+03\end{array}$ \\
\hline $\begin{array}{l}S B-129 \\
T E-121 \\
T E-121 M \\
T E-123 \\
T E-123 M\end{array}$ & 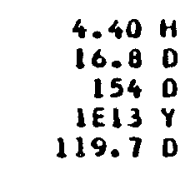 & $\begin{array}{l}8.76 E+03 \\
3.33 E+03 \\
1.21 E+03 \\
2.17 E+01 \\
8.20 E+02\end{array}$ & $\begin{array}{l}6.93 E+03 \\
2.52 E+03 \\
9.1 \theta E+02 \\
6.10 E+00 \\
6.20 E+02\end{array}$ & $\begin{array}{l}8.29 E * 03 \\
3.08 E+03 \\
1.15 E * 03 \\
1.30 E * 01 \\
7.96 E+02\end{array}$ & $\begin{array}{l}5.46 E+03 \\
2.00 E+03 \\
7.11 E+02 \\
1.41 E+00 \\
4.64 E+02\end{array}$ & $\begin{array}{l}7.50 E+03 \\
2.84 E+03 \\
1.05 E+03 \\
1.51 E+01 \\
7.20 E+02\end{array}$ & $\begin{array}{l}1.01 E+04 \\
3.85 E+03 \\
1.36 E+03 \\
4.50 E+01 \\
9.07 E+02\end{array}$ & $\begin{array}{l}2.43 E+03 \\
1.89 E+01 \\
6.94 E+01 \\
0.0 \\
8.17 E+01\end{array}$ & $\begin{array}{l}1.25 E+04 \\
3.87 E \bullet 03 \\
1.43 E+03 \\
4.50 E+01 \\
9.89 E+02\end{array}$ \\
\hline $\begin{array}{l}\text { TE-125M } \\
\text { TE-127 } \\
\text { TE-127M } \\
\text { TE-129 } \\
\text { IE-129M }\end{array}$ & $\begin{array}{rr}58 & 0 \\
9.35 & H \\
109 & 0 \\
69.6 & 4 \\
33.6 & 0\end{array}$ & $\begin{array}{l}6.84 E+01 \\
2.81 E+01 \\
2.19 E+01 \\
3.19 E+02 \\
2.05 E+02\end{array}$ & $\begin{array}{l}2.45 E+01 \\
2.12 E+01 \\
7.98 E+00 \\
2.40 E+02 \\
1.53 E+02\end{array}$ & $\begin{array}{l}4.46 E \bullet 01 \\
2.62 E * 01 \\
1.44 E \bullet 01 \\
2.95 E \bullet 02 \\
1.89 E+02\end{array}$ & $\begin{array}{l}7.14 E+00 \\
1.70 E+01 \\
2.56 E+00 \\
1.90 E+02 \\
1.17 E+02\end{array}$ & $\begin{array}{l}4.84 E+01 \\
2.42 E+01 \\
1.56 E+01 \\
2.12 E+02 \\
1.12 E+02\end{array}$ & $\begin{array}{l}1.28 E+02 \\
3.18 E+01 \\
4.09 E+01 \\
3.71 E+02 \\
2.48 E+02\end{array}$ & & $\begin{array}{l}2.15 E+02 \\
1.33 E+03 \\
8.88 E+01 \\
4.18 E+03 \\
1.90 E+03\end{array}$ \\
\hline $\begin{array}{l}\text { IE-131 } \\
\text { IE-131M } \\
T E-132 \\
T E-133 \\
T E-133 M\end{array}$ & $\begin{array}{r}25.0 \mathrm{M} \\
30 \mathrm{H} \\
78.2 \mathrm{H} \\
12.45 \mathrm{M} \\
55.4 \mathrm{M}\end{array}$ & $\begin{array}{l}2.50 E+03 \\
8.68 E+03 \\
1.26 E+03 \\
5.59 E+03 \\
1.36 E+04\end{array}$ & $\begin{array}{l}1.94 E+03 \\
6.85 E+03 \\
9.40 E+02 \\
4.37 E+03 \\
1.08 E+04\end{array}$ & $\begin{array}{l}2.38 E \bullet 03 \\
8.23 E * 03 \\
1.19 E * 03 \\
5.27 E * 03 \\
1.29 E \bullet 04\end{array}$ & $\begin{array}{l}1.51 E+03 \\
5.37 E+03 \\
7.22 E+02 \\
3.49 E+03 \\
8.47 E+03\end{array}$ & & & & $\begin{array}{l}8.15 E+03 \\
1.09 E \bullet 04 \\
1.59 E \bullet 03 \\
1.27 E \bullet 04 \\
2.10 E+04\end{array}$ \\
\hline $\begin{array}{l}1 E-134 \\
1-122 \\
1-123 \\
1-124 \\
1-125\end{array}$ & $\begin{array}{r}41.8 \mathrm{M} \\
3.62 \mathrm{M} \\
13.13 \mathrm{H} \\
4.18 \\
60.14 \\
0\end{array}$ & $\begin{array}{l}5.17 E+03 \\
5.72 E+03 \\
9.25 E+02 \\
6.41 E+03 \\
7.93 E+01\end{array}$ & $\begin{array}{l}3.99 E+03 \\
4.35 E+03 \\
6.90 E+02 \\
4.97 E+03 \\
2.72 E+01\end{array}$ & $\begin{array}{l}4.88 E+03 \\
5.31 E+03 \\
8.06 E+02 \\
5.97 E+03 \\
5.08 E+01\end{array}$ & $\begin{array}{l}3.12 E+03 \\
3.48 E+03 \\
5.18 E+02 \\
3.99 E+03 \\
7.17 E+00\end{array}$ & $\begin{array}{l}4.44 E+03 \\
4.89 E+03 \\
8.05 E+02 \\
5.49 E+03 \\
5.57 E+01\end{array}$ & $\begin{array}{l}5.91 E+03 \\
6.56 E+03 \\
1.04 E+03 \\
7.36 E+03 \\
1.51 E+02\end{array}$ & $\begin{array}{l}5.08 E+02 \\
8.2 \theta E+03 \\
6.23 E+01 \\
1.52 E+03 \\
0.0\end{array}$ & $\begin{array}{l}6.42 E+03 \\
1.48 E+04 \\
1.10 E+03 \\
8.88 E+03 \\
1.51 E+02\end{array}$ \\
\hline $\begin{array}{l}1-126 \\
1-128 \\
1-129 \\
1-130 \\
1-131\end{array}$ & $\begin{array}{r}12.93 \mathrm{O} \\
24.99 \mathrm{H} \\
1.57 \mathrm{Y} \\
12.36 \mathrm{H} \\
8.040 \mathrm{D}\end{array}$ & $\begin{array}{l}2.72 E+03 \\
4.39 E+02 \\
5.85 E+01 \\
1.28 E+04 \\
2.22 E+03\end{array}$ & $\begin{array}{l}2.09 E+03 \\
3.31 E+02 \\
2.61 E+01 \\
9.93 E+03 \\
1.08 E+03\end{array}$ & $\begin{array}{l}2.55 E+03 \\
4.07 E * 02 \\
4.15 E * 01 \\
1.20 E * 04 \\
2.07 E \nLeftarrow 03\end{array}$ & $\begin{array}{l}1.65 E+03 \\
2.65 E+02 \\
0.16 E+00 \\
7.82 E+03 \\
1.34 E+03\end{array}$ & $\begin{array}{l}2.33 E+03 \\
3.16 E+02 \\
4.17 E+01 \\
1.09 E+04 \\
1.91 E+03\end{array}$ & $\begin{array}{l}3.14 E \cdot 03 \\
5.02 E+02 \\
9.79 E+01 \\
1.47 E+04 \\
2.51 E+03\end{array}$ & $\begin{array}{l}8.14 E+02 \\
5.83 E+03 \\
1.78 E+01 \\
1.82 E+03 \\
1.01 E+03\end{array}$ & $\begin{array}{l}4.01 E+03 \\
6.34 E+03 \\
1.16 E+02 \\
1.65 E+04 \\
3.52 E+03\end{array}$ \\
\hline
\end{tabular}


OOSE-PATE FACTOPS FOR BODY URGANS ANO EFFECTIVE DOSE-RATE FACTOR FOR IMMERSION IN CONTAMINATEI AIR

\begin{tabular}{|c|c|c|c|c|c|c|c|c|c|c|}
\hline CLIDE & HALF-LIFE & ADRENALS & BLADDER & BRAIN & BREAST & HE AP.T & $\begin{array}{l}\text { SMALL } \\
\text { INTESTINE }\end{array}$ & $\begin{array}{c}\text { UPPER } \\
\text { LARGE } \\
\text { INTESTINEE }\end{array}$ & $\begin{array}{c}\text { LOHER } \\
\text { LARGE } \\
\text { INTESTINEE }\end{array}$ & KIONEYS \\
\hline $\begin{array}{l}1-132 \\
1-133 \\
1-134 \\
1-135 \\
1-136\end{array}$ & 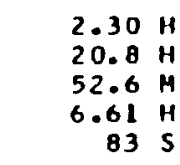 & $\begin{array}{l}1.02 E+04 \\
2.64 E+03 \\
1.18 E+04 \\
7.03 E+03 \\
1.17 E+04\end{array}$ & $\begin{array}{l}9.87 E+03 \\
2.52 E+03 \\
1.15 E+04 \\
1.01 E+03 \\
1.19 E+04\end{array}$ & $\begin{array}{l}1.11 E+04 \\
2.82 E+03 \\
1.29 E+04 \\
7.89 E+03 \\
1.33 E+04\end{array}$ & $\begin{array}{l}.26 E+04 \\
.30 E+03 \\
.45 E+04 \\
.78 E+03 \\
.49 E+04\end{array}$ & $\begin{array}{l}9.12 E+03 \\
2.47 E+03 \\
1.13 E+04 \\
6.95 E+03 \\
1.18 E+04\end{array}$ & $\begin{array}{l}8.01 E+03 \\
2.23 E+03 \\
1.03 E+04 \\
.40 E+03 \\
1.10 E+04\end{array}$ & $\begin{array}{l}1.03 E+04 \\
2.63 E+03 \\
1.21 E+04 \\
1.39 E+03 \\
1.26 E+04\end{array}$ & $\begin{array}{l}9.52 E+03 \\
2.42 E+03 \\
1.11 E+04 \\
6.81 E+03 \\
1.16 E * 04\end{array}$ & $\begin{array}{l}1.03 E+04 \\
2.63 E+03 \\
1.20 E+04 \\
7.39 E+03 \\
1.27 E * 04\end{array}$ \\
\hline 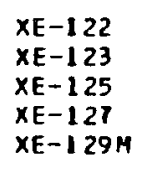 & $\begin{array}{r}20.1 H \\
2.14 \\
16.8 \\
36.406 \\
8.89 \\
8\end{array}$ & $\begin{array}{l}2.40 E+02 \\
2.68 E+03 \\
1.04 E+03 \\
1.10 E+03 \\
6.53 E+01\end{array}$ & $\begin{array}{l}2.28 E+02 \\
2.59 E+03 \\
9.70 E+02 \\
1.01 E+03 \\
7.16 E+01\end{array}$ & $\begin{array}{l}2.38 E+02 \\
2.87 E+03 \\
1.04 E+03 \\
1.08 E+03 \\
5.46 E * 01\end{array}$ & $\begin{array}{l}3.86 E+02 \\
3.54 E+03 \\
1.53 E+03 \\
1.62 E+03 \\
2.07 E+02\end{array}$ & $\begin{array}{l}2.14 E+02 \\
2.54 E+03 \\
9.35 E+02 \\
9.77 E+02 \\
5.10 E+01\end{array}$ & $\begin{array}{l}.92 E+02 \\
.30 E+03 \\
.39 E+02\end{array}$ & $\begin{array}{l}2.33 E+02 \\
2.71 E+03 \\
1.00 E+03 \\
1.04 E+03 \\
6.63 E+01\end{array}$ & $\begin{array}{l}2.14 E+02 \\
2.50 E+03 \\
9.30 E \div 02 \\
9.72 E \div 02 \\
5.62 E+01\end{array}$ & $\begin{array}{l}2.44 E+02 \\
2.71 E+03 \\
1.01 E+03 \\
1.05 E+03 \\
9.31 E+01\end{array}$ \\
\hline $\begin{array}{l}E-131 M \\
E-133 \\
E-133 M \\
E-135 \\
E-135 M\end{array}$ & $\begin{array}{rl}11.84 & 0 \\
5.245 & 0 \\
2.19 & 0 \\
9.11 & 4 \\
15.36 & M\end{array}$ & $\begin{array}{l}2.32 E+01 \\
1.13 E+02 \\
1.13 E+02 \\
1.06 E+03 \\
1.85 E+03\end{array}$ & $\begin{array}{l}2.55 E+01 \\
1.20 E+02 \\
1.08 E+02 \\
9.76 E+02 \\
1.76 E+03\end{array}$ & $\begin{array}{l}1.90 E * 01 \\
1.15 E * 02 \\
1.07 E * 02 \\
1.06 E * 03 \\
1.96 E * 03\end{array}$ & & $\begin{array}{l}1.79 E+01 \\
1.04 E+02 \\
9.16 E * 01 \\
9.51 E * 02 \\
1.71 E \cdot 03\end{array}$ & $\begin{array}{l}1.72 E+01 \\
9.31 E+01 \\
8.87 E+01\end{array}$ & $\begin{array}{l}2.38 E+01 \\
1.16 E+02 \\
1.08 E+02\end{array}$ & $\begin{array}{l}1.99 E \cdot 01 \\
1.01 E+02 \\
9.94 E+01 \\
9.45 E+02 \\
1.68 E+03\end{array}$ & $\begin{array}{l}\text { 3. } 38 E+01 \\
1.31 E+02 \\
1.19 E+02 \\
1.01 E+03 \\
1.82 E+03\end{array}$ \\
\hline $\begin{array}{l}X E-137 \\
X E-138 \\
C S-126 \\
C S-129 \\
C S-1131\end{array}$ & $\begin{array}{r}3.83 M \\
14.13 \mathrm{M} \\
1.64 \mathrm{M} \\
32.06 \mathrm{H} \\
9.6880\end{array}$ & $\begin{array}{l}8.16 E+02 \\
5.22 E+03 \\
4.87 E+03 \\
1.10 E+03 \\
1.28 E+01\end{array}$ & $\begin{array}{l}7.83 E+02 \\
5.25 E+03 \\
4.63 E+03 \\
1.03 E+03 \\
1.75 E+01\end{array}$ & $\begin{array}{l}0.72 E+02 \\
5.86 E+03 \\
5.16 E+03 \\
1.12 E \bullet 03 \\
7.56 E \bullet 00\end{array}$ & $\begin{array}{l}1.04 E+03 \\
6.11 E+03 \\
6.16 E+03 \\
1.51 E+03 \\
7.87 E+01\end{array}$ & $\begin{array}{l}7.68 E * 02 \\
5.22 E \cdot 03 \\
4.53 E+03 \\
9.95 E+02 \\
7.52 E * 00\end{array}$ & & & & \\
\hline $\begin{array}{l}C S-132 \\
C S-134 \\
C S-134 M \\
C S-135 \\
C S-136\end{array}$ & $\begin{array}{r}6.4750 \\
2.062 Y \\
2.90 \mathrm{H} \\
2.316 \% \\
13.160\end{array}$ & $\begin{array}{l}3.08 E+03 \\
6.90 E+03 \\
7.49 E+01 \\
0.0 \\
9.63 E+03\end{array}$ & $\begin{array}{l}2.96 E+03 \\
6.63 E+03 \\
7.28 E+01 \\
0.0 \\
9.34 E+03\end{array}$ & $\begin{array}{l}3.30 E+03 \\
7.43 E+03 \\
7.21 E+01 \\
0.0 \\
1.05 E+04\end{array}$ & $\begin{array}{l}3.86 E+03 \\
0.50 E+03 \\
1.49 E+02 \\
0.0 \\
1.20 E+04\end{array}$ & $\begin{array}{l}2.89 E * 03 \\
6.52 E * 03 \\
6.67 E * 01 \\
0.0 \\
9.21 E \bullet 03\end{array}$ & $\begin{array}{l}2.81 E+03 \\
5.89 E \cdot 03 \\
5.88 E+01 \\
0.0 \\
8.36 E+03\end{array}$ & $\begin{array}{l}3.08 E+03 \\
6.93 E \times 03 \\
7.44 E+01 \\
0.0 \\
9.80 E \bullet 03\end{array}$ & $\begin{array}{l}2.83 E \cdot 03 \\
6.38 E \cdot 03 \\
6.64 E \cdot 01 \\
0.0 \\
9.02 E \bullet 03\end{array}$ & $\begin{array}{l}\text { 3. } 09 E \cdot 03 \\
6.91 E+03 \\
1.88 E \div 01 \\
0.0 \\
9.77 E \bullet 03\end{array}$ \\
\hline $\begin{array}{l}C 5-137 \\
C S-138 \\
C S-139 \\
B A-131 \\
B A-133\end{array}$ & $\begin{array}{rl}30.17 Y \\
32.2 & M \\
9.40 & M \\
11.8 & 0 \\
10.5 & Y\end{array}$ & $\begin{array}{l}0.0 \\
1.07 E+04 \\
1.39 E+03 \\
1.92 E+03 \\
1.52 E+03\end{array}$ & $\begin{array}{l}0.0 \\
1.07 E+04 \\
1.41 E+03 \\
1.82 E+03 \\
1.43 E+03\end{array}$ & $\begin{array}{l}0.0 \\
1.20 E+04 \\
1.58 E+03 \\
1.99 E+03 \\
1.54 E * 03\end{array}$ & $\begin{array}{l}0.0 \\
1.35 E+04 \\
1.76 E+03 \\
2.62 E+03 \\
2.18 E+03\end{array}$ & $\begin{array}{l}0.0 \\
1.06 E+04 \\
1.40 E+03 \\
1.76 E+03 \\
1.37 E+03\end{array}$ & $\begin{array}{l}0.0 \\
9.82 E \bullet 03 \\
1.30 E \bullet 03 \\
1.5 B E \bullet 03 \\
1.23 E \bullet 03\end{array}$ & $\begin{array}{l}0.0 \\
1.13 E+04 \\
1.49 E+03 \\
1.88 E+03 \\
1.46 E+03\end{array}$ & $\begin{array}{l}0.0 \\
1.04 E+04 \\
1.3 .8 E \cdot 03 \\
1.74 E+03 \\
1.36 E+03\end{array}$ & $\begin{array}{l}0.0 \\
1.13 E+04 \\
1.50 E+03 \\
1.89 E+03 \\
1.50 E+03\end{array}$ \\
\hline $\begin{array}{l}B A-133 M \\
8 A-135 M \\
B A-137 M \\
B A-139 \\
B A-140\end{array}$ & $\begin{array}{r}38.9 \mathrm{H} \\
28.7 \mathrm{H} \\
2.552 \mathrm{M} \\
83.1 \mathrm{H} \\
12.789 \mathrm{O}\end{array}$ & $\begin{array}{l}2.27 E \cdot 02 \\
1.98 E+02 \\
2.64 E+03 \\
1.48 E+02 \\
8.04 E+02\end{array}$ & $\begin{array}{l}2.15 E+02 \\
1.08 E+02 \\
2.52 E+03 \\
1.37 E+02 \\
7.61 E+02\end{array}$ & $\begin{array}{l}2.21 E+02 \\
1.92 E+02 \\
2.63 E+03 \\
1.48 E+02 \\
8.42 E+02\end{array}$ & $\begin{array}{l}3.59 E+02 \\
3.21 E+02 \\
3.26 E+03 \\
2.20 E+02 \\
1.04 E+03\end{array}$ & $\begin{array}{l}2.00 E+02 \\
1.73 E+02 \\
2.48 E+03 \\
1.35 E+02 \\
1.42 E+02\end{array}$ & $\begin{array}{l}1.81 E+02 \\
1.58 E+02 \\
2.24 E+03 \\
1.20 E * 02 \\
6.6 \mathrm{VE} \cdot 02\end{array}$ & $\begin{array}{l}2.17 E+02 \\
1.89 E+02 \\
2.64 E+03 \\
1.44 E+02 \\
7.89 E+02\end{array}$ & $\begin{array}{l}2.01 E+02 \\
1.75 E+02 \\
2.43 E+03 \\
1.33 E+02 \\
7.29 E \cdot 02\end{array}$ & $\begin{array}{l}2.30 E+02 \\
2.03 E+02 \\
2.63 E * 03 \\
1.42 E+02 \\
1.90 E \cdot 02\end{array}$ \\
\hline $\begin{array}{l}B A-142 \\
L A-140 \\
L A-141 \\
L A-142\end{array}$ & $\begin{array}{r}18.27 \mathrm{H} \\
10.70 \mathrm{M} \\
40.22 \mathrm{H} \\
3.94 \\
95.4 \mathrm{H}\end{array}$ & $\begin{array}{l}3.90 E+03 \\
3.98 E+03 \\
1.03 E+04 \\
1.87 E+02 \\
1.30 E+04\end{array}$ & $\begin{array}{l}3.75 E \bullet 03 \\
3.87 E \bullet 03 \\
1.03 E \bullet 04 \\
1.88 E \bullet 02 \\
1.32 E * 04\end{array}$ & $\begin{array}{l}4.16 E+03 \\
4.33 E * 03 \\
1.16 E * 04 \\
2.12 E+02 \\
1.47 E+04\end{array}$ & $\begin{array}{l}5.04 E+03 \\
5.00 E+03 \\
1.30 E+04 \\
2.34 E+02 \\
1.67 E+04\end{array}$ & $\begin{array}{l}3.69 E+03 \\
3.01 E+03 \\
1.02 E+04 \\
1.86 E+02 \\
1.32 E+04\end{array}$ & $\begin{array}{l}3.35 E+03 \\
3.47 E 003 \\
9.43 E+03 \\
1.72 E 02 \\
1.23 E+04\end{array}$ & $\begin{array}{l}3.92 E+03 \\
4.06 E+03 \\
1.09 E+04 \\
1.98 E+02 \\
1.40 E+04\end{array}$ & $\begin{array}{l}3.63 E+03 \\
3.74 E+03 \\
1.00 E+04 \\
1.83 E+02 \\
1.30 E \div 04\end{array}$ & $\begin{array}{l}3.91 E \div 03 \\
4.05 E * 03 \\
1.09 E * 04 \\
1.98 E+02 \\
1.42 E * 04\end{array}$ \\
\hline
\end{tabular}


COSE-PATE FACTORS FOR BODY ORGANS AND EFFECTIVE DOSE-RATE FACTCR FOR IMMERSIOM IN CONT GAIVATEU AI?

\begin{tabular}{|c|c|c|c|c|c|c|c|c|c|c|}
\hline NUCL IDE & LF-LIFE & LIVER & LUNGS & MARROH & $\begin{array}{l}\text { RED } \\
\text { MARROW }\end{array}$ & OVARIES & INCREAS & ELETON & SPLEEN & stou \\
\hline $\begin{array}{l}1-132 \\
1-133 \\
1-134 \\
1-135 \\
1-136\end{array}$ & $\begin{array}{rl}2.30 & H \\
20.8 & H \\
52.6 & H \\
6.61 & H \\
83 & S\end{array}$ & $\begin{array}{l}9.81 E+03 \\
2.51 E+03 \\
1.14 E+04 \\
7.00 E+03 \\
1.19 E+04\end{array}$ & $\begin{array}{l}1.05 E * 04 \\
2.69 E * 03 \\
1.22 E * 04 \\
7.41 E * 03 \\
1.25 E * 04\end{array}$ & $\begin{array}{l}1.15 E+04 \\
3.04 E+03 \\
1.32 E \cdot 04 \\
7.82 E+03 \\
1.30 E+04\end{array}$ & $\begin{array}{l}1.06 E+04 \\
2.74 E+03 \\
1.23 E+04 \\
7.49 E+03 \\
1.27 E+04\end{array}$ & $\begin{array}{l}9.48 E * 03 \\
2.42 E+03 \\
1.10 E+04 \\
6.61 E+03 \\
1.10 E * 04\end{array}$ & $\begin{array}{l}8.47 E \cdot 03 \\
2.16 E \cdot 03 \\
9.87 E \cdot 03 \\
6.07 E * 03 \\
1.04 E \cdot 04\end{array}$ & $\begin{array}{l}1.16 E * 04 \\
3.08 E * 03 \\
1.34 E * 04 \\
7.95 E * 03 \\
1.32 E * 04\end{array}$ & $\begin{array}{l}9.52 E+03 \\
2.45 E+03 \\
1.11 E+04 \\
6.76 E+03 \\
1.15 E+04\end{array}$ & $\begin{array}{l}9.69 \mathrm{E} \\
2.48 \mathrm{E} \\
1.13 \mathrm{E} \\
6.85 \mathrm{E} \\
1.17 \mathrm{E}\end{array}$ \\
\hline $\begin{array}{l}X E-122 \\
X E-123 \\
\times E-125 \\
x E-127 \\
X E-129 M\end{array}$ & $\begin{array}{r}20.1 \mathrm{H} \\
2.14 \mathrm{H} \\
16.8 \mathrm{H} \\
36.406 \mathrm{D} \\
8.89 \mathrm{D}\end{array}$ & $\begin{array}{l}2.22 E+02 \\
2.58 E+03 \\
9.60 E+02 \\
1.00 E+03 \\
6.26 E+01\end{array}$ & $\begin{array}{l}2.43 E+02 \\
2.76 E+03 \\
1.04 E+03 \\
1.09 E+03 \\
1.29 E+01\end{array}$ & $\begin{array}{l}3.17 E * 02 \\
3.21 E \bullet 03 \\
1.38 E \bullet 03 \\
1.49 E \bullet 03 \\
1.12 E \bullet 02\end{array}$ & $\begin{array}{l}2.37 E * 02 \\
2.77 E \bullet 03 \\
1.03 E \bullet 03 \\
1.08 E \bullet 03 \\
5.18 E+01\end{array}$ & $\begin{array}{l}2.05 E+02 \\
2.42 E+03 \\
8.67 E+02 \\
8.94 E+02 \\
5.58 E+01\end{array}$ & $\begin{array}{l}1.8 B E \bullet 02 \\
2.22 E \bullet 03 \\
8.18 E \bullet 02 \\
8.54 E+02 \\
4.68 E \bullet 01\end{array}$ & $\begin{array}{l}3.23 E+02 \\
3.27 E+03 \\
1.41 E+03 \\
1.52 E+03 \\
1.15 E+02\end{array}$ & $\begin{array}{l}2.18 E+02 \\
2.51 E+03 \\
9.47 E+02 \\
9.93 E+02 \\
5.77 E+01\end{array}$ & $\begin{array}{l}2.191 \\
2.54 \\
9.47 \\
9.90 \\
6.02\end{array}$ \\
\hline $\begin{array}{l}X E-131 M \\
\times E-133 \\
\times E-133 M \\
\times E-135 \\
X E-135 M\end{array}$ & $\begin{array}{rl}11.84 & 0 \\
5.245 & 0 \\
2.19 & 0 \\
9.11 & H \\
15.36 & H\end{array}$ & $\begin{array}{l}2.21 E \cdot 01 \\
1.16 E \cdot 02 \\
1.04 E \cdot 02 \\
9.12 E \cdot 02 \\
1.74 E \cdot 03\end{array}$ & $\begin{array}{l}2.61 E * 01 \\
1.30 E+02 \\
1.15 E * 02 \\
1.05 E \cdot 03 \\
1.07 E \cdot 03\end{array}$ & $\begin{array}{l}4.10 E+01 \\
2.24 E+02 \\
1.58 E+02 \\
1.37 E \cdot 03 \\
2.15 E \cdot 03\end{array}$ & $\begin{array}{l}1.77 E+01 \\
8.49 E+01 \\
1.08 E+02 \\
1.07 E+03 \\
1.91 E+03\end{array}$ & $\begin{array}{l}1.98 E \cdot 01 \\
9.74 E \cdot 01 \\
9.27 E \cdot 01 \\
8.76 E+02 \\
1.68 E+03\end{array}$ & $\begin{array}{l}1.64 E+01 \\
8.12 E \cdot 01 \\
8.68 E \cdot 01 \\
8.37 E+02 \\
1.50 E+03\end{array}$ & & $\begin{array}{l}2.04 E+01 \\
1.08 E+02 \\
1.02 E+02 \\
9.03 E+02 \\
1.70 E+03\end{array}$ & \\
\hline $\begin{array}{l}X E-137 \\
X E-138 \\
C S-126 \\
C S-129 \\
C S-131\end{array}$ & $\begin{array}{r}3.83 \mathrm{M} \\
14.13 \mathrm{H} \\
1.64 \mathrm{H} \\
32.06 \mathrm{H} \\
9.68 \mathrm{D}\end{array}$ & $\begin{array}{l}1.00 E+02 \\
5.27 E+03 \\
4.61 E+03 \\
1.02 E+03 \\
1.30 E+01\end{array}$ & $\begin{array}{l}8.35 E+02 \\
5.56 E+03 \\
4.94 E+03 \\
1.10 E+03 \\
1.68 E+01\end{array}$ & $\begin{array}{l}9.50 E+02 \\
5.95 E+03 \\
5.68 E+03 \\
1.34 E+03 \\
2.83 E+01\end{array}$ & $\begin{array}{l}8.54 E * 02 \\
5.64 E \bullet 03 \\
5.05 E * 03 \\
1.12 E \bullet 03 \\
6.22 E \bullet 00\end{array}$ & $\begin{array}{l}7.39 E+02 \\
4.83 E+03 \\
4.40 E+03 \\
9.60 E+02 \\
1.21 E+01\end{array}$ & $\begin{array}{l}6.74 E+02 \\
4.57 E+03 \\
3.97 E+03 \\
8.77 E+02 \\
7.93 E+00\end{array}$ & & & \\
\hline $\begin{array}{l}\mathrm{CS}-132 \\
\mathrm{CS}-134 \\
\mathrm{CS}-134 \mathrm{1} \\
\mathrm{CS}-135 \\
\mathrm{CS}-136\end{array}$ & $\begin{array}{rl}t .475 & 0 \\
2.062 & Y \\
2.90 & H \\
2.3 E 6 & Y \\
13.16 & 0\end{array}$ & $\begin{array}{l}2.93 E \cdot 03 \\
6.5 B E \cdot 03 \\
7.04 E \cdot 01 \\
0.0 \\
9.29 E \cdot 03\end{array}$ & $\begin{array}{l}3.14 E \cdot 03 \\
7.04 E * 03 \\
7.07 E * 01 \\
0.0 \\
9.91 E * 03\end{array}$ & $\begin{array}{l}3.50 E+03 \\
7.78 E \cdot 03 \\
1.24 E+02 \\
0.0 \\
1.09 E \cdot 04\end{array}$ & $\begin{array}{l}3.19 E+03 \\
7.17 E+03 \\
6.51 E+01 \\
0.0 \\
1.00 E+04\end{array}$ & $\begin{array}{l}2.84 E+03 \\
6.38 E+03 \\
6.10 E+01 \\
0.0 \\
8.92 E+03\end{array}$ & $\begin{array}{l}2.52 E+03 \\
5.60 E+03 \\
5.60 E+01 \\
0.0 \\
0.01 E+03\end{array}$ & $\begin{array}{l}3.56 E+03 \\
7.91 E+03 \\
1.28 E+02 \\
0.0 \\
1.11 E+04\end{array}$ & $\begin{array}{l}2.85 E+03 \\
6.40 E+03 \\
6.8 B E+01 \\
0.0 \\
9.01 E+03\end{array}$ & $\begin{array}{l}0.0 \\
9.16 E\end{array}$ \\
\hline $\begin{array}{l}C S-137 \\
C S-139 \\
C S-139 \\
B A-131 \\
B A-133\end{array}$ & $\begin{array}{rl}30.17 & Y \\
32.2 & N \\
9.40 & M \\
11.80 & 0 \\
10.5 & Y\end{array}$ & $\begin{array}{l}1.07 E+04 \\
1.41 E+03 \\
1.80 E+03 \\
1.42 E+03\end{array}$ & $\begin{array}{l}1.13 E+04 \\
1.49 E \cdot 03 \\
1.95 E+03 \\
1.53 E+03\end{array}$ & $\begin{array}{l}0.0 \\
1.19 E+04 \\
1.55 E+03 \\
2.39 E+03 \\
1.97 E+03\end{array}$ & $\begin{array}{l}0.0 \\
1.15 E \cdot 04 \\
1.51 E \cdot 03 \\
1.95 E * 03 \\
1.52 E+03\end{array}$ & $\begin{array}{l}9.99 E+03 \\
1.31 E+03 \\
1.69 E+03 \\
1.30 E+03\end{array}$ & $\begin{array}{l}0.0 \\
9.31 E+03 \\
1.23 E+03 \\
1.54 E+03 \\
1.20 E \cdot 03\end{array}$ & $\begin{array}{l}0.0 \\
1.21 E+04 \\
1.57 E+03 \\
2.43 E+03 \\
2.011 E+03\end{array}$ & $\begin{array}{l}1.03 E+04 \\
1.36 E+03 \\
1.76 E+03 \\
1.39 E+03\end{array}$ & $\begin{array}{l}1.05 \mathrm{Eq} \\
1.38 \mathrm{Eq} \\
1.78 \mathrm{Eq} \\
1.39 \mathrm{Eq}\end{array}$ \\
\hline $\begin{array}{l}B A-133 M \\
B A-135 M \\
B A-137 M \\
B A-139 \\
B A-140\end{array}$ & $\begin{array}{r}38.9 \mathrm{H} \\
28.7 \mathrm{H} \\
2.552 \mathrm{M} \\
83.1 \mathrm{M} \\
12.789 \mathrm{O}\end{array}$ & $\begin{array}{l}2.09 E+02 \\
1.83 E+02 \\
2.51 E+03 \\
1.37 E+02 \\
1.56 E+02\end{array}$ & $\begin{array}{l}2.00 E+02 \\
2.68 E \cdot 03 \\
1.49 E \cdot 02 \\
8.13 E+02\end{array}$ & $\begin{array}{l}3.02 E+02 \\
2.67 E+02 \\
2.99 E+03 \\
2.08 E+02 \\
9.59 E+02\end{array}$ & $\begin{array}{l}2.24 E+02 \\
1.94 E+02 \\
2.74 E \bullet 03 \\
1.43 E \bullet 02 \\
8.27 E+02\end{array}$ & $\begin{array}{l}1.89 E+02 \\
1.65 E+02 \\
2.43 E+03 \\
1.21 E+02 \\
7.19 E+02\end{array}$ & $\begin{array}{l}1.71 E+02 \\
1.54 E+02 \\
2.16 E+03 \\
1.16 E+02 \\
6.49 E+02\end{array}$ & & & $\begin{array}{l}1.19 E \\
2.48 \mathrm{E} \\
1.35 \mathrm{E} \\
7.46 \mathrm{E}\end{array}$ \\
\hline $\begin{array}{l}B A-141 \\
B A-142 \\
L A-140 \\
L A-141 \\
L A-142\end{array}$ & $\begin{array}{r}10.27 \mathrm{M} \\
10.70 \mathrm{M} \\
40.22 \mathrm{H} \\
3.94 \mathrm{H} \\
95.4 \mathrm{M}\end{array}$ & $\begin{array}{l}3.74 E+03 \\
3.85 E+03 \\
1.03 E+04 \\
1.80 E+02 \\
1.33 E+04\end{array}$ & $\begin{array}{l}4.00 E+03 \\
4.10 E+03 \\
1.09 E+04 \\
1.98 E+02 \\
1.40 E+04\end{array}$ & $\begin{array}{l}4.61 E+03 \\
4.53 E+03 \\
1.16 E+04 \\
2.01 E+02 \\
1.45 E+04\end{array}$ & $\begin{array}{l}4.06 E+03 \\
4.14 E+03 \\
1.11 E+04 \\
2.00 E+02 \\
1.42 E * 04\end{array}$ & $\begin{array}{l}3.50 E+03 \\
3.67 E+03 \\
9.67 E+03 \\
1.77 E+02 \\
1.21 E+04\end{array}$ & $\begin{array}{l}3.23 E+03 \\
3.32 E+03 \\
8.95 E+03 \\
1.63 E+02 \\
1.16 E+04\end{array}$ & $\begin{array}{l}4.70 E+03 \\
4.01 E+03 \\
1.18 E+04 \\
2.11 E+02 \\
1.47 E+04\end{array}$ & $\begin{array}{l}3.65 E+03 \\
3.74 E+03 \\
9.96 E+03 \\
1.81 E+02 \\
1.28 E+04\end{array}$ & $\begin{array}{l}3.79 \mathrm{Eg} \\
1.01 \mathrm{E} \\
1.83 \mathrm{E} \\
1.31 \mathrm{Eg}\end{array}$ \\
\hline
\end{tabular}


DOSE-RATE FACTORS FOR BODY ORGANS AND EFFECTIVE DOSE-RATE FACTOR FOR IMMERSION IN CIJMTAMINATED MID

IN MREM/YR PER MICROCURIE/ICUBIC MI

\begin{tabular}{|c|c|c|c|c|c|c|c|c|c|}
\hline ICLIDE & HALF-LIFE & TESTES & THYMUS & THYROIO & UTERUS & EFFECTIVE & $\begin{array}{c}\text { SKIN } \\
\text { IPHOTONI }\end{array}$ & $\begin{array}{c}\text { SKIN } \\
\text { (ELECTRONI }\end{array}$ & $\begin{array}{l}\text { SKIN } \\
\text { IrOTALI }\end{array}$ \\
\hline $\begin{array}{l}1-132 \\
1-133 \\
1-134 \\
1-135 \\
1-136\end{array}$ & $\begin{array}{rl}2.30 & H \\
20.8 & H \\
52.6 & M \\
6.61 & H \\
83 & S\end{array}$ & $\begin{array}{l}.39 E \cdot 04 \\
.59 E+03 \\
.61 E+04 \\
.72 E+03 \\
.64 E+04\end{array}$ & $\begin{array}{l}28 E+04 \\
.74 E+03 \\
.30 E+04\end{array}$ & $\begin{array}{l}31 E+04 \\
35 E+03 \\
53 E+04 \\
17 E+03 \\
52 E+04\end{array}$ & $\begin{array}{l}57 E+03 \\
19 E+03 \\
00 E+04 \\
22 E+03 \\
06 E * 04\end{array}$ & $\begin{array}{l}19 E+04 \\
07 E+03 \\
38 E+04 \\
34 E+03 \\
41 E+04\end{array}$ & $\begin{array}{l}1.60 E+04 \\
4.12 E+03 \\
1.85 E+04 \\
1.11 E+04 \\
1.85 E+04\end{array}$ & $\begin{array}{l}3.52 E+03 \\
2.85 E+03 \\
4.52 E+03 \\
2.52 E+03 \\
1.67 E+04\end{array}$ & $\begin{array}{l}1.95 E+04 \\
6.97 E+03 \\
2.31 E+04 \\
1.37 E+04 \\
3.52 E+04\end{array}$ \\
\hline $\begin{array}{l}X E-122 \\
X E-123 \\
X E-125 \\
X E-127 \\
X E-129 M\end{array}$ & $\begin{array}{r}20.1 \mathrm{H} \\
2.14 \mathrm{H} \\
16.8 \mathrm{H} \\
36.406 \mathrm{O} \\
8.89 \mathrm{O}\end{array}$ & $\begin{array}{l}3.58 E+02 \\
3.71 E * 03 \\
1.46 E+03 \\
1.53 E+03 \\
1.52 E \cdot 02\end{array}$ & $2.52 E+02$ & & & $\begin{array}{l}3.02 E+02 \\
3.19 E+03 \\
1.26 E+03 \\
1.33 E+03 \\
1.17 E+02\end{array}$ & $\begin{array}{l}4.34 E \cdot 02 \\
4.24 E+03 \\
1.66 E \cdot 03 \\
1.72 E \cdot 03 \\
2.25 E \cdot 02\end{array}$ & $\begin{array}{l}6.74 E+00 \\
1.21 E+03 \\
8.53 E+01 \\
9.50 E+01 \\
7.31 E+02\end{array}$ & $\begin{array}{l}4.41 E * 02 \\
5.44 E * 03 \\
1.75 E \cdot 03 \\
1.82 E \cdot 03 \\
9.62 E \cdot 02\end{array}$ \\
\hline $\begin{array}{l}-131 M \\
-133 \\
-133 M \\
-1339 \\
=135 \\
-135 M\end{array}$ & 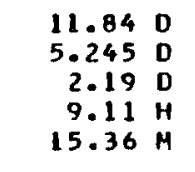 & $\begin{array}{l}5.62 E+01 \\
2.12 E+02 \\
1.78 E+02\end{array}$ & & & & & $\begin{array}{l}8.54 E \cdot 01 \\
2.49 E+02 \\
2.21 E+02 \\
1.60 E * 03 \\
2.89 E \cdot 03\end{array}$ & & $\begin{array}{l}5.61 E+02 \\
5.81 E+02 \\
1.21 E+03 \\
3.64 E+03 \\
3.5 T E * 03\end{array}$ \\
\hline $\begin{array}{l}X E-137 \\
X E-138 \\
C S-126 \\
C S-129 \\
C S-131\end{array}$ & $\begin{array}{r}3.83 \mathrm{M} \\
14.13 \mathrm{M} \\
1.64 \mathrm{M} \\
32.06 \mathrm{M} \\
9.688 \mathrm{D}\end{array}$ & $\begin{array}{l}1.11 E+03 \\
7.26 E+03 \\
6.63 E+03 \\
1.53 E+03 \\
5.07 E+01\end{array}$ & $\begin{array}{l}8.52 E+ \\
5.70 E \\
5.03 E\end{array}$ & & & & & & \\
\hline $\begin{array}{l}\text { CS-132 } \\
\text { CS-134 } \\
\text { CS-134M } \\
\text { CS-135 } \\
C S-136\end{array}$ & 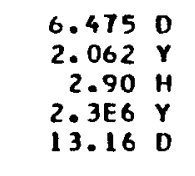 & $\begin{array}{l}4.21 E+03 \\
9.37 E+03 \\
1.23 E+02 \\
0.0 \\
1.31 E+04\end{array}$ & $\begin{array}{l}3.26 E+03 \\
7.32 E+03 \\
8.71 E+01 \\
0.0 \\
1.04 E+04\end{array}$ & $\begin{array}{l}8.83 E+03 \\
1.15 E+02 \\
0.0 \\
1.25 E \cdot 04\end{array}$ & $\begin{array}{l}2.55 E+03 \\
5.74 E+03 \\
5.88 E+01 \\
0.0 \\
8.12 E+03\end{array}$ & $\begin{array}{l}.59 E+03 \\
.01 E \cdot 03 \\
.04 E \cdot 02 \\
.0 \\
.13 E+04\end{array}$ & $\begin{array}{l}4.89 E \cdot 03 \\
1.08 E \cdot 04 \\
1.46 E+02 \\
0.0 \\
1.51 E+04\end{array}$ & & $\begin{array}{l}4.93 E+03 \\
1.17 E+04 \\
3.37 E+02 \\
6.95 E+01 \\
1.56 E+04\end{array}$ \\
\hline 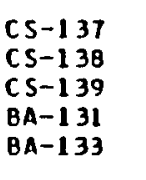 & $\begin{array}{rl}30.17 & Y \\
32.2 & M \\
9.40 & M \\
11.8 & 0 \\
10.5 & Y\end{array}$ & $\begin{array}{l}0.0 \\
1.48 E+04 \\
1.94 E+03 \\
2.67 E+03 \\
2.14 E+03\end{array}$ & $\begin{array}{l}0.0 \\
1.17 E+04 \\
1.54 E+03 \\
2.01 E+03 \\
1.59 E+03\end{array}$ & $\begin{array}{l}1.39 \mathrm{EA} \\
1.81 \mathrm{EA} \\
2.49 \mathrm{EA} \\
1.99 \mathrm{E}\end{array}$ & $\begin{array}{l}9.55 \mathrm{E} \\
1.26 \mathrm{E} \\
1.57 \mathrm{E} \\
1.23 \mathrm{E}\end{array}$ & $\begin{array}{l}1.27 \mathrm{E}+04 \\
1.67 \mathrm{E}+03 \\
2.29 \mathrm{E}+03 \\
1.84 \mathrm{E}+03\end{array}$ & $\begin{array}{l}1.69 E \cdot 04 \\
2.21 E+03 \\
3.06 E+03 \\
2.45 E+03\end{array}$ & $\begin{array}{l}8.75 E+02 \\
9.75 E+03 \\
1.35 E+04 \\
1.69 E+01 \\
5.16 E+01\end{array}$ & $\begin{array}{l}8.75 E+02 \\
2.66 E+04 \\
1.57 E+04 \\
3.13 E+03 \\
2.50 E+03\end{array}$ \\
\hline $\begin{array}{l}B A-133 M \\
B A-135 M \\
B A-137 M \\
B A-139 \\
B A-140\end{array}$ & $\begin{array}{r}38.9 \mathrm{H} \\
28.7 \mathrm{H} \\
2.552 \mathrm{M} \\
83.1 \mathrm{M} \\
12.789 \mathrm{D}\end{array}$ & $\begin{array}{l}3.33 E \cdot 02 \\
2.95 E \cdot 02 \\
3.58 E+03 \\
2.06 E+02 \\
1.10 E \cdot 03\end{array}$ & $\begin{array}{l}2.40 E \cdot 02 \\
2.11 E \cdot 02 \\
2.7 \theta E+03 \\
1.58 E+02 \\
8.31 E \cdot 02\end{array}$ & $\begin{array}{l}3.06 E \cdot 02 \\
2.69 E \cdot 02 \\
3.37 E * 03 \\
1.99 E \cdot 02 \\
1.02 E \cdot 03\end{array}$ & $\begin{array}{l}1.80 E \cdot 02 \\
1.57 E \cdot 02 \\
2.18 E \cdot 03 \\
1.21 E \cdot 02 \\
6.60 E \cdot 02\end{array}$ & $\begin{array}{l}2.83 E+02 \\
2.50 E+02 \\
3.06 E+03 \\
1.80 E+02 \\
9.41 E+02\end{array}$ & $\begin{array}{l}3.94 E+02 \\
3.51 E+02 \\
4.13 E+03 \\
2.26 E+02 \\
1.26 E+03\end{array}$ & $\begin{array}{l}1.20 E+03 \\
1.17 E+03 \\
4.71 E+02 \\
6.97 E+03 \\
1.16 E+03\end{array}$ & $\begin{array}{l}1.59 E+03 \\
1.52 E+03 \\
4.60 E+03 \\
1.20 E+03 \\
3.02 E \cdot 03\end{array}$ \\
\hline $\begin{array}{l}B A-141 \\
B A-142 \\
L A-140 \\
1 A-141 \\
1 A-142\end{array}$ & $\begin{array}{r}18.27 \mathrm{M} \\
10.70 \mathrm{M} \\
40.22 \mathrm{H} \\
3.94 \mathrm{H} \\
95.4 \mathrm{M}\end{array}$ & $\begin{array}{l}5.33 E+03 \\
5.45 E \cdot 03 \\
1.43 E+04 \\
2.59 E * 02 \\
1.82 E+04\end{array}$ & $\begin{array}{l}4.15 E+03 \\
4.32 E+03 \\
1.13 E+04 \\
2.07 E+02 \\
1.43 E \cdot 04\end{array}$ & $\begin{array}{l}5.03 E+03 \\
5.18 E 003 \\
1.34 E \cdot 04 \\
2.45 E+02 \\
1.67 E+04\end{array}$ & $\begin{array}{l}3.30 E+03 \\
3.38 E+03 \\
9.19 E+03 \\
1.67 E+02 \\
1.19 E+04\end{array}$ & $\begin{array}{l}4.59 E+03 \\
4.61 E+03 \\
1.23 E+04 \\
2.23 E+02 \\
1.57 E+04\end{array}$ & $\begin{array}{l}6.06 E+03 \\
6.26 E+03 \\
1.64 E+04 \\
2.97 E+02 \\
2.05 E+04\end{array}$ & $\begin{array}{l}6.57 E+03 \\
3.22 E 03 \\
3.87 E+03 \\
7.40+03 \\
6.58 E+03\end{array}$ & $\begin{array}{l}1.26 E \cdot 04 \\
9.48 E+03 \\
2.02 E+04 \\
7.70 E * 03 \\
2.70 E \cdot 04\end{array}$ \\
\hline
\end{tabular}




\begin{tabular}{|c|c|c|c|c|c|c|c|c|c|c|}
\hline NUCL IDE & HALF-LIFE & ADRENALS & BLADDER & BRAJN & OREAST & HE ART & $\begin{array}{l}\text { SMALL } \\
\text { INTES TINE }\end{array}$ & $\begin{array}{l}\text { UPPER } \\
\text { LARGE } \\
\text { INTESTINE }\end{array}$ & $\begin{array}{c}\text { LOWER } \\
\text { LARGE } \\
\text { INTESTINE }\end{array}$ & KIDNEYS \\
\hline $\begin{array}{l}C E-139 \\
C E-141 \\
C E-143 \\
C E-144 \\
P R-142\end{array}$ & $\begin{array}{r}137.66 \mathrm{O} \\
32.50 \mathrm{O} \\
33.0 \mathrm{H} \\
284.3 \mathrm{O} \\
19.13 \mathrm{H}\end{array}$ & $\begin{array}{l}5.93 E+02 \\
3.06 E+02 \\
1.10 E+03 \\
6.87 E+01 \\
2.59 E+02\end{array}$ & $\begin{array}{l}5.54 E+02 \\
2.85 E+02 \\
1.05 E+03 \\
6.60 E+01 \\
2.62 E+02\end{array}$ & $\begin{array}{l}5.75 E+02 \\
3.00 E+02 \\
1.13 E * 03 \\
6.75 E+01 \\
2.95 E+02\end{array}$ & $\begin{array}{l}9.73 E * 02 \\
4.97 E * 02 \\
1.52 E+03 \\
1.22 E * 02 \\
3.26 E * 02\end{array}$ & $\begin{array}{l}5.28 E+02 \\
2.76 E+02 \\
1.00 E+03 \\
6.22 E+01 \\
2.60 E+02\end{array}$ & $\begin{array}{l}4.68 E \cdot 02 \\
2.42 E \cdot 02 \\
9.07 E+02 \\
5.47 E+01 \\
2.42 E+02\end{array}$ & $\begin{array}{l}5.71 E+02 \\
2.97 E+02 \\
1.07 E+03 \\
6.78 E+01 \\
2.77 E+02\end{array}$ & $\begin{array}{l}5.25 E+02 \\
2.11 E+02 \\
9.93 E+02 \\
6.111 E+01 \\
2.56 E+02\end{array}$ & $\begin{array}{l}\text { 5. } 80 E+02 \\
2.93 E+02 \\
1.10 E+03 \\
6.88 E+01 \\
2.71 E+02\end{array}$ \\
\hline $\begin{array}{l}P R-143 \\
P R-144 \\
P R-144 M \\
N D-147 \\
N D-149\end{array}$ & $\begin{array}{r}13.56 \mathrm{D} \\
17.28 \mathrm{M} \\
7.2 \mathrm{M} \\
10.98 \mathrm{D} \\
1.73 \mathrm{H}\end{array}$ & $\begin{array}{l}3.98 E-05 \\
1.49 E+02 \\
1.12 E+01 \\
5.35 E+02 \\
1.60 E+03\end{array}$ & $\begin{array}{l}3.83 E-05 \\
1.51 E+02 \\
1.67 E+01 \\
5.22 E+02 \\
1.51 E \cdot 03\end{array}$ & $\begin{array}{l}4.30 E-05 \\
1.68 E \cdot 02 \\
8.58 E+00 \\
5.56 E \cdot 02 \\
1.63 E \cdot 03\end{array}$ & $\begin{array}{l}4.88 E-05 \\
1.89 E \cdot 02 \\
5.00 E \cdot 01 \\
7.95 E \cdot 02 \\
2.21 E \cdot 03\end{array}$ & $\begin{array}{l}3.17 E-05 \\
1.50 E+02 \\
6.04 E+00 \\
4.93 E \cdot 02 \\
1.46 E+03\end{array}$ & $\begin{array}{l}3.40 E-05 \\
1.38 E+02 \\
9.07 E+00 \\
4.44 E+02 \\
1.31 E+03\end{array}$ & $\begin{array}{l}4.01 E-05 \\
1.59 E+02 \\
1.28 E+01 \\
5.31 E+02 \\
1.55 E+03\end{array}$ & $\begin{array}{l}3.68 E-05 \\
1.47 E+02 \\
9.61 E+00 \\
4.84 E+02 \\
1.44 E+03\end{array}$ & $\begin{array}{l}4.00 E-05 \\
1.60 \mathrm{E}+02 \\
2.29 \mathrm{E}+01 \\
5.48 \mathrm{E}+02 \\
1.56 \mathrm{E}+03\end{array}$ \\
\hline $\begin{array}{l}P M-143 \\
P M-144 \\
P M-145 \\
P M-146 \\
P M-147\end{array}$ & $\begin{array}{r}2650 \\
36380 \\
17.78 \\
2020 \\
2.6234\end{array}$ & $\begin{array}{l}1.30 E+03 \\
6.75 E+03 \\
3.85 E+01 \\
3.25 E+03 \\
1.42 E-02\end{array}$ & $\begin{array}{l}1.27 E+03 \\
6.47 E \cdot 03 \\
5.44 E+01 \\
3.11 E+03 \\
1.33 E-02\end{array}$ & $\begin{array}{l}1.40 E * 03 \\
7.22 E+03 \\
3.24 E+01 \\
3.46 E+03 \\
1.42 E-02\end{array}$ & $\begin{array}{l}1.69 E+03 \\
8.46 E+03 \\
1.49 E+02 \\
4.11 E+03 \\
2.37 E-02\end{array}$ & $\begin{array}{l}1.23 E+03 \\
6.33 E+03 \\
2.98 E+01 \\
3.04 E+03 \\
1.31 E-02\end{array}$ & $\begin{array}{l}1.11 E+03 \\
5.71 E+03 \\
3.19 E+01 \\
2.74 E+03 \\
1.13 E-02\end{array}$ & $\begin{array}{l}1.31 E+03 \\
6.73 E+03 \\
4.30 E+01 \\
3.24 E+03 \\
1.40 E-02\end{array}$ & $\begin{array}{l}1.20 E+03 \\
6.20 E+03 \\
3.35 E+01 \\
2.90 E+03 \\
1.27 E-02\end{array}$ & $\begin{array}{l}1.34 E+03 \\
6.75 E+03 \\
7.05 E+01 \\
3.25 E+03 \\
1.35 E-02\end{array}$ \\
\hline $\begin{array}{l}P M-148 \\
P M-148 M \\
P M-149 \\
P M-151 \\
S M-147\end{array}$ & $\begin{array}{r}5.370 \\
41.30 \\
53.08 \\
28.40 \\
1.069811\end{array}$ & $\begin{array}{l}2.52 E \cdot 03 \\
8.76 E \cdot 03 \\
5.01 E \cdot 01 \\
1.42 E \cdot 03 \\
0.0\end{array}$ & $\begin{array}{l}2.49 E * 03 \\
8.38 E * 03 \\
4.66 E \cdot 01 \\
1.34 E \cdot 03 \\
0.0\end{array}$ & $\begin{array}{l}2 . B 0 E+03 \\
9.37 E+03 \\
5.09 E \cdot 01 \\
1.46 E+03 \\
0.0\end{array}$ & $\begin{array}{l}3.14 E \cdot 03 \\
1.09 E \cdot 04 \\
6.66 E \cdot 01 \\
1.92 E \cdot 03 \\
0.0\end{array}$ & $\begin{array}{l}2.46 E+03 \\
8.23 E+03 \\
4.54 E+01 \\
1.30 E+03 \\
0.0\end{array}$ & $\begin{array}{l}2.25 E+03 \\
7.42 E+03 \\
4.08 E+01 \\
1.17 E+03 \\
0.0\end{array}$ & $\begin{array}{l}2.62 E+03 \\
8.74 E+03 \\
4.81 E+01 \\
1.38 E+03 \\
0.0\end{array}$ & $\begin{array}{l}2.41 E * 03 \\
8.06 E+03 \\
4.50 E+01 \\
1.2 B E+03 \\
0.0\end{array}$ & $\begin{array}{l}2.01 E+03 \\
8.73 E+03 \\
4.82 E+01 \\
1.39 E+03 \\
0.0\end{array}$ \\
\hline $\begin{array}{l}S M-151 \\
S M-153 \\
E U-152 \\
E U-152 M \\
E U-154\end{array}$ & $\begin{array}{rl}90 & Y \\
46.7 & H \\
13.6 & Y \\
9.32 & H \\
8.8 & Y\end{array}$ & $\begin{array}{l}1.07 E-03 \\
1.71 E+02 \\
4.98 E+03 \\
1.3 B E+03 \\
5.51 E+03\end{array}$ & $\begin{array}{l}5.74 E-04 \\
1.81 E+02 \\
4.88 E+03 \\
1.34 E+03 \\
5.40 E+03\end{array}$ & $\begin{array}{l}3.24 E-04 \\
1.68 E+02 \\
5.44 E \bullet 03 \\
1.50 E+03 \\
6.05 E \bullet 03\end{array}$ & $\begin{array}{l}1.57 E-02 \\
3.74 E * 02 \\
6.36 E * 03 \\
1.74 E * 03 \\
6.93 E * 03\end{array}$ & $\begin{array}{l}3.81 E-04 \\
1.54 E+02 \\
4.79 E+03 \\
1.32 E+03 \\
5.32 E+03\end{array}$ & $\begin{array}{l}2.90 E-04 \\
1.39 E+02 \\
4.38 E+03 \\
1.20 E * 03 \\
4.86 E * 03\end{array}$ & $\begin{array}{l}9.46 E-04 \\
1.75 E+02 \\
5.11 E+03 \\
1.41 E+03 \\
5.67 E+03\end{array}$ & $\begin{array}{l}7.59 E-04 \\
1.52 E+02 \\
4.71 E+03 \\
1.29 E+03 \\
5.22 E+03\end{array}$ & $\begin{array}{l}1.45 \mathrm{E}-03 \\
1.96 \mathrm{E}+02 \\
5.13 \mathrm{E}+03 \\
1.41 \mathrm{E}+03 \\
5.66 \mathrm{E}+03\end{array}$ \\
\hline $\begin{array}{l}E U-155 \\
E U-156 \\
G D-152 \\
G D-153 \\
G D-159\end{array}$ & $\begin{array}{r}4.96 Y \\
15.19 \\
1.1 E 14 Y \\
241.60 \\
18.56 \mathrm{H}\end{array}$ & $\begin{array}{l}2.01 E+02 \\
6.16 E+03 \\
0.0 \\
2.8 B E \cdot 02 \\
1.53 E \cdot 02\end{array}$ & $\begin{array}{l}2.04 E+02 \\
6.17 E+03 \\
0.0 \\
3.12 E+02 \\
1.47 E+02\end{array}$ & $\begin{array}{l}2.04 E+02 \\
6.92 E+03 \\
0.0 \\
2.81 E \cdot 02 \\
1.55 E+02\end{array}$ & $\begin{array}{l}4.01 E+02 \\
7.76 E+03 \\
0.0 \\
6.61 E+02 \\
2.25 E+02\end{array}$ & $\begin{array}{l}1.86 E+02 \\
0.12 E+03 \\
0.0 \\
2.58 E+02 \\
1.38 E+02\end{array}$ & $\begin{array}{l}1.64 E+02 \\
5.64 E+03 \\
0.0 \\
2.35 E+02 \\
1.25 E+02\end{array}$ & $\begin{array}{l}2.04 E+02 \\
6.52 E+03 \\
0.0 \\
2.97 E+02 \\
1.48 E+02\end{array}$ & $\begin{array}{l}1.80 E+02 \\
6.01 E+03 \\
0.0 \\
2.57 E+02 \\
1.37 E+02\end{array}$ & $\begin{array}{l}2.12 E+02 \\
6.53 E+03 \\
0.0 \\
3.42 E+02 \\
1.55 E+02\end{array}$ \\
\hline $\begin{array}{l}60-162 \\
18-157 \\
18-160 \\
18-162 \\
0 Y-157\end{array}$ & $\begin{array}{rl}9.7 & H \\
150 & Y \\
72.3 & 0 \\
7.76 & 4 \\
8.06 & H\end{array}$ & $\begin{array}{l}1.81 E * 03 \\
6.28 E+00 \\
4.38 E+03 \\
4.86 E+03 \\
1.41 E+03\end{array}$ & $\begin{array}{l}1.70 E * 03 \\
8.5 B E+00 \\
4.66 E+03 \\
4.66 E+03 \\
1.33 E+03\end{array}$ & $\begin{array}{l}1.88 E+03 \\
5.70 E+00 \\
5.21 E+03 \\
5.19 E+03 \\
1.42 E+03\end{array}$ & $\begin{array}{l}2.33 E+03 \\
2.20 E+01 \\
5.98 E+03 \\
6.15 E+03 \\
1.99 E+03\end{array}$ & $\begin{array}{l}1.66 E+03 \\
5.15 E+00 \\
4.59 E+03 \\
4.58 E+03 \\
1.27 E+03\end{array}$ & $\begin{array}{l}1.49 E+03 \\
5.36 E+00 \\
4.18 E \cdot 03 \\
4.14 E+03 \\
1.14 E+03\end{array}$ & $\begin{array}{l}1.70 E+03 \\
6.84 E * 00 \\
4.88 E+03 \\
4.87 E * 03 \\
1.35 E+03\end{array}$ & $\begin{array}{l}1.63 E * 03 \\
5.52 E+00 \\
4.50 E * 03 \\
4.49 E+03 \\
1.26 E+03\end{array}$ & $\begin{array}{l}1.76 E+03 \\
1.05 E+01 \\
4.88 E+03 \\
4.86 E+03 \\
1.38 E+03\end{array}$ \\
\hline $\begin{array}{l}\text { DY }-165 \\
\text { OY-166 } \\
H 0-166 \\
110-166 \mathrm{M} \\
\text { ER-169 }\end{array}$ & $\begin{array}{r}2.334 \mathrm{H} \\
81.6 \mathrm{H} \\
26.80 \mathrm{H} \\
1.2063 \mathrm{Y} \\
9.40 \mathrm{D}\end{array}$ & $\begin{array}{l}9.91 E+01 \\
1.00 E+02 \\
1.09 E+02 \\
1.02 E+03 \\
6.43 E-03\end{array}$ & $\begin{array}{l}9.70 E+01 \\
1.12 E+02 \\
1.12 E+02 \\
6.71 E+03 \\
6.00 E-03\end{array}$ & $\begin{array}{l}1.04 E+02 \\
1.02 E+02 \\
1.21 E+02 \\
7.47 E+03 \\
6.35 E-03\end{array}$ & $\begin{array}{l}1.47 E+02 \\
2.35 E+02 \\
1.67 E+02 \\
8.92 E+03 \\
1.81 E-02\end{array}$ & $\begin{array}{l}9.17 E+01 \\
9.10 E+01 \\
1.07 E+02 \\
6.58 E+03 \\
5.86 E-03\end{array}$ & $\begin{array}{l}8.28 E+01 \\
8.45 E+01 \\
9.85 E+01 \\
5.94 E+03 \\
5.05 E-03\end{array}$ & $\begin{array}{l}9.86 E+01 \\
1.03 E+02 \\
1.15 E+02 \\
7.00 E+03 \\
6.31 E-03\end{array}$ & $\begin{array}{l}9.01 E+01 \\
9.00 E+01 \\
1.05 E+02 \\
6.45 E+03 \\
5.70 E-03\end{array}$ & $\begin{array}{l}1.02 E+02 \\
1.22 E+02 \\
1.19 E+02 \\
6.99 E+03 \\
6.09 E-03\end{array}$ \\
\hline
\end{tabular}


DOSE-RATE FACTORS FOR BODY ORGANS ANO EFFECTIVE DOSE-F.ATE FACTOR FOP IMMERSIUN IN CONTAMINATEI) AIR
IN MREM/YR PER MICROCURIE/ICUBIC MI

MUCL TOE MALF-LIFE LIVER

$\begin{array}{rrrr}C E-139 & 137.660 & 5.46 E+02 & 6.00 E+02 \\ C E-141 & 32.50 \mathrm{D} & 2.82 E+02 & 3.11 E \div 02\end{array}$

$\begin{array}{lrlll}C E-139 & 137.66 & 5.46 E+02 & 6.00 E+02 & 8.84 E+02 \\ C E-141 & 32.50 \mathrm{D} & 2.82 E+02 & 33.11 E+02 & 4.72 E+02 \\ C E-143 & 33.0 \mathrm{H} & 1.03 E+03 & 1.11 E+03 & 1.37 E+03 \\ C E-144 & 284.3 \mathrm{D} & 6.48 E+01 & 7.16 E+01 & 1.136+02 \\ P R-142 & 19.13 \mathrm{H} & 2.62 E+02 & 2.17 E+02 & 2.88 E+02\end{array}$

$\begin{array}{lll}284.3 & 0 & 6.48 E+01 \\ 19.13 \mathrm{H} & 2.62 E+02\end{array}$

$P R-143$
$P R-144$
$P R-144 M$

$\begin{array}{lll}13.56 \mathrm{D} & 3.80 E-05 & 4.06 E-05 \\ 17.28 \mathrm{M} & 1.51 E+02 & 1.59 E+02\end{array}$

$7.16 E+01$
$2.77 E+02$

MARROW

RED
MARROW

OVARIES PAN

ANCPEAS

SKELETON

SPLEEN

STOMACH

$5.72 E+02 \quad 2.82 E+02$
$1.37 E \cdot 03$

$4.74 E+02$
$2.44 E+02$

$4.53 E+02$
$2.33 E+02$

$0.05 E+02$
.02

$5.40 E+02$
$2.80 E+02$

5. $38 E+02$

1.11E.02 $9.63 E 402$ $8.33 E 002$ $4.35 E 402$

$2.79 \mathrm{E}+02 \quad 2.45 \mathrm{E}+02 \quad 2.28 \mathrm{E}+02 \quad 2.92 \mathrm{E}+02$

$1.01 E+03$

$1.02 E+03$

$4 \cdot 13 E-05$

3.70E-05

$3.28 E-05$

$4.53 E-05$
$1.70 E+02$
$2.68 E+01$

$2.53 E .02$

2. $55 \mathrm{E} \cdot 02$

$0.98 \mathrm{D} \quad 5.13 \mathrm{E}+02$

$1.49 E+01$
$5.55 E \cdot 02$

1.

$3.69 E-05$
$1.45 E+02$

3. $16 \mathrm{E}-05$

?.16E+02

$5.22 E+02$
$1.61 \mathrm{E}+03$

$4.77 E+02$

$7.17 E+00$
$4.23 E+02$
$1.27 E+03$

$2.68 E+01$
$7.31 E+02$

$1.03 E+0$

1. $14 \mathrm{E}+01$

$P M-143$

$\begin{array}{llll}265 \mathrm{D} & 1.25 E+03 & 1.34 E+03 \\ 363 \mathrm{D} & 6.42 E+03 & 6.88 E+03\end{array}$

$1.50 E+03$

$1.34 E+03$
$6.99 E+03$

$1.21 E \cdot 03$

$1.07 E+03$

$1.52 E+03$

$1.47 E+03$

$5.02 E+02$
$1.47 E * 03$

$2020 \mathrm{O} 3.09 \mathrm{E} \cdot 03$ 3.3LE०O3

$P M-146$
$P M-147$

$P M-148$

8. $85 \mathrm{E}+0$

6.21E.03 $5.52 E+03$

$2.22 E * 01$
$3.36 E * 03$
$1.27 E-02$

$3.65 E+0$
$2.98 E+03$

$2.52 E+01$
$2.65 E+03$

7.85E+03

$1.21 E+03$
$6.24 E+03$

1. $23 E+03$

2.33E-02

$\begin{array}{llll}5.37 & 0 & 2.48 E \cdot 03 & 2.63 E+03\end{array}$

$2.82 E+03$

$2.67 E \cdot 03$

$2.36 E+03$

3. $81 E+03$
$2.39 E-02$

$3.59 E+0$

6. $34 \mathrm{E}+03$

$.09 E+03 \quad 2.36 E+03 \quad 2.15 E+03$

3. $05 E+03$
$1.31 E-02$

\begin{tabular}{l}
$4.63 E+01 \quad 5.00 E+01 \quad 6.21 E+01$ \\
\hline
\end{tabular}

$P M-149$
$P M-151$
$S M-147$

$1.069 E 11$ Y $1.33 E+03$

$5.00 E+01$
$1.43 E+03$
0.0

$1.77 E+09$
0.0

$5.13 E+01$
$1.43 E+03$

$4.27 E \cdot 01 \quad 4.00 E+01$

$2.40 E+03$

$2.43 E+03$

$.13 E+03$

O. $33 E+0$
$0.01 E+0$

8.11E+0

8. $23 \mathrm{E}+03$

$5 M-151$

$\begin{array}{rrr}90 Y Y & 4.90 E-04 \\ 46.7 \mathrm{H} & 1.73 E \cdot 02\end{array}$

$1.26 E-03$
$1.91 E+02$

$1.83 E-03$

4. $31 E-04$

0.0

$E U-152 M$
$E U-154$

EU-155

$E U-156$
$G D-152$

GO- 153
GO- 159

60-162

$T 8-157$
$1 B-160$

TB-162
Dr- 157

DY -165
DY -166

HO-166

$M 0-166 M$
$E R-169$

9.32 H $\quad 1.33 E+03$
$8.8 Y \quad 5.37 E+03$

$1.17 E+03$
$1.42 E+03$
$5.72 E+03$

$5.72 E+03$

$4.96 Y Y \quad 2.00 E+02$

$2.22 E \cdot 02$

$15.190 \quad 6.17 E \cdot 0$

$\begin{array}{ll}241.60 & 2.94 E+02 \\ 18.56 & \end{array}$

$6.53 \mathrm{E} \cdot 03$

$5.196+03$

8.24E-04

$6.54 E-04$

2. $10 E-03$

1.

$0.08 E+03$

$4.62 E+03$
$1.28 E * 03$

$4.18 E+03$

3. $30 E+02$

$6.61 E-04$
$1.61 E+02$

$12.3 \mathrm{D} 4.63 \mathrm{E}+03$ 4.94E:03

$\begin{array}{lll}1.16 \mathrm{M} & 4.63 E+03 & 4.96 E+0 \\ 8.06 \mathrm{H} & 1.31 \mathrm{E}+03 & 1.42 \mathrm{E}+0\end{array}$

$2.334 \mathrm{H} \quad 9.56 \mathrm{E}+01 \quad 1.03 \mathrm{E} \cdot 02$

. $20.6 \mathrm{H}$ H $1.01 E+02$ $1.18 E+02$

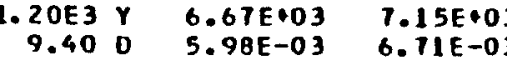

$1.61 E+02$
$6.59 E+03$

$1.60 E+03$
$G, 37 E+03$

$4.70 E \cdot 03$

7. $53 \mathrm{E}-04$

$1.64 E+02$

$1.31 E \cdot 03$
$5.2 B E+03$

$1.47 E \cdot 02$

$3.05 E+02$

$1.29 E \times 03$
$5.21 E \times 03$

T. OOE +03

$1.90 \mathrm{E}+02$

$5.94 E+03$
0.0

$1.92 E+02$

$5.56 E+02$
$2.02 E+02$

$\begin{array}{lll}0.21 E+02 & 2.47 E+02 & 2.08 E+02 \\ 1.51 E+02 & 1.33 E+02 & 1.20 E+02\end{array}$

$5.71 E+02$
$2.05 E+02$

$2.72 E+02$
$1.40 E * 02$

$2.17 E+03$

1. $87 E+03$

$1.60 E \cdot 03$

$1.46 E+03$

$2.20 \mathrm{E} \cdot 03$

2. $78 E+02$

$4.44 \mathrm{E}+03$

$3.99 E+03$

$1.51 E+01$
$5.51 E+03$

$1.66 E+03$
$5.99 E+00$

$1.67 E \cdot 03$

$1.82 \mathrm{E}+03$

$1.42 E+03 \quad 1.20 E+03$

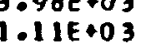

5. T4E+03
$1.85 E+03$

C

$6.48 E * 00$
$4.56 E * 03$

$1.33 E+02$

$9.63 E+01$

8.89E+01

T.

$1.36 E+02$

$4.51 E+33$
$1.28 E+03$

1.. $29 E+03$

1. $07 E+02$

$1.001 \mathrm{E}+02$

. 20E 01

$1.03 E+02$
$1.51 E \rightarrow 02$

$9.19 E \cdot 01$

9. $34 \mathrm{E}+01$

$9.93 E+01$
$1.07 E 002$

Q. $00 \mathrm{E}-02$

5.53E-03 5.17E-03

$\begin{array}{ll}4.81 E-03 & \text { 1.12E-02 }\end{array}$

$\begin{array}{ll}6.49 E+03 & 6.59 E+03 \\ 5.93 E-03 & 5.91 E-03\end{array}$ 


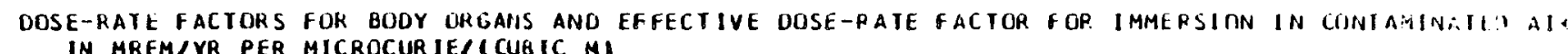

\begin{tabular}{|c|c|c|c|c|c|c|c|c|c|}
\hline NUCL IDE & HALF-LIFE & TESTES & THYMUS & THYROIO & UTERUS & EFFECTIVE & $\begin{array}{c}\text { SKIN } \\
\text { (PHOTONI }\end{array}$ & $\begin{array}{l}\text { SKIN } \\
\text { IELECIRONI }\end{array}$ & $\begin{array}{l}\text { SKIN } \\
\text { ITOTALI }\end{array}$ \\
\hline $\begin{array}{l}C E-139 \\
C E-141 \\
C E-143 \\
C E-144 \\
P R-142\end{array}$ & $\begin{array}{r}137.660 \\
32.50 \\
33.0 \mathrm{H} \\
284.3 \mathrm{O} \\
19.13 \mathrm{H}\end{array}$ & $\begin{array}{l}B .64 E+02 \\
4.41 E+02 \\
1.54 E+03 \\
1.05 E+02 \\
3.61 E+02\end{array}$ & $\begin{array}{l}6.45 E+02 \\
3.36 E+02 \\
1.16 E+03 \\
7.94 E+01 \\
2.87 E+02\end{array}$ & $\begin{array}{l}0.29 E \bullet 02 \\
4.31 E+C 2 \\
1.44 E \bullet 03 \\
1.02 E \bullet 02 \\
3.38 E \bullet 02\end{array}$ & $\begin{array}{l}4.74 E+02 \\
2.46 E+02 \\
8.97 E+02 \\
5.54 E+01 \\
2.36 E+02\end{array}$ & $\begin{array}{l}7.50 E+02 \\
3.86 E+02 \\
1.32 E+03 \\
9.09 E+01 \\
3.10 E+02\end{array}$ & $\begin{array}{l}9.69 E * 02 \\
4.80 E * 02 \\
1.76 E+03 \\
1.11 E+02 \\
4.12 E+02\end{array}$ & $\begin{array}{l}7.90 E+01 \\
7.11 E+02 \\
2.89 E+03 \\
2.29 E+02 \\
0.41 E+03\end{array}$ & $\begin{array}{l}1.05 E+03 \\
1.19 E+03 \\
4.66 E+03 \\
3.45 E+02 \\
6.83 E+03\end{array}$ \\
\hline $\begin{array}{l}P R-1<3 \\
P R-144 \\
P R-144 M \\
N D-147 \\
N D-1<9\end{array}$ & $\begin{array}{r}13.560 \\
17.28 \mathrm{M} \\
7.2 \mathrm{M} \\
10.98 \mathrm{D} \\
1.73 \mathrm{H}\end{array}$ & $\begin{array}{l}5.40 E-05 \\
2.08 E+02 \\
3.60 E+01 \\
7.84 E+02 \\
2.21 E+03\end{array}$ & $\begin{array}{l}4.24 E-05 \\
1.64 E+02 \\
2.10 E+01 \\
5.90 E+02 \\
1.68 E+03\end{array}$ & $\begin{array}{l}5.11 E-05 \\
1.93 E+02 \\
2.88 E+01 \\
7.33 E+02 \\
2.09 E+03\end{array}$ & $\begin{array}{l}3.31 E-05 \\
1.35 E+02 \\
7.76 E+00 \\
4.39 E+02 \\
1.31 E+03\end{array}$ & $\begin{array}{l}4.62 E-05 \\
1.79 E+02 \\
2.67 E+01 \\
6.65 E+02 \\
1.91 E+03\end{array}$ & $\begin{array}{l}0.24 E-05 \\
2.36 E+02 \\
5.20 E+01 \\
8.98 E+02 \\
2.49 E+03\end{array}$ & $\begin{array}{l}2.07 E+03 \\
9.63 E+03 \\
0.0 \\
1.41 E+03 \\
3.27 E+03\end{array}$ & $\begin{array}{l}2.07 E+03 \\
9.87 E+03 \\
5.20 E+01 \\
2.30 E+03 \\
5.76 E+03\end{array}$ \\
\hline $\begin{array}{l}P M-143 \\
P M-144 \\
P M-145 \\
P M-146 \\
P M-147\end{array}$ & $\begin{array}{r}2650 \\
3630 \\
17.7 \mathrm{Y} \\
2020 \mathrm{D} \\
2.6234 \mathrm{~V}\end{array}$ & $\begin{array}{l}1.82 E+03 \\
9.22 E+03 \\
1.10 E+02 \\
4.45 E+03 \\
2.08 E-02\end{array}$ & $\begin{array}{l}1.41 E+03 \\
7.12 E+03 \\
6.91 E+01 \\
3.43 E+03 \\
1.60 E-02\end{array}$ & $\begin{array}{l}1.71 E * 03 \\
8.64 E+03 \\
9.23 E+01 \\
4.17 E+03 \\
2.06 E-02\end{array}$ & & & $\begin{array}{l}2.13 E+03 \\
1.06 E+04 \\
1.51 E+02 \\
5.12 E+03 \\
2.21 E-02\end{array}$ & $\begin{array}{l}7.42 E+00 \\
5.87 E+01 \\
7.39 E-03 \\
5.81 E+02 \\
9.58 E+01\end{array}$ & $\begin{array}{l}2.14 E+03 \\
1.07 E+04 \\
1.51 E+02 \\
5.70 E \bullet 03 \\
9.59 E+01\end{array}$ \\
\hline $\begin{array}{l}P M-148 \\
P M-148 M \\
P M-149 \\
P M-151 \\
S H-147\end{array}$ & $\begin{array}{r}5.37 \mathrm{D} \\
41.3 \mathrm{D} \\
53.08 \mathrm{H} \\
28.40 \mathrm{H} \\
1.069 \mathrm{E} 11 \mathrm{Y}\end{array}$ & $\begin{array}{l}3.47 E+03 \\
1.19 E+04 \\
6.16 E+01 \\
1.95 E+03 \\
0.0\end{array}$ & $\begin{array}{l}2.74 E+03 \\
9.22 E+03 \\
5.14 E+01 \\
1.49 E+03 \\
0.0\end{array}$ & $\begin{array}{l}3.27 E+03 \\
1.12 E+04 \\
6.38 E * 01 \\
1.84 E * 03 \\
0.0\end{array}$ & $\begin{array}{l}2.19 E+03 \\
7.26 E+03 \\
4.08 E+01 \\
1.16 E+03 \\
0.0\end{array}$ & & $\begin{array}{l}3.98 E \cdot 03 \\
1.37 E \cdot 04 \\
7.61 E \cdot 01 \\
2.21 E+03 \\
0.0\end{array}$ & $\begin{array}{l}5.53 E+03 \\
7.80 E+02 \\
2.47 E+03 \\
1.81 E+03 \\
0.0\end{array}$ & $\begin{array}{l}9.52 E+03 \\
1.45 E+04 \\
2.55 E+03 \\
4.02 E+03 \\
0.0\end{array}$ \\
\hline $\begin{array}{l}S M-151 \\
S M-153 \\
E U-152 \\
E U-152 M \\
E U-154\end{array}$ & $\begin{array}{r}90 Y \\
46.7 \mathrm{H} \\
13.6 Y \\
9.32 \\
8.8 Y\end{array}$ & $\begin{array}{l}5.99 E-03 \\
3.08 E+02 \\
6.89 E+03 \\
1.90 E+03 \\
7.59 E+03\end{array}$ & $\begin{array}{l}5.35 E-04 \\
2.27 E+02 \\
5.44 E * 03 \\
1.51 E * 03 \\
6.03 E * 03\end{array}$ & & & & & & \\
\hline $\begin{array}{l}E U-155 \\
E U-156 \\
G D-152 \\
G D-153 \\
G D-159\end{array}$ & 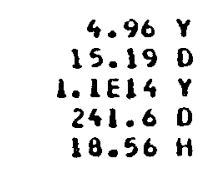 & $\begin{array}{l}3.36 E \cdot 02 \\
8.54 E+03 \\
0.0 \\
5.38 E \cdot 02 \\
2.21 E \cdot 02\end{array}$ & $\begin{array}{l}2.56 E+02 \\
6.78 E \div 03 \\
0.0 \\
3.92 E+02 \\
1.65 E+02\end{array}$ & $\begin{array}{l}3.30 E * 02 \\
8.01 E * 03 \\
0.0 \\
5.09 E * 02 \\
2.05 E * 02\end{array}$ & $\begin{array}{l}1.65 E+02 \\
5.49 E+03 \\
0.0 \\
2.32 E+02 \\
1.24 E * 02\end{array}$ & $\begin{array}{l}2.87 E+02 \\
7.34 E+03 \\
0.0 \\
4.46 E+02 \\
1.88 E+02\end{array}$ & $\begin{array}{l}3.6 \theta E+02 \\
9.77 E+03 \\
0.0 \\
6.23 E+02 \\
2.53 E+02\end{array}$ & $\begin{array}{l}3.93 E+01 \\
2.74 E+03 \\
0.0 \\
7.48 E+00 \\
2.03 E+03\end{array}$ & $\begin{array}{l}4.07 E+02 \\
1.25 E+04 \\
0.0 \\
0.31 E+02 \\
2.29 E+03\end{array}$ \\
\hline $\begin{array}{l}\text { GD-162 } \\
18-157 \\
1 B-160 \\
1 B-162 \\
O \gamma-157\end{array}$ & $\begin{array}{rl}9.7 & M \\
150 \% \\
72.3 & 0 \\
7.76 & M \\
8.06 \mathrm{H}\end{array}$ & $\begin{array}{l}2.46 E+03 \\
1.67 E * 01 \\
6.55 E+03 \\
6.62 E+03 \\
1.97 E+03\end{array}$ & $\begin{array}{l}1.85 E+03 \\
1.12 E+01 \\
5.21 E+03 \\
5.20 E+03 \\
1.47 E+03\end{array}$ & $\begin{array}{l}2.28 E+03 \\
1.46 E+01 \\
6.24 E+03 \\
6.30 E+03 \\
1.84 E+03\end{array}$ & $\begin{array}{l}1.48 E+03 \\
4.92 E+00 \\
4.05 E+03 \\
4.04 E+03 \\
1.14 E+03\end{array}$ & $\begin{array}{l}2.11 E+03 \\
1.29 E+01 \\
5.61 E+03 \\
5.68 E+03 \\
1.69 E+03\end{array}$ & $\begin{array}{l}2.79 E+03 \\
2.19 E \cdot 01 \\
7.54 E * 03 \\
7.59 E * 03 \\
2.23 E * 03\end{array}$ & $\begin{array}{l}2.14 E+03 \\
0.0 \\
1.35 E+03 \\
3.52 E+03 \\
2.13 E+01\end{array}$ & $\begin{array}{l}4.93 E+03 \\
2.19 E \div 01 \\
8.89 E \div 03 \\
1.11 E \div 04 \\
2.25 E+03\end{array}$ \\
\hline $\begin{array}{l}D \gamma-165 \\
\text { DY-166 } \\
H O-160 \\
H O-1684 \\
E P-169\end{array}$ & $\begin{array}{r}2.334 \mathrm{H} \\
81.6 \mathrm{H} \\
26.80 \mathrm{H} \\
1.20 \mathrm{Y} \\
9.40 \mathrm{O}\end{array}$ & $\begin{array}{l}1.46 E+02 \\
1.94 E+02 \\
1.66 E+02 \\
9.57 E+03 \\
1.01 E-02\end{array}$ & $\begin{array}{l}1.11 E+02 \\
1.42 E+02 \\
1.30 E+02 \\
1.47 E+03 \\
1.31 E-03\end{array}$ & $\begin{array}{l}1.37 E+02 \\
1.83 E+02 \\
1.5 B E+02 \\
9.07 E+03 \\
9.68 E-03\end{array}$ & $\begin{array}{l}0.14 E+01 \\
0.21 E+01 \\
9.59 E+01 \\
5.81 E+03 \\
5.14 E-03\end{array}$ & $\begin{array}{l}1.24 E+02 \\
1.59 E+02 \\
1.41 E+02 \\
8.21 E+03 \\
9.58 E-03\end{array}$ & $\begin{array}{l}1.67 E \bullet 02 \\
2.26 E+02 \\
1.90 E+02 \\
1.10 E+04 \\
2.54 E-02\end{array}$ & $\begin{array}{l}3.11 E+03 \\
4.68 E+02 \\
5.13 E+03 \\
3.63 E+02 \\
3.26 E+02\end{array}$ & $\begin{array}{l}3.28 E \bullet 03 \\
6.94 E+02 \\
5.32 E \bullet 03 \\
1.13 E \bullet 04 \\
3.26 E \bullet 02\end{array}$ \\
\hline
\end{tabular}




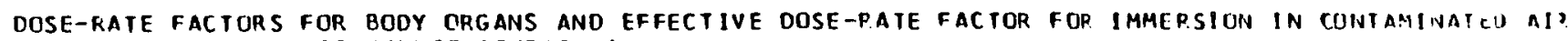

IN MREM/YR PER MICROCUR IE/ICUBIC MI

\begin{tabular}{|c|c|c|c|c|c|c|c|c|c|c|}
\hline LIOE & ALF-LIFE & DRENALS & ADDER & BRAIN & EAST & HE AR I & $\begin{array}{l}\text { SMALL } \\
\text { ITESTINE }\end{array}$ & $\begin{array}{c}\text { UPPER } \\
\text { LARGE } \\
\text { INTESTINE }\end{array}$ & $\begin{array}{c}\text { LOHER } \\
\text { LARGE } \\
\text { INIESTINE }\end{array}$ & R IONEYS \\
\hline $\begin{array}{l}E R-171 \\
T H-170 \\
Y M-171 \\
Y B-169 \\
Y B-175\end{array}$ & 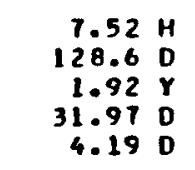 & $\begin{array}{l}1.54 E+03 \\
1.50 E+01 \\
1.53 E+00 \\
1.06 E+03 \\
1.65 E+02\end{array}$ & $\begin{array}{l}.44 E+03 \\
.62 E+01 \\
.79 E+00 \\
.05 E 003 \\
.55 E * 02\end{array}$ & $\begin{array}{l}1.55 E+03 \\
1.56 E+01 \\
1.58 E \cdot 00 \\
1.05 E+03 \\
1.68 E+02\end{array}$ & $\begin{array}{l}18 E+03 \\
.39 E+01 \\
07 E * 00 \\
.92 E+03 \\
26 E+02\end{array}$ & & & & $\begin{array}{l}1.38 E+03 \\
1.35 E+01 \\
1.37 E+00 \\
9.43 E+02 \\
1.4 B E+02\end{array}$ & $\begin{array}{l}1.49 E \cdot 03 \\
1.11 E+01 \\
1.97 E+00 \\
1.10 E+03 \\
1.60 E+02\end{array}$ \\
\hline $\begin{array}{l}L U-177 \\
L U-177 M \\
M F-181 \\
T A-182 \\
W-181\end{array}$ & 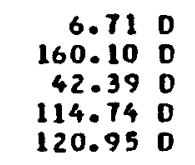 & $\begin{array}{l}1.41 E+02 \\
4.09 E+03 \\
2.30 E+03 \\
5.61 E \bullet 03 \\
9.80 E+01\end{array}$ & $\begin{array}{l}1.32 E+02 \\
3.82 E+03 \\
2.1 T E+03\end{array}$ & $\begin{array}{l}.39 E+02 \\
.10 E+03 \\
.39 E+03 \\
20 E+03 \\
.03 E \cdot 02\end{array}$ & $\begin{array}{l}2.20 E+02 \\
5.91 E+03 \\
3.07 E+03 \\
7.17 E+03 \\
2.47 E+02\end{array}$ & & & & & $\begin{array}{l}1.36 E+02 \\
3.95 E+03 \\
2.25 E+03 \\
5.82 E+03 \\
1.22 E+02\end{array}$ \\
\hline $\begin{array}{l}H-185 \\
H-187 \\
H-188 \\
R E-182 \\
R E-182 M\end{array}$ & $\begin{array}{rr}75.1 & \mathrm{D} \\
23.83 & \mathrm{H} \\
69.4 \mathrm{D} & \\
64.0 \mathrm{H} \\
12.7 \mathrm{H}\end{array}$ & $\begin{array}{l}1.09 E-01 \\
2.05 E+03 \\
7.46 E+00 \\
7.43 E+03\end{array}$ & $\begin{array}{l}1.02 E-01 \\
1.97 E+03 \\
6.88 E+00 \\
7.28 E \bullet 03 \\
5.11 E \bullet 03\end{array}$ & $\begin{array}{l}1.09 E-01 \\
2.18 E+03 \\
1.42 E+00 \\
8.05 E+03 \\
5.69 E+03\end{array}$ & $\begin{array}{l}1.80 E-01 \\
2.64 E+03 \\
1.04 E+01 \\
9.93 E+03 \\
6.69 E+03\end{array}$ & & & & & $\begin{array}{l}1.03 E-01 \\
2.05 E+03 \\
1.10 E+00 \\
1.62 E+03 \\
5.37 E+03\end{array}$ \\
\hline $\begin{array}{l}R E-183 \\
R E-184 \\
R E-184 M \\
R E-188 \\
R E-187\end{array}$ & $\begin{array}{rr}70 & \mathrm{D} \\
38.0 & \mathrm{D} \\
169 & \mathrm{D} \\
90.64 \mathrm{H} \\
4.7 \mathrm{E} 10 \mathrm{Y}\end{array}$ & $\begin{array}{l}5.14 E+02 \\
3.89 E+03 \\
1.61 E+03 \\
7.66 E+01 \\
0.0\end{array}$ & $\begin{array}{l}5.18 E+02 \\
3.79 E+03 \\
1.56 E+03 \\
7.42 E+01 \\
0.0\end{array}$ & $\begin{array}{l}5.20 E+02 \\
4.22 E+03 \\
1.70 E+03 \\
7.70 E+01\end{array}$ & & & & & & \\
\hline $\begin{array}{l}\text { RE-1 } 88 \\
0 S-185 \\
05-186 \\
05-1904 \\
05-191\end{array}$ & $\begin{array}{r}16.98 \mathrm{H} \\
93.6 \mathrm{D} \\
2.0 \mathrm{E} 15 \mathrm{Y} \\
9.9 \mathrm{M} \\
15.4 \mathrm{D}\end{array}$ & $\begin{array}{l}2.47 E: \\
3.05 E \\
0.0\end{array}$ & $\begin{array}{l}2.35 E+02 \\
2.94 E+03 \\
0.0 \\
6.48 E+03 \\
2.49 E+02\end{array}$ & $\begin{array}{l}2.58 E+02 \\
3.27 E+03 \\
0.0 \\
7.18 E+03 \\
2.53 E+02\end{array}$ & $\begin{array}{l}3.45 E+02 \\
3.90 E+03 \\
0.0 \\
8.82 E+03 \\
4.76 E+02\end{array}$ & & $\begin{array}{l}02 \\
03\end{array}$ & $\begin{array}{l}02 \\
03\end{array}$ & $\begin{array}{l}.02 \\
03 \\
03 \\
03 \\
02\end{array}$ & $\begin{array}{l}2.43 E+02 \\
3.07 E+03 \\
0.0 \\
6.12 E+03 \\
2.57 E+02\end{array}$ \\
\hline $\begin{array}{l}05-191 M \\
05-193 \\
1 R-190 \\
1 R-190 M \\
\text { IR-190M }\end{array}$ & $\begin{array}{r}13.03 \mathrm{H} \\
30.0 \mathrm{H} \\
11.78 \mathrm{O} \\
1.2 \mathrm{H} \\
3.2 \mathrm{H}\end{array}$ & $\begin{array}{l}1.28 E+01 \\
2.73 E * 02 \\
6.00 E * 03\end{array}$ & & & & $\begin{array}{l}1.20 E+01 \\
2.51 E+02 \\
5.56 E+03 \\
2.12 E-03 \\
1.24 E+02\end{array}$ & & & & \\
\hline $\begin{array}{l}1 R-192 \\
1 R-193 M \\
1 R-194 \\
1 R-194 M \\
P T-191\end{array}$ & 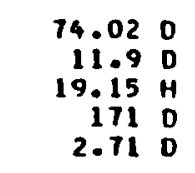 & $\begin{array}{l}3.52 E \\
9.73 E \\
3.97 E \\
1.01 E \\
1.11 E\end{array}$ & $\begin{array}{l}3.29 \\
1.06 \\
3.76 \\
9.61 \\
1.09\end{array}$ & $\begin{array}{l}3.6 \\
1.0 \\
4.1 \\
1.0 \\
1.1\end{array}$ & $\begin{array}{l}4.61 E \\
3.43 E \\
5.11 E \\
1.29 E \\
1.71 E\end{array}$ & $\begin{array}{l}3.21 \mathrm{E} \\
9.01 \mathrm{E} \\
3.68 \mathrm{E} \\
9.00 \mathrm{E} \\
1.03 \mathrm{E}\end{array}$ & $\begin{array}{l}2.88 E \\
8.21 E \\
3.33 E \\
8.46 E \\
9.25 E\end{array}$ & $\begin{array}{l}3.40 E \\
9.75 E \\
3.91 E \\
9.91 E \\
1.10 E\end{array}$ & & \\
\hline $\begin{array}{l}P T-193 \\
P T-193 M \\
P T-195 M \\
P T-197 \\
P T-197 M\end{array}$ & $\begin{array}{rl}50 & Y \\
4.33 & 0 \\
4.02 & 0 \\
18.3 & H \\
94.4 & H\end{array}$ & $\begin{array}{l}1.59 E-02 \\
3.13 E \cdot 01 \\
2.19 E \cdot 02 \\
8.28 E \cdot 01 \\
2.99 E+02\end{array}$ & $\begin{array}{l}2.86 E-09 \\
3.41 E \cdot 01 \\
2.32 E \cdot 02 \\
8.27 E \cdot 01 \\
2.93 E * 02\end{array}$ & $\begin{array}{l}1.26 E-03 \\
3.32 E+01 \\
2.29 E * 02 \\
8.50 E+01 \\
3.07 E * 02\end{array}$ & $\begin{array}{l}1.24 E+00 \\
7.12 E+01 \\
4.68 E+02 \\
1.54 E+02 \\
4.89 E+02\end{array}$ & $\begin{array}{l}2.06 E-03 \\
2.96 E+01 \\
2.06 E+02 \\
1.68 E+01 \\
2.14 E+02\end{array}$ & $\begin{array}{l}2.66 E-05 \\
2.67 E+01 \\
1.84 E+02 \\
6.82 E+01 \\
2.47 E+02\end{array}$ & $\begin{array}{l}3.92 E-05 \\
3.18 E+01 \\
2.22 E+02 \\
8.1 \theta E+01 \\
2.92 E+02\end{array}$ & & $\begin{array}{l}1.90 E-05 \\
3.56 E+C 1 \\
2.41 E+02 \\
8.50 E+01 \\
3.04 E+02\end{array}$ \\
\hline
\end{tabular}




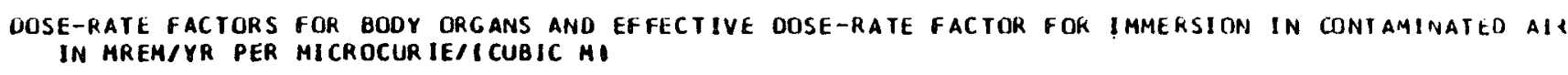

\begin{tabular}{|c|c|c|c|c|c|c|c|c|c|c|}
\hline NUCL IDE & HALF-LIFE & LIVER & LUNGS & MARROH & $\begin{array}{l}\text { REO } \\
\text { MARROW }\end{array}$ & OVARIES & PANCREAS & SKELETON & SPLEEN & STOMACH \\
\hline $\begin{array}{l}E R-171 \\
T H-170 \\
T H-171 \\
Y Q-169 \\
Y B-175\end{array}$ & 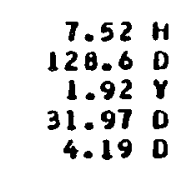 & $\begin{array}{l}1.43 E+03 \\
1.59 E+01 \\
1.72 E+00 \\
1.03 E+03 \\
1.54 E+02\end{array}$ & $\begin{array}{l}1.55 E+03 \\
1.16 E+01 \\
1.92 E+00 \\
1.13 E+03 \\
1.66 E+02\end{array}$ & $\begin{array}{l}2.04 E+03 \\
3.08 E+01 \\
3.45 E+00 \\
1.74 E+03 \\
2.11 E+02\end{array}$ & $\begin{array}{l}54 E+03 \\
10 E+01 \\
97 E-01 \\
36 E+02 \\
66 E+02\end{array}$ & & $\begin{array}{l}1.21 E+03 \\
1.07 E+01 \\
1.05 E+00 \\
1.95 E+02 \\
1.31 E * 02\end{array}$ & $\begin{array}{l}2.08 E+03 \\
3.17 E+01 \\
3.54 E+00 \\
1.78 E+03 \\
2.15 E+02\end{array}$ & & $\begin{array}{l}1.41 E+03 \\
1.49 E+01 \\
1.56 E+00 \\
9.93 E+02 \\
1.51 E+02\end{array}$ \\
\hline $\begin{array}{l}L U-177 \\
1 U-177 M \\
M F-181 \\
T A-182 \\
M-182\end{array}$ & $\begin{array}{rl}6.71 & 0 \\
160.10 & 0 \\
42.39 & 0 \\
114.74 & 0 \\
120.95 & 0\end{array}$ & $\begin{array}{l}1.31 E+02 \\
3.80 E+03 \\
2.16 E+03 \\
5.52 E+03 \\
1.10 E+02\end{array}$ & $\begin{array}{l}1.43 E+02 \\
4.12 E+03 \\
2.33 E+03 \\
5.87 E+03 \\
1.21 E+02\end{array}$ & $\begin{array}{l}2.08 E+02 \\
5.54 E \cdot 03 \\
2.87 E \bullet 03 \\
6.47 E+03 \\
2.10 E+02\end{array}$ & & & $\begin{array}{l}1.08 E+02 \\
3.20 E+03 \\
1.84 E+03 \\
4.73 E+03 \\
6.80 E+01\end{array}$ & & & $\begin{array}{l}1.29 E+02 \\
3.13 E+03 \\
2.13 E+03 \\
5.41 E+03 \\
9.99 E+01\end{array}$ \\
\hline $\begin{array}{l}H-185 \\
H-187 \\
M-188 \\
R E-182 \\
R E-182 M\end{array}$ & $\begin{array}{rl}75.1 & \mathrm{D} \\
23.83 & \mathrm{H} \\
69.4 \mathrm{D} \\
64.0 \mathrm{H} \\
12.1 \mathrm{H}\end{array}$ & $\begin{array}{l}1.02 E-01 \\
1.95 E+03 \\
6.05 E+00 \\
7.24 E+03 \\
5.08 E+03\end{array}$ & $\begin{array}{l}1.12 E-01 \\
2.10 E+03 \\
7.42 E+00 \\
1.74 E+03 \\
5.41 E+03\end{array}$ & $\begin{array}{l}1.77 E-01 \\
2.44 E+03 \\
9.78 E+00 \\
9.03 E \cdot 03 \\
6.02 E+03\end{array}$ & $\begin{array}{l}9.04 E-02 \\
2.10 E+03 \\
7.50 E+00 \\
7.61 E+03 \\
5.31 E \cdot 03\end{array}$ & $\begin{array}{l}\text { 9.77E-02 } \\
1.87 E \cdot 03 \\
6.16 \mathrm{E} \cdot 00 \\
6.17 \mathrm{E}+03 \\
4.80 \mathrm{E} \cdot 03\end{array}$ & $\begin{array}{l}8.29 E-02 \\
1.66 E+03 \\
5.06 E \cdot 00 \\
6.16 E+03 \\
4.33 E+03\end{array}$ & $\begin{array}{l}1.82 E-01 \\
2.48 E+03 \\
9.97 E+00 \\
9.20 E+03 \\
6.13 E+03\end{array}$ & $\begin{array}{l}1.01 E-01 \\
1.90 E \cdot 03 \\
6.77 E+00 \\
7.02 E+03 \\
4.90 E+03\end{array}$ & $\begin{array}{l}1.01 E-01 \\
1.93 E+03 \\
6.75 E+00 \\
7.09 E+03 \\
4.97 E+03\end{array}$ \\
\hline $\begin{array}{l}R E-183 \\
R E-184 \\
R E-184 M \\
R E-186 \\
R E-107\end{array}$ & 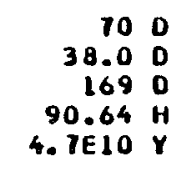 & $\begin{array}{l}5.11 E+02 \\
3.76 E+03 \\
1.55 E+03 \\
7.37 E+01 \\
0.0\end{array}$ & $\begin{array}{l}5.61 E+02 \\
4.02 E+03 \\
1.67 E+03 \\
8.12 E+01 \\
0.0\end{array}$ & $\begin{array}{l}.94 E+02 \\
.53 E+03 \\
.07 E+03 \\
.29 E \cdot 02 \\
.0\end{array}$ & $\begin{array}{l}4.37 E+02 \\
3.99 E+03 \\
1.60 E+03 \\
6.68 E+01 \\
0.0\end{array}$ & $\begin{array}{l}4.33 E+02 \\
3.62 E+03 \\
1.44 E+03 \\
6.29 E+01 \\
0.0\end{array}$ & $\begin{array}{l}3.81 E+02 \\
3.20 E+03 \\
1.29 E+03 \\
5.74 E \cdot 01 \\
0.0\end{array}$ & $\begin{array}{l}9.11 E+02 \\
4.61 E+03 \\
2.11 E+03 \\
1.33 E+02 \\
0.0\end{array}$ & $\begin{array}{l}4.82 E+02 \\
3.64 E+03 \\
1.50 E+03 \\
7.15 E+01 \\
0.0\end{array}$ & $\begin{array}{l}\text { 4. } 88 E+02 \\
3.71 E+03 \\
1.52 E+03 \\
7.17 E+01 \\
0.0\end{array}$ \\
\hline $\begin{array}{l}\text { RE-188 } \\
\text { OS-185 } \\
O S-186 \\
O S-1904 \\
O S-191\end{array}$ & $\begin{array}{r}16.98 \mathrm{H} \\
93.6 \mathrm{D} \\
2.0615 \mathrm{Y} \\
9.9 \mathrm{M} \\
15.4 \mathrm{D}\end{array}$ & $\begin{array}{l}2.34 E+02 \\
2.92 E \cdot 03 \\
0.0 \\
6.44 E+03 \\
2.47 E+02\end{array}$ & $\begin{array}{l}2.53 E+02 \\
3.13 E+03 \\
0.0 \\
6.93 E+03 \\
2.72 E+02\end{array}$ & $\begin{array}{l}3.24 E+02 \\
3.58 E+03 \\
0.0 \\
8.18 E+03 \\
4.53 E+02\end{array}$ & $\begin{array}{l}.45 E \cdot 02 \\
.12 E \cdot 03 \\
.0 \\
.05 E+03 \\
.03 E+02\end{array}$ & $\begin{array}{l}2.16 E+02 \\
2.81 E+03 \\
0.0 \\
6.12 E+03 \\
2.07 E+02\end{array}$ & $\begin{array}{l}1.98 E+02 \\
2.48 E * 03 \\
0.0 \\
5.54 E * 03 \\
1.82 E * 02\end{array}$ & $\begin{array}{l}3.31 E+02 \\
3.64 E+03 \\
0.0 \\
8.32 E+03 \\
4.65 E+02\end{array}$ & $\begin{array}{l}2.29 E+02 \\
2.83 E+03 \\
0.0 \\
6.30 E+03 \\
2.34 E+02\end{array}$ & $\begin{array}{l}2.31 E+02 \\
2.8 B E+03 \\
0.0 \\
6.36 E+03 \\
2.37 E+02\end{array}$ \\
\hline $\begin{array}{l}\text { OS-191M } \\
\text { OS-193 } \\
1 R-190 \\
\text { IR-190M } \\
\text { IR-190M }\end{array}$ & 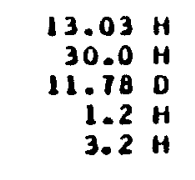 & $\begin{array}{l}1.40 E+01 \\
2.59 E \cdot 02 \\
5.67 E \cdot 03 \\
1.66 E-04 \\
1.44 E+02\end{array}$ & $\begin{array}{l}1.54 E+01 \\
2.81 E+02 \\
6.09 E+03 \\
1.29 E-02 \\
1.59 E+02\end{array}$ & & & & & & & $\begin{array}{l}1.29 E * 01 \\
2.54 E+02 \\
5.59 E+03 \\
3.32 E-03 \\
1.33 E * 02\end{array}$ \\
\hline $\begin{array}{l}\text { IR-1 } 92 \\
\text { IR-193M } \\
\text { IR-194 } \\
\text { IR-194M } \\
P I-191\end{array}$ & 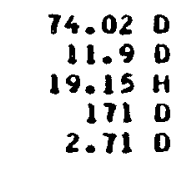 & $\begin{array}{l}1.04 \\
3.74 \\
9.55 \\
1.08\end{array}$ & $\begin{array}{l}1.16 \\
4.01 \\
1.03 \\
1.17\end{array}$ & $\begin{array}{l}4.72 E+02 \\
1.19 E+04 \\
1.59 E+03\end{array}$ & $\begin{array}{l}4.10 \mathrm{E} \\
1.05 \mathrm{E} \\
1.06 \mathrm{E}\end{array}$ & & & & & $\begin{array}{l}3.23 \mathrm{E}+03 \\
9.67 \mathrm{E}-01 \\
3.69 \mathrm{E}+02 \\
9.43 \mathrm{E}+03 \\
1.05 \mathrm{E}+03\end{array}$ \\
\hline $\begin{array}{l}P Y-193 \\
P Y-193 M \\
P T-195 M \\
P Y-197 \\
P T-197 M\end{array}$ & $\begin{array}{rl}50 & Y \\
4.33 & D \\
4.02 & 0 \\
18.3 & H \\
94.4 & H\end{array}$ & $\begin{array}{l}3.37 E \cdot 01 \\
2.29 E \cdot 02 \\
9.23 E+01 \\
2.91 E * 02\end{array}$ & $\begin{array}{l}3.72 E * 01 \\
2.53 \mathrm{E}+02 \\
9.03 E * 01 \\
3.16 E+02\end{array}$ & $\begin{array}{l}6.57 E+01 \\
4.39 E+02 \\
1.42 E+02 \\
4.56 E+02\end{array}$ & $\begin{array}{l}2.26 E+01 \\
1.66 E+02 \\
6.97 E+01 \\
2.76 E+02\end{array}$ & $\begin{array}{l}2.76 E+01 \\
1.89 E+02 \\
6.94 E+01 \\
2.57 E+02\end{array}$ & $\begin{array}{l}2.22 E+01 \\
1.58 E+02 \\
6.14 E+01 \\
2.29 E+02\end{array}$ & $\begin{array}{l}6.74 E+01 \\
4.51 E+02 \\
1.50 E+02 \\
4.66 E+02\end{array}$ & $\begin{array}{l}3.57 E-03 \\
3.05 E+01 \\
2.11 E+02 \\
7.82 E+01 \\
2.78 E+02\end{array}$ & $\begin{array}{l}3.23 E-03 \\
3.13 E+01 \\
2.16 E+02 \\
7.89 E+01 \\
2.81 E+02\end{array}$ \\
\hline
\end{tabular}


DOSE-R.ATE FACTORS TOR. BODY ORGANS ANU EFFECTIVE DOSE-PATE FACTUR FOR IMMERSION IN CIINTAMINAII:I R.1Q

\begin{tabular}{|c|c|c|c|c|c|c|c|c|c|}
\hline CLIDE & F-LIFE & STES & Hrimus & AY ROID & UTERUS & EFFECTIVE & $\begin{array}{c}\text { SKIN } \\
\text { I PHOTONI }\end{array}$ & $\begin{array}{l}\text { SKIN } \\
\text { ELECIRONI }\end{array}$ & $\begin{array}{l}\text { SKIN } \\
\text { IrOTALI }\end{array}$ \\
\hline $\begin{array}{l}E P-171 \\
Y M-170 \\
T M-171 \\
Y B-169 \\
Y B-175\end{array}$ & 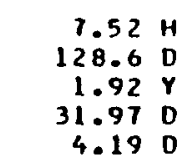 & $\begin{array}{l}14 E+03 \\
.78 E+01 \\
26 E+00 \\
68 E+03 \\
.27 E+02\end{array}$ & $\begin{array}{l}.62 E+03 \\
10 E+01 \\
38 E+00 \\
26 E+03 \\
11 E+02\end{array}$ & $\begin{array}{l}2.02 E+03 \\
2.71 E+01 \\
3.09 E+00 \\
1.61 E+03 \\
2.13 E+02\end{array}$ & $\begin{array}{l}1.25 E \cdot 03 \\
1.25 E \bullet 01 \\
1.25 E+00 \\
8.57 E+02 \\
1.34 E \bullet 02\end{array}$ & $\begin{array}{l}1.85 E+03 \\
2.33 E+01 \\
2.65 E+00 \\
1.43 E+03 \\
1.96 E+02\end{array}$ & $\begin{array}{l}.39 E+03 \\
.12 E+01 \\
.80 E+00 \\
.89 E+03 \\
.56 E+02\end{array}$ & $\begin{array}{l}2.49 E+03 \\
2.07 E+03 \\
3.59 E-01 \\
1.77 E+02 \\
5.57 E+02\end{array}$ & $\begin{array}{l}4.88 E+03 \\
2.10 E+03 \\
4.16 E+00 \\
2.07 E+03 \\
8.13 E+02\end{array}$ \\
\hline $\begin{array}{l}L U-177 \\
L U-177 M \\
H F-181 \\
T A-182 \\
W-181\end{array}$ & 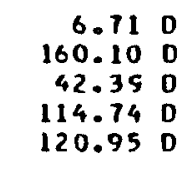 & $\begin{array}{l}.01 E+02 \\
.70 E+03 \\
.17 E+03 \\
.80 E+03 \\
.00 E+02\end{array}$ & $\begin{array}{l}.53 E+02 \\
.32 E \cdot 03 \\
.40 E+03 \\
.23 E+03 \\
.50 E+02\end{array}$ & $\begin{array}{l}.95 E+02 \\
.42 E+03 \\
.96 E+03 \\
.44 E+03 \\
.93 E+02\end{array}$ & $\begin{array}{l}1.14 E \cdot 02 \\
3.31 E+03 \\
1.89 E+03 \\
4.85 E+03 \\
8.18 E+01\end{array}$ & $\begin{array}{l}1.75 E+02 \\
4.94 E+03 \\
2.72 E * 03 \\
6.68 E+03 \\
1.65 E+02\end{array}$ & $\begin{array}{l}.21 E+02 \\
.35 E+03 \\
.58 E+03 \\
.95 E \cdot 03 \\
.29 E+02\end{array}$ & $\begin{array}{l}6.10 E * 02 \\
4.84 E+02 \\
6.53 E+02 \\
5.85 E * 02 \\
0.0\end{array}$ & $\begin{array}{l}8.31 E+02 \\
6.83 E+03 \\
4.23 E+03 \\
9.53 E+03 \\
2.29 E+02\end{array}$ \\
\hline $\begin{array}{l}W-185 \\
W-187 \\
W-188 \\
R E-182 \\
R E-182 M\end{array}$ & $\begin{array}{rr}75.1 & \mathrm{O} \\
23.83 & \mathrm{H} \\
69.4 & \mathrm{D} \\
64.0 & \mathrm{H} \\
12.7 & \mathrm{H}\end{array}$ & $\begin{array}{l}1.58 E-01 \\
2.83 E \bullet 03 \\
1.01 E \bullet 01 \\
1.04 E+04 \\
1.22 E+03\end{array}$ & $\begin{array}{l}.22 E-01 \\
.10 E+03 \\
.69 E+00 \\
.21 E+03 \\
.74 E+03\end{array}$ & & & & & & $\begin{array}{l}5.27 E+02 \\
4.94 E+03 \\
3.37 E+02 \\
1.21 E+04 \\
8.42 E+03\end{array}$ \\
\hline $\begin{array}{l}R E-183 \\
R E-184 \\
R E-184 M \\
R E-186 \\
R E-187\end{array}$ & $\begin{array}{r}70 \\
38.0 \\
169 \\
90 \\
90.64 \mathrm{H} \\
4.7 E 10 \mathrm{~V}\end{array}$ & $\begin{array}{l}8.40 E+02 \\
5.39 E+03 \\
2.28 E+03 \\
1.18 E+02 \\
0.0\end{array}$ & $\begin{array}{l}6.38 E \cdot 02 \\
4.27 E \cdot 03 \\
1.78 E+03 \\
9.05 E \cdot 01 \\
0.0\end{array}$ & $\begin{array}{l}8.13 E+02 \\
5.14 E+03 \\
2.18 E+03 \\
1.16 E \cdot 02 \\
0.0\end{array}$ & $\begin{array}{l}4.21 E+02 \\
3.26 E+03 \\
1.34 E+03 \\
6.26 E+01 \\
0.0\end{array}$ & $\begin{array}{l}7.14 E+02 \\
4.60 E+03 \\
1.96 E+03 \\
1.02 E+02 \\
0.0\end{array}$ & $\begin{array}{l}9.36 E \cdot 02 \\
6.22 E+03 \\
2.59 E+03 \\
1.28 E+02 \\
0.0\end{array}$ & $\begin{array}{l}7.4 T E * 01 \\
7.29 E+01 \\
8.25 E+01 \\
2.22 E+03 \\
0.0\end{array}$ & $\begin{array}{l}1.01 E+03 \\
6.29 E+03 \\
2.68 E+03 \\
2.35 E+03 \\
0.0\end{array}$ \\
\hline $\begin{array}{l}\text { RE-1 188 } \\
\text { OS-1 85 } \\
\text { OS-1 } 86 \\
\text { OS-190M } \\
\text { OS-191 }\end{array}$ & $\begin{array}{r}16.98 \mathrm{H} \\
93.6 \mathrm{O} \\
2.0 E 15 \mathrm{Y} \\
9.9 \mathrm{M} \\
15.4 \mathrm{O}\end{array}$ & $\begin{array}{l}3.44 E+02 \\
4.21 E+03 \\
0.0 \\
9.32 E+03 \\
4.05 E+02\end{array}$ & $\begin{array}{l}2.68 E+02 \\
3.27 E \cdot 03 \\
0.0 \\
7.10 E \cdot 03 \\
3.12 E \cdot 02\end{array}$ & $\begin{array}{l}3.31 E+02 \\
3.97 E+03 \\
0.0 \\
8.72 E \cdot 03 \\
3.99 E \cdot 02\end{array}$ & $\begin{array}{l}2.04 E+02 \\
2.53 E+03 \\
0.0 \\
5.63 E+03 \\
2.05 E+02\end{array}$ & $\begin{array}{l}2.98 E+02 \\
3.59 E+03 \\
0.0 \\
8.01 E+03 \\
3.47 E+02\end{array}$ & $\begin{array}{l}3.86 E+02 \\
4.85 E \cdot 03 \\
0.0 \\
1.06 E+04 \\
4.43 E+02\end{array}$ & $\begin{array}{l}5.90 E+03 \\
4.12 E+01 \\
0.0 \\
3.83 E+02 \\
5.57 E+01\end{array}$ & $\begin{array}{l}6.29 E+03 \\
4.09 E+03 \\
0.0 \\
1.10 E+04 \\
4.99 E+02\end{array}$ \\
\hline $\begin{array}{l}\text { OS }-191 \mathrm{M} \\
\text { OS-193 } \\
\text { IR-190 } \\
\text { IR-190M } \\
\text { IR-190M }\end{array}$ & $\begin{array}{r}13.03 \mathrm{H} \\
30.0 \mathrm{H} \\
11.78 \mathrm{D} \\
1.2 \mathrm{H} \\
3.2 \mathrm{H}\end{array}$ & & & & & & & $\begin{array}{l}4.14 E-01 \\
2.34 E+03 \\
2.52 E+02 \\
0.0 \\
1.85 E+01\end{array}$ & \\
\hline $\begin{array}{l}\text { IR-1 } 92 \\
\text { IR-1 } 93 \mathrm{M} \\
\text { IR-194 } \\
\text { IR-194M } \\
P T-191\end{array}$ & 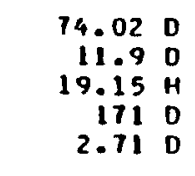 & $\begin{array}{l}4.76 E+03 \\
1.92 E+00 \\
5.38 E+02 \\
1.38 E+04 \\
1.65 E+03\end{array}$ & $\begin{array}{l}3.60 E \cdot 03 \\
1.39 E \cdot 00 \\
4.15 E+02 \\
1.05 E \bullet 04 \\
1.26 E+03\end{array}$ & & & $\begin{array}{l}4.11 E+03 \\
1.73 E+00 \\
4.63 E+02 \\
1.1 B E+04 \\
1.41 E+03\end{array}$ & & $\begin{array}{l}1.11 E+03 \\
3.42 E+00 \\
6.26 E+03 \\
5.41 E+02 \\
9.16 E+01\end{array}$ & $\begin{array}{l}6.55 E+03 \\
8.06 E+00 \\
6.07 E+03 \\
1.63 E \times 04 \\
1.96 E+03\end{array}$ \\
\hline $\begin{array}{l}P T-193 \\
P T-193 M \\
P T-195 M \\
P T-191 \\
P T-197 M\end{array}$ & $\begin{array}{rl}50 & Y \\
4.33 & 0 \\
4.02 & 0 \\
18.3 & H \\
94.4 & M\end{array}$ & $\begin{array}{l}1.15 E-01 \\
5.00 E+01 \\
3.80 E+02 \\
1.32 E+02 \\
4.53 E+02\end{array}$ & $\begin{array}{l}4.4 \theta E+01 \\
2.99 E+02 \\
1.03 E+02 \\
3.45 E+02\end{array}$ & $\begin{array}{l}7.93 \mathrm{E}-03 \\
5.70 \mathrm{E} * 01 \\
3.82 \mathrm{01} \\
1.30 \mathrm{02} \\
4.33 \mathrm{02}+02\end{array}$ & $\begin{array}{l}1.16 E-06 \\
2.66 E * 01 \\
1.84 E * 02 \\
6.89 E * 01 \\
2.46 E * 02\end{array}$ & $\begin{array}{l}2.20 E-01 \\
4.89 E+01 \\
3.29 E+02 \\
1.14 E+02 \\
3.87 E+02\end{array}$ & $\begin{array}{l}2.65 E \cdot 00 \\
6.65 E \cdot 01 \\
4.31 E \cdot 02 \\
1.46 E+02 \\
5.10 E \cdot 02\end{array}$ & $\begin{array}{l}0.0 \\
2.86 E+02 \\
2.65 E+02 \\
1.09 E+03 \\
1.67 E+03\end{array}$ & $\begin{array}{l}2.65 E \times 00 \\
3.53 E+02 \\
6.96 E+02 \\
1.24 E+03 \\
2.18 E+03\end{array}$ \\
\hline
\end{tabular}




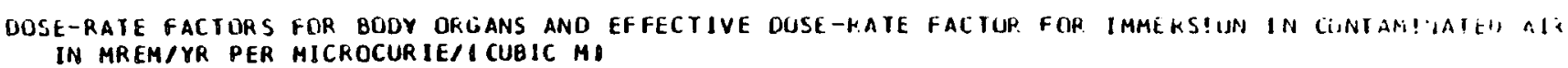

\begin{tabular}{|c|c|c|c|c|c|c|c|c|c|c|}
\hline CLIDE & HALF-LIFE & ADRENALS & BLADOER & BRAIN & BREAST & HE AR T & $\begin{array}{l}\text { SMALL } \\
\text { INTESTINE }\end{array}$ & $\begin{array}{c}\text { UPPER } \\
\text { LARGE } \\
\text { INTESIIVE }\end{array}$ & $\begin{array}{c}\text { LNWEH } \\
\text { LARGE } \\
\text { INIESTINE }\end{array}$ & KIDNEYS \\
\hline $\begin{array}{l}A U-194 \\
A U-195 \\
A U-195 A \\
A U-196 \\
A U-198\end{array}$ & 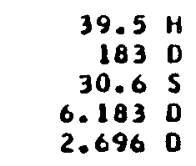 & $\begin{array}{l}4.14 E+03 \\
2.52 E+02 \\
8.34 E+02 \\
1.96 E+03 \\
1.74 E+03\end{array}$ & $\begin{array}{l}4.69 E+03 \\
2.69 E \cdot 02 \\
7.72 E \cdot 02 \\
1.85 E \cdot 03 \\
1.63 E \cdot 03\end{array}$ & $\begin{array}{l}21 E+03 \\
65 E+02 \\
28 E+02 \\
01 E * 03 \\
80 E+03\end{array}$ & $\begin{array}{l}20 E \cdot 03 \\
7 F E+02 \\
19 E+03 \\
24 E+03 \\
24 E \cdot 03\end{array}$ & $\begin{array}{l}4.62 E+03 \\
2.37 E+02 \\
7.47 E+02 \\
1.79 E+03 \\
1.59 E+03\end{array}$ & $\begin{array}{l}4.24 E+03 \\
2.13 E+02 \\
0.69 E+02 \\
1.61 E+03 \\
1.43 E+03\end{array}$ & $\begin{array}{l}4.91 E+03 \\
2.55 E+02 \\
7.91 E+02 \\
1.89 E+03 \\
1.69 E+03\end{array}$ & $\begin{array}{l}4.54 E+03 \\
2.27 E+02 \\
7.41 E+02 \\
1.76 E+03 \\
: .57 E+03\end{array}$ & $\begin{array}{l}4.93 E+03 \\
2.80 E+02 \\
7.96 E+02 \\
1.91 E+03 \\
1.09 E+03\end{array}$ \\
\hline $\begin{array}{l}A U-199 \\
\text { HU-197 } \\
H G-1914 \\
H G G-203 \\
\text { TL-200 }\end{array}$ & $\begin{array}{r}3.1390 \\
64.14 \mathrm{H} \\
23.8 \mathrm{H} \\
46.60 \mathrm{O} \\
26.1 \mathrm{H}\end{array}$ & $\begin{array}{l}3.63 E \cdot 02 \\
2.08 E \bullet 02 \\
3.47 E+02 \\
9.70 E+02 \\
5.69 E * 03\end{array}$ & $\begin{array}{l}3.38 E+02 \\
2.23 E+02 \\
3.34 E+02 \\
8.96 E+02 \\
5.54 E+03\end{array}$ & $\begin{array}{l}.58 E+02 \\
.20 E+02 \\
.50 E+02 \\
.67 E+02 \\
.18 E+03\end{array}$ & $\begin{array}{l}.73 E+02 \\
0.50 E+02 \\
0.97 E+02 \\
1.35 E+03 \\
.30 E+03\end{array}$ & $\begin{array}{l}3.28 E+02 \\
1.97 E+02 \\
3.19 E+02 \\
8.70 E+02 \\
5.44 E \cdot 03\end{array}$ & $\begin{array}{l}2.89 E+02 \\
1.76 E+02 \\
2.81 E \cdot 02 \\
7.80 E+02 \\
4.96 E \cdot 03\end{array}$ & $\begin{array}{l}3.49 E+02 \\
2.11 E+02 \\
3.41 E+02 \\
9.21 E+02 \\
5.78 E+03\end{array}$ & $\begin{array}{l}3.22 E+02 \\
1.08 E+02 \\
3.10 E+02 \\
8.63 E+02 \\
5.34 E \bullet 03\end{array}$ & $\begin{array}{l}3.45 E+02 \\
2.31 E+02 \\
3.42 E+02 \\
9.23 E+02 \\
5.79 E+03\end{array}$ \\
\hline $\begin{array}{l}T L-201 \\
\mathrm{~T} L-202 \\
\mathrm{I} L-204 \\
\mathrm{I} L-207 \\
\mathrm{I} L-208\end{array}$ & $\begin{array}{r}73.06 \mathrm{H} \\
12.23 \mathrm{D} \\
3.779 \mathrm{~V} \\
4.77 \mathrm{M} \\
3.053 \mathrm{M}\end{array}$ & $\begin{array}{l}3.05 E \cdot 02 \\
1.94 E * 03 \\
3.36 E * 00 \\
9.80 E+00 \\
1.64 E * 04\end{array}$ & $\begin{array}{l}3.13 E \bullet 02 \\
1.85 E \bullet 03 \\
3.60 E \bullet 00 \\
9.50 E \bullet 00 \\
1.67 E+04\end{array}$ & $\begin{array}{l}3.16 E+02 \\
2.02 E 03 \\
3.57 E+00 \\
1.07 E+01 \\
1.05 E+04\end{array}$ & $\begin{array}{l}0.04 E+02 \\
2.63 E+03 \\
1.29 E+00 \\
1.20 E+01 \\
2.11 E+04\end{array}$ & $\begin{array}{l}2.85 E+02 \\
1.78 E+03 \\
3.19 E+00 \\
9.39 E+00 \\
1.66 E+04\end{array}$ & $\begin{array}{l}2.53 E+02 \\
1.60 E+03 \\
2.85 E+00 \\
8.51 E \cdot 00 \\
1.55 E+04\end{array}$ & $\begin{array}{l}3.04 E+02 \\
1.89 E+03 \\
3.41 E+00 \\
9.99 E+00 \\
1.71 E+04\end{array}$ & $\begin{array}{l}2.74 E+02 \\
1.75 E+03 \\
3.04 E+00 \\
9.18 E+00 \\
1.64 E \cdot 04\end{array}$ & $\begin{array}{l}3.23 \mathrm{E} * 02 \\
1.91 \mathrm{E} * 03 \\
3.73 \mathrm{E} * 00 \\
9.96 \mathrm{E} * 00 \\
1.79 \mathrm{E} * 04\end{array}$ \\
\hline $\begin{array}{l}\mathrm{IL}-209 \\
\mathrm{IL}-210 \\
\mathrm{PQ}-203 \\
\mathrm{PQ}-204 \mathrm{M} \\
\mathrm{PQ}-205\end{array}$ & $\begin{array}{r}2.20 \mathrm{M} \\
1.30 \mathrm{M} \\
52.02 \mathrm{H} \\
66.9 \mathrm{M} \\
1.51 \mathrm{~V}\end{array}$ & $\begin{array}{l}9.26 E+03 \\
1.26 E+04 \\
1.22 E+03 \\
9.34 E+03 \\
2.17 E-02\end{array}$ & $\begin{array}{l}9.22 E+03 \\
1.24 E+04 \\
1.16 E+03 \\
9.00 E+03 \\
1.02 E-08\end{array}$ & $\begin{array}{l}1.03 E+04 \\
1.39 E+04 \\
1.23 E+03 \\
1.01 E+04 \\
1.79 E-03\end{array}$ & $\begin{array}{l}1.19 E+04 \\
1.58 E+04 \\
1.82 E+03 \\
1.15 E+04 \\
1.59 E+00\end{array}$ & $\begin{array}{l}1.11 E+03 \\
8.87 E+03 \\
2.91 E-03\end{array}$ & & $\begin{array}{l}9.70 E+03 \\
1.31 E+04 \\
1.18 E+03 \\
9.44 E+03 \\
3.87 E-05\end{array}$ & & $\begin{array}{l}9.69 E \cdot 03 \\
1.31 E \bullet 04 \\
1.19 E+03 \\
9.41 E+03 \\
3.57 E-05\end{array}$ \\
\hline $\begin{array}{l}P B-209 \\
P B-210 \\
P B-211 \\
P B-212 \\
P B-214\end{array}$ & $\begin{array}{r}3.253 \mathrm{H} \\
22.26 \mathrm{Y} \\
36.1 \mathrm{M} \\
10.643 \mathrm{H} \\
26.8 \mathrm{M}\end{array}$ & $\begin{array}{l}0.0 \\
3.26 E+00 \\
2.25 E+02 \\
6.04 E+02 \\
1.05 E+03\end{array}$ & $\begin{array}{l}0.0 \\
4.24 E+00 \\
2.14 E+02 \\
5.64 E+02 \\
9.84 E \bullet 02\end{array}$ & & & $\begin{array}{l}2.11 E+02 \\
5.43 E+02 \\
9.56 E+02\end{array}$ & & & & $\begin{array}{l}0.0 \\
5.01 E+00 \\
2.23 E+02 \\
5.81 E+02 \\
1.02 E+03\end{array}$ \\
\hline $\begin{array}{l}81-206 \\
61-207 \\
01-208 \\
81-210 \\
01-211\end{array}$ & $\begin{array}{r}6.2430 \\
33.4 \mathrm{Y} \\
3.68 \mathrm{H} \mathrm{Y} \\
5.013 \mathrm{D} \\
2.13 \mathrm{M}\end{array}$ & $\begin{array}{l}1.45 E+04 \\
6.76 E+03 \\
1.31 E+04 \\
0.0 \\
2.02 E \cdot 02\end{array}$ & $\begin{array}{l}1.42 E+04 \\
6.59 E+03 \\
1.36 E+04 \\
0.0 \\
1.8 B E+02\end{array}$ & $\begin{array}{l}1.59 E+04 \\
7.38 E+03 \\
1.51 E+04 \\
0.0 \\
2.06 E+02\end{array}$ & $\begin{array}{l}1.83 E+04 \\
8.52 E+03 \\
1.72 E+04 \\
0.0 \\
2.70 E+02\end{array}$ & $\begin{array}{l}6.49 E+03 \\
1.36 E+04 \\
0.0 \\
1.83 E+02\end{array}$ & $\begin{array}{l}5.90 E+03 \\
1.28 E+04 \\
0.0 \\
1.64 E+02\end{array}$ & $\begin{array}{l}1.49 E+04 \\
6.90 E+03 \\
1.45 E+04 \\
0.0 \\
1.94 E+02\end{array}$ & $\begin{array}{l}1.37 E+04 \\
6.35 E+03 \\
1.34 E+04 \\
0.0 \\
1.81 E+02\end{array}$ & $\begin{array}{l}1.49 E+04 \\
6.90 E+03 \\
1.48 E+04 \\
0.0 \\
1.94 E+02\end{array}$ \\
\hline $\begin{array}{l}81-212 \\
81-213 \\
8 I-214 \\
P 0-209 \\
90-210\end{array}$ & $\begin{array}{r}60.55 M \\
45.65 \mathrm{M} \\
19.9 \mathrm{H} \\
102 \mathrm{Y} \\
138.378 \mathrm{D}\end{array}$ & $\begin{array}{l}5.97 E+02 \\
0.03 E+03 \\
1.51 E+01 \\
3.02 E-02\end{array}$ & $\begin{array}{l}5.64 E+02 \\
6.81 E+03 \\
1.45 E+01 \\
3.69 E-02\end{array}$ & & & $\begin{array}{l}5.50 E+02 \\
6.75 E+03 \\
1.42 E+01 \\
3.64 E-02\end{array}$ & & $\begin{array}{l}5.83 E+02 \\
7.18 E+03 \\
1.51 E+01 \\
3.87 E-02\end{array}$ & $\begin{array}{l}5.40 E+02 \\
6.62 E+03 \\
1.39 E+01 \\
3.56 E-02\end{array}$ & $\begin{array}{l}8.41 E+02 \\
5.84 E+02 \\
7.19 E+03 \\
1.51 E+01 \\
3.86 E-02\end{array}$ \\
\hline $\begin{array}{l}P O-211 \\
P O-212 \\
P O-213 \\
P O-214 \\
P O-215\end{array}$ & $\begin{array}{rr}0.516 & 5 \\
2.98 E-7 & S \\
4.2 E-6 & S \\
1.637 E-4 & S \\
1.778 E-3 & S\end{array}$ & $\begin{array}{l}3.46 E+01 \\
0.0 \\
1.37 E-01 \\
3.74 E-01 \\
0.41 E-01\end{array}$ & $\begin{array}{l}3.33 E+01 \\
0.0 \\
1.32 E-01 \\
3.61 E-01 \\
6.03 E-01\end{array}$ & $\begin{array}{l}3.74 E+01 \\
0.0 \\
1.48 E-01 \\
4.06 E-01 \\
6.68 E-01\end{array}$ & $\begin{array}{l}4.26 E+01 \\
0.0 \\
1.67 E-01 \\
4.58 E-01 \\
8.20 E-01\end{array}$ & $\begin{array}{l}3.28 E+01 \\
0.0 \\
1.30 E-01 \\
3.56 E-01 \\
5.89 E-01\end{array}$ & $\begin{array}{l}2.97 E \cdot 01 \\
0.0 \\
1.17 E-01 \\
3.22 E-01 \\
5.29 E-01\end{array}$ & $\begin{array}{l}3.49 E \cdot 01 \\
0.0 \\
1.38 E-01 \\
3.79 E-01 \\
6.24 E-01\end{array}$ & $\begin{array}{l}3.21 E+01 \\
0.0 \\
1.27 E-01 \\
3.48 E-01 \\
5.79 E-01\end{array}$ & $\begin{array}{l}3.48 E+01 \\
0.0 \\
1.38 E-01 \\
3.77 E-01 \\
6.24 E-01\end{array}$ \\
\hline
\end{tabular}


DOSE-RATE FACTORS FOR BODY ORGANS AND EFFECTIVE DOSE-PATE FACTOR FOR. IMMERSION IM COMTAATHATLU AlP

\begin{tabular}{|c|c|c|c|c|c|c|c|c|c|c|}
\hline KCLIDE & HALF-LIFE & LIVER & LUNGS & MARROH & $\begin{array}{c}\text { REO } \\
\text { MARROH }\end{array}$ & IARIES & ANCREAS & SKELETON & SP LEEN & STOMA \\
\hline $\begin{array}{l}J 194 \\
j-195 \\
j-195 M \\
-196 \\
j-198\end{array}$ & $\begin{array}{rr}39.5 & H \\
183 & 0 \\
30.6 & 5 \\
6.183 & 0 \\
2.696 & D\end{array}$ & & & & & & & & & 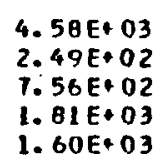 \\
\hline $\begin{array}{l}-199 \\
-197 \\
-1974 \\
-203\end{array}$ & $\begin{array}{r}64 . \\
23 \\
46 . \\
26\end{array}$ & & & & & & & & & 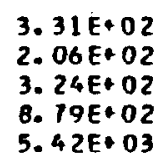 \\
\hline-2 & $\begin{array}{r}73.06 \mathrm{H} \\
12.23 \mathrm{D} \\
3.719 \mathrm{Y} \\
4.77 \mathrm{M} \\
3.053 \mathrm{H}\end{array}$ & $\begin{array}{l}3.10 E+02 \\
1.83 E \cdot 03 \\
3.57 E+00 \\
9.45 E+00\end{array}$ & 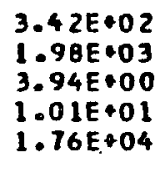 & e & & & & & & $E+C$ \\
\hline $\begin{array}{l}-209 \\
-210 \\
-203 \\
-204 M \\
-205\end{array}$ & $\begin{array}{r}2.20 \mathrm{M} \\
1.30 \mathrm{M} \\
52.02 \mathrm{H} \\
66.9 \mathrm{M} \\
1.51 E 7 \mathrm{Y}\end{array}$ & $\begin{array}{l}1.24 \\
1.19 \\
8.95 \\
2.52\end{array}$ & $\begin{array}{l}3 \\
4 \\
3\end{array}$ & $\begin{array}{l}1 . \\
1 . \\
1 .\end{array}$ & $\begin{array}{l}9 . \\
1 . \\
1 . \\
9 . \\
5 .\end{array}$ & & & & & 4. \\
\hline-2 & $\begin{array}{r}3.253 \mathrm{H} \\
22.26 \mathrm{Y} \\
36.1 \mathrm{H} \\
10.643 \mathrm{H} \\
26.8 \mathrm{H}\end{array}$ & 2 & & $\begin{array}{l}7.6 \\
2.5 \\
8.5 \\
1.3\end{array}$ & & & & & & \\
\hline $\begin{array}{l}-206 \\
-207 \\
-208 \\
-210\end{array}$ & $\begin{array}{r}6.2 \\
33 \\
3.68 \\
5.0 \\
2.0\end{array}$ & $\begin{array}{l}1.42 E+04 \\
6.56 E+03 \\
1.37 E+04 \\
0.0 \\
1.07 E+02\end{array}$ & & $\begin{array}{l}1 . \\
7 . \\
0 . \\
2 .\end{array}$ & $\begin{array}{l}1.52 E \\
7.02 E \\
1.46 E \\
0.0 \\
2.06 E\end{array}$ & & & & & \\
\hline $\begin{array}{l}81-212 \\
01-213 \\
81-214 \\
P 0-209 \\
P 0-210\end{array}$ & $\begin{array}{r}60.55 \mathrm{M} \\
45.65 \mathrm{M} \\
19.9 \mathrm{M} \\
102 \mathrm{Y} \\
138.370 \mathrm{D}\end{array}$ & $\begin{array}{l}5.61 \\
6.81 \\
1.44 \\
3.66\end{array}$ & & & & & & & & $\begin{array}{l}1.42 E+ \\
3.63 E-C\end{array}$ \\
\hline $\begin{array}{l}b-211 \\
-212 \\
b-213 \\
-214 \\
-215\end{array}$ & $\begin{array}{r}2.98 E-15 \\
4.2 E-65 \\
.637 E-45 \\
.178 E-3\end{array}$ & $\begin{array}{l}3.31 E \cdot 01 \\
0.0 \\
1.31 E-01 \\
3.59 E-01 \\
6.00 E-01\end{array}$ & $\begin{array}{l}3.54 E+01 \\
0.0 \\
1.40 E-01 \\
3.83 E-01 \\
6.45 E-01\end{array}$ & $\begin{array}{l}3.89 E+01 \\
0.0 \\
1.53 E-01 \\
4.17 E-01 \\
7.62 E-01\end{array}$ & $\begin{array}{l}3.60 E+01 \\
0.0 \\
1.42 E-01 \\
3.89 E-01 \\
6.64 E-01\end{array}$ & $\begin{array}{l}3.21 E+01 \\
0.0 \\
1.27 E-01 \\
3.49 E-01 \\
5.69 E-01\end{array}$ & $\begin{array}{l}2.05 E \cdot 01 \\
0.0 \\
1.13 E-01 \\
3.09 E-01 \\
5.17 E-01\end{array}$ & $\begin{array}{l}3.95 E-01 \\
0.0 \\
1.55 E-01 \\
4.24 E-01 \\
7.74 E-01\end{array}$ & $\begin{array}{l}3.21 E+01 \\
0.0 \\
1.27 E-01 \\
3.48 E-01 \\
5.88 E-01\end{array}$ & $\begin{array}{l}1.295- \\
3.55 \mathrm{E}- \\
5.92 \mathrm{E}-\end{array}$ \\
\hline
\end{tabular}


DUSE-KATE FACTORS FOR BDOY ORGANS AND EFFECTIVE DOSE-RATE FACTOR FOK IMMERSIUM IN CTINIAMIHATED alY
IN MREM/YR PER MICROCUR IE/ICUBIC MI

\begin{tabular}{|c|c|c|c|c|c|c|c|c|c|}
\hline NUCL IDE & HALF-LIFE & TESTES & THYMUS & THYROID & UTERUS & EFFECTIVE & $\begin{array}{c}\text { SK1N } \\
\text { (PHOTONI }\end{array}$ & $\begin{array}{l}\text { SKIN } \\
\text { (ELECTRON) }\end{array}$ & $\begin{array}{l}\text { SKIN } \\
\text { GTOTALI }\end{array}$ \\
\hline $\begin{array}{l}A U-194 \\
A U-195 \\
A U-195 M \\
A U-198 \\
A U-198\end{array}$ & $\begin{array}{rr}39.5 & H \\
183 & 0 \\
30.6 & 5 \\
6.183 & D \\
2.696 & D\end{array}$ & $\begin{array}{l}6.61 E+03 \\
4.53 E+02 \\
1.14 E+03 \\
2.72 E+03 \\
2.35 E+03\end{array}$ & $\begin{array}{l}5.18 E \bullet 03 \\
3.50 E+02 \\
8.69 E \bullet 02 \\
2.06 E \bullet 03 \\
1.78 E \bullet 03\end{array}$ & $\begin{array}{l}6.21 E+03 \\
4.47 E+02 \\
1.09 E+03 \\
2.56 E+03 \\
2.19 E+03\end{array}$ & $\begin{array}{l}4.16 E+03 \\
2.12 E+02 \\
6.75 E+02 \\
1.61 E+03 \\
1.43 E+03\end{array}$ & $\begin{array}{l}5.69 E+03 \\
3.83 E+02 \\
9.97 E+02 \\
2.35 E+03 \\
2.03 E+03\end{array}$ & $\begin{array}{l}7.51 E+03 \\
5.01 E+02 \\
1.27 E \cdot 03 \\
3.07 E+03 \\
2.67 E+03\end{array}$ & $\begin{array}{l}1.77 E+02 \\
1.02 E+01 \\
2.96 E+02 \\
1.48 E+02 \\
2.16 E+03\end{array}$ & $\begin{array}{l}7.69 E+03 \\
5.11 E+02 \\
1.51 E+03 \\
3.21 E+03 \\
4.81 E+03\end{array}$ \\
\hline $\begin{array}{l}A U-199 \\
H G-197 \\
H G-197 M \\
H G-203 \\
Y L-200\end{array}$ & $\begin{array}{r}3.139 \mathrm{D} \\
64.14 \mathrm{H} \\
23.8 \mathrm{H} \\
46.60 \mathrm{D} \\
26.1 \mathrm{H}\end{array}$ & $\begin{array}{l}5.17 E \cdot 02 \\
3.73 E \cdot 02 \\
5.24 E+02 \\
1.32 E+03 \\
7.85 E+03\end{array}$ & $\begin{array}{l}3.97 E \bullet 02 \\
2.90 E \bullet 02 \\
4.04 E \bullet 02 \\
9.99 E \bullet 02 \\
6.16 E \bullet 03\end{array}$ & $\begin{array}{l}5.06 E+02 \\
3.69 E+02 \\
5.15 E+02 \\
1.25 E+03 \\
1.43 E+03\end{array}$ & $\begin{array}{l}2.94 E \cdot 02 \\
1.77 E+02 \\
2.85 E+02 \\
7.85 E+02 \\
4.85 E+03\end{array}$ & $\begin{array}{l}4.53 E+02 \\
3.16 E+02 \\
4.55 E+02 \\
1.15 E+03 \\
6.73 E+03\end{array}$ & $\begin{array}{l}5.61 E+02 \\
4.11 E+02 \\
5.73 E+02 \\
1.47 E+03 \\
8.98 E \cdot 03\end{array}$ & $\begin{array}{l}4.01 E+02 \\
1.95 E+00 \\
6.19 E+02 \\
3.34 E+02 \\
1.63 E+02\end{array}$ & $\begin{array}{l}9.63 E+02 \\
4.13 E+02 \\
1.19 E+03 \\
1.80 E+03 \\
9.14 E+03\end{array}$ \\
\hline $\begin{array}{l}\mathrm{TL}-201 \\
\mathrm{IL}-202 \\
\mathrm{I} L-204 \\
\mathrm{~T} L-207 \\
\mathrm{IL}-208\end{array}$ & $\begin{array}{rl}73.06 & H \\
12.23 & D \\
3.779 & Y \\
4.77 & M \\
3.053 & M\end{array}$ & $\begin{array}{l}5.11 E+02 \\
2.70 E+03 \\
6.04 E+00 \\
1.33 E+01 \\
2.29 E+04\end{array}$ & $\begin{array}{l}3.96 E \cdot 02 \\
2.04 E \bullet 03 \\
4.70 E \bullet 00 \\
1.06 E \bullet 01 \\
1.79 E+04\end{array}$ & $\begin{array}{l}5.05 E+02 \\
2.52 E+03 \\
5.98 E+00 \\
1.27 E+01 \\
2.09 E+04\end{array}$ & $\begin{array}{l}2.55 E+02 \\
1.59 E+03 \\
2.06 E+00 \\
8.23 E+00 \\
1.50 E+04\end{array}$ & $\begin{array}{l}4.37 E+02 \\
2.31 E+03 \\
5.12 E+00 \\
1.14 E+01 \\
1.98 E+04\end{array}$ & $\begin{array}{l}5.60 E+02 \\
3.06 E+03 \\
6.65 E+00 \\
1.54 E+01 \\
2.56 E+04\end{array}$ & $\begin{array}{l}3.17 E+01 \\
8.83 E+01 \\
1.62 E+03 \\
3.57 E+03 \\
4.32 E+03\end{array}$ & $\begin{array}{l}5.91 E+02 \\
3.15 E+03 \\
1.63 E+03 \\
3.58 E+03 \\
3.00 E+04\end{array}$ \\
\hline $\begin{array}{l}I L-209 \\
1 L-210 \\
P B-203 \\
P B-204 A \\
P B-205\end{array}$ & $\begin{array}{r}2.20 \mathrm{M} \\
1.30 \mathrm{M} \\
52.02 \mathrm{H} \\
66.9 \mathrm{M} \\
1.51 E 7 \mathrm{Y}\end{array}$ & $\begin{array}{l}1.29 E+04 \\
1.73 E+04 \\
1.74 E+03 \\
1.27 E+04 \\
1.57 E-01\end{array}$ & $\begin{array}{l}1.01 E+04 \\
1.37 E+04 \\
1.32 E+03 \\
1.00 E+04 \\
5.59 E-06\end{array}$ & $\begin{array}{l}1.21 E+04 \\
1.63 E+04 \\
1.66 E+03 \\
1.21 E+04 \\
1.10 E-02\end{array}$ & $\begin{array}{l}8.21 E+03 \\
1.10 E+04 \\
9.99 E+02 \\
7.81 E+03 \\
1.47 E-06\end{array}$ & $\begin{array}{l}1.11 E+04 \\
1.49 E+04 \\
1.50 E+03 \\
1.09 E+04 \\
2.86 E-01\end{array}$ & $\begin{array}{l}1.47 E+04 \\
1.98 E+04 \\
1.93 E+03 \\
1.46 E+04 \\
3.34 E+00\end{array}$ & $\begin{array}{l}5.10 E \cdot 03 \\
5.19 E+03 \\
2.67 E+02 \\
7.32 E+02 \\
0.0\end{array}$ & $\begin{array}{l}1.98 E+04 \\
2.50 E+04 \\
2.20 E+03 \\
1.54 E+04 \\
3.34 E+00\end{array}$ \\
\hline $\begin{array}{l}P B-209 \\
P B-210 \\
P B-211 \\
P B-212 \\
P B-214\end{array}$ & $\begin{array}{r}3.253 H \\
22.26 \mathrm{H} \\
36.1 \mathrm{H} \\
10.643 \mathrm{H} \\
26.8 \mathrm{H}\end{array}$ & $\begin{array}{l}0.0 \\
8.32 E+00 \\
3.05 E+02 \\
8.49 E+02 \\
1.44 E+03\end{array}$ & $\begin{array}{l}0.0 \\
5.59 E+00 \\
2.37 E+02 \\
6.46 E+02 \\
1.09 E+03\end{array}$ & $\begin{array}{l}0.0 \\
7.30 E+00 \\
2.87 E+02 \\
0.17 E \bullet 02 \\
1.36 E+03\end{array}$ & $\begin{array}{l}0.0 \\
2.56 E+00 \\
1.86 E+02 \\
4.90 E+02 \\
8.59 E+02\end{array}$ & $\begin{array}{l}0.0 \\
6.70 E+00 \\
2.61 E+02 \\
7.40 E+02 \\
1.25 E+03\end{array}$ & $\begin{array}{l}0.0 \\
1.40 E \cdot 01 \\
3.50 E \cdot 02 \\
9.37 E \cdot 02 \\
1.62 E \cdot 03\end{array}$ & $\begin{array}{l}1.09 E+03 \\
0.0 \\
3.24 E+03 \\
6.36 E+02 \\
1.03 E+03\end{array}$ & $\begin{array}{l}1.09 E \cdot 03 \\
1.40 E \bullet 01 \\
3.59 E+03 \\
1.57 E+03 \\
3.25 E \cdot 03\end{array}$ \\
\hline $\begin{array}{l}81-200 \\
8 I-207 \\
8 I-208 \\
8 I-210 \\
8 I-211\end{array}$ & $\begin{array}{r}6.243 \mathrm{D} \\
33.4 Y \\
3.6085 Y \\
5.013 \mathrm{D} \\
2.13 \mathrm{M}\end{array}$ & $\begin{array}{l}2.00 E+04 \\
9.30 E+03 \\
1.86 E+04 \\
0.0 \\
2.74 E+02\end{array}$ & $\begin{array}{l}1.57 E+04 \\
7.33 E+03 \\
1.46 E+04 \\
0.0 \\
2.07 E+02\end{array}$ & $\begin{array}{l}1.89 E+04 \\
8.80 E+03 \\
1.68 E+04 \\
0.0 \\
2.57 E+02\end{array}$ & $\begin{array}{l}1.24 E+04 \\
5.74 E+03 \\
1.24 E+04 \\
0.0 \\
1.65 E+02\end{array}$ & $\begin{array}{l}1.71 E+04 \\
7.96 E+03 \\
1.61 E+04 \\
0.0 \\
2.37 E+02\end{array}$ & $\begin{array}{l}2.30 E+04 \\
1.07 E \bullet 04 \\
2.06 E+04 \\
0.0 \\
3.09 E+02\end{array}$ & $\begin{array}{l}6.19 E+02 \\
8.05 E+02 \\
3.34 E+01 \\
2.69 E+03 \\
5.91 E+01\end{array}$ & $\begin{array}{l}2.36 E \cdot 04 \\
1.15 E \cdot 04 \\
2.07 E+04 \\
2.69 E+03 \\
3.68 E \cdot 02\end{array}$ \\
\hline $\begin{array}{l}81-212 \\
81-213 \\
81-214 \\
P 0-209 \\
P 0-210\end{array}$ & $\begin{array}{r}60.55 \mathrm{M} \\
45.65 \mathrm{M} \\
19.9 \mathrm{M} \\
102 \mathrm{Y} \\
138.318 \mathrm{D}\end{array}$ & $\begin{array}{l}1.12 E+03 \\
8.12 E+02 \\
9.45 E+03 \\
2.08 E+01 \\
5.19 E-02\end{array}$ & $\begin{array}{l}8.85 E+02 \\
6.15 E+02 \\
7.47 E+03 \\
1.64 E+01 \\
4.11 E-02\end{array}$ & $\begin{array}{l}1.06 E+03 \\
7.56 E+02 \\
8.05 E+03 \\
1.99 E \cdot 01 \\
4.93 E-02\end{array}$ & $\begin{array}{l}7.02 E+02 \\
4.91 E \cdot 02 \\
6.05 E \cdot 03 \\
1.26 E \cdot 01 \\
3.19 E-02\end{array}$ & $\begin{array}{l}9.60 E \bullet 02 \\
6.97 E \bullet 02 \\
8.11 E * 03 \\
1.79 E+01 \\
4.44 E-02\end{array}$ & $\begin{array}{l}1.29 E \cdot 03 \\
9.23 E \cdot 02 \\
1.08 E \cdot 04 \\
2.37 E+01 \\
6.00 E-02\end{array}$ & $\begin{array}{l}3.52 E * 03 \\
3.09 E * 03 \\
4.86 E+03 \\
1.00 E+00 \\
0.0\end{array}$ & $\begin{array}{l}4.81 E+03 \\
4.01 E+03 \\
1.57 E+04 \\
2.47 E+01 \\
6.00 E-02\end{array}$ \\
\hline $\begin{array}{l}P O-211 \\
P O-212 \\
P D-213 \\
P 0-214 \\
P 0-215\end{array}$ & $\begin{array}{rr}0.516 & 5 \\
2.98 E-7 & 5 \\
4.2 E-6 & 5 \\
1.637 E-4 & 5 \\
1.778 E-3 & S\end{array}$ & $\begin{array}{l}4.10 E \cdot 01 \\
0.0 \\
1.86 E-01 \\
5.08 E-01 \\
8.68 E-01\end{array}$ & $\begin{array}{l}3.69 E \cdot 01 \\
0.0 \\
1.46 E-01 \\
4.02 E-01 \\
6.54 E-01\end{array}$ & $\begin{array}{l}4.44 E+01 \\
0.0 \\
1.76 E-01 \\
4.83 E-01 \\
8.05 E-01\end{array}$ & $\begin{array}{l}2.88 E \cdot 01 \\
0.0 \\
1.14 E-01 \\
3.12 E-01 \\
5.26 E-01\end{array}$ & $\begin{array}{l}4.02 E+01 \\
0.0 \\
1.59 E-01 \\
4.34 E-01 \\
7.45 E=01\end{array}$ & $\begin{array}{l}5.42 E+01 \\
0.0 \\
2.84 E-01 \\
5.88 E-01 \\
9.87 E-01\end{array}$ & $\begin{array}{l}0.0 \\
0.0 \\
0.0 \\
0.0 \\
0.0\end{array}$ & $\begin{array}{l}5.42 E+01 \\
0.0 \\
2.14 E-01 \\
5.88 E-01 \\
9.87 E-01\end{array}$ \\
\hline
\end{tabular}


DUSE-RATE FACTORS FOR BODY ORGANS ANO EFFECTIVE DUS:-PATE FACTOR FOR IMMERSION IN CONI AMINATE" MIR

IN MREMIYR PER MICROCURIE/ICUBIC MI

\begin{tabular}{|c|c|c|c|c|c|c|c|c|c|c|}
\hline$\angle I D E$ & LIFE & ADRENALS & BLADDER & BRAIN & BREAST & HE AR T & $\begin{array}{l}\text { SMALL } \\
\text { NTESTINE }\end{array}$ & $\begin{array}{c}\text { UPPER } \\
\text { LARGE } \\
\text { INTESIINE }\end{array}$ & $\begin{array}{c}\text { LOHER } \\
\text { IARGE } \\
\text { INIESTIINE }\end{array}$ & K JONEYS \\
\hline $\begin{array}{l}P O-216 \\
P O-218 \\
A T-211 \\
A T-217 \\
R N-218\end{array}$ & $\begin{array}{r}0.146 \\
3.05 \\
7.014 \\
7.214 \mathrm{H} \\
0.0323 \mathrm{~S} \\
0.035 \mathrm{~S}\end{array}$ & $\begin{array}{l}6.51 E-02 \\
0.0 \\
1.29 E+02 \\
1.04 E * 00 \\
3.32 E \cdot 00\end{array}$ & $\begin{array}{l}E-02 \\
E+02 \\
E-01 \\
E+00\end{array}$ & $\begin{array}{l}7.06 E-02 \\
0.0 \\
1.36 E+02 \\
1.11 E+00 \\
3.54 E+00\end{array}$ & $\begin{array}{l}7.96 E-02 \\
0.0 \\
2.55 E+02 \\
1.29 E+00 \\
4.11 E+00\end{array}$ & $\begin{array}{l}6.20 E-02 \\
0.0 \\
1.22 E+02 \\
9.74 E-01 \\
3.11 E+00\end{array}$ & $\begin{array}{l}.61 E-02 \\
.0 \\
.08 E+02 \\
.77 E-01 \\
.80 E+00\end{array}$ & $\begin{array}{l}6.59 E-02 \\
0.0 \\
1.31 E+02 \\
1.03 E+00 \\
3.30 E+00\end{array}$ & $\begin{array}{l}6.06 E-02 \\
0.0 \\
1.17 E+02 \\
9.53 E-01 \\
3.04 E+00\end{array}$ & $\begin{array}{l}6.57 E-02 \\
0.0 \\
1.36 E+02 \\
1.03 E+00 \\
3.29 E+00\end{array}$ \\
\hline $\begin{array}{l}R N-219 \\
R N-220 \\
R N-222 \\
F R-221 \\
F R-223\end{array}$ & $\begin{array}{r}3.96 \mathrm{~S} \\
55.61 \mathrm{~S} \\
3.8235 \mathrm{D} \\
4.0 \mathrm{M} \\
21.0 \mathrm{M}\end{array}$ & $\begin{array}{l}2.45 E+02 \\
2.27 E+00 \\
1.6 B E+00 \\
1.30 E+02 \\
1.69 E * 02\end{array}$ & $\begin{array}{l}28 E+02 \\
16 E+00 \\
59 E+00 \\
19 E+02 \\
70 E+02\end{array}$ & $\begin{array}{l}2.48 E+02 \\
2.41 E+00 \\
1.77 E+00 \\
1.27 E+02 \\
1.72 E+02\end{array}$ & & $\begin{array}{l}2.22 E+02 \\
2.11 E+00 \\
1.56 E+00 \\
1.16 E+02 \\
1.54 E+02\end{array}$ & $\begin{array}{l}1.99 E+02 \\
1.90 E+00 \\
1.40 E+00 \\
1.03 E+02 \\
1.39 E+02\end{array}$ & $\begin{array}{l}2.35 E+02 \\
2.24 E+00 \\
1.65 E+00 \\
1.23 E+02 \\
1.67 E+02\end{array}$ & $\begin{array}{l}2.19 E+02 \\
2.07 E+00 \\
1.53 E+00 \\
1.15 E+02 \\
1.52 E+02\end{array}$ & $\begin{array}{l}2.35 E+02 \\
2.24 E+00 \\
1.65 E+00 \\
1.23 E+02 \\
1.79 E+02\end{array}$ \\
\hline $\begin{array}{l}\text { RA-222 } \\
\text { RA-223 } \\
\text { RA-224 } \\
\text { RA-225 } \\
\text { RA-226 }\end{array}$ & $\begin{array}{r}38.0 \mathrm{~S} \\
11.434 \mathrm{D} \\
3.62 \mathrm{D} \\
14.8 \mathrm{O} \\
1600 \mathrm{Y}\end{array}$ & $\begin{array}{l}.94 E \cdot 01 \\
38 E+02 \\
.36 E+01 \\
.26 E+01\end{array}$ & & & & & $\begin{array}{l}3.19 E+01 \\
4.35 E+02 \\
3.40 E+01\end{array}$ & $\begin{array}{l}3.76 E+01 \\
5.22 E+02 \\
4.03 E+01 \\
1.70 E * 01 \\
2.68 E+01\end{array}$ & $\begin{array}{l}3.52 E+01 \\
4.80 E+02 \\
3.78 E+01 \\
1.31 E+01 \\
2.49 E \cdot 01\end{array}$ & $\begin{array}{l}3.77 E+01 \\
5.23 E+02 \\
4.02 E+01 \\
2.87 E+01 \\
2.65 E+01\end{array}$ \\
\hline $\begin{array}{l}R A-228 \\
A C-225 \\
A C-227 \\
A C-228 \\
T H-226\end{array}$ & 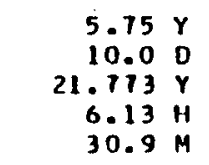 & $\begin{array}{l}2.30 E-0 B \\
5.19 E+01 \\
4.64 E-01 \\
4.12 E+03 \\
3.10 E+01\end{array}$ & $\begin{array}{l}4.15 E-15 \\
4.95 E+01 \\
4.33 E-01 \\
4.01 E+03 \\
2.91 E+01\end{array}$ & $\begin{array}{l}1.84 E-09 \\
5.21 E \bullet 01 \\
4.60 E-01 \\
4.49 E+03 \\
3.09 E+01\end{array}$ & $\begin{array}{l}1.00 E-0 E \\
9.02 E \bullet 01 \\
9.38 E-01 \\
5.14 E+03 \\
5.10 E+01\end{array}$ & $\begin{array}{l}3.00 E-09 \\
4.76 E+01 \\
4.25 E-01 \\
3.96 E+03 \\
2.83 E+01\end{array}$ & & $\begin{array}{l}5.69 E-11 \\
5.10 E+01 \\
4.57 E-01 \\
4.21 E+03 \\
3.03 E+01\end{array}$ & $\begin{array}{l}9.45 E-09 \\
4.63 E \bullet 01 \\
4.13 E-01 \\
3.8 B \cdot 03 \\
2.77 E \bullet 01\end{array}$ & $\begin{array}{l}2.76 E-11 \\
5.05 E+01 \\
4.40 E-01 \\
4.20 E+03 \\
2.97 E+01\end{array}$ \\
\hline $\begin{array}{l}T H-227 \\
T H-228 \\
T H-229 \\
T H-230 \\
T H-231\end{array}$ & $\begin{array}{r}18.718 \mathrm{O} \\
1.9132 \mathrm{Y} \\
7.34 E 3 \mathrm{Y} \\
7.7 E 4 \mathrm{Y} \\
25.52 \mathrm{H}\end{array}$ & $\begin{array}{l}4.34 E+02 \\
7.40 E+00 \\
3.24 E+02 \\
1.27 E+00 \\
3.88 E+01\end{array}$ & $\begin{array}{l}7.12 E+00 \\
3.13 E+02 \\
1.28 E+00 \\
3.89 E+01\end{array}$ & $\begin{array}{l}4.31 E+02 \\
7.45 E+00 \\
3.27 E * 02 \\
1.29 E \bullet 00 \\
3.92 E+01\end{array}$ & $\begin{array}{l}6.31 E+02 \\
1.37 E+01 \\
5.80 E+02 \\
3.38 E+00 \\
9.16 E+01\end{array}$ & $\begin{array}{l}3.89 E+02 \\
6.78 E+00 \\
2.99 E+02 \\
1.16 E+00 \\
3.57 E+01\end{array}$ & & $\begin{array}{l}4.13 E+02 \\
1.23 E \bullet 00 \\
3.20 E+02 \\
1.24 E+00 \\
3.92 E+01\end{array}$ & & $\begin{array}{l}4.16 E+02 \\
7.28 E+00 \\
3.20 E+02 \\
1.33 E+00 \\
4.08 E+01\end{array}$ \\
\hline $\begin{array}{l}T H-232 \\
T H-233 \\
T H-234 \\
P A-230 \\
P A-231\end{array}$ & $\begin{array}{r}1.405 E 10 Y \\
22.3 Y \\
24.10 \\
17.40 \\
3.276 E 4 Y\end{array}$ & $\begin{array}{l}5.22 E-01 \\
1.42 E+02 \\
2.69 E+01 \\
2.88 E+03 \\
1.25 E+02\end{array}$ & $\begin{array}{l}5.33 E-01 \\
1.35 E+02 \\
2.74 E \cdot 01 \\
2.79 E \cdot 03 \\
1.16 E .02\end{array}$ & $\begin{array}{l}5.19 E-01 \\
1.49 E * 02 \\
2.77 E * 01 \\
3.12 E \cdot 03 \\
1.24 E * 02\end{array}$ & $\begin{array}{l}1.93 E+02 \\
5.48 E+01 \\
3.70 E+03 \\
1.85 E+02\end{array}$ & $\begin{array}{l}4.69 E-01 \\
1.31 E+02 \\
2.51 E+01 \\
2.15 E+03 \\
1.12 E+02\end{array}$ & & & $\begin{array}{l}4.61 E-01 \\
1.29 E \cdot 02 \\
2.42 E \cdot 01 \\
2.68 E+03 \\
1.11 E+02\end{array}$ & $\begin{array}{l}1.40 E * 02 \\
2.82 E+01 \\
2.92 E * 03 \\
1.20 E * 02\end{array}$ \\
\hline $\begin{array}{l}\text { PA-233 } \\
\text { PA-234 } \\
\text { PA-234M } \\
U-230 \\
U-231\end{array}$ & $\begin{array}{rl}21.0 & D \\
6.70 & H \\
1.17 & M \\
20.8 & 0 \\
4.2 & 0\end{array}$ & $\begin{array}{l}8.90 E+02 \\
8.67 E+03 \\
5.10 E+01 \\
4.04 E+00 \\
2.52 E+02\end{array}$ & $\begin{array}{l}8.29 E+02 \\
8.41 E+03 \\
4.96 E+01 \\
3.88 E+00 \\
2.45 E+02\end{array}$ & $\begin{array}{l}8.98 E+02 \\
9.41 E+C 3 \\
5.57 E+01 \\
4.05 E+00 \\
2.55 E+02\end{array}$ & $\begin{array}{l}1.26 E+03 \\
1.09 E+04 \\
6.32 E+01 \\
8.38 E+00 \\
4.78 E+02\end{array}$ & $\begin{array}{r}8.06 E+02 \\
8.29 E+03 \\
4.90 E+01 \\
3.67 E+00 \\
2.34 E+02\end{array}$ & & & $\begin{array}{l}8.11 E+03 \\
4.79 E+01 \\
3.59 E+00 \\
2.26 E+02\end{array}$ & $\begin{array}{l}\text { 8. } 79 E+03 \\
5.20 E+01 \\
3.99 E+00 \\
2.51 E+02\end{array}$ \\
\hline $\begin{array}{l}u-234 \\
u-235 \\
u-236\end{array}$ & $\begin{array}{r}12 Y \\
1.592 E 5 Y \\
2.445 E 5 Y \\
7.038 E 8 Y \\
2.3415 E 7 Y\end{array}$ & $\begin{array}{l}7.62 E-01 \\
8.56 E-01 \\
3.69 E-01 \\
6.31 E+02 \\
2.57 E-01\end{array}$ & $\begin{array}{l}7.39 E-01 \\
7.95 E-01 \\
3.52 E-01 \\
5.79 E+02 \\
2.50 E-01\end{array}$ & $\begin{array}{l}7.39 E-01 \\
0.45 E-01 \\
3.44 E-01 \\
6.18 E+02 \\
2.46 E-01\end{array}$ & $\begin{array}{l}2.95 E * 00 \\
1.94 E * 00 \\
2.05 E+00 \\
9.56 E+02 \\
1.17 E \cdot 00\end{array}$ & $\begin{array}{l}1.00 E-01 \\
3.14 E-01 \\
5.66 E+02 \\
2.21 E-01\end{array}$ & $\begin{array}{l}6.71 E-01 \\
2.78 E-01 \\
5.00 E 002 \\
1.95 E-01\end{array}$ & $\begin{array}{l}8.38 E-01 \\
3.39 E-01 \\
6.01 E+02 \\
2.32 E-01\end{array}$ & $\begin{array}{l}7.60 E-01 \\
3.19 E-01 \\
5.59 E 002 \\
2.21 E-01\end{array}$ & $\begin{array}{l}\text { 8.07E-01 } \\
3.74 \mathrm{E}-01 \\
5.92 \mathrm{E} 02 \\
2.61 \mathrm{E}-01\end{array}$ \\
\hline
\end{tabular}




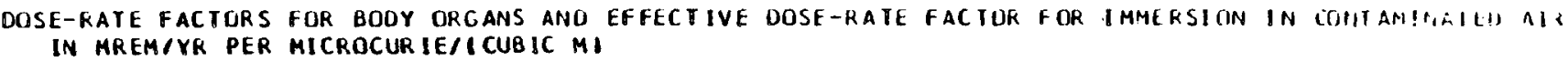

\begin{tabular}{|c|c|c|c|c|c|c|c|c|c|c|}
\hline CLI & HALF-LIFE & LIVER & LUNGS & MARROW & $\begin{array}{c}\text { RED } \\
\text { MARROH }\end{array}$ & OVARIES & ANCREAS & SKELETON & SPLEEN & STOMAC \\
\hline $\begin{array}{l}P O-216 \\
P 0-218 \\
A T-211 \\
A T-217 \\
R N-218\end{array}$ & $\begin{array}{r}0.146 \mathrm{~S} \\
3.05 \mathrm{H} \\
7.214 \mathrm{H} \\
0.0323 \mathrm{~S} \\
0.035 \mathrm{~S}\end{array}$ & $\begin{array}{l}0.24 E-02 \\
0.0 \\
1.32 E+02 \\
9.87 E-01 \\
3.15 E+00\end{array}$ & $\begin{array}{l}6.67 E-02 \\
0.0 \\
1.45 E+02 \\
1.06 E+00 \\
3.37 E+00\end{array}$ & $\begin{array}{l}7.26 E-02 \\
0.0 \\
2.46 E+02 \\
1.19 E \cdot 00 \\
3.79 E+00\end{array}$ & $\begin{array}{l}6.77 E-02 \\
0.0 \\
1.02 E+02 \\
1.08 E+00 \\
3.44 E \bullet 00\end{array}$ & $\begin{array}{l}6.07 E-02 \\
0.0 \\
1.11 E+02 \\
9.55 E-01 \\
3.05 E+00\end{array}$ & $\begin{array}{l}5.39 E-02 \\
0.0 \\
9.46 E+01 \\
8.50 E-01 \\
2.71 E+00\end{array}$ & $\begin{array}{l}7.38 E-02 \\
0.0 \\
2.53 E \cdot 02 \\
1.21 E \cdot 00 \\
3.85 E+00\end{array}$ & $\begin{array}{l}0.06 E-02 \\
0.0 \\
1.24 E+02 \\
9.61 E-01 \\
3.06 E+00\end{array}$ & $\begin{array}{l}0.18 E-02 \\
0.0 \\
1.20 E+02 \\
9.75 E-01 \\
3.11 E+00\end{array}$ \\
\hline $\begin{array}{l}N-219 \\
N-220 \\
N-222 \\
R-221 \\
R-223\end{array}$ & 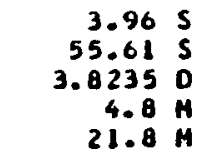 & $\begin{array}{l}2.26 E+02 \\
2.15 E+00 \\
1.58 E+00 \\
1.19 E+02 \\
1.67 E+02\end{array}$ & $\begin{array}{l}2.45 E+02 \\
2.30 E+00 \\
1.70 E+00 \\
1.29 E+02 \\
1.83 E+02\end{array}$ & $\begin{array}{l}3.09 E+02 \\
2.62 E \bullet 00 \\
1.96 E+00 \\
1.79 E+02 \\
2.74 E+02\end{array}$ & $\begin{array}{l}.50 E+02 \\
.35 E+00 \\
.74 E+0 Q \\
.28 E+02 \\
.49 E+02\end{array}$ & $\begin{array}{l}2.08 E+02 \\
2.07 E+00 \\
1.52 E+00 \\
1.05 E+02 \\
1.46 E+02\end{array}$ & $\begin{array}{l}1.95 E+02 \\
1.85 E+00 \\
1.36 E+00 \\
1.01 E+02 \\
1.28 E+02\end{array}$ & $\begin{array}{l}3.14 E+02 \\
2.66 E+00 \\
1.99 E+00 \\
1.83 E+02 \\
2.81 E+02\end{array}$ & $\begin{array}{l}2.23 E+02 \\
2.09 E+00 \\
1.55 E+00 \\
1.18 E \bullet 02 \\
1.5 B E+02\end{array}$ & $\begin{array}{l}2.23 E+02 \\
2.12 E+00 \\
1.56 E+00 \\
1.17 E+02 \\
1.60 E+02\end{array}$ \\
\hline $\begin{array}{l}R A-222 \\
R A-223 \\
R A-224 \\
R A-225 \\
R A-226\end{array}$ & 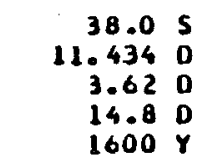 & $\begin{array}{l}3.63 E+01 \\
5.07 E+02 \\
3.89 E+01 \\
1.79 E+01 \\
2.5 B E+01\end{array}$ & $\begin{array}{l}3.92 E+01 \\
5.54 E+02 \\
4.23 E+01 \\
2.00 E+01 \\
2.82 E+01\end{array}$ & $\begin{array}{l}4.92 E+01 \\
7.95 E+02 \\
5.68 E+01 \\
3.55 E+01 \\
4.05 E+01\end{array}$ & $\begin{array}{l}4.04 E+01 \\
5.97 E+02 \\
4.26 E+01 \\
8.33 E+00 \\
2.71 E+01\end{array}$ & & $\begin{array}{l}3.13 E+01 \\
4.15 E+02 \\
3.33 E+01 \\
9.74 E+00 \\
2.17 E+01\end{array}$ & $\begin{array}{l}5.01 E+01 \\
8.14 E+02 \\
5.80 E+01 \\
3.62 E+01 \\
4.15 E+01\end{array}$ & $\begin{array}{l}3.59 E+01 \\
4.96 E+02 \\
3.86 E+01 \\
1.41 E+01 \\
2.56 E+01\end{array}$ & $\begin{array}{l}3.59 E+01 \\
4.97 E+02 \\
3.84 E+01 \\
1.55 E+01 \\
2.54 E+01\end{array}$ \\
\hline $\begin{array}{l}R A-228 \\
A C-225 \\
A C-227 \\
A C-228 \\
T H-226\end{array}$ & 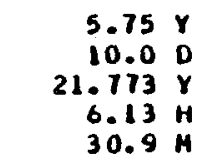 & $\begin{array}{l}2.24 E-10 \\
4.93 E \times 01 \\
4.32 E-01 \\
3.99 E \cdot 03 \\
2.90 E \times 01\end{array}$ & $\begin{array}{l}1.82 E-08 \\
5.43 E+01 \\
4.83 E-01 \\
4.25 E+03 \\
3.19 E+01\end{array}$ & $\begin{array}{l}1.09 E-08 \\
8.52 E+01 \\
1.71 E-01 \\
4.65 E+03 \\
4.86 E+01\end{array}$ & $\begin{array}{l}5.97 E-09 \\
4.00 E+01 \\
4.06 E-01 \\
4.30 E+03 \\
2.84 E+01\end{array}$ & $\begin{array}{l}9.01 E-09 \\
4.24 E+01 \\
3.73 E-01 \\
3.82 E+03 \\
2.52 E+01\end{array}$ & & & $\begin{array}{l}5.19 E-09 \\
4.83 E+01 \\
4.30 E-01 \\
3.87 E+03 \\
2.86 E+01\end{array}$ & $\begin{array}{l}4.69 E-09 \\
4.82 E+01 \\
4.28 E-01 \\
3.93 E+03 \\
2.85 E+01\end{array}$ \\
\hline $\begin{array}{l}T H-227 \\
T H-228 \\
T H-229 \\
T H-230 \\
T H-231\end{array}$ & $\begin{array}{r}18.718 \mathrm{O} \\
1.9132 \mathrm{Y} \\
7.34 E 3 \mathrm{Y} \\
7.7 E 4 \mathrm{Y} \\
25.52 \mathrm{H}\end{array}$ & $\begin{array}{l}4.01 E+02 \\
7.09 E+00 \\
3.11 E+02 \\
1.27 E+00 \\
3.82 E+01\end{array}$ & $\begin{array}{l}4.35 E \cdot 02 \\
7.81 E+00 \\
3.44 E+02 \\
1.41 E+00 \\
4.30 E+01\end{array}$ & $\begin{array}{l}5.87 E+02 \\
1.23 E+01 \\
5.52 E+02 \\
2.34 E+00 \\
7.25 E+01\end{array}$ & $\begin{array}{l}0.51 E+00 \\
2.80 E+02 \\
1.02 E \bullet 00 \\
3.07 E \bullet 01\end{array}$ & $\begin{array}{l}6.07 E+00 \\
2.66 E+02 \\
1.07 E+00 \\
3.25 E+01\end{array}$ & $\begin{array}{l}.39 E+02 \\
.56 E \cdot 00 \\
.43 E \cdot 02 \\
.27 E-01 \\
.83 E+01\end{array}$ & $\begin{array}{l}0.00 E+02 \\
1.26 E+01 \\
5.61 E+02 \\
2.41 E+00 \\
1.48 E+01\end{array}$ & & $\begin{array}{l}6.90 E+00 \\
3.03 E+02 \\
1.21 E+00 \\
3.70 E+01\end{array}$ \\
\hline $\begin{array}{l}I H-232 \\
I H-233 \\
I H-234 \\
P A-230 \\
P A-231\end{array}$ & $\begin{array}{r}1.405 E 10 \mathrm{Y} \\
22.3 \\
24.10 \\
17.4 \\
3.276 E 4 \mathrm{Y}\end{array}$ & $\begin{array}{l}5.24 E-01 \\
1.34 E+02 \\
2.71 E+01 \\
2.78 E+03 \\
1.15 E+02\end{array}$ & $\begin{array}{l}5.96 E-01 \\
1.45 E+02 \\
3.01 E+01 \\
2.97 E+03 \\
1.25 E+02\end{array}$ & $\begin{array}{l}1.00 E \bullet 00 \\
1.78 E \bullet 02 \\
5.14 E * 01 \\
3.39 E \bullet 03 \\
1.64 E \bullet 02\end{array}$ & & & & & $\begin{array}{l}4.8 B E-01 \\
1.31 E+02 \\
2.56 E+01 \\
2.69 E * 03 \\
1.13 E+02\end{array}$ & $\begin{array}{l}4.98 E-01 \\
1.32 E+02 \\
2.60 E+01 \\
2.74 E+03 \\
1.13 E+02\end{array}$ \\
\hline $\begin{array}{l}P A-233 \\
P A-234 \\
P A-234 M \\
U-230 \\
U-231\end{array}$ & $\begin{array}{rll}27.0 & 0 \\
6.70 & H \\
1.17 & H \\
20.8 & 0 \\
4.2 & 0\end{array}$ & $\begin{array}{l}0.25 E+02 \\
8.37 E+03 \\
4.93 E+01 \\
3.86 E+00 \\
2.43 E+02\end{array}$ & $\begin{array}{l}8.95 E \times 02 \\
8.94 E * 03 \\
5.26 E * 01 \\
4.26 E * 00 \\
2.71 E+02\end{array}$ & $\begin{array}{l}1.19 E+03 \\
9.97 E+03 \\
5.74 E+01 \\
6.57 E+00 \\
4.48 E+02\end{array}$ & $\begin{array}{l}5.296 \\
3.58 E \\
2.10 E\end{array}$ & $\begin{array}{l}4.76 E \\
3.32 E \\
2.07 E\end{array}$ & $\begin{array}{l}.99 E+02 \\
.20 E+03 \\
.25 E+01 \\
.04 E \bullet 00 \\
.87 E+02\end{array}$ & & & $\begin{array}{l}8.13 E+02 \\
8.25 E+03 \\
4.87 E+01 \\
3.75 E+00 \\
2.37 E+02\end{array}$ \\
\hline $\begin{array}{l}u-232 \\
u-233 \\
u-234 \\
u-235 \\
u-236\end{array}$ & $\begin{array}{r}72 Y \\
1.592 E 5 Y \\
2.445 E S Y \\
7.038 E 8 Y \\
2.3415 E 7 Y\end{array}$ & $\begin{array}{l}7.30 E-01 \\
7.93 E-01 \\
3.40 E-01 \\
5.77 E+02 \\
2.48 E-01\end{array}$ & $\begin{array}{l}8.37 E-01 \\
0.90 E-01 \\
4.11 E-01 \\
6.31 E+02 \\
2.99 E-01\end{array}$ & $\begin{array}{l}1.35 E+00 \\
1.42 E+00 \\
6.71 E-01 \\
9.14 E+02 \\
5.07 E-01\end{array}$ & $\begin{array}{l}6.08 E-01 \\
1.45 E-01 \\
2.76 E-01 \\
6.06 E+02 \\
1.72 E-01\end{array}$ & $\begin{array}{l}6.29 E-01 \\
6.87 E-01 \\
3.03 E-01 \\
5.03 E+02 \\
2.15 E-01\end{array}$ & $\begin{array}{l}5.56 E-01 \\
6.43 E-01 \\
2.64 E-01 \\
4.86 E+02 \\
1.79 E-01\end{array}$ & $\begin{array}{l}1.41 E+00 \\
1.47 E+00 \\
7.09 E-01 \\
9.36 E+02 \\
5.39 E-01\end{array}$ & $\begin{array}{l}6.90 E-01 \\
7.90 E-01 \\
3.31 E-01 \\
5.75 E+02 \\
2.32 E-01\end{array}$ & $\begin{array}{l}7.07 E-01 \\
7.06 E-01 \\
3.37 E-01 \\
5.70 E+02 \\
2.39 E-01\end{array}$ \\
\hline
\end{tabular}



DOSE-RATE FACTORS FOR BDOY ORGANS AND EFFECTIVE DOSE-P.ATE FACTOR FOR IMMERSION IN CONIAMINATfG AIR

\begin{tabular}{|c|c|c|c|c|c|c|c|c|c|}
\hline JCL IDE & IFE & STES & THYMUS & IYROIO & UTERUS & EFFECTIVE & $\begin{array}{l}\text { SKIN } \\
\text { PHOTONI }\end{array}$ & $\begin{array}{l}\text { SKIN } \\
=L E C T R O N I\end{array}$ & $\begin{array}{l}\text { SKIN } \\
\text { ITOTALI }\end{array}$ \\
\hline $\begin{array}{l}P O-216 \\
P O-218 \\
A T-211 \\
A T-217 \\
R N-21 B\end{array}$ & $\begin{array}{r}0.146 \mathrm{~S} \\
3.05 \mathrm{M} \\
7.214 \mathrm{H} \\
0.0323 \mathrm{~S} \\
0.035 \mathrm{~S}\end{array}$ & $\begin{array}{l}\text { 8. } 84 E-02 \\
0.0 \\
2.16 E \cdot 02 \\
1.41 E \cdot 00 \\
4.50 E \cdot 00\end{array}$ & $\begin{array}{l}.00 E-02 \\
.0 \\
.69 E+02 \\
.09 E+00 \\
.47 E+00\end{array}$ & $\begin{array}{l}8.40 E-02 \\
0.0 \\
2.15 E \cdot 02 \\
1.32 E \cdot 00 \\
4.21 E+00\end{array}$ & $\begin{array}{l}5.43 E-02 \\
0.0 \\
1.09 E \cdot 02 \\
8.60 E-01 \\
2.74 E+00\end{array}$ & $\begin{array}{l}7.56 E-02 \\
0.0 \\
1.85 E+02 \\
1.21 E+00 \\
3.85 E+00\end{array}$ & $\begin{array}{l}1.02 E-01 \\
0.0 \\
2.36 E+02 \\
1.62 E+00 \\
5.18 E \cdot 00\end{array}$ & $\begin{array}{l}0.0 \\
0.0 \\
0.0 \\
0.0 \\
0.0\end{array}$ & $\begin{array}{l}1.02 E-01 \\
0.0 \\
2.36 E+02 \\
1.62 E+00 \\
5.18 E+00\end{array}$ \\
\hline $\begin{array}{l}R N-219 \\
R N-220 \\
R N-222 \\
F R-221 \\
F R-223\end{array}$ & $\begin{array}{r}3.96 \mathrm{~S} \\
55.61 \mathrm{~S} \\
3.8235 \mathrm{D} \\
4.8 \mathrm{M} \\
21.8 \mathrm{M}\end{array}$ & $\begin{array}{l}3.32 E+02 \\
3.08 E+00 \\
2.28 E+00 \\
1.78 E+02 \\
2.73 E+02\end{array}$ & $\begin{array}{l}2.51 E+02 \\
2.35 E+0 a \\
1.73 E+00 \\
1.36 E+02 \\
2.04 E+02\end{array}$ & $\begin{array}{l}3.12 E+02 \\
2 . \theta 7 E+00 \\
2.11 E+00 \\
1.71 E+02 \\
2.60 E+02\end{array}$ & $\begin{array}{l}1.99 E+02 \\
1.87 E+00 \\
1.38 E+00 \\
1.05 E+02 \\
1.37 E+02\end{array}$ & $\begin{array}{l}2.8 B E+02 \\
2.63 E+00 \\
1.95 E+00 \\
1.56 E+02 \\
2.31 E+02\end{array}$ & $\begin{array}{l}3.72 E+02 \\
3.53 E \cdot 00 \\
2.61 E+00 \\
1.96 E+02 \\
3.16 E+02\end{array}$ & $\begin{array}{l}3.28 E+01 \\
0.0 \\
0.0 \\
3.73 E+01 \\
2.33 E+03\end{array}$ & $\begin{array}{l}4.05 E+02 \\
3.53 E+00 \\
2.61 E+00 \\
2.33 E+02 \\
2.64 E+03\end{array}$ \\
\hline $\begin{array}{l}R A-222 \\
R A-223 \\
R A-224 \\
R A-225 \\
R A-226\end{array}$ & $\begin{array}{r}38.0 \mathrm{~S} \\
11.434 \mathrm{D} \\
3.62 \mathrm{D} \\
14.8 \mathrm{D} \\
1600 \mathrm{Y}\end{array}$ & $\begin{array}{l}5.31 E \cdot 01 \\
7.74 E+02 \\
5.78 E+01 \\
4.48 E \cdot 01 \\
3.90 E \cdot 01\end{array}$ & $\begin{array}{l}4.01 E+01 \\
5.92 E+02 \\
4.39 E+01 \\
2.83 E+01 \\
2.99 E+01\end{array}$ & $\begin{array}{l}4.99 E \cdot 01 \\
7.48 E+02 \\
3.52 E+01 \\
3.73 E+01 \\
3.79 E \cdot 01\end{array}$ & $\begin{array}{l}3.20 E+01 \\
4.40 E+02 \\
3.44 E+01 \\
1.13 E+01 \\
2.27 E+01\end{array}$ & $\begin{array}{l}4.61 \mathrm{E}+01 \\
6.72 \mathrm{E}+02 \\
5.06 \mathrm{E}+01 \\
3.40 \mathrm{E}+01 \\
3.43 \mathrm{O}+01\end{array}$ & $\begin{array}{l}5.96 E+01 \\
8.54 E \div 02 \\
6.40 E \cdot 01 \\
6.35 E+01 \\
4.26 E+01\end{array}$ & $\begin{array}{l}4.17 E+00 \\
1.99 E+02 \\
1.01 E+01 \\
2.8 B E+02 \\
1.29 E+01\end{array}$ & $\begin{array}{l}6.38 E \div 01 \\
1.05 E \cdot 03 \\
7.46 E \bullet 01 \\
3.52 E \cdot 02 \\
5.55 E * 01\end{array}$ \\
\hline $\begin{array}{l}R A-228 \\
A C-225 \\
A C-227 \\
A C-228 \\
T H-226\end{array}$ & 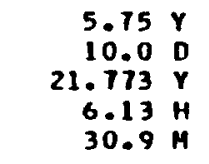 & $\begin{array}{l}1.67 E-07 \\
7.78 E \bullet 01 \\
7.07 E-01 \\
5.63 E+03 \\
4.50 E+01\end{array}$ & $\begin{array}{l}3.84 E-12 \\
5.97 E \cdot 01 \\
5.25 E-01 \\
4.46 E+03 \\
3.45 E+01\end{array}$ & $\begin{array}{l}1.15 E-08 \\
7.64 E+01 \\
6.81 E-01 \\
5.35 E+03 \\
4.41 E * 01\end{array}$ & $\begin{array}{l}1.68 E-12 \\
4.23 E+01 \\
3.74 E-01 \\
3.50 E+03 \\
2.52 E+01\end{array}$ & $\begin{array}{l}3.20 E-01 \\
6.79 E+01 \\
6.29 E-01 \\
4.83 E+03 \\
3.95 E+01\end{array}$ & $\begin{array}{l}3.84 E-06 \\
0.76 E+01 \\
1.00 E \cdot 00 \\
6.48 E \cdot 03 \\
5.00 E \cdot 01\end{array}$ & $\begin{array}{l}0.0 \\
3.00 E+00 \\
0.0 \\
2.60 E \cdot 03 \\
2.02 E+01\end{array}$ & $\begin{array}{l}3.84 E-06 \\
9.06 E+01 \\
1.00 E \cdot 00 \\
9.00 E \cdot 03 \\
7.02 E \cdot 01\end{array}$ \\
\hline $\begin{array}{l}T H-227 \\
T H-228 \\
T H-229 \\
T H-230 \\
T H-231\end{array}$ & $\begin{array}{r}18.718 \mathrm{D} \\
1.9132 \mathrm{Y} \\
7.34 \mathrm{Y} \\
7.7 E 4 \mathrm{Y} \\
25.52 \mathrm{H}\end{array}$ & $\begin{array}{l}4.97 E+02 \\
2.22 E+00 \\
6.81 E+01\end{array}$ & $\begin{array}{l}3.82 E \cdot 02 \\
1.62 E+00 \\
4.86 E+01\end{array}$ & $\begin{array}{l}5.72 E * 02 \\
1.10 E * 01 \\
4.90 E * 02 \\
2.07 E+00 \\
6.40 E * 01\end{array}$ & & & & $\begin{array}{l}5.68 E+01 \\
1.29 E+00 \\
7.77 E+01 \\
0.0 \\
1.93 E+02\end{array}$ & $\begin{array}{l}1.33 E+02 \\
1.53 E+01 \\
6.29 E+02 \\
4.03 E+00 \\
2 . \theta 8 E \cdot 02\end{array}$ \\
\hline $\begin{array}{l}T H-232 \\
T H-233 \\
T H-234 \\
P A-230 \\
P A-231\end{array}$ & 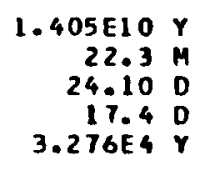 & $\begin{array}{l}1.03 E+00 \\
1.97 E+02 \\
4.52 E+01 \\
3.97 E+03 \\
1.74 E+02\end{array}$ & $\begin{array}{l}3.48 E \cdot 01 \\
3.14 E * 03 \\
1.29 E+02\end{array}$ & & $\begin{array}{l}4.16 E-01 \\
1.17 E+02 \\
2.23 E+01 \\
2.42 E+03 \\
1.01 E+02\end{array}$ & & & & $\begin{array}{l}3.03 E+03 \\
8.81 E+01 \\
4.68 E+03 \\
2.37 E+02\end{array}$ \\
\hline $\begin{array}{l}P A-233 \\
P A-234 \\
P A-234 M \\
U-230 \\
U-231\end{array}$ & $\begin{array}{rl}27.0 & 0 \\
6.70 & H \\
1.17 & M \\
20.8 & 0 \\
4.2 & 0\end{array}$ & $\begin{array}{l}1.23 E+03 \\
1.19 E+04 \\
6.97 E+01 \\
6.32 E+00 \\
3.98 E+02\end{array}$ & $\begin{array}{l}9.30 E+02 \\
9.38 E+03 \\
5.57 E \bullet 01 \\
4.68 E \bullet 00 \\
3.03 E+02\end{array}$ & $\begin{array}{l}1.17 E+03 \\
1.13 E+04 \\
6.67 E+01 \\
5.98 E+00 \\
3.92 E+02\end{array}$ & & & & $\begin{array}{l}2.10 E \cdot 03 \\
6.41 E+03 \\
6.55 E-01 \\
7.7 E-01\end{array}$ & \\
\hline $\begin{array}{l}u-232 \\
u-233 \\
u-234 \\
u-235 \\
u-236\end{array}$ & $\begin{array}{r}72 Y \\
1.592 E 5 Y \\
2.445 E S Y \\
7.038 E \theta Y \\
2.3415 E 7 Y\end{array}$ & $\begin{array}{l}1.46 E+00 \\
1.33 E+00 \\
8.12 E-01 \\
8.73 E+02 \\
6.27 E-01\end{array}$ & $\begin{array}{l}9.64 E-01 \\
4.46 E-01 \\
6.69 E \cdot 02 \\
3.2 \mathrm{BE}-01\end{array}$ & $\begin{array}{l}1.22 E+00 \\
1.26 E+00 \\
6.08 E-01 \\
8.51 E+02 \\
4.47 E-01\end{array}$ & $\begin{array}{l}6.85 E-01 \\
2.74 E-01 \\
5.09 E+02 \\
1.94 E-01\end{array}$ & $\begin{array}{l}1.33 E+00 \\
1.20 E+00 \\
7.65 E-01 \\
7.70 E+02 \\
6.05 E-01\end{array}$ & $\begin{array}{l}4.12 E+00 \\
2.23 E+00 \\
3.09 E+00 \\
9.55 E+02 \\
2.76 E+00\end{array}$ & $\begin{array}{l}3.43 E-01 \\
1.66 E-02 \\
1.03 E-01 \\
2.10 E-01 \\
1.19 E-01\end{array}$ & $\begin{array}{l}4.46 E+00 \\
2.25 E * 00 \\
3.27 E \cdot 00 \\
9.76 E \cdot 02 \\
2.88 E * 00\end{array}$ \\
\hline
\end{tabular}




\begin{tabular}{|c|c|c|c|c|c|c|c|c|c|c|}
\hline NUCL IDE & ALF-LIFE & ADRENALS & ADDER & BRAIN & REAST & HE ART & $\begin{array}{l}\text { SMALL } \\
\text { INTESTINE }\end{array}$ & $\begin{array}{c}\text { UPPER } \\
\text { LARGE } \\
\text { INIEST INE }\end{array}$ & $\begin{array}{c}\text { LOHER } \\
\text { LARGE } \\
\text { INTESTINE }\end{array}$ & r IONEYS \\
\hline $\begin{array}{l}y-237 \\
y-238 \\
y-239 \\
u-240 \\
N P-235\end{array}$ & $\begin{array}{r}6.75 \mathrm{D} \\
4.46 \mathrm{BE9} \\
23.40 \mathrm{M} \\
14.1 \mathrm{H} \\
396.1 \mathrm{D}\end{array}$ & $\begin{array}{l}5.15 E+02 \\
2.14 E-01 \\
1.65 E+02 \\
1.29 E+00 \\
6.80 E+00\end{array}$ & $\begin{array}{l}.93 E+02 \\
08 E-01 \\
.71 E \bullet 02 \\
.53 E+00 \\
.43 E+00\end{array}$ & $\begin{array}{l}14 E+02 \\
04 E-01 \\
75 E+02 \\
03 E+00 \\
75 E+00\end{array}$ & $\begin{array}{l}84 E+02 \\
55 E+00 \\
14 E+02 \\
03 E+01 \\
.74 E+01\end{array}$ & $\begin{array}{l}4.68 E+02 \\
1.83 E-01 \\
1.56 E+02 \\
9.44 E-01 \\
6.21 E+00\end{array}$ & $\begin{array}{l}4.14 E+02 \\
1.62 E-01 \\
1.40 E+02 \\
9.58 E-01 \\
5.35 E \cdot 00\end{array}$ & $\begin{array}{l}5.03 E+02 \\
1.92 E-01 \\
1.67 E+02 \\
1.22 E+00 \\
6.69 E+00\end{array}$ & $\begin{array}{l}4.59 E+02 \\
1.84 E-01 \\
1.50 E+02 \\
1.08 E+00 \\
6.05 E+00\end{array}$ & $\begin{array}{l}5.08 E+02 \\
2.19 E-01 \\
1.78 E+02 \\
1.87 E+00 \\
6.55 E+00\end{array}$ \\
\hline $\begin{array}{l}N P-236 \\
N P-2364 \\
N P-237 \\
N P-238 \\
N P-239\end{array}$ & $\begin{array}{rl}1.15 E 6 & Y \\
22.5 & H \\
2.14 E 6 & Y \\
2.117 & 0 \\
2.355 & 0\end{array}$ & $\begin{array}{l}5.03 E+02 \\
1.98 E+02 \\
8.12 E+01 \\
2.48 E+03 \\
6.74 E+02\end{array}$ & $\begin{array}{l}.75 E+02 \\
.90 E+02 \\
.02 E * 01 \\
42 E * 03 \\
29 E+02\end{array}$ & $\begin{array}{l}.02 E+02 \\
.03 E+02 \\
.17 E+01 \\
.72 E+03 \\
.71 E+02\end{array}$ & $\begin{array}{l}.72 E+02 \\
.32 E+02 \\
.66 E+02 \\
.03 E \cdot 03 \\
.06 E+03\end{array}$ & $\begin{array}{l}.61 E+02 \\
.84 E+02 \\
.46 E+01 \\
.39 E+03 \\
.11 E+02\end{array}$ & $\begin{array}{l}4.01 E+02 \\
1.61 E \cdot 02 \\
6.54 E \cdot 01\end{array}$ & $\begin{array}{l}4.96 E+02 \\
1.98 E+02 \\
0.12 E+01 \\
2.55 E+03 \\
6.54 E+02\end{array}$ & $\begin{array}{l}4.49 E+02 \\
1.78 E+02 \\
7.25 E+01 \\
2.34 E+03 \\
6.01 E+02\end{array}$ & $\begin{array}{l}4.84 E+02 \\
1.95 E+02 \\
8.37 E+01 \\
2.54 E+03 \\
6.45 E+02\end{array}$ \\
\hline $\begin{array}{l}N P-240 \\
N P-240 M \\
P U-236 \\
P U-237 \\
P U-238\end{array}$ & 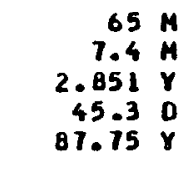 & $\begin{array}{l}5.08 E+03 \\
1.44 E+03 \\
1.81 E-01 \\
1.78 E+02 \\
1.04 E-01\end{array}$ & $\begin{array}{l}88 E+03 \\
39 E+03 \\
5 E-01\end{array}$ & & & & & & & \\
\hline $\begin{array}{l}P U-239 \\
P U-240 \\
P U-241 \\
P U-242 \\
P U-243\end{array}$ & $\begin{array}{r}24131 Y \\
6537 Y \\
14.4 Y \\
3.75855 Y \\
4.956 \mathrm{H}\end{array}$ & $\begin{array}{l}2.33 E-01 \\
1.05 E-01 \\
0.0 \\
9.37 E-02 \\
8.35 E+01\end{array}$ & $\begin{array}{l}.05 E-01 \\
.67 E-02 \\
.0 \\
.03 E-02 \\
.40 E+01\end{array}$ & $\begin{array}{l}19 E-01 \\
16 E-02 \\
0 \\
67 E-02 \\
67 E+01\end{array}$ & $\begin{array}{l}9.98 E-01 \\
1.74 E+00 \\
0.0 \\
1.45 E+00 \\
1.59 E+02\end{array}$ & $\begin{array}{l}2.03 E-01 \\
6.64 E-02 \\
0.0 \\
6.15 E-02 \\
7.84 E+01\end{array}$ & -02 & $\begin{array}{l}.16 E-01 \\
.65 E-02 \\
.0 \\
15 E-02\end{array}$ & $\begin{array}{l}E-01 \\
E-02 \\
E-02\end{array}$ & $\begin{array}{l}\text { 8.62E-02 } \\
0.0 \\
7.18 E-02 \\
8.61 E .01\end{array}$ \\
\hline $\begin{array}{l}P U-244 \\
P U-245 \\
P U-246 \\
A M-241 \\
A M-242\end{array}$ & $\begin{array}{r}0.26 E 7 \mathrm{Y} \\
10.51 \mathrm{H} \\
10.85 \mathrm{O} \\
432.2 \mathrm{Y} \\
16.02 \mathrm{H}\end{array}$ & $\begin{array}{l}4.82 E-02 \\
1.82 E+03 \\
3.89 E+02 \\
5.59 E+01 \\
5.14 E+01\end{array}$ & 80000 & & & & & & & \\
\hline$A 4-246$ & $\begin{array}{r}152 \mathrm{Y} \\
7.38 \mathrm{H} \mathrm{Y} \\
10.1 \mathrm{H} \\
122.4 \mathrm{M} \\
25.0 \mathrm{M}\end{array}$ & & & & & & & & & \\
\hline $\begin{array}{l}C M-242 \\
C M-243 \\
C H-244 \\
C M-245 \\
C M-246\end{array}$ & $\begin{array}{r}163.2 \mathrm{O} \\
28.5 Y \\
18.11 Y \\
8.5 E 3 Y \\
4.75 E 3 Y\end{array}$ & & & & & & & & & $\begin{array}{l}7.58 \mathrm{E}-02 \\
4.91 \mathrm{E}+02 \\
5.26 \mathrm{E}-02 \\
2.62 \mathrm{E}+02 \\
3.42 \mathrm{E}-02\end{array}$ \\
\hline $\begin{array}{l}C M-247 \\
C M-248 \\
C M-249 \\
C M-250 \\
\theta K-249\end{array}$ & $\begin{array}{r}3.39 E 5 Y \\
64.15 Y \\
6.9 E 3 \\
320 \\
32\end{array}$ & $\begin{array}{l}6.96 E-02 \\
0.22 E+01 \\
0.0 \\
0.0\end{array}$ & $\begin{array}{l}4.14 E-02 \\
7.82 E+01 \\
0.0 \\
0.0\end{array}$ & $\begin{array}{l}4.13 E-02 \\
8.73 E+01 \\
0.0 \\
0.0\end{array}$ & $\begin{array}{l}1.26 E+00 \\
1.03 E+02 \\
0.0 \\
0.0\end{array}$ & $\begin{array}{l}3.92 \mathrm{E}-02 \\
7.67 \mathrm{E} \cdot 01 \\
0.0 \\
0.0\end{array}$ & $\begin{array}{l}3.12 E-02 \\
6.90 E \cdot 01 \\
0.0 \\
0.0\end{array}$ & $\begin{array}{l}3.63 E-02 \\
0.15 E+01 \\
0.0 \\
0.0\end{array}$ & $\begin{array}{l}1.22 E+03 \\
5.08 E-02 \\
7.51 E+01 \\
0.0 \\
0.0\end{array}$ & $\begin{array}{l}1.31 E+03 \\
4.71 E-02 \\
8.13 E+01 \\
0.0 \\
0.0\end{array}$ \\
\hline
\end{tabular}


DOSE-RATE FACTORS FOR BODY URGANS AND EFFECTIVE DOSE-PATE FACTOR FOR. IMMERSION IN CUNTAHIINAIE!! AIR

\begin{tabular}{|c|c|c|c|c|c|c|c|c|c|c|}
\hline NUCL & LIFE & LIVER & LUNGS & MARROH & $\begin{array}{c}\text { RED } \\
\text { MARROH }\end{array}$ & OVARIES & PANCREAS & KELETOV & SP LEEN & STOMAC.H \\
\hline $\begin{array}{l}U-237 \\
U-238 \\
U-239 \\
U-240 \\
N P-235\end{array}$ & $\begin{array}{r}6.75 \mathrm{D} \\
4.468 E 9 \mathrm{~V} \\
23.40 \mathrm{M} \\
14.1 \mathrm{H} \\
396.1 \mathrm{D}\end{array}$ & $\begin{array}{l}89 E+02 \\
07 E-01 \\
69 E+02\end{array}$ & $\begin{array}{l}9 E+02 \\
1 E-01 \\
5 E+02 \\
3 E+00\end{array}$ & $\begin{array}{l}30 E+02 \\
25 E-01 \\
96 E+02 \\
80 E+00 \\
19 E+01\end{array}$ & $\begin{array}{l}62 E+02 \\
41 E-01 \\
36 E+02 \\
01 E-01\end{array}$ & $\begin{array}{l}4.22 E+02 \\
1.80 E-01 \\
1.46 E+02 \\
1.15 E+00 \\
5.53 E+00\end{array}$ & $\begin{array}{l}.89 E+02 \\
.49 E-01 \\
.23 E+02 \\
.39 E-01 \\
.05 E+00\end{array}$ & $\begin{array}{l}52 E+02 \\
53 E-01 \\
03 E+02 \\
96 E+00 \\
.23 E+01\end{array}$ & $\begin{array}{l}4.17 E+02 \\
1.93 E-01 \\
1.58 E+02 \\
1.13 E+00 \\
6.31 E+00\end{array}$ & $\begin{array}{l}4.78 E+02 \\
1.99 E-01 \\
1.61 E+02 \\
1.22 E+00 \\
6.32 E+00\end{array}$ \\
\hline $\begin{array}{l}\text { NP-236 } \\
\text { NP-236M } \\
\text { NP-237 } \\
\text { NP-238 } \\
\text { NP-239 }\end{array}$ & $\begin{array}{r}1.15 E 6 Y \\
22.5 \mathrm{H} \\
2.14 E 6 \mathrm{Y} \\
2.117 \mathrm{O} \\
2.355 \mathrm{O}\end{array}$ & $\begin{array}{l}4.73 E \cdot 02 \\
1.89 E+02 \\
7.91 E+01\end{array}$ & $\begin{array}{l}5.24 E+02 \\
2.08 E \cdot 02 \\
8.81 E+01\end{array}$ & $\begin{array}{l}.30 E \cdot 02 \\
.17 E \cdot 02 \\
.46 E+02 \\
.73 E+03 \\
.00 E+03\end{array}$ & $\begin{array}{l}.46 E+02 \\
.76 E+02 \\
.70 E+01 \\
.59 E+03\end{array}$ & $\begin{array}{l}4.07 E+02 \\
1.67 E+02 \\
6.73 E+01 \\
2.33 E+03\end{array}$ & $\begin{array}{l}.80 E+02 \\
.51 E+02 \\
.99 E+01 \\
.08 E+03\end{array}$ & $\begin{array}{l}.54 E+02 \\
.25 E+02 \\
.50 E+02 \\
.18 E+03 \\
.03 E \cdot 03\end{array}$ & $\begin{array}{l}4.67 E+02 \\
1.85 E+02 \\
7.62 E+01 \\
2.33 E+03 \\
6.19 E+02\end{array}$ & $\begin{array}{l}4.66 E+02 \\
1.86 E+02 \\
7.67 E+01 \\
2.37 E+03 \\
6.17 E+02\end{array}$ \\
\hline $\begin{array}{l}N P-240 \\
N P-240 M \\
P U-236 \\
P U-237 \\
P U-238\end{array}$ & $\begin{array}{r}65 M \\
7.4 M \\
2.851 Y \\
45.30 \\
87.75 Y\end{array}$ & $\begin{array}{l}4.86 E+03 \\
1.38 E+03 \\
1.51 E-01 \\
1.70 E+02 \\
7.16 E-02\end{array}$ & $\begin{array}{l}5.20 E+03 \\
1.48 E+03 \\
2.09 E-01 \\
1.90 E+02 \\
1.18 E-01\end{array}$ & $\begin{array}{l}5.86 E * 03 \\
1.65 E * 03 \\
3.38 E-01 \\
3.13 E+02 \\
1.79 E-01\end{array}$ & $\begin{array}{l}5.24 E+03 \\
.50 E+03 \\
.05 E-01 \\
.50 E+02 \\
5.31 E-02\end{array}$ & $\begin{array}{l}4.66 \mathrm{E}+03 \\
1.33 \mathrm{E} * 03 \\
1.44 \mathrm{E}-01 \\
1.46 \mathrm{E}+02 \\
1.77 \mathrm{E}-02\end{array}$ & $\begin{array}{l}4.18 E+03 \\
1.19 E+03 \\
.22 E-01 \\
.33 E+02\end{array}$ & $\begin{array}{l}5.97 E+03 \\
1.68 E+03 \\
3.76 \mathrm{E}-01 \\
3.22 \mathrm{E}+02 \\
2.09 \mathrm{E}-01\end{array}$ & $\begin{array}{l}4.72 E+03 \\
1.34 E+03 \\
1.47 E-01 \\
1.67 E+02 \\
1.50 E-02\end{array}$ & $\begin{array}{l}4.80 E+03 \\
1.36 E+03 \\
1.53 E-01 \\
1.67 E+02 \\
1.83 E-02\end{array}$ \\
\hline $\begin{array}{l}P U-239 \\
P U-240 \\
P U-241 \\
P U-242 \\
P U-243\end{array}$ & $\begin{array}{r}24131 \mathrm{Y} \\
6537 \mathrm{Y} \\
14.4 \mathrm{Y} \\
.75 \mathrm{BE} 5 \mathrm{Y} \\
4.956 \mathrm{H}\end{array}$ & $\begin{array}{l}2.05 E-01 \\
7.59 E-02 \\
0.0 \\
6.98 E-02 \\
8.35 E+01\end{array}$ & $\begin{array}{l}2.42 E-01 \\
1.21 E-01 \\
0.0 \\
1.07 E-01 \\
9.21 E+01\end{array}$ & $\begin{array}{l}3.80 E-01 \\
1.86 E-01 \\
0.0 \\
1.67 E-01 \\
1.52 E+02\end{array}$ & $\begin{array}{l}.95 E-01 \\
.45 E-02 \\
.0 \\
.00 E-02 \\
.85 E+01\end{array}$ & $\begin{array}{l}1.64 E-01 \\
0.02 E-02 \\
0.0 \\
7.20 E-02 \\
1.08 E+01\end{array}$ & $\begin{array}{l}1.73 E-01 \\
6.90 E-02 \\
0.0 \\
6.18 E-02 \\
6.17 E+01\end{array}$ & $\begin{array}{l}9.01 E-01 \\
2.15 E-01 \\
0.0 \\
1.92 E-01 \\
1.56 E+02\end{array}$ & $\begin{array}{l}2.08 E-01 \\
1.79 E-02 \\
0.0 \\
7.09 E-02 \\
7.95 E+01\end{array}$ & $\begin{array}{l}2.07 E-01 \\
8.14 E-02 \\
0.0 \\
7.39 E-02 \\
8.02 E+01\end{array}$ \\
\hline $\begin{array}{l}P U-244 \\
P U-245 \\
P U-246 \\
A M-241 \\
A M-242\end{array}$ & $\begin{array}{l}10.57 \mathrm{H} \\
10.85 \mathrm{O} \\
432.2 \mathrm{Y} \\
16.02 \mathrm{H}\end{array}$ & $\begin{array}{l}1.72 E+03 \\
3.59 E+02 \\
6.23 E+01 \\
4.86 E+01\end{array}$ & $\begin{array}{l}3.92 E+02 \\
6.92 E \cdot 01 \\
5.42 E+01\end{array}$ & $\begin{array}{l}7.20 E-02 \\
2.17 E+03 \\
5.49 E \cdot 02 \\
1.24 E \cdot 02 \\
8.85 E \cdot 01\end{array}$ & $\begin{array}{l}2.03 E-02 \\
1.87 E \bullet 03 \\
3.77 E \bullet 02 \\
3.74 E \bullet 01 \\
4.40 E \bullet 01\end{array}$ & $\begin{array}{l}3.29 E-02 \\
1.63 E+03 \\
3.15 E+02 \\
5.06 E+01 \\
4.18 E+01\end{array}$ & $\begin{array}{l}3.03 E-02 \\
1.48 E+03 \\
3.00 E+02 \\
3.8 B E+01 \\
3.84 E+01\end{array}$ & & & $\begin{array}{l}3.03 E-02 \\
1.70 E \cdot 03 \\
3.52 E \cdot 02 \\
5.69 E \cdot 01 \\
4.79 E * 01\end{array}$ \\
\hline $\begin{array}{l}A M-242 M \\
A M-243 \\
A M-244 \\
A M-245 \\
A M-246\end{array}$ & $\begin{array}{r}152 \mathrm{Y} \\
7.38 \mathrm{Y} \\
10.1 \mathrm{H} \\
122.4 \mathrm{M} \\
25.0 \mathrm{M}\end{array}$ & $\begin{array}{l}3.40 E+03 \\
1.18 E+02 \\
4.24 E+03\end{array}$ & $\begin{array}{l}3.64 E+C \\
1.29 E+C \\
4.51 E+C\end{array}$ & $\begin{array}{l}3.43 E \cdot 02 \\
4.05 E+03 \\
1.87 E+02 \\
4.84 E \cdot 03\end{array}$ & $\begin{array}{l}3.68 E+03 \\
1.21 E+02 \\
4.56 E+03\end{array}$ & & & & & $\begin{array}{l}9.20 E-01 \\
1.67 E+02 \\
3.37 E .03 \\
1.16 E .02 \\
4.18 E+03\end{array}$ \\
\hline $\begin{array}{l}C M-242 \\
C M-243 \\
C M-244 \\
C M-245 \\
C M-246\end{array}$ & $\begin{array}{r}163.2 \text { O } \\
28.5 Y \\
18.11 Y \\
8.5 E 3 Y \\
4.75 E 3 Y\end{array}$ & $\begin{array}{l}4.76 E+02 \\
4.79 E-02 \\
2.57 E+02 \\
2.60 E-02\end{array}$ & $\begin{array}{l}5.22 E \cdot 02 \\
9.69 E-02 \\
2.84 E \cdot 02 \\
6.7 \theta E-02\end{array}$ & & & & & & & \\
\hline $\begin{array}{l}C M-247 \\
C M-248 \\
C M-249 \\
C M-250 \\
\theta K-249\end{array}$ & 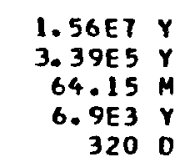 & $\begin{array}{l}1.26 E+03 \\
4.24 E-02 \\
1.78 E+01 \\
0.0 \\
0.0\end{array}$ & $\begin{array}{l}1.36 E+03 \\
7.80 E-02 \\
8.34 E+01 \\
0.0 \\
0.0\end{array}$ & $\begin{array}{l}1.65 E \cdot 03 \\
1.15 E-01 \\
9.51 E \cdot 01 \\
0.0 \\
0.0\end{array}$ & $\begin{array}{l}1.40 E \cdot 03 \\
3.43 E-02 \\
8.50 E \cdot 01 \\
0.0 \\
0.0\end{array}$ & $\begin{array}{l}5.06 E-02 \\
7.49 E+01 \\
0.0 \\
0.0\end{array}$ & $\begin{array}{l}4.52 E-02 \\
6.69 E+01 \\
0.0 \\
0.0\end{array}$ & $\begin{array}{l}1.371 \\
9.678 \\
0.0 \\
0.0\end{array}$ & $\begin{array}{l}1.24 \\
4.78 \\
7.58 \\
0.0 \\
0.0\end{array}$ & $\begin{array}{l}4.99 \\
7.69 \\
0.0 \\
0.0\end{array}$ \\
\hline
\end{tabular}




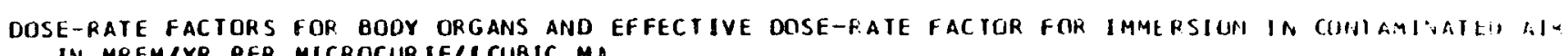

\begin{tabular}{|c|c|c|c|c|c|c|c|c|c|}
\hline ICL IOE & $F-L I F E$ & ESTES & THYMUS & THYROID & UTERUS & EFFECTIVE & $\begin{array}{c}\text { SKIN } \\
\text { (PHOTON) }\end{array}$ & $\begin{array}{l}\text { SKIN } \\
\text { IELECTRON }\end{array}$ & $\begin{array}{l}\text { SKIN } \\
\text { IIOIAL. }\end{array}$ \\
\hline $\begin{array}{l}U-237 \\
U-238 \\
U-239 \\
U-240 \\
N P-235\end{array}$ & $\begin{array}{r}0.75 \mathrm{D} \\
4.468 \mathrm{BE} 9 \mathrm{Y} \\
23.40 \mathrm{M} \\
14.1 \mathrm{H} \\
396.1 \mathrm{D}\end{array}$ & $\begin{array}{l}1.76 E+02 \\
5.37 E-01 \\
2.15 E+02 \\
4.16 E+00 \\
1.12 E+01\end{array}$ & $\begin{array}{l}91 E+02 \\
.75 E-01 \\
14 E+02 \\
.00 E .00\end{array}$ & $\begin{array}{l}7.56 E+02 \\
3.76 E-01 \\
2.69 E+02 \\
2.80 E+00 \\
1.04 E+01\end{array}$ & $\begin{array}{l}4.18 E+02 \\
1.60 E-01 \\
1.39 E+02 \\
8.79 E-01 \\
5.44 E+00\end{array}$ & $\begin{array}{l}6.72 E+02 \\
5.19 E-01 \\
2.34 E+02 \\
3.61 E+00 \\
1.01 E+01\end{array}$ & $\begin{array}{l}0.62 E+02 \\
2.43 E+00 \\
3.06 E+02 \\
1.51 E+01 \\
2.04 E+01\end{array}$ & $\begin{array}{l}\text { 3. } 11 E+02 \\
8.57 E-02 \\
2.76 E+03 \\
5.20 E+02 \\
9.98 E-04\end{array}$ & $\begin{array}{l}1.17 E+03 \\
2.51 E+00 \\
3.07 E+03 \\
5.35 E+02 \\
2.04 E+01\end{array}$ \\
\hline $\begin{array}{l}N P-236 \\
N P-236 M \\
N P-237 \\
N P-238 \\
N P-239\end{array}$ & 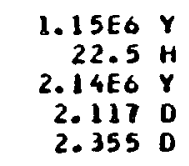 & $\begin{array}{l}7.48 E+02 \\
2.96 E+02 \\
1.34 E+02 \\
3.30 E+03 \\
9.61 E+02\end{array}$ & $\begin{array}{l}5.74 E \bullet 02 \\
2.2 B E+02 \\
9.92 E+01 \\
2.71 E \bullet 03 \\
1.33 E \bullet 02\end{array}$ & $\begin{array}{l}7.38 E+C 2 \\
2.91 E+02 \\
1.29 E+02 \\
3.23 E+03 \\
9.32 E+02\end{array}$ & $\begin{array}{l}4.08 E+02 \\
1.62 E+02 \\
6.59 E+01 \\
2.10 E+03 \\
5.45 E+02\end{array}$ & $\begin{array}{l}6.56 E+02 \\
2.57 E+02 \\
1.15 E+02 \\
2.89 E+03 \\
8.40 E+02\end{array}$ & $\begin{array}{l}8.32 E+02 \\
3.28 E+02 \\
1.63 E+02 \\
3.92 E+03 \\
1.06 E+03\end{array}$ & $\begin{array}{l}3.11 E+02 \\
3.67 E+02 \\
6.04 E+00 \\
1.411 E+03 \\
7.73 E+02\end{array}$ & $\begin{array}{l}1.14 E+03 \\
0.95 E+02 \\
1.69 E \times 02 \\
5.33 E+03 \\
1.84 E+03\end{array}$ \\
\hline $\begin{array}{l}N P-240 \\
N P-240 M \\
P U-236 \\
P U-237 \\
P U-236\end{array}$ & $\begin{array}{rr}65 & M \\
7.4 & M \\
2.851 & Y \\
45.3 & 0 \\
07.75 & Y\end{array}$ & $\begin{array}{l}6.93 E+03 \\
1.97 E+03 \\
5.95 E-01 \\
2.76 E+02 \\
4.23 E-01\end{array}$ & $\begin{array}{l}5.44 E \bullet 03 \\
1.53 E \bullet 03 \\
2.02 E-01 \\
2.11 E \cdot 02 \\
9.64 E-02\end{array}$ & $\begin{array}{l}6.57 E+03 \\
1.85 E+03 \\
3.10 E-01 \\
2.73 E+02 \\
1.71 E-01\end{array}$ & $\begin{array}{l}4.24 E+03 \\
1.21 E+03 \\
1.12 E-01 \\
1.45 E+02 \\
5.01 E-02\end{array}$ & $\begin{array}{l}5.94 E+03 \\
1.69 E+03 \\
5.96 E-01 \\
2.41 E+02 \\
4.41 E-01\end{array}$ & $\begin{array}{l}7.97 E+03 \\
2.27 E+03 \\
3.58 E+00 \\
3.07 E+02 \\
3.06 E+00\end{array}$ & $\begin{array}{l}1.84 E+03 \\
4.45 E+03 \\
0.0 \\
4.04 E-02 \\
0.0\end{array}$ & $\begin{array}{l}9.82 E+03 \\
6.72 E+03 \\
3.58 E+00 \\
3.07 E+02 \\
3.06 E+00\end{array}$ \\
\hline $\begin{array}{l}P U-239 \\
P U-240 \\
P U-241 \\
P U-242 \\
P U-243\end{array}$ & $\begin{array}{r}24131 Y \\
6537 y \\
14.4 Y \\
3.75865 Y \\
4.956 \%\end{array}$ & $\begin{array}{l}4.34 E-01 \\
4.19 E-01 \\
0.0 \\
3.58 E-01 \\
1.36 E+02\end{array}$ & $\begin{array}{l}2.49 E-01 \\
1.03 E-01 \\
0.0 \\
9.41 E-02 \\
1.05 E+02\end{array}$ & $\begin{array}{l}3.39 E-01 \\
1.37 E-01 \\
0.0 \\
1.58 E-01 \\
1.34 E+02\end{array}$ & $\begin{array}{l}1.76 E-01 \\
5.28 E-02 \\
0.0 \\
4.96 E-02 \\
6.97 E+01\end{array}$ & $\begin{array}{l}4.11 E-01 \\
4.32 E-01 \\
0.0 \\
3.67 E-01 \\
1.17 E+02\end{array}$ & $\begin{array}{l}1.45 E+00 \\
2.93 E+00 \\
0.0 \\
2.44 E+00 \\
1.49 E+02\end{array}$ & $\begin{array}{l}0.0 \\
0.0 \\
0.0 \\
0.0 \\
8.03 E+02\end{array}$ & $\begin{array}{l}1.45 E+00 \\
2.93 E+00 \\
0.0 \\
2.44 E+00 \\
9.53 E+02\end{array}$ \\
\hline $\begin{array}{l}P U-244 \\
P Y-245 \\
P U-246 \\
A M-241 \\
A M-242\end{array}$ & $\begin{array}{r}8.26 E 7 \mathrm{Y} \\
10.57 \mathrm{H} \\
10.85 \mathrm{O} \\
432.2 \mathrm{Y} \\
16.02 \mathrm{H}\end{array}$ & $\begin{array}{l}2.51 E-01 \\
2.40 E+03 \\
5.55 E+02 \\
1.15 E+02 \\
7.89 E+01\end{array}$ & $\begin{array}{l}3.44 E-02 \\
1.92 E \cdot 03 \\
4.18 E \bullet 02 \\
8.53 E+01 \\
5.99 E+01\end{array}$ & & $\begin{array}{l}1.49 E-02 \\
1.50 E+03 \\
3.13 E+02 \\
4.66 E+01 \\
4.16 E+01\end{array}$ & & $\begin{array}{l}2.06 E+00 \\
2.83 E+03 \\
6.22 E+02 \\
1.41 E+02 \\
9.23 E \bullet 01\end{array}$ & $\begin{array}{l}0.0 \\
1.91 E+03 \\
8.06 E+01 \\
1.06 E-01 \\
8.1 I E+02\end{array}$ & $\begin{array}{l}2.06 E+00 \\
4.74 E+03 \\
7.08 E+02 \\
1.41 E+02 \\
9.63 E+02\end{array}$ \\
\hline $\begin{array}{l}A M-242 M \\
A M-243 \\
A M-244 \\
A M-245 \\
A M-246\end{array}$ & $\begin{array}{r}152 \mathrm{Y} \\
7.38 \mathrm{~B} \mathrm{Y} \\
10.1 \mathrm{H} \\
122.4 \mathrm{M} \\
25.0 \mathrm{M}\end{array}$ & $\begin{array}{l}3.01 E+02 \\
4.85 E+03 \\
1.80 E+02 \\
5.96 E+03\end{array}$ & $\begin{array}{l}2.33 E+02 \\
3.82 E \cdot 03 \\
1.37 E \cdot 02 \\
4.76 E+03\end{array}$ & & $\begin{array}{l}7.24 E-01 \\
1.43 E+02 \\
2.96 E+03 \\
1.03 E+02 \\
3.71 E+03\end{array}$ & & $\begin{array}{l}1.04 E+01 \\
3.36 E+02 \\
5.61 E+03 \\
1.99 E+02 \\
6.89 E+03\end{array}$ & $\begin{array}{l}0.0 \\
6.45 E-02 \\
9.26 E+02 \\
1.70 E+03 \\
3.07 E+03\end{array}$ & $\begin{array}{l}3.36 E+02 \\
6.54 E+03 \\
1.90 E+03 \\
9.96 E+03\end{array}$ \\
\hline $\begin{array}{l}C M-242 \\
C M-243 \\
C M-244 \\
C M-245 \\
C M-246\end{array}$ & $\begin{array}{r}163.2 \mathrm{D} \\
28.5 Y \\
18.11 Y \\
8.5 E 3 Y \\
4.75 E 3 Y\end{array}$ & $\begin{array}{l}7.29 E+02 \\
4.11 E-01 \\
4.06 E+02 \\
3.44 E-01\end{array}$ & $\begin{array}{l}5.55 E+02 \\
6.19 E-02 \\
3.11 E+02 \\
3.51 E-02\end{array}$ & $\begin{array}{l}7.05 E+02 \\
1.38 E-01 \\
4.01 E+02 \\
9.16 E-02\end{array}$ & $\begin{array}{l}4.16 E+02 \\
3.25 E-02 \\
2.21 E+02 \\
1.52 E-02\end{array}$ & $\begin{array}{l}6.37 E+02 \\
4.18 E-01 \\
3.57 E+02 \\
3.51 E-01\end{array}$ & $\begin{array}{l}3.33 E+00 \\
8.10 E+02 \\
2.95 E+00 \\
4.50 E+02 \\
2.60 E+00\end{array}$ & $\begin{array}{l}0.0 \\
3.16 E+02 \\
0.0 \\
1.07 E+02 \\
0.0\end{array}$ & $\begin{array}{l}1.13 E+03 \\
2.95 E+00 \\
5.57 E+02 \\
2.60 E+00\end{array}$ \\
\hline $\begin{array}{l}C M-247 \\
C M-248 \\
C H-249 \\
C H-250 \\
B K-249\end{array}$ & $\begin{array}{r}1.56 E 7 \\
3.39 E 5 \\
64.15 \\
6.9 E 3 \\
320 \\
32\end{array}$ & $\begin{array}{l}3.07 E-01 \\
1.12 E+02 \\
0.0 \\
0.0\end{array}$ & $\begin{array}{l}1.38 E+03 \\
5.57 E-02 \\
8.58 E+01 \\
0.0 \\
0.0\end{array}$ & $\begin{array}{l}1.13 E-01 \\
1.05 E+02 \\
0.0 \\
0.0\end{array}$ & $\begin{array}{l}1.11 E \cdot 03 \\
2.91 E-02 \\
6.78 E+01 \\
0.0 \\
0.0\end{array}$ & $\begin{array}{l}1.58 E+03 \\
3.08 E-01 \\
9.55 E \circ 01 \\
0.0 \\
0.0\end{array}$ & $\begin{array}{l}2.08 E+03 \\
2.10 E+00 \\
1.28 E * 02 \\
0.0 \\
0.0\end{array}$ & $\begin{array}{l}0.03 E+01 \\
0.0 \\
1.72 E+03 \\
0.0 \\
5.87 E+00\end{array}$ & $\begin{array}{l}2.10 E+00 \\
1.85 E+03 \\
0.0 \\
5.87 E+00\end{array}$ \\
\hline
\end{tabular}


DOSE-RATE FACTORS FOR BOOY ORGANS AND EFFECTIVE DOSE-RATE FACTOR FOR IMMERSION IN CUNTAMIVATE! AIR
IN MREM/YR PER MICROCUR IE/ICUBIC MI

\begin{tabular}{|c|c|c|c|c|c|c|c|c|c|c|}
\hline NUCL IDE & HALF-LIFE & AORENALS & BLA DDER & BRAIN & BREAST & HE ART & $\begin{array}{c}\text { SMALL } \\
\text { INTESTINE }\end{array}$ & $\begin{array}{c}\text { UPPEP } \\
\text { LARGE } \\
\text { INTESTINE }\end{array}$ & $\begin{array}{l}\text { LOHER } \\
\text { LARGE } \\
\text { INTESTINEE }\end{array}$ & X IDNEYS \\
\hline $\begin{array}{l}B K-250 \\
B K-252 \\
C F-248 \\
C F-249 \\
C F-250\end{array}$ & $\begin{array}{r}3.222 \mathrm{H} \\
57.0 \mathrm{H} \\
333.5 \mathrm{O} \\
350.6 \mathrm{Y} \\
13.08 \mathrm{Y}\end{array}$ & $\begin{array}{l}3.98 E+03 \\
0.0 \\
5.12 E-02 \\
1.41 E+03 \\
1.28 E-01\end{array}$ & $\begin{array}{l}3.89 E+03 \\
0.0 \\
1.41 E-02 \\
1.31 E+03 \\
8.57 E-02\end{array}$ & $\begin{array}{l}4.38 E+03 \\
0.0 \\
1.58 E-02 \\
1.44 E+03 \\
9.29 E-02\end{array}$ & $\begin{array}{l}4.87 E+03 \\
0.0 \\
1.47 E+00 \\
1.85 E+03 \\
1.59 E+00\end{array}$ & $\begin{array}{l}3.84 E+03 \\
0.0 \\
1.78 E-02 \\
1.28 E+03 \\
0.70 E-02\end{array}$ & $\begin{array}{l}50 E+03 \\
0 \\
00 E-02\end{array}$ & $\begin{array}{l}4.09 E+03 \\
0.0 \\
1.12 E-02 \\
1.35 E+03 \\
8.28 E-02\end{array}$ & $\begin{array}{l}3.76 E+03 \\
0.0 \\
3.59 E-02 \\
1.26 E+03 \\
1.00 E-01\end{array}$ & $\begin{array}{l}4.08 E \cdot 03 \\
0.0 \\
2.14 E-02 \\
1.35 E+03 \\
9.21 E-02\end{array}$ \\
\hline $\begin{array}{l}\text { CF-25I } \\
\text { CF-252 } \\
\text { CF-253 } \\
\text { CF-254 } \\
\text { ES }-253\end{array}$ & 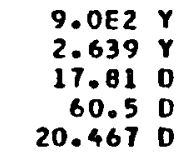 & $\begin{array}{l}4.68 E+02 \\
9.86 E-02 \\
1.01 E-03 \\
3.08 E-05 \\
1.28 E+00\end{array}$ & $\begin{array}{l}4.36 E+02 \\
6.06 E-02 \\
2.37 E-04 \\
4.33 E-05 \\
1.14 E+00\end{array}$ & $\begin{array}{l}4.64 E+02 \\
6.51 E-02 \\
3.35 E-04\end{array}$ & $\begin{array}{l}1.55 E+02 \\
1.45 E+00 \\
2.48 E-02 \\
1.10 E-04 \\
2.68 E+00\end{array}$ & $\begin{array}{l}4.25 E+02 \\
6.14 E-02 \\
3.59 E-04 \\
2.48 E-05 \\
1.11 E+00\end{array}$ & $\begin{array}{l}3.72 E+02 \\
4.86 E-02 \\
2.03 E-04 \\
2.63 E-05 \\
9.90 E-01\end{array}$ & $\begin{array}{l}4.55 E+02 \\
5.64 E-02 \\
2.14 E-04\end{array}$ & & $\begin{array}{l}4.45 E+02 \\
6.69 E-02 \\
3.21 E-04 \\
5.35 E-05 \\
1.17 E+00\end{array}$ \\
\hline $\begin{array}{l}E S-254 \\
E S-254 M \\
E S-255 \\
F M-254 \\
F M-255\end{array}$ & $\begin{array}{r}275.7 \mathrm{O} \\
39.3 \mathrm{H} \\
39.8 \mathrm{O} \\
3.240 \mathrm{H} \\
20.07 \mathrm{H}\end{array}$ & $\begin{array}{l}1.05 E \cdot 01 \\
2.47 E * 03 \\
4.70 E-03 \\
1.97 E-01 \\
5.76 E \cdot 00\end{array}$ & $\begin{array}{l}9.76 E+00 \\
2.37 E+03 \\
1.38 E-03 \\
1.42 E-01 \\
5.54 E+00\end{array}$ & $\begin{array}{l}1.00 E+01 \\
2.65 E+03 \\
1.41 E-03 \\
1.54 E-01 \\
5.58 E+00\end{array}$ & $\begin{array}{l}3.61 E+01 \\
3.06 E+03 \\
1.12 E-01 \\
1.86 E+00 \\
2.34 E+01\end{array}$ & $\begin{array}{l}9.05 E+00 \\
2.32 E * 03 \\
1.57 E-03 \\
1.44 E-01 \\
5.06 E+00\end{array}$ & $\begin{array}{l}0.08 E+00 \\
2.10 E \cdot 03 \\
9.59 E-04 \\
1.16 E-01 \\
4.44 E+00\end{array}$ & $\begin{array}{l}9.54 E+00 \\
2.47 E+03 \\
1.10 E-03 \\
1.41 E-01 \\
5.37 E+00\end{array}$ & $\begin{array}{l}9.13 E+00 \\
2.27 E+03 \\
3.02 E-03 \\
1.60 E-01 \\
5.05 E+00\end{array}$ & $\begin{array}{l}1.03 E+01 \\
2.47 E+03 \\
2.10 E-03 \\
1.52 E-01 \\
5.83 E+00\end{array}$ \\
\hline & 7.61 & .0 & & & & - & 0.0 & .0 & 0.0 & 0.0 \\
\hline
\end{tabular}




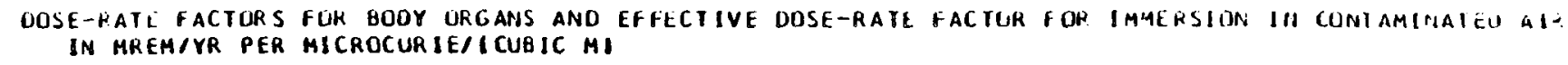

\begin{tabular}{|c|c|c|c|c|c|c|c|c|c|c|}
\hline NUCL JOE & HALF-LIFE & Liver & LUNGS & MARPOH & $\begin{array}{c}\text { REO } \\
\text { MARROW }\end{array}$ & OVARIES & PANCREAS & SKELETON & SPLEEN & STOMACH \\
\hline $\begin{array}{l}8 K-250 \\
8 K-251 \\
C F-248 \\
C F-249 \\
C F-250\end{array}$ & $\begin{array}{r}3.222 \mathrm{H} \\
57.0 \mathrm{H} \\
333.5 \mathrm{O} \\
350.6 \mathrm{Y} \\
13.08 \mathrm{Y}\end{array}$ & $\begin{array}{l}3.87 E \cdot 03 \\
0.0 \\
1.63 E-02 \\
1.30 E+03 \\
8.94 E-02\end{array}$ & $\begin{array}{l}4.12 E \cdot 03 \\
0.0 \\
6.05 E-02 \\
1.41 E .03 \\
1.41 E-01\end{array}$ & $\begin{array}{l}4.39 E+03 \\
0.0 \\
3.55 E-02 \\
1.72 E+03 \\
2.15 E-08\end{array}$ & $\begin{array}{l}4.16 E+03 \\
0.0 \\
2.16 E-02 \\
1.44 E+03 \\
7.71 E-02\end{array}$ & $\begin{array}{l}3.74 E+03 \\
0.0 \\
3.59 E-02 \\
1.22 E+03 \\
9.62 E-02\end{array}$ & $\begin{array}{l}3.34 E+03 \\
0.0 \\
3.54 E-02 \\
1.12 E+03 \\
8.71 E-02\end{array}$ & $\begin{array}{l}4.47 \mathrm{E}+03 \\
0.0 \\
1.02 \mathrm{E}-01 \\
1.76 \mathrm{E} .03 \\
2.45 \mathrm{E}-01\end{array}$ & $\begin{array}{l}3.14 E \cdot 03 \\
0.0 \\
2.88 E-02 \\
1.28 E+03 \\
9.79 E-02\end{array}$ & $\begin{array}{l}3.82 E+03 \\
0.0 \\
3.00 E-02 \\
1.29 E+03 \\
1.00 E-01\end{array}$ \\
\hline $\begin{array}{l}\text { CF-251 } \\
\text { CF-252 } \\
\text { CF-253 } \\
\text { CF-254 } \\
\text { ES-253 }\end{array}$ & 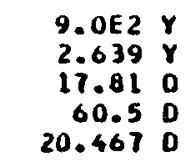 & $\begin{array}{l}4.34 E+02 \\
6.38 E-02 \\
3.04 E-04 \\
3.66 E-05 \\
1.14 E-00\end{array}$ & $\begin{array}{l}4.18 E+02 \\
1.09 E-01 \\
1.06 E-03 \\
4.05 E-05 \\
1.26 E+00\end{array}$ & $\begin{array}{l}1.20 E \cdot 02 \\
1.65 E-01 \\
1.34 E-03 \\
1.27 E-05 \\
1.74 E+00\end{array}$ & $\begin{array}{l}4.34 E+02 \\
5.41 E-02 \\
4.09 E-04 \\
1.76 E-05 \\
1.24 E+00\end{array}$ & $\begin{array}{l}3.11 E+02 \\
7.30 E-02 \\
6.32 E-04 \\
2.94 E-05 \\
1.01 E+00\end{array}$ & $\begin{array}{l}3.58 E+02 \\
6.57 E-02 \\
6.32 E-04 \\
2.01 E-05 \\
9.87 E-01\end{array}$ & $\begin{array}{l}7.39 E+02 \\
1.91 E-01 \\
1.78 E-03 \\
1.42 E-05 \\
1.80 E+00\end{array}$ & $\begin{array}{l}4.31 E+02 \\
7.17 E-02 \\
5.29 E-04 \\
2.92 E-05 \\
1.14 E+00\end{array}$ & $\begin{array}{l}4.28 \mathrm{E}+02 \\
7.41 \mathrm{E}-02 \\
5.54 \mathrm{E}-04 \\
3.18 \mathrm{E}-05 \\
1.13 \mathrm{E}+00\end{array}$ \\
\hline $\begin{array}{l}E S-254 \\
\text { ES-254M } \\
\text { ES-255 } \\
F M-254 \\
F M-255\end{array}$ & $\begin{array}{r}275.7 \mathrm{D} \\
39.3 \mathrm{H} \\
39.8 \mathrm{D} \\
3.240 \mathrm{H} \\
20.07 \mathrm{H}\end{array}$ & $\begin{array}{l}9.71 E+00 \\
2.35 E+03 \\
1.53 E-03 \\
1.47 E-01 \\
5.53 E+00\end{array}$ & $\begin{array}{l}1.12 E \cdot 01 \\
2.52 E \cdot 03 \\
5.06 E-03 \\
2.15 E-01 \\
6.52 E+00\end{array}$ & $\begin{array}{l}1.67 E+01 \\
2.80 E+03 \\
6.52 E-03 \\
3.35 E-01 \\
1.09 E+01\end{array}$ & $\begin{array}{l}0.95 E+00 \\
2.56 E+03 \\
1.02 E-03 \\
1.30 E-01 \\
4.26 E+00\end{array}$ & $\begin{array}{l}8.67 E+00 \\
2.28 E+03 \\
3.05 E-03 \\
1.52 E-01 \\
4.81 E+00\end{array}$ & $\begin{array}{l}7.79 E+00 \\
2.02 E+03 \\
2.92 E-03 \\
1.37 E-01 \\
4.12 E+00\end{array}$ & $\begin{array}{l}1.74 E+01 \\
2.85 E+03 \\
8.53 E-03 \\
3.72 E-01 \\
1.14 E+01\end{array}$ & $\begin{array}{l}9.41 E+00 \\
2.29 E+03 \\
2.48 E-03 \\
1.59 E-01 \\
5.29 E+00\end{array}$ & $\begin{array}{l}9.53 E+00 \\
2.32 E+03 \\
2.66 E-03 \\
1.62 E-01 \\
5.39 E+00\end{array}$ \\
\hline$M-256$ & $157.6 \mathrm{M}$ & 0.0 & 0.0 & 0.0 & 0.0 & 0.0 & 0.0 & 0.0 & 0.0 & 0.0 \\
\hline
\end{tabular}




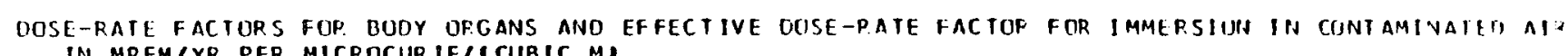

\begin{tabular}{|c|c|c|c|c|c|c|c|c|c|}
\hline NUCL IOE & HALF & TESTES & THYMUS & THYROID & UTERUS & EFFECTIVE & $\begin{array}{c}\text { SKIN } \\
\text { I PHOT ONI }\end{array}$ & $\begin{array}{l}\text { SKIN } \\
\text { IELECIRON I }\end{array}$ & $\begin{array}{l}\text { SKIN } \\
\text { ITOTALI }\end{array}$ \\
\hline $\begin{array}{l}B K-250 \\
8 K-251 \\
C F-248 \\
C F-249 \\
C F-250\end{array}$ & $\begin{array}{r}3.222 \mathrm{H} \\
57.0 \mathrm{M} \\
333.5 \mathrm{D} \\
350.6 \mathrm{~V} \\
13.08 \mathrm{Y}\end{array}$ & $\begin{array}{l}5.43 E+03 \\
0.0 \\
3.42 E-01 \\
1.90 E+03 \\
4.52 E-01\end{array}$ & $\begin{array}{l}4.36 E+03 \\
0.0 \\
1.92 E-02 \\
1.43 E+03 \\
1.12 E-01\end{array}$ & $\begin{array}{l}5.19 E+03 \\
0.0 \\
0.13 E-02 \\
1.71 E+03 \\
2.04 E-01\end{array}$ & $\begin{array}{l}3.38 E \cdot 03 \\
0.0 \\
7.71 E-03 \\
1.15 E+03 \\
6.90 E-02\end{array}$ & $\begin{array}{l}4.65 E+03 \\
0.0 \\
3.36 E-01 \\
1.64 E+03 \\
4.30 E-01\end{array}$ & $\begin{array}{l}6.29 E+03 \\
0.0 \\
2.43 E \cdot 00 \\
2.15 E+03 \\
2.52 E+00\end{array}$ & $\begin{array}{l}1.78 E+03 \\
2.46 E+03 \\
0.0 \\
7.10 E+01 \\
9.92 E-03\end{array}$ & $\begin{array}{l}8.07 E \cdot 03 \\
2.46 E+03 \\
2.43 E+00 \\
2.22 E+03 \\
2.53 E+00\end{array}$ \\
\hline $\begin{array}{l}\text { CF-251 } \\
\text { CF-252 } \\
\text { CF- } 253 \\
\text { CF-254 } \\
E S-253\end{array}$ & 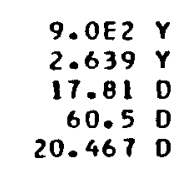 & $\begin{array}{l}6.71 E+02 \\
3.92 E-01 \\
5.74 E-03 \\
8.41 E-05 \\
1.89 E+00\end{array}$ & $\begin{array}{l}5.13 E+02 \\
8.05 E-02 \\
3.31 E-04 \\
5.62 E-05 \\
1.29 E+00\end{array}$ & $\begin{array}{l}6.57 E+02 \\
1.60 E-01 \\
1.48 E-03 \\
7.31 E-05 \\
1.67 E+00\end{array}$ & $\begin{array}{l}3.78 E+02 \\
4.69 E-02 \\
1.72 E-04 \\
2.41 E-05 \\
1.00 E+00\end{array}$ & $\begin{array}{l}5.89 E+02 \\
3.75 E-01 \\
5.68 E-03 \\
6.47 E-05 \\
1.68 E+00\end{array}$ & & $\begin{array}{l}5.20 E \\
1.11 E \\
1.92 E \\
0.0 \\
0.0\end{array}$ & $\begin{array}{l}1.26 E+03 \\
2.33 E \cdot 00 \\
1.92 E+02 \\
1.08 E-04 \\
3.30 E+00\end{array}$ \\
\hline $\begin{array}{l}E S-254 \\
E S-254 M \\
E S-255 \\
F M-254 \\
F M-255\end{array}$ & $\begin{array}{rr}275.7 & \mathrm{D} \\
39.3 & \mathrm{H} \\
39.8 & \mathrm{D} \\
3.240 & \mathrm{H} \\
20.07 & \mathrm{H}\end{array}$ & $\begin{array}{l}1.98 E+01 \\
3.36 E+03 \\
2.74 E-02 \\
6.14 E-01 \\
1.22 E+01\end{array}$ & $\begin{array}{l}1.18 E+01 \\
2.61 E+03 \\
1.87 E-03 \\
1.82 E-01 \\
7.10 E+00\end{array}$ & $\begin{array}{l}1.58 E+01 \\
3.16 E+03 \\
7.55 E-03 \\
3.16 E-01 \\
9.76 E+00\end{array}$ & $\begin{array}{l}0.05 E \bullet 00 \\
2.04 E+03 \\
7.43 E-04 \\
1.17 E-01 \\
4.43 E \bullet 00\end{array}$ & $\begin{array}{l}1.73 E+01 \\
2.87 E+03 \\
2.61 E-02 \\
5.57 E-01 \\
1.06 E+01\end{array}$ & $\begin{array}{l}4.83 E+01 \\
3.88 E+03 \\
1.82 E-01 \\
2.83 E+00 \\
3.02 E+01\end{array}$ & $\begin{array}{l}4.62 E-02 \\
9.57 E+02 \\
1.65 E \cdot 02 \\
1.85 E-01 \\
1.41 E \cdot 00\end{array}$ & $\begin{array}{l}4.83 E+01 \\
4.84 E+03 \\
1.65 E * 02 \\
3.01 E \cdot 00 \\
3.16 E * 01\end{array}$ \\
\hline & & & & 0 & 0.0 & 0,0 & 0.0 & 0.0 & 0.0 \\
\hline
\end{tabular}




\section{A. 2 Dose-Rate Factors for Immersion in Contaminated Water}

The following tables give the dose-rate conversion factors for external exposure to photons and electrons for immersion in an infinite, uniformly contaminated water medium. In lieu of site-specific data, the dose-rate factors for this exposure mode should be reduced by a factor of 100 in estimating dose equivalents to members of the public. Dosereduction factors which can be applied to these results to take into account external exposure to contaminated water during boating activities are discussed in Section 3.2.3. 
DOSE-RATE FACTORS FOR BOOY ORGANS AND EFFECTIVE DOSE-P.ATE FACTOR FOR IMMERSION IN CONTAMINATLO WATER

IN MREM/YR PER MICROCUR IE/I CUBIC MI

\begin{tabular}{|c|c|c|c|c|c|c|c|c|c|c|}
\hline VUCLIDE & HALF-LIFE & ADRENALS & BLADDER & BRAIN & BREAST & HE AR T & $\begin{array}{c}\text { SMALL } \\
\text { INTESTINE }\end{array}$ & $\begin{array}{c}\text { UPPER } \\
\text { LARGE } \\
\text { INTESTINE }\end{array}$ & $\begin{array}{c}\text { LOWER } \\
\text { LARGE } \\
\text { INTESTINE }\end{array}$ & r IONEYS \\
\hline $\begin{array}{l}H-3 \\
B E-7 \\
B E-10 \\
C-11 \\
C-14\end{array}$ & $\begin{array}{r}12.28 \mathrm{Y} \\
53.44 \mathrm{D} \\
1.6 E 6 \mathrm{Y} \\
20.48 \mathrm{M} \\
5.73 E 3 \mathrm{Y}\end{array}$ & $\begin{array}{l}0.0 \\
4.65 E-01 \\
0.0 \\
9.55 E+00 \\
0.0\end{array}$ & $\begin{array}{l}0.0 \\
4.40 E-01 \\
0.0 \\
9.07 E+00 \\
0.0\end{array}$ & $\begin{array}{l}0.0 \\
4.09 E-01 \\
0.0 \\
1.01 E+01 \\
0.0\end{array}$ & $\begin{array}{l}0.0 \\
5.89 E-01 \\
0.0 \\
1.20 E+01 \\
0.0\end{array}$ & $\begin{array}{l}0.0 \\
4.29 E-01 \\
0.0 \\
8.86 E+00 \\
0.0\end{array}$ & $\begin{array}{l}.0 \\
.86 E-01 \\
.0 \\
.97 E \cdot 00 \\
.0\end{array}$ & $\begin{array}{l}0.0 \\
4.56 E-01 \\
0.0 \\
9.40 E+00 \\
0.0\end{array}$ & $\begin{array}{l}0.0 \\
4.21 E-01 \\
0.0 \\
8.68 E+00 \\
0.0\end{array}$ & $\begin{array}{l}0.0 \\
4.56 E-01 \\
0.0 \\
9.40 E+00 \\
0.0\end{array}$ \\
\hline $\begin{array}{l}N-13 \\
N-16 \\
O-15 \\
F-18 \\
N A-22\end{array}$ & $\begin{array}{r}9.97 \mathrm{M} \\
7.13 \mathrm{~S} \\
122.24 \mathrm{~S} \\
109.74 \mathrm{M} \\
2.602 \mathrm{Y}\end{array}$ & $\begin{array}{l}9.55 E+00 \\
5.31 E+01 \\
9.56 E+00 \\
9.26 E+00 \\
2.07 E+01\end{array}$ & $\begin{array}{l}9.07 E \bullet 00 \\
5.37 E \bullet 01 \\
9.08 E \bullet 00 \\
8.79 E \bullet 00 \\
2.02 E \bullet 01\end{array}$ & $\begin{array}{l}1.01 E+01 \\
5.68 E+01 \\
1.01 E+01 \\
9.80 E+00 \\
2.27 E+01\end{array}$ & $\begin{array}{l}1.20 E+01 \\
6.84 E+01 \\
1.21 E+01 \\
1.17 E+01 \\
2.58 E+01\end{array}$ & $\begin{array}{l}8.86 E+00 \\
5.29 E+01 \\
8.87 E+00 \\
8.59 E+00 \\
1.99 E+01\end{array}$ & $\begin{array}{l}7.97 E \cdot 00 \\
5.06 E+01 \\
7.98 E+00 \\
7.73 E+00 \\
1.81 E+01\end{array}$ & $\begin{array}{l}9.41 E+00 \\
5.65 E \bullet 01 \\
9.42 E+00 \\
9.12 E+00 \\
2.11 E+01\end{array}$ & $\begin{array}{l}8.69 E+00 \\
5.30 E+01 \\
8.70 E+00 \\
8.42 E+00 \\
1.95 E+01\end{array}$ & $\begin{array}{l}9.41 E+00 \\
6.00 E+01 \\
9.41 E+00 \\
9.12 E+00 \\
2.11 E+01\end{array}$ \\
\hline $\begin{array}{l}N A-24 \\
M G-27 \\
M G-28 \\
A L-26 \\
A L-28\end{array}$ & $\begin{array}{l}15.00 \mathrm{H} \\
9.458 \mathrm{H} \\
20.91 \mathrm{H} \\
7.2 \mathrm{H} 5 \mathrm{Y} \\
2.240 \mathrm{M}\end{array}$ & $\begin{array}{l}4.26 E+01 \\
8.69 E+00 \\
1.29 E+01 \\
2.63 E+01 \\
1.78 E+01\end{array}$ & $\begin{array}{l}4.37 E * 01 \\
8.43 E * 00 \\
1.27 E * 01 \\
2.64 E * 01 \\
1.82 E * 01\end{array}$ & $\begin{array}{l}4.86 E+01 \\
9.4 \theta E+00 \\
1.43 E+01 \\
2.95 E+01 \\
2.04 E+01\end{array}$ & $\begin{array}{l}5.49 E+01 \\
1.06 E+01 \\
1.62 E+01 \\
3.34 E+01 \\
2.26 E+01\end{array}$ & $\begin{array}{l}4.36 E+01 \\
0.32 E+00 \\
1.26 E+01 \\
2.61 E+01 \\
1.81 E+01\end{array}$ & $\begin{array}{l}4.09 E+01 \\
7.55 E+00 \\
1.15 E+01 \\
2.41 E+01 \\
1.69 E+01\end{array}$ & $\begin{array}{l}4.64 E+01 \\
8.86 E+00 \\
1.34 E+01 \\
2.78 E+01 \\
1.93 E+01\end{array}$ & $\begin{array}{l}4.30 E+01 \\
8.14 E+00 \\
1.23 E+01 \\
2.56 E+01 \\
1.78 E+01\end{array}$ & $\begin{array}{l}4.71 E+01 \\
8.83 E+00 \\
1.34 E+01 \\
2.78 E+01 \\
1.93 E+01\end{array}$ \\
\hline $\begin{array}{l}S 1-31 \\
51-32 \\
p-32 \\
p-33 \\
S-35\end{array}$ & $\begin{array}{l}157.3 \mathrm{M} \\
3.322 \mathrm{Y} \\
14.29 \\
25.4 \\
87.44 \\
87.44\end{array}$ & $\begin{array}{l}8.41 E-03 \\
0.0 \\
0.0 \\
0.0 \\
0.0\end{array}$ & $\begin{array}{l}8.36 E-03 \\
0.0 \\
0.0 \\
0.0 \\
0.0\end{array}$ & $\begin{array}{l}9.43 E-03 \\
0.0 \\
0.0 \\
0.0\end{array}$ & $\begin{array}{l}1.04 E-02 \\
0.0 \\
0.0 \\
0.0 \\
0.0\end{array}$ & $\begin{array}{l}8.27 E-03 \\
0.0 \\
0.0 \\
0.0 \\
0.0\end{array}$ & $\begin{array}{l}7.62 E-03 \\
0.0 \\
0.0 \\
0.0 \\
0.0\end{array}$ & $\begin{array}{l}8.81 E-03 \\
0.0 \\
0.0 \\
0.0 \\
0.0\end{array}$ & $\begin{array}{l}8.12 E-03 \\
0.0 \\
0.0 \\
0.0 \\
0.0\end{array}$ & $\begin{array}{l}\text { 8. } 79 E-03 \\
0.0 \\
0.0 \\
0.0 \\
0.0\end{array}$ \\
\hline $\begin{array}{l}C L-36 \\
C L-38 \\
A R-37 \\
A R-39 \\
A R-41\end{array}$ & $\begin{array}{r}3.01 E 5 \\
37.21 \mathrm{M} \\
35.02 \\
269 \mathrm{Y} \\
1.827 \mathrm{H}\end{array}$ & $\begin{array}{l}7.47 E-09 \\
1.53 E+01 \\
1.19 E-06 \\
0.0 \\
1.22 E+01\end{array}$ & $\begin{array}{l}1.35 E-15 \\
1.51 E+01 \\
2.15 E-13 \\
0.0 \\
1.21 E+01\end{array}$ & $\begin{array}{l}5.96 E-10 \\
1.76 E+01 \\
9.51 E-08 \\
0.0 \\
1.37 E+01\end{array}$ & $\begin{array}{l}5.85 E-07 \\
1.96 E+01 \\
9.34 E-05 \\
0.0 \\
1.51 E+01\end{array}$ & $\begin{array}{l}9.73 E-10 \\
1.57 E+01 \\
1.55 E-07 \\
0.0 \\
1.20 E \cdot 01\end{array}$ & $\begin{array}{l}1.25 E-11 \\
1.46 E+01 \\
2.00 E-09 \\
0.0 \\
1.11 E \cdot 01\end{array}$ & $\begin{array}{l}1.85 E-11 \\
1.67 E+01 \\
2.95 E-09 \\
0.0 \\
1.28 E+01\end{array}$ & $\begin{array}{l}3.07 E-09 \\
1.54 E+01 \\
4.09 E-07 \\
0.0 \\
1.18 E+01\end{array}$ & $\begin{array}{l}\text { 0. } 95 E-12 \\
1.68 E+01 \\
1.43 E-09 \\
0.0 \\
1.27 E+01\end{array}$ \\
\hline $\begin{array}{l}K-40 \\
K-42 \\
K-43 \\
C A-41 \\
C A-45\end{array}$ & $\begin{array}{r}1.277 E 9 \mathrm{Y} \\
12.36 \mathrm{H} \\
22.6 \mathrm{H} \\
1.03 E 5 \mathrm{Y} \\
162.7 \mathrm{D}\end{array}$ & $\begin{array}{l}1.47 E+00 \\
2.62 E \cdot 00 \\
9.16 E+00 \\
2.78 E-06 \\
2.24 E-11\end{array}$ & $\begin{array}{l}1.48 E+00 \\
2.65 E * 00 \\
8.67 E * 00 \\
5.01 E-13 \\
2.07 E-15\end{array}$ & & $\begin{array}{l}1.05 E+00 \\
3.30 E * 00 \\
1.16 E * 01 \\
2.18 E-04 \\
1.07 E-09\end{array}$ & & & & & \\
\hline $\begin{array}{l}\text { CA-4T } \\
\text { CAA-49 } \\
\text { SC-44 } \\
\text { SC-46 } \\
\text { SC-46M }\end{array}$ & $\begin{array}{l}4.536 \\
8.719 \\
3.927 \\
83.80 \\
18.72 \\
18.72\end{array}$ & $\begin{array}{l}3.42 E+01 \\
2.03 E+01 \\
1.95 E+01 \\
8.52 E-01\end{array}$ & $\begin{array}{l}1.00 E+01 \\
3.53 E+01 \\
1.97 E \cdot 01 \\
1.90 E \cdot 01 \\
7.05 E-01\end{array}$ & & $\begin{array}{l}1.25 E+01 \\
4.4 B E+01 \\
2.52 E+01 \\
2.3 B E+01 \\
1.34 E+00\end{array}$ & & & $\begin{array}{l}1.05 E+01 \\
3.75 E+01 \\
2.06 E+01 \\
2.00 E+01 \\
8.27 E-01\end{array}$ & $\begin{array}{l}9.71 E+00 \\
3.50 E+01 \\
1.90 E+01 \\
1.84 E+01 \\
7.58 E-01\end{array}$ & $\begin{array}{l}1.05 E+01 \\
3.86 E+01 \\
2.06 E+01 \\
2.00 E+01 \\
7.97 E-01\end{array}$ \\
\hline $\begin{array}{l}S C-47 \\
S C-48 \\
S C-49 \\
T I-44 \\
T 1-45\end{array}$ & $\begin{array}{rl}3.422 & D \\
43.67 & \mathrm{H} \\
57.4 & \mathrm{M} \\
47.3 \mathrm{Y} \\
3.08 \mathrm{H}\end{array}$ & $\begin{array}{l}3.23 E+01 \\
1.03 E-02 \\
1.06 E+00 \\
8.15 E \cdot 00\end{array}$ & $\begin{array}{l}3.18 E+01 \\
1.06 E-02 \\
1.13 E+00 \\
1.74 E+00\end{array}$ & $\begin{array}{l}1.02 E+00 \\
3.59 E+01 \\
1.19 E-02 \\
1.13 E+00 \\
8.63 E+00\end{array}$ & $\begin{array}{l}1.58 E+00 \\
3.98 E+01 \\
1.31 E-02 \\
2.27 E+00 \\
1.03 E+01\end{array}$ & $\begin{array}{l}9.35 E-01 \\
3.15 E+01 \\
1.05 E-02 \\
1.01 E+00 \\
7.57 E+00\end{array}$ & $\begin{array}{l}8.20 E-01 \\
2.88 E \cdot 01 \\
9.78 E-03 \\
9.00 E-01 \\
6.81 E+00\end{array}$ & $\begin{array}{l}9.95 E-01 \\
3.35 E+01 \\
1.12 E-02 \\
1.08 E+00 \\
8.03 E+00\end{array}$ & $\begin{array}{l}9.21 E-01 \\
3.08 E+01 \\
1.03 E-02 \\
9.61 E-01 \\
7.42 E+00\end{array}$ & $\begin{array}{l}9.67 E-01 \\
3.34 E+01 \\
1.12 E-02 \\
1.17 E+00 \\
0.03 E+00\end{array}$ \\
\hline
\end{tabular}




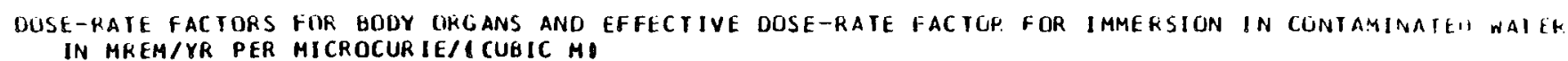

\begin{tabular}{|c|c|c|c|c|c|c|c|c|c|c|}
\hline NUCLIDE & HALF-LIFE & LIVER & LUNGS & MARROW & $\begin{array}{c}\text { PEED } \\
\text { MARROH }\end{array}$ & OVARIES & PANCREAS & SKELETON & SPLEEN & S JOMACH \\
\hline $\begin{array}{l}H-3 \\
B E-7 \\
B E-10 \\
C-11 \\
C-14\end{array}$ & $\begin{array}{r}12.28 Y \\
53.44 \\
1.6 E 6 \\
20.48 \\
5.73 E 3 Y\end{array}$ & $\begin{array}{l}.0 \\
.37 E-01 \\
.0 \\
.01 E+00 \\
0\end{array}$ & $\begin{array}{l}.0 \\
.10 E-01 \\
.0 \\
.67 E+00 \\
.0\end{array}$ & $\begin{array}{l}0.0 \\
5.47 E-01 \\
0.0 \\
1.011 E+01 \\
0.0\end{array}$ & $\begin{array}{l}.0 \\
02 E-01 \\
00 \\
00 E+00 \\
.0\end{array}$ & $\begin{array}{l}0.0 \\
4.18 E-01 \\
0.0 \\
0.66 E \diamond 00 \\
0.0\end{array}$ & $\begin{array}{l}0 \\
76 E-01 \\
0 \\
76 E \cdot 00 \\
0\end{array}$ & $\begin{array}{l}0.0 \\
5.55 E-01 \\
0.0 \\
1.13 E+01 \\
0.0\end{array}$ & $\begin{array}{l}0.0 \\
4.28 E-01 \\
0.0 \\
8.80 E+00 \\
0.0\end{array}$ & $\begin{array}{l}0.0 \\
4.31 E-01 \\
0.0 \\
8.89 E+00 \\
0.0\end{array}$ \\
\hline $\begin{array}{l}N-13 \\
N-16 \\
0-15 \\
F-18 \\
N A-22\end{array}$ & $\begin{array}{r}9.97 \mathrm{M} \\
7.13 \mathrm{~S} \\
122.24 \mathrm{~S} \\
109.74 \mathrm{M} \\
2.602 \mathrm{~V}\end{array}$ & $\begin{array}{l}.02 E+00 \\
35 E+01 \\
03 E+C 0 \\
6 F+00\end{array}$ & $\begin{array}{l}9.60 E+00 \\
5.49 E+01\end{array}$ & $\begin{array}{l}1.11 E+01 \\
5.77 E+01 \\
1.12 E+01 \\
1.08 E+01 \\
2.34 E+01\end{array}$ & $\begin{array}{l}9.90 E+00 \\
5.96 E \bullet 01 \\
9.91 E \bullet 00 \\
9.60 E+00 \\
2.17 E * 01\end{array}$ & & & $\begin{array}{l}1.13 E+01 \\
5.84 E+01 \\
1.13 E+01 \\
1.10 E+01 \\
2.38 E+01\end{array}$ & $\begin{array}{l}.80 E * 00 \\
23 E+01 \\
01 E+00 \\
03 E+00 \\
.95 E \cdot 01\end{array}$ & $\begin{array}{l}\text { 8. } 90 E+00 \\
5.46 E+01 \\
8.91 E+00 \\
8.62 E+00 \\
1.97 E+01\end{array}$ \\
\hline $\begin{array}{l}N A-24 \\
M G-27 \\
M G-28 \\
A L-26 \\
A L-28\end{array}$ & $\begin{array}{l}15.00 \mathrm{H} \\
9.458 \mathrm{H} \\
20.91 \mathrm{H} \\
7.2 E 5 \mathrm{Y} \\
2.240 \mathrm{H}\end{array}$ & $\begin{array}{l}4.39 E+01 \\
8.38 E+00 \\
1.27 E+01 \\
2.64 E+01 \\
1.83 E+01\end{array}$ & $\begin{array}{l}4.60 E+01 \\
8.93 E+00 \\
1.35 E+01 \\
2.79 E+01 \\
1.92 E+01\end{array}$ & $\begin{array}{l}4.73 E+01 \\
9.63 E+00 \\
1.44 E+01 \\
2.97 E+01 \\
1.98 E+01\end{array}$ & $\begin{array}{l}4.69 E+01 \\
9.05 E+00 \\
1.36 E+01 \\
2.83 E+01 \\
1.94 E+01\end{array}$ & & & & & $\begin{array}{l}\text { 4. } 33 E+01 \\
8.28 E \bullet 00 \\
1.24 E+01 \\
2.58 E+01 \\
1.78 E+01\end{array}$ \\
\hline $\begin{array}{l}S I-31 \\
S 1-32 \\
p-32 \\
p-33 \\
S-35\end{array}$ & 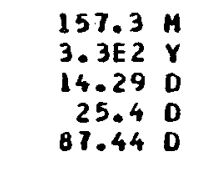 & $\begin{array}{l}8.34 E-03 \\
0.0 \\
0.0 \\
0.0\end{array}$ & $\begin{array}{l}8.83 E-03 \\
0.0 \\
0.0 \\
0.0 \\
0.0\end{array}$ & $\begin{array}{l}9.29 E-03 \\
0.0 \\
0.0 \\
0.0 \\
0.0\end{array}$ & $\begin{array}{l}0.91 E-03 \\
0.0 \\
0.0 \\
0.0 \\
0.0\end{array}$ & $\begin{array}{l}7.94 E-03 \\
0.0 \\
0.0 \\
0.0 \\
0.0\end{array}$ & $\begin{array}{l}7.23 E-0.3 \\
0.0 \\
0.0 \\
0.0 \\
0.0\end{array}$ & $\begin{array}{l}9.44 E-03 \\
0.0 \\
0.0 \\
0.0 \\
0.0\end{array}$ & $\begin{array}{l}.06 \text { E-03 } \\
.0 \\
.00 \\
0.0\end{array}$ & $\begin{array}{l}0.16 E-03 \\
0.0 \\
0.0 \\
0.0 \\
0.0\end{array}$ \\
\hline $\begin{array}{l}C L-36 \\
C L-38 \\
A R-37 \\
A R-39 \\
A R-41\end{array}$ & $\begin{array}{r}3.01 E 5 \\
37.21 \\
35.02 \\
269 \\
269 \\
1.827 \mathrm{H}\end{array}$ & $\begin{array}{l}1.26 E-11 \\
1.58 E+01 \\
1.16 E-08 \\
0.0 \\
1.21 E+01\end{array}$ & $\begin{array}{l}1.66 E+01 \\
9.43 E-07 \\
0.0 \\
1.28 E+01\end{array}$ & $\begin{array}{l}3.55 E-09 \\
1.70 E+01 \\
5.66 E-07 \\
0.0 \\
1.34 E \cdot 01\end{array}$ & $\begin{array}{l}1.94 E-09 \\
1.68 E+01 \\
3.09 E-07 \\
0.0 \\
1.29 E+01\end{array}$ & $\begin{array}{l}2.92 E-09 \\
1.44 E+01 \\
4.67 E-07 \\
0.0 \\
1.15 E+01\end{array}$ & $\begin{array}{l}4.36 E-09 \\
1.38 E+01 \\
6.96 E-07 \\
0.0 \\
1.05 E+01\end{array}$ & $\begin{array}{l}9.59 E-09 \\
1.73 E+01 \\
1.53 E-06 \\
0.0 \\
1.37 E+01\end{array}$ & $\begin{array}{l}1.68 E-09 \\
1.51 E+01 \\
2.69 E-07 \\
0.0 \\
1.17 E+01\end{array}$ & $\begin{array}{l}1.52 E-09 \\
1.54 E+01 \\
2.43 E-07 \\
0.0 \\
1.18 E+01\end{array}$ \\
\hline $\begin{array}{l}k-40 \\
k-42 \\
k-43 \\
C A-41 \\
C A-45\end{array}$ & $\begin{array}{r}1.277 E 9 Y \\
12.36 \mathrm{H} \\
22.6 \mathrm{H} \\
1.03 E 5 \mathrm{Y} \\
162.7 \mathrm{D}\end{array}$ & $\begin{array}{l}2.65 E+00 \\
8.62 E+00 \\
2.70 E-00 \\
7.68 E-13\end{array}$ & $\begin{array}{l}2.80 E+00 \\
9.25 E+00 \\
2.20 E-06 \\
2.03 E-11\end{array}$ & $\begin{array}{l}1.63 E+00 \\
2.92 E+00 \\
1.07 E+01 \\
1.32 E-06 \\
1.75 E-11\end{array}$ & & $\begin{array}{l}1.39 E+00 \\
2.48 E+00 \\
8.23 E+00 \\
1.09 E-06 \\
1.04 E-11\end{array}$ & & & & $\begin{array}{l}1.44 E+00 \\
2.58 E+00 \\
6.52 E+00 \\
5.66 E-07 \\
6.94 E-12\end{array}$ \\
\hline $\begin{array}{l}C A-49 \\
S C-44 \\
S C-46 \\
S C-46.4\end{array}$ & $\begin{array}{l}8.719 \mathrm{H} \\
3.927 \mathrm{H} \\
83.80 \mathrm{O} \\
18.72 \mathrm{~S}\end{array}$ & & & & & & & & & $\begin{array}{l}\text { 9. } 16 E+00 \\
3.54 E+01 \\
1.93 E+01 \\
1.87 E+01 \\
1.76 E-01\end{array}$ \\
\hline $\begin{array}{l}S C-47 \\
S C-48 \\
S C-49 \\
I I-44 \\
I I-45\end{array}$ & $\begin{array}{r}3.422 \mathrm{O} \\
43.67 \mathrm{H} \\
57.4 \mathrm{M} \\
47.3 \mathrm{Y} \\
3.08 \mathrm{H}\end{array}$ & $\begin{array}{l}3.17 E+01 \\
1.06 E-02 \\
1.12 E+00 \\
7.70 E+00\end{array}$ & $\begin{array}{l}3.37 E+01 \\
1.12 E-02 \\
1.24 E \cdot 00 \\
8.26 E+00\end{array}$ & $\begin{array}{l}3.57 E+01 \\
1.15 E-02 \\
2.17 E+00 \\
9.51 E+00\end{array}$ & $\begin{array}{l}3.40 E+01 \\
1.13 E-02 \\
1.69 E-01 \\
8.45 E+00\end{array}$ & $\begin{array}{l}0.24 E-01 \\
3.04 E+01 \\
9.76 E-03 \\
9.24 E-01 \\
7.39 E+00\end{array}$ & $\begin{array}{l}2.74 \mathrm{E} \cdot 01 \\
9.21 \mathrm{E}-03 \\
7.62 \mathrm{E}-01 \\
6.62 \mathrm{E}+00\end{array}$ & $\begin{array}{l}1.57 E+00 \\
3.63 E+01 \\
1.17 E-02 \\
2.23 E+00 \\
9.66 E+00\end{array}$ & $\begin{array}{l}9.48 E-01 \\
3.07 E+01 \\
1.02 E-02 \\
1.03 E+00 \\
7.52 E+00\end{array}$ & $\begin{array}{l}9.39 E-01 \\
3.11 E+01 \\
1.03 E-02 \\
1.05 E+00 \\
1.59 E+00\end{array}$ \\
\hline
\end{tabular}


OOSE-RATE FACTORS FOR BODY OPGANS ANO EFFECTIVE DOSE-PATE FACTOR FOR IMMERSION IN CUNTAMINATEI WATEH

\begin{tabular}{|c|c|c|c|c|c|c|c|c|c|}
\hline NUCL IDE & HALF-LIFE & TESTES & THYMUS & THY ROI D & UTERUS & FFECTIVE & $\begin{array}{l}\text { SKIN } \\
\text { HOIONI }\end{array}$ & $\begin{array}{l}\text { SKIN } \\
\text { (ELECIRON) }\end{array}$ & $\begin{array}{l}\text { SKIN } \\
\text { IIOTALI }\end{array}$ \\
\hline $\begin{array}{l}H-3 \\
B E-7 \\
B E-10 \\
C-11 \\
C-14\end{array}$ & 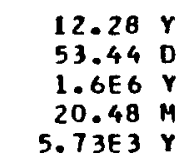 & $\begin{array}{l}0.0 \\
6.31 E-01 \\
0.0 \\
1.30 E+01 \\
0.0\end{array}$ & $\begin{array}{l}0.0 \\
4.76 E-01 \\
0.0 \\
9.83 E+00 \\
0.0\end{array}$ & $\begin{array}{l}0.0 \\
5.84 E-01 \\
0.0 \\
1.20 E+01 \\
0.0\end{array}$ & $\begin{array}{l}0.0 \\
3.83 E-01 \\
0.0 \\
7.87 E+00 \\
0.0\end{array}$ & $\begin{array}{l}0.0 \\
5.41 E-01 \\
0.0 \\
1.11 E+01 \\
0.0\end{array}$ & $\begin{array}{l}0.0 \\
7.20 E-01 \\
0.0 \\
1.40 E+01 \\
0.0\end{array}$ & $\begin{array}{l}0.0 \\
0.0 \\
1.16 E+00 \\
2.80 E \cdot 00 \\
3.05 E-02\end{array}$ & $\begin{array}{l}0.0 \\
7.20 E-01 \\
1.16 E+00 \\
1.17 E+01 \\
3.05 E-02\end{array}$ \\
\hline $\begin{array}{l}N-13 \\
N-16 \\
0-15 \\
F-18 \\
N A-22\end{array}$ & $\begin{array}{r}9.97 \mathrm{M} \\
7.13 \mathrm{~S} \\
122.24 \mathrm{~S} \\
109.74 \mathrm{M} \\
2.602 \mathrm{Y}\end{array}$ & $\begin{array}{l}1.30 E+01 \\
7.35 E+01 \\
1.30 E+01 \\
1.26 E+01 \\
2.84 E+01\end{array}$ & $\begin{array}{l}9.83 E+00 \\
5.97 E \bullet 01 \\
9.84 E+00 \\
9.53 E+00 \\
2.22 E+01\end{array}$ & $\begin{array}{l}1.20 E+01 \\
6.66 E+01 \\
1.20 E+01 \\
1.17 E+01 \\
2.61 E+01\end{array}$ & $\begin{array}{l}7.88 E+00 \\
4.14 E \div 01 \\
1.88 E+00 \\
1.63 E+00 \\
1.117 E+01\end{array}$ & $\begin{array}{l}1.11 E * 01 \\
6.36 E+01 \\
1.11 E+01 \\
1.08 E+01 \\
2.43 E * 01\end{array}$ & $\begin{array}{l}1.49 E+01 \\
1.11 E+01 \\
1.49 E+01 \\
1.44 E+01 \\
3.25 E \cdot 01\end{array}$ & $\begin{array}{l}3.16 E+00 \\
2.43 E+01 \\
5.94 E+00 \\
1.54 E+00 \\
1.16 E+00\end{array}$ & $\begin{array}{l}1.86 E+01 \\
1.01 E+02 \\
2.08 E+01 \\
1.59 E \cdot 01 \\
3.37 E * 01\end{array}$ \\
\hline $\begin{array}{l}N A-24 \\
M G-27 \\
M G-28 \\
A L-26 \\
A L-28\end{array}$ & $\begin{array}{l}15.00 \mathrm{H} \\
9.458 \mathrm{M} \\
20.91 \mathrm{H} \\
1.2 \mathrm{ES} \mathrm{Y} \\
2.240 \mathrm{M}\end{array}$ & $\begin{array}{l}5.99 E+01 \\
1.18 E+01 \\
1.78 E+01 \\
3.66 E+01 \\
2.49 E+01\end{array}$ & $\begin{array}{l}4.73 E+01 \\
9.42 E+00 \\
1.41 E \bullet 01 \\
2.86 E+01 \\
1.97 E+01\end{array}$ & $\begin{array}{l}5.48 \mathrm{E}+01 \\
1.13 \mathrm{E}+01 \\
1.68 \mathrm{E}+01 \\
3.39 \mathrm{E} \cdot 01 \\
2.32 \mathrm{E}+\mathrm{Cl}\end{array}$ & $\begin{array}{l}3.94 E+01 \\
7.30 E+00 \\
1.12 E+01 \\
2.36 E+01 \\
1.65 E+01\end{array}$ & $\begin{array}{l}5.16 E+01 \\
1.01 E+01 \\
1.53 E+01 \\
3.14 E+01 \\
2.15 E+01\end{array}$ & $\begin{array}{l}6.69 E \cdot 01 \\
1.37 E+01 \\
2.05 E+01 \\
4.16 E+01 \\
2.83 E+01\end{array}$ & $\begin{array}{l}4.30 E \cdot 00 \\
5.63 E+00 \\
7.43 E-01 \\
3.05 E+00 \\
1.05 E+01\end{array}$ & $\begin{array}{l}1.12 E+01 \\
1.93 E+01 \\
2.13 E+01 \\
4.47 E+01 \\
3.89 E+01\end{array}$ \\
\hline $\begin{array}{l}S 1-31 \\
S 1-32 \\
p-32 \\
p-33 \\
S-35\end{array}$ & 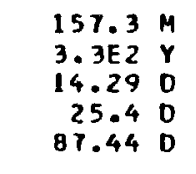 & $\begin{array}{l}1.16 \mathrm{E}-02 \\
0.0 \\
0.0 \\
0.0 \\
0.0\end{array}$ & $\begin{array}{l}9.29 E-03 \\
0.0 \\
0.0 \\
0.0 \\
0.0\end{array}$ & $\begin{array}{l}1.10 E-02 \\
0.0 \\
0.0 \\
0.0 \\
0.0\end{array}$ & $\begin{array}{l}7.38 E-03 \\
0.0 \\
0.0 \\
0.0 \\
0.0\end{array}$ & $\begin{array}{l}9.94 E-03 \\
0.0 \\
0.0 \\
0.0 \\
0.0\end{array}$ & $\begin{array}{l}1.33 E-02 \\
0.0 \\
0.0 \\
0.0 \\
0.0\end{array}$ & & $\begin{array}{l}4.6 \theta E+00 \\
1.02 E-01 \\
5.57 E+00 \\
1.71 E-01 \\
3.7 T E-02\end{array}$ \\
\hline $\begin{array}{l}C L-36 \\
C L-38 \\
A R-37 \\
A R-39 \\
A R-41\end{array}$ & $\begin{array}{r}3.01 E 5 \\
37.21 \mathrm{M} \\
35.02 \mathrm{O} \\
269 \mathrm{Y} \\
1.827 \mathrm{H}\end{array}$ & $\begin{array}{l}5.42 E-08 \\
2.15 E+01 \\
8.65 E-06 \\
0.0 \\
1.68 E+01\end{array}$ & $\begin{array}{l}1.25 E-12 \\
1.70 E+01 \\
1.99 E-10 \\
0.0 \\
1.34 E+01\end{array}$ & $\begin{array}{l}3.74 E-09 \\
1.98 E+01 \\
5.96 E-07 \\
0.0 \\
1.59 E+01\end{array}$ & $\begin{array}{l}5.45 E-13 \\
1.43 E+01 \\
e .70 E-11 \\
0.0 \\
1.07 E \cdot 01\end{array}$ & $\begin{array}{l}1.04 E-07 \\
1.85 E+01 \\
1.66 E-05 \\
0.0 \\
1.44 E+01\end{array}$ & $\begin{array}{l}1.25 E-06 \\
2.43 E+01 \\
1.99 E-04 \\
0.0 \\
1.93 E+01\end{array}$ & $\begin{array}{l}1.59 E+00 \\
1.33 E+01 \\
0.0 \\
1.33 E+00 \\
3.50 E+00\end{array}$ & $\begin{array}{l}1.59 E+00 \\
3.76 E+01 \\
1.99 E-04 \\
1.33 E+00 \\
2.2 B E+01\end{array}$ \\
\hline $\begin{array}{l}K-40 \\
K-42 \\
K-43 \\
C A-41 \\
C A-45\end{array}$ & $\begin{array}{r}1.277 E 9 Y \\
12.36 \mathrm{H} \\
22.6 \mathrm{H} \\
1.03 E 5 \mathrm{Y} \\
162.7 \mathrm{O}\end{array}$ & $\begin{array}{l}2.05 E+00 \\
3.66 E+00 \\
1.24 E+01 \\
2.02 E-05 \\
1.56 E-10\end{array}$ & $\begin{array}{l}1.64 E+00 \\
2.91 E+00 \\
9.49 E+00 \\
4.64 E-10 \\
7.08 E-14\end{array}$ & & & & & $\begin{array}{l}3.4 B E+00 \\
1.23 E+01 \\
2.0 B E+00 \\
0.0 \\
1.19 E-01\end{array}$ & \\
\hline $\begin{array}{l}C A-47 \\
C A-49 \\
S C-44 \\
S C-46 \\
S C-464\end{array}$ & $\begin{array}{l}4.536 \mathrm{O} \\
8.719 \mathrm{M} \\
3.927 \mathrm{H} \\
83.80 \mathrm{D} \\
18.72 \mathrm{~S}\end{array}$ & $\begin{array}{l}1.39 E+01 \\
4.83 E+01 \\
2.77 E+01 \\
2.66 E+01 \\
1.20 E+00\end{array}$ & $\begin{array}{l}1.11 E+01 \\
3.80 E+01 \\
2.17 E+01 \\
2.13 E \cdot 01 \\
9.27 E-01\end{array}$ & $\begin{array}{l}1.32 E+01 \\
4.33 E+01 \\
2.61 E+01 \\
2.54 E+01 \\
1.19 E+00\end{array}$ & $\begin{array}{l}8.84 E \cdot 00 \\
3.18 E \cdot 01 \\
1.12 E \cdot 01 \\
1.66 E+01 \\
6.89 E-01\end{array}$ & $\begin{array}{l}1.19 E+01 \\
4.17 E+01 \\
2.37 E+01 \\
2.28 E+01 \\
1.06 E+00\end{array}$ & $\begin{array}{l}1.60 E \bullet 01 \\
5.28 E+01 \\
3.18 E+01 \\
3.07 E \bullet 01 \\
1.29 E \bullet 00\end{array}$ & $\begin{array}{l}2.44 E+00 \\
1.17 E+00 \\
4.75 E+00 \\
4.25 E-01 \\
1.81 E-01\end{array}$ & $\begin{array}{l}1.84 E \cdot 01 \\
5.99 E+01 \\
3.66 E+01 \\
3.11 E+01 \\
1.47 E+00\end{array}$ \\
\hline $\begin{array}{l}S C-4 T \\
5 C-48 \\
S C-49 \\
T 1-44 \\
T 1-45\end{array}$ & $\begin{array}{r}3.422 \mathrm{D} \\
43.67 \mathrm{H} \\
57.4 \mathrm{M} \\
47.3 \mathrm{Y} \\
3.08 \mathrm{H}\end{array}$ & $\begin{array}{l}1.44 E+00 \\
4.43 E+01 \\
1.45 E-02 \\
1.89 E \cdot 00 \\
1.11 E 401\end{array}$ & $\begin{array}{l}1.11 E+00 \\
3.55 E+01 \\
1.15 E-02 \\
1.47 E+00 \\
8.40 E+00\end{array}$ & $\begin{array}{l}1.41 E+00 \\
4.22 E+01 \\
1.35 E-02 \\
1.08 E+00 \\
1.03 E+01\end{array}$ & $\begin{array}{l}0.37 E-01 \\
2.79 E+01 \\
5.55 E-03 \\
9.04 E-01 \\
6.72 E+00\end{array}$ & $\begin{array}{l}1.27 E+00 \\
3.80 E+01 \\
1.25 E-02 \\
1.61 E+00 \\
9.4 B E+00\end{array}$ & $\begin{array}{l}1.55 E \bullet 00 \\
5.11 E \bullet 01 \\
1.65 E-02 \\
2.05 E \bullet 00 \\
1.27 E \cdot 01\end{array}$ & $\begin{array}{l}8.41 E-01 \\
1.33 E+00 \\
6.72 E+00 \\
1.41 E-04 \\
2.79 E+00\end{array}$ & $\begin{array}{l}2.39 E \cdot 00 \\
5.24 E \cdot 01 \\
6.74 E+00 \\
2.05 E \cdot 00 \\
1.55 E \cdot 01\end{array}$ \\
\hline
\end{tabular}




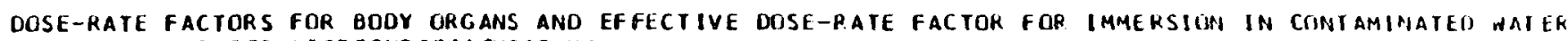

IN MREM/YR PER MICROCUR IE/ICUBIC MI

\begin{tabular}{|c|c|c|c|c|c|c|c|c|c|c|}
\hline NUCL IDE & HALF-LIFE & ADRENALS & BL ADDER & BRAIN & BREAST & HE ART & $\begin{array}{l}\text { SMALL } \\
\text { JTESTINE }\end{array}$ & $\begin{array}{c}\text { UPPER } \\
\text { LARGE } \\
\text { INTESTIVE }\end{array}$ & $\begin{array}{c}\text { LOWER } \\
\text { LARGE } \\
\text { INIESTINE }\end{array}$ & rIONEYS \\
\hline $\begin{array}{l}V 1-51 \\
v-48 \\
v-49 \\
v-52 \\
C R-49\end{array}$ & $\begin{array}{r}5.752 \\
15.971 \\
330 \\
3.75 \mathrm{H} \\
42.09 \mathrm{H}\end{array}$ & $\begin{array}{l}3.50 E * 00 \\
2.79 E * 01 \\
8.40 E-06 \\
1.37 E+01 \\
9.74 E+00\end{array}$ & $\begin{array}{l}27 E \cdot 00 \\
74 E \cdot 01 \\
51 E-12 \\
37 E+01 \\
26 E \cdot 00\end{array}$ & $\begin{array}{l}.59 E * 00 \\
.08 E * 01 \\
.70 E-07 \\
.55 E+01 \\
.03 E * 01\end{array}$ & $\begin{array}{l}58 E+00 \\
46 E+01 \\
58 E+04 \\
71 E+01 \\
27 E+01\end{array}$ & $\begin{array}{r}.19 E+00 \\
.71 E+01 \\
.09 E-06 \\
.36 E+01 \\
.03 E+00\end{array}$ & $\begin{array}{l}2.87 E+00 \\
2.48 E+01 \\
1.41 E-08 \\
1.26 E+01 \\
8.10 E+00\end{array}$ & $\begin{array}{l}3.38 E+00 \\
2.88 E+01 \\
2.08 E-00 \\
1.45 E+01 \\
9.59 E+00\end{array}$ & $\begin{array}{l}3.16 E+00 \\
2.65 E+01 \\
3.45 E-06 \\
1.33 E+01 \\
8.84 E+00\end{array}$ & $\begin{array}{l}\text { 3. } 38 E+00 \\
2.88 E+01 \\
1.01 E-08 \\
1.45 E+01 \\
9.59 E .00\end{array}$ \\
\hline $\begin{array}{l}C R-51 \\
M N-52 \\
M N-52 M \\
M N-53 \\
M N-54\end{array}$ & $\begin{array}{rl}27.704 & D \\
5.591 & D \\
21.4 & H \\
3.7 E 6 & Y \\
312.7 & D\end{array}$ & $\begin{array}{l}2.97 E-01 \\
3.30 E+01 \\
2.27 E+01 \\
1.56 E-05 \\
8.12 E+00\end{array}$ & $\begin{array}{l}.74 E-01 \\
.25 E+01 \\
.23 E+01 \\
.82 E-12 \\
04 E+00\end{array}$ & & & & & & & $\begin{array}{l}2.83 E-01 \\
3.41 E+01 \\
2.33 E+01 \\
1.87 E-08 \\
8.21 E+00\end{array}$ \\
\hline $\begin{array}{l}M N-56 \\
M N-57 \\
F E-52 \\
F E-55 \\
F E-59\end{array}$ & $\begin{array}{r}2.5785 \mathrm{H} \\
1.47 \mathrm{H} \\
8.275 \mathrm{H} \\
2.7 \mathrm{Y} \\
44.63 \mathrm{D}\end{array}$ & $\begin{array}{l}1.69 E+01 \\
6.80 E-01 \\
6.92 E+00 \\
2.06 E-05 \\
1.14 E+01\end{array}$ & $\begin{array}{l}1.69 E+01 \\
6.54 E-01 \\
6.51 E+00 \\
3.71 E-12 \\
1.12 E+01\end{array}$ & $\begin{array}{l}1.90 E+01 \\
7.25 E-01 \\
7.19 E+00 \\
1.64 E-06 \\
1.27 E+01\end{array}$ & & & & & & $\begin{array}{l}1.79 E+01 \\
6.80 E-01 \\
6.73 E+00 \\
2.47 E-08 \\
1.18 E+01\end{array}$ \\
\hline $\begin{array}{l}C 0-56 \\
C 0-51 \\
C 0-58 \\
C 0-584 \\
C 0-60\end{array}$ & $\begin{array}{rr}70.76 & D \\
270.9 & 0 \\
70.80 & 0 \\
9.15 & H \\
5.271 & Y\end{array}$ & $\begin{array}{l}3.61 E \cdot 01 \\
1.13 E+00 \\
9.40 E \bullet 00 \\
3.69 E-05 \\
2.38 E \cdot 01\end{array}$ & $\begin{array}{l}9.06 E+00 \\
7.01 E-00 \\
2.37 E+01\end{array}$ & & & & & & & $\begin{array}{l}3.84 E+01 \\
1.07 E+00 \\
9.46 E+00 \\
1.51 E-05 \\
2.49 E+01\end{array}$ \\
\hline $\begin{array}{l}C O-604 \\
C O-61 \\
N I-56 \\
N I-57 \\
N I-59\end{array}$ & $\begin{array}{r}10.47 \mathrm{H} \\
1.650 \mathrm{H} \\
6.10 \mathrm{O} \\
36.08 \mathrm{H} \\
7.564 \mathrm{Y}\end{array}$ & $\begin{array}{l}3.84 E-02 \\
7.33 E-01 \\
1.65 E+01 \\
1.84 E+01\end{array}$ & & & & & & & & \\
\hline $\begin{array}{l}\text { NI-63 } \\
\text { NI-65 } \\
\text { CU-61 } \\
\text { CU-62 } \\
C U-64\end{array}$ & $\begin{array}{r}100.1 \mathrm{Y} \\
2.520 \mathrm{H} \\
3.408 \mathrm{H} \\
9.74 \mathrm{H} \\
12.701 \mathrm{H}\end{array}$ & $\begin{array}{l}0.0 \\
5.22 E \bullet 00 \\
7.73 E+00 \\
9.43 E+00 \\
1.77 E * 00\end{array}$ & $\begin{array}{l}0.0 \\
5.22 E \bullet 00 \\
7.36 E \bullet 00 \\
8.96 E \bullet 00 \\
1.69 E \bullet 00\end{array}$ & $\begin{array}{l}0 \\
87 E+00 \\
21 E+00 \\
98 E+00\end{array}$ & $\begin{array}{l}.0 \\
52 E+00 \\
76 E+00 \\
.19 E+01 \\
.23 E+00\end{array}$ & $\begin{array}{l}0.0 \\
5.16 E+00 \\
7.20 E+00 \\
8.76 E+00 \\
1.65 E+00\end{array}$ & & & & \\
\hline $\begin{array}{l}C U-67 \\
2 N-62 \\
2 N-65 \\
2 N-69 \\
2 N-694\end{array}$ & $\begin{array}{r}61.88 \mathrm{D} \\
9.26 \mathrm{H} \\
244.4 \mathrm{D} \\
55.6 \mathrm{H} \\
13.76 \mathrm{H}\end{array}$ & $\begin{array}{l}1.07 E \cdot 00 \\
4.19 E \cdot 00 \\
5.59 E \bullet 00 \\
5.61 E-05 \\
3.89 E+00\end{array}$ & $\begin{array}{l}9.93 E-01 \\
3.99 E+00 \\
5.50 E+00 \\
5.26 E-05 \\
3.66 E+00\end{array}$ & $\begin{array}{l}1.06 E \bullet 00 \\
4.43 E \bullet 00 \\
6.20 E+00 \\
5.81 E-05 \\
4.05 E+00\end{array}$ & $\begin{array}{l}1.65 E+00 \\
5.36 E+00 \\
6.87 E * 00 \\
7.21 E-05 \\
4.97 E+00\end{array}$ & $\begin{array}{l}9.67 E-01 \\
3.89 E+00 \\
5.44 E+00 \\
5.13 E-05 \\
3.57 E+00\end{array}$ & $\begin{array}{l}4.97 E+0 \\
4.01 E-0 \\
3.21 E \cdot 0\end{array}$ & $\begin{array}{l}1.03 E+00 \\
4.14 E+00 \\
5.79 E+00 \\
5.44 E-05 \\
3.18 E+00\end{array}$ & $\begin{array}{l}9.53 E-01 \\
3.81 E+00 \\
5.33 E+00 \\
5.05 E-05 \\
3.51 E+00\end{array}$ & $\begin{array}{l}1.02 E+00 \\
4.16 E+00 \\
5.77 E+00 \\
5.44 E-05 \\
3.79 E+00\end{array}$ \\
\hline $\begin{array}{l}G A-66 \\
G A-67 \\
G A-6 B \\
G A-72 \\
G E-6 B\end{array}$ & $\begin{array}{rl}9.40 & H \\
3.261 & 0 \\
68.0 & M \\
14.1 & H \\
288 & 0\end{array}$ & $\begin{array}{l}2.54 E+01 \\
1.34 E+00 \\
0.91 E+00 \\
2.73 E+01 \\
8.31 E-05\end{array}$ & $\begin{array}{l}2.55 E \bullet 01 \\
1.25 E \bullet 00 \\
8.47 E \bullet 00 \\
2.14 E \bullet 01 \\
1.88 E-11\end{array}$ & $\begin{array}{l}9.44 E+00 \\
3.07 E+01 \\
6.66 E-06\end{array}$ & $\begin{array}{l}3.25 E+01 \\
2.01 E * 00 \\
1.12 E * 01 \\
3.44 E * 01 \\
6.44 E-03\end{array}$ & $\begin{array}{l}2.53 E \bullet 01 \\
1.22 E+00 \\
8.28 E \bullet 00 \\
2.12 E+01 \\
1.09 E-05\end{array}$ & $\begin{array}{l}7.46 E+00 \\
2.51 E+01 \\
1.45 E-07\end{array}$ & $\begin{array}{l}2.69 E * 01 \\
1.29 E+00 \\
8.79 E+00 \\
2.90 E+01 \\
1.96 E-07\end{array}$ & $\begin{array}{l}2.49 E+01 \\
1.20 E+00 \\
8.12 E+00 \\
2.67 E+01 \\
3.42 E-05\end{array}$ & $\begin{array}{l}2.73 E+01 \\
1.29 E+00 \\
8.79 E+00 \\
2.91 E+01 \\
1.05 E-07\end{array}$ \\
\hline
\end{tabular}


DOSE-PATE FACTORS FOR BODY ORGANS ANO EFFECTIVE DOSE-RATE FACTOR FOR IMMERSIUN IN CONIMMIMATEII WATER
IN MREM/YR PER MICPOCUR IE/ICUBIC MI

\begin{tabular}{|c|c|c|c|c|c|c|c|c|c|c|}
\hline ICL $10 E$ & HALF-LIFE & LIVER & LUNGS & MARROH & $\begin{array}{l}\text { RED } \\
\text { MARROH }\end{array}$ & OVARIES & PANCREAS & SKELETON & $S^{D} L E E N$ & STOMACH \\
\hline $\begin{array}{l}T 1-51 \\
V-48 \\
V-49 \\
V-52 \\
C R-49\end{array}$ & $\begin{array}{r}5.752 \mathrm{M} \\
15.971 \mathrm{D} \\
330 \mathrm{D} \\
3.75 \mathrm{M} \\
42.09 \mathrm{M}\end{array}$ & 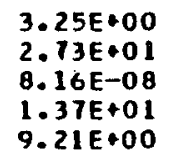 & $\begin{array}{l}50 E \bullet 00 \\
90 E+01 \\
64 E-06 \\
45 E+01 \\
91 E \bullet 00\end{array}$ & & $\begin{array}{l}-60 E+00 \\
94 E+01 \\
18 E-06\end{array}$ & $\begin{array}{l}3.02 E+00 \\
2.62 E+01 \\
3.29 E-06 \\
1.29 E+01 \\
8.75 E+00\end{array}$ & $\begin{array}{l}2.81 E+00 \\
2.36 E+01 \\
4.91 E-06 \\
1.19 E+01 \\
7.85 E+00\end{array}$ & $\begin{array}{l}4.34 E+00 \\
3.17 E+01 \\
1.08 E-05 \\
1.54 E+01 \\
1.21 E+01\end{array}$ & $\begin{array}{l}.20 E+00 \\
.65 E+01 \\
.09 E-06 \\
.32 E+01 \\
.99 E+00\end{array}$ & $\begin{array}{l}21 E+00 \\
68 E+01 \\
71 E-06 \\
34 E+01 \\
08 E+00\end{array}$ \\
\hline $\begin{array}{l}C R-51 \\
M N-52 \\
M N-52 M \\
M N-53 \\
M N-54\end{array}$ & $\begin{array}{rr}27.704 & 0 \\
5.591 & 0 \\
21.4 & M \\
3.7 E 6 & r \\
312.7 & 0\end{array}$ & $\begin{array}{l}2.73 E-01 \\
3.23 E+01 \\
2.22 E+01 \\
1.52 E-07 \\
7.79 E+00\end{array}$ & $\begin{array}{l}.95 E-01 \\
.44 E+01 \\
36 E+01 \\
.24 E-05 \\
.32 E+00\end{array}$ & & & & & & & $\begin{array}{l}\text { 2. 10E-OI } \\
3.18 E+01 \\
2.18 E+01 \\
3.18 E-06 \\
7.11 E+00\end{array}$ \\
\hline $\begin{array}{l}M N-56 \\
M N-57 \\
F E-52 \\
F E-55 \\
F E-59\end{array}$ & $\begin{array}{r}2.5785 \mathrm{H} \\
1.47 \mathrm{M} \\
8.275 \mathrm{H} \\
2.7 \mathrm{Y} \\
44.63 \mathrm{D}\end{array}$ & $\begin{array}{l}1.69 E+01 \\
6.51 E-01 \\
6.48 E+00 \\
2.00 E-01 \\
1.12 E+01\end{array}$ & $\begin{array}{l}79 E+01 \\
00 E-01 \\
99 E+00 \\
63 E-05\end{array}$ & $\begin{array}{l}1.88 E+01 \\
8.39 E-01 \\
6.51 E+00 \\
9.78 E-06 \\
1.26 E+01\end{array}$ & & & & & & $\begin{array}{l}1.66 E * 01 \\
6.42 E-01 \\
6.40 E+00 \\
4.20 E-06 \\
1.10 E+01\end{array}$ \\
\hline $\begin{array}{l}C 0-56 \\
C 0-51 \\
C 0-58 \\
C 0-58 M \\
C 0-60\end{array}$ & 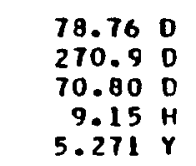 & $\begin{array}{l}3.61 \mathrm{E} \\
1.05 \mathrm{E} \\
9.00 \mathrm{E} \\
5.50 \mathrm{E} \\
2.36 \mathrm{E}\end{array}$ & $\begin{array}{l}.626 E- \\
.50 E+4 \\
.50 E\end{array}$ & $\begin{array}{l}.01 E+01 \\
.82 E+00 \\
.05 E+01 \\
.86 E-05\end{array}$ & & $\begin{array}{l}3.36 E+01 \\
9.06 E-01 \\
8.73 E+00 \\
1.79 E-05 \\
2.25 E+01\end{array}$ & $\begin{array}{l}3.14 E+01 \\
8.54 E-01 \\
7.76 E+00 \\
2.16 E-05 \\
2.05 E+01\end{array}$ & $\begin{array}{l}4.07 E+01 \\
1.88 E+00 \\
1.07 E+01 \\
5.36 E-05 \\
2.68 E+01\end{array}$ & & $\begin{array}{l}3.56 E+01 \\
1.04 E * 00 \\
8.90 E+00 \\
1.21 E-05 \\
2.31 E+01\end{array}$ \\
\hline $\begin{array}{l}C 0-604 \\
C 0-61 \\
N 1-56 \\
N 1-57 \\
N 1-59\end{array}$ & $\begin{array}{r}10.47 \mathrm{M} \\
1.650 \mathrm{H} \\
6.10 \mathrm{O} \\
36.08 \mathrm{H} \\
7.5 E 4 \mathrm{Y}\end{array}$ & $\begin{array}{l}3.91 \mathrm{E}-0 \\
7.57 \mathrm{E}-\mathrm{C} \\
1.5 \mathrm{EE}+\mathrm{C} \\
1.83 \mathrm{E}+\mathrm{C} \\
3.38 \mathrm{E}-\mathrm{C}\end{array}$ & $\begin{array}{l}4.18 E-02 \\
8.23 E-01 \\
1.69 E+01 \\
1.94 E+01 \\
2.75 E-05\end{array}$ & $\begin{array}{l}5.07 E-02 \\
1.23 E+00 \\
1.92 E+01 \\
2.07 E+01 \\
1.65 E-05\end{array}$ & & $\begin{array}{l}3.60 E-02 \\
6.65 E-01 \\
1.51 E .01 \\
1.72 E \cdot 01 \\
1.36 E-05\end{array}$ & & & & $\begin{array}{l}\text { 3. } 77 E-02 \\
7.20 E-01 \\
1.56 E+01 \\
1.79 E+01 \\
7.09 E-06\end{array}$ \\
\hline $\begin{array}{l}N 1-63 \\
N 1-65 \\
C U-61 \\
C U-62 \\
C U-64\end{array}$ & $\begin{array}{r}100.1 \mathrm{Y} \\
2.520 \mathrm{H} \\
3.408 \mathrm{H} \\
9.74 \mathrm{H} \\
12.701 \mathrm{H}\end{array}$ & $\begin{array}{l}0.0 \\
5.21 E \\
7.32 E \\
8.91 E \\
1.68 E\end{array}$ & $\begin{array}{l}5.51 E+00 \\
7.85 E+00 \\
9.56 E+00 \\
1.80 E+00\end{array}$ & $\begin{array}{l}5.80 E+00 \\
9.01 E+00 \\
1.10 E+01 \\
2.06 E+00\end{array}$ & & & & & & \\
\hline $\begin{array}{l}C U-67 \\
2 N-62 \\
2 N-65 \\
2 N-69 \\
2 N-694\end{array}$ & $\begin{array}{rr}61.8 B & 0 \\
9.26 & H \\
244.4 & D \\
55.6 & M \\
13.76 & H\end{array}$ & $\begin{array}{l}3.96 \mathrm{E}+1 \\
5.47 \mathrm{E} \\
5.23 \mathrm{E}-\mathrm{C} \\
3.64 \mathrm{E}+\end{array}$ & $\begin{array}{l}4.25 E \cdot 00 \\
5.81 E \cdot 00 \\
5.63 E-05 \\
3.91 E+00\end{array}$ & $\begin{array}{l}4.91 E+00 \\
6.17 E+00 \\
6.71 E-05 \\
4.62 E+00\end{array}$ & & & & & & \\
\hline $\begin{array}{l}G A-66 \\
G A-67 \\
G A-68 \\
G A-72 \\
G E-68\end{array}$ & $\begin{array}{r}9.40 \mathrm{H} \\
3.261 \\
68.0 \mathrm{D} \\
14.1 \mathrm{H} \\
288 \mathrm{D}\end{array}$ & $\begin{array}{l}2.55 \mathrm{E} \\
1.25 \mathrm{E} \\
8.42 \mathrm{E}\end{array}$ & $\begin{array}{l}1.36 \\
9.03\end{array}$ & $\begin{array}{l}1.9 \\
1.0\end{array}$ & $\begin{array}{l}2.76 E+01 \\
1.29 E+00 \\
9.24 E+09 \\
2.94 E 001 \\
2.16 E-05\end{array}$ & $\begin{array}{l}1.11 E+00 \\
8.09 E+00 \\
2.55 E+01 \\
3.26 E-05\end{array}$ & $\begin{array}{l}1.04 E \cdot 00 \\
7.25 E+00 \\
2.38 E+01 \\
4.86 E-05\end{array}$ & $\begin{array}{l}2.88 E+01 \\
1.95 E+00 \\
1.05 E+01 \\
3.10 E+01 \\
1.07 E-04\end{array}$ & $\begin{array}{l}2.46 E+01 \\
1.23 E+00 \\
0.22 E+00 \\
2.64 E+01 \\
1.89 E-05\end{array}$ & $\begin{array}{l}2.53 E+01 \\
1.23 E+00 \\
8.31 E+00 \\
2.70 E+01 \\
1.71 E-05\end{array}$ \\
\hline
\end{tabular}




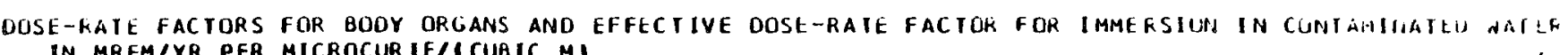

\begin{tabular}{|c|c|c|c|c|c|c|c|c|c|}
\hline NUCL IDE & HALF-LIFE & TESTES & THYMUS & THYROID & UTERUS & EFFECTIVE & $\begin{array}{c}\text { SKIN } \\
\text { I PHOTONI }\end{array}$ & $\begin{array}{c}\text { SKIN } \\
\text { IELECTRON I }\end{array}$ & $\begin{array}{l}\text { SKIN } \\
\text { ITORALI }\end{array}$ \\
\hline $\begin{array}{l}V 1-51 \\
V-48 \\
V-49 \\
V-52 \\
C R-49\end{array}$ & $\begin{array}{r}5.752 \\
15.971 \\
330 \\
330 \\
3.75 \mathrm{M} \\
42.09 \mathrm{M}\end{array}$ & $\begin{array}{l}4.71 E+00 \\
3.83 E+01 \\
6.09 E-05 \\
1.90 E \times 01 \\
1.34 E \cdot 01\end{array}$ & $\begin{array}{l}3.60 E \cdot 00 \\
3.04 E+01 \\
1.40 E-09 \\
1.51 E+01 \\
1.02 E+01\end{array}$ & $\begin{array}{l}4.44 E+00 \\
3.63 E+01 \\
4.20 E-06 \\
1.79 E+01 \\
1.25 E+01\end{array}$ & $\begin{array}{l}2.86 E+00 \\
2.40 E+01 \\
6.13 E-10 \\
1.22 E+01 \\
8.03 E+00\end{array}$ & $\begin{array}{l}4.08 E+00 \\
3.28 E+01 \\
1.17 E-04 \\
1.63 E+01 \\
1.15 E+01\end{array}$ & $\begin{array}{l}5.32 E+00 \\
4.41 E+01 \\
1.40 E-03 \\
2.17 E+01 \\
1.52 E+01\end{array}$ & $\begin{array}{l}7.11 E+00 \\
9.84 E-01 \\
0.0 \\
8.95 E+00 \\
4.73 E+00\end{array}$ & $\begin{array}{l}1.24 E+01 \\
4.51 E+01 \\
1.40 E-03 \\
3.07 E+01 \\
2.00 E+01\end{array}$ \\
\hline $\begin{array}{l}\text { CR-51 } \\
M N-52 \\
M N-52 M \\
M N-53 \\
M N-54\end{array}$ & $\begin{array}{rr}27.704 & 0 \\
5.591 & D \\
21.4 & M \\
3.7166 & Y \\
312.7 & D\end{array}$ & $\begin{array}{l}3.99 E-01 \\
4.53 E+01 \\
3.12 E+01 \\
1.13 E-04 \\
1.10 E+01\end{array}$ & $\begin{array}{l}3.01 E-01 \\
3.59 E \cdot 01 \\
2.44 E \cdot 01 \\
2.61 E-09 \\
8.75 E+00\end{array}$ & $\begin{array}{l}3.74 E-01 \\
4.29 E+01 \\
2.92 E+01 \\
7.81 E-06 \\
1.05 E+01\end{array}$ & $\begin{array}{l}2.41 E-01 \\
2.85 E+01 \\
1.97 E+01 \\
1.14 E-09 \\
6.78 E+00\end{array}$ & $\begin{array}{l}3.47 E-01 \\
3.88 E+01 \\
2.68 E+01 \\
2.17 E-04 \\
9.42 E+00\end{array}$ & $\begin{array}{l}4.50 E-01 \\
5.22 E+01 \\
3.57 E+01 \\
2.61 E-03 \\
1.28 E+01\end{array}$ & $\begin{array}{l}0.0 \\
4.45 E-01 \\
9.56 E+00 \\
0.0 \\
0.0\end{array}$ & $\begin{array}{l}4.50 E-01 \\
5.26 E+01 \\
4.53 E+01 \\
2.61 E-03 \\
1.28 E+01\end{array}$ \\
\hline $\begin{array}{l}M N-56 \\
M N-57 \\
F E-52 \\
F E-55 \\
F E-59\end{array}$ & $\begin{array}{r}2.5785 \mathrm{H} \\
1.47 \mathrm{H} \\
0.275 \mathrm{H} \\
2.7 \mathrm{Y} \\
44.63 \mathrm{D}\end{array}$ & $\begin{array}{l}2.34 E+01 \\
9.41 E-01 \\
9.43 E+00 \\
1.50 E-04 \\
1.56 E+01\end{array}$ & $\begin{array}{l}1.85 E+01 \\
7.33 E-01 \\
1.16 E+00 \\
3.44 E-09 \\
1.25 E+01\end{array}$ & $\begin{array}{l}2.19 E+01 \\
8.95 E-01 \\
0.84 E+00 \\
1.03 E-05 \\
1.49 E+01\end{array}$ & $\begin{array}{l}1.50 E+01 \\
5.71 E-01 \\
5.68 E+00 \\
1.50 E-09 \\
9.88 E+00\end{array}$ & $\begin{array}{l}2.01 E+01 \\
8.11 E-01 \\
8.13 E+00 \\
2.86 E-04 \\
1.34 E+01\end{array}$ & $\begin{array}{l}2.68 E+01 \\
1.07 E+00 \\
1.07 E+01 \\
3.44 E-03 \\
1.80 E+01\end{array}$ & $\begin{array}{l}0.81 E+03 \\
9.25 E+00 \\
1.37 E+00 \\
0.0 \\
4.89 E-01\end{array}$ & $\begin{array}{l}3.36 E+01 \\
1.03 E+01 \\
1.20 E+01 \\
3.44 E-03 \\
1.85 E+01\end{array}$ \\
\hline $\begin{array}{l}C 0-56 \\
C 0-57 \\
C O-58 \\
C 0-584 \\
C O-60\end{array}$ & $\begin{array}{rr}78.76 & D \\
270.9 & D \\
70.80 & 0 \\
9.15 & H \\
5.271 & Y\end{array}$ & $\begin{array}{l}5.00 E+01 \\
1.64 E+00 \\
1.20 E+01 \\
2.50 E-04 \\
3.29 E * 01\end{array}$ & $\begin{array}{l}3.95 E+01 \\
1.26 E+00 \\
1.00 E * 01 \\
7.22 E-06 \\
2.63 E \cdot 01\end{array}$ & $\begin{array}{l}4.66 E+01 \\
1.62 E+00 \\
1.21 E+01 \\
3.41 E-05 \\
3.12 E+01\end{array}$ & $\begin{array}{l}3.20 E+01 \\
9.14 E-01 \\
7.03 E+00 \\
1.37 E-06 \\
2.09 E+01\end{array}$ & & $\begin{array}{l}5.67 E+01 \\
1.76 E+00 \\
1.47 E+01 \\
5.01 E-03 \\
3.78 E+01\end{array}$ & $\begin{array}{l}9.50 E-01 \\
1.18 E-02 \\
1.71 E-01 \\
0.0 \\
3.08 E-01\end{array}$ & $\begin{array}{l}5.77 E+01 \\
1.77 E \bullet 00 \\
1.49 E+01 \\
5.01 E-03 \\
3.81 E+01\end{array}$ \\
\hline $\begin{array}{l}\text { CO-6OM } \\
\text { CO-61 } \\
N I-56 \\
N I-57 \\
N I-59\end{array}$ & $\begin{array}{r}10.47 \mathrm{M} \\
1.650 \mathrm{H} \\
6.10 \mathrm{D} \\
36.08 \mathrm{H} \\
7.5 \mathrm{E} \\
\mathrm{Y}\end{array}$ & $\begin{array}{l}5.82 E-02 \\
1.21 E+00 \\
2.25 E+01 \\
2.55 E+01 \\
2.52 E-04\end{array}$ & $\begin{array}{l}4.56 E-02 \\
9.45 E-01 \\
1.76 E+01 \\
2.01 E+01 \\
5.01 E-09\end{array}$ & $\begin{array}{l}5.52 E-02 \\
1.18 E+00 \\
2.14 E+01 \\
2.39 E+01 \\
1.74 E-05\end{array}$ & $\begin{array}{l}3.36 E-02 \\
6.21 E-01 \\
1.38 E+01 \\
1.63 E+01 \\
2.54 E-09\end{array}$ & $\begin{array}{l}4.95 E-02 \\
1.02 E+00 \\
1.93 E \bullet 01 \\
2.19 E+01 \\
4.84 E-04\end{array}$ & $\begin{array}{l}7.15 E-02 \\
1.35 E+00 \\
2.58 E+01 \\
2.91 E+01 \\
5.81 E-03\end{array}$ & $\begin{array}{l}1.11 E-02 \\
3.48 E+00 \\
9.17 E-03 \\
9.80 E-01 \\
0.0\end{array}$ & $\begin{array}{l}8.27 E-02 \\
4.83 E+00 \\
2.58 E+01 \\
3.01 E+01 \\
5.81 E-03\end{array}$ \\
\hline $\begin{array}{l}N I-63 \\
N I-65 \\
C U-61 \\
C U-62 \\
C U-64\end{array}$ & $\begin{array}{r}100.1 \mathrm{Y} \\
2.520 \mathrm{H} \\
3.408 \mathrm{H} \\
9.74 \mathrm{H} \\
12.701 \mathrm{H}\end{array}$ & $\begin{array}{l}0.0 \\
1.23 E+00 \\
1.05 E+01 \\
1.20 E+01 \\
2.41 E+00\end{array}$ & $\begin{array}{l}0.0 \\
5.76 E * 00 \\
0.03 E+00 \\
9.71 E * 00 \\
1.83 E+00\end{array}$ & $\begin{array}{l}0.0 \\
6.83 E+00 \\
9.79 E+00 \\
1.19 E+01 \\
2.24 E \bullet 00\end{array}$ & $\begin{array}{l}0.0 \\
4.63 E+00 \\
6.40 E+00 \\
7.78 E+00 \\
1.47 E+00\end{array}$ & $\begin{array}{l}0.0 \\
6.20 E+00 \\
9.01 E+00 \\
1.10 E+01 \\
2.06 E+00\end{array}$ & $\begin{array}{l}0.0 \\
8.29 E+00 \\
1.20 E+01 \\
1.47 E+01 \\
2.76 E+00\end{array}$ & $\begin{array}{l}0.0 \\
5.02 E+00 \\
2.36 E+00 \\
1.09 E+01 \\
7.31 E-01\end{array}$ & $\begin{array}{l}0.0 \\
1.33 E+01 \\
1.44 E+01 \\
2.56 E+01 \\
3.49 E+00\end{array}$ \\
\hline $\begin{array}{l}\text { CU-67 } \\
2 N-62 \\
2 N-65 \\
2 N-69 \\
2 N-694\end{array}$ & $\begin{array}{r}61.88 \mathrm{O} \\
9.26 \mathrm{H} \\
244.4 \mathrm{D} \\
55.6 \mathrm{H} \\
13.76 \mathrm{H}\end{array}$ & $\begin{array}{l}1.51 E+00 \\
5.74 E+00 \\
7.66 E+00 \\
7.58 E-05 \\
5.26 E \cdot 00\end{array}$ & $\begin{array}{l}1.16 E+00 \\
4.37 E+00 \\
6.14 E+00 \\
5.71 E-05 \\
3.97 E+00\end{array}$ & $\begin{array}{l}1.47 E+00 \\
5.33 E+00 \\
7.30 E+00 \\
7.04 E-05 \\
4.88 E+00\end{array}$ & $\begin{array}{l}8.68 E-01 \\
3.45 E+00 \\
4.81 E+00 \\
4.59 E-05 \\
3.19 E+00\end{array}$ & $\begin{array}{l}1.32 E+00 \\
4.90 E+00 \\
6.56 E+00 \\
6.52 E-05 \\
4.52 E+00\end{array}$ & $\begin{array}{l}1.64 E \cdot 00 \\
6.60 E+00 \\
8.85 E+00 \\
8.60 E-05 \\
5.99 E+00\end{array}$ & $\begin{array}{l}6.79 E-01 \\
1.27 E-01 \\
8.80 E-03 \\
2.23 E+00 \\
1.62 E-01\end{array}$ & $\begin{array}{l}2.32 E+00 \\
6.73 E+00 \\
8.85 E+00 \\
2.23 E+00 \\
6.15 E+00\end{array}$ \\
\hline $\begin{array}{l}\text { GA-66 } \\
G A-67 \\
G A-68 \\
G A-72 \\
G E-68\end{array}$ & $\begin{array}{r}9.40 \mathrm{H} \\
3.261 \mathrm{O} \\
68.0 \mathrm{M} \\
14.1 \mathrm{H} \\
288 \mathrm{D}\end{array}$ & $\begin{array}{l}3.53 E+01 \\
1.89 E+00 \\
1.21 E * 01 \\
3.79 E+01 \\
6.02 E-04\end{array}$ & $\begin{array}{l}2.77 E+01 \\
1.44 E+00 \\
9.19 E \cdot 00 \\
2.99 E \bullet 01 \\
1.51 E-08\end{array}$ & $\begin{array}{l}3.24 E+01 \\
1.02 E+00 \\
1.12 E+01 \\
3.53 E+01 \\
4.21 E-05\end{array}$ & $\begin{array}{l}2.26 E+01 \\
1.09 E+00 \\
1.36 E+00 \\
2.44 E+01 \\
5.99 E-09\end{array}$ & $\begin{array}{l}3.04 E+01 \\
1.64 E+00 \\
1.04 E+01 \\
3.26 E+01 \\
1.14 E-03\end{array}$ & $\begin{array}{l}3.95 E+01 \\
2.09 E+00 \\
1.39 E+01 \\
4.31 E+01 \\
1.37 E-02\end{array}$ & $\begin{array}{l}8.31 E+00 \\
1.45 E-02 \\
6.04 E \cdot 00 \\
3.85 E+00 \\
0.0\end{array}$ & $\begin{array}{l}4.78 E+01 \\
2.10 E \bullet 00 \\
1.99 E+01 \\
4.70 E+01 \\
1.37 E-02\end{array}$ \\
\hline
\end{tabular}




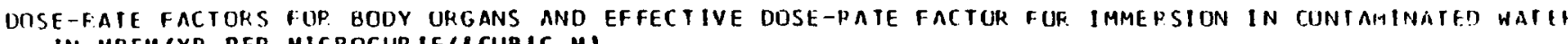

IN MREM/YR PER MICROCUP.IE/ICUBIC MI

\begin{tabular}{|c|c|c|c|c|c|c|c|c|c|c|}
\hline CLIOE & ILF-LIFE & DRENALS & BLADDER & BP.AIN & BREAST & HE AP.T & $\begin{array}{l}\text { SMALL } \\
\text { NTESTINE }\end{array}$ & $\begin{array}{c}\text { UPPER } \\
\text { LARGE } \\
\text { INTESTIVE }\end{array}$ & $\begin{array}{l}\text { LOWER } \\
\text { LARGE } \\
\text { INTESTINEE }\end{array}$ & XIONEYS \\
\hline $\begin{array}{l}S E-71 \\
G E-77 \\
A S-72 \\
A S-73 \\
A S-74\end{array}$ & 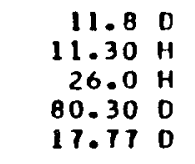 & $\begin{array}{l}8.40 E-05 \\
1.02 E+01 \\
1.71 E+01 \\
3.04 E-02 \\
7.21 E+00\end{array}$ & $\begin{array}{l}.90 E-11 \\
.71 E+00 \\
.65 E+01 \\
.60 E-02 \\
.87 E+00\end{array}$ & $\begin{array}{l}6.74 E-06 \\
1.08 E+01 \\
1.84 E+01 \\
3.09 E-02 \\
1.68 E+00\end{array}$ & $\begin{array}{l}6.51 E-03 \\
1.31 E+01 \\
2.13 E+01 \\
1.01 E-01 \\
9.01 E+00\end{array}$ & $\begin{array}{l}1.10 E-05 \\
9.54 E+00 \\
1.62 E * 01 \\
2.74 E-02 \\
6.13 E+00\end{array}$ & $\begin{array}{l}.47 E-07 \\
.64 E+00 \\
.46 E+01 \\
.59 E-02 \\
.06 E+00\end{array}$ & $\begin{array}{l}1.98 E-07 \\
1.01 E+01 \\
1.12 E+01 \\
3.10 E-02 \\
1.15 E+00\end{array}$ & $\begin{array}{l}.46 E-05 \\
.39 E+00 \\
.58 E+01 \\
.11 E-02 \\
.59 E+00\end{array}$ & $\begin{array}{l}1.01 \mathrm{E}-01 \\
1.01 \mathrm{E} 001 \\
1.12 \mathrm{E} 01 \\
4.00 \mathrm{E}-02 \\
1.14 \mathrm{E}+00\end{array}$ \\
\hline $\begin{array}{l}A S-76 \\
A S-77 \\
S E-73 \\
S E-75 \\
S E-79\end{array}$ & 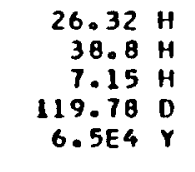 & $\begin{array}{l}.10 E \cdot 00 \\
011 E-02 \\
.01 E+01 \\
.65 E+00 \\
.0\end{array}$ & $\begin{array}{l}3.96 E+00 \\
7.53 E-02 \\
9.62 E+00 \\
3.36 E+00 \\
0.0\end{array}$ & $\begin{array}{l}4.44 E+00 \\
8.21 E-02 \\
1.06 E+01 \\
3.64 E+00 \\
0.0\end{array}$ & $\begin{array}{l}5.11 E+00 \\
1.10 E-01 \\
1.33 E+01 \\
5.22 E+00 \\
0.0\end{array}$ & $\begin{array}{l}3.90 E+00 \\
7.35 E-02 \\
9.35 E+00 \\
3.29 E+00 \\
0.0\end{array}$ & & $\begin{array}{r}14 E+00 \\
.79 E-02 \\
.92 E+00\end{array}$ & & $\begin{array}{l}\text { 4. } 14 E+00 \\
7.78 E-02 \\
9.97 E+00 \\
3.45 E+00 \\
0.0\end{array}$ \\
\hline $\begin{array}{l}B R-77 \\
8 R-80 \\
B R-804 \\
B R-82 \\
B R-83\end{array}$ & $\begin{array}{r}57.04 \mathrm{H} \\
17.4 \mathrm{M} \\
4.42 \mathrm{H} \\
35.30 \mathrm{H} \\
2.39 \mathrm{H}\end{array}$ & $\begin{array}{l}2.97 E \cdot 00 \\
7.06 E-01 \\
3.78 E-02 \\
2.53 E+01 \\
6.99 E-02\end{array}$ & $\begin{array}{l}.80 E+00 \\
.74 E-01 \\
.61 E-02 \\
.46 E+01 \\
.65 E-02\end{array}$ & & & & & & & $\begin{array}{l}2.90 E+00 \\
7.00 E-01 \\
7.67 E-02 \\
2.57 E+01 \\
6.90 E-02\end{array}$ \\
\hline $\begin{array}{l}K R-79 \\
K R-81 \\
K R-834\end{array}$ & 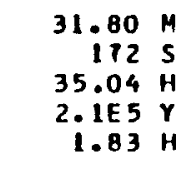 & & & & & & & & & $\begin{array}{l}2.00 E+01 \\
6.60 E-01 \\
2.32 E+00 \\
8.89 E-02 \\
6.48 E-05\end{array}$ \\
\hline $\begin{array}{l}K R-85 \\
K R-85 M \\
K R-87 \\
K R-8 B \\
K R-89\end{array}$ & $\begin{array}{r}10.72 \mathrm{Y} \\
4.48 \mathrm{H} \\
76.3 \mathrm{M} \\
2.84 \mathrm{H} \\
3.16 \mathrm{M}\end{array}$ & $\begin{array}{l}2.09 E-02 \\
1.50 E+00 \\
0.05 E+00 \\
2.02 E+01 \\
1.84 E+01\end{array}$ & $\begin{array}{l}.98 E-02 \\
.38 E \cdot 00 \\
.08 E+00\end{array}$ & & & & & & & \\
\hline $\begin{array}{l}K R-90 \\
R B-81 \\
R B-82 \\
R B-83\end{array}$ & $\begin{array}{rl}32.32 & \mathrm{~S} \\
4.58 & \mathrm{H} \\
1.25 & \mathrm{M} \\
86.2 & \mathrm{D} \\
32.9 & \mathrm{D}\end{array}$ & $\begin{array}{l}1.23 E+01 \\
5.69 E+00 \\
1.03 E+01 \\
4.68 E+00 \\
8.63 E+00\end{array}$ & $\begin{array}{l}1 . \\
5 . \\
9 .\end{array}$ & & & & & & & \\
\hline $\begin{array}{l}R B-86 \\
R B-87 \\
R B-88 \\
R B-89 \\
R B-90\end{array}$ & $\begin{array}{r}18.66 \\
4.73 E 10 \mathrm{Y} \\
17.8 \mathrm{M} \\
15.44 \mathrm{M} \\
157 \mathrm{~S}\end{array}$ & $\begin{array}{l}0.0 \\
6.44 E A \\
2.07 E E \\
2.30 E \%\end{array}$ & & & & & & $\begin{array}{l}9.47 E-01 \\
0.0 \\
6.93 E+00 \\
2.19 E+01 \\
2.47 E+01\end{array}$ & & $\begin{array}{l}9.44 E-01 \\
0.0 \\
6.91 E+00 \\
2.20 E+01 \\
2.55 E+01\end{array}$ \\
\hline $\begin{array}{l}R B-904 \\
S R-82 \\
S R-85 \\
S R-854 \\
S R-87 M\end{array}$ & $\begin{array}{rl}258 & S \\
25.0 & \mathrm{D} \\
64.84 & \mathrm{D} \\
67.66 & \mathrm{M} \\
2.805 & \mathrm{H}\end{array}$ & $\begin{array}{l}5.61 E-04 \\
4.78 E+00 \\
2.04 E \cdot 00 \\
2.99 E \cdot 00\end{array}$ & $\begin{array}{l}1.01 E-06 \\
4.54 E+00 \\
1.86 E+00 \\
2.80 E+00\end{array}$ & $\begin{array}{l}3.76 E+01 \\
7.21 E-05 \\
5.06 E+00 \\
2.00 E+00 \\
3.08 E * 00\end{array}$ & $\begin{array}{l}2.11 E-02 \\
2.05 E+00 \\
2.89 E+00 \\
3.88 E+00\end{array}$ & $\begin{array}{l}3.37 E+01 \\
1.05 E-04 \\
4.44 E+00 \\
1.82 E+00 \\
2.73 E+00\end{array}$ & $\begin{array}{l}3.14 E+01 \\
1.11 E-05 \\
3.99 E+00 \\
1.62 E+00 \\
2.45 E+00\end{array}$ & $\begin{array}{l}3.58 E+01 \\
2.13 E-06 \\
4.11 E * 00 \\
1.92 E+00 \\
2.89 E * 00\end{array}$ & $\begin{array}{l}3.32 E+01 \\
3.03 E-04 \\
4.35 E \cdot 00 \\
1.011 E \cdot 00 \\
2.69 E+00\end{array}$ & $\begin{array}{l}3.64 E+01 \\
2.41 E-05 \\
4.71 E+00 \\
1.92 E+00 \\
2.89 E+00\end{array}$ \\
\hline
\end{tabular}




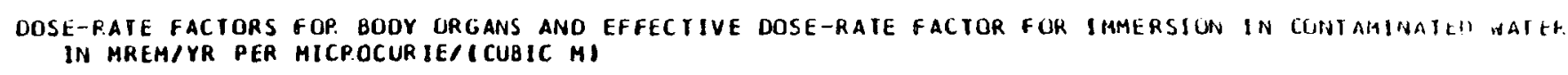

\begin{tabular}{|c|c|c|c|c|c|c|c|c|c|c|}
\hline CLIOE & HALF-LIFE & LIVER & LUNGS & MARROH & $\begin{array}{c}\text { RED } \\
\text { MARRRH }\end{array}$ & ovaries & PANCREAS & SKELETON & SPLEEN & STOMACH \\
\hline $\begin{array}{l}\text { GE }-71 \\
G E-77 \\
A S-72 \\
A S-73 \\
A S-74\end{array}$ & 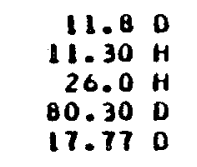 & $\begin{array}{l}8.42 E-01 \\
9.67 E+00 \\
1.64 E+01 \\
3.44 E-02 \\
6.83 E+00\end{array}$ & $\begin{array}{l}6 E-05 \\
4 E+01 \\
15 E+01 \\
35 E-02\end{array}$ & $\begin{array}{l}4.04 E-05 \\
1.20 E+01 \\
1.95 E+01 \\
6.94 E-02 \\
8.31 E+00\end{array}$ & $\begin{array}{l}2.19 E-05 \\
1.05 E+01 \\
1.78 E+01 \\
1.92 E-02 \\
7.48 E+00\end{array}$ & $\begin{array}{l}3.30 E-05 \\
9.08 E+00 \\
1.57 E+01 \\
2.79 E-02 \\
6.59 E+00\end{array}$ & $\begin{array}{l}4.91 E-05 \\
8.35 E+00 \\
1.41 E+01 \\
2.07 E-02 \\
5.88 E+00\end{array}$ & $\begin{array}{l}1.08 E-04 \\
1.22 E+01 \\
1.98 E+01 \\
7.14 E-02 \\
8.44 E+00\end{array}$ & $\begin{array}{l}1.91 E-05 \\
9.45 E+00 \\
1.59 E+01 \\
2.95 E-02 \\
6.66 E+00\end{array}$ & $\begin{array}{l}1.73 E-05 \\
9.53 E+00 \\
1.62 E+01 \\
3.11 E-02 \\
6.14 E+00\end{array}$ \\
\hline $\begin{array}{l}\text { AS-76 } \\
\text { AS-77 } \\
\text { SE-73 } \\
S E-75 \\
S E-79\end{array}$ & $\begin{array}{r}26.32 \mathrm{H} \\
38.8 \mathrm{H} \\
7.15 \mathrm{H} \\
119.78 \mathrm{O} \\
6.5 \mathrm{E} Y\end{array}$ & $\begin{array}{l}3.94 E+00 \\
7.50 E-02 \\
9.56 E+00 \\
3.35 E+00 \\
0.0\end{array}$ & $\begin{array}{l}4.21 E+00 \\
0.11 E-02 \\
1.03 E+01 \\
3.65 E+00 \\
0.0\end{array}$ & $\begin{array}{l}4.66 E+00 \\
1.03 E-01 \\
1.23 E+01 \\
4.95 E+00 \\
0.0\end{array}$ & $\begin{array}{l}4.29 E+00 \\
8.21 E-02 \\
1.04 E+01 \\
3.61 E+00 \\
0.0\end{array}$ & $\begin{array}{l}3.78 E+00 \\
6.87 E-02 \\
9.04 E+00 \\
3.00 E+00 \\
0.0\end{array}$ & $\begin{array}{l}3.40 E+00 \\
6.43 E-02 \\
8.16 E+00 \\
2.85 E+00 \\
0.0\end{array}$ & $\begin{array}{l}4.73 E+00 \\
1.05 E-01 \\
1.20 E+01 \\
5.06 E+00 \\
0.0\end{array}$ & $\begin{array}{l}3.33 E+00 \\
7.40 E-02 \\
9.33 E+00 \\
3.32 E+00 \\
0.0\end{array}$ & $\begin{array}{l}\text { 3. } 8 B E+00 \\
7.40 E-C 2 \\
9.41 E+00 \\
3.31 E+00 \\
0.0\end{array}$ \\
\hline $\begin{array}{l}B R-77 \\
8 R-80 \\
B R-8 O M \\
8 R-82 \\
B R-83\end{array}$ & $\begin{array}{r}57.04 \mathrm{H} \\
17.4 \mathrm{M} \\
4.42 \mathrm{H} \\
35.30 \mathrm{H} \\
2.39 \mathrm{H}\end{array}$ & $\begin{array}{l}2.79 E+00 \\
6.69 E-01 \\
4.37 E-02 \\
2.45 E+01 \\
6.61 E-02\end{array}$ & $\begin{array}{l}3.00 E+00 \\
7.17 E-01 \\
5.00 E-02 \\
2.61 E .01 \\
7.09 E-02\end{array}$ & $\begin{array}{l}3.57 E+00 \\
8.09 E-01 \\
8.71 E-02 \\
2.84 E+01 \\
8.12 E-02\end{array}$ & $\begin{array}{l}3.00 E+00 \\
7.32 E-01 \\
2.01 E-02 \\
2.65 E+01 \\
7.25 E-02\end{array}$ & $\begin{array}{l}2.63 E+00 \\
6.47 E-01 \\
3.63 E-02 \\
2.36 E+01 \\
6.36 E-02\end{array}$ & $\begin{array}{l}2.40 E \bullet 00 \\
5.77 E-01 \\
2.41 E-02 \\
2.11 E+01 \\
5.69 E-02\end{array}$ & $\begin{array}{l}3.63 E+00 \\
8.22 E-01 \\
8.98 E-02 \\
2.89 E+01 \\
8.25 E-02\end{array}$ & $\begin{array}{l}2.73 E+00 \\
6.52 E-01 \\
3.43 E-02 \\
2.37 E+01 \\
6.45 E-02\end{array}$ & $\begin{array}{l}2.75 E+00 \\
6.61 E-01 \\
3.80 E-02 \\
2.41 E+01 \\
6.52 E-02\end{array}$ \\
\hline $\begin{array}{l}B R-84 \\
B R-85 \\
K R-79 \\
K R-81 \\
K R-834\end{array}$ & $\begin{array}{r}31.80 \mathrm{M} \\
172 \mathrm{~S} \\
35.04 \mathrm{H} \\
2.1 E S \mathrm{~V} \\
1.83 \mathrm{H}\end{array}$ & $\begin{array}{l}1.86 E+01 \\
6.26 E-01 \\
2.23 E+00 \\
8.58 E-02 \\
3.50 E-05\end{array}$ & & $\begin{array}{l}2.04 \mathrm{E}+01 \\
7.16 \mathrm{E}-01 \\
2.03 \mathrm{E} \cdot 00 \\
1.20 \mathrm{E}-01 \\
1.65 \mathrm{E}-04\end{array}$ & & & & & & $\begin{array}{l}1.84 E+01 \\
6.18 E-01 \\
2.20 E+00 \\
8.49 E-02 \\
6.74 E-05\end{array}$ \\
\hline $\begin{array}{l}K R-85 \\
K R-85 M \\
K R-87 \\
K R-88 \\
K R-89\end{array}$ & $\begin{array}{r}10.72 \mathrm{Y} \\
4.48 \mathrm{H} \\
76.3 \mathrm{H} \\
2.84 \mathrm{H} \\
3.16 \mathrm{H}\end{array}$ & $\begin{array}{l}1.97 E-02 \\
1.37 E+00 \\
8.10 E+00 \\
2.08 E+01 \\
1.85 E+01\end{array}$ & $\begin{array}{l}1.50 E+00 \\
8.54 E+00 \\
2.18 E+01 \\
1.95 E .01\end{array}$ & & & & & & & $\begin{array}{l}1.95 E-02 \\
1.36 E+00 \\
1.97 E+00 \\
2.03 E+01 \\
1.82 E+01\end{array}$ \\
\hline $\begin{array}{l}K R-90 \\
R \theta-81 \\
R \theta-82 \\
R \theta-83 \\
R \theta-84\end{array}$ & 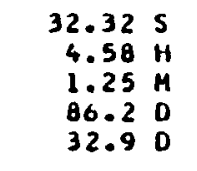 & $\begin{array}{l}1.21 E * 01 \\
5.33 E \bullet 00 \\
9.73 E * 00 \\
4.42 E * 00 \\
8.28 E * 00\end{array}$ & $\begin{array}{l}1.29 E+01 \\
5.74 E \cdot 00 \\
1.04 E+01 \\
4.74 E+00 \\
8.84 E+00\end{array}$ & $\begin{array}{l}1.40 E+01 \\
6.92 E+00 \\
1.19 E+01 \\
5.43 E+00 \\
9.72 E+00\end{array}$ & $\begin{array}{l}\text { 1. } 30 E * 01 \\
5.83 E+00 \\
1.07 E \bullet 01 \\
4.85 E+00 \\
8.99 E+00\end{array}$ & & $\begin{array}{l}1.05 E+01 \\
4.58 E+00 \\
8.37 E+00 \\
3.81 E * 00 \\
7.14 E+00\end{array}$ & & & $\begin{array}{l}1.19 E+01 \\
5.26 E+00 \\
9.60 E+00 \\
4.36 E+00 \\
8.18 E+00\end{array}$ \\
\hline $\begin{array}{l}R B-86 \\
R B-87 \\
R B-8 B \\
R B-89 \\
R B-90\end{array}$ & 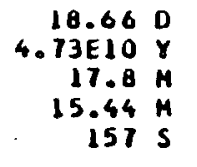 & $\begin{array}{l}8.95 E-01 \\
0.0 \\
6.56 E+00 \\
2.07 E+01 \\
2.34 E+01\end{array}$ & $\begin{array}{l}9.5 \mid E-01 \\
0.0 \\
6.91 E \cdot 00 \\
2.19 E+01 \\
2.43 E+01\end{array}$ & $\begin{array}{l}1.01 E+00 \\
0.0 \\
1.16 E+00 \\
2.29 E+01 \\
2.52 E+01\end{array}$ & $\begin{array}{l}9.61 E-01 \\
0.0 \\
7.01 E+00 \\
2.22 E+01 \\
2.54 E+01\end{array}$ & $\begin{array}{l}8.62 E-01 \\
0.0 \\
6.05 E+00 \\
1.94 E+01 \\
2.09 E+01\end{array}$ & $\begin{array}{l}7.74 E-01 \\
0.0 \\
5.71 E+00 \\
1.80 E+01 \\
2.09 E+01\end{array}$ & $\begin{array}{l}1.03 E+00 \\
0.0 \\
7.29 E+00 \\
2.33 E+01 \\
2.56 E \cdot 01\end{array}$ & $\begin{array}{l}0.65 E-01 \\
0.0 \\
6.31 E+00 \\
2.00 E+01 \\
2.26 E+01\end{array}$ & $\begin{array}{l}8.81 E-01 \\
0.0 \\
6.43 E+00 \\
2.04 E+01 \\
2.35 E+01\end{array}$ \\
\hline $\begin{array}{l}R B-904 \\
S R-82 \\
S R-85 \\
S R-85 M \\
S R-87 M\end{array}$ & $\begin{array}{rr}258 & 5 \\
25.0 & 0 \\
64.84 & 0 \\
67.66 & M \\
2.805 & H\end{array}$ & $\begin{array}{l}3.39 E+01 \\
3.54 E-05 \\
4.51 E+00 \\
1.86 E+00 \\
2.78 E+00\end{array}$ & $\begin{array}{l}3.56 E+01 \\
5.38 E-04 \\
4.85 E+00 \\
2.02 E \cdot 00 \\
2.99 E \cdot 00\end{array}$ & $\begin{array}{l}3.72 E+01 \\
5.35 E-04 \\
5.57 E+00 \\
2.74 E+00 \\
3.61 E+00\end{array}$ & $\begin{array}{l}3.65 E+01 \\
1.01 E-04 \\
4.96 E+00 \\
2.03 E+00 \\
3.09 E+00\end{array}$ & $\begin{array}{l}3.10 E+01 \\
2.88 E-04 \\
4.34 E+00 \\
1.65 E+00 \\
2.61 E+00\end{array}$ & $\begin{array}{l}2.97 E+01 \\
3.42 E-04 \\
3.89 E+00 \\
1.59 E+00 \\
2.40 E+00\end{array}$ & $\begin{array}{l}\text { 3. } 77 E \cdot 01 \\
8.79 E-04 \\
5.66 E+00 \\
2.80 E+00 \\
3.67 E+00\end{array}$ & $\begin{array}{l}3.27 E+01 \\
2.05 E-04 \\
4.41 E+00 \\
1.85 E+00 \\
2.73 E+00\end{array}$ & $\begin{array}{l}\text { 3. } 36 E+01 \\
2.10 E-04 \\
4.45 E+00 \\
1.83 E+00 \\
3.74 E+00\end{array}$ \\
\hline
\end{tabular}


DOSE-RATE FACTORS FOR BODY ORGANS ANO EFFECTIVE OOSE-RATE FACTOR FOR IMMERSION IN CONTAMINATEU WATEF

\begin{tabular}{|c|c|c|c|c|c|c|c|c|c|}
\hline CIOE & HALF-LIFE & TESTES & THYMUS & THYROID & TERUS & FECTIVE & $\begin{array}{c}\text { SKIN } \\
\text { I PHOTONI }\end{array}$ & $\begin{array}{l}\text { SKIN } \\
\text { IELECTRON }\end{array}$ & $\begin{array}{l}\text { SKIN } \\
\text { ITOTALI }\end{array}$ \\
\hline $\begin{array}{l}\text { GE-71 } \\
G E-77 \\
A S-72 \\
A S-13 \\
A S-74\end{array}$ & $\begin{array}{rl}11.8 & 0 \\
11.30 & H \\
26.0 & H \\
80.30 & 0 \\
17.77 & 0\end{array}$ & $\begin{array}{l}6.09 E-04 \\
1.38 E+01 \\
2.33 E+01 \\
6.82 E-02 \\
9.80 E+00\end{array}$ & $\begin{array}{l}.52 E-08 \\
07 E+01 \\
00 E+01 \\
.80 E+01 \\
.81 E-02 \\
.50 E+00\end{array}$ & & & $\begin{array}{l}1.16 E-03 \\
1.19 E+01 \\
1.99 E+01 \\
5.68 E-02 \\
8.38 E+00\end{array}$ & $\begin{array}{l}1.39 E-02 \\
1.5 \mathrm{TE}+01 \\
2.67 E+01 \\
1.14 E-01 \\
1.12 E .01\end{array}$ & $\begin{array}{l}0.0 \\
5.18 E+00 \\
8.74 E+00 \\
0.0 \\
2.00 E+00\end{array}$ & $\begin{array}{l}1.39 E-02 \\
2.09 E+01 \\
3.55 E+01 \\
1.14 E-01 \\
1.32 E+01\end{array}$ \\
\hline $\begin{array}{l}A S-76 \\
A S-77 \\
S E-73 \\
S E-75 \\
S E-79\end{array}$ & 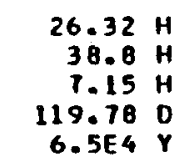 & $\begin{array}{l}5.59 E+00 \\
1.10 E-01 \\
1.39 E+01 \\
5.00 E+00 \\
0.0\end{array}$ & $\begin{array}{l}4.34 E \cdot 00 \\
8.36 E-02 \\
1.05 E \cdot 01 \\
3.80 E \cdot 00 \\
0.0\end{array}$ & $\begin{array}{l}5.23 E * 00 \\
1.04 E-01 \\
1.30 E+01 \\
4.78 E+00 \\
0.0\end{array}$ & $\begin{array}{l}3.46 E+00 \\
6.60 E-02 \\
8.34 E+00 \\
2.95 E+00 \\
0.0\end{array}$ & $\begin{array}{l}4.79 E+00 \\
9.56 E-02 \\
1.19 E+01 \\
4.37 E+00 \\
0.0\end{array}$ & $\begin{array}{l}6.42 E+00 \\
1.23 E-01 \\
1.5 B E+01 \\
5.53 E+00 \\
0.0\end{array}$ & $\begin{array}{l}8.99 E+00 \\
1.41 E+00 \\
2.93 E+00 \\
1.79 E-02 \\
4.12 E-02\end{array}$ & $\begin{array}{l}1.54 E+01 \\
1.54 E+00 \\
1.88 E+01 \\
5.55 E+00 \\
4.12 E-02\end{array}$ \\
\hline $\begin{array}{l}B R-77 \\
8 R-80 \\
B R-80 M \\
B R-82 \\
B R-B 3\end{array}$ & $\begin{array}{r}57.04 \mathrm{H} \\
17.4 \mathrm{H} \\
4.42 \mathrm{H} \\
35.30 \mathrm{H} \\
2.39 \mathrm{H}\end{array}$ & $\begin{array}{l}4.03 E+00 \\
9.59 E-01 \\
1.23 E-01 \\
3.46 E-01 \\
9.51 E-02\end{array}$ & $\begin{array}{l}3.08 E+00 \\
7.37 E-01 \\
7.03 E-02 \\
2.72 E+01 \\
7.22 E-02\end{array}$ & $\begin{array}{l}3.7 \theta E+00 \\
8.96 E-01 \\
9.63 E-02 \\
3.26 E+01 \\
8.62 E-02\end{array}$ & $\begin{array}{l}2.44 E+00 \\
5.83 E-01 \\
2.60 E-02 \\
2.14 E+01 \\
5.77 E-02\end{array}$ & $\begin{array}{l}3.47 E+00 \\
6.20 E-01 \\
9.27 E-02 \\
2.96 E+01 \\
0.13 E-02\end{array}$ & $\begin{array}{l}4.60 E \cdot 00 \\
1.10 E+00 \\
2.11 E-01 \\
3.98 E+01 \\
1.09 E-01\end{array}$ & $\begin{array}{l}1.36 E-02 \\
5.90 E+00 \\
0.0 \\
6.29 E-01 \\
2.22 E+00\end{array}$ & $\begin{array}{l}4.62 E+00 \\
1.00 E+00 \\
2.11 E-01 \\
4.04 E+01 \\
2.33 E+00\end{array}$ \\
\hline $\begin{array}{l}8 R-84 \\
B R-85 \\
K R-79 \\
K R-81 \\
K R-839\end{array}$ & 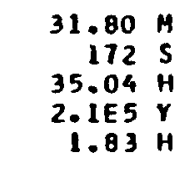 & $\begin{array}{l}2.56 E+01 \\
8.80 E-01 \\
3.22 E+00 \\
1.28 E-01 \\
9.67 E-04\end{array}$ & $\begin{array}{l}2.03 E+01 \\
6.99 E-01 \\
2.46 E+00 \\
9.54 E-02 \\
4.86 E-05\end{array}$ & $\begin{array}{l}2.36 E+01 \\
8.35 E-01 \\
3.01 E+00 \\
1.20 E-01 \\
1.92 E-04\end{array}$ & & & $\begin{array}{l}2.87 E * 01 \\
1.02 E * 00 \\
3.69 E * 00 \\
1.67 E-01 \\
1.09 E-02\end{array}$ & $\begin{array}{l}1.08 E+01 \\
0.39 E+00 \\
1.23 E-01 \\
0.0 \\
0.0\end{array}$ & $\begin{array}{l}3.95 E+01 \\
9.41 E \cdot 00 \\
3.01 E+00 \\
1.67 E-01 \\
1.09 E-02\end{array}$ \\
\hline $\begin{array}{l}K R-85 \\
K R-85.4 \\
K R-87 \\
K R-88 \\
K R-89\end{array}$ & $\begin{array}{r}10.72 \mathrm{Y} \\
4.48 \mathrm{H} \\
76.3 \mathrm{M} \\
2.84 \mathrm{H} \\
3.16 \mathrm{M}\end{array}$ & $\begin{array}{l}2.84 E-02 \\
2.07 E * 00 \\
1.12 E \bullet 01 \\
2.83 E+01 \\
2.56 E * 01\end{array}$ & $\begin{array}{l}2.15 E-02 \\
1.59 E+00 \\
8.74 E+00 \\
2.23 E+01 \\
2.01 E+01\end{array}$ & $\begin{array}{l}2.63 E-02 \\
2.01 E+00 \\
1.03 E+01 \\
2.61 E+01 \\
2.37 E+01\end{array}$ & $\begin{array}{l}1.12 E-02 \\
1.21 E+00 \\
7.24 E+00 \\
1.87 E+01 \\
1.64 E+01\end{array}$ & & $\begin{array}{l}3.25 E-02 \\
2.25 E+00 \\
1.26 E \cdot 01 \\
3.19 E \cdot 01 \\
2 . \theta 8 E+01\end{array}$ & & $\begin{array}{l}1.69 E+00 \\
3.95 E+00 \\
2.40 E+01 \\
3.46 E+01 \\
4.06 E+01\end{array}$ \\
\hline $\begin{array}{l}K R-90 \\
R B-81 \\
R B-82 \\
R B-83 \\
R B-84\end{array}$ & 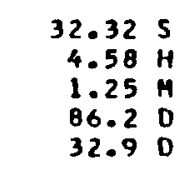 & $\begin{array}{l}1.70 E * 01 \\
7.74 E * 00 \\
1.40 E * 01 \\
6.36 E * 00 \\
1.17 E * 01\end{array}$ & $\begin{array}{l}1.34 E * 01 \\
5.88 E+00 \\
1.06 E+01 \\
4.83 E+00 \\
9.21 E * 00\end{array}$ & $\begin{array}{l}1.60 E+01 \\
7.24 E+00 \\
1.30 E+01 \\
5.90 E+00 \\
1.11 E+01\end{array}$ & $\begin{array}{l}1.08 E+01 \\
4.67 E+00 \\
8.50 E+00 \\
3.06 E+00 \\
7.22 E+00\end{array}$ & & $\begin{array}{l}1.94 E+01 \\
8.79 E+00 \\
1.60 E+01 \\
1.31 E+00 \\
1.36 E+01\end{array}$ & $\begin{array}{l}1.12 E+01 \\
1.39 E+00 \\
1.21 E+01 \\
0.0 \\
1.26 E \bullet 00\end{array}$ & $\begin{array}{l}3.06 E+01 \\
1.02 E+01 \\
2.81 E+01 \\
1.31 E+00 \\
1.40 E+01\end{array}$ \\
\hline $\begin{array}{l}R \theta-86 \\
R B-87 \\
R B-88 \\
R B-89 \\
R B-90\end{array}$ & $\begin{array}{r}18.66 \\
4.73 \mathrm{E10} \\
17.8 \mathrm{Y} \\
15.44 \mathrm{M} \\
157 \mathrm{~S}\end{array}$ & $\begin{array}{l}1.25 E \cdot 00 \\
0.0 \\
0.99 E \bullet 00 \\
2.86 E * 01 \\
3.22 E+01\end{array}$ & $\begin{array}{l}1.01 E+00 \\
0.0 \\
1.12 E+00 \\
2.28 E+01 \\
2.55 E+01\end{array}$ & $\begin{array}{l}1.20 E+00 \\
0.0 \\
8.35 E+00 \\
2.68 E+01 \\
2.91 E+01\end{array}$ & $\begin{array}{l}7.04 E-01 \\
0.0 \\
5.88 E+00 \\
1.84 E+01 \\
2.07 E+01\end{array}$ & $\begin{array}{l}1.07 E+00 \\
0.0 \\
1.74 E+00 \\
2.46 E+01 \\
2.77 E+01\end{array}$ & $\begin{array}{l}1.45 E \cdot 00 \\
0.0 \\
1.02 E+01 \\
3.26 E+01 \\
3.50 E \times 01\end{array}$ & $\begin{array}{l}5.42 E \cdot 00 \\
1.96 E-01 \\
1.85 E+01 \\
8.59 E+00 \\
1.75 E+01\end{array}$ & $\begin{array}{l}6.86 E+00 \\
1.96 E-01 \\
2.87 E+01 \\
4.12 E+01 \\
5.25 E+01\end{array}$ \\
\hline $\begin{array}{l}\text { RB-90M } \\
\text { SR-82 } \\
\text { SR-85 } \\
\text { SR-85M } \\
\text { SR-87M }\end{array}$ & $\begin{array}{rr}258 & S \\
25.0 & 0 \\
64.84 & 0 \\
67.66 & M \\
2.805 & H\end{array}$ & $\begin{array}{l}4.67 E+01 \\
3.82 E-03 \\
6.51 E+00 \\
2.76 E+00 \\
4.04 E+00\end{array}$ & $\begin{array}{l}3.69 E+01 \\
7.10 E-06 \\
4.92 E+00 \\
2.10 E+00 \\
3.04 E+00\end{array}$ & $\begin{array}{l}4.30 E+01 \\
6.29 E-04 \\
6.02 E+00 \\
2.64 E+00 \\
3.76 E+00\end{array}$ & $\begin{array}{l}3.01 E+01 \\
2.00 E-07 \\
3.94 E+00 \\
1.64 E+00 \\
2.44 E+00\end{array}$ & $\begin{array}{l}4.02 E+01 \\
4.38 E-03 \\
5.56 E+00 \\
2.42 E+00 \\
3.48 E * 00\end{array}$ & $\begin{array}{l}5.23 E+01 \\
3.74 E-02 \\
7.47 E+00 \\
3.05 E+00 \\
4.58 E+00\end{array}$ & $\begin{array}{l}1.20 E * 01 \\
0.0 \\
2.38 E-02 \\
3.24 E-02 \\
4.69 E-01\end{array}$ & $\begin{array}{l}6.44 E \times 01 \\
3.74 E-02 \\
7.50 E+00 \\
3.08 E+00 \\
5.05 E \div 00\end{array}$ \\
\hline
\end{tabular}




\begin{tabular}{|c|c|c|c|c|c|c|c|c|c|c|}
\hline IOE & ILF-LIFE & DRENALS & ADDER & BP.AIN & EAST & HE AR T & $\begin{array}{l}\text { SMALL } \\
\text { INTESTINE }\end{array}$ & $\begin{array}{c}\text { UPPER } \\
\text { LARGE } \\
\text { INIESTINE }\end{array}$ & $\begin{array}{l}\text { LOWEF } \\
\text { LARGE } \\
\text { INIESTINE }\end{array}$ & IONEYS \\
\hline $\begin{array}{l}-89 \\
-90 \\
-91 \\
-92 \\
-93\end{array}$ & $\begin{array}{rl}50.55 & \mathrm{D} \\
28.6 \mathrm{Y} & \\
9.5 \mathrm{H} \\
2.71 \mathrm{H} \\
7.3 \mathrm{M}\end{array}$ & $\begin{array}{l}1.33 E-03 \\
0.0 \\
0.65 E \bullet 00 \\
1.27 E+01 \\
2.18 E+01\end{array}$ & $\begin{array}{l}29 E-03 \\
0 \\
46 E+00 \\
27 E \bullet 01 \\
.15 E+01\end{array}$ & $\begin{array}{l}46 E-03 \\
0 \\
26 E+00 \\
43 E+01 \\
41 E+01\end{array}$ & $\begin{array}{l}63 E-03 \\
0 \\
16 E+00 \\
58 E+01 \\
73 E+01\end{array}$ & $\begin{array}{l}1.28 E-03 \\
0.0 \\
6.38 E+00 \\
1.25 E+01 \\
2.12 E+01\end{array}$ & $\begin{array}{l}1.16 E-03 \\
0.0 \\
5.79 E+00 \\
1.16 E+01 \\
1.94 E+01\end{array}$ & $\begin{array}{l}1.36 E-03 \\
0.0 \\
6.79 E+00 \\
1.33 E+01 \\
2.26 E+01\end{array}$ & $\begin{array}{l}1.25 E-03 \\
0.0 \\
6.24 E+00 \\
1.23 E+01 \\
2.08 E+01\end{array}$ & $\begin{array}{l}1.36 E-03 \\
0.0 \\
6.77 E+00 \\
1.33 E+01 \\
2.26 E+01\end{array}$ \\
\hline $\begin{array}{l}86 \\
87 \\
88 \\
90 \\
904\end{array}$ & $\begin{array}{r}14.74 \mathrm{H} \\
80.3 \mathrm{H} \\
106.60 \mathrm{D} \\
64.1 \mathrm{H} \\
3.19 \mathrm{H}\end{array}$ & $\begin{array}{l}3.50 E+01 \\
4.27 E+00 \\
2.68 E+01 \\
0.0 \\
5.95 E+00\end{array}$ & 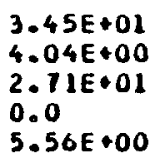 & $\begin{array}{l}.87 E+01 \\
.50 E+00 \\
.04 E+01 \\
.0 \\
.11 E+00\end{array}$ & $\begin{array}{l}37 E+01 \\
43 E+00 \\
3 B E+01 \\
0 \\
87 E+00\end{array}$ & $\begin{array}{l}3.41 E+01 \\
3.95 E+00 \\
2.69 E+01 \\
0.0 \\
5.43 E+00\end{array}$ & $\begin{array}{l}.12 E+01 \\
.55 E \cdot 00 \\
.49 E+01 \\
.0 \\
.87 E+00\end{array}$ & $\begin{array}{l}.63 E+01 \\
.19 E+00 \\
.86 E+01 \\
.0 \\
.76 E+00\end{array}$ & $\begin{array}{l}3.35 E+01 \\
3.87 E+00 \\
2.64 E+01 \\
0.0 \\
5.35 E+00\end{array}$ & $\begin{array}{l}\text { 3. } 63 E+01 \\
4.19 E+00 \\
2.87 E+01 \\
0.0 \\
5.75 E+00\end{array}$ \\
\hline $\begin{array}{l}-91 \\
-91 M \\
-92 \\
-93 \\
2-86\end{array}$ & $\begin{array}{rr}58.51 & 0 \\
49.71 & M \\
3.54 & H \\
10.1 & H \\
16.5 & M\end{array}$ & $\begin{array}{l}3.45 E-02 \\
5.00 E+00 \\
2.43 E+00 \\
8.79 E-01 \\
2.57 E+00\end{array}$ & $\begin{array}{l}.41 E-02 \\
.76 E+00 \\
38 E+00 \\
.69 E-01 \\
.36 E+00\end{array}$ & $\begin{array}{l}.85 E-02 \\
.31 E+00 \\
.68 E+00 \\
.69 E-01 \\
.55 E+00\end{array}$ & & & & & & $\begin{array}{l}3.59 \mathrm{E}-02 \\
4.94 \mathrm{E}+00 \\
2.50 \mathrm{E}+00 \\
9.16 \mathrm{E}-01 \\
2.44 \mathrm{E}+00\end{array}$ \\
\hline $\begin{array}{l}2 R-88 \\
2 R-89 \\
2 R-93 \\
2 R-95 \\
2 R-97\end{array}$ & $\begin{array}{r}83.4 \mathrm{D} \\
78.43 \mathrm{H} \\
1.53 E 6 \mathrm{~V} \\
64.02 \mathrm{O} \\
16.90 \mathrm{H}\end{array}$ & $\begin{array}{l}3.58 E+00 \\
1.12 E+01 \\
0.0 \\
7.07 E+00 \\
1.13 E+00\end{array}$ & $\begin{array}{l}.35 E \cdot 00 \\
.08 E+01 \\
.0 \\
.80 E+00 \\
.70 E+00\end{array}$ & $\begin{array}{l}.69 E+00 \\
.21 E+01 \\
.0 \\
.04 E+00 \\
.90 E+00\end{array}$ & $\begin{array}{l}.66 E * 00 \\
37 E+01 \\
0 \\
07 E+00 \\
677 E * 00 \\
177 E 0\end{array}$ & $\begin{array}{l}3.27 E+00 \\
1.07 E+01 \\
0.0 \\
6.69 E+00 \\
1.67 E+00\end{array}$ & $\begin{array}{l}3 E+00 \\
6 E+00 \\
5 E+00\end{array}$ & $\begin{array}{l}3.46 E+00 \\
1.13 E+01 \\
0.0 \\
7.12 E+00 \\
1.78 E+00\end{array}$ & $\begin{array}{l}3.22 E+00 \\
1.04 E+01 \\
0.0 \\
6.55 E+00 \\
1.64 E+00\end{array}$ & $\begin{array}{l}3.46 E+00 \\
1.13 E+01 \\
0.0 \\
1.10 E+00 \\
1.78 E+00\end{array}$ \\
\hline $\begin{array}{l}-90 \\
-90 \\
-91 \\
-914 \\
-92 \\
-92.4\end{array}$ & 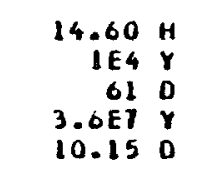 & $\begin{array}{l}1.71 E-02 \\
4.09 E-01 \\
1.44 E+01 \\
9.38 E+00\end{array}$ & $\begin{array}{l}26 E+01 \\
49 E-02 \\
04 E-01 \\
.39 E+01 \\
12 E+00\end{array}$ & $\begin{array}{l}.76 E+01 \\
.69 E-02 \\
55 E-01 \\
.56 E+01 \\
.03 E+01\end{array}$ & & & & & & $\begin{array}{l}4.53 E+01 \\
1.58 E-02 \\
4.24 \mathrm{E}-01 \\
1.45 \mathrm{E}+01 \\
9.57 \mathrm{E}+00\end{array}$ \\
\hline $\begin{array}{l}-934 \\
-934 \\
-94 M \\
-95 \\
-954\end{array}$ & 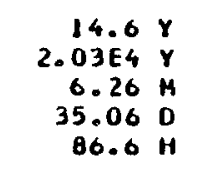 & $\begin{array}{l}4.15 E-02 \\
7.3 \mathrm{BE}+00 \\
5.62 \mathrm{E}-01\end{array}$ & $\begin{array}{l}1.95 E-05 \\
1.47 E+01 \\
3.91 E-02 \\
1.10 E+00 \\
5.12 E-01\end{array}$ & & & & & & & $\begin{array}{l}1.05 E-04 \\
1.53 E+01 \\
4.13 E-02 \\
7.42 E+00 \\
5.28 E-01\end{array}$ \\
\hline $\begin{array}{l}-96 \\
-97 \\
-97 M \\
-91 \\
-93\end{array}$ & $\begin{array}{r}23.35 \mathrm{H} \\
72.1 \mathrm{H} \\
60 \mathrm{~S}\end{array}$ & $\begin{array}{l}6.33 E+00 \\
7.01 E+00 \\
9.17 E+00 \\
1.79 E-03\end{array}$ & $\begin{array}{l}30 E+01 \\
06 E+00 \\
74 E+00 \\
72 E+00 \\
09 E-04\end{array}$ & & & & & & & $\begin{array}{l}2.40 E+01 \\
6.32 E+00 \\
7.04 E+00 \\
9.05 E+00 \\
5.90 E-04\end{array}$ \\
\hline $\begin{array}{l}-99 \\
-101 \\
-105 \\
-95 \\
-954 \\
-96\end{array}$ & $\begin{array}{r}14.61 \mathrm{M} \\
20.0 \mathrm{H} \\
61 \mathrm{O} \\
4.28 \mathrm{D}\end{array}$ & $\begin{array}{l}1.45 E+01 \\
7.58 E+00 \\
6.36 E+00 \\
2.42 E+01\end{array}$ & $\begin{array}{l}1.44 E+01 \\
7.30 E+00 \\
6.06 E+00 \\
2.34 E+01\end{array}$ & $\begin{array}{l}1.61 E+01 \\
0.20 E+00 \\
6.74 E+00 \\
2.63 E+01\end{array}$ & $\begin{array}{l}8.13 E+00 \\
2.96 E+01\end{array}$ & $\begin{array}{l}1.42 E+01 \\
7.20 E+00 \\
5.95 E * 00 \\
2.30 E+01\end{array}$ & $2.09 E+01$ & $\begin{array}{l}6.33 E+00 \\
2.45 E+01\end{array}$ & $\begin{array}{l}1.39 E+01 \\
1.04 E+00 \\
5.84 E+00 \\
2.25 E+01\end{array}$ & $\begin{array}{l}1.51 E+00 \\
3.63 E+00 \\
6.31 E+00 \\
2.44 E+01\end{array}$ \\
\hline
\end{tabular}


DOSE-PATE FACIORS FOR BODY ORGANS AND EFFECTIVE DOSE-RATE FACTOR FUR IMMERSION IN CONTAMIHATE') HAI I.
IV MREM/YR PER MICROCURIE/(CUBIC MI

\begin{tabular}{|c|c|c|c|c|c|c|c|c|c|c|}
\hline NUCLIOE & HALF-LIFE & LIVER & LUNGS & MARROH & $\begin{array}{c}\text { REO } \\
\text { MARRROH }\end{array}$ & OVARIES & PANCREAS & SKELETON & SPLEEN & $5 \mathrm{TIMACH}$ \\
\hline $\begin{array}{l}\text { SR-89 } \\
\text { SR-90 } \\
\text { SR-91 } \\
\text { SR-92 } \\
\text { SR-93 }\end{array}$ & $\begin{array}{r}50.55 \mathrm{O} \\
28.6 \mathrm{Y} \\
9.5 \mathrm{H} \\
2.71 \mathrm{H} \\
7.3 \mathrm{M}\end{array}$ & $\begin{array}{l}1.29 E-03 \\
0.0 \\
6.42 E+00 \\
1.26 E \cdot 01 \\
2.14 E \cdot 01\end{array}$ & $\begin{array}{l}1.37 E-03 \\
0.0 \\
6.85 E+00 \\
1.34 E+01 \\
2.28 E+01\end{array}$ & $\begin{array}{l}1.47 E-03 \\
0.0 \\
7.40 E \bullet 00 \\
1.40 E+01 \\
2.46 E+01\end{array}$ & $\begin{array}{l}1.39 E-03 \\
0.0 \\
6.94 E+00 \\
1.35 E+01 \\
2.31 E+04\end{array}$ & $\begin{array}{l}1.25 E-03 \\
0.0 \\
6.21 \mathrm{E}+00 \\
1.20 \mathrm{E}+01 \\
2.03 \mathrm{E}+01\end{array}$ & $\begin{array}{l}1.11 E-03 \\
0.0 \\
5.55 E+00 \\
1.10 E \cdot 01 \\
1.86 E \cdot 01\end{array}$ & $\begin{array}{l}1.50 E-03 \\
0.0 \\
7.53 E+00 \\
1.43 E \cdot 01 \\
2.50 E+01\end{array}$ & $\begin{array}{l}1.25 E-03 \\
0.0 \\
6.23 E \cdot 00 \\
1.22 E \cdot 01 \\
2.07 E \cdot 01\end{array}$ & $\begin{array}{l}1.27 E-03 \\
0.0 \\
6.34 E+00 \\
1.23 E \cdot 01 \\
2.11 E+01\end{array}$ \\
\hline $\begin{array}{l}\gamma-86 \\
\gamma-87 \\
\gamma-88 \\
\gamma-90 \\
\gamma-904\end{array}$ & $\begin{array}{r}14.74 \mathrm{H} \\
80.3 \mathrm{H} \\
106.60 \mathrm{O} \\
64.1 \mathrm{H} \\
3.19 \mathrm{H}\end{array}$ & $\begin{array}{l}3.44 E+01 \\
4.02 E+00 \\
2.71 E+01 \\
0.0 \\
5.54 E+00\end{array}$ & $\begin{array}{l}3.65 E+01 \\
4.32 E+00 \\
2.66 E+01 \\
0.0 \\
5.97 E+00\end{array}$ & $\begin{array}{l}3.92 E+01 \\
5.01 E+00 \\
2.99 E+01 \\
0.0 \\
7.35 E \cdot 00\end{array}$ & $\begin{array}{l}3.71 E \cdot 01 \\
4.42 E \cdot 00 \\
2.90 E \cdot 01 \\
0.0 \\
6.07 E+00\end{array}$ & $\begin{array}{l}3.26 E+01 \\
3.85 E+00 \\
2.53 E+01 \\
0.0 \\
5.16 E+00\end{array}$ & $\begin{array}{l}2.98 E+01 \\
3.46 E+00 \\
2.35 E+01 \\
0.0 \\
4.76 E+00\end{array}$ & $\begin{array}{l}3.99 E \bullet 01 \\
5.09 E \bullet 00 \\
3.04 E+01 \\
0.0 \\
7.49 E \cdot 00\end{array}$ & $\begin{array}{l}3.33 E+01 \\
3.93 E+00 \\
2.61 E+01 \\
0.0 \\
5.44 E+00\end{array}$ & $\begin{array}{l}\text { 3. } 38 E \cdot 01 \\
3.97 E+00 \\
2.65 E+01 \\
0.0 \\
5.46 E+00\end{array}$ \\
\hline $\begin{array}{l}r-91 \\
r-91 M \\
r-92 \\
r-93 \\
Z R-86\end{array}$ & 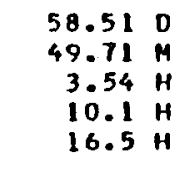 & $\begin{array}{l}3.40 E-02 \\
4.73 E+00 \\
2.37 E+00 \\
0.69 E-01 \\
2.35 E+00\end{array}$ & $\begin{array}{l}3.61 E-02 \\
5.07 E+00 \\
2.52 E \cdot 00 \\
9.22 E-01 \\
2.55 E+00\end{array}$ & $\begin{array}{l}3.80 E-02 \\
5.76 E+00 \\
2.70 E+00 \\
1.01 E+00 \\
3.32 E+00\end{array}$ & $\begin{array}{l}3.64 E-02 \\
5.18 E+00 \\
2.55 E \bullet 00 \\
9.36 E-01 \\
2.59 E+00\end{array}$ & $\begin{array}{l}3.25 E-02 \\
4.56 E+00 \\
2.27 E+00 \\
6.06 E-01 \\
2.12 E+00\end{array}$ & $\begin{array}{l}2.95 E-02 \\
4.07 E+00 \\
2.05 E+00 \\
1.53 E-01 \\
2.02 E+00\end{array}$ & $\begin{array}{l}3.87 E-02 \\
5.85 E+00 \\
2.75 E+00 \\
1.03 E+00 \\
3.39 E+00\end{array}$ & $\begin{array}{l}3.29 E-02 \\
4.61 E+00 \\
2.29 E+00 \\
8.41 E-01 \\
2.33 E+00\end{array}$ & $\begin{array}{l}3.33 E-02 \\
4.67 E+00 \\
2.33 E+00 \\
8.52 E-01 \\
2.32 E+00\end{array}$ \\
\hline $\begin{array}{l}2 R-8 \theta \\
2 R-89 \\
2 R-93 \\
2 R-95 \\
2 R-97\end{array}$ & $\begin{array}{r}83.4 \mathrm{D} \\
78.43 \mathrm{H} \\
1.53 E 6 \mathrm{Y} \\
64.02 \mathrm{D} \\
16.90 \mathrm{H}\end{array}$ & $\begin{array}{l}3.33 E+00 \\
1.07 E+01 \\
0.0 \\
6.16 E+00 \\
1.69 E+00\end{array}$ & $\begin{array}{l}3.58 E+00 \\
1.15 E+01 \\
0.0 \\
7.22 E \cdot 00 \\
1.80 E \cdot C 0\end{array}$ & $\begin{array}{l}4.32 E+00 \\
1.25 E+01 \\
0.0 \\
7.92 E \cdot 00 \\
1.96 E+00\end{array}$ & $\begin{array}{l}3.70 E+00 \\
1.16 E+01 \\
0.0 \\
3.35 E+00 \\
1.83 E+00\end{array}$ & $\begin{array}{l}3.13 E+00 \\
1.04 E+01 \\
0.0 \\
6.57 E+00 \\
1.61 E+00\end{array}$ & $\begin{array}{l}2.87 E+00 \\
9.27 E \cdot 00 \\
0.0 \\
5.82 E+00 \\
1.46 E+00\end{array}$ & $\begin{array}{l}4.39 E+00 \\
1.27 E+01 \\
0.0 \\
8.06 E+00 \\
1.99 E+00\end{array}$ & $\begin{array}{l}3.27 E+00 \\
1.04 E+01 \\
0.0 \\
6.56 E+00 \\
1.64 E+00\end{array}$ & $\begin{array}{l}\text { 3. } 29 E+00 \\
1.06 E+01 \\
0.0 \\
6.68 E+00 \\
1.66 E+00\end{array}$ \\
\hline $\begin{array}{l}N B-90 \\
N B-91 \\
N B-914 \\
N B-92 \\
N B-92 M\end{array}$ & 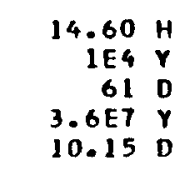 & $\begin{array}{l}4.27 E \cdot 01 \\
1.50 E-02 \\
4.02 E-01 \\
1.38 E+01 \\
9.07 E+00\end{array}$ & $\begin{array}{l}4.51 E+01 \\
1.74 E-02 \\
4.28 E-01 \\
1.48 E+01 \\
9.66 E \cdot 00\end{array}$ & $\begin{array}{l}4.16 E+01 \\
2.01 E-02 \\
4.55 E-01 \\
1.62 E+01 \\
1.04 E+01\end{array}$ & $\begin{array}{l}4.5 T E+01 \\
1.68 E-02 \\
4.30 E-01 \\
1.50 E+01 \\
9.79 E .00\end{array}$ & $\begin{array}{l}3.92 E+01 \\
1.51 E-02 \\
3.05 E-01 \\
1.34 E+01 \\
8.79 E+00\end{array}$ & $\begin{array}{l}3.11 E+01 \\
1.36 E-02 \\
3.49 E-01 \\
1.19 E \cdot 01 \\
7.83 E \times 00\end{array}$ & $\begin{array}{l}4.84 E+01 \\
2.10 E-02 \\
4.64 E-01 \\
1.64 E+01 \\
1.05 E+01\end{array}$ & $\begin{array}{l}4.11 E+01 \\
1.51 E-02 \\
3.90 E-01 \\
1.34 E+01 \\
8.78 E+00\end{array}$ & $\begin{array}{l}4.19 \mathrm{E}+01 \\
1.53 \mathrm{E}-02 \\
3.95 \mathrm{E}-01 \\
1.31 \mathrm{E}+01 \\
8.96 \mathrm{E}+00\end{array}$ \\
\hline $\begin{array}{l}N B-934 \\
\text { NB-94 } \\
\text { NB-94M } \\
\text { NB-95 } \\
\text { NB-954 }\end{array}$ & 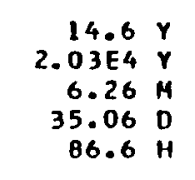 & $\begin{array}{l}6.03 E-05 \\
1.46 E \cdot 01 \\
3.90 E-02 \\
7.06 E \cdot 00 \\
5.10 E-01\end{array}$ & $\begin{array}{l}3.43 E-04 \\
1.56 E+01 \\
4.27 E-02 \\
7.54 E+00 \\
5.55 E-01\end{array}$ & $\begin{array}{l}4.27 E-04 \\
1.70 E \cdot 01 \\
4.64 E-02 \\
8.25 E+00 \\
7.43 E-01\end{array}$ & $\begin{array}{l}1.16 E-04 \\
1.50 E+01 \\
4.23 E-02 \\
7.67 E+00 \\
5.64 E-01\end{array}$ & $\begin{array}{l}2.03 E-04 \\
1.42 E+01 \\
3.84 E-02 \\
6.86 E+00 \\
4.55 E-01\end{array}$ & $\begin{array}{l}1.96 E-04 \\
1.26 E+01 \\
3.42 E-02 \\
6.08 E+00 \\
4.40 E-01\end{array}$ & $\begin{array}{l}5.63 E-04 \\
1.73 E+01 \\
4.77 E-02 \\
6.39 E+00 \\
7.59 E-01\end{array}$ & $\begin{array}{l}1.55 E-04 \\
1.42 E+01 \\
3.82 E-02 \\
6.85 E+00 \\
5.08 E-01\end{array}$ & $\begin{array}{l}1.70 E-04 \\
1.44 E+01 \\
3.90 E-02 \\
6.98 E+00 \\
5.05 E-01\end{array}$ \\
\hline $\begin{array}{l}\text { NB-96 } \\
\text { NB-97 } \\
\text { NB-97M } \\
M 0-91 \\
M D-93\end{array}$ & 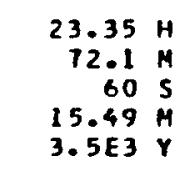 & $\begin{array}{l}2.29 E+01 \\
6.03 E+00 \\
6.69 E+00 \\
8.67 E+00 \\
3.38 E-04\end{array}$ & $\begin{array}{l}2.44 E+01 \\
6.45 E+00 \\
1.16 E+00 \\
9.30 E+00 \\
1.92 E-03\end{array}$ & $\begin{array}{l}2.66 E+01 \\
7.17 E+00 \\
1.05 E \bullet 00 \\
1.07 E+01 \\
2.39 E-03\end{array}$ & $\begin{array}{l}2.48 E+01 \\
6.57 E+00 \\
1.28 E+00 \\
9.52 E+00 \\
6.48 E-04\end{array}$ & $\begin{array}{l}2.21 E+01 \\
5.85 E+00 \\
6.51 E+00 \\
8.32 E+00 \\
1.14 E-03\end{array}$ & $\begin{array}{l}1.97 E \cdot 01 \\
5.19 E \times 00 \\
5.77 E+00 \\
7.46 E * 00 \\
1.10 E-03\end{array}$ & $\begin{array}{l}2.71 E+01 \\
7.29 E \bullet 00 \\
7.98 E+00 \\
1.09 E+01 \\
3.15 E-03\end{array}$ & $\begin{array}{l}2.22 E+01 \\
5.86 E+00 \\
6.50 E+00 \\
8.46 E+00 \\
8.68 E-04\end{array}$ & $\begin{array}{l}\text { 2. } 25 E+01 \\
5.96 E+00 \\
6.62 E+00 \\
8.55 E+00 \\
9.50 E-04\end{array}$ \\
\hline $\begin{array}{l}M O-99 \\
M O-101 \\
T C-95 \\
T C-95 M \\
\text { IC-96 }\end{array}$ & 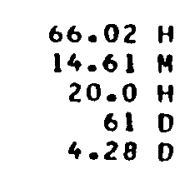 & $\begin{array}{l}1.41 E+00 \\
1.43 E+01 \\
7.26 E * 00 \\
6.02 E+00 \\
2.32 E+01\end{array}$ & $\begin{array}{l}1.51 E+00 \\
1.52 E+01 \\
7.75 E+00 \\
6.46 E+00 \\
2.48 E+01\end{array}$ & $\begin{array}{l}1.72 E+00 \\
1.64 E+01 \\
8.47 E+00 \\
1.45 E+00 \\
2.69 E+01\end{array}$ & $\begin{array}{l}1.53 E+00 \\
1.54 E+01 \\
7.88 E+00 \\
6.55 E+00 \\
2.52 E+01\end{array}$ & $\begin{array}{l}1.35 E \cdot 00 \\
1.35 E \cdot 01 \\
1.05 E \bullet 00 \\
5.74 E+00 \\
2.26 E+01\end{array}$ & $\begin{array}{l}1.21 E \times 00 \\
1.24 E \times 01 \\
6.26 E+00 \\
5.19 E+00 \\
2.00 E \times 01\end{array}$ & $\begin{array}{l}1.75 E+00 \\
1.67 E+01 \\
8.61 E+00 \\
7.58 E+00 \\
2.74 E+01\end{array}$ & $\begin{array}{l}1.37 E+00 \\
1.39 E \cdot 01 \\
7.04 E+00 \\
5.80 E+00 \\
2.25 E+01\end{array}$ & $\begin{array}{l}1.39 E+00 \\
1.41 E+01 \\
7.18 E+00 \\
5.96 E+00 \\
2.30 E+01\end{array}$ \\
\hline
\end{tabular}




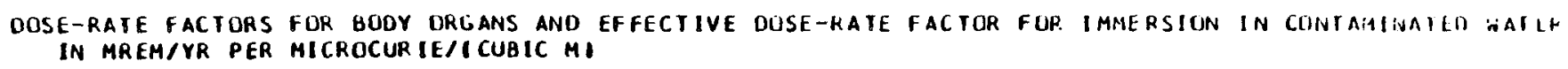

\begin{tabular}{|c|c|c|c|c|c|c|c|c|c|}
\hline NUCL IDE & HALF-LIFE & TESTES & IHYMUS & THYROID & UTERUS & EFFECIIVE & $\begin{array}{c}\text { SKIN } \\
\text { IPHOIONI }\end{array}$ & $\begin{array}{l}\text { SKIN } \\
\text { IELECTRONI }\end{array}$ & $\begin{array}{l}\text { SKIN } \\
\text { (roTALI }\end{array}$ \\
\hline $\begin{array}{l}S R-89 \\
S R-90 \\
S R-91 \\
S R-92 \\
S R-93\end{array}$ & $\begin{array}{rl}50.55 & 0 \\
28.6 & Y \\
9.5 & H \\
2.71 & H \\
i .3 & H\end{array}$ & $\begin{array}{l}1.01 E-03 \\
0.0 \\
9.06 E+00 \\
1.75 E+01 \\
3.00 E+01\end{array}$ & $\begin{array}{l}1.45 E-03 \\
0.0 \\
7.20 E+00 \\
1.40 E+01 \\
2.36 E+01\end{array}$ & $\begin{array}{l}1.13 E-03 \\
0.0 \\
0.62 E+00 \\
1.66 E+01 \\
2.82 E+01\end{array}$ & $\begin{array}{l}1.12 E-03 \\
0.0 \\
5.61 E+00 \\
1.12 E+01 \\
1.89 E+01\end{array}$ & $\begin{array}{l}1.55 E-03 \\
0.0 \\
7.75 E+00 \\
1.50 E+01 \\
2.57 E+01\end{array}$ & $\begin{array}{l}2.10 E-03 \\
0.0 \\
1.05 E+01 \\
2.01 E+01 \\
3.43 E+O 1\end{array}$ & $\begin{array}{l}4.66 E+00 \\
1.17 E+00 \\
5.26 E+00 \\
1.18 E+00 \\
7.48 E \cdot 00\end{array}$ & $\begin{array}{l}4.66 E+00 \\
1.17 E+00 \\
1.57 E+01 \\
2.13 E+01 \\
4.1 B E+01\end{array}$ \\
\hline $\begin{array}{l}y-86 \\
y-87 \\
\gamma-88 \\
y-90 \\
\gamma-90 M\end{array}$ & $\begin{array}{r}14.74 \mathrm{H} \\
00.3 \mathrm{H} \\
106.60 \mathrm{D} \\
64.1 \mathrm{H} \\
3.19 \mathrm{H}\end{array}$ & $\begin{array}{l}4.81 E+01 \\
5.81 E+00 \\
3.73 E+01 \\
0.0 \\
8.08 E+00\end{array}$ & $\begin{array}{l}3.80 E+01 \\
4.38 E+00 \\
2.96 E+01 \\
0.0 \\
6.12 E+00\end{array}$ & $\begin{array}{l}4.52 E+01 \\
5.37 E \bullet 00 \\
3.49 E+01 \\
0.0 \\
7.57 E+00\end{array}$ & $\begin{array}{l}3.04 E+01 \\
3.52 E+00 \\
2.42 E+01 \\
0.0 \\
4.86 E+00\end{array}$ & $\begin{array}{l}4.13 E+01 \\
4.97 E+00 \\
3.21 E+01 \\
0.0 \\
6.97 E+00\end{array}$ & $\begin{array}{l}5.52 E+01 \\
0.66 E+00 \\
4.27 E \times 01 \\
0.0 \\
9.11 E+00\end{array}$ & $\begin{array}{l}1.19 E+00 \\
1.01 E-02 \\
5.61 E-03 \\
7.86 E+00 \\
3.34 E-01\end{array}$ & $\begin{array}{l}5.70 E+01 \\
6.67 E+00 \\
4.27 E+01 \\
7.86 E+00 \\
9.45 E+00\end{array}$ \\
\hline $\begin{array}{l}\gamma-91 \\
r-914 \\
y-92 \\
y-93 \\
2 R-86\end{array}$ & $\begin{array}{r}58.51 \\
49.71 \\
3.54 \\
10.1 \mathrm{H} \\
16.5 \mathrm{H}\end{array}$ & $\begin{array}{l}4.74 E-02 \\
6.79 E+00 \\
3.33 E+00 \\
1.21 E+00 \\
3.49 E+00\end{array}$ & $\begin{array}{l}3.80 E-02 \\
5.18 E+00 \\
2.64 E+00 \\
9.54 E-01 \\
2.64 E \bullet 00\end{array}$ & $\begin{array}{l}4.51 E-02 \\
6.31 E+00 \\
3.16 E+00 \\
1.14 E+00 \\
3.30 E+00\end{array}$ & $\begin{array}{l}3.00 E-02 \\
4.12 E+00 \\
2.08 E+00 \\
7.14 E-01 \\
2.07 E+00\end{array}$ & $\begin{array}{l}4.06 E-02 \\
5.80 E \cdot 00 \\
2.85 E+00 \\
1.05 E+00 \\
3.05 E+00\end{array}$ & $\begin{array}{l}5.46 E-02 \\
7.79 E+00 \\
3.83 E+00 \\
1.38 E+00 \\
3.99 E+00\end{array}$ & $\begin{array}{l}4.84 E+00 \\
2.07 E-01 \\
1.26 E+01 \\
1.01 E+01 \\
5.60 E-02\end{array}$ & $\begin{array}{l}4.89 E \times 00 \\
7.99 E \bullet 00 \\
1.64 E+01 \\
1.14 E+01 \\
4.05 E+00\end{array}$ \\
\hline $\begin{array}{l}2 R-8 \theta \\
2 R-89 \\
2 R-93 \\
2 R-95 \\
2 R-97\end{array}$ & $\begin{array}{r}83.4 \mathrm{O} \\
78.43 \mathrm{H} \\
1.53 \mathrm{HE} \\
64.02 \mathrm{O} \\
16.90 \mathrm{H}\end{array}$ & $\begin{array}{l}4.04 E+00 \\
1.52 E+01 \\
0.0 \\
9.60 E+00 \\
2.38 E+00\end{array}$ & $\begin{array}{l}3.64 E+00 \\
1.20 E+01 \\
0.0 \\
7.54 E+00 \\
1.87 E * 00\end{array}$ & $\begin{array}{l}4.49 E+00 \\
1.44 E+01 \\
0.0 \\
9.08 E+00 \\
2.24 E+00\end{array}$ & $\begin{array}{l}2.93 E+00 \\
9.37 E+00 \\
0.0 \\
5.87 E+00 \\
1.49 E+00\end{array}$ & $\begin{array}{l}4.17 E+00 \\
1.30 E+01 \\
0.0 \\
8.20 E+00 \\
2.04 E+00\end{array}$ & $\begin{array}{l}5.52 E+00 \\
1.76 E \cdot 01 \\
0.0 \\
1.11 E+01 \\
2.72 E \cdot 00\end{array}$ & $\begin{array}{l}7.30 E-02 \\
7.23 E-01 \\
0.0 \\
4.69 E-01 \\
5.60 E+00\end{array}$ & $\begin{array}{l}5.59 E+00 \\
1.83 E \bullet 01 \\
0.0 \\
1.16 E+01 \\
8.33 E+00\end{array}$ \\
\hline $\begin{array}{l}N B-90 \\
N B-91 \\
N B-91 M \\
N B-92 \\
N B-92 M\end{array}$ & 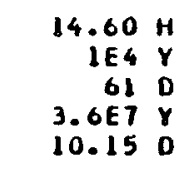 & $\begin{array}{l}5.88 E \cdot 01 \\
3.04 E-02 \\
5.71 E-01 \\
1.96 E \cdot 01 \\
1.28 E+01\end{array}$ & $\begin{array}{l}4.63 E+01 \\
1.63 E-02 \\
4.50 E-01 \\
1.54 E+01 \\
1.02 E+01\end{array}$ & $\begin{array}{l}5.45 E+01 \\
2.20 E-02 \\
5.37 E-01 \\
1.85 E+01 \\
1.22 E+01\end{array}$ & $\begin{array}{l}3.82 E \cdot 01 \\
1.29 E-02 \\
3.55 E-01 \\
1.20 E+01 \\
1.91 E \cdot 00\end{array}$ & $\begin{array}{l}5.07 E+01 \\
2.64 E-02 \\
4.89 E-01 \\
1.68 E+01 \\
1.09 E+01\end{array}$ & $\begin{array}{l}6.66 E+01 \\
8.10 E-02 \\
6.96 E-01 \\
2.27 E+01 \\
1.48 E+01\end{array}$ & $\begin{array}{l}2.93 E+00 \\
5.23 E-04 \\
7.20 E-02 \\
1.10 E-02 \\
0.0\end{array}$ & $\begin{array}{l}6.95 E+01 \\
8.16 E-02 \\
1.68 E-01 \\
2.27 E+01 \\
1.48 E+01\end{array}$ \\
\hline $\begin{array}{l}N B-934 \\
N B-94 \\
N B-94 A \\
N B-95 \\
N B-954\end{array}$ & 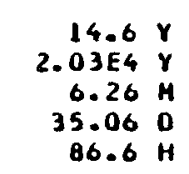 & $\begin{array}{l}2.03 E-03 \\
2.07 E \cdot 01 \\
6.26 E-02 \\
1.00 E+01 \\
7.63 E-01\end{array}$ & $\begin{array}{l}3.38 E-05 \\
1.63 E+01 \\
4.38 E-02 \\
7.89 E+00 \\
5.74 E-01\end{array}$ & $\begin{array}{l}5.51 E-04 \\
1.96 E+01 \\
5.44 E-02 \\
9.49 E+00 \\
7.24 E-01\end{array}$ & $\begin{array}{l}1.76 E-0 E \\
1.27 E+01 \\
3.37 E-02 \\
6.13 E+00 \\
4.52 E-01\end{array}$ & $\begin{array}{l}1.75 E-03 \\
1.17 E+01 \\
5.34 E-02 \\
8.56 E+00 \\
6.69 E-01\end{array}$ & & $\begin{array}{l}0.0 \\
7.15 E-01 \\
1.56 E-02 \\
3.30 E-02 \\
1.07 E+00\end{array}$ & $\begin{array}{l}1.11 E-02 \\
2.46 E+01 \\
1.21 E-01 \\
1.16 E+01 \\
1.95 E+00\end{array}$ \\
\hline $\begin{array}{l}\mathrm{NB}-96 \\
\mathrm{NB}-97 \\
\mathrm{NB}-97 \mathrm{M} \\
\mathrm{MO}-91 \\
\mathrm{MO}-93\end{array}$ & $\begin{array}{r}23.35 \mathrm{H} \\
12.1 \mathrm{M} \\
60 \mathrm{~S} \\
15.49 \mathrm{H} \\
3.5 \mathrm{E} \mathrm{Y}\end{array}$ & $\begin{array}{l}3.23 E+01 \\
8.60 E+00 \\
9.51 E+00 \\
1.25 E+01 \\
1.13 E-02\end{array}$ & $\begin{array}{l}2.55 E+01 \\
6.60 E+00 \\
7.47 E+00 \\
9.45 E+00 \\
1.89 E-04\end{array}$ & $\begin{array}{l}3.06 E+01 \\
8.08 E+00 \\
9.00 E+00 \\
1.16 E+01 \\
3.09 E-03\end{array}$ & $\begin{array}{l}2.00 E+01 \\
5.24 E+00 \\
5.02 E+00 \\
7.58 E+00 \\
9.86 E-06\end{array}$ & $\begin{array}{l}2.77 E+01 \\
1.34 E+00 \\
0.13 E+00 \\
1.07 E+01 \\
9.78 E-03\end{array}$ & & $\begin{array}{l}1.61 E * 00 \\
3.53 E * 00 \\
1.15 E-01 \\
1.25 E * 01 \\
0.0\end{array}$ & $\begin{array}{l}3.09 E \cdot 01 \\
1.34 E+01 \\
1.11 E+01 \\
2.60 E+01 \\
0.20 E-02\end{array}$ \\
\hline $\begin{array}{l}M 0-99 \\
M 0-101 \\
1 C-95 \\
I C-954 \\
I C-96\end{array}$ & 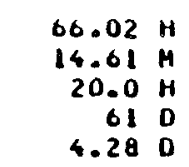 & $\begin{array}{l}2.02 E+00 \\
2.00 E+01 \\
1.03 E+01 \\
0.65 E+00 \\
3.29 E+01\end{array}$ & $\begin{array}{l}1.58 E * 00 \\
1.58 E * 01 \\
8.12 E * 00 \\
6.74 E * 00 \\
2.60 E * 01\end{array}$ & $\begin{array}{l}1.91 E+00 \\
1.88 E+01 \\
9.76 E+00 \\
8.20 E+00 \\
3.12 E+01\end{array}$ & $\begin{array}{l}1.23 E+00 \\
1.27 E+01 \\
6.31 E+00 \\
5.26 E+00 \\
2.02 E+01\end{array}$ & $\begin{array}{l}1.73 E+00 \\
1.72 E+01 \\
B .80 E+00 \\
7.43 E+00 \\
2.81 E+01\end{array}$ & $\begin{array}{l}2.32 E+00 \\
2.29 E+01 \\
1.20 E+01 \\
9.95 E+00 \\
3.80 E+01\end{array}$ & $\begin{array}{l}2.88 E+00 \\
4.03 E+00 \\
7.21 E-03 \\
4.17 E-02 \\
7.57 E-03\end{array}$ & $\begin{array}{l}5.19 E+00 \\
2.70 E+01 \\
1.20 E+01 \\
9.99 E+00 \\
3.81 E+01\end{array}$ \\
\hline
\end{tabular}




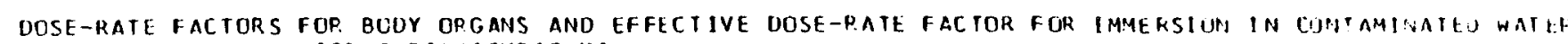

IV MREM/YR PER MICROCURIEIICUBIC MI

\begin{tabular}{|c|c|c|c|c|c|c|c|c|c|c|}
\hline CLIDE & HALF-LIFE & AORENALS & BLADDER & BRAIN & BREAST & HE AR T & $\begin{array}{c}\text { SMALL } \\
\text { INTESTINE }\end{array}$ & $\begin{array}{c}\text { UPPER } \\
\text { LARGE } \\
\text { INTESTINE }\end{array}$ & $\begin{array}{l}\text { LOHER } \\
\text { LARGE } \\
\text { NTESTINE }\end{array}$ & KIONEYS \\
\hline $\begin{array}{l}\text { TC-96M } \\
\text { TC-97 } \\
\text { TC-97M } \\
\text { CC-98 } \\
\text { IC-99 }\end{array}$ & $\begin{array}{r}51.5 \\
2.6 E 6 \\
89 \\
89 \\
4.2 E 6 Y \\
2.13 E 5 Y\end{array}$ & $\begin{array}{l}3.98 E-01 \\
2.40 E-03 \\
5.05 E-03 \\
1.34 E+01 \\
4.43 E-06\end{array}$ & $\begin{array}{l}3.86 E-01 \\
2.79 E-04 \\
3.13 E-03 \\
1.28 E+01\end{array}$ & $\begin{array}{l}4.35 E-01 \\
5.18 E-04 \\
3.34 E-03 \\
1.44 E+01 \\
4.57 E-06\end{array}$ & $\begin{array}{l}5.16 E-01 \\
4.76 E-02 \\
4.56 E-02 \\
1.64 E+01 \\
0.44 E-06\end{array}$ & $\begin{array}{l}3.81 E-01 \\
6.65 E-04 \\
3.25 E-03 \\
1.26 E-01 \\
4.16 E-06\end{array}$ & $\begin{array}{l}.47 E-01 \\
.02 E-04 \\
.57 E-03 \\
14 E \cdot 01 \\
.62 E-06\end{array}$ & $\begin{array}{l}4.06 E-01 \\
4.16 E-04 \\
3.48 E-03 \\
1.34 E-01 \\
4.47 E-06\end{array}$ & $\begin{array}{l}3.74 E-01 \\
1.56 E-03 \\
4.01 E-03 \\
1.23 E+01 \\
3.98 E-06\end{array}$ & $\begin{array}{l}4.05 \mathrm{E}-01 \\
1.20 \mathrm{E}-03 \\
4.35 \mathrm{E}-03 \\
1.34 \mathrm{E} .01 \\
4.50 \mathrm{E}-06\end{array}$ \\
\hline $\begin{array}{l}\mathrm{TC}-994 \\
\mathrm{TC}-101 \\
\mathrm{RU}-97 \\
R U-103 \\
\mathrm{RU}-105\end{array}$ & $\begin{array}{r}6.02 \mathrm{H} \\
14.2 \mathrm{M} \\
2.99 \\
39.35 \\
4.44 \mathrm{O}\end{array}$ & $\begin{array}{l}1.19 E+00 \\
3.24 E+00 \\
2.15 E+00 \\
4.52 E+00 \\
7.48 E+00\end{array}$ & $\begin{array}{l}1.10 E+00 \\
3.00 E+00 \\
1.97 E+00 \\
4.29 E+00 \\
7.14 E+00\end{array}$ & $\begin{array}{l}1.18 E+00 \\
3.29 E+00 \\
2.12 E+00 \\
4.78 E+00 \\
7.98 E+00\end{array}$ & $\begin{array}{l}1.90 E+00 \\
4.29 E+00 \\
3.08 E+00 \\
5.71 E+00 \\
9.33 E+00\end{array}$ & $\begin{array}{l}1.08 E+00 \\
2.93 E+00 \\
1.92 E+00 \\
4.20 E+00 \\
7.02 E+00\end{array}$ & $\begin{array}{l}9.44 E-01 \\
2.63 E+00 \\
1.72 E+00 \\
3.77 E+00 \\
6.33 E+00\end{array}$ & $\begin{array}{l}1.16 E+00 \\
3.11 E+00 \\
2.03 E+00 \\
4.45 E+00 \\
7.45 E+00\end{array}$ & $\begin{array}{l}1.06 E * 00 \\
2.90 E * 00 \\
1.91 E * 00 \\
4.11 E * 00 \\
6.8 \theta E * 00\end{array}$ & $\begin{array}{l}1.12 E * 00 \\
3.10 E * 00 \\
2.03 E * 00 \\
4.45 E+00 \\
7.44 E * 00\end{array}$ \\
\hline $\begin{array}{l}R U-106 \\
R H+103 M \\
R H-105 \\
R H-105 M \\
R H-105\end{array}$ & $\begin{array}{r}368.20 \\
56.119 \mathrm{M} \\
35.36 \mathrm{H} \\
45 \mathrm{~S} \\
29.92 \mathrm{~S}\end{array}$ & $\begin{array}{l}0.0 \\
6.61 E-04 \\
1.33 E-01 \\
2.53 E-01 \\
1.96 E+00\end{array}$ & $\begin{array}{l}0.0 \\
3.83 E-04 \\
6.77 E-01 \\
2.33 E-01 \\
1.8 B E+00\end{array}$ & $\begin{array}{l}0.0 \\
2.37 E-04 \\
7.38 E-01 \\
2.49 E-01 \\
2.10 E+00\end{array}$ & $\begin{array}{l}0.0 \\
8.46 E-03 \\
5.76 E-01 \\
4.50 E-01 \\
2.45 E+00\end{array}$ & $\begin{array}{l}0.0 \\
2.63 E-04 \\
6.60 E-01 \\
2.29 E-01 \\
1.84 E+00\end{array}$ & $\begin{array}{l}0.0 \\
2.12 E-04 \\
5.92 E-01 \\
1.99 E-01 \\
1.66 E+00\end{array}$ & $\begin{array}{l}0.0 \\
5.27 E-04 \\
6.98 E-01 \\
2.47 E-01 \\
1.96 E+00\end{array}$ & $\begin{array}{l}0.0 \\
4.80 E-04 \\
6.54 E-01 \\
2.25 E-01 \\
1.80 E+00\end{array}$ & $\begin{array}{l}0.0 \\
8.60 E-04 \\
6.98 E-01 \\
2.39 E-01 \\
1.95 E+00\end{array}$ \\
\hline $\begin{array}{l}P D-103 \\
P D-107 \\
P D-109 \\
A G-105 M \\
A G-108\end{array}$ & $\begin{array}{r}16.961 \\
6.5 \mathrm{D} \\
13.453 \mathrm{H} \\
8.46 \mathrm{D} \\
2.37 \mathrm{M}\end{array}$ & $\begin{array}{l}6.24 E-03 \\
0.0 \\
6.45 E-03 \\
2.61 E+01 \\
1.65 E-01\end{array}$ & $\begin{array}{l}3.30 E-03 \\
0.0 \\
6.12 E-03 \\
2.59 E+01 \\
1.57 E-01\end{array}$ & $\begin{array}{l}2.57 E-03 \\
0.0 \\
6.82 E-03 \\
2.91 E \cdot 01 \\
1.76 E-01\end{array}$ & $\begin{array}{l}7.42 E-02 \\
0.0 \\
8.13 E-03 \\
3.33 E+01 \\
2.07 E-01\end{array}$ & $\begin{array}{l}2.74 E-03 \\
0.0 \\
5.98 E-03 \\
2.55 E+01 \\
1.54 E-01\end{array}$ & $\begin{array}{l}2.12 E-03 \\
0.0 \\
5.38 E-03 \\
2.32 E+01 \\
1.39 E-01\end{array}$ & $\begin{array}{l}4.89 E-03 \\
0.0 \\
6.35 E-03 \\
2.72 E+01 \\
1.64 E-01\end{array}$ & $\begin{array}{l}4.60 E-03 \\
0.0 \\
5.86 E-03 \\
2.50 E+01 \\
1.51 E-01\end{array}$ & $\begin{array}{l}7.29 E-03 \\
0.0 \\
6.34 E-03 \\
2.71 E+01 \\
1.64 E-01\end{array}$ \\
\hline $\begin{array}{l}A G-10 B M \\
A G-109 M \\
A G-110 \\
A G-110 M \\
A G-111\end{array}$ & $\begin{array}{r}127 \% \\
39.65 \\
24.575 \\
249.850 \\
7.460\end{array}$ & $\begin{array}{l}1.52 E \cdot 01 \\
3.1 \theta E-02 \\
2.91 E-01 \\
2.63 E \cdot 01 \\
2.48 E-01\end{array}$ & $\begin{array}{l}1.45 E \cdot 01 \\
3.09 E-02 \\
2.79 E-01 \\
2.56 E+01 \\
2.30 E-01\end{array}$ & & & & & & & $\begin{array}{l}1.51 E+01 \\
3.51 E-02 \\
2.90 E-01 \\
2.68 E+01 \\
2.37 E-01\end{array}$ \\
\hline $\begin{array}{l}C D-109 \\
C D-111 M \\
C D-113 \\
C D-113 M \\
C D-115\end{array}$ & $\begin{array}{r}464 \mathrm{O} \\
48.1 \mathrm{M} \\
9.3 E 15 \mathrm{~V} \\
13.7 \mathrm{Y} \\
53.46 \mathrm{H}\end{array}$ & $\begin{array}{l}7.62 E-03 \\
2.63 E+00 \\
0.0 \\
0.0 \\
1.91 E+00\end{array}$ & $\begin{array}{l}5.75 E-03 \\
2.41 E \cdot 00 \\
0.0 \\
0.0 \\
1.81 E \cdot 00\end{array}$ & $\begin{array}{l}2.65 E-03 \\
2.59 E+00 \\
0.0 \\
0.0 \\
2.02 E+00\end{array}$ & $\begin{array}{l}8.67 E-02 \\
3.10 E+00 \\
0.0 \\
0.0 \\
2.42 E+00\end{array}$ & $\begin{array}{l}3.02 E-03 \\
2.35 E+00 \\
0.0 \\
0.0 \\
1.77 E+00\end{array}$ & $\begin{array}{l}2.10 E+00 \\
0.0 \\
0.0 \\
1.59 E+00\end{array}$ & $\begin{array}{l}7 E-03 \\
9 E+00\end{array}$ & $\begin{array}{l}5.61 E-03 \\
2.33 E+00 \\
0.0 \\
0.0 \\
1.13 E+00\end{array}$ & $\begin{array}{l}\text { 1. } 27 E-02 \\
2.48 E+00 \\
0.0 \\
0.0 \\
1.88 E+00\end{array}$ \\
\hline $\begin{array}{l}C D-115 M \\
C D-117 \\
C D-117 M \\
I N-111 \\
I N-113 M\end{array}$ & $\begin{array}{l}44.60 \\
2.49 \mathrm{H} \\
3.36 \mathrm{H} \\
2.8330 \\
1.658 \mathrm{H}\end{array}$ & $\begin{array}{l}2.11 E-01 \\
1.04 E+01 \\
2.04 E+01 \\
3.67 E+00 \\
2.39 E+00\end{array}$ & $\begin{array}{l}1.03 E \cdot 01 \\
2.05 E \cdot 01 \\
3.35 E \cdot 00 \\
2.23 E+00\end{array}$ & & & & & & & $\begin{array}{l}2.17 E-01 \\
1.08 E+01 \\
2.17 E+01 \\
3.45 E+00 \\
2.31 E+00\end{array}$ \\
\hline $\begin{array}{l}1 N-114 \\
\text { IN-114M } \\
1 N-115 \\
1 N-115 M \\
\text { IN-116M }\end{array}$ & $\begin{array}{r}71.9 \mathrm{~S} \\
49.511 \mathrm{D} \\
4.6 \mathrm{E15} \mathrm{Y} \\
4.36 \mathrm{H} \\
54.15 \mathrm{M}\end{array}$ & $\begin{array}{l}3.00 E-01 \\
8.43 E-01 \\
0.0 \\
1.49 E+00 \\
2.38 E+01\end{array}$ & $\begin{array}{l}2.87 E-01 \\
7.94 E-01 \\
0.0 \\
1.38 E+00 \\
2.37 E+01\end{array}$ & $\begin{array}{l}3.21 E-01 \\
8.71 E-01 \\
0.0 \\
1.51 E+00 \\
2.66 E+01\end{array}$ & $\begin{array}{l}3.75 E-01 \\
1.16 E+00 \\
0.0 \\
2.01 E+00 \\
2.97 E+01\end{array}$ & $\begin{array}{l}2.81 E-01 \\
7.75 E-01 \\
0.0 \\
1.34 E+00 \\
2.34 E+01\end{array}$ & $\begin{array}{l}2.54 E-01 \\
6.95 E-01 \\
0.0 \\
1.21 E+00 \\
2.16 E+01\end{array}$ & $\begin{array}{l}2.99 E-01 \\
8.28 E-01 \\
0.0 \\
1.43 E+00 \\
2.50 E+01\end{array}$ & $\begin{array}{l}2.15 E-01 \\
1.64 E-01 \\
0.0 \\
1.33 E+00 \\
2.30 E+01\end{array}$ & $\begin{array}{l}2.99 E-01 \\
9.29 E-01 \\
0.0 \\
1.43 E+00 \\
2.49 E+01\end{array}$ \\
\hline
\end{tabular}




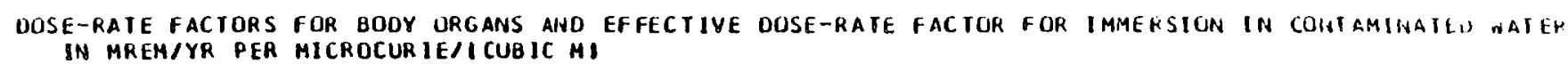

\begin{tabular}{|c|c|c|c|c|c|c|c|c|c|c|}
\hline NUCL IDE & HALF-LIFE & LIVER & LUNGS & MARROW & $\begin{array}{c}\text { RED } \\
\text { MARRRW }\end{array}$ & OVARIES & PANCREAS & SKELETON & SPLEEN & STOMACH \\
\hline $\begin{array}{l}\text { TC-9CM } \\
\text { IC }-97 \\
\text { IC-97M } \\
\text { IC-98 } \\
\text { IC }-99\end{array}$ & $\begin{array}{r}51.5 \% \\
2.6 E 6 Y \\
898 \\
4.2 E 6 Y \\
2.13 E 5 Y\end{array}$ & $\begin{array}{l}3.84 E-01 \\
5.13 E-04 \\
3.34 E-03 \\
1.27 E+01 \\
4.40 E-06\end{array}$ & $\begin{array}{l}11 E-01 \\
34 E-03 \\
55 E-03 \\
36 E+01 \\
7 E-06\end{array}$ & $\begin{array}{l}4 E-01 \\
4 E-03 \\
4 E-03 \\
0 E+01\end{array}$ & $\begin{array}{l}15 E-01 \\
94 E-04 \\
16 E-03 \\
39 E+01\end{array}$ & $\begin{array}{l}3.72 E-01 \\
1.61 E-03 \\
3.88 E-03 \\
1.24 E+01 \\
3.70 E-06\end{array}$ & $\begin{array}{l}3.33 E-01 \\
1.47 E-03 \\
3.45 E-03 \\
1.10 E+01 \\
3.26 E-06\end{array}$ & $\begin{array}{l}52 E-01 \\
35 E-03 \\
36 E-03 \\
53 E+01 \\
50 E-06\end{array}$ & $\begin{array}{l}73 E-01 \\
24 E-03 \\
05 E-03 \\
24 E+01 \\
22 E-06\end{array}$ & $\begin{array}{l}\text { 3. } 79 \mathrm{E}-01 \\
1.38 \mathrm{E}-03 \\
4.01 \mathrm{E}-03 \\
1.26 \mathrm{EO} 01 \\
4.24 \mathrm{E}-06\end{array}$ \\
\hline $\begin{array}{l}\text { rC-994 } \\
\text { RC-101 } \\
R U-97 \\
R U-103 \\
R U-105\end{array}$ & $\begin{array}{r}6.02 \mathrm{H} \\
14.2 \mathrm{H} \\
2.9 \mathrm{D} \\
39.35 \mathrm{O} \\
4.44 \mathrm{H}\end{array}$ & $\begin{array}{l}1.10 E \cdot 00 \\
2.99 E \cdot 00 \\
1.96 E+00 \\
4.27 E+00 \\
7.10 E+00\end{array}$ & $\begin{array}{l}1.21 E \cdot 00 \\
3.23 E+00 \\
2.13 E \cdot 00 \\
4.58 E+00 \\
7.61 E+00\end{array}$ & $\begin{array}{l}1.85 E+00 \\
4.01 E+00 \\
2.86 E+00 \\
5.29 E+00 \\
8.57 E+00\end{array}$ & $\begin{array}{l}1.10 E+00 \\
3.32 E+00 \\
2.15 E+00 \\
4.69 E+00 \\
7.76 E+00\end{array}$ & $\begin{array}{l}9.51 E-01 \\
2.76 E+00 \\
1.75 E+00 \\
4.10 E+00 \\
6.84 E+00\end{array}$ & $\begin{array}{l}9.10 E-01 \\
2.58 E+00 \\
1.69 E+00 \\
3.67 E+00 \\
6.12 E+00\end{array}$ & $\begin{array}{l}1.90 E+00 \\
4.09 E+00 \\
2.92 E+00 \\
5.37 E+00 \\
8.72 E+00\end{array}$ & $\begin{array}{l}1.10 E+00 \\
2.95 E+00 \\
1.95 E+00 \\
4.17 E+00 \\
6.92 E+00\end{array}$ & $\begin{array}{l}1.09 E+00 \\
2.95 E+00 \\
1.94 E+00 \\
4.21 E+00 \\
7.02 E+00\end{array}$ \\
\hline $\begin{array}{l}R H-106 \\
R H+103 H \\
R H-105 \\
R H-105 H \\
R H-106\end{array}$ & 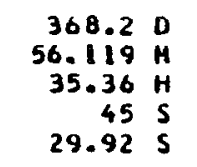 & $\begin{array}{l}0.0 \\
3.45 E-04 \\
6.73 E-01 \\
2.33 E-01 \\
1.87 E+00\end{array}$ & $\begin{array}{l}0.0 \\
7.88 E-04 \\
7.27 E-01 \\
2.59 E-01 \\
2.00 E \cdot 00\end{array}$ & $\begin{array}{l}0.0 \\
1.17 E-03 \\
9.14 E-01 \\
4.04 E-01 \\
2.25 E+00\end{array}$ & $\begin{array}{l}0.0 \\
2.77 E-04 \\
1.51 E-01 \\
2.28 E-01 \\
2.04 E+00\end{array}$ & $\begin{array}{l}0.0 \\
5.20 E-04 \\
6.18 E-01 \\
2.02 E-01 \\
1.80 E+00\end{array}$ & $\begin{array}{l}0.0 \\
4.08 E-04 \\
5.82 E-01 \\
1.92 E-01 \\
1.61 E .09\end{array}$ & $\begin{array}{l}0.0 \\
1.33 E-03 \\
9.30 E-01 \\
4.15 E-01 \\
2.28 E+00\end{array}$ & $\begin{array}{l}0.0 \\
4.29 E-04 \\
6.66 E-01 \\
2.33 E-01 \\
1.02 E+00\end{array}$ & $\begin{array}{l}0.0 \\
4.85 E-04 \\
6.65 E-01 \\
2.31 E-01 \\
1.84 E+00\end{array}$ \\
\hline $\begin{array}{l}P D-103 \\
P D-107 \\
P D-109 \\
A G-106 M \\
A G-108\end{array}$ & $\begin{array}{r}16.961 \mathrm{D} \\
6.5 \mathrm{E} 6 \mathrm{Y} \\
13.453 \mathrm{H} \\
8.46 \mathrm{D} \\
2.31 \mathrm{M}\end{array}$ & $\begin{array}{l}3.16 E-03 \\
0.0 \\
6.00 E-03 \\
2.58 E+01 \\
1.56 E-01\end{array}$ & $\begin{array}{l}1.13 E-03 \\
0.0 \\
6.53 E-03 \\
2.15 E+01 \\
1.67 E-01\end{array}$ & $\begin{array}{l}1.00 E-02 \\
0.0 \\
7.52 E-03 \\
3.02 E+01 \\
1.09 E-01\end{array}$ & $\begin{array}{l}3.15 E-03 \\
0.0 \\
6.68 E-03 \\
2.00 E+01 \\
1.11 E-01\end{array}$ & $\begin{array}{l}4.85 E-03 \\
0.0 \\
5.84 E-03 \\
2.47 E+01 \\
1.51 E-01\end{array}$ & $\begin{array}{l}4.01 E-03 \\
0.0 \\
5.23 E-01 \\
2.23 E+01 \\
1.35 E-01\end{array}$ & $\begin{array}{l}1.14 E-02 \\
0.0 \\
7.64 E-03 \\
3.07 E 001 \\
1.92 E-01\end{array}$ & $\begin{array}{l}4.11 E-03 \\
0.0 \\
5.94 E-03 \\
2.51 E+01 \\
1.52 E-01\end{array}$ & $\begin{array}{l}4.54 E-03 \\
0.0 \\
6.00 E-03 \\
2.54 E+01 \\
1.54 E-01\end{array}$ \\
\hline $\begin{array}{l}A G-100 M \\
A G-109 M \\
A G-110 \\
A G-110 M \\
A G-111\end{array}$ & $\begin{array}{rr}127 & Y \\
39.6 & S \\
24.57 & S \\
249.85 & 0 \\
7.46 & D\end{array}$ & $\begin{array}{l}1.44 E+01 \\
3.00 E-02 \\
2.77 E-01 \\
2.54 E+01 \\
2.29 E-01\end{array}$ & & & & & & & & \\
\hline $\begin{array}{l}C D-109 \\
C D-111 M \\
C D-113 \\
C D-1134 \\
C D-115\end{array}$ & $\begin{array}{r}464 \mathrm{O} \\
48.7 \mathrm{H} \\
9.3 E 15 \mathrm{Y} \\
13.7 \mathrm{Y} \\
53.46 \mathrm{H}\end{array}$ & $\begin{array}{l}4.29 E-03 \\
2.40 E+00 \\
0.0 \\
0.0 \\
1.80 E+00\end{array}$ & $\begin{array}{l}9.25 E-03 \\
2.61 E \bullet 00 \\
0.0 \\
0.0 \\
1.93 E \bullet 00\end{array}$ & $\begin{array}{l}1.40 E-02 \\
3.54 E+00 \\
0.0 \\
0.0 \\
2.23 E+00\end{array}$ & $\begin{array}{l}3.20 E-03 \\
2.62 E+00 \\
0.0 \\
0.0 \\
1.98 E+00\end{array}$ & $\begin{array}{l}6.20 E-03 \\
2.13 E+00 \\
0.0 \\
0.0 \\
1.73 E+00\end{array}$ & $\begin{array}{l}4.67 E-03 \\
2.06 E+00 \\
0.0 \\
0.0 \\
1.55 E+00\end{array}$ & $\begin{array}{l}1.55 E-02 \\
3.62 E+00 \\
0.0 \\
0.0 \\
2.27 E+00\end{array}$ & $\begin{array}{l}5.07 E-03 \\
2.39 E+00 \\
0.0 \\
0.0 \\
1.76 E+00\end{array}$ & $\begin{array}{l}5.81 E-03 \\
2.37 E+00 \\
0.0 \\
0.0 \\
1.78 E+00\end{array}$ \\
\hline $\begin{array}{l}C D-115 M \\
C 0-117 \\
C D-117 M \\
1 N-111 \\
8 N-113 M\end{array}$ & $\begin{array}{r}44.6 \mathrm{D} \\
2.49 \mathrm{H} \\
3.36 \mathrm{H} \\
2.83 \mathrm{D} \\
1.658 \mathrm{H}\end{array}$ & $\begin{array}{l}2.06 E-01 \\
1.03 E+01 \\
2.05 E+01 \\
3.34 E+00 \\
2.22 E+00\end{array}$ & $\begin{array}{l}2.19 E-01 \\
1.09 E \bullet 01 \\
2.17 E \bullet 01 \\
3.64 E+00 \\
2.39 E+00\end{array}$ & $\begin{array}{l}2.34 E-01 \\
1.18 E+01 \\
2.27 E+01 \\
5.01 E+00 \\
2.08 E+00\end{array}$ & $\begin{array}{l}2.22 E-01 \\
1.10 E+01 \\
2.20 E+01 \\
3.62 E+00 \\
2.46 E+00\end{array}$ & $\begin{array}{l}1.98 E-01 \\
9.67 E+00 \\
1.91 E+01 \\
2.96 E+00 \\
2.08 E+00\end{array}$ & $\begin{array}{l}1.78 E-01 \\
8.88 E+00 \\
1.78 E+01 \\
2.86 E+00 \\
1.91 E+00\end{array}$ & $\begin{array}{l}2.38 E-01 \\
1.20 E+01 \\
2.31 E+01 \\
5.13 E+00 \\
2.93 E+00\end{array}$ & $\begin{array}{l}2.00 E-01 \\
9.94 E+00 \\
1.98 E+01 \\
3.33 E+00 \\
2.18 E+00\end{array}$ & $\begin{array}{l}2.03 E-01 \\
1.00 E+01 \\
2.01 E+01 \\
3.30 E+00 \\
2.19 E+00\end{array}$ \\
\hline $\begin{array}{l}I N-114 \\
\text { IN-114M } \\
I N-115 \\
I N-115 M \\
I N-116 M\end{array}$ & $\begin{array}{r}71.9 \mathrm{~S} \\
49.51 \mathrm{O} \\
4.6 \mathrm{E15} \mathrm{Y} \\
4.36 \mathrm{H} \\
54.15 \mathrm{M}\end{array}$ & $\begin{array}{l}2.85 E-01 \\
7.88 E-01 \\
0.0 \\
1.37 E+00 \\
2.36 E+01\end{array}$ & $\begin{array}{l}3.05 E-01 \\
8.52 E-01 \\
0.0 \\
1.48 E+00 \\
2.50 E+01\end{array}$ & $\begin{array}{l}3.44 E-01 \\
1.04 E+00 \\
0.0 \\
1.04 E \cdot 00 \\
2.65 E+01\end{array}$ & $\begin{array}{l}3.12 E-01 \\
0.54 E-01 \\
0.0 \\
1.53 E+00 \\
2.53 E+01\end{array}$ & $\begin{array}{l}2.75 E-01 \\
7.40 E-01 \\
0.0 \\
1.27 E+00 \\
2.23 E+01\end{array}$ & $\begin{array}{l}2.46 E-01 \\
6.76 E-01 \\
0.0 \\
1.19 E+00 \\
2.05 E+01\end{array}$ & $\begin{array}{l}3.49 E-01 \\
1.06 E+00 \\
0.0 \\
1.87 E+00 \\
2.69 E+01\end{array}$ & $\begin{array}{l}2.78 E-01 \\
1.73 E-01 \\
0.0 \\
1.35 E+00 \\
2.28 E+01\end{array}$ & $\begin{array}{l}\text { 2. } 82 E-01 \\
7.79 E-01 \\
0.0 \\
1.35 E+00 \\
2.31 E+01\end{array}$ \\
\hline
\end{tabular}




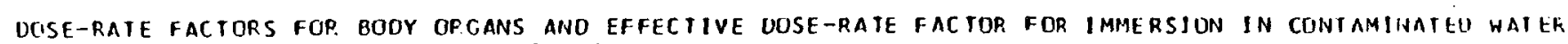

\begin{tabular}{|c|c|c|c|c|c|c|c|c|c|}
\hline NUCL IOE & HALF-LIFE & TESTES & THYMUS & THYROIO & UTERUS & EFFECTIVE & $\begin{array}{c}\text { SKIN } \\
\text { I PHOT ONI }\end{array}$ & $\begin{array}{l}\text { SKIN } \\
\text { (ELECTRON }\end{array}$ & $\begin{array}{l}\text { SKIN } \\
\text { ITOTALI }\end{array}$ \\
\hline $\begin{array}{l}\text { TC-964 } \\
\text { IC }-97 \\
\text { IC- }-974 \\
\text { IC-98 } \\
\text { IC }-99\end{array}$ & $\begin{array}{r}51.5 \mathrm{M} \\
2.6 E 6 \mathrm{Y} \\
89 \mathrm{D} \\
4.2 E 6 \mathrm{Y} \\
2.13 E 5 \mathrm{Y}\end{array}$ & $\begin{array}{l}5.51 E-01 \\
1.49 E-02 \\
1.82 E-02 \\
1.81 E+01 \\
7.15 E-06\end{array}$ & $\begin{array}{l}4.30 E-01 \\
3.89 E-04 \\
3.86 E-03 \\
1.42 E+01 \\
5.56 E-06\end{array}$ & $\begin{array}{l}5.17 E-01 \\
4.51 E-03 \\
8.95 E-03 \\
1.71 E+01 \\
7.15 E-06\end{array}$ & $\begin{array}{l}3.36 E-01 \\
2.64 E-05 \\
2.30 E-03 \\
1.11 E+01 \\
3.68 E-06\end{array}$ & $\begin{array}{l}4.11 E-01 \\
1.22 E-02 \\
1.45 E-02 \\
1.55 E+01 \\
6.10 E-06\end{array}$ & $\begin{array}{l}6.66 E-01 \\
7.14 E-02 \\
6.37 E-02 \\
2.09 E \cdot 01 \\
7.63 E-06\end{array}$ & $\begin{array}{l}0.0 \\
0.0 \\
3.68 E-02 \\
5.03 E-01 \\
2.35 E-01\end{array}$ & $\begin{array}{l}6.66 E-01 \\
7.14 E-02 \\
1.00 E-01 \\
2.14 E-01 \\
2.35 E-01\end{array}$ \\
\hline $\begin{array}{l}\text { TC-994 } \\
T C-101 \\
R U-97 \\
R U-103 \\
R U-105\end{array}$ & $\begin{array}{r}6.02 \mathrm{H} \\
14.2 \mathrm{M} \\
2.9 \mathrm{O} \\
39.35 \mathrm{O} \\
4.44 \mathrm{H}\end{array}$ & $\begin{array}{l}1.69 E+00 \\
4.36 E \bullet 00 \\
2.93 E \cdot 00 \\
6.15 E+00 \\
1.02 E+01\end{array}$ & $\begin{array}{l}1.30 E+00 \\
3.31 E+00 \\
2.21 E+00 \\
4.65 E+00 \\
7.88 E+00\end{array}$ & $\begin{array}{l}1.67 E+00 \\
4.10 E+00 \\
2.78 E+00 \\
5.70 E+00 \\
9.56 E+00\end{array}$ & $\begin{array}{l}9.65 E-01 \\
2.63 E+00 \\
1.13 E+00 \\
3.13 E+00 \\
6.49 E+00\end{array}$ & $\begin{array}{l}1.49 E \cdot 00 \\
3.78 E+00 \\
2.56 E+00 \\
5.26 E+00 \\
8.69 E \cdot 00\end{array}$ & $\begin{array}{l}1.01 E \cdot 00 \\
4.91 E+00 \\
3.30 E+00 \\
7.03 E * 00 \\
1.17 E \cdot 01\end{array}$ & $\begin{array}{l}3.59 E-02 \\
3.57 E+00 \\
3.71 E-02 \\
1.65 E-01 \\
2.95 E+00\end{array}$ & $\begin{array}{l}1.85 E+00 \\
8.48 E+00 \\
3.34 E * 00 \\
7.20 E * 00 \\
1.46 E+01\end{array}$ \\
\hline $\begin{array}{l}R U-106 \\
R H-103 M \\
R H-105 \\
R H-105 M \\
R H-106\end{array}$ & 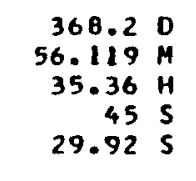 & $\begin{array}{l}0.0 \\
3.53 E-03 \\
9.85 E-01 \\
3.76 E-01 \\
2.67 E \cdot 00\end{array}$ & $\begin{array}{l}0.0 \\
4.11 E-04 \\
7.43 E-01 \\
2.78 E-01 \\
2.05 E+00\end{array}$ & $\begin{array}{l}0.0 \\
1.52 E-03 \\
9.24 E-01 \\
3.63 E-01 \\
2.49 E+00\end{array}$ & $\begin{array}{l}0.0 \\
9.45 E-05 \\
5.94 E-01 \\
2.03 E-01 \\
1.63 E+00\end{array}$ & $\begin{array}{l}0.0 \\
2.59 E-03 \\
8.55 E-01 \\
3.29 E-01 \\
2.28 E+00\end{array}$ & $\begin{array}{l}0.0 \\
1.16 E-02 \\
1.10 E+00 \\
4.42 E-01 \\
3.06 E+00\end{array}$ & $\begin{array}{l}0.0 \\
0.0 \\
7.85 E-01 \\
2.29 E-01 \\
1.22 E+01\end{array}$ & $\begin{array}{l}0.0 \\
1.16 E-02 \\
1.89 E+00 \\
6.71 E-01 \\
1.52 E+01\end{array}$ \\
\hline $\begin{array}{l}P D-103 \\
P D-107 \\
P D-109 \\
A G-106 M \\
A G-108\end{array}$ & $\begin{array}{r}16.961 \mathrm{D} \\
6.5 E 6 \mathrm{Y} \\
13.453 \mathrm{H} \\
8.46 \mathrm{D} \\
2.37 \mathrm{M}\end{array}$ & $\begin{array}{l}3.10 E-02 \\
0.0 \\
8.76 E-03 \\
3.65 E+01 \\
2.24 E-01\end{array}$ & $\begin{array}{l}3.32 E-03 \\
0.0 \\
6.63 E-03 \\
2.86 E+01 \\
1.72 E-01\end{array}$ & $\begin{array}{l}1.32 E-02 \\
0.0 \\
B .11 E-03 \\
3.44 E=01 \\
2.09 E-01\end{array}$ & $\begin{array}{l}1.13 E-03 \\
0.0 \\
5.31 E-03 \\
2.27 E+01 \\
1.36 E-01\end{array}$ & $\begin{array}{l}2.28 E-02 \\
0.0 \\
7.49 E-03 \\
3.13 E+01 \\
1.92 E-01\end{array}$ & $\begin{array}{l}1.02 E-01 \\
0.0 \\
1.00 E-02 \\
4.20 E+01 \\
2.59 E-01\end{array}$ & $\begin{array}{l}0.0 \\
0.0 \\
2.58 E+C \\
2.20 E-C \\
4.83 E+C\end{array}$ & $\begin{array}{l}1.02 E-01 \\
0.0 \\
2.59 E+00 \\
4.20 E+01 \\
5.09 E+00\end{array}$ \\
\hline $\begin{array}{l}A G-108 M \\
A G-109 M \\
A G-110 \\
A G-110 M \\
A G-111\end{array}$ & $\begin{array}{r}127 \% \\
39.65 \\
24.575 \\
249.850 \\
7.460\end{array}$ & $\begin{array}{l}2.07 E+01 \\
6.66 E-02 \\
3.95 E-01 \\
3.59 E+01 \\
3.34 E-01\end{array}$ & $\begin{array}{l}1.60 E \cdot 01 \\
3.80 E-02 \\
3.07 E-01 \\
2.83 E \cdot 01 \\
2.52 E-01\end{array}$ & $\begin{array}{l}1.94 E \cdot 01 \\
5.52 E-02 \\
3.71 E-01 \\
3.40 E+01 \\
3.13 E-01\end{array}$ & $\begin{array}{l}1.26 E \cdot 01 \\
2.36 E-02 \\
2.41 E-01 \\
2.23 E+01 \\
2.02 E-01\end{array}$ & $\begin{array}{l}1.77 E+01 \\
5.41 E-02 \\
3.37 E-01 \\
3.07 E+01 \\
2.90 E-01\end{array}$ & $\begin{array}{l}2.38 E+01 \\
1.08 E-01 \\
4.55 E-01 \\
4.13 E+01 \\
3.76 E-01\end{array}$ & $\begin{array}{l}4.32 E-02 \\
1.97 E-02 \\
1.00 E+01 \\
2.01 E-01 \\
2.49 E+00\end{array}$ & $\begin{array}{l}2.39 E+01 \\
1.20 E-01 \\
1.05 E+01 \\
4.16 E+01 \\
2.07 E+00\end{array}$ \\
\hline $\begin{array}{l}C D-109 \\
C D-111 M \\
C D-113 \\
C D-113 M \\
C D-115\end{array}$ & $\begin{array}{r}464 \mathrm{D} \\
48.7 \mathrm{M} \\
9.3 E 15 \mathrm{Y} \\
13.7 \mathrm{Y} \\
53.46 \mathrm{H}\end{array}$ & $\begin{array}{l}4.03 E-02 \\
3.59 E+00 \\
0.0 \\
0.0 \\
2.60 E+00\end{array}$ & $\begin{array}{l}5.27 E-03 \\
2.71 E \cdot 00 \\
0.0 \\
0.0 \\
1.97 E \cdot 00\end{array}$ & $\begin{array}{l}1.08 E-02 \\
3.42 E \cdot 00 \\
0.0 \\
0.0 \\
2.41 E * 00\end{array}$ & $\begin{array}{l}0.38 E-04 \\
2.12 E \bullet 00 \\
0.0 \\
0.0 \\
1.57 E \bullet 00\end{array}$ & $\begin{array}{l}2.65 E-02 \\
3.14 E+00 \\
0.0 \\
0.0 \\
2.22 E+00\end{array}$ & $\begin{array}{l}1.13 E-01 \\
4.00 E \cdot 00 \\
0.0 \\
0.0 \\
2.97 E \cdot 00\end{array}$ & $\begin{array}{l}0.0 \\
3.75 E-01 \\
2.96 E-01 \\
1.04 E+00 \\
2.16 E+00\end{array}$ & $\begin{array}{l}1.13 E-01 \\
4.37 E+00 \\
2.96 E-01 \\
1.04 E \cdot 00 \\
5.13 E \cdot 00\end{array}$ \\
\hline $\begin{array}{l}C O-115 M \\
C D-117 \\
C D-117 M \\
I N-111 \\
I N-113 M\end{array}$ & $\begin{array}{r}44.6 \mathrm{D} \\
2.49 \mathrm{H} \\
3.36 \mathrm{H} \\
2.83 \mathrm{D} \\
1.658 \mathrm{H}\end{array}$ & $\begin{array}{l}2.89 E-01 \\
1.44 E+01 \\
2.83 E+01 \\
5.03 E+00 \\
3.23 E+00\end{array}$ & $\begin{array}{l}2.31 E-01 \\
1.14 E+01 \\
2.24 E+01 \\
3.80 E+00 \\
2.42 E+00\end{array}$ & $\begin{array}{l}2.75 E-01 \\
1.36 E+01 \\
2.64 E+01 \\
4.81 E+00 \\
3.00 E+00\end{array}$ & $\begin{array}{l}1.81 E-01 \\
9.09 E+00 \\
1.83 E+01 \\
2.95 E+00 \\
1.95 E+00\end{array}$ & $\begin{array}{l}2.48 E-01 \\
1.23 E+01 \\
2.43 E+01 \\
4.40 E+00 \\
2.18 E+00\end{array}$ & $\begin{array}{l}3.34 E-01 \\
1.64 E \cdot 01 \\
3.23 E * 01 \\
5.61 E \cdot 00 \\
3.69 E \bullet 00\end{array}$ & $\begin{array}{l}4.78 E+00 \\
3.22 E+00 \\
1.21 E+00 \\
1.34 E-01 \\
9.25 E-01\end{array}$ & $\begin{array}{l}5.12 E+00 \\
1.97 E * 01 \\
3.35 E * 01 \\
5.74 E * 00 \\
4.61 E * 00\end{array}$ \\
\hline $\begin{array}{l}N-114 \\
N-114 M \\
N-115 \\
\mid N-115 M \\
N-116 M\end{array}$ & $\begin{array}{r}71.9 \mathrm{~S} \\
49.51 \mathrm{D} \\
4.6 E 15 \mathrm{Y} \\
4.36 \mathrm{H} \\
54.15 \mathrm{M}\end{array}$ & $\begin{array}{l}4.00 E-01 \\
1.1 T E * 00 \\
0.0 \\
2.02 E * 00 \\
3.28 E * 01\end{array}$ & $\begin{array}{l}3.14 E-01 \\
8.84 E-01 \\
0.0 \\
1.51 E+00 \\
2.61 E+01\end{array}$ & $\begin{array}{l}3.81 E-01 \\
1.10 E+00 \\
0.0 \\
1.88 E+00 \\
3.10 E+01\end{array}$ & $\begin{array}{l}2.49 E-01 \\
6.87 E-01 \\
0.0 \\
1.21 E+00 \\
2.10 E+01\end{array}$ & $\begin{array}{l}3.49 E-01 \\
1.00 E+00 \\
0.0 \\
1.75 E+00 \\
2.82 E \cdot 01\end{array}$ & $\begin{array}{l}4.70 E-01 \\
1.36 E+00 \\
0.0 \\
2.31 E \cdot 00 \\
3.76 E \cdot 01\end{array}$ & $\begin{array}{l}6.28 E+00 \\
6.61 E-01 \\
7.61 E-01 \\
1.13 E * 00 \\
2.06 E \cdot 00\end{array}$ & $\begin{array}{l}6.74 E+00 \\
2.03 E+00 \\
7.61 E-01 \\
3.44 E \cdot 00 \\
3.97 E \cdot 01\end{array}$ \\
\hline
\end{tabular}


DOSE-RATE FACTORS FOR BOOY ORGANS ANO EFFECTIVE DOSE-RATE FACTOR FOR IMMERSIUN IN CONIAII'TAIEH GNI it
IN MREM/YR PER MICROCUR IE/ICUEIC MI

\begin{tabular}{|c|c|c|c|c|c|c|c|c|c|c|}
\hline OE & IfE & RENAL $S$ & ADOER & BP.AIN & BREAST & HE AR T & $\begin{array}{l}\text { SMALL } \\
\text { NTESTINE }\end{array}$ & $\begin{array}{c}\text { UPPER } \\
\text { LARGE } \\
\text { INTESTINE }\end{array}$ & $\begin{array}{c}\text { LOWER } \\
\text { LARGE } \\
\text { INTESTINEE }\end{array}$ & IDN \\
\hline $\begin{array}{l}1 N-117 \\
I N-117 M \\
S N-113 \\
S N-1174 \\
S N-119 M\end{array}$ & $\begin{array}{r}43.8 \mathrm{M} \\
116.5 \mathrm{M} \\
115.1 \mathrm{D} \\
13.60 \mathrm{D} \\
293.0 \mathrm{D}\end{array}$ & $\begin{array}{l}0.49 E+00 \\
8.27 E-01 \\
5.96 E-02 \\
1.36 E+00 \\
8.47 E-03\end{array}$ & $\begin{array}{l}6.13 E+00 \\
7.62 E-01 \\
5.62 E-02 \\
1.25 E+00 \\
9.17 E-03\end{array}$ & $\begin{array}{l}6.19 E+00 \\
8.25 E-01 \\
5.15 E-02 \\
1.32 E+00 \\
3.68 E-03\end{array}$ & $\begin{array}{l}8.50 E+00 \\
1.18 E+00 \\
1.05 E-01 \\
2.18 E+00 \\
7.59 E-02\end{array}$ & $\begin{array}{l}6.01 E+00 \\
7.43 E-01 \\
4.75 E-02 \\
1.22 E+00 \\
3.95 E-03\end{array}$ & $\begin{array}{l}.38 E+00 \\
.63 E-01 \\
.31 E-02 \\
.07 E+00 \\
.00 E-03\end{array}$ & $\begin{array}{l}6.38 E+00 \\
7.91 E-01 \\
6.00 E-02 \\
1.31 E-00 \\
1.08 E-02\end{array}$ & $\begin{array}{l}5.89 E+00 \\
7.36 E-01 \\
5.13 E-02 \\
1.20 E+00 \\
6.53 E-03\end{array}$ & $\begin{array}{l}0.34 E+00 \\
7.87 E-01 \\
7.00 E-02 \\
1.28 E+00 \\
1.73 E-02\end{array}$ \\
\hline $\begin{array}{l}S N-123 \\
S N-125 \\
S N-126 \\
S B-117 \\
S B-122\end{array}$ & $\begin{array}{r}129.2 \mathrm{D} \\
9.64 \mathrm{D} \\
1.0 \mathrm{Y} 5 \mathrm{Y} \\
2.80 \mathrm{H} \\
2.70 \mathrm{O}\end{array}$ & $\begin{array}{l}6.67 E-02 \\
2.97 E+00 \\
3.91 E-01 \\
1.58 E+00 \\
4.19 E+00\end{array}$ & $\begin{array}{l}6.55 E-02 \\
2.93 E+00 \\
4.00 E-01 \\
1.46 E+00 \\
3.99 E+00\end{array}$ & $\begin{array}{l}7.38 E-02 \\
3.29 E+00 \\
4.02 E-01 \\
1.57 E+00 \\
4.46 E .00\end{array}$ & $\begin{array}{l}0.18 E-02 \\
3.68 E \cdot 00 \\
8.28 E-01 \\
2.47 E+00 \\
5.22 E+00\end{array}$ & $\begin{array}{l}6.48 E-02 \\
2.90 E+00 \\
3.65 E-01 \\
1.43 E+00 \\
3.91 E+00\end{array}$ & $\begin{array}{l}.92 E-02 \\
.65 E 000 \\
.21 E-01 \\
.26 E+00 \\
.53 E+00\end{array}$ & $\begin{array}{l}6.90 E-02 \\
3.09 E+00 \\
3.98 E-01 \\
1.53 E+00 \\
4.16 E+00\end{array}$ & $\begin{array}{l}0.34 E-02 \\
2.84 E+00 \\
3.52 E-01 \\
1.41 E+00 \\
3.83 E+00\end{array}$ & $\begin{array}{l}6.88 E-02 \\
3.08 E+00 \\
4.18 E-01 \\
1.51 E+00 \\
4.15 E+00\end{array}$ \\
\hline $\begin{array}{l}S B-124 \\
S B-125 \\
S B-126 \\
S B-126 M \\
S B-127\end{array}$ & $\begin{array}{rl}60.20 & 0 \\
2.77 & Y \\
12.4 & 0 \\
19.0 & H \\
3.85 & 0\end{array}$ & $\begin{array}{l}1.81 E+01 \\
3.96 E+00 \\
2.63 E+01 \\
1.49 E+01 \\
6.29 E+00\end{array}$ & $\begin{array}{l}1.80 E+01 \\
3.76 E+00 \\
2.51 E+01 \\
1.43 E+01 \\
6.00 E+00\end{array}$ & $\begin{array}{l}2.02 E+01 \\
4.16 E+00 \\
2.02 E \cdot 01 \\
1.59 E+01 \\
6.70 E+00\end{array}$ & $\begin{array}{l}2.28 E+01 \\
5.10 E+00 \\
3.25 E+01 \\
1.86 E+01 \\
1.05 E+00\end{array}$ & & $\begin{array}{l}.64 E+01 \\
.30 E+00 \\
.23 E+01 \\
.26 E+01 \\
.31 E+00\end{array}$ & $\begin{array}{l}1.89 E+01 \\
3.90 E+00 \\
2.63 E+01 \\
1.49 E+01 \\
6.26 E+00\end{array}$ & & $\begin{array}{l}1.90 E+01 \\
3.91 E+00 \\
2.62 E+01 \\
1.48 E+01 \\
6.25 E+00\end{array}$ \\
\hline $\begin{array}{l}S B-129 \\
\text { IE-121 } \\
\text { TE-1 } 121 \mathrm{H} \\
\text { TE-123 } \\
\text { TE-1 } 123 \mathrm{M}\end{array}$ & 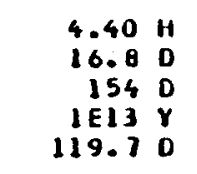 & $\begin{array}{l}1.39 E+01 \\
5.27 E+00 \\
1.94 E+00 \\
1.25 E-02 \\
1.30 E+00\end{array}$ & $\begin{array}{l}1.35 E+01 \\
5.02 E+00 \\
1.39 E+00\end{array}$ & $\begin{array}{l}1.52 E+01 \\
5.59 E+00 \\
1.92 E+00 \\
6.02 E-03 \\
1.26 E+00\end{array}$ & $\begin{array}{l}1.71 E+01 \\
6.71 E+00 \\
2.81 E+00 \\
9.75 E-02 \\
2.07 E+00\end{array}$ & & & & & $\begin{array}{l}1.86 E+00 \\
2.73 E-02 \\
1.23 E+00\end{array}$ \\
\hline $\begin{array}{l}T E-125 M \\
T E-127 \\
T E-127 M \\
\text { IE-129 } \\
\text { TE-129M }\end{array}$ & $\begin{array}{rl}58 & 0 \\
9.35 & H \\
109 & 0 \\
69.6 & H \\
33.6 & D\end{array}$ & $\begin{array}{l}4.37 E-02 \\
4.53 E-02 \\
1.46 E-02\end{array}$ & $\begin{array}{l}5.68 E-02 \\
4.24 E-02 \\
1.83 E-02 \\
4.78 E-01 \\
3.00 E-01\end{array}$ & & & & & & & $\begin{array}{l}2.96 E-02 \\
5.01 E-01 \\
3.21 E-01\end{array}$ \\
\hline 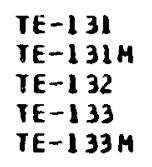 & $\begin{array}{r}25.0 \mathrm{M} \\
30 \mathrm{H} \\
18.2 \mathrm{H} \\
12.45 \mathrm{M} \\
55.4 \mathrm{M}\end{array}$ & $\begin{array}{l}3.98 E+00 \\
1.37 E+01 \\
2.00 E+00 \\
8.90 E+00 \\
2.16 E+01\end{array}$ & $\begin{array}{l}3.79 E+00 \\
1.33 E+01 \\
1.84 E+00 \\
8.61 E+00 \\
2.10 E+01\end{array}$ & $\begin{array}{l}4.20 E+00 \\
1.49 E+01 \\
1.95 E+00 \\
9.59 E+00 \\
2.35 E+01\end{array}$ & $\begin{array}{l}5.29 E+00 \\
1.72 E+01 \\
3.00 E+00 \\
1.13 E+01 \\
2.68 E+01\end{array}$ & & & & $\begin{array}{l}3.60 E * 00 \\
1.29 E * 01 \\
1.77 E * 00 \\
8.33 E * 00 \\
2.03 E * 01\end{array}$ & $\begin{array}{l}3.94 E+0 \\
1.40 E \theta 0 \\
1.92 E+0 \\
9.00 E+0 \\
2.20 E+0\end{array}$ \\
\hline $\begin{array}{l}\text { TE-134 } \\
1-122 \\
1-123 \\
1-124 \\
1-125\end{array}$ & 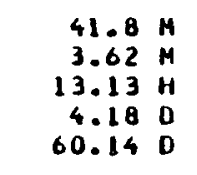 & $\begin{array}{l}8.23 E+00 \\
9.12 E+00 \\
1.44 E+00 \\
1.01 E+01 \\
4.85 E-02\end{array}$ & $\begin{array}{l}7.82 E+00 \\
8.70 E \bullet 00 \\
1.34 E \bullet 00 \\
9.84 E \bullet 00 \\
6.40 E-02\end{array}$ & $\begin{array}{l}8.66 E+00 \\
9.69 E \cdot 00 \\
1.41 E+00 \\
1.10 E+01 \\
2.62 E-02\end{array}$ & $\begin{array}{l}1.06 E+01 \\
1.15 E+01 \\
2.32 E+00 \\
1.27 E+01 \\
3.36 E-01\end{array}$ & $\begin{array}{l}7.65 E+00 \\
8.51 E+00 \\
1.30 E+00 \\
9.69 E+00 \\
2.66 E-02\end{array}$ & & $\begin{array}{l}8.13 E+00 \\
9.03 E+00 \\
1.40 E+00 \\
1.03 E+01 \\
5.93 E-02\end{array}$ & $\begin{array}{l}7.51 E+00 \\
8.34 E+00 \\
1.28 E+00 \\
9.50 E+00 \\
3.89 E-02\end{array}$ & $\begin{array}{l}8.14 E+00 \\
9.04 E+00 \\
1.39 E+00 \\
1.03 E+01 \\
1.08 E-01\end{array}$ \\
\hline $\begin{array}{l}1-126 \\
1-128 \\
1-129 \\
1-130 \\
1-131\end{array}$ & $\begin{array}{r}12.93 \\
24.99 \\
1.5167 \\
12.36 \\
12.36 \\
8.040\end{array}$ & $\begin{array}{l}4.31 E+00 \\
7.01 E-01 \\
4.00 E-02 \\
2.03 E+01 \\
3.58 E+00\end{array}$ & $\begin{array}{l}4.11 E+00 \\
6.63 E-01 \\
5.56 E-02 \\
1.95 E+01 \\
3.34 E+00\end{array}$ & $\begin{array}{l}4.58 E+00 \\
7.34 E-01 \\
2.56 E-02 \\
2.18 E+01 \\
3.68 E+00\end{array}$ & $\begin{array}{l}5.47 E+00 \\
8.99 E-01 \\
2.27 E-01 \\
2.52 E+01 \\
4.66 E+00\end{array}$ & $\begin{array}{l}4.02 E+00 \\
6.46 E-01 \\
2.49 E-02 \\
1.91 E+01 \\
3.26 E+00\end{array}$ & $\begin{array}{l}3.63 E+00 \\
5.82 E-01 \\
2.82 E-02 \\
1.73 E+01 \\
2.93 E 000\end{array}$ & $\begin{array}{l}4.28 E * 00 \\
6.86 E-01 \\
4.51 E-02 \\
2.03 E * 01 \\
3.46 E+00\end{array}$ & $\begin{array}{l}3.95 E \bullet 00 \\
6.35 E-01 \\
3.31 E-02 \\
1.87 E \bullet 01 \\
3.22 E \bullet 00\end{array}$ & $\begin{array}{l}4.29 \mathrm{E}- \\
6.88 \mathrm{E}- \\
8.65 \mathrm{E}- \\
2.03 \mathrm{Et} \\
3.46 \mathrm{Et}\end{array}$ \\
\hline
\end{tabular}


DOSE-RATE FACTORS FOR BDOY OPGANS AND EFFECTIVE OUSE-RATE FAC TUR FOP IMMERSION IN CUNIAMITATEU AATER
IN MREM/YR PER MICROCURIE/ICUBIC MI

\begin{tabular}{|c|c|c|c|c|c|c|c|c|c|c|}
\hline CLIDE & ALF-LIFE & LIVER & LUNGS & ARROH & $\mathrm{OH}$ & OVARIES & ANCREAS & ELETON & SPLEEN & S TOMAC \\
\hline $\begin{array}{l}1 N-117 \\
1 N-117 M \\
S N-113 \\
S N-111 M \\
S N-119 M\end{array}$ & $\begin{array}{rl}43.8 & 4 \\
116.5 & 4 \\
115.1 & 0 \\
13.60 & 0 \\
293.0 & 0\end{array}$ & $\begin{array}{l}0 \\
\text { a }\end{array}$ & & & & & & & $\begin{array}{l}5.97 E+00 \\
1.52 E-01 \\
5.16 E-0.2 \\
1.24 E+00 \\
6.22 E-03\end{array}$ & $\begin{array}{l}.02 E+00 \\
.50 E-01 \\
.27 E-02 \\
.23 E \cdot 00 \\
.12 E-03\end{array}$ \\
\hline $\begin{array}{l}S N-123 \\
S N-125 \\
S N-126 \\
S B-117 \\
S B-122\end{array}$ & $\begin{array}{r}129.20 \\
9.64 \\
1.065 \\
2.80 \\
2.70 \\
2.70\end{array}$ & $6.52 E-02$ & $\begin{array}{l}.93 E-02 \\
.10 E+00 \\
.40 E-01 \\
\end{array}$ & & & & & $\begin{array}{r}7.48 E-02 \\
3.36 E+00 \\
7.74 E-01 \\
7.3 E-000\end{array}$ & $\begin{array}{l}6.31 E-02 \\
2.82 E+00 \\
3.73 E-01 \\
1.45 E+00 \\
3.87 E+00\end{array}$ & $\begin{array}{l}6.42 E-02 \\
2.87 E+00 \\
3.78 E-01 \\
1.44 E+00 \\
3.92 E+00\end{array}$ \\
\hline $\begin{array}{l}S B-124 \\
S B-125 \\
S B-126 \\
S B-126 M \\
S B-127\end{array}$ & $\begin{array}{rl}60.20 & 0 \\
2.77 & Y \\
12.4 & 0 \\
19.0 & 4 \\
3.85 & 0\end{array}$ & $\begin{array}{l}1.80 E+01 \\
3.73 E+00 \\
2.50 E+01 \\
1.42 E+01 \\
5.96 E+00\end{array}$ & $\begin{array}{l}.91 E+01 \\
01 E+00 \\
067 E+01 \\
.52 E+01 \\
.39 E+00 \\
39 E+00\end{array}$ & $\begin{array}{l}.04 E+01 \\
.65 E+00 \\
.99 E \cdot 01 \\
.71 E+01 \\
.21 E+00\end{array}$ & $\begin{array}{l}93 E+01 \\
08 E+00 \\
13 E \bullet 01 \\
55 E \bullet 01 \\
51 E \bullet 00\end{array}$ & $\begin{array}{l}1.69 E+01 \\
3.56 E+00 \\
2.42 E+01 \\
1.37 E+01 \\
5.75 E+00\end{array}$ & $\begin{array}{l}.56 E+01 \\
.21 E \cdot 00 \\
2.15 E+01 \\
1.22 E+01 \\
.14 E+00\end{array}$ & & & $\begin{array}{l}1.76 E+01 \\
3.6 B E+00 \\
2.47 E+01 \\
1.40 E+01 \\
5.89 E+00\end{array}$ \\
\hline $\begin{array}{l}S B-129 \\
T E-121 \\
T E-121 M \\
T E-123 \\
T E-123 M\end{array}$ & $\begin{array}{rl}4.40 & H \\
16.88 & 0 \\
1540 \\
1113 \\
119.7 & 0\end{array}$ & b & & & & & & & & $\begin{array}{l}1.76 E+ \\
1.10 E-0 \\
1.17 E+C\end{array}$ \\
\hline $\begin{array}{l}T E-125 M \\
T E-127 \\
T E-127 M \\
T E-129 \\
T E-129 M\end{array}$ & $\begin{array}{rl}58 & 0 \\
9.35 & H \\
109 & 0 \\
69.6 & H \\
33.6 & 0\end{array}$ & $\begin{array}{l}4.09 E-02 \\
4.22 E-02 \\
1.36 E-02\end{array}$ & & & & & & & & $2.92 \mathrm{E}-1$ \\
\hline $\begin{array}{l}T E-131 \\
T E-131 M \\
T E-132 \\
T E-133 \\
T E-133 M\end{array}$ & $\begin{array}{r}25.0 \mathrm{M} \\
30 \mathrm{H} \\
78.2 \mathrm{H} \\
12.45 \mathrm{M} \\
55.4 \mathrm{M}\end{array}$ & $\begin{array}{l}3.77 E+00 \\
1.33 E+01 \\
1.82 E+00\end{array}$ & & & & & & & & $\begin{array}{l}8.44 \mathrm{Ea} \\
2.06 \mathrm{E}\end{array}$ \\
\hline $\begin{array}{l}T E-134 \\
1-122 \\
1-123 \\
1-124 \\
1-125\end{array}$ & $\begin{array}{rl}41.8 & M \\
3.62 & M \\
13.13 & H \\
4.18 & 0 \\
60.14 & 0\end{array}$ & $\begin{array}{l}7.77 \mathrm{E} \\
8.65 \mathrm{E} \\
1.32 \mathrm{E} \\
9.81 \mathrm{E} \\
4.52 \mathrm{E}\end{array}$ & $\begin{array}{l}8.35 E \\
9.21 E \\
1.46 E \\
1.04 E \\
6.29 E\end{array}$ & $\begin{array}{l}1.00 E \\
2.11 E \\
1.15 E \\
1.04 E\end{array}$ & $\begin{array}{l}1.37 E \\
1.06 E \\
2.29 E-\end{array}$ & $\begin{array}{l}7.38 E+00 \\
8.30 E+00 \\
1.17 E+00 \\
9.32 E+00 \\
4.47 E-02\end{array}$ & $\begin{array}{l}6.67 E+00 \\
7.44 E+00 \\
1.11 E+00 \\
6.47 E+00 \\
2.99 E-02\end{array}$ & $\begin{array}{l}9.91 E \bullet 00 \\
1.0 B E+01 \\
2.16 E+00 \\
1.16 E+01 \\
1.09 E-01\end{array}$ & $\begin{array}{l}1.59 E+00 \\
8.43 E+00 \\
1.32 E+00 \\
9.50 E+00 \\
3.91 E-02\end{array}$ & $\begin{array}{l}7.67 E+C \\
8.53 E+C \\
1.31 E+C \\
9.64 E+C \\
4.45 E-C\end{array}$ \\
\hline $\begin{array}{l}1-126 \\
1-128 \\
1-129 \\
1-130 \\
1-131\end{array}$ & $\begin{array}{r}12.930 \\
24.99 \\
1.57 E 7 \\
12.36 \\
8.040 \\
8.04\end{array}$ & $\begin{array}{l}4.08 E+00 \\
6.58 E-01 \\
4.21 E-02 \\
1.94 E+01 \\
3.32 E+00\end{array}$ & $\begin{array}{l}4.3 \theta E+00 \\
7.08 E-01 \\
5.25 E-02 \\
2.01 E+01 \\
3.58 E+00\end{array}$ & $\begin{array}{l}4.97 E+00 \\
8.28 E-01 \\
8.96 E-02 \\
2.31 E+01 \\
4.33 E+00\end{array}$ & $\begin{array}{l}4.46 E+00 \\
7.26 E-01 \\
2.00 E-02 \\
2.11 E+01 \\
3.68 E+00\end{array}$ & $\begin{array}{l}3.93 E+00 \\
6.27 E-01 \\
3.79 E-02 \\
1.87 E+01 \\
3.11 E+00\end{array}$ & $\begin{array}{l}3.52 E+00 \\
5.67 E-01 \\
2.50 E-02 \\
1.67 E+01 \\
2.87 E+00\end{array}$ & $\begin{array}{l}8.42 E-01 \\
9.26 E-02 \\
2.35 E+01 \\
4.41 E+00\end{array}$ & $\begin{array}{l}3.98 E+00 \\
6.44 E-01 \\
3.43 E-02 \\
1.89 E+01 \\
3.27 E+00\end{array}$ & $\begin{array}{l}6.50 \mathrm{E}-\mathrm{C} \\
3.86 \mathrm{E}-\mathrm{C} \\
1.91 \mathrm{E} C \\
3.28 \mathrm{E} \cdot \mathrm{C}\end{array}$ \\
\hline
\end{tabular}


DOSE-RATE FACTORS FUR BOOY ORGANS ANO EFFECTIVE DOSE-RATE FACTOR FOR IMMERSIGN IN CONTAMINATLU WAILK
IN MREMTYR PER MICROCUR IE/ICUEIC MI

\begin{tabular}{|c|c|c|c|c|c|c|c|c|c|}
\hline NUCL IOE & HALF-LIFE & TESTES & THYAUS & THY ROID & UTERUS & FFECTIVE & $\begin{array}{c}\text { SKIN } \\
\text { (PHOTON) }\end{array}$ & $\begin{array}{c}\text { SKIV } \\
\text { IELECIRON I }\end{array}$ & $\begin{array}{l}\text { SKIN } \\
\text { ITOTALI }\end{array}$ \\
\hline $\begin{array}{l}1 N-117 \\
1 N-117 M \\
S N-113 \\
S N-117 M \\
S N-119 M\end{array}$ & 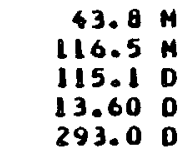 & $\begin{array}{l}8.86 E+00 \\
1.13 E * 00 \\
1.26 E-01 \\
1.93 E \cdot 00 \\
4.04 E-02\end{array}$ & $\begin{array}{l}6.77 E+00 \\
8.51 E-01 \\
6.03 E-02 \\
1.45 E+00 \\
8.86 E-03\end{array}$ & $\begin{array}{l}8.33 E+00 \\
1.07 E+00 \\
9.32 E-02 \\
1.87 E+00 \\
2.21 E-02\end{array}$ & $\begin{array}{l}5.33 E+00 \\
6.00 E-01 \\
4.02 E-02 \\
1.09 E+00 \\
1.86 E-03\end{array}$ & $\begin{array}{l}7.63 E+00 \\
9.87 E-01 \\
9.87 E-02 \\
1.69 E+00 \\
2.82 E-02\end{array}$ & $\begin{array}{l}1.00 E+01 \\
1.28 E \cdot 00 \\
2.20 E-01 \\
2.16 E+00 \\
9.37 E-02\end{array}$ & $\begin{array}{l}1.64 E \bullet 00 \\
3.31 E \bullet 00 \\
0.0 \\
5.06 E-01 \\
0.0\end{array}$ & $\begin{array}{l}1.17 E+0 ! \\
4.59 E+00 \\
2.20 E-01 \\
2.60 E+00 \\
9.37 E-02\end{array}$ \\
\hline $\begin{array}{l}S N-123 \\
S N-125 \\
S N-126 \\
S B-117 \\
S B-122\end{array}$ & $\begin{array}{r}129.20 \\
9.640 \\
1.065 \mathrm{Y} \\
2.80 \mathrm{H} \\
2.70 \mathrm{O}\end{array}$ & $\begin{array}{l}9.13 E-02 \\
4.08 E+00 \\
6.77 E-01 \\
2.25 E \cdot 00 \\
5.69 E \cdot 00\end{array}$ & $\begin{array}{l}1.33 E-02 \\
3.25 E \bullet 00 \\
5.06 E-01 \\
1.69 E * 00 \\
4.36 E \cdot 00\end{array}$ & $\begin{array}{l}8.72 E-02 \\
3.86 E+00 \\
6.58 E-01 \\
2.15 E+00 \\
5.31 E+00\end{array}$ & $\begin{array}{l}5.72 E-02 \\
2.57 \mathrm{E}+00 \\
3.22 \mathrm{E}-01 \\
1.27 \mathrm{E}+00 \\
3.46 \mathrm{E}+00\end{array}$ & $\begin{array}{l}7.82 E-02 \\
3.50 E+00 \\
5.75 E-01 \\
1.96 E+00 \\
4.86 E+00\end{array}$ & $\begin{array}{l}1.05 E-01 \\
4.69 E \bullet 00 \\
7.70 E-01 \\
2.54 E \cdot 00 \\
6.53 E+00\end{array}$ & $\begin{array}{l}4.16 E+00 \\
6.78 E+00 \\
1.48 E-01 \\
8.64 E-02 \\
4.45 E+00\end{array}$ & $\begin{array}{l}4.26 E+00 \\
1.15 E+01 \\
9.17 E-01 \\
2.63 E+00 \\
1.10 E+01\end{array}$ \\
\hline $\begin{array}{l}S B-124 \\
S B-125 \\
S B-126 \\
S B-126 A \\
S B-127\end{array}$ & $\begin{array}{r}60.20 \\
2.77 \\
12.40 \\
19.0 \mathrm{H} \\
3.85 \mathrm{D}\end{array}$ & $\begin{array}{l}2.50 E+01 \\
5.42 E+00 \\
3.57 E+01 \\
2.03 E+01 \\
8.54 E+00\end{array}$ & $\begin{array}{l}1.97 E+01 \\
4.11 E+00 \\
2.77 E+01 \\
1.57 E+01 \\
6.60 E+00\end{array}$ & $\begin{array}{l}2.34 E+01 \\
5.04 E+00 \\
3.36 E+01 \\
1.90 E+01 \\
8.02 E+00\end{array}$ & $\begin{array}{l}1.60 E+01 \\
3.25 E+00 \\
2.10 E+01 \\
1.24 E+01 \\
5.20 E+00\end{array}$ & $\begin{array}{l}2.15 E+01 \\
4.63 E+00 \\
3.05 E+01 \\
1.73 E+01 \\
7.30 E+00\end{array}$ & $\begin{array}{l}2.87 E+01 \\
6.24 E+00 \\
4.10 E+01 \\
2.33 E+01 \\
9.80 E+00\end{array}$ & $\begin{array}{l}2.80 E+00 \\
3.54 E-01 \\
2.10 E+00 \\
4.72 E+00 \\
2.16 E+00\end{array}$ & $\begin{array}{l}3.14 E+01 \\
6.59 E+00 \\
4.31 E+01 \\
2.80 E+01 \\
1.20 E+01\end{array}$ \\
\hline $\begin{array}{l}S B-129 \\
T E-121 \\
T E-121 H \\
T E-123 \\
T E-123 H\end{array}$ & $\begin{array}{rl}4.40 & H \\
16.8 & 0 \\
154 & 0 \\
1 E 13 & \gamma \\
119.7 & 0\end{array}$ & $\begin{array}{l}1.89 E \bullet 01 \\
1.22 E \bullet 00 \\
2.67 E \bullet 00 \\
5.60 E-02 \\
1.84 E \bullet 00\end{array}$ & $\begin{array}{l}1.50 E+01 \\
5.47 E+00 \\
2.02 E+00 \\
1.57 E-02 \\
1.39 E+00\end{array}$ & $\begin{array}{l}1.79 E+01 \\
6.69 E+00 \\
2.54 E+00 \\
3.34 E-02 \\
1.78 E+00\end{array}$ & $\begin{array}{l}1.18 E+01 \\
4.33 E+00 \\
1.57 E+00 \\
3.64 E-03 \\
1.04 E+00\end{array}$ & $\begin{array}{l}1.62 E+01 \\
6.15 E+00 \\
2.33 E+00 \\
3.91 E-02 \\
1.61 E+00\end{array}$ & $\begin{array}{l}2.18 E \cdot 01 \\
8.36 E+00 \\
3.02 E+00 \\
1.16 E-01 \\
2.04 E+00\end{array}$ & $\begin{array}{l}2.58 E+00 \\
2.00 E-02 \\
7.29 E-02 \\
0.0 \\
8.57 E-02\end{array}$ & $\begin{array}{l}2.44 E+01 \\
8.38 E+00 \\
3.10 E+00 \\
1.16 E-01 \\
2.13 E+00\end{array}$ \\
\hline $\begin{array}{l}\text { TE-125M } \\
\text { TE-127 } \\
\text { TE-127M } \\
\text { TE-129 } \\
\text { TE-129M }\end{array}$ & $\begin{array}{rl}58 & D \\
9.35 & H \\
109 & D \\
69.6 & A \\
33.6 & D\end{array}$ & $\begin{array}{l}1.75 E-01 \\
6.12 E-02 \\
5.59 E-02 \\
6.97 E-01 \\
4.47 E-01\end{array}$ & $\begin{array}{l}6.26 E-02 \\
4.62 E-02 \\
2.02 E-02 \\
5.22 E-01 \\
3.32 E-01\end{array}$ & $\begin{array}{l}1.14 E-01 \\
5.70 E-02 \\
3.67 E-02 \\
6.43 E-01 \\
4.10 E-01\end{array}$ & $\begin{array}{l}1.81 E-02 \\
3.70 E-02 \\
6.37 E-03 \\
4.13 E-01 \\
2.53 E-01\end{array}$ & $\begin{array}{l}1.24 E-01 \\
5.20 E-02 \\
3.97 E-02 \\
5.94 E-01 \\
3.75 E-01\end{array}$ & $\begin{array}{l}3.29 E-01 \\
6.93 E-02 \\
1.05 E-01 \\
8.13 E-01 \\
5.45 E-01\end{array}$ & $\begin{array}{l}9.12 E-02 \\
1.37 E+00 \\
5.05 E-02 \\
4.05 E+00 \\
1.76 E+00\end{array}$ & $\begin{array}{l}4.20 E-01 \\
1.44 E * 00 \\
1.55 E-01 \\
4.86 E * 00 \\
2.30 E * 00\end{array}$ \\
\hline $\begin{array}{l}\text { TE- } 131 \\
T E-131 M \\
T E-132 \\
T E-133 \\
T E-133 M\end{array}$ & $\begin{array}{r}25.0 \mathrm{M} \\
30 \mathrm{H} \\
78.2 \mathrm{H} \\
12.45 \mathrm{M} \\
55.4 \mathrm{H}\end{array}$ & $\begin{array}{l}5.47 E+00 \\
1.88 E+01 \\
2.79 E+00 \\
1.22 E+01 \\
2.95 E+01\end{array}$ & $\begin{array}{l}4.25 E+00 \\
1.48 E+01 \\
2.08 E+00 \\
9.48 E+00 \\
2.33 E+01\end{array}$ & $\begin{array}{l}5.21 E+00 \\
1.78 E+01 \\
2.64 E+00 \\
1.14 E+01 \\
2.79 E+01\end{array}$ & & & & & \\
\hline $\begin{array}{l}T E-134 \\
1-122 \\
1-123 \\
1-124 \\
1-125\end{array}$ & $\begin{array}{r}41.8 \mathrm{M} \\
3.62 \\
13.13 \\
4.18 \\
60.14 \\
60\end{array}$ & $\begin{array}{l}1.12 E+01 \\
1.24 E+01 \\
2.08 E+00 \\
1.39 E+01 \\
2.04 E-01\end{array}$ & $\begin{array}{l}9.67 E+00 \\
9.44 E+00 \\
1.54 E+00 \\
1.07 E+01 \\
6.99 E-02\end{array}$ & $\begin{array}{l}1.06 E * 01 \\
1.15 E+01 \\
1.99 E \cdot 00 \\
1.29 E \cdot 01 \\
1.31 E-01\end{array}$ & & & & $\begin{array}{l}5.34 E-01 \\
8.84 E+00 \\
6.54 E-02 \\
1.62 E+00 \\
0.0\end{array}$ & \\
\hline $\begin{array}{l}1-120 \\
1-128 \\
1-129 \\
1-130 \\
1-131\end{array}$ & $\begin{array}{r}12.93 \mathrm{O} \\
24.99 \mathrm{M} \\
1.57 \mathrm{~V} \\
12.36 \mathrm{H} \\
8.040 \mathrm{D}\end{array}$ & $\begin{array}{l}5.89 E+00 \\
9.54 E-01 \\
1.49 E-01 \\
2.76 E+01 \\
4.83 E+00\end{array}$ & $\begin{array}{l}4.52 E+00 \\
7.20 E-01 \\
6.67 E-02 \\
2.14 E+01 \\
3.66 E+00\end{array}$ & $\begin{array}{l}5.50 E+00 \\
8.84 E-01 \\
1.06 E-01 \\
2.60 E+01 \\
4.51 E+00\end{array}$ & $\begin{array}{l}3.55 E+00 \\
5.76 E-01 \\
2.08 E-02 \\
1.69 E+01 \\
2.92 E+00\end{array}$ & $\begin{array}{l}5.03 E+00 \\
8.17 E-01 \\
1.07 E-01 \\
2.36 E+01 \\
4.17 E+00\end{array}$ & $\begin{array}{l}6.00 E+00 \\
1.09 E+00 \\
2.50 E-01 \\
3.18 E+01 \\
5.47 E+00\end{array}$ & $\begin{array}{l}9.25 E-01 \\
6.21 E+00 \\
1.87 E-02 \\
1.92 E+00 \\
1.06 E+00\end{array}$ & $\begin{array}{l}7.73 E+00 \\
7.30 E+00 \\
2.69 E-01 \\
3.37 E * 01 \\
6.53 E * 00\end{array}$ \\
\hline
\end{tabular}




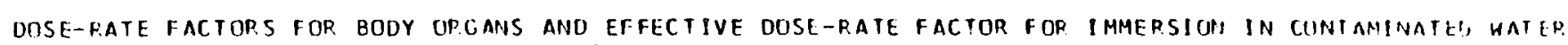

IN MREM/YR PER MICROCURIEIICUBIC MI

\begin{tabular}{|c|c|c|c|c|c|c|c|c|c|c|}
\hline$C L 10 E$ & ILF-LIFE & DRENALS & BL ADDER & BRAIN & BREAST & HE AP.T & $\begin{array}{l}\text { SMALL } \\
\text { INTESTINE }\end{array}$ & $\begin{array}{c}\text { UPPER } \\
\text { LAPGE } \\
\text { INTESTINE }\end{array}$ & $\begin{array}{c}\text { LOWER } \\
\text { LARGE } \\
\text { INTESTINEE }\end{array}$ & K IONEYS \\
\hline $\begin{array}{l}1-132 \\
1-133 \\
1-134 \\
1-135 \\
1-136\end{array}$ & $\begin{array}{rl}2.30 & H \\
20.8 & H \\
52.6 & M \\
6.61 & H \\
83 & S\end{array}$ & $\begin{array}{l}.20 E+01 \\
.73 E+00 \\
.55 E+01 \\
.52 E+01 \\
.54 E+01\end{array}$ & $\begin{array}{l}13 E+01 \\
48 E+00 \\
48 E+01 \\
52 E+01 \\
57 E+01\end{array}$ & $\begin{array}{l}39 E \bullet 01 \\
12 E+00 \\
.79 E+01 \\
11 E+01 \\
87 E \bullet 01\end{array}$ & $\begin{array}{l}1 E+01 \\
6 E+00 \\
4 E+01 \\
0 E+01 \\
2 E+01\end{array}$ & $\begin{array}{l}10 E+01 \\
37 E+00 \\
45 E+01 \\
51 E+01 \\
56 E+01\end{array}$ & $\begin{array}{l}1.90 E+01 \\
4.84 E+00 \\
2.23 E+01 \\
1.39 E+01 \\
2.38 E+01\end{array}$ & $\begin{array}{l}2.23 E+01 \\
5.70 E * 00 \\
2.61 E+01 \\
1.60 E+01 \\
2.72 E+01\end{array}$ & $\begin{array}{l}2.05 E+01 \\
5.26 E+00 \\
2.40 E+01 \\
1.48 E+01 \\
2.52 E+01\end{array}$ & $\begin{array}{l}2.23 E+01 \\
5.70 E+00 \\
2.60 E+01 \\
1.60 E+01 \\
2.75 E+01\end{array}$ \\
\hline $\begin{array}{l}X E-122 \\
X E-123 \\
X E-125 \\
X E-127 \\
X E-129 M\end{array}$ & $\begin{array}{r}20.1 \mathrm{H} \\
2.14 \mathrm{H} \\
16.8 \mathrm{H} \\
36.406 \mathrm{D} \\
8.89 \mathrm{D}\end{array}$ & $\begin{array}{l}5.31 E-01 \\
5.65 E+00 \\
2.29 E+00 \\
2.42 E+00 \\
1.53 E-01\end{array}$ & $\begin{array}{l}.06 E-01 \\
.65 E+00 \\
.14 E+00 \\
.24 E+00 \\
10 E-01\end{array}$ & & $\begin{array}{l}.76 E-01 \\
.76 E+00 \\
.40 E+00 \\
62 E+00 \\
.10 E-01\end{array}$ & $\begin{array}{l}.71 E-01 \\
.54 E+00 \\
.06 E+00 \\
16 E+00 \\
.1 B E-01\end{array}$ & & & $\begin{array}{l}4.73 E-01 \\
5.45 E \cdot 00 \\
2.05 E \bullet 00 \\
2.15 E+00 \\
1.32 E-01\end{array}$ & $\begin{array}{l}5.46 E-01 \\
5.91 E+00 \\
2.24 E+00 \\
2.33 E+00 \\
2.25 E-01\end{array}$ \\
\hline $\begin{array}{l}E-131 M \\
E-133 \\
E-133 M \\
E-135 \\
E-135 M\end{array}$ & $\begin{array}{r}11.84 \\
5.245 \\
2.19 \\
9.11 \\
15.36 \\
15\end{array}$ & & & & & & & & & $\begin{array}{l}24 \mathrm{E}-02 \\
12 \mathrm{E}-01 \\
69 \mathrm{E}-01 \\
21 \mathrm{E}+00 \\
96 \mathrm{E}+00\end{array}$ \\
\hline$X E-137$ & $\begin{array}{r}3.83 \mathrm{M} \\
14.13 \mathrm{M} \\
1.64 \mathrm{M} \\
32.06 \mathrm{H} \\
9.688 \mathrm{D}\end{array}$ & $\begin{array}{l}1.77 E+00 \\
1.13 E+01 \\
1.06 E+01\end{array}$ & $\begin{array}{l}1.70 E \bullet 00 \\
1.14 E \cdot 01 \\
1.01 E+01 \\
2.26 E+00\end{array}$ & & & & & & & $\begin{array}{l}1.11 E+00 \\
1.21 E+01 \\
1.05 E+01 \\
2.38 E+00 \\
1.33 E-02\end{array}$ \\
\hline 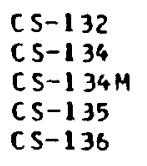 & $\begin{array}{r}6.475 \mathrm{O} \\
2.062 \mathrm{Y} \\
2.90 \mathrm{H} \\
2.3 E 6 \mathrm{Y} \\
13.16 \mathrm{D}\end{array}$ & $\begin{array}{l}6.64 E+00 \\
1.49 E+01 \\
1.11 E-01 \\
0.0 \\
2.09 E+01\end{array}$ & $\begin{array}{l}6.37 E+00 \\
1.43 E+01 \\
1.67 E-01 \\
0.0 \\
2.02 E+01\end{array}$ & $\begin{array}{l}7.11 E+00 \\
1.60 E+01 \\
1.64 E-01 \\
0.0 \\
2.27 E+01\end{array}$ & $\begin{array}{l}8.34 E+0 C \\
1.83 E+01 \\
3.48 E-01 \\
0.0 \\
2.60 E+01\end{array}$ & $\begin{array}{l}6.23 E \cdot 00 \\
1.41 E+01 \\
1.52 E-01 \\
0.0 \\
2.00 E+01\end{array}$ & $5.63 E+00$ & & $\begin{array}{l}0.11 E \cdot 00 \\
1.38 E+01 \\
1.52 E-01 \\
0.0 \\
1.96 E+01\end{array}$ & $\begin{array}{l}6.67 E \cdot 00 \\
1.49 E+01 \\
1.83 E-01 \\
0.0 \\
2.12 E+01\end{array}$ \\
\hline $\begin{array}{l}C S-137 \\
C S-138 \\
C S-139 \\
B A-131 \\
B A-133\end{array}$ & $\begin{array}{r}30.17 \mathrm{Y} \\
32.2 \mathrm{M} \\
9.40 \mathrm{M} \\
11.8 \mathrm{O} \\
10.5 \mathrm{Y}\end{array}$ & $\begin{array}{l}0.0 \\
2.31 E+01 \\
3.02 E+00 \\
4.20 E * 00 \\
3.35 E+00\end{array}$ & $\begin{array}{l}2.32 E \mathrm{Et} \\
3.05 \mathrm{E} \\
3.98 \mathrm{EE} \\
3.16 \mathrm{E}\end{array}$ & $\begin{array}{l}0.0 \\
2.60 E+01 \\
3.42 E+00\end{array}$ & $\begin{array}{l}0.0 \\
2.92 E+01 \\
3.81 E+00 \\
5.70 E+00 \\
4.86 E+00\end{array}$ & & & & $\begin{array}{l}0.0 \\
2.26 E \cdot 01 \\
2.98 E+00 \\
3.80 E+00 \\
2.99 E+00\end{array}$ & \\
\hline $\begin{array}{l}8 A-133 M \\
8 A-135 M \\
B A-137 M \\
B A-139 \\
B A-140\end{array}$ & $\begin{array}{r}38.9 \mathrm{H} \\
28.7 \mathrm{H} \\
2.552 \mathrm{M} \\
83.1 \mathrm{M} \\
12.789 \mathrm{O}\end{array}$ & $\begin{array}{l}5.02 E-01 \\
4.39 E-01 \\
5.68 E+00 \\
3.28 E-01 \\
1.75 E \cdot 00\end{array}$ & $\begin{array}{l}4.78 E-01 \\
4.19 E-01 \\
5.43 E+00 \\
3.05 E-01 \\
1.66 E+00\end{array}$ & $\begin{array}{l}4.88 E-01 \\
4.24 E-01 \\
6.09 E+00 \\
3.28 E-01 \\
1.83 E+00\end{array}$ & $\begin{array}{l}0.12 E-01 \\
7.29 E-01 \\
1.02 E+00 \\
4.91 E-01 \\
2.28 E+00\end{array}$ & $\begin{array}{l}4.41 E-01 \\
3.84 E-01 \\
5.34 E+00 \\
2.99 E-01 \\
1.62 E+00\end{array}$ & $\begin{array}{l}4.01 E-01 \\
3.49 E-01 \\
4.81 E 000 \\
2.66 E-01 \\
1.45 E+00\end{array}$ & $\begin{array}{l}4.81 E-01 \\
4.21 E-01 \\
5.67 E+00 \\
3.19 E-01 \\
1.72 E+00\end{array}$ & $\begin{array}{l}4.44 E-01 \\
3.80 E-01 \\
5.22 E+00 \\
2.95 E-01 \\
1.59 E+00\end{array}$ & $\begin{array}{l}5.15 E-01 \\
4.55 E-01 \\
5.67 E-00 \\
3.15 E-01 \\
1.72 E+00\end{array}$ \\
\hline $\begin{array}{l}B A-141 \\
B A-142 \\
L A-140 \\
L A-141 \\
L A-142\end{array}$ & $\begin{array}{r}18.27 \mathrm{M} \\
10.70 \mathrm{M} \\
40.22 \mathrm{H} \\
3.94 \mathrm{H} \\
95.4 \mathrm{M}\end{array}$ & $\begin{array}{l}8.50 E * 00 \\
8.64 E+00 \\
2.24 E+01 \\
4.06 E-01 \\
2.81 E+01\end{array}$ & $\begin{array}{l}8.16 E+00 \\
8.41 E+00 \\
2.24 E+01 \\
4.07 E-01 \\
2.86 E+01\end{array}$ & $\begin{array}{l}9.05 E+00 \\
9.40 E+00 \\
2.51 E+01 \\
4.59 E-01 \\
3.18 E+01\end{array}$ & $\begin{array}{l}1.10 E+01 \\
1.09 E+01 \\
2.82 E+01 \\
5.07 E-01 \\
3.59 E+01\end{array}$ & $\begin{array}{l}8.03 E+00 \\
8.28 E+00 \\
2.21 E+01 \\
4.03 E-01 \\
2.85 E+01\end{array}$ & $\begin{array}{l}7.29 E+00 \\
7.54 E+00 \\
2.04 E+01 \\
3.73 E-01 \\
2.65 E \cdot 01\end{array}$ & $\begin{array}{l}8.53 E+00 \\
8.81 E+00 \\
2.36 E+01 \\
4.30 E-01 \\
3.03 E+01\end{array}$ & $\begin{array}{l}7.90 E+00 \\
8.12 E+00 \\
2.17 E+01 \\
3.96 E-01 \\
2.80 E+01\end{array}$ & $\begin{array}{l}\text { B. } 81 E+00 \\
2.36 E+01 \\
4.29 E-01 \\
3.06 E+01\end{array}$ \\
\hline
\end{tabular}




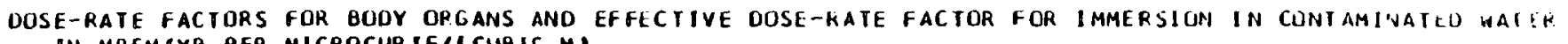

IN MREM/YR PER HICROCURIE/ICUBIC HI

\begin{tabular}{|c|c|c|c|c|c|c|c|c|c|c|}
\hline $30 \mathrm{E}$ & ALF-LIFE & LIVER & LUNGS & ARROH & $\begin{array}{c}\text { RED } \\
\text { MARROH }\end{array}$ & OVARIES & ANCREAS & EELETON & SPLEEN & S TOMA \\
\hline $\begin{array}{l}1-132 \\
1-133 \\
1-134 \\
1-135\end{array}$ & $\begin{array}{rl}2.30 & H \\
20.8 & H \\
52.6 & H \\
6.61 & H \\
B 3 & S\end{array}$ & $\begin{array}{l}1 \\
0 \\
1 \\
1\end{array}$ & & & $\begin{array}{l}30 E+01 \\
95 E+00 \\
67 E+01 \\
62 E+01\end{array}$ & & & $\begin{array}{l}51 E+01 \\
69 E+00 \\
89 E+01 \\
72 E+01 \\
86 E+01\end{array}$ & $\begin{array}{l}.06 E+01 \\
.30 E+00 \\
.39 E+01 \\
.46 E+01 \\
.48 E+01\end{array}$ & $\begin{array}{l}2.09 E+01 \\
5.37 E+00 \\
.44 E+01\end{array}$ \\
\hline $\begin{array}{l}X E-122 \\
\times E-123 \\
\times E-125 \\
\times E-127 \\
\times E-1294\end{array}$ & 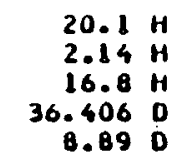 & $\begin{array}{l}4.92 E-01 \\
5.62 E+00 \\
2.12 E+00 \\
2.22 E+00 \\
1.48 E-01\end{array}$ & $\begin{array}{l}.40 E-01 \\
.03 E+00 \\
.30 E+00 \\
.42 E+00 \\
.13 E-01\end{array}$ & $\begin{array}{l}7.06 E-01 \\
7.03 E+00 \\
3.05 E+00 \\
3.29 E+00 \\
2.67 E-01\end{array}$ & $\begin{array}{l}5.21 E-01 \\
6.04 E+00 \\
2.27 E+00 \\
2.39 E+00 \\
1.19 E-01\end{array}$ & $\begin{array}{l}4.54 E-01 \\
5.27 E+00 \\
1.91 E+00 \\
1.98 E+00 \\
1.32 E-01\end{array}$ & $\begin{array}{l}4.16 E-01 \\
4.83 E+00 \\
1.80 E+00 \\
1.89 E+00 \\
1.09 E-01\end{array}$ & & & $\begin{array}{l}4.85 \mathrm{E}- \\
5.53 \mathrm{Et}\end{array}$ \\
\hline $\begin{array}{l}E-131 M \\
E-133 \\
E-133 M \\
E-135 \\
E-135 M\end{array}$ & $\begin{array}{rl}11.84 & 0 \\
5.245 & 0 \\
2.19 & 0 \\
9.11 & H \\
15.36 & H\end{array}$ & $\begin{array}{l}5.25 E-02 \\
2.76 E-01 \\
2.32 E-01 \\
2.13 E+00 \\
3.79 E \cdot 00\end{array}$ & $\begin{array}{l}6.23 E-02 \\
3.08 E-01 \\
2.57 E-01 \\
2.31 E+00 \\
4.07 E \cdot 00\end{array}$ & $\begin{array}{l}9.83 E-02 \\
5.32 E-01 \\
3.56 E-01 \\
3.01 E+00 \\
4.67 E+00\end{array}$ & $\begin{array}{l}4.10 E-02 \\
2.01 E-01 \\
2.40 E-01 \\
2.36 E+00 \\
4.15 E+00\end{array}$ & $\begin{array}{l}4.71 E-02 \\
2.31 E-01 \\
2.07 E-01 \\
1.92 E+00 \\
3.64 E+00\end{array}$ & & & & $\begin{array}{l}5.06 E-C \\
2.62 E-C \\
2.28 E-C \\
2.11 E+C \\
3.74 E+C\end{array}$ \\
\hline 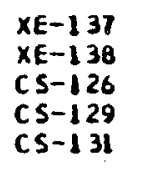 & $\begin{array}{rl}3.83 & H \\
14.13 & H \\
1.64 & H \\
32.06 & H \\
9.688 & D\end{array}$ & $\begin{array}{l}\text { G9E+00 } \\
14 E+01 \\
00 E+01\end{array}$ & & & & & & & & $\begin{array}{l}\text { 1. 11E+ } \\
9.87 E E \\
2.20 E+ \\
3.11 E-\end{array}$ \\
\hline $\begin{array}{l}\text { CS }-132 \\
C S-134 \\
C S-134 M \\
C S-135 \\
C S-136\end{array}$ & 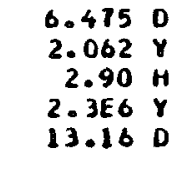 & $\begin{array}{l}0.32 E+00 \\
1.42 E+01 \\
1.61 E-01 \\
0.0 \\
2.01 E+01\end{array}$ & $\begin{array}{l}6.77 E \cdot 00 \\
1.52 E \cdot 01 \\
1.81 E-01 \\
0.0 \\
2.15 E \cdot 01\end{array}$ & $\begin{array}{l}7.54 E+00 \\
1.68 E+01 \\
2.86 E-01 \\
0.0 \\
2.36 E+01\end{array}$ & $\begin{array}{l}6.87 E+00 \\
1.55 E+01 \\
1.48 E-01 \\
0.0 \\
2.17 E+01\end{array}$ & $\begin{array}{l}6.13 E+00 \\
1.38 E+01 \\
1.40 E-01 \\
0.0 \\
1.93 E+01\end{array}$ & $\begin{array}{l}5.44 E+00 \\
1.23 E+01 \\
1.28 E-01 \\
0.0 \\
1.74 E+01\end{array}$ & $\begin{array}{l}7.67 E+00 \\
1.71 E+01 \\
2.94 E-01 \\
0.0 \\
2.40 E+01\end{array}$ & $\begin{array}{l}0.14 E+00 \\
1.38 E+01 \\
1.57 E-01 \\
0.0 \\
1.95 E+01\end{array}$ & $\begin{array}{l}1.40 E+ \\
1.58 E- \\
0.0 \\
1.99 E+\end{array}$ \\
\hline $\begin{array}{l}C S-137 \\
C S-138 \\
C 5-139 \\
B A-131 \\
8 A-133\end{array}$ & $\begin{array}{rl}30.11 & Y \\
32.2 & H \\
9.40 & M \\
11.8 & 0 \\
10.5 & Y\end{array}$ & & & & & & & & & $\begin{array}{l}0.0 \\
2.27 \mathrm{E} \\
2.99 \mathrm{Et} \\
3.89 \mathrm{E} \\
3.07 \mathrm{E}\end{array}$ \\
\hline $\begin{array}{l}B A-133 M \\
B A-135 M \\
B A-1374 \\
8 A-139 \\
B A-140\end{array}$ & $\begin{array}{r}38.9 \mathrm{H} \\
28.7 \mathrm{H} \\
2.552 \mathrm{H} \\
83.1 \mathrm{H} \\
12.789 \mathrm{D}\end{array}$ & & & & & & & & & $\begin{array}{l}3.98 \mathrm{E}- \\
5.34 \mathrm{EF} \\
3.00 \mathrm{E}- \\
1.62 \mathrm{EO}\end{array}$ \\
\hline $\begin{array}{l}B A-142 \\
1 A-140 \\
1 A-141 \\
L A-142\end{array}$ & $\begin{array}{rl}18.27 & H \\
10.70 & H \\
40.22 & H \\
33.94 & H \\
95.4 H\end{array}$ & $\begin{array}{l}8.13 E+00 \\
0.36 E+00 \\
2.23 E+01 \\
4.07 E-01 \\
2.07 E+01\end{array}$ & $\begin{array}{l}8.92 E \cdot 00 \\
2.36 E+01 \\
4.30 E-01 \\
3.01 E \cdot 01\end{array}$ & $\begin{array}{l}9.84 E+0 \\
2.51 E+0 \\
4.50 E-0 \\
3.13 E+0\end{array}$ & $\begin{array}{l}9.00 E 0 \\
2.39 \mathrm{E} \\
4.34 \mathrm{E} \\
3.07 \mathrm{E}\end{array}$ & $\begin{array}{l}7.61 \mathrm{EO} \\
7.98 \mathrm{E} \\
2.09 \mathrm{E} \\
3.04 \mathrm{E} \\
2.01 \mathrm{E}\end{array}$ & $\begin{array}{l}7.02 E+00 \\
7.21 E+00 \\
1.94 E+01 \\
3.53 E-01 \\
2.51 E+01\end{array}$ & $\begin{array}{l}1.02 E+01 \\
1.00 E+01 \\
2.55 E+01 \\
4.57 E-01 \\
3.18 E+08\end{array}$ & $\begin{array}{l}.94 E+00 \\
3.12 E+00 \\
2.16 E+01 \\
3.93 E-01 \\
.76 E+01\end{array}$ & 2. $82 \mathrm{E}$ \\
\hline
\end{tabular}



DOSE-KATE FACTORS FOR BODY ORGANS AND EFFECTIVE OUSE-RATE FACTOR FOR IMMERSIUN IN CUNTAMIMATE') WAITR
IN MREM/YR PER MICROCUR IE/(CU日IC MI

\begin{tabular}{|c|c|c|c|c|c|c|c|c|c|}
\hline CLIDE & HALF-LIFE & TESTES & THYMUS & THYROID & UTERUS & EFFECTIVE & $\begin{array}{c}\text { SKIN } \\
\text { (PHOTON) }\end{array}$ & $\begin{array}{l}\text { SKIN } \\
\text { (ELECIRON) }\end{array}$ & $\begin{array}{l}\text { SKIN } \\
\text { ITOTALI }\end{array}$ \\
\hline $\begin{array}{l}1-132 \\
1-133 \\
1-134 \\
1-135 \\
1-136\end{array}$ & $\begin{array}{rl}2.30 & H \\
20.8 & H \\
52.6 & M \\
6.61 & H \\
83 & 5\end{array}$ & $\begin{array}{l}3.00 E+01 \\
7.79 E+00 \\
3.48 E+01 \\
2.11 E+01 \\
3.54 E+01\end{array}$ & $\begin{array}{l}2.35 E+01 \\
5.98 E+00 \\
2.76 E+01 \\
1.60 E * 01 \\
2.81 E * 01\end{array}$ & $\begin{array}{l}2.83 E+01 \\
7.27 E+00 \\
3.30 E+01 \\
1.99 E+01 \\
3.28 E \bullet 01\end{array}$ & $\begin{array}{l}1.85 E+01 \\
4.76 E+00 \\
2.16 E+01 \\
1.35 E+01 \\
2.30 E+01\end{array}$ & $\begin{array}{l}2.56 E+01 \\
6.66 E+00 \\
2.98 E+01 \\
1.81 E+01 \\
3.05 E+01\end{array}$ & $\begin{array}{l}3.45 E+01 \\
8.94 E+00 \\
4.01 E+01 \\
2.41 E+01 \\
3.99 E+01\end{array}$ & $\begin{array}{l}3.74 E \cdot 00 \\
3.02 E \bullet 00 \\
4.80 E+00 \\
2.67 E+00 \\
1.80 E+01\end{array}$ & $\begin{array}{l}3.83 E+01 \\
1.20 E+01 \\
4.49 E+01 \\
2.60 E+01 \\
5.79 E+01\end{array}$ \\
\hline $\begin{array}{l}X E-1 \\
X E-122 \\
X E-125 \\
X E-127 \\
X E-129 M\end{array}$ & $\begin{array}{r}20.1 \mathrm{H} \\
2.14 \mathrm{H} \\
16.8 \mathrm{H} \\
36.406 \mathrm{O} \\
8.89 \mathrm{O}\end{array}$ & $\begin{array}{l}8.03 E-01 \\
8,11 E+00 \\
3.23 E+00 \\
3.39 E+00 \\
3.69 E-01\end{array}$ & $\begin{array}{l}5.61 E-01 \\
6.25 E+00 \\
2.41 E+00 \\
2.52 E \bullet 00 \\
1.99 E-01\end{array}$ & $\begin{array}{l}7.23 E-01 \\
7.61 E+00 \\
3.05 E+00 \\
3.20 E+00 \\
2.90 E-01\end{array}$ & $\begin{array}{l}4.20 E-01 \\
4.95 E+00 \\
1.85 E+00 \\
1.94 E+00 \\
1.03 E-01\end{array}$ & $\begin{array}{l}6.75 E-01 \\
6.96 E+00 \\
2.19 E+00 \\
2.94 E+00 \\
2.02 E-01\end{array}$ & $\begin{array}{l}9.85 E-01 \\
9.27 E+00 \\
3.71 E+00 \\
3.84 E+00 \\
5.55 E-01\end{array}$ & $\begin{array}{l}7.10 E-03 \\
1.28 E+00 \\
8.96 E-02 \\
9.98 E-02 \\
7.74 E-01\end{array}$ & $\begin{array}{l}9.92 E-01 \\
1.06 E+01 \\
3.80 E=00 \\
3.93 E+00 \\
1.33 E \times 00\end{array}$ \\
\hline $\begin{array}{l}X E-131 M \\
X E-133 \\
X E-133 M \\
X E-135 \\
X E-135 M\end{array}$ & $\begin{array}{rl}11.84 & 0 \\
5.245 & 0 \\
2.19 & 0 \\
9.11 & H \\
15.36 & 4\end{array}$ & $\begin{array}{l}1.38 E-01 \\
5.06 E-01 \\
4.05 E-01 \\
3.14 E+00 \\
5.47 E+00\end{array}$ & $\begin{array}{l}7.13 E-02 \\
3.64 E-01 \\
2.73 E-01 \\
2.38 E+00 \\
4.14 E+00\end{array}$ & $\begin{array}{l}1.07 E-01 \\
4.77 E-01 \\
3.60 E-01 \\
2.99 E+00 \\
5.06 E+00\end{array}$ & $\begin{array}{l}3.58 E-02 \\
2.18 E-01 \\
1.94 E-01 \\
1.8 B E+00 \\
3.30 E+00\end{array}$ & $\begin{array}{l}1.04 E-01 \\
4.19 E-01 \\
3.37 E-01 \\
2.74 E+00 \\
4.67 E \cdot 00\end{array}$ & $\begin{array}{l}2.12 E-01 \\
5.9 B E-01 \\
5.10 E-01 \\
3.50 E \cdot 00 \\
6.27 E+00\end{array}$ & & $\begin{array}{l}7.11 E-01 \\
9.47 E-01 \\
1.55 E+00 \\
5.66 E \bullet 00 \\
7.00 E \bullet 00\end{array}$ \\
\hline $\begin{array}{l}X E-137 \\
X E-136 \\
C S-126 \\
C S-129 \\
C S-131\end{array}$ & $\begin{array}{r}3.83 \mathrm{M} \\
14.13 \mathrm{M} \\
1.64 \mathrm{M} \\
32.06 \mathrm{H} \\
9.688 \mathrm{D}\end{array}$ & $\begin{array}{l}2.42 E+00 \\
1.57 E+01 \\
1.44 E+01 \\
3.36 E+00 \\
1.30 E-01\end{array}$ & $\begin{array}{l}1.85 E \bullet 00 \\
1.23 E \cdot 01 \\
1.09 E \cdot 01 \\
2.47 E \cdot 00 \\
5.27 E-02\end{array}$ & $\begin{array}{l}2.25 E+00 \\
1.46 E+01 \\
1.34 E+01 \\
3.09 E+00 \\
0.87 E-02\end{array}$ & & & $\begin{array}{l}2.75 E \cdot 00 \\
1.78 E+01 \\
1.65 E+01 \\
3.92 E+00 \\
2.26 E-01\end{array}$ & $\begin{array}{l}1.56 E+01 \\
4.96 E+00 \\
1.16 E+01 \\
2.30 E-02 \\
0.0\end{array}$ & $\begin{array}{l}1.83 E+01 \\
2.28 E+01 \\
2.80 E+01 \\
3.94 E+00 \\
2.26 E-01\end{array}$ \\
\hline $\begin{array}{l}C S-132 \\
C S-134 \\
C S-134 M \\
C S-135 \\
C S-136\end{array}$ & $\begin{array}{l}6.475 \\
2.062 \mathrm{Y} \\
2.90 \mathrm{H} \\
2.3 \mathrm{H} 6 \mathrm{Y} \\
13.16 \mathrm{D}\end{array}$ & $\begin{array}{l}9.09 E+00 \\
2.02 E+01 \\
2.86 E-01 \\
0.0 \\
2.85 E+01\end{array}$ & $\begin{array}{l}7.02 E+00 \\
1.58 E+01 \\
2.00 E-01 \\
0.0 \\
2.26 E+01\end{array}$ & $\begin{array}{l}8.52 E+00 \\
1.91 E+01 \\
2.66 E-01 \\
0.0 \\
2.71 E+01\end{array}$ & $\begin{array}{l}5.48 E+00 \\
1.24 E+01 \\
1.34 E-01 \\
0.0 \\
1.76 E+01\end{array}$ & $\begin{array}{l}7.75 E \cdot 00 \\
1.73 E+01 \\
2.40 E-01 \\
0.0 \\
2.44 E+01\end{array}$ & $\begin{array}{l}1.06 E+01 \\
2.33 E \cdot 01 \\
3.43 E-01 \\
0.0 \\
3.28 E+01\end{array}$ & & $\begin{array}{l}1.06 E \bullet 01 \\
2.42 E \cdot 01 \\
5.43 E-01 \\
7.29 E-02 \\
3.33 E+01\end{array}$ \\
\hline $\begin{array}{l}C S-137 \\
C S-138 \\
C S-139 \\
8 A-139 \\
B A-133\end{array}$ & $\begin{array}{r}30.17 Y \\
32.2 Y \\
9.40 \\
11.8 \\
10.5 \\
10.5\end{array}$ & $\begin{array}{l}0.0 \\
3.21 E+01 \\
4.20 E+00 \\
5.86 E+00 \\
4.75 E+00\end{array}$ & $\begin{array}{l}0.0 \\
2.54 E+01 \\
3.34 E+00 \\
4.40 E+00 \\
3.52 E+00\end{array}$ & $\begin{array}{l}0.0 \\
3.00 E+01 \\
3.92 E+00 \\
5.46 E+00 \\
4.41 E+00\end{array}$ & $\begin{array}{l}0.0 \\
2.07 E+01 \\
2.73 E+00 \\
3.43 E+00 \\
2.11 E+00\end{array}$ & $\begin{array}{l}0.0 \\
2.76 E \cdot 01 \\
3.61 E+00 \\
5.02 E+00 \\
4.06 E+00\end{array}$ & $\begin{array}{l}0.0 \\
3.66 E+01 \\
4.78 E+00 \\
6.72 E+00 \\
5.45 E+00\end{array}$ & $\begin{array}{l}9.23 E-01 \\
1.04 E+01 \\
1.45 E \cdot 01 \\
8.08 E-02 \\
5.44 E-02\end{array}$ & $\begin{array}{l}9.23 E-01 \\
4.70 E \times 01 \\
1.93 E \bullet 01 \\
6.80 E \times 00 \\
5.50 E \bullet 00\end{array}$ \\
\hline $\begin{array}{l}B A-133 M \\
B A-135 M \\
B A-137 M \\
B A-139 \\
B A-140\end{array}$ & $\begin{array}{r}38.9 \mathrm{H} \\
28.7 \mathrm{H} \\
2.552 \mathrm{M} \\
83.1 \mathrm{M} \\
12.789 \mathrm{D}\end{array}$ & $\begin{array}{l}7.48 E-01 \\
6.64 E-01 \\
1.71 E+00 \\
4.58 E-01 \\
2.40 E+00\end{array}$ & $\begin{array}{l}5.34 E-01 \\
4.70 E-01 \\
5.99 E+00 \\
3.52 E-01 \\
1.81 E+00\end{array}$ & $\begin{array}{l}6.82 E-01 \\
6.04 E-01 \\
7.25 E+00 \\
4.44 E-01 \\
2.23 E+00\end{array}$ & $\begin{array}{l}3.98 E-01 \\
3.46 E-01 \\
4.69 E+00 \\
2.60 E-01 \\
1.44 E+00\end{array}$ & $\begin{array}{l}6.33 E-01 \\
5.60 E-01 \\
6.5 B E+00 \\
4.01 E-01 \\
2.05 E+00\end{array}$ & $\begin{array}{l}8.91 E-01 \\
7.96 E-01 \\
8.89 E+00 \\
5.03 E-01 \\
2.75 E+00\end{array}$ & $\begin{array}{l}1.26 E+00 \\
1.23 E+00 \\
4.99 E-01 \\
1.43 E+00 \\
1.86 E+00\end{array}$ & $\begin{array}{l}2.15 E+00 \\
2.03 E+00 \\
9.39 E+00 \\
1.93 E+00 \\
4.61 E+00\end{array}$ \\
\hline $\begin{array}{l}B A-141 \\
B A-142 \\
L A-140 \\
L A-141 \\
L A-142\end{array}$ & $\begin{array}{l}18.27 \mathrm{H} \\
10.70 \mathrm{M} \\
40.22 \mathrm{H} \\
3.94 \mathrm{H} \\
95.4 \mathrm{H}\end{array}$ & $\begin{array}{l}1.16 E+01 \\
1.18 E+01 \\
3.10 E+01 \\
5.63 E-01 \\
3.93 E+01\end{array}$ & $\begin{array}{l}9.03 E+00 \\
9.38 E \cdot 00 \\
2.45 E+01 \\
4.50 E-01 \\
3.09 E+01\end{array}$ & $\begin{array}{l}1.10 E \bullet 01 \\
1.13 E \bullet 01 \\
2.90 E+01 \\
5.31 E-01 \\
3.61 E+01\end{array}$ & $\begin{array}{l}1.18 E+00 \\
1.33 E+00 \\
1.99 E+01 \\
3.63 E-01 \\
2.57 E+01\end{array}$ & $\begin{array}{l}1.00 E+01 \\
1.02 E+01 \\
2.66 E+01 \\
4.83 E-01 \\
3.38 E+01\end{array}$ & $\begin{array}{l}1.32 E+01 \\
1.36 E+01 \\
3.54 E+01 \\
6.45 E-01 \\
4.41 E+01\end{array}$ & $\begin{array}{l}7.00 E+00 \\
3.42 E+00 \\
4.11 E+00 \\
7.89 E+00 \\
7.02 E+00\end{array}$ & $\begin{array}{l}2.02 E+01 \\
1.70 E+01 \\
3.95 E+01 \\
0.53 E+00 \\
5.12 E+01\end{array}$ \\
\hline
\end{tabular}


DOSE-RATE FACTORS FOR BODY ORGANS AND EFFECTIVE DOSE-PATE FACTOR FOR IMMERSION IN CONTAMINATEU WATES.

IN MREM/YR PER MICROCUR IE/ ICUBIC MI

\begin{tabular}{|c|c|c|c|c|c|c|c|c|c|c|}
\hline NUCL IDE & HALF-LIFE & ADRENALS & BLADOER & BRAIN & BREAST & HE AR T & $\begin{array}{l}\text { SMALL } \\
\text { INTESTINE }\end{array}$ & $\begin{array}{l}\text { UPPER } \\
\text { LARGE } \\
\text { INTESTINE }\end{array}$ & $\begin{array}{l}\text { LOWER } \\
\text { LARGE } \\
\text { INTESTINE }\end{array}$ & KIONEYS \\
\hline $\begin{array}{l}C E-139 \\
C E-141 \\
C E-143 \\
C E-144 \\
P R-142\end{array}$ & $\begin{array}{r}137.66 \mathrm{D} \\
32.50 \mathrm{O} \\
33.0 \mathrm{H} \\
284.3 \mathrm{O} \\
19.13 \mathrm{H}\end{array}$ & $\begin{array}{l}1.33 E+00 \\
6.88 E-01 \\
2.41 E+00 \\
1.56 E-01 \\
5.61 E-01\end{array}$ & $\begin{array}{l}1.25 E+00 \\
6.42 E-01 \\
2.30 E+00 \\
1.51 E-01 \\
5.68 E-01\end{array}$ & $\begin{array}{l}1.29 E+00 \\
6.75 E-01 \\
2.47 E+00 \\
1.54 E-01 \\
6.39 E-01\end{array}$ & $\begin{array}{l}2.21 E+00 \\
1.13 E+00 \\
3.36 E+00 \\
2.81 E-01 \\
7.07 E-01\end{array}$ & $\begin{array}{l}1.18 E+00 \\
6.21 E-01 \\
2.20 E+00 \\
1.41 E-01 \\
5.64 E-01\end{array}$ & $\begin{array}{l}.05 E+00 \\
45 E-01 \\
99 E+00 \\
24 E-01\end{array}$ & $\begin{array}{l}1.28 E * 00 \\
6.69 E-01 \\
2.35 E+00 \\
1.55 E-01 \\
6.00 E-01\end{array}$ & $\begin{array}{l}1.18 E+00 \\
6.11 E-0.1 \\
2.17 E+00 \\
1.39 E-01 \\
5.54 E-01\end{array}$ & $\begin{array}{l}1.31 E \bullet 00 \\
6.61 E-01 \\
2.41 E+00 \\
1.58 E-01 \\
6.01 E-01\end{array}$ \\
\hline $\begin{array}{l}P R-143 \\
P R-144 \\
P R-144 M \\
N D-147 \\
N D-149\end{array}$ & $\begin{array}{r}13.56 \mathrm{D} \\
17.28 \mathrm{M} \\
7.2 \mathrm{M} \\
10.98 \mathrm{D} \\
1.73 \mathrm{H}\end{array}$ & $\begin{array}{l}8.57 E-08 \\
3.22 E-01 \\
2.86 E-02 \\
1.18 E+00 \\
3.51 E+00\end{array}$ & $\begin{array}{l}8.24 E-08 \\
3.25 E-01 \\
4.25 E-02 \\
1.16 E+00 \\
3.30 E+00\end{array}$ & $\begin{array}{l}9.25 E-08 \\
3.63 E-01 \\
2.18 E-02 \\
1.23 E+00 \\
3.58 E+00\end{array}$ & $\begin{array}{l}1.05 E-07 \\
4.08 E-01 \\
1.27 E-01 \\
1.79 E+00 \\
4.87 E+00\end{array}$ & $\begin{array}{l}8.11 E-08 \\
3.23 E-01 \\
2.04 E-02 \\
1.09 E+00 \\
3.20 E+00\end{array}$ & $\begin{array}{l}7.32 E-08 \\
2.98 E-01 \\
2.31 E-02 \\
9.83 E-01 \\
2.87 E+00\end{array}$ & $\begin{array}{l}8.62 E-08 \\
3.43 E-01 \\
3.26 E-02 \\
1.18 E+00 \\
3.41 E+00\end{array}$ & $\begin{array}{l}7.93 E-08 \\
3.17 E-01 \\
2.44 E-02 \\
1.07 E+00 \\
3.16 E+00\end{array}$ & $\begin{array}{l}8.60 E-08 \\
3.45 E-01 \\
5.83 E-02 \\
1.22 E+00 \\
3.43 E+00\end{array}$ \\
\hline $\begin{array}{l}P M-143 \\
P M-144 \\
P M-145 \\
P M-146 \\
P M-147\end{array}$ & $\begin{array}{r}265 \mathrm{D} \\
363 \mathrm{D} \\
17.7 \mathrm{Y} \\
2020 \mathrm{D} \\
2.6234 \mathrm{Y}\end{array}$ & $\begin{array}{l}2.82 E+00 \\
1.46 E+01 \\
9.69 E-02 \\
7.03 E+00 \\
3.23 E-05\end{array}$ & $\begin{array}{l}2.75 E \bullet 00 \\
1.40 E+01 \\
1.37 E-01 \\
6.74 E+00 \\
3.02 E-05\end{array}$ & $\begin{array}{l}3.02 E+00 \\
1.56 E+01 \\
8.11 E-02 \\
7.48 E+00 \\
3.22 E-05\end{array}$ & $\begin{array}{l}3.69 E+00 \\
1.83 E+01 \\
3.76 E-01 \\
8.90 E+00 \\
5.39 E-05\end{array}$ & $\begin{array}{l}2.65 \mathrm{E}+00 \\
1.36 \mathrm{E}+01 \\
7.48 \mathrm{E}-02 \\
6.57 \mathrm{E}+00 \\
2.97 \mathrm{E}-05\end{array}$ & $\begin{array}{l}2.40 E+00 \\
1.23 E+01 \\
8.02 E-02 \\
5.94 E+00 \\
2.57 E-05\end{array}$ & $\begin{array}{l}2.84 E+00 \\
1.45 E+01 \\
1.08 E-01 \\
7.00 E+00 \\
3.19 E-05\end{array}$ & $\begin{array}{l}2.60 E+00 \\
1.34 E+01 \\
8.41 E-02 \\
6.45 E+00 \\
2.89 E-05\end{array}$ & $\begin{array}{l}2.90 E+00 \\
1.46 E+01 \\
1.78 E-01 \\
7.03 E+00 \\
3.07 E-05\end{array}$ \\
\hline $\begin{array}{l}P M-148 \\
P M-148 M \\
P M-149 \\
P M-151 \\
S M-147\end{array}$ & $\begin{array}{r}5.37 \mathrm{D} \\
41.33 \mathrm{D} \\
53.08 \mathrm{H} \\
28.40 \mathrm{H} \\
1.069 \mathrm{E11} \mathrm{Y}\end{array}$ & $\begin{array}{l}5.46 E+00 \\
1.89 E+01 \\
1.10 E-01 \\
3.10 E+00 \\
0.0\end{array}$ & $\begin{array}{l}5.39 E+00 \\
1.81 E+01 \\
1.02 E-01 \\
2.93 E+00 \\
0.0\end{array}$ & $\begin{array}{l}6.06 E+00 \\
2.03 E+01 \\
1.11 E-01 \\
3.19 E+00 \\
0.0\end{array}$ & $\begin{array}{l}6.80 E+00 \\
2.36 E+01 \\
1.46 E-01 \\
4.22 E+00 \\
0.0\end{array}$ & $\begin{array}{l}5.33 E+00 \\
1.78 E+01 \\
9.93 E-02 \\
2.84 E+00 \\
0.0\end{array}$ & $\begin{array}{l}4.89 E+00 \\
1.61 E+01 \\
8.93 E-02\end{array}$ & $\begin{array}{l}5.67 E+00 \\
1.89 E+01 \\
1.05 E-01 \\
3.02 E+00 \\
0.0\end{array}$ & $\begin{array}{l}5.22 E+00 \\
1.74 E+01 \\
9.83 E-02 \\
2.80 E+00 \\
0.0\end{array}$ & $\begin{array}{l}5.66 E+00 \\
1.89 E+01 \\
1.05 E-01 \\
3.04 E+00 \\
0.0\end{array}$ \\
\hline $\begin{array}{l}S M-151 \\
S M-153 \\
E U-152 \\
E U-152 M \\
E U-154\end{array}$ & $\begin{array}{r}90 Y \\
46.7 \mathrm{H} \\
13.6 \mathrm{Y} \\
9.32 \mathrm{H} \\
8.8 \mathrm{Y}\end{array}$ & $\begin{array}{l}2.77 E-06 \\
4.02 E-01 \\
1.08 E+01 \\
2.99 E+00 \\
1.20 E+01\end{array}$ & $\begin{array}{l}1.49 E-06 \\
4.29 E-01 \\
1.06 E+01 \\
2.92 E+00 \\
1.17 E+01\end{array}$ & $\begin{array}{l}8.40 E-07 \\
3.94 E-01 \\
1.18 E+01 \\
3.25 E+00 \\
1.31 E+01\end{array}$ & $\begin{array}{l}4.06 E-05 \\
E .93 E-01 \\
1.39 E+01 \\
3.80 E+00 \\
1.51 E+01\end{array}$ & $\begin{array}{l}9.89 E-07 \\
3.61 E-01 \\
1.04 E+01 \\
2.86 E+00 \\
1.16 E+01\end{array}$ & $\begin{array}{l}7.53 E-07 \\
3.28 E-01 \\
9.52 E+00 \\
2.60 E+00 \\
1.05 E+01\end{array}$ & $\begin{array}{l}2.46 E-06 \\
4.12 E-01 \\
1.11 E+01 \\
3.05 E+00 \\
1.23 E+01\end{array}$ & $\begin{array}{l}1.97 E-06 \\
3.58 E-01 \\
1.02 E+01 \\
2.80 E+00 \\
1.13 E+01\end{array}$ & $\begin{array}{l}3.76 E-06 \\
4.67 E-01 \\
1.12 E+01 \\
3.07 E+00 \\
1.23 E+01\end{array}$ \\
\hline $\begin{array}{l}E U-155 \\
E U-156 \\
60-152 \\
6 D-153 \\
6 D-159\end{array}$ & $\begin{array}{r}4.96 Y \\
15.19 \mathrm{D} \\
1.1 E 14 \mathrm{Y} \\
241.6 \mathrm{D} \\
18.56 \mathrm{H}\end{array}$ & $\begin{array}{l}\text { 4.69E-01 } \\
1.33 E+01 \\
0.0 \\
6.82 E-01 \\
3.38 E-01\end{array}$ & $\begin{array}{l}4.78 E-01 \\
1.34 E+01 \\
0.0 \\
7.44 E-01 \\
3.27 E-01\end{array}$ & $\begin{array}{l}4.75 E-01 \\
1.50 E+01 \\
0.0 \\
6.64 E-01 \\
3.43 E-01\end{array}$ & $\begin{array}{l}9.41 E-01 \\
1.68 E+01 \\
0.0 \\
1.59 E+00 \\
5.06 E-01\end{array}$ & $\begin{array}{l}4.34 E-01 \\
1.33 E+01 \\
0.0 \\
6.09 E-01 \\
3.05 E-01\end{array}$ & $\begin{array}{l}3.83 E-01 \\
1.22 E+01 \\
0.0 \\
5.57 E-01 \\
2.77 E-01\end{array}$ & $\begin{array}{l}4.76 E-01 \\
1.41 E+01 \\
0.0 \\
7.05 E-01 \\
3.28 E-01\end{array}$ & $\begin{array}{l}4.21 E-01 \\
1.30 E+01 \\
0.0 \\
6.07 E-01 \\
3.02 E-01\end{array}$ & $\begin{array}{l}4.98 E-01 \\
1.41 E+01 \\
0.0 \\
8.18 E-01 \\
3.44 E-01\end{array}$ \\
\hline $\begin{array}{l}60-162 \\
T B-157 \\
18-160 \\
1 B-162 \\
D Y-157\end{array}$ & $\begin{array}{rl}9.7 & M \\
150 & Y \\
72.3 & 0 \\
7.76 & M \\
8.06 & H\end{array}$ & $\begin{array}{l}3.94 E+00 \\
1.58 E-02 \\
1.04 E+01 \\
1.05 E+01 \\
3.11 E+00\end{array}$ & $\begin{array}{l}3.70 E+00 \\
2.16 E-02 \\
1.01 E+01 \\
1.01 E+01 \\
2.94 E+00\end{array}$ & $\begin{array}{l}4.09 E+00 \\
1.43 E-02 \\
1.13 E+01 \\
1.12 E+01 \\
3.13 E+00\end{array}$ & $\begin{array}{l}5.08 E+00 \\
5.54 E-02 \\
1.30 E+01 \\
1.34 E+01 \\
4.43 E+00\end{array}$ & $\begin{array}{l}3.61 E+00 \\
1.30 E-02 \\
9.96 E+00 \\
9.93 E+00 \\
2.79 E+00\end{array}$ & $\begin{array}{l}3.25 E+00 \\
1.35 E-02 \\
9.07 E+00 \\
8.98 E+00 \\
2.52 E+00\end{array}$ & $\begin{array}{l}3.83 E+00 \\
1.72 E-02 \\
1.06 E+01 \\
1.06 E+01 \\
2.98 E+00\end{array}$ & $\begin{array}{l}3.55 E+00 \\
1.39 E-02 \\
9.76 E+00 \\
9.74 E+00 \\
2.77 E+00\end{array}$ & $\begin{array}{l}3.84 E+00 \\
2.63 E-02 \\
1.06 E+01 \\
1.05 E+01 \\
3.07 E+00\end{array}$ \\
\hline $\begin{array}{l}\text { DY }-165 \\
\text { DY-166 } \\
\text { HO-166 } \\
\text { HO-166M } \\
\text { ER-169 }\end{array}$ & $\begin{array}{r}2.334 \mathrm{H} \\
81.66 \mathrm{H} \\
26.80 \mathrm{H} \\
1.20 E 3 \mathrm{Y} \\
9.40 \mathrm{O}\end{array}$ & $\begin{array}{l}2.19 E-01 \\
2.39 E-01 \\
2.43 E-01 \\
1.52 E+01 \\
1.47 E-05\end{array}$ & $\begin{array}{l}2.15 E-01 \\
2.68 E-01 \\
2.51 E-01 \\
1.45 E+01 \\
1.37 E-05\end{array}$ & $\begin{array}{l}2.29 E-01 \\
2.42 E-01 \\
2.69 E-01 \\
1.62 E+01 \\
1.45 E-05\end{array}$ & $\begin{array}{l}\text { 3. } 30 E-01 \\
5.68 E-01 \\
3.78 E-01 \\
1.94 E+01 \\
4.32 E-05\end{array}$ & $\begin{array}{l}2.03 E-01 \\
2.17 E-01 \\
2.37 E-01 \\
1.43 E+01 \\
1.34 E-05\end{array}$ & $\begin{array}{l}1.83 E-01 \\
2.01 E-01 \\
2.19 E-01 \\
1.29 E+01 \\
1.15 E-05\end{array}$ & $\begin{array}{l}2.18 E-01 \\
2.45 E-01 \\
2.55 E-01 \\
1.52 E+01 \\
1.44 E-05\end{array}$ & $\begin{array}{l}1.99 E-01 \\
2.14 E-01 \\
2.33 E-01 \\
1.40 E+01 \\
1.31 E-05\end{array}$ & $\begin{array}{l}2.26 \mathrm{E}-01 \\
2.93 \mathrm{E}-01 \\
2.66 \mathrm{E}-01 \\
1.52 \mathrm{E}+01 \\
1.39 \mathrm{E}-05\end{array}$ \\
\hline
\end{tabular}


DOSE-RATE FACTORS FOR BOOY ORGANS AND EFFECTIVE OOSE-RATE FACTOR FOR IMMERSION IN CONTAMINATEO WAT LR.
IN MREM/YR PER MICROCUR IE/ICUBIC MI

\begin{tabular}{|c|c|c|c|c|c|c|c|c|c|c|}
\hline CLIDE & HALF-LIFE & LIVER & LUNGS & MARROW & $\begin{array}{c}\text { RED } \\
\text { MARROH }\end{array}$ & OVARIES & PANCPEAS & SKELETON & SPLEEN & STOMACH \\
\hline $\begin{array}{l}C E-139 \\
C E-141 \\
C E-143 \\
C E-144 \\
P R-142\end{array}$ & $\begin{array}{r}137.66 \mathrm{D} \\
32.50 \mathrm{O} \\
33.0 \mathrm{H} \\
284.3 \mathrm{D} \\
19.13 \mathrm{H}\end{array}$ & $\begin{array}{l}1.22 E * 00 \\
6.36 E-01 \\
2.26 E+00 \\
1.48 E-01 \\
5.69 E-01\end{array}$ & $\begin{array}{l}1.35 E+00 \\
7.01 E-01 \\
2.44 E+00 \\
1.63 E-01 \\
6.00 E-01\end{array}$ & $\begin{array}{l}1.99 E+00 \\
1.06 E+00 \\
3.01 E+00 \\
2.5 B E-01 \\
6.23 E-01\end{array}$ & $\begin{array}{l}1.25 E+00 \\
6.35 E-01 \\
2.43 E+00 \\
1.38 E-01 \\
6.05 E-01\end{array}$ & $\begin{array}{l}1.06 E+00 \\
5.50 E-01 \\
2.11 E+00 \\
1.27 E-01 \\
5.30 E-01\end{array}$ & $\begin{array}{l}1.02 E+00 \\
5.24 E-01 \\
1.92 E+00 \\
1.18 E-01 \\
4.94 E-01\end{array}$ & $\begin{array}{l}2.04 E+00 \\
1.09 E+00 \\
3.07 E+00 \\
2.65 E-01 \\
6.33 E-01\end{array}$ & $\begin{array}{l}21 E+00 \\
.32 E-01 \\
21 E+00 \\
.45 E-01 \\
48 E-01\end{array}$ & $\begin{array}{l}1.21 E+00 \\
6.28 E-01 \\
2.23 E+00 \\
1.45 E-01 \\
5.53 E-01\end{array}$ \\
\hline $\begin{array}{l}P R-143 \\
P R-144 \\
P R-144 M \\
N D-147 \\
N D-149\end{array}$ & $\begin{array}{r}13.56 \mathrm{D} \\
17.28 \mathrm{M} \\
7.2 \mathrm{M} \\
10.98 \mathrm{D} \\
1.73 \mathrm{H}\end{array}$ & $\begin{array}{l}8.18 E-08 \\
3.25 E-01 \\
3.32 E-02 \\
1.14 E+00 \\
3.28 E+00\end{array}$ & $\begin{array}{l}8.75 E-08 \\
3.43 E-01 \\
3.79 E-02 \\
1.23 E+00 \\
3.54 E+00\end{array}$ & $\begin{array}{l}9.60 E-08 \\
3.60 E-01 \\
6.66 E-02 \\
1.60 E+00 \\
4.52 E+00\end{array}$ & $\begin{array}{l}8.90 E-08 \\
3.49 E-01 \\
1.53 E-02 \\
1.15 E+00 \\
3.53 E+0 C\end{array}$ & $\begin{array}{l}7.96 E-08 \\
3.01 E-01 \\
2.75 E-02 \\
1.05 E+00 \\
3.02 E+00\end{array}$ & $\begin{array}{l}7.06 E-08 \\
2.83 E-01 \\
1.82 E-02 \\
9.35 E-01 \\
2.79 E \cdot 00\end{array}$ & $\begin{array}{l}9.76 E-08 \\
3.66 E-01 \\
6.80 E-02 \\
1.63 E+00 \\
4.61 E+00\end{array}$ & $\begin{array}{l}7.95 E-08 \\
3.13 E-01 \\
2.61 E-02 \\
1.10 E+00 \\
3.22 E+00\end{array}$ & $\begin{array}{l}\text { 8. } 10 \mathrm{E}-08 \\
3.19 \mathrm{E}-01 \\
2.89 \mathrm{E}-02 \\
1.11 \mathrm{E}+00 \\
3.23 \mathrm{E}+00\end{array}$ \\
\hline $\begin{array}{l}P M-143 \\
P M-144 \\
P M-145 \\
P M-146 \\
P M-147\end{array}$ & $\begin{array}{r}2650 \\
363 \\
17.7 Y \\
2020 \mathrm{D} \\
2.6234 \mathrm{Y}\end{array}$ & $\begin{array}{l}1.12 E-01 \\
6.68 \mathrm{E}+00 \\
3.02 E-05\end{array}$ & $\begin{array}{l}2.90 E+00 \\
1.48 E+01 \\
1.26 E-01 \\
7.16 E+00 \\
3.34 E-05\end{array}$ & & $\begin{array}{l}2.88 E+00 \\
1.51 E+01 \\
5.56 E-02 \\
7.27 E+00 \\
2.89 E-05\end{array}$ & & & & & $\begin{array}{l}2.67 E+00 \\
1.37 E+01 \\
9.83 E-02 \\
6.59 E+00 \\
2.98 E-05\end{array}$ \\
\hline $\begin{array}{l}P M-148 \\
P M-148 M \\
P M-149 \\
P M-151 \\
S M-147\end{array}$ & $\begin{array}{r}5.37 \mathrm{D} \\
41.3 \mathrm{D} \\
53.08 \mathrm{H} \\
28.40 \mathrm{H} \\
1.069 \mathrm{EII} \mathrm{Y}\end{array}$ & $\begin{array}{l}5.38 E+00 \\
1.80 E+01 \\
1.01 E-01 \\
2.90 E+00 \\
0.0\end{array}$ & $\begin{array}{l}5.71 E+00 \\
1.93 E+01 \\
1.09 E-01 \\
3.13 E+00 \\
0.0\end{array}$ & $\begin{array}{l}6.11 E+00 \\
2.16 E+01 \\
1.36 E-01 \\
3.89 E+00 \\
0.0\end{array}$ & & & & & & $\begin{array}{l}5.27 E+00 \\
1.78 E+01 \\
1.00 E-01 \\
2.86 E+00 \\
0.0\end{array}$ \\
\hline $\begin{array}{l}S M-151 \\
S M-153 \\
E U-152 \\
E U-152 M \\
E U-154\end{array}$ & $\begin{array}{rl}90 & Y \\
46.7 & H \\
13.6 Y & Y \\
9.32 & H \\
8.8 Y & Y\end{array}$ & $\begin{array}{l}1.27 E-06 \\
4.07 E-01 \\
1.06 E+01 \\
2.89 E+00 \\
1.17 E+01\end{array}$ & & $\begin{array}{l}4.75 E-06 \\
7.61 E-01 \\
1.25 E+01 \\
3.41 E+C 0 \\
1.36 E+01\end{array}$ & & & & & & $\begin{array}{l}1.95 E-06 \\
3.86 E-01 \\
1.04 E+01 \\
2.85 E+00 \\
1.15 E+01\end{array}$ \\
\hline $\begin{array}{l}E U-155 \\
E U-156 \\
G D-152 \\
G D-153 \\
G O-159\end{array}$ & 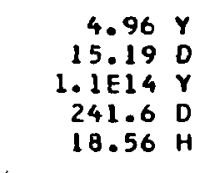 & $\begin{array}{l}4.68 E-01 \\
1.34 E+01 \\
0.0 \\
6.99 E-01 \\
3.21 E-01\end{array}$ & $\begin{array}{l}5.19 E-01 \\
1.41 E \cdot 01 \\
0.0 \\
7.77 E-01 \\
3.47 E-01\end{array}$ & $\begin{array}{l}8.77 E-01 \\
1.49 E+01 \\
0.0 \\
1.32 E+00 \\
4.51 E-01\end{array}$ & $\begin{array}{l}3.76 E-01 \\
1.43 E+01 \\
0.0 \\
5.21 E-01 \\
3.33 E-01\end{array}$ & $\begin{array}{l}3.94 E-01 \\
1.25 E+01 \\
0.0 \\
5.86 E-01 \\
2.94 E-01\end{array}$ & $\begin{array}{l}3.44 E-01 \\
1.16 E+01 \\
0.0 \\
4.90 E-01 \\
2.66 E-01\end{array}$ & $\begin{array}{l}9.02 E-01 \\
1.51 E+01 \\
0.0 \\
1.36 E+00 \\
4.59 E-01\end{array}$ & $\begin{array}{l}4.45 E-01 \\
1.29 E+01 \\
0.0 \\
6.43 E-01 \\
3.10 E-01\end{array}$ & $\begin{array}{l}\text { 4. } 50 E-01 \\
1.31 E+01 \\
0.0 \\
6.59 E-01 \\
3.13 E-01\end{array}$ \\
\hline $\begin{array}{l}\text { GD-1 } 62 \\
18-157 \\
T \theta-160 \\
T \theta-162 \\
\text { OY-157 }\end{array}$ & $\begin{aligned} 9.7 \mathrm{M} \\
150 \mathrm{Y} \\
72.3 \mathrm{D} \\
7.76 \mathrm{M} \\
8.06 \mathrm{H}\end{aligned}$ & $\begin{array}{l}3.68 E+00 \\
1.87 E-02 \\
1.01 E+01 \\
1.00 E+01 \\
2.90 E+00\end{array}$ & $\begin{array}{l}3.96 E+00 \\
2.07 E-02 \\
1.07 E+01 \\
1.08 E+01 \\
3.14 E+00\end{array}$ & $\begin{array}{l}4.71 E+00 \\
3.72 E-02 \\
1.18 E+01 \\
1.22 E+01 \\
4.03 E+00\end{array}$ & & & & & & $\begin{array}{l}3.63 E+00 \\
1.63 E-02 \\
9.90 E+00 \\
9.92 E+00 \\
2.84 E \cdot 00\end{array}$ \\
\hline $\begin{array}{l}\text { DY }-165 \\
\text { DY-166 } \\
\text { HO-166 } \\
\text { HO-165M } \\
\text { ER-169 }\end{array}$ & $\begin{array}{r}2.334 \mathrm{H} \\
81.6 \mathrm{H} \\
26.80 \mathrm{H} \\
1.20 \mathrm{H} \\
9.40 \mathrm{Y}\end{array}$ & $\begin{array}{l}2.12 \mathrm{E}-01 \\
2.56 \mathrm{E}-01 \\
2.49 \mathrm{E}-01 \\
1.45 \mathrm{E}-01 \\
1.37 \mathrm{E}-05\end{array}$ & $\begin{array}{l}2.83 E-01 \\
2.67 E-01 \\
1.55 E \cdot 01 \\
1.54 E-05\end{array}$ & $\begin{array}{l}2.98 E-01 \\
4.78 E-01 \\
3.35 E-01 \\
1.78 E+01 \\
2.47 E-05\end{array}$ & $\begin{array}{l}1.78 E-01 \\
2.37 E-01 \\
1.56 E+01 \\
1.27 E-05\end{array}$ & $\begin{array}{l}2.14 E-01 \\
2.26 E-01 \\
1.38 E+01 \\
1.18 E-05\end{array}$ & $\begin{array}{l}2.01 E-01 \\
1.24 E+01 \\
1.10 E-05\end{array}$ & $\begin{array}{l}3.04 E-01 \\
4.90 E-01 \\
3.42 E-01 \\
1.81 E+01 \\
2.56 E-05\end{array}$ & $\begin{array}{l}2.03 E-01 \\
2.29 E-01 \\
2.35 E-01 \\
1.41 E+01 \\
1.36 E-05\end{array}$ & $\begin{array}{l}2.07 \mathrm{E}-01 \\
2.37 \mathrm{E}-01 \\
2.39 \mathrm{E}-01 \\
1.43 \mathrm{E}+01 \\
1.35 \mathrm{E}-05\end{array}$ \\
\hline
\end{tabular}


DOSE-RATE FACTORS FOR BODY ORGANS ANO EFFECTIVE DOSE-RATE FACTUR FOR IMMERSION IN CONTAMINATL') WATt.K

\begin{tabular}{|c|c|c|c|c|c|c|c|c|c|}
\hline NUCLIDE & HALF & TESTES & THYMUS & IYROIO & UTERUS & EFFECTIVE & $\begin{array}{c}\text { SKIN } \\
\text { (PHOTONI }\end{array}$ & $\begin{array}{l}\text { SKIN } \\
\text { (ELECTRON) }\end{array}$ & $\begin{array}{l}\text { SKIN } \\
\text { GOTALS }\end{array}$ \\
\hline $\begin{array}{l}\text { CE-139 } \\
C E-141 \\
C E-143 \\
\text { CE-144 } \\
P R-142\end{array}$ & $\begin{array}{r}137.66 \mathrm{D} \\
32.50 \mathrm{O} \\
33.0 \mathrm{H} \\
284.3 \mathrm{O} \\
19.13 \mathrm{H}\end{array}$ & $\begin{array}{l}1.95 E+00 \\
9.96 E-01 \\
3.39 E+00 \\
2.42 E-01 \\
1.82 E-01\end{array}$ & $\begin{array}{l}1.45 E+00 \\
7.58 E-01 \\
2.56 E+00 \\
1.01 E-01 \\
6.22 E-01\end{array}$ & $\begin{array}{l}1.87 E+00 \\
9.73 E-01 \\
3.17 E+00 \\
2.34 E-01 \\
1.33 E-01\end{array}$ & $\begin{array}{l}1.06 E+00 \\
5.55 E-01 \\
1.96 E+00 \\
1.26 E-01 \\
5.10 E-01\end{array}$ & $\begin{array}{l}1.69 E+00 \\
8.71 E-01 \\
2.89 E+00 \\
2.08 E-01 \\
6.72 E-01\end{array}$ & $\begin{array}{l}2.20 E+00 \\
1.09 E+00 \\
3.89 E+00 \\
2.68 E-01 \\
8.93 E-01\end{array}$ & $\begin{array}{l}8.29 E-02 \\
7.49 E-01 \\
3.06 E+00 \\
2.40 E-01 \\
6.83 E+00\end{array}$ & $\begin{array}{l}2.28 E+00 \\
1.84 E+00 \\
6.96 E+00 \\
5.08 E-01 \\
7.72 E+00\end{array}$ \\
\hline $\begin{array}{l}P R-143 \\
P R-144 \\
P R-144 M \\
N D-147 \\
N D-149\end{array}$ & $\begin{array}{r}13.56 \mathrm{O} \\
17.28 \mathrm{M} \\
7.2 \mathrm{M} \\
10.98 \mathrm{O} \\
1.73 \mathrm{H}\end{array}$ & $\begin{array}{l}1.16 E-07 \\
4.48 E-01 \\
9.15 E-02 \\
1.75 E+00 \\
4.86 E+00\end{array}$ & $\begin{array}{l}9.13 E-08 \\
3.53 E-01 \\
5.33 E-02 \\
1.31 E+00 \\
3.69 E+00\end{array}$ & $\begin{array}{l}1.10 E-07 \\
4.16 E-01 \\
7.31 E-02 \\
1.64 E+00 \\
4.59 E+00\end{array}$ & $\begin{array}{l}1.11 E-08 \\
2.91 E-01 \\
1.97 E-02 \\
9.71 E-01 \\
2.86 E+00\end{array}$ & $\begin{array}{l}9.93 E-08 \\
3.86 E-01 \\
6.78 E-02 \\
1.48 E+00 \\
4.19 E+00\end{array}$ & $\begin{array}{l}1.34 E-07 \\
5.09 E-01 \\
1.32 E-01 \\
2.01 E 00 \\
5.47 E+00\end{array}$ & $\begin{array}{l}2.18 E+00 \\
1.03 E+01 \\
0.0 \\
1.48 E+00 \\
3.47 E \cdot 00\end{array}$ & $\begin{array}{l}2.18 E+00 \\
1.08 E+01 \\
1.32 E-01 \\
3.49 E+00 \\
8.94 E+00\end{array}$ \\
\hline $\begin{array}{l}P M-143 \\
P M-144 \\
P M-145 \\
P M-146 \\
P M-147\end{array}$ & $\begin{array}{r}2650 \\
3630 \\
17.7 y \\
2020 \\
2.6234 y\end{array}$ & $\begin{array}{l}3.96 E+00 \\
1.99 E+01 \\
2.79 E-01 \\
9.63 E+00 \\
4.71 E-05\end{array}$ & $\begin{array}{l}.07 E+00 \\
.54 E \bullet 01 \\
.74 E-01 \\
.42 E+00 \\
.64 E-05\end{array}$ & $\begin{array}{l}3.72 E+00 \\
1.87 E+01 \\
2.33 E-O I \\
9.02 E+00 \\
4.69 E-05\end{array}$ & & & $\begin{array}{l}4.63 E+00 \\
2.30 E+01 \\
3.81 E-01 \\
1.11 E+01 \\
5.02 E-05\end{array}$ & $\begin{array}{l}7.07 E-03 \\
6.22 E-02 \\
7.75 E-06 \\
6.14 E-01 \\
1.01 E-01\end{array}$ & $\begin{array}{l}4.64 E+00 \\
2.30 E+01 \\
3.81 E-01 \\
1.17 E+01 \\
1.01 E-01\end{array}$ \\
\hline $\begin{array}{l}P M-148 \\
P M-148 M \\
P M-149 \\
P M-151 \\
S M-147\end{array}$ & $\begin{array}{r}5.370 \\
41.30 \\
53.08 \mathrm{O} \\
28.40 \mathrm{H} \\
1.069 \mathrm{E} 1 \mathrm{Y}\end{array}$ & $\begin{array}{l}7.52 E+00 \\
2.57 E+01 \\
1.48 E-01 \\
4.28 E+00 \\
0.0\end{array}$ & $\begin{array}{l}5.95 E+00 \\
1.99 E \cdot 01 \\
1.13 E-01 \\
3.26 E+00 \\
0.0\end{array}$ & $\begin{array}{l}7.09 E+00 \\
2.42 E \cdot 01 \\
1.39 E-01 \\
4.03 E+00 \\
0.0\end{array}$ & $\begin{array}{l}4.76 E+00 \\
1.57 E+01 \\
0.91 E-02 \\
2.53 E+00 \\
0.0\end{array}$ & $\begin{array}{l}6.45 E+00 \\
2.20 E+01 \\
1.28 E-01 \\
3.68 E+00 \\
0.0\end{array}$ & $\begin{array}{l}8.63 E+00 \\
2.96 E+01 \\
1.67 E-01 \\
4.85 E+00 \\
0.0\end{array}$ & $\begin{array}{l}5.89 E+00 \\
8.22 E-01 \\
2.62 E+00 \\
1.91 E \cdot 00 \\
0.0\end{array}$ & $\begin{array}{l}1.45 E+01 \\
3.04 E+01 \\
2.79 E+00 \\
6.76 E+00 \\
0.0\end{array}$ \\
\hline $\begin{array}{l}S M-151 \\
S M-153 \\
E U-152 \\
E U-152 M \\
E U-154\end{array}$ & $\begin{array}{r}90 Y \\
46.7 Y H \\
13.6 Y \\
9.32 Y \\
8.8 Y\end{array}$ & $\begin{array}{l}1.55 E-05 \\
7.33 E-01 \\
1.50 E+01 \\
4.13 E+00 \\
1.65 E+01\end{array}$ & $\begin{array}{l}1.39 \mathrm{E}-06 \\
5.37 \mathrm{E}-01 \\
1.1 \mathrm{BE}+01 \\
3.27 \mathrm{E}+00 \\
1.31 \mathrm{E}+01\end{array}$ & $\begin{array}{l}6.42 \mathrm{E}-06 \\
6.96 \mathrm{E}-01 \\
1.42 \mathrm{E} * 01 \\
3.93 \mathrm{0} \cdot 00 \\
1.57 \mathrm{E}+01\end{array}$ & $\begin{array}{l}1.80 E-07 \\
3.24 E-01 \\
9.26 E+00 \\
2.51 E+00 \\
1.02 E+01\end{array}$ & & & & $\begin{array}{l}6.34 E-05 \\
2.23 E+00 \\
1.79 E+01 \\
8.69 E+00 \\
2.04 E+01\end{array}$ \\
\hline $\begin{array}{l}E U-155 \\
E U-156 \\
G 0-152 \\
G 0-153 \\
G D-159\end{array}$ & 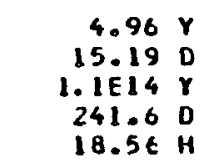 & $\begin{array}{l}7.89 E-01 \\
1.85 E+01 \\
0.0 \\
1.29 E+00 \\
4.94 E-01\end{array}$ & $\begin{array}{l}6.00 E-01 \\
1.47 E+01 \\
0.0 \\
9.36 E-01 \\
3.61 E-01\end{array}$ & $\begin{array}{l}7.74 E-01 \\
1.73 E+01 \\
0.0 \\
1.21 E+00 \\
4.58 E-01\end{array}$ & $\begin{array}{l}3.86 E-01 \\
1.19 E+01 \\
0.0 \\
5.49 E-01 \\
2.75 E-01\end{array}$ & $\begin{array}{l}6.72 E-01 \\
1.59 E+01 \\
0.0 \\
1.06 E+00 \\
4.19 E-01\end{array}$ & $\begin{array}{l}8.65 E-01 \\
2.11 E+01 \\
0.0 \\
1.50 E+00 \\
5.67 E-01\end{array}$ & $\begin{array}{l}4.13 E-02 \\
2.92 E+00 \\
0.0 \\
7.84 E-03 \\
2.15 E+00\end{array}$ & $\begin{array}{l}9.06 E-01 \\
2.41 E+01 \\
0.0 \\
1.51 E+00 \\
2.72 E+00\end{array}$ \\
\hline $\begin{array}{l}60-162 \\
18-157 \\
18-160 \\
1 B-162 \\
D Y-157\end{array}$ & $\begin{array}{rl}9.7 & M \\
150 & Y \\
72.3 & 0 \\
7.76 & M \\
8.06 & H\end{array}$ & $\begin{array}{l}5.34 E+00 \\
4.19 E-02 \\
1.42 E+01 \\
1.44 E+01 \\
4.31 E+00\end{array}$ & $\begin{array}{l}4.02 E+00 \\
2.82 E-02 \\
1.13 E+01 \\
1.13 E+01 \\
3.27 E \cdot 00\end{array}$ & $\begin{array}{l}4.95 E \cdot 00 \\
3.68 E-02 \\
1.36 E+01 \\
1.37 E \cdot 01 \\
4.08 E+00\end{array}$ & $\begin{array}{l}3.23 E * 00 \\
8.24 E-02 \\
8.80 E+00 \\
8.76 E+00 \\
2.52 E+00\end{array}$ & $\begin{array}{l}4.59 E+00 \\
3.26 E-02 \\
1.22 E 001 \\
1.23 E+01 \\
3.75 E+00\end{array}$ & $\begin{array}{l}6.07 E+00 \\
5.52 E-02 \\
1.64 E+01 \\
1.65 E 001 \\
4.96 E+00\end{array}$ & $\begin{array}{l}2.26 E+00 \\
0.0 \\
1.43 E+00 \\
3.73 E+00 \\
2.25 E-02\end{array}$ & $\begin{array}{l}8.33 E+00 \\
5.52 E-02 \\
1.78 E 001 \\
2.02 E \bullet 01 \\
4.98 E+00\end{array}$ \\
\hline $\begin{array}{l}\text { DY-165 } \\
\text { DY-166 } \\
H O-166 \\
M O-166 M \\
\text { ER-1 } 169\end{array}$ & $\begin{array}{r}2.334 \mathrm{H} \\
81.6 \mathrm{H} \\
26.80 \mathrm{H} \\
1.20 E 3 \mathrm{Y} \\
9.40 \mathrm{D}\end{array}$ & $\begin{array}{l}3.25 E-01 \\
4.68 E-01 \\
3.74 E-01 \\
2.08 E+01 \\
2.34 E-05\end{array}$ & $\begin{array}{l}2.47 \mathrm{E}-01 \\
3.42 \mathrm{E}-01 \\
2.92 \mathrm{E}-01 \\
1.62 \mathrm{E}+01 \\
1.67 \mathrm{E}-05\end{array}$ & $\begin{array}{l}3.06 E-01 \\
4.40 E-01 \\
3.55 E-01 \\
8.97 E+01 \\
2.17 E-05\end{array}$ & $\begin{array}{l}1.80 E-01 \\
1.96 E-01 \\
2.13 E-01 \\
1.26 E-01 \\
1.18 E-05\end{array}$ & $\begin{array}{l}2.75 E-01 \\
3.84 E-01 \\
3.16 E-01 \\
1.78 E+01 \\
2.22 E-05\end{array}$ & $\begin{array}{l}3.72 E-01 \\
5.46 E-01 \\
4.27 E-01 \\
2.38 E+01 \\
6.17 E-05\end{array}$ & $\begin{array}{l}3.30 E+00 \\
4.92 E-01 \\
5.46 E+00 \\
3.83 E-01 \\
3.42 E-01\end{array}$ & $\begin{array}{l}3.67 E+00 \\
1.04 E+00 \\
5.89 E+00 \\
2.42 E+01 \\
3.42 E-01\end{array}$ \\
\hline
\end{tabular}


DOSE-RATE FACTORS FOR BODY URGANS ANO EFFECTIVE DOSE-RATE FACTOR FOR IMMERSION IN CUNT AMINATED hateR

\begin{tabular}{|c|c|c|c|c|c|c|c|c|c|c|}
\hline NUCLIDE & HALF-LIFE & ADRENALS & BLADDER & BRAIN & BREAST & HE ART T & $\begin{array}{l}\text { SMALL } \\
\text { INTESTINE }\end{array}$ & $\begin{array}{l}\text { UPPER } \\
\text { LARGE } \\
\text { INTESIIVE }\end{array}$ & $\begin{array}{l}\text { LOWER } \\
\text { LARGE } \\
\text { INTESTINE }\end{array}$ & K IONEYS \\
\hline $\begin{array}{l}E R-171 \\
T M-170 \\
T M-171 \\
Y B-169 \\
Y B-175\end{array}$ & 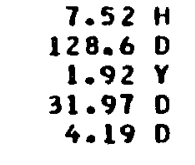 & $\begin{array}{l}3.41 E+00 \\
3.59 E-02 \\
3.16 E-03 \\
2.42 E+00 \\
3.62 E-01\end{array}$ & $\begin{array}{l}3.19 E+00 \\
3.88 E-02 \\
4.41 E-03 \\
2.41 E+00 \\
3.40 E-01\end{array}$ & $\begin{array}{l}3.44 E+00 \\
3.72 E-02 \\
3.8 B E-03 \\
2.42 E+00\end{array}$ & $\begin{array}{l}4.85 E+00 \\
8.13 E-02 \\
1.00 E-02 \\
4.46 E+00 \\
4.99 E-01\end{array}$ & $\begin{array}{l}3.08 E+00 \\
3.35 E-02 \\
3.44 E-03 \\
2.19 E+00 \\
3.29 E-01\end{array}$ & $\begin{array}{l}2.76 E+00 \\
3.02 E-02 \\
3.23 E-03 \\
1.97 E+00\end{array}$ & $\begin{array}{l}3.28 E+00 \\
3.66 E-02 \\
3.86 E-03 \\
2.37 E+00 \\
3.49 E-01\end{array}$ & $\begin{array}{l}3.05 E+00 \\
3.23 E-02 \\
3.38 E-03 \\
2.16 E+00 \\
3.24 E-01\end{array}$ & $\begin{array}{l}\text { 3. } 30 E+00 \\
4.11 E-02 \\
4.85 E-03 \\
2.54 E+00 \\
3.52 E-01\end{array}$ \\
\hline $\begin{array}{l}2 U-177 \\
2 U-177 M \\
\text { WF-181 } \\
\text { TA-182 } \\
M-181\end{array}$ & $\begin{array}{rl}6.71 & 0 \\
160.10 & 0 \\
42.39 & 0 \\
114.74 & 0 \\
120.95 & 0\end{array}$ & $\begin{array}{l}3.16 E-01 \\
9.04 E+00 \\
5.03 E+00 \\
1.22 E+01 \\
2.40 E-01\end{array}$ & $\begin{array}{l}2.95 E-01 \\
B .46 E+00 \\
4.76 E+00 \\
1.21 E+01 \\
2.76 E-01\end{array}$ & $\begin{array}{l}3.11 E-01 \\
9.08 E+00 \\
5.24 E+00 \\
1.35 E+01 \\
2.52 E-01\end{array}$ & $\begin{array}{l}4.95 E-01 \\
1.31 E+01 \\
6.75 E+00 \\
1.57 E+01 \\
6.06 E-01\end{array}$ & $\begin{array}{l}2.84 E-01 \\
8.16 E+00 \\
4.63 E+00 \\
1.19 E+01 \\
2.23 E-01\end{array}$ & $\begin{array}{l}2.52 E-01 \\
7.30 E+00 \\
4.15 E+00 \\
1.09 E+01 \\
2.07 E-01\end{array}$ & $\begin{array}{l}3.03 E-01 \\
8.68 E+00 \\
4.92 E+00 \\
1.27 E+01 \\
2.45 E-01\end{array}$ & $\begin{array}{l}2.80 E-01 \\
8.01 E+00 \\
4.55 E+00 \\
1.17 E+01 \\
2.16 E-01\end{array}$ & $\begin{array}{l}3.04 E-01 \\
8.75 E+00 \\
4.93 E+00 \\
1.27 E+01 \\
2.98 E-01\end{array}$ \\
\hline $\begin{array}{l}H-185 \\
H-187 \\
H-188 \\
R E-182 \\
R E-182 M\end{array}$ & $\begin{array}{r}75.1 \\
23.83 \\
69.4 \mathrm{H} \\
64.0 \mathrm{H} \\
12.7 \mathrm{H}\end{array}$ & $\begin{array}{l}2.48 E-04 \\
4.45 E+00 \\
1.64 E-02 \\
1.63 E+01 \\
1.12 E+01\end{array}$ & $\begin{array}{l}2.31 E-04 \\
4.27 E+00 \\
1.52 E-02 \\
1.60 E \cdot 01 \\
1.12 E \cdot 01\end{array}$ & $\begin{array}{l}2.46 E-04 \\
4.74 E+00 \\
1.63 E-02 \\
1.76 E+01 \\
1.24 E+01\end{array}$ & $\begin{array}{l}4.08 E-04 \\
5.76 E+00 \\
2.30 E-02 \\
2.19 E+01 \\
1.47 E+01\end{array}$ & $\begin{array}{l}2.27 E-04 \\
4.17 E+00 \\
1.47 E-02 \\
1.56 E+01 \\
1.09 E+01\end{array}$ & $\begin{array}{l}1.97 E-04 \\
3.75 E+00 \\
1.32 E-02 \\
1.42 E+01 \\
1.00 E+01\end{array}$ & $\begin{array}{l}2.44 E-04 \\
4.43 E+00 \\
1.56 E-02 \\
1.66 E+01 \\
1.17 E+01\end{array}$ & $\begin{array}{l}2.21 E-04 \\
4.07 E+00 \\
1.46 E-02 \\
1.53 E+01 \\
1.07 E+01\end{array}$ & $\begin{array}{l}2.34 E-04 \\
4.44 E+00 \\
1.56 E-02 \\
1.67 E+01 \\
1.17 E+01\end{array}$ \\
\hline $\begin{array}{l}R E-183 \\
R E-184 \\
R E-184 M \\
R E-186 \\
R E-187\end{array}$ & $\begin{array}{r}700 \\
38.00 \\
1690 \\
90.64 \mathrm{H} \\
4.7 E 10 \mathrm{Y}\end{array}$ & $\begin{array}{l}1.19 E+00 \\
8.46 E+00 \\
3.54 E+00 \\
1.75 E-01 \\
0.0\end{array}$ & $\begin{array}{l}1.20 E+00 \\
8.23 E+00 \\
3.44 E+00 \\
1.70 E-01 \\
0.0\end{array}$ & $\begin{array}{l}1.20 E+00 \\
9.17 E+00 \\
3.74 E+00 \\
1.76 E-01 \\
0.0\end{array}$ & $\begin{array}{l}2.27 E \bullet 00 \\
1.09 E+01 \\
5.00 E+00 \\
3.12 E-01 \\
0.0\end{array}$ & $\begin{array}{l}1.09 E+00 \\
8.06 E+00 \\
3.32 E+00 \\
1.61 E-01 \\
0.0\end{array}$ & $\begin{array}{l}9.77 E-01 \\
7.30 E+00 \\
3.00 E+00 \\
1.42 E-01 \\
0.0\end{array}$ & $\begin{array}{l}1.17 E+00 \\
8.59 E+00 \\
3.54 E+00 \\
1.73 E-01 \\
0.0\end{array}$ & $\begin{array}{l}1.06 E+00 \\
7.86 E+00 \\
3.25 E+00 \\
1.57 E-01 \\
0.0\end{array}$ & $\begin{array}{l}1.26 E+00 \\
8.62 E+00 \\
3.59 E+00 \\
1.75 E-01 \\
0.0\end{array}$ \\
\hline $\begin{array}{l}\text { RE-188 } \\
0 S-185 \\
0 S-186 \\
0 S-1904 \\
O S-191\end{array}$ & $\begin{array}{r}16.98 \mathrm{H} \\
93.6 \mathrm{D} \\
2.0 E 15 \mathrm{Y} \\
9.9 \mathrm{M} \\
15.4 \mathrm{D}\end{array}$ & $\begin{array}{l}5.44 E-01 \\
6.60 E+00 \\
0.0 \\
1.49 E+01 \\
5.16 E-01\end{array}$ & $\begin{array}{l}5.18 E-01 \\
6.37 E+00 \\
0.0 \\
1.41 E+01 \\
5.01 E-01\end{array}$ & $\begin{array}{l}5.67 E-01 \\
7.08 E+00 \\
0.0 \\
1.56 E+01 \\
5.90 E-01\end{array}$ & $\begin{array}{l}7.63 E-01 \\
0.49 E+00 \\
0.0 \\
1.92 E+01 \\
1.12 E+00\end{array}$ & $\begin{array}{l}5.07 E-01 \\
6.21 E+00 \\
0.0 \\
1.38 E+01 \\
5.35 E-01\end{array}$ & $\begin{array}{l}4.53 E-01 \\
5.61 E+00 \\
0.0 \\
1.24 E+01 \\
4.74 E-01\end{array}$ & $\begin{array}{l}5.40 E-01 \\
6.61 E+00 \\
0.0 \\
1.46 E+01 \\
5.75 E-01\end{array}$ & $\begin{array}{l}4.96 E-01 \\
6.07 E+00 \\
0.0 \\
1.35 E+01 \\
5.17 E-01\end{array}$ & $\begin{array}{l}5.36 E-01 \\
6.65 E+00 \\
0.0 \\
1.46 E+01 \\
6.00 E-01\end{array}$ \\
\hline $\begin{array}{l}0 S-191 M \\
0 S-193 \\
1 R-190 \\
\text { IR-190M } \\
\text { IR-190M }\end{array}$ & $\begin{array}{r}13.03 \\
30.0 \\
11.78 \\
1.2 \\
1.2 \\
3.2 \mathrm{H}\end{array}$ & $\begin{array}{l}3.10 E-02 \\
6.04 E-01 \\
1.31 E+01 \\
4.12 E-05 \\
3.19 E-01\end{array}$ & $\begin{array}{l}3.45 E-02 \\
5.79 E-01 \\
1.24 E+01 \\
2.91 E-08 \\
3.57 E-01\end{array}$ & $\begin{array}{l}3.28 E-02 \\
6.26 E-01 \\
1.37 E+01 \\
3.29 E-06 \\
3.39 E-01\end{array}$ & & & & & & $\begin{array}{l}3.65 E-02 \\
5.99 \mathrm{E}-01 \\
1.29 \mathrm{E}-01 \\
1.02 \mathrm{E}-07 \\
3.76 \mathrm{E}-01\end{array}$ \\
\hline $\begin{array}{l}1 R-192 \\
\text { IR-193M } \\
\text { IR-194 } \\
\text { IR-194M } \\
P T-191\end{array}$ & 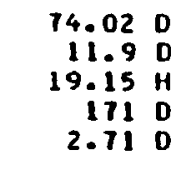 & $\begin{array}{l}7.69 E+00 \\
2.35 E-03 \\
8.65 E-01 \\
2.20 E+01 \\
2.49 E+00\end{array}$ & $\begin{array}{l}7.18 E+00 \\
2.56 E-03 \\
8.19 E-01 \\
2.09 E+01 \\
2.44 E+00\end{array}$ & $\begin{array}{l}1.90 E+00 \\
2.46 E-03 \\
9.06 E-01 \\
2.32 E+01 \\
2.60 E+00\end{array}$ & $\begin{array}{l}1.01 E+01 \\
8.43 E-03 \\
1.11 E+00 \\
2.80 E+01 \\
3.87 E+00\end{array}$ & $\begin{array}{l}1.01 E+00 \\
2.19 E-03 \\
8.03 E-01 \\
2.04 E+01 \\
2.30 E+00\end{array}$ & $\begin{array}{l}6.30 E+00 \\
1.98 E-03 \\
7.26 E-01 \\
1.04 E+01 \\
2.07 E \cdot 00\end{array}$ & $\begin{array}{l}7.42 E+00 \\
2.36 E-03 \\
8.52 E-01 \\
2.16 E+01 \\
2.45 E+00\end{array}$ & $\begin{array}{l}6.91 E+00 \\
2.11 E-03 \\
7.91 E-01 \\
2.00 E+01 \\
2.25 E+00\end{array}$ & $\begin{array}{l}7.43 E+00 \\
2.68 E-03 \\
8.52 E-01 \\
2.16 E+01 \\
2.54 E+00\end{array}$ \\
\hline $\begin{array}{l}P T-193 \\
P T-193 M \\
P T-195 M \\
P T-197 \\
P T-197 M\end{array}$ & $\begin{array}{rl}50 & Y \\
4.33 & 0 \\
4.02 & 0 \\
18.3 & H \\
94.4 & M\end{array}$ & $\begin{array}{l}4.01 E-05 \\
7.53 E-02 \\
5.21 E-01 \\
1.91 E-01 \\
6.75 E-01\end{array}$ & $\begin{array}{l}7.23 \mathrm{E}-12 \\
6.21 \mathrm{E}-02 \\
5.51 \mathrm{E}-01 \\
1.92 \mathrm{E}-01 \\
6.66 \mathrm{E}-01\end{array}$ & $\begin{array}{l}3.20 E-06 \\
7.99 E-02 \\
5.45 E-01 \\
1.97 E-01 \\
6.96 E-01\end{array}$ & $\begin{array}{l}3.14 E-03 \\
1.12 E-01 \\
1.12 E+00 \\
3.58 E-01 \\
1.12 E+00\end{array}$ & $\begin{array}{l}5.22 \mathrm{E}-06 \\
7.12 \mathrm{E}-02 \\
4.90 \mathrm{E}-01 \\
1.70 \mathrm{E}-01 \\
6.20 \mathrm{E}-01\end{array}$ & $\begin{array}{l}6.74 E-08 \\
6.42 E-02 \\
4.37 E-01 \\
1.58 E-01 \\
5.58 E-01\end{array}$ & $\begin{array}{l}9.92 \mathrm{E}-00 \\
7.65 \mathrm{E}-02 \\
5.27 \mathrm{E}-01 \\
1.89 \mathrm{E}-01 \\
6.60 \mathrm{E}-01\end{array}$ & $\begin{array}{l}1.65 E-05 \\
6.80 E-02 \\
4.69 E-01 \\
1.72 E-01 \\
6.06 E-01\end{array}$ & $\begin{array}{l}4.81 E-08 \\
8.57 E-02 \\
5.73 E-01 \\
1.97 E-01 \\
6.91 E-01\end{array}$ \\
\hline
\end{tabular}


DOSE-RATE FAC TORS FOR BOOY URGANS AND EFFECTIVE DOSE-RATE FACTCR FOR IMMERSIUN IN COMTAMINATED WATER
IN MREM/YR PER MICROCURIE/ICUB IC MI

\begin{tabular}{|c|c|c|c|c|c|c|c|c|c|c|}
\hline CLIOE & IFE & LIVER & LUNGS & RROW & $\begin{array}{l}\text { o } \\
\text { ow }\end{array}$ & VARIES & NCREAS & KELETON & SPLEEN & STOMACH \\
\hline $\begin{array}{l}E R-171 \\
T M-170 \\
T M-171 \\
Y B B-169 \\
Y B-175\end{array}$ & $\begin{array}{r}7.52 \mathrm{H} \\
128.6 \mathrm{D} \\
1.92 \mathrm{Y} \\
31.97 \mathrm{D} \\
4.19 \mathrm{D}\end{array}$ & $\begin{array}{l}3.17 E+00 \\
3.80 E-02 \\
4.24 E-03 \\
2.37 E+00 \\
3.38 E-01\end{array}$ & $\begin{array}{l}3.44 E+00 \\
4.22 E-02 \\
4.72 E-03 \\
2.61 E+00 \\
3.65 E-01\end{array}$ & $\begin{array}{l}4.52 E+00 \\
7.39 E-02 \\
8.50 E-03 \\
4.03 E+00\end{array}$ & $\begin{array}{l}3.39 E+00 \\
2.63 E-02 \\
2.45 E-03 \\
2.12 E+00 \\
3.64 E-01\end{array}$ & $\begin{array}{l}.88 E+00 \\
.14 E-02 \\
.44 E-03 \\
.03 E+00\end{array}$ & $\begin{array}{l}2.68 E+00 \\
2.56 E-02 \\
2.59 E-03 \\
1.82 E+00 \\
2.87 E-01\end{array}$ & $\begin{array}{l}4.61 E+00 \\
7.59 E-02 \\
8.72 E-03 \\
4.13 E+00 \\
4.74 E-01\end{array}$ & $\begin{array}{l}3.11 E+00 \\
3.47 E-02 \\
3.67 E-03 \\
2.25 E+00 \\
3.31 E-01\end{array}$ & $\begin{array}{l}3.12 \mathrm{E}+00 \\
3.56 \mathrm{E}-02 \\
3.85 \mathrm{E}-03 \\
2.28 \mathrm{E}+00 \\
3.32 \mathrm{E}-01\end{array}$ \\
\hline $\begin{array}{l}1 U-177 \\
L U-17 M \\
H F-181 \\
T A-182 \\
H-181\end{array}$ & 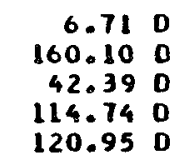 & $\begin{array}{l}2.93 E-01 \\
8.41 E \bullet 00 \\
4.73 E \bullet 00 \\
1.20 E+01 \\
2.68 E-01\end{array}$ & $\begin{array}{l}3.21 E-01 \\
9.14 E+00 \\
5.11 E+00 \\
1.28 E+01 \\
2.97 E-01\end{array}$ & $\begin{array}{l}4.68 E-01 \\
1.23 E+01 \\
6.30 E+00 \\
1.41 E+01 \\
5.34 E-01\end{array}$ & $\begin{array}{l}.99 E-01 \\
.90 E+00 \\
.09 E \bullet 00 \\
.27 E+01 \\
.61 E-01\end{array}$ & $\begin{array}{l}.56 E-01 \\
057 E+00 \\
.44 E+00 \\
.14 E+01 \\
.18 E-01\end{array}$ & $\begin{array}{l}2.42 E-01 \\
7.07 E+00 \\
4.03 E+00 \\
1.03 E+01 \\
1.66 E-01\end{array}$ & $\begin{array}{l}4.79 E-01 \\
1.26 E+01 \\
6.41 E+00 \\
1.44 E+01 \\
5.47 E-01\end{array}$ & $\begin{array}{l}2.89 E-01 \\
8.26 E+00 \\
4.63 E+00 \\
1.16 E+01 \\
2.35 E-01\end{array}$ & $\begin{array}{l}2.88 E-01 \\
8.27 E+00 \\
4.66 E+00 \\
1.18 E+01 \\
2.45 E-01\end{array}$ \\
\hline $\begin{array}{l}H-185 \\
H-187 \\
H-188 \\
R E-182 \\
R E-182 M\end{array}$ & $\begin{array}{rl}75.1 & \mathrm{D} \\
23.83 & \mathrm{H} \\
69.4 \mathrm{D} \\
64.0 \mathrm{H} \\
12.7 \mathrm{H}\end{array}$ & $\begin{array}{l}2.30 E-04 \\
4.24 E+00 \\
1.51 E-02 \\
1.59 E+01 \\
1.11 E+01\end{array}$ & $\begin{array}{l}2.55 E-04 \\
4.55 E \cdot 00 \\
1.64 E-02 \\
1.70 E \cdot 01 \\
1.18 E \cdot 01\end{array}$ & $\begin{array}{l}4.00 E-04 \\
5.31 E+00 \\
2.16 E-02 \\
1.99 E+01 \\
1.32 E+01\end{array}$ & & & & & & $\begin{array}{l}2.28 \mathrm{E}-04 \\
4.18 \mathrm{E}+00 \\
1.49 \mathrm{E}-02 \\
1.55 \mathrm{E}+01 \\
1.09 \mathrm{E}+01\end{array}$ \\
\hline $\begin{array}{l}R E-183 \\
R E-184 \\
R E-184 M \\
R E-186 \\
R E-187\end{array}$ & $\begin{array}{r}70 \\
38.0 \\
169 \\
90 \\
90.64 \mathrm{H} \\
4.7 E 10 \mathrm{Y}\end{array}$ & $\begin{array}{l}1.19 E+00 \\
8.18 E+00 \\
3.41 E+00 \\
1.69 E-01 \\
0.0\end{array}$ & $\begin{array}{l}1.30 E+00 \\
8.75 E+00 \\
3.6 \theta E+00 \\
1.87 E-01 \\
0.0\end{array}$ & $\begin{array}{l}2.09 E+00 \\
9.88 E+00 \\
4.60 E+00 \\
2.97 E-01 \\
0.0\end{array}$ & $\begin{array}{l}1.00 E+00 \\
8.65 E+00 \\
3.51 E+00 \\
1.52 E-01 \\
0.0\end{array}$ & & & $\begin{array}{l}2.14 E+00 \\
1.01 E+01 \\
4.69 E \bullet 00 \\
3.05 E-01 \\
0.0\end{array}$ & & $\begin{array}{l}1.13 E+00 \\
B .06 E+00 \\
3.35 E+00 \\
1.64 E-01 \\
0.0\end{array}$ \\
\hline $\begin{array}{l}\text { RE- } 188 \\
\text { OS-185 } \\
\text { OS-186 } \\
\text { OS-190M } \\
\text { OS-191 }\end{array}$ & $\begin{array}{r}16.99 \mathrm{H} \\
93.6 \mathrm{D} \\
2.0 \mathrm{E} 15 \mathrm{Y} \\
9.9 \mathrm{M} \\
15.4 \mathrm{O}\end{array}$ & $\begin{array}{l}5.15 E-01 \\
6.33 E+00 \\
0.0 \\
1.40 E+01\end{array}$ & $\begin{array}{l}5.57 E-01 \\
6.78 E+00 \\
0.0 \\
1.51 E+01 \\
6.36 E-01\end{array}$ & $\begin{array}{l}7.16 E-01 \\
7.78 E+00 \\
0.0\end{array}$ & $\begin{array}{l}5.38 E-01 \\
6.74 E+00 \\
0.0 \\
1.53 E+01\end{array}$ & $\begin{array}{l}4.74 E-01 \\
6.08 E+00 \\
0.0 \\
1.33 E+01\end{array}$ & $\begin{array}{l}4.35 E-01 \\
5.38 E+00 \\
0.0 \\
1.20 E+01\end{array}$ & $\begin{array}{l}7.32 E-01 \\
7.92 E+00 \\
0.0 \\
1.81 E+01 \\
1.09 E+00\end{array}$ & $\begin{array}{l}5.05 E-01 \\
6.12 E+00 \\
0.0 \\
1.37 E \cdot 01 \\
5.46 E-01\end{array}$ & $\begin{array}{l}5.08 E-01 \\
6.23 E \cdot 00 \\
0.0 \\
1.38 E+01 \\
5.52 E-01\end{array}$ \\
\hline $\begin{array}{l}O S-191 M \\
O S-193 \\
1 R-190 \\
1 R-190 M \\
\text { IR-190M }\end{array}$ & $\begin{array}{r}13.03 \mathrm{H} \\
30.0 \mathrm{H} \\
11.78 \mathrm{D} \\
1.2 \mathrm{H} \\
3.2 \mathrm{H}\end{array}$ & $\begin{array}{l}3.39 E-02 \\
5.75 E-01 \\
1.23 E+01 \\
4.19 E-07 \\
3.50 E-01\end{array}$ & $\begin{array}{l}3.75 E-02 \\
6.23 E-01 \\
1.33 E+01\end{array}$ & & & $\begin{array}{l}2.76 E-02 \\
5.27 \mathrm{E}-01 \\
1.17 \mathrm{E}+01 \\
1.61 \mathrm{E}-05\end{array}$ & & & & $\begin{array}{l}3.13 E-02 \\
5.63 E-01 \\
8.22 E+01 \\
8.41 E-06 \\
3.23 E-01\end{array}$ \\
\hline $\begin{array}{l}1 R-192 \\
1 R-193 M \\
1 R-194 \\
1 R-194 M \\
P T-191\end{array}$ & 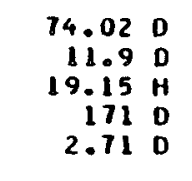 & $\begin{array}{l}7.15 E+00 \\
2.52 E-03 \\
8.15 E-01 \\
2.07 E+01 \\
2.42 E+00\end{array}$ & $\begin{array}{l}7.70 E+00 \\
2.81 E-03 \\
8.75 E-01 \\
2.23 E+01 \\
2.63 E+00\end{array}$ & $\begin{array}{l}9.39 E+00 \\
4.95 E-03 \\
1.03 E+00 \\
2.59 E+01 \\
3.61 E+00\end{array}$ & $\begin{array}{l}7.89 E+00 \\
1.65 E-03 \\
8.94 E-01 \\
2.28 E+01 \\
2.34 E+00\end{array}$ & $\begin{array}{l}6.67 E+00 \\
2.07 E-03 \\
7.65 E-01 \\
1.98 E+01 \\
2.19 E+00\end{array}$ & $\begin{array}{l}6.15 E+00 \\
1.66 E-03 \\
7.04 E-01 \\
1.78 E+01 \\
1.93 E+00\end{array}$ & $\begin{array}{l}9.56 E+00 \\
5.11 E-03 \\
1.05 E+00 \\
2.64 E+01 \\
3.69 E+00\end{array}$ & $\begin{array}{l}7.02 E \bullet 00 \\
2.27 E-03 \\
1.98 E-0 I \\
2.03 E * 0 I \\
2.32 E \bullet 00\end{array}$ & $\begin{array}{l}7.05 E+00 \\
2.34 E-03 \\
8.04 E-00 \\
2.05 E+01 \\
2.35 E+00\end{array}$ \\
\hline $\begin{array}{l}\text { PY }-193 \\
\text { PT-193M } \\
\text { PT-195M } \\
\text { PT-1 } 197 \\
\text { PT-197M }\end{array}$ & $\begin{array}{rl}50 & Y \\
4.33 & D \\
4.02 & D \\
18.3 & H \\
94.4 & M\end{array}$ & $\begin{array}{l}3.90 \mathrm{E}-07 \\
0.11 \mathrm{E}-02 \\
5.45 \mathrm{E}-01 \\
1.91 \mathrm{E}-01 \\
6.60 \mathrm{E}-01\end{array}$ & $\begin{array}{l}3.17 E-05 \\
8.94 E-02 \\
6.02 E-01 \\
2.09 E-01 \\
7.19 E-01\end{array}$ & $\begin{array}{l}1.90 E-05 \\
1.58 E-01 \\
1.05 E+00 \\
3.41 E-01 \\
1.04 E+00\end{array}$ & $\begin{array}{l}1.04 E-05 \\
5.43 E-02 \\
3.93 E-01 \\
1.60 E-01 \\
6.19 E-01\end{array}$ & $\begin{array}{l}1.57 E-05 \\
6.63 E-02 \\
4.50 E-01 \\
1.61 E-01 \\
5.82 E-01\end{array}$ & $\begin{array}{l}2.34 E-05 \\
5.34 E-02 \\
3.75 E-01 \\
1.42 E-01 \\
5.17 E-01\end{array}$ & $\begin{array}{l}5.15 E-05 \\
1.62 E-01 \\
1.07 E+00 \\
3.50 E-01 \\
1.07 E+00\end{array}$ & $\begin{array}{l}9.05 E-06 \\
7.34 E-02 \\
5.03 E-01 \\
1.81 E-01 \\
6.30 E-01\end{array}$ & $\begin{array}{l}\text { B. } 18 E-06 \\
7.53 E-02 \\
5.13 E-01 \\
1.83 E-01 \\
6.37 E-01\end{array}$ \\
\hline
\end{tabular}


DUSE-KATE FACTORS FOR BODY ORGANS AND EFFECTIVE DOSE-RATE FACTOR FOR IMMEFSION IN CUNTAMITATEIS WAI E.

\begin{tabular}{|c|c|c|c|c|c|c|c|c|c|}
\hline$\angle 10 E$ & ALF-LIFE & TESTES & THYMUS & IYROID & UTERUS & EFFECTIVE & $\begin{array}{c}\text { SKIN } \\
\text { (PHOTON) }\end{array}$ & $\begin{array}{c}\text { SKIN } \\
\text { (ELECTRONI }\end{array}$ & $\begin{array}{l}\text { SKIN } \\
\text { ITOTAL I }\end{array}$ \\
\hline $\begin{array}{l}E R-171 \\
Y M-170 \\
T M-171 \\
Y B-169 \\
Y B-175\end{array}$ & 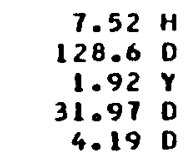 & $\begin{array}{l}4.74 E+00 \\
6.67 E-02 \\
9.04 E-03 \\
3.90 E+00 \\
5.00 E-01\end{array}$ & $\begin{array}{l}3.58 E+00 \\
5.04 E-02 \\
5.88 E-03 \\
2.92 E+00 \\
3.7 T E-01\end{array}$ & $\begin{array}{l}4.49 E+00 \\
6.49 E-02 \\
7.61 E-03 \\
3.73 E+00 \\
4.70 E-01\end{array}$ & $\begin{array}{l}2.77 E+00 \\
2.98 E-02 \\
3.08 E-03 \\
1.96 E+00 \\
2.95 E-01\end{array}$ & $\begin{array}{l}4.10 E+00 \\
5.59 E-02 \\
6.54 E-03 \\
3.31 E+00 \\
4.31 E-01\end{array}$ & $\begin{array}{l}5.31 E+00 \\
7.49 E-02 \\
9.37 E-03 \\
4.38 E+00 \\
5.63 E-01\end{array}$ & $\begin{array}{l}2.63 E+00 \\
2.19 E+00 \\
3.76 E-04 \\
1.86 E-01 \\
5.86 E-01\end{array}$ & $\begin{array}{l}7.94 E+00 \\
2.26 E+00 \\
9.15 E-03 \\
4.56 E+00 \\
1.15 E+00\end{array}$ \\
\hline $\begin{array}{l}1 U-177 \\
1 U-177 M \\
H F-181 \\
T A-182 \\
H-181\end{array}$ & 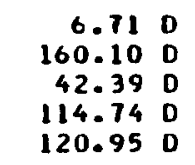 & $\begin{array}{l}4.51 E-01 \\
1.26 E+01 \\
6.94 E+00 \\
1.70 E+01 \\
4.91 E-01\end{array}$ & $\begin{array}{l}3.44 E-01 \\
9.58 E+00 \\
5.25 E+00 \\
1.36 E+01 \\
3.68 E-01\end{array}$ & $\begin{array}{l}4.37 E-01 \\
1.20 E+01 \\
6.50 E+00 \\
1.63 E+01 \\
4.72 E-01\end{array}$ & $\begin{array}{l}2.55 E-01 \\
7.33 E+00 \\
4.13 E+00 \\
1.06 E+01 \\
2.00 E-01\end{array}$ & $\begin{array}{l}3.94 E-01 \\
1.10 E+01 \\
5.96 E \cdot 00 \\
1.46 E \cdot 01 \\
4.04 E-01\end{array}$ & $\begin{array}{l}4.95 E-01 \\
1.41 E+01 \\
7.85 E+00 \\
1.95 E \circ 01 \\
5.61 E-01\end{array}$ & $\begin{array}{l}6.42 E-01 \\
5.08 E-01 \\
6.87 E-01 \\
6.16 E-01 \\
0.0\end{array}$ & $\begin{array}{l}1.14 E+00 \\
1.46 E * 01 \\
8.54 E * 00 \\
2.02 E * 01 \\
5.61 E-01\end{array}$ \\
\hline $\begin{array}{l}W-185 \\
H-187 \\
H-188 \\
R E-182 \\
R E-182 M\end{array}$ & $\begin{array}{r}75.1 \mathrm{D} \\
23.83 \mathrm{H} \\
69.4 \mathrm{D} \\
64.0 \mathrm{H} \\
12.7 \mathrm{H}\end{array}$ & $\begin{array}{l}3.58 E-04 \\
6.14 E+00 \\
2.24 E-02 \\
2.29 E+01 \\
1.58 E+01\end{array}$ & $\begin{array}{l}2.77 E-04 \\
4.74 E+00 \\
1.70 E-02 \\
1.80 E+01 \\
1.26 E+01\end{array}$ & $\begin{array}{l}3.56 E-04 \\
5.78 E+00 \\
2.12 E-02 \\
2.18 E+01 \\
1.50 E+01\end{array}$ & $\begin{array}{l}2.01 E-04 \\
3.68 E+00 \\
1.33 E-02 \\
1.39 E+01 \\
9.73 E+00\end{array}$ & $\begin{array}{l}3.16 \mathrm{E}-04 \\
5.25 \mathrm{E}+00 \\
1.95 \mathrm{E}-02 \\
1.96 \mathrm{E} \cdot 01 \\
1.35 \mathrm{E} \cdot 01\end{array}$ & $\begin{array}{l}3.02 E-04 \\
7.03 E+00 \\
2.49 E-02 \\
2.60 E+01 \\
1.81 E+01\end{array}$ & $\begin{array}{l}5.54 E-01 \\
1.80 E+00 \\
3.43 E-01 \\
2.79 E-01 \\
1.56 E-01\end{array}$ & $\begin{array}{l}5.55 E-01 \\
8.83 E+00 \\
3.67 E-01 \\
2.63 E+01 \\
1.83 E+01\end{array}$ \\
\hline $\begin{array}{l}R E-183 \\
R E-184 \\
R E-184 M \\
R E-186 \\
R E-187\end{array}$ & $\begin{array}{r}700 \\
38.00 \\
1690 \\
90.64 \mathrm{H} \\
4 . J E 10 \mathrm{~V}\end{array}$ & $\begin{array}{l}1.96 E \bullet 00 \\
1.17 E \bullet 01 \\
5.05 E \bullet 00 \\
2.71 E-01 \\
0.0\end{array}$ & $\begin{array}{l}1.49 E+00 \\
9.29 E+00 \\
3.93 E+00 \\
2.08 E-01 \\
0.0\end{array}$ & $\begin{array}{l}1.90 E+00 \\
1.12 E+01 \\
4.83 E+00 \\
2.66 E-01 \\
0.0\end{array}$ & $\begin{array}{l}9.75 E-01 \\
1.07 E+00 \\
2.94 E \bullet 00 \\
1.43 E-01 \\
0.0\end{array}$ & $\begin{array}{l}1.66 E+00 \\
1.00 E \bullet 01 \\
4.32 E+00 \\
2.34 E-01 \\
0.0\end{array}$ & $\begin{array}{l}2.19 E+00 \\
1.35 E+01 \\
5.73 E+00 \\
2.95 E-01 \\
0.0\end{array}$ & $\begin{array}{l}7.84 E-02 \\
7.67 E-02 \\
8.66 E-02 \\
2.35 E+00 \\
0.0\end{array}$ & $\begin{array}{l}2.27 E+00 \\
1.36 E+01 \\
5.82 E+00 \\
2.64 E+00 \\
0.0\end{array}$ \\
\hline $\begin{array}{l}\text { RE-1 } 88 \\
O S-185 \\
C S-186 \\
O S-190 M \\
O S-191\end{array}$ & $\begin{array}{r}16.98 \mathrm{H} \\
93.6 \mathrm{O} \\
2.0 \mathrm{E} 15 \mathrm{Y} \\
9.9 \mathrm{M} \\
15.4 \mathrm{D}\end{array}$ & $\begin{array}{l}7.59 E-01 \\
9.14 E \cdot 00 \\
0.0 \\
2.03 E \cdot 01 \\
9.46 E-01\end{array}$ & $\begin{array}{l}5.91 E-01 \\
7.11 E+00 \\
0.0 \\
1.54 E+01 \\
7.30 E-01\end{array}$ & $\begin{array}{l}1.30 E-01 \\
8.62 E+00 \\
0.0 \\
1.90 E+01 \\
9.33 E-01\end{array}$ & $\begin{array}{l}4.50 E-01 \\
5.47 E+00 \\
0.0 \\
1.22 E \cdot 01 \\
4.77 E-01\end{array}$ & $\begin{array}{l}6.57 E-01 \\
7.79 E+00 \\
0.0 \\
1.74 E+01 \\
8.11 E-01\end{array}$ & $\begin{array}{l}8.50 E-01 \\
1.05 E+01 \\
0.0 \\
2.31 E+01 \\
1.04 E+00\end{array}$ & $\begin{array}{l}6.28 E+00 \\
4.37 E-02 \\
0.0 \\
4.04 E-01 \\
5.84 E-02\end{array}$ & $\begin{array}{l}1.13 E+00 \\
1.06 E+01 \\
0.0 \\
2.35 E+01 \\
1.10 E+00\end{array}$ \\
\hline $\begin{array}{l}O S-191 \mathrm{M} \\
0 S-193 \\
1 R-190 \\
\text { IR-190M } \\
\text { IR-190M }\end{array}$ & 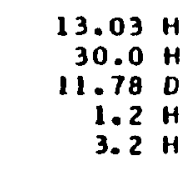 & $\begin{array}{l}5.98 E-02 \\
0.62 E-01 \\
1.79 E+01 \\
2.99 E-04 \\
6.15 E-01\end{array}$ & $\begin{array}{l}4.57 E-02 \\
6.56 E-01 \\
1.37 E+01 \\
3.52 E-08 \\
4.72 E-01\end{array}$ & $\begin{array}{l}5.83 E-02 \\
8.16 E-01 \\
1.68 E+01 \\
2.07 E-05 \\
6.01 E-01\end{array}$ & $\begin{array}{l}2.62 E-02 \\
4.97 E-01 \\
1.08 E+01 \\
9.06 E-09 \\
2.71 E-01\end{array}$ & $\begin{array}{l}5.02 \mathrm{E}-02 \\
7.40 \mathrm{E}-01 \\
1.54 \mathrm{E}+01 \\
5.72 \mathrm{E}-04 \\
5.13 \mathrm{E}-01\end{array}$ & $\begin{array}{l}7.24 E-02 \\
9.69 E-01 \\
2.04 E+01 \\
6.88 E-03 \\
6.88 E-01\end{array}$ & $\begin{array}{l}4.33 E-04 \\
2.48 E+00 \\
2.65 E-01 \\
0.0 \\
1.94 E-02\end{array}$ & $\begin{array}{l}7.28 E-02 \\
3.45 E+00 \\
2.07 E+01 \\
6.88 E-03 \\
7.07 E-01\end{array}$ \\
\hline $\begin{array}{l}\text { IR-192 } \\
\text { IR-193M } \\
1 R-194 \\
\text { IR-194M } \\
\text { PT-191 }\end{array}$ & 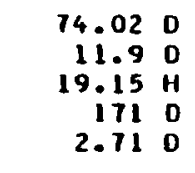 & $\begin{array}{l}1.04 E+01 \\
4.66 E-03 \\
1.17 E+00 \\
2.99 E+01 \\
3.12 E+00\end{array}$ & $\begin{array}{l}7.87 E \cdot 00 \\
3.37 E-03 \\
9.05 E-01 \\
2.28 E+01 \\
2.84 E+00\end{array}$ & $\begin{array}{l}9.73 E+00 \\
4.31 E-03 \\
1.11 E+00 \\
2.79 E+01 \\
3.53 E \bullet 00\end{array}$ & $\begin{array}{l}6.27 E \cdot 00 \\
1.97 E-03 \\
1.17 E-01 \\
1.81 E+01 \\
2.06 E+00\end{array}$ & $\begin{array}{l}4.21 E-03 \\
1.01 E+00 \\
2.56 E+01 \\
3.17 E+00\end{array}$ & $\begin{array}{l}1.18 E+01 \\
1.15 E-02 \\
1.33 E+00 \\
3.41 E+01 \\
4.18 E+00\end{array}$ & & $\begin{array}{l}1.30 E+01 \\
1.51 E-02 \\
7.99 E+00 \\
3.47 E+01 \\
4.29 E+00\end{array}$ \\
\hline $\begin{array}{l}\text { PT-193 } \\
\text { PT-193M } \\
\text { PT-195M } \\
\text { PT-197 } \\
\text { PT-197M }\end{array}$ & $\begin{array}{rl}50 & Y \\
4.33 & 0 \\
4.02 & D \\
18.3 & H \\
94.4 & M\end{array}$ & $\begin{array}{l}2.91 E-04 \\
1.39 E-01 \\
9.24 E-01 \\
3.07 E-01 \\
1.03 E+00\end{array}$ & $\begin{array}{l}6.70 E-09 \\
1.08 E-01 \\
7.12 E-01 \\
2.38 E-01 \\
7.86 E-01\end{array}$ & $\begin{array}{l}2.01 \mathrm{E}-05 \\
1.37 \mathrm{E}-01 \\
9.10 \mathrm{E}-01 \\
3.03 \mathrm{E}-01 \\
9.87 \mathrm{E}-01\end{array}$ & $\begin{array}{l}2.93 E-09 \\
6.40 E-02 \\
4.38 E-01 \\
1.59 E-01 \\
5.58 E-01\end{array}$ & $\begin{array}{l}5.58 E-04 \\
1.18 E-01 \\
7.83 E-01 \\
2.64 E-01 \\
8.82 E-01\end{array}$ & $\begin{array}{l}6.70 E-03 \\
1.60 E-01 \\
1.03 E+00 \\
3.39 E-01 \\
1.16 E .00\end{array}$ & $\begin{array}{l}0.0 \\
3.00 E-01 \\
2.77 E-01 \\
1.15 E+00 \\
1.76 E+00\end{array}$ & $\begin{array}{l}6.70 E-03 \\
4.61 E-01 \\
1.31 E+00 \\
1.49 E+00 \\
2.92 E+00\end{array}$ \\
\hline
\end{tabular}


DOSE-RATE FACTORS FOR BODY ORGANS AND EFFECTIVE DOSE-RATE FACTOR FOR IMMEFSION IN CONIAMINATEQ HATLA

\begin{tabular}{|c|c|c|c|c|c|c|c|c|c|c|}
\hline NUCL IDE & HALF-LIFE & ADRENALS & BLADDER & BRAIN & BREAST & HE ART & $\begin{array}{l}\text { SHALL } \\
\text { INTESTINE }\end{array}$ & $\begin{array}{c}\text { UPPER } \\
\text { LARGE } \\
\text { INTESTINE }\end{array}$ & $\begin{array}{c}\text { LOWER } \\
\text { IARGE } \\
\text { INTESTINE }\end{array}$ & KIONEYS \\
\hline $\begin{array}{l}A U-194 \\
A U-195 \\
A U-195 \mathrm{M} \\
A U-196 \\
A U-198\end{array}$ & $\begin{array}{rl}39.5 & H \\
183 & 0 \\
30.6 & 5 \\
6.183 & 0 \\
2.696 & 0\end{array}$ & $\begin{array}{l}1.03 E+01 \\
6.01 E-01 \\
1.04 E+00 \\
4.33 E \cdot 00 \\
3.19 E \cdot 00\end{array}$ & $\begin{array}{l}02 E+01 \\
44 E-01 \\
70 E+00 \\
0 B E \cdot 00 \\
55 E \cdot 00\end{array}$ & $\begin{array}{l}14 E+01 \\
33 E-01 \\
83 E+00 \\
43 E+00 \\
92 E+00\end{array}$ & $\begin{array}{l}1.35 E+01 \\
1.31 E+00 \\
2.63 E+00 \\
6.02 E+00 \\
4.88 E+00\end{array}$ & $\begin{array}{l}1.01 E+01 \\
5.67 E-01 \\
1.65 E+00 \\
3.94 E+00 \\
3.47 E+00\end{array}$ & $\begin{array}{l}9.22 E+00 \\
5.00 E-01 \\
1.48 E+00 \\
3.54 E+00 \\
3.11 E+00\end{array}$ & $\begin{array}{l}1.07 E+01 \\
6.10 E-01 \\
1.75 E+00 \\
4.17 E+00 \\
3.67 E+00\end{array}$ & $\begin{array}{l}9.88 E+00 \\
5.42 E-01 \\
1.64 E+00 \\
3.88 E+00 \\
3.41 E+00\end{array}$ & $\begin{array}{l}1.0 B E+01 \\
6.70 E-01 \\
1.76 E+00 \\
4.22 E+00 \\
3.67 E+00\end{array}$ \\
\hline $\begin{array}{l}A U-199 \\
H G-197 \\
H G-197 M \\
H G-203 \\
T L-200\end{array}$ & $\begin{array}{rl}3.139 & 0 \\
64.14 & H \\
23.8 \mathrm{H} & \mathrm{H} \\
46.60 & 0 \\
26.1 & \mathrm{H}\end{array}$ & $\begin{array}{l}8.15 E-01 \\
4.96 E-01 \\
7.92 E-01 \\
2.14 E+00 \\
1.24 E+01\end{array}$ & $\begin{array}{l}7.60 E-01 \\
5.32 E-01 \\
7.64 E-01 \\
1.97 E \bullet 00 \\
1.21 E+01\end{array}$ & $\begin{array}{l}8.06 \mathrm{E}-01 \\
5.26 \mathrm{E}-01 \\
8.00 \mathrm{E}-01 \\
2.13 \mathrm{E}+00 \\
1.35 \mathrm{E}+01\end{array}$ & $\begin{array}{l}1.29 E+00 \\
1.08 E+00 \\
1.37 E+00 \\
2.97 E+00 \\
1.59 E+01\end{array}$ & $\begin{array}{l}7.37 E-01 \\
4.70 E-01 \\
7.28 E-01 \\
1.92 E+00 \\
1.18 E+01\end{array}$ & $\begin{array}{l}6.50 E-01 \\
4.21 E-01 \\
6.41 E-01 \\
1.72 E+00 \\
1.08 E+01\end{array}$ & $\begin{array}{l}7.84 E-01 \\
5.03 E-01 \\
7.78 E-01 \\
2.03 E \cdot 00 \\
1.26 E+01\end{array}$ & $\begin{array}{l}7.24 E-01 \\
4.48 E-01 \\
7.08 E-01 \\
1.90 E-00 \\
1.16 E+01\end{array}$ & $\begin{array}{l}1.77 E-01 \\
5.51 E-01 \\
1.82 E-01 \\
2.03 E+00 \\
1.26 E+01\end{array}$ \\
\hline $\begin{array}{l}T L-201 \\
T L-202 \\
T L-204 \\
T L-207 \\
T L-208\end{array}$ & $\begin{array}{r}73.06 \mathrm{H} \\
12.23 \mathrm{O} \\
3.779 \\
4.77 \\
3.053\end{array}$ & $\begin{array}{l}1.14 E-01 \\
4.24 E \cdot 00 \\
8.03 E-03 \\
2.12 E-02 \\
3.53 E \cdot 01\end{array}$ & $\begin{array}{l}7.35 E-01 \\
4.05 E \bullet 00 \\
8.61 E-03 \\
2.05 E-02 \\
3.60 E \bullet 01\end{array}$ & $\begin{array}{l}1.42 \mathrm{E}-01 \\
4.43 \mathrm{E}+00 \\
8.52 \mathrm{E}-03 \\
2.31 \mathrm{E}-02 \\
3.99 \mathrm{E}+01\end{array}$ & $\begin{array}{l}1.42 E \cdot 00 \\
5.01 E \cdot 00 \\
1.74 E-02 \\
2.58 E-02 \\
4.56 E \cdot 01\end{array}$ & $\begin{array}{l}6.68 E-01 \\
3.91 E+00 \\
7.63 E-03 \\
2.03 E-02 \\
3.59 E+01\end{array}$ & $\begin{array}{l}5.95 E-01 \\
3.52 E+00 \\
6.82 E-03 \\
1.84 E-02 \\
3.35 E+01\end{array}$ & $\begin{array}{l}7.14 E-01 \\
4.15 E+00 \\
8.16 E-03 \\
2.16 E-02 \\
3.81 E+01\end{array}$ & $\begin{array}{l}6.42 E-01 \\
3.83 E+00 \\
7.26 E-03 \\
1.98 E-02 \\
3.53 E .01\end{array}$ & $\begin{array}{l}\text { 7. } 58 \mathrm{E}-01 \\
4.19 \mathrm{E}+00 \\
8.92 \mathrm{E}-03 \\
2.15 \mathrm{E}-02 \\
3.87 \mathrm{E} 01\end{array}$ \\
\hline $\begin{array}{l}I L-209 \\
T L-210 \\
P B-203 \\
P B-204 M \\
P B-205\end{array}$ & $\begin{array}{r}2.20 \mathrm{M} \\
1.30 \mathrm{M} \\
52.02 \mathrm{H} \\
66.9 \mathrm{M} \\
1.51 E 7 \mathrm{Y}\end{array}$ & $\begin{array}{l}2.01 E+01 \\
2.72 E \bullet 01 \\
2.72 E \bullet 00 \\
2.02 E+01 \\
5.53 E-05\end{array}$ & $\begin{array}{l}2.00 E+01 \\
2.69 E+01 \\
2.58 E+00 \\
1.95 E \bullet 01 \\
2.60 E-11\end{array}$ & $\begin{array}{l}2.24 E+01 \\
3.01 E+01 \\
2.75 E \bullet 00 \\
2.18 E+01 \\
4.56 E-06\end{array}$ & $\begin{array}{l}2.59 E+01 \\
3.43 E+01 \\
4.09 E+00 \\
2.50 E+01 \\
4.05 E-03\end{array}$ & $\begin{array}{l}1.98 E+01 \\
2.67 E+01 \\
2.47 E+00 \\
1.92 E+01 \\
7.38 E-06\end{array}$ & $\begin{array}{l}1.82 E+01 \\
2.45 E+01 \\
2.21 E+00 \\
1.74 E+01 \\
1.16 E-07\end{array}$ & $\begin{array}{l}2.11 E+01 \\
2.84 E+01 \\
2.62 E+00 \\
2.04 E+01 \\
9.84 E-08\end{array}$ & $\begin{array}{l}1.94 E \bullet 01 \\
2.62 E+01 \\
2.43 E \bullet 00 \\
1.88 E \bullet 01 \\
2.33 E-05\end{array}$ & $\begin{array}{l}2.11 E+01 \\
2.84 E+01 \\
2.66 E+00 \\
2.04 E+01 \\
9.08 E-08\end{array}$ \\
\hline $\begin{array}{l}P B-209 \\
P B-210 \\
P B-211 \\
P B-212 \\
P B-214\end{array}$ & $\begin{array}{r}3.253 \mathrm{H} \\
22.26 \mathrm{Y} \\
36.1 \mathrm{M} \\
10.643 \mathrm{H} \\
26.8 \mathrm{M}\end{array}$ & $\begin{array}{l}0.0 \\
8.17 E-03 \\
4.86 E-01 \\
1.34 E \bullet 00 \\
2.31 E+00\end{array}$ & $\begin{array}{l}0.0 \\
1.06 E-02 \\
4.64 E-01 \\
1.26 E .00 \\
2.16 E+00\end{array}$ & $\begin{array}{l}0.0 \\
7.67 E-03 \\
5.18 E-01 \\
1.34 E \bullet 00 \\
2.35 E+00\end{array}$ & $\begin{array}{l}0.0 \\
3.11 E-02 \\
t .07 E-01 \\
2.03 E+00 \\
3.16 E+00\end{array}$ & $\begin{array}{l}0.0 \\
6.88 E-03 \\
4.56 E-01 \\
1.21 E+00 \\
2.10 E+00\end{array}$ & $\begin{array}{l}0.0 \\
6.96 E-03 \\
4.11 E-01 \\
1.08 E+00 \\
1.89 E+00\end{array}$ & & $\begin{array}{l}0.0 \\
7.21 E-03 \\
4.47 E-01 \\
1.19 E+00 \\
2.07 E+00\end{array}$ & $\begin{array}{l}0.0 \\
1.26 E-02 \\
4.84 E-01 \\
1.29 E+00 \\
2.23 E+00\end{array}$ \\
\hline $\begin{array}{l}81-206 \\
81-201 \\
B I-208 \\
81-210 \\
8 I-211\end{array}$ & $\begin{array}{r}6.2430 \\
33.4 \mathrm{Y} \\
3.68 \mathrm{Y} 5 \mathrm{Y} \\
5.013 \mathrm{O} \\
2.13 \mathrm{H}\end{array}$ & $\begin{array}{l}3.15 E+01 \\
1.47 E+01 \\
2.83 E+01 \\
0.0 \\
4.43 E-01\end{array}$ & $\begin{array}{l}3.08 E \cdot 01 \\
1.43 E+01 \\
2.94 E \cdot 01 \\
0.0 \\
4.13 E-01\end{array}$ & $\begin{array}{l}3.45 E \bullet 01 \\
1.60 E+01 \\
3.25 E+01 \\
0.0 \\
4.51 E-01\end{array}$ & $\begin{array}{l}3.98 E+01 \\
1.85 E+01 \\
3.71 E+01 \\
0.0 \\
5.92 E-01\end{array}$ & $\begin{array}{l}3.04 E+01 \\
1.41 E+01 \\
2.94 E+01 \\
0.0 \\
4.01 E-01\end{array}$ & $\begin{array}{l}2.76 E \bullet 01 \\
1.28 E \bullet 01 \\
2.76 E \cdot 01 \\
0.0 \\
3.60 E-01\end{array}$ & $\begin{array}{l}3.23 E+01 \\
1.50 E+01 \\
3.12 E+01 \\
0.0 \\
4.25 E-01\end{array}$ & $\begin{array}{l}2.97 E+01 \\
1.38 E+01 \\
2.90 E 001 \\
0.0 \\
3.90 E-01\end{array}$ & $\begin{array}{l}\text { 3. } 23 E+01 \\
1.50 E O 01 \\
3.18 E+01 \\
0.0 \\
4.26 E-01\end{array}$ \\
\hline $\begin{array}{l}81-212 \\
81-213 \\
81-214 \\
P 0-209 \\
P 0-210\end{array}$ & $\begin{array}{r}60.55 \mathrm{M} \\
45.65 \mathrm{M} \\
19.9 \mathrm{M} \\
102 \mathrm{Y} \\
138.378 \mathrm{O}\end{array}$ & $\begin{array}{l}1.76 E \cdot 00 \\
1.30 E+00 \\
1.48 E+01 \\
3.30 E-02 \\
8.25 E-05\end{array}$ & $\begin{array}{l}1.73 E \cdot 00 \\
1.23 E \cdot 00 \\
1.47 E \bullet 01 \\
3.17 E-02 \\
1.96 E-05\end{array}$ & $\begin{array}{l}1.94 E+00 \\
1.36 E+00 \\
1.65 E+01 \\
3.50 E-02 \\
8.94 E-05\end{array}$ & $\begin{array}{l}2.19 E+00 \\
1.60 E+00 \\
1.05 E+01 \\
4.32 E-02 \\
1.01 E-04\end{array}$ & & & $\begin{array}{l}1.82 E+00 \\
1.27 E+00 \\
1.55 E+01 \\
3.30 E-02 \\
8.34 E-05\end{array}$ & $\begin{array}{l}1.67 E+00 \\
1.18 E+00 \\
1.43 E+01 \\
3.04 E-02 \\
7.67 E-05\end{array}$ & $\begin{array}{l}1.82 E * 00 \\
1.27 E+00 \\
1.56 E+01 \\
3.30 E-02 \\
8.32 E-05\end{array}$ \\
\hline $\begin{array}{l}P O-211 \\
P O-212 \\
P O-213 \\
P O-214 \\
P O-215\end{array}$ & $\begin{array}{r}0.5165 \\
2.98 E-15 \\
4.2 E-6 \\
1.637 E-45 \\
1.778 E-35\end{array}$ & $\begin{array}{l}1.48 E-02 \\
0.0 \\
2.95 E-04 \\
8.08 E-04 \\
1.39 E-03\end{array}$ & $\begin{array}{l}7.20 E-02 \\
0.0 \\
2.04 E-04 \\
7.79 E-04 \\
1.31 E-03\end{array}$ & $\begin{array}{l}8.08 E-02 \\
0.0 \\
3.19 E-04 \\
8.75 E-04 \\
1.45 E-03\end{array}$ & $\begin{array}{l}9.20 E-02 \\
0.0 \\
3.60 E-04 \\
9.87 E-04 \\
1.78 E-03\end{array}$ & $\begin{array}{l}7.09 E-02 \\
0.0 \\
2.00 E-04 \\
7.68 E-04 \\
1.28 E-03\end{array}$ & $\begin{array}{l}6.41 E-02 \\
0.0 \\
2.53 E-04 \\
6.94 E-04 \\
1.15 E-03\end{array}$ & $\begin{array}{l}7.54 E-02 \\
0.0 \\
2.97 E-04 \\
8.17 E-04 \\
1.36 E-03\end{array}$ & $\begin{array}{l}6.94 E-02 \\
0.0 \\
2.74 E-04 \\
7.51 E-04 \\
1.26 E-03\end{array}$ & $\begin{array}{l}\text { 7. } 52 E-02 \\
0.0 \\
2.97 E-04 \\
8.14 E-04 \\
8.36 E-03\end{array}$ \\
\hline
\end{tabular}


DOSE-RATE FACTORS FOR BOOY ORGANS ANO EFFECTIVE DOSE-RATE FACTOR FOR IMMERS!ON IN CONTANINATEU WATER

IN MREM/YR PER MICROCUR IE/I CUBIC MI

\begin{tabular}{|c|c|c|c|c|c|c|c|c|c|c|}
\hline NUCL IDE & HALF-LIFE & LIVER & LUNGS & MARROH & $\begin{array}{c}\text { RED } \\
\text { MARROW }\end{array}$ & OVARIES & PANCREAS & SKELETON & SP LEEN & STOMA \\
\hline $\begin{array}{l}A U-194 \\
A U-195 \\
A U-195 M \\
A U-196 \\
A U-198\end{array}$ & 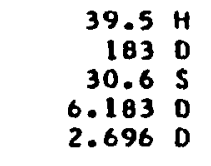 & $\begin{array}{l}1.02 E+01 \\
6.37 E-01 \\
1.70 E+00 \\
4.06 E+00 \\
3.53 E+00\end{array}$ & $\begin{array}{l}1.09 E+01 \\
7.03 E-01 \\
1.84 E+00 \\
4.39 E+00 \\
3.80 E+00\end{array}$ & $\begin{array}{l}1.22 E+01 \\
1.23 E+00 \\
2.47 E+00 \\
5.62 E+00 \\
4.54 E+00\end{array}$ & $\begin{array}{l}1.08 E+01 \\
4.45 E-01 \\
1.83 E+00 \\
4.34 E+00 \\
3.91 E+00\end{array}$ & & $\begin{array}{l}8.76 E+00 \\
4.30 E-01 \\
1.44 E+00 \\
3.42 E+00 \\
3.05 E+00\end{array}$ & $\begin{array}{l}1.24 E+01 \\
1.26 E+00 \\
2.53 E+00 \\
5.73 E+00 \\
4.61 E+00\end{array}$ & $\begin{array}{l}9.87 E+00 \\
5.83 E-01 \\
1.67 E+00 \\
3.97 E+00 \\
3.47 E+00\end{array}$ & $\begin{array}{l}9.99 E+00 \\
5.96 E-01 \\
1.67 E+00 \\
3.98 E+00 \\
3.49 E+00\end{array}$ \\
\hline $\begin{array}{l}A U-199 \\
H G-197 \\
H G-197 M \\
H G-203 \\
T L-200\end{array}$ & $\begin{array}{r}3.139 \mathrm{D} \\
64.14 \mathrm{H} \\
23.8 \mathrm{H} \\
46.60 \mathrm{O} \\
26.1 \mathrm{H}\end{array}$ & $\begin{array}{l}7.58 \mathrm{E} \\
5.27 \mathrm{E} \\
7.61 \mathrm{E} \\
1.96 \mathrm{E} \\
1.20 \mathrm{E}\end{array}$ & $\begin{array}{l}8.3 \\
5.8 \\
8.3 \\
2.1 \\
1.2\end{array}$ & $\begin{array}{l}1.24 E+00 \\
1.02 E+00 \\
1.31 E+00 \\
2.79 E+00 \\
1.45 E+01\end{array}$ & & & & & $\begin{array}{l}7.48 E-01 \\
4.82 E-01 \\
7.39 E-01 \\
1.94 E+00 \\
1.17 E+01\end{array}$ & $\begin{array}{l}4 E-01 \\
4 E-01 \\
0 E-01 \\
4 E-00\end{array}$ \\
\hline $\begin{array}{l}T L-201 \\
T L-202 \\
T L-204 \\
T L-207 \\
T L-208\end{array}$ & $\begin{array}{r}73.06 \mathrm{H} \\
12.23 \mathrm{D} \\
3.779 \mathrm{Y} \\
4.77 \mathrm{M} \\
3.053 \mathrm{M}\end{array}$ & $\begin{array}{l}7.29 E-01 \\
4.03 E+00 \\
8.53 E-03 \\
2.04 E-02 \\
3.61 E+01\end{array}$ & $\begin{array}{l}8.03 E-01 \\
4.34 E+00 \\
9.41 E-03 \\
2.18 E-02 \\
3.79 E+01\end{array}$ & $\begin{array}{l}1.36 E+00 \\
5.41 E+00 \\
1.65 E-02\end{array}$ & & $\begin{array}{l}6.07 E-01 \\
3.76 E+00 \\
7.00 E-03 \\
1.98 E-02 \\
3.26 E+01\end{array}$ & & & $\begin{array}{l}6.83 E-01 \\
3.92 E+00 \\
7.81 E-03 \\
1.98 E-02 \\
3.47 E+01\end{array}$ & $\begin{array}{l}7.9 \\
2.0 \\
3.5\end{array}$ \\
\hline $\begin{array}{l}\mathrm{IL}-209 \\
\mathrm{IL}-210 \\
\mathrm{~PB}-203 \\
\mathrm{~PB}-204 \mathrm{M} \\
\mathrm{PB}-205\end{array}$ & $\begin{array}{r}2.20 \mathrm{M} \\
1.30 \mathrm{M} \\
52.02 \mathrm{H} \\
66.9 \mathrm{H} \\
1.51 \mathrm{E} \mathrm{Y}\end{array}$ & $\begin{array}{l}2.00 E+01 \\
2.69 E+01 \\
2.57 E+00 \\
1.94 E+01 \\
6.40 E-07\end{array}$ & $\begin{array}{l}2.12 E+01 \\
2.85 E+01 \\
2.79 E+00 \\
2.07 E+01 \\
4.45 E-05\end{array}$ & $\begin{array}{l}2.32 E+01 \\
3.07 E+01 \\
3.86 E+00 \\
2.28 E+01 \\
2.83 E-05\end{array}$ & $\begin{array}{l}2.13 E \cdot 01 \\
2.89 E \bullet 01 \\
2.63 E \bullet 00 \\
2.10 E \cdot 01 \\
1.47 E-05\end{array}$ & $\begin{array}{l}1.87 E+01 \\
2.52 E+01 \\
2.29 E+00 \\
1.87 E+01 \\
2.21 E-05\end{array}$ & $\begin{array}{l}1.73 E+01 \\
2.33 E+01 \\
2.11 E+00 \\
1.67 E+01 \\
3.25 E-05\end{array}$ & $\begin{array}{l}2.36 E+01 \\
3.12 E+01 \\
3.94 E+00 \\
2.32 E+01 \\
7.23 E-05\end{array}$ & $\begin{array}{l}1.94 E+01 \\
2.60 E+01 \\
2.50 E+00 \\
1.88 E+01 \\
1.31 E-05\end{array}$ & $\begin{array}{l}2.51 \\
1.91 \\
1.20\end{array}$ \\
\hline $\begin{array}{l}P B-209 \\
P B-210 \\
P B-211 \\
P B-212 \\
P B-214\end{array}$ & $\begin{array}{r}3.253 \mathrm{H} \\
22.26 \mathrm{Y} \\
36.1 \mathrm{M} \\
10.643 \mathrm{H} \\
26.8 \mathrm{H}\end{array}$ & $\begin{array}{l}0.0 \\
9.51 E-03 \\
4.61 E-01 \\
1.25 E+00 \\
2.15 E+00\end{array}$ & $\begin{array}{l}0.0 \\
1.06 E-02 \\
4.94 E-01 \\
1.36 E+00 \\
2.32 E+00\end{array}$ & $\begin{array}{l}0.0 \\
1.91 E-02 \\
5.58 E-01 \\
1.92 E+00 \\
2.96 E+00\end{array}$ & $\begin{array}{l}0.0 \\
4.03 E-03 \\
5.04 E-01 \\
1.30 E+00 \\
2.34 E+00\end{array}$ & $\begin{array}{l}0.0 \\
7.69 E-03 \\
4.44 E-01 \\
1.10 E+00 \\
1.98 E+00\end{array}$ & $\begin{array}{l}5.43 E-0 \\
3.98 E-0 \\
1.04 E+0 \\
1.83 E+0\end{array}$ & $\begin{array}{l}0.0 \\
1.96 E-02 \\
5.68 E-01 \\
1.96 E+00 \\
3.01 E+00\end{array}$ & $\begin{array}{l}7.83 E-03 \\
4.49 E-01 \\
1.23 E+00 \\
2.11 E+00\end{array}$ & $\begin{array}{l}4.56 \mathrm{E}- \\
1.23 \mathrm{Et} \\
2.12 \mathrm{E}\end{array}$ \\
\hline $\begin{array}{l}81-206 \\
81-207 \\
81-208 \\
81-210 \\
81-211\end{array}$ & $\begin{array}{r}6.243 \mathrm{D} \\
33.4 \mathrm{Y} \\
3.6065 \mathrm{Y} \\
5.013 \mathrm{O} \\
2.13 \mathrm{M}\end{array}$ & $\begin{array}{l}3.07 E+01 \\
1.43 E+01 \\
2.96 E+01 \\
0.0 \\
4.11 E-01\end{array}$ & $\begin{array}{l}3.27 E+01 \\
1.52 E+01 \\
3.09 E+01 \\
0.0 \\
4.43 E-01\end{array}$ & $\begin{array}{l}3.61 E+01 \\
1.69 E+01 \\
3.17 E+01 \\
0.0 \\
5.54 E-01\end{array}$ & $\begin{array}{l}3.29 E+01 \\
1.52 E+01 \\
3.15 E+01 \\
0.0 \\
4.51 E-01\end{array}$ & $\begin{array}{l}2.92 E+01 \\
1.36 E+01 \\
2.62 E+01 \\
0.0 \\
3.00 E-01\end{array}$ & $\begin{array}{l}2.64 E \cdot 01 \\
1.22 E \cdot 01 \\
2.59 E \cdot 01 \\
0.0 \\
3.52 E-01\end{array}$ & $\begin{array}{l}3.67 E+01 \\
1.71 E+01 \\
3.23 E+01 \\
0.0 \\
5.64 E-01\end{array}$ & $\begin{array}{l}2.97 E+01 \\
1.38 E+01 \\
2.83 E+01 \\
0.0 \\
4.04 E-01\end{array}$ & $\begin{array}{l}1.40 E A \\
2.92 E+ \\
0.0 \\
4.05 E-\end{array}$ \\
\hline $\begin{array}{l}81-212 \\
81-213 \\
81-214 \\
P 0-209 \\
P 0-210\end{array}$ & $\begin{array}{r}60.55 \mathrm{H} \\
45.65 \mathrm{M} \\
19.9 \mathrm{M} \\
102 \mathrm{Y} \\
138.378 \mathrm{D}\end{array}$ & $\begin{array}{l}1.72 E \bullet 00 \\
1.22 E \bullet 00 \\
1.47 E \bullet 01 \\
3.15 E-02 \\
7.90 E-05\end{array}$ & $\begin{array}{l}1.83 E+00 \\
1.31 E+00 \\
1.56 E+01 \\
3.38 E-02 \\
8.44 E-05\end{array}$ & $\begin{array}{l}1.98 E+00 \\
1.56 E+00 \\
1.65 E+01 \\
3.99 E-02 \\
9.19 E-05\end{array}$ & $\begin{array}{l}1.86 E+00 \\
1.34 E+00 \\
1.58 E+01 \\
3.35 E-02 \\
8.57 E-05\end{array}$ & & $\begin{array}{l}1.05 E+00 \\
1.28 E \cdot 01 \\
2.68 E-02 \\
6.82 E-05\end{array}$ & & & $\begin{array}{l}1.20 E \\
1.44 E \\
3.10 E \\
7.82 E\end{array}$ \\
\hline $\begin{array}{l}P O-211 \\
P O-212 \\
P O-213 \\
P O-214 \\
P O-215\end{array}$ & $\begin{array}{r}0.5165 \\
2.98 E-75 \\
4.2 E-65 \\
1.637 E-45 \\
1.778 E-35\end{array}$ & $\begin{array}{l}7.16 E-02 \\
0.0 \\
2.82 E-04 \\
7.74 E-04 \\
1.30 E-03\end{array}$ & $\begin{array}{l}1.65 E-02 \\
0.0 \\
3.01 E-04 \\
8.26 E-04 \\
1.40 E-03\end{array}$ & $\begin{array}{l}0.40 E-02 \\
0.0 \\
3.29 E-04 \\
9.00 E-04 \\
1.65 E-03\end{array}$ & $\begin{array}{l}1.78 E-02 \\
0.0 \\
3.06 E-04 \\
8.39 E-04 \\
1.44 E-03\end{array}$ & $\begin{array}{l}6.93 E-02 \\
0.0 \\
2.74 E-04 \\
7.52 E-04 \\
1.24 E-03\end{array}$ & $\begin{array}{l}6.17 E-02 \\
0.0 \\
2.43 E-04 \\
6.67 E-04 \\
1.12 E-03\end{array}$ & $\begin{array}{l}8.54 E-02 \\
0.0 \\
3.34 E-04 \\
9.15 E-04 \\
1.68 E-03\end{array}$ & $\begin{array}{l}6.95 E-02 \\
0.0 \\
2.74 E-04 \\
1.51 E-04 \\
1.28 E-03\end{array}$ & $\begin{array}{l}2.79 \mathrm{E}- \\
1.66 \mathrm{E}- \\
1.29 \mathrm{E}\end{array}$ \\
\hline
\end{tabular}




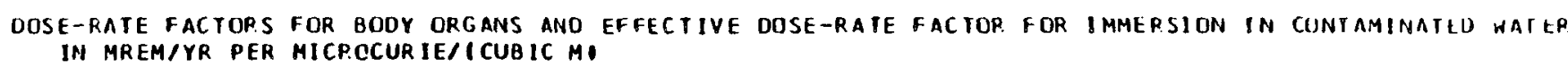

\begin{tabular}{|c|c|c|c|c|c|c|c|c|c|}
\hline NUCL IDE & HALF-LIFE & TESTES & THYMUS & THYROIO & UTERUS & EFFECTIVE & $\begin{array}{c}\text { SKIN } \\
\text { (PHOTONI }\end{array}$ & $\begin{array}{c}\text { SKIV } \\
\text { (ELECTRONI }\end{array}$ & $\begin{array}{l}\text { SKIN } \\
\text { ITOTALI }\end{array}$ \\
\hline $\begin{array}{l}A U-194 \\
A U-195 \\
A U-1954 \\
A U-196 \\
A U-198\end{array}$ & $\begin{array}{rl}39.5 & H \\
183 & 0 \\
30.6 & 5 \\
6.183 & 0 \\
2.696 & 0\end{array}$ & $\begin{array}{l}1.44 E+01 \\
1.08 E+00 \\
2.53 E+00 \\
6.02 E+00 \\
5.12 E+00\end{array}$ & $\begin{array}{l}.13 E+01 \\
38 E-01 \\
92 E+00 \\
55 E+00 \\
86 E+00\end{array}$ & $\begin{array}{l}1.35 E+01 \\
1.07 E+00 \\
2.41 E+00 \\
5.65 E+00 \\
4.76 E \bullet 00\end{array}$ & $\begin{array}{l}9.05 E * 00 \\
5.08 E-01 \\
1.49 E+00 \\
3.54 E+00 \\
3.10 E+00\end{array}$ & $\begin{array}{l}1.24 E+01 \\
9.17 E-01 \\
2.21 E+00 \\
5.18 E+00 \\
4.41 E+00\end{array}$ & $\begin{array}{l}1.64 E+01 \\
1.20 E \bullet 00 \\
2.82 E+00 \\
6.77 E+00 \\
5.81 E+00\end{array}$ & $\begin{array}{l}1.07 E-01 \\
1.07 E-02 \\
3.11 E-01 \\
1.56 E-01 \\
2.26 E+00\end{array}$ & $\begin{array}{l}1.66 E+01 \\
1.21 E+00 \\
3.13 E+00 \\
6.93 E+00 \\
8.07 E+00\end{array}$ \\
\hline $\begin{array}{l}U-199 \\
=197 \\
-197 M \\
-203 \\
-200\end{array}$ & $\begin{array}{rl}3.139 & 0 \\
64.14 & H \\
23.8 & H \\
46.60 & 0 \\
26.1 & H\end{array}$ & $\begin{array}{l}1.16 E+00 \\
8.91 E-01 \\
1.20 E+00 \\
2.91 E+00 \\
1.71 E+01\end{array}$ & $\begin{array}{l}9.95 E-01 \\
6.93 E-01 \\
9.26 E-01 \\
2.20 E+00 \\
1.34 E+01\end{array}$ & & $\begin{array}{l}6.61 E-01 \\
4.22 E-01 \\
6.50 E-01 \\
1.73 E+00 \\
1.06 E+01\end{array}$ & & $\begin{array}{l}1.26 E+00 \\
9.84 E-01 \\
1.31 E+00 \\
3.24 E+00 \\
1.96 E+01\end{array}$ & & $\begin{array}{l}1.69 E+00 \\
9.86 E-01 \\
1.96 E+00 \\
3.59 E .00 \\
1.97 E+01\end{array}$ \\
\hline $\begin{array}{l}\mathrm{IL}-201 \\
\mathrm{TL}-202 \\
\mathrm{TL}-204 \\
\mathrm{TL}-207 \\
\mathrm{IL}-208\end{array}$ & $\begin{array}{lll}13.06 & H \\
12.23 & 0 \\
3.779 & Y \\
4.77 & 4 \\
3.053 & H\end{array}$ & $\begin{array}{l}1.20 E \bullet 00 \\
5.94 E \cdot 00 \\
1.44 E-02 \\
2.88 E-02 \\
4.95 E \cdot 01\end{array}$ & $\begin{array}{l}9.33 \mathrm{E}-01 \\
4.49 \mathrm{E}-00 \\
1.12 \mathrm{E}-02\end{array}$ & $\begin{array}{l}1.19 E \bullet 00 \\
5.55 E \bullet 00 \\
1.43 E-02 \\
2.75 E-02 \\
4.51 E+01\end{array}$ & $\begin{array}{l}5.99 E-01 \\
3.50 E+00 \\
6.84 E-03 \\
1.78 E-02 \\
3.24 E \bullet 01\end{array}$ & & & & $\begin{array}{l}1.35 E+00 \\
6.82 E+00 \\
1.73 E+00 \\
3.82 E+00 \\
5.99 E+01\end{array}$ \\
\hline $\begin{array}{l}\mathrm{T} L-209 \\
\mathrm{~T} L-210 \\
\mathrm{~PB}-203 \\
\mathrm{~PB}-204 \mathrm{M} \\
\mathrm{PB}-205\end{array}$ & $\begin{array}{r}2.20 \mathrm{M} \\
1.30 \mathrm{H} \\
52.02 \mathrm{H} \\
66.9 \mathrm{H} \\
1.51 E 7 \mathrm{Y}\end{array}$ & $\begin{array}{l}2.80 E+01 \\
3.75 E \bullet 01 \\
3.88 E \bullet 00 \\
2.75 E+01 \\
3.99 E-04\end{array}$ & $\begin{array}{l}2.20 E \cdot 01 \\
2.96 E \cdot 01 \\
2.96 E \cdot 00 \\
2.17 E+01 \\
1.42 E-08\end{array}$ & $\begin{array}{l}2.63 E+01 \\
3.52 E+01 \\
3.71 E+00 \\
2.61 E+01 \\
3.00 E-05\end{array}$ & $\begin{array}{l}1.78 E \cdot 01 \\
2.38 E \bullet 01 \\
2.22 E \bullet 00 \\
1.69 E+01 \\
3.73 E-09\end{array}$ & $\begin{array}{l}2.41 E+01 \\
3.22 E+01 \\
3.36 E+00 \\
2.35 E+01 \\
7.27 E-04\end{array}$ & $\begin{array}{l}3.19 E+01 \\
4.28 E+01 \\
4.31 E+00 \\
3.17 E+01 \\
8.49 E-03\end{array}$ & $\begin{array}{l}5.43 E+00 \\
5.52 E+00 \\
2.81 E-01 \\
7.78 E-01 \\
0.0\end{array}$ & $\begin{array}{l}3.73 E+01 \\
4.83 E+01 \\
4.60 E+00 \\
3.24 E+01 \\
8.49 E-03\end{array}$ \\
\hline $\begin{array}{l}P B-209 \\
P B-210 \\
P B-211 \\
P B-212 \\
P B-214\end{array}$ & $\begin{array}{r}3.253 \mathrm{H} \\
22.26 \mathrm{Y} \\
36.1 \mathrm{H} \\
10.643 \mathrm{H} \\
26.8 \mathrm{H}\end{array}$ & $\begin{array}{l}0.0 \\
2.09 E-02 \\
6.60 E-01 \\
1.89 E \bullet 00 \\
3.17 E \bullet 00\end{array}$ & $\begin{array}{l}1.40 E-02 \\
5.13 E-01 \\
1.45 E 00 \\
2.40 E \cdot 00\end{array}$ & $\begin{array}{l}1.83 E-02 \\
6.22 E-01 \\
1.02 E 000 \\
2.98 E+00\end{array}$ & $\begin{array}{l}0.0 \\
6.44 E-03 \\
4.03 E-01 \\
1.09 E+00 \\
1.89 E+00\end{array}$ & $\begin{array}{l}0.0 \\
1.68 E-02 \\
5.65 E-01 \\
1.65 E+00 \\
2.74 E .00\end{array}$ & $\begin{array}{l}0.0 \\
3.52 E-02 \\
7.57 E-01 \\
2.09 E \cdot 00 \\
3.56 E \cdot 00\end{array}$ & $\begin{array}{l}1.15 E+00 \\
0.0 \\
3.44 E+00 \\
6.68 E-01 \\
1.72 E+00\end{array}$ & $\begin{array}{l}1.15 E+00 \\
3.52 E-02 \\
4.19 E+00 \\
2.76 E \cdot 00 \\
5.28 E \cdot 00\end{array}$ \\
\hline $\begin{array}{l}81-206 \\
81-207 \\
81-208 \\
81-210 \\
81-211\end{array}$ & $\begin{array}{r}6.2430 \\
33.4 y \\
3.68 E 5 y \\
5.0130 \\
2.13\end{array}$ & $\begin{array}{l}4.34 E+01 \\
2.02 E+01 \\
4.01 E+01 \\
0.0 \\
6.02 E-01\end{array}$ & $\begin{array}{l}1.59 E+01 \\
3.15 E \cdot 01 \\
0.0 \\
4.54 E-01\end{array}$ & $\begin{array}{l}4.10 E+01 \\
1.91 E+01 \\
3.64 E+01 \\
0.0 \\
5.63 E-01\end{array}$ & $\begin{array}{l}2.69 E \bullet 01 \\
1.25 E \bullet 01 \\
2.67 E+01 \\
0.0 \\
3.61 E-01\end{array}$ & $\begin{array}{l}3.72 E+01 \\
1.73 E+01 \\
3.47 E+01 \\
0.0 \\
5.20 E-01\end{array}$ & $\begin{array}{l}4.98 E+01 \\
2.32 E+01 \\
4.45 E * 01 \\
0.0 \\
6.77 E-01\end{array}$ & & $\begin{array}{l}5.04 E+01 \\
2.41 E+01 \\
4.46 E+01 \\
2.85 E+00 \\
7.39 E-01\end{array}$ \\
\hline $\begin{array}{l}81-212 \\
81-213 \\
81-214 \\
P 0-209 \\
P 0-210\end{array}$ & $\begin{array}{r}60.55 \mathrm{M} \\
45.65 \mathrm{M} \\
19.99 \mathrm{M} \\
102 \mathrm{Y} \\
138.378 \mathrm{D}\end{array}$ & $\begin{array}{l}2.42 E+00 \\
1.17 E \bullet 00 \\
2.04 E \cdot 01 \\
4.54 E-02 \\
1.12 E-04\end{array}$ & $\begin{array}{l}1.34 E+00 \\
1.62 E+01 \\
3.51-02 \\
8.86 E-05\end{array}$ & $\begin{array}{l}2.28 E+00 \\
1.65 E \cdot 00 \\
1.91 E+01 \\
4.34 E-02 \\
1.06 E-04\end{array}$ & $\begin{array}{l}1.52 E+00 \\
1.07 E+00 \\
1.31 E+01 \\
2.14 E-02 \\
6.87 E-05\end{array}$ & $\begin{array}{l}1.52 E 000 \\
1.75 E+01 \\
3.900-02 \\
9.57 E-05\end{array}$ & $\begin{array}{l}2.79 E+00 \\
2.01 E+00 \\
2.34 E+01 \\
5.18 E-02 \\
1.29 E-04\end{array}$ & $\begin{array}{l}3.75 E+00 \\
3.28 E \cdot 00 \\
5.17 E+00 \\
1.05 E-03 \\
0.0\end{array}$ & $\begin{array}{l}5.29 E+00 \\
2.85 E+01 \\
5.28 E-02 \\
1.29 E-04\end{array}$ \\
\hline $\begin{array}{l}P O-211 \\
P Q-2112 \\
P O-211 \\
P O-214 \\
P O-215\end{array}$ & 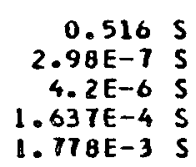 & $\begin{array}{l}1.02 E-01 \\
0.0 \\
4.00 E-04 \\
1.10 E-03 \\
1.89 E-03\end{array}$ & $\begin{array}{l}3.16 E-04 \\
0.67 E-04 \\
1.42 E-03\end{array}$ & $\begin{array}{l}9.61 E-02 \\
0.0 \\
3.79 E-04 \\
1.04 E-03 \\
1.75 E-03\end{array}$ & $\begin{array}{l}6.23 E-02 \\
0.0 \\
2.45 E-04 \\
6.13 E-04 \\
1.14 E-03\end{array}$ & $\begin{array}{l}0.69 E-02 \\
0.0 \\
3.42 E-04 \\
9.37 E-04 \\
1.62 E-03\end{array}$ & $\begin{array}{l}1.17 E-01 \\
0.0 \\
4.62 E-04 \\
1.27 E-03 \\
2.14 E-03\end{array}$ & $\begin{array}{l}0.0 \\
0.0 \\
0.0 \\
0.0\end{array}$ & $\begin{array}{l}0.0 \\
4.62 E-04 \\
1.27 E-03 \\
2.14 E-03\end{array}$ \\
\hline
\end{tabular}




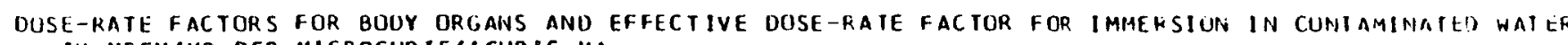

IN MREM/YR PER MICROCURIE/ICUBIC MI

\begin{tabular}{|c|c|c|c|c|c|c|c|c|c|c|}
\hline NUCL IOE & HALF-LIFE & ADRENAL S & BLADOER & BRAIN & BREAST & HE ART & $\begin{array}{l}\text { SMALL } \\
\text { INTESTINE }\end{array}$ & $\begin{array}{l}\text { UPPER } \\
\text { LARGE } \\
\text { INTESTINE }\end{array}$ & $\begin{array}{l}\text { LOWER } \\
\text { LARGE } \\
\text { INTESTINE }\end{array}$ & $\times$ IONEYS \\
\hline $\begin{array}{l}P O-218 \\
P O-218 \\
A T-211 \\
A T-217 \\
R N-218\end{array}$ & $\begin{array}{r}0.146 \mathrm{~S} \\
3.05 \mathrm{M} \\
7.214 \mathrm{H} \\
0.0323 \mathrm{~S} \\
0.035 \mathrm{~S}\end{array}$ & $\begin{array}{l}1.41 E-04 \\
0.0 \\
3.03 E-01 \\
2.25 E-03 \\
7.15 E-03\end{array}$ & $\begin{array}{l}1.36 E-04 \\
0.0 \\
3.11 E-01 \\
2.14 E-03 \\
6.83 E-03\end{array}$ & $\begin{array}{l}1.52 E-04 \\
0.0 \\
3.18 E-01 \\
2.40 E-03 \\
7.64 E-03\end{array}$ & $\begin{array}{l}1.72 E-04 \\
0.0 \\
6.00 E-01 \\
2.79 E-03 \\
8.87 E-03\end{array}$ & $\begin{array}{l}1.34 E-04 \\
0.0 \\
2.87 E-01 \\
2.10 E-03 \\
6.69 E-03\end{array}$ & $\begin{array}{l}1.21 E-04 \\
0.0 \\
2.53 E-01 \\
1.89 E-03 \\
6.03 E-03\end{array}$ & $\begin{array}{l}1.42 E-04 \\
0.0 \\
3.01 E-01 \\
2.23 E-03 \\
7.11 E-03\end{array}$ & $\begin{array}{l}1.31 E-04 \\
0.0 \\
2.74 E-01 \\
2.06 E-03 \\
6.55 E-03\end{array}$ & $\begin{array}{l}1.42 \mathrm{E}-04 \\
0.0 \\
3.19 \mathrm{E}-01 \\
2.23 \mathrm{E}-03 \\
7.10 \mathrm{E}-03\end{array}$ \\
\hline $\begin{array}{l}R N-219 \\
R N-220 \\
R N-222 \\
F R-221 \\
F R-223\end{array}$ & $\begin{array}{r}3.96 \mathrm{~S} \\
55.61 \mathrm{~S} \\
3.8235 \mathrm{O} \\
4.8 \mathrm{M} \\
21.8 \mathrm{H}\end{array}$ & $\begin{array}{l}5.37 E-01 \\
4.92 E-03 \\
3.65 E-03 \\
2.87 E-01 \\
3.06 E-01\end{array}$ & $\begin{array}{l}4.98 E-01 \\
4.68 E-03 \\
3.46 E-03 \\
2.64 E-01 \\
3.90 E-01\end{array}$ & $\begin{array}{l}.43 E-01 \\
.22 E-03 \\
.86 E-03 \\
8.82 E-01 \\
.93 E-01\end{array}$ & $\begin{array}{l}7.21 E-01 \\
6.15 E-03 \\
4.59 E-03 \\
4.20 E-01 \\
7.20 E-01\end{array}$ & $\begin{array}{l}4.85 E-01 \\
4.58 E-03 \\
3.38 E-03 \\
2.56 E-01 \\
3.52 E-01\end{array}$ & $\begin{array}{l}4.35 E-01 \\
4.12 E-03 \\
3.04 E-03 \\
2.29 E-01 \\
3.19 E-01\end{array}$ & $\begin{array}{l}5.14 E-01 \\
4.86 E-03 \\
3.59 E-03 \\
2.72 E-01 \\
3.82 E-01\end{array}$ & $\begin{array}{l}4.80 E-01 \\
4.48 E-03 \\
3.31 E-03 \\
2.55 E-01 \\
3.46 E-01\end{array}$ & $\begin{array}{l}5.14 \mathrm{E}-01 \\
4.86 \mathrm{E}-03 \\
3.59 \mathrm{E}-03 \\
2.72 \mathrm{E}-01 \\
4.13 \mathrm{E}-01\end{array}$ \\
\hline $\begin{array}{l}R A-222 \\
R A-223 \\
R A-224 \\
R A-225 \\
R A-226\end{array}$ & 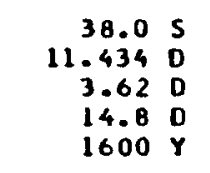 & $\begin{array}{l}8.65 E-02 \\
1.21 E+00 \\
9.39 E-02 \\
3.83 E-02 \\
6.27 E-02\end{array}$ & $\begin{array}{l}8.01 E-02 \\
1.14 E+00 \\
8.61 E-02 \\
5.61 E-02 \\
5.76 E-02\end{array}$ & $\begin{array}{l}8.74 E-02 \\
1.22 E+00 \\
9.25 E-02 \\
3.15 E-02 \\
6.14 E-02\end{array}$ & $\begin{array}{l}1.15 E-01 \\
1.88 E+00 \\
1.33 E-01 \\
1.56 E-01 \\
9.45 E-02\end{array}$ & $\begin{array}{l}8.38 E-02 \\
2.91 E-02 \\
5.61 E-02\end{array}$ & $\begin{array}{l}7.00 E-02 \\
9.76 E-01 \\
7.50 E-02 \\
3.21 E-02 \\
4.97 E-02\end{array}$ & $\begin{array}{l}8.26 E-02 \\
1.17 E+00 \\
B .87 E-02 \\
4.32 E-02 \\
5.96 E-02\end{array}$ & $\begin{array}{l}7.12 E-02 \\
1.08 E+00 \\
8.33 E-02 \\
3.31 E-02 \\
5.55 E-02\end{array}$ & $\begin{array}{l}\text { 8. 26E-02 } \\
1.17 E+00 \\
\text { 8.87E-02 } \\
1.27 E-02 \\
5.90 E-02\end{array}$ \\
\hline $\begin{array}{l}R A-228 \\
A C-225 \\
A C-227 \\
A C-228 \\
T H-226\end{array}$ & $\begin{array}{r}5.75 \mathrm{Y} \\
10.0 \mathrm{D} \\
21.773 \mathrm{Y} \\
6.13 \mathrm{H} \\
30.9 \mathrm{H}\end{array}$ & $\begin{array}{l}5.77 E-11 \\
1.18 E-01 \\
1.06 E-03 \\
8.92 E+00 \\
7.00 E-02\end{array}$ & $\begin{array}{l}1.04 E-17 \\
1.13 E-01 \\
9.88 E-04 \\
8.69 E+00 \\
6.57 E-02\end{array}$ & $\begin{array}{l}4.60 E-12 \\
1.19 E-01 \\
1.05 E-03 \\
9.74 E+00 \\
6.97 E-02\end{array}$ & $\begin{array}{l}4.52 E-09 \\
2.07 E-01 \\
2.18 E-03 \\
1.11 E+01 \\
1.16 E-01\end{array}$ & $\begin{array}{l}7.51 E-12 \\
1.08 E-01 \\
9.68 E-04 \\
0.57 E+00 \\
6.38 E-02\end{array}$ & $\begin{array}{l}9.69 E-14 \\
9.50 E-02 \\
8.35 E-04 \\
7.80 E+00 \\
5.59 E-02\end{array}$ & $\begin{array}{l}1.43 E-13 \\
1.16 E-01 \\
1.04 E-03 \\
9.12 E+00 \\
6.84 E-02\end{array}$ & & $\begin{array}{l}6.91 E-14 \\
1.15 E-01 \\
1.00 E-03 \\
9.10 E+00 \\
6.71 E-02\end{array}$ \\
\hline $\begin{array}{l}T H-227 \\
T H-228 \\
T H-229 \\
T H-230 \\
T H-231\end{array}$ & $\begin{array}{rl}18.718 & 0 \\
1.9132 & Y \\
7.34 E 3 & Y \\
7.7 E 4 Y Y & Y \\
25.52 & H\end{array}$ & $\begin{array}{l}9.60 E-01 \\
1.69 E-02 \\
7.43 E-01 \\
2.97 E-03 \\
9.09 E-02\end{array}$ & $\begin{array}{l}8.92 E-01 \\
1.63 E-02 \\
7.18 E-01 \\
3.01 E-03 \\
9.10 E-02\end{array}$ & $\begin{array}{l}9.54 E-01 \\
1.10 E-02 \\
1.49 E-01 \\
3.02 E-03 \\
9.15 E-02\end{array}$ & & & & & & $\begin{array}{l}9.22 \mathrm{E}-01 \\
1.66 \mathrm{E}-02 \\
1.35 \mathrm{E}-01 \\
3.12 \mathrm{E}-03 \\
9.59 \mathrm{E}-02\end{array}$ \\
\hline $\begin{array}{l}T H-232 \\
T H-233 \\
T H-234 \\
P A-230 \\
P A-231\end{array}$ & $\begin{array}{r}1.405 E 10 \mathrm{Y} \\
22.3 \\
24.10 \\
17.4 \\
3.276 E 4 \mathrm{Y}\end{array}$ & $\begin{array}{l}1.24 E-03 \\
3.10 E-01 \\
6.31 E-02 \\
6.28 E .00 \\
2.76 E-01\end{array}$ & $\begin{array}{l}1.27 E-03 \\
2.96 E-01 \\
6.44 E-02 \\
6.06 E \cdot 00 \\
2.56 E-01\end{array}$ & $\begin{array}{l}1.23 E-03 \\
3.25 E-01 \\
6.51 E-02 \\
6.78 E+00 \\
2.75 E-01\end{array}$ & $\begin{array}{l}4.94 E-03 \\
4.26 E-01 \\
1.29 E-01 \\
8.07 E+00 \\
4.13 E-01\end{array}$ & & & & & $\begin{array}{l}1.34 \mathrm{E}-03 \\
3.07 \mathrm{E}-01 \\
6.63 \mathrm{E}-02 \\
6.35 \mathrm{E} 00 \\
2.66 \mathrm{E}-01\end{array}$ \\
\hline $\begin{array}{l}P A-233 \\
P A-234 \\
P A-234 M \\
U-230 \\
U-231\end{array}$ & $\begin{array}{rl}27.0 & 0 \\
6.70 & \mathrm{H} \\
1.17 & \mathrm{M} \\
20.8 & 0 \\
4.2 & 0\end{array}$ & $\begin{array}{l}1.97 E+00 \\
1.88 E+01 \\
1.11 E-01 \\
9.22 E-03 \\
5.82 E-01\end{array}$ & $\begin{array}{l}1.83 E+00 \\
1.82 E+01 \\
1.07 E-01 \\
8.87 E-03 \\
5.66 E-01\end{array}$ & $\begin{array}{l}1.99 E+00 \\
2.04 E * 01 \\
1.21 E-01 \\
9.23 E-03 \\
5.89 E-01\end{array}$ & $\begin{array}{l}2.81 E+00 \\
2.38 E+01 \\
1.37 E-01 \\
1.96 E-02 \\
1.11 E+00\end{array}$ & $\begin{array}{l}1.78 E+00 \\
1.80 E+01 \\
1.06 E-01 \\
8.37 E-03 \\
5.40 E-01\end{array}$ & $\begin{array}{l}1.59 E+00 \\
1.63 E+01 \\
9.63 E-02 \\
1.43 E-03 \\
4.68 E-01\end{array}$ & $\begin{array}{l}1.89 E+00 \\
1.91 E+01 \\
1.13 E-01 \\
8.89 E-03 \\
5.84 E-01\end{array}$ & $\begin{array}{l}1.76 E+00 \\
1.76 E+01 \\
1.04 E-01 \\
8.20 E-03 \\
5.21 E-01\end{array}$ & $\begin{array}{l}1.89 E+00 \\
1.91 E+01 \\
1.13 E-01 \\
9.12 E-03 \\
5.80 E-01\end{array}$ \\
\hline $\begin{array}{l}u-232 \\
u-233 \\
u-234 \\
u-235 \\
u-236\end{array}$ & $\begin{array}{r}12 Y \\
1.592 E 5 Y \\
2.445 E 5 Y \\
7.038 E B Y \\
2.3415 E Y Y\end{array}$ & $\begin{array}{l}1.79 E-03 \\
1.96 E-03 \\
8.76 E-04 \\
1.41 E+00 \\
6.24 E-04\end{array}$ & $\begin{array}{l}1.73 E-03 \\
1.81 E-03 \\
8.34 E-04 \\
1.29 E .00 \\
6.011 E-04\end{array}$ & $\begin{array}{l}1.73 E-03 \\
1.93 E-03 \\
8.12 E-04 \\
1.38 E+00 \\
5.93 E-04\end{array}$ & $\begin{array}{l}7.27 E-03 \\
4.55 E-03 \\
5.11 E-03 \\
2.13 E 00 \\
4.47 E-03\end{array}$ & $\begin{array}{l}1.57 E-03 \\
1.78 E-03 \\
7.41 E-04 \\
1.26 E+00 \\
5.33 E-04\end{array}$ & $\begin{array}{l}1.39 E-03 \\
1.53 E-03 \\
6.56 E-04 \\
1.11 E+00 \\
4.71 E-04\end{array}$ & $\begin{array}{l}1.68 E-03 \\
1.91 E-03 \\
8.00 E-04 \\
1.34 E+00 \\
5.58 E-04\end{array}$ & $\begin{array}{l}1.56 E-03 \\
1.74 E-03 \\
7.54 E-04 \\
1.25 E+00 \\
5.35 E-04\end{array}$ & $\begin{array}{l}1.82 E-03 \\
1.84 E-03 \\
8.88 E-04 \\
1.32 E+00 \\
6.29 E-04\end{array}$ \\
\hline
\end{tabular}




\begin{tabular}{|c|c|c|c|c|c|c|c|c|c|c|}
\hline C. 10 & HALF & LIVER & LUNGS & MARROH & $\begin{array}{l}\text { ED } \\
\text { ROH }\end{array}$ & OVARIES & ANCREAS & ELETON & SPLEEN & STOMA \\
\hline $\begin{array}{l}P O-216 \\
P O-218 \\
A T-211 \\
A T-217 \\
R N-218\end{array}$ & $\begin{array}{r}0.1465 \\
3.05 \mathrm{M} \\
7.214 \mathrm{H} \\
0.0323 \mathrm{~S} \\
0.035 \mathrm{~S}\end{array}$ & $\begin{array}{l}1.35 E-04 \\
0.0 \\
3.09 E-01 \\
2.13 E-03 \\
6.78 E-03\end{array}$ & $\begin{array}{l}1.44 E-04 \\
0.0 \\
3.41 E-01 \\
2.20 E-03 \\
7.27 E-03\end{array}$ & $\begin{array}{l}57 E-04 \\
0 \\
79 E-01 \\
57 E-03 \\
17 E-03\end{array}$ & $\begin{array}{l}.46 E-04 \\
.0 \\
.39 E-01 \\
.33 E-03 \\
.42 E-03\end{array}$ & $\begin{array}{l}1.31 E-04 \\
0.0 \\
2.59 E-01 \\
2.06 E-03 \\
6.57 E-03\end{array}$ & $\begin{array}{l}1.16 E-04 \\
0.0 \\
2.22 E-01 \\
1.83 E-03 \\
5.84 E-03\end{array}$ & $\begin{array}{l}1.59 E-04 \\
0.0 \\
5.95 E-01 \\
2.61 E-03 \\
8.30 E-03\end{array}$ & $\begin{array}{l}1.31 E-04 \\
0.0 \\
2.91 E-01 \\
2.08 E-03 \\
6.60 E-03\end{array}$ & $\begin{array}{l}1.33 E-04 \\
0.0 \\
2.95 E-08 \\
2.11 E-03 \\
6.70 E-03\end{array}$ \\
\hline $\begin{array}{l}R N-219 \\
R N+220 \\
R N-222 \\
F R-221 \\
F R-223\end{array}$ & $\begin{array}{r}3.96 \mathrm{~S} \\
55.61 \mathrm{~S} \\
3.8235 \mathrm{D} \\
4.0 \mathrm{M} \\
21.0 \mathrm{M}\end{array}$ & $\begin{array}{l}4.96 E-01 \\
4.65 E-03 \\
3.44 E-03 \\
2.63 E-01 \\
3.03 E-01\end{array}$ & $\begin{array}{l}.35 E-01 \\
.99 E-03 \\
.69 E-03 \\
.86 E-01 \\
.21 E-01\end{array}$ & $\begin{array}{l}.16 E-01 \\
.68 E-03 \\
.25 E-03 \\
.97 E-01 \\
.36 E-01\end{array}$ & $\begin{array}{l}46 E-01 \\
10 E-03 \\
78 E-03 \\
82 E-01 \\
37 E-01\end{array}$ & $\begin{array}{l}4.56 E-01 \\
4.48 E-03 \\
3.31 E-03 \\
2.32 E-01 \\
3.35 E-01\end{array}$ & & & $\begin{array}{l}4.89 E-01 \\
4.53 E-03 \\
3.36 E-03 \\
2.61 E-01 \\
3.61 E-01\end{array}$ & $\begin{array}{l}\text { 4. } 09 \mathrm{E}-01 \\
4.59 \mathrm{E}-03 \\
3.39 \mathrm{E}-03 \\
2.59 \mathrm{E}-01 \\
3.67 \mathrm{E}-01\end{array}$ \\
\hline $\begin{array}{l}\text { RA-222 } \\
R A-223 \\
R A-224 \\
R A-225 \\
\text { RA-226 }\end{array}$ & 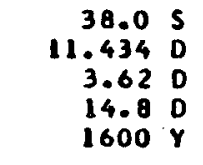 & $\begin{array}{l}7.97 E-02 \\
1.14 E \cdot 00 \\
8.57 E-02 \\
4.54 E-02 \\
5.74 E-02\end{array}$ & $\begin{array}{r}.60 E-02 \\
.24 E+00 \\
.31 E-02\end{array}$ & & & & & & & $\begin{array}{l}\text { 7. } 87 \mathrm{E}-02 \\
1.11 \mathrm{E}+00 \\
8.46 \mathrm{E}-02 \\
3.92 \mathrm{E}-02 \\
5.66 \mathrm{E}-02\end{array}$ \\
\hline $\begin{array}{l}A C-227 \\
A C-228 \\
T H-226\end{array}$ & $\begin{array}{r}5.75 \mathrm{Y} \\
10.0 \mathrm{O} \\
21.773 \mathrm{Y} \\
6.13 \mathrm{H} \\
30.9 \mathrm{M}\end{array}$ & $\begin{array}{l}5.60 E-13 \\
1.12 E-01 \\
9.86 E-04 \\
8.65 E+00 \\
6.54 E-02\end{array}$ & & $\begin{array}{l}2.74 E-11 \\
1.95 E-01 \\
1.76 E-03 \\
1.01 E+01 \\
1.10 E-01\end{array}$ & & & & & & $\begin{array}{l}1.10 E-11 \\
1.10 E-01 \\
9.75 E-04 \\
8.52 E+00 \\
6.44 E-02\end{array}$ \\
\hline $\begin{array}{l}T H-22 T \\
T H-228 \\
T H-229 \\
T H-230 \\
T H-231\end{array}$ & 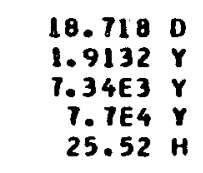 & $\begin{array}{l}8.87 E-01 \\
1.62 E-02 \\
1.14 E-01 \\
2.98 E-03 \\
8.94 E-02\end{array}$ & & $\begin{array}{l}1.30 E+00 \\
2.81 E-02 \\
1.27 E+00 \\
5.50 E-03\end{array}$ & & & & & & \\
\hline $\begin{array}{l}T H+232 \\
I H-233 \\
T H-234 \\
P A-230 \\
P A-231\end{array}$ & $\begin{array}{rr}1.405 E 10 & Y \\
22.3 & H \\
24.10 & 0 \\
17.4 & 0 \\
3.276 E 4 & Y\end{array}$ & $\begin{array}{l}1.25 E-03 \\
2.94 E-01 \\
6.38 E-02 \\
6.04 E+00 \\
2.54 E-01\end{array}$ & & & & & & & & \\
\hline $\begin{array}{l}P A-233 \\
P A-234 \\
P A-234 M \\
U-230 \\
U-231\end{array}$ & $\begin{array}{rl}27.0 & 0 \\
6.70 & H \\
1.17 & H \\
20.8 & 0 \\
4.2 & 0\end{array}$ & $\begin{array}{l}1.83 E+00 \\
1.81 E+01 \\
1.07 E-01 \\
8.83 E-03 \\
5.63 E-01\end{array}$ & & & & & & & & $\begin{array}{l}1.80 E+00 \\
1.79 E+01 \\
1.06 E-01 \\
8.57 E-03 \\
5.49 E-01\end{array}$ \\
\hline $\begin{array}{l}u-232 \\
u-233 \\
u-234 \\
u-235 \\
u-236\end{array}$ & $\begin{array}{r}12 Y \\
1.592 E 5 Y \\
2.445 E 5 y \\
7.038 E 8 Y \\
2.3415 E 7 Y\end{array}$ & $\begin{array}{l}1.71 E-03 \\
1.81 E-03 \\
8.18 E-04 \\
1.29 E+00 \\
5.97 E-04\end{array}$ & $\begin{array}{l}2.03 \mathrm{E}- \\
9.79 \mathrm{E}- \\
1.41 \mathrm{E} \\
7.25 \mathrm{E}-\end{array}$ & $\begin{array}{l}3.24 \mathrm{E}- \\
1.60 \mathrm{E}- \\
2.04 \mathrm{EE} \\
1.23 \mathrm{E}-\end{array}$ & $\begin{array}{l}1.70 \mathrm{E}- \\
6.48 \mathrm{E}- \\
1.35 \mathrm{E} \\
4.16 \mathrm{E}-\end{array}$ & $\begin{array}{l}1.57 E-03 \\
7.20 E-04 \\
1.82 E+00 \\
5.21 E-04\end{array}$ & $\begin{array}{l}1.30 E-03 \\
1.47 E-03 \\
6.25 E-04 \\
1.09 E 000 \\
4.35 E-04\end{array}$ & $\begin{array}{l}3.33 E-03 \\
3.36 E-03 \\
1.69 E-03 \\
2.09 E-00 \\
1.31 E-03\end{array}$ & $\begin{array}{l}1.03 E-03 \\
1.00 E-03 \\
7.033 E-04 \\
1.28 E+00 \\
5.61 E-04\end{array}$ & 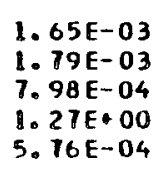 \\
\hline
\end{tabular}


DOSE-RATE FACTOPS FOR BODY URGANS AND EFFECTIVE DOSE-FATE FACTOR FOR IMHERSIOM IN CONTAMIMATE! WATFP

IN MREM/YR PER MICROCURIE/ICUBIC MI

\begin{tabular}{|c|c|c|c|c|c|c|c|c|c|}
\hline CLIDE & HALF-LIFE & TESTES & THYMUS & THYROIO & TERUS & EFFECTIVE & $\begin{array}{c}\text { SKIN } \\
\text { (PHOT ONI }\end{array}$ & $\begin{array}{c}\text { SKIN } \\
\text { IELECTROVI }\end{array}$ & $\begin{array}{l}\text { SKIN } \\
\text { ITOTALI }\end{array}$ \\
\hline $\begin{array}{l}P O-216 \\
P O-218 \\
A T-211 \\
A T-217 \\
R N-218\end{array}$ & $\begin{array}{r}0.146 \mathrm{~S} \\
3.05 \mathrm{H} \\
7.214 \mathrm{H} \\
0.0323 \mathrm{~S} \\
0.035 \mathrm{~S}\end{array}$ & $\begin{array}{l}1.91 E-04 \\
0.0 \\
5.07 E-01 \\
3.05 E-03 \\
9.71 E-03\end{array}$ & $\begin{array}{l}51 E-04 \\
0 \\
96 E-01 \\
35 E-03 \\
48 E-03\end{array}$ & $\begin{array}{l}81 E-04 \\
0 \\
04 E-01 \\
85 E-03 \\
08 E-03\end{array}$ & $\begin{array}{l}17 E-04 \\
0 \\
55 E-01 \\
86 E-03 \\
91 E-03\end{array}$ & $\begin{array}{l}1.63 E-04 \\
0.0 \\
4.35 E-01 \\
2.61 E-03\end{array}$ & $\begin{array}{l}2.21 E-04 \\
0.0 \\
5.54 E-01 \\
3.51 E-03 \\
1.12 E-02\end{array}$ & $\begin{array}{l}0.0 \\
0.0 \\
0.0 \\
0.0 \\
0.0\end{array}$ & $\begin{array}{l}2.21 E-04 \\
0.0 \\
5.54 E-01 \\
3.51 E-03 \\
1.12 E-02\end{array}$ \\
\hline $\begin{array}{l}R N-219 \\
R N-220 \\
R N-222 \\
F R-221 \\
F R-223\end{array}$ & $\begin{array}{r}3.96 \mathrm{~S} \\
55.61 \mathrm{~S} \\
3.8235 \mathrm{D} \\
4.8 \mathrm{M} \\
21.8 \mathrm{M}\end{array}$ & $\begin{array}{l}7.27 E-01 \\
6.68 E-03 \\
4.96 \mathrm{E}-03 \\
3.94 \mathrm{E}-01 \\
6.32 \mathrm{E}-01\end{array}$ & $.49 E-01$ & $\begin{array}{l}5.83 E-01 \\
.21 E-03 \\
6.59 E-03 \\
.80 E-01 \\
.01 E-01\end{array}$ & $\begin{array}{l}4.36 E-01 \\
4.06 E-03 \\
3.01 E-03 \\
2.32 E-01 \\
3.14 E-01\end{array}$ & $\begin{array}{l}6.30 E-01 \\
5.71 E-03 \\
4.24 E-03 \\
3.46 E-01\end{array}$ & $\begin{array}{l}0.15 E-01 \\
7.66 E-03 \\
5.67 E-03 \\
4.35 E-01 \\
7.33 E-01\end{array}$ & $\begin{array}{l}3.45 E-02 \\
0.0 \\
0.0 \\
3.91 E-02 \\
2.47 E+00\end{array}$ & $\begin{array}{l}8.50 E-01 \\
7.66 E-03 \\
5.67 E-03 \\
4.74 E-01 \\
3.20 E+00\end{array}$ \\
\hline $\begin{array}{l}R A-222 \\
R A-223 \\
R A-224 \\
R A-225 \\
R A-226\end{array}$ & $\begin{array}{r}38.0 \mathrm{~S} \\
11.434 \mathrm{D} \\
3.62 \mathrm{D} \\
14.8 \mathrm{D} \\
1600 \mathrm{Y}\end{array}$ & $\begin{array}{l}1.17 E-01 \\
1.74 E+00 \\
1.27 E-01 \\
1.14 E-01 \\
8.68 E-02\end{array}$ & $\begin{array}{l}9.79 E-02 \\
1.33 E+00 \\
9.67 E-02 \\
7.17 E-02 \\
6.65 E-02\end{array}$ & $\begin{array}{l}1.09 E-01 \\
1.60 E+00 \\
1.22 E-01 \\
9.47 E-02 \\
8.44 E-02\end{array}$ & $\begin{array}{l}7.02 \mathrm{E}-02 \\
9.86 \mathrm{E}-01 \\
7.57 \mathrm{E}-02 \\
2.87 \mathrm{E}-02 \\
5.06 \mathrm{E}-02\end{array}$ & $\begin{array}{l}1.01 E-01 \\
1.51 E+00 \\
1.11 E-01 \\
8.61 E-02 \\
7.64 E-02\end{array}$ & $\begin{array}{l}1.31 E-01 \\
1.92 E-00 \\
1.41 E-01 \\
1.61 E-01 \\
9.49 E-02\end{array}$ & $\begin{array}{l}4.40 E-03 \\
2.09 E-01 \\
1.12 E-02 \\
3.03 E-01 \\
1.36 E-02\end{array}$ & $\begin{array}{l}1.35 E-01 \\
2.13 E+00 \\
1.52 E-01 \\
4.64 E-01 \\
1.08 E-01\end{array}$ \\
\hline $\begin{array}{l}R A-228 \\
A C-225 \\
A C-227 \\
A C-228 \\
T H-226\end{array}$ & $\begin{array}{r}5.75 \mathrm{Y} \\
10.0 \mathrm{D} \\
21.773 \mathrm{Y} \\
6.13 \mathrm{H} \\
30.9 \mathrm{H}\end{array}$ & $\begin{array}{l}4.18 \mathrm{E}-10 \\
1.78 \mathrm{E}-01 \\
1.62 \mathrm{E}-03 \\
1.22 \mathrm{E}+01 \\
1.02 \mathrm{E}-01\end{array}$ & $\begin{array}{l}9.63 E-15 \\
1.36 E-01 \\
1.20 E-03 \\
9.67 E+00 \\
7.79 E-02\end{array}$ & $\begin{array}{l}2.68 \mathrm{E}-11 \\
1.75 \mathrm{E}-01 \\
1.55 \mathrm{E}-03 \\
1.16 \mathrm{E}+01 \\
9.97 \mathrm{E}-02\end{array}$ & $\begin{array}{l}4.21 E-15 \\
5.64 E-02 \\
8.52 E-04 \\
7.58 E+00 \\
5.68 E-02\end{array}$ & $\begin{array}{l}8.02 E-10 \\
1.55 E-01 \\
1.44 E-03 \\
1.05 E .01 \\
8.93 E-02\end{array}$ & $\begin{array}{l}9.63 E-09 \\
2.01 E-01 \\
2.37 E-03 \\
1.40 E-01 \\
1.13 E-01\end{array}$ & $\begin{array}{l}0.0 \\
3.14 E-03 \\
0.0 \\
2.76 E+00 \\
2.12 E-02\end{array}$ & $\begin{array}{l}9.63 \mathrm{E}-09 \\
2.04 \mathrm{E}-01 \\
2.37 \mathrm{E}-03 \\
1.6 \mathrm{BE}+01 \\
1.35 \mathrm{E}-01\end{array}$ \\
\hline $\begin{array}{l}T H-227 \\
T H-228 \\
T H-229 \\
T H-230 \\
T H-231\end{array}$ & $\begin{array}{r}1.9132 Y \\
7.3453 \\
7.7 E 4 Y \\
25.52 \mathrm{H}\end{array}$ & $\begin{array}{l}1.33 E+00 \\
2.59 E-02 \\
1.14 E+00 \\
5.26 E-03 \\
1.61 E-01\end{array}$ & $\begin{array}{l}1.01 E+00 \\
1.97 E-02 \\
8.78 E-01 \\
3.80 E-03 \\
1.14 E-01\end{array}$ & $\begin{array}{l}1.27 E \cdot 00 \\
2.52 E-02 \\
1.13 E+00 \\
4.88 E-03 \\
1.50 E-01\end{array}$ & $\begin{array}{l}1.76 E-01 \\
1.38 E-02 \\
6.08 E-01 \\
2.44 E-03 \\
7.33 E-02\end{array}$ & $\begin{array}{l}2.27 E-02 \\
9.93 E-01 \\
4.64 E-03 \\
1.371 E-01\end{array}$ & $\begin{array}{l}1.50 E+00 \\
3.26 E-02 \\
1.27 E+00 \\
9.84 E-03 \\
2.28 E-01\end{array}$ & $\begin{array}{l}5.97 E-02 \\
1.35 E-03 \\
8.15 E-02 \\
0.0 \\
2.02 E-01\end{array}$ & $\begin{array}{l}1.56 E+00 \\
3.39 E-02 \\
1.35 E+00 \\
9.84 E-03 \\
4.31 E-01\end{array}$ \\
\hline $\begin{array}{l}T H+232 \\
T H-233 \\
T H-234 \\
P A-230 \\
P A-231\end{array}$ & $\begin{array}{r}1.405 E 10 \\
22.3 \\
24.10 \\
17.4 \\
3.276 E 4 \\
3.278\end{array}$ & $\begin{array}{l}2.49 E-03 \\
4.33 E-01 \\
1.07 E-01 \\
8.66 E+00 \\
3.06 E-01\end{array}$ & & $\begin{array}{l}2.13 E-03 \\
4.07 E-01 \\
1.05 E-01 \\
0.26 E+00 \\
3.60 E-01\end{array}$ & $\begin{array}{l}9.06 E-04 \\
2.55 E-01 \\
5.24 E-02 \\
5.26 E+00 \\
2.22 E-01\end{array}$ & $\begin{array}{l}2.26 E-03 \\
3.72 E-01 \\
9.13 E-02 \\
7.42 E+00 \\
3.34 E-01\end{array}$ & & & $\begin{array}{l}1.03 E-03 \\
3.46 E+00 \\
1.59 E-01 \\
1.01 E+01 \\
4.94 E-01\end{array}$ \\
\hline $\begin{array}{l}P A-233 \\
P A-234 \\
P A-234 M \\
U-230 \\
U-231\end{array}$ & $\begin{array}{rl}27.0 & 0 \\
6.70 & H \\
1.17 & M \\
20.8 & 0 \\
4.2 & 0\end{array}$ & $\begin{array}{l}2.72 E+00 \\
2.58 E+01 \\
1.51 E-01 \\
1.45 E-02 \\
9.21 E-01\end{array}$ & & $\begin{array}{l}2.58 E+00 \\
2.45 E+01 \\
1.45 E-01 \\
1.37 E-02 \\
9.07 E-01\end{array}$ & & $\begin{array}{l}2.37 E * 00 \\
2.21 E * 01 \\
1.29 E-01 \\
1.20 E-02 \\
7.99 E-01\end{array}$ & & & \\
\hline $\begin{array}{l}u-232 \\
u-233 \\
u-234 \\
u-235 \\
u-236\end{array}$ & $\begin{array}{r}72 Y \\
1.592 E 5 Y \\
2.445 E 5 Y \\
7.038 E 8 Y \\
2.3415 E Y Y\end{array}$ & $\begin{array}{l}3.49 \mathrm{E}-03 \\
3.06 \mathrm{E}-03 \\
1.97 \mathrm{E}-03 \\
1.95 \mathrm{E}+00 \\
1.54 \mathrm{E}-03\end{array}$ & $\begin{array}{l}2.20 E-03 \\
1.06 E-03 \\
1.49 E+00 \\
7.91 E-04\end{array}$ & $\begin{array}{l}2.88 E-03 \\
2.87 E-03 \\
1.45 E-03 \\
1.90 E+00 \\
1.08 E-03\end{array}$ & $\begin{array}{l}1.56 E-03 \\
6.46 E-04 \\
1.13 E+00 \\
4.68 E-04\end{array}$ & $\begin{array}{l}3.20 E-03 \\
2.76 E-03 \\
1.86 E-03 \\
1.72 E+00 \\
1.50 E-03\end{array}$ & $\begin{array}{l}5.35 E-03 \\
7.80 E-03 \\
2.13 E+00 \\
7.01 E-03\end{array}$ & $\begin{array}{l}1.74 E-05 \\
1.92 E-04 \\
2.21 E-02 \\
1.25 E-04\end{array}$ & $\begin{array}{l}1.06 E-02 \\
5.3 T E-03 \\
7.99 E-03 \\
2.15 E+00 \\
7.14 E-03\end{array}$ \\
\hline
\end{tabular}




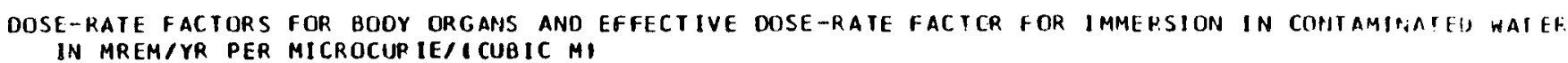

\begin{tabular}{|c|c|c|c|c|c|c|c|c|c|c|}
\hline NUCLIDE & HALF-LIFE & DRENALS & BL ADDER & BR AIN & BREAST & HE AR T & $\begin{array}{l}\text { SMALL } \\
\text { INTESTINE }\end{array}$ & $\begin{array}{l}\text { UPPER } \\
\text { LARGE } \\
\text { INTESTINE }\end{array}$ & $\begin{array}{c}\text { LOWER } \\
\text { LARGE } \\
\text { INIESIINE }\end{array}$ & KIONEYS \\
\hline $\begin{array}{l}U-237 \\
U-238 \\
U-239 \\
U-240 \\
N P-235\end{array}$ & $\begin{array}{r}6.750 \\
4.468 E 9 \\
23.40 \mathrm{M} \\
14.1 \mathrm{H} \\
396.1 \mathrm{D}\end{array}$ & $\begin{array}{l}1.17 E+00 \\
5.22 E-04 \\
3.83 E-01 \\
3.26 E-03 \\
1.57 E-02\end{array}$ & $\begin{array}{l}12 E \cdot 00 \\
.04 E-04 \\
.99 E-01 \\
.86 E-03 \\
.48 E-02\end{array}$ & $\begin{array}{l}17 E+00 \\
92 E-04 \\
06 E-01 \\
60 E-03 \\
56 E-02\end{array}$ & $\begin{array}{l}03 E+0 C \\
91 E-03 \\
38 E-01 \\
63 E-02 \\
15 E-02\end{array}$ & $\begin{array}{l}07 E \cdot 00 \\
43 E-04 \\
62 E-01 \\
38 E-03 \\
43 E-02\end{array}$ & $\begin{array}{l}.42 E-01 \\
02 E-04 \\
024 E-01 \\
041 E-03 \\
.23 E-02\end{array}$ & $\begin{array}{l}1.14 E+00 \\
4.64 E-04 \\
3.88 E-01 \\
3.07 E-03 \\
1.54 E-02\end{array}$ & $\begin{array}{l}1.04 E-00 \\
4.46 E-04 \\
3.48 E-01 \\
2.71 E-03 \\
1.40 E-02\end{array}$ & $\begin{array}{l}1.16 E+00 \\
5.30 E-04 \\
4.14 E-01 \\
4.72 E-03 \\
1.51 E-02\end{array}$ \\
\hline $\begin{array}{l}N P-236 \\
N P-236 M \\
N P-237 \\
N P-236 \\
N P-239\end{array}$ & $\begin{array}{rl}1.15 E 6 & Y \\
22.5 & H \\
2.14 E 6 & Y \\
2.117 & D \\
2.355 & D\end{array}$ & $\begin{array}{l}1.15 E+00 \\
4.48 E-01 \\
1.80 E-01 \\
5.37 E+00 \\
1.51 E+00\end{array}$ & $\begin{array}{l}.08 E+00 \\
.31 E-01 \\
.86 E-01 \\
.24 E \cdot 00 \\
.41 E+00\end{array}$ & $\begin{array}{l}.14 E+00 \\
.59 E-01 \\
.09 E-01 \\
.90 E+00 \\
51 E+00\end{array}$ & & & & & $\begin{array}{l}1.02 E+00 \\
4.04 E-01 \\
1.68 E-01 \\
5.07 E+00 \\
1.35 E+00\end{array}$ & $\begin{array}{l}1.10 E+00 \\
4.42 \mathrm{E}-01 \\
1.95 \mathrm{E}-01 \\
5.50 \mathrm{E}+00 \\
1.45 \mathrm{E}+00\end{array}$ \\
\hline $\begin{array}{l}N P-240 \\
N P-240 M \\
P U-236 \\
P U-237 \\
P U-238\end{array}$ & $\begin{array}{r}65 \mathrm{M} \\
7.4 \mathrm{M} \\
2.851 \mathrm{Y} \\
45.3 \mathrm{D} \\
87.75 \mathrm{Y}\end{array}$ & $\begin{array}{l}1.10 E+01 \\
3.13 E+00 \\
4.48 E-04 \\
4.10 E-01 \\
2.60 E-04\end{array}$ & $\begin{array}{r}.06 E+01 \\
.01 E+00 \\
3.70 E-04\end{array}$ & $\begin{array}{l}.18 E+01 \\
.37 E+00 \\
.58 E-04 \\
.13 E-01 \\
.69 E-04\end{array}$ & & & & & $\begin{array}{l}1.02 E+01 \\
2.90 E+00 \\
3.59 E-04 \\
3.67 E-01 \\
1.94 E-04\end{array}$ & $\begin{array}{l}1.11 E+01 \\
3.14 E+00 \\
3.99 E-04 \\
4.03 E-01 \\
1.99 E-04\end{array}$ \\
\hline $\begin{array}{l}P U-239 \\
P U-240 \\
P U-241 \\
P U-242 \\
P U-243\end{array}$ & $\begin{array}{r}24131 Y \\
6537 Y \\
14.4 Y \\
3.75865 Y \\
4.956 \mathrm{H}\end{array}$ & $\begin{array}{l}5.37 E-04 \\
2.64 E-04 \\
0.0 \\
2.34 E-04 \\
1.94 E-01\end{array}$ & $\begin{array}{l}.68 E-04 \\
.90 E-04 \\
.0 \\
.73 E-04\end{array}$ & $\begin{array}{l}5.00 E-04 \\
1.78 E-04 \\
0.0 \\
1.65 E-04 \\
2.01 E-01\end{array}$ & $\begin{array}{l}.46 E-03 \\
.44 E-03 \\
.0 \\
.7 E-03\end{array}$ & $\begin{array}{l}.64 E-04 \\
.65 E-04 \\
.0 \\
.52 E-04\end{array}$ & $\begin{array}{l}3.95 E-04 \\
1.40 E-04 \\
0.0\end{array}$ & $\begin{array}{l}4.92 \mathrm{E}-04 \\
1.64 \mathrm{E}-04 \\
0.0 \\
1.52 \mathrm{E}-04 \\
1.95 \mathrm{E}-01\end{array}$ & $\begin{array}{l}4.65 E-04 \\
2.00 E-04 \\
0.0 \\
1.79 E-04 \\
1.75 E-01\end{array}$ & $\begin{array}{l}4.76 \mathrm{E}-04 \\
2.13 \mathrm{E}-04 \\
0.0 \\
1.92 \mathrm{E}-04 \\
2.00 \mathrm{E}-01\end{array}$ \\
\hline $\begin{array}{l}P U-244 \\
P U-245 \\
P U-246 \\
A M-241 \\
A M-242\end{array}$ & $\begin{array}{r}8.26 E 7 \mathrm{Y} \\
10.57 \mathrm{H} \\
10.85 \mathrm{O} \\
432.2 \mathrm{Y} \\
16.02 \mathrm{H}\end{array}$ & $\begin{array}{l}1.23 E-04 \\
3.96 E+00 \\
8.66 E-01 \\
1.37 E-01 \\
1.18 E-01\end{array}$ & $\begin{array}{l}6.59 E-05 \\
3.77 E+00 \\
8.14 E-01 \\
1.57 E-01 \\
1.12 E-01\end{array}$ & $\begin{array}{l}5.25 E-05 \\
4.18 E+00 \\
8.42 E-01 \\
1.43 E-01 \\
1.18 E-01\end{array}$ & $\begin{array}{l}3.05 E-03 \\
5.13 E+00 \\
1.35 E+00 \\
351 E-01 \\
2.22 E-01\end{array}$ & $\begin{array}{l}5.28 E-05 \\
3.70 E+00 \\
7.67 E-01 \\
1.27 E-01 \\
1.09 E-01\end{array}$ & & & & $\begin{array}{l}8.27 E-05 \\
3.92 E+00 \\
8.51 E-01 \\
1.69 E-01 \\
1.14 E-01\end{array}$ \\
\hline $\begin{array}{l}A M-242 M \\
A M-243 \\
A M-244 \\
A M-245 \\
A M-246\end{array}$ & $\begin{array}{r}152 \mathrm{Y} \\
1.3863 \mathrm{Y} \\
10.1 \mathrm{H} \\
122.4 \mathrm{M} \\
25.0 \mathrm{M}\end{array}$ & $\begin{array}{l}2.50 E-03 \\
4.03 E-01 \\
1.68 E+00 \\
2.85 E-01 \\
9.44 E+00\end{array}$ & $\begin{array}{l}2.12 \mathrm{E}-03 \\
4.29 \mathrm{E}-01 \\
7.39 \mathrm{E}+00 \\
2.64 \mathrm{E}-01 \\
9.22 \mathrm{E}+00\end{array}$ & $\begin{array}{l}2.15 \mathrm{E}-03 \\
4.25 \mathrm{E}-01 \\
8.29 \mathrm{E} \cdot 00 \\
2.82 \mathrm{E}-01 \\
1.04 \mathrm{E}+01\end{array}$ & $\begin{array}{l}12 \\
1 \\
11 \\
11\end{array}$ & $\begin{array}{l}2.00 E-03 \\
3.81 E-01 \\
7.29 E+00 \\
2.57 E-01 \\
9.11 E+00\end{array}$ & $\begin{array}{l}1.71 \\
3.40 \\
6.58 \\
2.27 \\
8.30\end{array}$ & & & $\begin{array}{l}2.26 E-03 \\
4.44 E-01 \\
7.72 E+00 \\
2.70 E-01 \\
9.61 E+00\end{array}$ \\
\hline $\begin{array}{l}C M-242 \\
C M-243 \\
C M-244 \\
C M-245 \\
C M-246\end{array}$ & $\begin{array}{r}163.28 \\
28.5 Y \\
18.11 Y \\
8.5 E 3 Y \\
4.75 E 3 Y\end{array}$ & $\begin{array}{l}2.84 E-04 \\
1.15 E+00 \\
2.22 E-04 \\
6.23 E-01 \\
1.60 E-04\end{array}$ & $\begin{array}{l}1.06 E-04 \\
1.07 E+00 \\
1.13 E-04 \\
5.87 E-01 \\
6.63 E-05\end{array}$ & $\begin{array}{l}1.70 E-04 \\
1.14 E+00 \\
1.18 E-04 \\
6.21 E-01 \\
5.95 E-05\end{array}$ & $\begin{array}{l}5.14 E-03 \\
1.79 E+00 \\
4.52 E-03 \\
1.08 E+00 \\
3.97 E-03\end{array}$ & $\begin{array}{l}1.61 E-04 \\
1.04 E+00 \\
1.14 E-04 \\
5.11 E-01 \\
6.19 E-05\end{array}$ & $\begin{array}{l}1.27 E-04 \\
9.19 E-01 \\
8.63 E-05 \\
4.95 E-01 \\
4.43 E-05\end{array}$ & $\begin{array}{l}1.47 E-04 \\
1.11 E+00 \\
9.93 E-05 \\
6.15 E-01 \\
5.32 E-05\end{array}$ & $\begin{array}{l}2.08 E-04 \\
1.02 E+00 \\
1.57 E-04 \\
5.56 E-08 \\
1.05 E-04\end{array}$ & $\begin{array}{l}1.08 E-04 \\
1.10 E+00 \\
1.30 E-04 \\
5.98 E-01 \\
8.63 E-05\end{array}$ \\
\hline $\begin{array}{l}C M-247 \\
C M-248 \\
C M-249 \\
C M-250 \\
B K-249\end{array}$ & 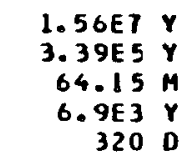 & $\begin{array}{l}2.96 E+00 \\
1.75 E-04 \\
1.78 E-01 \\
0.0 \\
0.0\end{array}$ & $\begin{array}{l}2.77 E+00 \\
1.02 E-04 \\
1.69 E-01 \\
0.0 \\
0.0\end{array}$ & $\begin{array}{l}3.05 E+00 \\
1.02 E-04 \\
1.89 E-01 \\
0.0 \\
0.0\end{array}$ & $\begin{array}{l}3.85 E+00 \\
3.24 E-03 \\
2.23 E-01 \\
0.0 \\
0.0\end{array}$ & $\begin{array}{l}2.70 E+00 \\
9.73 E-05 \\
1.66 E-01 \\
0.0 \\
0.0\end{array}$ & $\begin{array}{l}2.43 E+00 \\
7.71 E-05 \\
1.49 E-01 \\
0.0 \\
0.0\end{array}$ & $\begin{array}{l}2.86 E+03 \\
8.94 E-05 \\
1.16 E-01 \\
0.0 \\
0.0\end{array}$ & $\begin{array}{l}2.66 E+00 \\
1.27 E-04 \\
1.62 E-01 \\
0.0 \\
0.0\end{array}$ & $\begin{array}{l}2.86 E+00 \\
1.16 E-04 \\
1.76 E-01 \\
0.0 \\
0.0\end{array}$ \\
\hline
\end{tabular}



DOSE-RATE FAC TORS FOR BOOY ORGANS AND EFFECTIVE OUSE-RATE FACTOR FOP IMMERSIUN IN CUNTAMITATEL WATER
IN MREM/YR PER MICROCUR IE/ICUBIC MI

\begin{tabular}{|c|c|c|c|c|c|c|c|c|c|c|}
\hline NUCLIDE & HALF-LIFE & LIVER & LUNGS & MARROW & $\begin{array}{c}\text { REO } \\
\text { HARROH }\end{array}$ & OVARIES & PANCREAS & SKELETON & SPLEEN & STOMACH \\
\hline $\begin{array}{l}U-237 \\
U-238 \\
U-239 \\
U-240 \\
N P-235\end{array}$ & 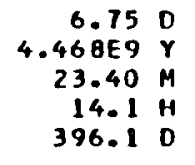 & $\begin{array}{l}11 E+00 \\
00 E-04 \\
94 E-01 \\
35 E-03 \\
48 E-02\end{array}$ & $\begin{array}{l}3 E+00 \\
10 E-04 \\
32 E-01 \\
12 E-03\end{array}$ & $\begin{array}{l}10 E+00 \\
13 E-03 \\
4 E-01 \\
6 E-03 \\
5 E-02\end{array}$ & $\begin{array}{l}05 E+00 \\
3 E-04 \\
3 E-01 \\
37 E-03 \\
31 E-02\end{array}$ & $\begin{array}{l}9.61 E-01 \\
4.37 E-04 \\
3.38 E-01 \\
2.91 E-03 \\
1.28 E-02\end{array}$ & $\begin{array}{l}8.84 E-01 \\
3.62 E-04 \\
2.84 E-01 \\
2.12 E-03 \\
1.17 E-02\end{array}$ & $\begin{array}{l}1.95 E+00 \\
1.10 E-03 \\
7.11 E-01 \\
7.47 E-03 \\
2.85 E-02\end{array}$ & $\begin{array}{l}1.39 E+00 \\
4.6 B E-04 \\
3.67 E-01 \\
2.84 E-03 \\
1.46 E-02\end{array}$ & $\begin{array}{l}1.09 E-00 \\
4.82 E-04 \\
3.15 E-01 \\
3.07 E-03 \\
1.46 E-02\end{array}$ \\
\hline $\begin{array}{l}N P-236 \\
N P-236 M \\
N P-237 \\
N P-238 \\
N P-239\end{array}$ & 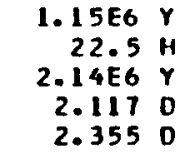 & $\begin{array}{l}08 E+00 \\
29 E-01 \\
83 E-01 \\
21 E+00 \\
41 E+00\end{array}$ & $\begin{array}{l}19 E \cdot 00 \\
73 E-01 \\
05 E-01 \\
54 E+00 \\
54 E \cdot 00\end{array}$ & $\begin{array}{l}9 E+00 \\
2 E-01 \\
8 E-01 \\
2 E+00 \\
6 E+00\end{array}$ & $\begin{array}{l}01 E+00 \\
99 E-01 \\
55 E-01 \\
61 E+00\end{array}$ & $\begin{array}{l}9.28 E-01 \\
3.78 E-01 \\
1.56 E-01 \\
5.04 E+00 \\
1.24 E+00\end{array}$ & $\begin{array}{l}0.65 E-01 \\
3.42 E-01 \\
1.39 E-01 \\
4.50 E+00 \\
1.16 E+00\end{array}$ & $\begin{array}{l}1.95 E+00 \\
7.42 E-01 \\
3.40 E-01 \\
6.03 E+00 \\
2.32 E+00\end{array}$ & $\begin{array}{l}1.07 E+00 \\
4.19 E-01 \\
1.77 E-01 \\
5.04 E+00 \\
1.39 E+00\end{array}$ & $\begin{array}{l}1.06 E+00 \\
4.21 \mathrm{E}-01 \\
1.78 \mathrm{E}-01 \\
5.14 \mathrm{E}+00 \\
1.39 \mathrm{E}+00\end{array}$ \\
\hline $\begin{array}{l}N P-240 \\
N P-240 M \\
P U-236 \\
P U-231 \\
P U-238\end{array}$ & $\begin{array}{r}65 \mathrm{M} \\
7.4 \mathrm{M} \\
2.851 \mathrm{Y} \\
45.3 \mathrm{D} \\
87.75 \mathrm{Y}\end{array}$ & $\begin{array}{l}1.05 E+01 \\
2.99 E+00 \\
3.68 E-04 \\
3.93 E-01 \\
1.77 E-04\end{array}$ & $\begin{array}{l}1.13 E \cdot 01 \\
3.20 E \cdot 00 \\
5.17 E-04 \\
4.37 E-01 \\
2.94 E-04\end{array}$ & & & & & & $\begin{array}{l}1.03 E+01 \\
2.91 E+00 \\
3.62 E-04 \\
3.05 E-01 \\
1.87 E-04\end{array}$ & $\begin{array}{l}1.04 E+01 \\
2.95 E+00 \\
3.75 E-04 \\
3.85 E-01 \\
1.95 E-04\end{array}$ \\
\hline $\begin{array}{l}P U-239 \\
P U-240 \\
P U-241 \\
P U-242 \\
P U-243\end{array}$ & $\begin{array}{r}24131 \mathrm{Y} \\
6531 \mathrm{Y} \\
14.4 \mathrm{Y} \\
3.758 E 5 \mathrm{Y} \\
4.956 \mathrm{H}\end{array}$ & $\begin{array}{l}4.68 E-04 \\
1.88 E-04 \\
0.0 \\
1.72 E-04 \\
1.94 E-01\end{array}$ & $\begin{array}{l}5.57 E-04 \\
3.02 E-04 \\
0.0 \\
2.68 E-04 \\
2.14 E-01\end{array}$ & $\begin{array}{l}8.72 E-04 \\
4.64 E-04 \\
0.0 \\
4.16 E-04 \\
3.55 E-01\end{array}$ & $\begin{array}{l}.47 E-04 \\
1.36 E-04 \\
.0 \\
1.24 E-04 \\
.59 E-01\end{array}$ & $\begin{array}{l}4.22 E-04 \\
2.00 E-04 \\
0.0 \\
1.79 E-04 \\
1.64 E-01\end{array}$ & $\begin{array}{l}3.98 E-04 \\
1.73 E-04 \\
0.0 \\
1.54 E-04 \\
1.43 E-01\end{array}$ & $\begin{array}{l}9.22 E-04 \\
5.38 E-04 \\
0.0 \\
4.78 E-04 \\
3.64 E-01\end{array}$ & $\begin{array}{l}4.76 E-04 \\
1.94 E-04 \\
0.0 \\
1.76 E-04 \\
1.85 E-01\end{array}$ & $\begin{array}{l}4.15 E-04 \\
2.03 E-04 \\
0.0 \\
1.83 E-04 \\
1.86 E-01\end{array}$ \\
\hline $\begin{array}{l}P U-244 \\
P U-245 \\
P U-246 \\
A M-241 \\
A M-242\end{array}$ & $\begin{array}{r}0.26 E 7 \\
10.57 \\
10.85 \\
432 \\
132.2 Y \\
16.02 \mathrm{H}\end{array}$ & $\begin{array}{l}6.09 E-05 \\
3.75 E \cdot 00 \\
8.01 E-01 \\
1.53 E-01 \\
1.12 E-01\end{array}$ & $\begin{array}{l}1.34 E-04 \\
4.03 E+00 \\
0.73 E-01 \\
1.69 E-01 \\
1.25 E-01\end{array}$ & $\begin{array}{l}1.83 E-04 \\
4.74 E+00 \\
1.23 E+00 \\
3.04 E-01 \\
2.03 E-01\end{array}$ & $\begin{array}{l}5 \\
0 \\
1 \\
2 \\
1\end{array}$ & $\begin{array}{l}8.36 E-05 \\
3.55 E \cdot 00 \\
7.02 E-01 \\
1.24 E-01 \\
9.59 E-02\end{array}$ & $\begin{array}{l}7.72 E-05 \\
3.22 E \cdot 00 \\
6.67 E-01 \\
9.49 E-02 \\
8.83 E-02\end{array}$ & $\begin{array}{l}2.32 E-04 \\
4.02 E+00 \\
1.26 E+00 \\
3.12 E-01 \\
2.10 E-01\end{array}$ & $\begin{array}{l}7.26 E-05 \\
3.66 E 000 \\
7.86 E-01 \\
1.34 E-01 \\
1.10 E-01\end{array}$ & $\begin{array}{l}7.60 E-05 \\
3.70 E-00 \\
7.85 E-01 \\
1.39 E-01 \\
1.10 E-01\end{array}$ \\
\hline $\begin{array}{l}A M-242 M \\
A M-243 \\
A M-244 \\
A M-245 \\
A M-246\end{array}$ & $\begin{array}{r}152 \mathrm{Y} \\
1.38 \mathrm{Y} \mathrm{Y} \\
10.1 \mathrm{H} \\
122.4 \mathrm{M} \\
25.0 \mathrm{M}\end{array}$ & $\begin{array}{l}2.11 E-03 \\
4.24 E-01 \\
7.35 E+00 \\
2.63 E-01 \\
9.1 B E \bullet 00\end{array}$ & $\begin{array}{l}2.72 E-03 \\
4.68 E-01 \\
7.86 E+00 \\
2.88 E-01 \\
9.77 E+00\end{array}$ & $\begin{array}{l}4.30 E-03 \\
8.18 E-01 \\
8.75 E+00 \\
4.19 E-01 \\
1.05 E+01\end{array}$ & $\begin{array}{l}1.03 E-03 \\
3.00 E-01 \\
7.94 E+00 \\
2.10 E-01 \\
9.08 E+00\end{array}$ & $\begin{array}{l}1.98 E-03 \\
3.49 E-01 \\
7.10 E+00 \\
2.32 E-01 \\
8.85 E+00\end{array}$ & $\begin{array}{l}1.78 E-03 \\
2.89 E-01 \\
6.33 E 000 \\
2.19 E-01 \\
1.93 E 000\end{array}$ & $\begin{array}{l}4.64 E-03 \\
8.40 E-01 \\
8.90 E+00 \\
4.29 E-01 \\
1.07 E+01\end{array}$ & $\begin{array}{l}2.13 E-03 \\
3.90 E-01 \\
1.14 E+00 \\
2.61 E-01 \\
8.89 E \cdot 00\end{array}$ & $\begin{array}{l}2.17 \mathrm{E}-03 \\
3.98 \mathrm{E}-01 \\
7.21 \mathrm{E}+00 \\
2.60 \mathrm{E}-01 \\
9.05 \mathrm{E}+00\end{array}$ \\
\hline $\begin{array}{l}C M-242 \\
C M-243 \\
C M-244 \\
C M-245 \\
C M-246\end{array}$ & $\begin{array}{r}163.2 \mathrm{O} \\
28.5 Y \\
18.11 Y \\
8.5 E 3 Y \\
4.75 E 3 Y\end{array}$ & $\begin{array}{l}1.12 E-04 \\
1.07 E+00 \\
1.18 E-04 \\
5.85 E-01 \\
6.57 E-05\end{array}$ & $\begin{array}{l}3.16 E-04 \\
1.17 E+00 \\
2.44 E-04 \\
6.48 E-01 \\
1.73 E-04\end{array}$ & $\begin{array}{l}4.64 E-04 \\
1.70 E+00 \\
3.46 E-04 \\
1.03 E+00 \\
2.28 E-04\end{array}$ & $\begin{array}{l}1.42 E-04 \\
1.09 E+00 \\
1.05 E-04 \\
5.50 E-01 \\
6.50 E-05\end{array}$ & $\begin{array}{l}2.05 E-04 \\
9.39 E-01 \\
1.55 E-04 \\
5.03 E-01 \\
1.06 E-04\end{array}$ & $\begin{array}{l}1.85 E-04 \\
8.06 E-01 \\
1.43 E-04 \\
4.69 E-01 \\
1.00 E-04\end{array}$ & $\begin{array}{l}5.53 E-04 \\
1.74 E+00 \\
4.24 E-04 \\
1.06 E+00 \\
2.96 E-04\end{array}$ & $\begin{array}{l}1.95 E-04 \\
1.06 E+00 \\
1.43 E-04 \\
5.79 E-01 \\
8.94 E-05\end{array}$ & $\begin{array}{l}2.03 E-04 \\
1.05 E+00 \\
1.49 E-04 \\
5.76 E-01 \\
9.46 E-05\end{array}$ \\
\hline $\begin{array}{l}C M-247 \\
C M-248 \\
C M-249 \\
C M-250 \\
8 K-249\end{array}$ & $\begin{array}{r}1.56 E T Y \\
3.39 E 5 Y \\
64.15 Y \\
6.9 E 3 Y \\
320 Y\end{array}$ & $\begin{array}{l}2.75 E+00 \\
1.05 E-04 \\
1.68 E-01 \\
0.0 \\
0.0\end{array}$ & $\begin{array}{l}2.97 E+00 \\
1.96 E-04 \\
1.80 E-01 \\
0.0 \\
0.0\end{array}$ & $\begin{array}{l}3.59 E+00 \\
2.07 E-04 \\
2.06 E-01 \\
0.0 \\
0.0\end{array}$ & $\begin{array}{l}3.05 E+00 \\
8.57 E-05 \\
1.84 E-01 \\
0.0 \\
0.0\end{array}$ & $\begin{array}{l}2.58 E+00 \\
1.27 E-04 \\
1.62 E-01 \\
0.0 \\
0.0\end{array}$ & $\begin{array}{l}2.37 E+00 \\
1.14 E-04 \\
1.45 E-01 \\
0.0 \\
0.0\end{array}$ & $\begin{array}{l}3.65 E+00 \\
3.43 E-04 \\
2.09 E-01 \\
0.0 \\
0.0\end{array}$ & $\begin{array}{l}2.71 E+00 \\
1.19 E-04 \\
1.64 E-01 \\
0.0 \\
0.0\end{array}$ & $\begin{array}{l}2.72 E+00 \\
1.24 E-04 \\
1.66 E-01 \\
0.0 \\
0.0\end{array}$ \\
\hline
\end{tabular}




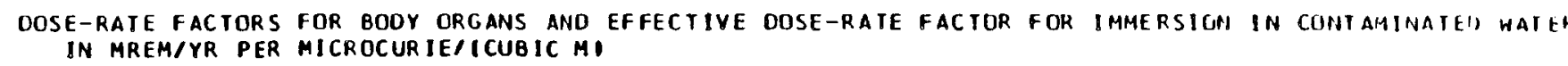

\begin{tabular}{|c|c|c|c|c|c|c|c|c|c|}
\hline NUCL IDE & HALF-LIFE & TESTES & THYMUS & THYROID & UTERUS & EFFECTIVE & $\begin{array}{c}\text { SKIN } \\
\text { S PHOTONI }\end{array}$ & $\begin{array}{c}\text { SKIN } \\
\text { (ELECIRON ) }\end{array}$ & $\begin{array}{l}\text { SKIN } \\
\text { ITOIAL, }\end{array}$ \\
\hline $\begin{array}{l}U-237 \\
U-238 \\
U-239 \\
U-240 \\
N P-235\end{array}$ & $\begin{array}{r}6.75 \mathrm{O} \\
4.468 \mathrm{Y} 9 \\
23.40 \mathrm{H} \\
14.1 \mathrm{H} \\
396.1 \mathrm{D}\end{array}$ & $\begin{array}{l}1.77 E+00 \\
1.32 E-03 \\
6.43 E-01 \\
1.05 E-02 \\
2.62 E-02\end{array}$ & $\begin{array}{l}1.35 E+00 \\
6.65 E-04 \\
4.99 E-01 \\
5.05 E-03 \\
1.83 E-02\end{array}$ & $\begin{array}{l}1.73 E+00 \\
9.12 E-04 \\
6.30 E-01 \\
7.07 E-03 \\
2.41 E-02\end{array}$ & $\begin{array}{l}9.50 E-01 \\
3.88 E-04 \\
3.23 E-01 \\
2.21 E-03 \\
1.25 E-02\end{array}$ & $\begin{array}{l}1.53 E+00 \\
1.29 E-03 \\
5.46 E-01 \\
9.16 E-03 \\
2.35 E-02\end{array}$ & $\begin{array}{l}1.98 E-00 \\
6.17 E-03 \\
7.15 E-01 \\
3.85 E-02 \\
4.96 E-02\end{array}$ & $\begin{array}{l}3.27 E-01 \\
8.98 E-05 \\
2.92 E+00 \\
5.47 E-01 \\
1.05 E-06\end{array}$ & $\begin{array}{l}2.30 E-00 \\
6.25 E-03 \\
3.64 E+00 \\
5.85 E-01 \\
4.96 E-02\end{array}$ \\
\hline $\begin{array}{l}\text { NP-236 } \\
N P-236 M \\
\text { NP-237 } \\
\text { NP-238 } \\
\text { NP-239 }\end{array}$ & $\begin{array}{r}1.15 E 6 \\
22.5 \\
2.14 E 6 \\
2.117 \\
2.355 \\
\end{array}$ & $\begin{array}{l}1.71 E+00 \\
6.73 E-01 \\
3.12 E-01 \\
7.32 E \cdot 00 \\
2.16 E+00\end{array}$ & $\begin{array}{l}1.31 E+00 \\
5.19 E-01 \\
2.30 E-01 \\
5.81 E \cdot 00 \\
1.65 E+00\end{array}$ & $\begin{array}{l}1.68 E+00 \\
6.61 E-01 \\
3.00 E-01 \\
7.00 E+00 \\
2.09 E+00\end{array}$ & $\begin{array}{l}9.31 \mathrm{E}-01 \\
3.67 \mathrm{E}-01 \\
1.53 \mathrm{E}-01 \\
4.55 \mathrm{E} \cdot 00 \\
1.22 \mathrm{E}+00\end{array}$ & $\begin{array}{l}1.50 E+00 \\
5.85 E-01 \\
2.68 E-01 \\
6.27 E+00 \\
1.89 E+00\end{array}$ & $\begin{array}{l}1.91 E+00 \\
7.47 E-01 \\
3.84 E-01 \\
8.49 E+00 \\
2.39 E \times 00\end{array}$ & $\begin{array}{l}3.26 E-01 \\
3.87 E-01 \\
6.33 E-03 \\
1.49 E+00 \\
8.13 E-08\end{array}$ & $\begin{array}{l}2.23 E * 00 \\
1.13 E * 00 \\
3.90 E-01 \\
9.98 E+00 \\
3.20 E+00\end{array}$ \\
\hline $\begin{array}{l}N P-240 \\
N P-240 M \\
P U-236 \\
P U-237 \\
P U-238\end{array}$ & $\begin{array}{r}65 \mathrm{M} \\
7.4 \mathrm{M} \\
2.851 \mathrm{Y} \\
45.3 \mathrm{O} \\
87.75 \mathrm{Y}\end{array}$ & $\begin{array}{l}1.50 E+01 \\
4.26 E+00 \\
1.49 E-03 \\
6.37 E-01 \\
1.07 E-03\end{array}$ & $\begin{array}{l}1.18 E+01 \\
3.31 E+00 \\
4.93 E-04 \\
4.88 E-01 \\
2.38 E-04\end{array}$ & $\begin{array}{l}1.43 E+01 \\
4.00 E+00 \\
7.64 E-04 \\
6.31 E-01 \\
4.27 E-04\end{array}$ & $\begin{array}{l}9.20 E+00 \\
2.62 E+00 \\
2.73 E-04 \\
3.34 E-01 \\
1.24 E-04\end{array}$ & $\begin{array}{l}1.29 E+01 \\
3.65 E+00 \\
1.50 E-03 \\
5.55 E-01 \\
1.12 E-03\end{array}$ & $\begin{array}{l}1.73 E+01 \\
4.91 E \cdot 00 \\
9.14 E-03 \\
1.12 E-01 \\
7.84 E-03\end{array}$ & $\begin{array}{l}1.95 E+00 \\
4.73 E+00 \\
0.0 \\
4.23 E-05 \\
0.0\end{array}$ & $\begin{array}{l}1.93 E \bullet 01 \\
9.65 E \bullet 00 \\
9.14 E-03 \\
7.12 E-01 \\
1.84 E-03\end{array}$ \\
\hline $\begin{array}{l}P U-239 \\
P U-240 \\
P U-241 \\
P U-242 \\
P U-243\end{array}$ & $\begin{array}{r}24131 Y \\
6537 \gamma \\
14.4 Y \\
3.75865 Y \\
4.956 \mathrm{H}\end{array}$ & $\begin{array}{l}1.02 E-03 \\
1.06 E-03 \\
0.0 \\
9.05 E-04 \\
3.16 E-01\end{array}$ & $\begin{array}{l}5.60 E-04 \\
2.55 E-04 \\
0.0 \\
2.32 E-04 \\
2.44 E-01\end{array}$ & $\begin{array}{l}7.79 E-04 \\
4.43 E-04 \\
0.0 \\
3.93 E-04 \\
3.12 E-01\end{array}$ & $\begin{array}{l}4.02 E-04 \\
1.31 E-04 \\
0.0 \\
1.22 E-04 \\
1.62 E-01\end{array}$ & $\begin{array}{l}9.74 E-04 \\
1.10 E-03 \\
0.0 \\
9.32 E-04 \\
2.71 E-01\end{array}$ & $\begin{array}{l}3.63 E-03 \\
7.51 \mathrm{IE}-03 \\
0.0 \\
6.25 E-03 \\
3.48 E-01\end{array}$ & $\begin{array}{l}0.0 \\
0.0 \\
0.0 \\
0.0 \\
8.47 E-01\end{array}$ & $\begin{array}{l}3.63 E-03 \\
7.51 E-03 \\
0.0 \\
6.25 E-03 \\
1.19 E+00\end{array}$ \\
\hline $\begin{array}{l}P U-244 \\
P U-245 \\
P U-246 \\
P M-241 \\
A M-242\end{array}$ & $\begin{array}{r}8.26 E 7 Y \\
10.51 \mathrm{H} \\
10.85 \mathrm{D} \\
432.2 \mathrm{Y} \\
16.02 \mathrm{H}\end{array}$ & $\begin{array}{l}6.41 E-04 \\
5.41 E+00 \\
1.24 E+00 \\
2.82 E-01 \\
1.82 E-01\end{array}$ & $\begin{array}{l}8.66 E-05 \\
4.19 E 000 \\
9.34 E-01 \\
2.09 E-01 \\
1.38 E-01\end{array}$ & $\begin{array}{l}1.94 E-04 \\
5.12 E+00 \\
1.18 E+00 \\
2.69 E-01 \\
1.78 E-01\end{array}$ & $\begin{array}{l}\text { 3. } 71 E-05 \\
3.28 E * 00 \\
6.95 E-01 \\
1.14 E-01 \\
9.55 E-02\end{array}$ & $\begin{array}{l}6.87 E-04 \\
4.65 E+00 \\
1.08 E \bullet 00 \\
2.33 E-01 \\
1.59 E-01\end{array}$ & $\begin{array}{l}5.28 E-03 \\
6.17 E+00 \\
1.40 E+00 \\
3.46 E-01 \\
2.15 E-01\end{array}$ & $\begin{array}{l}0.0 \\
2.02 E+00 \\
9.09 E-02 \\
1.11 E-04 \\
9.19 E-01\end{array}$ & $\begin{array}{l}5.28 E-03 \\
8.19 E+00 \\
1.49 E+00 \\
3.46 E-01 \\
1.13 E+00\end{array}$ \\
\hline $\begin{array}{l}A M-242 M \\
A M-243 \\
A M-244 \\
A M-245 \\
A M-246\end{array}$ & $\begin{array}{r}152 Y \\
7.38 E 3 Y \\
10.1 Y \\
122.4 \mathrm{M} \\
25.0 \mathrm{M}\end{array}$ & $\begin{array}{l}6.29 E-03 \\
7.18 E-01 \\
1.05 E+01 \\
4.02 E-01 \\
1.29 E+01\end{array}$ & $\begin{array}{l}2.66 E-03 \\
5.56 E-01 \\
8.25 E+00 \\
3.06 E-01 \\
1.03 E+01\end{array}$ & $\begin{array}{l}3.97 E-03 \\
7.09 E-01 \\
9.95 E+00 \\
3.90 E-01 \\
1.23 E+01\end{array}$ & $\begin{array}{l}1.69 E-03 \\
3.41 E-01 \\
6.39 E+00 \\
2.30 E-01 \\
0.03 E+00\end{array}$ & $\begin{array}{l}5.83 E-03 \\
6.09 E-01 \\
8.91 E+00 \\
3.52 E-01 \\
1.10 E+01\end{array}$ & $\begin{array}{l}2.64 E-02 \\
0.03 E-01 \\
1.21 E+01 \\
4.45 E-01 \\
1.49 E .01\end{array}$ & $\begin{array}{l}0.0 \\
6.76 E-05 \\
9.75 E-01 \\
1.80 E+00 \\
3.26 E+00\end{array}$ & $\begin{array}{l}2.64 E-02 \\
8.03 E-01 \\
1.31 E+01 \\
2.24 E+00 \\
1.02 E+01\end{array}$ \\
\hline $\begin{array}{l}C M-242 \\
C M-243 \\
C M-244 \\
C M-245 \\
C M-246\end{array}$ & $\begin{array}{r}163.2 \mathrm{D} \\
28.5 Y \\
18.11 Y \\
8.5 E 3 Y \\
4.75 E 3 Y\end{array}$ & $\begin{array}{l}1.24 E-03 \\
1.63 E+00 \\
1.05 E-03 \\
9.27 E-01 \\
8.81 E-04\end{array}$ & $\begin{array}{l}2.23 E-04 \\
1.24 E+00 \\
1.53 E-04 \\
7.10 E-01 \\
8.83 E-05\end{array}$ & $\begin{array}{l}4.54 E-04 \\
1.58 E+00 \\
3.47 E-04 \\
9.16 E-01 \\
2.49 E-04\end{array}$ & $\begin{array}{l}1.20 E-04 \\
9.30 E-01 \\
0.00 E-05 \\
5.04 E-01 \\
3.83 E-05\end{array}$ & $\begin{array}{l}1.25 E-03 \\
1.43 E-00 \\
1.07 E-03 \\
8.14 E-01 \\
9.01 E-04\end{array}$ & $\begin{array}{l}8.54 E-03 \\
1.02 E+00 \\
1.56 E-03 \\
1.03 E \otimes 00 \\
6.69 E-03\end{array}$ & $\begin{array}{l}0.0 \\
3.32 E-01 \\
0.0 \\
1.12 E-01 \\
0.0\end{array}$ & $\begin{array}{l}8.54 E-03 \\
2.15 E+00 \\
7.56 E-03 \\
1.14 E+00 \\
6.69 E-03\end{array}$ \\
\hline $\begin{array}{l}C M-247 \\
C M-248 \\
C M-249 \\
C M-250 \\
B K-249\end{array}$ & $\begin{array}{r}1.56 E 7 Y \\
3.39 E 5 Y \\
64.15 \mathrm{M} \\
6.9 E 3 \mathrm{Y} \\
320 \mathrm{D}\end{array}$ & $\begin{array}{l}4.00 E+00 \\
7.01 E-04 \\
2.41 E-01 \\
0.0 \\
0.0\end{array}$ & $\begin{array}{l}3.02 E+00 \\
1.37 E-04 \\
1.86 E-01 \\
0.0 \\
0.0\end{array}$ & $\begin{array}{l}\text { 3. } 73 E+00 \\
2.83 E-04 \\
2.26 E-01 \\
0.0 \\
0.0\end{array}$ & $\begin{array}{l}2.42 \mathrm{E}+00 \\
7.17 \mathrm{E}-05 \\
1.47 \mathrm{E}-01 \\
0.0 \\
0.0\end{array}$ & $\begin{array}{l}3.45 E+00 \\
3.84 E-04 \\
2.07 E-01 \\
0.0 \\
0.0\end{array}$ & $\begin{array}{l}4.53 E+00 \\
5.38 E-03 \\
2.77 E-01 \\
0.0 \\
0.0\end{array}$ & $\begin{array}{l}6.35 E-02 \\
0.0 \\
1.82 E \cdot 00 \\
0.0 \\
6.16 E-03\end{array}$ & $\begin{array}{l}4.60 E+00 \\
5.38 E-03 \\
2.09 E+00 \\
0.0 \\
6.16 E-03\end{array}$ \\
\hline
\end{tabular}


DOSE-RATE FACTORS FOP BODY URGANS ANU EFFECTIVE DOSE-PATE FACTOR. FOR IMMLGSION IN CONTAMIIHATLS hateH.

\begin{tabular}{|c|c|c|c|c|c|c|c|c|c|c|}
\hline NUCL IOE & HALF-LIFE & AORENALS & BLADOER & BRAIN & BREAST & HE ART $T$ & $\begin{array}{c}\text { SMALL } \\
\text { I NTESTIINE }\end{array}$ & $\begin{array}{c}\text { UPPER } \\
\text { LARGE } \\
\text { INTESTINE }\end{array}$ & $\begin{array}{c}\text { IOHER } \\
\text { LARGE } \\
\text { INIESTINE }\end{array}$ & K IONEYS \\
\hline $\begin{array}{l}B K-250 \\
B K-251 \\
C F-248 \\
C F-249 \\
C F-250\end{array}$ & 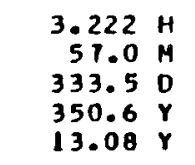 & $\begin{array}{l}8.63 E+00 \\
0.0 \\
1.47 E-04 \\
3.07 E+00 \\
3.13 E-04\end{array}$ & $\begin{array}{l}8.42 E \cdot 00 \\
0.0 \\
3.55 E-05 \\
2.86 E+00 \\
2.03 E-04\end{array}$ & $\begin{array}{l}9.49 E+00 \\
0.0 \\
4.02 E-05 \\
3.14 E+00 \\
2.22 E-04\end{array}$ & $\begin{array}{l}1.06 E+01 \\
0.0 \\
3.79 E-03 \\
4.04 E+00 \\
4.05 E-03\end{array}$ & $\begin{array}{l}8.33 E+00 \\
0.0 \\
4.55 E-05 \\
2.79 E+00 \\
2.08 E-04\end{array}$ & $\begin{array}{l}7.58 E+00 \\
0.0 \\
2.54 E-05 \\
2.51 E \cdot 00 \\
1.67 E-04\end{array}$ & $\begin{array}{l}\text { 8. 87E+00 } \\
0.0 \\
2.84 E-05 \\
2.96 E+00 \\
1.96 E-04\end{array}$ & $\begin{array}{l}8.15 E+00 \\
0.0 \\
9.20 E-05 \\
2.75 E+00 \\
2.43 E-04\end{array}$ & $\begin{array}{l}8.84 E+00 \\
0.0 \\
5.43 E-05 \\
2.96 E+00 \\
2.19 E-04\end{array}$ \\
\hline $\begin{array}{l}\text { CF-251 } \\
\text { CF-252 } \\
\text { CF-253 } \\
\text { CF-254 } \\
\text { ES-253 }\end{array}$ & 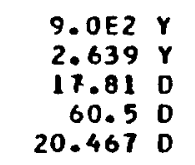 & $\begin{array}{l}1.05 E+00 \\
2.45 E-04 \\
2.56 E-06 \\
7.77 E-08 \\
2.83 E-03\end{array}$ & $\begin{array}{l}9.81 E-01 \\
1.46 E-04 \\
5.19 E-07 \\
1.09 E-07 \\
2.51 E-03\end{array}$ & $\begin{array}{l}1.04 E+00 \\
1.58 E-04 \\
8.34 E-07 \\
6.88 E-08 \\
2.69 E-03\end{array}$ & $\begin{array}{l}1.71 E+00 \\
3.10 E-03 \\
6.38 E-05 \\
2.78 E-07 \\
6.23 E-03\end{array}$ & $\begin{array}{l}9.57 E-01 \\
1.49 E-04 \\
8.99 E-01 \\
6.25 E-08 \\
2.46 E-03\end{array}$ & $\begin{array}{l}8.38 E-01 \\
1.17 E-04 \\
5.00 E-07 \\
6.65 E-08 \\
2.19 E-03\end{array}$ & $\begin{array}{l}1.02 E+00 \\
1.36 E-04 \\
5.25 E-07 \\
8.59 E-0 B \\
2.60 E-03\end{array}$ & $\begin{array}{l}9.38 E-01 \\
1.85 E-04 \\
1.64 E-06 \\
6.81 E-08 \\
2.48 E-03\end{array}$ & $\begin{array}{l}1.00 E+00 \\
1.62 \mathrm{E}-04 \\
7.95 \mathrm{E}-07 \\
1.35 \mathrm{E}-07 \\
2.59 \mathrm{E}-03\end{array}$ \\
\hline $\begin{array}{l}E S-254 \\
E S-254 M \\
E S-255 \\
F M-254 \\
F M-255\end{array}$ & $\begin{array}{r}275.7 \mathrm{D} \\
39.3 \mathrm{H} \\
39.8 \mathrm{D} \\
3.240 \mathrm{H} \\
20.07 \mathrm{H}\end{array}$ & $\begin{array}{l}2.41 E-02 \\
5.32 E+00 \\
1.21 E-05 \\
4.76 E-04 \\
1.37 E-02\end{array}$ & $\begin{array}{l}2.24 E-02 \\
5.09 E+00 \\
3.48 E-06 \\
3.32 E-04 \\
1.31 E-02\end{array}$ & $\begin{array}{l}2.30 E-02 \\
5.71 E+00 \\
3.60 E-06 \\
3.64 E-04 \\
1.32 E-02\end{array}$ & $\begin{array}{l}8.86 E-02 \\
6.59 E \cdot 00 \\
2.88 E-04 \\
4.14 E-0 ? \\
5.81 E-02\end{array}$ & $\begin{array}{l}2.08 E-02 \\
5.00 E \cdot 00 \\
4.00 E-06 \\
3.41 E-04 \\
1.20 E-02\end{array}$ & $\begin{array}{l}1.85 E-02 \\
4.51 E \cdot 00 \\
2.43 E-06 \\
2.76 E-04 \\
1.05 E-02\end{array}$ & $\begin{array}{l}2.19 \mathrm{E}-02 \\
5.32 \mathrm{E} \cdot 00 \\
2.77 \mathrm{E}-06 \\
3.31 \mathrm{E}-04 \\
1.27 \mathrm{E}-02\end{array}$ & $\begin{array}{l}2.10 E-02 \\
4.90 E+00 \\
7.75 E-06 \\
3.83 E-04 \\
1.20 E-02\end{array}$ & $\begin{array}{l}2.36 E-02 \\
5.31 E+00 \\
5.32 E-06 \\
3.58 E-04 \\
1.38 E-02\end{array}$ \\
\hline & & & & & & & & & & \\
\hline
\end{tabular}




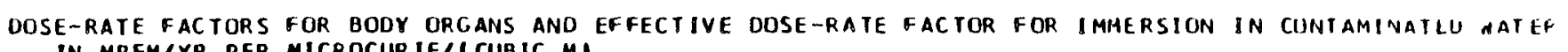

IN MREM/YR PER MICROCUR IE/ICUBIC MI

\begin{tabular}{|c|c|c|c|c|c|c|c|c|c|c|}
\hline NUCL IDE & HALF-LIFE & LIVER & LUNGS & MARROH & $\begin{array}{c}\text { RED } \\
\text { HARROW }\end{array}$ & OVARIES & PANCREAS & SKELETON & SPLEEN & S TOMACH \\
\hline $\begin{array}{l}8 K-250 \\
8 K-251 \\
C F-248 \\
C F-249 \\
C F-250\end{array}$ & $\begin{array}{r}3.222 \mathrm{H} \\
57.0 \mathrm{M} \\
333.5 \mathrm{D} \\
350.6 \mathrm{Y} \\
13.08 \mathrm{Y}\end{array}$ & $\begin{array}{l}8.38 E+00 \\
0.0 \\
4.13 E-05 \\
2.85 E+00 \\
2.13 E-04\end{array}$ & $\begin{array}{l}8.92 E+00 \\
0.0 \\
1.55 E-04 \\
3.07 E+00 \\
3.43 E-04\end{array}$ & $\begin{array}{l}9.52 E+00 \\
0.0 \\
1.93 E-04 \\
3.71 E+00 \\
5.20 E-04\end{array}$ & $\begin{array}{l}9.02 E+00 \\
0.0 \\
5.54 E-05 \\
3.15 E+00 \\
1.86 E-04\end{array}$ & $\begin{array}{l}8.10 E+00 \\
0.0 \\
9.18 E-05 \\
2.65 E+00 \\
2.33 E-04\end{array}$ & $\begin{array}{l}7.24 E+00 \\
0.0 \\
9.09 E-05 \\
2.45 E+00 \\
2.13 E-04\end{array}$ & $\begin{array}{l}9.68 E+00 \\
0.0 \\
2.60 E-04 \\
3.83 E+00 \\
5.96 E-04\end{array}$ & $\begin{array}{l}8.11 E+00 \\
0.0 \\
1.37 E-05 \\
2.80 E+00 \\
2.36 E-04\end{array}$ & $\begin{array}{l}\text { o. } 27 E+00 \\
0.0 \\
1.84 E-05 \\
2.81 E+00 \\
2.42 E-04\end{array}$ \\
\hline $\begin{array}{l}\text { CF-251 } \\
\text { CF-252 } \\
\text { CF-253 } \\
\text { CF-254 } \\
\text { ES-253 }\end{array}$ & 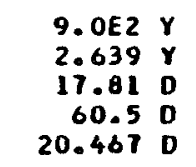 & $\begin{array}{l}9.17 E-01 \\
1.54 E-04 \\
7.54 E-07 \\
9.24 E-08 \\
2.51 E-03\end{array}$ & $\begin{array}{l}1.08 E+00 \\
2.71 E-04 \\
2.69 E-06 \\
1.02 E-07 \\
2.80 E-03\end{array}$ & $\begin{array}{l}1.62 E+00 \\
4.05 E-04 \\
3.38 E-06 \\
1.84 E-07 \\
3.86 E-03\end{array}$ & $\begin{array}{l}9.75 E-01 \\
1.33 E-04 \\
1.04 E-06 \\
4.44 E-08 \\
2.75 E-03\end{array}$ & $\begin{array}{l}8.50 E-01 \\
1.80 E-04 \\
1.60 E-06 \\
7.43 E-08 \\
2.24 E-03\end{array}$ & & & & $\begin{array}{l}9.64 \mathrm{E}-01 \\
1.82 \mathrm{E}-04 \\
1.40 \mathrm{E}-0 \mathrm{0} \\
8.02 \mathrm{E}-08 \\
2.51 \mathrm{E}-03\end{array}$ \\
\hline $\begin{array}{l}\text { ES-254 } \\
\text { ES-254M } \\
\text { ES- } 255 \\
F M-254 \\
F M-255\end{array}$ & $\begin{array}{r}275.1 \mathrm{O} \\
39.3 \mathrm{H} \\
39.8 \mathrm{O} \\
3.240 \mathrm{H} \\
20.07 \mathrm{H}\end{array}$ & $\begin{array}{l}2.23 E-02 \\
5.06 E+00 \\
3.90 E-06 \\
3.47 E-04 \\
1.31 E-02\end{array}$ & $\begin{array}{l}2.59 E-02 \\
5.42 E+00 \\
1.30 E-05 \\
5.19 E-04 \\
1.55 E-02\end{array}$ & $\begin{array}{l}3.89 E-02 \\
6.03 E-00 \\
1.67 E-05 \\
8.02 E-04 \\
2.60 E-02\end{array}$ & $\begin{array}{l}2.03 E-02 \\
5.52 E+00 \\
4.67 E-06 \\
3.09 E-04 \\
1.01 E-02\end{array}$ & $\begin{array}{l}2.00 E-02 \\
4.91 E+00 \\
7.81 E-06 \\
3.63 E-04 \\
1.14 E-02\end{array}$ & $\begin{array}{l}1.79 E-02 \\
4.36 E+00 \\
7.49 E-06 \\
3.30 E-04 \\
9.79 E-03\end{array}$ & & & $\begin{array}{l}2.19 \mathrm{E}-02 \\
5.01 \mathrm{E}+00 \\
6.80 \mathrm{E}-06 \\
3.85 \mathrm{E}-04 \\
1.28 \mathrm{E}-02\end{array}$ \\
\hline $1-256$ & $57.6 \mathrm{M}$ & 0.0 & 0.0 & 0.0 & 0.0 & .0 & 0.0 & .0 & .0 & 0.0 \\
\hline
\end{tabular}


DOSE-RATE FACTORS FOR BODY ORGANS AND EFFECTIVE DOSE-RATE FACTOR FOP IMMERSION IN CONTAMIIIATLO WATEM
IN MREM/YR PER MICROCURIE/ICUOIC MI

\begin{tabular}{|c|c|c|c|c|c|c|c|c|c|}
\hline NUCLIDE & HALF-LIFE & TESTES & THYMUS & THYROID & UTERUS & EFFECTIVE & $\begin{array}{c}\text { SKIN } \\
\text { IPHOIONI }\end{array}$ & $\begin{array}{l}\text { SKIN } \\
\text { IELECTRONI }\end{array}$ & $\begin{array}{l}\text { SKIN } \\
\text { ITOTALI }\end{array}$ \\
\hline $\begin{array}{l}8 K-250 \\
8 K-251 \\
C F-248 \\
C F-249 \\
C F-250\end{array}$ & $\begin{array}{r}3.222 \mathrm{H} \\
57.0 \mathrm{M} \\
333.5 \mathrm{O} \\
350.6 \mathrm{~V} \\
13.08 \mathrm{Y}\end{array}$ & $\begin{array}{l}1.18 E+01 \\
0.0 \\
8.19 E-04 \\
4.15 E+00 \\
1.14 E-03\end{array}$ & $\begin{array}{l}9.44 E+00 \\
0.0 \\
4.86 E-05 \\
3.13 E+00 \\
2.66 E-04\end{array}$ & $\begin{array}{l}1.13 E \cdot 01 \\
0.0 \\
2.24 E-04 \\
3.87 E+00 \\
4.96 E-04\end{array}$ & $\begin{array}{l}7.32 E+00 \\
0.0 \\
1.95 E-05 \\
2.50 E+00 \\
1.64 E-04\end{array}$ & $\begin{array}{l}1.01 E+01 \\
0.0 \\
8.64 E-04 \\
3.59 E+00 \\
1.08 E-03\end{array}$ & $\begin{array}{l}1.36 E \cdot 01 \\
0.0 \\
6.26 E-03 \\
4.70 E \cdot 00 \\
6.45 E-03\end{array}$ & $\begin{array}{l}1.0 B E+00 \\
2.61 E+00 \\
0.0 \\
7.47 E-02 \\
1.04 E-05\end{array}$ & $\begin{array}{l}1.55 E+01 \\
2.61 E+00 \\
6.26 E-03 \\
4.7 \theta E+00 \\
6.46 E-03\end{array}$ \\
\hline $\begin{array}{l}\text { CF-251 } \\
\text { CF-252 } \\
\text { CF-253 } \\
\text { CF-254 } \\
\text { ES-253 }\end{array}$ & 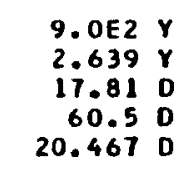 & $\begin{array}{l}1.51 E+00 \\
9.94 E-04 \\
1.47 E-05 \\
2.12 E-07 \\
4.26 E-03\end{array}$ & $\begin{array}{l}1.16 E+00 \\
1.94 E-04 \\
8.12 E-07 \\
1.42 E-07 \\
2.86 E-03\end{array}$ & $\begin{array}{l}1.48 E+00 \\
3.95 E-04 \\
3.75 E-06 \\
1.85 E-07 \\
3.72 E-03\end{array}$ & $\begin{array}{l}8.52 E-01 \\
1.13 E-04 \\
4.19 E-07 \\
6.09 E-08 \\
2.21 E-03\end{array}$ & $\begin{array}{l}1.33 E+00 \\
9.51 E-04 \\
1.46 E-05 \\
1.63 E-07 \\
3.79 E-03\end{array}$ & $\begin{array}{l}1.67 E+00 \\
5.95 E-03 \\
1.06 E-04 \\
2.74 E-07 \\
7.82 E-03\end{array}$ & $\begin{array}{l}5.46 E-01 \\
1.16 E-05 \\
2.02 E-01 \\
0.0 \\
0.0\end{array}$ & $\begin{array}{l}2.22 E \cdot 00 \\
5.96 E-03 \\
2.02 E-01 \\
2.74 E-07 \\
7.82 E-03\end{array}$ \\
\hline $\begin{array}{l}E S-254 \\
E S-254 M \\
E S-255 \\
F M-254 \\
F H-255\end{array}$ & $\begin{array}{r}275.7 \mathrm{D} \\
39.3 \mathrm{H} \\
39.8 \mathrm{O} \\
3.240 \mathrm{H} \\
20.07 \mathrm{H}\end{array}$ & $\begin{array}{l}4.68 E-02 \\
7.23 E+00 \\
7.06 E-05 \\
1.53 E-03 \\
2.96 E-02\end{array}$ & $\begin{array}{l}2.71 E-02 \\
5.62 E+00 \\
4.74 E-06 \\
4.26 E-04 \\
1.60 E-02\end{array}$ & $\begin{array}{l}3.66 E-02 \\
6.80 E+00 \\
1.93 E-05 \\
1.61 E-04 \\
2.33 E-02\end{array}$ & $\begin{array}{l}1.04 E-02 \\
4.40 E+00 \\
1.88 E-06 \\
2.73 E-04 \\
1.05 E-02\end{array}$ & $\begin{array}{l}4.11 E-02 \\
6.17 E+00 \\
6.73 E-05 \\
1.39 E-03 \\
2.57 E-02\end{array}$ & $\begin{array}{l}1.20 E-01 \\
8.36 E+00 \\
4.69 E-04 \\
7.23 E-03 \\
7.58 E-02\end{array}$ & $\begin{array}{l}4.84 E-05 \\
1.01 E+00 \\
1.73 E-01 \\
1.94 E-04 \\
1.48 E-03\end{array}$ & $\begin{array}{l}1.20 E-01 \\
9.37 E \cdot 00 \\
1.74 E-01 \\
7.43 E-03 \\
7.73 E-02\end{array}$ \\
\hline $1-256$ & $157.6 \mathrm{M}$ & 0.0 & 0.0 & 0.0 & 0.0 & 0.0 & 0.0 & 0.0 & 0.0 \\
\hline
\end{tabular}




\section{A.3 Dose-Rate Factors for Exposure to a \\ Contaminated Ground Surface}

The following tables give the dose-rate conversion factors for external exposure to photons and electrons from an infinite, uniformiy contaminated ground surface. Exposure is assumed to occur at a height of $1 \mathrm{~m}$ above ground. Dose-reduction factors which can be applied to these results to take into account external exposure to a contaminated ground surface during indoor residence, the effects of ground roughness on external dose equivalents, and external exposure to contaminated shorelines are discussed in Sections 3.2.1, 3.2.2, and 3.2.4, respectively. Dose-rate factors for external exposure to radionuclides that are distributed with depth in soil are discussed in Section 3.3. 
DOSE-RATE FACTORS FOR BOOY ORgans AND EFFECTIVE DOSE-RATE FACTOR FOR EXPOSURE 1 M above CIJNTAYIVATED jROUNU SJRF ACF IN MREMTYR PI

\begin{tabular}{|c|c|c|c|c|c|c|c|c|c|c|}
\hline NUCL IOE & HALF-LIFE & AORENALS & BL AODER & BRAIN & BREAST & HEART & $\begin{array}{l}\text { SMALL } \\
\text { INTESTINE }\end{array}$ & $\begin{array}{c}\text { UPPER } \\
\text { LARGE } \\
\text { INTESTJNE }\end{array}$ & $\begin{array}{c}\text { LOWER } \\
\text { LARGE } \\
\text { INTESTINE }\end{array}$ & KIONEYS \\
\hline $\begin{array}{l}H-3 \\
B E-7 \\
B E-10 \\
C-11 \\
C-14\end{array}$ & 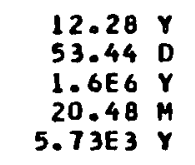 & $\begin{array}{l}0.0 \\
4.44 E+00 \\
0.0 \\
9.05 E+01 \\
0.0\end{array}$ & $\begin{array}{l}0.0 \\
4.20 E \cdot 00 \\
0.0 \\
8.59 E+01 \\
0.0\end{array}$ & $\begin{array}{l}0.0 \\
4.67 E \cdot 00 \\
0.0 \\
9.57 E \cdot 01 \\
0.0\end{array}$ & $\begin{array}{l}0.0 \\
5.63 E \cdot 00 \\
0.0 \\
1.14 E+02 \\
0.0\end{array}$ & $\begin{array}{l}0.0 \\
4.10 E+00 \\
0.0 \\
8.40 E \cdot 01 \\
0.0\end{array}$ & $\begin{array}{l}0.0 \\
3.69 E+00 \\
0.0 \\
7.55 E \cdot 01 \\
0.0\end{array}$ & $\begin{array}{l}0.0 \\
4.35 E+00 \\
0.0 \\
8.91 E+01 \\
0.0\end{array}$ & $\begin{array}{l}0.0 \\
4.03 E+00 \\
0.0 \\
8.23 E+01 \\
0.0\end{array}$ & $\begin{array}{l}0.0 \\
4.35 E+00 \\
0.0 \\
8.91 E+01 \\
0.0\end{array}$ \\
\hline $\begin{array}{l}N-13 \\
N-16 \\
0-15 \\
f-18 \\
N A-22\end{array}$ & $\begin{array}{r}9.97 \mathrm{M} \\
7.13 \mathrm{~S} \\
122.24 \mathrm{~S} \\
109.74 \mathrm{M} \\
2.602 \mathrm{Y}\end{array}$ & $\begin{array}{l}9.05 E+01 \\
2.711 E+02 \\
9.06 E+01 \\
8.77 E+01 \\
1.79 E+02\end{array}$ & $\begin{array}{l}8.60 E \bullet 01 \\
2.75 E \cdot 02 \\
8.60 E+01 \\
8.33 E+01 \\
1.74 E+02\end{array}$ & $\begin{array}{l}9.58 E+01 \\
2.91 E+02 \\
9.59 E+01 \\
9.28 E+01 \\
1.95 E+02\end{array}$ & $\begin{array}{l}1.14 E+02 \\
3.50 E+02 \\
1.14 E+02 \\
1.11 E+02 \\
2.23 E+02\end{array}$ & $\begin{array}{l}8.40 E+01 \\
2.70 E+02 \\
0.41 E+01 \\
8.14 E+01 \\
1.71 E+02\end{array}$ & $\begin{array}{l}7.56 E+01 \\
2.59 E+02 \\
7.56 E+01 \\
7.32 E+01 \\
1.56 E+02\end{array}$ & $\begin{array}{l}8.91 E+01 \\
2.89 E+02 \\
8.92 E+01 \\
8.64 E+01 \\
1.82 E+02\end{array}$ & $\begin{array}{l}8.23 E+01 \\
2.71 E+02 \\
8.24 E+01 \\
7.98 E+01 \\
1.68 E+02\end{array}$ & $\begin{array}{l}\text { 8. } 91 E+01 \\
3.07 E+02 \\
8.92 E+01 \\
8.64 E+01 \\
1.82 E+02\end{array}$ \\
\hline $\begin{array}{l}N A-24 \\
M G-27 \\
M G-28 \\
A L-26 \\
A L-28\end{array}$ & $\begin{array}{l}15.00 \mathrm{H} \\
9.458 \mathrm{H} \\
20.91 \mathrm{H} \\
7.2 \mathrm{H} \mathrm{Y} \\
2.240 \mathrm{H}\end{array}$ & $\begin{array}{l}2.94 E+02 \\
7.63 E+01 \\
1.08 E+02 \\
2.10 E+02 \\
1.31 E+02\end{array}$ & $\begin{array}{l}3.01 E+02 \\
7.39 E \bullet 01 \\
1.06 E \bullet 02 \\
2.10 E \bullet 02 \\
1.34 E+02\end{array}$ & $\begin{array}{l}3.35 E+02 \\
8.32 E+01 \\
1.19 E+02 \\
2.35 E+02 \\
1.50 E+02\end{array}$ & $\begin{array}{l}3.78 E+02 \\
5.31 E \cdot 01 \\
1.38 E \cdot 02 \\
2.66 E \cdot 02 \\
1.67 E+02\end{array}$ & $\begin{array}{l}3.00 E+02 \\
7.30 E+01 \\
1.05 E+02 \\
2.07 E+02 \\
1.34 E+02\end{array}$ & $\begin{array}{l}2.61 E+02 \\
6.63 E+01 \\
9.60 E+01 \\
1.91 E+02 \\
1.24 E+02\end{array}$ & $\begin{array}{l}3.19 E+02 \\
7.77 E+01 \\
1.12 E+02 \\
2.21 E+02 \\
1.42 E+02\end{array}$ & $\begin{array}{l}2.96 E+02 \\
7.14 E+01 \\
1.03 E+02 \\
2.04 E+02 \\
1.31 E+02\end{array}$ & $\begin{array}{l}\text { 3. } 24 E+02 \\
7.75 E+01 \\
1.12 E+02 \\
2.21 E+02 \\
1.43 E+02\end{array}$ \\
\hline $\begin{array}{l}s 1-31 \\
S 1-32 \\
p-32 \\
p-33 \\
s-35\end{array}$ & $\begin{array}{rl}157.3 & M \\
3.3 E 2 & Y \\
14.29 & D \\
25.4 & D \\
87.44 & D\end{array}$ & $\begin{array}{l}0.77 E-02 \\
0.0 \\
0.0 \\
0.0 \\
0.0\end{array}$ & $\begin{array}{l}6.73 E-02 \\
0.0 \\
0.0 \\
0.0 \\
0.0\end{array}$ & $\begin{array}{l}7.60 E-02 \\
0.0 \\
0.0 \\
0.0 \\
0.0\end{array}$ & $\begin{array}{l}8.39 E-02 \\
0.0 \\
0.0 \\
0.0 \\
0.0\end{array}$ & $\begin{array}{l}6.66 E-02 \\
0.0 \\
0.0 \\
0.0 \\
0.0\end{array}$ & $\begin{array}{l}6.14 E-02 \\
0.0 \\
0.0 \\
0.0 \\
0.0\end{array}$ & $\begin{array}{l}7.10 E-02 \\
0.0 \\
0.0 \\
0.0\end{array}$ & $\begin{array}{l}6.54 E-02 \\
0.0 \\
0.0 \\
0.0 \\
0.0\end{array}$ & $\begin{array}{l}7.08 E-02 \\
0.0 \\
0.0 \\
0.0 \\
0.0\end{array}$ \\
\hline $\begin{array}{l}C L-36 \\
C L-38 \\
A R-37 \\
A R-39 \\
A R-41\end{array}$ & $\begin{array}{r}3.01 E 5 Y \\
37.21 \mathrm{M} \\
35.02 \mathrm{O} \\
269 \mathrm{Y} \\
1.827 \mathrm{H}\end{array}$ & $\begin{array}{l}3.36 E-07 \\
1.09 E+02 \\
5.66 E-05 \\
0.0 \\
9.74 E+01\end{array}$ & $\begin{array}{l}6.05 E-14 \\
1.12 E+02 \\
1.02 E-11 \\
0.0 \\
9.70 E+01\end{array}$ & $\begin{array}{l}2.67 E-08 \\
1.26 E+02 \\
4.52 E-06 \\
0.0 \\
1.09 E \bullet 02\end{array}$ & $\begin{array}{l}2.63 E-05 \\
1.40 E+02 \\
4.44 E-03 \\
0.0 \\
1.21 E \cdot 02\end{array}$ & $\begin{array}{l}4.37 E-08 \\
1.12 E+02 \\
7.37 E-06 \\
0.0 \\
9.60 E+01\end{array}$ & $\begin{array}{l}5.63 E-10 \\
1.05 E+02 \\
9.51 E-08 \\
0.0 \\
8.86 E+01\end{array}$ & $\begin{array}{l}8.29 E-10 \\
1.19 E+02 \\
1.40 E-07 \\
0.0 \\
1.02 E+02\end{array}$ & $\begin{array}{l}1.38 E-07 \\
1.10 E+02 \\
2.32 E-05 \\
0.0 \\
9.42 E+01\end{array}$ & $\begin{array}{l}4.02 E-10 \\
1.20 E+02 \\
6.79 E-08 \\
0.0 \\
1.02 E+02\end{array}$ \\
\hline $\begin{array}{l}k-40 \\
k-42 \\
k-43 \\
C A-41 \\
C A-45\end{array}$ & $\begin{array}{r}1.277 E 9 Y \\
12.36 \mathrm{H} \\
22.6 \mathrm{H} \\
1.03 E 5 \mathrm{Y} \\
162.7 \mathrm{O}\end{array}$ & $\begin{array}{l}1.15 E+01 \\
2.02 E+01 \\
8.68 E+01 \\
1.48 E-04 \\
2.30 E-09\end{array}$ & $\begin{array}{l}1.15 E \bullet 01 \\
2.04 E \bullet 01 \\
8.21 E \bullet 01 \\
2.67 E-11 \\
2.12 E-13\end{array}$ & & $\begin{array}{l}1.44 E \cdot 01 \\
2.54 E \cdot 01 \\
1.10 E \cdot 02 \\
1.16 E-02 \\
1.10 E-01\end{array}$ & $\begin{array}{l}1.14 E+01 \\
2.02 E+01 \\
8.04 E+01 \\
1.93 E-05 \\
3.75 E-10\end{array}$ & & & & $\begin{array}{l}1.22 E+01 \\
2.16 E+01 \\
8.52 E+01 \\
1.78 E-07 \\
2.86 E-11\end{array}$ \\
\hline $\begin{array}{l}C A-47 \\
C A-49 \\
\text { SC }-44 \\
S C-46 \\
S C-46.4\end{array}$ & 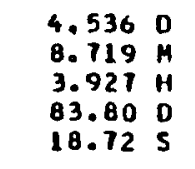 & $\begin{array}{l}0.17 E+01 \\
2.12 E+02 \\
1.18 E+02 \\
1.66 E+02 \\
8.40 E+00\end{array}$ & $\begin{array}{l}8.11 E+01 \\
2.19 E+02 \\
1.73 E+02 \\
1.62 E+02 \\
7.74 E+00\end{array}$ & $\begin{array}{l}9.14 E+01 \\
2.41 E+02 \\
1.93 E+02 \\
1.83 E+02 \\
8.29 E+00\end{array}$ & $\begin{array}{l}1.01 E * 02 \\
2.78 E \bullet 02 \\
2.22 E \bullet 02 \\
2.03 E+02 \\
1.33 E \bullet 01\end{array}$ & $\begin{array}{l}8.02 E+01 \\
2.19 E+02 \\
1.70 E+02 \\
1.60 E+02 \\
7.64 E+00\end{array}$ & $\begin{array}{l}7.38 E+01 \\
2.07 E+02 \\
1.54 E+02 \\
1.46 E+02 \\
6.65 E+00\end{array}$ & $\begin{array}{l}8.54 E+01 \\
2.33 E+02 \\
1.80 E+02 \\
1.71 E+02 \\
8.15 E+00\end{array}$ & $\begin{array}{l}7.86 E+01 \\
2.17 E+02 \\
1.66 E+02 \\
1.57 E+02 \\
7.47 E+00\end{array}$ & $\begin{array}{l}\text { 8. } 52 E+01 \\
2.40 E+02 \\
1.80 E+02 \\
1.70 E+02 \\
\text { 7. } 86 E+00\end{array}$ \\
\hline $\begin{array}{l}S C-47 \\
\text { SC-48 } \\
\text { SC-49 } \\
T I-44 \\
T I-45\end{array}$ & $\begin{array}{r}3.422 \mathrm{D} \\
43.67 \mathrm{H} \\
57.4 \mathrm{M} \\
47.3 \mathrm{Y} \\
3.08 \mathrm{H}\end{array}$ & $\begin{array}{l}1.02 E+01 \\
2.69 E+02 \\
7.67 E-02 \\
1.12 E+01 \\
7.72 E+01\end{array}$ & $\begin{array}{l}9.38 E+00 \\
2.64 E+02 \\
7.84 E-02 \\
1.19 E+01 \\
7.33 E+01\end{array}$ & $\begin{array}{l}1.01 E+01 \\
2.98 E+02 \\
8.00 E-02 \\
1.18 E+01 \\
8.17 E+01\end{array}$ & $\begin{array}{l}1.57 E+01 \\
3.31 E+02 \\
5.74 E-02 \\
2.39 E+01 \\
9.73 E+01\end{array}$ & $\begin{array}{l}9.23 E+00 \\
2.62 E+02 \\
7.60 E-02 \\
1.06 E+01 \\
7.17 E+01\end{array}$ & $\begin{array}{l}8.10 E+00 \\
2.39 E \cdot 02 \\
7.25 E-02 \\
9.45 E+00 \\
6.45 E \bullet 01\end{array}$ & $\begin{array}{l}9.83 E+00 \\
2.79 E+02 \\
8.30 E-02 \\
1.13 E+01 \\
7.61 E+01\end{array}$ & $\begin{array}{l}9.09 E+00 \\
2.56 E+02 \\
7.66 E-02 \\
1.01 E+01 \\
7.03 E+01\end{array}$ & $\begin{array}{l}9.55 E+00 \\
2.78 E+02 \\
8.32 E-02 \\
1.23 E+01 \\
7.61 E+01\end{array}$ \\
\hline
\end{tabular}




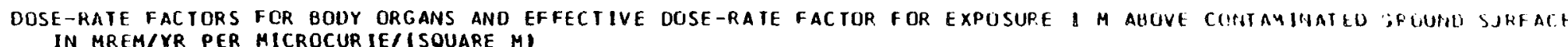

\begin{tabular}{|c|c|c|c|c|c|c|c|c|c|c|}
\hline NUCLIDE & HALF-LIFE & CIVER & LUNGS & MARROH & $\begin{array}{c}\text { REO } \\
\text { MARROH }\end{array}$ & OVARIES & ANCREAS & KELETON & SPLEEN & STOMACH \\
\hline $\begin{array}{l}H-3 \\
B E-7 \\
B E-10 \\
C-11 \\
C-14\end{array}$ & $\begin{array}{r}12.28 Y \\
53.44 \\
1.6 E 6 Y \\
20.48 \mathrm{Y} \\
5.73 E 3 \mathrm{Y}\end{array}$ & $\begin{array}{l}0.0 \\
4.18 E+00 \\
0.0 \\
8.54 E \cdot 01 \\
0.0\end{array}$ & $\begin{array}{l}.0 \\
.49 E+00 \\
.0 \\
.17 E+01 \\
.0\end{array}$ & $\begin{array}{l}0.0 \\
5.22 E+00 \\
0.0 \\
1.06 E \cdot 02 \\
0.0\end{array}$ & $\begin{array}{l}0.0 \\
4.60 E \bullet 00 \\
0.0 \\
9.38 E \bullet 01 \\
0.0\end{array}$ & $\begin{array}{l}0.0 \\
3.99 E+00 \\
0.0 \\
8.20 E+01 \\
0.0\end{array}$ & $\begin{array}{l}0.0 \\
3.60 E \times 00 \\
0.0 \\
7.35 E \cdot 01 \\
0.0\end{array}$ & $\begin{array}{l}0.0 \\
5.31 E+00 \\
0.0 \\
1.07 E+02 \\
0.0\end{array}$ & $\begin{array}{l}0.0 \\
4.09 E \cdot 00 \\
0.0 \\
8.34 E+01 \\
0.0\end{array}$ & $\begin{array}{l}0.0 \\
4.12 E \cdot 00 \\
0.0 \\
8.43 E+01 \\
0.0\end{array}$ \\
\hline $\begin{array}{l}N-13 \\
N-16 \\
0-15 \\
F-18 \\
N A-22\end{array}$ & $\begin{array}{r}9.97 \mathrm{M} \\
7.13 \mathrm{~S} \\
122.24 \mathrm{~S} \\
109.74 \mathrm{M} \\
2.602 \mathrm{~V}\end{array}$ & $\begin{array}{l}8.55 E+01 \\
2.73 E+02 \\
8.55 E+01 \\
8.28 E+01 \\
1.73 E+02\end{array}$ & $\begin{array}{l}9.17 E+01 \\
2.81 E+02 \\
9.18 E+01 \\
8.89 E+01 \\
1.85 E+02\end{array}$ & & $\begin{array}{l}39 E+01 \\
05 E+02 \\
39 E+01 \\
10 E+01 \\
01 E+02\end{array}$ & & $\begin{array}{l}.35 E+01 \\
.52 E+02 \\
.36 E+01 \\
133 E+01 \\
.50 E+02\end{array}$ & $\begin{array}{l}.07 E+02 \\
2.98 E+02 \\
1.07 E+02 \\
1.04 E+02 \\
2.06 E+02\end{array}$ & $\begin{array}{l}8.34 E+01 \\
2.67 E+02 \\
8.35 E+01 \\
8.09 E+01 \\
1.68 E+02\end{array}$ & $\begin{array}{l}0.43 E+01 \\
2.79 E+02 \\
0.44 E+01 \\
8.17 E+01 \\
1.70 E+02\end{array}$ \\
\hline $\begin{array}{l}M A-24 \\
M G-27 \\
M G-28 \\
A L-26 \\
A L-28\end{array}$ & $\begin{array}{l}15.00 \mathrm{H} \\
9.458 \mathrm{H} \\
20.91 \mathrm{H} \\
7.265 \\
2.240 \mathrm{H}\end{array}$ & $\begin{array}{l}3.02 E+02 \\
7.35 E * 01 \\
1.06 E+02 \\
2.10 E+02\end{array}$ & $\begin{array}{l}3.16 E+02 \\
1.84 E+01 \\
1.13 E+02 \\
2.22 E+02 \\
1.42 E+02\end{array}$ & $\begin{array}{l}3.26 E+02 \\
8.45 E+01 \\
1.21 E+02 \\
2.38 E+02 \\
1.46 E+02\end{array}$ & & $\begin{array}{l}2.13 E+02 \\
1.13 E+01 \\
1.01 E+02 \\
1.95 E+02 \\
1.24 E+02\end{array}$ & $\begin{array}{l}.65 E+02 \\
0.35 E+01 \\
9.14 E+01 \\
882 E+02 \\
.17 E+02\end{array}$ & 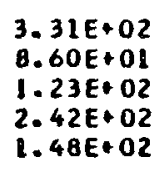 & & $\begin{array}{l}2.98 E+02 \\
7.27 E+01 \\
1.04 E+02 \\
2.05 E+02 \\
1.31 E+02\end{array}$ \\
\hline $\begin{array}{l}S I-31 \\
S I-32 \\
p-32 \\
p-33 \\
S-35\end{array}$ & 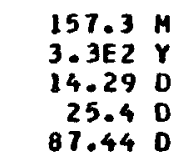 & $\begin{array}{l}0.72 E-02 \\
0.0 \\
0.0 \\
0.0 \\
0.0\end{array}$ & $\begin{array}{l}11 E-02 \\
0 \\
0 \\
0 \\
0\end{array}$ & $\begin{array}{l}7.4 B E-02 \\
0.0 \\
0.0 \\
0.0 \\
0.0\end{array}$ & $\begin{array}{l}7.18 E-02 \\
0.0 \\
0.0 \\
0.0 \\
0.0\end{array}$ & $\begin{array}{l}6.40 E-02 \\
0.0 \\
0.0 \\
0.0 \\
0.0\end{array}$ & $\begin{array}{l}5.82 E-02 \\
0.0 \\
0.0 \\
0.0 \\
0.0\end{array}$ & $\begin{array}{l}7.61 E-02 \\
0.0 \\
0.0 \\
0.0 \\
0.0\end{array}$ & $\begin{array}{l}6.49 E-02 \\
0.0 \\
0.0 \\
0.0 \\
0.0\end{array}$ & $\begin{array}{l}6.57 E-02 \\
0.0 \\
0.0 \\
0.0 \\
0.0\end{array}$ \\
\hline $\begin{array}{l}C L-36 \\
C L-38 \\
A R-37 \\
A R-39 \\
A R-41\end{array}$ & 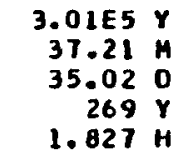 & $\begin{array}{l}3.26 E-09 \\
1.13 E+02 \\
5.50 E-07 \\
0.0 \\
9.68 E+01\end{array}$ & $\begin{array}{l}2.65 E-07 \\
1.19 E+02 \\
4.4 B E-05 \\
0.0 \\
1.02 E+02\end{array}$ & $\begin{array}{l}1.59 E-07 \\
1.22 E+02 \\
2.69 E-05 \\
0.0 \\
1.08 E+02\end{array}$ & $\begin{array}{l}\text { E. } 70 E-08 \\
1.20 E+02 \\
1.47 E-05 \\
0.0 \\
1.03 E+02\end{array}$ & $\begin{array}{l}1.31 E-07 \\
1.03 E+02 \\
2.22 E-05 \\
0.0 \\
9.20 E+01\end{array}$ & $\begin{array}{l}1.96 E-07 \\
9.84 E+01 \\
3.31 E-05 \\
0.0 \\
8.39 E+01\end{array}$ & $\begin{array}{l}\text { 4. } 30 E-07 \\
1.24 E+02 \\
7.27 E-05 \\
0.0 \\
1.09 E+02\end{array}$ & $\begin{array}{l}1.57 E-08 \\
1.08 E+02 \\
1.28 E-05 \\
0.0 \\
9.35 E+01\end{array}$ & $\begin{array}{l}6.84 E-08 \\
1.10 E+02 \\
1.15 E-05 \\
0.0 \\
9.46 E+01\end{array}$ \\
\hline $\begin{array}{l}K-40 \\
K-42 \\
K-43 \\
C A-41 \\
C A-45\end{array}$ & $\begin{array}{r}1.277 E 9 Y \\
12.36 \mathrm{H} \\
22.6 \mathrm{H} \\
1.03 E 5 \mathrm{Y} \\
162.10\end{array}$ & $\begin{array}{l}1.15 E+01 \\
2.04 E+01 \\
0.16 E+01 \\
1.44 E-06 \\
1.88 E-11\end{array}$ & $\begin{array}{l}1.22 E+01 \\
2.16 E+01 \\
0.77 E+01 \\
1.17 E-04 \\
2.08 E-09\end{array}$ & & $\begin{array}{l}1.23 E+01 \\
2.17 E+01 \\
8.99 E+01 \\
3.85 E-05 \\
6.95 E-10\end{array}$ & $\begin{array}{l}1.08 E+01 \\
1.91 E+01 \\
7.80 E+01 \\
5.81 E-05 \\
1.07 E-09\end{array}$ & & & & $\begin{array}{l}1.12 E+01 \\
1.99 E+01 \\
8.07 E+01 \\
3.02 E-05 \\
1.12 E-10\end{array}$ \\
\hline $\begin{array}{l}C A-47 \\
C A-49 \\
\text { SC-44 } \\
\text { SC-46 } \\
\text { SC-46M }\end{array}$ & $\begin{array}{l}4.5360 \\
8.719 \\
3.927 \\
83.80 \\
18.72 \\
18.75\end{array}$ & $\begin{array}{l}8.08 E \bullet 01 \\
2.20 E+02 \\
1.72 E+02 \\
1.61 E \bullet 02 \\
7.73 E \cdot 00\end{array}$ & $\begin{array}{l}2.29 E+02 \\
1.83 E+02 \\
1.72 E+02 \\
8.51 E+00\end{array}$ & $\begin{array}{l}2.35 E+C 2 \\
2.02 E+02 \\
1.83 E+02 \\
1.29 E \cdot 01\end{array}$ & $\begin{array}{l}2.37 E+02 \\
1.86 E+02 \\
1.74 E+02 \\
1.80 E+00\end{array}$ & $\begin{array}{l}\text { 7. } 70 E+01 \\
1.94 E+02 \\
1.65 E+02 \\
1.56 E+02 \\
6.69 E+00\end{array}$ & & & & $\begin{array}{l}\text { 7. } 91 E+01 \\
2.20 E * 02 \\
1.69 E * 02 \\
1.59 E * 02 \\
7.65 E * 00\end{array}$ \\
\hline $\begin{array}{l}S C-47 \\
S C-48 \\
S C-49 \\
T I-44 \\
J I-45\end{array}$ & $\begin{aligned} & 3.242 \\
& 43.67 \mathrm{H} \\
& 57.4 \mathrm{H} \\
& 47.3 \mathrm{Y} \\
& 3.08 \mathrm{H}\end{aligned}$ & $\begin{array}{l}2.63 E+02 \\
7.86 E-02 \\
1.18 E+01 \\
7.29 E+01\end{array}$ & $\begin{array}{l}2.80 E \cdot 02 \\
8.28 E-02 \\
1.30 E+01 \\
7.83 E+01\end{array}$ & $\begin{array}{l}2.97 \mathrm{E}+02 \\
8.54 \mathrm{E}-02 \\
2.28 \mathrm{E} * 01 \\
9.01 \mathrm{E} * 01\end{array}$ & $\begin{array}{l}2.83 E+02 \\
8.35 E-02 \\
8.28 E+00 \\
8.01 E * 01\end{array}$ & $\begin{array}{l}2.53 E+02 \\
7.24 E-02 \\
9.70 E+00 \\
7.00 E+01\end{array}$ & $\begin{array}{l}7.87 E+00 \\
2.28 E \div 02 \\
6.83 E-02 \\
8.00 E * 00 \\
6.27 E * 01\end{array}$ & $\begin{array}{l}1.56 E+01 \\
3.02 E+02 \\
8.69 E-02 \\
2.34 E+01 \\
9.15 E+01\end{array}$ & $\begin{array}{l}2.55 E+02 \\
7.55 E-02 \\
1.08 E+01 \\
7.12 E+01\end{array}$ & $\begin{array}{l}9.27 E+00 \\
2.59 E+02 \\
7.65 \mathrm{E}-02 \\
1.11 \mathrm{E}+01 \\
7.19 \mathrm{E}+01\end{array}$ \\
\hline
\end{tabular}




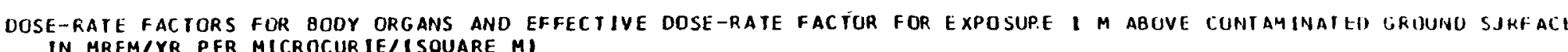

\begin{tabular}{|c|c|c|c|c|c|c|c|c|c|}
\hline NUCL IDE & HALF-LIFE & TESTES & THYMUS & THY ROIO & UTERUS & EFFECTIVE & $\begin{array}{c}\text { SKIN } \\
\text { ( PHOTONI }\end{array}$ & $\begin{array}{l}\text { SKIN } \\
\text { (ELECTRON) }\end{array}$ & $\begin{array}{l}\text { SKIN } \\
\text { I TOTALI }\end{array}$ \\
\hline $\begin{array}{l}H-3 \\
\theta E-7 \\
B E-10 \\
C-11 \\
C-14\end{array}$ & 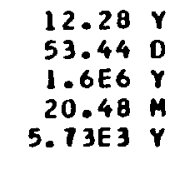 & $\begin{array}{l}0.0 \\
6.03 E+00 \\
0.0 \\
1.23 E+02 \\
0.0\end{array}$ & $\begin{array}{l}0.0 \\
4.55 E+00 \\
0.0 \\
9.31 E \cdot 01 \\
0.0\end{array}$ & $\begin{array}{l}0.0 \\
5.58 E+00 \\
0.0 \\
1.14 E+02 \\
0.0\end{array}$ & $\begin{array}{l}0.0 \\
3.66 E+00 \\
0.0 \\
7.46 E+01 \\
0.0\end{array}$ & $\begin{array}{l}0.0 \\
5.1 \text { TE } 00 \\
0.0 \\
1.05 E+02 \\
0.0\end{array}$ & $\begin{array}{l}0.0 \\
6.88 E \cdot 00 \\
0.0 \\
1.41 E+02 \\
0.0\end{array}$ & $\begin{array}{l}0.0 \\
0.0 \\
5.07 E+01 \\
3.15 E+02 \\
0.0\end{array}$ & $\begin{array}{l}0.0 \\
6.86 E+00 \\
5.07 E \bullet 01 \\
4.56 E+02 \\
0.0\end{array}$ \\
\hline $\begin{array}{l}N-13 \\
N-16 \\
0-15 \\
F-18 \\
N A-22\end{array}$ & $\begin{array}{r}9.97 \mathrm{M} \\
7.13 \mathrm{~S} \\
122.24 \mathrm{~S} \\
109.74 \mathrm{M} \\
2.602 \mathrm{Y}\end{array}$ & $\begin{array}{l}1.23 E+02 \\
3.76 E+02 \\
1.23 E+02 \\
1.19 E+02 \\
2.45 E+02\end{array}$ & $\begin{array}{l}9.32 E+01 \\
3.05 E \cdot 02 \\
9.33 E+01 \\
9.03 E+01 \\
1.91 E \div 02\end{array}$ & $\begin{array}{l}1.14 E+02 \\
3.40 E \bullet 02 \\
1.14 E+02 \\
1.10 E+02 \\
2.30 E+02\end{array}$ & $\begin{array}{l}7.47 E+01 \\
2.42 E+02 \\
7.47 E+01 \\
7.24 E+01 \\
1.53 E+02\end{array}$ & & & $\begin{array}{l}4.63 E+02 \\
2.86 E+03 \\
7.68 E+02 \\
1.03 E+02 \\
4.98 E+01\end{array}$ & $\begin{array}{l}6.03 E+02 \\
3.26 E+03 \\
9.09 E+02 \\
2.39 E+02 \\
3.31 E+02\end{array}$ \\
\hline $\begin{array}{l}\text { NA-24 } \\
M G-27 \\
M G-28 \\
A L-26 \\
A L-28\end{array}$ & $\begin{array}{l}15.00 \mathrm{H} \\
9.458 \mathrm{M} \\
20.91 \mathrm{H} \\
7.2 \mathrm{Y} \\
2.240 \mathrm{M}\end{array}$ & $\begin{array}{l}4.12 E+02 \\
1.04 E+02 \\
1.50 E+02 \\
2.91 E+02 \\
1.04 E+02\end{array}$ & $\begin{array}{l}3.26 E+02 \\
0.26 E+01 \\
1.18 E+02 \\
2.27 E+02 \\
1.45 E+02\end{array}$ & $\begin{array}{l}3.78 E+02 \\
9.89 E+01 \\
1.41 E+02 \\
2.70 E+02 \\
1.71 E+02\end{array}$ & $\begin{array}{l}2.11 E+02 \\
6.40 E \bullet 01 \\
9.31 E+01 \\
1.67 E+02 \\
1.21 E+02\end{array}$ & $\begin{array}{l}3.56 E+02 \\
8.87 E+01 \\
1.28 E+02 \\
2.50 E+02 \\
1.58 E+02\end{array}$ & $\begin{array}{l}4.62 E+02 \\
1.20 E+02 \\
1.74 E+02 \\
3.32 E+02 \\
2.09 E+02\end{array}$ & $\begin{array}{l}5.41 E+02 \\
7.23 E+02 \\
1.06 E+0 i \\
3.75 E+02 \\
1.34 E+03\end{array}$ & $\begin{array}{l}1.00 E+03 \\
8.43 E+02 \\
1.84 E+02 \\
1.07 E+02 \\
1.55 E+03\end{array}$ \\
\hline $\begin{array}{l}S 1-31 \\
S 1-32 \\
p-32 \\
p-33 \\
S-35\end{array}$ & $\begin{array}{r}157.3 \mathrm{M} \\
3.3 \mathrm{E} 2 \mathrm{Y} \\
14.29 \mathrm{O} \\
25.4 \mathrm{D} \\
87.44 \mathrm{D}\end{array}$ & $\begin{array}{l}9.34 E-02 \\
0.0 \\
0.0 \\
0.0 \\
0.0\end{array}$ & $\begin{array}{l}7.48 E-02 \\
0.0 \\
0.0 \\
0.0 \\
0.0\end{array}$ & $\begin{array}{l}8.87 E-02 \\
0.0 \\
0.0 \\
0.0 \\
0.9\end{array}$ & $\begin{array}{l}5.95 E-02 \\
0.0 \\
0.0 \\
0.0 \\
0.0\end{array}$ & $\begin{array}{l}8.01 E-02 \\
0.0 \\
0.0 \\
0.0 \\
0.0\end{array}$ & $\begin{array}{l}1.07 E-01 \\
0.0 \\
0.0 \\
0.0 \\
0.0\end{array}$ & $\begin{array}{l}5.93 E+02 \\
0.0 \\
7.15 E+02 \\
0.0 \\
0.0\end{array}$ & $\begin{array}{l}5.94 E+02 \\
0.0 \\
7.15 E+02 \\
0.0 \\
0.0\end{array}$ \\
\hline $\begin{array}{l}C L-36 \\
C L-38 \\
A R-37 \\
A R-39 \\
A R-41\end{array}$ & $\begin{array}{r}3.01 E 5 Y \\
37.21 \mathrm{M} \\
35.02 \mathrm{O} \\
269 \mathrm{Y} \\
1.827 \mathrm{H}\end{array}$ & $\begin{array}{l}2.43 E-06 \\
1.54 E+02 \\
4.11 E-04 \\
0.0 \\
1.35 E+02\end{array}$ & $\begin{array}{l}5.60 E-11 \\
1.21 E+02 \\
9.46 E-09 \\
0.0 \\
1.08 E+02\end{array}$ & $\begin{array}{l}1.68 E-07 \\
1.42 E+02 \\
2.83 E-05 \\
0.0 \\
1.28 E \cdot 02\end{array}$ & $\begin{array}{l}2.45 E-11 \\
1.02 E+02 \\
4.13 E-09 \\
0.0 \\
8.58 E+01\end{array}$ & $\begin{array}{l}4.66 E-06 \\
1.33 E+02 \\
7.87 E-04 \\
0.0 \\
1.15 E+02\end{array}$ & $\begin{array}{l}.60 E-05 \\
.74 E+02 \\
0.46 E-03 \\
.0 \\
.55 E+02\end{array}$ & $\begin{array}{l}1.26 E+02 \\
1.62 E \cdot 03 \\
0.0 \\
7.66 E+01 \\
4.25 E+02\end{array}$ & $\begin{array}{l}1.26 E+02 \\
1.79 E+03 \\
9.46 E-03 \\
7.66 E+01 \\
5.80 E+02\end{array}$ \\
\hline $\begin{array}{l}k-40 \\
x-42 \\
k-43 \\
C A-41 \\
C A-45\end{array}$ & $\begin{array}{r}1.277 E 9 Y \\
12.36 \mathrm{H} \\
22.6 \mathrm{H} \\
1.03 E 5 \mathrm{Y} \\
162.7 \mathrm{O}\end{array}$ & $\begin{array}{l}1.59 E+01 \\
2.82 E+01 \\
1.18 E+02 \\
1.08 E-03 \\
1.60 E-08\end{array}$ & $\begin{array}{l}1.27 E+01 \\
2.24 E+01 \\
8.99 E+01 \\
2.48 E-08 \\
1.26 E-12\end{array}$ & $\begin{array}{l}1.50 E+01 \\
2.65 E+01 \\
1.10 E+02 \\
7.42 E-05 \\
2.02 E-09\end{array}$ & $\begin{array}{l}1.03 E+01 \\
1.03 E+01 \\
7.14 E+01 \\
1.08 E-08 \\
2.70 E-13\end{array}$ & $\begin{array}{l}1.37 E+01 \\
2.42 E=01 \\
1.01 E+02 \\
2.06 E-03 \\
2.14 E-08\end{array}$ & $\begin{array}{l}1.82 E+01 \\
3.22 E+01 \\
1.34 E \cdot 02 \\
2.48 E-02 \\
2.06 E-07\end{array}$ & $\begin{array}{l}4.32 E+02 \\
1.55 E+03 \\
2.06 E+02 \\
0.0 \\
0.0\end{array}$ & $\begin{array}{l}4.50 E+02 \\
1.58 E \cdot 03 \\
3.40 E \cdot 02 \\
2.48 E-02 \\
2.06 E-07\end{array}$ \\
\hline $\begin{array}{l}C A-47 \\
C A-49 \\
S C-44 \\
S C-46 \\
S C-464\end{array}$ & $\begin{array}{l}4.536 \\
8.719 \\
3.927 \\
83.80 \\
18.72 \\
18\end{array}$ & $\begin{array}{l}1.13 E+02 \\
3.00 E+02 \\
2.43 E+02 \\
2.27 E+02 \\
1.18 E+01\end{array}$ & $\begin{array}{l}8.99 E \cdot 01 \\
2.36 E+02 \\
1.90 E+02 \\
1.81 E+02 \\
9.14 E+00\end{array}$ & $\begin{array}{l}1.07 E \cdot 02 \\
2.69 E+02 \\
2.29 E * 02 \\
2.16 E * 02 \\
1.17 E \cdot 01\end{array}$ & $\begin{array}{l}1.16 E \cdot 01 \\
1.97 E+02 \\
1.51 E+02 \\
1.41 E+02 \\
6.80 E+00\end{array}$ & $\begin{array}{l}9.65 E+01 \\
2.59 E+02 \\
2.08 E+02 \\
1.94 E+02 \\
1.05 E+01\end{array}$ & & $\begin{array}{l}2.48 E+02 \\
9.21 E+02 \\
6.10 E+02 \\
2.07 E-02 \\
0.0\end{array}$ & $\begin{array}{l}3.77 E+02 \\
1.25 E+03 \\
8.09 E+02 \\
2.62 E+02 \\
1.27 E+01\end{array}$ \\
\hline $\begin{array}{l}S C-47 \\
S C-48 \\
S C-49 \\
T I-44 \\
T I-45\end{array}$ & $\begin{array}{r}3.422 \mathrm{D} \\
43.67 \mathrm{H} \\
57.4 \mathrm{H} \\
47.3 \mathrm{Y} \\
3.08 \mathrm{H}\end{array}$ & $\begin{array}{l}1.42 E+01 \\
3.68 E+02 \\
1.07 E-01 \\
1.99 E+01 \\
1.05 E+02\end{array}$ & $\begin{array}{l}1.09 E \bullet 01 \\
2.95 E \bullet 02 \\
8.52 E-02 \\
1.55 E \bullet 01 \\
7.95 E \cdot 01\end{array}$ & $\begin{array}{l}1.39 E+01 \\
3.51 E+02 \\
1.00 E-01 \\
1.97 E+01 \\
9.73 E+01\end{array}$ & $\begin{array}{l}0.27 E+00 \\
2.31 E+02 \\
7.09 E-02 \\
9.48 E+00 \\
6.37 E+01\end{array}$ & $\begin{array}{l}1.26 E+01 \\
3.16 E+02 \\
9.25 E-02 \\
1.69 E+01 \\
8.98 E+01\end{array}$ & $\begin{array}{l}1.53 E+01 \\
4.25 E+02 \\
1.22 E-01 \\
2.16 E+01 \\
1.20 E+02\end{array}$ & $\begin{array}{l}2.49 E+01 \\
8.66 E+01 \\
8.66 E+02 \\
0.0 \\
3.31 E+02\end{array}$ & $\begin{array}{l}4.02 E+01 \\
5.11 E+02 \\
8.66 E+02 \\
2.16 E+01 \\
4.52 E \cdot 02\end{array}$ \\
\hline
\end{tabular}




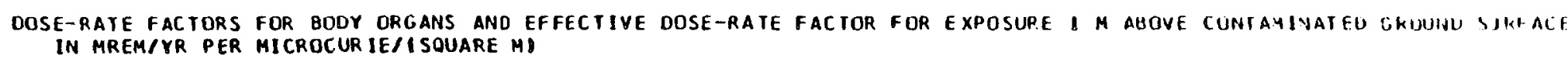

\begin{tabular}{|c|c|c|c|c|c|c|c|c|c|c|}
\hline IDE & IfE & RENALS 5 & ADDER & BRAIN & EAST & HE AR T & $\begin{array}{l}\text { SMALL } \\
\text { TIESTINE }\end{array}$ & $\begin{array}{c}\text { UPPER } \\
\text { LARGE } \\
\text { INTESTINE }\end{array}$ & $\begin{array}{c}\text { LOHER } \\
\text { LARGE } \\
\text { INIESTINEE }\end{array}$ & SONEY \\
\hline $\begin{array}{l}V I-51 \\
V-48 \\
V-49 \\
V-52 \\
C R-49\end{array}$ & 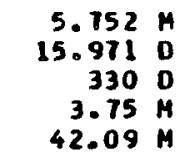 & $\begin{array}{l}3.36 E+01 \\
2.36 E+02 \\
5.42 E-04 \\
1.07 E+02 \\
9.28 E+01\end{array}$ & $\begin{array}{l}E E+01 \\
E+02 \\
E+11 \\
E+02\end{array}$ & & & & & $\begin{array}{l}3.24 E+01 \\
2.43 E+02 \\
1.34 E-06 \\
1.13 E+02\end{array}$ & & $\begin{array}{l}24 E+01 \\
42 E+02 \\
50 E-07 \\
13 E+02\end{array}$ \\
\hline $\begin{array}{l}C R-51 \\
M N-52 \\
M N-52 M \\
M N-53 \\
M N-54\end{array}$ & $\begin{array}{r}27.704 \\
5.591 \\
21.6 \\
3.7 E 6 \\
312.7 \\
30\end{array}$ & $\begin{array}{l}2.92 E \cdot 00 \\
2.00 E \cdot 02 \\
1.93 E+02 \\
1.14 E-03 \\
1.24 E+01\end{array}$ & $\begin{array}{l}.69 E+00 \\
.74 E \cdot 02 \\
.89 E+02 \\
.05 E-10 \\
.00 E+01\end{array}$ & $\begin{array}{l}2.94 E+00 \\
3.08 E+02 \\
2.12 E+02 \\
9.08 E-05 \\
7.87 E+01\end{array}$ & $\begin{array}{l}3.93 E+00 \\
.46 E+02 \\
.42 E+02 \\
0.92 E-02 \\
.95 E+01\end{array}$ & $\begin{array}{l}63 E+00 \\
.71 E+02 \\
06 E+02 \\
.48 E-04 \\
.48 E+01 \\
.90 E+01\end{array}$ & & $\begin{array}{l}2.78 E+00 \\
2.88 E+02 \\
1.98 E+02 \\
2.82 E-06 \\
7.34 E+01\end{array}$ & $\begin{array}{l}2.60 E+00 \\
2.65 E+02 \\
1.02 E+02 \\
4.67 E-04 \\
6.75 E+01\end{array}$ & $\begin{array}{l}\text { 2. } 78 E+00 \\
\text { 2. } 88 E+02 \\
1.98 E+02 \\
1.36 E-06 \\
\text { 1. } 32 E+01\end{array}$ \\
\hline 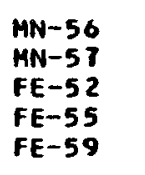 & $\begin{array}{r}2.5785 \mathrm{H} \\
1.47 \mathrm{H} \\
\mathrm{B.275} \mathrm{H} \\
2.7 \mathrm{Y} \\
44.63 \mathrm{O}\end{array}$ & $\begin{array}{l}1.35 E+02 \\
6.18 E+00 \\
6.62 E \bullet 01 \\
1.59 E-03 \\
9.32 E+01\end{array}$ & $\begin{array}{l}23 E+01 \\
87 E-10 \\
.22 E+01\end{array}$ & $\begin{array}{l}1.51 E+02 \\
6.56 E * 00 \\
6.8 B E \bullet 01 \\
1.27 E-04 \\
1.04 E+02\end{array}$ & & & & & & $\begin{array}{l}1.42 \mathrm{E} \cdot 02 \\
6.14 \mathrm{E}+00 \\
6.43 \mathrm{E}+01 \\
1.91 \mathrm{E}-06 \\
9.68 \mathrm{E}+01\end{array}$ \\
\hline $\begin{array}{l}C 0-56 \\
C 0-57 \\
C 0-58 \\
C 0-584 \\
C 0-60\end{array}$ & $\begin{array}{rr}78.76 & 0 \\
270.9 & 0 \\
70.80 & 0 \\
9.15 & \mathrm{H} \\
5.271 & \mathrm{Y}\end{array}$ & $\begin{array}{l}2.80 E+02 \\
1.11 E+01 \\
6.49 E \cdot 01 \\
.00 E-01\end{array}$ & $\begin{array}{l}2.79 E+02 \\
1.04 E+01 \\
8.10 E+01\end{array}$ & $\begin{array}{l}3.12 E+02 \\
1.11 E+01 \\
9.18 E+01 \\
2.66 E-04 \\
2.16 E+02\end{array}$ & $\begin{array}{l}3.51 E+02 \\
1.90 E+01 \\
1.04 E * 02 \\
2.00 E-01 \\
2.38 E+02\end{array}$ & & & & & $\begin{array}{l}2.96 E+02 \\
1.05 E+01 \\
8.54 E+01 \\
3.18 E-04 \\
2.01 E+02\end{array}$ \\
\hline $\begin{array}{l}C O-604 \\
C O-61 \\
N 1-56 \\
N I-57 \\
N I-59\end{array}$ & $\begin{array}{r}10.47 \mathrm{M} \\
1.650 \mathrm{H} \\
6.10 \mathrm{O} \\
36.08 \mathrm{H} \\
7.5 \mathrm{H} \mathrm{Y}\end{array}$ & $\begin{array}{l}3.35 E-01 \\
7.24 E+00 \\
1.49 E+02\end{array}$ & $\begin{array}{l}3.45 E-01 \\
7.59 E+00 \\
1.43 E+02\end{array}$ & $\begin{array}{l}3.68 \mathrm{E}-01 \\
7.78 \mathrm{E} * 00 \\
1.59 \mathrm{E}: 02 \\
1.66 \mathrm{E} \cdot 02 \\
2.39 \mathrm{E}-04\end{array}$ & & & & & & \\
\hline $\begin{array}{l}N I-63 \\
N I-65 \\
C U-61 \\
C U-62 \\
C U-64\end{array}$ & $\begin{array}{r}100.1 \mathrm{Y} \\
2.520 \mathrm{H} \\
3.400 \mathrm{H} \\
9.74 \mathrm{H} \\
12.701 \mathrm{H}\end{array}$ & $\begin{array}{l}0.0 \\
4.17 E+01 \\
1.25 E+01 \\
8.93 E+01 \\
1.67 E+01\end{array}$ & $\begin{array}{l}0.0 \\
4.16 E+01 \\
6.90 E+01 \\
8.40 E+01\end{array}$ & $\begin{array}{l}0.0 \\
9.068 \mathrm{E} \\
7.69 \mathrm{ES} \\
9.45 \mathrm{EG} \\
1.77 \mathrm{ES}\end{array}$ & $\begin{array}{l}0.0 \\
5.21 E+01 \\
9.17 E+01 \\
1.13 E+02 \\
2.12 E \cdot 01\end{array}$ & $\begin{array}{l}0.0 \\
4.1 \\
6.9 \\
8.2 \\
1.9\end{array}$ & & & & $\begin{array}{l}0.0 \\
4.38 E+01 \\
7.17 E+01 \\
8.19 E+01 \\
1.65 E+01\end{array}$ \\
\hline $\begin{array}{l}\text { CU-67 } \\
2 N-62 \\
2 N-65 \\
2 N-69 \\
2 N-69 N\end{array}$ & $\begin{array}{r}61.88 \mathrm{D} \\
9.26 \mathrm{H} \\
244.4 \mathrm{O} \\
55.6 \mathrm{H} \\
13.76 \mathrm{H}\end{array}$ & $\begin{array}{l}1.06 E * 01 \\
3.98 E * 01 \\
4.66 E+01 \\
5.44 E-04 \\
3.75 E+01\end{array}$ & $\begin{array}{l}9.85 E \\
3.80 E \\
4.5 B E \\
5.10 E-1 \\
3.53 E+\end{array}$ & $\begin{array}{l}1.05 E \\
4.02 E \\
5.16 E \\
5.64 E \\
3.91 E\end{array}$ & $\begin{array}{l}1.65 E \\
5.19 E \\
5.77 E \\
6.99 E \\
4.80 E\end{array}$ & $\begin{array}{l}9.58 E \\
3.69 E \\
4.53 E \\
4.98 E \\
3.44 E\end{array}$ & $\begin{array}{l}8.47 E+00 \\
3.33 E+01 \\
4.15 E+01 \\
4.47 E-04 \\
3.10 E+01\end{array}$ & $\begin{array}{l}1.02 E+01 \\
3.93 E+01 \\
4.83 E+01 \\
5.27 E-04 \\
3.65 E+01\end{array}$ & $\begin{array}{l}9.45 E \bullet 00 \\
3.62 E+01 \\
4.44 E+01 \\
4.90 E-04 \\
3.39 E 001\end{array}$ & $\begin{array}{l}1.01 E+01 \\
3.97 E+01 \\
4.81 E+01 \\
5.28 E-04 \\
3.65 E+01\end{array}$ \\
\hline $\begin{array}{l}G A-66 \\
G A-67 \\
G A-68 \\
G A-72 \\
G E-68\end{array}$ & $\begin{array}{r}9.40 \\
3.261 \\
68.0 \mathrm{H} \\
14.1 \\
280\end{array}$ & $\begin{array}{l}1.08 E+02 \\
1.33 E+01 \\
8.39 E+01 \\
2.14 E+02 \\
0.38 E-03\end{array}$ & $\begin{array}{l}1.87 E+02 \\
1.24 E+01 \\
1.98 E+01 \\
2.14 E+02 \\
1.91 E-09\end{array}$ & $\begin{array}{l}2.00 E+02 \\
1.33 E+01 \\
8.90 E+01 \\
2.39 E+02 \\
6.72 E-04\end{array}$ & $\begin{array}{l}2.39 E+02 \\
2.05 E+01 \\
1.06 E+02 \\
2.69 E+02 \\
6.50 E-01\end{array}$ & $\begin{array}{l}1.86 E * 02 \\
1.20 \mathrm{E} * 01 \\
7.80 \mathrm{E}+01 \\
2.12 \mathrm{E} \cdot 02 \\
1.10 \mathrm{E}-03\end{array}$ & $\begin{array}{l}1.72 E \cdot 02 \\
1.07 E+01 \\
7.02 E+01 \\
1.95 E+02 \\
1.47 E-05\end{array}$ & $\begin{array}{l}8.97 E \cdot 02 \\
1.28 E+01 \\
8.28 E+01 \\
2.26 E+02 \\
1.98 E-05\end{array}$ & $\begin{array}{l}1.83 E+02 \\
1.18 E+01 \\
7.65 E+01 \\
2.08 E+02 \\
3.46 E-03\end{array}$ & $\begin{array}{l}\text { 2. } 00 E+02 \\
1.27 E+01 \\
B .28 E * 01 \\
2.26 E * 02 \\
1.07 E-05\end{array}$ \\
\hline
\end{tabular}


DOSE-RATE FACTORS FOR BODY ORGANS AND EFFECTIVE DOSE-PATE FACTOR FOR EXPOSURE I M ABOVE CUNTAMINAIED SROUNO SJRFACE
IN MREM/YR PER MICROCUR JE/I SOUARE MI

\begin{tabular}{|c|c|c|c|c|c|c|c|c|c|c|}
\hline NUCL IDE & HALF-LIFE & LIVER & LUNGS & MARROH & $\begin{array}{c}\text { RED } \\
\text { MARROW }\end{array}$ & OVARIES & PANCREAS & KELETON & SPLEEN & $S$ TOMACH \\
\hline $\begin{array}{l}T I-51 \\
V-48 \\
V-49 \\
v-52 \\
C R-49\end{array}$ & $\begin{array}{r}5.752 \\
15.971 \\
330 \\
3.75 \\
32.09 \\
42.09 \mathrm{M}\end{array}$ & $\begin{array}{l}3.12 E+01 \\
2.30 E+02 \\
5.27 E-06 \\
1.07 E+02 \\
8.78 E+01\end{array}$ & $\begin{array}{l}3.36 E+01 \\
2.45 E+02 \\
4.29 E-04 \\
1.13 E+02 \\
9.45 E+01\end{array}$ & $\begin{array}{l}4.10 E+01 \\
2.63 E+02 \\
2.57 E-04 \\
1.18 E+02 \\
1.13 E+02\end{array}$ & $\begin{array}{l}.46 E+01 \\
.48 E+02 \\
.41 E-04 \\
.14 E+02 \\
.46 E+01\end{array}$ & $\begin{array}{l}.90 E \bullet 01 \\
.21 E+02 \\
12 E-04 \\
.01 E+02 \\
.34 E+01\end{array}$ & $\begin{array}{l}2.69 E+01 \\
1.99 E+02 \\
3.17 E-04 \\
9.30 E+01 \\
7.48 E+01\end{array}$ & $\begin{array}{l}4.17 E+01 \\
2.60 E+02 \\
6.96 E-04 \\
1.20 E+02 \\
1.15 E+02\end{array}$ & $\begin{array}{l}3.07 E+01 \\
2.23 E+02 \\
1.22 E-04 \\
1.03 E+02 \\
8.57 E+01\end{array}$ & $\begin{array}{l}\text { 3. } 08 E+01 \\
2.26 E+02 \\
1.10 E-04 \\
1.04 E+02 \\
8.65 E+01\end{array}$ \\
\hline $\begin{array}{l}C R-51 \\
M N-52 \\
M N-52 M \\
M N-53 \\
M N-54\end{array}$ & 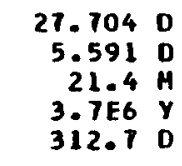 & $\begin{array}{l}2.68 E+00 \\
2.73 E+02 \\
1.88 E+02 \\
1.11 E-05 \\
6.95 E+01\end{array}$ & $\begin{array}{l}2.89 E+00 \\
2.90 E+02 \\
2.00 E+02 \\
9.00 E-04 \\
7.42 E+01\end{array}$ & $\begin{array}{l}3.62 E+00 \\
3.12 E+02 \\
2.19 E+02 \\
5.40 E-04 \\
8.05 E+01\end{array}$ & $\begin{array}{l}2.99 E+00 \\
2.94 E+02 \\
2.03 E+02 \\
2.95 E-04 \\
1.53 E+01\end{array}$ & $\begin{array}{l}2.46 E+00 \\
2.62 E+02 \\
1.79 E+02 \\
4.46 E-04 \\
6.16 E+01\end{array}$ & $\begin{array}{l}2.32 E+00 \\
2.36 E+02 \\
1.63 E+02 \\
6.65 E-04 \\
6.00 E+01\end{array}$ & $\begin{array}{l}3.69 E+00 \\
3.17 E+02 \\
2.22 E+02 \\
1.46 E-03 \\
8.18 E+01\end{array}$ & $\begin{array}{l}2.65 E+00 \\
2.65 E+02 \\
1.83 E+02 \\
2.57 E-04 \\
6.76 E+01\end{array}$ & $\begin{array}{l}2.65 E+00 \\
2.68 E+02 \\
1.84 E+02 \\
2.32 E-04 \\
6.8 B E+01\end{array}$ \\
\hline $\begin{array}{l}M N-56 \\
M N-57 \\
F E-52 \\
F E-55 \\
F E-59\end{array}$ & $\begin{array}{r}2.5785 \mathrm{H} \\
1.47 \mathrm{H} \\
8.275 \mathrm{H} \\
2.7 \mathrm{Y} \\
44.63 \mathrm{D}\end{array}$ & $\begin{array}{l}1.35 E+02 \\
5.89 E+00 \\
6.20 E+01 \\
1.55 E-05 \\
9.18 E+01\end{array}$ & $\begin{array}{l}1.43 E+02 \\
6.35 E+00 \\
6.60 E+01 \\
1.26 E-03 \\
9.74 E+01\end{array}$ & $\begin{array}{l}1.51 E+02 \\
7.68 E+00 \\
8.16 E+01 \\
7.56 E-04 \\
1.03 E+02\end{array}$ & $\begin{array}{l}1.45 E \cdot 02 \\
6.25 E \cdot 00 \\
6.74 E+01 \\
4.13 E-04 \\
9.83 E+01\end{array}$ & $\begin{array}{l}1.27 E+02 \\
5.53 E+00 \\
5.02 E+01 \\
6.23 E-04 \\
8.79 E+01\end{array}$ & $\begin{array}{l}1.17 E+02 \\
5.03 E+00 \\
5.31 E * 01 \\
9.30 E-04 \\
7.95 E+01\end{array}$ & $\begin{array}{l}1.53 E+02 \\
7.85 E+00 \\
8.31 E+01 \\
2.04 E-03 \\
1.05 E \cdot 02\end{array}$ & $\begin{array}{l}1.30 E+02 \\
5.76 E+00 \\
6.08 E+01 \\
3.59 E-04 \\
8.8 B E+01\end{array}$ & $\begin{array}{l}1.32 E+02 \\
5 . B 2 E+00 \\
6.12 E+01 \\
3.24 E-04 \\
9.01 E+01\end{array}$ \\
\hline $\begin{array}{l}c 0-56 \\
c 0-57 \\
C 0-58 \\
C 0-584 \\
00-60\end{array}$ & $\begin{array}{rr}78.76 & 0 \\
270.9 & 0 \\
70.80 & 0 \\
9.15 & \mathrm{H} \\
5.271 & \mathrm{r}\end{array}$ & $\begin{array}{l}2.78 E+02 \\
1.04 E+01 \\
8.13 E+01 \\
1.34 E-04 \\
1.91 E+02\end{array}$ & $\begin{array}{l}2.94 E+02 \\
1.15 E+01 \\
8.69 E+01\end{array}$ & $\begin{array}{l}3.11 E+02 \\
1 . B 0 E+01 \\
9.53 E+01 \\
1.51 E-03 \\
2.12 E+02\end{array}$ & $\begin{array}{l}2.99 E+02 \\
9.99 E+00 \\
8.03 E+01 \\
7.27 E-04\end{array}$ & & & & & $\begin{array}{l}2.74 E+02 \\
1.02 E+01 \\
8.04 E+01 \\
6.45 E-04 \\
1.86 E+02\end{array}$ \\
\hline $\begin{array}{l}\mathrm{CO}-60 \mathrm{OM} \\
\mathrm{CO}-61 \\
\mathrm{~N} 1-56 \\
\mathrm{NI}-57 \\
\mathrm{NI}-59\end{array}$ & $\begin{array}{r}10.47 \\
1.650 \\
6.10 \\
36.08 \\
7.564 \\
7.5 E\end{array}$ & $\begin{array}{l}3.42 E-01 \\
7.51 E+00 \\
1.42 E+02 \\
1.48 E+02 \\
2.91 E-05\end{array}$ & $\begin{array}{l}3.69 E-01 \\
8.19 E+00 \\
1.52 E+02 \\
1.57 E+02 \\
2.37 E-03\end{array}$ & $\begin{array}{l}4.65 E-01 \\
1.25 E+01 \\
1.74 E * 02 \\
1.60 E \cdot 02 \\
1.42 E-03\end{array}$ & $\begin{array}{l}3.20 E-01 \\
6.09 E \cdot 00 \\
1.54 E \cdot 02 \\
1.58 E \cdot 02 \\
7 . T 7 E-04\end{array}$ & $\begin{array}{l}3.12 E-01 \\
6.55 E+00 \\
1.35 E+02 \\
1.39 E+02 \\
1.17 E-03\end{array}$ & $\begin{array}{l}2.74 E-01 \\
5.47 E+00 \\
1.22 E+02 \\
1.28 E+02 \\
1.75 E-03\end{array}$ & $\begin{array}{l}4.77 E-01 \\
1.28 E+01 \\
1.77 E+02 \\
1.71 E+02 \\
3.84 E-03\end{array}$ & $\begin{array}{l}3.22 E-01 \\
6.95 E+00 \\
1.39 E+02 \\
1.43 E+02 \\
6.75 E-04\end{array}$ & $\begin{array}{l}3.28 E-01 \\
1.13 E+00 \\
1.40 E+02 \\
1.44 E+02 \\
6.10 E-04\end{array}$ \\
\hline $\begin{array}{l}N 1-63 \\
N 1-65 \\
C U-61 \\
C U-62 \\
C U-64\end{array}$ & $\begin{array}{r}100.1 \mathrm{Y} \\
2.520 \mathrm{H} \\
3.408 \mathrm{H} \\
9.74 \mathrm{M} \\
12.701 \mathrm{H}\end{array}$ & $\begin{array}{l}0.0 \\
4.15 E+01 \\
6.86 E+01 \\
0.43 E+01 \\
1.58 E+01\end{array}$ & $\begin{array}{l}0.0 \\
4.40 E+01 \\
7.36 E+01 \\
9.05 E+01 \\
1.69 E+01\end{array}$ & $\begin{array}{l}0.0 \\
4.64 E+01 \\
8.46 E+01 \\
1.04 E+02 \\
1.95 E+01\end{array}$ & $\begin{array}{l}0.0 \\
4.44 E+01 \\
7.52 E+01 \\
9.26 E+01 \\
1.73 E+01\end{array}$ & $\begin{array}{l}0.0 \\
3.93 E+01 \\
6.58 E+01 \\
8.10 E+01 \\
1.52 E+01\end{array}$ & $\begin{array}{l}0.0 \\
3.60 E+01 \\
5.91 E+01 \\
7.26 E+01 \\
1.36 E+01\end{array}$ & $\begin{array}{l}0.0 \\
4.71 E+01 \\
0.60 E \cdot 01 \\
1.06 E+02 \\
1.98 E+01\end{array}$ & $\begin{array}{l}0.0 \\
4.01 E+01 \\
6.10 E+01 \\
0.23 E+01 \\
1.54 E+01\end{array}$ & $\begin{array}{l}0.0 \\
4.06 E+01 \\
6.77 E+01 \\
8.32 E+01 \\
1.56 E+01\end{array}$ \\
\hline $\begin{array}{l}\mathrm{CU}-67 \\
2 N-62 \\
2 N-65 \\
2 N-69 \\
2 N-69 M\end{array}$ & $\begin{array}{r}61.88 \mathrm{D} \\
9.26 \mathrm{H} \\
244.4 \mathrm{O} \\
55.6 \mathrm{H} \\
13.76 \mathrm{H}\end{array}$ & $\begin{array}{l}9.81 E+00 \\
3.77 E+01 \\
4.56 E+01 \\
5.07 E-04 \\
3.51 E+01\end{array}$ & $\begin{array}{l}1.07 E+01 \\
4.05 E+01 \\
4.85 E+01 \\
5.46 E-04 \\
3.77 E+01\end{array}$ & $\begin{array}{l}1.57 E+01 \\
4.69 E+01 \\
5.15 E+01 \\
6.51 E-04 \\
4.46 E+01\end{array}$ & $\begin{array}{l}1.01 E+01 \\
4.09 E+01 \\
4.90 E+01 \\
5.62 E-04 \\
3.08 E+01\end{array}$ & $\begin{array}{l}8.54 E+00 \\
3.61 E+01 \\
4.39 E+01 \\
4.79 E-04 \\
3.33 E+01\end{array}$ & $\begin{array}{l}8.17 E+00 \\
3.22 E+01 \\
3.95 E+01 \\
4.30 E-04 \\
3.03 E+01\end{array}$ & & & $\begin{array}{l}9.67 E+00 \\
3.71 E+01 \\
4.49 E+01 \\
5.01 E-04 \\
3.46 E+01\end{array}$ \\
\hline $\begin{array}{l}\text { GA-66 } \\
G A-67 \\
6 A-68 \\
G A-72 \\
\text { GE-68 }\end{array}$ & 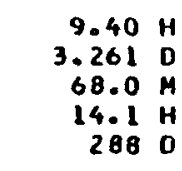 & $\begin{array}{l}1.87 E+02 \\
1.23 E+01 \\
7.93 E+01 \\
2.14 E+02 \\
8.40 E-05\end{array}$ & $\begin{array}{l}1.98 E+02 \\
1.35 E \cdot 01 \\
8.51 E \cdot 01 \\
2.26 E+02 \\
6.65 E-03\end{array}$ & $\begin{array}{l}2.11 E+02 \\
1.89 E+01 \\
9.77 E+01 \\
2.38 E+02 \\
4.03 E-03\end{array}$ & $\begin{array}{l}2.03 E+02 \\
1.27 E+01 \\
8.71 E+01 \\
2.30 E+02 \\
2.18 E-03\end{array}$ & $\begin{array}{l}1.73 E+02 \\
1.10 E+01 \\
7.62 E+01 \\
2.00 E+02 \\
3.29 E-03\end{array}$ & $\begin{array}{l}1.64 E+02 \\
1.03 E+01 \\
6.83 E+01 \\
1.85 E+02 \\
4.90 E-03\end{array}$ & $\begin{array}{l}2.14 E+02 \\
1.94 E+01 \\
9.93 E+01 \\
2.42 E+02 \\
1.08 E-02\end{array}$ & $\begin{array}{l}1.81 E+02 \\
1.22 E+01 \\
7.74 E+01 \\
2.06 E+02 \\
1.91 E-03\end{array}$ & $\begin{array}{l}1.86 E+02 \\
1.21 E+01 \\
7.83 E+01 \\
2.10 E+02 \\
1.73 E-03\end{array}$ \\
\hline
\end{tabular}




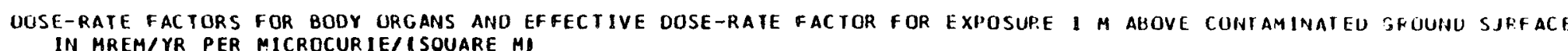

\begin{tabular}{|c|c|c|c|c|c|c|c|c|c|}
\hline NUCL IDE & HALF-LIFE & TESTES & THYMUS & THYROID & UTERUS & FFECIIVE & $\begin{array}{c}\text { SKIN } \\
\text { (PHOTON) }\end{array}$ & $\begin{array}{l}\text { SKIN } \\
\text { IELECIRON }\end{array}$ & $\begin{array}{l}\text { SKIN } \\
\text { ITOTALI }\end{array}$ \\
\hline $\begin{array}{l}V I-51 \\
V-48 \\
V-49 \\
V-52 \\
C R-49\end{array}$ & $\begin{array}{r}5.752 \mathrm{M} \\
15.971 \mathrm{O} \\
330 \mathrm{D} \\
3.75 \mathrm{M} \\
42.09 \mathrm{M}\end{array}$ & $\begin{array}{l}4.53 E+01 \\
3.23 E+02 \\
3.93 E-03 \\
1.48 E+02 \\
1.28 E \cdot 02\end{array}$ & $\begin{array}{l}3.45 E+01 \\
2.56 E+02 \\
9.05 E-08 \\
1.18 E+02 \\
9.70 E \cdot 01\end{array}$ & $\begin{array}{l}4.26 E+01 \\
3.06 E+02 \\
2.71 E-04 \\
1.40 E+02 \\
1.19 E \cdot 02\end{array}$ & $\begin{array}{l}2.74 E+01 \\
2.03 E+02 \\
3.95 E-08 \\
9.56 E+01 \\
7.65 E+01\end{array}$ & $\begin{array}{l}3.92 E+01 \\
2.17 E+02 \\
7.54 E-03 \\
1.27 E+02 \\
1.10 E+02\end{array}$ & $\begin{array}{l}5.11 E+01 \\
3.72 E+02 \\
9.05 E-02 \\
1.70 E+02 \\
1.45 E+02\end{array}$ & $\begin{array}{l}9.15 E+02 \\
8.18 E+01 \\
0.0 \\
1.15 E+03 \\
6.07 E+02\end{array}$ & $\begin{array}{l}9.66 E+02 \\
4.54 E+02 \\
9.05 E-02 \\
1.32 E+03 \\
7.52 E+02\end{array}$ \\
\hline $\begin{array}{l}C R-51 \\
M N-52 \\
M N-52 M \\
M N-53 \\
M N-54\end{array}$ & 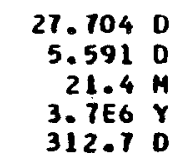 & $\begin{array}{l}3.92 E+00 \\
3.83 E+02 \\
2.65 E+02 \\
8.26 E-03 \\
9.83 E+01\end{array}$ & $\begin{array}{l}2.95 E+00 \\
3.04 E+02 \\
2.07 E+02 \\
1.90 E-07 \\
7.00 E \cdot 01\end{array}$ & $\begin{array}{l}3.67 E+00 \\
3.63 E+02 \\
2.4 E E+02 \\
5.69 E-04 \\
9.36 E+01\end{array}$ & $\begin{array}{l}2.36 E+00 \\
2.40 E+02 \\
1.66 E+02 \\
8.31 E-01 \\
6.05 E+01\end{array}$ & $\begin{array}{l}3.41 E+00 \\
3.28 E+02 \\
2.27 E+02 \\
1.58 E-02 \\
8.41 E+01\end{array}$ & $\begin{array}{l}4.53 E+00 \\
4.41 E+02 \\
3.03 E+02 \\
1.90 E-01 \\
1.14 E+02\end{array}$ & $\begin{array}{l}0.0 \\
2.39 E+01 \\
1.23 E+03 \\
0.0 \\
0.0\end{array}$ & $\begin{array}{l}4.53 E+00 \\
4.65 E+02 \\
1.53 E+03 \\
1.90 E-01 \\
1.14 E+02\end{array}$ \\
\hline $\begin{array}{l}M N-56 \\
M N-57 \\
F E-52 \\
F E-55 \\
F E-59\end{array}$ & $\begin{array}{r}2.5785 \mathrm{H} \\
1.41 \mathrm{H} \\
8.275 \mathrm{H} \\
2.7 \mathrm{Y} \\
44.63 \mathrm{O}\end{array}$ & $\begin{array}{l}1.87 E+02 \\
8.62 E \bullet 00 \\
9.02 E+01 \\
1.16 E-02 \\
1.28 E+02\end{array}$ & $\begin{array}{l}1.48 E+02 \\
6.64 E+00 \\
6.85 E+01 \\
2.66 E-07 \\
1.03 E+02\end{array}$ & $\begin{array}{l}1.75 E \cdot 02 \\
8.15 E+00 \\
8.46 E+01 \\
7.96 E-04 \\
1.22 E+02\end{array}$ & $\begin{array}{l}1.19 E \bullet 02 \\
5.16 E \bullet 00 \\
5.43 E \bullet 01 \\
1.16 E-07 \\
8.10 E \bullet 01\end{array}$ & $\begin{array}{l}1.60 E+02 \\
7.46 E+00 \\
7.78 E+01 \\
2.21 E-02 \\
1.10 E+02\end{array}$ & $\begin{array}{l}2.14 E+02 \\
1.06 E+01 \\
1.02 E+02 \\
2.66 E-01 \\
1.48 E+02\end{array}$ & $\begin{array}{l}8.46 E+02 \\
1.18 E+03 \\
1.35 E+02 \\
0.0 \\
1.00 E+00\end{array}$ & $\begin{array}{l}1.06 E+03 \\
1.19 E+03 \\
2.37 E+02 \\
2.66 E-01 \\
1.55 E+02\end{array}$ \\
\hline $\begin{array}{l}\mathrm{CO}-56 \\
\mathrm{CO}-57 \\
\mathrm{CO}-58 \\
\mathrm{CO}-584 \\
\mathrm{CO}-60\end{array}$ & $\begin{array}{rl}78.76 & 0 \\
270.9 & 0 \\
70.80 & 0 \\
9.15 & H \\
5.271 & Y\end{array}$ & $\begin{array}{l}3.06 \mathrm{E}+02 \\
1.62 \mathrm{E}+01 \\
1.15 \mathrm{E}+02 \\
1.92 \mathrm{E}-02 \\
2.65 \mathrm{E}+02\end{array}$ & $\begin{array}{l}3.06 E+02 \\
1.24 E+01 \\
9.07 E+01 \\
1.51 E-04 \\
2.12 E+02\end{array}$ & $\begin{array}{l}3.61 E * 02 \\
1.60 E * 01 \\
1.09 E * 02 \\
1.67 E-03 \\
2.52 E+02\end{array}$ & $\begin{array}{l}2.47 E+02 \\
9.01 E \cdot 00 \\
1.08 E * 01 \\
2.88 E-05 \\
1.69 E+02\end{array}$ & $\begin{array}{l}3.32 E+02 \\
1.43 E+01 \\
9.87 E+01 \\
3.58 E-02 \\
2.27 E+02\end{array}$ & $\begin{array}{l}4.40 E+02 \\
1.85 E+01 \\
1.33 E+02 \\
4.26 E-01 \\
3.05 E \cdot 02\end{array}$ & $\begin{array}{l}1.21 E+02 \\
0.0 \\
3.65 E+00 \\
0.0 \\
0.0\end{array}$ & $\begin{array}{l}5.61 E+02 \\
1.05 E+01 \\
1.37 E+02 \\
4.26 E-01 \\
3.05 E+02\end{array}$ \\
\hline $\begin{array}{l}C O-60 M \\
C O-61 \\
N I-56 \\
N I-57 \\
N I-59\end{array}$ & $\begin{array}{r}10.47 \mathrm{H} \\
1.650 \mathrm{H} \\
6.10 \mathrm{O} \\
36.08 \mathrm{H} \\
T .5 \mathrm{E} 4 \mathrm{~V}\end{array}$ & $\begin{array}{l}5.36 E-01 \\
1.21 E+01 \\
2.03 E+02 \\
2.06 E+02 \\
2.17 E-02\end{array}$ & $\begin{array}{l}4.05 E-01 \\
9.45 E+00 \\
1.59 E \cdot 02 \\
1.62 E+02 \\
5.00 E-07\end{array}$ & $\begin{array}{l}4.94 E-01 \\
1.18 E+01 \\
1.93 E+02\end{array}$ & & & & $\begin{array}{l}1.41 E+00 \\
4.24 E+02 \\
0.0 \\
1.00 E+02 \\
0.0\end{array}$ & $\begin{array}{l}2.44 E+00 \\
4.37 E+02 \\
2.32 E+02 \\
3.36 E+02 \\
5.00 E-01\end{array}$ \\
\hline $\begin{array}{l}N I-63 \\
N I-65 \\
C U-61 \\
C U-62 \\
C U-64\end{array}$ & $\begin{array}{r}100.1 \mathrm{Y} \\
2.520 \mathrm{H} \\
3.408 \mathrm{H} \\
9.74 \mathrm{H} \\
12.701 \mathrm{H}\end{array}$ & $\begin{array}{l}0.0 \\
5.77 E+01 \\
9.07 E+01 \\
1.21 E \bullet 02 \\
2.27 E \cdot 01\end{array}$ & $\begin{array}{l}0.0 \\
4.60 E+01 \\
7.52 E+01 \\
9.19 E+01 \\
1.72 E+01\end{array}$ & $\begin{array}{l}0.0 \\
5.45 E+01 \\
9.18 E+01 \\
1.12 E * 02 \\
2.11 E+01\end{array}$ & $\begin{array}{l}0.0 \\
3.69 E * 01 \\
6.00 E * 01 \\
1.37 E+01 \\
1.38 E+01\end{array}$ & $\begin{array}{l}0.0 \\
4.95 E+01 \\
8.45 E+01 \\
1.04 E+02 \\
1.95 E+01\end{array}$ & $\begin{array}{l}0.0 \\
6.01 E \cdot 01 \\
1.13 E \cdot 02 \\
1.39 E+02 \\
2.63 E+01\end{array}$ & $\begin{array}{l}0.0 \\
6.18 E \cdot 02 \\
2.90 E \cdot 02 \\
1.39 E+03 \\
4.37 E+01\end{array}$ & $\begin{array}{l}0.0 \\
6.84 E+02 \\
4.03 E+02 \\
1.53 E+03 \\
7.00 E+01\end{array}$ \\
\hline $\begin{array}{l}C U-67 \\
2 N-62 \\
2 N-65 \\
2 N-69 \\
2 N-69 M\end{array}$ & $\begin{array}{r}61.80 \\
9.26 \mathrm{H} \\
244.4 \mathrm{O} \\
55.6 \mathrm{M} \\
13.76 \mathrm{H}\end{array}$ & $\begin{array}{l}1.50 E+01 \\
5.49 E+01 \\
6.39 E+01 \\
7.35 E-04 \\
5.00 E+01\end{array}$ & $\begin{array}{l}1.15 E+01 \\
4.16 E+01 \\
5.12 E+01 \\
5.54 E-04 \\
3.83 E+01\end{array}$ & $\begin{array}{l}1.46 E+01 \\
5.09 E+01 \\
6.09 E+01 \\
6.82 E-04 \\
4.71 E+01\end{array}$ & $\begin{array}{l}6.60 E+00 \\
3.27 E+01 \\
4.01 E+01 \\
4.45 E-04 \\
3.08 E+01\end{array}$ & $\begin{array}{l}1.31 E+01 \\
4.68 E+01 \\
5.48 E+01 \\
6.32 E-04 \\
4.36 E+01\end{array}$ & $\begin{array}{l}1.64 E+01 \\
6.40 E+01 \\
7.45 E+01 \\
0.34 E-04 \\
5.78 E+01\end{array}$ & $\begin{array}{l}1.35 E+01 \\
7.9 B E+00 \\
0.0 \\
2.31 E+02 \\
2.40 E+01\end{array}$ & $\begin{array}{l}2.99 E+01 \\
7.20 E+01 \\
7.45 E+01 \\
2.31 E+02 \\
8.18 E+01\end{array}$ \\
\hline $\begin{array}{l}G A-66 \\
G A-67 \\
G A-68 \\
G A-72 \\
G E-6 B\end{array}$ & $\begin{array}{rl}9.40 & H \\
3.261 & D \\
68.0 & H \\
14.1 & H \\
288 & 0\end{array}$ & $\begin{array}{l}2.60 E \cdot 02 \\
1.07 E+01 \\
1.14 E+02 \\
2.96 E+02 \\
6.08 E-02\end{array}$ & $\begin{array}{l}2.04 E+02 \\
1.42 E+01 \\
8.66 E+01 \\
2.34 E+02 \\
1.52 E-06\end{array}$ & $\begin{array}{l}2.40 E+02 \\
1.80 E * 01 \\
1.06 E+02 \\
2.76 E * 02 \\
4.25 E-03\end{array}$ & $\begin{array}{l}1.66 E+02 \\
1.08 E+01 \\
6.93 E+01 \\
8.90 E+02 \\
6.04 E-07\end{array}$ & $\begin{array}{l}2.24 E+02 \\
1.64 E+01 \\
9.76 E+01 \\
2.54 E+02 \\
1.15 E-01\end{array}$ & $\begin{array}{l}2.93 E+02 \\
2.20 E+01 \\
1.31 E+02 \\
3.37 E+02 \\
1.38 E+00\end{array}$ & $\begin{array}{l}1.03 E+03 \\
0.0 \\
7.84 E+02 \\
4.46 E+02 \\
0.0\end{array}$ & $\begin{array}{l}1.32 E+03 \\
2.20 E+01 \\
9.14 E+02 \\
1.84 E+02 \\
1.38 E+00\end{array}$ \\
\hline
\end{tabular}




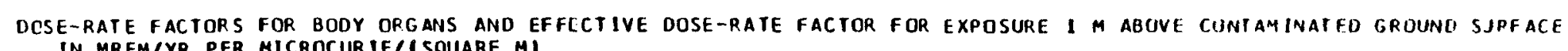

\begin{tabular}{|c|c|c|c|c|c|}
\hline NUCL IOE & HALF-LIFE & ADRENALS & BLADDER & BRAIN & BREAST \\
\hline $\begin{array}{l}\text { GE-71 } \\
G E-77 \\
A S-72 \\
A S-73 \\
A S-74\end{array}$ & 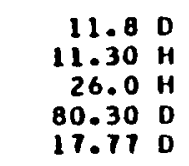 & $\begin{array}{l}8.48 E-03 \\
9.23 E+01 \\
1.55 E+02 \\
3.93 E-01 \\
6.78 E+01\end{array}$ & $\begin{array}{l}1.93 E-09 \\
8.79 E+01 \\
1.49 E+02 \\
4.40 E-01 \\
6.45 E+01\end{array}$ & $\begin{array}{l}6.80 E-04 \\
9.74 E+01 \\
1.66 E+02 \\
3.80 E-01 \\
7.21 E+01\end{array}$ & $\begin{array}{l}6.57 E-01 \\
1.19 E+02 \\
1.93 E+02 \\
2.80 E \bullet 00 \\
1.49 E \bullet 01\end{array}$ \\
\hline $\begin{array}{l}A S-76 \\
A S-77 \\
S E-73 \\
S E-75 \\
S E-79\end{array}$ & $\begin{array}{r}26.32 \mathrm{H} \\
38.8 \mathrm{H} \\
7.15 \mathrm{H} \\
119.70 \mathrm{O} \\
6.5 \mathrm{Y}\end{array}$ & $\begin{array}{l}3.66 E+01 \\
7.09 E-01 \\
9.74 E+01 \\
3.59 E+01 \\
0.0\end{array}$ & $\begin{array}{l}3.53 E+01 \\
7.33 E-01 \\
9.24 E+01 \\
3.31 E+01 \\
0.0\end{array}$ & $\begin{array}{l}3.95 E+01 \\
7.98 E-01 \\
1.02 E+02 \\
3.58 E+01 \\
0.0\end{array}$ & $\begin{array}{l}4.56 E * 01 \\
1.07 E+00 \\
1.29 E+02 \\
5.24 E+01 \\
0.0\end{array}$ \\
\hline $\begin{array}{l}\theta R-77 \\
B R-80 \\
B R-80 M \\
B R-82 \\
B R-83\end{array}$ & $\begin{array}{r}57.04 \mathrm{H} \\
17.4 \mathrm{H} \\
4.42 \mathrm{H} \\
35.30 \mathrm{H} \\
2.39 \mathrm{H}\end{array}$ & $\begin{array}{l}2.83 E+01 \\
6.60 E \cdot 00 \\
5.66 E-01 \\
2.23 E * 02 \\
6.61 E-01\end{array}$ & $\begin{array}{l}2.66 E+01 \\
6.29 E+00 \\
7.83 E-01 \\
2.16 E+02 \\
6.28 E-01\end{array}$ & $\begin{array}{l}2.94 E+01 \\
7.04 E+00 \\
4.03 E-01 \\
2.42 E+02 \\
7.00 E-01\end{array}$ & $\begin{array}{l}3.79 E+01 \\
8.29 E+00 \\
4.49 E+00 \\
2.75 E+02 \\
8.30 E-01\end{array}$ \\
\hline $\begin{array}{l}8 R-84 \\
8 R-85 \\
K R-79 \\
K R-81 \\
K R-834\end{array}$ & $\begin{array}{r}31.80 \mathrm{M} \\
172 \mathrm{~S} \\
35.04 \mathrm{H} \\
2.1 E 5 \mathrm{Y} \\
1.83\end{array}$ & $\begin{array}{l}1.33 E+02 \\
5.5 B E+00 \\
2.25 E+01 \\
9.54 E-01 \\
1.29 E-02\end{array}$ & $\begin{array}{l}1.34 E+02 \\
5.44 E+00 \\
2.12 E+01 \\
8.49 E-01 \\
8.08 E-04\end{array}$ & $\begin{array}{l}1.49 E+02 \\
6.12 E+00 \\
2.35 E \cdot 01 \\
9.23 E-01 \\
1.74 E-03\end{array}$ & $\begin{array}{l}1.69 E+02 \\
6.85 E+00 \\
3.04 E+01 \\
2.68 E+00 \\
5.77 E-01\end{array}$ \\
\hline $\begin{array}{l}K R-85 \\
K R-85.4 \\
K R-87 \\
K R-8 B \\
K R-89\end{array}$ & $\begin{array}{r}10.72 \mathrm{Y} \\
4.48 \mathrm{H} \\
76.3 \mathrm{M} \\
2.84 \mathrm{H} \\
3.16 \mathrm{M}\end{array}$ & $\begin{array}{l}1.9 B E-01 \\
1.48 E+01 \\
6.20 E \div 01 \\
1.46 E+02 \\
1.40 E+02\end{array}$ & $\begin{array}{l}1.88 E-01 \\
1.36 E+01 \\
6.17 E+01 \\
1.49 E+02 \\
1.40 E+02\end{array}$ & $\begin{array}{l}2.10 E-01 \\
1.46 E+01 \\
6.86 E \cdot 01 \\
1.66 E+02 \\
1.56 E+02\end{array}$ & $\begin{array}{l}2.49 E-01 \\
2.23 E+01 \\
1.96 E+01 \\
1.80 E+02 \\
1.78 E+02\end{array}$ \\
\hline $\begin{array}{l}K R-90 \\
R B-81 \\
R B-82 \\
R B-83 \\
R B-84\end{array}$ & 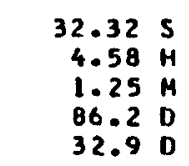 & $\begin{array}{l}1.01 E+02 \\
5.45 E+01 \\
9.68 E+01 \\
4.42 E+01 \\
7.76 E+01\end{array}$ & $\begin{array}{l}9.97 E+01 \\
5.12 E+01 \\
9.21 E+01 \\
4.20 E+01 \\
7.47 E+01\end{array}$ & $\begin{array}{l}1.12 E+02 \\
5.65 E+01 \\
1.03 E+02 \\
4.68 E+01 \\
8.38 E+01\end{array}$ & $\begin{array}{l}1.29 E+02 \\
7.28 E+01 \\
1.22 E+02 \\
5.12 E+01 \\
9.69 E+01\end{array}$ \\
\hline $\begin{array}{l}R \theta-86 \\
R B-87 \\
R B-8 B \\
R B-89 \\
R B-90\end{array}$ & $\begin{array}{r}18.66 \mathrm{O} \\
4.73810 \mathrm{Y} \\
17.8 \mathrm{M} \\
15.44 \mathrm{M} \\
157 \mathrm{~S}\end{array}$ & $\begin{array}{l}7.68 E+00 \\
0.0 \\
4.81 E+01 \\
1.60 E+02 \\
1.46 E+02\end{array}$ & $\begin{array}{l}7.53 E \cdot 00 \\
0.0 \\
4.87 E+01 \\
1.60 E+02 \\
1.4 B E+02\end{array}$ & $\begin{array}{l}B .49 E \cdot 00 \\
0.0 \\
5.46 E+02 \\
1.80 E * 02 \\
1.63 E+02\end{array}$ & $\begin{array}{l}9.40 E+00 \\
0.0 \\
6.09 E+01 \\
2.00 E+02 \\
1.09 E+02\end{array}$ \\
\hline $\begin{array}{l}R \theta-90 M \\
S R-82 \\
S R-85 \\
S R-85 M \\
S R-87 M\end{array}$ & $\begin{array}{rr}258 & \mathrm{~S} \\
25.0 & \mathrm{D} \\
64.84 & \mathrm{O} \\
67.66 \mathrm{M} \\
2.805 \mathrm{H}\end{array}$ & $\begin{array}{l}2.43 E+02 \\
5.25 E-02 \\
4.53 E+01 \\
2.02 E+01 \\
2.91 E+01\end{array}$ & $\begin{array}{l}2.44 E+02 \\
8.59 E-05 \\
4.30 E+01 \\
1.04 E+01 \\
2.72 E+01\end{array}$ & $\begin{array}{l}2.12 E+02 \\
6.69 E-03 \\
4.79 E+01 \\
1.98 E+01 \\
3.00 E 001\end{array}$ & $\begin{array}{l}3.08 E+02 \\
1.99 E+00 \\
5.91 E+01 \\
2.89 E+01 \\
3.81 E+01\end{array}$ \\
\hline
\end{tabular}

$\begin{array}{ccc}\text { SMPPE } & \text { LOHER } \\ \text { HEART INTELTINE } & \text { LARGE } & \text { LARGE } \\ \text { INTSTIME }\end{array}$

$1.11 E-03 \quad 1.48 E-05$

INTESTINE INTESTINE KIONEYS

3.50E-03

1.08E-05

1.32E+02 $1.55 \mathrm{E}+02$

$\begin{array}{lll}1.55 E+02 & 1.43 E+02 & 9.15 E+01\end{array}$

3.38E-01 $3.17 E-01$

3.79E-01
$6.71 E+01$

3.41E-01 4. $90 E-0$

$3.47 E+01 \quad 3.15 E+01$

$3.69 E+01$

$6.19 E+01$

6. $71 E+01$

$\begin{array}{lll} & \\ 9.45 E-01 & 6.40 E-01 & 7.57 E-01 \\ 980.08 E+01 & 9.53 E+0\end{array}$

$2.88 E+01$

$3.44 E+0$

$3.40 E+01$
$7.07 E-01$

$7.56 E-0$

$2.60 E+01 \quad 2.34 E+01$

$6.17 E+00 \quad 5.56 E+00$

3. BOE-01 4.24E-O

6. $55 E+00$
$5.98 E-01$

O.

9.58E+ 01 0.0

$\begin{array}{ll}.56 E+01 & 2.76 E+01 \\ 6.04 E+00 & 6.55 E+00\end{array}$

$1.55 E+00$
$1.07 E+00$

$\begin{array}{lllll}2.13 E+02 & 1.93 E+02 & 2.26 E+02 & 2.08 E+02 & 2.26 E+02 \\ 6.14 E-01 & 5.53 E-01 & 6.52 E-01 & 6.02 E-01 & 6.52 E-01\end{array}$

$\begin{array}{ll}1.24 E+02 \\ .37 E+00\end{array} \quad 4.89 E+00$

$5.72 E+00$

$1.31 E+02$

$1.43 E+02$
$5.70 E+00$

$2.08 E+01$ 1.87E+01 2.20E+01 2.04E+01 2.20E+0I

. $32 E-01$ 1.42E-0I $8.75 E-01$

$8.36 E-01$

8. $515-0$

$1.04 E-01 \quad 1.65 E-01$

$1.95 \mathrm{E}-01$

$1.00 E-01$

1.

1.

b.65et01

$1.39 E+02 \quad 1.28 E+02 \quad 1.47 E+02 \quad 1.36 E+02 \quad 1.49 E+02$

$\begin{array}{lll}9.06 E+01 & 9.03 E+01 & 1.05 E+02 \\ 5.00 E+01 & 4.49 E+01 & 5.31 E+01\end{array}$

\begin{tabular}{ll}
$9.67 E+01$ & $1.05 E+02$ \\
\hline $.92 E+01$ & $5.30 E * 01$
\end{tabular}

.01E+01 B.11E+01 9.56E+01

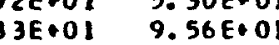

$7.45 E+00 \quad 6.81 E+00$
0.0

$7.30 E+00$

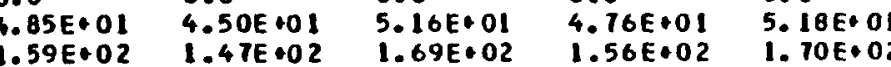

$\begin{array}{lllll}1.59 E+02 & 1.47 E+02 & 1.69 E+02 & 1.56 E+02 & 1.70 E+02 \\ 1.48 E+02 & 1.39 E+02 & 1.57 E+02 & 1.46 E+02 & 1.62 E+02\end{array}$

$2.43 E+02 \quad 2.26 E+02$

$2.58 \mathrm{E}+02$

$2.39 \mathrm{E}+02 \quad 2.62 \mathrm{E}+02$

$9.76 E-03 \quad 1.01 E-0$

1. BOE+01 $1.61 \mathrm{E}+01$

$2.83 \mathrm{E}-02 \quad 2.16 \mathrm{E}-03$

$1.79 E+01$ 1.89E+O

3. $81 E+01$

2.39E+01 2.81E+01 


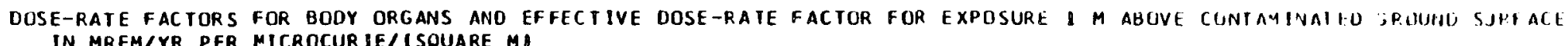
IN MREM/YR PER MICROCUR IE/I SOUARE MI

\begin{tabular}{|c|c|c|c|c|c|c|c|c|c|c|}
\hline NUCLIOE & HALF-LIFE & LIVER & LUNGS & HARROW & $\begin{array}{c}\text { P.EO } \\
\text { MARROH }\end{array}$ & OVARIES & ANCREAS & ELLERON & SPLEEN & cromacy \\
\hline $\begin{array}{l}\text { GE-71 } \\
\text { GE } 77 \\
A S-72 \\
A S-73 \\
\text { AS }-74\end{array}$ & 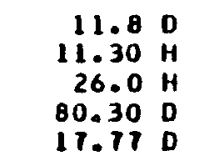 & $\begin{array}{l}-05 \\
01 \\
01 \\
02 \\
-01 \\
01 \\
01\end{array}$ & $\begin{array}{l}3 E-03 \\
9 E+01 \\
8 E+02 \\
8 E-01\end{array}$ & $\begin{array}{l}.08 E-03 \\
.10 E+02 \\
.76 E+02 \\
.60 E-01\end{array}$ & $\begin{array}{l}21 E-03 \\
55 E+01 \\
61 E+02 \\
41 E-01 \\
03 E+01\end{array}$ & $\begin{array}{l}3 E-03 \\
1 E+01 \\
2 E+02 \\
0 E-01\end{array}$ & $\begin{array}{l}6 E-03 \\
6 E+01 \\
6 E+02 \\
6 E-01 \\
6 E+01\end{array}$ & $\begin{array}{l}9 E-02 \\
2 E+02 \\
9 E+02 \\
1 E-01 \\
3 E+01\end{array}$ & $\begin{array}{l}93 E-03 \\
56 E+01 \\
44 E+02 \\
.66 E-01 \\
.25 E+01\end{array}$ & $\begin{array}{l}5 E-03 \\
3 E+01 \\
6 E+02 \\
5 E-01 \\
4 E+01\end{array}$ \\
\hline $\begin{array}{l}\text { AS-76 } \\
\text { AS-71 } \\
\text { SE-73 } \\
\text { SE-75 } \\
\text { SE-79 }\end{array}$ & $\begin{array}{r}26.32 \mathrm{H} \\
38.0 \mathrm{H} \\
7.15 \mathrm{H} \\
119.78 \mathrm{O} \\
6.5 \mathrm{~F}\end{array}$ & $\begin{array}{l}3.51 E+01 \\
1.29 E-01 \\
9.19 E+01 \\
3.30 E+01 \\
0.0\end{array}$ & $\begin{array}{l}3.75 E \bullet 01 \\
7.89 E-01 \\
9.89 E+01 \\
3.59 E+01 \\
0.0\end{array}$ & $\begin{array}{l}4.16 E \cdot 01 \\
1.01 E+00 \\
1.19 E+02 \\
4.88 E+01 \\
0.0\end{array}$ & $\begin{array}{l}3.82 E+01 \\
7.99 E-01 \\
9.94 E+01 \\
3.55 E+01 \\
0.0\end{array}$ & $\begin{array}{l}3.37 E+01 \\
6.68 E-01 \\
8.6 B E 01\end{array}$ & $\begin{array}{l}3.03 E+01 \\
6.26 E-01 \\
7.83 E \cdot 01 \\
2.01 E \cdot 01 \\
0.0\end{array}$ & $\begin{array}{l}4.23 E+01 \\
1.03 E+00 \\
1.21 E+02 \\
4.98 E+01 \\
0.0\end{array}$ & & $\begin{array}{l}3.46 E+01 \\
7.20 E-01 \\
9.04 E+01 \\
3.26 E+01 \\
0.0\end{array}$ \\
\hline $\begin{array}{l}B R-77 \\
B R-80 \\
B R-8 O M \\
B R-82 \\
B R-83\end{array}$ & $\begin{array}{r}57.04 \mathrm{H} \\
17.4 \mathrm{H} \\
4.42 \mathrm{H} \\
35.30 \mathrm{H} \\
2.39 \mathrm{H}\end{array}$ & $\begin{array}{l}2.65 E \cdot 01 \\
6.26 E+00 \\
6.12 E-01 \\
2.15 E+02 \\
6.24 E-01\end{array}$ & $\begin{array}{l}2.05 E \cdot 01 \\
6.70 E \cdot 00 \\
7.32 E-01 \\
2.29 E+02 \\
6.70 E-01\end{array}$ & $\begin{array}{l}3.40 \mathrm{E}+01 \\
7.56 \mathrm{E}+00 \\
1.25 \mathrm{E}+00 \\
2.50 \mathrm{0}+02 \\
7.67 \mathrm{E}-01\end{array}$ & $\begin{array}{l}2.91 E+01 \\
6.85 E+00 \\
2.92 E-01 \\
2.33 E+02 \\
6.85 E-01\end{array}$ & $\begin{array}{l}2.50 E+01 \\
6.05 E+00 \\
5.24 E-01 \\
2.07 E+02 \\
6.01 E-01\end{array}$ & $\begin{array}{l}2.2 B E+01 \\
5.39 E+00 \\
3.59 E-01 \\
1.85 E+02 \\
5.37 E-01\end{array}$ & $\begin{array}{l}3.47 E+01 \\
7.69 E+00 \\
1.31 E+00 \\
2.54 E+02 \\
7.79 E-01\end{array}$ & & $\begin{array}{l}2.62 E+01 \\
6.18 E+00 \\
5.42 E-01 \\
2.12 E+02 \\
6.16 E-01\end{array}$ \\
\hline $\begin{array}{l}B R-84 \\
B R-85 \\
K R-79 \\
K R-81 \\
K R-834\end{array}$ & $\begin{array}{r}31.80 \mathrm{M} \\
112 \\
35.04 \\
2.1 E 5 \\
1.83 \\
\mathrm{H}\end{array}$ & $\begin{array}{l}1.34 E+02 \\
5.41 E+00 \\
2.11 E+01 \\
8.46 E-01 \\
1.12 E-03\end{array}$ & $\begin{array}{l}1.41 E \bullet 02 \\
5.76 E \bullet 00 \\
2.27 E \bullet 01 \\
9.40 E-01 \\
1.20 E-02\end{array}$ & $\begin{array}{l}1.47 E+02 \\
6.20 E+00 \\
2.69 E+01 \\
1.20 E \bullet 00 \\
1.14 E-02\end{array}$ & $\begin{array}{l}1.44 E+02 \\
5.85 E+00 \\
2.32 E+01 \\
9.49 E-01 \\
4.06 E-03\end{array}$ & & $\begin{array}{l}1.17 E+02 \\
4.67 E+00 \\
1.82 E+01 \\
7.47 E-01 \\
7.82 E-03\end{array}$ & $\begin{array}{l}1.50 E+02 \\
6.31 E+00 \\
2.74 E+01 \\
1.25 E+00 \\
1.97 E-02\end{array}$ & $\begin{array}{l}1.29 E+02 \\
5.24 E+00 \\
2.07 E+01 \\
6.47 E-01 \\
4.50 E-03\end{array}$ & $\begin{array}{l}5.34 E+00 \\
2.09 E+01 \\
8.43 E-01 \\
4.54 E-03\end{array}$ \\
\hline $\begin{array}{l}K R-85 \\
K R-85 M \\
K R-87 \\
K R-88 \\
K R-89\end{array}$ & $\begin{array}{r}10.72 \mathrm{Y} \\
4.48 \mathrm{H} \\
76.3 \mathrm{M} \\
2.84 \mathrm{H} \\
3.16 \mathrm{M}\end{array}$ & $\begin{array}{l}1.07 E-01 \\
1.35 E+01 \\
6.18 E+01 \\
1.50 E+02 \\
1.40 E+02\end{array}$ & $\begin{array}{l}6.54 E+01 \\
1.57602 \\
1.48 E+02\end{array}$ & $\begin{array}{l}2.31 E-01 \\
2.13 E+01 \\
7.06 E+01 \\
1.64 E+02 \\
1.58 E+02\end{array}$ & & & & & & $\begin{array}{l}\text { 1. } 34 E+08 \\
6.09 E+01 \\
1.47 E+02 \\
1.38 E+02\end{array}$ \\
\hline $\begin{array}{l}K R-90 \\
R B-81 \\
R B-82 \\
R B-83 \\
R B-84\end{array}$ & 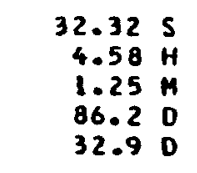 & $\begin{array}{l}9.96 E+01 \\
5.09 E+01 \\
9.16 E+01 \\
4.17 E+01 \\
7.43 E+01\end{array}$ & & $\begin{array}{l}1.16 E+02 \\
6.63 E+01 \\
1.12 E \cdot 02 \\
5.13 E+01 \\
8.74 E \cdot 01\end{array}$ & $\begin{array}{l}1.07 E+02 \\
5.57 E+01 \\
1.00 E+02 \\
4.58 E+01 \\
0.07 E \cdot 01\end{array}$ & & & & & $\begin{array}{l}5.03 \mathrm{E}+01 \\
9.04 \mathrm{E}+01 \\
4.12 \mathrm{E}+01 \\
7.34 \mathrm{E}+01\end{array}$ \\
\hline $\begin{array}{l}R B-86 \\
R B-87 \\
R B-88 \\
R B-89 \\
R B-90\end{array}$ & $\begin{array}{r}18.66 \\
4.73 E 10 \\
17.8 \mathrm{H} \\
15.44 \mathrm{M} \\
157 \mathrm{~S}\end{array}$ & $\begin{array}{l}1.50 E+00 \\
0.0 \\
4.88 E+01 \\
1.60 E+02 \\
1.48 E+02\end{array}$ & $\begin{array}{l}0.0 \\
5.15 E * 01 \\
1.69 E * 02 \\
1.55 E * 02\end{array}$ & $\begin{array}{l}8.46 E+00 \\
0.0 \\
5.34 E+01 \\
1.78 E \cdot 02 \\
1.61 E+02\end{array}$ & $\begin{array}{l}8.05 E \cdot 00 \\
0.0 \\
5.21 E+01 \\
1.72 E+02 \\
1.61 E+02\end{array}$ & $\begin{array}{l}1.23 E+00 \\
0.0 \\
4.51 E+01 \\
1.50 E+02 \\
1.34 E+02\end{array}$ & $\begin{array}{l}6.48 E+00 \\
0.0 \\
4.25 E+01 \\
1.39 E+02 \\
1.32 E+02\end{array}$ & $\begin{array}{l}0.61 E+00 \\
0.0 \\
5.43 E+01 \\
1.81 E+02 \\
1.63 E+02\end{array}$ & $\begin{array}{l}7.25 E+00 \\
0.0 \\
4.69 E+01 \\
8.54 E+02 \\
1.44 E+02\end{array}$ & $\begin{array}{l}0.0 \\
4.78 E+0 \\
8.57 E+0 \\
\text { B. } 49 E+0\end{array}$ \\
\hline $\begin{array}{l}R B-90 M \\
S R-82 \\
S R-85 \\
S R-85 M \\
S R-874\end{array}$ & $\begin{array}{rl}258 & \mathrm{~S} \\
25.0 & \mathrm{D} \\
64.84 & \mathrm{D} \\
67.66 & \mathrm{H} \\
2.805 & \mathrm{H}\end{array}$ & $\begin{array}{l}2.45 E+02 \\
3.24 E-03 \\
4.28 E+01 \\
1.84 E+01 \\
2.71 E+01\end{array}$ & $\begin{array}{l}2.57 E \cdot 02 \\
5.02 E-02 \\
4.59 E+01 \\
2.00 E+01 \\
2.92 E+01\end{array}$ & $\begin{array}{l}2.70 E \cdot 02 \\
4.97 E-02 \\
5.28 E+01 \\
2.71 E+01 \\
3.52 E \cdot 01\end{array}$ & $\begin{array}{l}2.63 E+02 \\
1.69 E-02 \\
4.70 E+01 \\
2.01 E * 01 \\
3.01 E+01\end{array}$ & $\begin{array}{l}2.25 E+02 \\
2.68 E-02 \\
4.11 E * 01 \\
1.63 E+01 \\
2.54 E+01\end{array}$ & $\begin{array}{l}2.14 E+02 \\
3.20 E-02 \\
3.68 E+01 \\
1.58 E+01 \\
2.34 E+01\end{array}$ & $\begin{array}{l}2.74 E+02 \\
8.20 E-02 \\
5.37 E+01 \\
2.77 E+01 \\
3.58 E+01\end{array}$ & $\begin{array}{l}2.36 E+02 \\
1.91 E-02 \\
4.18 E+01 \\
1.83 E+01 \\
2.66 E+01\end{array}$ & $\begin{array}{l}2.42 E+02 \\
1.95 E-02 \\
4.22 E+01 \\
1.81 E+01 \\
2.67 E+01\end{array}$ \\
\hline
\end{tabular}




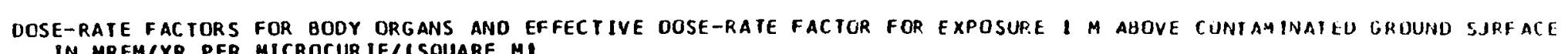

\begin{tabular}{|c|c|c|c|c|c|c|c|c|c|}
\hline NUCLIDE & HALF-LIFE & TESTES & THYMUS & THYROID & TERUS & EFFECTIVE & $\begin{array}{c}\text { SKIN } \\
\text { (PHOTONI }\end{array}$ & $\begin{array}{l}\text { SKIN } \\
\text { IELECTRONI }\end{array}$ & $\begin{array}{l}\text { SKIN } \\
\text { ITOTALI }\end{array}$ \\
\hline $\begin{array}{l}\text { GE-71 } \\
\text { GE-77 } \\
A S-72 \\
A S-73 \\
A S-74\end{array}$ & 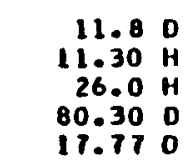 & $\begin{array}{l}6.15 E-02 \\
1.26 E+02 \\
2.11 E+02 \\
9.86 E-01 \\
9.21 E \bullet 01\end{array}$ & $\begin{array}{l}1.54 E-06 \\
9.73 E+01 \\
1.63 E+02 \\
5.89 E-01 \\
7.04 E+01\end{array}$ & $\begin{array}{l}4.30 E-03 \\
1.19 E+02 \\
1.97 E+02 \\
1.78 E-01 \\
8.58 E+01\end{array}$ & $\begin{array}{l}6.11 E-07 \\
7.70 E+01 \\
1.29 E+02 \\
2.99 E-01 \\
5.59 E+01\end{array}$ & $\begin{array}{l}1.17 E-01 \\
1.00 E .02 \\
1.00 E+02 \\
9.73 E-01 \\
1.07 E+01\end{array}$ & $\begin{array}{l}1.40 E+00 \\
1.43 E+02 \\
2.42 E+02 \\
4.72 E+00 \\
1.06 E+02\end{array}$ & $\begin{array}{l}0.0 \\
6.43 E+02 \\
1.12 E+03 \\
0.0 \\
2.31 E+02\end{array}$ & $\begin{array}{l}1.40 E+00 \\
7.86 E+02 \\
1.36 E+03 \\
4.72 E+00 \\
3.37 E+02\end{array}$ \\
\hline $\begin{array}{l}\text { AS }-76 \\
A S-77 \\
S E-73 \\
S E-75 \\
S E-79\end{array}$ & $\begin{array}{r}26.32 \mathrm{H} \\
38.8 \mathrm{H} \\
7.15 \mathrm{H} \\
119.78 \mathrm{O} \\
6.5 E 4\end{array}$ & $\begin{array}{l}4.99 E+01 \\
1.07 E+00 \\
1.34 E+02 \\
4.93 E+01 \\
0.0\end{array}$ & $\begin{array}{l}3.87 E+01 \\
8.14 E-01 \\
1.01 E+02 \\
3.74 E+01 \\
0.0\end{array}$ & $\begin{array}{l}4.67 E+01 \\
1.01 E+00 \\
1.25 E+02 \\
4.71 E+01 \\
0.0\end{array}$ & $\begin{array}{l}3.08 E+01 \\
6.42 E-01 \\
8.01 E+01 \\
2.91 E+01 \\
0.0\end{array}$ & $\begin{array}{l}4.27 E+01 \\
9.30 E-01 \\
1.15 E+02 \\
4.32 E+01 \\
0.0\end{array}$ & $\begin{array}{l}5.73 E+01 \\
1.20 E \cdot 00 \\
1.53 E+02 \\
5.65 E \cdot 01 \\
0.0\end{array}$ & $\begin{array}{l}1.14 E+03 \\
1.03 E+02 \\
3.68 E+02 \\
0.0 \\
0.0\end{array}$ & $\begin{array}{l}1.20 E+03 \\
1.05 E+02 \\
5.22 E+02 \\
5.65 E \cdot 01 \\
0.0\end{array}$ \\
\hline $\begin{array}{l}B R-77 \\
B R-80 \\
B R-80 M \\
B R-82 \\
B R-83\end{array}$ & $\begin{array}{r}57.04 \mathrm{H} \\
17.4 \mathrm{H} \\
4.42 \mathrm{H} \\
35.30 \mathrm{H} \\
2.39 \mathrm{H}\end{array}$ & $\begin{array}{l}3.85 E+01 \\
0.97 E+00 \\
1.98 E+00 \\
3.04 E+02 \\
8.98 E-01\end{array}$ & $\begin{array}{l}2.93 E+01 \\
6.89 E \cdot 00 \\
9.82 E-01 \\
2.39 E+02 \\
6.82 E-01\end{array}$ & $\begin{array}{l}3.60 E+01 \\
8.37 E+00 \\
1.38 E+00 \\
2.87 E+02 \\
8.33 E-01\end{array}$ & $\begin{array}{l}2.32 E+01 \\
5.45 E+00 \\
3.63 E-01 \\
1.8 B E+02 \\
5.45 E-01\end{array}$ & $\begin{array}{l}3.33 E+01 \\
1.67 E+00 \\
1.65 E+00 \\
2.60 E+02 \\
7.68 E-01\end{array}$ & $\begin{array}{l}4.60 E+01 \\
1.04 E+01 \\
6.54 E \bullet 00 \\
3.50 E \cdot 02 \\
1.03 E \cdot 00\end{array}$ & $\begin{array}{l}0.0 \\
7.57 E+02 \\
0.0 \\
5.98 E+00 \\
2.32 E+02\end{array}$ & $\begin{array}{l}4.60 E+01 \\
7.67 E+02 \\
6.54 E+00 \\
3.56 E+02 \\
2.33 E+02\end{array}$ \\
\hline $\begin{array}{l}B R-84 \\
B R-85 \\
K R-79 \\
K R-81 \\
K R-834\end{array}$ & $\begin{array}{r}31.00 \\
172 \mathrm{M} \\
35.04 \mathrm{H} \\
2.165 \mathrm{Y} \\
1.03 \mathrm{H}\end{array}$ & $\begin{array}{l}1.85 E+02 \\
7.61 E+00 \\
3.07 E+01 \\
1.44 E+00 \\
8.78 E-02\end{array}$ & $\begin{array}{l}1.46 E+02 \\
6.05 E+00 \\
2.33 E \times 01 \\
9.40 E-01 \\
1.04 E-03\end{array}$ & $\begin{array}{l}1.71 E+02 \\
1.23 E+00 \\
2.86 E+01 \\
1.20 E+00 \\
1.30 E-02\end{array}$ & $\begin{array}{l}1.19 E+02 \\
4.13 E+00 \\
1.05 E+01 \\
7.48 E-01 \\
2.95 E-04\end{array}$ & $\begin{array}{l}1.59 E+02 \\
6.52 E+00 \\
2.65 E+01 \\
1.36 E+00 \\
1.14 E-01\end{array}$ & $\begin{array}{l}2.08 E+02 \\
8.79 E+00 \\
3.74 E+01 \\
4.08 E+00 \\
1.08 E+00\end{array}$ & $\begin{array}{l}1.32 E+03 \\
1.07 E+03 \\
7.78 E+00 \\
0.0 \\
0.0\end{array}$ & $\begin{array}{l}1.53 E+03 \\
1.08 E+03 \\
4.52 E+01 \\
4.08 E+00 \\
1.08 E+00\end{array}$ \\
\hline $\begin{array}{l}K R-85 \\
K R-85 M \\
K R-87 \\
K R-88 \\
K R-89\end{array}$ & $\begin{array}{r}10.72 \mathrm{Y} \\
4.48 \mathrm{H} \\
76.3 \mathrm{H} \\
2.84 \mathrm{H} \\
3.16 \mathrm{M}\end{array}$ & $\begin{array}{l}2.69 E-01 \\
2.05 E * 01 \\
0.59 E+01 \\
2.05 E \cdot 02 \\
1.94 E+02\end{array}$ & $\begin{array}{l}2.04 E-01 \\
1.57 E+01 \\
6.69 E+01 \\
1.61 E+02 \\
1.53 E+02\end{array}$ & $\begin{array}{l}2.49 \mathrm{E}-01 \\
1.99 \mathrm{E}+01 \\
1.92 \mathrm{E}+01 \\
1.89 \mathrm{E}+02 \\
1.80 \mathrm{O}+02\end{array}$ & $\begin{array}{l}1.63 E-01 \\
1.19 E+01 \\
5.51 E+01 \\
1.34 E+02 \\
1.24 E+02\end{array}$ & $\begin{array}{l}2.30 E-01 \\
1.00 E+01 \\
1.40 E+01 \\
1.77 E+02 \\
1.67 E+02\end{array}$ & $\begin{array}{l}3.08 E-01 \\
2.25 E \cdot 01 \\
9.69 E+01 \\
2.31 E+02 \\
2.20 E \cdot 02\end{array}$ & $\begin{array}{l}1.41 E+02 \\
1.49 E+02 \\
1.42 E+03 \\
2.69 E+02 \\
1.46 E+03\end{array}$ & $\begin{array}{l}1.42 E \cdot 02 \\
1.12 E+02 \\
1.52 E+03 \\
5.00 E+02 \\
1.68 E+03\end{array}$ \\
\hline $\begin{array}{l}K R-90 \\
R B-81 \\
R B-82 \\
R B-83 \\
R B-84\end{array}$ & 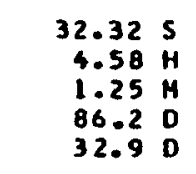 & $\begin{array}{l}1.40 E+02 \\
7.42 E+01 \\
1.32 E+02 \\
6.03 E+01 \\
1.06 E+02\end{array}$ & $\begin{array}{l}1.10 E+02 \\
5.62 E+01 \\
1.00 E+02 \\
4.56 E+01 \\
0.27 E+01\end{array}$ & $\begin{array}{l}1.32 E+02 \\
6.93 E+01 \\
1.22 E+02 \\
5.58 E+01 \\
9.96 E+01\end{array}$ & $\begin{array}{l}8.01 E+01 \\
4.46 E+01 \\
0.00 E+01 \\
3.64 E+01 \\
6.48 E+01\end{array}$ & $\begin{array}{l}1.20 E+02 \\
6.40 E+01 \\
1.13 E+02 \\
5.17 E+01 \\
9.04 E+01\end{array}$ & $\begin{array}{l}1.60 E+02 \\
8.69 E+01 \\
1.51 E+02 \\
1.20 E+01 \\
1.24 E+02\end{array}$ & $\begin{array}{l}1.40 E+03 \\
1.33 E+02 \\
1.53 E \bullet 03 \\
0.0 \\
1.51 E \bullet 02\end{array}$ & $\begin{array}{l}1.56 E * 03 \\
2.20 E \bullet 02 \\
1.6 B E \bullet 03 \\
7.20 E+01 \\
2.75 E+02\end{array}$ \\
\hline $\begin{array}{l}R B-86 \\
R \theta-87 \\
R B-88 \\
R B-89 \\
R B-90\end{array}$ & $\begin{array}{r}18.66 \mathrm{O} \\
4.73610 \mathrm{Y} \\
27.8 \mathrm{H} \\
15.44 \mathrm{H} \\
157 \mathrm{~S}\end{array}$ & $\begin{array}{l}1.05 E+01 \\
0.0 \\
6.70 E+01 \\
2.22 E+02 \\
2.04 E+02\end{array}$ & $\begin{array}{l}8.43 E+00 \\
0.0 \\
5.31 E+01 \\
1.76 E+02 \\
1.62 E+02\end{array}$ & $\begin{array}{l}1.00 E+01 \\
0.0 \\
6.23 E+01 \\
2.08 E+02 \\
1.85 E+02\end{array}$ & $\begin{array}{l}6.57 E+00 \\
0.0 \\
4.37 E+01 \\
1.42 E+02 \\
1.31 E+02\end{array}$ & $\begin{array}{l}8.99 E+00 \\
0.0 \\
5.77 E+01 \\
1.90 E+02 \\
1.76 E+02\end{array}$ & $\begin{array}{l}1.21 E+01 \\
0.0 \\
7.61 E+01 \\
2.53 E+02 \\
2.24 E+02\end{array}$ & $\begin{array}{l}6.86 E+02 \\
0.0 \\
2.24 E+03 \\
1.07 E+03 \\
2.10 E+03\end{array}$ & $\begin{array}{l}6.9 B E+02 \\
0.0 \\
2.32 E+03 \\
1.32 E+03 \\
2.33 E+03\end{array}$ \\
\hline $\begin{array}{l}R B-90 M \\
S R-82 \\
S R-85 \\
S R-85 M \\
S R-87 M\end{array}$ & $\begin{array}{r}258 \mathrm{~S} \\
25.0 \mathrm{D} \\
64.84 \mathrm{D} \\
67.66 \mathrm{M} \\
2.805 \mathrm{H}\end{array}$ & $\begin{array}{l}3.38 E+02 \\
3.58 E-01 \\
6.20 E+01 \\
2.73 E+01 \\
3.94 E+01\end{array}$ & $\begin{array}{l}2.67 E+02 \\
6.32 E-04 \\
4.66 E+01 \\
2.06 E+01 \\
2.96 E+01\end{array}$ & $\begin{array}{l}3.12 E+02 \\
5.83 E-02 \\
5.71 E+01 \\
2.61 E+01 \\
3.66 E+01\end{array}$ & $\begin{array}{l}2.17 E+02 \\
1.78 E-0 E \\
3.73 E+01 \\
1.62 E+01 \\
2.38 E+01\end{array}$ & $\begin{array}{l}2.91 E+02 \\
4.11 E-01 \\
5.31 E+01 \\
2.40 E+01 \\
3.40 E+01\end{array}$ & $\begin{array}{l}3.80 E+02 \\
3.53 E+00 \\
7.40 E+01 \\
3.06 E+01 \\
4.51 E+01\end{array}$ & $\begin{array}{l}1.49 E+03 \\
0.0 \\
3.43 E+00 \\
0.0 \\
7.17 E+01\end{array}$ & $\begin{array}{l}1.87 E+03 \\
3.53 E+00 \\
7.75 E+01 \\
3.06 E+01 \\
1.17 E+02\end{array}$ \\
\hline
\end{tabular}



DOSE-RATE FACTORS FOR BODY ORGANS AND EFFECTIVE DOSE-RATE FAC TOR FOR EXPOSURE I H ABOVE CONTAMINATEO SFOUNO SJRFACE
IN MREM/YR PER HICROCUR IE/ISOUARE MI

\begin{tabular}{|c|c|c|c|c|c|c|c|c|c|c|}
\hline NUCL IDE & HALF-LIFE & ADRENALS & BL ADDER & BP.AIN & BREAST & HE AR T & $\begin{array}{l}\text { SHALL } \\
\text { INTESTINE }\end{array}$ & $\begin{array}{c}\text { UPPER } \\
\text { LARGE } \\
\text { INTESTINE }\end{array}$ & $\begin{array}{c}\text { LOWER } \\
\text { LARGE } \\
\text { INTESTINE }\end{array}$ & K IONEYS \\
\hline $\begin{array}{l}S R-89 \\
S R-90 \\
S R-91 \\
S R-92 \\
S R-93\end{array}$ & $\begin{array}{rl}50.55 & \mathrm{D} \\
20.6 & \mathrm{Y} \\
9.5 & \mathrm{H} \\
2.71 & \mathrm{H} \\
7.3 \mathrm{M}\end{array}$ & $\begin{array}{l}1.17 E-02 \\
0.0 \\
5.81 E+01 \\
1.01 E+02 \\
1.83 E+02\end{array}$ & $\begin{array}{l}1.13 E-02 \\
0.0 \\
5.64 E \bullet 01 \\
1.01 E+02 \\
1.79 E+02\end{array}$ & $\begin{array}{l}1.27 E-02 \\
0.0 \\
6.34 E \cdot 01 \\
1.13 E \bullet 02 \\
2.01 E+02\end{array}$ & $\begin{array}{l}1.42 E-02 \\
0.0 \\
7.13 E+01 \\
1.25 E+02 \\
2.29 E+02\end{array}$ & $\begin{array}{l}.12 E-02 \\
.0 \\
051 E+01 \\
0.95 E+01 \\
.77 E+02\end{array}$ & $\begin{array}{l}1.02 E-02 \\
0.0 \\
5.06 E+01 \\
9.19 E+01 \\
1.62 E+02\end{array}$ & $\begin{array}{l}1.19 E-02 \\
0.0 \\
5.93 E+01 \\
1.06 E+02 \\
1.88 E+02\end{array}$ & $\begin{array}{l}1.09 E-02 \\
0.0 \\
5.45 E+01 \\
9.77 E+01 \\
1.74 E+02\end{array}$ & $\begin{array}{l}1.19 E-02 \\
0.0 \\
5.91 E+01 \\
1.06 E+02 \\
1 . B 8 E+02\end{array}$ \\
\hline $\begin{array}{l}Y-86 \\
Y-87 \\
Y-88 \\
r-90 \\
Y-90 M\end{array}$ & $\begin{array}{r}14.74 \mathrm{H} \\
80.3 \mathrm{H} \\
106.60 \mathrm{D} \\
64.1 \mathrm{H} \\
3.19 \mathrm{H}\end{array}$ & $\begin{array}{l}2.92 E+02 \\
4.08 E+01 \\
2.08 E+02 \\
0.0 \\
5.75 E+01\end{array}$ & $\begin{array}{l}2.87 E+02 \\
3.86 E+01 \\
2.09 E+02 \\
0.0 \\
5.30 E+01\end{array}$ & $\begin{array}{l}3.21 E+02 \\
4.29 E+01 \\
2.35 E+02 \\
0.0 \\
5.90 E \bullet 01\end{array}$ & $\begin{array}{l}3.65 E+02 \\
5.38 E+01 \\
2.64 E+02 \\
0.0 \\
7.64 E+01\end{array}$ & $\begin{array}{l}2.83 E+02 \\
3.77 E+01 \\
2.08 E+02 \\
0.0 \\
5.25 E+01\end{array}$ & $\begin{array}{l}2.59 E+02 \\
3.39 E+01 \\
1.92 E+02 \\
0.0 \\
4.71 E+01\end{array}$ & $\begin{array}{l}3.01 E+02 \\
4.00 E+01 \\
2.21 E+02 \\
0.0 \\
5.56 E+01\end{array}$ & $\begin{array}{l}2.78 E+02 \\
3.70 E+01 \\
2.04 E+02 \\
0.0 \\
5.17 E+01\end{array}$ & $\begin{array}{l}3.01 E+02 \\
4.00 E \cdot 01 \\
2.22 E \cdot 02 \\
0.0 \\
5.56 E+01\end{array}$ \\
\hline $\begin{array}{l}\gamma-91 \\
\gamma-91 M \\
\gamma-92 \\
\gamma-93 \\
2 R-86\end{array}$ & $\begin{array}{r}58.51 \mathrm{D} \\
49.71 \mathrm{M} \\
3.54 \mathrm{H} \\
10.1 \mathrm{H} \\
16.5 \mathrm{H}\end{array}$ & $\begin{array}{l}2.81 E-01 \\
4.70 E+01 \\
2.07 E+01 \\
7.20 E+00 \\
2.54 E+01\end{array}$ & $\begin{array}{l}2.78 E-01 \\
4.47 E+01 \\
2.02 E+01 \\
7.08 E+00 \\
2.33 E+01\end{array}$ & $\begin{array}{l}3.14 E-01 \\
5.00 E+01 \\
2.28 E+01 \\
7.89 E+00 \\
2.51 E+01\end{array}$ & $\begin{array}{l}3.47 E-01 \\
5.88 E+01 \\
2.55 E+01 \\
9.23 E+00 \\
3.99 E+01\end{array}$ & $\begin{array}{l}2.75 E-01 \\
4.38 E+01 \\
2.00 E+01 \\
7.01 E+00 \\
2.26 E+01\end{array}$ & $\begin{array}{l}2.53 E-01 \\
3.94 E+01 \\
1.82 E+01 \\
6.42 E+00 \\
2.03 E .401\end{array}$ & $\begin{array}{l}2.93 E-01 \\
4.65 E+01 \\
2.13 E+01 \\
1.45 E+00 \\
2.40 E+01\end{array}$ & $\begin{array}{l}2.70 E-01 \\
4.29 E+01 \\
1.96 E+01 \\
6.89 E+00 \\
2.26 E+01\end{array}$ & $\begin{array}{l}2.92 E-01 \\
4.65 E+01 \\
2.12 E+01 \\
7.45 E+00 \\
2.42 E+01\end{array}$ \\
\hline $\begin{array}{l}2 R-88 \\
2 R-89 \\
2 R-93 \\
2 R-95 \\
2 R-97\end{array}$ & $\begin{array}{r}83.44 \\
78.43 \mathrm{H} \\
1.53 E 6 \\
64.02 \\
64 \\
16.90 \mathrm{H}\end{array}$ & $\begin{array}{l}3.49 E \cdot 01 \\
9.92 E \cdot 01 \\
0.0 \\
6.45 E+01 \\
1.48 E+01\end{array}$ & $\begin{array}{l}3.26 E+01 \\
9.59 E+01 \\
0.0 \\
6.20 E+01 \\
1.44 E+01\end{array}$ & $\begin{array}{l}3.59 E+01 \\
1.08 E+02 \\
0.0 \\
6.96 E+01 \\
1.62 E+01\end{array}$ & $\begin{array}{l}4.74 E \cdot 01 \\
1.24 E \cdot 02 \\
0.0 \\
7.90 E+01 \\
1.85 E+01\end{array}$ & $\begin{array}{l}3.18 E+01 \\
9.45 E+01 \\
0.0 \\
6.10 E+01 \\
1.42 E+01\end{array}$ & $\begin{array}{l}2.85 E+01 \\
8.56 E+01 \\
0.0 \\
5.51 E+01 \\
1.30 E+01\end{array}$ & $\begin{array}{l}3.36 E+01 \\
1.01 E+02 \\
0.0 \\
6.49 E+01 \\
1.51 E+01\end{array}$ & $\begin{array}{l}3.14 E+01 \\
9.25 E+01 \\
0.0 \\
5.97 E+01 \\
1.40 E+01\end{array}$ & $\begin{array}{l}\text { 3. } 37 E+01 \\
1.00 E+02 \\
0.0 \\
6.47 E+01 \\
1.51 E+01\end{array}$ \\
\hline $\begin{array}{l}N B-90 \\
N B-91 \\
N B-91 M \\
N B-92 \\
N B-92 M\end{array}$ & 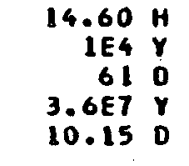 & $\begin{array}{l}3.21 E+02 \\
2.43 E-01 \\
3.42 E+00 \\
1.29 E+02 \\
8.14 E+01\end{array}$ & $\begin{array}{l}3.22 \\
1.44 \\
3.30 \\
1.24 \\
7.91\end{array}$ & $\begin{array}{l}3.60 E \\
3.74 \mathrm{E} \\
3.13 \mathrm{E} \\
1.40 E \\
8.90 E\end{array}$ & $\begin{array}{l}2.6 \\
6.0 \\
1.6 \\
1.0\end{array}$ & $\begin{array}{l}1.6 \\
3.2 \\
1.2 \\
7.8\end{array}$ & & & & $\begin{array}{l}\text { 3. } 42 \mathrm{E}+02 \\
\text { 1. } 65 \mathrm{E}-01 \\
\text { 3. } 49 \mathrm{E}+00 \\
1.30 \mathrm{E}+02 \\
\text { 8. } 30 \mathrm{E}+01\end{array}$ \\
\hline $\begin{array}{l}N B-93 M \\
N B-94 \\
N B-94 M \\
N B-95 \\
N 8-95 M\end{array}$ & 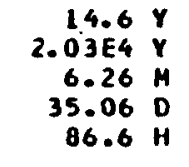 & $\begin{array}{l}1.87 E-02 \\
1.37 E+02 \\
4.28 E-01 \\
6.69 E+01 \\
5.62 E+00\end{array}$ & $\begin{array}{l}1.03 E-03 \\
1.32 E+02 \\
3.50 E-01 \\
6.44 E+01 \\
5.07 E+00\end{array}$ & $\begin{array}{l}3.59 E- \\
1.48 E 9 \\
4.02 E- \\
7.23 E 9 \\
5.46 E 4\end{array}$ & $\begin{array}{l}4.25 \\
1.68 \\
2.05 \\
8.15 \\
9.32\end{array}$ & $\begin{array}{l}4.74 \\
1.34 \\
3.55 \\
6.34 \\
4.99\end{array}$ & $\begin{array}{l}1.5 \\
1.1 \\
3.1 \\
5.1 \\
4.4\end{array}$ & $\begin{array}{l}1.3 \\
1.3 \\
3.6 \\
6.7 \\
5.2\end{array}$ & $\begin{array}{l}2 \\
2 \\
11 \\
1 \\
12\end{array}$ & $\begin{array}{l}5.83 E-03 \\
1.38 E+02 \\
3.86 E-01 \\
6.73 E+01 \\
5.24 E+00\end{array}$ \\
\hline $\begin{array}{l}N B-96 \\
N B-97 \\
N B-97 M \\
M O-91 \\
M O-93\end{array}$ & $\begin{array}{r}23.35 \mathrm{H} \\
72.1 \mathrm{M} \\
60 \mathrm{~S} \\
15.49 \mathrm{M} \\
3.5 \mathrm{E}\end{array}$ & $\begin{array}{l}2.10 E+02 \\
5.86 E+01 \\
6.39 E+01 \\
8.64 E+01 \\
1.05 E-01\end{array}$ & $\begin{array}{l}2.03 E+02 \\
5.61 E+01 \\
6.14 E+01 \\
8.22 E+01 \\
5.79 E-03\end{array}$ & $\begin{array}{l}2.28 E+02 \\
6.29 E+01 \\
6.89 E+01 \\
9.16 E+01 \\
2.01 E-02\end{array}$ & $\begin{array}{l}2.59 E+02 \\
7.22 E+01 \\
7.83 E+01 \\
1.09 E \cdot 02 \\
2.38 E+00\end{array}$ & $\begin{array}{l}2.00 E+02 \\
5.51 E+01 \\
6.04 E+01 \\
0.03 E+01 \\
2.66 E-02\end{array}$ & $\begin{array}{l}1.82 E+02 \\
4.97 E \cdot 01 \\
5.46 E+01 \\
7.23 E+01 \\
6.65 E-03\end{array}$ & $\begin{array}{l}2.13 E+02 \\
5.85 E+01 \\
6.43 E+01 \\
8.53 E+01 \\
7.59 E-03\end{array}$ & $\begin{array}{l}1.96 E \cdot 02 \\
5.39 E \cdot 01 \\
5.91 E \cdot 01 \\
7.87 E+01 \\
6.58 E-02\end{array}$ & $\begin{array}{l}2.13 E+02 \\
5.84 E+01 \\
6.41 E+01 \\
8.53 E+01 \\
3.27 E-02\end{array}$ \\
\hline $\begin{array}{l}M O-99 \\
M O-101 \\
\text { TC-95 } \\
\text { TC-95M } \\
\text { TC-96 }\end{array}$ & $\begin{array}{r}66.02 \mathrm{H} \\
14.61 \mathrm{M} \\
20.0 \mathrm{H} \\
61 \\
4.28 \mathrm{D}\end{array}$ & $\begin{array}{l}1.36 E+01 \\
1.20 E+02 \\
6.85 E+01 \\
5.88 E+01 \\
2.16 E+02\end{array}$ & $\begin{array}{l}1.30 E \bullet 01 \\
1.19 E+02 \\
6.59 E \bullet 01 \\
5.59 E \bullet 01 \\
2.08 E+02\end{array}$ & $\begin{array}{l}1 \cdot 45 E+01 \\
1.33 E+02 \\
7.41 E+01 \\
6.21 E+01 \\
2.34 E+02\end{array}$ & $\begin{array}{l}1.74 E \bullet 01 \\
1.52 E+02 \\
9.62 E+01 \\
7.32 E \bullet 01 \\
2.66 E \bullet 02\end{array}$ & $\begin{array}{l}1.28 E+01 \\
1.17 E+02 \\
6.49 E+01 \\
5.49 E+01 \\
2.06 E+02\end{array}$ & $\begin{array}{l}1.16 E+01 \\
1.07 E+02 \\
5.87 E+01 \\
4.95 E+01 \\
1.86 E+02\end{array}$ & $\begin{array}{l}1.36 E+01 \\
1.25 E+02 \\
6.91 E+01 \\
5.83 E+01 \\
2.19 E+02\end{array}$ & $\begin{array}{l}1.26 E+01 \\
1.15 E+02 \\
6.36 E 001 \\
5.39 E * 01 \\
2.01 E+02\end{array}$ & $\begin{array}{l}1.36 E+01 \\
1.25 E+02 \\
6.89 E+01 \\
5.82 E+01 \\
2.18 E+02\end{array}$ \\
\hline
\end{tabular}


DOSE-RATE FAC TORS FOR BODY URGANS AND EFFECTIVE DOSE-RATE FACTOR FOR EXPOSUP.E I M ABOUE CunTAMINATEU GKIUUND SJFFACE

\begin{tabular}{|c|c|c|c|c|c|c|c|c|c|c|}
\hline NUCL $10 \mathrm{E}$ & ALF-LIFE & LIVER & LUNGS & MARROH & $\begin{array}{c}\text { REO } \\
\text { MARROW }\end{array}$ & OVARIES & ANCREAS & SKELETON & SPLEEN & STOMAC \\
\hline $\begin{array}{l}-89 \\
-99 \\
-91 \\
i-91 \\
i-92 \\
-93\end{array}$ & $\begin{array}{r}50.55 \mathrm{D} \\
28.6 \mathrm{Y} \\
9.5 \mathrm{H} \\
2.71 \mathrm{H} \\
7.3 \mathrm{M}\end{array}$ & $\begin{array}{l}.13 E-02 \\
.0 \\
61 E \cdot 01\end{array}$ & $\begin{array}{l}1.20 E-02 \\
0.0 \\
5.98 E+01 \\
1.06 E+02 \\
1.90 E+02\end{array}$ & $\begin{array}{l}1.29 E-02 \\
0.0 \\
6.47 E+01 \\
1.12 E+02 \\
2.06 E+02\end{array}$ & $\begin{array}{l}1.22 E-02 \\
0.0 \\
6.07 E+01 \\
1.07 E+02 \\
1.93 E+02\end{array}$ & $\begin{array}{l}1.09 E-02 \\
0.09 \\
5.43 E+01 \\
9.50 E+01 \\
1.69 E+02\end{array}$ & $\begin{array}{l}9.72 E-03 \\
0.0 \\
4.84 E+01 \\
8.70 E+01 \\
1.55 E+02\end{array}$ & $\begin{array}{l}1.31 E-02 \\
0.0 \\
6.58 E+01 \\
1.13 E+02 \\
2.09 E+02\end{array}$ & $\begin{array}{l}1.09 E-02 \\
0.0 \\
5.44 E+01 \\
9.70 E+01 \\
1.73 E+02\end{array}$ & $\begin{array}{l}5.54 E+01 \\
9.80 E+01 \\
1.76 E+02\end{array}$ \\
\hline $\begin{array}{l}-86 \\
-87 \\
-88 \\
-90 \\
-90 M\end{array}$ & $\begin{array}{r}14.74 \mathrm{H} \\
80.3 \mathrm{H} \\
106.60 \mathrm{O} \\
64.1 \mathrm{H} \\
3.19 \mathrm{H}\end{array}$ & $\begin{array}{l}.86 E+02 \\
.83 E+01 \\
.10 E+02 \\
.0 \\
.35 E+01\end{array}$ & $\begin{array}{l}.04 E+02 \\
.12 E+01 \\
.22 E+02 \\
.0 \\
.77 E .01\end{array}$ & $\begin{array}{l}3.27 E+02 \\
4.79 E+01 \\
2.31 E+02 \\
0.0 \\
7.11 E+01\end{array}$ & $\begin{array}{l}3.08 E+02 \\
4.22 E+01 \\
2.24 E+02 \\
0.0 \\
5.87 E+01\end{array}$ & $\begin{array}{l}2.72 E+02 \\
3.67 E+01 \\
1.96 E+02 \\
0.0 \\
4.98 E+02\end{array}$ & $\begin{array}{l}2.47 E+02 \\
3.30 E+01 \\
1.02 E+02 \\
0.0 \\
4.59 E+01\end{array}$ & $\begin{array}{l}3.33 E+02 \\
4.87 E+01 \\
2.35 E+02 \\
0.0 \\
3.24 E+01\end{array}$ & $\begin{array}{l}2.77 E+02 \\
3.75 E+01 \\
2.02 E+02 \\
0.0 \\
5.26 E+01\end{array}$ & $\begin{array}{l}2.81 E+0 \\
3.78 E+0 \\
2.05 E+0\end{array}$ \\
\hline $\begin{array}{l}-91 \\
-91 M \\
-92\end{array}$ & $\begin{array}{rrr}58.51 & \mathrm{O} \\
49.71 & \mathrm{H} \\
3.54 & \mathrm{H} \\
10.1 & \mathrm{H} \\
16.5 \mathrm{H}\end{array}$ & $\begin{array}{l}2.17 E-01 \\
2.45 E+01 \\
2.01 E+01\end{array}$ & $\begin{array}{l}.94 E-01 \\
17 E+01 \\
14 E+01 \\
.52 E+00\end{array}$ & & & & & & & $2.29 E *$ \\
\hline $\begin{array}{l}R-88 \\
R-89 \\
R-93 \\
R-95 \\
R-97\end{array}$ & $\begin{array}{r}83.4 \mathrm{D} \\
78.43 \mathrm{H} \\
1.53 E 6 \mathrm{~V} \\
64.02 \mathrm{D} \\
16.90 \mathrm{H}\end{array}$ & $\begin{array}{l}3.24 E+01 \\
9.53 E+01 \\
0.0 \\
6.16 E+01 \\
1.44 E+01\end{array}$ & $\begin{array}{l}.49 E+01 \\
.02 E+02 \\
.0 \\
.58 E+01 \\
.53 E+01\end{array}$ & $\begin{array}{l}4.21 E+01 \\
1.11 E+02 \\
0.0 \\
7.22 E+01 \\
1.68 E+01\end{array}$ & $\begin{array}{l}3.60 E+01 \\
1.03 E * 02 \\
0.0 \\
6.69 E+01 \\
1.56 E * 01\end{array}$ & $\begin{array}{l}3.05 E+01 \\
9.23 E+01 \\
0.0 \\
5.99 E+01 \\
1.37 E+01\end{array}$ & $\begin{array}{l}.80 E+01 \\
.23 E+01 \\
.0 \\
.31 E+01 \\
.24 E+01\end{array}$ & $\begin{array}{l}4.28 E+01 \\
1.13 E+02 \\
0.0 \\
7.34 E+01 \\
1.71 E+01\end{array}$ & $\begin{array}{l}3.19 E+01 \\
9.25 E+01 \\
0.0 \\
5.98 E+01 \\
1.40 E+01\end{array}$ & $\begin{array}{l}0.0 \\
6.09 \mathrm{EF} \\
1.41 \mathrm{E}\end{array}$ \\
\hline $\begin{array}{l}-90 \\
-91 \\
3-914 \\
-92 \\
-924\end{array}$ & $\begin{array}{r}14.60 \mathrm{H} \\
1 E 4 \mathrm{Y} \\
61 \mathrm{D} \\
3.6 \mathrm{EY} Y \\
10.15 \mathrm{D}\end{array}$ & & & & & & & & & $\begin{array}{l}3.22 \mathrm{E} \\
1.78 \mathrm{E}+\end{array}$ \\
\hline $\begin{array}{l}-934 \\
-94 \\
-94 M \\
-05\end{array}$ & $\begin{array}{r}14.6 \mathrm{Y} \\
2.03 E 4 \\
6.26 \mathrm{M} \\
35.06 \mathrm{D} \\
86.6 \mathrm{H}\end{array}$ & & & & & & & & & $5.02 \mathrm{E}+$ \\
\hline $\begin{array}{l}8-974 \\
0-91 \\
0-93\end{array}$ & 72 & $\begin{array}{l}5.57 E . \\
6.10 E . \\
8.17 E \\
1.92 E\end{array}$ & -12E & & & & & & & $5.52 \mathrm{E}^{-}$ \\
\hline $\begin{array}{l}-99 \\
-101 \\
-95 \\
-95 \\
-954 \\
-96\end{array}$ & 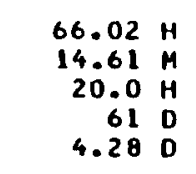 & $\begin{array}{l}1.30 E+01 \\
1.18 E+02 \\
6.55 E+01 \\
5.56 E+01 \\
2.07 E+02\end{array}$ & $\begin{array}{l}.39 E+01 \\
1.26 E+02 \\
0.01 E+01 \\
5.97 E+01 \\
.21 E+02\end{array}$ & $\begin{array}{l}1.59 E+01 \\
1.36 E+02 \\
7.65 E+01 \\
6.91 E+01 \\
2.40 E+02\end{array}$ & $\begin{array}{l}1.27 E+02 \\
7.11 E+01 \\
6.04 E+01 \\
2.25 E+02\end{array}$ & $\begin{array}{l}1.24 E+01 \\
1.12 E+02 \\
6.3 T E+01 \\
5.30 E+01 \\
2.01 E+02\end{array}$ & $\begin{array}{l}1.11 E+01 \\
1.02 E+02 \\
5.65 E+01 \\
4.79 E+01 \\
1.79 E+02\end{array}$ & $\begin{array}{l}1.62 E+01 \\
1.39 E+02 \\
7.79 E+01 \\
7.04 E+01 \\
2.44 E+02\end{array}$ & $\begin{array}{l}1.26 E+01 \\
1.15 E+02 \\
6.36 E+01 \\
5.43 E+01 \\
2.01 E \cdot 02\end{array}$ & $\begin{array}{l}1.16 \mathrm{EA} \\
6.4 \mathrm{BE} \\
5.50 \mathrm{E} \\
2.05 \mathrm{E}\end{array}$ \\
\hline
\end{tabular}




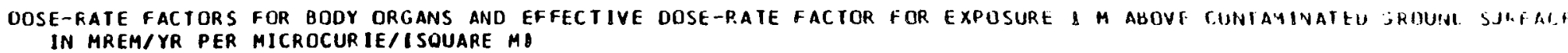

\begin{tabular}{|c|c|c|c|c|c|c|c|c|c|}
\hline NUCL IDE & HALF-LIFE & TESTES & THYMUS & THYRDID & UTERUS & EFFECTIVE & $\begin{array}{c}\text { SKIN } \\
\text { (PHOTON) }\end{array}$ & $\begin{array}{l}\text { SKIN } \\
\text { LECTRONI }\end{array}$ & $\begin{array}{l}\text { SKIN } \\
\text { (TOTALI) }\end{array}$ \\
\hline $\begin{array}{l}S R-89 \\
S R-90 \\
S R-91 \\
S R-92 \\
S R-93\end{array}$ & $\begin{array}{rl}50.55 & \mathrm{D} \\
28.6 & \mathrm{Y} \\
9.5 \mathrm{H} \\
2.71 \mathrm{H} \\
7.3 \mathrm{H}\end{array}$ & $\begin{array}{l}1.59 E-02 \\
0.0 \\
7.92 E+01 \\
1.39 E+02 \\
2.51 E+02\end{array}$ & $\begin{array}{l}1.27 E-02 \\
0.0 \\
6.28 E+01 \\
1.11 E+02 \\
1.97 E+02\end{array}$ & $\begin{array}{l}1.51 E-02 \\
0.0 \\
7.53 E+01 \\
1.32 E+02 \\
2.36 E+02\end{array}$ & $\begin{array}{l}9.80 E-03 \\
0.0 \\
4.90 E+01 \\
8.93 E+01 \\
1.57 E+02\end{array}$ & $\begin{array}{l}1.36 E-02 \\
0.0 \\
6.77 E+01 \\
1.19 E+02 \\
2.15 E+02\end{array}$ & $\begin{array}{l}1.84 E-02 \\
0.0 \\
9.14 E+01 \\
1.60 E+02 \\
2.88 E+02\end{array}$ & $\begin{array}{l}5.89 E+02 \\
5.84 E+01 \\
6.52 E+02 \\
6.85 E+01 \\
9.37 E+02\end{array}$ & $\begin{array}{l}5.89 E+02 \\
5.84 E+01 \\
1.44 E+02 \\
2.28 E+02 \\
1.22 E+03\end{array}$ \\
\hline $\begin{array}{l}\gamma-86 \\
y-87 \\
\gamma-88 \\
\gamma-90 \\
\gamma-904\end{array}$ & $\begin{array}{r}14.74 \mathrm{H} \\
80.3 \mathrm{H} \\
106.60 \mathrm{D} \\
64.11 \mathrm{H} \\
3.19 \mathrm{H}\end{array}$ & $\begin{array}{l}4.01 E+02 \\
5.58 E+01 \\
2.89 E+02 \\
0.0 \\
7.81 E+01\end{array}$ & $\begin{array}{l}3.15 E+02 \\
4.18 E+01 \\
2.29 E+02 \\
0.0 \\
5.91 E+01\end{array}$ & $\begin{array}{l}3.77 E+02 \\
5.13 E+01 \\
2.71 E+02 \\
0.0 \\
7.32 E+01\end{array}$ & $\begin{array}{l}2.52 E * 02 \\
3.35 E+01 \\
1.87 E+02 \\
0.0 \\
4.69 E+01\end{array}$ & $\begin{array}{l}3.44 E+02 \\
4.78 E+01 \\
2.49 E+02 \\
0.0 \\
6.75 E+01\end{array}$ & $\begin{array}{l}4.62 E+02 \\
6.69 E+01 \\
3.34 E+02 \\
0.0 \\
8.84 E+01\end{array}$ & $\begin{array}{l}2.27 E+02 \\
1.24 E+00 \\
5.91 E-01 \\
1.01 E+03 \\
4.44 E+01\end{array}$ & $\begin{array}{l}6.89 E+02 \\
6.82 E+01 \\
3.35 E+02 \\
1.01 E+03 \\
1.33 E+02\end{array}$ \\
\hline $\begin{array}{l}Y-91 \\
Y-91 M \\
Y-92 \\
Y-93 \\
2 R-86\end{array}$ & $\begin{array}{rr}58.51 & \mathrm{D} \\
49.71 & \mathrm{H} \\
3.54 & \mathrm{H} \\
10.1 & \mathrm{H} \\
16.5 \mathrm{H}\end{array}$ & $\begin{array}{l}3.81 E-01 \\
6.39 E+01 \\
2.83 E+01 \\
9.91 E+00 \\
3.55 E+01\end{array}$ & $\begin{array}{l}3.10 E-01 \\
4.87 E+01 \\
2.25 E+01 \\
7.78 E+00 \\
2.60 E+01\end{array}$ & $\begin{array}{l}3.68 E-01 \\
5.94 E+01 \\
2.68 E+01 \\
9.31 E+00 \\
3.28 E+01\end{array}$ & $\begin{array}{l}2.45 E-01 \\
3.88 E+01 \\
1.77 E+01 \\
6.30 E+00 \\
2.04 E+01\end{array}$ & $\begin{array}{l}3.31 E-01 \\
5.46 E+01 \\
2.42 E+01 \\
8.55 E+00 \\
3.09 E+01\end{array}$ & $\begin{array}{l}4.45 E-01 \\
7.34 E+01 \\
3.26 E+01 \\
1.13 E+01 \\
4.61 E+01\end{array}$ & $\begin{array}{l}6.14 E+02 \\
2.93 E+01 \\
1.57 E+03 \\
1.27 E+03 \\
0.0\end{array}$ & $\begin{array}{l}6.14 E+02 \\
1.03 E+02 \\
1.60 E+03 \\
1.28 E+03 \\
4.61 E+01\end{array}$ \\
\hline $\begin{array}{l}2 R-88 \\
2 R-89 \\
2 R-93 \\
2 R-95 \\
2 R-97\end{array}$ & $\begin{array}{r}63.4 \mathrm{D} \\
78.43 \mathrm{H} \\
1.53 E 6 \mathrm{Y} \\
64.02 \mathrm{D} \\
16.90 \mathrm{H}\end{array}$ & $\begin{array}{l}4.75 E+01 \\
1.35 E+02 \\
0.0 \\
8.75 E+01 \\
2.03 E+01\end{array}$ & $\begin{array}{l}3.54 E+01 \\
1.07 E+02 \\
0.0 \\
6.07 E+01 \\
1.59 E+01\end{array}$ & $\begin{array}{l}4.38 E \cdot 01 \\
1.28 E \cdot 02 \\
0.0 \\
8.28 E+01 \\
1.91 E+01\end{array}$ & $\begin{array}{l}2.85 E+01 \\
8.31 E \cdot 01 \\
0.0 \\
5.35 E+01 \\
1.27 E+01\end{array}$ & $\begin{array}{l}4.10 E+01 \\
1.16 E+02 \\
0.0 \\
7.47 E+01 \\
1.74 E+01\end{array}$ & $\begin{array}{l}5.72 E+01 \\
1.59 E+02 \\
0.0 \\
1.01 E+02 \\
2.32 E \cdot 01\end{array}$ & $\begin{array}{l}1.11 E+01 \\
8.22 E+01 \\
0.0 \\
2.74 E+00 \\
1.12 E+02\end{array}$ & $\begin{array}{l}6.83 E+01 \\
2.41 E+02 \\
0.0 \\
1.04 E+02 \\
7.35 E+02\end{array}$ \\
\hline $\begin{array}{l}N B-90 \\
N B-91 \\
N B-91 M \\
N B-92 \\
N B-92 M\end{array}$ & $\begin{array}{r}14.60 \\
\text { IE4 Y } \\
61 \mathrm{D} \\
3.6 E 7 \mathrm{Y} \\
10.15 \mathrm{D}\end{array}$ & $\begin{array}{l}4.47 E+02 \\
8.14 E-01 \\
5.14 E+00 \\
1.76 E+02 \\
1.11 E+02\end{array}$ & $\begin{array}{l}3.51 E+02 \\
1.59 E-01 \\
3.69 E+00 \\
1.38 E+02 \\
8.85 E+01\end{array}$ & $\begin{array}{l}4.15 E+02 \\
3.34 E-01 \\
4.52 E+00 \\
1.66 E \bullet 02 \\
1.06 E+02\end{array}$ & $\begin{array}{l}2.89 E+02 \\
1.23 E-01 \\
2.90 E+00 \\
1.08 E+02 \\
6.86 E+01\end{array}$ & $\begin{array}{l}3.85 E+02 \\
7.33 E-01 \\
4.40 E+00 \\
1.51 E+02 \\
9.53 E+01\end{array}$ & $\begin{array}{l}5.09 E+02 \\
4.12 E+00 \\
8.30 E+00 \\
2.06 E+02 \\
1.32 E+02\end{array}$ & $\begin{array}{l}3.63 E+02 \\
0.0 \\
0.0 \\
1.56 E+00 \\
0.0\end{array}$ & $\begin{array}{l}8.12 E+02 \\
4.12 E+00 \\
8.30 E+00 \\
2.08 E+02 \\
1.32 E+02\end{array}$ \\
\hline $\begin{array}{l}N B-934 \\
N B-94 \\
N B-944 \\
N B-95 \\
N B-954\end{array}$ & $\begin{array}{r}14.6 \mathrm{Y} \\
2.03 E 4 \mathrm{Y} \\
6.26 \mathrm{M} \\
35.06 \mathrm{D} \\
86.6 \mathrm{H}\end{array}$ & $\begin{array}{l}1.19 E-01 \\
1.86 E+02 \\
9.41 E-01 \\
9.08 E+01 \\
1.91 E+00\end{array}$ & $\begin{array}{l}1.87 E-03 \\
1.47 E+02 \\
3.95 E-01 \\
7.15 E+01 \\
5.60 E+00\end{array}$ & $\begin{array}{l}3.20 E-02 \\
1.76 E+02 \\
5.86 E-01 \\
8.61 E \cdot 01 \\
1.25 E \cdot 00\end{array}$ & $\begin{array}{l}9.33 E-05 \\
1.14 E+02 \\
2.99 E-01 \\
5.56 E \cdot 01 \\
4.47 E \cdot 00\end{array}$ & $\begin{array}{l}1.59 E+02 \\
8.04 E-01 \\
7.66 E 01 \\
6.92 E+00\end{array}$ & $\begin{array}{l}6.60 E-01 \\
2.15 E \cdot 02 \\
3.07 E+00 \\
1.05 E+02 \\
1.07 E+01\end{array}$ & $\begin{array}{l}0.0 \\
1.26 E+01 \\
1.88 E+00 \\
1.12 E+00 \\
2.24 E+01\end{array}$ & $\begin{array}{l}6.60 E-01 \\
2.27 E+02 \\
4.96 E+00 \\
1.06 E+02 \\
3.30 E+01\end{array}$ \\
\hline $\begin{array}{l}N B-96 \\
N B-97 \\
N B-97 M \\
M D-91 \\
M O-93\end{array}$ & 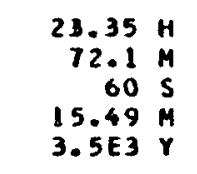 & $\begin{array}{l}2.86 E+02 \\
7.95 E+01 \\
8.67 E+01 \\
1.18 E+02 \\
6.67 E-01\end{array}$ & $\begin{array}{l}2.25 E+02 \\
6.18 E+01 \\
6.81 E+01 \\
8.91 E+01 \\
1.05 E-02\end{array}$ & $\begin{array}{l}2.71 E+02 \\
7.47 E+01 \\
8.20 E+01 \\
1.09 E+02 \\
1.79 E-01\end{array}$ & $\begin{array}{l}1.17 E+02 \\
4.85 E+01 \\
5.30 E+01 \\
7.14 E+01 \\
5.23 E-04\end{array}$ & $\begin{array}{l}2.45 E+02 \\
6.79 E+01 \\
7.41 E+01 \\
1.01 E+02 \\
5.78 E-01\end{array}$ & $\begin{array}{l}3.30 E * 02 \\
9.16 E * 01 \\
1.00 E * 02 \\
1.35 E * 02 \\
3.69 E * 00\end{array}$ & $\begin{array}{l}1.35 E+02 \\
4.31 E+02 \\
1.57 E+01 \\
1.58 E+03 \\
0.0\end{array}$ & $\begin{array}{l}4.65 E+02 \\
5.22 E+02 \\
1.16 E * 02 \\
1.71 E * 03 \\
3.69 E * 00\end{array}$ \\
\hline $\begin{array}{l}M 0-99 \\
M 0-101 \\
T C-95 \\
T C-954 \\
T C-96\end{array}$ & 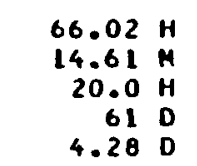 & $\begin{array}{l}1.86 E+01 \\
1.66 E+02 \\
9.36 E+01 \\
8.05 E+01 \\
2.94 E+02\end{array}$ & $\begin{array}{l}1.45 E+01 \\
1.31 E+02 \\
7.32 E+01 \\
6.22 E+01 \\
2.32 E+02\end{array}$ & $\begin{array}{l}1.77 E+01 \\
1.56 E+02 \\
B .83 E+01 \\
7.59 E+01 \\
2.79 E+02\end{array}$ & $\begin{array}{l}1.13 E+01 \\
1.05 E+02 \\
5.69 E+01 \\
4.85 E+01 \\
1.80 E+02\end{array}$ & $\begin{array}{l}1.60 E+01 \\
1.42 E+02 \\
8.00 E+01 \\
6.91 E+01 \\
2.51 E+02\end{array}$ & $\begin{array}{l}2.15 E+01 \\
1.90 E \bullet 02 \\
1.11 E+02 \\
9.4 \mathrm{EE}+01 \\
3.43 E \bullet 02\end{array}$ & $\begin{array}{l}3.33 E+02 \\
4.74 E+02 \\
9.85 E-01 \\
0.14 E-01 \\
1.03 E+00\end{array}$ & $\begin{array}{l}3.55 E+02 \\
6.64 E+02 \\
1.12 E+02 \\
9.57 E+01 \\
3.44 E+02\end{array}$ \\
\hline
\end{tabular}




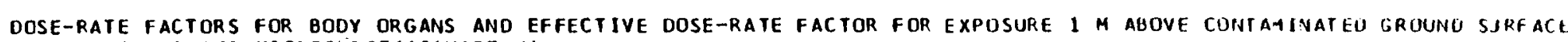

IN MREM/YR PER MICROCUR IE/ISQUARE HE

\begin{tabular}{|c|c|c|c|c|c|c|c|c|c|c|}
\hline NUCL $10 E$ & ALF-LIFE & RENALS & BLADDER & BRAIN & BREAST & HE AR T & $\begin{array}{l}\text { SHALL } \\
\text { NTESTINE }\end{array}$ & $\begin{array}{c}\text { UPPER } \\
\text { LARGE } \\
\text { INTESTINE }\end{array}$ & $\begin{array}{c}\text { LOHER } \\
\text { IARGE } \\
\text { INTESTINE }\end{array}$ & K IONEYS \\
\hline $\begin{array}{l}1 C-96 M \\
1 C-97 \\
1 C-9 M \\
1 C-98 \\
1 C-99\end{array}$ & $\begin{array}{r}51.5 \mathrm{H} \\
2.6 E 6 \mathrm{Y} \\
89 \mathrm{D} \\
4.2 E 6 \mathrm{Y} \\
2.13 E 5 \mathrm{Y}\end{array}$ & $\begin{array}{l}3.53 E+00 \\
1.26 E-01 \\
1.37 E-01 \\
1.23 E+02 \\
4.49 E-05\end{array}$ & $\begin{array}{l}3.37 E+00 \\
1.42 E-02 \\
4.06 E-02 \\
1.18 E+02 \\
4.48 E-05\end{array}$ & $\begin{array}{l}3.79 E+00 \\
2.70 E-02 \\
5.43 E-02 \\
1.32 E+02 \\
4.63 E-05\end{array}$ & $\begin{array}{l}5.62 E+00 \\
2.50 E \bullet 00 \\
1.98 E \cdot 00 \\
1.51 E \cdot 02 \\
8.56 E-05\end{array}$ & $\begin{array}{l}3.33 E+00 \\
3.47 E-02 \\
5.85 E-02 \\
1.16 E+02 \\
4.22 E-05\end{array}$ & $\begin{array}{l}3.02 E+00 \\
1.46 E-02 \\
3.92 E-02 \\
1.04 E+02 \\
3.67 E-05\end{array}$ & $\begin{array}{l}3.55 E+00 \\
2.10 E-02 \\
6.19 E-02 \\
1.23 E+02 \\
4.53 E-05\end{array}$ & $\begin{array}{l}3.29 E+00 \\
8.16 E-02 \\
9.64 E-02 \\
1.13 E+02 \\
4.04 E-05\end{array}$ & $\begin{array}{l}3.57 E+00 \\
6.16 E-02 \\
1.05 E-01 \\
1.23 E+02 \\
4.56 E-05\end{array}$ \\
\hline $\begin{array}{l}T C-994 \\
T C-101 \\
R U-97 \\
R U-103 \\
R U-105\end{array}$ & $\begin{array}{r}6.02 \mathrm{H} \\
14.2 \mathrm{H} \\
2.9 \\
39.35 \mathrm{D} \\
4.44 \mathrm{H}\end{array}$ & $\begin{array}{l}1.18 E+01 \\
3.15 E+01 \\
2.14 E+01 \\
4.30 E+01 \\
6.93 E+01\end{array}$ & $\begin{array}{l}1.08 E+01 \\
2.92 E+01 \\
1.95 E+01 \\
4.08 E+01 \\
6.61 E+01\end{array}$ & $\begin{array}{l}1.16 E+01 \\
3.20 E+01 \\
2.09 E+01 \\
4.54 E+01 \\
7.39 E+01\end{array}$ & $\begin{array}{l}1.09 E+01 \\
4.19 E+01 \\
3.26 E+01 \\
5.43 E+01 \\
8.65 E+01\end{array}$ & $\begin{array}{l}1.07 E+01 \\
2.85 E+01 \\
1.90 E+01 \\
3.98 E+01 \\
6.50 E+01\end{array}$ & $\begin{array}{l}9.30 E+00 \\
2.56 E \bullet 01 \\
1.70 E+01 \\
3.58 E+01 \\
5.86 E+01\end{array}$ & $\begin{array}{l}1.14 E+01 \\
3.02 E+01 \\
2.01 E+01 \\
4.23 E+01 \\
6.90 E+01\end{array}$ & $\begin{array}{l}1.05 E+01 \\
2.82 E+01 \\
1.89 E+01 \\
3.90 E+01 \\
6.37 E+01\end{array}$ & $\begin{array}{l}1.10 E+01 \\
3.02 E+01 \\
2.01 E+01 \\
4.23 E+01 \\
6.89 E+01\end{array}$ \\
\hline $\begin{array}{l}\text { RU-1 06 } \\
\text { RH-1 103 } \\
\text { RH-1 05 } \\
\text { RH-1 05 M } \\
\text { RH-1 06 }\end{array}$ & 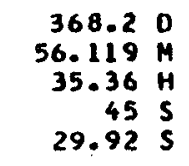 & $\begin{array}{l}0.0 \\
2.78 E-02 \\
7.19 E+00 \\
2.60 E+00 \\
1.81 E+01\end{array}$ & $\begin{array}{l}0.0 \\
1.31 E-02 \\
6.64 E+00 \\
2.34 E+00 \\
1.73 E+01\end{array}$ & $\begin{array}{l}0.0 \\
8.65 E-03 \\
7.24 E+00 \\
2.48 E+00 \\
1.93 E+01\end{array}$ & $\begin{array}{l}0.0 \\
3.85 E-01 \\
9.58 E+00 \\
6.03 E+00 \\
2.25 E+01\end{array}$ & $\begin{array}{l}0.0 \\
1.01 E-02 \\
6.47 E+00 \\
2.30 E+00 \\
1.69 E+01\end{array}$ & $\begin{array}{l}0.0 \\
7.39 E-03 \\
5.81 E+00 \\
1.98 E+00 \\
1.53 E+01\end{array}$ & $\begin{array}{l}0.0 \\
2.04 E-02 \\
6.85 E+00 \\
2.51 E+00 \\
1.80 E+01\end{array}$ & $\begin{array}{l}0.0 \\
1.98 E-02 \\
6.42 E+00 \\
2.29 E+00 \\
1.66 E+01\end{array}$ & $\begin{array}{l}0.0 \\
3.34 E-02 \\
6.85 E+00 \\
2.48 E+00 \\
1.80 E+01\end{array}$ \\
\hline $\begin{array}{l}P D-103 \\
P D-107 \\
P D-109 \\
A G-106 M \\
A G-108\end{array}$ & $\begin{array}{r}16.961 \\
6.5 E 6 \\
13.453 \mathrm{H} \\
8.46 \mathrm{D} \\
2.31 \mathrm{M}\end{array}$ & $\begin{array}{l}2.49 E-01 \\
0.0 \\
6.11 E-02 \\
2.35 E+02 \\
1.55 E+00\end{array}$ & $\begin{array}{l}1.09 E-01 \\
0.0 \\
5.80 E-02 \\
2.28 E+02 \\
1.47 E+00\end{array}$ & $\begin{array}{l}7.83 E-02 \\
0.0 \\
6.46 E-02 \\
2.55 E \cdot 02 \\
1.65 E \cdot 00\end{array}$ & $\begin{array}{l}3.42 E+00 \\
0.0 \\
1.71 E-02 \\
2.95 E+02 \\
1.99 E+00\end{array}$ & $\begin{array}{l}9.06 E-02 \\
0.0 \\
5.67 E-02 \\
2.24 E+02 \\
1.44 E+00\end{array}$ & & & $\begin{array}{l}1.77 E-01 \\
0.0 \\
5.56 E-02 \\
2.20 E+02 \\
1.42 E+00\end{array}$ & $\begin{array}{l}2.88 E-01 \\
0.0 \\
6.01 E-02 \\
2.38 E+02 \\
1.54 E+00\end{array}$ \\
\hline $\begin{array}{l}A G-108 M \\
A G-109 M \\
A G-110 \\
A G-110 M \\
A G-111\end{array}$ & $\begin{array}{rr}127 & Y \\
39.6 & 5 \\
24.57 & S \\
249.85 & 0 \\
7.46 & 0\end{array}$ & $\begin{array}{l}1.42 E+02 \\
4.15 E-01 \\
2.69 E+00 \\
2.30 E+02 \\
2.43 E+00\end{array}$ & $\begin{array}{l}1.36 E+02 \\
3.78 E-01 \\
2.57 E+00 \\
2.23 E+02 \\
2.25 E+00\end{array}$ & $\begin{array}{l}1.52 E+02 \\
3.37 E-01 \\
2.89 E+00 \\
2.51 E+02 \\
2.46 E+00\end{array}$ & $\begin{array}{l}1.80 E+02 \\
2.09 E+00 \\
3.32 E+00 \\
2.83 E+02 \\
3.22 E+00\end{array}$ & $\begin{array}{l}1.33 E+02 \\
3.18 E-01 \\
2.53 E+00 \\
2.20 E+02 \\
2.20 E+00\end{array}$ & $\begin{array}{l}1.20 E+02 \\
2.76 E-01 \\
2.28 E+00 \\
2.00 E+02 \\
1.9 \mathrm{TE}+00\end{array}$ & & & $\begin{array}{l}1.41 E+02 \\
5.03 E-01 \\
2.68 E+00 \\
2.34 E+02 \\
2.32 E+00\end{array}$ \\
\hline $\begin{array}{l}\text { CD-109 } \\
\text { CD-111M } \\
\text { CD-113 } \\
\text { CD-113M } \\
C D-115\end{array}$ & $\begin{array}{r}464 \mathrm{D} \\
48.7 \mathrm{M} \\
9.3 \mathrm{E} 15 \mathrm{Y} \\
13.7 \mathrm{Y} \\
53.46 \mathrm{H}\end{array}$ & $\begin{array}{l}2.49 E-01 \\
2.61 E+01 \\
0.0 \\
0.0 \\
1.81 E+01\end{array}$ & $\begin{array}{l}1.78 E-01 \\
2.39 E+01 \\
0.0 \\
0.0 \\
1.72 E+01\end{array}$ & $\begin{array}{l}8.48 E-02 \\
2.56 E+01 \\
0.0 \\
0.0 \\
1.91 E+01\end{array}$ & $\begin{array}{l}2.91 E+00 \\
3.84 E+01 \\
0.0 \\
0.0 \\
2.30 E+01\end{array}$ & $\begin{array}{l}9.70 E-02 \\
2.33 E+01 \\
0.0 \\
0.0 \\
1.68 E+01\end{array}$ & $\begin{array}{l}8.25 E-02 \\
2.07 E+01 \\
0.0 \\
0.0 \\
1.51 E+01\end{array}$ & $\begin{array}{l}2.66 E-01 \\
2.48 E+01 \\
0.0 \\
0.0 \\
1.78 E+01\end{array}$ & $\begin{array}{l}1.83 E-01 \\
2.31 E+01 \\
0.0 \\
0.0 \\
1.65 E+01\end{array}$ & $\begin{array}{l}4.03 E-01 \\
2.47 E+01 \\
0.0 \\
0.0 \\
1.78 E+01\end{array}$ \\
\hline $\begin{array}{l}C D-115 M \\
C D-117 \\
C D-111 M \\
1 N-111 \\
1 N-113 M\end{array}$ & $\begin{array}{r}44.6 \mathrm{D} \\
2.49 \mathrm{H} \\
3.36 \mathrm{H} \\
2.83 \mathrm{O} \\
1.658 \mathrm{H}\end{array}$ & $\begin{array}{l}1.80 E+00 \\
8.69 E+01 \\
1.58 E+02 \\
3.64 E+01 \\
2.33 E+01\end{array}$ & $\begin{array}{l}1.76 E+00 \\
8.54 E+01 \\
1.59 E+02 \\
3.33 E+01 \\
2.17 E+01\end{array}$ & $\begin{array}{l}1.98 E+00 \\
9.56 \mathrm{E} * 01 \\
1.78 E * 02 \\
3.56 \mathrm{E} * 01 \\
2.39 \mathrm{E} \bullet 01\end{array}$ & $\begin{array}{l}2.21 E+00 \\
1.10 E+02 \\
1.99 E+02 \\
5.52 E+01 \\
3.09 E+01\end{array}$ & $\begin{array}{l}1.74 E+00 \\
8.43 E+01 \\
1.58 E+02 \\
3.24 E+01 \\
2.12 E+01\end{array}$ & $\begin{array}{l}1.59 E+00 \\
7.73 E+01 \\
1.45 E+02 \\
2.00 E+01 \\
1.90 E+01\end{array}$ & $\begin{array}{l}1.85 E+00 \\
8.97 E+01 \\
1.68 E+02 \\
3.46 E+01 \\
2.25 E+01\end{array}$ & & $\begin{array}{l}1.05 E+00 \\
8.96 E+01 \\
1.68 E+02 \\
3.45 E+01 \\
2.26 E+01\end{array}$ \\
\hline $\begin{array}{l}\text { IN-114 } \\
\text { IN-114H } \\
\text { IN-115 } \\
\text { IN-115M } \\
\text { IN-116M }\end{array}$ & $\begin{array}{r}71.9 \mathrm{~S} \\
49.51 \mathrm{D} \\
4.6 \mathrm{E15} \mathrm{Y} \\
4.36 \mathrm{H} \\
54.15 \mathrm{M}\end{array}$ & $\begin{array}{l}2.78 E+00 \\
8.07 E+00 \\
0.0 \\
1.47 E+01 \\
1.91 E+02\end{array}$ & $\begin{array}{l}2.66 E+00 \\
7.60 E+00 \\
0.0 \\
1.36 E+01 \\
1.90 E+02\end{array}$ & $\begin{array}{l}2.97 E \bullet 00 \\
8.27 E+00 \\
0.0 \\
1.48 E+01 \\
2.14 E+02\end{array}$ & $\begin{array}{l}3.49 E+00 \\
1.19 E+01 \\
0.0 \\
2.04 E+01 \\
2.39 E+02\end{array}$ & $\begin{array}{l}2.61 E+00 \\
7.37 E+00 \\
0.0 \\
1.32 E+01 \\
1.88 E+02\end{array}$ & $\begin{array}{l}6.62 E \cdot 00 \\
0.0 \\
1.18 E+01 \\
1.73 E+02\end{array}$ & $\begin{array}{l}2.77 E+00 \\
7.94 E+00 \\
0.0 \\
1.40 E+01 \\
2.00 E+02\end{array}$ & $\begin{array}{l}2.55 E+00 \\
1.30 E+00 \\
0.0 \\
1.31 E+01 \\
1.85 E+02\end{array}$ & $\begin{array}{l}2.77 E+00 \\
8.01 E+00 \\
0.0 \\
1.41 E+01 \\
2.00 E+02\end{array}$ \\
\hline
\end{tabular}




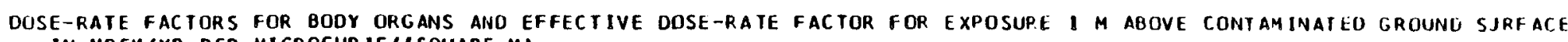

IN MREM

\begin{tabular}{|c|c|c|c|c|c|c|c|c|c|c|}
\hline CLIOE & ALF-LIFE & LIVER & LUNGS & MAPROH & $\begin{array}{c}\text { RED } \\
\text { MARROH }\end{array}$ & VARIES & ANCREAS & KELETON & SPLEEN & M \\
\hline $\begin{array}{l}-964 \\
-97 \\
-974 \\
-98 \\
-99\end{array}$ & 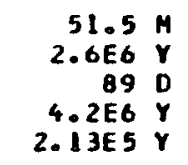 & $\begin{array}{l}36 E+00 \\
97 E-02 \\
91 E-02 \\
17 E+02 \\
46 E-05\end{array}$ & & $\begin{array}{l}E+00 \\
E-01 \\
E-01 \\
E+02 \\
E-05\end{array}$ & & & & & $\begin{array}{l}28 E+00 \\
48 E-02 \\
62 E-02 \\
14 E+02 \\
27 E-05\end{array}$ & $\begin{array}{l}4 E+00 \\
7 E-02 \\
4 E-02 \\
6 E+02 \\
0 E-05\end{array}$ \\
\hline $\begin{array}{l}-103 \\
-105\end{array}$ & $\begin{array}{r}6.02 \mathrm{H} \\
14.2 \mathrm{M} \\
2.9 \mathrm{D} \\
39.35 \mathrm{O} \\
4.44 \mathrm{H}\end{array}$ & 1 & & & & & & & & $\begin{array}{l}2 E+0 \\
0 E+0 \\
0 E+0\end{array}$ \\
\hline $\begin{array}{l}-103 M \\
-105 \\
-105 M \\
-106\end{array}$ & 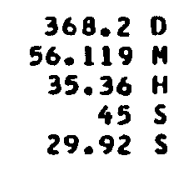 & $\begin{array}{l}6.6 \\
2.3 \\
1.72\end{array}$ & $\begin{array}{l}0.0 \\
3.27 E-02 \\
7.14 E+00 \\
2.68 E \bullet 00 \\
1.84 E+01\end{array}$ & $\begin{array}{l}0.0 \\
4.75 E-02 \\
8.97 E+00 \\
4.16 E+00 \\
2.07 E+01\end{array}$ & & & & & & 1. $70 \mathrm{E}$ \\
\hline $\begin{array}{l}-107 \\
-109 \\
-106 M \\
-108\end{array}$ & $\begin{array}{r}16.961 \mathrm{D} \\
6.5 \mathrm{E} 6 \mathrm{Y} \\
13.453 \mathrm{H} \\
8.46 \mathrm{D} \\
2.31 \mathrm{H}\end{array}$ & EE-01 & $\begin{array}{l}9 E-01 \\
9 E-02 \\
2 E+02\end{array}$ & $\begin{array}{l}4.12 E-01 \\
0.0 \\
1.13 E-02 \\
2.66 E+02 \\
1.78 E+00\end{array}$ & & & & & & \\
\hline $\begin{array}{l}-109 M \\
-110 \\
-110 M \\
-111\end{array}$ & $\begin{array}{rr}127 & Y \\
39.6 & S \\
24.57 & S \\
249.85 & 0 \\
7.46 & D\end{array}$ & $\begin{array}{l}3.54 \\
2.56 \\
2.22 \\
2.24\end{array}$ & $\begin{array}{l}2 \\
1 \\
0 \\
2\end{array}$ & $\begin{array}{l}1.6 \\
7.7 \\
3.0 \\
2.5 \\
3.0\end{array}$ & $\begin{array}{l}2.8 \\
2.8 \\
2.4 \\
2.4\end{array}$ & $\begin{array}{l}1 . \\
3 . \\
2.1 \\
2 .\end{array}$ & $\begin{array}{l}b_{2} \\
b 1 \\
b 0 \\
b 2\end{array}$ & & & \\
\hline $\begin{array}{l}-109 \\
-111 M \\
-113 \\
-113 M \\
-115\end{array}$ & $\begin{array}{r}464 \mathrm{D} \\
48.7 \mathrm{M} \\
9.3 \mathrm{EI} 5 \mathrm{Y} \\
13.7 \mathrm{Y} \\
53.46 \mathrm{H}\end{array}$ & $\begin{array}{l}1.36 E-01 \\
2.38 E+01 \\
0.0 \\
0.0 \\
1 . T 1 E+01\end{array}$ & $\begin{array}{l}3.02 E-01 \\
2.59 E+01 \\
0.0 \\
0.0 \\
1.03 E+01\end{array}$ & $\begin{array}{l}4.54 E-01 \\
3.52 E+01 \\
0.0 \\
0.0 \\
2.12 E \cdot 01\end{array}$ & $\begin{array}{l}1.04 E-01 \\
2.59 E+01 \\
0.0 \\
0.0 \\
1.01 E+01\end{array}$ & $\begin{array}{l}2.01 E-01 \\
2.12 E+01 \\
0.0 \\
0.0 \\
1.64 E+01\end{array}$ & $\begin{array}{l}3 E-01 \\
G E \cdot 01 \\
B E \cdot 01\end{array}$ & $\begin{array}{l}b E-01 \\
9 E \cdot 01 \\
5 E+01\end{array}$ & $\begin{array}{l}4 E-01 \\
6 E+01 \\
T E+01\end{array}$ & $\begin{array}{l}2.35 E \\
0.0 \\
0.0 \\
1.68 E\end{array}$ \\
\hline $\begin{array}{l}C D-115 M \\
C D-117 \\
C D-117 M \\
1 N-111 \\
1 N-113 A\end{array}$ & $\begin{array}{r}44.6 \mathrm{O} \\
2.49 \mathrm{H} \\
3.36 \mathrm{H} \\
2.83 \mathrm{O} \\
1.658 \mathrm{H}\end{array}$ & $\begin{array}{l}1.75 E+00 \\
8.52 E+01 \\
1.59 E+02 \\
3.31 E+01 \\
2.16 E+01\end{array}$ & $\begin{array}{l}1.86 E+00 \\
9.05 E+01 \\
1.68 E+02 \\
3.62 E+01 \\
2.33 E+01\end{array}$ & $\begin{array}{l}1.99 E+00 \\
9.09 E+01 \\
1.77 E+02 \\
4.99 E+01 \\
2.81 E+01\end{array}$ & $\begin{array}{l}1.88 E+00 \\
9.17 E+01 \\
1.70 E+02 \\
3.59 E+01 \\
2.40 E+01\end{array}$ & $\begin{array}{l}1.69 E+00 \\
8.03 E+01 \\
1.48 E+02 \\
2.94 E \bullet 01 \\
2.03 E+01\end{array}$ & $\begin{array}{l}1.51 E * 00 \\
1.37 E * 01 \\
1.38 E \cdot 02 \\
2.83 E \cdot 01 \\
1.86 E+01\end{array}$ & $\begin{array}{l}2.03 E+00 \\
1.01 E+02 \\
1.00 E+02 \\
5.10 E+01 \\
2.86 E+01\end{array}$ & & 3. $28 E+$ \\
\hline $\begin{array}{l}1 N-114 \\
1 N-114 M \\
I N-115 \\
1 N-115 M\end{array}$ & $\begin{array}{r}71.9 \mathrm{~S} \\
49.51 \mathrm{D} \\
4.615 \mathrm{Y} \\
4.36 \mathrm{H}\end{array}$ & $\begin{array}{l}2.64 E+00 \\
7.51 E \cdot 00 \\
0.0 \\
1.35 E+01\end{array}$ & $\begin{array}{l}2.83 E+00 \\
8.17 E+00 \\
0.0 \\
1.46 E+01\end{array}$ & $\begin{array}{l}3.19 E \bullet 00 \\
1.00 E \bullet 01 \\
0.0 \\
1.02 E+01\end{array}$ & $\begin{array}{l}2.89 E+00 \\
0.12 E+00 \\
0.0 \\
1.50 E+01\end{array}$ & $\begin{array}{l}2.55 \mathrm{E} \\
7.06 \mathrm{E} \\
0.0 \\
1.25 \mathrm{E}\end{array}$ & $\begin{array}{l}2.28 E+00 \\
6.45 E+00 \\
0.0 \\
1.16 E \cdot 01\end{array}$ & $\begin{array}{l}3.24 E+00 \\
1.02 E+01 \\
0.0 \\
1.05 E+01\end{array}$ & $\begin{array}{l}2.58 E+00 \\
7.3 B E+00 \\
0.0 \\
1.33 E+01\end{array}$ & $\begin{array}{l}0.0 \\
1.33\end{array}$ \\
\hline
\end{tabular}


DOSE-RATE FACTORS FOR BOOY ORGANS ANO EFFECTIVE DOSE-RATE FACTOR fOR EXPOSURE I M above CONTAMINATED ground SJRFACE IN MREM/YR PER MICROCUR IE/ISOUARE MI

\begin{tabular}{|c|c|c|c|c|c|c|c|c|c|}
\hline NUCL IDE & HALF-LIFE & TESTES & THYMUS & THYROID & UTERUS & FECTIVE & $\begin{array}{c}\text { SKIN } \\
\text { I PHOTONI }\end{array}$ & $\begin{array}{l}\text { SKIV } \\
\text { (ELECTRONI }\end{array}$ & $\begin{array}{l}\text { SKIN } \\
\text { (rOTAL) }\end{array}$ \\
\hline $\begin{array}{l}T C-96 M \\
T C-97 \\
T C-97 M \\
\text { TC-98 } \\
\text { TC-99 }\end{array}$ & $\begin{array}{r}51.5 \mathrm{M} \\
2.6 E 6 \mathrm{Y} \\
89 \mathrm{D} \\
4.2 E 6 \mathrm{Y} \\
2.13 E 5 \mathrm{Y}\end{array}$ & $\begin{array}{l}5.18 E+00 \\
7.80 E-01 \\
7.07 E-01 \\
1.66 E+02 \\
7.25 E-05\end{array}$ & $\begin{array}{l}0 \\
02 \\
2 \\
2 \\
5\end{array}$ & $\begin{array}{l}00 \\
01 \\
01 \\
02 \\
05\end{array}$ & $\begin{array}{l}00 \\
03 \\
02 \\
05\end{array}$ & $\begin{array}{l}30 \\
01 \\
01 \\
32 \\
05\end{array}$ & $\begin{array}{l}7.45 E+00 \\
3.76 E \cdot 00 \\
2 . B 6 E \cdot 00 \\
1.92 E+02 \\
7.73 E-05\end{array}$ & $\begin{array}{l}0.0 \\
0.0 \\
0.0 \\
3.32 E+00 \\
0.0\end{array}$ & $\begin{array}{l}7.45 \mathrm{E}+00 \\
3.76 \mathrm{7}+00 \\
2.86 \mathrm{E} \cdot 00 \\
1.95 \mathrm{E}+02 \\
1.73 \mathrm{E}-05\end{array}$ \\
\hline $\begin{array}{l}T C-99 M \\
T C-101 \\
R U-97 \\
R U-103 \\
R U-105\end{array}$ & $\begin{array}{r}6.02 \mathrm{H} \\
14.2 \mathrm{M} \\
2.9 \mathrm{D} \\
39.35 \mathrm{D} \\
4.44 \mathrm{H}\end{array}$ & $\begin{array}{l}1.67 E+01 \\
4.25 E+01 \\
2.96 E+01 \\
5.84 E+01 \\
9.40 E+01\end{array}$ & $\begin{array}{l}28 E+01 \\
22 E+01 \\
19 E+01 \\
42 E+01\end{array}$ & $\begin{array}{l}.64 E+01 \\
.99 E+01 \\
.77 E+01 \\
.41 E+01 \\
.85 E+01\end{array}$ & $\begin{array}{l}E+00 \\
E+01 \\
E+01 \\
E+01 \\
E+01\end{array}$ & & & $\begin{array}{l}0.0 \\
4.32 E+02 \\
0.0 \\
6.31 E+00 \\
3.43 E+02\end{array}$ & $\begin{array}{l}1.82 E+01 \\
4.80 E+02 \\
3.57 E+01 \\
7.31 E+01 \\
4.51 E+02\end{array}$ \\
\hline $\begin{array}{l}\text { RU-1 } 06 \\
R H-103 M \\
R H-105 \\
R H-105 M \\
R H-106\end{array}$ & 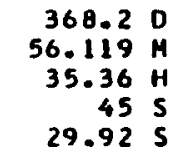 & $\begin{array}{l}0.0 \\
1.56 E-01 \\
9.66 E+00 \\
4.35 E+00 \\
2.46 E+01\end{array}$ & $\begin{array}{l}0.0 \\
1.35 E-02 \\
7.29 E+00 \\
2.79 E+00 \\
1.89 E+01\end{array}$ & $\begin{array}{l}.0 \\
.32 E-02 \\
0.07 E+00 \\
02 E+00 \\
.29 E \bullet 01\end{array}$ & $\begin{array}{l}2.0 \\
2.32 E-03 \\
5.83 E+00 \\
2.00 E+00 \\
.50 E+01\end{array}$ & $\begin{array}{l}.0 \\
15 E-01 \\
.39 E+00 \\
.70 E+00\end{array}$ & $\begin{array}{l}0.0 \\
5.32 E-01 \\
1.09 E+01 \\
6.58 E+00 \\
2.82 E+01\end{array}$ & $\begin{array}{l}0.0 \\
0.0 \\
3.21 E+01 \\
0.0 \\
1.53 E+03\end{array}$ & $\begin{array}{l}0.0 \\
5.32 E-01 \\
4.29 E+01 \\
6.58 E+00 \\
1.56 E+03\end{array}$ \\
\hline $\begin{array}{l}P D-103 \\
P D-107 \\
P D-109 \\
A G-109 \\
A G-106 \\
A G-108\end{array}$ & $\begin{array}{r}16.961 \mathrm{O} \\
6.5 E 6 \mathrm{Y} \\
13.453 \mathrm{H} \\
8.46 \mathrm{O} \\
2.37 \mathrm{M}\end{array}$ & $\begin{array}{l}1.38 E+00 \\
0.0 \\
8.31 E-02 \\
3.22 E+02 \\
2.13 E+00\end{array}$ & $\begin{array}{l}1.09 E-01 \\
0.0 \\
6.29 E-02 \\
2.51 E+02 \\
1.61 E+00\end{array}$ & $\begin{array}{l}5.52 E-01 \\
0.0 \\
7.69 E-02 \\
3.02 E+02 \\
1.97 E+00\end{array}$ & $\begin{array}{l}2.00 E-02 \\
0.0 \\
5.04 E-02 \\
1.99 E+02 \\
1.27 E+00\end{array}$ & $\begin{array}{l}1.02 E+00 \\
0.0 \\
1.11 E-02 \\
2.75 E+02 \\
1.81 E+00\end{array}$ & $\begin{array}{l}4.73 E \cdot 00 \\
0.0 \\
9.50 E-02 \\
3.72 E+02 \\
2.50 E+00\end{array}$ & $\begin{array}{l}0.0 \\
0.0 \\
2.89 E+02 \\
2.82 E+00 \\
6.14 E+02\end{array}$ & $\begin{array}{l}4.73 E+00 \\
0.0 \\
2.89 E+02 \\
3.75 E+02 \\
6.16 E+02\end{array}$ \\
\hline $\begin{array}{l}A G-108 M \\
A G-109 M \\
A G-110 \\
A G-110 M \\
A G-111\end{array}$ & $\begin{array}{rr}127 & Y \\
39.6 & S \\
24.57 & S \\
249.85 & 0 \\
7.46 & 0\end{array}$ & $\begin{array}{l}1.94 E+02 \\
1.17 E+00 \\
3.65 E+00 \\
3.13 E+02 \\
3.27 E+00\end{array}$ & $\begin{array}{l}1.49 E+02 \\
4.44 E-01 \\
2.84 E \cdot 00 \\
2.48 E \cdot 02 \\
2.47 E+00\end{array}$ & $\begin{array}{l}1.82 E+02 \\
7.84 E-01 \\
3.43 E * 00 \\
2.91 E+02 \\
3.07 E+00\end{array}$ & $\begin{array}{l}1.18 E+02 \\
2.49 E-01 \\
2.23 E+00 \\
1.94 E+02 \\
1.98 E+00\end{array}$ & $\begin{array}{l}1.66 \\
8.97 \\
3.12 \\
2.68 \\
2.84\end{array}$ & $\begin{array}{l}2.26 E \\
2.52 E \\
4.22 E \\
3.61 E \\
3.68 E\end{array}$ & $\begin{array}{l}6.22 E+00 \\
0.0 \\
1.28 E+03 \\
1.05 E+01 \\
2.74 E+02\end{array}$ & $\begin{array}{l}2.32 E+02 \\
2.52 E+00 \\
1.28 E+03 \\
3.72 E+02 \\
2.78 E+02\end{array}$ \\
\hline $\begin{array}{l}C D-109 \\
C D-1114 \\
C D-113 \\
C D-113 M \\
C D-115\end{array}$ & $\begin{array}{r}464 \mathrm{D} \\
48.7 \mathrm{H} \\
9.3 \mathrm{i} 5 \mathrm{Y} \\
13.7 \mathrm{Y} \\
53.46 \mathrm{H}\end{array}$ & $\begin{array}{l}1.33 E+00 \\
3.60 E+01 \\
0.0 \\
0.0 \\
2.47 E+01\end{array}$ & $\begin{array}{l}1.63 E-01 \\
2.69 E \bullet 01 \\
0.0 \\
0.0 \\
1.87 E \bullet 01\end{array}$ & $\begin{array}{l}6.11 E-01 \\
3.41 E+01 \\
0.0 \\
0.0 \\
2.28 E+01\end{array}$ & $\begin{array}{l}2.49 E-02 \\
2.09 E+01 \\
0.0 \\
0.0 \\
1.49 E+01\end{array}$ & $\begin{array}{l}9.45 E-01 \\
3.14 E+01 \\
0.0 \\
0.0 \\
2.11 E+01\end{array}$ & $\begin{array}{l}3.82 E+00 \\
4.09 E+01 \\
0.0 \\
0.0 \\
2.83 E+01\end{array}$ & $\begin{array}{l}0.0 \\
0.0 \\
0.0 \\
5.01 E+01 \\
2.23 E+02\end{array}$ & $\begin{array}{l}3.82 E+00 \\
4.09 E+01 \\
0.0 \\
5.01 E+01 \\
2.51 E+02\end{array}$ \\
\hline $\begin{array}{l}C D-115 M \\
C D-117 \\
C D-117 M \\
1 N-111 \\
\text { IN-113M }\end{array}$ & $\begin{array}{lll}44.6 & \mathrm{D} \\
2.49 & \mathrm{H} \\
3.36 & \mathrm{H} \\
2.83 & \mathrm{D} \\
1.658 \mathrm{H}\end{array}$ & $\begin{array}{l}2.46 E+00 \\
1.20 E+02 \\
2.20 E+02 \\
5.07 E+01 \\
3.17 E+01\end{array}$ & $\begin{array}{l}1.96 E \cdot 00 \\
9.44 E \cdot 01 \\
1.74 E \cdot 02 \\
3.76 E \cdot 01 \\
2.36 E+01\end{array}$ & $\begin{array}{l}2.34 E+00 \\
1.13 E+02 \\
2.05 E+02 \\
4.80 E+01 \\
2.93 E+01\end{array}$ & $\begin{array}{l}1.53 E+00 \\
7.54 E+01 \\
1.42 E \bullet 02 \\
2.91 E * 01 \\
1.89 E+01\end{array}$ & $\begin{array}{l}2.10 E+00 \\
1.03 E+02 \\
1.89 E+02 \\
4.42 E+01 \\
2.73 E+01\end{array}$ & $\begin{array}{l}2.84 E+00 \\
1.37 E+02 \\
2.51 E+02 \\
5.82 E+01 \\
3.65 E+01\end{array}$ & $\begin{array}{l}6.06 E+02 \\
3.69 E+02 \\
8.65 E+01 \\
0.0 \\
2.92 E+01\end{array}$ & $\begin{array}{l}6.09 E+02 \\
5.06 E+02 \\
3.37 E+02 \\
5.02 E+01 \\
6.57 E+01\end{array}$ \\
\hline $\begin{array}{l}I N-114 \\
I N-114 M \\
I N-115 \\
I N-115 M \\
I N-1164\end{array}$ & $\begin{array}{r}71.9 \mathrm{~S} \\
49.51 \mathrm{D} \\
4.6 \mathrm{E} 15 \mathrm{Y} \\
4.36 \mathrm{H} \\
54.15 \mathrm{H}\end{array}$ & $\begin{array}{l}3.80 E+00 \\
1.15 E+01 \\
0.0 \\
2.02 E+01 \\
2.64 E+02\end{array}$ & $\begin{array}{l}2.91 E+00 \\
8.45 E+00 \\
0.0 \\
1.48 E+01 \\
2.10 E+02\end{array}$ & $\begin{array}{l}3.54 E+00 \\
1.06 E+01 \\
0.0 \\
1.86 E+01 \\
2.49 E+02\end{array}$ & $\begin{array}{l}2.31 E+00 \\
\epsilon .52 E+00 \\
0.0 \\
1.18 E+01 \\
1.68 E+02\end{array}$ & $\begin{array}{l}3.24 E+00 \\
9.80 E+00 \\
0.0 \\
1.74 E+01 \\
2.27 E+02\end{array}$ & $\begin{array}{l}4.38 E+00 \\
1.39 E+01 \\
0.0 \\
2.35 E+01 \\
3.03 E+02\end{array}$ & $\begin{array}{l}0.06 E+02 \\
0.0 \\
1.10 E+01 \\
6.93 E+00 \\
2.12 E+02\end{array}$ & $\begin{array}{l}8.10 E+02 \\
1.39 E+01 \\
1.70 E+01 \\
3.04 E+01 \\
5.15 E+02\end{array}$ \\
\hline
\end{tabular}



DOSE-RATE FACTORS FOR BOOY ORGANS ANO EFFECTIVE DOSE-RATE FACTOR FOR EXPOSURE I M ABOVE CIINI MAI IVAIEO GKIJUHO SJRFACE
IN MREM/YR PER MICROCURIE/ISOUARE MI

\begin{tabular}{|c|c|c|c|c|c|c|c|c|c|c|}
\hline NUCLIDE & HALF-LIFE & AORENALS & BL AODER & BRAIN & BREAST & HE AR T & $\begin{array}{l}\text { SMALL } \\
\text { INTES IINE }\end{array}$ & $\begin{array}{c}\text { UPPER } \\
\text { LARGE } \\
\text { INTESTINE }\end{array}$ & $\begin{array}{c}\text { LOHER } \\
\text { LARGE } \\
\text { INTESTINE }\end{array}$ & XIONEYS \\
\hline $\begin{array}{l}I N-117 \\
I N-117 M \\
S N-113 \\
S N-117 M \\
S N-119 M\end{array}$ & $\begin{array}{r}43.8 \mathrm{M} \\
116.5 \mathrm{M} \\
115.110 \mathrm{D} \\
13.60 \mathrm{D} \\
293.0 \mathrm{D}\end{array}$ & $\begin{array}{l}6.17 E+01 \\
8.17 E+00 \\
7.46 E-01 \\
1.35 E+01 \\
1.78 E-01\end{array}$ & $\begin{array}{l}5.83 E \cdot 01 \\
7.53 E+00 \\
7.11 E-01 \\
1.25 E+01 \\
1.89 E-01\end{array}$ & $\begin{array}{l}6.45 E+01 \\
8.12 E+00 \\
5.71 E-01 \\
1.31 E+01 \\
7.55 E-02\end{array}$ & $\begin{array}{l}\text { Q.11E*01 } \\
1.20 E \bullet 01 \\
3.35 E+00 \\
2.26 E * 01 \\
1.63 E \cdot 00\end{array}$ & $\begin{array}{l}5.71 E+01 \\
7.32 E+00 \\
5.37 E-01 \\
1.21 E+01 \\
0.15 E-02\end{array}$ & $\begin{array}{l}5.11 E+01 \\
6.53 E+00 \\
4.93 E-01 \\
1.06 E+01 \\
8.19 E-02\end{array}$ & $\begin{array}{l}6.07 E+01 \\
7.83 E+00 \\
7.98 E-01 \\
1.31 E+01 \\
2.27 E-01\end{array}$ & $\begin{array}{l}5.60 E+01 \\
1.27 E+00 \\
6.26 E-01 \\
1.20 E+01 \\
1.36 E-01\end{array}$ & $\begin{array}{l}6.03 E+01 \\
1.02 E+00 \\
9.99 E-01 \\
1.29 E+01 \\
3.60 E-01\end{array}$ \\
\hline $\begin{array}{l}S N-123 \\
S N-125 \\
S N-126 \\
S B-117 \\
S B-122\end{array}$ & 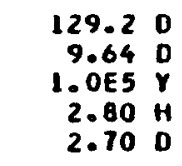 & $\begin{array}{l}5.58 E-01 \\
2.47 E+01 \\
4.11 E+00 \\
1.56 E+01 \\
3.91 E+01\end{array}$ & $\begin{array}{l}5.48 E-01 \\
2.43 E+01 \\
4.21 E+00 \\
1.45 E+01 \\
3.73 E+01\end{array}$ & $\begin{array}{l}6.18 E-01 \\
2.74 E+01 \\
4.17 E+00 \\
1.53 E+01 \\
4.17 E+01\end{array}$ & $\begin{array}{l}6.84 E-01 \\
3.06 E \cdot 01 \\
9.23 E+00 \\
2.56 E+01 \\
4.88 E+01\end{array}$ & $\begin{array}{l}5.42 E-01 \\
2.41 E+01 \\
3.79 E+00 \\
1.40 E+01 \\
3.65 E+01\end{array}$ & $\begin{array}{l}4.96 E-01 \\
2.20 E+01 \\
3.35 E+00 \\
1.24 E+01 \\
3.29 E \cdot 01\end{array}$ & $\begin{array}{l}5.77 E-01 \\
2.56 E+01 \\
4.20 E+00 \\
1.52 E+01 \\
3.80 E+01\end{array}$ & $\begin{array}{l}5.31 E-01 \\
2.36 E+01 \\
3.68 E+00 \\
1.39 E+01 \\
3.58 E+01\end{array}$ & $\begin{array}{l}5.76 E-01 \\
2.56 E+01 \\
4.48 E+00 \\
1.51 E+01 \\
\text { 3. 88E+01 }\end{array}$ \\
\hline $\begin{array}{l}S B-124 \\
S B-125 \\
S B-126 \\
S B-1264 \\
S B-127\end{array}$ & $\begin{array}{r}60.200 \\
2.77 \gamma \\
12.40 \\
19.0 \mathrm{~N} \\
3.85 \mathrm{D}\end{array}$ & $\begin{array}{l}1.49 E+02 \\
3.77 E+01 \\
2.42 E+022 \\
1.39 E+02 \\
5.84 E+01\end{array}$ & $\begin{array}{l}1.48 E+02 \\
3.58 E+01 \\
2.32 E \cdot 02 \\
1.33 E \bullet 02 \\
5.58 E \cdot 01\end{array}$ & $\begin{array}{l}1.65 \mathrm{E} * 02 \\
3.96 \mathrm{E} * 01 \\
2.60 \mathrm{01} \\
1.48 \mathrm{02} \\
6.23 \mathrm{E} * 01\end{array}$ & & $\begin{array}{l}1.46 E+02 \\
3.48 E+01 \\
2.28 E+02 \\
1.30 E+02 \\
5.47 E+01\end{array}$ & $\begin{array}{l}1.34 E+02 \\
3.14 E+01 \\
2.06 E+02 \\
1.17 E+02 \\
4.93 E+01\end{array}$ & $\begin{array}{l}1.55 E+02 \\
3.71 E+01 \\
2.42 E+02 \\
1.38 E+02 \\
5.81 E+01\end{array}$ & & $\begin{array}{l}1.55 E+02 \\
3.74 E+01 \\
2.42 E+02 \\
1.38 E+02 \\
5.81 E+01\end{array}$ \\
\hline $\begin{array}{l}58-129 \\
T E-121 \\
T E-121 M \\
T E-123 \\
T E-123 M\end{array}$ & $\begin{array}{r}4.40 \\
16.80 \\
1540 \\
1613 \% \\
119.10\end{array}$ & $\begin{array}{l}1.20 E+02 \\
4.97 E+01 \\
1.89 E+01 \\
2.29 E-01 \\
1.29 E+01\end{array}$ & $\begin{array}{l}1.17 E+02 \\
4.74 E \bullet 01 \\
1.75 E+01 \\
2.83 E-01 \\
1.19 E \bullet 01\end{array}$ & $\begin{array}{l}1.31 E+02 \\
5.26 E+01 \\
1.86 E+01 \\
1.12 E-01 \\
1.26 E+01\end{array}$ & $\begin{array}{l}1.48 E+02 \\
6.44 E+01 \\
2.82 E+01\end{array}$ & & & & & $\begin{array}{l}1.22 E+02 \\
4.96 E \cdot 01 \\
1.83 E+01 \\
5.02 E-01 \\
1.24 E+01\end{array}$ \\
\hline $\begin{array}{l}T E-125 M \\
T E-12 T \\
T E-12 T M \\
T E-129 \\
T E-129 M\end{array}$ & $\begin{array}{rl}58 & D \\
9.35 & H \\
109 & D \\
69.6 & N \\
33.6 & D\end{array}$ & $\begin{array}{l}7.34 E-01 \\
4.40 E-01 \\
2.38 E-01 \\
4.76 E+00 \\
2.92 E \cdot 00\end{array}$ & $\begin{array}{l}9.64 E-01 \\
4.11 E-01 \\
3.05 E-01 \\
9.54 E+00 \\
2.06 E+00\end{array}$ & & & & $\begin{array}{l}4.59 E-01 \\
3.61 E-01 \\
1.50 E-01 \\
3.96 E \cdot 00 \\
2.45 E \cdot 00\end{array}$ & & & $\begin{array}{l}1.60 E+00 \\
4.25 E-01 \\
5.02 E-01 \\
4.78 E+00 \\
3.12 E+00\end{array}$ \\
\hline $\begin{array}{l}\text { TE-1 } 31 \\
T E-131 M \\
T E-132 \\
T E-133 \\
T E-133 M\end{array}$ & $\begin{array}{r}25.0 \mathrm{M} \\
30 \mathrm{H} \\
78.2 \mathrm{H} \\
12.45 \mathrm{H} \\
55.4 \mathrm{H}\end{array}$ & $\begin{array}{l}3.67 E \cdot 01 \\
1.20 E+02 \\
2.01 E+01 \\
1.83 E+01 \\
1.87 E+02\end{array}$ & $\begin{array}{l}3.49 E \cdot 01 \\
1.17 E \bullet 02 \\
1.86 E * 01 \\
7.55 E+01 \\
1.82 E+02\end{array}$ & & $\begin{array}{l}01 \\
02 \\
01\end{array}$ & $\begin{array}{l}3.43 E+01 \\
1.15 E+02 \\
1.77 E+01 \\
1.43 E+01 \\
1.80 E+02\end{array}$ & & & & $\begin{array}{l}3.63 E+01 \\
1.22 E+02 \\
1.96 E+01 \\
1.88 E+01 \\
1.91 E+02\end{array}$ \\
\hline $\begin{array}{l}I E-134 \\
1-122 \\
1-123 \\
1-124 \\
1-125\end{array}$ & $\begin{array}{r}41.8 \mathrm{M} \\
3.62 \mathrm{M} \\
13.13 \mathrm{H} \\
4.18 \mathrm{O} \\
60.14 \mathrm{O}\end{array}$ & $\begin{array}{l}7.73 E+01 \\
8.54 E+01 \\
1.44 E+01 \\
8.76 E+01 \\
8.40 E-01\end{array}$ & & & & & & & & $\begin{array}{l}1.65 E+01 \\
8.46 E+01 \\
1.41 E+01 \\
8.96 E+01 \\
1.87 E+00\end{array}$ \\
\hline $\begin{array}{l}1-126 \\
1-128 \\
1-129 \\
1-130 \\
1-131\end{array}$ & $\begin{array}{r}12.93 \mathrm{O} \\
24.99 \mathrm{M} \\
1.57 E 7 \mathrm{Y} \\
12.36 \mathrm{H} \\
0.040 \mathrm{D}\end{array}$ & $\begin{array}{l}4.04 E+01 \\
6.72 E+00 \\
8.12 E-01 \\
1.87 E+02 \\
3.47 E+01\end{array}$ & $\begin{array}{l}3.86 E+01 \\
6.35 E+00 \\
1.12 E * 00 \\
1.19 E+02 \\
3.24 E+01\end{array}$ & $\begin{array}{l}4.28 E+01 \\
7.03 E+00 \\
5.07 E-01 \\
2.01 E+02 \\
3.56 E+01\end{array}$ & $\begin{array}{l}8.67 E+00 \\
4.74 E+00 \\
2.32 E+02 \\
4.53 E+01\end{array}$ & $\begin{array}{l}3.77 E \cdot 01 \\
6.19 E \cdot 00 \\
4.97 E-01 \\
1.76 E * 02 \\
3.16 E+01\end{array}$ & $\begin{array}{l}3.40 E+01 \\
5.5 \mathrm{TE}+00 \\
5.61 E-01 \\
1.59 E+02 \\
2.84 E+01\end{array}$ & $\begin{array}{l}4.01 E \cdot 01 \\
6.51 E+00 \\
9.15 E-01 \\
1.87 E+02 \\
3.35 E+01\end{array}$ & $\begin{array}{l}3.70 E \cdot 01 \\
6.08 E+00 \\
6.69 E-01 \\
8.73 E+02 \\
3.12 E+01\end{array}$ & $\begin{array}{l}\text { 4. } 04 E+01 \\
6.60 E+00 \\
1.77 E+00 \\
1.87 E+02 \\
\text { 3. } 36 E+01\end{array}$ \\
\hline
\end{tabular}


DOSE-RATE FACTORS FOR BODY ORGANS AND EFFECTIVE DOSE-RATE FACTOR FOR EXPOSURE I M ABOVE CONTAMINATED ;ROUND SJRF ACE
IN MREM/YR PER MICROCUR IE/ISOUARE MI

\begin{tabular}{|c|c|c|c|c|}
\hline JCL IDE & HALF-LIFE & LIVER & LUNGS & MARROW \\
\hline $\begin{array}{l}\text { IN-117 } \\
\text { IN-117M } \\
\text { SN-113 } \\
\text { SN-117M } \\
\text { SN-119M }\end{array}$ & $\begin{array}{rl}43.8 & M \\
116.5 & M \\
115.1 & 0 \\
13.60 & D \\
293.0 & 0\end{array}$ & $\begin{array}{l}5.80 E+01 \\
7.48 E+00 \\
6.17 E-01 \\
1.23 E+01 \\
1.29 E-01\end{array}$ & $\begin{array}{l}6.25 E+01 \\
8.15 E+00 \\
8.10 E-01 \\
1.36 E+01 \\
2.23 E-01\end{array}$ & $\begin{array}{l}7.56 E+01 \\
1.08 E+01 \\
1.16 E+00 \\
2.02 E+01 \\
3.53 E-01\end{array}$ \\
\hline $\begin{array}{l}S N-123 \\
S N-125 \\
S N-126 \\
S B-117 \\
S B-122\end{array}$ & $\begin{array}{r}129.20 \\
9.64 \\
1.065 \mathrm{~V} \\
2.80 \mathrm{H} \\
2.70 \mathrm{D}\end{array}$ & $\begin{array}{l}5.46 E-01 \\
2.43 E+01 \\
4.13 E+00 \\
1.43 E+01 \\
3.71 E+01\end{array}$ & $\begin{array}{l}5.80 E-01 \\
2.58 E+01 \\
4.63 E+00 \\
1.58 E+01 \\
3.97 E+01\end{array}$ & $\begin{array}{l}6.16 E-01 \\
2.75 E+01 \\
7.91 E+00 \\
2.25 E+01 \\
4.49 E+01\end{array}$ \\
\hline $\begin{array}{l}S B-124 \\
S B-125 \\
S B-126 \\
S B-126 M \\
S B-127\end{array}$ & $\begin{array}{r}60.20 \mathrm{D} \\
2.77 \mathrm{~V} \\
12.4 \mathrm{D} \\
19.0 \mathrm{H} \\
3.85 \mathrm{D}\end{array}$ & $\begin{array}{l}1.47 E+02 \\
3.55 E+01 \\
2.30 E+02 \\
1.32 E+02 \\
5.54 E+01\end{array}$ & $\begin{array}{l}1.56 E+02 \\
3.82 E+01 \\
2.47 E+02 \\
1.41 E+02 \\
5.94 E+01\end{array}$ & $\begin{array}{l}1.68 E+02 \\
4.45 E+01 \\
2.76 E+02 \\
1.59 E+02 \\
6.72 E+01\end{array}$ \\
\hline $\begin{array}{l}S B-129 \\
T E-121 \\
T E-121 M \\
T E-123 \\
T E-123 M\end{array}$ & $\begin{array}{r}4.40 \mathrm{H} \\
16.8 \mathrm{O} \\
154 \mathrm{D} \\
1 \mathrm{E} 13 \mathrm{Y} \\
119.7 \mathrm{D}\end{array}$ & $\begin{array}{l}1.16 E+02 \\
4.70 E+01 \\
1.73 E+01 \\
1.96 E-01 \\
1.18 E+01\end{array}$ & $\begin{array}{l}1.24 E+02 \\
5.05 E+01 \\
1.89 E+01 \\
2.94 E-01 \\
1.30 E+01\end{array}$ & $\begin{array}{l}1.34 E+02 \\
5.77 E+01 \\
2.52 E+01 \\
4.79 E-01 \\
1.93 E+01\end{array}$ \\
\hline $\begin{array}{l}\text { TE-1 } 25 \mathrm{M} \\
\text { TE-1 } 27 \\
\text { TE-1 } 27 \mathrm{M} \\
\text { TE-1 } 29 \\
\text { TE-1 } 29 \mathrm{M}\end{array}$ & $\begin{array}{rl}58 & 0 \\
9.35 & H \\
109 & 0 \\
69.6 & H \\
33.6 & 0\end{array}$ & $\begin{array}{l}6.93 E-01 \\
4.09 E-01 \\
2.24 E-01 \\
4.48 E+00 \\
2.78 E+00\end{array}$ & $\begin{array}{l}9.46 E-01 \\
4.41 E-01 \\
3.05 E-01 \\
4.84 E+00 \\
3.02 E+00\end{array}$ & $\begin{array}{l}1.57 E+00 \\
5.32 E-01 \\
5.04 E-01 \\
5.66 E+00 \\
3.46 E+00\end{array}$ \\
\hline $\begin{array}{l}\text { TE- } 131 \\
T E-131 M \\
T E-132 \\
T E-133 \\
T E-133 M\end{array}$ & $\begin{array}{r}25.0 \mathrm{M} \\
30 \mathrm{H} \\
78.2 \mathrm{H} \\
12.45 \mathrm{M} \\
55.4 \mathrm{H}\end{array}$ & $\begin{array}{l}3.47 E+01 \\
1.16 E+02 \\
1.84 E+01 \\
1.52 E+01 \\
1.81 E+02\end{array}$ & $\begin{array}{l}3.74 E+01 \\
1.24 E+02 \\
2.01 E+01 \\
0.03 E+01 \\
1.93 E+02\end{array}$ & $\begin{array}{l}4.55 E+01 \\
1.37 E+02 \\
2.76 E+01 \\
9.09 E+01 \\
2.12 E+02\end{array}$ \\
\hline $\begin{array}{l}T E-134 \\
1-122 \\
1-123 \\
1-124 \\
1-125\end{array}$ & 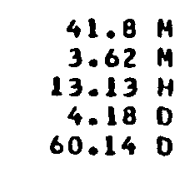 & $\begin{array}{l}1.29 E+01 \\
8.09 E+01 \\
1.32 E+01 \\
8.49 E+01 \\
7.90 E-01\end{array}$ & $\begin{array}{l}7.84 E+01 \\
8.68 E+01 \\
1.46 E+01 \\
9.06 E+01 \\
1.09 E+00\end{array}$ & $\begin{array}{l}9.24 E+01 \\
9.93 E+01 \\
2.12 E+01 \\
9.99 E+01 \\
1.81 E+00\end{array}$ \\
\hline $\begin{array}{l}1-126 \\
1-128 \\
1-129 \\
1-130 \\
1-131\end{array}$ & $\begin{array}{r}12.93 \mathrm{D} \\
24.99 \mathrm{M} \\
1.57 E 7 \mathrm{Y} \\
12.36 \mathrm{H} \\
8.040 \mathrm{O}\end{array}$ & $\begin{array}{l}3.83 E+01 \\
6.30 E+00 \\
8.43 E-01 \\
1.78 E+02 \\
3.22 E+01\end{array}$ & $\begin{array}{l}4.11 E+01 \\
6.78 E+00 \\
1.07 E+00 \\
1.91 E+02 \\
3.47 E+01\end{array}$ & $\begin{array}{l}4.68 E+01 \\
7.95 E+00 \\
1.81 E+00 \\
2.13 E+02 \\
4.20 E+01\end{array}$ \\
\hline
\end{tabular}

6. 29E+01

$5.48 E+01$
$6.78 E+00$

PANCREAS

SKELETON

SPLEEN

STOMACH

$6.21 E-01$
$1.00 E+01$

$4.97 E+01$
$5.31 E 000$

1. $21 E$ E -02

$1.00 \mathrm{E}+01$
$1.54 \mathrm{E}-01$

$1.04 E+01$
$1.09 E-01$

$7.70 E+01$
$1.10 E+01$

$1.21 E+00$

$5.68 \mathrm{E}+01$
$7.42 \mathrm{E}+00$

$5.73 E+01$

$5.86 E-01$

$5.26 \mathrm{E}-01$

4. $12 E-01$

2. $79 \mathrm{E}-01$

C. $48 \mathrm{E}-01$

3.48E+00 2.98E+00

6.26E-01

$1.23 E+01$
$1.29 E-01$

$1.22 \mathrm{E}+01$
$1.48 \mathrm{E}-01$

$1,48 E+0$

$2.98 E+00$

8. $15 E+00$

$5.28 E-01$

5. 37E-0I

3. $58 \mathrm{E}+01$

$1.21 E+01$
$3.19 E+01$

2. $31 E+01$
$4.56 E+01$

3.90E+00

3. $96 \mathrm{E}+00$

$\begin{array}{llll}1.59 E+02 & 1.39 E+02 & 1.28 E+02 & 1.70 E+02\end{array}$

$\begin{array}{lll}1.88 E+01 & 3.39 E+01 & 3.05 E+01 \\ 2.52 E+02 & 2.23 E+02 & 1.99 E+02\end{array}$

4. $32 \mathrm{E}+01$
$2.80 E+02$

3. $66 E+01$

$1.27 E+02 \quad 1.14 E+02$

$1.62 E+02$

$1.42 E+02$

$1.44 E+02$

$\begin{array}{llllll}1.26 E+02 & 1.12 E+02 & 1.00 E+02 & 1.37 E+02 & 1.13 E+02 & 1.15 E+02\end{array}$

$\begin{array}{lllllll}1.26 E+02 & 1.12 E+02 & 1.00 E+02 & 1.37 E+02 & 1.13 E+02 & 1.15 E+02\end{array}$

$\begin{array}{ll}1.29 E+02 & 1.30 \mathrm{EH}+02 \\ 5.40 \mathrm{OE}+01 & 5.48 \mathrm{E}+01\end{array}$

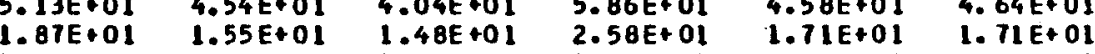

$\begin{array}{llllll}1.05 E-01 & 2.07 E-01 & 1.41 E-01 & 5.05 E-01 & 1.78 E-01 & 2.03 E-01 \\ 1.05 E+01 & 1.03 E+01 & 9.88 E+00 & 1.98 E+01 & 1.18 E+01 & 1.17 E+01\end{array}$

$\begin{array}{llll}3.59 E-01 & 6.76 E-01 & 4.57 E-01 & 1.64 E+00\end{array}$

$5.99 E-01$

6. $717 \mathrm{E}-01$

$\begin{array}{llllll} & \\ 4.21 E-01 & 2.85 E-01 & 3.53 E-01 & 5.46 E-01 & 4.02 E-01 & 4.04 E-01 \\ & 1.96 E-01 & 2.20 E-01\end{array}$

$\begin{array}{llllll}4.89 E+00 & 4.28 E+00 & 3.85 E+00 & 5.76 E+00 & 4.38 E+00 & 4.42 E+00 \\ 2.95 E+00 & 2.71 E+00 & 2.37 E+00 & 3.52 E+00 & 2.69 E+00 & 2.75 E+00\end{array}$

3. $52 E+00$

$2.69 \mathrm{E}+00$

$75 E+00$

3. $72 E+01$

3.25E+01 2.97E +01

$4.64 E+01$

$3.40 E+01$
$1.13 E+02$

$3.43 E+01$

$1.96 \mathrm{E}+01$ 1.63E+01 $1.55 \mathrm{E}+01$ 2.82E+01 1.81E+01 $1.81 \mathrm{E}+01$

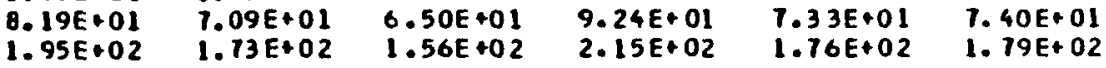

1. $95 \mathrm{E} \cdot 02$

$1.90 E+01$
$0.86 E+01$

$1.36 E+01$
$9.20 E+01$

$6.92 E+01 \quad 6.25 E+01$

$9.41 E+01$
$1.01 E+02$
$2: 102+01$

3. $98 \mathrm{E}-01$

i. $17 \mathrm{H}^{2}+01$

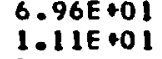

$2.18 E+01$
$1.02 E \rightarrow 02$

$7.12 E+01$

7. $20 E+01$

B.77E-01 $5.3,19 \mathrm{E}-01$

1. $89 \mathrm{EE}+00$

$1.31 E+01 \quad 1.31 E+01$

$\begin{array}{ll}\text { 8.24E+01 } & 8.36 E+01 \\ 6.80 E-01 & 7.73 E-01\end{array}$

4.. $18 E+01$

$3.68 E+01 \quad 3.29 E+01$

4. $76 \mathrm{EE} 01$
8.07E+ 01

(3)

$4.02 E-01$

$7.68 \mathrm{E}-01$

$5.43 \mathrm{E}+00$

O. $07 E+00$
$1.87 E+00$

$3.73 E+01$
$6.17 \mathrm{E}+00$
1.00

3. $78 \mathrm{EF} 01$
$6.22 \mathrm{E}+00$

$1.95 E+02$
$3.57 E+01$ 


\begin{tabular}{|c|c|c|c|c|c|c|c|c|c|}
\hline $.10 E$ & IFE & ES & inus & I ROID & US & fIVE & $\begin{array}{l}\text { SKIN } \\
\text { I PHOTONI }\end{array}$ & SKIN & Nat \\
\hline 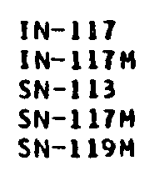 & $\begin{array}{ll}116.5 & M \\
115.1 & 0 \\
113.60 & 0 \\
293.0 & 0\end{array}$ & 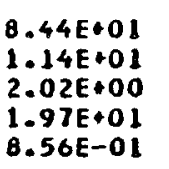 & & & & & & 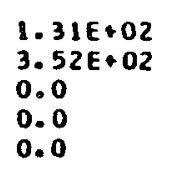 & \\
\hline & $\begin{array}{r}129.20 \\
9.640 \\
1.065 \mathrm{D} \\
2.060 \mathrm{Y} \\
2.70 \mathrm{O}\end{array}$ & 1 & & & & & & & \\
\hline & $\begin{array}{r}60.200 \\
2.77 y \\
12.40 \\
19.0 \\
3.850\end{array}$ & $\begin{array}{l}3.296 \\
1.896 \\
1.944\end{array}$ & $\begin{array}{l}2 \\
1 \\
2 \\
2 \\
1\end{array}$ & & & & & & \\
\hline $\begin{array}{l}-129 \\
121 \\
121 M \\
12123 \\
1123 M\end{array}$ & $\begin{array}{r}4.40 \mathrm{H} \\
16.8 \mathrm{O} \\
154 \mathrm{O} \\
1 \mathrm{E13} Y \\
119.7 \mathrm{D}\end{array}$ & $\begin{array}{l}6.871 \\
2.864 \\
1.022 \\
1.878\end{array}$ & 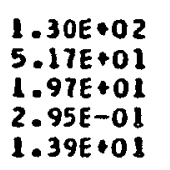 & & & & & 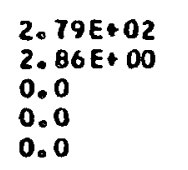 & \\
\hline $\begin{array}{l}27 \mathrm{r} \\
29 \\
29 \mathrm{M}\end{array}$ & $\begin{array}{r}58 \text { O } \\
9.35 \% \\
109 \\
69.6 \\
33.6 \\
33.60\end{array}$ & $\begin{array}{l}2.99 E+00 \\
5.94 E-01 \\
9.46-01 \\
6.696-01 \\
4.42 E+00\end{array}$ & $\begin{array}{l}1.07 E+00 \\
4.48 E-01 \\
3.38 E-01 \\
4.956+00 \\
3.16 E+00\end{array}$ & & & & & & \\
\hline $\begin{array}{l}131 \\
1314 \\
1322 \\
132\end{array}$ & $\begin{array}{r}25.0 \mathrm{M} \\
30 \mathrm{H} \\
78.2 \mathrm{H} \\
12.45 \mathrm{H} \\
55.4 \mathrm{M}\end{array}$ & & & & & & & & \\
\hline-13 & $\begin{array}{r}41.8 \mathrm{~N} \\
33.62 \mathrm{M} \\
13.13 \mathrm{H} \\
4.18 \mathrm{D} \\
60.14 \mathrm{O}\end{array}$ & & $\begin{array}{l}8.84 E+08 \\
1.55 E+01 \\
9.32 E+001 \\
1.23 E+00\end{array}$ & & & & & & \\
\hline 28 & $\begin{aligned} 12.930 \\
24.999 \\
1.579 \mathrm{H} \\
12.36 \mathrm{H} \\
0.040 \mathrm{D}\end{aligned}$ & $\begin{array}{l}9.17 \mathrm{E} \\
3.09 \mathrm{E}\end{array}$ & $\begin{array}{l}248 \\
496 \\
36\end{array}$ & $\begin{array}{l}5.18 \mathrm{~B} \\
8.486 \\
2.171 \\
2.396 \\
4.396\end{array}$ & $\begin{array}{l}.33 E+01 \\
.51 E+00 \\
05 E-01 \\
.55 E+02 \\
03 E+01 \\
03 E 01\end{array}$ & $\begin{array}{l}0 \\
1 \\
1\end{array}$ & $\begin{array}{l}6.45 E \\
1.05 E \\
5.28 \mathrm{E} \\
2.93 \mathrm{E} \\
5.32 \mathrm{E}\end{array}$ & & $\begin{array}{l}\begin{array}{l}8 E+00 \\
0 E+02 \\
6 E+02\end{array}\end{array}$ \\
\hline
\end{tabular}




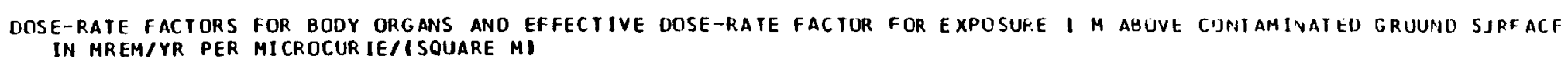

nuclide half-life adrenals

BLADOER

BRAIN BREAST

HE ART

SMALL
INTESIINE

UPPER

LOWER

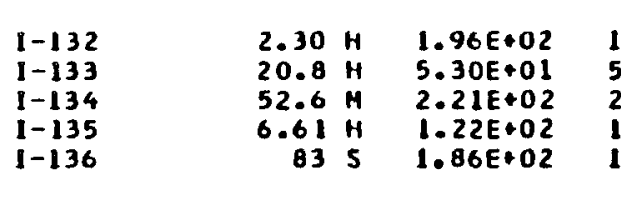

$1.90 E \div 02$

2. $13 E \cdot 02$

$2.42 E+02$

$1.87 E+02$

$1.69 E+02$

INTESTINE

LARGE
LESTINE

$2.15 E+02$

$2.42 E+02$
$1.37 E+02$

$2.12 E+02$

$2.12 E+02$

$1.93 E+02 \quad 5.27 E+01$

$20.1 \mathrm{H} \quad 5.45 E+00$

$5.29 \mathrm{E}+00$

$2.10 E+02$

2. $35 E+02$

$1.20 \mathrm{E}+02$
1.02

$1.11 E+02$

$2.26 E+02$
$1.28 E+02$

$1.83 E+02 \quad 1.98 E+02$

$X E-122$
$X E-123$

$X E-125$
$X E-125$
$X E-127$

$\begin{array}{rrr}16.8 & \mathrm{H} & 2.25 E+0 \\ 36.406 & \mathrm{D} & 2.42 E+0\end{array}$

$1.02 E+01$
$7.11 E+01$

$4.76 E+00$
$4.93 E+01$

प.

1. $99 E+02$

$\begin{array}{lll}2.01 E+01 & 1.81 E+01 & 2.18 E+01 \\ 2.14 E+01 & 1.92 E+01 & 2.31 E+01\end{array}$

$X E-129 M$

$\begin{array}{ll}2.23 E+00 & 2.24 E+01 \\ & \end{array}$

$2.36 E+01$
$1.68 E+00$

$3.75 E+01$

$2.14 E \cdot 01$

$2.31 E \cdot 0$

$4.83 E+00 \quad 5.91 E+00$

$X E-131 M$
$X E-133$

$X E-133$
$X E-133 M$
$X E-135$

$11.840 \quad 8.24 E-01$

$9.04 E-01$

$2.19 \mathrm{D} \quad 2.79 \mathrm{E}+00 \quad 2.78 E+00$

$6.01 E-01$

3. $70 E+00$

$5.76 E-01$

$1.61 \mathrm{E} \cdot 00$

$2.35 E \cdot 00$

B. $13 E \bullet 00$

$6.51 E+00$
$3.18 E+01$

$15.36 \mathrm{M} 3.80 \mathrm{E}+01$

$2.11 \mathrm{E} \bullet 01$
$3.61 \mathrm{E} \bullet 01$

$2.28 E+01$
$4.02 E+01$

$X E-137$
$X E-138$

$x S-126$
CS-129

$3.83 \mathrm{M} \quad 1.62 \mathrm{E}+01$
$14.13 \mathrm{M} \quad 8.79 \mathrm{E}+0$

$1.55 E \cdot 01$
$8.800 \cdot 01$

8. $76 E-01$

2. 36E.OI

4. $84 \mathrm{E}+01$

(2)

$2.14 E+00$
$1.84 E+01$

$2.72 E+00$
$2.17 E+01$

$6.93 E-01 \quad 1.45 E+00$

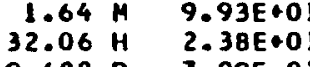

$2.05 E+01$
$1.13 E+02$

3. $52 \mathrm{E}+01$

3.17E.01

$2.17 E+01$
$3.75 E+01$

$3.84 E+00$
$3.32 E+00$

$2.04 E+01 \quad 2.18 E+0$

$.27 E+01$

$1.05 E * 02$
$2.41 E * 01$

$1.26 E+02$
$3.59 E+01$

$0.73 E+01$

$1.37 E+01$
$8.05 E+01$

$1.61 E+01$
$9.28 E+0$

$3.46 E+01$ 3. $76 E+01$

6.475 O $6.17 \mathrm{E}+0$

$5.94 E+01$

$4.14 E-01$

$4.41 \mathrm{E}+00$

$2.14 E+01$

$0.32 E+01$
$1.94 E+01$

$9.80 E+01$
$2.32 E+01$

$.49 E+01 \quad 1.61 E+01$
$0.58 E+01 \quad 9.31 E+0$

CS-132

CS-134

CS-135

$2.062 \mathrm{Y} \quad 1.36 \mathrm{E}+02$

$2.90 \mathrm{H}$ I.87E+00 $1.90 \mathrm{E}+00$

$\begin{array}{lll}2.3 E 6 & Y & 0.0 \\ 13.16 & 0 & 1.83 E+02\end{array}$

0.0

$6.59 E+01$

$7.96 E+01$

$4.13 E-01 \quad 4.68 E-01$

$9.06 E+01 \quad 9.82 E+01$

$\begin{array}{ll}2.13 E+01 & 2.44 E+01 \\ 5.78 E-01 & 1.58 E+00\end{array}$

$58 \mathrm{~F}+01$
$.28 \mathrm{O}+02$
1.61

$5.22 E+01$

6. $18 E+01$

$5.78 \mathrm{E}-01 \quad 1.58 \mathrm{E}+0$

$1.73 E+00 \quad 4.55 E+00$

0.0

0.0

Cs-137

CS-137
CS-138
CS-139

CS -139
$B A-131$

$30.17 Y \quad 0.0$

$0.0 \quad 0.0$

0.0

$1.61 E+00$
0.0

$1.45 \mathrm{E}+00$

$32.2 \mathrm{M} \quad 1.01 \mathrm{E}+02$

$9.40 \mathrm{M}$
$11.8 \mathrm{D}$
$4.08 \mathrm{2}+0 \mathrm{E}+01$

$1.81 E+02 \quad 2.03 E+02$

0.0
$2.27 E$

$.75 E+02$

0.0
$1.59 E+02$

1. $36 E \bullet 02$
$1.80 E \bullet 00$

$.67 E+01 \quad 6.24 E+0$

$\begin{array}{ll}1.26 E+02 & 1.36 E+02 \\ 1.64 E+00 & 2.21 E+00\end{array}$

$0.0 \quad 0.0$

0.0
$1.86 E+02$

0.0

0.0

0.0

$\begin{array}{ll}3.09 E+01 & 2.59 E+01 \\ 3.19 E+01 & \\ 3.21 E+01 & 3.37 E+01\end{array}$

$2.8 B E+01$
$5.8 B E+01$

$2.29 \mathrm{E} O 01$
$3.12 \mathrm{EOOI}$
$3.35 \mathrm{E}+01$

$1.90 E+02$
$2.44 E+01$

$10.5 \mathrm{Y}$ 3.36E+O

$5.11 E+00$

4. $99 E+00$

$9.61 E \cdot 00$

3. $01 E+01$

$3.35 E \cdot 01$
$2.72 E \cdot 01$

$4.00 E+01$
$3.25 E+01$

$\begin{array}{ll}0.0 & 0.0\end{array}$

$B A-133 M$
$B A-135 M$

$B A-137 M$
$B A-139$

$\begin{array}{ll}38.9 \mathrm{H} & 5.23 \mathrm{2}+00 \\ 28.7 \mathrm{H} & 4.61 \mathrm{1}+00\end{array}$

$4.55 \mathrm{E} \cdot 00$

4. $36 E+00$

$9.61 E+00$
$8.78 E+00$

4.52E+00

$4.15 E \cdot 00$
$3.65 E+00$

5. $06 E+00$

.

$2.94 E+00$ 3. $14 E+00$

$83.1 \mathrm{H}$
$12.789 \mathrm{O}$

$6.52 E+01$
$4.80 E+00$

$4.94 \mathrm{E} \in 01$
$2.86 \rightarrow 00$

4. $46 E+01$

$4.47 \mathrm{E}+00$

3.

$2.54 \mathrm{E}$
1.3900

3. 06E*0

4.06E+00 5.13E+00

2.24E.01

$7.15 \mathrm{E} \cdot 01$
$7.16 \mathrm{E}+01$
3.1600

$6.48 \mathrm{E}+01$

$7.59 \mathrm{E} \cdot 0$

$4.83 E+00 \quad 3.04 E+00$
$0.52 E+01$

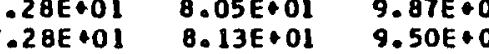

2. $26 \mathrm{E}+02$

40.22 H $1.49 \mathrm{EE} .02$

$95.4 \mathrm{H} \quad 2.03 E+02$

$3.20 E+00$
$2.06 E+02$

$2.30 E+02$

$2.59 E+02$

$3.17 E+00$
$2.05 E+02$

.91E.02 3.37E+00

$7.03 E+01 \quad 7.59 E+01$

$1.03 E+01 \quad 7.64 E+01$

3.11E+00 3. 37E+0O 


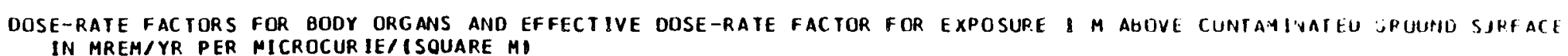

\begin{tabular}{|c|c|c|c|c|c|c|c|c|c|c|}
\hline NUCL IDE & HALF-LIFE & LIVER & LUNGS & MARROW & $\begin{array}{c}\text { RED } \\
\text { MARROH }\end{array}$ & OVARIES & PANCREAS & SKELETON & SPLEEN & STOMACH \\
\hline $\begin{array}{l}1-132 \\
1-133 \\
1-134 \\
1-135 \\
1-136\end{array}$ & $\begin{array}{rl}2.30 & H \\
20.8 & H \\
52.6 & H \\
6.61 & H \\
83 & S\end{array}$ & $\begin{array}{l}1.89 E+02 \\
5.04 E+01 \\
2.14 E+02 \\
1.21 E+02 \\
1.88 E+02\end{array}$ & $\begin{array}{l}2.01 E+02 \\
5.39 E+01 \\
2.28 E+02 \\
1.29 E+02 \\
1.98 E+02\end{array}$ & $\begin{array}{l}2.21 E+02 \\
6.10 E+01 \\
2.47 E+02 \\
1.36 E+02 \\
2.06 E+02\end{array}$ & $\begin{array}{l}2.05 E+02 \\
5.51 E+01 \\
2.31 E+02 \\
1.30 E+02 \\
2.01 E+02\end{array}$ & $\begin{array}{l}1.82 E+02 \\
4.85 E+01 \\
2.06 E+02 \\
1.15 E+02 \\
1.74 E+02\end{array}$ & $\begin{array}{l}1.63 E+02 \\
4.34 E+01 \\
1.85 E+02 \\
1.05 E+02 \\
1.64 E+02\end{array}$ & $\begin{array}{l}2.24 E+02 \\
6.20 E+01 \\
2.51 E+02 \\
1.38 E+02 \\
2.09 E+02\end{array}$ & $\begin{array}{l}1.83 E+02 \\
4.91 E+01 \\
2.07 E+02 \\
1.17 E+02 \\
1.81 E+02\end{array}$ & $\begin{array}{l}1.86 E+02 \\
4.97 E+01 \\
2.11 E+02 \\
1.19 E+02 \\
1.85 E+02\end{array}$ \\
\hline 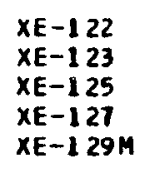 & 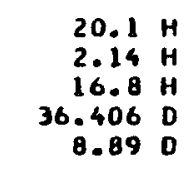 & $\begin{array}{l}5.07 E \bullet 00 \\
5.01 E \bullet 01 \\
2.08 E \bullet 01 \\
2.21 E \bullet 01 \\
2.18 E \bullet 00\end{array}$ & $\begin{array}{l}5.62 E+00 \\
5.39 E+01 \\
2.27 E+01 \\
2.42 E+01 \\
2.64 E+00\end{array}$ & $\begin{array}{l}7.46 E+00 \\
6.36 E+01 \\
3.04 E+01 \\
3.31 E+01 \\
4.22 E+00\end{array}$ & $\begin{array}{l}5.22 E \cdot 00 \\
5.37 E+01 \\
2.21 E * 01 \\
2.37 E \cdot 01 \\
1.52 E \cdot 00\end{array}$ & $\begin{array}{l}4.67 E+00 \\
4.70 E+01 \\
1.88 E+01 \\
1.90 E+01 \\
1.97 E+00\end{array}$ & $\begin{array}{l}4.22 E+00 \\
4.30 E+01 \\
1.76 E+01 \\
1.88 E+01 \\
1.52 E+00\end{array}$ & $\begin{array}{l}7.63 E+00 \\
6.48 E+01 \\
3.10 E+01 \\
3.38 E+01 \\
4.35 E+00\end{array}$ & $\begin{array}{l}4.92 E+00 \\
4.89 E+01 \\
2.05 E+01 \\
2.19 E+01 \\
1.93 E+00\end{array}$ & $\begin{array}{l}\text { 4. } 99 E+00 \\
\text { 4. } 94 E+01 \\
2.05 E+01 \\
2.10 E+01 \\
2.08 E+00\end{array}$ \\
\hline $\begin{array}{l}X E-131 M \\
X E-133 \\
X E-133 M \\
X E-135 \\
X E-135 M\end{array}$ & 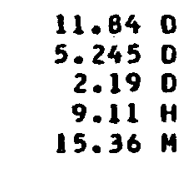 & $\begin{array}{l}8.00 E-01 \\
3.15 E+00 \\
2.58 E+00 \\
2.10 E+01 \\
3.59 E+01\end{array}$ & $\begin{array}{l}9.85 E-01 \\
3.56 E \cdot 00 \\
2.92 E+00 \\
2.27 E+01 \\
3.85 E \cdot 01\end{array}$ & $\begin{array}{l}1.59 E+00 \\
6.12 E+00 \\
4.16 E+00 \\
2.97 E+01 \\
4.43 E+01\end{array}$ & $\begin{array}{l}5.41 E-01 \\
2.22 E+00 \\
2.51 E+00 \\
2.32 E+01 \\
3.93 E+01\end{array}$ & $\begin{array}{l}7.31 E-01 \\
2.66 E+00 \\
2.32 E+00 \\
1.89 E+01 \\
3.45 E+01\end{array}$ & $\begin{array}{l}5.55 E-01 \\
2.16 E+00 \\
2.09 E+00 \\
1.81 E+01 \\
3.08 E+01\end{array}$ & $\begin{array}{l}1.65 E+00 \\
6.30 E+00 \\
4.27 E+00 \\
3.03 E+01 \\
4.50 E+01\end{array}$ & $\begin{array}{l}7.10 E-01 \\
2.8 B E+00 \\
2.48 E+00 \\
2.08 E+01 \\
3.50 E+01\end{array}$ & $\begin{array}{l}\text { 7. } 66 E-01 \\
2.97 E+00 \\
2.53 E+00 \\
2.07 E+01 \\
3.54 E+01\end{array}$ \\
\hline $\begin{array}{l}X E-137 \\
X E-138 \\
C S-126 \\
C S-129 \\
C S-1131\end{array}$ & 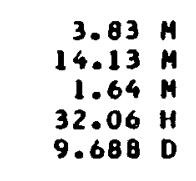 & $\begin{array}{l}1.54 E+01 \\
8.81 E+01 \\
9.39 E+01 \\
2.22 E+01 \\
7.13 E-01\end{array}$ & $\begin{array}{l}1.65 E+01 \\
9.33 E+01 \\
1.01 E+02 \\
2.42 E+01 \\
9.29 E-01\end{array}$ & $\begin{array}{l}1.09 E \bullet 01 \\
1.01 E \bullet 02 \\
1.16 E \bullet 02 \\
2.98 E \bullet 01 \\
1.56 E \bullet 00\end{array}$ & $\begin{array}{l}1.69 E * 01 \\
9.46 E * 01 \\
1.03 E * 02 \\
2.40 E * 01 \\
3.43 E-01\end{array}$ & $\begin{array}{l}1.46 E+01 \\
8.09 E+01 \\
8.98 E+01 \\
2.09 E+01 \\
6.67 E-01\end{array}$ & $\begin{array}{l}1.33 E+01 \\
7.65 E+01 \\
8.08 E+01 \\
1.89 E+01 \\
4.39 E-01\end{array}$ & & & $\begin{array}{l}1.52 E+01 \\
8.62 E+01 \\
9.26 E+01 \\
2.19 E+01 \\
6.70 E-01\end{array}$ \\
\hline $\begin{array}{l}C S-132 \\
C S-134 \\
C S-134 M \\
C S-135 \\
C S-136\end{array}$ & 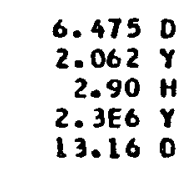 & $\begin{array}{l}5.87 E+01 \\
1.30 E+02 \\
1.78 E+00 \\
0.0 \\
1.76 E+02\end{array}$ & $\begin{array}{l}0.30 E+01 \\
1.39 E+02 \\
2.02 E+00 \\
0.0 \\
1.88 E+02\end{array}$ & $\begin{array}{l}7.05 E+01 \\
1.53 E+02 \\
3.22 E+00 \\
0.0 \\
2.08 E+02\end{array}$ & $\begin{array}{l}6.36 E+01 \\
1.41 E+02 \\
1.55 E+00 \\
0.0 \\
1.90 E+02\end{array}$ & $\begin{array}{l}5.70 E+01 \\
1.26 E+02 \\
1.55 E+00 \\
0.0 \\
1.69 E+02\end{array}$ & $\begin{array}{l}5.04 E+01 \\
1.12 E+02 \\
1.37 E+00 \\
0.0 \\
1.52 E+02\end{array}$ & $\begin{array}{l}7.17 E+01 \\
1.56 E+02 \\
3.32 E+00 \\
0.0 \\
2.12 E+02\end{array}$ & $\begin{array}{l}5.70 E+01 \\
1.26 E+02 \\
1.71 E+00 \\
0.0 \\
1.71 E+02\end{array}$ & $\begin{array}{l}\text { 5. } 80 E+01 \\
1.28 E+02 \\
1.73 E+00 \\
0.0 \\
1.74 E+02\end{array}$ \\
\hline $\begin{array}{l}C S-137 \\
C S-138 \\
C S-139 \\
B A-131 \\
B A-133\end{array}$ & $\begin{array}{r}30.17 \mathrm{Y} \\
32.2 \mathrm{M} \\
9.40 \mathrm{M} \\
11.8 \mathrm{D} \\
10.5 \mathrm{Y}\end{array}$ & $\begin{array}{l}0.0 \\
1.81 E+02 \\
2.31 E \cdot 01 \\
3.83 E+01 \\
3.15 E+01\end{array}$ & $\begin{array}{l}0.0 \\
1.91 E+02 \\
2.43 E+01 \\
4.15 E+01 \\
3.43 E+01\end{array}$ & $\begin{array}{l}0.0 \\
2.02 E+02 \\
2.54 E+01 \\
5.16 E+01 \\
4.46 E+01\end{array}$ & $\begin{array}{l}0.0 \\
1.93 E+02 \\
2.46 E+01 \\
4.10 E+01 \\
3.32 E+01\end{array}$ & $\begin{array}{l}0.0 \\
1.69 E+02 \\
2.15 E+01 \\
3.59 E+01 \\
2.89 E+01\end{array}$ & $\begin{array}{l}0.0 \\
1.58 E+02 \\
2.01 E+01 \\
3.25 E+01 \\
2.64 E+01\end{array}$ & & $\begin{array}{l}0.0 \\
1.74 E+02 \\
2.22 E+01 \\
3.74 E+01 \\
3.07 E+01\end{array}$ & $\begin{array}{l}\text { D. } 0 \\
1.71 E+02 \\
2.26 E+01 \\
3.78 E+01 \\
3.09 E+08\end{array}$ \\
\hline $\begin{array}{l}B A-133 H \\
B A-135 M \\
B A-137 H \\
B A-139 \\
B A-140\end{array}$ & 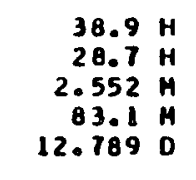 & $\begin{array}{l}4.80 E+00 \\
4.31 E+00 \\
5.00 E+01 \\
2.92 E+00 \\
1.58 E+01\end{array}$ & $\begin{array}{l}5.37 E+00 \\
4.76 E+00 \\
5.36 E+01 \\
3.19 E+00 \\
1.70 E+01\end{array}$ & $\begin{array}{l}7.27 E \bullet 00 \\
6.52 E \bullet 00 \\
5.97 E \bullet 01 \\
4.50 E+00 \\
2.01 E \bullet 01\end{array}$ & $\begin{array}{l}5.01 E \cdot 00 \\
4.37 E \bullet 00 \\
5.46 E \bullet 01 \\
3.04 E+00 \\
1.72 E+01\end{array}$ & $\begin{array}{l}4.40 E+00 \\
3.80 E+00 \\
4.86 E+01 \\
2.57 E+00 \\
1.50 E+01\end{array}$ & $\begin{array}{l}4.03 E+00 \\
3.53 E+00 \\
4.31 E+01 \\
2.46 E+00 \\
1.35 E+01\end{array}$ & $\begin{array}{l}7.43 E+00 \\
6.66 E+00 \\
6.06 E+01 \\
4.60 E+00 \\
2.05 E+01\end{array}$ & $\begin{array}{l}4.71 E+00 \\
4.15 E+00 \\
4.87 E+01 \\
2.89 E+00 \\
1.54 E+01\end{array}$ & $\begin{array}{l}\text { 4. } 76 E+00 \\
4.20 E+00 \\
4.95 E+01 \\
2.88 E+00 \\
1.56 E+01\end{array}$ \\
\hline $\begin{array}{l}B A-141 \\
B A-142 \\
1 A-140 \\
1 A-141 \\
L A-142\end{array}$ & $\begin{array}{c}18.21 \mathrm{M} \\
10.70 \mathrm{M} \\
40.22 \mathrm{H} \\
3.94 \mathrm{H} \\
95.4 \mathrm{H}\end{array}$ & $\begin{array}{l}7.25 E+01 \\
7.24 E+01 \\
1.79 E+02 \\
3.19 E+00 \\
2.07 E+02\end{array}$ & $\begin{array}{l}7.76 E+01 \\
7.73 E+01 \\
1.89 E+02 \\
3.37 E+00 \\
2.17 E+02\end{array}$ & $\begin{array}{l}9.05 E+01 \\
8.59 E+01 \\
2.02 E+02 \\
3.53 E+00 \\
2.27 E+02\end{array}$ & $\begin{array}{l}7.87 E+01 \\
7.70 E+01 \\
1.91 E+02 \\
3.40 E+00 \\
2.22 E+02\end{array}$ & $\begin{array}{l}6.70 E+01 \\
6.90 E+01 \\
1.68 E+02 \\
3.01 E+00 \\
1.89 E+02\end{array}$ & $\begin{array}{l}6.25 E+01 \\
6.24 E+01 \\
1.55 E+02 \\
2.77 E+00 \\
1.80 E+02\end{array}$ & $\begin{array}{l}9.21 E,+01 \\
8.74 E+01 \\
2.05 E+02 \\
3.59 E+00 \\
2.30 E+02\end{array}$ & $\begin{array}{l}7.08 E+01 \\
7.03 E+01 \\
1.72 E+02 \\
3.08 E+00 \\
1.99 E+02\end{array}$ & $\begin{array}{l}7.13 E+01 \\
7.13 E+01 \\
1.75 E+02 \\
3.11 E+00 \\
2.03 E+02\end{array}$ \\
\hline
\end{tabular}


DOSE-RATE FACTORS FOR BODY ORG ANS AND EFFECTIVE DOSE-RATE FACTOR FOR EXPOSURE I M ABOVE CONTAMINATEO GROUND SURFACE
IN MREM/YR PER MICROCUR IE/ISQUARE MI

\begin{tabular}{|c|c|c|c|c|c|c|c|c|c|}
\hline NUCL IDE & HALF-LIFE & TESTES & THYMUS & THY ROID & UTERUS & EFFECTIVE & $\begin{array}{c}\text { SKIN } \\
\text { I PHOTONI }\end{array}$ & $\begin{array}{c}\text { SKIN } \\
\text { (ELECTRON) }\end{array}$ & $\begin{array}{l}\text { SKIN } \\
\text { ITOTALI }\end{array}$ \\
\hline $\begin{array}{l}1-132 \\
1-133 \\
1-134 \\
1-135 \\
1-136\end{array}$ & $\begin{array}{rl}2.30 & H \\
20.8 & H \\
52.6 & H \\
6.61 & H \\
B 3 & S\end{array}$ & $\begin{array}{l}2.67 E+02 \\
7.21 E+01 \\
3.02 E+02 \\
1.69 E+02 \\
2.59 E+02\end{array}$ & $\begin{array}{l}2.10 E+02 \\
5.53 E+01 \\
2.39 E+02 \\
1.34 E+02 \\
2.05 E+02\end{array}$ & $\begin{array}{l}2.53 E+02 \\
6.72 E+01 \\
2.86 E+02 \\
1.59 E+02 \\
2.41 E+02\end{array}$ & $\begin{array}{l}1.65 E+02 \\
4.40 E+01 \\
1.07 E+02 \\
1.08 E+02 \\
1.68 E+02\end{array}$ & $\begin{array}{l}2.29 E \bullet 02 \\
6.16 E \bullet 01 \\
2.5 B E+02 \\
1.45 E \bullet 02 \\
2.22 E \bullet 02\end{array}$ & $\begin{array}{l}3.08 E+02 \\
6.27 E+01 \\
3.48 E+02 \\
1.93 E+02 \\
2.93 E+02\end{array}$ & $\begin{array}{l}4.50 E+02 \\
3.56 E+02 \\
6.03 E+02 \\
3.01 E+02 \\
2.19 E+03\end{array}$ & $\begin{array}{l}7.58 E+02 \\
4.38 E+02 \\
9.51 E+02 \\
4.95 E+02 \\
2.48 E+03\end{array}$ \\
\hline $\begin{array}{l}X E-122 \\
X E-123 \\
x E-125 \\
x E-127 \\
X E-129 M\end{array}$ & 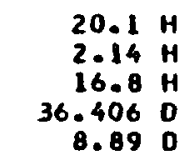 & $\begin{array}{l}8.89 E+00 \\
7.33 E+01 \\
3.27 E+01 \\
3.46 E+01 \\
6.45 E+00\end{array}$ & $\begin{array}{l}5.87 E+00 \\
5.59 E+01 \\
2.39 E+01 \\
2.53 E+01 \\
3.11 E+00\end{array}$ & $\begin{array}{l}7.76 E+00 \\
6.85 E+01 \\
3.04 E+01 \\
3.24 E+01 \\
4.79 E+00\end{array}$ & $\begin{array}{l}4.22 E+00 \\
4.40 E+01 \\
1.80 E+01 \\
1.93 E+01 \\
1.35 E+00\end{array}$ & $\begin{array}{l}7.32 E \bullet 00 \\
6.27 E \bullet 01 \\
2.80 E \bullet 01 \\
2.98 E \bullet 01 \\
4.76 E \bullet 00\end{array}$ & $\begin{array}{l}1.15 E+01 \\
8.44 E+01 \\
3.84 E+01 \\
3.99 E+01 \\
1.04 E+01\end{array}$ & $\begin{array}{l}0.0 \\
1.54 E+02 \\
3.35 E-01 \\
0.0 \\
0.0\end{array}$ & $\begin{array}{l}1.15 E+01 \\
2.38 E+02 \\
3.87 E+01 \\
3.99 E+01 \\
1.04 E+01\end{array}$ \\
\hline $\begin{array}{l}X E-131 M \\
X E-133 \\
X E-133 H \\
X E-135 \\
X E-135 M\end{array}$ & 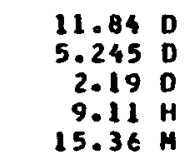 & $\begin{array}{l}2.49 E+00 \\
6.29 E+00 \\
5.17 E+00 \\
3.10 E+01 \\
5.20 E+01\end{array}$ & $\begin{array}{l}1.15 E+00 \\
4.22 E+00 \\
3.16 E+00 \\
2.35 E+01 \\
3.93 E+01\end{array}$ & $\begin{array}{l}1.82 E+00 \\
5.68 E+00 \\
4.35 E+00 \\
2.94 E+01 \\
4.81 E+01\end{array}$ & $\begin{array}{l}4.77 E-01 \\
2.40 E+00 \\
2.05 E+00 \\
1.85 E+01 \\
3.13 E+01\end{array}$ & $\begin{array}{l}1.83 E+00 \\
5.0 B E+00 \\
4.15 E+00 \\
2.71 E+01 \\
4.43 E+01\end{array}$ & $\begin{array}{l}4.11 E+00 \\
7.97 E+00 \\
7.13 E+00 \\
3.46 E+01 \\
5.98 E * 01\end{array}$ & $\begin{array}{l}0.0 \\
0.0 \\
0.0 \\
2.10 E+02 \\
1.04 E+02\end{array}$ & $\begin{array}{l}4.11 E+00 \\
7.97 E+00 \\
1.13 E \bullet 00 \\
2.44 E+02 \\
1.64 E+02\end{array}$ \\
\hline $\begin{array}{l}X E-137 \\
X E-138 \\
C S-126 \\
C S-129 \\
C S-131\end{array}$ & $\begin{array}{r}3.83 \mathrm{M} \\
14.13 \mathrm{M} \\
1.64 \mathrm{M} \\
32.06 \mathrm{H} \\
9.688 \mathrm{O}\end{array}$ & $\begin{array}{l}2.20 E+01 \\
1.22 E+02 \\
1.35 E+02 \\
3.49 E+01 \\
2.82 E+00\end{array}$ & $\begin{array}{l}1.60 E+01 \\
9.56 E+01 \\
1.03 E+02 \\
2.49 E+01 \\
1.13 E+00\end{array}$ & $\begin{array}{l}2.05 E \bullet 01 \\
1.14 E+02 \\
1.25 E+02 \\
3.15 E+01 \\
1.92 E \cdot 00\end{array}$ & $\begin{array}{l}1.35 E+01 \\
7.90 E+01 \\
0.21 E+01 \\
1.91 E+01 \\
3.17 E-01\end{array}$ & $\begin{array}{l}1.89 E+01 \\
1.05 E+02 \\
1.16 E+02 \\
2.94 E+01 \\
1.99 E+00\end{array}$ & $\begin{array}{l}2.51 E+01 \\
1.38 E+02 \\
1.55 E+02 \\
4.20 E+01 \\
4.97 E+00\end{array}$ & $\begin{array}{l}1.92 E+03 \\
5.96 E+02 \\
1.45 E+03 \\
1.36 E+00 \\
0.0\end{array}$ & $\begin{array}{l}1.95 E+03 \\
7.34 E+02 \\
1.60 E+03 \\
4.34 E+01 \\
4.97 E+00\end{array}$ \\
\hline $\begin{array}{l}C S-132 \\
C S-134 \\
C S-1344 \\
C S-135 \\
C S-136\end{array}$ & $\begin{array}{r}6.475 \mathrm{D} \\
2.062 \mathrm{Y} \\
2.90 \mathrm{H} \\
2.356 \mathrm{~V} \\
13.16 \mathrm{O}\end{array}$ & $\begin{array}{l}8.56 E+01 \\
1.85 E+02 \\
3.52 E+00 \\
0.0 \\
2.50 E+02\end{array}$ & $\begin{array}{l}6.55 E+01 \\
1.44 E+02 \\
2.28 E+00 \\
0.0 \\
1.98 E+02\end{array}$ & $\begin{array}{l}7.97 E+01 \\
1.74 E+02 \\
3.11 E+00 \\
0.0 \\
2.38 E+02\end{array}$ & $\begin{array}{l}5.08 E+01 \\
1.13 E+02 \\
1.41 E+00 \\
0.0 \\
1.54 E+02\end{array}$ & $\begin{array}{l}7.26 E+01 \\
1.58 E+02 \\
2.87 E+00 \\
0.0 \\
2.14 E+02\end{array}$ & $\begin{array}{l}1.00 E+02 \\
2.13 E+02 \\
4.66 E+00 \\
0.0 \\
2.88 E+02\end{array}$ & $\begin{array}{l}5.07 E+00 \\
6.30 E+01 \\
0.0 \\
0.0 \\
5.37 E+00\end{array}$ & $\begin{array}{l}1.05 E+02 \\
2.76 E+02 \\
4.66 E+00 \\
0.0 \\
2.94 E+02\end{array}$ \\
\hline $\begin{array}{l}C S-137 \\
C S-138 \\
C S-139 \\
B A-131 \\
B A-133\end{array}$ & $\begin{array}{r}30.17 \mathrm{Y} \\
32.2 \mathrm{M} \\
9.40 \mathrm{M} \\
11.8 \mathrm{O} \\
10.5 \mathrm{Y}\end{array}$ & $\begin{array}{l}0.0 \\
2.50 E+02 \\
3.10 E+01 \\
5.84 E+01 \\
4.94 E+01\end{array}$ & $\begin{array}{l}0.0 \\
1.98 E+02 \\
2.52 E \cdot 01 \\
4.30 E+01 \\
3.58 E+01\end{array}$ & $\begin{array}{l}0.0 \\
2.34 E * 02 \\
2.97 E \cdot 01 \\
5.36 E * 01 \\
4.53 E * 01\end{array}$ & $\begin{array}{l}0.0 \\
1.61 E+02 \\
2.06 E+01 \\
3.31 E+01 \\
2.70 E+01\end{array}$ & $\begin{array}{l}0.0 \\
2.15 E+02 \\
2.73 E+01 \\
4.96 E+01 \\
4.19 E+01\end{array}$ & $\begin{array}{l}0.0 \\
2.85 E+02 \\
3.62 E+01 \\
6.81 E+01 \\
5.82 E+01\end{array}$ & $\begin{array}{l}3.99 E+01 \\
1.32 E+03 \\
1.79 E+03 \\
2.56 E+00 \\
0.0\end{array}$ & $\begin{array}{l}3.99 E+01 \\
1.60 E+03 \\
1.83 E+03 \\
7.07 E+01 \\
5.82 E+01\end{array}$ \\
\hline $\begin{array}{l}B A-133 M \\
B A-135 M \\
B A-137 M \\
B A-139 \\
B A-140\end{array}$ & $\begin{array}{r}38.9 H \\
28.7 H \\
2.552 M \\
83.1 \% \\
12.789 \mathrm{H}\end{array}$ & $\begin{array}{l}8.42 E+00 \\
7.61 E+00 \\
7.16 E+01 \\
4.43 E+00 \\
2.32 E+01\end{array}$ & $\begin{array}{l}5.75 E+00 \\
5.13 E \cdot 00 \\
5.55 E \cdot 01 \\
3.39 E \cdot 00 \\
1.74 E \cdot 01\end{array}$ & $\begin{array}{l}7.4 B E+00 \\
6.72 E+00 \\
6.72 E+01 \\
4.29 E+00 \\
2.15 E+01\end{array}$ & $\begin{array}{l}4.07 E+00 \\
3.57 E+00 \\
4.35 E+01 \\
2.57 E+00 \\
1.37 E+01\end{array}$ & $\begin{array}{l}6.98 E+00 \\
6.27 E+00 \\
6.11 E+01 \\
3.8 B E+00 \\
1.98 E+01\end{array}$ & $\begin{array}{l}1.06 E+01 \\
9.60 E+00 \\
8.26 E+01 \\
4.90 E+00 \\
2.70 E+01\end{array}$ & $\begin{array}{l}0.0 \\
0.0 \\
6.94 E+01 \\
9.51 E+02 \\
1.82 E+02\end{array}$ & $\begin{array}{l}1.06 E+01 \\
9.60 E+00 \\
1.52 E+02 \\
9.56 E+02 \\
2.09 E+02\end{array}$ \\
\hline $\begin{array}{l}B A-141 \\
B A-142 \\
L A-140 \\
L A-141 \\
L A-142\end{array}$ & $\begin{array}{r}18.27 \mathrm{M} \\
10.70 \mathrm{M} \\
40.22 \mathrm{H} \\
3.94 \mathrm{H} \\
95.4 \mathrm{H}\end{array}$ & $\begin{array}{l}1.04 E+02 \\
1.03 E+02 \\
2.48 E+02 \\
4.42 E+00 \\
2.84 E+02\end{array}$ & $\begin{array}{l}8.05 E+01 \\
8.13 E+01 \\
1.96 E * 02 \\
3.53 E * 00 \\
2.23 E \bullet 02\end{array}$ & $\begin{array}{l}9.80 E+01 \\
9.78 E+01 \\
2.33 E+02 \\
4.17 E+00 \\
2.61 E+02\end{array}$ & $\begin{array}{l}6.39 E+01 \\
6.35 E+01 \\
1.59 E+02 \\
2.84 E+00 \\
1.85 E+02\end{array}$ & $\begin{array}{l}8.94 E+01 \\
0.82 E \cdot 01 \\
2.13 E+02 \\
3.79 E+00 \\
2.44 E+02\end{array}$ & $\begin{array}{l}1.18 E+02 \\
1.18 E+02 \\
2.84 E \cdot 02 \\
5.06 E+00 \\
3.20 E+02\end{array}$ & $\begin{array}{l}8.82 E+02 \\
4.04 E+02 \\
5.08 E+02 \\
1.01 E+03 \\
8.81 E+02\end{array}$ & $\begin{array}{l}1.00 E+03 \\
5.22 E+02 \\
1.92 E+02 \\
1.01 E+03 \\
1.20 E+03\end{array}$ \\
\hline
\end{tabular}




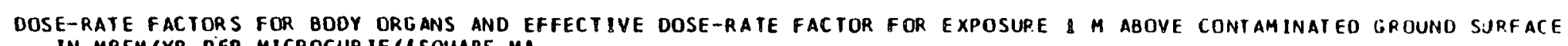

\begin{tabular}{|c|c|c|c|c|c|c|c|c|c|c|}
\hline CLIDE & HALF-LIFE & DRENALS & BLADDER & BRAIN & BREAST & HE ART & $\begin{array}{l}\text { SMALL } \\
\text { NTESTINE }\end{array}$ & $\begin{array}{l}\text { UPPER } \\
\text { LARGE } \\
\text { NTESTINE }\end{array}$ & $\begin{array}{l}\text { LOHER } \\
\text { LARGE } \\
\text { NIESTINE }\end{array}$ & $K$ IONE $Y$ \\
\hline $\begin{array}{l}-139 \\
-141 \\
-143 \\
-144 \\
-142\end{array}$ & $\begin{array}{r}137.66 \mathrm{D} \\
32.50 \mathrm{D} \\
33.0 \mathrm{H} \\
284.3 \mathrm{O} \\
19.13 \mathrm{H}\end{array}$ & $\begin{array}{l}.34 \mathrm{E} \\
.59 \mathrm{E} \\
.28 \mathrm{E}\end{array}$ & $\begin{array}{l}1 \\
0 \\
1 \\
0 \\
0\end{array}$ & $\begin{array}{l}11 \\
10 \\
10 \\
10\end{array}$ & $\begin{array}{l}1 \\
1 \\
10 \\
0\end{array}$ & & & & $\begin{array}{l}19 E+01 \\
10 E+00 \\
111 E+01 \\
011 E+00 \\
.22 E+00\end{array}$ & $\begin{array}{l}1.38 E+01 \\
6.69 E+00 \\
2.38 E+01 \\
1.65 E+00 \\
4.58 E+00\end{array}$ \\
\hline $\begin{array}{l}-143 \\
-144 \\
-1444 \\
-147 \\
-149\end{array}$ & $\begin{array}{r}13.56 \mathrm{O} \\
17.28 \mathrm{H} \\
7.2 \mathrm{M} \\
10.98 \mathrm{D} \\
1.73 \mathrm{H}\end{array}$ & $\begin{array}{l}7.81 E-01 \\
2.50 E+00 \\
4.25 E-01 \\
1.16 E+01 \\
3.41 E+01\end{array}$ & $\begin{array}{l}7.51 E-07 \\
2.50 E+00 \\
6.32 E-01 \\
1.15 E+01 \\
3.22 E \cdot 01\end{array}$ & $\begin{array}{l}8.43 E-07 \\
2.00 E+00 \\
3.25 E-01 \\
1.20 E+01 \\
3.4 T E+01\end{array}$ & $\begin{array}{l}9.57 E-d 7 \\
3.15 E+00 \\
1.91 E+00 \\
1.83 E+01 \\
4.79 E+01\end{array}$ & & & & & $\begin{array}{l}84 E-07 \\
66 E+00 \\
66 E-01 \\
22 E 01 \\
34 E+01\end{array}$ \\
\hline $\begin{array}{l}-143 \\
-144 \\
-145 \\
-146 \\
-147\end{array}$ & $\begin{array}{r}265 \mathrm{D} \\
363 \mathrm{D} \\
17.7 \mathrm{Y} \\
2020 \mathrm{O} \\
2.6234 \mathrm{Y}\end{array}$ & $\begin{array}{l}2.61 E+01 \\
1.36 E+02 \\
1.32 E+00 \\
6.51 E+01 \\
3.19 E-04\end{array}$ & $\begin{array}{l}2.57 E \bullet 01 \\
1.30 E \bullet 02 \\
1.88 E \bullet 00 \\
6.31 E \bullet 01 \\
2.99 E-04\end{array}$ & $\begin{array}{l}2.18 E+01 \\
1.45 E+02 \\
1.09 E+00 \\
6.99 E+01 \\
3.18 E-04\end{array}$ & $\begin{array}{l}5.24 E \\
8.40 E \\
5.32 E\end{array}$ & & & & & $\begin{array}{l}2.73 E+01 \\
1.36 E+02 \\
2.46 E+00 \\
6.60 E+01 \\
3.03 E-04\end{array}$ \\
\hline $\begin{array}{l}4-148 \\
4-148 M \\
4-149 \\
4-151 \\
-141\end{array}$ & $\begin{array}{r}5.37 \\
41.3 \\
53.08 \\
28.40 \mathrm{H} \\
1.069 \mathrm{E} 11 \mathrm{~V}\end{array}$ & $\begin{array}{l}4.56 E+01 \\
1.75 E+02 \\
1.06 E+00 \\
3.00 E+01 \\
0.0\end{array}$ & $\begin{array}{l}4.49 E+01 \\
1.67 E+02 \\
9.89 E-01 \\
2.84 E+01 \\
0.0\end{array}$ & $\begin{array}{l}5.05 E \\
1.07 E \\
1.00 E \\
3.08 E\end{array}$ & $\begin{array}{l}1 \\
2 \\
0 \\
3 \\
3\end{array}$ & $\begin{array}{l}4.43 E \\
1.64 E \\
9.63 E \\
2.74 E\end{array}$ & $\begin{array}{l}01 \\
02 \\
01\end{array}$ & $\begin{array}{l}01 \\
02 \\
00\end{array}$ & & $\begin{array}{l}\text { 4. } 71 \mathrm{E}+01 \\
1.74 \mathrm{E}+02 \\
1.02 \mathrm{E}+00 \\
2.96 \mathrm{E} 01 \\
0.0\end{array}$ \\
\hline $\begin{array}{l}S M-151 \\
S M-153 \\
E U-152 \\
E U-152 M \\
E U-154\end{array}$ & $\begin{array}{rl}90 & Y \\
46.7 & H \\
13.6 & Y \\
9.32 & H \\
8.8 & Y\end{array}$ & $\begin{array}{l}1.16 E-04 \\
4.48 E+00 \\
9.35 E+01 \\
2.65 E+01 \\
1.02 E+02\end{array}$ & $\begin{array}{l}6.10 E-05 \\
4.94 E+00 \\
9.18 E+01 \\
2.59 E+01 \\
1.00 E+02\end{array}$ & $\begin{array}{l}3.46 E \\
4.35 E \\
1.02 E \\
2.87 E \\
1.12 E\end{array}$ & $\begin{array}{l}1.87 E \\
1.06 E \\
1.22 E \\
3.43 E \\
1.30 E\end{array}$ & $\begin{array}{l}4.09 \\
3.98 \\
8.96 \\
2.52 \\
9.87\end{array}$ & $\begin{array}{l}05 \\
0.0 \\
b 1 \\
b 1 \\
b 1\end{array}$ & $\begin{array}{l}04 \\
00 \\
01 \\
01 \\
02\end{array}$ & & $\begin{array}{l}1.54 E-04 \\
5.4 B E+00 \\
9.66 E+01 \\
2.73 E+01 \\
1.05 E+02\end{array}$ \\
\hline $\begin{array}{l}E U-155 \\
E U-156 \\
G D-152 \\
G D-153 \\
G D-159\end{array}$ & 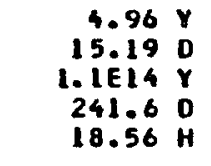 & $\begin{array}{l}4.91 E+00 \\
1.05 E+02 \\
0.0 \\
7.71 E+00 \\
3.42 E+00\end{array}$ & $\begin{array}{l}5.06 E+00 \\
1.05 E+02 \\
0.0 \\
0.7 T E+00 \\
3.35 E+00\end{array}$ & $\begin{array}{l}4.96 E+00 \\
1.18 E+02 \\
0.0 \\
1.47 E+00 \\
3.46 E+00\end{array}$ & $\begin{array}{l}1.01 E+01 \\
1.33 E+02 \\
0.0 \\
1.94 E+01 \\
5.35 E+00\end{array}$ & $\begin{array}{l}4.53 E * 00 \\
1.04 E \cdot 02 \\
0.0 \\
6.84 E+00 \\
3.08 E+00\end{array}$ & $\begin{array}{l}4.02 E \cdot 00 \\
9.60 E \cdot 01 \\
0.0 \\
6.37 E+00 \\
2.80 E+00\end{array}$ & $\begin{array}{l}5.00 E+00 \\
1.11 E+02 \\
0.0 \\
8.09 E+00 \\
3.33 E+00\end{array}$ & $\begin{array}{l}4.40 E+00 \\
1.02 E \cdot 02 \\
0.0 \\
6.90 E+00 \\
3.06 E+00\end{array}$ & $\begin{array}{l}1.11 E+02 \\
0.0 \\
9.84 E+00 \\
3.55 E+00\end{array}$ \\
\hline $\begin{array}{l}: 0-162 \\
88-157 \\
8-160 \\
8-160 \\
8 \gamma-157 \\
0-157\end{array}$ & $\begin{array}{rl}9.7 & H \\
150 & Y \\
72.3 & D \\
7.76 & H \\
8.06 & H\end{array}$ & $\begin{array}{l}3.82 E+01 \\
2.23 E-01 \\
9.01 E+01 \\
9.59 E+01 \\
3.10 E+01\end{array}$ & $\begin{array}{l}3.59 E+01 \\
3.05 E-01 \\
8.77 E+01 \\
9.18 E+01 \\
2.96 E+01\end{array}$ & $\begin{array}{l}3.96 E+01 \\
2.02 E-01 \\
9.00 E+01 \\
1.02 E+02 \\
3.12 E+01\end{array}$ & $\begin{array}{l}4.93 E+01 \\
8.39 E-01 \\
1.14 E+02 \\
1.22 E+02 \\
4.55 E+01\end{array}$ & $\begin{array}{l}3.50 E+01 \\
1.82 \mathrm{E}-01 \\
8.63 \mathrm{E}+01 \\
9.02 \mathrm{E}+01 \\
2.79 \mathrm{E}+01\end{array}$ & & $\begin{array}{l}9.19 \mathrm{E}+\text { ol } \\
9.59 \mathrm{O} \text {. Ol } \\
2.99 \mathrm{E}+0 \mathrm{l}\end{array}$ & $\begin{array}{l}3.44 E+01 \\
1.96 E-01 \\
8.46 E+01 \\
8.85 E+01 \\
2.77 E+01\end{array}$ & $\begin{array}{l}9.19 E+01 \\
9.59 E+01 \\
3.10 E+01\end{array}$ \\
\hline $\begin{array}{l}-165 \\
-166 \\
-166 \\
-166 M \\
-169\end{array}$ & 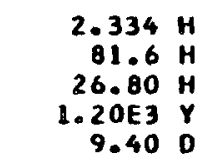 & $\begin{array}{l}2.71 E \\
2.13 E \\
1.40 E \\
1.66 E\end{array}$ & $\begin{array}{l}2.13 E \\
3.08 E \\
2.22 E \\
1.34 E \\
1.37 E\end{array}$ & $\begin{array}{l}2.73 E \\
2.33 E \\
1.49 E \\
1.46 E\end{array}$ & $\begin{array}{l}3.36 E \\
6.4 E \\
3.57 E \\
1.006 \\
1.99 E-\end{array}$ & $\begin{array}{l}1.98 E \\
2.44 E \\
2.06 E \\
1.32 E \\
1.36 E\end{array}$ & $\begin{array}{l}1.79 E+00 \\
2.2 B E+00 \\
1.90 E+00 \\
1.19 E+02 \\
1.15 E-04\end{array}$ & $\begin{array}{l}2.38 E+00 \\
2.23 E+00 \\
1.40 E+02 \\
1.44 E-04\end{array}$ & $\begin{array}{l}1.95 E+00 \\
2.42 E+00 \\
2.02 E+00 \\
1.29 E+02 \\
1.38 E-04\end{array}$ & $\begin{array}{l}3.39 \mathrm{E}+\mathrm{C} \\
2.36 \mathrm{E}+\mathrm{C} \\
8.40 \mathrm{E} \\
1.39 \mathrm{E}-\mathrm{C}\end{array}$ \\
\hline
\end{tabular}




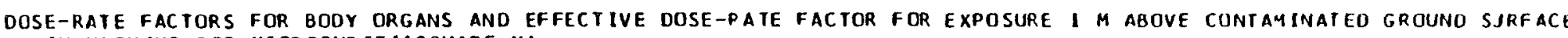

IN MREMIYR PER MICROCUR IESISOUARE MI

\begin{tabular}{|c|c|c|c|c|c|c|c|c|c|c|}
\hline CLL IOE & IFE & VER & LUNGS & RROH & $\begin{array}{l}\text { D } \\
\text { OH }\end{array}$ & ARIES & NCREAS & ELETON & LEEN & TOMA \\
\hline $\begin{array}{l}C E-139 \\
C E-141 \\
C E-143 \\
C E-144 \\
P R-142\end{array}$ & $\begin{array}{r}137.66 \mathrm{O} \\
32.50 \\
33.0 \mathrm{H} \\
284.3 \mathrm{O} \\
19.13 \mathrm{H}\end{array}$ & $\begin{array}{l}.25 E+01 \\
.37 F+00 \\
21 E+01 \\
51 E+00 \\
34 E+00\end{array}$ & $\begin{array}{l}8 E+01 \\
22 E+00 \\
9 E E+01 \\
b E E+00 \\
S T E+00\end{array}$ & $\begin{array}{l}.05 E+01 \\
.07 E+01 \\
.98 E+01 \\
.65 E+00 \\
.75 E+00\end{array}$ & $\begin{array}{l}5 E+01 \\
1 E+00 \\
5 E+01 \\
9 E+00 \\
1 E+00\end{array}$ & 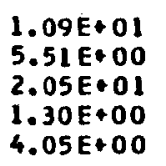 & & & & $\begin{array}{l}3 E+01 \\
B E+00 \\
17 E+01 \\
B E+00 \\
2 E+00\end{array}$ \\
\hline $\begin{array}{l}R-143 \\
R-144 \\
R-144 M \\
D-147 \\
D-149\end{array}$ & $\begin{array}{r}13.56 \mathrm{D} \\
17.28 \mathrm{M} \\
7.2 \mathrm{M} \\
10.98 \mathrm{O} \\
1.73 \mathrm{H}\end{array}$ & $1.46 E-01$ & $\begin{array}{l}97 E-07 \\
65 E+00 \\
64 E-01\end{array}$ & $.75 E-07$ & $\begin{array}{l}.11 E-07 \\
.69 E+00 \\
.27 E-01 \\
.12 E+01 \\
.41 E+01\end{array}$ & & & & & $\begin{array}{l}.38 E-07 \\
.46 E+00 \\
30 E-01 \\
10 E+01 \\
14 E+01\end{array}$ \\
\hline $\begin{array}{l}\mathrm{PH}-143 \\
\mathrm{PPM}-14 \\
\mathrm{PM}-14\end{array}$ & $\begin{array}{r}265 \mathrm{D} \\
363 \mathrm{D} \\
17.7 \mathrm{Y} \\
2020 \mathrm{D} \\
2.6234 \mathrm{Y}\end{array}$ & $2.52 E+01$ & & & & & & & & $\begin{array}{l}2.47 E+01 \\
1.28 E+02 \\
1.34 E+00 \\
6.16 E+01 \\
2.94 E-04\end{array}$ \\
\hline & $\begin{array}{r}5.37 \mathrm{D} \\
41.3 \mathrm{O} \\
53.08 \mathrm{H} \\
28.40 \mathrm{H} \\
069 E 11 \mathrm{~V}\end{array}$ & & & & & & & & & $\begin{array}{l}\text { 4. } 39 E+01 \\
1.64 E+02 \\
9.70 E-01 \\
2.77 E+01 \\
0.0\end{array}$ \\
\hline 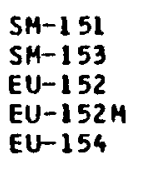 & $\begin{array}{rl}90 Y & Y \\
46.7 & H \\
13.6 & Y \\
9.32 & H \\
8.8 Y\end{array}$ & tenco & $\begin{array}{l}5.1 \\
9 .\end{array}$ & & & & & & & \\
\hline $\begin{array}{l}E U-155 \\
\text { EU-156 } \\
G D-152 \\
G O-153 \\
G 0-159\end{array}$ & $\begin{array}{r}4.96 Y \\
15.19 \\
1.1 E 14 \\
241.6 \\
18.56 \mathrm{H}\end{array}$ & 12 & 12 & $\begin{array}{l}9.27 E+0 O \\
1.18 E+02 \\
0.0 \\
1.54 E+01 \\
4.68 E+00\end{array}$ & 3. 32E 800 & $\begin{array}{l}+\infty 0 \\
+01 \\
00\end{array}$ & $\begin{array}{l}E E+00 \\
B E+01 \\
D E+00\end{array}$ & & $\begin{array}{l}00 \\
02 \\
.00\end{array}$ & $\begin{array}{l}E+00 \\
E+02 \\
E+00 \\
E+00\end{array}$ \\
\hline $\begin{array}{l}C D-162 \\
1 B-157 \\
1 B-160 \\
1 B-162 \\
D Y-157\end{array}$ & $\begin{array}{rl}9.7 & M \\
150 & Y \\
12.3 & D \\
7.76 & M \\
8.06 & H\end{array}$ & B. & & & & & & & & \\
\hline $\begin{array}{l}\text { OY-165 } \\
\text { OY-166 } \\
\text { HO-166 } \\
\text { HO-166M } \\
\text { ER-169 }\end{array}$ & $\begin{array}{r}2.334 \mathrm{H} \\
81.6 \mathrm{H} \\
26.80 \mathrm{H} \\
1.20 E 3 \mathrm{Y} \\
9.40 \mathrm{O}\end{array}$ & $.36 E-04$ & $.68 E-$ & $\begin{array}{l}5.52 E+00 \\
3.10 E+00 \\
1.65 E+02 \\
2.55 E-04\end{array}$ & $\begin{array}{l}1.97 \mathrm{EA} \\
2.01 \mathrm{E} \\
1.44 \mathrm{EA} \\
1.31 \mathrm{E}\end{array}$ & $\begin{array}{l}2.43 \mathrm{EE} \\
1.97 \mathrm{E}+ \\
1.27 \mathrm{E} \\
1.25 \mathrm{E}-\end{array}$ & $\begin{array}{l}1.69 E+00 \\
1.92 E+00 \\
1.73 E+00 \\
1.14 E+02 \\
1.21 E-04\end{array}$ & $\begin{array}{l}3.06 E+00 \\
5.65 E+00 \\
3.17 E+00 \\
1.68 E+02 \\
2.80 E-04\end{array}$ & $\begin{array}{l}2.00 E+00 \\
2.59 E+00 \\
2.06 E+00 \\
1.30 E+02 \\
1.39 E-04\end{array}$ & $\begin{array}{l}2.03 E+00 \\
2.70 E+00 \\
2.10 E+00 \\
1.32 E+02 \\
1.39 E-04\end{array}$ \\
\hline
\end{tabular}




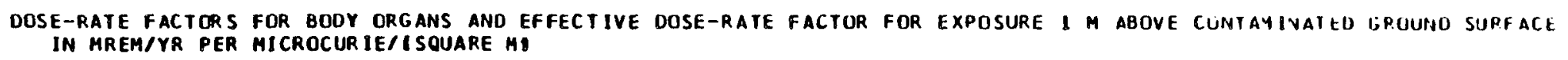

\begin{tabular}{|c|c|c|c|c|c|c|c|c|c|}
\hline NUCL IDE & HALF-LIFE & TESTES & THYMUS & THYROID & UTERUS & EFFECTIVE & $\begin{array}{c}\text { SKIN } \\
\text { IPHOTONI }\end{array}$ & $\begin{array}{c}\text { SKIN } \\
\text { IELECTRON }\end{array}$ & $\begin{array}{l}\text { SKIN } \\
\text { (TOTAL, }\end{array}$ \\
\hline $\begin{array}{l}C E-139 \\
C E-141 \\
C E-143 \\
C E-144 \\
P R-142\end{array}$ & 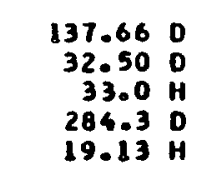 & $\begin{array}{l}2.06 E+01 \\
1.01 E+01 \\
3.35 E+01 \\
2.53 E+00 \\
5.97 E+00\end{array}$ & $\begin{array}{l}1.50 E+01 \\
7.63 E \bullet 00 \\
2.51 E \cdot 01 \\
1.88 E+00 \\
4.75 E+00\end{array}$ & $\begin{array}{l}1.94 E+01 \\
9.80 E+00 \\
3.12 E+01 \\
2.43 E+00 \\
5.59 E+00\end{array}$ & $\begin{array}{l}1.07 E+01 \\
5.53 E+00 \\
8.90 E * 01 \\
1.28 E+00 \\
3.89 E+00\end{array}$ & $\begin{array}{l}E E+01 \\
E+00 \\
E+01 \\
E+00 \\
E+00 \\
E+00\end{array}$ & $\begin{array}{l}39 E \cdot 01 \\
11 E+01 \\
80 E+01 \\
86 E+00 \\
81 E+00\end{array}$ & $\begin{array}{l}0.0 \\
1.64 E+01 \\
3.53 E+02 \\
0.0 \\
8.70 E+02\end{array}$ & $\begin{array}{l}2.39 E \cdot 01 \\
2.75 E+01 \\
3.92 E+02 \\
2.86 E+00 \\
8.7 T E \cdot 02\end{array}$ \\
\hline $\begin{array}{l}P R-143 \\
P R-144 \\
P R-144 M \\
N D-147 \\
N D-149\end{array}$ & $\begin{array}{rr}13.56 & 0 \\
17.28 & M \\
7.2 & M \\
10.98 & 0 \\
1.73 & H\end{array}$ & $\begin{array}{l}1.06 E-06 \\
3.46 E+00 \\
1.36 E+00 \\
1.76 E+01 \\
4.75 E+01\end{array}$ & $\begin{array}{l}8.33 E-07 \\
2.72 E \bullet 00 \\
7.936-01 \\
1.31 E \bullet 01 \\
3.60 E+01\end{array}$ & $\begin{array}{l}1.00 E-06 \\
3.22 E+00 \\
1.09 E+00 \\
1.64 E+01 \\
4.48 E+01\end{array}$ & $\begin{array}{l}6.4 \theta E-07 \\
2.24 E+00 \\
2.94 E-01 \\
9.53 E+00 \\
2.7 \theta E+01\end{array}$ & $\begin{array}{l}9.06 E-07 \\
2.98 E+00 \\
1.01 E+00 \\
1.40 E+01 \\
4.09 E \bullet 01\end{array}$ & $\begin{array}{l}1.22 E-06 \\
3.94 E+00 \\
2.01 E+00 \\
2.04 E+01 \\
5.36 E+01\end{array}$ & $\begin{array}{l}2.27 E+02 \\
1.31 E+03 \\
0.0 \\
1.27 E+02 \\
4.07 E+02\end{array}$ & $\begin{array}{l}2.27 E+02 \\
1.31 E+03 \\
2.01 E+00 \\
1.4 B+02 \\
4.61 E+02\end{array}$ \\
\hline $\begin{array}{l}P M-143 \\
P H-144 \\
P H-145 \\
P M-146 \\
P M-147\end{array}$ & $\begin{array}{r}2650 \\
363 \% \\
17.7 Y \\
20200 \\
2.6234 \gamma\end{array}$ & $\begin{array}{l}3.74 E+01 \\
1.86 E+02 \\
3.85 E+00 \\
9.04 E+01 \\
4.65 E-04\end{array}$ & $\begin{array}{l}2.87 E+01 \\
1.44 E+02 \\
2.39 E+00 \\
6.95 E+01 \\
3.59 E-04\end{array}$ & $\begin{array}{l}3.49 E+01 \\
1.74 E+02 \\
3.20 E+00 \\
8.46 E+01 \\
4.63 E-04\end{array}$ & $\begin{array}{l}2.15 E+01 \\
1.12 E+02 \\
9.72 E-01 \\
5.42 E+01 \\
2.59 E-04\end{array}$ & $\begin{array}{l}3.15 E+01 \\
1.59 E+02 \\
2.91 E+00 \\
1.71 E+01 \\
4.10 E-04\end{array}$ & $\begin{array}{l}4.41 E \cdot 01 \\
2.15 E \cdot 02 \\
5.36 E \bullet 00 \\
1.04 E \cdot 02 \\
4.96 E-04\end{array}$ & $\begin{array}{l}1.08 E+00 \\
8.81 E+00 \\
0.0 \\
5.64 E+01 \\
0.0\end{array}$ & $\begin{array}{l}4.52 E+01 \\
2.24 E \cdot 02 \\
5.36 E+00 \\
1.61 E+02 \\
4.96 E-04\end{array}$ \\
\hline $\begin{array}{l}P M-148 \\
P M-148 M \\
P M-149 \\
P M-151 \\
S M-147\end{array}$ & $\begin{array}{r}5.370 \\
41.330 \\
53.08 \mathrm{H} \\
28.40 \mathrm{H} \\
1.069 \mathrm{E11} Y\end{array}$ & $\begin{array}{l}6.27 E+01 \\
2.38 E+02 \\
1.44 E+00 \\
4.17 E+01 \\
0.0\end{array}$ & $\begin{array}{l}4.95 E \bullet 01 \\
1.84 E \bullet 02 \\
1.09 E \bullet 00 \\
3.17 E \bullet 01 \\
0.0\end{array}$ & $\begin{array}{l}5.90 E+01 \\
2.23 E+02 \\
1.36 E+00 \\
3.93 E+01 \\
0.0\end{array}$ & $\begin{array}{l}3.95 E+01 \\
1.45 E+02 \\
8.65 E-01 \\
2.45 E+01 \\
0.0\end{array}$ & $\begin{array}{l}5.37 E+01 \\
2.03 E+02 \\
1.25 E+00 \\
3.59 E+01 \\
0.0\end{array}$ & $\begin{array}{l}7.20 E+01 \\
2.73 E+02 \\
1.62 E \cdot 00 \\
4.74 E \cdot 01 \\
0.0\end{array}$ & $\begin{array}{l}7.38 E+02 \\
3.83 E+01 \\
2.94 E \cdot 02 \\
1.81 E \cdot 02 \\
0.0\end{array}$ & $\begin{array}{l}0.10 E+02 \\
3.12 E+02 \\
2.96 E+02 \\
2.34 E+02 \\
0.0\end{array}$ \\
\hline $\begin{array}{l}S M-151 \\
S M-153 \\
E U-152 \\
E U-152 M \\
E U-154\end{array}$ & $\begin{array}{r}90 Y \\
46.7 \mathrm{Y} \\
13.6 \mathrm{Y} \\
9.32 \mathrm{H} \\
0.8 \mathrm{Y}\end{array}$ & $\begin{array}{l}6.56 E-04 \\
8.60 E \bullet 00 \\
1.30 E \bullet 02 \\
3.70 E+01 \\
1.41 E \bullet 02\end{array}$ & $\begin{array}{l}5.69 E-05 \\
6.21 E+00 \\
1.03 E+02 \\
2.91 E+01 \\
1.12 E+02\end{array}$ & $\begin{array}{l}2.64 E-04 \\
9.05 E+00 \\
1.23 E+02 \\
3.51 E+01 \\
1.34 E+02\end{array}$ & $\begin{array}{l}1.36 E-06 \\
3.60 E+00 \\
7.97 E+01 \\
2.22 E+01 \\
8.74 E+01\end{array}$ & $\begin{array}{l}5.20 E-04 \\
1.08 E+00 \\
1.11 E+02 \\
3.15 E+01 \\
1.21 E+02\end{array}$ & $\begin{array}{l}2.87 E-03 \\
1.02 E+01 \\
1.50 E \bullet 02 \\
4.29 E \bullet 01 \\
1.62 E+02\end{array}$ & $\begin{array}{l}0.0 \\
1.01 E+02 \\
6.05 E+01 \\
4.97 E+02 \\
1.35 E+02\end{array}$ & $\begin{array}{l}2.87 E-03 \\
1.11 E+02 \\
2.11 E * 02 \\
5.40 E+02 \\
2.97 E * 02\end{array}$ \\
\hline $\begin{array}{l}E U-155 \\
E U-156 \\
G D-152 \\
G D-153 \\
G D-159\end{array}$ & $\begin{array}{r}4.96 Y \\
15.19 \\
1.1 E 14 Y \\
241.6 \mathrm{O} \\
18.56 \mathrm{H}\end{array}$ & $\begin{array}{l}0.43 E+00 \\
1.46 E+02 \\
0.0 \\
1.55 E+01 \\
5.12 E+00\end{array}$ & $\begin{array}{l}6.37 E+00 \\
1.16 E+02 \\
0.0 \\
1.11 E+01 \\
3.79 E+00\end{array}$ & $\begin{array}{l}8.22 E+00 \\
1.37 E+02 \\
0.0 \\
1.44 E+01 \\
4.74 E+00\end{array}$ & $\begin{array}{l}4.03 E+00 \\
9.34 E+01 \\
0.0 \\
6.22 E+00 \\
2.78 E+00\end{array}$ & $\begin{array}{l}7.15 E+00 \\
1.26 E+02 \\
0.0 \\
1.27 E+01 \\
4.33 E+00\end{array}$ & $\begin{array}{l}9.39 E \bullet 00 \\
1.67 E+02 \\
0.0 \\
1.86 E+01 \\
5.94 E \bullet 00\end{array}$ & $\begin{array}{l}0.0 \\
3.36 E+02 \\
0.0 \\
0.0 \\
2.22 E+02\end{array}$ & $\begin{array}{l}9.39 E+00 \\
5.03 E+02 \\
0.0 \\
1.86 E+01 \\
2.28 E+02\end{array}$ \\
\hline $\begin{array}{l}60-162 \\
18-157 \\
18-160 \\
18-162 \\
0 Y-157\end{array}$ & $\begin{array}{rl}9.7 & M \\
150 & Y \\
72.3 & 0 \\
8.96 & M \\
8.06 & H\end{array}$ & $\begin{array}{l}5.18 E+01 \\
5.98 E-01 \\
1.24 E \bullet 02 \\
1.31 E+02 \\
4.43 E+01\end{array}$ & $\begin{array}{l}3.90 E+01 \\
3.99 E-01 \\
9.02 E \bullet 01 \\
1.03 E \bullet 02 \\
3.30 E+01\end{array}$ & $\begin{array}{l}4.80 E+01 \\
5.20 E-01 \\
1.18 E+02 \\
1.24 E+02 \\
4.13 E+01\end{array}$ & $\begin{array}{l}3.13 \mathrm{E}+01 \\
1.75 \mathrm{E}-01 \\
7.62 \mathrm{E}+01 \\
1.96 \mathrm{E}+01 \\
2.52 \mathrm{E}+01\end{array}$ & $\begin{array}{l}4.45 E+01 \\
4.70 E-01 \\
1.06 E+02 \\
1.12 E+02 \\
3.79 E+01\end{array}$ & $\begin{array}{l}5.90 E \cdot 01 \\
8.96 E-01 \\
1.42 E+02 \\
8.50 E+02 \\
5.06 E \cdot 01\end{array}$ & $\begin{array}{l}2.38 E+02 \\
0.0 \\
1.10 E+02 \\
4.56 E+02 \\
0.0\end{array}$ & $\begin{array}{l}2.97 E+02 \\
0.96 E-01 \\
2.53 E+02 \\
6.06 E+02 \\
5.06 E+01\end{array}$ \\
\hline $\begin{array}{l}\text { DY }-165 \\
\text { OY-166 } \\
H O-166 \\
M O-166 M \\
E R-169\end{array}$ & $\begin{array}{r}2.334 \mathrm{H} \\
81.6 \mathrm{H} \\
26.80 \mathrm{H} \\
1.20 E 3 \mathrm{Y} \\
9.40 \mathrm{O}\end{array}$ & $\begin{array}{l}3.25 E+00 \\
5.44 E+00 \\
3.38 E+00 \\
1.92 E+02 \\
3.17 E-04\end{array}$ & $\begin{array}{l}2.46 E+00 \\
3.95 E+00 \\
2.62 E+00 \\
1.50 E+02 \\
1.66 E-04\end{array}$ & $\begin{array}{l}3.06 E+00 \\
5.09 E+00 \\
3.21 E+00 \\
1.82 E+02 \\
2.26 E-04\end{array}$ & $\begin{array}{l}1.16 E+00 \\
2.21 E+00 \\
1.85 E+00 \\
1.16 E+02 \\
1.11 E-04\end{array}$ & $\begin{array}{l}2.74 E+00 \\
4.45 E+00 \\
2.86 E+00 \\
1.65 E+02 \\
4.96 E-04\end{array}$ & $\begin{array}{l}3.75 E+00 \\
6.58 E \cdot 00 \\
3.97 E+00 \\
2.20 E+02 \\
3.94 E-03\end{array}$ & $\begin{array}{l}3.97 E+02 \\
1.72 E+00 \\
6.95 E+02 \\
2.22 E+01 \\
0.0\end{array}$ & $\begin{array}{l}4.01 E+02 \\
8.30 E+00 \\
6.99 E+02 \\
2.42 E+02 \\
3.94 E-03\end{array}$ \\
\hline
\end{tabular}




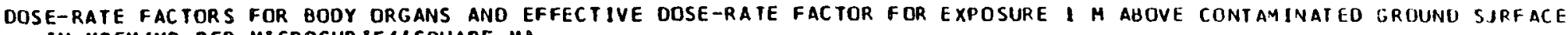

IN MREM/YR PER MICROCUR IE/ISOUARE MI

\begin{tabular}{|c|c|c|c|c|c|c|c|c|c|c|}
\hline NUCLIDE & HALF-LIFE & ADRENALS & BLADOER & BRAIN & BREAST & HEART & $\begin{array}{l}\text { SMALL } \\
\text { INTESTINE }\end{array}$ & $\begin{array}{c}\text { UPPER } \\
\text { LARGEE } \\
\text { INTESTINE }\end{array}$ & $\begin{array}{c}\text { LOHER } \\
\text { LARGE } \\
\text { INTESTINE }\end{array}$ & K IONEY \\
\hline $\begin{array}{l}E R-171 \\
T M-170 \\
Y M-171 \\
Y \theta-169 \\
Y 8-175\end{array}$ & $\begin{array}{r}1.52 \\
128.6 \\
1.92 \\
31.97 \\
3.97 \\
4.190\end{array}$ & $\begin{array}{l}3.37 E+01 \\
3.97 E-01 \\
4.52 E-02 \\
2.56 E+01 \\
3.56 E+00\end{array}$ & $\begin{array}{l}16 E+01 \\
31 E-01 \\
31 E-02 \\
5 B E+01 \\
35 E+00\end{array}$ & $\begin{array}{l}40 E+01 \\
10 E-01 \\
66 E-02 \\
55 E+01 \\
63 E+00\end{array}$ & $\begin{array}{l}4.86 E+01 \\
9.40 E-01 \\
1.24 E-01 \\
4.90 E+01 \\
4.96 E+00\end{array}$ & $\begin{array}{l}3.05 E+01 \\
3.68 E-01 \\
4.13 E-02 \\
2.31 E+01 \\
3.24 E+00\end{array}$ & $\begin{array}{l}73 E+01 \\
33 E-01 \\
88 E-02 \\
09 E+01 \\
.91 E+00\end{array}$ & $\begin{array}{l}24 E+01 \\
.03 E-01 \\
64 E-02 \\
.51 E+01 \\
.44 E+00\end{array}$ & $\begin{array}{l}.01 E+01 \\
.57 E-01 \\
.06 E-02 \\
.28 E+01 \\
3.19 E+00\end{array}$ & $\begin{array}{l}\text { 3. } 28 E+01 \\
4.58 E-01 \\
5.85 E-02 \\
2.73 E+01 \\
3.47 E+00\end{array}$ \\
\hline $\begin{array}{l}L U-117 \\
L U-1 T H \\
H F-181 \\
T A-182 \\
U-181\end{array}$ & 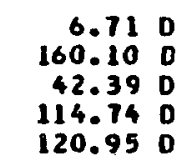 & $\begin{array}{l}3.17 E+00 \\
8.97 E+01 \\
4.85 E+01 \\
1.03 E \bullet 02 \\
2.76 E \bullet 00\end{array}$ & $\begin{array}{l}2.97 E+00 \\
8.42 E+01 \\
4.60 E+01 \\
1.01 E+02 \\
3.17 E+00\end{array}$ & $\begin{array}{l}3.12 E+00 \\
9.00 E+01 \\
5.05 E+01 \\
1.13 E+02 \\
2.89 E+00\end{array}$ & $\begin{array}{l}5.02 E+00 \\
1.32 E+02 \\
6.55 E+01 \\
1.33 E+02 \\
1.18 E+00\end{array}$ & $\begin{array}{l}2.84 E+00 \\
0.10 E+01 \\
4.47 E+01 \\
9.96 E+01 \\
2.56 E+00\end{array}$ & $\begin{array}{l}2.53 E+00 \\
7.25 E+01 \\
4.01 E \bullet 01 \\
9.12 E+01 \\
2.3 \mathrm{TE}+00\end{array}$ & $\begin{array}{l}3.03 E+00 \\
8.61 E \cdot 01 \\
4.75 E \cdot 01 \\
1.06 E+02 \\
2.82 E+00\end{array}$ & & $\begin{array}{l}\text { 3. } 06 E+00 \\
\text { 8. T0E+01 } \\
4.76 E+01 \\
1.06 E+02 \\
3.43 E+00\end{array}$ \\
\hline $\begin{array}{l}W-185 \\
W-187 \\
H-18 B \\
R E-182 \\
R E-182 M\end{array}$ & $\begin{array}{rl}75.1 & 0 \\
23.83 & H \\
69.4 & 0 \\
64.0 & H \\
12.1 & H\end{array}$ & $\begin{array}{l}2.44 E-03 \\
4.18 E+01 \\
1.63 E-01 \\
1.42 E \cdot 02 \\
9.43 E \cdot 01\end{array}$ & $\begin{array}{l}2.28 E-03 \\
4.02 E+01 \\
1.50 E-01 \\
1.40 E+02 \\
9.40 E+01\end{array}$ & $\begin{array}{l}1.62 \mathrm{E}- \\
1.53 \mathrm{EO} \\
1.04 \mathrm{EO}\end{array}$ & & $\begin{array}{l}2.24 E-03 \\
3.91 E+01 \\
1.46 E-01 \\
1.36 E+02 \\
9.17 E+01\end{array}$ & & $\begin{array}{l}2.40 E-03 \\
4.16 E+01 \\
1.54 E-01 \\
1.45 E+02 \\
9.78 E+01\end{array}$ & & $\begin{array}{l}2.31 \mathrm{E}-03 \\
4.18 \mathrm{E}+01 \\
1.55 \mathrm{E}-01 \\
1.46 \mathrm{E}+02 \\
9.88 \mathrm{E}+01\end{array}$ \\
\hline $\begin{array}{l}\text { RE-1 } 83 \\
\text { RE-1 B4 } \\
\text { RE-1 B4M } \\
\text { RE-1 } 86 \\
\text { RE-1 } 187\end{array}$ & $\begin{array}{r}70 \\
38.0 \\
169 \\
90.64 \mathrm{H} \\
4.7 \mathrm{E} 10 \mathrm{Y}\end{array}$ & $\begin{array}{l}1.25 E \bullet 01 \\
1.61 E+01 \\
3.33 E+01 \\
1.78 E+00 \\
0.0\end{array}$ & $\begin{array}{l}1.28 E+01 \\
7.43 E+01 \\
3.23 E+01 \\
1.74 E+00 \\
0.0\end{array}$ & $\begin{array}{l}1.27 E+01 \\
8.25 E+01 \\
3.50 E+01 \\
1.79 E+00 \\
0.0\end{array}$ & $\begin{array}{l}2.50 E+01 \\
9.92 E+01 \\
4.83 E+01 \\
3.26 E+00 \\
0.0\end{array}$ & $\begin{array}{l}1.14 E+01 \\
1.25 E+01 \\
3.11 E+01 \\
1.63 E+00 \\
0.0\end{array}$ & $\begin{array}{l}1.03 E+01 \\
6.5 T E+01 \\
2.01 E+01 \\
1.44 E+00 \\
0.0\end{array}$ & $\begin{array}{l}1.24 E+01 \\
7.73 E+01 \\
3.32 E+01 \\
1.76 E+00 \\
0.0\end{array}$ & & $\begin{array}{l}1.34 E+01 \\
7.78 E+01 \\
3.37 E+01 \\
1.79 E+00 \\
0.0\end{array}$ \\
\hline $\begin{array}{l}\text { RE-1 } 88 \\
O S-185 \\
O S-186 \\
O S-190 \mathrm{M} \\
\text { OS-191 }\end{array}$ & $\begin{array}{r}16.98 \mathrm{H} \\
93.6 \mathrm{D} \\
2.0 E 15 \mathrm{~V} \\
9.9 \mathrm{M} \\
15.4 \mathrm{D}\end{array}$ & $\begin{array}{l}5.12 E \cdot 00 \\
6.14 E \cdot 01 \\
0.0 \\
1.42 E+02 \\
5.95 E+00\end{array}$ & $\begin{array}{l}4.87 E+00 \\
5.94 E+01 \\
0.0 \\
1.34 E+02 \\
6.02 E+00\end{array}$ & $\begin{array}{l}5.31 E+00 \\
6.59 E+01 \\
0.0 \\
1.49 E+02 \\
6.09 E+00\end{array}$ & $\begin{array}{l}7.20 E+00 \\
8.00 E+01 \\
0.0 \\
1.84 E+02 \\
1.21 E+01\end{array}$ & $\begin{array}{l}4.76 E+00 \\
5.78 E+01 \\
0.0 \\
1.31 E+02 \\
5.52 E+00\end{array}$ & $\begin{array}{l}4.25 E \cdot 00 \\
5.22 E+01 \\
0.0 \\
1.18 E+02 \\
4.89 E \cdot 00\end{array}$ & $\begin{array}{l}5.06 E+00 \\
6.15 E+01 \\
0.0 \\
1.39 E+02 \\
5.93 E+00\end{array}$ & $\begin{array}{l}4.66 E+00 \\
5.65 E+01 \\
0.0 \\
1.29 E+02 \\
5.33 E+00\end{array}$ & $\begin{array}{l}\text { 6. } 20 E+01 \\
0.0 \\
1.39 E+02 \\
6.22 E+00\end{array}$ \\
\hline $\begin{array}{l}\text { OS-191M } \\
0 S-193 \\
\text { IR-190 } \\
\text { IR-190M } \\
\text { IR-190M }\end{array}$ & $\begin{array}{rl}13.03 & H \\
30.0 & H \\
11.78 & 0 \\
1.22 & H \\
3.2 & H\end{array}$ & $\begin{array}{l}3.46 E-01 \\
5.92 E+00 \\
1.24 E+02 \\
4.12 E-03 \\
3.54 E+00\end{array}$ & $\begin{array}{l}3.82 E-01 \\
5.68 E+00 \\
1.18 E+02 \\
4.77 E-07 \\
3.95 E+00\end{array}$ & $\begin{array}{l}1.30 E+02 \\
3.29 E-04 \\
3.75 E+00\end{array}$ & $\begin{array}{l}0.70 E+0 \\
1.63 E+0 \\
3.23 E-0 \\
8.62 E+0\end{array}$ & $\begin{array}{l}3.23 E-01 \\
5.45 E+00 \\
1.15 E+02 \\
5.37 E-04 \\
3.33 E+00\end{array}$ & & $\begin{array}{l}3.49 E-01 \\
5.19 E+00 \\
1.22 E+02 \\
1.07 E-05 \\
3.60 E+00\end{array}$ & & $\begin{array}{l}\text { 5. } 88 E+00 \\
1.23 E+02 \\
5.80 E-06 \\
4.17 E+00\end{array}$ \\
\hline $\begin{array}{l}\text { IR-192 } \\
\text { IR-193M } \\
\text { IR-194 } \\
\text { IR-194M } \\
\text { PT-191 }\end{array}$ & $\begin{array}{rl}14.02 & 0 \\
11.9 & 0 \\
19.15 & 0 \\
171 & 0 \\
2.71 & 0\end{array}$ & $\begin{array}{l}7.45 E \\
2.91 E- \\
8.02 E \\
2.09 E \\
2.47 E\end{array}$ & & & & & & 12 & & $\begin{array}{l}2.05 E+02 \\
2.53 E+01\end{array}$ \\
\hline $\begin{array}{l}\text { PT-193 } \\
\text { PT-1 93M } \\
\text { PT-195M } \\
\text { PT-197 } \\
\text { PT-197M }\end{array}$ & $\begin{array}{rl}50 & Y \\
4.33 & D \\
4.02 & D \\
18.3 & H \\
94.4 & H\end{array}$ & $\begin{array}{l}4.02 E-03 \\
8.09 E-01 \\
5.50 E \cdot 00 \\
1.96 E \cdot 00 \\
6.84 E+00\end{array}$ & $\begin{array}{l}7.24 E-10 \\
8.78 E-01 \\
5.83 E+00 \\
1.96 E+00 \\
6.76 E+00\end{array}$ & $\begin{array}{l}0.54 E-01 \\
5.74 E+00 \\
2.01 E \bullet 00 \\
1.05 E \bullet 00\end{array}$ & $\begin{array}{l}2.19 \mathrm{E} \\
1.28 \mathrm{E} \\
3.96 \mathrm{E} \\
1.21 \mathrm{E}\end{array}$ & $\begin{array}{l}5.23 E-04 \\
7.62 E-01 \\
5.16 E+00 \\
1.81 E+00 \\
6.28 E+00\end{array}$ & $\begin{array}{l}6.74 E-06 \\
6.86 E-01 \\
4.61 E+00 \\
1.61 E+00 \\
5.65 E+00\end{array}$ & $\begin{array}{l}9.93 E-06 \\
8.17 E-01 \\
5.56 E+00 \\
1.93 E+00 \\
6.69 E+00\end{array}$ & $\begin{array}{l}1.65 E-03 \\
7.28 E-01 \\
4.95 E+00 \\
1.75 E+00 \\
6.13 E+00\end{array}$ & $\begin{array}{l}4.81 E-06 \\
9.16 E-01 \\
6.07 E * 00 \\
2.01 E * 00 \\
7.02 E * 00\end{array}$ \\
\hline
\end{tabular}




\begin{tabular}{|c|c|c|c|c|c|c|c|c|c|c|}
\hline IUCLIDE & HALF-LIFE & LIVER & LUNGS & MARROH & $\begin{array}{c}\text { RED } \\
\text { MARROH }\end{array}$ & OVARIES & PANCREAS & SKELETON & SPLEEN & $S$ TOMACH \\
\hline $\begin{array}{l}E R-171 \\
I M-170 \\
Y H-171 \\
Y B-169 \\
Y B-175\end{array}$ & $\begin{array}{r}7.52 \mathrm{H} \\
128.6 \mathrm{D} \\
1.92 \mathrm{Y} \\
31.97 \mathrm{D} \\
4.19 \mathrm{D}\end{array}$ & $\begin{array}{l}3.14 E+01 \\
4.22 E-01 \\
5.10 E-02 \\
2.53 E+01 \\
3.33 E+00\end{array}$ & $\begin{array}{l}3.41 E+01 \\
4.68 E-01 \\
5.68 E-02 \\
2.78 E+01 \\
3.60 E \bullet 00\end{array}$ & $\begin{array}{l}51 E+01 \\
21 E-01 \\
02 E-01 \\
36 E+01 \\
.61 E+00\end{array}$ & $\begin{array}{l}34 E+01 \\
88 E-01 \\
94 E-02 \\
20 E+01 \\
57 E+00\end{array}$ & $\begin{array}{l}2.85 E+01 \\
3.48 E-01 \\
4.84 E-02 \\
2.16 E+01 \\
3.06 E+00\end{array}$ & $\begin{array}{l}2.65 E+01 \\
2.82 E-01 \\
3.11 E-02 \\
1.91 E+01 \\
2.82 E+00\end{array}$ & $\begin{array}{l}4.60 E+01 \\
0.44 E-01 \\
1.05 E-01 \\
4.47 E+01 \\
4.70 E+00\end{array}$ & $\begin{array}{l}3.00 E+01 \\
3.83 E-01 \\
4.42 E-02 \\
2.39 E+01 \\
3.26 E+00\end{array}$ & $\begin{array}{l}3.09 E+01 \\
3.94 E-01 \\
4.63 E-02 \\
2.42 E+01 \\
3.27 E+00\end{array}$ \\
\hline $\begin{array}{l}1 U-177 \\
1 U-177 M \\
M F-1 \quad 101 \\
T A-1 \quad 82 \\
W-181\end{array}$ & $\begin{array}{rr}6.71 & D \\
160.10 & D \\
42.39 & D \\
114.74 & D \\
120.95 & D\end{array}$ & $\begin{array}{l}2.95 E+00 \\
8.36 E+01 \\
4.57 E+01 \\
1.01 E+02 \\
3.08 E+00\end{array}$ & $\begin{array}{l}3.23 E+00 \\
9.08 E+01 \\
4.93 E+01 \\
1.08 E+02 \\
3.42 E+00\end{array}$ & $\begin{array}{l}72 E+00 \\
23 E+02 \\
10 E+01 \\
20 E+02 \\
13 E+00\end{array}$ & & & & & & 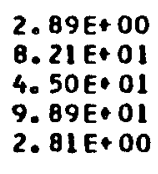 \\
\hline $\begin{array}{l}W-185 \\
H-187 \\
H-188 \\
\text { RE-182 } \\
\text { RE-182M }\end{array}$ & $\begin{array}{rl}75.1 & \mathrm{D} \\
23.83 & \mathrm{H} \\
69.4 \mathrm{D} \\
64.0 \mathrm{H} \\
12.7 \mathrm{H}\end{array}$ & $\begin{array}{l}2.27 E-03 \\
3.99 E+01 \\
1.49 E-01 \\
1.39 E+02 \\
9.35 E+01\end{array}$ & $\begin{array}{l}2.51 E-03 \\
4.28 E+01 \\
1.62 E-01 \\
1.49 E+02 \\
9.97 E+01\end{array}$ & $\begin{array}{l}3.94 E-03 \\
5.02 E+01 \\
2.14 E-01 \\
1.77 E+02 \\
1.13 E+02\end{array}$ & $\begin{array}{l}.20 E-03 \\
0.26 E+01 \\
.63 E-01 \\
.44 E+02 \\
.06 E+01\end{array}$ & & & & & $\begin{array}{l}2.24 \mathrm{E}-03 \\
3.93 \mathrm{E}+01 \\
1.47 \mathrm{E}-01 \\
1.36 \mathrm{E}+02 \\
9.13 \mathrm{O}+01\end{array}$ \\
\hline $\begin{array}{l}R E-183 \\
R E-184 \\
R E-184 M \\
R E-186 \\
R E-187\end{array}$ & $\begin{array}{r}70 \\
38.0 \\
169 \\
169 \\
90.64 \mathrm{H} \\
4.7 \mathrm{E} 10 \mathrm{Y}\end{array}$ & $\begin{array}{l}1.26 E+01 \\
7.37 E+01 \\
3.21 E+01 \\
1.72 E+00 \\
0.0\end{array}$ & $\begin{array}{l}1.39 E \bullet 01 \\
7.89 E+01 \\
3.46 E+01 \\
1.90 E \bullet 00 \\
0.0\end{array}$ & $\begin{array}{l}2.24 E+01 \\
9.00 E+01 \\
4.40 E+01 \\
3.05 E+00 \\
0.0\end{array}$ & & & & & & $\begin{array}{l}1.20 E+01 \\
T .26 E+01 \\
3.14 E+01 \\
1.67 E+00 \\
0.0\end{array}$ \\
\hline $\begin{array}{l}\text { RE-1 } 88 \\
O S-185 \\
O S-186 \\
O S-190 \mathrm{H} \\
O S-191\end{array}$ & $\begin{array}{r}16.98 \mathrm{H} \\
93.6 \mathrm{D} \\
2.0 \mathrm{E} 15 \mathrm{Y} \\
9.9 \mathrm{M} \\
15.4 \mathrm{D}\end{array}$ & $\begin{array}{l}4.84 E+00 \\
5.90 E+01 \\
0.0 \\
1.33 E+02 \\
5.97 E+00\end{array}$ & $\begin{array}{l}5.24 E+00 \\
6.33 E+01 \\
0.0 \\
1.44 E+02 \\
6.59 E+00\end{array}$ & $\begin{array}{l}6.01 E+00 \\
7.31 E+01 \\
0.0 \\
1.70 E+02 \\
1.10 E+01\end{array}$ & $\begin{array}{l}04 E+00 \\
25 E+01 \\
0 \\
46 E+02\end{array}$ & $\begin{array}{l}4.44 E+00 \\
5.66 E+01 \\
0.0 \\
1.27 E+02 \\
5.00 E+00\end{array}$ & $\begin{array}{l}4.07 E+00 \\
5.00 E \cdot 01 \\
0.0 \\
1.15 E+02 \\
4.36 E+00\end{array}$ & $\begin{array}{l}6.96 \mathrm{E}+00 \\
7.44 \mathrm{E} \cdot 01 \\
0.0 \\
1.73 \mathrm{~T}+02 \\
1.13 \mathrm{1} \cdot 01\end{array}$ & $\begin{array}{l}4.74 E+00 \\
5.70 E+01 \\
0.0 \\
1.31 E * 02 \\
5.64 E+00\end{array}$ & $\begin{array}{l}5.01 E+01 \\
0.0 \\
1.32 E+02 \\
5.70 E+00\end{array}$ \\
\hline $\begin{array}{l}O S-191 M \\
0 S-193 \\
I R-190 \\
I R-190 M \\
I R-190 M\end{array}$ & $\begin{array}{r}13.03 \mathrm{H} \\
30.0 \mathrm{H} \\
11.78 \mathrm{O} \\
1.2 \mathrm{H} \\
3.2 \mathrm{H}\end{array}$ & $\begin{array}{l}3.75 E-01 \\
5.65 E+00 \\
1.17 E+02 \\
4.04 E-05 \\
3.88 E \cdot 00\end{array}$ & $\begin{array}{l}1.26 E \\
3.26 E \\
4.28 E\end{array}$ & $\begin{array}{l}7.41 E-01 \\
8.11 E+00 \\
8.51 E+02 \\
1.96 E-03 \\
7.64 E+00\end{array}$ & $\begin{array}{l}1.27 E \\
1.07 E \\
2.46 E\end{array}$ & $\begin{array}{l}3.07 E-01 \\
5.16 E+00 \\
1.11 E+02 \\
1.61 E-03 \\
3.15 E+00\end{array}$ & $\begin{array}{l}4.0 \\
1.0 \\
2.0 \\
2 .\end{array}$ & $\begin{array}{l}7.63 E-01 \\
8.27 E+00 \\
1.54 E+02 \\
5.29 E-03 \\
7.84 E+00\end{array}$ & $\begin{array}{l}3.36 E-01 \\
5.48 E+00 \\
1.14 E+02 \\
9.30 E-04 \\
3.46 E+00\end{array}$ & $\begin{array}{l}3.47 E-01 \\
5.52 E+00 \\
1.16 E+02 \\
0.40 E-04 \\
3.57 E+00\end{array}$ \\
\hline $\begin{array}{l}\text { IR-192 } \\
\text { IR-193M } \\
\text { IR-194 } \\
\text { IR-194M } \\
\text { PT-191 }\end{array}$ & 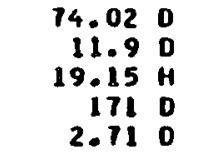 & $\begin{array}{l}6.92 E+01 \\
2.74 E-02 \\
7.55 E+00 \\
1.97 E+02 \\
2.41 E+01\end{array}$ & $\begin{array}{l}7.46 E+01 \\
3.33 E-02 \\
8.10 E+00 \\
2.11 E+02 \\
2.62 E+01\end{array}$ & $\begin{array}{l}9.11 E+01 \\
5.55 E-02 \\
9.59 E+00 \\
2.46 E+02 \\
3.64 E+01\end{array}$ & & $\begin{array}{l}6.46 E+01 \\
2.39 E-02 \\
7.08 E+00 \\
1.87 E+02 \\
2.18 E+01\end{array}$ & $\begin{array}{l}5.96 E+01 \\
2.01 E-02 \\
6.52 E+00 \\
1.69 E+02 \\
1.92 E+01\end{array}$ & & & $\begin{array}{l}6.03 E+01 \\
2.61 E-02 \\
1.44 E+00 \\
1.94 E+02 \\
2.34 E+01\end{array}$ \\
\hline $\begin{array}{l}P T-193 \\
P T-193 M \\
P T-195 M \\
P T-197 \\
P T-197 M\end{array}$ & $\begin{array}{rl}50 Y \\
4.33 & 0 \\
4.02 & 0 \\
18.3 & H \\
94.4 & M\end{array}$ & $\begin{array}{l}3.90 E-05 \\
8.66 E-01 \\
5.75 E+00 \\
1.95 E+00 \\
6.70 E \cdot 00\end{array}$ & $\begin{array}{l}3.17 E-03 \\
9.59 E-01 \\
6.37 E+00 \\
2.14 E \cdot 00 \\
7.31 E: 00\end{array}$ & $\begin{array}{l}1.69 E+00 \\
1.11 E+01 \\
3.49 E * 00 \\
1.07 E+01\end{array}$ & $\begin{array}{l}1.04 E-03 \\
5.80 E-01 \\
4.13 E+00 \\
1.63 E+00 \\
6.22 E * 00\end{array}$ & $\begin{array}{l}1.57 E-03 \\
7.10 E-01 \\
4.76 E+00 \\
1.64 E+00 \\
5.90 E+00\end{array}$ & $\begin{array}{l}2.34 E-03 \\
5.74 E-01 \\
3.95 E+00 \\
1.45 E+00 \\
5.22 E+00\end{array}$ & $\begin{array}{l}5.15 E-03 \\
1.74 E+00 \\
1.14 E+01 \\
3.59 E+00 \\
1.09 E+01\end{array}$ & $\begin{array}{l}9.05 E-04 \\
7.05 E-01 \\
5.30 E+00 \\
1.85 E+00 \\
6.39 E+00\end{array}$ & $\begin{array}{l}\text { 8. } 18 \mathrm{E}-04 \\
8.06 \mathrm{E}-01 \\
5.41 \mathrm{E} \cdot 00 \\
8.86 \mathrm{E}+00 \\
6.46 \mathrm{E}+00\end{array}$ \\
\hline
\end{tabular}




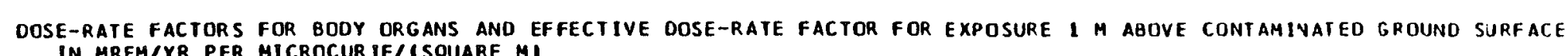

\begin{tabular}{|c|c|c|c|c|c|c|c|c|c|}
\hline CLEE & ILF-LIFE & TESTES & THYMUS & THYROID & UTERUS & FFECTIVE & $\begin{array}{l}\text { KIN } \\
\text { TOT ON }\end{array}$ & $\begin{array}{l}\text { SKIN } \\
\text { LECTRONI }\end{array}$ & $\begin{array}{l}\text { SKIN } \\
\text { SOTALI }\end{array}$ \\
\hline $\begin{array}{l}-171 \\
-170 \\
-171 \\
-169\end{array}$ & 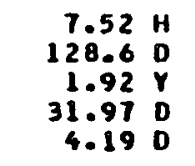 & $\begin{array}{l}72 E+01 \\
48 E-01\end{array}$ & $\begin{array}{l}56 E+01 \\
61 E-01\end{array}$ & & & & & $\begin{array}{l}2.88 E+02 \\
2.28 E+02 \\
0.0 \\
0.0 \\
8.19 E+00\end{array}$ & $\begin{array}{l}.41 E+02 \\
.29 E+02 \\
19 E-01 \\
.03 E+01 \\
.36 E+01\end{array}$ \\
\hline $\begin{array}{l}-181 \\
-182 \\
-182\end{array}$ & $\begin{array}{rl}6.71 & D \\
160.10 & D \\
42.39 & D \\
114.74 & D \\
120.95 & 0\end{array}$ & $\begin{array}{l}4.5 \\
1.2 \\
6.7 \\
1.4 \\
5.6\end{array}$ & $\begin{array}{l}0 \\
1 \\
1 \\
2 \\
0\end{array}$ & $\begin{array}{l}4.4 \\
1.2 \\
6.2\end{array}$ & $\begin{array}{l}0 \\
1 \\
1 \\
1 \\
0\end{array}$ & & & & $\begin{array}{l}E+01 \\
E+02 \\
E E+01 \\
E+02 \\
E+00\end{array}$ \\
\hline $\begin{array}{l}-185 \\
-187 \\
-188 \\
E-182 \\
E-182 M\end{array}$ & $\begin{array}{rr}75.1 & \mathrm{D} \\
23.83 & \mathrm{H} \\
69.4 & \mathrm{D} \\
64.0 & \mathrm{H} \\
12.7 & \mathrm{H}\end{array}$ & $\begin{array}{l}5.7 \\
2.2 \\
2.0\end{array}$ & 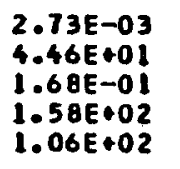 & $\begin{array}{l}3 \\
1 \\
1 \\
12\end{array}$ & & & & $\begin{array}{l}E+00 \\
E+02 \\
E+01\end{array}$ & $\begin{array}{l}E-01 \\
E+02 \\
E+02\end{array}$ \\
\hline $\begin{array}{l}-183 \\
-184 \\
-184 M\end{array}$ & $\begin{array}{r}10 \\
38.0 \\
169 \\
169 \\
90.64 \mathrm{H} \\
4.7 E 10 \mathrm{Y}\end{array}$ & $\begin{array}{l}2.10 E+01 \\
1.06 E+02 \\
4.78 E+01 \\
2.78 E+00 \\
0.0\end{array}$ & $\begin{array}{l}1.59 E+01 \\
8.40 E+01 \\
3.72 E+01\end{array}$ & & & & & & \\
\hline $\begin{array}{l}-188 \\
-185 \\
-185 \\
-186 \\
0-1904 \\
-191\end{array}$ & $\begin{array}{r}16.98 \mathrm{H} \\
93.6 \mathrm{D} \\
2.0 E 15 \mathrm{Y} \\
9.9 \mathrm{H} \\
15.4 \mathrm{D}\end{array}$ & $\begin{array}{l}7.17 E \\
8.55 E \\
0.0 \\
1.93 E \\
9.88 E\end{array}$ & $\begin{array}{l}0 \\
1 \\
2\end{array}$ & $\begin{array}{l}6 . \\
8 . \\
0 . \\
1 .\end{array}$ & $\begin{array}{l}0 \\
11 \\
12 \\
10\end{array}$ & $\begin{array}{l}0 \\
1 \\
2 \\
0\end{array}$ & $\begin{array}{l}00 \\
01 \\
02 \\
01\end{array}$ & $\begin{array}{l}02 \\
00 \\
01\end{array}$ & $5 E+02$ \\
\hline $\begin{array}{l}\text { OS-191M } \\
\text { OS-193 } \\
\text { IR-190 } \\
\text { IR-190M } \\
\text { IR-190M }\end{array}$ & $\begin{array}{r}13.03 \mathrm{H} \\
30.0 \mathrm{H} \\
11.78 \mathrm{O} \\
1.2 \mathrm{H} \\
3.2 \mathrm{H}\end{array}$ & $\begin{array}{l}6.87 E-01 \\
8.50 E \cdot 00 \\
1.71 E+02 \\
2.99 E-02 \\
6.84 E+00\end{array}$ & $\begin{array}{l}5.05 E-01 \\
6.46 E+00 \\
1.31 E+02 \\
1.15 E-06 \\
5.22 E+00\end{array}$ & $\begin{array}{l}6.47 E-01 \\
0.04 E+00 \\
1.60 E+02 \\
2.06 E-03 \\
6.66 E+00\end{array}$ & $\begin{array}{l}1 \\
12 \\
17 \\
10\end{array}$ & & & $\begin{array}{l}I E+02 \\
D E+00\end{array}$ & $8.26 E+0$ \\
\hline $\begin{array}{l}\text { IR-192 } \\
1 R-193 \mathrm{M} \\
1 R-194 \\
1 R-194 \mathrm{H} \\
P T-191\end{array}$ & 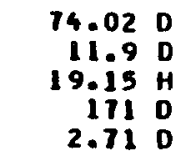 & $\begin{array}{l}1.01 E+02 \\
7.67 E-02 \\
1.09 E+01 \\
2.84 E+02 \\
3.73 E+01\end{array}$ & $\begin{array}{l}7.63 E+01 \\
3.66 E-02 \\
8.37 E+00 \\
2.17 E \cdot 02 \\
2.84 E+01\end{array}$ & $\begin{array}{l}9.43 \mathrm{E}+01 \\
4.86 \mathrm{E}-02 \\
1.02 \mathrm{E}+01 \\
2.65 \mathrm{E}+02 \\
3.54 \mathrm{E}+01\end{array}$ & $\begin{array}{l}6.08 E+01 \\
2.14 E-02 \\
6.64 E+00 \\
1.72 E+02 \\
2.04 E+01\end{array}$ & & & & TiE*C \\
\hline $\begin{array}{l}-193 \\
-193 M \\
-195 M \\
-197 \\
-197 M\end{array}$ & $\begin{array}{rl}50 & Y \\
4.33 & 0 \\
4.02 & 0 \\
18.3 & H \\
94.4 & H\end{array}$ & $\begin{array}{l}2.91 E-02 \\
1.53 E+00 \\
9.89 E+00 \\
3.17 E+00 \\
1.06 E+01\end{array}$ & $\begin{array}{l}0.71 E-07 \\
1.15 E+00 \\
7.53 E+00 \\
2.43 E+00 \\
\theta .02 E+00\end{array}$ & $\begin{array}{l}2.01 E-03 \\
1.47 E+00 \\
9.64 E+00 \\
3.10 E+00 \\
1.01 E+01\end{array}$ & $\begin{array}{l}2.93 E-07 \\
6.04 E-01 \\
4.61 E+00 \\
1.63 E+00 \\
5.65 E+00\end{array}$ & $\begin{array}{l}5.58 E-02 \\
1.32 E+00 \\
8.46 E+00 \\
2.75 E+00 \\
9.10 E+00\end{array}$ & $\begin{array}{l}6.71 E-01 \\
2.47 E+00 \\
1.30 E+01 \\
4.10 E+00 \\
1.33 E+01\end{array}$ & $\begin{array}{l}0.0 \\
0.0 \\
0.0 \\
6.76 E+01 \\
3.29 E \bullet 00\end{array}$ & $1.65 E+0$ \\
\hline
\end{tabular}



DCSE-RATE FACTORS FOR BODY ORGANS AND EFFECTIVE DOSE-RATE FACTOR FOR EXPOSUP.E 1 M ABOVE COHIAMINATEO GROUNG SJRFACE
IN MREM/YR PER MICROCUR IE/ISOUARE MI

\begin{tabular}{|c|c|c|c|c|c|c|c|c|c|c|}
\hline$E$ & IFE & RENALS & ADDER & RAIN & BREAST & HE AR $T$ & $\begin{array}{l}\text { SHALL } \\
\text { NTESTINE }\end{array}$ & $\begin{array}{c}\text { UPPER } \\
\text { LARGE } \\
\text { INTESTINE }\end{array}$ & $\begin{array}{c}\text { LOWER } \\
\text { LARGE } \\
\text { INTESTINE }\end{array}$ & IDNEYS \\
\hline $\begin{array}{l}\text { AU-194 } \\
\text { AU -195 } \\
A U-195 \mathrm{M} \\
\text { AU-196 }\end{array}$ & $\begin{array}{rr}39.5 & H \\
183 & D \\
30.6 & 5 \\
6.183 & D \\
2.696 & D\end{array}$ & $\begin{array}{l}8.68 E+01 \\
6.30 E+00 \\
1.82 E+01 \\
4.26 E+01 \\
3.67 E+01\end{array}$ & $\begin{array}{l}6 E+01 \\
3 E+00 \\
B E \bullet 01 \\
2 E+01 \\
E E \bullet 01\end{array}$ & & & & & & & $\begin{array}{l}9 E+01 \\
2 E+00 \\
4 E+01 \\
6 E+01 \\
6 E+01\end{array}$ \\
\hline $\begin{array}{l}A U-199 \\
A U G 199 \\
H G-197 \\
H G-197 M \\
H G-203 \\
T L-200\end{array}$ & $\begin{array}{r}3.1390 \\
64.14 \mathrm{H} \\
23.8 \mathrm{H} \\
46.60 \mathrm{O} \\
26.1 \mathrm{H}\end{array}$ & $\begin{array}{l}8.11 E+00 \\
5.22 E+00 \\
7.94 E+00 \\
2.11 E+01 \\
1.09 E+02\end{array}$ & $\begin{array}{l}.57 E+00 \\
.59 E+00 \\
.68 E+00 \\
.95 E+01 \\
.06 E+02\end{array}$ & $\begin{array}{l}8.02 E+00 \\
5.52 E+00 \\
8.03 E+00 \\
2.10 E+01 \\
1.18 E+02\end{array}$ & $\begin{array}{l}1 E+01 \\
D E+01 \\
E+01 \\
E E+01 \\
E E+02\end{array}$ & $\begin{array}{l}4.9 \\
7.3 \\
1.8 \\
1.0\end{array}$ & & & & $\begin{array}{l}75 E+00 \\
79 E+00 \\
B 6 E+00 \\
O 1 E+01 \\
11 E+02\end{array}$ \\
\hline $\begin{array}{l}T L-201 \\
T L-202 \\
T L-204 \\
T L-207 \\
T L-200\end{array}$ & $\begin{array}{r}73.06 \mathrm{H} \\
12.23 \mathrm{D} \\
3.779 \mathrm{Y} \\
4.77 \\
3.053 \mathrm{M}\end{array}$ & $\begin{array}{l}7.37 E+00 \\
4.13 E+01 \\
8.43 E-02 \\
1.86 E-01 \\
2.51 E+02\end{array}$ & $\begin{array}{l}.59 E+00 \\
.94 E \cdot 01 \\
.03 E-02 \\
.80 E-01 \\
.55 E \cdot 02\end{array}$ & $\begin{array}{l}7.66 E+00 \\
4.31 E+01 \\
8.93 E-02 \\
2.03 E-01 \\
2.03 E+02\end{array}$ & $\begin{array}{l}1.54 E+01 \\
5.72 E+01 \\
1.94 E-01 \\
2.27 E-01 \\
3.24 E+02\end{array}$ & $\begin{array}{l}6.9 \\
3.9 \\
7.9 \\
1.7 \\
2.5\end{array}$ & $\begin{array}{l}+00 \\
+01 \\
-02 \\
-01 \\
01 \\
002\end{array}$ & $\begin{array}{l}7.37 E \\
4.04 E \\
8.55 E \\
1.90 E \\
2.70 E\end{array}$ & & $\begin{array}{l}4 E+00 \\
8 E+01 \\
5 E-02 \\
9 E-01 \\
4 E+02\end{array}$ \\
\hline $\begin{array}{l}\mathrm{TL}-209 \\
\mathrm{IL}-210 \\
\mathrm{~PB}-203 \\
\mathrm{PBB}-204 \mathrm{M} \\
\mathrm{PB}-205\end{array}$ & $\begin{array}{r}2.20 \mathrm{H} \\
1.30 \mathrm{M} \\
52.02 \mathrm{H} \\
66.9 \mathrm{M} \\
1.51 \mathrm{E}\end{array}$ & $\begin{array}{l}1.64 E+02 \\
2.23 E+02 \\
2.71 E+01 \\
1.81 E+02 \\
5.74 E-03\end{array}$ & $\begin{array}{l}1.63 E+02 \\
2.20 E+02 \\
2.56 E+01 \\
1.74 E+02 \\
2.69 E-09\end{array}$ & $\begin{array}{l}1.8 \\
2.4 \\
2.7 \\
1.9 \\
4.7\end{array}$ & $\begin{array}{l}2.1 \\
2.8 \\
4.1 \\
2.2 \\
4.2\end{array}$ & $\begin{array}{l}1.61 \\
2.17 \\
2.45 \\
1.71 \\
1.67\end{array}$ & & $\begin{array}{l}1.71 \\
2.31 \\
2.60 \\
1.82 \\
1.02\end{array}$ & & $\begin{array}{l}1.11 E+02 \\
2.31 E+02 \\
2.65 E 001 \\
1.82 E \cdot 02 \\
9.43 E-06\end{array}$ \\
\hline $\begin{array}{l}P B-211 \\
P B-212 \\
P B-214\end{array}$ & $\begin{array}{r}3.253 \mathrm{H} \\
22.26 \mathrm{Y} \\
36.1 \mathrm{H} \\
10.643 \mathrm{H} \\
26.8 \mathrm{H}\end{array}$ & $\begin{array}{l}0.0 \\
1.13 E-01 \\
4.49 E+00 \\
1.34 E+01 \\
2.26 E+01\end{array}$ & $\begin{array}{l}0.0 \\
1.38 E-01 \\
4.29 E+00 \\
1.25 E+01 \\
2.11 E+01\end{array}$ & & & & & & & $\begin{array}{l}0.0 \\
1.63 E-01 \\
4.47 E * 00 \\
1.29 E+01 \\
2.18 E+01\end{array}$ \\
\hline $\begin{array}{l}81-206 \\
81-206 \\
81-208 \\
81-210 \\
81-211\end{array}$ & $\begin{array}{r}6.243 \mathrm{D} \\
33.4 \mathrm{Y} \\
3.68 \mathrm{Y} \mathrm{Y} \\
5.013 \mathrm{D} \\
2.13 \mathrm{M}\end{array}$ & $\begin{array}{l}2.74 E+02 \\
1.28 E+02 \\
1.07 E+02 \\
0.0 \\
4.33 E+00\end{array}$ & $\begin{array}{l}2.67 E * 02 \\
1.25 E * 02 \\
1.94 E * 02 \\
0.0 \\
4.04 E+00\end{array}$ & $\begin{array}{l}2.99 E+02 \\
1.40 E+02 \\
2.14 E+02 \\
0.0 \\
4.42 E+00\end{array}$ & $\begin{array}{l}1.63 E+02 \\
2.46 E+02 \\
0.0 \\
5.82 E+00\end{array}$ & $\begin{array}{l}2.63 E+02 \\
1.23 E * 02 \\
1.94 E+02 \\
0.0 \\
3.93 E+00\end{array}$ & $\begin{array}{l}1.12 E+02 \\
1.02 E+02 \\
0.00 \\
3.53 E+00\end{array}$ & $\begin{array}{l}1.31 E+02 \\
2.06 E+02 \\
0.0 \\
4.16 E+00\end{array}$ & $\begin{array}{l}1.20 E+02 \\
1.91 E+02 \\
0.0 \\
3.88 E+00\end{array}$ & $\begin{array}{l}1.31 E+02 \\
2.10 E+02 \\
0.0 \\
4.10 E+00\end{array}$ \\
\hline $\begin{array}{l}81-214 \\
P O-209 \\
P D-210\end{array}$ & $\begin{array}{r}60.55 \mathrm{M} \\
45.65 \mathrm{M} \\
19.9 \mathrm{M} \\
102 \mathrm{Y} \\
138.378 \mathrm{D}\end{array}$ & $\begin{array}{l}1.51 \\
1.24 \\
1.19 \\
3.02 \\
7.41\end{array}$ & & $\begin{array}{l}1.30 E+ \\
1.32 E+ \\
3.20 E- \\
8.04 E-\end{array}$ & $\begin{array}{l}1.61 E+01 \\
1.49 E+02 \\
4.01 E-01 \\
9.06 E-04\end{array}$ & & & & & \\
\hline $\begin{array}{l}P O-211 \\
P O-212 \\
P O-213 \\
P O-214 \\
P O-215\end{array}$ & $\begin{array}{rr}0.516 & 5 \\
2.98 E-7 & 5 \\
4.2 E-6 & 5 \\
1.637 E-4 & 5 \\
1.178 E-3 & 5\end{array}$ & $\begin{array}{l}0.0 \\
2.66 \mathrm{E}-03 \\
7.27 \mathrm{E}-03 \\
1.34 \mathrm{E}-02\end{array}$ & $\begin{array}{l}6.49 E-01 \\
0.0 \\
2.57 E-03 \\
1.01 E-03 \\
1.26 E-02\end{array}$ & $\begin{array}{l}0.0 \\
2.88 E-03 \\
8.88 E-03 \\
1.40 E-02\end{array}$ & $\begin{array}{l}0.0 \\
3.26 E-03 \\
0.08 E-03 \\
1.12 E-02\end{array}$ & $\begin{array}{l}0.0 \\
2.53 E-0 \\
6.91 E-0 \\
1.23 E-0\end{array}$ & & $\begin{array}{l}6.80 E-01 \\
0.0 \\
2.69 E-03 \\
7.35 E-03 \\
1.31 E-02\end{array}$ & $\begin{array}{l}01 \\
03\end{array}$ & $\begin{array}{l}6.78 E-01 \\
0.0 \\
2.68 E-03 \\
7.33 E-03 \\
1.31 E-02\end{array}$ \\
\hline
\end{tabular}




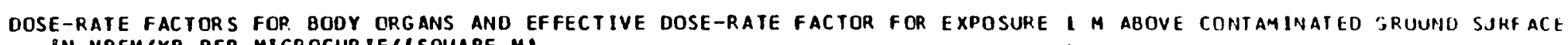

\begin{tabular}{|c|c|c|c|c|c|c|c|c|c|c|}
\hline ID & HALF & LIVER & LUNGS & ARROH & MAI & VARIES & P ANCREAS & KELETON & SPLEEN & 5 TOMACH \\
\hline $\begin{array}{l}A U-194 \\
A U-195 \\
A U-195 M \\
A U-196 \\
A U-198\end{array}$ & 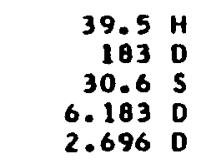 & $\begin{array}{l}6 \\
1\end{array}$ & 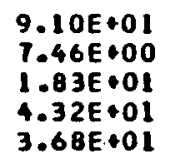 & & & & & & & $\begin{array}{l}8.36 \mathrm{E}+01 \\
6.32 \mathrm{E}+00 \\
1.65 \mathrm{E}+01 \\
3.92 \mathrm{E}+01 \\
3.38 \mathrm{E}+01\end{array}$ \\
\hline $\begin{array}{l}U-199 \\
G-197 \\
G-197 M \\
G-203 \\
L-200\end{array}$ & $\begin{array}{r}3.139 \mathrm{D} \\
64.14 \mathrm{H} \\
23.8 \mathrm{H} \\
46.60 \mathrm{D} \\
26.1 \mathrm{H}\end{array}$ & $\begin{array}{l}1.5 \\
5.5 \\
7.6 \\
1.9\end{array}$ & $\begin{array}{l}0 \\
0 \\
0 \\
1\end{array}$ & $\begin{array}{l}E \in 01 \\
E+01 \\
E+01 \\
E+C O\end{array}$ & & & $\begin{array}{l}6.19 E \cdot 00 \\
3.73 E+00 \\
5.97 E+00\end{array}$ & & & \\
\hline $\begin{array}{l}T L-201 \\
T L-202 \\
T L-204 \\
T L-207 \\
T L-208\end{array}$ & $\begin{array}{r}73.06 \mathrm{H} \\
12.23 \mathrm{O} \\
3.779 \\
4.77 \mathrm{M} \\
3.053 \mathrm{M}\end{array}$ & 2.561 & $\begin{array}{l}0 \\
1 \\
2\end{array}$ & & & & & & & $\begin{array}{l}E+01 \\
E-02 \\
E-01 \\
E+02\end{array}$ \\
\hline $\begin{array}{l}-210 \\
-203 \\
-204 M \\
-205\end{array}$ & $\begin{array}{r}2.20 \mathrm{M} \\
1.30 \mathrm{M} \\
52.02 \mathrm{H} \\
66.9 \mathrm{M} \\
1.51 \mathrm{Y}\end{array}$ & $\begin{array}{l}2.1 \\
2.5 \\
1.7 \\
6.6\end{array}$ & $\begin{array}{l}2.3 \\
2.7 \\
1.8 \\
4.6\end{array}$ & $\begin{array}{l}2.5 \\
3.8 \\
2.0 \\
2.9\end{array}$ & & & $\begin{array}{l}1.4 \\
1.8 \\
2.1 \\
1.4 \\
3.3\end{array}$ & & & $\begin{array}{l}2.16 E+02 \\
2.49 E+01 \\
1.11 E+02 \\
1.24 E-03\end{array}$ \\
\hline $\begin{array}{l}P B-209 \\
P B-210 \\
P B-211 \\
P B-212 \\
P B-214\end{array}$ & $\begin{array}{r}3.253 \mathrm{H} \\
22.26 \mathrm{Y} \\
36.1 \mathrm{M} \\
10.643 \mathrm{H} \\
26.8 \mathrm{H}\end{array}$ & $\begin{array}{l}1.23 E \\
4.26 E \\
1.25 E \\
2.10 E\end{array}$ & $\begin{array}{l}0.0 \\
1.43 \mathrm{E} \\
4.57 \mathrm{E} \\
1.36 \mathrm{E}\end{array}$ & $\begin{array}{l}0.0 \\
2.52 E- \\
5.17 E \\
1.92 E\end{array}$ & $\begin{array}{l}E-02 \\
E+00 \\
E+01 \\
E * 01\end{array}$ & $\begin{array}{l}1 \\
0 \\
1 \\
1\end{array}$ & & & & $\begin{array}{l}1.10 E-01 \\
4.21 E+00 \\
1.22 E+01 \\
2.07 E+01\end{array}$ \\
\hline $\begin{array}{l}81-206 \\
81-207 \\
81-208 \\
81-210 \\
81-211\end{array}$ & $\begin{array}{r}6.2430 \\
33.4 \mathrm{Y} \\
3.60 E 5 \mathrm{Y} \\
5.013 \mathrm{D} \\
2.13 \mathrm{M}\end{array}$ & $\begin{array}{l}2.66 E+02 \\
1.24 E+02 \\
1.95 E+02 \\
0.0 \\
4.02 E+00\end{array}$ & 2.0 & 12 & 12 & $\begin{array}{l}2.54 E+02 \\
1.19 E+02 \\
1.73 E+02 \\
0.0 \\
3.72 E+00\end{array}$ & $\begin{array}{l}2.29 E \cdot 02 \\
1.07 E+02 \\
1.71 E+02 \\
0.0 \\
3.45 E+00\end{array}$ & $\begin{array}{l}3.21 E+02 \\
1.51 E+02 \\
2.14 E+02 \\
0.0 \\
5.53 E+00\end{array}$ & $\begin{array}{l}1.20 E+02 \\
1.87 E+02 \\
0.0 \\
3.96 E+00\end{array}$ & $\begin{array}{l}\text { 1. } 22 E+02 \\
1.92 E+02 \\
0.0 \\
3.96 E+00\end{array}$ \\
\hline $\begin{array}{l}81-212 \\
81-213 \\
81-214 \\
P 0-209 \\
P 0-210\end{array}$ & $\begin{array}{r}60.55 \mathrm{M} \\
45.65 \mathrm{M} \\
19.9 \mathrm{M} \\
102 \mathrm{Y} \\
138.378 \mathrm{D}\end{array}$ & $\begin{array}{l}1.48 E+01 \\
1.17 E+01 \\
1.18 E+02 \\
2.88 E-01 \\
7.10 E-04\end{array}$ & $\begin{array}{l}1.57 E+01 \\
1.26 E+01 \\
1.25 E+02 \\
3.09 E-01 \\
7.59 E-04\end{array}$ & $\begin{array}{l}1.70 E+01 \\
1.50 E+01 \\
1.33 E+02 \\
3.68 E-01 \\
8.26 E-04\end{array}$ & $\begin{array}{l}1.59 E+01 \\
1.28 E+01 \\
1.26 E+02 \\
3.06 E-01 \\
1.71 E-04\end{array}$ & $\begin{array}{l}1.41 E+01 \\
1.11 E+01 \\
1.11 E+02 \\
2.71 E-01 \\
6.91 E-04\end{array}$ & & & & \\
\hline $\begin{array}{l}-211 \\
-212 \\
-213 \\
-214 \\
-215\end{array}$ & $\begin{array}{r}0 . \\
2.98\end{array}$ & $\begin{array}{l}6.45 E-01 \\
0.0 \\
2.55 E-03 \\
6.96 E-03 \\
1.26 E-02\end{array}$ & $\begin{array}{l}6.90 E-01 \\
0.0 \\
2.72 E-03 \\
7.44 E-03 \\
1.35 E-02\end{array}$ & $\begin{array}{l}7.59 E-01 \\
0.0 \\
2.97 E-03 \\
8.10 E-03 \\
1.60 E-02\end{array}$ & $\begin{array}{l}7.01 E-01 \\
0.0 \\
2.77 E-03 \\
7.56 E-03 \\
1.39 E-02\end{array}$ & $\begin{array}{l}6.25 E-01 \\
0.0 \\
2.48 E-03 \\
6.77 E-03 \\
1.19 E-02\end{array}$ & $\begin{array}{l}5.57 E-01 \\
0.0 \\
2.20 E-03 \\
6.01 E-03 \\
1.08 E-02\end{array}$ & $\begin{array}{l}7.71 E-01 \\
0.0 \\
3.02 E-03 \\
8.24 E-03 \\
1.62 E-02\end{array}$ & $\begin{array}{l}0.27 E-01 \\
0.0 \\
2.47 E-03 \\
6.76 E-03 \\
1.23 E-02\end{array}$ & $\begin{array}{l}6.38 E-01 \\
0.0 \\
2.52 E-03 \\
6.89 E-03 \\
1.24 E-02\end{array}$ \\
\hline
\end{tabular}




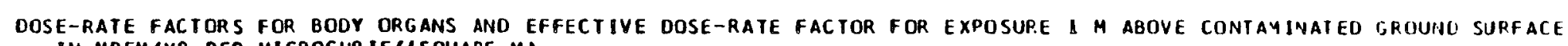
IN MREMIYR PER MICROCUR IE/ISOUAPE MI

\begin{tabular}{|c|c|c|c|c|c|c|c|c|c|}
\hline E LOE & LIFE & TESTES & THYMUS & HYROID & TERUS & FECTIVE & $\begin{array}{c}\text { SKIN } \\
\text { IPHOTONI }\end{array}$ & $\begin{array}{l}\text { SKIN } \\
\text { IELEC TRON }\end{array}$ & $\begin{array}{l}\text { SKIN } \\
\text { ITOTALI }\end{array}$ \\
\hline $\begin{array}{l}A U-194 \\
A U-195 \\
A U-1954 \\
A U-198 \\
\text { AU-198 }\end{array}$ & $\begin{array}{r}39.5 \mathrm{H} \\
183 \mathrm{D} \\
30.6 \mathrm{~S} \\
6.183 \mathrm{D} \\
2.696 \mathrm{D}\end{array}$ & $\begin{array}{l}1.21 E+02 \\
1.16 E+01 \\
2.52 E+01 \\
5.94 E+01 \\
4.96 E+01\end{array}$ & $\begin{array}{l}9.48 E+01 \\
0.89 E+00 \\
1.91 E+01 \\
4.49 E+01 \\
3.74 E+01\end{array}$ & $\begin{array}{l}14 E+02 \\
14 E+01 \\
39 E+01 \\
58 E+01\end{array}$ & $\begin{array}{l}7.55 E+01 \\
5.38 E+00 \\
1.48 E+01 \\
3.48 E+01\end{array}$ & & $\begin{array}{l}1.39 E+02 \\
1.44 E+01 \\
2.90 E+01 \\
6.76 E+01 \\
5.63 E+01\end{array}$ & $\begin{array}{l}1.07 E+01 \\
0.0 \\
0.0 \\
0.0 \\
2.31 E+02\end{array}$ & $\begin{array}{l}1.49 E+02 \\
1.44 E+01 \\
2.90 E+01 \\
6.76 E+01 \\
? .87 E+02\end{array}$ \\
\hline $\begin{array}{l}A U-199 \\
H G-197 \\
H G-197 M \\
H G-203 \\
T L-200\end{array}$ & $\begin{array}{r}3.1390 \\
64.14 H \\
23.80 H \\
46.60 \\
26.11\end{array}$ & $\begin{array}{l}1.16 E+01 \\
9.43 E+00 \\
1.21 E+01 \\
2.87 E+01 \\
1.51 E+02\end{array}$ & $\begin{array}{l}8.93 E+00 \\
7.2 B E * 00 \\
9.31 E+00 \\
2.17 E+01 \\
1.18 E+02\end{array}$ & $\begin{array}{l}1.14 E+01 \\
9.27 E+00 \\
1.19 E+01 \\
2.72 E+01 \\
1.42 E+02\end{array}$ & $\begin{array}{l}6.58 E+00 \\
4.43 E+00 \\
6.52 E+00 \\
1.71 E+01 \\
9.24 E+01\end{array}$ & $\begin{array}{l}1.02 E+01 \\
8.06 E+00 \\
1.06 E+01 \\
2.50 E+01 \\
1.29 E+02\end{array}$ & $\begin{array}{l}1.30 E+01 \\
1.19 E+01 \\
1.47 E+01 \\
3.22 E+01 \\
1.73 E .02\end{array}$ & $\begin{array}{l}8.31 E-01 \\
0.0 \\
0.0 \\
0.0 \\
9.59 E+00\end{array}$ & $\begin{array}{l}1.39 E+01 \\
1.19 E+01 \\
1.47 E+01 \\
3.22 E+01 \\
1.82 E+02\end{array}$ \\
\hline $\begin{array}{l}Y L-201 \\
T L-202 \\
T L-204 \\
T L-207 \\
T L-208\end{array}$ & $\begin{array}{r}73.06 \mathrm{H} \\
12.23 \mathrm{D} \\
3.779 \mathrm{Y} \\
4.77 \mathrm{M} \\
3.053 \mathrm{M}\end{array}$ & $\begin{array}{l}1.25 E+01 \\
5.79 E+01 \\
1.52 E-01 \\
2.53 E-01 \\
3.52 E+02\end{array}$ & $\begin{array}{l}9.65 E+00 \\
4.38 E+01 \\
1.18 E-01 \\
2.02 E-01 \\
2.75 E+02\end{array}$ & $\begin{array}{l}1.23 E+01 \\
5.42 E+01 \\
1.50 E-01 \\
2.41 E-01 \\
3.21 E+02\end{array}$ & $\begin{array}{l}6.18 E+00 \\
3.40 E+01 \\
1.16 E-02 \\
1.56 E-01 \\
2.29 E+02\end{array}$ & $\begin{array}{l}1.07 E+01 \\
4.97 E+01 \\
1.30 E-01 \\
2.16 E-01 \\
3.03 E+02\end{array}$ & $\begin{array}{l}1.51 E+01 \\
6.65 E+01 \\
1.91 E-01 \\
2.93 E-01 \\
3.94 E+02\end{array}$ & $\begin{array}{l}0.0 \\
4.91 E+00 \\
1.59 E+02 \\
4.66 E+02 \\
5.71 E+02\end{array}$ & $\begin{array}{l}1.51 E+01 \\
1.15 E+01 \\
1.59 E+02 \\
4.66 E+02 \\
9.65 E+02\end{array}$ \\
\hline $\begin{array}{l}Y L-209 \\
Y L-210 \\
P B-203 \\
P Q-204 M \\
P B-205\end{array}$ & $\begin{array}{r}2.20 \mathrm{M} \\
1.30 \mathrm{M} \\
52.02 \mathrm{H} \\
66.9 \mathrm{M} \\
1.511 \mathrm{Y}\end{array}$ & $\begin{array}{l}2.28 E+02 \\
3.07 E+02 \\
3.87 E+01 \\
2.45 E+02 \\
4.14 E-02\end{array}$ & $\begin{array}{l}1.79 E \cdot 02 \\
2.42 E+022 \\
2.95 E+01 \\
1.94 E+02 \\
1.47 E-06\end{array}$ & $\begin{array}{l}2.14 E+02 \\
2.88 E+02 \\
3.70 E+01 \\
2.33 E+02 \\
3.11 E-03\end{array}$ & $\begin{array}{l}1.45 E+02 \\
1.94 E+02 \\
2.21 E+01 \\
1.51 E+02 \\
3.87 E-01\end{array}$ & $\begin{array}{l}1.96 E+02 \\
2.63 E+02 \\
3.36 E+01 \\
2.10 E+02 \\
1.55 E-02\end{array}$ & $\begin{array}{l}2.60 E+02 \\
3.51 E 002 \\
4.43 E \cdot 01 \\
2.83 E \cdot 02 \\
B .82 E-01\end{array}$ & $\begin{array}{l}6.92 E+02 \\
6.77 E+02 \\
0.0 \\
8.99 E+01 \\
0.0\end{array}$ & $\begin{array}{l}9.52 E+02 \\
1.03 E+03 \\
4.43 E+01 \\
3.13 E+02 \\
8.82 E-01\end{array}$ \\
\hline $\begin{array}{l}P B-209 \\
P B-210 \\
P B-211 \\
P B-212 \\
P B-214\end{array}$ & $\begin{array}{r}3.253 \mathrm{H} \\
22.26 \mathrm{Y} \\
36.1 \mathrm{M} \\
10.643 \mathrm{H} \\
26.8 \mathrm{M}\end{array}$ & $\begin{array}{l}0.0 \\
3.20 E-02 \\
6.10 E+00 \\
1.89 E \times 01 \\
3.10 E+01\end{array}$ & $\begin{array}{l}0.0 \\
1.82 E-01 \\
4.74 E+00 \\
1.44 E+01 \\
2.35 E+01\end{array}$ & $\begin{array}{l}2.42 E-01 \\
5.75 \mathrm{E} \cdot 00 \\
1.02 \mathrm{~B} \cdot 01 \\
2.92 \mathrm{E} \cdot 01\end{array}$ & $\begin{array}{l}0.34 E-02 \\
3.72 E+00 \\
1.09 E+01 \\
1.84 E+01\end{array}$ & & & $\begin{array}{l}6.88 E+01 \\
0.0 \\
4.14 E+02 \\
3.92 E+00 \\
1.01 E+02\end{array}$ & $\begin{array}{l}6.88 E+01 \\
1.37 E+00 \\
4.21 E+02 \\
2.54 E+01 \\
1.36 E+02\end{array}$ \\
\hline $\begin{array}{l}81-206 \\
81-207 \\
81-208 \\
81-210 \\
81-211\end{array}$ & $\begin{array}{r}6.243 \mathrm{D} \\
33.4 \mathrm{Y} \\
3.68 E 5 \mathrm{Y} \\
5.013 \mathrm{O} \\
2.13 \mathrm{M}\end{array}$ & $\begin{array}{l}3.78 E+02 \\
1.77 E+02 \\
2.65 E+02 \\
0.0 \\
5.90 E+00\end{array}$ & $\begin{array}{l}2.9 T E+02 \\
1.39 E+02 \\
2.08 E+02 \\
0.0 \\
4.45 E+00\end{array}$ & $\begin{array}{l}3.57 E+02 \\
1.67 E+02 \\
2.40 E+02 \\
0.0 \\
5.52 E+00\end{array}$ & $\begin{array}{l}2.33 E+02 \\
1.09 E+02 \\
1.76 E+02 \\
0.0 \\
3.53 E+00\end{array}$ & $\begin{array}{l}3.24 E+02 \\
1.51 \mathrm{E}+02 \\
2.29 E+02 \\
0.0 \\
5.10 E+00\end{array}$ & $\begin{array}{l}4.35 E+02 \\
2.04 E \cdot 02 \\
2.95 E \cdot 02 \\
0.0 \\
6.67 E+00\end{array}$ & & \\
\hline $\begin{array}{l}B 1-212 \\
81-213 \\
81-214 \\
P 0-209 \\
P 0-210\end{array}$ & $\begin{array}{r}60.55 \mathrm{M} \\
45.65 \mathrm{H} \\
19.99 \mathrm{M} \\
102 \mathrm{Y} \\
138.378 \mathrm{O}\end{array}$ & $\begin{array}{l}2.08 E+01 \\
1.70 E+01 \\
1.64 E+02 \\
4.17 E-01 \\
1.01 E-03\end{array}$ & & & & & & $\begin{array}{l}4.73 E+02 \\
3.78 E+02 \\
6.42 E+02 \\
0.0 \\
0.0\end{array}$ & $\begin{array}{l}4.97 E+02 \\
3.98 E+02 \\
8.29 E+02 \\
4.80 E-01 \\
1.16 E-03\end{array}$ \\
\hline $\begin{array}{l}P O-211 \\
P Q-212 \\
P O-213 \\
P O-214 \\
P O-215\end{array}$ & $\begin{array}{rr}0.516 & 5 \\
2.98 E-7 & 5 \\
4.2 E-6 & 5 \\
1.637 E-4 & 5 \\
1.778 E-3 & S\end{array}$ & $\begin{array}{l}9.17 E-01 \\
0.0 \\
3.62 E-03 \\
9.87 E-03 \\
1.82 E-02\end{array}$ & $\begin{array}{l}7.19 E-01 \\
0.0 \\
2.85 E-03 \\
7.80 E-03 \\
1.37 E-02\end{array}$ & $\begin{array}{l}0.66 E-01 \\
0.0 \\
3.43 E-03 \\
9.37 E-03 \\
1.69 E-02\end{array}$ & $\begin{array}{l}5.62 E-01 \\
0.0 \\
2.22 E-03 \\
6.05 E-03 \\
1.10 E-02\end{array}$ & $\begin{array}{l}7.84 E-01 \\
0.0 \\
3.09 E-03 \\
8.43 E-03 \\
1.56 E-02\end{array}$ & $\begin{array}{l}1.06 E+00 \\
0.0 \\
4.18 E-03 \\
1.14 E-02 \\
2.07 E-02\end{array}$ & $\begin{array}{l}0.0 \\
0.0 \\
0.0 \\
0.0 \\
0.0\end{array}$ & $\begin{array}{l}1.06 E \cdot 00 \\
0.0 \\
4.18 E-03 \\
1.14 E-02 \\
2.07 E-02\end{array}$ \\
\hline
\end{tabular}




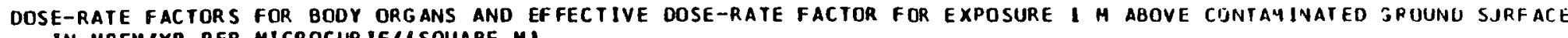
IN MREM/YR PER HICROCUR IE/ISOUARE MI

\begin{tabular}{|c|c|c|c|c|c|c|c|c|c|c|}
\hline NUCL IDE & HALF-LIFE & ADRENALS & BLADDER & BRAIN & BREAST & HE ART & $\begin{array}{l}\text { SMALL } \\
\text { NTESTINE }\end{array}$ & $\begin{array}{l}\text { LARGE } \\
\text { NTESTINE }\end{array}$ & $\begin{array}{l}\text { LARGE } \\
\text { NIESTINE }\end{array}$ & K IONEYS \\
\hline $\begin{array}{l}P O-216 \\
P O-218 \\
A T-211 \\
A T-217 \\
R N-218\end{array}$ & $\begin{array}{r}0.146 \mathrm{~S} \\
3.05 \mathrm{H} \\
7.214 \mathrm{H} \\
0.0323 \mathrm{~S} \\
0.035 \mathrm{~S}\end{array}$ & $\begin{array}{l}1.26 E-03 \\
0.0 \\
3.11 E+00 \\
2.11 E-02 \\
6.68 E-02\end{array}$ & $\begin{array}{l}1.22 E-03 \\
0.0 \\
3.19 E+00 \\
2.01 E-02 \\
6.38 E-02\end{array}$ & $\begin{array}{l}1.37 E-03 \\
0.0 \\
3.26 E+00 \\
2.25 E-02 \\
7.14 E-02\end{array}$ & $\begin{array}{l}.54 E-03 \\
0.0 \\
05 E+00 \\
57 E-02 E-02 \\
.29 E-02\end{array}$ & $\begin{array}{l}1.20 E-03 \\
0.0 \\
2.94 E+00 \\
1.97 E-02 \\
6.26 E-02\end{array}$ & $\begin{array}{l}.09 E-03 \\
.0 \\
.59 E-000 \\
.77 E-02 \\
.64 E-02\end{array}$ & $\begin{array}{l}28 E-03 \\
0 \\
14 E E+00 \\
09 E-02 \\
65 E-02\end{array}$ & $\begin{array}{l}1.17 E-03 \\
0.0 \\
2.01 E+00 \\
1.93 E-02 \\
6.12 E-02\end{array}$ & $\begin{array}{l}1.27 E-03 \\
0.0 \\
3.27 E+00 \\
2.09 E-02 \\
6.64 E-02\end{array}$ \\
\hline $\begin{array}{l}R N-219 \\
R N-220 \\
R N-222 \\
F R-221 \\
F R-223\end{array}$ & 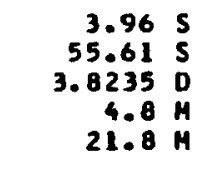 & $\begin{array}{l}5.26 E+00 \\
4.63 E-02 \\
3.45 E-02 \\
2.85 E+00 \\
4.09 E+00\end{array}$ & $\begin{array}{l}4.88 E+00 \\
4.41 E-02 \\
3.28 E-02 \\
2.62 E+00 \\
4.18 E+00\end{array}$ & $\begin{array}{l}5.32 E+00 \\
4.92 E-02 \\
3.65 E-02 \\
2.80 E+00 \\
4.14 E+00\end{array}$ & $\begin{array}{l}7.10 E+00 \\
5.79 E-02 \\
4.35 E-02 \\
4.22 E+00 \\
8.83 E+00\end{array}$ & $\begin{array}{l}4.75 E+00 \\
4.31 E-02 \\
3.20 E-02 \\
2.55 E+00 \\
3.71 E \bullet 00\end{array}$ & & & $\begin{array}{l}4.70 E+00 \\
4.22 E-02 \\
3.14 E-02 \\
2.53 E+00 \\
3.66 E+00\end{array}$ & $\begin{array}{l}5.04 E+00 \\
4.58 E-02 \\
3.40 E-02 \\
2.70 E+00 \\
4.45 E+00\end{array}$ \\
\hline $\begin{array}{l}\text { RA-222 } \\
\text { RA-223 } \\
R A-224 \\
\text { RA-225 } \\
\text { RA-226 }\end{array}$ & 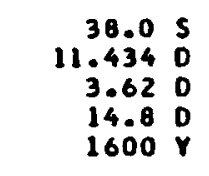 & $\begin{array}{l}8.48 E-01 \\
1.20 E+01 \\
9.29 E-01 \\
6.43 E-01 \\
6.21 E-01\end{array}$ & $\begin{array}{l}7.85 E-01 \\
1.14 E+01 \\
8.52 E-01 \\
9.30 E-01 \\
5.71 E-01\end{array}$ & $\begin{array}{l}8.57 E-01 \\
1.21 E+01 \\
9.15 E-01 \\
5.23 E-01 \\
6.08 E-01\end{array}$ & $\begin{array}{l}1.13 E+00 \\
1.94 E+01 \\
1.32 E+00 \\
2.99 E+00 \\
9.56 E-01\end{array}$ & $\begin{array}{l}7.65 E-01 \\
1.10 E+01 \\
0.29 E-01 \\
4.83 E-01 \\
5.56 E-01\end{array}$ & & & & $\begin{array}{l}\text { 0. 10E-01 } \\
1.17 E+01 \\
8.77 E-01 \\
1.20 E+00 \\
5.05 E-01\end{array}$ \\
\hline $\begin{array}{l}R A-228 \\
A C-225 \\
A C-227 \\
A C-228 \\
T H-226\end{array}$ & $\begin{array}{r}5.75 \mathrm{Y} \\
10.0 \mathrm{O} \\
21.773 \mathrm{Y} \\
6.13 \mathrm{H} \\
30.9 \mathrm{M}\end{array}$ & $\begin{array}{l}4.80 E-09 \\
1.19 E+00 \\
1.13 E-02 \\
7.75 E+01 \\
7.00 E-01\end{array}$ & $\begin{array}{l}8.65 E-16 \\
1.13 E+00 \\
9.79 E-03 \\
7.53 E+01 \\
6.53 E-01\end{array}$ & $\begin{array}{l}3.83 E-10 \\
1.19 E+00 \\
1.05 E-02 \\
8.43 E+01 \\
6.94 E-01\end{array}$ & $\begin{array}{l}3.76 E-07 \\
2.60 E+00 \\
5.62 E-02 \\
9.80 E+01 \\
1.35 E+00\end{array}$ & $\begin{array}{l}6.25 E-10 \\
1.09 E+00 \\
9.74 E-03 \\
1.43 E+01 \\
6.35 E-01\end{array}$ & & & & $\begin{array}{l}5.75 E-12 \\
1.16 E+00 \\
9.97 E-03 \\
7.88 E+01 \\
6.67 E-01\end{array}$ \\
\hline $\begin{array}{l}T H-227 \\
T H-228 \\
T H-229 \\
T H-230 \\
T H-231\end{array}$ & $\begin{array}{r}18.718 \mathrm{O} \\
1.9132 \mathrm{Y} \\
7.34 E 3 \mathrm{Y} \\
7.7 \mathrm{Y} \\
25.52 \mathrm{H}\end{array}$ & $\begin{array}{l}9.58 E+00 \\
1.75 E-01 \\
7.54 E+00 \\
3.53 E-02 \\
1.01 E+00\end{array}$ & $\begin{array}{l}8.911 \\
1.64 \\
7.27 \\
3.131 \\
9.591\end{array}$ & $\begin{array}{l}9.5 \\
1.7 \\
7.5 \\
3.1 \\
9.5\end{array}$ & & $\begin{array}{l}8.57 E+00 \\
1.57 E-01 \\
6.91 E+00 \\
2.90 E-02 \\
8.74 E-01\end{array}$ & $\begin{array}{l}E-01 \\
E+00 \\
E \rightarrow 02 \\
E-01\end{array}$ & & $\begin{array}{l}0.51 E+00 \\
1.54 E-01 \\
6.72 E+00 \\
2.97 E-02 \\
8.76 E-01\end{array}$ & $\begin{array}{l}9.21 E+00 \\
1.68 E-01 \\
7.46 E+00 \\
3.25 E-02 \\
1.03 E+00\end{array}$ \\
\hline $\begin{array}{l}T H-232 \\
T H-233 \\
T H-234 \\
P A-230 \\
P A-231\end{array}$ & $\begin{array}{r}1.405 E 10 Y \\
22.3 Y \\
24.10 \\
17.4 \\
3.27684 Y\end{array}$ & $\begin{array}{l}1.80 E-02 \\
2.98 E+00 \\
6.61 E-01 \\
5.63 E+01 \\
2.76 E+00\end{array}$ & $\begin{array}{l}1.39 E-02 \\
2.84 E+00 \\
6.69 E-01 \\
5.44 E+01 \\
2.54 E+00\end{array}$ & $\begin{array}{l}1.39 E-02 \\
3.11 E+00 \\
6.76 E-01 \\
6.07 E+01 \\
2.72 E+00\end{array}$ & $\begin{array}{l}2.72 E-01 \\
4.45 E+00 \\
1.63 E+00 \\
7.45 E+01 \\
5.40 E+00\end{array}$ & $\begin{array}{l}1.28 E-02 \\
2.75 E+00 \\
6.14 E-01 \\
5.35 E+01 \\
2.45 E+00\end{array}$ & $\begin{array}{l}1.09 E-02 \\
2.47 E+00 \\
5.40 E-01 \\
4.83 E+01 \\
2.19 E+00\end{array}$ & $\begin{array}{l}1.31 E-02 \\
2.94 E+00 \\
6.61 E-01 \\
5.70 E+01 \\
2.62 E+00\end{array}$ & & $\begin{array}{l}1.48 E-02 \\
2.96 E+00 \\
6.90 E-01 \\
5.68 E+01 \\
2.66 E+00\end{array}$ \\
\hline $\begin{array}{l}P A-233 \\
P A-234 \\
P A-234 M \\
U-230 \\
U-231\end{array}$ & $\begin{array}{rll}27.0 & 0 \\
6.70 & H \\
1.17 & H \\
20.8 & D \\
4.2 & D\end{array}$ & $\begin{array}{l}1.94 E+01 \\
1.66 E+02 \\
9.63 E-01 \\
1.01 E-01 \\
5.95 E+00\end{array}$ & $\begin{array}{l}1.81 E \cdot 01 \\
1.61 E * 02 \\
9.36 E-01 \\
8.94 E-02 \\
5.72 E \cdot 00\end{array}$ & $\begin{array}{l}1.96 E+01 \\
1.80 E+02 \\
1.05 E+00 \\
9.39 E-02 \\
5.94 E+00\end{array}$ & $\begin{array}{l}2.92 E+01 \\
2.14 E+02 \\
1.21 E \cdot 00 \\
5.53 E-01 \\
1.43 E+01\end{array}$ & $\begin{array}{l}1.76 E+01 \\
1.58 E+02 \\
9.24 E-01 \\
8.57 E-02 \\
5.45 E+00\end{array}$ & $\begin{array}{l}1.57 E+01 \\
1.44 E+02 \\
0.38 E-01 \\
7.49 E-02 \\
4.73 E+00\end{array}$ & & & \\
\hline $\begin{array}{l}u-232 \\
U-233 \\
u-234 \\
U-235 \\
U-236\end{array}$ & $\begin{array}{r}72 Y \\
1.592 E 5 Y \\
2.445 E 5 Y \\
7.038 E 8 Y \\
2.3415 E 7 Y\end{array}$ & $\begin{array}{l}2.70 E-02 \\
2.20 E-02 \\
1.66 E-02 \\
1.39 E+01 \\
1.34 E-02\end{array}$ & $\begin{array}{l}1.05 E-02 \\
1.80 E-02 \\
9.22 E-03 \\
1.28 E+01 \\
6.40 E-03\end{array}$ & $\begin{array}{l}1.93 E-02 \\
1.94 E-02 \\
9.69 E-03 \\
1.36 E .01 \\
1.09 E-03\end{array}$ & $\begin{array}{l}4.25 E-01 \\
1.59 E-01 \\
3.59 E-01 \\
2.20 E \div 01 \\
3.36 E-01\end{array}$ & $\begin{array}{l}1.80 E-02 \\
1.81 E-02 \\
9.30 E-03 \\
1.25 E+01 \\
6.84 E-03\end{array}$ & $\begin{array}{l}1.49 E-02 \\
1.52 E-02 \\
1.27 E-03 \\
1.10 E+01 \\
5.09 E-03\end{array}$ & $\begin{array}{l}1.79 E-02 \\
1.89 E-02 \\
8.76 E-03 \\
1.33 E+01 \\
5.95 E-03\end{array}$ & $\begin{array}{l}1.86 E-02 \\
1.19 E-02 \\
1.23 E+01 \\
9.17 E-03\end{array}$ & $\begin{array}{l}1.97 E-02 \\
1.83 E-02 \\
1.00 E-02 \\
1.30 E+01 \\
6.83 E-03\end{array}$ \\
\hline
\end{tabular}


DOSE-RAIE FACTORS FOR BODY ORGANS AND EFFECTIVE DOSE-RATE FACTOR FOR EXPOSURE I M ABOVE CONTAYINATED SROUNO SURFACE

\begin{tabular}{|c|c|c|c|c|c|c|c|c|c|c|}
\hline & HALF & LIVER & LUNGS & MARROH & $\begin{array}{c}\text { RED } \\
\text { MARROH }\end{array}$ & IVARIES & PANCREAS & SKELETON & SPLEEN & $S$ TOMACH \\
\hline $\begin{array}{r}21 \\
-21 \\
21 \\
21 \\
21\end{array}$ & $\begin{array}{r}0.1465 \\
3.05 \\
7.214 \\
0.0323 \\
0.035 \\
\end{array}$ & $\begin{array}{l}1.21 E-03 \\
0.0 \\
3.17 E+00 \\
2.00 E-02 \\
6.34 E-02\end{array}$ & $\begin{array}{l}1.29 E-03 \\
0.0 \\
3.51 E+00 \\
2.14 E-02 \\
6.79 E-02\end{array}$ & $\begin{array}{l}41 E-03 \\
0 \\
95 E+00 \\
11 E-02 \\
63 E-02\end{array}$ & $\begin{array}{l}31 E-03 \\
0 \\
45 E \cdot 00 \\
18 E-02 \\
93 E-02\end{array}$ & $\begin{array}{l}18 E-03 \\
0 \\
66 E+00 \\
93 E-02 \\
14 E-02\end{array}$ & $\begin{array}{l}1.04 E-03 \\
0.0 \\
2.27 E+00 \\
1.72 E-02 \\
5.46 E-02\end{array}$ & $\begin{array}{l}1.43 E-03 \\
0.0 \\
6.12 E+00 \\
2.45 E-02 \\
1.16 E-02\end{array}$ & $\begin{array}{l}1.17 E-03 \\
0.0 \\
2.99 E+00 \\
1.94 E-02 \\
6.17 E-02\end{array}$ & $\begin{array}{l}1.20 E-03 \\
0.0 \\
3.03 E+00 \\
1.97 E-02 \\
6.27 E-02\end{array}$ \\
\hline 22 & $\begin{array}{r}3.96 \mathrm{~S} \\
55.61 \mathrm{~S} \\
3.8235 \mathrm{O} \\
4.8 \mathrm{M} \\
21.8 \mathrm{M}\end{array}$ & $\begin{array}{l}4.86 E+00 \\
4.38 E-02 \\
3.26 E-02 \\
2.61 E+00 \\
4.09 E+00\end{array}$ & $\begin{array}{l}5.25 E+00 \\
4.70 E-02 \\
3.50 E-02 \\
2.84 E+00 \\
4.51 E+00\end{array}$ & $\begin{array}{l}6.63 E+00 \\
5.35 E-02 \\
4.03 E-02 \\
3.95 E+00 \\
6.94 E+00\end{array}$ & $\begin{array}{l}5.35 E+00 \\
4.80 E-02 \\
3.58 E-02 \\
2.80 E+00 \\
3.49 E+00\end{array}$ & $\begin{array}{l}4.46 E+00 \\
4.22 E-02 \\
3.13 E-02 \\
2.30 E+00 \\
3.55 E+00\end{array}$ & $\begin{array}{l}4.1 \mathrm{TE}+00 \\
3.7 \mathrm{TE}-02 \\
2.81 E-02 \\
2.21 E+00 \\
3.06 E+00\end{array}$ & $\begin{array}{l}6.16 E+00 \\
5.44 E-02 \\
4.09 E-02 \\
4.04 E+00 \\
7.13 E+00\end{array}$ & & $\begin{array}{l}\text { 4. } 19 E+00 \\
4.32 E-02 \\
3.22 E-02 \\
2.56 E+00 \\
3.91 E+00\end{array}$ \\
\hline 226 & 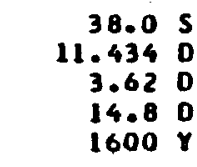 & $\begin{array}{l}7.01 E-01 \\
1.13 E+01 \\
0.48 E-01 \\
1.52 E-01 \\
5.69 E-01\end{array}$ & $\begin{array}{l}8.44 E-01 \\
1.24 E+01 \\
9.21 E-01 \\
0.47 E-01 \\
6.22 E-01\end{array}$ & $\begin{array}{l}1.06 E+00 \\
1.79 E+01 \\
1.24 E+00 \\
1.50 E+00 \\
8.95 E-01\end{array}$ & $\begin{array}{l}9.68 E-01 \\
1.13 E 01 \\
9.28 E-01 \\
3.52 E-01 \\
5.97 E-01\end{array}$ & $\begin{array}{l}7.19 \mathrm{E}-01 \\
1.00 \mathrm{E}+01 \\
7.56 \mathrm{E}-01 \\
6.14 \mathrm{E}-01 \\
4.96 \mathrm{E}-01\end{array}$ & $\begin{array}{l}6.74 E-01 \\
9.25 E+00 \\
7.26 E-01 \\
4.14 E-01 \\
4.79 E-01\end{array}$ & $\begin{array}{l}1.08 E+00 \\
1.84 E+01 \\
1.26 E+00 \\
1.53 E+00 \\
9.17 E-01\end{array}$ & $\begin{array}{l}7.71 E-01 \\
1.11 E+01 \\
6.42 E-01 \\
5.94 E-01 \\
5.65 E-01\end{array}$ & $\begin{array}{l}\text { 7. } 71 E-01 \\
1.111 E+01 \\
0.31 E-01 \\
6.52 E-01 \\
5.61 E-01\end{array}$ \\
\hline 226 & $\begin{array}{r}5.15 Y \\
10.0 \\
21.773 \\
6.13 \\
30.9\end{array}$ & $\begin{array}{l}4.66 E-11 \\
1.13 E+00 \\
9.81 E-03 \\
7.49 E+01 \\
6.51 E-01\end{array}$ & $\begin{array}{l}3.79 E-09 \\
1.25 E+00 \\
1.17 E-02 \\
7.99 E+01 \\
1.20 E-01\end{array}$ & $\begin{array}{l}2.28 E-09 \\
1.97 E+00 \\
1.62 E-02 \\
8.79 E+01 \\
1.10 E+00\end{array}$ & $\begin{array}{l}1.25 E-09 \\
1.05 E+00 \\
9.45 E-03 \\
0.08 E+01 \\
6.37 E-01\end{array}$ & $\begin{array}{l}1.88 E-09 \\
9.74 E-01 \\
8.85 E-03 \\
7.17 E+01 \\
5.67 E-01\end{array}$ & $\begin{array}{l}2.80 E-09 \\
0.98 E-01 \\
8.40 E-03 \\
6.47 E+01 \\
5.32 E-01\end{array}$ & & & $\begin{array}{l}9.78 E-10 \\
1.10 E+00 \\
9.95 E-03 \\
7.38 E+01 \\
6.42 E-01\end{array}$ \\
\hline 231 & $\begin{array}{r}10.718 \\
1.9132 \\
7.3453 \\
7.7 E 4 \\
25.52 \\
25\end{array}$ & $\begin{array}{l}8.05 E \cdot 00 \\
1.64 E-01 \\
7.22 E+00 \\
3.12 E-02 \\
9.36 E-01\end{array}$ & $\begin{array}{l}9.64 E \cdot 00 \\
1.85 E-01 \\
8.02 E+00 \\
3.86 E-02 \\
1.11 E+00\end{array}$ & $\begin{array}{l}1.31 E \bullet 01 \\
2.88 E-01 \\
1.29 E \bullet 01 \\
6.09 E-02 \\
1.84 E \cdot 00\end{array}$ & & $\begin{array}{l}7.91 E+00 \\
1.42 E-01 \\
6.18 E+00 \\
2.82 E-02 \\
8.26 E-01\end{array}$ & & & & $\begin{array}{l}\text { 8. } 70 E+00 \\
1.60 E-01 \\
7.04 E+00 \\
3.09 E-02 \\
9.25 E-01\end{array}$ \\
\hline & $\begin{array}{r}1.405 E 10 Y \\
22.3 \\
24.10 \\
17.4 \\
3.276 E 4\end{array}$ & $\begin{array}{l}1.38 E-02 \\
2.82 E+00 \\
6.63 E-01 \\
5.41 E+01 \\
2.51 E+00\end{array}$ & $\begin{array}{l}1.96 E-0 \\
3.05 E+0 \\
7.41 E-0 \\
5.80 E+0 \\
2.76 E+0\end{array}$ & $\begin{array}{l}2.99 E-02 \\
3.79 E \bullet 00 \\
1.26 E \bullet 00 \\
6.67 E+01 \\
3.64 E+00\end{array}$ & & $\begin{array}{l}1.36 E-02 \\
2.65 E+00 \\
5.58 E-01 \\
5.17 E+01 \\
2.29 E+00\end{array}$ & $\begin{array}{l}1.23 E-02 \\
2.38 E+00 \\
4.80 E-01 \\
4.62 E+01 \\
2.16 E+00\end{array}$ & & & $\begin{array}{l}1.43 E-02 \\
2.38 E+00 \\
6.36 E-01 \\
5.34 E+01 \\
2.49 E+00\end{array}$ \\
\hline & $\begin{array}{rl}27.0 & 0 \\
6.70 & H \\
1.17 & H \\
20.8 & 0 \\
4.2 & 0\end{array}$ & $\begin{array}{l}1.00 E+01 \\
1.60 E+02 \\
9.31 E-01 \\
8.93 E-02 \\
5.68 E+00\end{array}$ & $\begin{array}{l}1.96 E+01 \\
1.71 E+02 \\
9.94 E-01 \\
1.06 E-01 \\
6.39 E+00\end{array}$ & $\begin{array}{l}2.61 E * 01 \\
1.92 E \cdot 02 \\
1.09 E \cdot 00 \\
1.60 E-01 \\
1.05 E \cdot 01\end{array}$ & $\begin{array}{l}1.92 E+01 \\
1.72 E+02 \\
9.98 E-01 \\
0.42 E-02 \\
4.92 E \cdot 00\end{array}$ & $\begin{array}{l}1.63 E+01 \\
1.53 E+02 \\
8.99 E-01 \\
8.05 E-02 \\
4.88 E+00\end{array}$ & $\begin{array}{l}1.52 E+01 \\
1.36 E+02 \\
8.01 E-01 \\
8.47 E-02 \\
4.40 E+00\end{array}$ & & & $\begin{array}{l}\text { 1. } 77 E+01 \\
1.50 E+02 \\
9.20 E-01 \\
8.91 E-02 \\
5.57 E+00\end{array}$ \\
\hline & $\begin{array}{r}72 Y \\
1.592 E 5 Y \\
2.445 E 5 Y \\
7.038 E \theta Y \\
2.3415 E Y Y\end{array}$ & $\begin{array}{l}1.06 E-02 \\
1.81 E-02 \\
9.34 E-03 \\
1.27 E+01 \\
6.66 E-03\end{array}$ & $\begin{array}{l}2.26 E-02 \\
1.74 E-02 \\
1.39 E+01 \\
1.40 E-02\end{array}$ & $\begin{array}{l}4.13 E-02 \\
3.44 E-02 \\
2.38 E-02 \\
2.02 E+01 \\
1.88 E-02\end{array}$ & $\begin{array}{l}1.17 E-02 \\
9.15 E-03 \\
1.33 E+01 \\
6.53 E-03\end{array}$ & $\begin{array}{l}1.68 E-02 \\
8.14 E-02 \\
1.11 E+01 \\
8.82 E-03\end{array}$ & $\begin{array}{l}1.62 E-02 \\
1.11 E-02 \\
1.07 E+01 \\
8.72 E-03\end{array}$ & $\begin{array}{l}4.82 E-02 \\
3.13 E-02 \\
2.95 E-02 \\
2.07 E+01 \\
2.41 E-02\end{array}$ & $\begin{array}{l}2.01 E-02 \\
1.88 E-02 \\
1.09 E-02 \\
1.28 E+01 \\
8.24 E-03\end{array}$ & $\begin{array}{l}2.04 E-02 \\
1.81 E-02 \\
1.11 E-02 \\
1.26 E+01 \\
8.41 E-03\end{array}$ \\
\hline
\end{tabular}




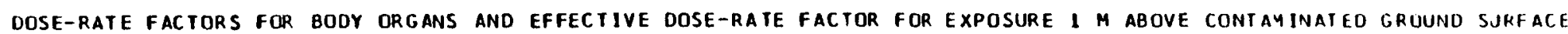

IN MREM/YR PER MICROCUR IE/ISOUARE MI

\begin{tabular}{|c|c|c|c|c|c|c|c|c|c|}
\hline LIDE & IFE & STES & THYMUS & THYROID & UTERUS & FECTIVE & $\begin{array}{c}\text { SKIN } \\
\text { (PHOTON) }\end{array}$ & $\begin{array}{l}\text { SKIN } \\
\text { (ELECTRON I }\end{array}$ & $\begin{array}{l}\text { SKIN } \\
\text { IIOTALI }\end{array}$ \\
\hline $\begin{array}{l}P O-216 \\
P O-218 \\
A T-211 \\
A T-217 \\
R N-218\end{array}$ & 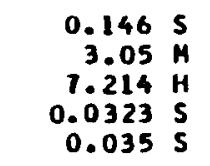 & $\begin{array}{l}1.71 E-03 \\
0.0 \\
5.26 E+00 \\
2.86 E-02 \\
9.07 E-02\end{array}$ & $\begin{array}{l}.36 E-03 \\
.0 \\
.06 E+00 \\
.20 E-02 \\
.99 E-02\end{array}$ & $\begin{array}{l}.63 E-03 \\
.0 \\
16 E+00 \\
.67 E-02 \\
.49 E-02\end{array}$ & $\begin{array}{l}.05 E-03 \\
.0 \\
.62 E+00 \\
.74 E-02 \\
.52 E-02\end{array}$ & $\begin{array}{l}\{7 E-03 \\
54 E+00 \\
54 E-02 \\
75 E-02\end{array}$ & $\begin{array}{l}1.98 E-03 \\
0.0 \\
6.49 E+00 \\
3.28 E-02 \\
1.04 E-01\end{array}$ & $\begin{array}{l}0.0 \\
0.0 \\
0.0 \\
0.0 \\
0.0\end{array}$ & $\begin{array}{l}1.98 E-03 \\
0.0 \\
6.49 E+00 \\
3.28 E-02 \\
1.04 E-01\end{array}$ \\
\hline $\begin{array}{l}R N-219 \\
R N-220 \\
R N-222 \\
F R-221 \\
F R-223\end{array}$ & $\begin{array}{r}3.96 \mathrm{~S} \\
55.61 \\
3.8235 \\
4.8 \mathrm{M} \\
21.8 \mathrm{M}\end{array}$ & $\begin{array}{l}7.12 E+00 \\
6.29 E-02 \\
4.70 E-02 \\
3.92 E+00 \\
7.00 E+00\end{array}$ & $\begin{array}{l}5.38 E+00 \\
4.80 E-02 \\
3.56 E-02 \\
2.99 E+00 \\
5.09 E+00\end{array}$ & $\begin{array}{l}.70 E+00 \\
.85 E-02 \\
0.35 E-02 \\
.17 E+00 \\
.53 E+00\end{array}$ & & & $\begin{array}{l}8.03 E+00 \\
7.21 E-02 \\
5.37 E-02 \\
4.42 E \cdot 00 \\
9.69 E \cdot 00\end{array}$ & $\begin{array}{l}4.26 E-01 \\
0.0 \\
0.0 \\
0.0 \\
2.61 E=02\end{array}$ & $\begin{array}{l}8.46 E+00 \\
7.21 E-02 \\
5.37 E-02 \\
4.42 E+00 \\
2.77 E+02\end{array}$ \\
\hline $\begin{array}{l}R A-222 \\
R A-223 \\
R A-224 \\
R A-225 \\
R A-226\end{array}$ & 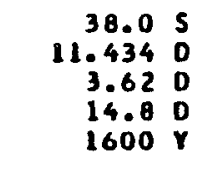 & $\begin{array}{l}1.14 E+00 \\
1.74 E \cdot 01 \\
1.26 E \cdot 00 \\
1.94 E+00 \\
8.63 E-01\end{array}$ & $\begin{array}{l}8.63 E-01 \\
1.33 E+01 \\
9.5 T E-01 \\
1.19 E+00 \\
6.59 E-01\end{array}$ & $\begin{array}{l}.07 E+00 \\
1.68 E+01 \\
1.20 E+00 \\
1.58 E+00 \\
.37 E-01\end{array}$ & & & & & $\begin{array}{l}1.29 E+00 \\
2.03 E+01 \\
1.41 E+00 \\
3.42 E+00 \\
9.7 T E-01\end{array}$ \\
\hline $\begin{array}{l}R A-228 \\
A C-225 \\
A C-227 \\
A C-228 \\
T H-226\end{array}$ & 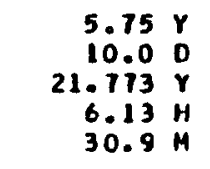 & $\begin{array}{l}3.48 E-08 \\
1.85 E+00 \\
2.18 E-02 \\
1.06 E+02 \\
1.04 E+00\end{array}$ & $\begin{array}{l}8.02 E-13 \\
1.37 E+00 \\
1.19 E-02 \\
8.38 E+01\end{array}$ & $\begin{array}{l}2.40 E-09 \\
1.76 E+00 \\
1.63 E-02 \\
1.01 E+02 \\
9.95 E-01\end{array}$ & & & & $\begin{array}{l}0.0 \\
0.0 \\
0.0 \\
3.07 E+02 \\
0.0\end{array}$ & \\
\hline $\begin{array}{l}T H-227 \\
T H-228 \\
T H-229 \\
T H-230 \\
T H-231\end{array}$ & $\begin{array}{r}18.718 \mathrm{D} \\
1.9132 \text { Y } \\
7.34 E 3 \\
i .7 E 4 \\
25.52 \mathrm{H}\end{array}$ & $\begin{array}{l}1.35 E+01 \\
2.97 E-01 \\
1.19 E+01\end{array}$ & & & & & & & \\
\hline $\begin{array}{l}T H-232 \\
T H-233 \\
T H-234 \\
P A-230 \\
P A-231\end{array}$ & $\begin{array}{r}1.405 E 10 Y \\
22.3 \\
24.10 \\
17.4 \\
17.0 \\
3.276 E 4 Y\end{array}$ & $\begin{array}{l}5.86 E-02 \\
4.24 E+00 \\
1.16 E+00 \\
7.79 E+01 \\
4.06 E+00\end{array}$ & $\begin{array}{l}1.80 E-02 \\
3.17 E+00 \\
8.52 E-01 \\
6.12 E+01 \\
2.83 E+00\end{array}$ & $\begin{array}{l}2.73 E-02 \\
3.93 E+00 \\
1.10 E+00 \\
7.42 E+01 \\
3.62 E \cdot 00\end{array}$ & $\begin{array}{l}1.08 E-02 \\
2.44 E+00 \\
5.43 E-01 \\
4.70 E+01 \\
2.19 E+00\end{array}$ & & $\begin{array}{l}4.85 \\
5.37 \\
1.71 \\
9.25 \\
6.94\end{array}$ & $\begin{array}{l}0.0 \\
3.441 \\
0.0 \\
4.401 \\
0.0\end{array}$ & $\begin{array}{l}4.85 E-01 \\
3.49 E+02 \\
1.77 E+00 \\
9.69 E+01 \\
6.94 E+00\end{array}$ \\
\hline $\begin{array}{l}P A-233 \\
P A-234 \\
P A-234 H \\
U-230 \\
U-231\end{array}$ & $\begin{array}{rll}27.0 & 0 \\
6.70 & H \\
1.17 & H \\
20.8 & 0 \\
4.2 & 0\end{array}$ & $\begin{array}{l}2.71 E \\
2.28 E \\
1.32 E \\
2.04 E \\
9.87 E\end{array}$ & $\begin{array}{l}2.03 E \\
1.80 \\
1.05 \\
1.08 \\
7.08 E\end{array}$ & $\begin{array}{l}2.17 \mathrm{E} \\
1.26 \mathrm{E} \\
1.46 \mathrm{E} \\
9.26 \mathrm{E}\end{array}$ & $\begin{array}{l}1.58 E+01 \\
1.40 E+02 \\
8.11 E-01 \\
7.56 E-02 \\
4.79 E+00\end{array}$ & $\begin{array}{l}2.36 E+01 \\
1.96 E+02 \\
1.13 E+00 \\
2.00 E-01 \\
8.71 E+00\end{array}$ & $\begin{array}{l}3.28 E * 01 \\
2.68 E+02 \\
1.55 E+00 \\
8.72 E-01 \\
1.62 E+01\end{array}$ & $\begin{array}{l}0.0 \\
1.52 E+02 \\
8.72 E+02 \\
0.0 \\
0.0\end{array}$ & $\begin{array}{l}3.28 E+01 \\
4.20 E+02 \\
8.74 E+02 \\
0.72 E-01 \\
1.62 E+01\end{array}$ \\
\hline $\begin{array}{l}U-232 \\
U-233 \\
U-234 \\
U-235 \\
U-236\end{array}$ & $\begin{array}{r}72 Y \\
1.592 E 5 Y \\
2.445 E 5 Y \\
7.038 E 8 Y \\
2.3415 E Y Y\end{array}$ & $\begin{array}{l}4.87 E-02 \\
7.07 E-02 \\
1.94 E+01 \\
6.30 E-02\end{array}$ & $\begin{array}{l}2.18 E-02 \\
1.18 E-02 \\
1.48 E+01 \\
8.45 E-03\end{array}$ & $\begin{array}{l}3.89 E-02 \\
3.10 E-02 \\
2.31 E-02 \\
1.88 E+01 \\
1.81 E-02\end{array}$ & $\begin{array}{l}1.47 \mathrm{E}-02 \\
1.55 \mathrm{E}-02 \\
7.04 \mathrm{E}-03 \\
1.12 \mathrm{E}+01 \\
4.98 \mathrm{E}-03\end{array}$ & $\begin{array}{l}1.03 E-01 \\
5.00 E-02 \\
8.07 E-02 \\
1.71 E+01 \\
1.33 E-02\end{array}$ & $\begin{array}{l}7.42 E-01 \\
2.61 E-01 \\
6.36 E-01 \\
2.27 E+01 \\
5.99 E-01\end{array}$ & $\begin{array}{l}0.0 \\
0.0 \\
0.0 \\
0.0 \\
0.0\end{array}$ & $\begin{array}{l}1.42 E-01 \\
2.61 E-01 \\
6.36 E-01 \\
2.27 E+01 \\
5.99 E-01\end{array}$ \\
\hline
\end{tabular}




\begin{tabular}{|c|c|c|c|c|c|c|c|c|c|c|}
\hline IUCL IDE & HALF-LIFE & AORENALS & BLADDER & BRAIN & BREAST & HE AR T & $\begin{array}{c}\text { SMALL } \\
\text { INTESTINE }\end{array}$ & $\begin{array}{c}\text { UPPER } \\
\text { LARGE } \\
\text { INIESTIVE }\end{array}$ & $\begin{array}{c}\text { LOWER } \\
\text { LARGE } \\
\text { INTESTINE }\end{array}$ & K IONEYS \\
\hline $\begin{array}{l}U-237 \\
U-238 \\
U-239 \\
U-240 \\
N P-235\end{array}$ & $\begin{array}{r}6.75 \\
4.46869 \\
23.40 \mathrm{H} \\
14.1 \mathrm{H} \\
396.1 \mathrm{O}\end{array}$ & $\begin{array}{l}1.19 E+01 \\
1.16 E-02 \\
3.88 E+00 \\
8.10 E-02 \\
1.88 E-01\end{array}$ & $\begin{array}{l}.14 E \cdot 01 \\
.45 E-03 \\
.04 E \cdot 00 \\
.34 E-02 \\
.49 E-01\end{array}$ & $\begin{array}{l}19 E+01 \\
0 E-03 \\
20 E+00 \\
0 E-02 \\
0 E-01\end{array}$ & $\begin{array}{l}2.31 E+01 \\
2.97 E-01 \\
7.99 E+00 \\
1.60 E+00 \\
1.59 E+00\end{array}$ & & & $\begin{array}{l}1.16 E+01 \\
5.03 E-03 \\
3.92 E \cdot 00 \\
4.25 E-02 \\
1.55 E-01\end{array}$ & $\begin{array}{l}1.06 E+01 \\
7.90 E-03 \\
3.52 E \cdot 00 \\
5.71 E-02 \\
1.56 E-01\end{array}$ & $\begin{array}{l}1.18 E+01 \\
5.85 E-03 \\
4.21 E+00 \\
6.67 E-02 \\
1.53 E-01\end{array}$ \\
\hline $\begin{array}{l}N P-236 \\
N P-236 M \\
N P-237 \\
N P-238 \\
N P-239\end{array}$ & $\begin{array}{rl}1.15 E 6 & Y \\
22.5 & H \\
2.14 E 6 & Y \\
2.117 & D \\
2.355 & D\end{array}$ & $\begin{array}{l}1.15 E+01 \\
4.44 E+00 \\
2.01 E+00 \\
4.59 E+01 \\
1.51 E+01\end{array}$ & $\begin{array}{l}1.08 E+01 \\
4.25 E+00 \\
1.96 E+00 \\
4.48 E+01 \\
1.40 E+01\end{array}$ & $\begin{array}{l}1.14 E+01 \\
4.52 E+00 \\
1.95 E+00 \\
5.04 E+01 \\
1.50 E+01\end{array}$ & $\begin{array}{l}2.43 E+01 \\
8.41 E+00 \\
6.13 E+00 \\
5.74 E+01 \\
2.57 E+01\end{array}$ & $\begin{array}{l}1.05 E+01 \\
4.11 E+00 \\
1.79 E+00 \\
4.43 E+01 \\
1.36 E+01\end{array}$ & $\begin{array}{l}9.11 E+00 \\
3.59 E+00 \\
1.57 E+00 \\
4.03 E+01 \\
1.20 E \bullet 01\end{array}$ & $\begin{array}{l}1.13 E+01 \\
4.42 E+00 \\
1.97 E+00 \\
4.71 E+01 \\
1.46 E+01\end{array}$ & $\begin{array}{l}1.03 E+01 \\
3.99 E+00 \\
1.77 E+00 \\
4.33 E+01 \\
1.34 E+01\end{array}$ & $\begin{array}{l}1.10 E+01 \\
4.35 E+00 \\
2.111 E+00 \\
4.70 E+01 \\
1.44 E+01\end{array}$ \\
\hline $\begin{array}{l}N P-240 \\
N P-240 M \\
P U-236 \\
P U-237 \\
P U-238\end{array}$ & $\begin{array}{r}65 \mathrm{M} \\
7.4 \mathrm{M} \\
2.851 \mathrm{Y} \\
45.3 \mathrm{D} \\
07.75 \mathrm{Y}\end{array}$ & $\begin{array}{l}9.95 E+01 \\
2.84 E+01 \\
1.55 E-02 \\
4.18 E+00 \\
1.23 E-02\end{array}$ & $\begin{array}{l}9.55 E+01 \\
2.72 E+01 \\
4.27 E-03 \\
3.99 E+00 \\
2.12 E-03\end{array}$ & $\begin{array}{l}1.07 E+02 \\
3.05 E+01 \\
5.43 E-03 \\
4.16 E+00 \\
3.17 E-03\end{array}$ & $\begin{array}{l}1.30 E+02 \\
3.66 E+01 \\
4.62 E-01 \\
9.36 E+00 \\
4.07 E-01\end{array}$ & $\begin{array}{l}9.40 E+01 \\
2.68 E+01 \\
5.66 E-03 \\
3.83 E+00 \\
3.56 E-03\end{array}$ & $\begin{array}{l}0.49 E \cdot 01 \\
2.42 E+01 \\
3.43 E-03 \\
3.31 E \cdot 00 \\
1.72 E-03\end{array}$ & $\begin{array}{l}9.99 E+01 \\
2.84 E+01 \\
3.85 E-03 \\
4.14 E+00 \\
1.86 E-03\end{array}$ & & $\begin{array}{l}9.96 E+01 \\
2.84 E+01 \\
4.95 E-03 \\
4.07 E+00 \\
2.67 E-03\end{array}$ \\
\hline $\begin{array}{l}P U-239 \\
P U-240 \\
P U-241 \\
P U-242 \\
P U-243\end{array}$ & $\begin{array}{r}24131 Y \\
6537 Y \\
14.4 Y \\
3.75855 Y \\
4.956 \mathrm{H}\end{array}$ & $\begin{array}{l}\text { 8.91E-03 } \\
1.19 E-02 \\
0.0 \\
1.00 E-02 \\
2.00 E+00\end{array}$ & $\begin{array}{l}4.64 E-03 \\
2.30 E-03 \\
0.0 \\
2.04 E-03 \\
2.00 E \cdot 00\end{array}$ & $\begin{array}{l}5.42 E-03 \\
3.23 E-03 \\
0.0 \\
2.84 E-03 \\
2.06 E+00\end{array}$ & $\begin{array}{l}1.62 E-01 \\
3.8 B E-01 \\
0.0 \\
3.22 E-01 \\
4.21 E+00\end{array}$ & $\begin{array}{l}5.26 E-03 \\
3.59 E-03 \\
0.0 \\
3.12 E-03 \\
1.87 E+00\end{array}$ & $\begin{array}{l}3.98 E-03 \\
1.83 E-03 \\
0.0 \\
1.64 E-03 \\
1.64 E+00\end{array}$ & $\begin{array}{l}4.90 E-03 \\
2.01 E-03 \\
0.0 \\
1.00 E-03 \\
2.00 E-00\end{array}$ & $\begin{array}{l}6.53 E-03 \\
7.07 E-03 \\
0.0 \\
5.99 E-03 \\
1.79 E+00\end{array}$ & $\begin{array}{l}\text { 4. } 84 E-03 \\
2.86 E-03 \\
0.0 \\
2.50 E-03 \\
2.05 E+00\end{array}$ \\
\hline $\begin{array}{l}P U-244 \\
P U-245 \\
P U-246 \\
A M-241 \\
A M-242\end{array}$ & $\begin{array}{r}8.26 E 7 \mathrm{Y} \\
10.57 \mathrm{H} \\
10.85 \mathrm{D} \\
432.2 \mathrm{Y} \\
16.02 \mathrm{H}\end{array}$ & $\begin{array}{l}7.84 E-03 \\
3.69 E+01 \\
8.78 E+00 \\
1.63 E+00 \\
1.21 E+00\end{array}$ & $\begin{array}{l}9.18 E-04 \\
3.50 E+01 \\
8.35 E+00 \\
1.83 E+00 \\
1.12 E+00\end{array}$ & $\begin{array}{l}1.50 E-03 \\
3.88 E+01 \\
8.52 E+00 \\
1.67 E+00 \\
1.19 E+00\end{array}$ & $\begin{array}{l}2.78 E-01 \\
4.04 E+01 \\
1.41 E+01 \\
5.55 E+00 \\
3.30 E+00\end{array}$ & $\begin{array}{l}1.86 E-03 \\
3.44 E+01 \\
7.75 E+00 \\
1.49 E+00 \\
1.10 E+00\end{array}$ & $\begin{array}{l}6.90 E-04 \\
3.09 E \cdot 01 \\
7.01 E+00 \\
1.37 E+00 \\
9.40 E-01\end{array}$ & $\begin{array}{l}7.43 E-04 \\
3.65 E+01 \\
8.39 E+00 \\
1.63 E+00 \\
1.18 E+00\end{array}$ & $\begin{array}{l}4.43 E-03 \\
3.37 E+01 \\
7.76 E+00 \\
1.45 E+00 \\
1.07 E+00\end{array}$ & $\begin{array}{l}\text { 1. } 35 E-03 \\
3.64 E+01 \\
8.28 E+00 \\
1.98 E+00 \\
1015 E+00\end{array}$ \\
\hline $\begin{array}{l}A M-242 M \\
A M-243 \\
A M-244 \\
A M-245 \\
A M-246\end{array}$ & $\begin{array}{r}152 Y \\
7.38 E 3 Y \\
10.1 \mathrm{H} \\
122.4 \mathrm{H} \\
25.0 \mathrm{H}\end{array}$ & $\begin{array}{l}5.14 E-02 \\
4.22 E+00 \\
6.96 E+01 \\
2.83 E+00 \\
8.09 E+01\end{array}$ & $\begin{array}{l}6.69 E \\
2.62 E \\
7.89 E\end{array}$ & $\begin{array}{l}2.12 E-02 \\
4.41 E+00 \\
7.49 E+01 \\
2.80 E+00 \\
8.88 E+01\end{array}$ & $\begin{array}{l}1.04 E+01 \\
9.10 E \cdot 01 \\
4.74 E+00 \\
1.01 E \bullet 02\end{array}$ & $\begin{array}{l}3.96 \mathrm{EH} \\
6.59 \mathrm{EH} \\
2.55 \mathrm{EH} \\
7.80 \mathrm{E}+\mathrm{C}\end{array}$ & & & & \\
\hline $\begin{array}{l}C M-242 \\
C M-243 \\
C H-244 \\
C M-245 \\
C M-246\end{array}$ & $\begin{array}{r}163.2 \mathrm{O} \\
28.5 \mathrm{Y} \\
18.11 \mathrm{Y} \\
8.5 E 3 \mathrm{Y} \\
4.75 E 3 \mathrm{~V}\end{array}$ & $\begin{array}{l}1.42 E-02 \\
1.15 E+01 \\
1.23 E-02 \\
6.26 E+00 \\
1.06 E-02\end{array}$ & $\begin{array}{l}1.06 E+01 \\
1.34 E-03 \\
5.85 E+00 \\
9.22 E-04\end{array}$ & $\begin{array}{l}3.47 E-03 \\
1.14 E+01 \\
2.71 E-03 \\
6.19 E+00 \\
1.97 E-03\end{array}$ & $\begin{array}{l}4.30 E-01 \\
1.98 E+01 \\
3.83 E-01 \\
1.29 E+01 \\
3.41 E-01\end{array}$ & $\begin{array}{l}4.02 E-03 \\
1.03 E+01 \\
3.24 E-03 \\
5.70 E+00 \\
2.50 E-03\end{array}$ & $\begin{array}{l}9.12 E+00 \\
1.25 E-03 \\
4.93 E+00 \\
8.12 E-04\end{array}$ & $\begin{array}{l}1.10 E+01 \\
1.21 E-03 \\
6.13 E+00 \\
7.64 E-04\end{array}$ & & \\
\hline $\begin{array}{l}C M-247 \\
C H-248 \\
C M-249 \\
C M-250 \\
\theta K-249\end{array}$ & $\begin{array}{r}1.56 E 7 \mathrm{Y} \\
3.39 E 5 \mathrm{Y} \\
64.15 \mathrm{M} \\
6.9 E 3 \mathrm{Y} \\
320 \mathrm{D}\end{array}$ & $\begin{array}{l}2.08 E+01 \\
8.90 E-03 \\
1.67 E+00 \\
0.0 \\
0.0\end{array}$ & $\begin{array}{l}1.22 E-03 \\
1.59 E \cdot 00 \\
0.0 \\
0.0\end{array}$ & $\begin{array}{l}2.14 E-03 \\
1.77 E+00 \\
0.0 \\
0.0\end{array}$ & $\begin{array}{l}3.77 E+01 \\
2.71 E-01 \\
2.11 E \cdot 00 \\
0.0 \\
0.0\end{array}$ & $\begin{array}{l}2.63 E+01 \\
2.49 E-03 \\
1.56 E+00 \\
0.0 \\
0.0\end{array}$ & $\begin{array}{l}1.08 E-03 \\
1.40 E+00 \\
0.0 \\
0.0\end{array}$ & $\begin{array}{l}\text { 2. } 78 E+01 \\
1.00 E-03 \\
1.65 E+00 \\
0.0 \\
0.0\end{array}$ & $\begin{array}{l}2.59 E+01 \\
5.31 E-03 \\
1.53 E+00 \\
0.0 \\
0.0\end{array}$ & $\begin{array}{l}\text { 2. } 78 E+01 \\
1.76 E-03 \\
1.65 E+00 \\
0.0 \\
0.0\end{array}$ \\
\hline
\end{tabular}




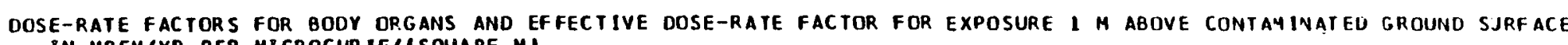

\begin{tabular}{|c|c|c|c|c|c|c|c|c|c|c|}
\hline$L 10 E$ & LF-LIFE & LIVER & LUNGS & MARROH & $\begin{array}{c}\text { RED } \\
\text { MARROW }\end{array}$ & OVARIES & PANCREAS & SKELETON & SPLEEN & STOMACH \\
\hline $\begin{array}{l}U-237 \\
U-238 \\
U-239 \\
U-240 \\
N P-235\end{array}$ & $\begin{array}{r}6.75 \\
.468 E 9 \\
23.40 \mathrm{Y} \\
14.1 \mathrm{H} \\
396.1 \mathrm{D}\end{array}$ & $\begin{array}{l}1.14 E+01 \\
5.67 E-03 \\
4.00 E+00 \\
4.87 E-02 \\
1.50 E-01\end{array}$ & $\begin{array}{l}.26 E+01 \\
1.21 E-02 \\
4.39 E+00 \\
9.17 E-02 \\
1.97 E-01\end{array}$ & $\begin{array}{l}95 E+01 \\
63 E-02 \\
11 E+00 \\
33 E-01 \\
04 E-01\end{array}$ & & $\begin{array}{l}.80 E+00 \\
.82 E-03 \\
.42 E+00 \\
.88 E-02 \\
.44 E-01\end{array}$ & & & & $\begin{array}{l}1 E+01 \\
2 E-03 \\
9 E+00 \\
1 E-02 \\
T E-01\end{array}$ \\
\hline $\begin{array}{l}N P-2 \\
N P-2 \\
N P-2 \\
N P-2\end{array}$ & $\begin{array}{rl}1.15 E 6 & Y \\
22.5 & H \\
2.14 E 6 & Y \\
2.117 & 0 \\
2.355 & D\end{array}$ & $\begin{array}{l}4.2 \\
1.9 \\
4.49 \\
1.40\end{array}$ & $\begin{array}{l}1 \\
0 \\
0 \\
1 \\
1\end{array}$ & & & & & & & $\begin{array}{l}6 E+01 \\
6 E+00 \\
7 E+00 \\
0 E+01 \\
B E+01\end{array}$ \\
\hline $\begin{array}{l}N P-240 \\
N P-240 H \\
P U-236 \\
P U-237 \\
P U-238\end{array}$ & $\begin{array}{rr}65 & M \\
7.4 & M \\
2.851 & Y \\
45.3 & 0 \\
87.75 & Y\end{array}$ & $\begin{array}{l}9.50 E+01 \\
2.71 E+01 \\
4.84 E-03 \\
3.91 E+00 \\
2.63 E-03\end{array}$ & $\begin{array}{l}.02 E+02 \\
.90 E+01 \\
.59 E-02 \\
.45 E+00\end{array}$ & $\begin{array}{l}.15 E+02 \\
.24 E+01 \\
.93 E-02 \\
.32 E+00\end{array}$ & & & & & & \\
\hline $\begin{array}{l}P U-239 \\
P U-240 \\
P U-241 \\
O U-247\end{array}$ & $\begin{array}{r}24131 Y \\
6537 Y \\
14.4 Y \\
.758 E 5 Y \\
4.956 \mathrm{H}\end{array}$ & $\begin{array}{l}4.85 E-03 \\
2.77 E-03 \\
0.0 \\
2.44 E-03 \\
1.99 E+00\end{array}$ & $\begin{array}{l}.94 E-03 \\
1.19 E-02 \\
0.0 \\
.01 E-02 \\
.21 E+00\end{array}$ & $\begin{array}{l}.20 E-02 \\
.37 E-02 \\
.0 \\
.17 E-02\end{array}$ & & $\begin{array}{l}6.00 E-03 \\
6.84 E-03 \\
0.0 \\
5.79 E-03 \\
1.69 E+00\end{array}$ & $\begin{array}{l}6.13 E-03 \\
7.40 E-03 \\
0.0 \\
6.23 E-03 \\
1.47 E+00\end{array}$ & $\begin{array}{l}1.47 E-02 \\
2.00 E-02 \\
0.0 \\
1.69 E-02 \\
3.76 E+00\end{array}$ & $\begin{array}{l}0.02 E-03 \\
.07 E-03 \\
0.0 \\
0.67 E-03 \\
.90 E+00\end{array}$ & $\begin{array}{l}.02 E-05 \\
.03 E-03 \\
.0 \\
0.81 E-03 \\
.91 E+00\end{array}$ \\
\hline $\begin{array}{l}P U-244 \\
P U-245 \\
P U-246 \\
A H-241 \\
A H-242\end{array}$ & 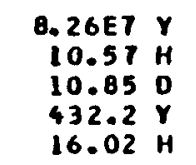 & $\begin{array}{l}1.20 \\
3.48 \\
8.17\end{array}$ & $\begin{array}{l}3 \\
1 \\
0 \\
0\end{array}$ & $\begin{array}{l}3 \\
1 \\
1 \\
0 \\
0\end{array}$ & & $\begin{array}{l}4.21 \\
3.31 \\
7.1 \\
1.4 \\
9.7\end{array}$ & $\begin{array}{l}2.9 \\
6.7 \\
1.1 \\
9.0\end{array}$ & & & \\
\hline $\begin{array}{l}A M-242 M \\
A M-243 \\
A M-244 \\
A M-245 \\
A M-246\end{array}$ & $\begin{array}{r}152 \mathrm{Y} \\
7.38 E 3 \mathrm{Y} \\
10.1 \mathrm{H} \\
122.4 \mathrm{H} \\
25.0 \mathrm{M}\end{array}$ & $\begin{array}{l}2.531 \\
4.411 \\
6.651 \\
2.611 \\
7.851\end{array}$ & $\begin{array}{l}2 \\
0 \\
1 \\
0\end{array}$ & $\begin{array}{l}7.90 E-02 \\
8.55 E+00 \\
7.94 E+01 \\
4.16 E+00 \\
8.98 E+01\end{array}$ & $\begin{array}{l}2 \\
0 \\
1 \\
0\end{array}$ & & $\begin{array}{l}3 . \\
5 . \\
2 .\end{array}$ & & & \\
\hline $\begin{array}{l}C M-242 \\
C M-243 \\
C M-244 \\
C M-245 \\
C M-246\end{array}$ & $\begin{array}{r}163.20 \\
28.5 Y \\
18.11 Y \\
B .5 E 3 Y \\
4.75 E 3 Y\end{array}$ & $\begin{array}{l}2.82 E- \\
1.06 E \\
2.12 E- \\
5.83 \mathrm{EE} \\
1.53 \mathrm{E}-\end{array}$ & $\begin{array}{l}1.43 E-02 \\
1.17 E+01 \\
1.24 E-02 \\
6.52 E \cdot 00 \\
1.06 E-02\end{array}$ & $\begin{array}{l}1.65 E-02 \\
1.69 E+01 \\
1.40 E-02 \\
1.03 E+01 \\
1.17 E-02\end{array}$ & & & & & & \\
\hline $\begin{array}{l}C M-247 \\
C M-248 \\
C M-249 \\
C M-250 \\
8 K-249\end{array}$ & $\begin{array}{r}1.56 E T Y \\
3.39 E 5 Y \\
64.15 Y \\
6.9 E 3 Y \\
320 \mathrm{O}\end{array}$ & $\begin{array}{l}2.68 E+01 \\
1.75 E-03 \\
1.58 E+00 \\
0.0 \\
0.0\end{array}$ & $\begin{array}{l}2.89 E+01 \\
9.01 E-03 \\
1.09 E+00 \\
0.0 \\
0.0\end{array}$ & $\begin{array}{l}3.49 E+01 \\
1.04 E-02 \\
1.93 E+00 \\
0.0 \\
0.0\end{array}$ & $\begin{array}{l}2.97 E+01 \\
3.26 E-03 \\
1.73 E+00 \\
0.0 \\
0.0\end{array}$ & $\begin{array}{l}2.51 E+01 \\
5.14 E-03 \\
1.52 E+00 \\
0.0 \\
0.0\end{array}$ & $\begin{array}{l}2.31 E+01 \\
5.52 E-03 \\
1.36 E \cdot 00 \\
0.0 \\
0.0\end{array}$ & $\begin{array}{l}1.50 E-02 \\
1.97 E+00 \\
0.0 \\
0.0\end{array}$ & $\begin{array}{l}2.63 E+01 \\
4.04 E-03 \\
1.54 E+00 \\
0.0 \\
0.0\end{array}$ & $\begin{array}{l}1.56 \\
0.0 \\
0.0\end{array}$ \\
\hline
\end{tabular}


DOSE-RATE FACTORS FOR BODY ORGANS AND EFFECTIVE DOSE-RATE FACTOR FOR EXPOSURE I M ABOVE CONTAMINATEO GROUND SURFACE
IN MREM/YR PER MICROCUR IE/ISOUARE MI

\begin{tabular}{|c|c|c|c|c|c|c|c|c|c|}
\hline NUCL IOE & HALF-LIFE & TESTES & THYMUS & THYROIO & UTERUS & EFFECTIVE & $\begin{array}{c}\text { SKIN } \\
\text { (PHOTON) }\end{array}$ & $\begin{array}{c}\text { SKIN } \\
\text { IELECTRON }\end{array}$ & $\begin{array}{l}\text { SKIN } \\
\text { ITOTALI }\end{array}$ \\
\hline $\begin{array}{l}U-237 \\
U-238 \\
U-239 \\
U-240 \\
N P-235\end{array}$ & $\begin{array}{r}6.75 \\
4.46859 \\
23.40 \mathrm{H} \\
14.1 \mathrm{H} \\
396.1 \mathrm{D}\end{array}$ & $\begin{array}{l}1.85 E+01 \\
5.54 E-02 \\
6.64 E+00 \\
3.91 E-01 \\
4.71 E-01\end{array}$ & $\begin{array}{l}1.38 E \bullet 01 \\
7.23 E-03 \\
5.09 E+00 \\
7.02 E-02 \\
1.84 E-01\end{array}$ & $\begin{array}{l}1.77 E+01 \\
1.57 E-02 \\
6.43 E+00 \\
1.39 E-01 \\
2.75 E-01\end{array}$ & $\begin{array}{l}9.65 E \bullet 00 \\
4.20 E-03 \\
3.27 E \bullet 00 \\
3.06 E-02 \\
1.26 E-01\end{array}$ & $\begin{array}{l}1.61 E+01 \\
6.46 E-02 \\
5.65 E+00 \\
3.98 E-01 \\
4.74 E-01\end{array}$ & $\begin{array}{l}2.42 E+01 \\
5.30 E-01 \\
B .04 E \cdot 00 \\
2.83 E \cdot 00 \\
2.57 E+00\end{array}$ & $\begin{array}{l}0.0 \\
0.0 \\
3.41 E+02 \\
3.69 E+00 \\
0.0\end{array}$ & $\begin{array}{l}2.42 E+01 \\
5.30 E-01 \\
3.49 E+02 \\
6.51 E+00 \\
2.57 E+00\end{array}$ \\
\hline $\begin{array}{l}N P-236 \\
N P-236 M \\
N P-237 \\
N P-238 \\
N P-239\end{array}$ & 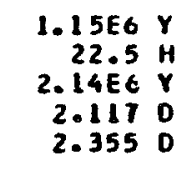 & $\begin{array}{l}1.78 E+01 \\
6.81 E+00 \\
3.71 E+00 \\
6.28 E+01 \\
2.19 E+01\end{array}$ & $\begin{array}{l}1.30 E \bullet 01 \\
5.12 E+00 \\
2.42 E \bullet 00 \\
5.01 E \bullet 01 \\
1.63 E \bullet 01\end{array}$ & $\begin{array}{l}1.69 E+01 \\
6.56 E+00 \\
3.25 E+00 \\
5.98 E+01 \\
2.09 E+01\end{array}$ & $\begin{array}{l}9.28 E+00 \\
3.61 E+00 \\
1.57 E+00 \\
3.89 E+01 \\
1.21 E+01\end{array}$ & $\begin{array}{l}1.58 E+01 \\
5.96 E+00 \\
3.24 E+00 \\
5.38 E+01 \\
1.92 E+01\end{array}$ & $\begin{array}{l}2.68 E+01 \\
8.98 E+00 \\
7.54 E+00 \\
7.47 E+01 \\
2.72 E+01\end{array}$ & $\begin{array}{l}0.0 \\
1.22 E+01 \\
0.0 \\
1.67 E+02 \\
5.78 E+00\end{array}$ & $\begin{array}{l}2.68 E+01 \\
2.12 E+01 \\
7.54 E+00 \\
2.41 E+02 \\
3.30 E+01\end{array}$ \\
\hline $\begin{array}{l}N P-240 \\
N P-240 M \\
P U-236 \\
P U-237 \\
P U-238\end{array}$ & $\begin{array}{r}65 \mathrm{H} \\
7.4 \mathrm{M} \\
2.851 \mathrm{Y} \\
45.3 \mathrm{D} \\
67.75 \mathrm{Y}\end{array}$ & $\begin{array}{l}1.36 E+02 \\
3.8 B E+01 \\
0.82 E-02 \\
6.74 E+00 \\
7.55 E-02\end{array}$ & $\begin{array}{l}1.06 E+02 \\
2.99 E+01 \\
5.78 E-03 \\
4.92 E+00 \\
2.92 E-03\end{array}$ & $\begin{array}{l}8.29 E \cdot 02 \\
3.62 E \cdot 01 \\
2.01 E-02 \\
6.42 E \cdot 00 \\
1.51 E-02\end{array}$ & $\begin{array}{l}0.29 E+01 \\
2.37 E+01 \\
3.15 E-03 \\
3.36 E+00 \\
1.47 E-03\end{array}$ & $\begin{array}{l}1.17 E \cdot 02 \\
3.33 E+01 \\
9.92 E-02 \\
5.95 E+00 \\
0.58 E-02\end{array}$ & $\begin{array}{l}1.62 E \cdot 02 \\
4.64 E+01 \\
8.11 E-01 \\
1.01 E+01 \\
7.1 \mathrm{TE}-01\end{array}$ & $\begin{array}{l}1.48 E \cdot 02 \\
5.92 E \cdot 02 \\
0.0 \\
0.0 \\
0.0\end{array}$ & $\begin{array}{l}3.11 E+02 \\
6.39 E+02 \\
0.11 E-01 \\
1.01 E+01 \\
7.17 E-01\end{array}$ \\
\hline $\begin{array}{l}P U-239 \\
P U-240 \\
P U-241 \\
P U-242 \\
P U-243\end{array}$ & $\begin{array}{r}24131 Y \\
6537 Y \\
14.4 Y \\
3.758 E 5 Y \\
4.956 H\end{array}$ & $\begin{array}{l}3.46 E-02 \\
7.24 E-02 \\
0.0 \\
6.02 E-02 \\
3.32 E+00\end{array}$ & $\begin{array}{l}5.67 E-03 \\
3.15 E-03 \\
0.0 \\
2.79 E-03 \\
2.51 E+00\end{array}$ & $\begin{array}{l}1.16 E-02 \\
1.49 E-02 \\
0.0 \\
1.25 E-02 \\
3.22 E+00\end{array}$ & $\begin{array}{l}3.99 E-03 \\
1.58 E-03 \\
0.0 \\
1.44 E-03 \\
1.66 E \bullet 00\end{array}$ & $\begin{array}{l}3.78 E-02 \\
8.20 E-02 \\
0.0 \\
6.82 E-02 \\
2.87 E+00\end{array}$ & $\begin{array}{l}2.80 E-01 \\
6.83 E-01 \\
0.0 \\
5.67 E-01 \\
4.24 E+00\end{array}$ & $\begin{array}{l}0.0 \\
0.0 \\
0.0 \\
0.0 \\
3.08 E+01\end{array}$ & $\begin{array}{l}2.80 E-01 \\
6.83 E-01 \\
0.0 \\
5.67 E-01 \\
3.51 E+01\end{array}$ \\
\hline $\begin{array}{l}P U-244 \\
P U-245 \\
P U-246 \\
A M-241 \\
A M-242\end{array}$ & $\begin{array}{r}8.26 E 7 Y \\
10.57 \mathrm{H} \\
10.85 \mathrm{O} \\
432.2 Y \\
16.02 \mathrm{H}\end{array}$ & $\begin{array}{l}5.09 E-02 \\
5.04 E+01 \\
1.29 E \bullet 01 \\
3.54 E+00 \\
2.04 E \bullet 00\end{array}$ & $\begin{array}{l}1.26 E-03 \\
3.90 E+01 \\
9.61 E+00 \\
2.43 E+00 \\
1.38 E+00\end{array}$ & $\begin{array}{l}9.39 E-03 \\
4.76 E+01 \\
1.22 E+01 \\
3.17 E+00 \\
1.83 E+00\end{array}$ & $\begin{array}{l}5.24 E-04 \\
3.04 E+01 \\
7.04 E+00 \\
1.33 E+00 \\
9.55 E-01\end{array}$ & $\begin{array}{l}5.80 E-02 \\
4.34 E+01 \\
1.11 E+01 \\
2.99 E+00 \\
1.82 E+00\end{array}$ & $\begin{array}{l}4.91 E-01 \\
5.83 E+01 \\
8.46 E+01 \\
6.39 E+00 \\
3.96 E+00\end{array}$ & $\begin{array}{l}0.0 \\
1.57 E+02 \\
0.0 \\
0.0 \\
5.28 E+01\end{array}$ & $\begin{array}{l}4.91 E-01 \\
2.15 E+02 \\
1.46 E+01 \\
6.39 E+00 \\
5.68 E+01\end{array}$ \\
\hline $\begin{array}{l}A M-242 M \\
A M-243 \\
A M-244 \\
A M-245 \\
A M-246\end{array}$ & 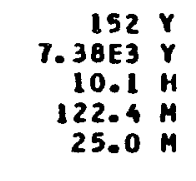 & $\begin{array}{l}2.74 E-01 \\
7.73 E+00 \\
9.56 E+01 \\
4.06 E \bullet 00 \\
1.11 E+02\end{array}$ & $\begin{array}{l}2.95 E-02 \\
5.80 E+00 \\
7.46 E+01 \\
3.04 E \cdot 00 \\
8.81 E+01\end{array}$ & $\begin{array}{l}8.22 E-02 \\
7.43 E+00 \\
9.02 E+01 \\
3.88 E+00 \\
1.05 E+02\end{array}$ & $\begin{array}{l}1.80 E-02 \\
3.55 E+00 \\
5.78 E+01 \\
2.28 E+00 \\
6.87 E+01\end{array}$ & $\begin{array}{l}2.77 E-01 \\
6.61 E+00 \\
8.20 E+01 \\
3.57 E+00 \\
9.48 E+01\end{array}$ & $\begin{array}{l}1.97 E+00 \\
1.06 E+01 \\
1.16 E+02 \\
5.03 E+00 \\
1.30 E+02\end{array}$ & $\begin{array}{l}0.0 \\
0.0 \\
4.21 E+01 \\
1.55 E+02 \\
3.01 E+02\end{array}$ & $\begin{array}{l}1.97 E+00 \\
1.06 E+01 \\
1.59 E+02 \\
1.60 E+02 \\
5.11 E+02\end{array}$ \\
\hline $\begin{array}{l}C M-242 \\
C M-243 \\
C M-244 \\
C M-245 \\
C M-246\end{array}$ & $\begin{array}{r}163.2 Y \\
28.5 Y \\
18.11 Y \\
8.5 E 3 Y \\
4.75 E 3 Y\end{array}$ & $\begin{array}{l}8.79 E-02 \\
1.66 E+01 \\
7.78 E-02 \\
9.65 E+00 \\
6.89 E-02\end{array}$ & $\begin{array}{l}2.77 E-03 \\
1.23 E+01 \\
1.95 E-03 \\
7.07 E+00 \\
1.34 E-03\end{array}$ & $\begin{array}{l}1.06 E-02 \\
1.58 E+01 \\
1.59 E-02 \\
9.20 E+00 \\
1.37 E-02\end{array}$ & $\begin{array}{l}1.40 E-03 \\
9.23 E+00 \\
9.40 E-04 \\
5.02 E+00 \\
5.26 E-04\end{array}$ & $\begin{array}{l}9.34 E-02 \\
1.46 E+01 \\
0.29 E-02 \\
8.54 E+00 \\
7.34 E-02\end{array}$ & $\begin{array}{l}7.36 E-01 \\
2.15 E+01 \\
6.56 E-01 \\
1.39 E \cdot 01 \\
5.84 E-01\end{array}$ & $\begin{array}{l}0.0 \\
0.0 \\
0.0 \\
0.0 \\
0.0\end{array}$ & $\begin{array}{l}7.36 E-01 \\
2.15 E+01 \\
6.56 E-01 \\
1.39 E+01 \\
5.84 E-01\end{array}$ \\
\hline $\begin{array}{l}C M-247 \\
C M-248 \\
C M-249 \\
C M-250 \\
B K-249\end{array}$ & 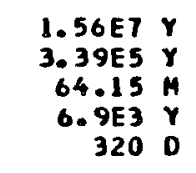 & $\begin{array}{l}3.90 E+01 \\
5.54 E-02 \\
2.27 E+00 \\
0.0 \\
0.0\end{array}$ & $\begin{array}{l}2.93 E+01 \\
1.73 E-03 \\
1.74 E+00 \\
0.0 \\
0.0\end{array}$ & $\begin{array}{l}3.63 E+01 \\
1.17 E-02 \\
2.12 E+00 \\
0.0 \\
0.0\end{array}$ & $\begin{array}{l}2.35 E+01 \\
8.47 E-04 \\
1.38 E+00 \\
0.0 \\
0.0\end{array}$ & $\begin{array}{l}3.36 E \cdot 01 \\
5.88 E-02 \\
1.94 E \div 00 \\
0.0 \\
0.0\end{array}$ & $\begin{array}{l}4.44 E+01 \\
4.63 E-01 \\
2.62 E+00 \\
0.0 \\
0.0\end{array}$ & $\begin{array}{l}1.02 E+00 \\
0.0 \\
1.74 E+02 \\
0.0 \\
0.0\end{array}$ & $\begin{array}{l}4.55 E \cdot 01 \\
4.63 E-01 \\
1.77 E+02 \\
0.0 \\
0.0\end{array}$ \\
\hline
\end{tabular}




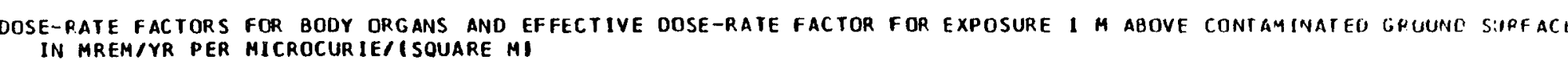

\begin{tabular}{|c|c|c|c|c|c|c|c|c|c|c|}
\hline NUCL IDE & HALF-LIFE & AORENALS & BLAODER & BRAIN & BREAST & HEAR T & $\begin{array}{l}\text { SMALL } \\
\text { INTESTINE }\end{array}$ & $\begin{array}{c}\text { UPPER } \\
\text { IARRGE } \\
\text { INTESTIINE }\end{array}$ & $\begin{array}{c}\text { LOHER } \\
\text { LARGEE } \\
\text { INTESTINEE }\end{array}$ & K IONEYS \\
\hline $\begin{array}{l}B K-250 \\
8 K-251 \\
C F-248 \\
C F-249 \\
C F-250\end{array}$ & 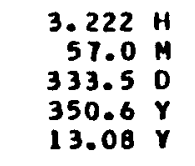 & $\begin{array}{l}7.36 E+01 \\
0.0 \\
1.03 E-02 \\
3.00 E+01 \\
1.18 E-02\end{array}$ & $\begin{array}{l}7.18 E+01 \\
0.0 \\
5.62 E-04 \\
2.79 E+01 \\
2.15 E-03\end{array}$ & $\begin{array}{l}8.09 E+01 \\
0.0 \\
1.81 E-03 \\
3.07 E+01 \\
3.58 E-03\end{array}$ & $\begin{array}{l}9.11 E+01 \\
0.0 \\
3.03 E-01 \\
4.05 E+01 \\
3.02 E-01\end{array}$ & $\begin{array}{l}7.10 E+01 \\
0.0 \\
2.38 E-03 \\
2.72 E+01 \\
3.96 E-03\end{array}$ & $\begin{array}{l}6.47 E+01 \\
0.0 \\
6.53 E-04 \\
2.44 E+01 \\
2.02 E-03\end{array}$ & $\begin{array}{l}7.56 E+01 \\
0.0 \\
4.83 E-04 \\
2.80 E+01 \\
2.10 E-03\end{array}$ & $\begin{array}{l}6.95 E+01 \\
0.0 \\
6.06 E-03 \\
2.69 E+01 \\
7.46 E-03\end{array}$ & $\begin{array}{l}\text { 7. } 54 E+01 \\
0.0 \\
1.54 E-03 \\
2.88 E+01 \\
3.05 E-03\end{array}$ \\
\hline $\begin{array}{l}\text { CF-251 } \\
C F-252 \\
C F-253 \\
C F-254 \\
E S-253\end{array}$ & 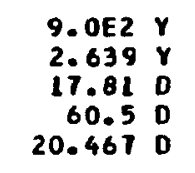 & $\begin{array}{l}1.05 E+01 \\
1.06 E-02 \\
1.74 E-04 \\
1.12 E-06 \\
3.34 E-02\end{array}$ & $\begin{array}{l}9.72 E+00 \\
1.60 E-03 \\
7.52 E-06 \\
1.57 E-06 \\
2.50 E-02\end{array}$ & $\begin{array}{l}1.04 E+01 \\
2.88 E-03 \\
3.14 E-05 \\
9.92 E-07 \\
2.75 E-02\end{array}$ & $\begin{array}{l}1.89 E-01 \\
2.81 E-01 \\
5.12 E-03 \\
4.01 E-06 \\
2.15 E-01\end{array}$ & $\begin{array}{l}9.50 E+00 \\
3.27 E-03 \\
4.09 E-05 \\
9.01 E-07 \\
2.56 E-02\end{array}$ & $\begin{array}{l}8.31 E+00 \\
1.53 E-03 \\
1.08 E-05 \\
9.58 E-07 \\
2.19 E-02\end{array}$ & $\begin{array}{l}1.02 E+01 \\
1.52 E-03 \\
1.32 E-06 \\
1.24 E-06 \\
2.5 B E-02\end{array}$ & $\begin{array}{l}9.34 E+00 \\
6.56 E-03 \\
1.02 E-04 \\
9.82 E-07 \\
2.77 E-02\end{array}$ & $\begin{array}{l}\text { 9. } 93 E+00 \\
2.45 E-03 \\
2.24 E-05 \\
1.95 E-06 \\
2.63 E-02\end{array}$ \\
\hline $\begin{array}{l}\text { ES-254 } \\
\text { ES-254M } \\
\text { ES-255 } \\
\text { FM-254 } \\
\text { FM-255 }\end{array}$ & $\begin{array}{r}275.1 \mathrm{D} \\
39.3 \mathrm{H} \\
39.8 \mathrm{D} \\
3.240 \mathrm{H} \\
20.07 \mathrm{H}\end{array}$ & $\begin{array}{l}3.65 E-01 \\
4.93 E+01 \\
7.90 E-04 \\
1.44 E-02 \\
2.19 E-01\end{array}$ & $\begin{array}{l}2.37 E-01 \\
4.71 E+01 \\
5.66 E-05 \\
3.53 E-03 \\
1.42 E-01\end{array}$ & $\begin{array}{l}2.58 E-01 \\
5.28 E+01 \\
1.49 E-04 \\
5.29 E-03 \\
1.53 E-01\end{array}$ & $\begin{array}{l}4.22 E+00 \\
6.23 E+01 \\
2.19 E-02 \\
3.07 E-01 \\
2.61 E+00\end{array}$ & $\begin{array}{l}2.41 E-01 \\
4.63 E+01 \\
1.92 E-04 \\
5.64 E-03 \\
1.44 E-01\end{array}$ & $\begin{array}{l}1.98 E-01 \\
4.17 E+01 \\
6.04 E-05 \\
3.29 E-03 \\
1.16 E-01\end{array}$ & $\begin{array}{l}2.30 E-01 \\
4.92 E+01 \\
4.80 E-05 \\
3.56 E-03 \\
1.37 E-01\end{array}$ & $\begin{array}{l}2.86 E-01 \\
4.53 E+01 \\
4.73 E-04 \\
9.60 E-03 \\
1.71 E-01\end{array}$ & $\begin{array}{l}2.62 \mathrm{E}-01 \\
4.91 \mathrm{E}+01 \\
1.47 \mathrm{E}-04 \\
5.04 \mathrm{E}-03 \\
1.60 \mathrm{E}-01\end{array}$ \\
\hline
\end{tabular}




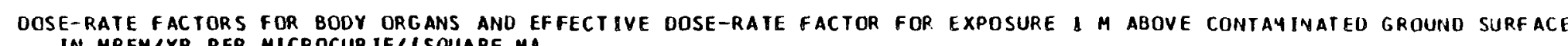

\begin{tabular}{|c|c|c|c|c|c|c|c|c|c|c|}
\hline NUCL IDE & HALF-LIFE & LIVER & LUNGS & MARROH & $\begin{array}{c}\text { RED } \\
\text { HARROH }\end{array}$ & OVARIES & PANCREAS & SKELETON & SPLEEN & S TOMACH \\
\hline $\begin{array}{l}8 K-250 \\
8 K-251 \\
C F-248 \\
C F-249 \\
C F-250\end{array}$ & $\begin{array}{rl}3.222 & H \\
57.0 & 4 \\
333.5 & 0 \\
350.6 & Y \\
13.08 & Y\end{array}$ & $\begin{array}{l}7.14 E+01 \\
0.0 \\
1.40 E-03 \\
2.70 E+01 \\
3.03 E-03\end{array}$ & $\begin{array}{l}7.61 E+01 \\
0.0 \\
1.05 E-02 \\
3.00 E+01 \\
1.22 E-02\end{array}$ & $\begin{array}{l}0.12 E+01 \\
0.0 \\
1.19 E-02 \\
3.68 E+01 \\
1.49 E-02\end{array}$ & $\begin{array}{l}7.69 E+01 \\
0.0 \\
3.58 E-03 \\
3.08 E+01 \\
4.83 E-03\end{array}$ & $\begin{array}{l}6.91 E+01 \\
0.0 \\
5.91 E-03 \\
2.59 E+01 \\
7.20 E-03\end{array}$ & $\begin{array}{l}6.17 E+01 \\
0.0 \\
6.33 E-03 \\
2.40 E+01 \\
7.44 E-03\end{array}$ & $\begin{array}{l}8.27 E+01 \\
0.0 \\
1.72 E-02 \\
3.75 E+01 \\
2.03 E-02\end{array}$ & $\begin{array}{l}6.92 E+01 \\
0.0 \\
4.44 E-03 \\
2.74 E+01 \\
5.97 E-03\end{array}$ & $\begin{array}{l}7.05 E+01 \\
0.0 \\
4.68 E-03 \\
2.74 E+01 \\
6.21 E-03\end{array}$ \\
\hline $\begin{array}{l}C F-251 \\
C F-252 \\
C F-253 \\
C F-254 \\
E S-253\end{array}$ & 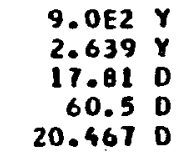 & $\begin{array}{l}9.70 E+00 \\
2.42 E-03 \\
2.28 E-05 \\
1.33 E-06 \\
2.55 E-02\end{array}$ & $\begin{array}{l}1.07 E+01 \\
1.09 E-02 \\
1.77 E-04 \\
1.47 E-06 \\
3.32 E-02\end{array}$ & $\begin{array}{l}1.62 E+01 \\
1.31 E-02 \\
1.99 E-04 \\
2.65 E-06 \\
4.44 E-02\end{array}$ & $\begin{array}{l}9.60 E+00 \\
4.11 E-03 \\
6.09 E-05 \\
6.41 E-01 \\
2.91 E-02\end{array}$ & $\begin{array}{l}0.46 E+00 \\
6.37 E-03 \\
9.94 E-05 \\
1.07 E-06 \\
2.52 E-02\end{array}$ & $\begin{array}{l}8.02 E+00 \\
6.61 E-03 \\
1.07 E-04 \\
7.33 E-07 \\
2.49 E-02\end{array}$ & $\begin{array}{l}1.67 E+01 \\
1.81 E-02 \\
2.90 E-04 \\
2.70 E-06 \\
4.84 E-02\end{array}$ & $\begin{array}{l}9.64 E+00 \\
5.15 E-03 \\
7.50 E-05 \\
1.06 E-06 \\
2.73 E-02\end{array}$ & $\begin{array}{l}9.59 E \cdot 00 \\
5.38 E-03 \\
7.88 E-05 \\
1.16 E-06 \\
2.72 E-02\end{array}$ \\
\hline $\begin{array}{l}E S-254 \\
E S-254 M \\
E S-255 \\
F M-254 \\
F M-255\end{array}$ & $\begin{array}{r}275.7 \mathrm{D} \\
39.3 \mathrm{H} \\
39.8 \mathrm{D} \\
3.240 \mathrm{H} \\
20.01 \mathrm{H}\end{array}$ & $\begin{array}{l}2.46 E-01 \\
4.68 E+01 \\
1.24 E-04 \\
4.73 E-03 \\
1.49 E-01\end{array}$ & $\begin{array}{l}3.87 E-01 \\
5.02 E+01 \\
8.14 E-01 \\
1.51 E-02 \\
2.42 E-01\end{array}$ & $\begin{array}{l}5.42 E-01 \\
5.59 E+01 \\
9.46 E-04 \\
1.95 E-02 \\
3.66 E-01\end{array}$ & $\begin{array}{l}2.49 E-01 \\
5.11 E+01 \\
2.79 E-04 \\
6.47 E-03 \\
1.32 E-01\end{array}$ & $\begin{array}{l}2.74 E-01 \\
4.54 E-01 \\
4.64 E-04 \\
9.29 E-03 \\
1.65 E-01\end{array}$ & $\begin{array}{l}2.55 E-01 \\
4.04 E+01 \\
4.06 E-04 \\
9.22 E-03 \\
1.49 E-01\end{array}$ & $\begin{array}{l}6.20 E-01 \\
5.68 E+01 \\
1.34 E-03 \\
2.52 E-02 \\
4.16 E-01\end{array}$ & $\begin{array}{l}2.74 E-01 \\
4.56 E+01 \\
3.52 E-04 \\
8.03 E-03 \\
1.66 E-01\end{array}$ & $\begin{array}{l}\text { 2. } 80 E-01 \\
4.63 E+01 \\
3.74 E-04 \\
8.39 E-03 \\
1.71 E-01\end{array}$ \\
\hline$F M-256$ & $151.6 \mathrm{H}$ & 0.0 & 0.0 & 0.0 & 0.0 & 0.0 & 0.0 & 0.0 & 0.0 & 0.0 \\
\hline
\end{tabular}


DOSE-RATE FACTORS FOR BOOY ORGANS AND EFFECTIVE DOSE-RATE FACTOR FOR EXPOSURE I A AGOVE CONTAMINATEO GROUNO SUPFACE IN MREM/YR PER MICROCUR IE/ISOUARE MI

\begin{tabular}{|c|c|c|c|c|c|c|c|c|c|}
\hline 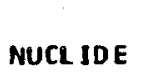 & ALF-LIFE & TESTES & IYMUS & IY RO10 & TERUS & EFECT IVE & $\begin{array}{c}\text { SKIN } \\
\text { (PHOTON I }\end{array}$ & $\begin{array}{l}\text { SKIN } \\
\text { (ELECTRON) }\end{array}$ & $\begin{array}{l}\text { SKIN } \\
\text { ITOTALI }\end{array}$ \\
\hline-28 & $\begin{array}{rl}3.222 & H \\
577.0 & H \\
333.5 & \mathrm{O} \\
350.6 & Y \\
13.08 & Y\end{array}$ & $\begin{array}{l}1.01 E+02 \\
0.0 \\
6.72 E-02 \\
4.07 E+01 \\
6.88 E-02\end{array}$ & $\begin{array}{l}8.05 E \times 01 \\
0.0 \\
9.35 E-04 \\
3.05 E+01\end{array}$ & $\begin{array}{l}9.60 E \cdot 01 \\
0.0 \\
1.43 E-02 \\
3.78 E+01 \\
1.67 E-02\end{array}$ & $\begin{array}{l}6.24 E \cdot 01 \\
0.0 \\
2.88 E-04 \\
2.44 E+01 \\
1.70 E-03\end{array}$ & $\begin{array}{l}0.61 E+01 \\
0.0 \\
6.73 E-02 \\
3.52 E+01\end{array}$ & $\begin{array}{l}1.18 E+02 \\
0.0 \\
5.05 E-01 \\
4.76 E+01 \\
5.01 E-01\end{array}$ & $\begin{array}{l}1.76 E+02 \\
2.93 E+02 \\
0.0 \\
0.0 \\
0.0\end{array}$ & \\
\hline $\begin{array}{l}-251 \\
-252 \\
-253 \\
-254 \\
-253\end{array}$ & $\begin{array}{r}9.0 E 2 \text { Y } \\
2.639 \\
17.81 \\
60.5 \\
20.467 \\
D\end{array}$ & $\begin{array}{l}1.54 E+01 \\
6.36 E-02 \\
1.13 E-03 \\
3.06 E-06 \\
7.73 E-02\end{array}$ & $\begin{array}{l}1.15 \\
2.25 \\
1.3 \\
2.05 \\
2.85\end{array}$ & $\begin{array}{l}1.48 \\
1.50 \\
2.39 \\
2.66 \\
4.43\end{array}$ & $\begin{array}{l}10 \\
13 \\
6 \\
17 \\
12\end{array}$ & $\begin{array}{l}01 \\
02 \\
06\end{array}$ & & $\begin{array}{l}0 \\
0 \\
0 \\
0\end{array}$ & $\begin{array}{l}1.99 E+01 \\
4.66 E-01 \\
8.54 E-03 \\
3.95 E-06 \\
3.30 E-01\end{array}$ \\
\hline $\begin{array}{l}-254 \\
-254 M \\
-255 \\
-255 \\
-254 \\
-255\end{array}$ & $\begin{array}{r}275.7 \mathrm{O} \\
39.3 \mathrm{H} \\
39.8 \mathrm{O} \\
3.240 \mathrm{H} \\
20.07 \mathrm{H}\end{array}$ & $\begin{array}{l}1.25 E+00 \\
6.72 E+01 \\
5.09 E-03 \\
7.80 E-02 \\
7.99 E-01\end{array}$ & $\begin{array}{l}2.91 E-01 \\
5.20 E+01 \\
9.12 E-05 \\
4.60 E-03 \\
1.04 E-01\end{array}$ & $\begin{array}{l}5.47 E \\
6.30 E \\
1.15 E \\
2.19 E \\
3.59 E\end{array}$ & $\begin{array}{l}1.92 \\
4.07 \\
2.84 \\
2.01 \\
1.11\end{array}$ & $\begin{array}{l}1.16 E \\
5.74 E \\
4.95 E \\
7.32 E \\
7.26 E\end{array}$ & & $\begin{array}{l}0.0 \\
6.46 E+01 \\
0.0 \\
0.0 \\
0.0\end{array}$ & $\begin{array}{l}6.6 B E \cdot 00 \\
1.44 E+02 \\
3.61 E-02 \\
4.93 E-01 \\
4.04 E+00\end{array}$ \\
\hline & & & & & & & & & \\
\hline
\end{tabular}




\section{APPENDIX B}

DIAGRAMS OF RADIOACTIVE DECAY CHAINS

The following diagrams show the parent-daughter relationships for all decay chains involving radionuclides considered in this report. (7) Each diagram gives the half-life, modes of decay, and decay branching fractions for each radionuclide in a decay chain. The decay modes include $\alpha, \beta^{-}, \beta^{+}$ and electron capture (EC), and isomeric transitions (IT). (7) The distinction between EC and $\beta^{+}$decay is that the latter involves a total decay energy for a transition greater than $1022 \mathrm{keV}$, so that positron emission may occur. (7) Branching fractions are given in percent, i.e., number of decays per 100 atoms of the parent radionuclide, and modes of decay with branching fractions less than 0.18 are omitted. The branching fraction for spontaneous fission decay, which is discussed in Section 3.1 .1 , is not indicated in the diagrams.
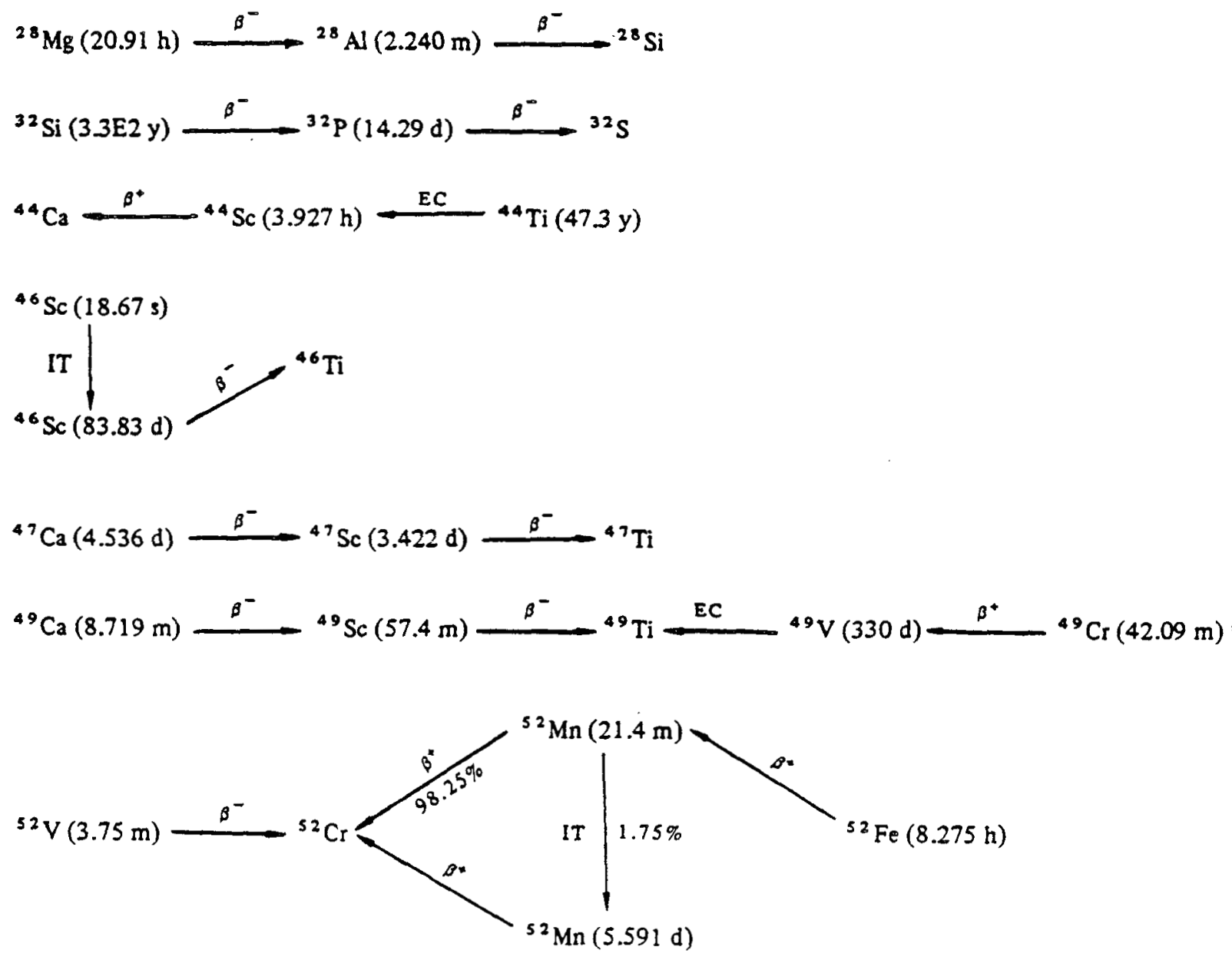

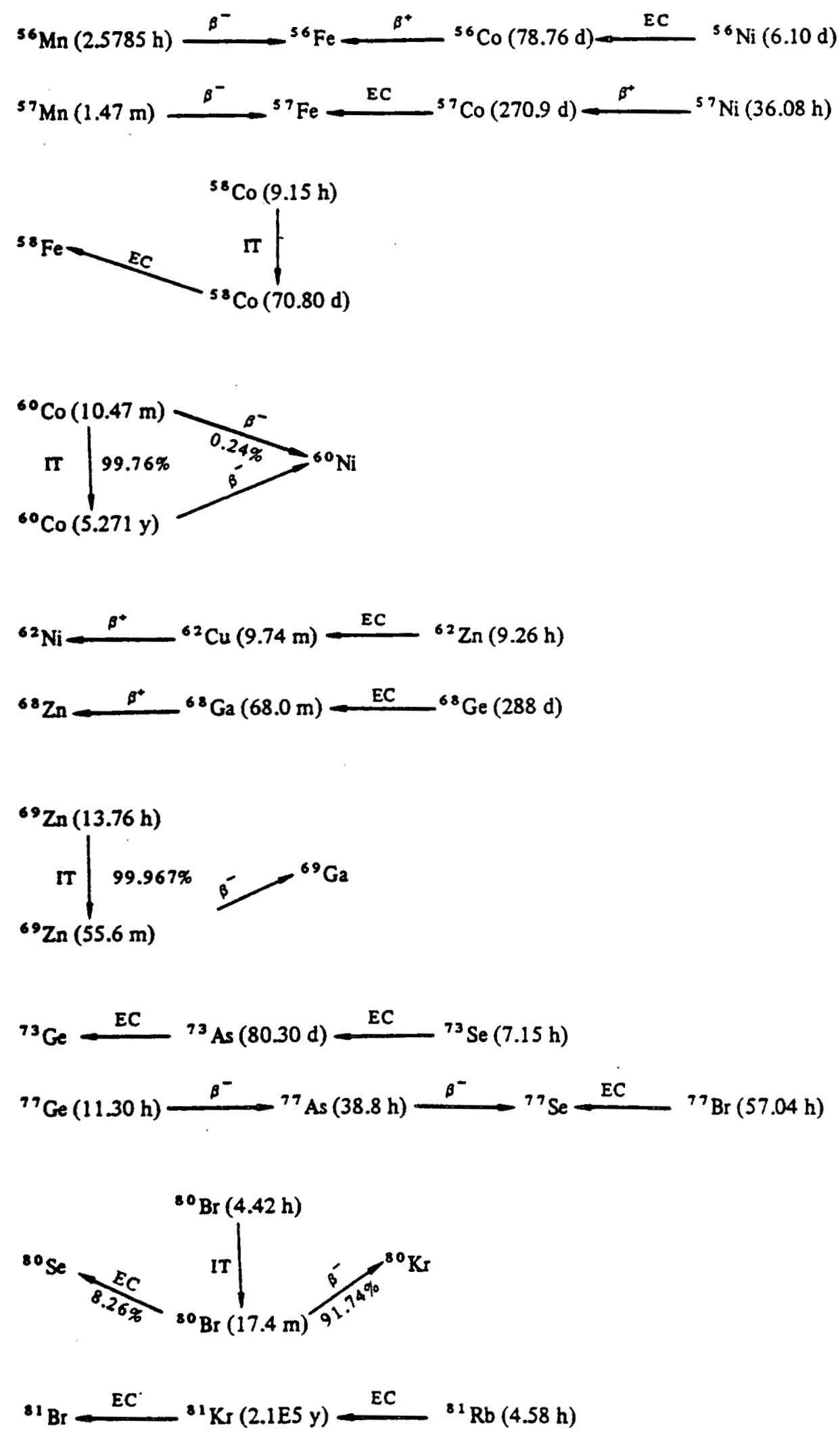


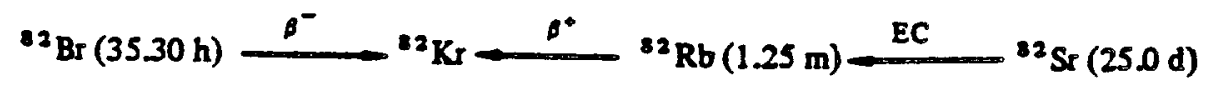
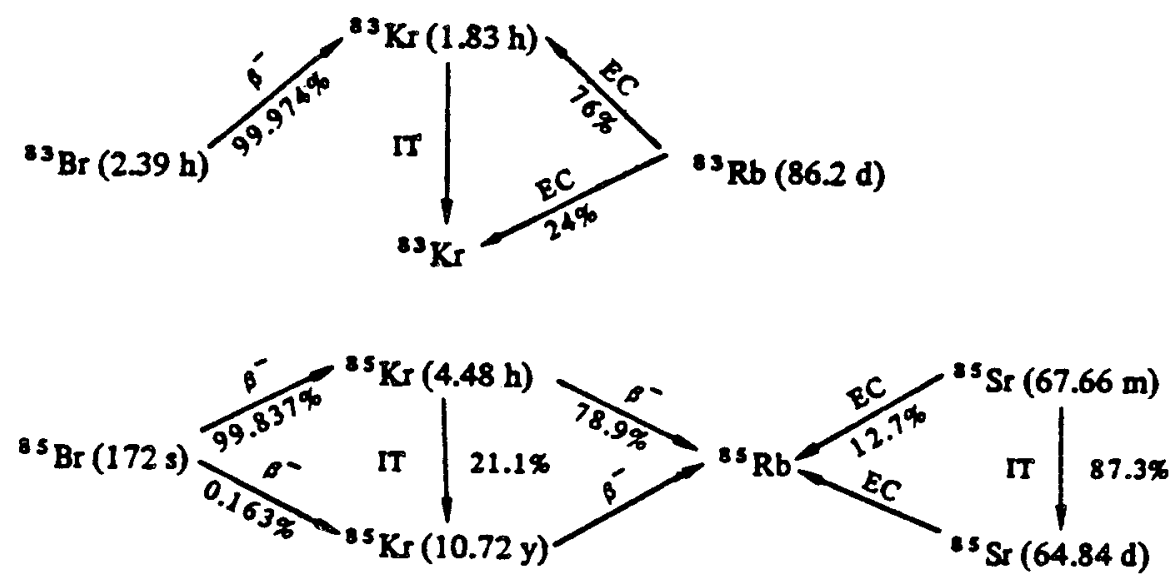

${ }^{86} \mathrm{Rb}(18.66 \mathrm{~d}) \stackrel{B^{-}}{-86 \mathrm{Sr}} \underset{\beta^{*}}{ }{ }^{86} \mathrm{Y}(14.74 \mathrm{~h})-\mathrm{EC}{ }^{86} \mathrm{Zr}(16.5 \mathrm{~h})$

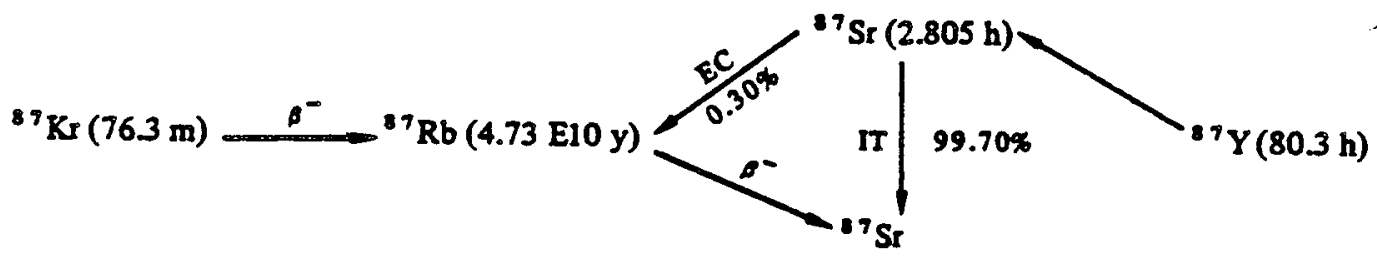

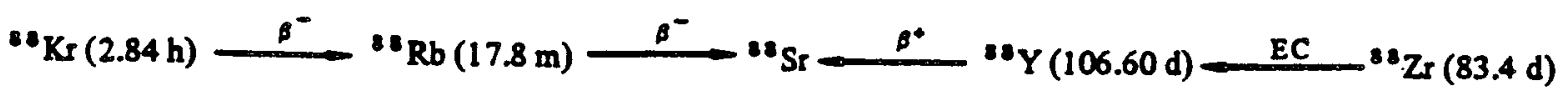

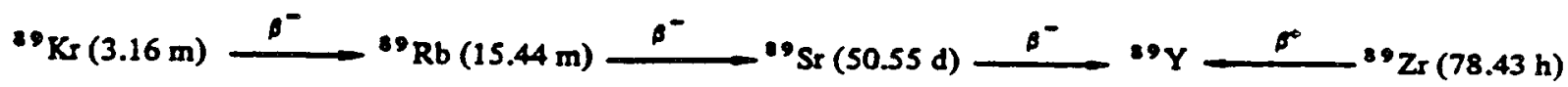

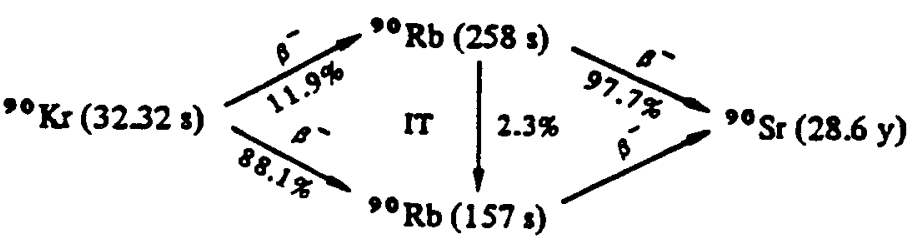

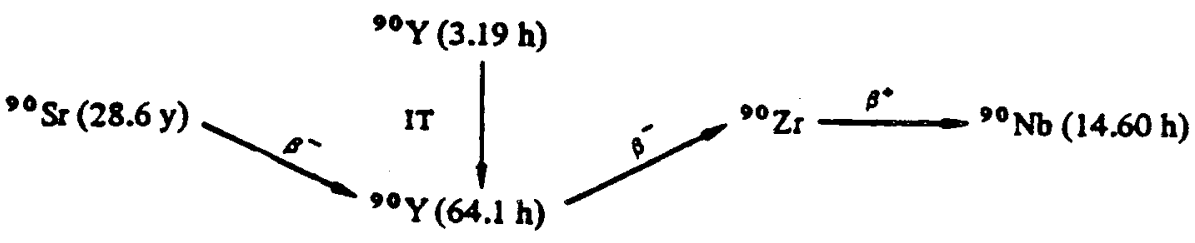



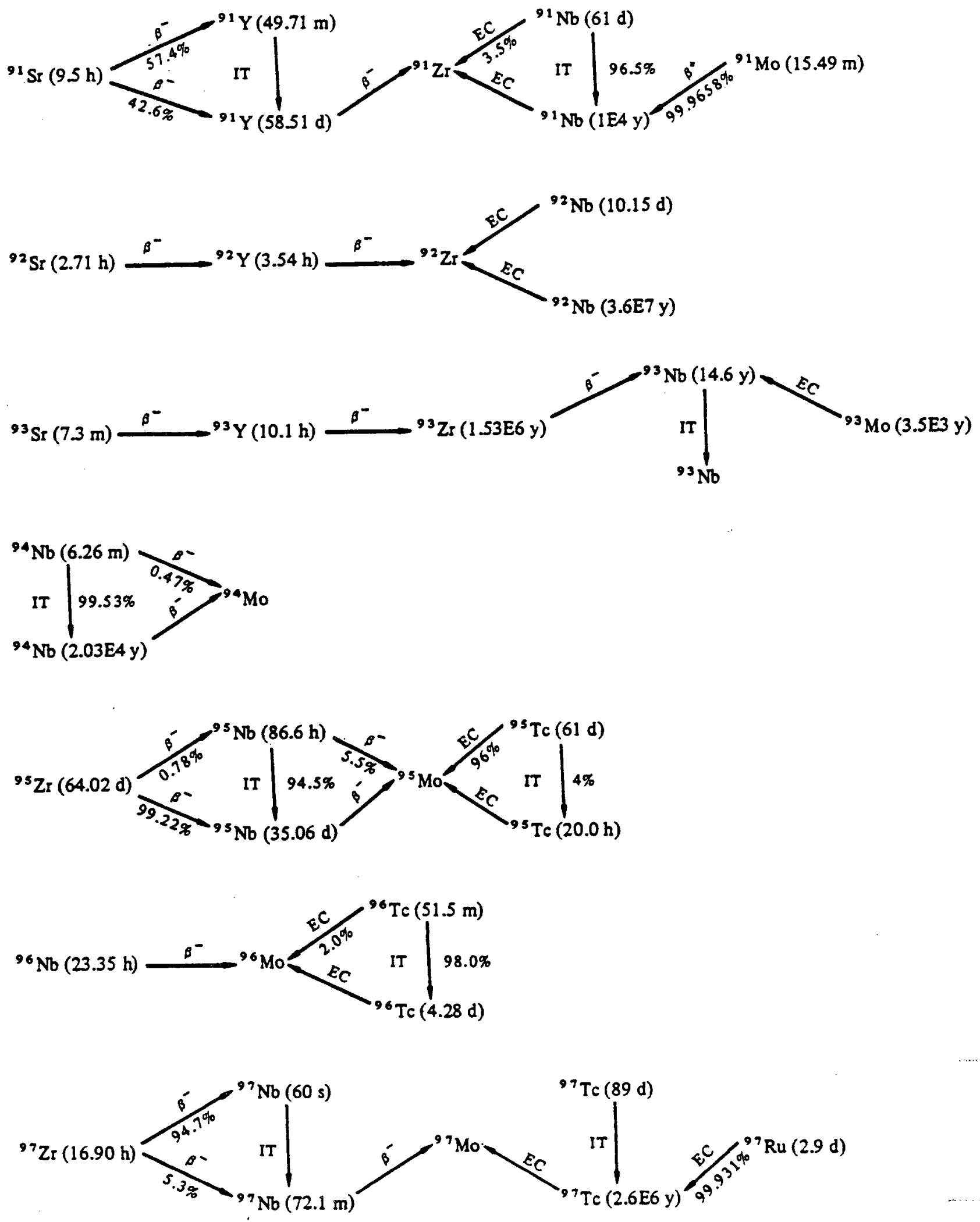


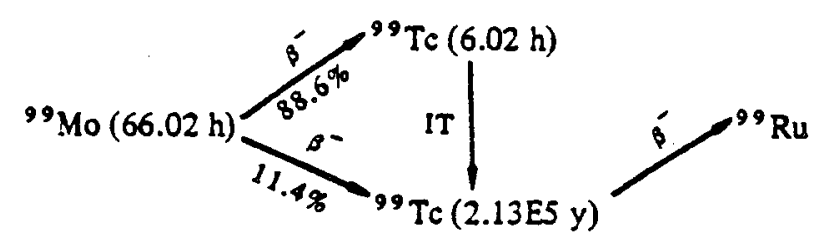

${ }^{101} \mathrm{Mo}(14.61 \mathrm{~m})-\mathrm{s}^{-}{ }^{101} \mathrm{Tc}(14.2 \mathrm{~m}) \mathrm{s}^{-}{ }^{101} \mathrm{Ru}$
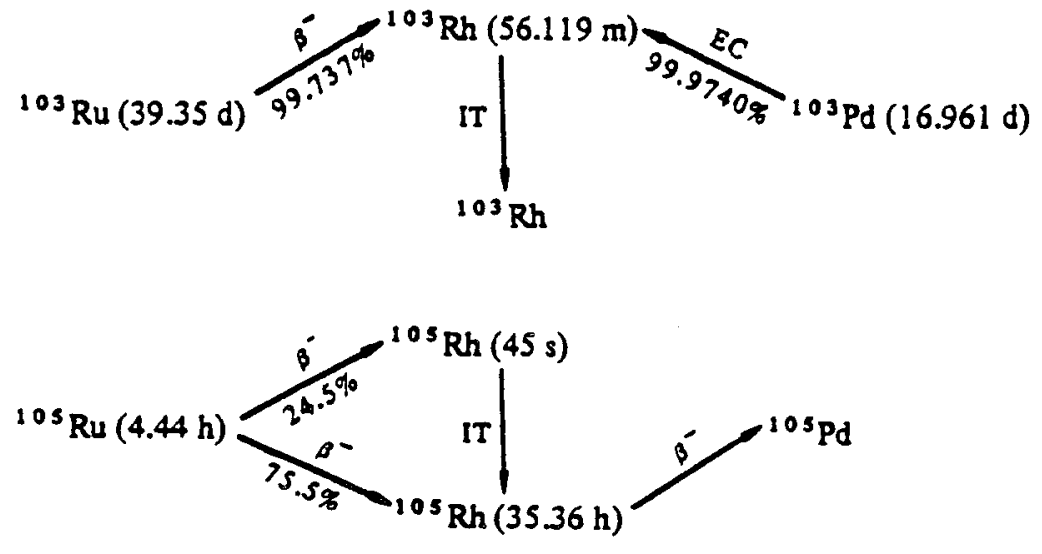

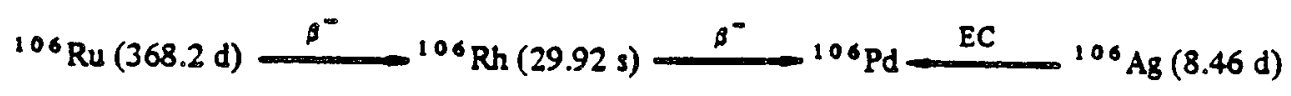

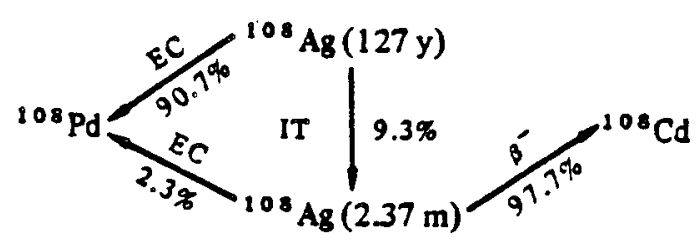

$\left.\left.{ }^{109} \mathrm{Pd}(13.53 \mathrm{~h})\right|_{999^{49^{40}}} ^{109} \mathrm{Ag}(39.6 \mathrm{~s})\right|_{109} ^{\mathrm{Ag}} \mathrm{Cd}(464 \mathrm{~d})$

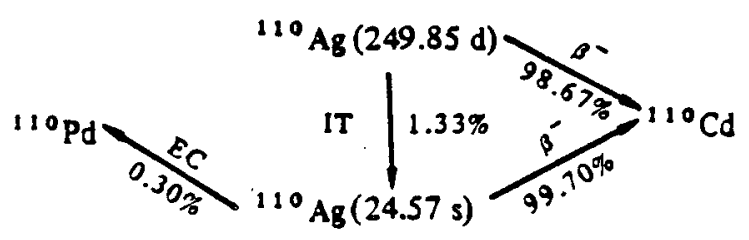



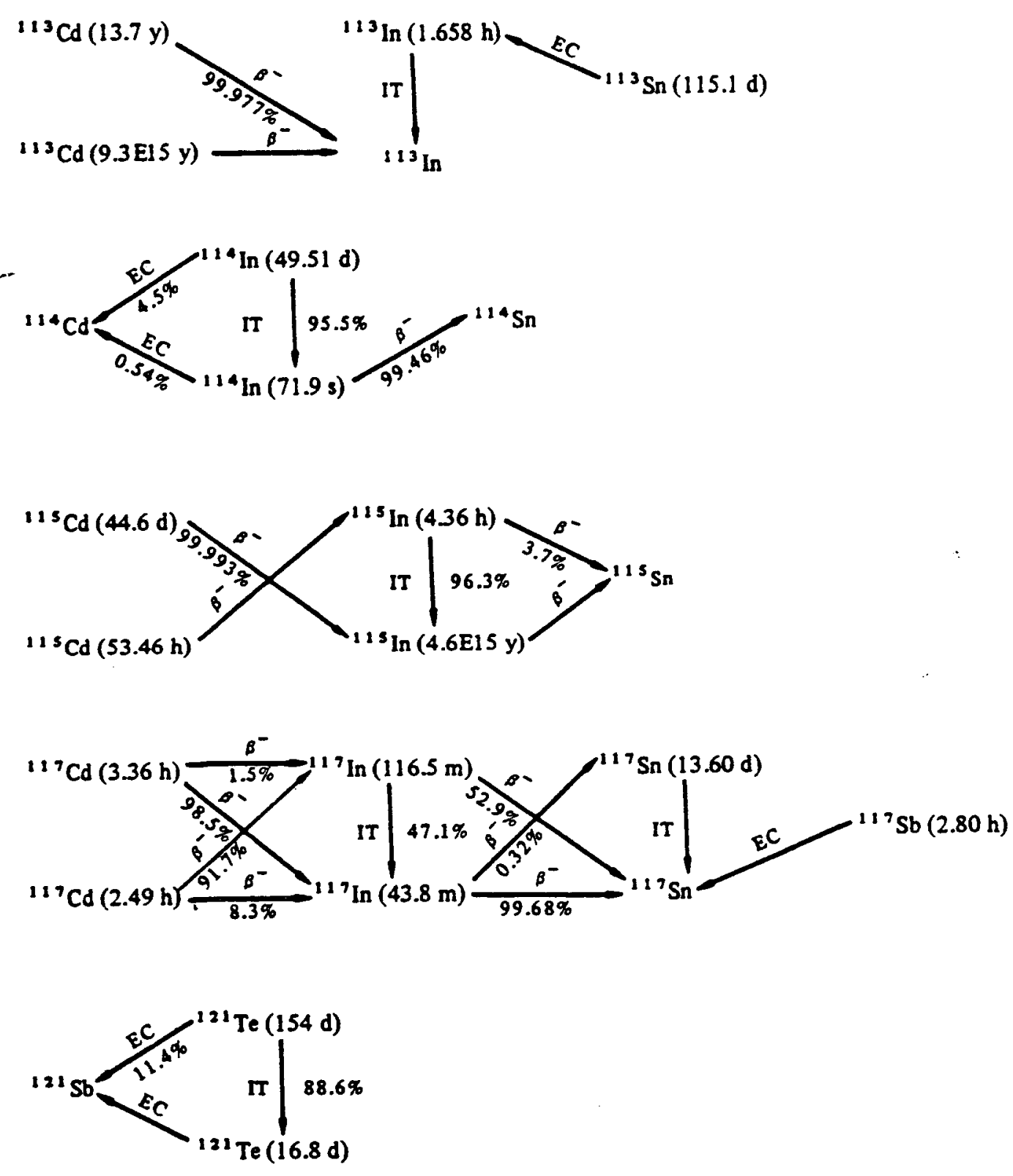

$122 \mathrm{Sn} \frac{\mathrm{EC}}{2.42 \%}{ }^{122} \mathrm{Sb}(2.70 \mathrm{~d}) \frac{\beta^{-}}{97.58 \%}{ }^{122} \mathrm{Te}-B^{*}{ }^{122} \mathrm{I}(3.62 \mathrm{~m})-E C \quad 122 \mathrm{Xe}(20.1 \mathrm{~h})$

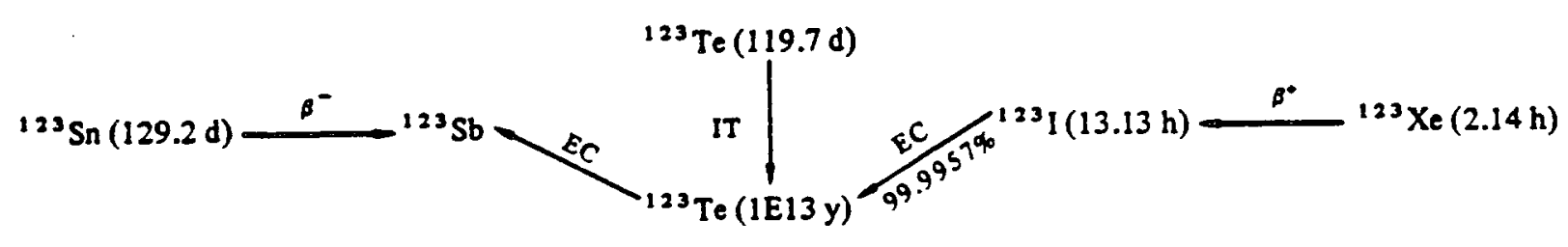



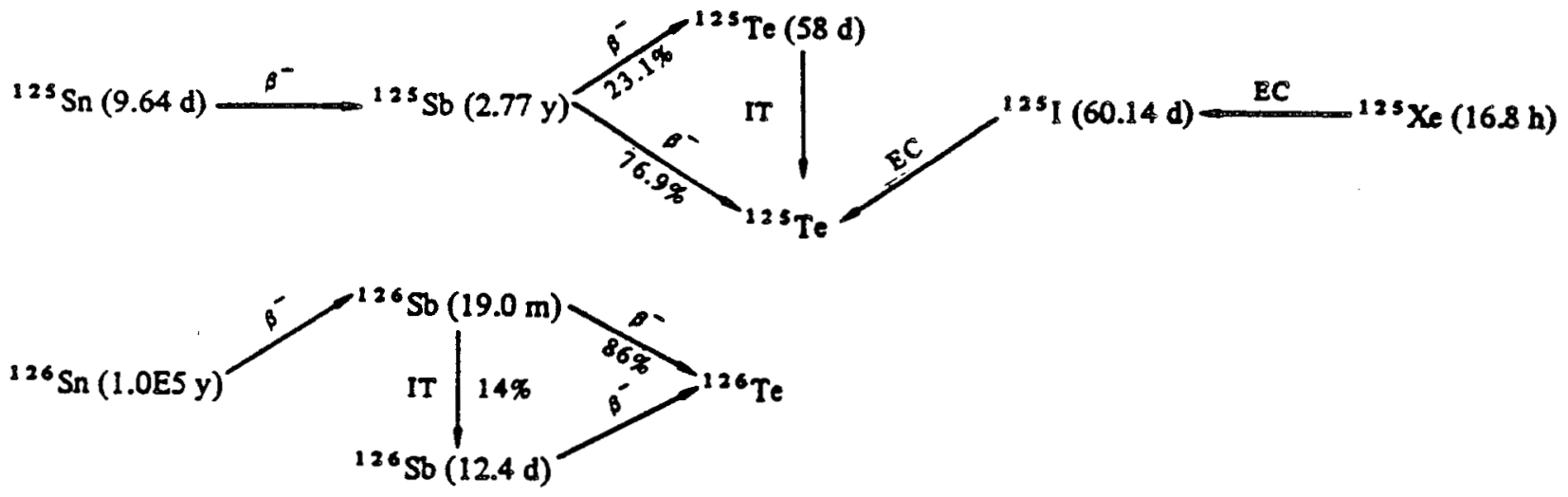

$$
{ }^{126} \mathrm{Te} \frac{E C}{61 \%}{ }^{126} \mathrm{I}(12.93 \mathrm{~d}) \frac{\beta^{-}}{39 \%}{ }^{126} \mathrm{Xe}{\stackrel{\beta^{\circ}}{ }}^{126} \mathrm{Cs}(1.64 \mathrm{~m})
$$

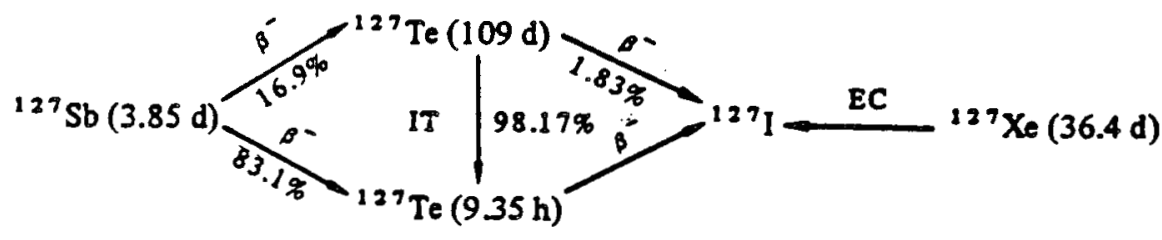
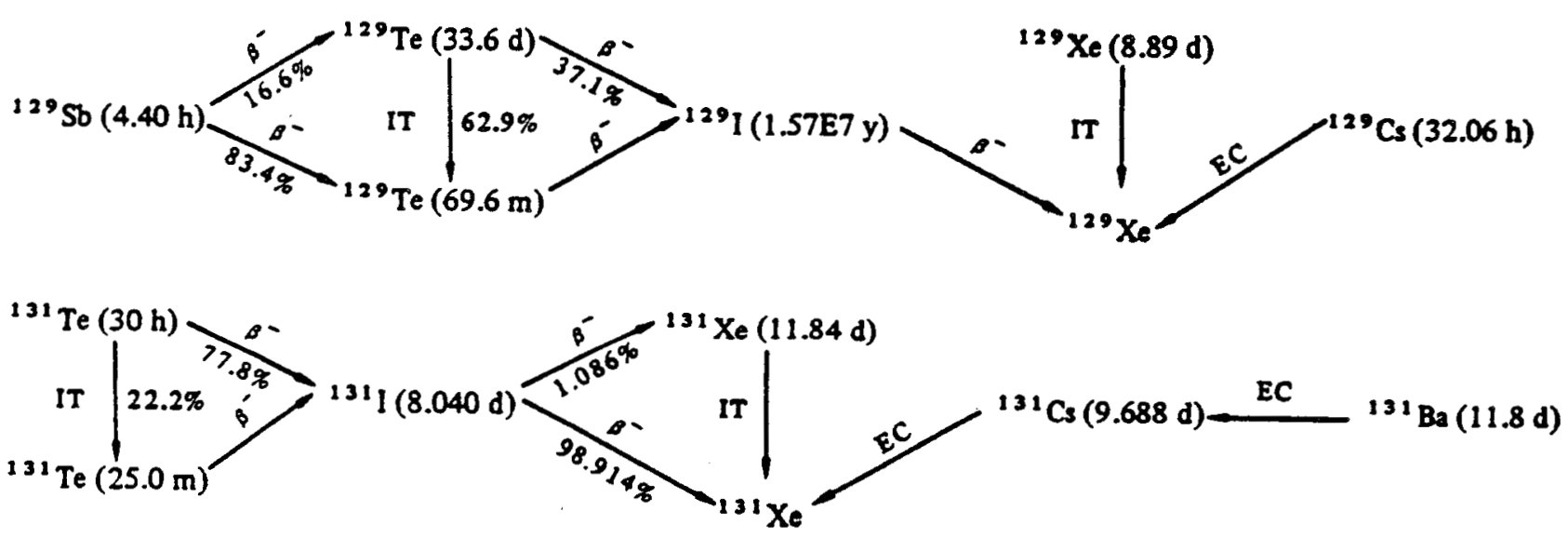

${ }^{132} \mathrm{Te}(78.2 \mathrm{~h}) \stackrel{A^{-}}{ }{ }^{132} \mathrm{I}(2.30 \mathrm{~h}) \stackrel{B^{-}}{{ }^{132} \mathrm{Xe}} \frac{\mathrm{EC}}{97.96 \%}{ }^{132} \mathrm{Cs}(6.475 \mathrm{~d}) \frac{A^{-}}{2.04 \%}{ }^{132} \mathrm{Ba}$

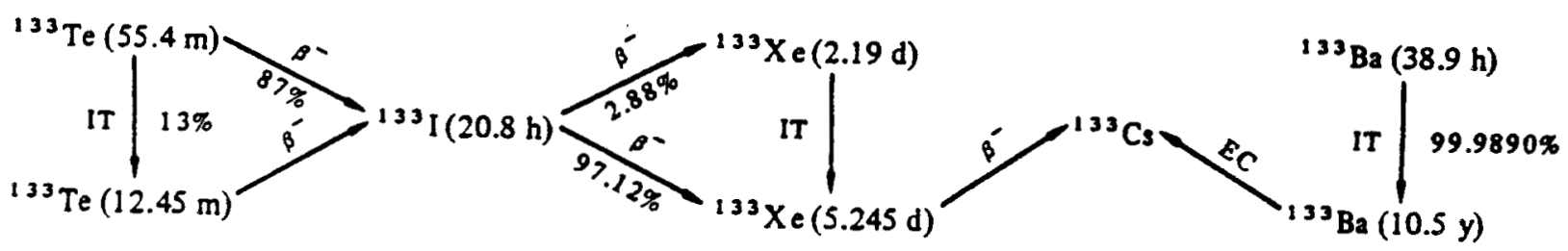


${ }^{34} \mathrm{Te}(41.8 \mathrm{~m}) \stackrel{\beta^{-}}{ }{ }^{134} \mathrm{I}(52.6 \mathrm{~m}) \stackrel{\beta^{-}}{\longrightarrow}{ }^{134} \mathrm{Xe}$
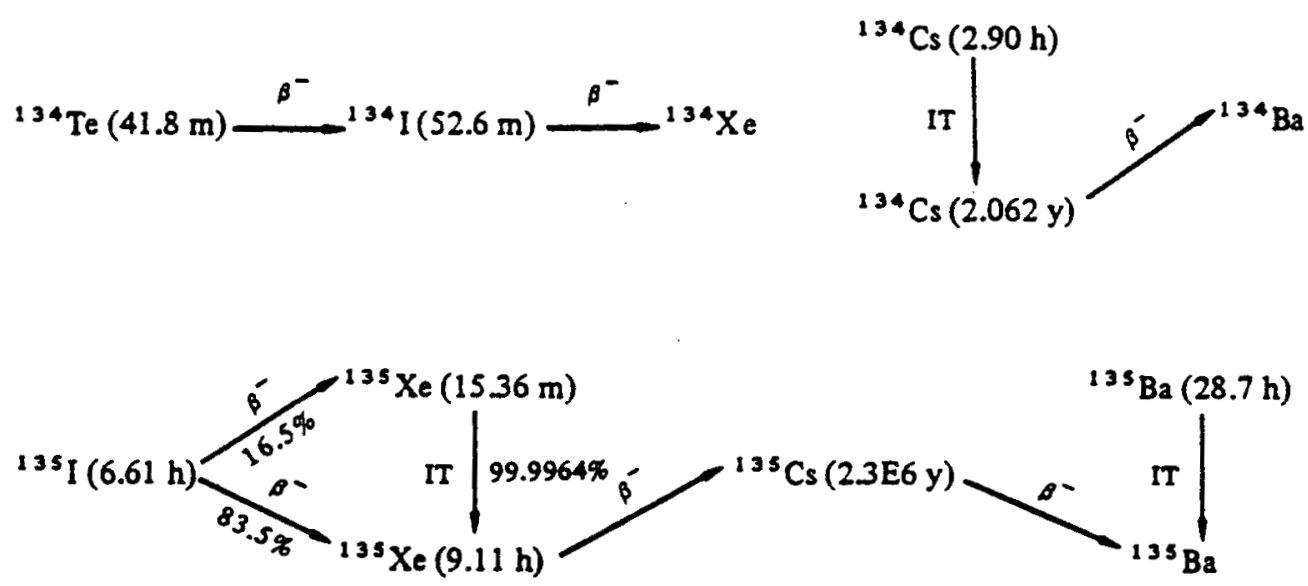

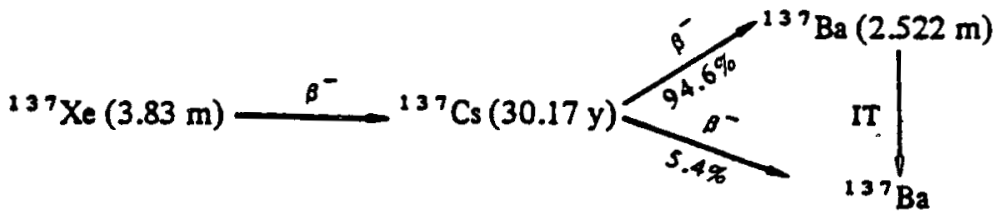

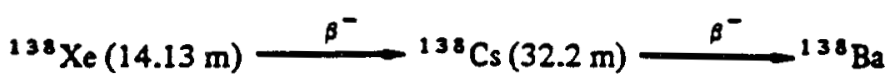

${ }^{139} \mathrm{Cs}(9.40 \mathrm{~m}) \stackrel{\mathrm{B}^{-}}{-139} \mathrm{Ba}(83.1 \mathrm{~m}) \mathrm{B}^{-}{ }^{139} \mathrm{La} \underset{\mathrm{EC}}{ }{ }^{139} \mathrm{Ce}(137.66 \mathrm{~d})$

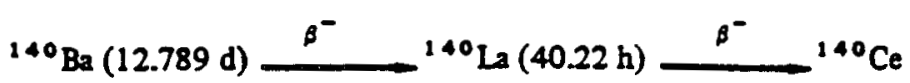

${ }^{141} \mathrm{Ba}(18.27 \mathrm{~m}) \stackrel{\beta^{-}}{ }{ }^{141} \mathrm{La}(3.94 \mathrm{~h}) \mathrm{B}^{-}{ }^{141} \mathrm{Ce}(32.50 \mathrm{~d}) \mathrm{B}^{-}{ }^{141} \mathrm{Pr}$

${ }^{142} \mathrm{Ba}(10.70 \mathrm{~m}) \mathrm{S}^{-}{ }^{142 \mathrm{La}(95.4 \mathrm{~m}) \mathrm{A}^{-}}{ }^{142} \mathrm{Ce} \quad{ }^{142} \mathrm{Pr}(19.13 \mathrm{~h}) \frac{\mathrm{s}^{-}}{99.9836 \%}{ }^{142} \mathrm{Nd}$

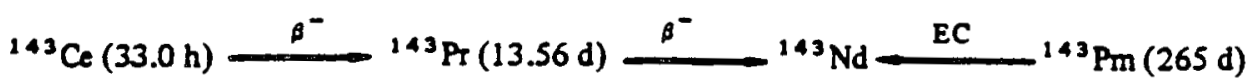



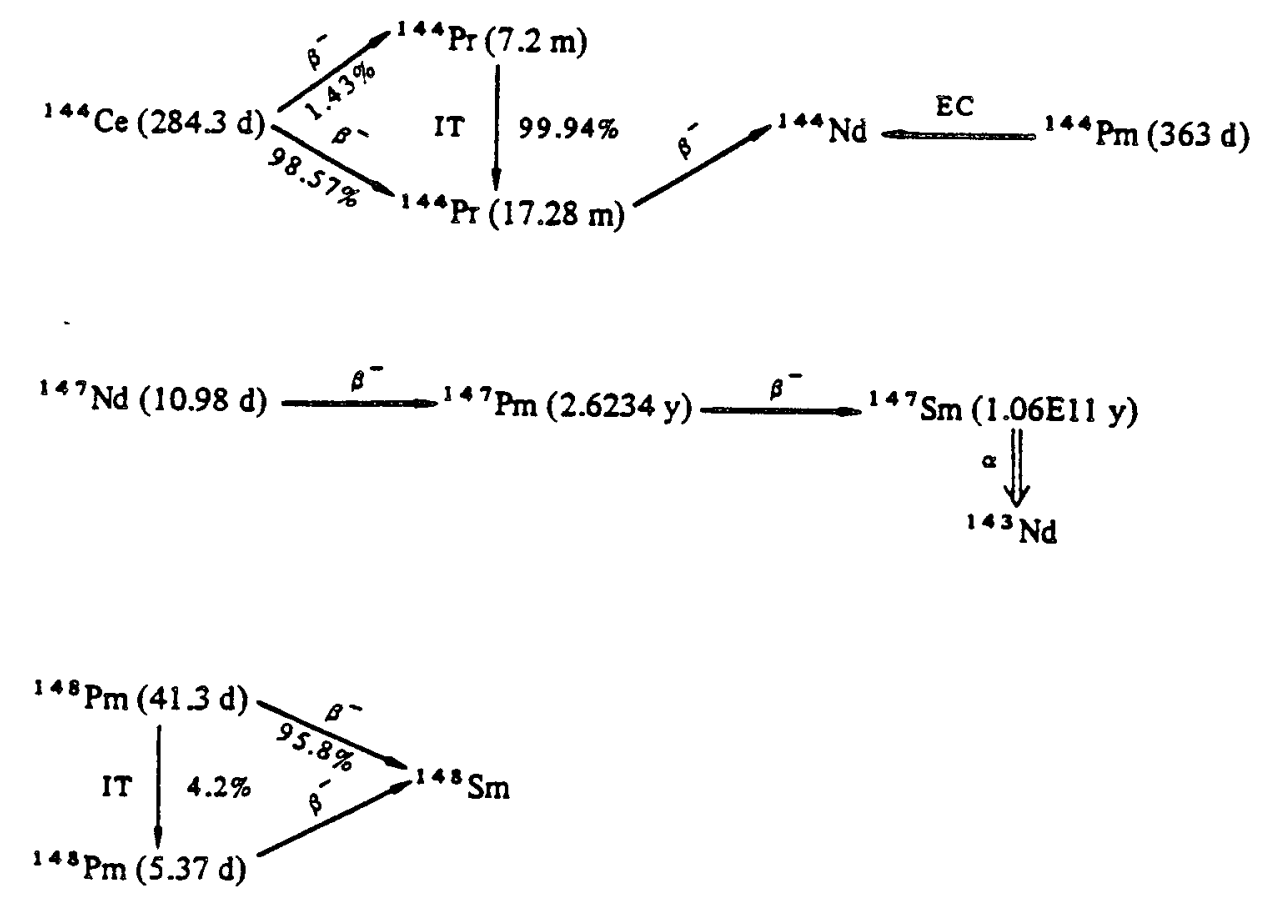

${ }^{149} \mathrm{Nd}(1.73 \mathrm{~h}) \stackrel{\beta^{-}}{\longrightarrow}{ }^{149} \mathrm{Pm}(53.08 \mathrm{~h}) \stackrel{\beta^{-}}{-149} \mathrm{Sm}$

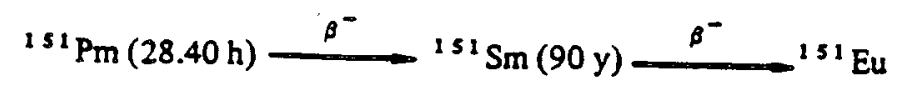

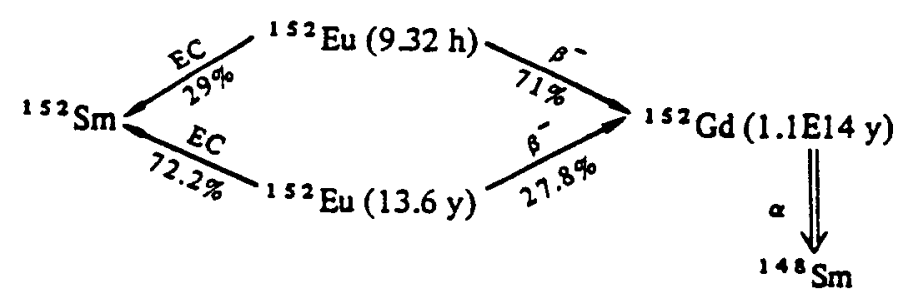

${ }^{157} \mathrm{Gd}-\frac{\mathrm{EC}}{{ }^{157} \mathrm{~Tb}(150 \mathrm{y})-\mathrm{EC}}{ }^{157} \mathrm{Dy}(8.06 \mathrm{~h})$

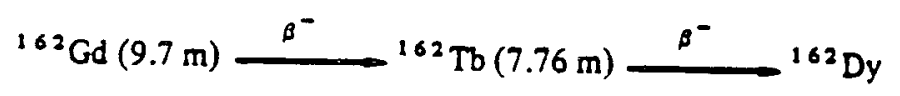




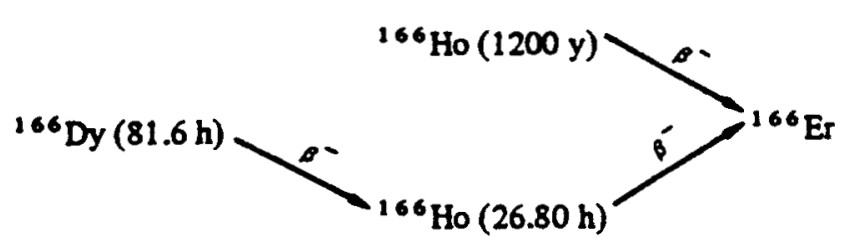

${ }^{171} \mathrm{Er}(7.52 \mathrm{~h}) \stackrel{\beta^{-}}{ }{ }^{171} \mathrm{Tm}(1.92 \mathrm{y}){\stackrel{\beta^{-}}{ }}^{171} \mathrm{Yb}$
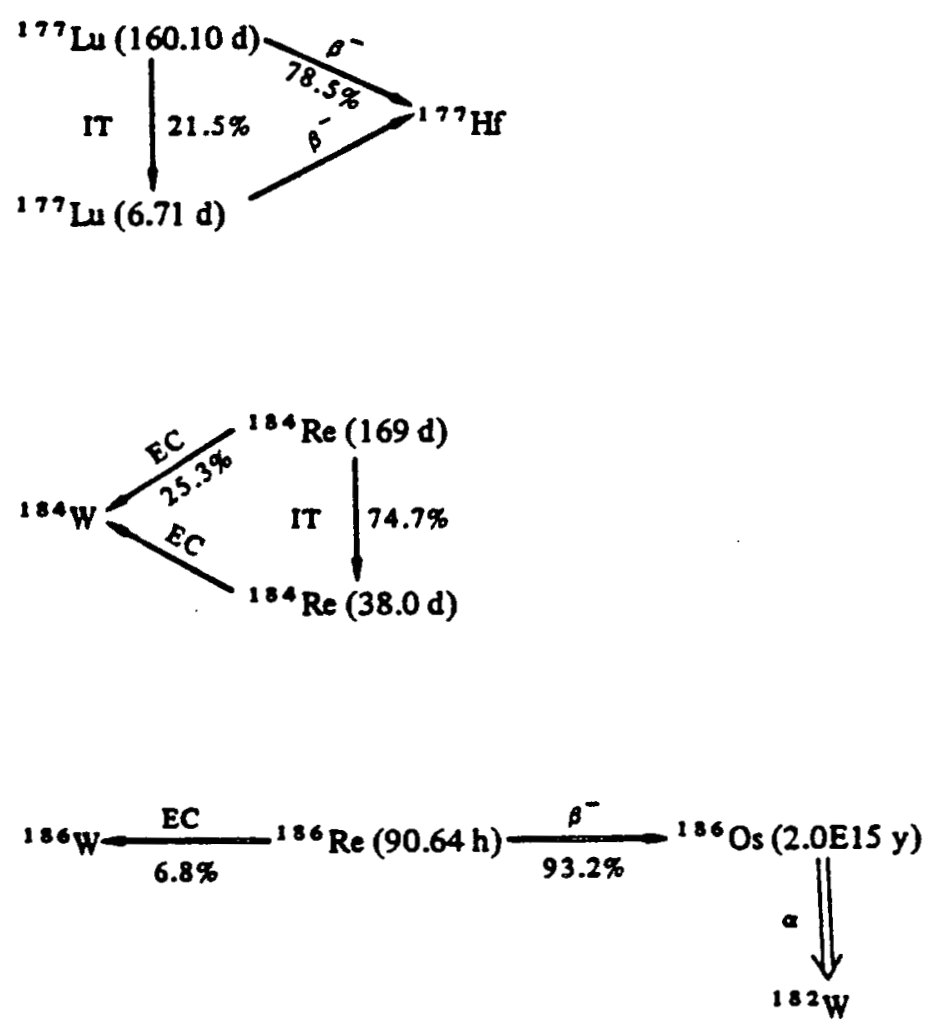

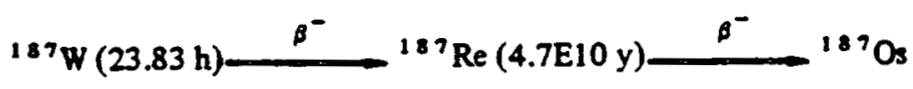

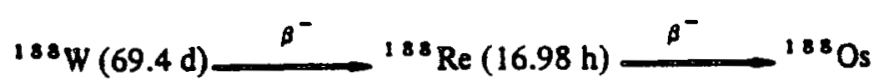



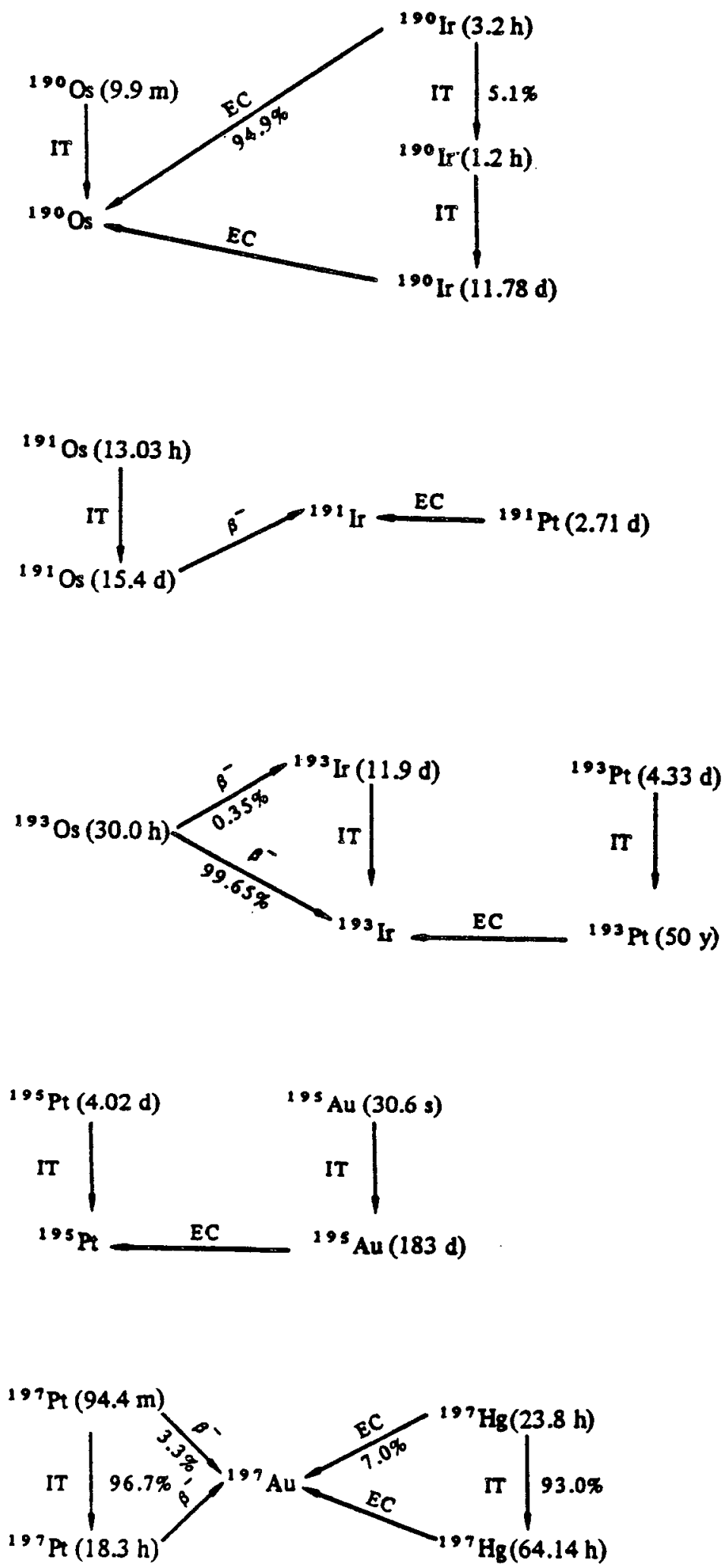
Neptunium Series

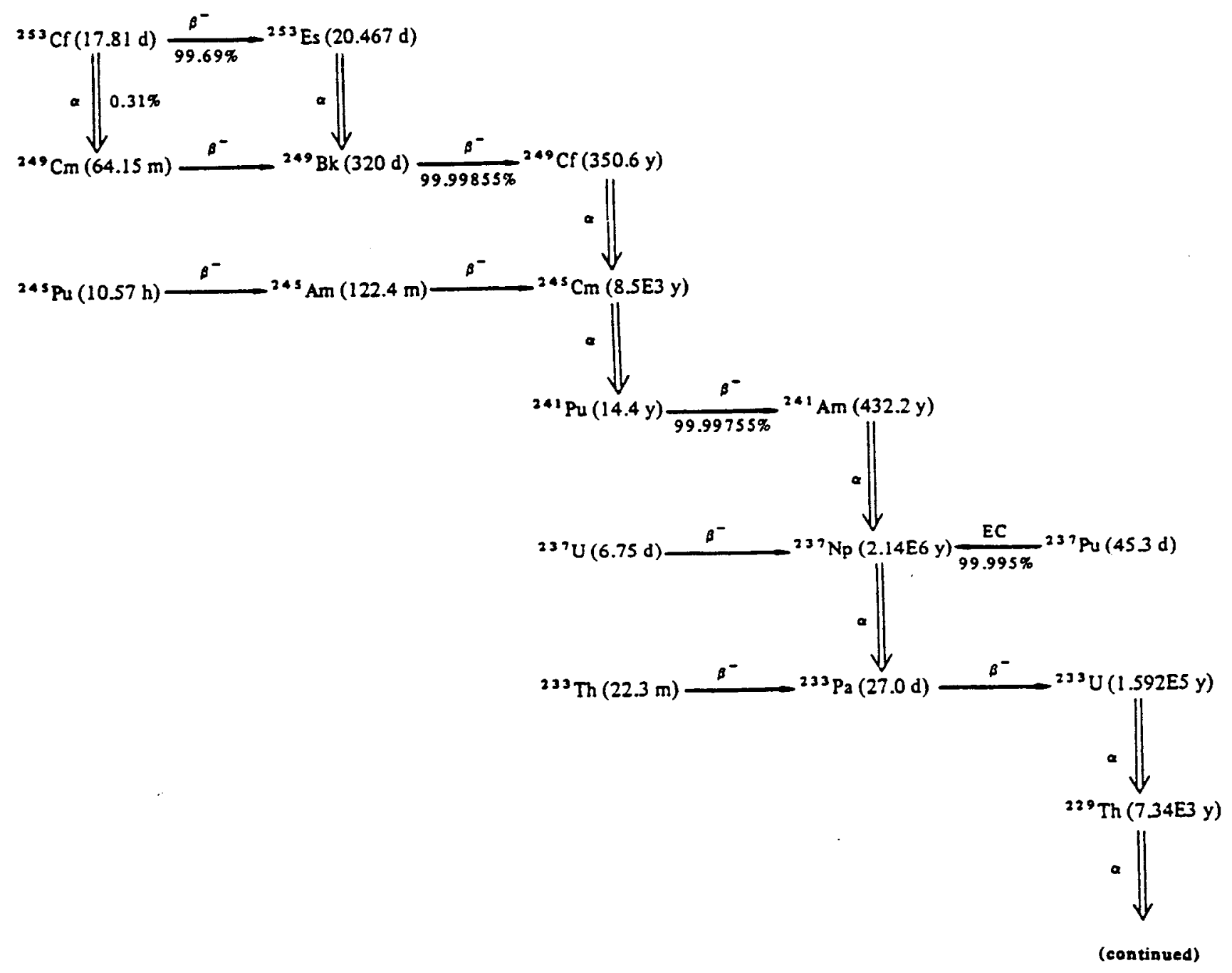




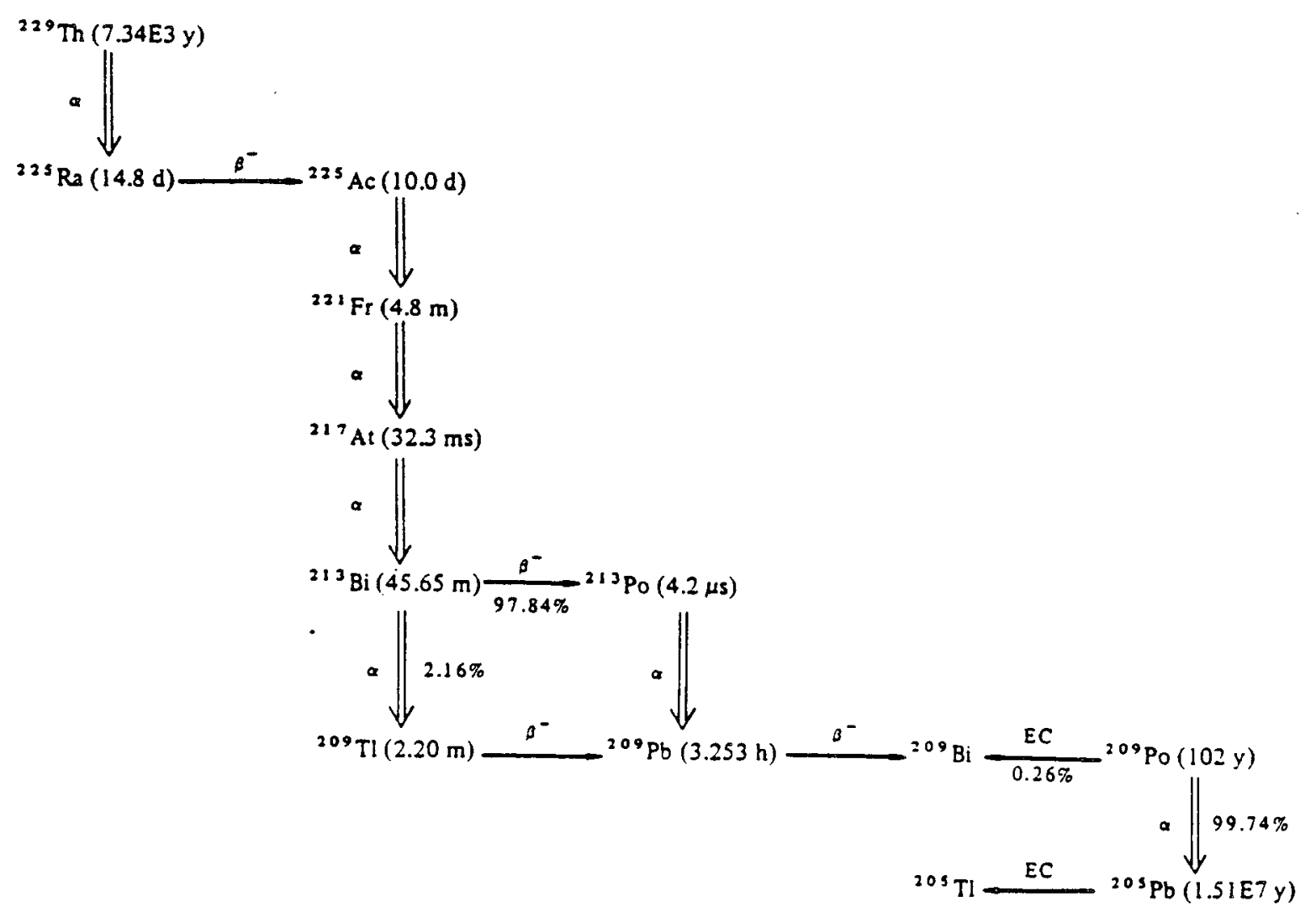


Unnium Serie
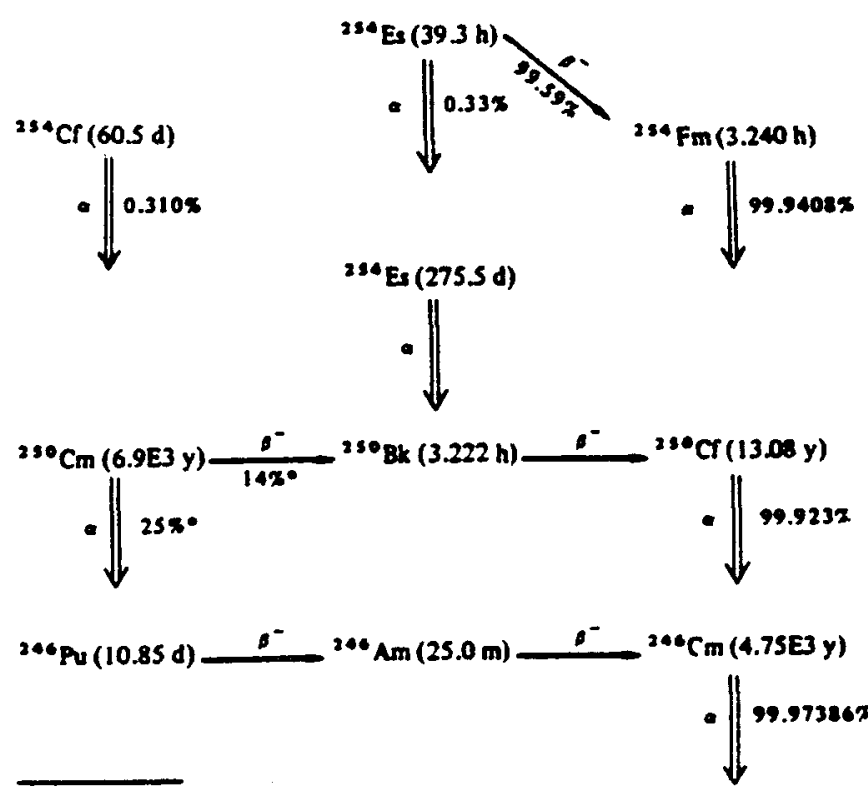

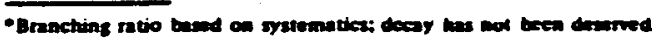
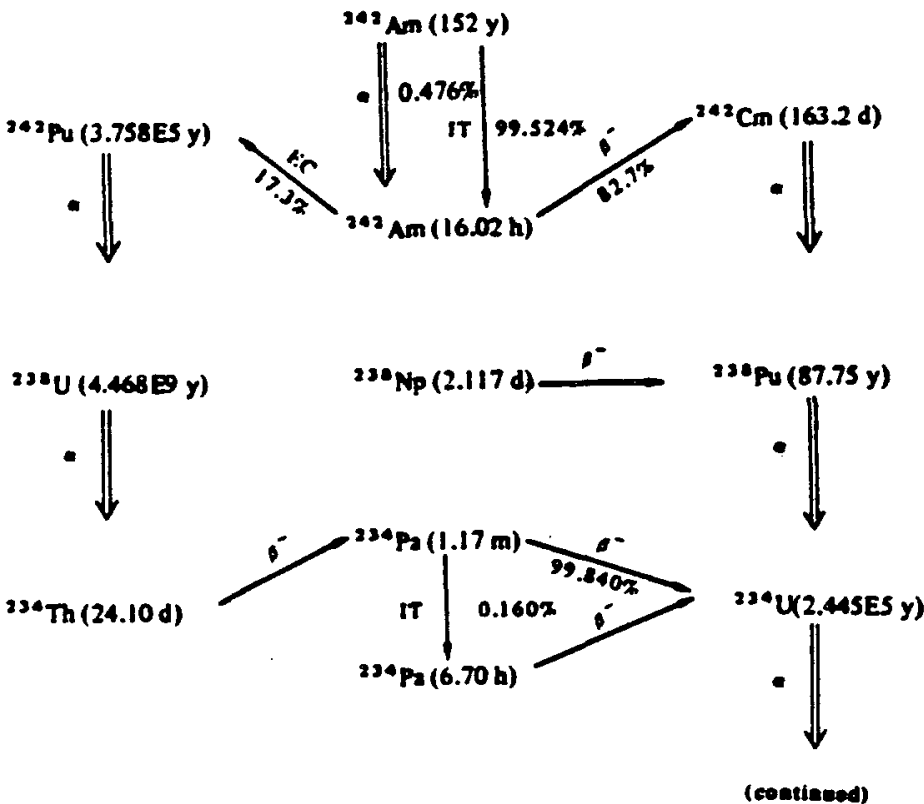


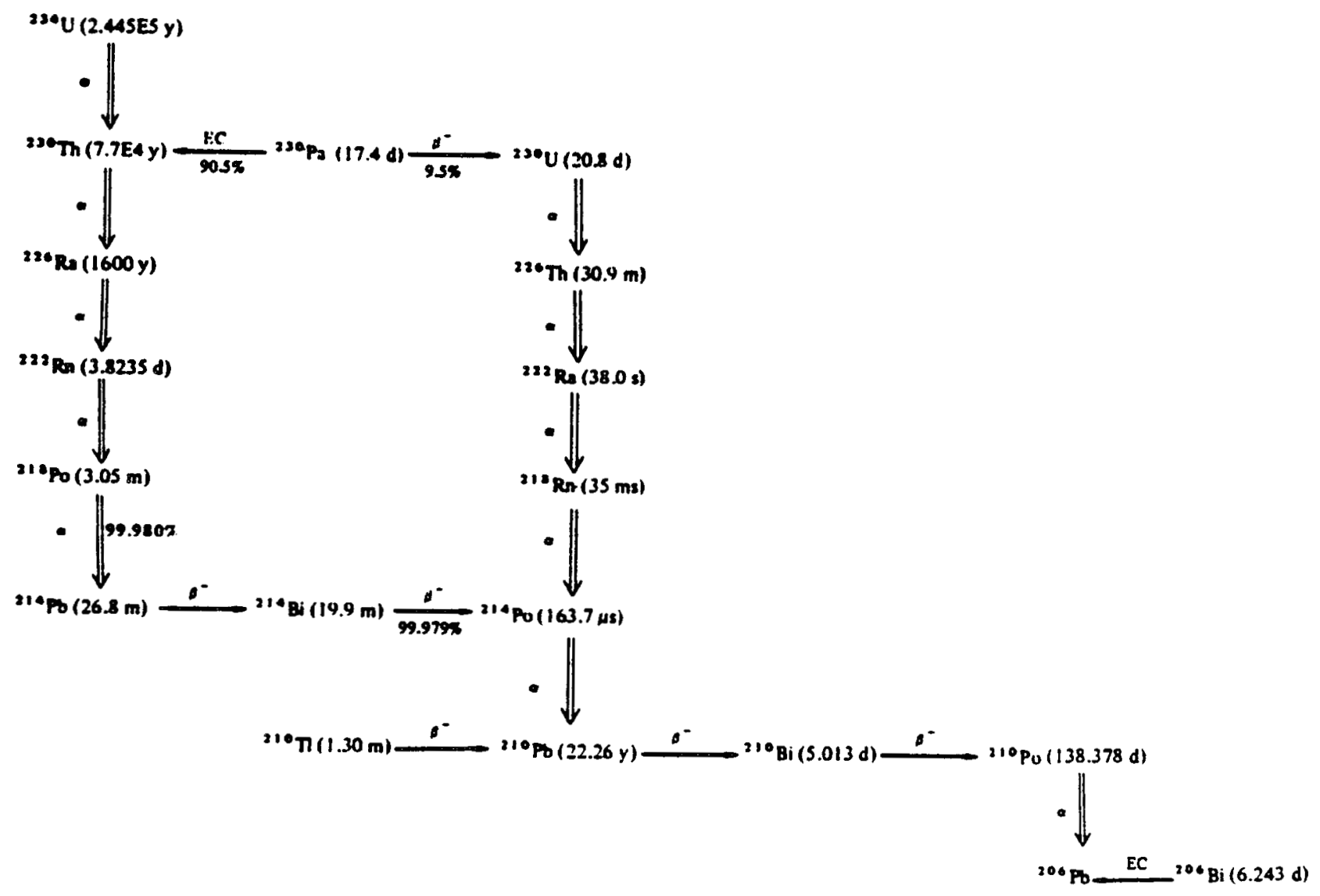




\section{Actinium Series}

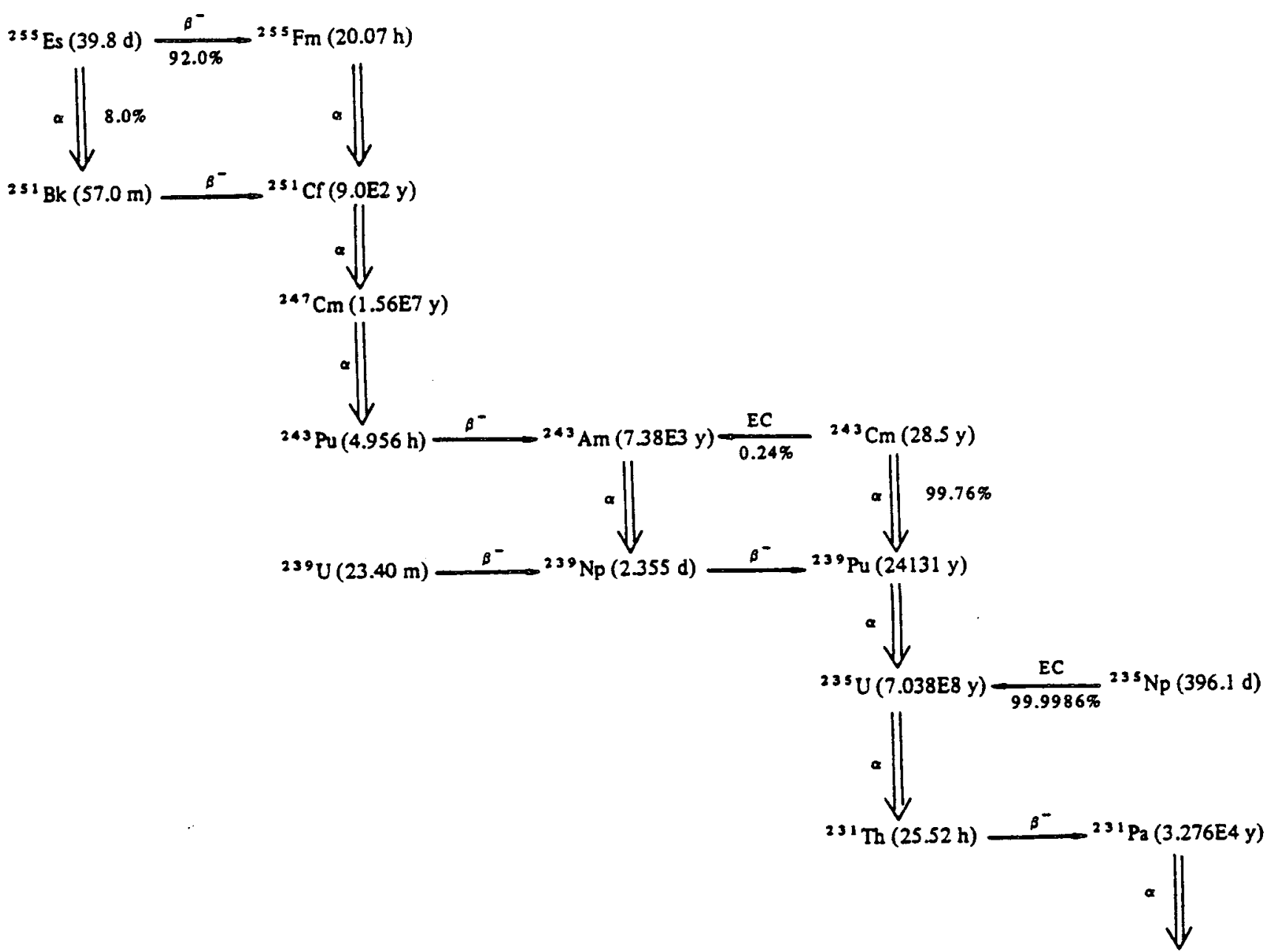

(continued) 


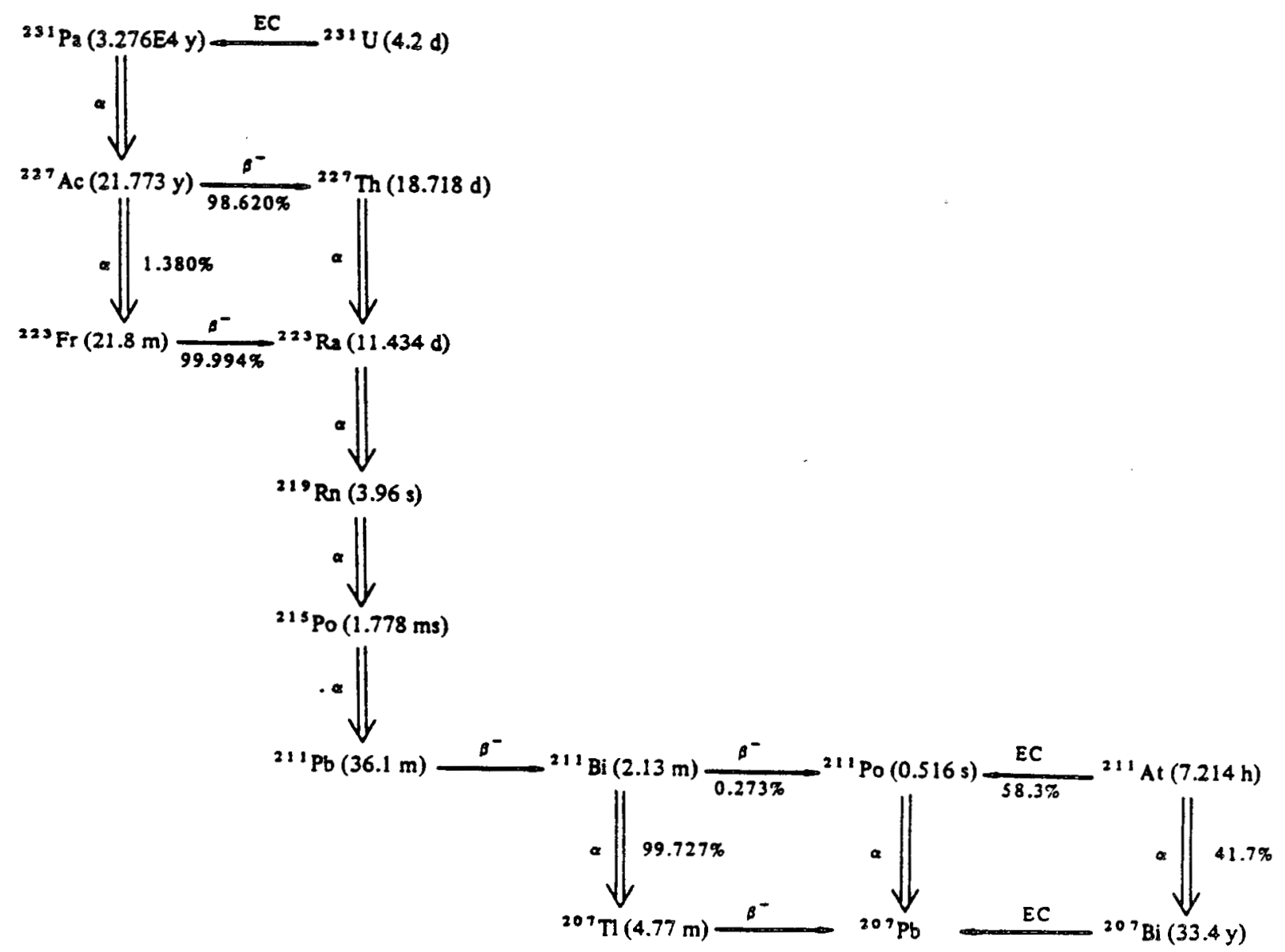


Thorium Seriea

${ }^{256} \mathrm{Fm}(157.6 \mathrm{~m})$

- $8.1 \%$

${ }^{252} \mathrm{Cf}(2.639 \mathrm{y})$

a

${ }^{248} \mathrm{Cm}$ (3.39E5 y)

- $\Downarrow 91.74 \%$

${ }^{244} \mathrm{Pu}(8.26 \mathrm{E} 7 \mathrm{y})$

$\propto$ 99.875\%

${ }^{248} \mathrm{Cr}(333.5 \mathrm{~d})$

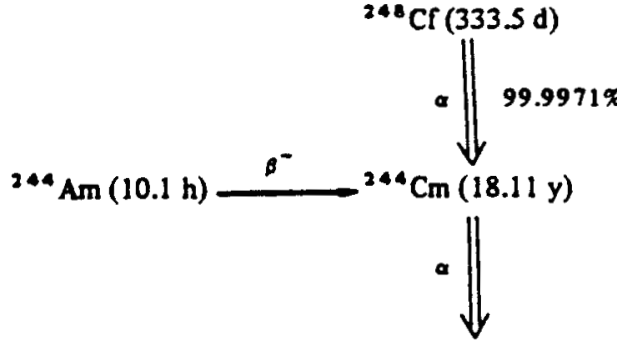

${ }^{240} \mathrm{U}(14.1 \mathrm{~h})$
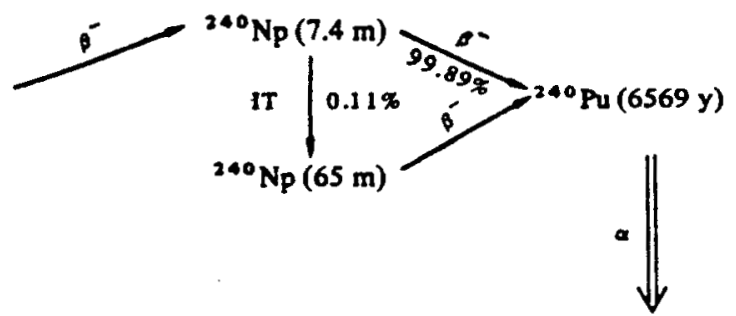

${ }^{236} \mathrm{U}(2.3415 \mathrm{E} 7 \mathrm{y})$

-

हो

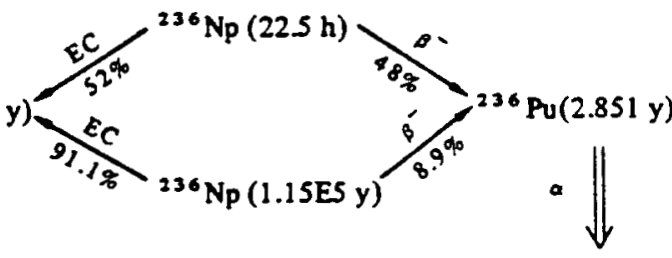

${ }^{232} \mathrm{Th}(1.405 E 10 \mathrm{y})$

a

${ }^{228} \mathrm{Ra}(5.75 \mathrm{y})-\mathrm{B}^{-}{ }^{228} \mathrm{Ac}(6.13 \mathrm{~h})$

${ }^{232} \mathrm{U}(72 \mathrm{y})$

a

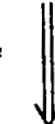

B- $^{228} \mathrm{Th}(1.9132 \mathrm{y})$

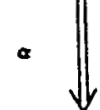

(continued) 
${ }_{189} \mid 90$

${ }^{28} \mathrm{Th}(1.9132 \mathrm{y})$

a

${ }^{224} \mathrm{Ra}(3.62 \mathrm{~d})$

a

${ }^{220} \mathrm{Rn}(55.61 \mathrm{~s})$

a

${ }^{216} \mathrm{PO}(0.146 \mathrm{~s})$

$\therefore$

$212 \mathrm{~Pb}(10.643 \mathrm{~h})$

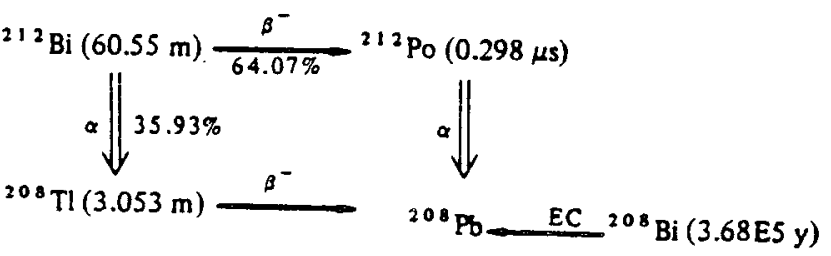




\section{APPENDIX $C$}

IISTING OF THE DOSFACTER COMPUTER CODE

This appendix gives a complete listing of the version of the DOSFACTER computer code that was used to generate the tables of external dose-rate conversion factors for photons and electrons given in Appendix A. 


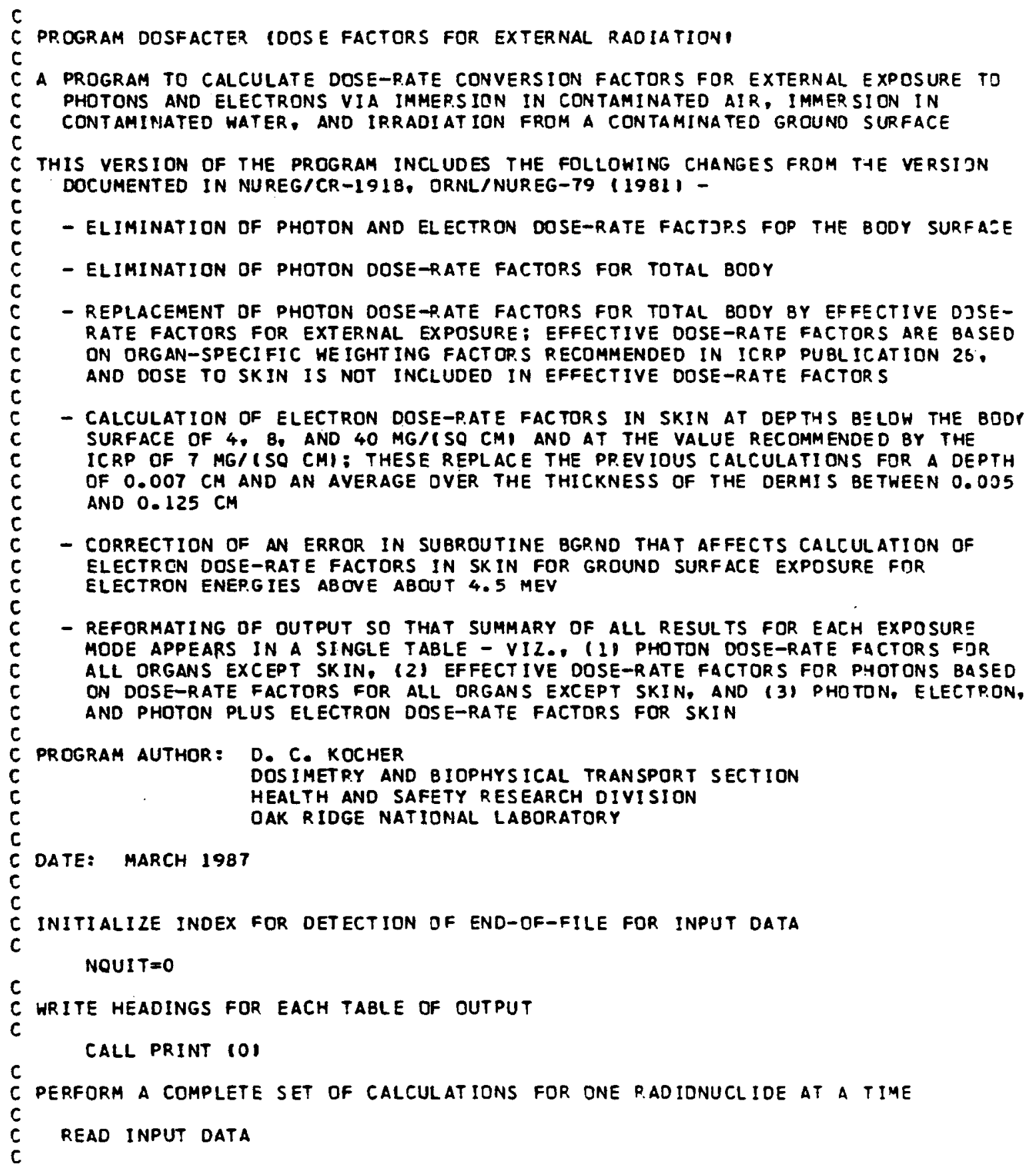


1 CALL DATIN INOUITI

C TEST FOR END-OF-FILE FOR INPUT OATA TO END CALCULATIONS

IF INOUIT . NE. OI GO TO 2

C WRITE INPUT DATA

CALL DATOUT

C Calculate doje-rate conversion factors

C CALL CALC

C HRITE DOSE-RATE CONYERSION FACTORS

CALL DOSOUT

GO TO 1

2 STOP END 
C HP.ITES HEADINGS FOR EACH TABLE DF OUTPUT

NTYPE - INDEX FOR EACH TYPE OF TABLE

= O FOR FIRST TIME THROUGH SUBROUTINE WHEN ALL TABLE HEADINGS ARE WRITTEN

$=1$ TO WRITE HEADINGS FOR RADIONUCLIDE AND SPECTRAL IDENTIFICATION INPUT DATA TABLE ONLY

$=2$ TO WRITE HEADINGS FDR BETA-PARTICLE INPUT DATA TABLE OMYY

$=3$ TO WRITE HEAOINGS FOR POSITRON INPUT DATA TABLE ONLY

$=4$ TO HRITE HEADINGS FOR AUGER AND INTERNAL CONVERSION ELECTRON INPUT DATA TABLE ONLY

$=5$ TO HRITE HEAOINGS FOR PHOTON INPUT DATA TABLE ONLY

$=6$ TO WRITE HEADINGS FOR SUMMARY TABLES OF PHOTON DOSE-RATE FACTDRS FOR ALL. ORGANS EXCEPT SKIN. PHOTON PLUS ELECTRON DOSE-RATE FACTORS FOR SKIN, AND EFFECTIVE DOSE-RATE FACTORS FOR EACH EXPOSURE MODE

$=7$ TO HRI TE HEADINGS FOR TABLES OF ELECTRON DOSE-RATE FACTORS FOR DIFFERENT OEPTHS IN SKIN FOR EACH EXPOSURE MODE

- 8 TO HRITE HEAOINGS FOR. TABLES OF DOSE-RATE FACTOP.S FOR SKIN FOZ PHOTONS, ELECTRONS, ANO PHOTONS PLUS ELECTRONS FOR EACH EXPOSURE MODE

- 9 TO HRITE HEADINGS FOR TABLE OF EFFECTIVE OOSE-RATE faCtORS for all THREE EXPOSURE MODES

IF INTYPE .EQ. 01 GO TO 1

GO TO $11,2,3,4,5,6,7,8,91$, NTYPE

C

C

1 HRITE $(10,10$

10 FORMAT $(1 \mathrm{H} 1)$.

WRITE 110,20

20 FORMAT (IHO, 2X, 4OHRADIONUCLIDE AND SPECTRAL IDENTIFICATION, WRITE $(10,21)$

21 FORMAT (1HO, $42 X, 24 H N U M B E R$ OF SPECTRAL LINES)

WRITE $(10,22)$

22 FORMAT IIH: 34X, 39H WRITE $(10,23)$

23 FDRMAT IIH, 5X, THNUCLIDE, 8X, 9HHALF-LIFE, 5X, 5HBETAS, $4 X_{*}$ 2 9HPOSITRONS, $2 X$, 9HELECTRONS, $3 X_{\text {, THPHOTONS } 1}$

IF INTYPE. NE. OI GO TO 99

C BETA-PARTICLE DATA

2 WRITE $(11,10)$

WRITE $(11,24)$

24 FORMAT IIHO, $2 X, 4$ THENERGIES ANO INTENSITIES FOR EACH BETA PAOTICL 2EI

WRITE $(11.25)$

25 FORMAT IIHO, 36X, 7HMAXIMUM, 9X, 7HAVERAGE, 9X, 9HINTENSITYI WR ITE $(11,26)$

26 FORMAT IIH, 5X, THNUCLIDE, 8X, 9HHALF-LIFE, 5X, 12 2HENERGY (MEVI, 
$24 X$, 12HENERGY (MEV). 5X, 11H(PER DECAY))

$c$

IF INTYPE. NE. OI GO TO 99

POSITRON DATA

3 WP.ITE $(12,10$

WRITE $(12,271$

27 FORMAT 11 HO, $2 X$, 42HENERGIES ANO INTENSITIES FOR EACH POSITROYI

WRITE $(12,25)$

WRITE $(12.26)$

IF INTYPE . NE. 01 GO TO 99

C AUGER ANO INTERNAL CONVERSION ELECTRON DATA

C

4 WRITE $(13,10)$ WRITE $(13,28)$

28 FORMAT 1 IHO, $2 X$, T2HENERGIES AND INTENSITIES FOR EACH AUGER ANO IN 2TERNAL CDNVERSION ELECTRON

WRITE $(13,29)$

29 FORMAT (1HO, $37 X$, GHENERGY, 9X, 9HINTENSITY)

WRITE $(13.30)$

30. FORMAT IIH, 5X, THWUCLIDE, 8X, 9HHALF-LIFE, 9X, 5HQMEVI, $8 X_{0}$ 2 IIHIPER DECAYII

C PHOTON DATA IF INTYPE. NE. OI GO TO 99

5 WRITE $(14,10)$

WRITE (14,31)

31 FORMAT IHO, 2X, 4OHENERGIES AND INTENSITIES FOR EACH PHOTONI WRITE $(14,29)$

WRITE 114,30

IF INTYPE NE, OI GO TO 99

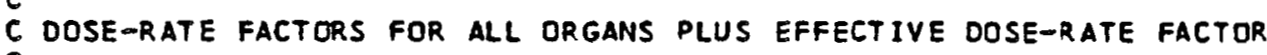

C AIR IMMERSION

6 WRITE $(15.10)$ WRITE $(15,41)$

41 FORMAT I 1HO, 2X, 98HDOSE-RATE FACTORS FOR BODY ORGANS AND EFFECTIV 2E DOSE-RATE FACTOR FOR IMMER SION IN CONTAMINATED AIRI HRITE $(15,42)$

42 FORMAT (IH, 5X, 35HIN MREM/YR PER MICROCURIE/(CUBIC M)) WRITE 115,431

43 FORMAT (1HO, 92X, 5HUPPER, 6X, 5HL OWERI WRITE $(15,44)$

44 FORMAT IIH, $B I X$, 5HSMALL, 6X, 5HLARGE, 6X, 5HLARGE WRITE 115,451

45 FORMAT IIH. 1X, THNUCLIDE, 4X, 9HHALF-LIFE, $3 X_{\text {, }} 8$ HADRENALS, $4 X$, 2 THBLADDER, 5X, 5HBRAIN, 5X, 6HBREAST, 6X, 5HHEART, $4 X_{0}$ 3 9HINTESTINE, $2 X$, 9HINTESTINE, $2 X$, 9HINTESTINE, $3 X$, THKIONEYSI HRITE 116,10 :

UR ITE $(16,41)$

WRITE $(16,42)$

WRITE 110.46

46 FORMAT (1HO, 6OX, 3HRED)

WRITE (16.4T)

47 FORMAT IIH, 1X, 7HNUCLIOE, 4X, 9HHALFLIFE, 5X, 5HLIVER, 6X, 
2 5HLUNGS, 5X, GHMARROH, 5X, GHMARROH, 5X, THOVARIES, $3 x$, 3 BHPANCREAS, $3 x$, BHSKELETON, $4 x$, 6HSPLEEN, $5 x$, THSTOMACH/I WRITE $(17.10)$

WRITE $(17,41)$

WRITE $(17,42)$

WPITE 117.601

60 FORMAT IIHO, 81X, 4HSKIN, 7X, 4HSKIN, 7X, 4HSKINI HRITE $(17.61)$

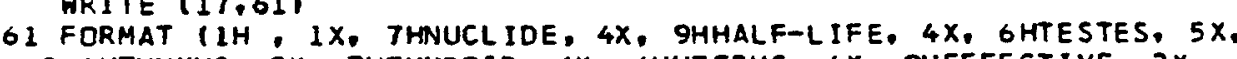
2 GHTHYMUS, $5 x$, 7HTHYROID, $4 x$, GHUTERUS, $4 x$, 9HEFFECTIVE, $2 x$. 3 BH(PHOTON, $2 x, 1$ OHIELECTRON), $3 x$, THITOTALI/1

$c$
$c$

HATER IMHERSION

WRITE $(20.101$

WRITE $(20,48)$

48 FORMAT 1 HO, $2 X, 100$ HOOSE-RATE FACTORS FOR BOOY ORGANS AND EFFECTI 2VE DOSE-RATE FACTOR FOR IMMERSION IN CONTAMINATED HATERI

WR I TE $(20,42)$

HRITE $(20.43)$

WRITE $(20.44)$

WRITE $(20,45)$

WRITE 121,101

WRITE (21.48)

HRITE $(21,421$

WRITE $(21,46)$

HRITE $(21.47)$

WRITE 122.10

WR.ITE 122.481

WRITE $(22,42)$

WRITE $(22,60)$

WRITE $(22,61)$

c

GROUND SURFACE EXPOSURE

\section{HRITE $(25,10)$}

WRITE $(25,49)$

49 FORMAT I1HO, 2X, I15HOOSE-RATE FACTORS FOR BODY ORGANS AND EFFECTI 2VE DOSE-RATE FACTOR FOR EXPOSURE I M ABOVE CONTAMINATED GROUND SUR 3FACE)

WRITE $(25,50)$

50 FORMAT IIH, 5X, 36HIN MREM/YR PER MICPOCURIE/ISQUARE MI)

WR ITE $(25,43$

UR ITE $(25,44)$

WRITE $(25,45)$

WRITE $(26,10)$

WRITE $(26.49)$

WRITE $(26,50)$

WRITE $(26,46)$

WRITE $(26,47)$

WRITE $(27,10)$

WRITE 127.491

WRITE $(27.50)$

WRITE $(27,60)$

WRITE $(27.61)$

IF INTYPE. NE. O) GO TO 99

c

ELECTRON DOSE-RATE FACTORS FOR DIFFERENT DEPTHS IN SKIN 


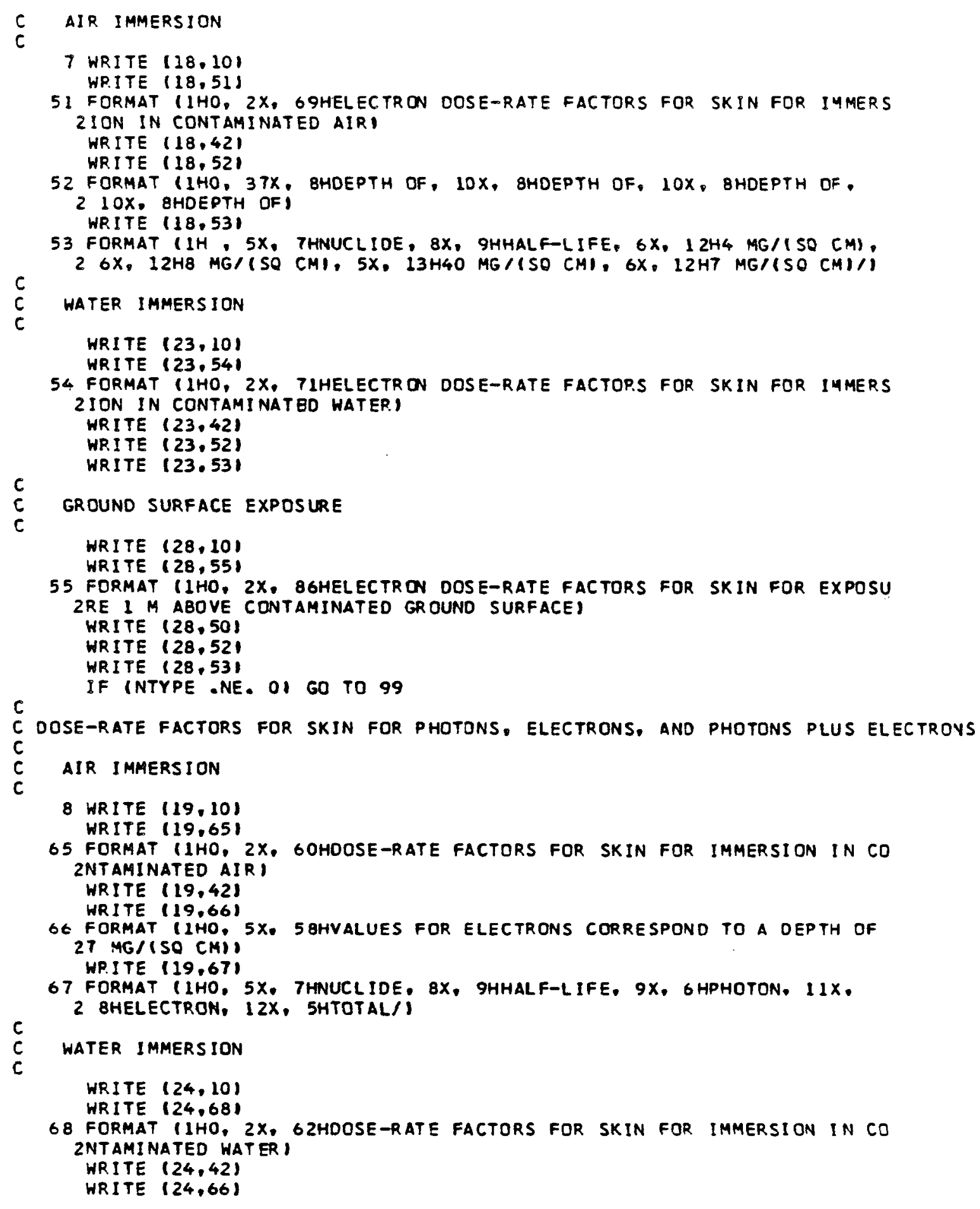


WRITE $(24,67)$

GROUNO SUP.FACE EXPOSURE

WRITE $(29,10)$

WRITE $(29,69)$

G9 FORMAT (1HO, $2 X$, TTHDOSE-RATE FACTORS FOR SKIN FOR EXPOSURE I M AB 2OVE CONTAMINATED GROUND SURFACEI

HRITE $(29.50)$

WRITE $(29,66)$

WRITE $(29,67)$

IF INTYPE .NE. OI GO TO 99

C EFFECTIVE DOSE-RATE fACTORS FOR AIR IMMERSION. HATER IMMERSION, AND GROUNO SURFACE EXPOSURE

9 WRITE $(30,10)$

WRITE $(30,32)$

32 FORMAT I1HO, 2X, 54HEFFECTIVE DOSE-RATE FACTORS FOR PHOTONS PL US E 2LECTRONSI

WRITE 130.331

33 FORMAT (1H, 5X, 67HIN MREM/YR PER MICROCURIE/ICUB IC M) FOR AIR AN 20 WATER IMMERSION ANDI

WRITE $(30,34)$

34 FORMAT (1H, 5X, 64HIN MREM/YR PER MICROCURIE/ISOUAP.E MI FOR GROUN 20 SURFACE EXPOSUREI

HR.ITE 130,351

35 FORMAT (1HO; 39X. 3HAIR, 14X, 5HWATER, 13X, 6HGROUND)

36 FORMAT IIH, 5X, THNUCLIDE, 8X, 9HHALF-LIFE, 7X. 9HIMMERSION, 9X, 2 9HIMMERS $10 \mathrm{~N}, 10 \mathrm{X}$, 7HSURFACE $/ 1$

99 RETURN

END 
C

$C$ C READS INPUT DATA FOR EACH NUCL IDE

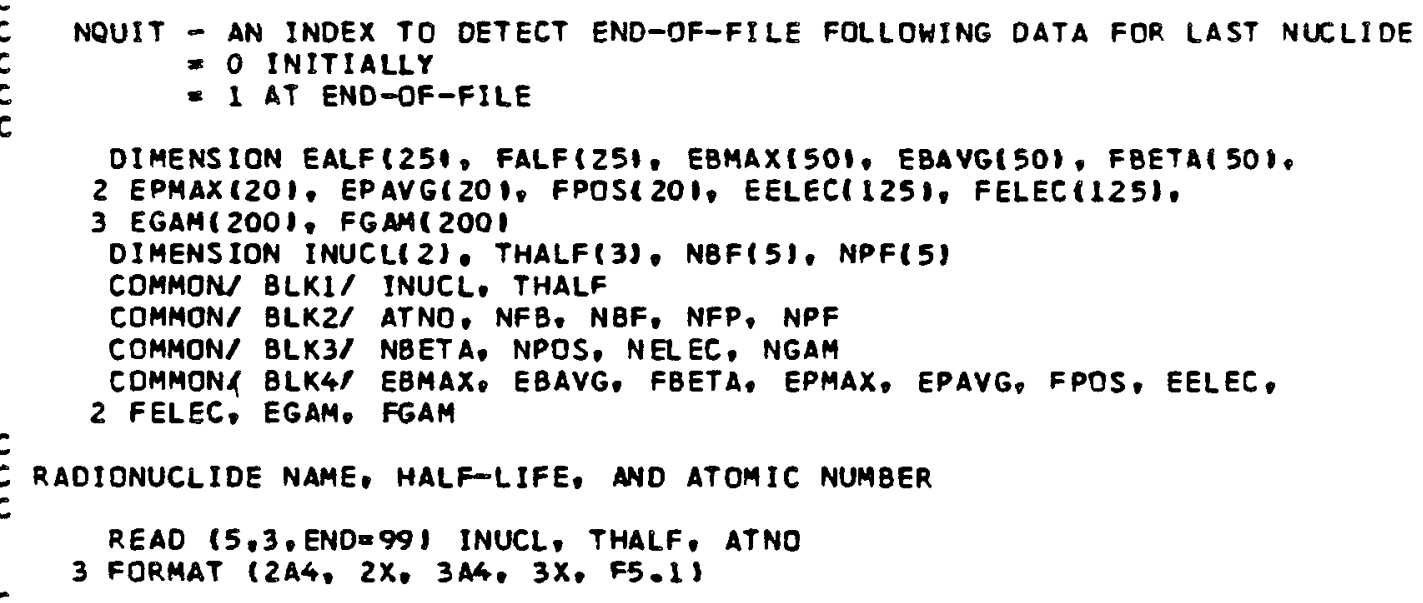


C DECAY

READ $(5,6)$ (EPMAX (J), EPAVG(J), FPOS(J), J=1,NPOS)

C NUMBER OF AUGER ANO INTERNAL CONVERSION ELECTRONS ILIMIT OF 1251

C T READ $(5,4)$ NELEC

IF INELEC. .EO. O) 60 TO 8

$c$

C AUGER AND INTERNAL CONVERSION ELECTRON ENERGIES IN MEV AND INTENSITIES IN NUMBER PER DECAY

C NUMBER OF PHOTONS ILIMIT OF 2001

READ (5.6) (EELECIJ). FELECIJ), J=1, NELEC)

8 READ 15,4$)$ NGAM

IF INGAM . EQ. OI GO TO 1

C $c$ PHOTON ENERGIES IN MEV ANO INTENSITIES IN NUMBER PER DECAY

READ (5.6) (EGAM(J), FGAM(J), J=1, NGAM)

GO TO 1

99 NOUIT $=1$

1 RETURN

END 
C

SUBROUTINE DATOUT

C WRITES INPUT DATA FOR EACH NUCLIDE

DIMENSION EBMAXI50). EBAVG (50), FBETA150), EPMAX1201. EPAVG(20). 2 FPOS (20), EELEC (125), FELEC (125), EGAM(200), FGAM(200)

DIMENSION INUCLI2I. THALFI3!

COMMON/ BLKL/ INUCL. THALF

COMMON/ BLK3L NBETA, NPOS, NELEC. NGAM

COMMON/ BLK4/ EBMAX, EBAVG, FBETA, EPMAX, EPAVG, FPOS, EELEC,

2 FELEC, EGAM, FGAM

C MAXIMUM NUMBER OF LINES PER PAge foR EACH tYPE OF dATA tABLE

DATA MAXLID/51/, MAXLSP/52/

c

LOGICAL FIRST/. TRUE.J

C TEST FOR FIRST TIME THROUGH SUBROUTINE

IF I.NOT. FIRSTI GO TO 9

C INITIALIZE LINE COUNT FOR EACH TABLE: RESET LOGICAL VARIABLE

LINID $=0$

LINBET $=0$

LINPOS $=0$

LINEL $=0$

LINGAM $=0$

FIR ST $=$. FALSE.

C RADIONUCLIDE AND SPECTRAL IDENTIFICATION

C HRITE $(10,10)$ INUCL. THALF, NBETA, NPOS, NELEC, NGAM

10 FORMAT (1H, 4X, 2A4, 5X, 3A4, 4X, 14, 3(7X, I4I)

LINID $=$ LINID+1

IF ILINIO LT. MAXLIDI GO TO 11

CALL PRINT (I)

IINID $=0$

11 IF INBETA .EO. OI GO TO 12

C BETA-PARTICLE DATA

CALL DATLIN (NBETA, EBMAX(1), EBAVG(1), fBeta(1), LINBET, MaXLSP, $21.11,21$

IF INBETA.EO. 1 I GO TO 12

DO $15 \mathrm{~J}=2$, NBETA

CALL DATLIN INBETA, EBMAXIJ), EBAVGIJ), FBETAIJ), LINBET, MAXL SP, $2 \mathrm{~J} .11 .21$

15 CONTINUE

C POSITRON DATA

12 IF INPOS.EO. OI GO TO 18 


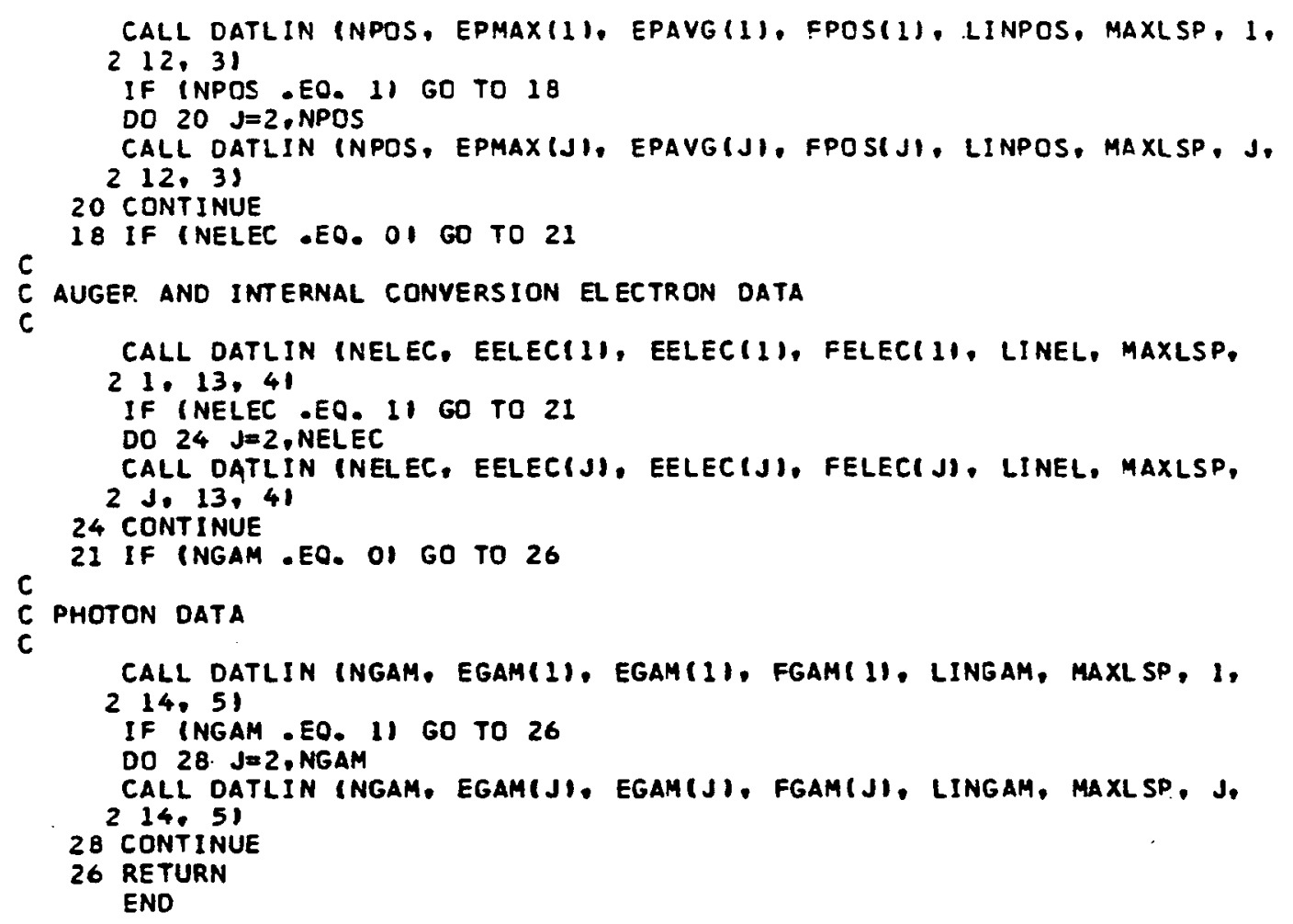


SUBROUTINE DATLIN INPART, EMAX, EAVG, FINT, LINE, MAXLIN, NLINE, 2 NPR INT, NTYPEI

C

C WRITES EACH LINE OF BETA-PARTICLE, POSITRON, AUGER AND INTERNAL CONVEPSIOV

C ELECTRON, OR PHOTON DATA

C NPART - NUMBER OF SPECTRAL LINES JF PARTICULAR RADIATION TYPE

C EMAX - ENDPOINT ENERGY OF PART ICULAR SPECTRAL LINE

C EAVG - $\triangle V E R A G E$ ENERGY OF PARTI CULAR SPECTRAL LINE

FINT - INTENSITY OF PARTICULAR SPECTRAL LINE

LINE - LINE COUNT FOR DATA TABLE FOR PARTICULAR, SPECTPAL LINE

MAXLIN - MAXIMUM LINE COUNT FOR DATA TABLE FOR PARTI CULAR RADIATION TYPE

NLINE - INDEX FOR. SPECTRAL LINE OF PARTICULAR RADIATION TYPE

NPRINT - DUTPUT DEVICE NUMBER

NTYPE - INDEX FOR RADIATION TYPE ISEE SUBROUTINE PRINT FOR LISTINGI

DIMENSION INUCL(2), THALF(3)

COMMON/ BLKL/ INUCL. THALF

C TEST FOR FIRST SPECTRAL LINE OF PARTICULAR RADIATION TYPE

IF INLINE. NE. I) GO TO 1

C WRITE FIRST SPECTRAL LINE

C TEST FOR CONTINUOUS DR DISCRETE RAOIATION

C IF IEMAX . EO. EAVGI GO TO 2

C BETA-PARTICLE OR POSITRON DATA

WRITE (NPRINT, 31 INUCL, THALF, EMAX, EAVG, FINT

3 FORMAT (1HO, 4X, 2A4, 5X, 3A4, 7X, 2(FT.4, 9X), FB.5) GO TO 4

C AUGER AND INTERNAL CONVERSION ELECTRON OR PHOTON DATA

C HRITE INPRINT, 51 INUCL. THALF, EMAX. FINT

5 FORMAT IIHO, 4X, 2A4, 5X, 3A4, 7X, F7.4, 9X, F8.51

4 LINE $=$ LINE+2

GO TO 6

C HRITE SECOND ANO SUBSEOUENT SPECTRAL LINES USING SAME PROCEDURE AS above

1 IF (EMAX EO, EAVGI GO TO 7

WRITE (NPRINT, B) EMAX, EAYG, FINT

8 FORMAT IIH, 36X, 2IFT.4, 9X), FB.5

GO TO 9

7 WRITE (NPRINT, 10$)$ EMAX, FINT

10 FORMAT (IH, 36X, F7.4, 9X, F8.5)

9 LINE $=L I N E+1$

C TEST FOR END OF PAGE 
6 IF (LINE IT. MAXLIN) GO TO 11

C WRITE TABLE heAoINGS ON NEH PAGE ANO SKIP a line after headings

C WRITE TABLE HEAOINGS ON

$C A L L$
LINE $=0$

IF INLINE .EQ. 1 . AND. NPART .NE. II WRITE INPP.INT.131

13 FORMAT IIH

IF INLINE .NE. 1 . AND. NLINE .NE. NPARTI HRITE INPRINT, 13 I

11 RETURN

END 
CALCULATES DOSE-RATE CONVERSION FACTORS FOR EXTERNAL EXPOSURE TO PHOTONS ANO ELECTRONS

DIMENSION EBMAX(50), EBAYG 50$)$. FBETA150). EPMAX1201. EPAVG1201. 2 FPOS (20). EELEC(125). FELEC $(125)$, EGAMI 2001. FGAM 12001

OIMENSION NBF(51. NPF(5)

DIMENS I ON ER (25). FMUEAR (25). FMUEHR (25). FMUETR(25), FMUAR 251 ,

2 SPAR (25), SPWR (25), SPTR (25), RNARI25), RNTRI 25). CARI25).

3 DAR $(25)$

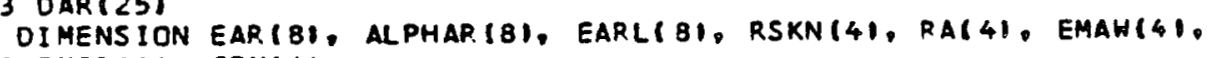

2 EMGR (4). CPM( 6 )

DIMENSION GTAR (25), GTWR (25), BTAR (25), BTHR (25)

DIMENSION EBSR(I201). RTAR $(600)$, RTHR (600), ALR 6001 . FIE(60)

DIMENSION SGAORG 231 , SGHORG(23), SGGORG1231, SBASKN(4).

2 SBWSKN(4), SBGSKN(4)

DIMENSION ORGF AC $(231$, GFAC14,600)

DI MENSI ON DOSORG $(3,23)$, DOSORB $(3,4)$, DOSSKN 31 , DOSEFF(3)

DIMENSION DEFF $(3,15)$, DEFFS $(3,15)$, DUMI $(15)$, DUM2 $(15)$

COMMON/ BLK2/ ATNO. NFB, NBF, NFP, NPF

COMMON BLK3/ NBET A, NPOS, NELEC, NGAM

COMMON/ BLK4/ EBMAX, EBAVG. FBETA, EPMAX, EPAYG, FPOS, EELEC,

COMMON/ BLK4/ EBMAX,
2 FELEC. EGAM, FGAM

COMMON/ BLKS/ DOSORG, DOSORB, DOSSKN, DOSEFF

DOUBLE PRECISION EI

C ENERGIES FOR REFER ENCE VALUES OF PHOTON ENERGY-ABSORPTION COEFFICIENTS, ATTENUATION COEFFICIENTS, BUILDUP FACTOR PARAMETERS. ANO ORGAN DOSE-RATE FACTORS; AND ELECTRON STOPPING POWEP.S AND RANGES - VALUES IN MEV

c

$\begin{array}{rrrrrrrr}\text { DATA ERI } & 0.010, & 0.015, & 0.020, & 0.030, & 0.040, & 0.050 \\ 2 & 0.060, & 0.080, & 0.100, & 0.150, & 0.200, & 0.300, & 0.400, \\ 3 & 0.500, & 0.600, & 0.800, & 1.0, & 1.5, & 2.0 \% & 3.0 \% \\ 4 & 4.0, & 5.0, & 6.0, & 8.0, & 10.0 \% & & \end{array}$

C REFERENCE VALUES OF PHOTON MASS ENERGY-ABSORPTION COEFFICIENTS IN AIR IN C ISO CMIIG

$\begin{array}{cccccccc}\text { DATA FMUEAR/ } & 4.61, & 1.27, & 0.511, & 0.148, & 0.0668, & 0.0406, \\ 2 & 0.0305, & 0.0243, & 0.0234, & 0.0250, & 0.0268, & 0.0287, & 0.0295, \\ 3 & 0.0296, & 0.0295, & 0.0289, & 0.0278, & 0.0254, & 0.0234, & 0.0205, \\ 4 & 0.0186, & 0.0174, & 0.0164, & 0.0152, & 0.0145, & & \end{array}$

C REFERENCE VALUES OF PHOTON MASS ENERGY-ABSORPTION COEFFICIENTS IN HATER IN C ISO CMI/G

$\begin{array}{ccccccccc}\text { CATA FMUEWR } & 4.79, & 1.28, & 0.512, & 0.149, & 0.0677, & 0.0418, \\ 2 & 0.0320, & 0.0262, & 0.0256, & 0.0277, & 0.0297, & 0.0319, & 0.0328, \\ 3 & 0.0330, & 0.0329, & 0.0321, & 0.0309, & 0.0282, & 0.0260, & 0.0227, \\ 4 & 0.0206, & 0.0191, & 0.0180, & 0.0166, & 0.0157, & & \end{array}$

C REFERENCE YALUES OF PHOTON MASS ENERGY-ABSORPTION COEFFICIENTS IN TISSUE

C (MUSCLE) IN ISO CMI/G 
c

$\begin{array}{cccccccc}\text { DATA FMUETRI } & 4.87, & 1.32, & 0.533, & 0.154, & 0.0701, & 0.0431, \\ 2 & 0.0328, & 0.0264, & 0.0256, & 0.0275, & 0.0294, & 0.0317, & 0.0325, \\ 3 & 0.0328, & 0.0325, & 0.0318, & 0.0308, & 0.0282, & 0.0259, & 0.0226, \\ 4 & 0.0203, & 0.0188, & 0.0178, & 0.0163, & 0.0154 ; & & \end{array}$

C REFERENCE VALUES OF PHOTON MASS ATTENUATION COEFFICIENTS IN AIR IN ISO CMIIG

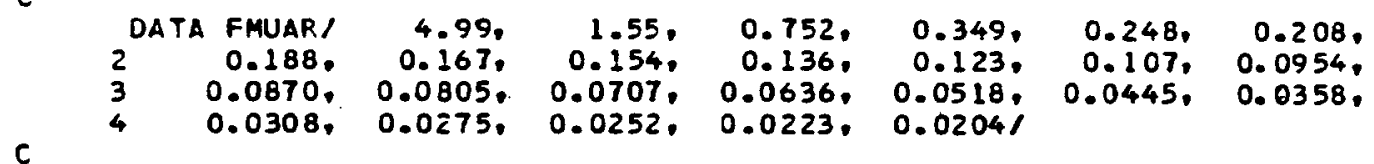

C REFERENCE VALUES OF PHOTON TISSUE-TO-AIR DOSE RATIO FDR IMMERSION IN CONTAMINATED AIR; VALUES ARE RATIO OF MASS ENERGY-ABSORPTION COEFFICIENTS AVERAGED OVER SPECTRUM OF PHOTONS IN AIR FROM MDNOENERGETIC SOURCES AND ARE OBTAINED FROM FIG. 6 OF DILLMAN, HEALTH PHYSICS 27, 571119741

$\begin{array}{llllllll}\text { DATA GTAR/ } & 0.954, & 0.954, & 0.956, & 0.961, & 0.973, & 0.985, \\ 2 & 0.998, & 1.018, & 1.032, & 1.054, & 1.066, & 1.078, & 1.085, \\ 3 & 1.089, & 1.091, & 1.094, & 1.096, & 1.099, & 1.00,100, & 1.098,\end{array}$

C REFERENCE VALUES OF ELECTRON MASS STOPPING POHERS IN A IR. IN (MEV-SO CMI/G C

$\begin{array}{cccccccc}\text { DATA SPARI } & 19.7, & 14.4, & 11.6, & 8.48, & 6.84, & 5.81 . \\ 2 & 5.11, & 4.20, & 3.63, & 2.86, & 2.47, & 2.09, & 1.91 . \\ 3 & 1.81 . & 1.75, & 1.70, & 1.68, & 1.68, & 1.71, & 1.79 .\end{array}$

C REFERENCE VALUES OF ELECTRON MASS STOPPING POWERS IN WATER IN (MEY-SO CMI/G C

$\begin{array}{lccccccc}\text { DATA } & \text { SPHR } & 23.2, & 16.9, & 13.5, & 9.88, & 7.96, & 6.75, \\ 2 & 5.92, & 4.86, & 4.20, & 3.30, & 2.85, & 2.40, & 2.19, \\ 3 & 2.07, & 2.00, & 1.93, & 1.89, & 1.88, & 1.89, & 1.93 . \\ 4 & 1.97, & 2.01, & 2.05, & 2.12, & 2.18 \% & & \end{array}$

C REFERENCE VALUES OF ELECTRON MASS STOPPING POWERS IN TISSUE IMUSCLEI IN C IMEV-SO CMI/G

$\begin{array}{rrrrrrrr}C \text { DATA SPTR/ } & 22.9, & 16.7, & 13.4, & 9.77, & 7.86, & 6.67 \% \\ 2 & 5.86, & 4.80, & 4.15, & 3.27, & 2.82, & 2.38, & 2.17 \% \\ 3 & 2.05, & 1.98, & 1.92, & 1.89, & 1.89, & 1.92, & 1.99, \\ 4 & 2.06, & 2.13, & 2.18, & 2.28, & 2.37 \% & & \end{array}$

C REFERENCE VALUES OF ELECTRON RANGES IN AIR IN G/(SO CM)

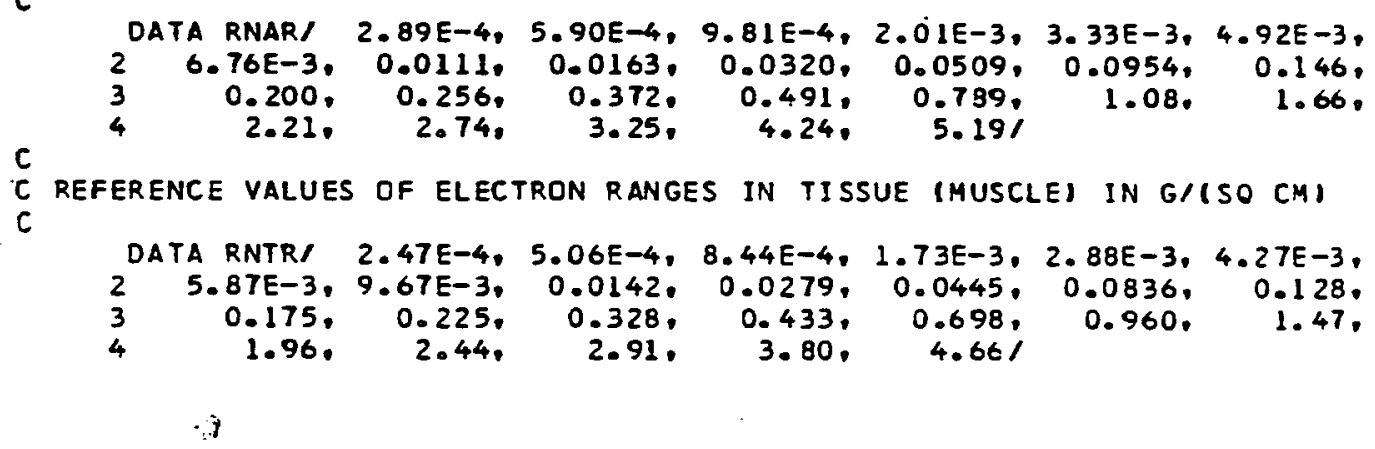


C REFERENCE VALUES OF BERGER C AND O COEFFICIENTS FOR PHOTON ENEPGY-ABSORPTION BUILDUP FACTORS IN AIR

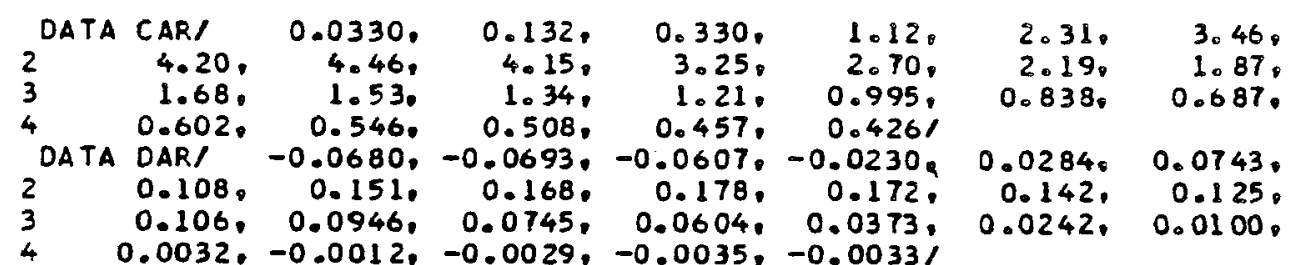

C

ENERgIES FOR. REFERENCE VALUES OF ELECTRON SCALING PARAMETER - ALPHA For aIR IMMERSION

$2_{2.01}^{\text {OATA EARI } 0.010,0.020,0.050,0.100,0.200,0.500,1.0,}$

C REFERENCE VALUES OF ELECTRON SCALING PARAMETER - ALPHA' for aIR IMMERSI ON

DATA ALPHAR/ 1.022,1.018, 1.018,1.018,1.017,1.018,1.009,

C FACTOR TO CONVERT ABSORBED ENERGY IN MEY TO DOSE EOUIVALENT IN REM IN (G-REM) /MEV

C NUMBER OF SECONOS PER YEAR

DATA FK, FS/ $1.60206 E-08,3.1536 E+07 /$

C DENSITY OF DRY AIR AT 20 DEG C AND 750 MM PRESSURE IN G/ICU CMI

$C$ OENSITY OF WATER AT 20 DEG C IN G/ICU CMI

C HEIGHT OF REFERENCE POSITION FOR GROUND SURFACE EXPOSURE IN CM

DATA RHOA. RHOW, $2 / 1.189 E-03,0.99823,100.0 /$

C DEPTHS OF SKIN BELON BODY SURFACE AT HHICH ELECTRON DOSE-RATE FACTORS ARE CALCULATED IN G/ ISO CMI

DATA RSKN/ 4.OE-3, 8. OE-3, 4.OE-2, 7.0E-3/

C PARTICLE-MEOIUM DOSE-RATE CORRECTION FACTORS: ORDER IS PHOTON DOSE-RATE
C CORRECTION FACTOR FOR AIR IMMERSION, HATER IMMERSION. AND GR OUND SURFACE

C EXPOSURE, AND ELECTRON DOSE-RATE CORRECTION FACTOR FOR THREE EXP OSURE MODES

DATA CPM $0.5,2 * 1.0 .3 * 0.5 /$

LOGICAL FIRSTI. TRUE.I

C TEST FOR FIRST TIME THROUGH SUBROUTINE

IF I.NOT. FIRSTI GC TO 19

C CALCULATE REFERENCE VALUES OF RATIOS OF PHOTON MASS ENERGY-ABSORPYION

C CDEFFICIENTS IN TISSUE TO VALUES IN HATER AND ELECTRON MASS STOPPING POWERS IN TISSUE TO VALUES IN AIR AND WATER AT ENERGIES GIYEN BY YALUES OF ER

$00 \quad 20 \quad I=1 \cdot 25$

GTWR (I)=FMUETR(I I/FMUEWR (I) 
BTAR $(I)=S P T R(I) / S P A R(I)$
BTHR $(I)=S P T R I I) / S P H R(I)$

20 CONTINUE

C CALCULATE CONSTANT FACTORS IN DOSE-RATE FACTOR EQUATIONS TO GIVE OUTPUT IN DESIRED UNITS

$F A C 1=37 . * F S * F K$

FACZ $=3700 . * F S * F K$

$C$

CALCULATE HEIGHT OF REFERENCE POSITION FOR GROUNO SURFACE EXPOSURE IN G/ (SO CM)

$R=R H O A * Z$

C CALCULATE MINIMUM ELECTRON ENERGIES CONTR IBUTING TO DOSE-RATE FACTORS FOR SKIV FOR DIFFERENT DEPTHS BELOW BODY SURFACE

AIR AND HATER IMMERSION

$00611=1.4$

EMAH I I) $=Y$ INTER (RSKNIII, RNTR, ER, 0, 3, 25)

61 CONT INUE

C GROUND SURface EXPOSURE; CALCULATIONS ASSUME THAT THICKNESS OF TISSUE IS REPLACED BY EQUIVALENT THICKNESS OF AIR

DO $62 \quad I=1.4$

RA $(I)=R+R S K N(I) * 1 \cdot 14$

EMGR I I I=Y INTER IRA I1), RNAR, ER, 0, 3, 25 I

62 CONTINUE

C DEFINE ENERGIES FOR CALCULATING SPECTRUM FOR BETA PARTICLE OR POSI TRON--VALUES C ARE EVERY $10 \mathrm{KEV}$ FROM 0 TO 12 MEV

EBSR I 1 I $=0.0$

DO $39 I=2,1201$

$\operatorname{EBSR}(I)=\operatorname{EBSR}(I-1)+0.010$

39 CONTINUE

c

AT ENERGIES GIVEN BY ALTERNATE VALUES OF EBSR. CALCULATE RATIOS OF ELECTRON MASS STOPPING POWER IN TISSUE TO VALUES IN AIR AND WATER, VALUE OF ELECTRDN SCALING PARAMETER 'ALPHA* AND GEOMETRICAL REDUCTION FACTOP.S FOR ELECTRON DOSE-RATE FACTORS FOR DIFFERENT DEPTHS BELOH BODY SURFACE FOR AIR ANO WATER IMMERSION

CALCULATE LOGAR ITHM OF REFERENCE ENERGIES FOR ELECTRON SCALING PARAMETER

DO $63 I=1.8$

EARL (I)=ALOG (EAR (I)

63 CDNTINUE

$0064 \mathrm{~J}=2,1200,2$

$\mathrm{K}=\mathrm{J} / 2$

CALCULATE RATIOS OF ELECTRON MASS STOPPING POWER IN TISSUE TO VALUES IN AIR AND HATER

RTAR (K)=YINTER (EBSR ( J), ER, BTAR, 0, 3, 25!

RTWR $(K)=Y$ INTER IEBSR (J), ER, BTHR, 0, 3, 25) 


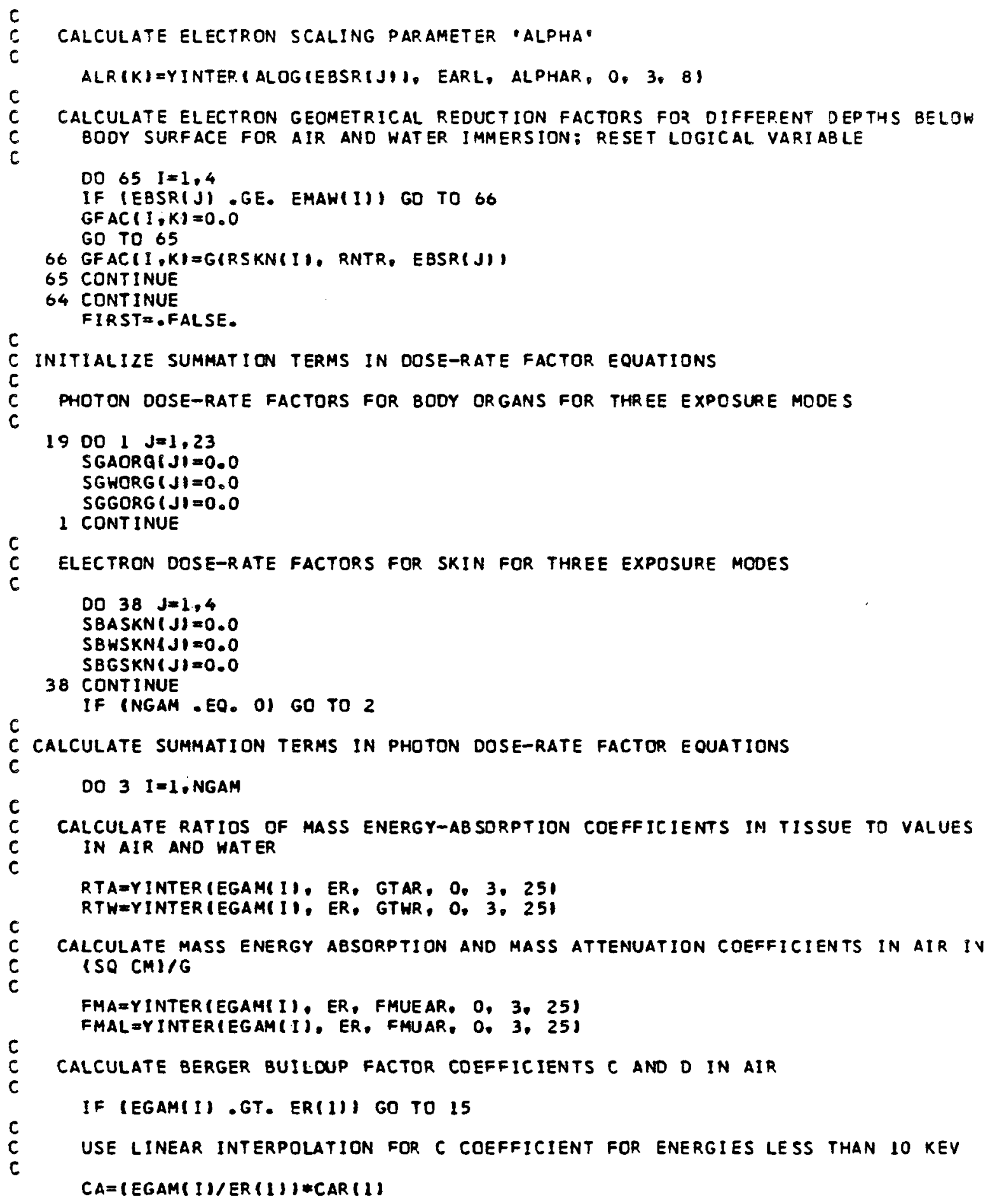


GO TO 16

15 CA $=$ YINTER (EGAMII), ER, CAR, 0, 3, 25)

16 DA=YINTER I EGAMII), ER, OAR, 0, 3, 251

c

CALCULATE RATIOS OF organ DOSE RATE TO DOSE RATE IN AIR FOR AIR IMMERSION

DO $4 K=1.23$

ORGFAC (K) =FACIK, EGAMII)

4 CONTINUE C CALCULATE SUMMATION TERMS FOR AIR IMMERSION, WATER IMMERSION, ANO GROUNO
C SURFACE EXPOSURE

C

TS=FGAM(I)*EGAM(I)

TGA=FMA*(E) (DBLE(FMAL), DBLE $(R))-(C A /(D A-1.0)) * E X P((D A-1.0) * F M A L *$

$2 R) 1$

DO $5 \mathrm{~K}=1.23$

SGAORG $(K)=S G A O R G(K)+T S * O R G F A C(K)$

SGWORG $(K)=S G H O R G(K)+T S * O R G F A C(K) * R T W / R T A$

$S G G O R G(K)=S G G O R G(K)+T S * T G A * O R G F A C(K)$

5 CONTINUE

$c$

3 CONTINUE

C CALCULATE PHOTON DOSE-RATE FACTORS FOR BOOY ORgANS FOR AIR IMMERSION, WATER IMMERSION, AND GROUNO SURFACE EXPOSURE

$200 \quad 6 \quad K=1.23$

DOSORG $(1, K)=$ CPMI 1$)$ \#AC 1 \&GAORG $(X) / R H O A$

DOSORG $(2, K)=C P M(2) * F A C) *$ SGWORG $(K) / R H O W$

DOSORG $(3 . K)=0.5 * C P M(3) * F A C 2 * S G G D R G(K)$

6 CONTINUE

c

CAlCulate summation terms In ElECTRON DOSE-RATE faCtOR EQUations

IF INBETA.EQ. OI GO TO 7

C BETA-PARTICLES

DO $8 I=1$, NBETA

IF (EBMAXII) :LT. EMAH (I) GO TO 8

c

CALCULATE Beta-PARTICLE SPECTRUM

SET INOEX FOR ALLOHED BETA TRANSITION

$N C D=1$

TEST FOR FIRST-FORBIDOEN UMIOUE TRANSITION AND RESET INDEX

IF INFB EQ. OI GO TO 24

DO $25 \mathrm{~J}=1$, NFB

IF (I . NE. NBF(J)) GO TO 25

$N C D=-1$

GO TO 24

25 CONTINUE

$\stackrel{c}{c}$

CALCULATE SPECTRUM

24 CALl BSPEC (EBMAX (11. FBeTAl 1), EBSR, ATNO, NCD, FIE, JSPEC, EMIDI 


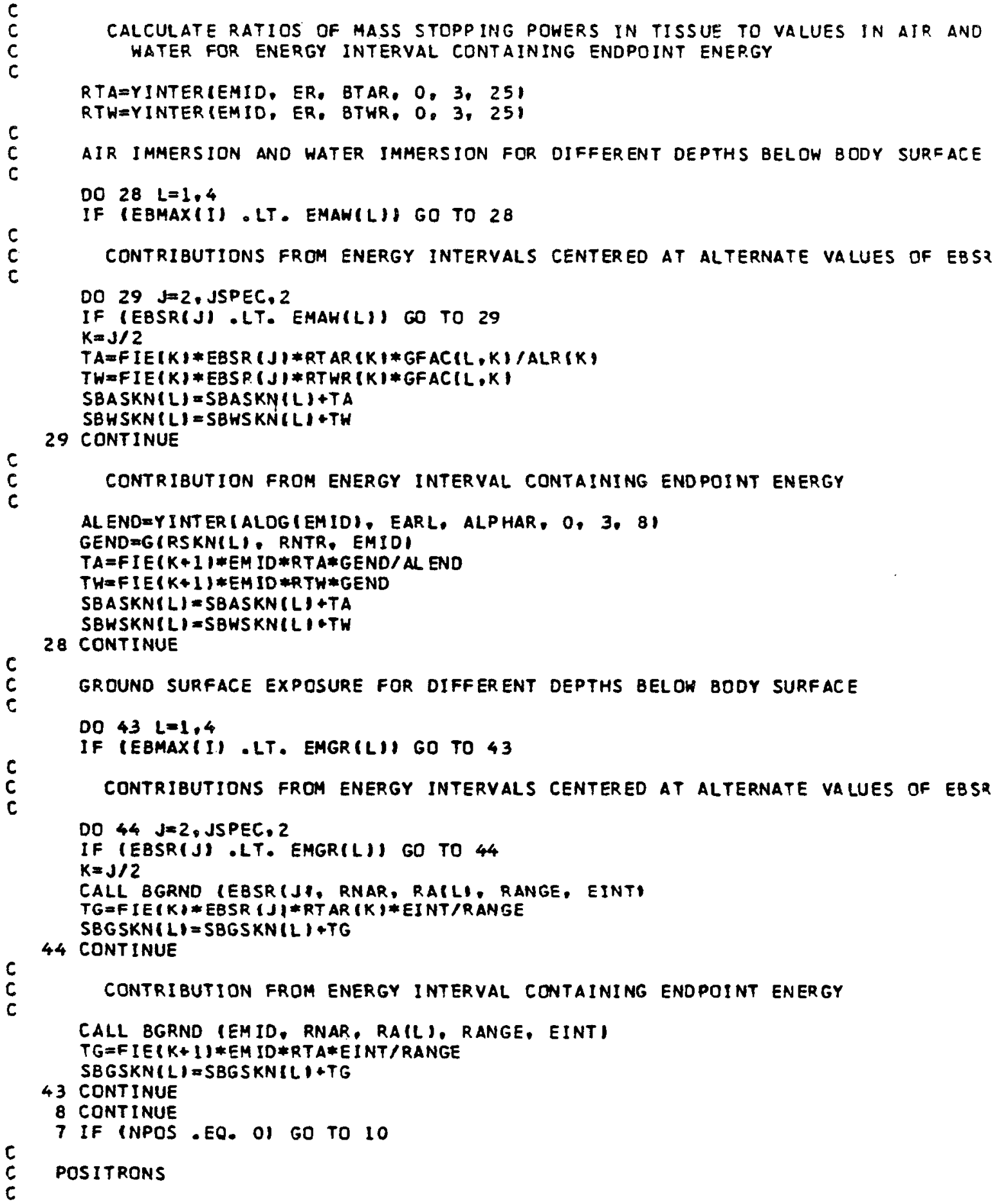


DO $11 I=1$.NPOS

IF (EPMAXII) - LT. EMAWIIII GO TO 11

CALCULATE POSITRON SPECTRUM

SET INDEX FOR ALLOWEO POSITRON TRANSITION

$N C D=2$

$c$
$c$

TEST FOR FIR.ST-FORBIDDEN UNIOUE TRANSITION ANO RESET INDEX

IF (NFP. EQ. OI GO TO 67

DO $68 \mathrm{~J}=1$. NFP

IF (I. NE. NPF (J)) GO TO 68

$\mathrm{NCO}=-2$

GO TO 67

68 CONTINUE

$c$
$c$

\section{CALCULATE SPECTRUM}

67 CALl BSPEC (EPMAX(II. FPOS(I), EBSR, ATNO, NCO, FIE, JSPEC, EMID)

c

CALCULATE RATIOS OF MASS STOPPING POWERS IN TISSUE TO VALUES IN AIR AND HATER FOR ENERGY INTERVAL CONTAINING ENDPOINT ENERGY

RTAEYINTER (EMID, ER, BTAR, 0, 3, 25)

RTH=YINTER (EMIO, ER, BTHR, 0, 3, 25 I

C AIR IMMERSION ANO WATER IMMERSION FOR OIFFERENT DEPTHS BELOW GODY SURFACE

DO $74 \quad L=1.4$

DO 74 LIFI:4

C CONTRIBUTIONS FROM ENERGY INTERVALS CENTERED AT ALTERNATE VALUES OF EBS?

CO 75 JE2.JSPEC, 2

IF (EBSRIJ) . LT. EMAHIL) GO TO 75

$K=J / 2$

$T A=F I E(K) * E B S R(J) * R T A R(K) * G F A C(L, K) / A L R(K)$

$T H=F I E(K) * E B S R(J) * R T H R(K) * G F A C(L, K)$

SBASKN(LI) =SBASKN(LI+TA

SBHSKN $(L)=$ SBHSKN $(L)+T H$

75 CONTINUE

$c$
$c$

CONTRIBUTION FROM ENERGY INTERVAL CONTAINING ENOPOINT ENEPGY

ALEND=YINTER (ALOG (EMID), EARL, ALPHAR, 0, 3. 81

GEND $=$ G(RSKN(L), RNTR, EMIO)

TA $=F I E(K+1) * E M I D * R T A * G E N D / A L$.END

$T W=F I E(K+1) \neq E M I D * R T W * G E N D$

SBASKN(L) = SBASKN(LI+TA

SBHSKN $(L)=S B H S K N(L)+T H$

74 CDNTINUE

c

GRQUND SURFACE EXPOSURE FOR DIFFERENT OEPTHS SELOW BOOY SURFACE

Do $78 \mathrm{~L}=1.4$

IF (EPMAXII) - LT. EMGR(LI) GO TO 78 
DO $79 \mathrm{~J}=2, J S P E C, 2$

IF (EBSR(J) - LT. EMGRIL) GO TO 79

$K=J / 2$

CALL BGRNO IEBSR (J), RNAR, RALLI, P.ANGE, EINT

TG=F IE (K) EEBSF ( ) $)$ RT AR $(K) \neq E I N T / R A N G E$

SBGSKN(L) $=$ SBGSKN $(L)+T G$

79 CONTINUE

$c$

CONTRIBUTION FROM ENERGY INTERVAL CONTAINING ENDPOINT ENERGY

CALL BGRND IEMID, RNAR, RAIL), RANGE, EINT)

$T G=F I E(K+1) * E M I D * R T A * E I N T / R A N G E$

SBGSKNILI =SBGSKN (LI ITG

78 CONTINUE

11 CONTINUE

c

10 IF INELEC .EQ. 01 GO TO 13

C AUGER ANO CONVERSION ELECTRONS

DO $14 I=1$, NELEC

IF (EELECII) - LT. EMAHIII) GO TO 14

TS $=F E L E C I I I \neq E E L E C I I)$

$c$
$c$
$c$
$c$

CALCULATE RATIOS OF MASS STOPPING POHERS IN TISSUE TO VALUES IN AIR AND WATER

RTA=YINTER IEELECII) ER, BTAR, 0, 3, 258

RTW=YINTER (EEL ECI I I, ER, BTHR, 0, 3, 25

$c$

AIR IMMERSION AND WATER IMMERSION FOR DIFFERENT DEPTHS BELOW BODY SURFACE

$0018 \quad L=1.4$

IF (EELECII) - LT. EMAN(L)) GO TO 18

ALEND=YINTER (ALDG (EELECI I) ), EARL, ALPHAR, 0, 3, 8 )

GEND $=G(R S K N I L)$. RNTR, EELECIIII

$T A=T S * R T A \# G E N O / A L E N D$

$T H=T S * R T W * G E N D$

SBASKN $(L)=S B A S K N(L)+T A$

SBHSKN(L) $=$ SBHSKN(L)+TH

18 CONTINUE

GR CUND SURFACE EXPOSURE FOR OIFFERENT DEPTHS BELOH BOOY SURFACE

DO $82 L=1,4$

IF (EELECIII - LT. EMGR ILII GO TO 82

CALL BGRND IEELECIII, RNAR, RA(L), RANGE, EINTI

$T G=T S * R T A * E I N T / R A N G E$

SBGSKN $(L)=$ SBGSKN $(L)+T G$

82 CONTINUE

14 CONTINUE

C CALCULATE ELECTRON DOSE-RATE FACTORS FOR SKIN FOR DIFFERENT DEPTHS BELOW BODY SURFACE FOR AIR IMMERSION, HATER IMMERSION, ANO GROUND SUP.FACE EXPOSURE

13 DO $83 K=1.4$

DOSORB $(1, K)=F A C)=S B A S K N(K) / R H O A$

DOSORB $(2, K)=F A C I$ SBHSKNIK I /RHOH 
DOSORB $(3, K)=0.5 * C P M(6) * F A C 2 * \operatorname{SBGSKN(K)}$ (c) 83 CONTINUE

C CALCULATE SUM OF PHOTON AND ELECTRON DOSE-RATE FACTORS FOR SKIN FOR AIR IMMERSION. WATER IMMERSION, ANO GROUNO SURFACE EXPOSURE USING VALUE AT DEPTH OF 7 MGJISO CMI FOR ELECTRONS

c

DO $59 \quad I=1.3$

DOSSKN $(I)=0 O S O R G(1,17)+\operatorname{DOSOR} B(1,4)$ 59 CONTINUE

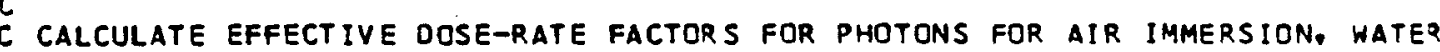
IMMERSION, AND GROUND SURFACE EXPOSURE USING ORGAN-SPECIFIC WEIGHTING FACTORS IN ICRP PUBLICATION 26

$0026 \quad I=1.3$

$c$

CONTRIBUTIONS FROM GONADS ITESTESI, BREAST, RED MARROW, LUNG, THYROID, AND BONE SURFACES

DOSEFF $(I)=0.25 *$ DOSORG $(I, 20+0.15 *$ DOSORG $(I, 4) * 0.12 * 100 S O P . G(1,13)$

2 DOSORGII, $1111+0.0$ \% (OOSORGII, $221+$ DOSORGII, 1611

c

REDEFINE ARRAYS OF DOSE-RATE FACTORS FOR BODY ORGANS TO EXCLUDE ORGANS ALREAOY CONSIOERED PLUS OVARIES AND SKIN

$0.071 \mathrm{~J}=1.3$

DEFF $(I, J]=\operatorname{DOSORG}(I, J)$

71 CONTINUE

DO $50 \quad J=5,10$

DEFF(I.J-I)=OOSORG (I, J)

50 CDNTINUE

DEFF $(1,10)=$ DOS ORG $(I .12)$

DEFF $(1,11)=00 S O R G(1.15)$

DO $51 \mathrm{~J}=18,19$

DEFF(I.J-6:=0OSORG (I.J)

51 CONTINUE

DEFF $(I, 14)=D O S O R G(I, 21)$

DEFF $(I, 15)=$ DOS ORG $(I, 23)$

c

SORT REDEFINED ARRAYS OF DOSE-RATE FACTORS FOR BODY ORGANS IN DECREASINJ NUMER ICAL CROER US ING DUMMY DNE-DIMENSIONAL ARRAYS

OO $52 \mathrm{~J}=1,15$

DUMI ( J) $=0$ OEFF ( I, J)

52 CONTINUE

CALL SORT (15, DUM1, DUM2)

$0053 \quad J=1.15$

DEFFS $(I, J)=$ OUMZ $(J)$

53 CONTINUE

$c$
$c$

ADO CONTRIBUTIONS FROM FIVE ORGANS IN REMAINDER CATEGOPY

$0054 \mathrm{~J}=1.5$

DOSEFF $(I)=$ DOSEFF $(I)+0.06 *$ DEFFS $(1 . \mathrm{J})$

54 CONT INUE

26 CONTINUE

RETURN

END 
FUNCTION YINTER(XI, $X, Y$, INDI, NI, IMAX)

c

C

C BASED ON DOUBLE PRECISION INTERPOLATION FUNCTION YLAG DESCRIBED IN UNION CARBIDE PUBL ICAT ION CTC-39

XI - ABCISSA OF OESIRED INTERPOLATION POINT

$X$ - SINGLY DIMENSIONED ARRAY OF DISTINCT. MONOTONICALLY INCPEASI NG ABCISSAS $Y$ - SINGLY DIMENSIONED ARRAY OF CORRESPONOING ORDINATES

IND? - SET TO ZERO

N1 - NUMBER OF POINTS FOR THE INTERPOLATION POLYNOMIAL

IMAX - NUMBER OF ELEMENTS FOR THE $X$ - OR $Y$-ARRAYS

DIMENSION $X(1), Y(1)$

IND=INDI

$N=N 1$

I EX $=0$

IF [N.LE. IMAX] GO TO 10

$N=$ I MAX

$I E X=N$

10 IF IIND .GT. 0 I GO TO 40

DO $20 \mathrm{~J}=1$. I MAX

IF $(X I-X \mid J)) 30,130,20$

20 CONTINUE

$I E X=1$

GQ TO 70

30 I $N D=3$

40 IF IIND.GT. 1) GO TO 50 .

IEX $=-1$

$50 I N L=I N O-1 N+11 / 2$

IF IINL.GT. OI GO TO 60

I $N L=I$

$60 I N U=I N L+N-1$

IF (INU.LE. IMAX) GO TO 80

70 INL $=1$ MAX $-N=1$

INU $=$ I MAX

$80 \quad \mathrm{~S}=0$.

DO $110 \mathrm{~J}=I \mathrm{NL}, 1 \mathrm{NU}$

$P=P=(X I-X(J))$

$D=1$.

DO $100 \quad I=I N L$. INU

IF (I.NE. J) GO TO 90

$X D=X I$

GO TO 100

$90 \times D=X(1)$

$100 \quad 0=0 *(\times 0-X(1)$

$110 S=S+Y(J) / D$

$Y I N T E R=S * P$

120 RETURN

130 YINTER=YIJ!

GO TO 120

END 
c

FUNCTION GIR, RNGTR, EBI

CALCULATES GEOMETRICAL REDUCTION FACTOR FOR ELECTRON DOSE-RATE FACTOR FOR SKIN FOR AIR AND HATER IMMERSION: SEE EQS. (15)-(18) OF BERGER. HEALTH PHYSICS 26. 1 (1974)

R. - DISTANCE FRoM BODY SURFACE TO SKIN IN G/ISO CM)

RNGTR - REFERENCE ARRAY OF ELECTRON RANGES IN TISSUE IN G/ISO CMI AT ENERGIES GIYEN BY YALUES OF EGR BELOW

EB - ELECTRON ENERGY .FOR EVALUATION OF GEOMETRICAL REDUCTION FACTOR

DIMENSION RNGTRII:

DIMENSION ZDRNGI21), EGR (25), GR 11 25), GR 2125), GR3(25), GR4(25),

2 GR5 (25), GR6(25). GR7(25). GR8(25), GR9(25), GR10(25). GR 11 (25).

3 GRI2(25i, GRI 3(25), GR14(25), GRI5(25), GR16(25), GR17(25).

4 GR18125), GR19(25). GR20125), GR21(25). GR(25.21)

DIMENSION ELR (17). QIRI1T). ALRI17), ZOALR(15), QZR(15)

$C$

C RATIOS OF DISTANCE FROM BODY SURFACE TO ELECTRON RANGE FOR REFERENCE VALUES OF ELECTRON GEDMETRICAL REDUCTION FACTORS

DATA ZDRNG $0.0,0.05,0.10,0.15,0.20,0.25,0.30,0.35,0.40$. $20.45,0.50,0.55,0.60,0.65,0.70,0.75,0.80,0.85,0.90 .0 .95$. $31.00 \%$

c

C ENERGIES FOR REFERENCE VALUES OF ELECTRON GEOMETRICAL REDUCTION FACTORS

$$
\begin{array}{rrrrrrrrr}
\text { DATA EGR/ } & 0.010, & 0.015, & 0.020, & 0.030, & 0.040, & 0.050, & 0.060 . \\
2 & 0.080, & 0.100, & 0.150, & 0.200, & 0.300, & 0.400, & 0.500, & 0.600, \\
3 & 0.800, & 1.0, & 1.5, & 2.0, & 3.0, & 4.0, & 5.0 . & 6.0, \\
4 & 8.0, & 10.01 & & & & & &
\end{array}
$$

C

\begin{tabular}{|c|c|c|c|c|c|c|c|}
\hline $\begin{array}{l}\text { DATA } \\
2 \\
3 \\
4\end{array}$ & $\begin{array}{l}\text { GR1/ } \\
0.5 . \\
0.5 \\
0.5\end{array}$ & $\begin{array}{l}0.5 \\
0.5 \\
0.5 \\
0.5\end{array}$ & $\begin{array}{l}0.50 \\
0.5, \\
0.5 .\end{array}$ & $\begin{array}{l}0.5, \\
0.5, \\
0.5 .\end{array}$ & $\begin{array}{l}0.5, \\
0.5, \\
0.5,\end{array}$ & $\begin{array}{l}0.5, \\
0.50 \\
0.5 .\end{array}$ & $\begin{array}{l}0.5, \\
0.5, \\
0.5 .\end{array}$ \\
\hline
\end{tabular}

C REFERENCE VALUES OF ELECTRON GEOMETRICAL REDUCTION FACTORS

ZDRNG $=0.0$

C ZDRNG $=0.05$

DATA GR2 $0.4188,0.4195,0.4200,0.4201,0.4200,0.4199,0.4197$, $20.4192,0.4187,0.4177,0.4168,0.4153,0.4143,0.4133,0.4123$.

$30.4109,0.4098,0.4077,0.4063,0.4051,0.4045,0.4041,0.4039$. $40.4039,0.4041 /$

C ZDRNG $=0.10$

DATA GR3/ $0.3575,0.3587,0.3595,0.3598,0.3599,0.3599,0.3597$, $20.3591,0.3586,0.3573,0.3561,0.3540,0.3526,0.3512,0.3500$. $30.3480,0.3465,0.3436,0.3419,0.3403,0.3396,0.3393,0.3391$, $40.3392,0.33951$ 
ZORNG $=0.15$

DATA GR4/ $0.3047,0.3063,0.3073,0.3079,0.3081,0.3081,0.3079$,

$20.3074,0.3069,0.3054,0.3041,0.3018, C .3002,0.2987,0.2974$,

$30.2952,0.2937,0.2906,0.2887,0.2871,0.2865,0.2863,0.2862$.

ZOR.NG $=0.20$

DATA GRS/ $0.2580,0.2600,0.2611,0.2618,0.2621,0.2621,0.2620$, $20.2614,0.2609,0.2592,0.2582,0.2559,0.2544,0.2529,0.2516$, $30.2494,0.2479,0.2449,0.2431,0.2418,0.2414,0.2414,0.2414$, $40.2420,0.24271$

DATA GR6/ 0.2165, 0.2186, 0.2197, 0.2205, 0.2207, 0.2208, 0.2207, $20.2202,0.2197,0.2181,0.2171,0.2150,0.2136,0.2122,0.2109$, $30.2089,0.2077,0.2050,0.2034,0.2025,0.2023,0.2025,0.2027_{\text {。 }}$ $40.2036 .0 .2046 /$

ZDRNG $=0.30$

DATA GRTI $0.1793,0.1815,0.1826,0.1833,0.1835,0.1835,0.1835$, $20.1830,0.1817,0.1812,0.1802,0.1783,0.1771,0.1759,0.1748$, $30.1731,0.1720,0.1697,0.1685,0.1681,0.1682,0.1687,0.1691$. $40.1701,0.17131$

ZDRNG $=0.35$

DATA GR8R $0.1464,0.1485,0.1495,0.1501,0.1502,0.1501,0.1500$, $20.1495,0.1483,0.1479,0.1470,0.1454,0.1444,0.1434,0.1425$. 3 0.1411, $0.1403,0.1386,0.1378,0.1378,0.1383,0.1390,0.1395$ 。 $40.1408,0.14231$

ZDRNG $=0.40$

DATA GR9/ $0.1173,0.1192,0.1201,0.1205,0.1204,0.1203,0.1202$, $20.1197,0.1192,0.1182,0.1175,0.1162,0.1154,0.1146,0.1138$, $30.1128,0.1122,0.1111,0.1107,0.1112,0.1120,0.1130,0.1137$. $40.1152,0.11681$

C ZDRNG $=0.45$

DATA GR 10/0.0919,0.0937, 0.0944, $0.0946,0.0943,0.0941,0.0939$, DATA GR 10/0.0919, 0.0937, 0.0944, 0.0946, 0.0943, 0.094, 0.0939, $\begin{array}{llll}2 & 0.0934,0.0929,0.0920,0.0915,0.0904,0.0898,0.0891,0.0886, \\ 3 & 0.0879,0.0876,0.0869,0.0869,0.0879,0.0890,0.0902,0.0910,\end{array}$ $40.0928,0.0946$ i

C ZDRNG $=0.50$

DATA GR11/0.0702, $0.0717,0.0722,0.0722,0.0718,0.0714,0.0712$, $20.0707,0.0702,0.0694,0.0690,0.0681,0.0677,0.0672,0.0668$, $\begin{array}{lllll}2 & 0.0707,0.0702,0.0694,0.0690,0.0681,0.0677,0.0672,0.0668,\end{array}$ $40.0733,0.07521$

ZDP.NG $=0.55$ 
DATA GR1210.0520, $0.0531,0.0535,0.0532,0.0527,0.0523,0.0521$ $20.0518,0.0512,0.0504,0.0501,0.0494,0.0490,0.0487,0.0485$, $30.0482,0.0482,0.0484,0.0489,0.0505,0.0519,0.0533,0.0544$, $40.0564,0.0584 /$

\section{$Z D R N G=0.60$}

DATA GR13/0.0371, $0.0380,0.0382,0.0377,0.0372,0.0368,0.0365$ $20.0361,0.0356,0.0349,0.0347,0.0341,0.0339,0.0337,0.0335$, $30.0334,0.0335,0.0338,0.0345,0.0361,0.0376,0.0389,0.0400$. $40.0421,0.0441 /$

$\stackrel{c}{c}$

ZDRNG $=0.65$

DATA GR14/0.0253, 0.0259,0.0260, $0.0255,0.0250,0.0246,0.0243$, $20.0239,0.0235,0.0229,0.0226,0.0221,0.0220,0.0219,0.0218$, $30.0218,0.0219,0.0223,0.0230,0.0245,0.0259,0.0271,0.0281$, $40.0301,0.0320 /$

$c$
$c$

ZDRNG $=0.70$

DATA GR15/0.0164, $0.0168,0.0168,0.0163,0.0159,0.0155,0.0153$, $20.0149,0.0145,0.0140,0.0138,0.0134,0.0133,0.0132,0.0132$, $30.0132,0.0133,0.0137,0.0143,0.0155,0.0167,0.0178,0.0187$, $40.0205,0.02221$

C ZDRNG $=0.75$

DATA GR16/0.0100, $0.0102,0.0102,0.0098,0.0094,0.0091,0.0089$ $20.0086,0.0083,0.0078,0.0077,0.0074,0.0073,0.0072,0.0072$, $30.0072,0.0073,0.0076,0.0081,0.0090,0.0099,0.0108,0.0115$, $40.0130,0.01441$

$c$ ZDRNG $=0.80$

DATA GR17/0.0056, 0.0057, 0.0057, 0.0054, 0.0051,0.0049,0.0048, $20.0045,0.0043,0.0040,0.0038,0.0036,0.0035,0.0035,0.0035$. $30.0035,0.0036,0.0037,0.0040,0.0047,0.0053,0.0059,0.0064$, $40.0075,0.0086 /$

C ZDRNG $=0.85$

DATA GR18/0.0029, $0.0029,0.0029,0.0027,0.0026,0.0024,0.0023$, $20.0022,0.0020,0.0018,0.0017,0.0015,0.0015,0.0014,0.0014$, $30.0014,0.0015,0.0016,0.0018,0.0021,0.0025,0.0028,0.0032$, $40.0039,0.0046 /$

$\mathbf{C}$
$\mathbf{C}$ ZORNG $=0.90$

DATA GR19/0.0013, $0.0013,0.0013,0.0012,0.0011,0.0011,0.0010$, $20.0009,0.0008,0.0007,0.0006,0.0005,0.0005,0.0005,0.0005$, $30.0005,0.0005,0.0005,0.0006,0.0008,0.0009,0.0011,0.0013$, $40.0017,0.0021 /$

c ZDRNG $=0.95$

OATA GR20/0.0005, $0.0005,0.0005,0.0005,0.0004,0.0004,0.0004$. $20.0003,0.0003,0.0002,0.0002,0.0001,0.0001,0.0001,0.0001$. $30.0001,0.0001,0.0001,0.0002,0.0002,0.0003,0.0004,0.0005$. 
$40.0006,0.0008 /$

C ZDRNG $=1.00$

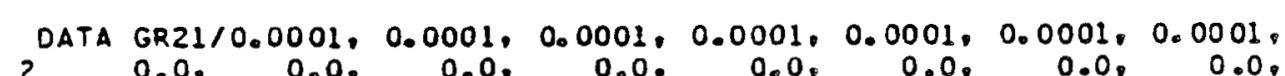

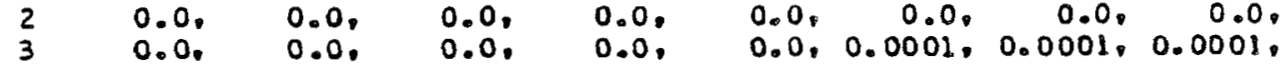

$40.0002,0.00021$

c

ENERGIES FOR REFERENCE VALUES OF LEAKAGE CORRECTION PARAMETERS

DATA ELR/ $0.020,0.030,0.040,0.050,0.060,0.080$,

$20.100,0.200,0.300,0.400,0.500,0.600,0.800$,

C REFERENCE VALUeS of Leakage CORRECTION PARAMETER $Q 1$

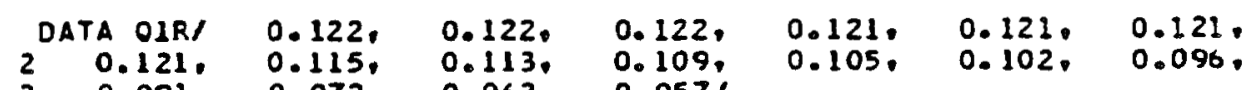

C Reference values of leakage CORRection parameter a IN CM

C

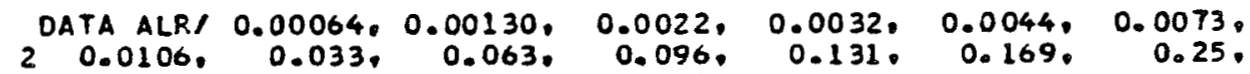

$c$

C SCALED DISTANCE I/A FOR REFERENCE VALUES OF LEAKAGE CDRRECTION PARAMETER 22

$\begin{array}{rrrrrr}\text { DATA ZOALR/ } 0.0,0.00625, & 0.0125, & 0.025, & 0.050, & 0.075 . \\ 2 & 0.15,0.0 .50, & 0.40, & 0.50, & 0.60 .\end{array}$

C REFEP.ENCE VALUES of leakage correction PARAMETER 02

DATA Q2R/ 1.0, 0.752, $0.639,0.497,0.352,0.281$.

$20.229,0.165$

C DENSITY OF SKIN TISSUE IN GIICU CMI

C DENSITY OF SKIN TISSUE

C DEFINE EOUIVALENT THO-DIMENSIONAL ARRAY DF GEOMETR ICAL REDUCTIONS FACTORS

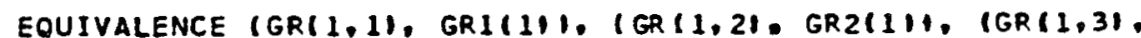

2 GR3 (1), IGRI1.41, GR4(1), IGR (1,5), GRS(1), (GR(1.6), GR.6(1)),

3 IGRII,Ti, GRTIIII, IGRIJ,BI, GR BI II), IGRII,9I, GR9III),

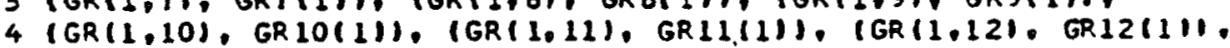

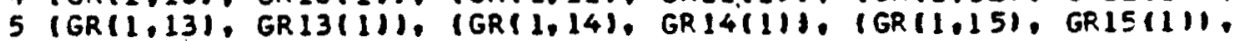

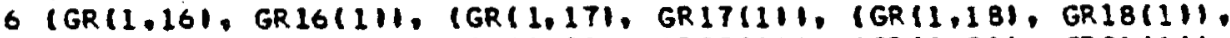

c

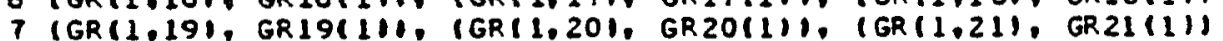

CALCULATE ELECTRON RANGE IN TISSUE

RANGE=YINTERIEB, EGR, RNGTR, 0, 3, 251

C CALCULATE RATIO OF DISTANCE FROM BODY SURFACE TO ELECTRON RANGE 


\section{ZOVR=R/RANGE}

C CALCULATE GEOMETRICAL REOUCTION FACTOR FOR GIVEN ENERGY ANO SCALED THICKNESS C IN TISSUE WITHOUT LEAKAGE CORRECTION

G=DINTERIEB, ZOVR, EGR, ZDRNG, GR, 25, 21, 4, 25I

C

C CALCULATE LEAKAGE CORRECTION TO GEOMETRICAL REDUCTION FACTOR

C calculate parameters ol ano a

QI $=Y I N T E R$ IEB, ELR, OIR, $0,3,171$

$A L=Y I N T E R I E B, E L R, A L R, 0,3,17)$

$c$
$c$
$c$

Calculate scaled oIstance z/a ano parameter 02

$Z O A L=R /(A L \# R H O S K)$

Q2 =YINTER I ZOAL, ZOALR, Q2R, 0, 3, 15)

C LEAKAGE CORRECTION

$Q L=1.0-Q 1 * 02$

c CALCULATE GEOMETRICAL REDUCTION: FACTOR HITH LEAKAGE CORRECTION

C

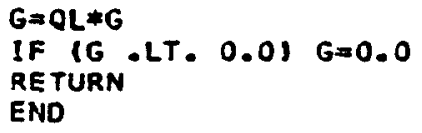


FUNCTION FACIK, EI

C CALCULATES RAT IO OF ORgan DOSE RATE TO DOSE RATE IN AIR FOR PHOTONS AT O DEg 6 ANO 760 MM PRESSURE USING ORGAN DOSE-RATE FACTORS FOR MONOENERGETIC SOUR.CES AT THE BOOY SURFACE GIVEN BY O'BRIEN ANO SANNA, HEALTH PHYSICS 30, 76 119761. AND SPECTRA OF SCATTERED PHOTONS FOR MONOENERGETIC SOUREES IN AY INFINITE ATMOSPH RIC CLOUD GIVEN BY DILLMAN, HEALTH PHYSICS 27, 571 (19741: SEE ECKERMAN, KERR, ANO RARIDON, HEALTH PHYSICS 39, 1054119801

K - INDEX FOR PARTICULAR ORGAN: SEE REFERENCE VALUES OF RATIOS SIVEN BELOW E - PHOTON ENERGY

OIMENSION EORG (15), ORG1 (15), ORG2 (15), ORG3(15), ORG4(15),

2 ORG5 (15), ORG6(15), ORG71 15), ORG8(15), ORG9(15), ORG101151,

3 ORG11(15), ORGI 2(15), ORG13115), ORG14(15), ORG15115), ORG16(15),

4 ORG171151, ORGI81 15), ORG19(15), ORG 20(15), ORG 21(15), ORG221 15),

5 ORG23(15). ORG(15.23), ORGF(15). EORGL(15)

c

ENERGIES FOR REFER ENCE VALUES OF RATIOS OF ORGAN DOSE RATE TO DOSE RATE IN AIV C IN MEY

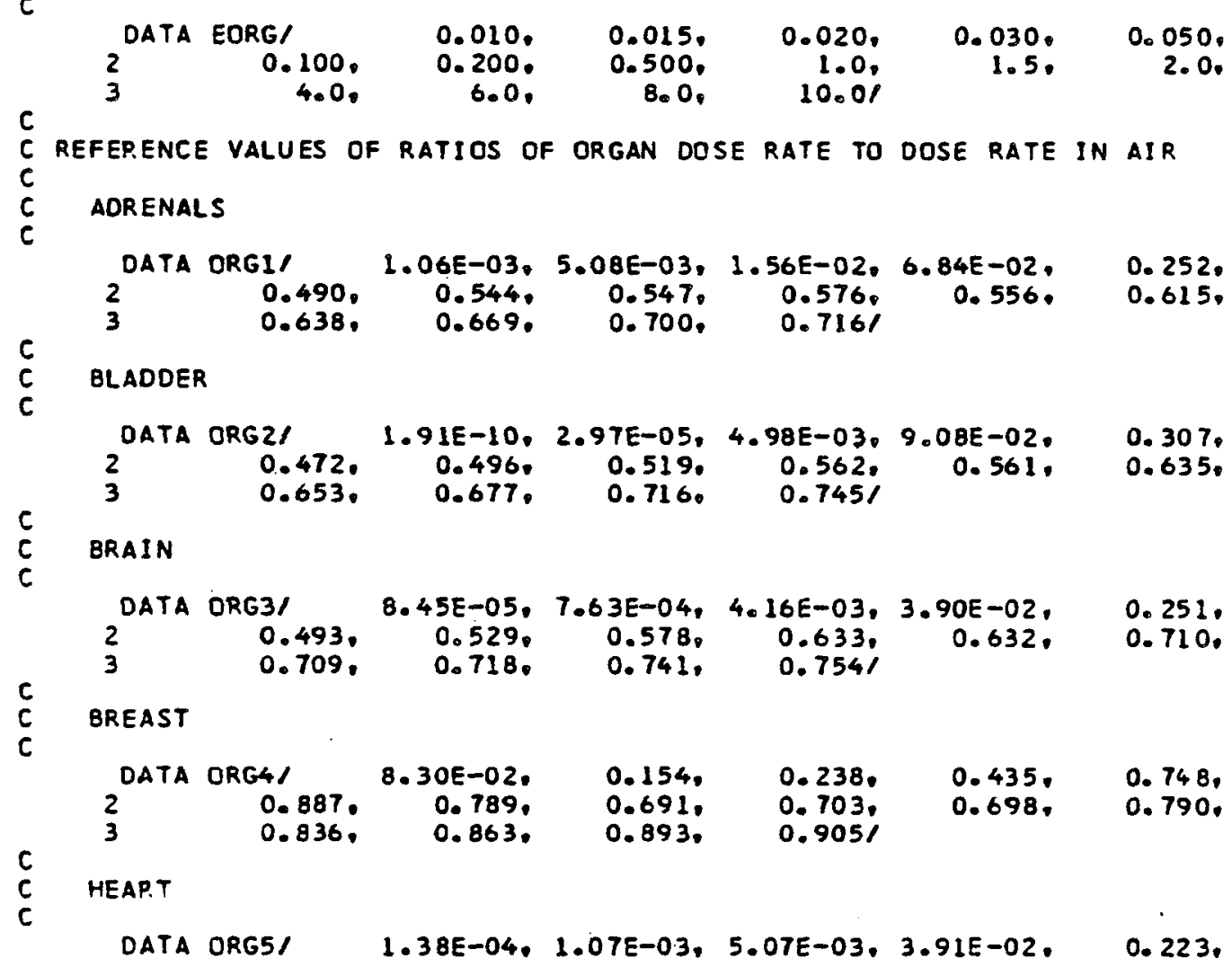




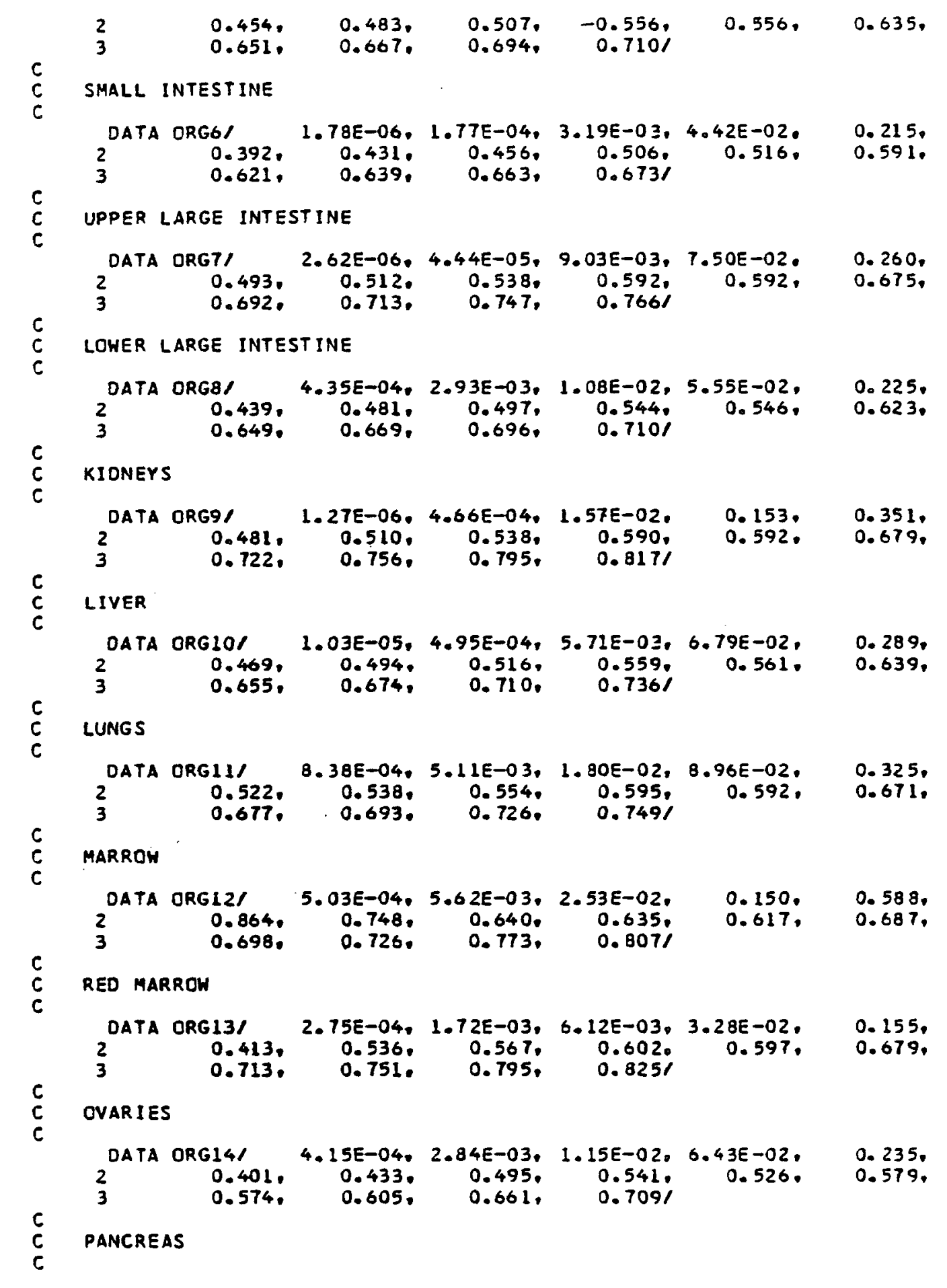




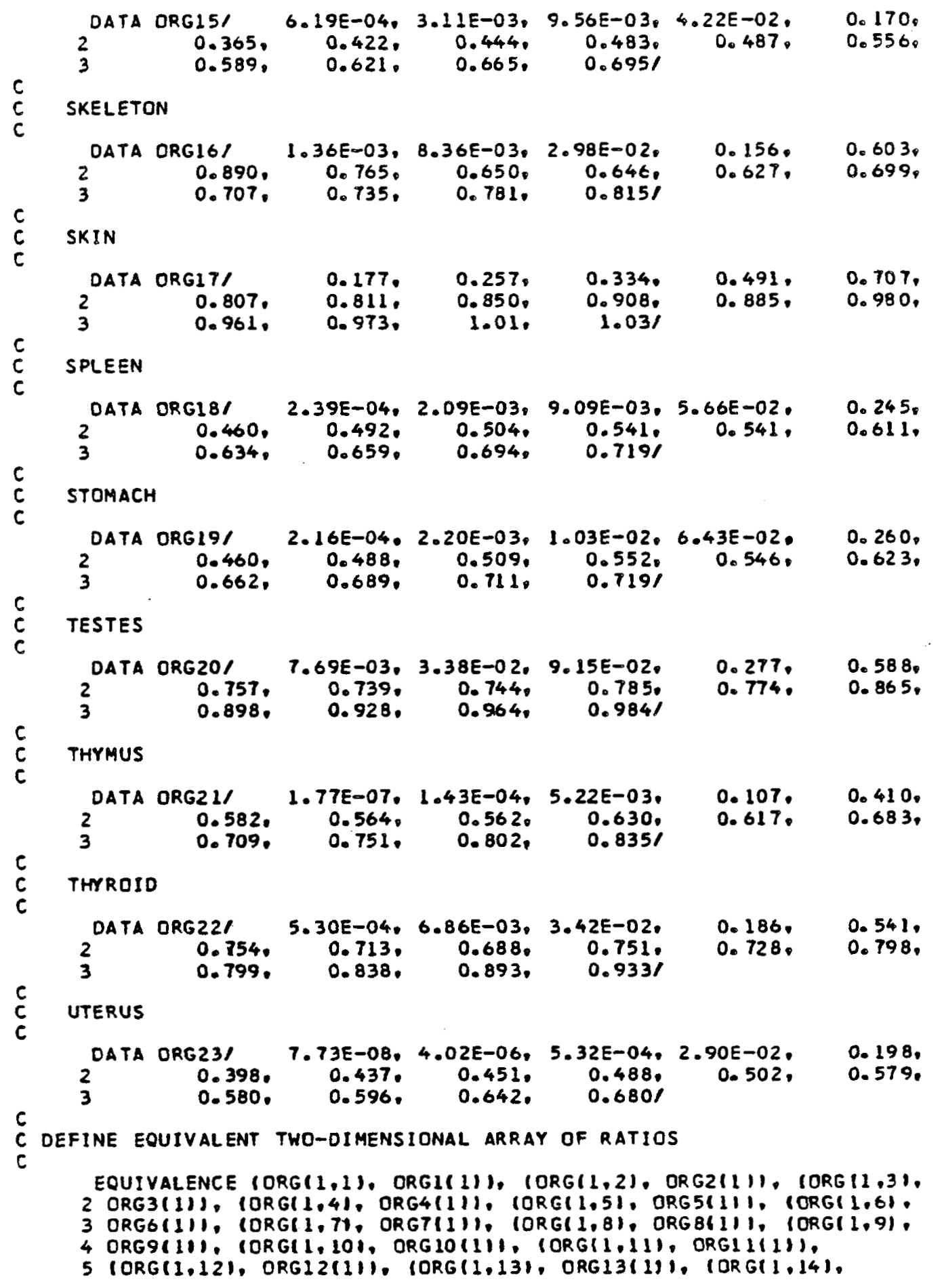


6 URG14(1)1, IORG(1.151, ORG15111), IORG11,16), ORG16(1)),

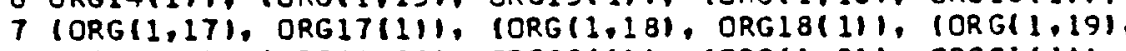

8 ORGI9(1), (ORG (1.20), ORG2O(1)), IORG(1,21), ORG $21(11)$.

(ORG $(1,22)$, ORG $22(1))$, IORG $(1,23)$, ORG $3(1))$

C CALCULATE RATIO OF ORGAN DOSE RATE TO DOSE RATE IN AIR USING LINEAR

C INTERPOLATION FOR ENERGIES LESS THAN $10 \mathrm{KEV}$

$F A C=(E / E O R G(1) \mid \equiv O R G(1, K)$

GO TO 3

C CALCULATE LOGARITHMS OF REFERENCE ENERGIES ANO REFERENCE RATIOS FOR PARTICULA?

C ORGAN

$20011=1.15$

EORGL (I I=ALOG ( EORG II)

ORGF(I)=ALOGIORG $(1, K)$

1 CONTINUE

C CALCULATE RATIO USING CUBIC INTERPOLATION OF LOGARITHMS

FAC $=E X P(Y I N T E R(A L O G(E)$, EORGL, ORGF, 0, 4, 15) )

3 RETURN

END 
FUNCTION EIIFM, $X\}$

C CALCULATES EXPONENTIAL INTEGRALS USING POLYNOMIAL AND RATIONAL APPROXIMATIONS:

$C$ SEE EOS. 5.1 .53 AND 5.1 .56 IN HANOBOOK OF MATHEMATICAL FUNCTIONS. PG. 231

DOUBLE PRECISI ON ARG, ALO, ALI, AL 2, AL 3, AL4, AL5

DOUBLE PRECISI ON AGI, AGZ, AG3, AG4, BI, B2, B3, B4

DOUBLE PRECISION DNUM, DNOM, FM, $X_{0} E 1$

DATA ALO. ALI. AL2, AL3, AL4, AL5/, $-0.5772156600,0.9999919300$,

$2-0.2499105500,0.0551996800,-0.0097600400,0.00107857001$

DATA AG1, AG2, AG3, AG4/8.573328740100, 18.059016973000.

$28.634760892500,0.267773734300 /$

DATA B1, B2, B3, 84/ 9.573322345400, 25.632956148600,

$221.099653082700 .3 .958496922800 /$

$A R G=F M * X$

IF IARG .GT. 1.000 , GO TO 1

$E I=A L O+A L 1 * A R G+A L 2 *(A R G * * 2.1+A L 3 *(A R G * 3 \cdot 1+A L 4 *(A R G * 4.1+A L 5 *$

2 (ARG**5.)-DLOG(ARG)

2 RETURN

1 ONUM=ARG $* 40 * A G) *(A R G * 3.1 * A G 2 * 1$ AR G**2.1+AG3*ARG+AG4

$D N C M=A R G * * 4 .+B 1 *(A R G * * 3)+.B 2 *(A R G * * 2)+.B 3 * A R G * B 4$

$E I=D N U M /(D N O M * A R G *$ OEXP (ARG)

GO TO 2

ENO 
SUBRDUTINE BSPEC (EN, FI, EBSR, ATNO, NCD, FIE, JSPEC, EMIDI

CALCULATES NUMBER OF ELECTRONS AS A FUNCTION OF ENERGY FOR CONTINUOUS SPECTRA FROM BETA OECAY; SEE EQS. $(3.121-13.141$ AND 13.221 OF EVANS. THE ATOMIC NUCLEUS

EN - ENDPOINT ENERGY FOR CONTINUOUS SPECTRUM

FI - TOTAL INTENSITY FOR CONTINUOUS SPECTRUM

EBSR - ARRAY OF ENERGIES FOR CALCULATION OF CONTINUOUS SPECTRUM

ATNO - ATOMIC NUMBER OF PARENT RADIONUCLIDE

NCD - INDEX FOR TYPE OF ELECTRON

- I FOR BETA PARTICLE (-1 FOR FIRST-FORBIDOEN UNIOUE TRANSITIONI

$=2$ FOR POSITRON (-2 FOR FIRST-FORBIDDEN UNIQUE TRANSITI ON)

FIE - ARRAY CF ELECTRON INTENSITIES AT ENERGIES GIVEN BY ALTERNATE VALUES OF EBSR

JSPEC - INDEX SPECIFYING HIGHEST FULL ENERGY INTERYAL CENTERED AT AN

ALTERNATE VALUE OF EBSR FOR HHICH INTENSITY IS NON-ZERO

EMID - ENERGY SPECIFYING LAST ENERGY INTERVAL IN SPECTRUM INCLUDING ENDPOINT

OIMENSION EBSR (1), FIE(1), FIBSI 12011 , ELOHI 31, FLOH 31

COMPLEX*16 Z1, DCGAM, GAM

$c$

C ELECTRON REST MASS IN MEY

FINE-STRUCTURE CONSTANT

C

OATA EMO, FSC/ $0.510976,7.29729 E-03 /$

C DEFINE FUNCTIONS FOR CONTINUOUS ELECTRON SPECTRUM

C STATISTICAL FACTCR FOR ALLOHED SPECTRUM

FO(H. ETA, HO $)=W * E T A *((W O-W) * 2$. $)$

FIRST-FORBIDOEN UNIQUE CORRECTION FACTOR

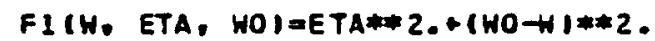

COULOMB CORRECTI ON FACTOR

FCIETA, S, Y, GAMSQ) $=($ ETA*\#(2.*SII*EXPI3.14159*Y)*GAMSO

c

C

FI BSI I I $=0.0$

IF (I.GT. 600) GO TO 10

$F I E(I)=0.0$

C calculate atomic number of daughter nuClide

10 CDNTINUE

IF IIABSINCD) -EO. II ZD=ATNO+1.

C 
C CALCULATE STATISTICAL FACTOR FOR ELECTRON SPECTRUM AT ENERGIES GIVEN BY VALUES OF EBSR: CALCULATE INTEGRAL OVER ENERGY OF STATISTICAL FACTDRS USING TRAPEZOIDAL RULE

INITIALIZE INTEGRAL AND SET FIP.ST-FORBIDDEN UNIOUE CORPECTION FACTOR FOR AN ALLOHED TRANSITION

$A R E A=0.0$

$F 1 C=1.0$

C CALCULATE ENOPOINT ENERGY IN UNITS OF ELECTRON REST MASS

WO $=E N / E M O+1$.

CALCULATE CONSTANT TERM IN COULOMB CORRECTION FACTOR

$S=S O R T(1 .-1 A B S(2 D) * F S C) * 2.1-1$.

CALCULATE STATISTICAL FACTORS AS A FUNCTION OF ENERGY

DO $1 I=2,1201$

IF IEBSRIII .GT. ENI GO TO 2

C CALCULATE ELECTRON TOTAL ENERGY aNo MOMENTUM

WEEBSR (I) / EMO+1.

ETA=SORT $(H * H-10)$

c CALCULATE FIRST-FORBIDDEN UNIOUE CORRECTION FACTOR

IF (NCD . LT. O) FIC=FICH, ETA, HOI

C CALCULATE TERMS IN COULOMB CORRECTION FACTOR

$Y=20 * F S C * H / E T A$

$21=D C M P L X$ CDBLE (1.+S), DBLE(Y))

GAM=DCGAML 211

GAMSO=GAM\#DCONJG (GAM)

$c$
$c$

CALCULATE STATISTICAL FACTOR

FIBSIII=FO(H, ETA, HOI*FIC*FCIETA, S, Y, GAMSOI

1 CONTINUE

SET INDEX FOR LAST ENTRY IN ENERGY ANO STATISTICAL FACTOR ARRAYS

2 NSPEC $=I-1$

c

CALCULATE STATISTICAL FACTOR AT ZEP.O ENERGY USING OUADRATIC EXTRAPOLATION

DO $31=1,3$

ELOW $(I)=E B S R(I+1)$

FLOWIIIIIFIES II+1)

3 CONTINUE

FIBSIII=Y INTER IEBSR(1), ELOH, FLOW, 0,3, 3)

C CALCULATE INTEGRAL OF STATISTICAL FACTORS

DO $4 I=2$, NSPEC 


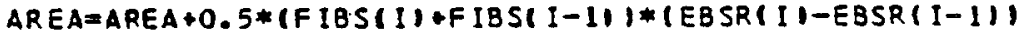

4 CONTINUE

$c$
$c$

ADO CONTRIBUTION FROM ENERGY INTERVAL CONTAINING ENOPOINT ENERGY

AREA $=A R E A+0.5 * F I B S$ (NSPEC) * (EN-EB SR (NSPEC)

C

C CALCULATE NUMBER OF ELECTRONS PER DI SINTEGRATION AT ENERGIES GIVEN BY

C ALTERNATE VALUES OF EBSR USING SIMPSON'S RULE

c

CALCULATE INTERVAL HIOTH AND SPECIFY INDEX FOR ENERGY FOR LAST FULL INTERVAL

OELEN=EBSR (2)-EBSR (1)

JSPEC $=$ NSPEC -1

C CAlCulate spectrum

C

DO $5 \quad I=2, J S P E C, 2$

DAREA =DELEN* $(F I B S(I-1)+F I B S(I+1)+4 . * F I B S(I)) / 3$.

$J=1 / 2$

FIEC $J I=F I$ OOAREA/ AREA

5 CONTINUE

C CALCULATE CONTRIBUTION TO SPECTRUM FROM ENERGY INTER VAL CONTAINING ENOPOINT

C ENERGY BY BISECT ING INTERVAL AND USING SIMPSON'S RULE

C IF II EO. JSPECI GO TO 6

EMID IEBSR IJSPECD AENIIT

FBMIO=YINTER IEMIO, EBSR, FIBS, 0,3, NSPECI

OAREA $=$ (EN-EMIO) $*$ (FIBSIJSPECI +4.*FBMIDI/3.

GO TO 7

6 EM $10=(E B S R(N S P E C)+E N) / 2$.

FBMID=YINTER IEMID, EBSR, FIBS, 0,3 , NSPECI

OAREA $=(E N-E M I D) *(F) B S(N S P E C)+4 \ldots * F B M I D) / 3$.

7 FIE $(J+1)=F I * D A R E A / A R E A$

RETURN

ENO 
C CALCULATES INTEGRAL OVER CONTAMINATED GROUND SURFACE OF ELECTRON SCALED POINT KERNEL IN AIR

EB - ELECTRON ENERGY FOR EVALUATION OF INTEGRAL

RNGAR - REFERENCE ARRAY OF ELECTRON RANGES IN AIR IN G/ISO CMI AT ENERGIES GIVEN BY VALUES OF EBR BELOH

$R$ - DISTANCE ABOVE GROUND FOR EVALUATION OF INTEGRAL IN G/ISO CMI

RANGE - ELECTRON RANGE IN AIR IN G/ISO CMI FOR ENERGY EB

EINT - INTEGRAL OVER GROUND SURFACE OF ELECTRON SCALED POINT KERNEL IN AIR FOR ENERGY EB ANO DISTANCE ABOVE GROUNO R

DIMENSION RNGAR(1), EAR (B), ALPHAR 181 , EARLI 81

OIMENSION REDISRI25), EBRI25), SPKR II 25), SPKR $2(25)$, SPKR3 1251,

2 SPKR $4(25)$. SPKR $5(25)$, SPKR6 (25), SPKRT $(25)$, SPKRB $(25)$, SPKR9(25),

3 SPKRIO(25). SPKR 11(25). SPKR12(25). SPKRI3(25), SPKR $14(25)$.

4 SPKR 151251, SPKR 16125). SPKRIT125), SPKR $18(25)$, SPKR 191251.

5 SPKR201251, SPKR211251, SPKR221251, SPKR231251, SPKR 241251.

6 SPKR 251251. SPKR $(25.25$ i

OIMENSION ALPHER (25). FAIRR(25,25), RINTZE 25,281 , OISR 28$)$. 2 DISLR (5)

C RATIOS OF DISTANCE FROM SOURCE POINT TO ELECTRON RANGE For REFERENCE VALUES OF C ELECTRON SCALED POINT KERNELS

DATA REDISR/ $0.0,0.05,0.10,0.15,0.20,0.25,0.30,0.35,0.40$, $20.45,0.50,0.55,0.60,0.65,0.70,0.75,0.80,0.85,0.90,0.95$, $31.00,1.05,1.10,1.15,1.20 \%$

C RATIOS OF OISTANCE FROM SOURCE POINT TO ELECTRON RANGE BETWEEN 0.0 AND 0.05 AT C WHICH REFERENCE ARRAY OF INTEGRALS IS TO BE CALCULATED

C DATA DISLR/ $0.001,0.002,0.005,0.01,0.021$

C

c

\begin{tabular}{|c|c|c|c|c|c|c|c|}
\hline $\begin{array}{l}2^{\text {DATA }} \\
3 \\
4\end{array}$ & $\begin{array}{l}\text { EBR/ } \\
0.060, \\
0.500 . \\
4.0 .\end{array}$ & $\begin{array}{r}0.010 \\
0.080 \\
0.600 \\
5.0\end{array}$ & $\begin{array}{r}0.015 . \\
0.100 \\
0.800 \\
6.0 .\end{array}$ & $\begin{array}{r}0.020 \\
0.150 \\
1.0 \\
8.0\end{array}$ & $\begin{array}{r}0.030 \\
0.200 \\
1.5 \\
10.0 \%\end{array}$ & $\begin{array}{l}0.040 . \\
0.300 . \\
2.0 .\end{array}$ & $\begin{array}{r}0.050 \\
0.400 \\
3.0\end{array}$ \\
\hline \multicolumn{8}{|c|}{$\begin{array}{l}\text { REFERENCE VALUES OF } \\
\text { REDISR }=0.0\end{array}$} \\
\hline $\begin{array}{l}2^{\text {DATA }} \\
3 \\
4\end{array}$ & $\begin{array}{l}\text { SPKR 1/ } \\
0.544 . \\
0.6740 \\
0.835\end{array}$ & $\begin{array}{l}0.508 \\
0.559 \\
0.689 \\
0.841\end{array}$ & $\begin{array}{l}0.508 \\
0.572 . \\
0.714 . \\
0.841 .\end{array}$ & $\begin{array}{l}0.510 \\
0.595 \\
0.736 \\
0.839\end{array}$ & $\begin{array}{l}0.521 . \\
0.611 . \\
0.711 \% \\
0.8331\end{array}$ & $\begin{array}{l}0.528, \\
0.640 . \\
0.798 .\end{array}$ & $\begin{array}{l}0.536, \\
0.660 \\
0.826\end{array}$ \\
\hline \multicolumn{8}{|c|}{ כ.4. } \\
\hline DATA & SPKR $2 /$ & 0.588 & 0.582. & 0.576 & 0.579. & 0.582 . & 0.586. \\
\hline
\end{tabular}




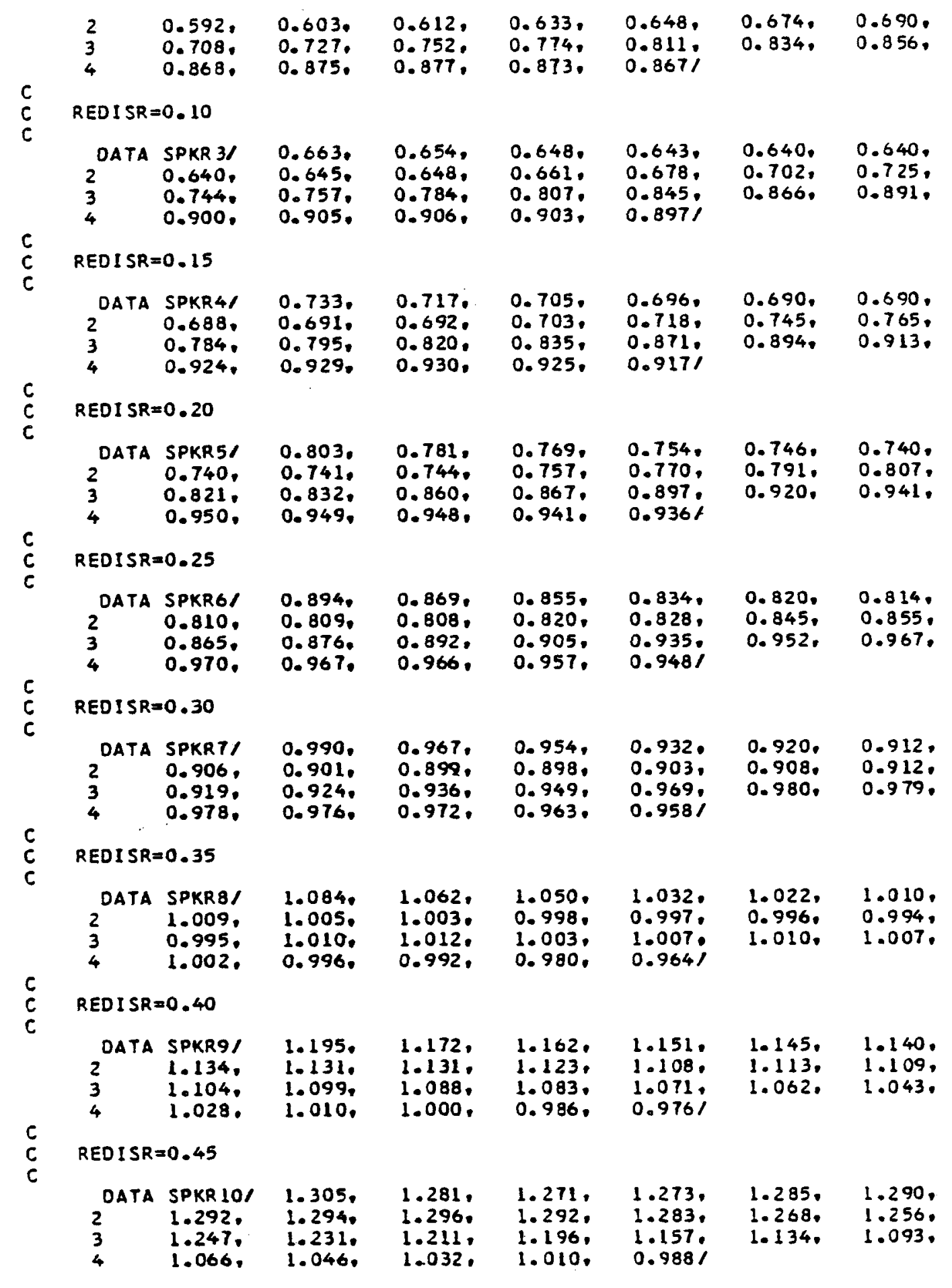


DATA SPKR II/$$
2
$$

c

$\begin{array}{llll} & 1.428, & 1.430, & 1.391, \\ 3 & 1.384, & 1.372, & 1.432,\end{array}$

REOISR=0.55 $\begin{array}{llllllll}\text { DATA SPKRL121 } & 1.472, & 1.475, & 1.479, & 1.503, & 1.525, & 1.5500 \\ 2 & 1.556, & 1.562, & 1.561, & 1.557, & 1.536, & 1.522, & 1.507, \\ 3 & 1.498, & 1.484, & 1.457, & 1.435, & 1.380, & 1.325, & 1.246, \\ 4 & 1.193, & 1.149, & 1.119, & 1.066, & 1.024 \% & & \end{array}$

C REDISR $=0.60$

$\begin{array}{llllllll}\text { OATA SPKR 13, } & 1.504, & 1.519, & 1.540, & 1.578, & 1.607, & 1.622, \\ 2 & 1.628, & 1.638, & 1.645, & 1.643, & 1.639, & 1.622, & 1.604, \\ 3 & 1.579, & 1.563, & 1.529, & 1.501, & 1.438, & 1.393, & 1.318, \\ 4 & 1.259, & 1.211, & 1.173, & 1.110, & 1.054 ; & & \end{array}$

C REDISR $=0.65$

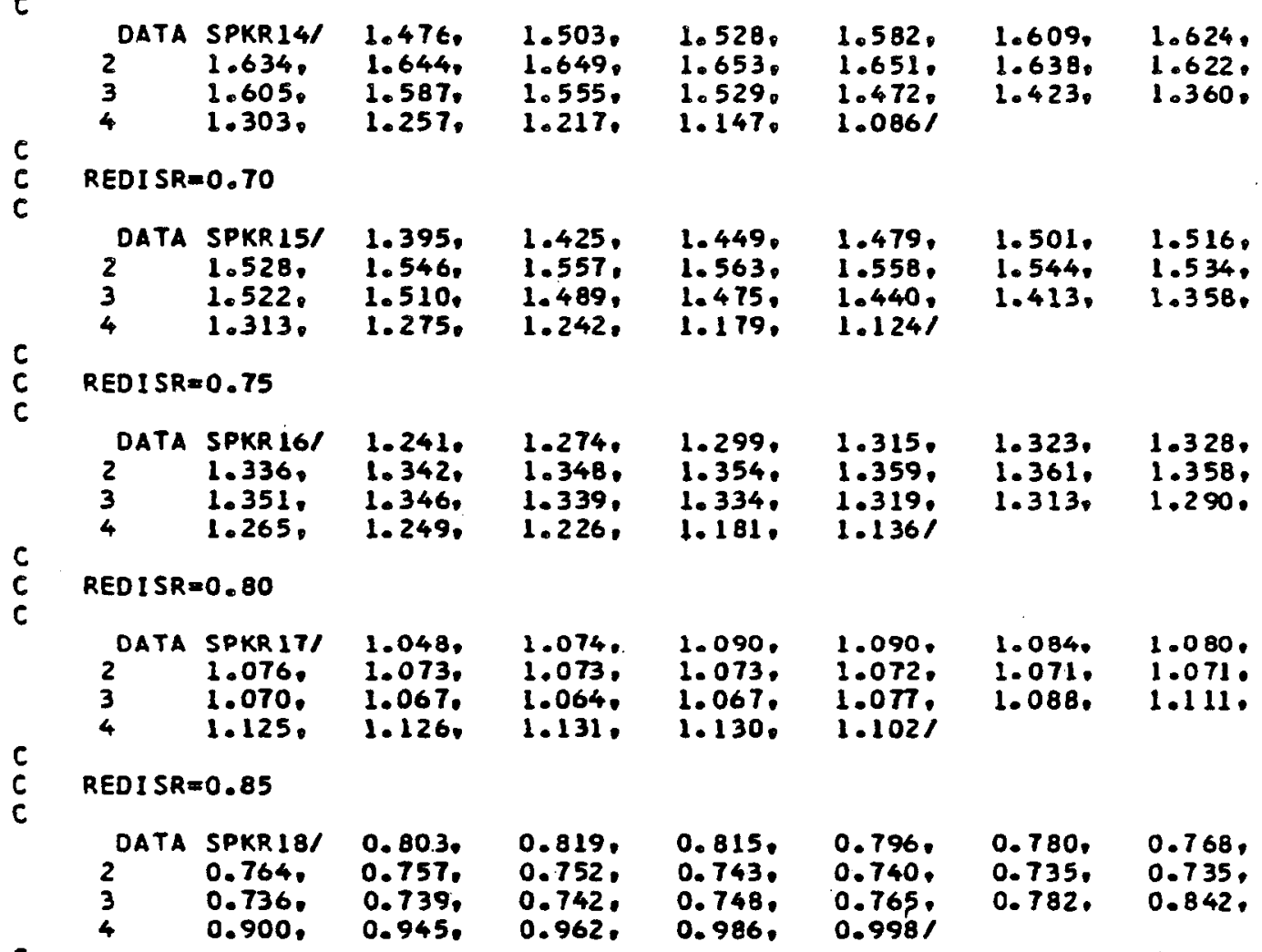

C REDISR $=0.90$ 


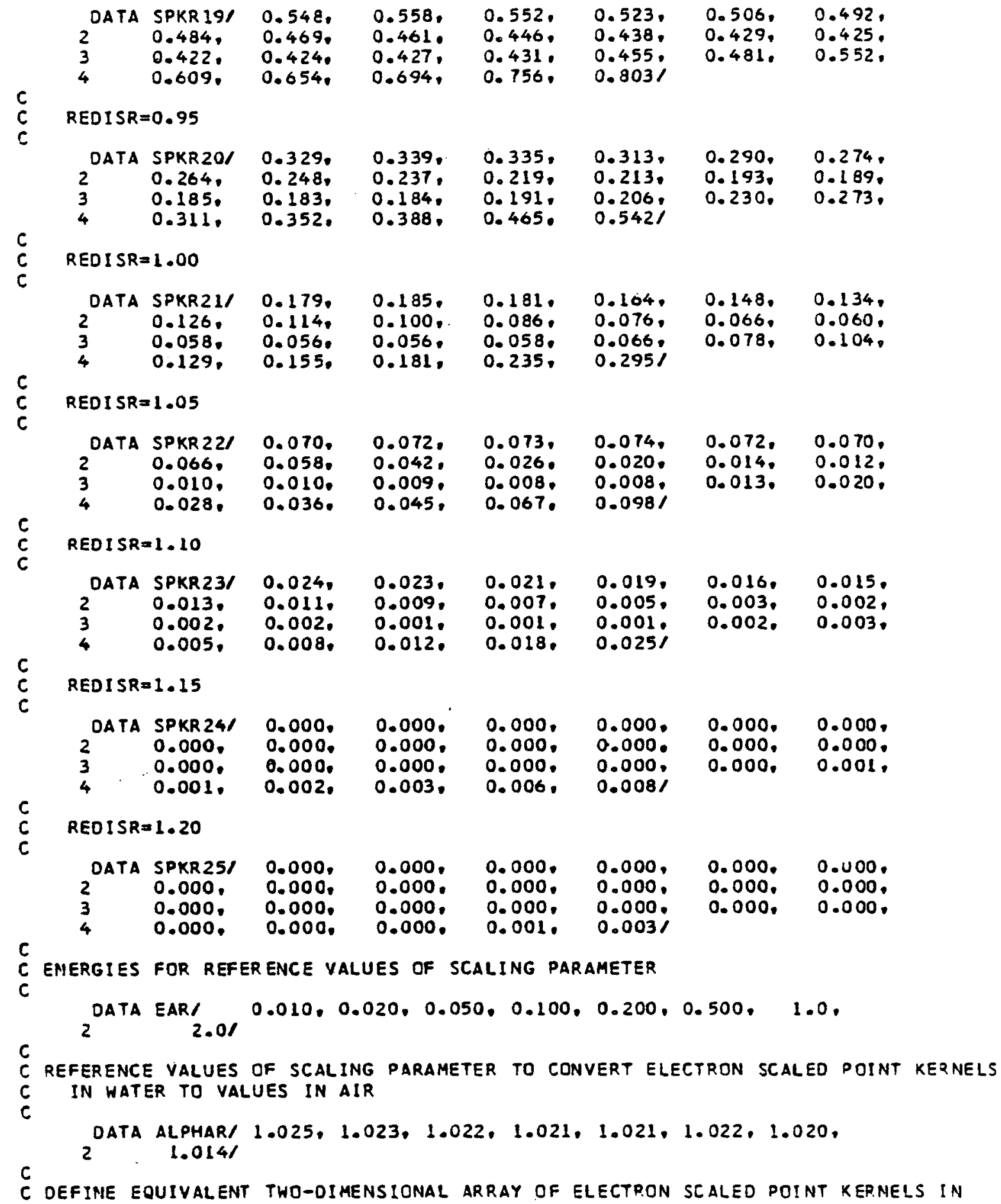




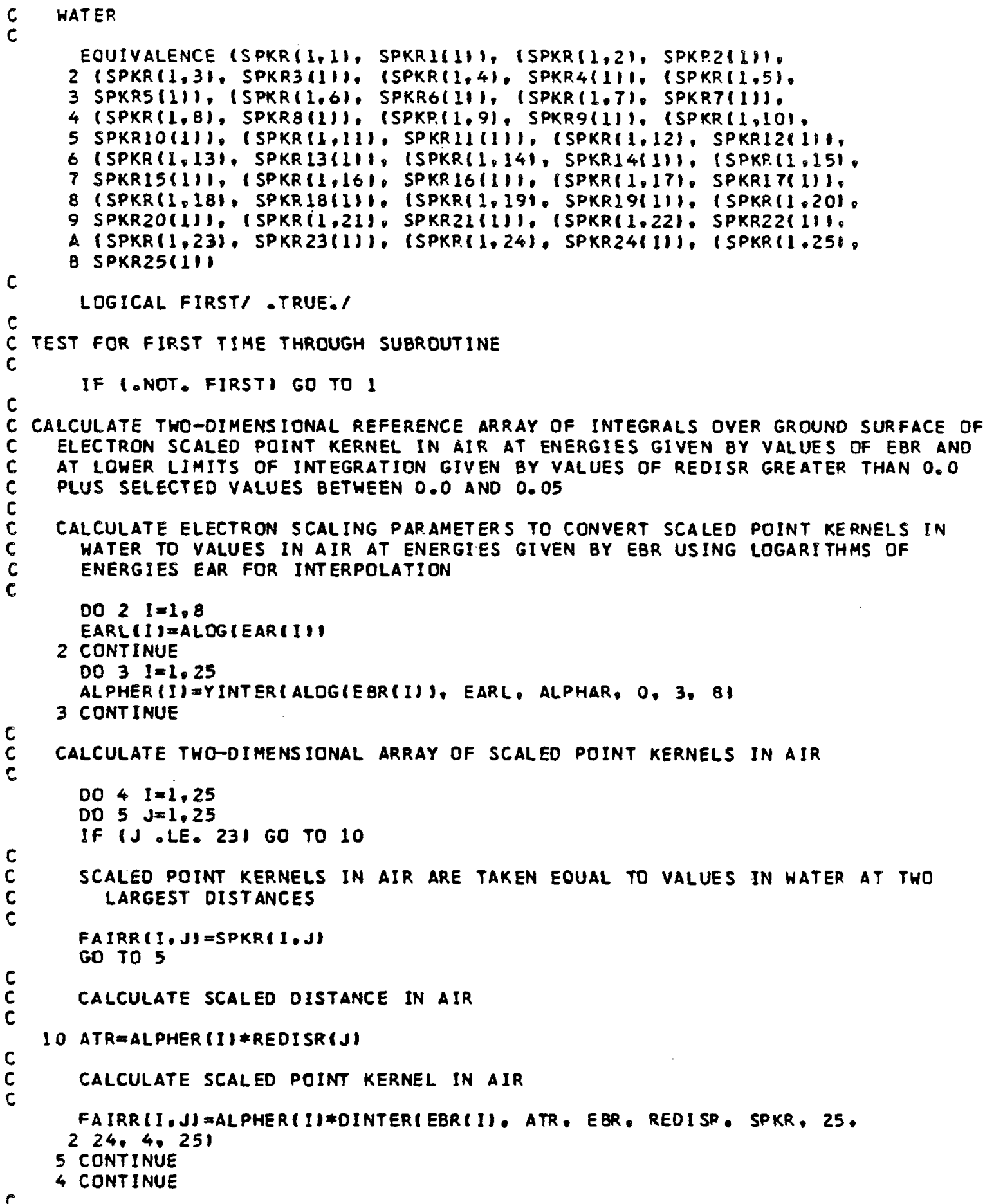


C

CALCULATE INTEGRALS AT REDISR=0.05, $0.15,0.25, \ldots \ldots 1.15$

$007 \mathrm{~J}=2.24,2$

INITIALIZE INNER TERMS IN SIMPSON'S RULE

$T 2=0.0$

$T 3=0.0$

C

CALCULATE OUTER TERM ASSUMING SCALED POINT KERNEL IS ZERO AT REDISR=1.25 TI=FAIRRI I , J)/REDISR (J)

C CALCULATE INNER TERMS

$J \mathbf{l}=\mathbf{J}+\mathbf{l}$

DO $8 \quad k=J 1.25,2$

$T 2=T 2+F A I R R(I, K) / R E D I S R(K)$

8 CONTINUE

IF (J.EQ. 24) GO TO 11

$J 2=j+2$

DO $\quad K=J 2,24,2$

DO $9 \quad K=J 2,24,2$
$T 3=T 3+F A I R R(I, K) / R E D I S R(K)$

9 CONTINUE

$c$
$c$
$c$
$c$
$C$
$c$
$C$
$C$
$C$
$C$
$C$
$C$

REDEFINE INOEX FOR VARIABLE REDISR SO THAT VALUE IS SIX AT REDISR=0.05; THIS ALLOWS ROOM FOR CALCULATING INTEGRALS FOR FIVE VALUES OF REDISP. BETWEEN 0.0 AND 0.05

$11 K=J+4$

COMBINE TERMS TO OBTAIN INTEGRAL; INTERVAL WIDTH IS 0.05

R.INTZE $(1, K)=0.05 *(T 1+4.0 * T 2+2.0 * T 31 / 3$.

7 CONTINUE

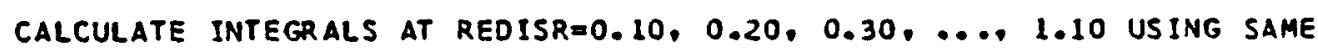

PROCEDURE AS ABOVE. EXCEPT FOR ASSUMPTION FOR OUTER TERM

DO $12 \mathrm{~J}=3.23 .2$

$\mathrm{T} 2=0.0$

$T 3=0.0$

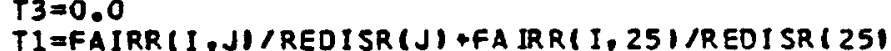

$J 1=J+1$

DO $13 \mathrm{~K}=\mathrm{J1}, 24,2$

$T 2=T 2+F A I R R(I, K) / R E D I S R(K)$

13 CONTINUE

IF IJ.EQ. 231 GO TO 14

$\mathrm{J} 2=\mathrm{J}+\dot{2}$

OO $15 \quad K=J 2,23,2$

T $3=T 3$ *FAIRR I I KI /REDISR (K)

15 CONTINUE

$14 K=J+4$

RINTIE $(I, K)=0.05 *(T 1+4.0 * T 2+2.0 * T 3) / 3$.

12 CONTINUE 


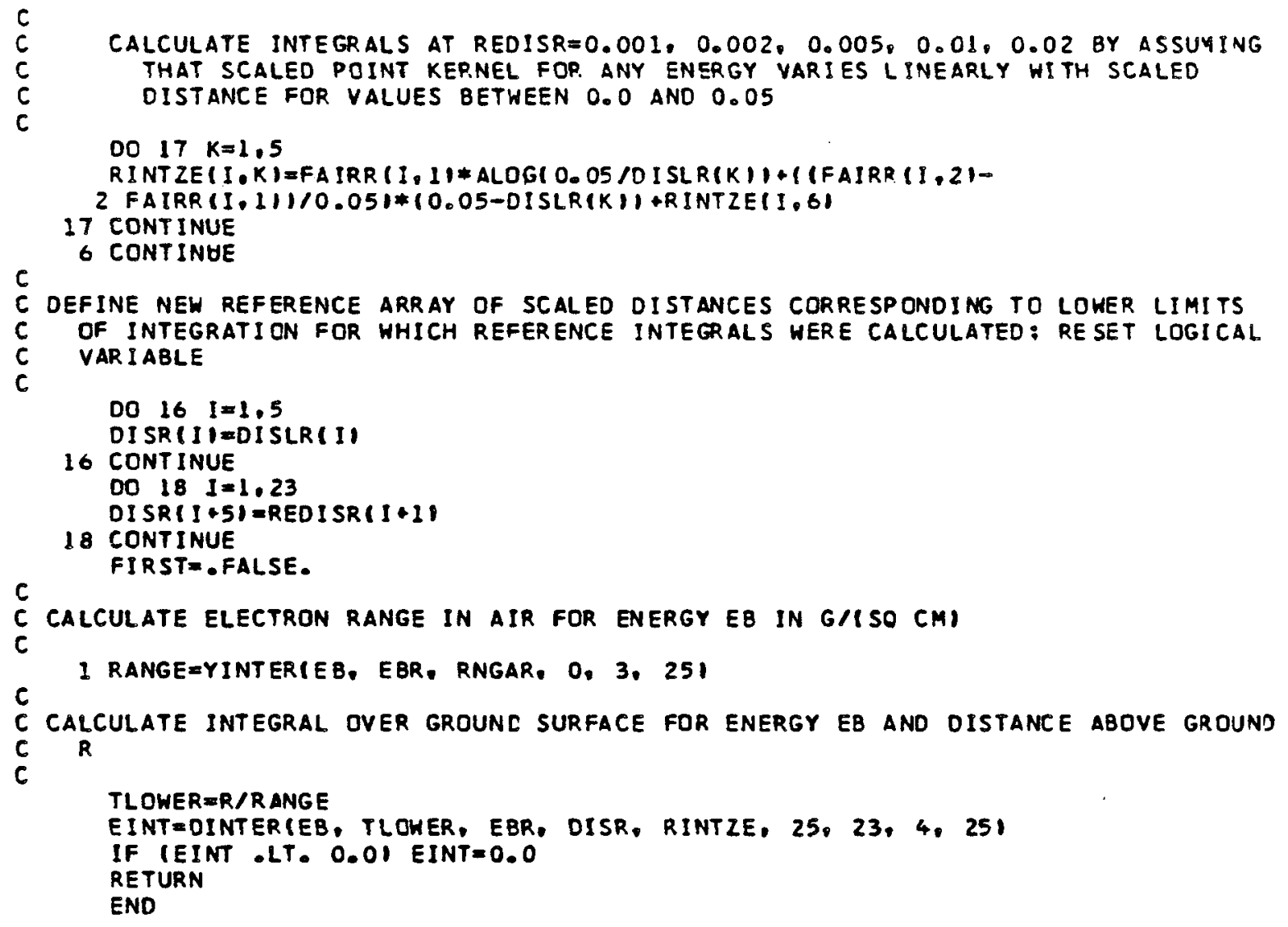


FUNCTION DINTERIXX, YY, X, Y, I, NX, NY, M, IDI

C PERFORMS LAGRAMGIAN INTERPOLATION OF A FUNCTION OF TWO VARIABLES

$C$ C BASED ON DOUBLE PRECISION INTERPOLATION FUNCTION OLAG DESCRIBED IN UNION CARB IDE PUBLICATION CTC-39

$X X$ - ABCISSA OF DESIRED INTERPOLATION POINT

$Y Y$ - DRDINATE OF DESIRED INTERPOLATION POINT

$X$ - SINGLY DIMENSIONED ARRAY OF ABCISSAS

$Y$ - SINGLY DIMENSIONED ARRAY OF ORDINATES

$Z$ - DOUBLY DIMENSIONED ARRAY OF FUNCTION VALUES GIVEN BY $211 . \mathrm{J} 1=$ $F(X(I), Y I J)$

NX - NUMBER OF ELEMENTS FOR THE X-ARRAY

NY - NUMBER OF ELEMENTS FOR THE Y-ARRAY

$M$ - NUMBER OF POINTS IS M\#M FOR THE INTERPOLATION

ID - FIRST DIMENSION OF $Z$ IN CALLING PROGRAM

DIMENSION Z(10,1), X(1), Y(1), XLAG(100)

LOGICAL OUITX, OUITY

C INITIALIZE

OUITX=,FALSE.

QUITY=.FALSE.

$M l=M$

$1 E X T X=0$

IEXTY $=0$

C FINO THE RANGE OF INTERPOLATION ALONG $x$

IF $|M|$.LE. $N X \mid$ GO TO 10

$M I=N X$

$100020 \quad I=1, N X$

If $(x x-x|I|) 30,90,20$

20 CONTINUE

IEXTX $=1$

GO TO 80

$30 \mathrm{MIOX}=1$

IF (MIDX . GT I) GO TO 40

IEXTX $=-1$

GO TO 50

40 IF (ABS(XX-X(MIOX)).GE. ABS(XX-X(MIDX-1)) GO TO 50 ISXPT $=M I O X-(M 1 / 2)$

GO TO 60

50 I $S X P T=M I D X-((M)+1) / 2)$

60 IF (ISXPT.GT. 0 ) GO TO 70

I $S X P T=1$

70 IEXPT $=$ ISXPT $+M 1-1$

IF (IEXPT LE. NX) GO TO 100

80 ISXPT $=N X-M i+1$

IEXPT $=N X$

GO TO 100

90 OUITX=.TRUE. 


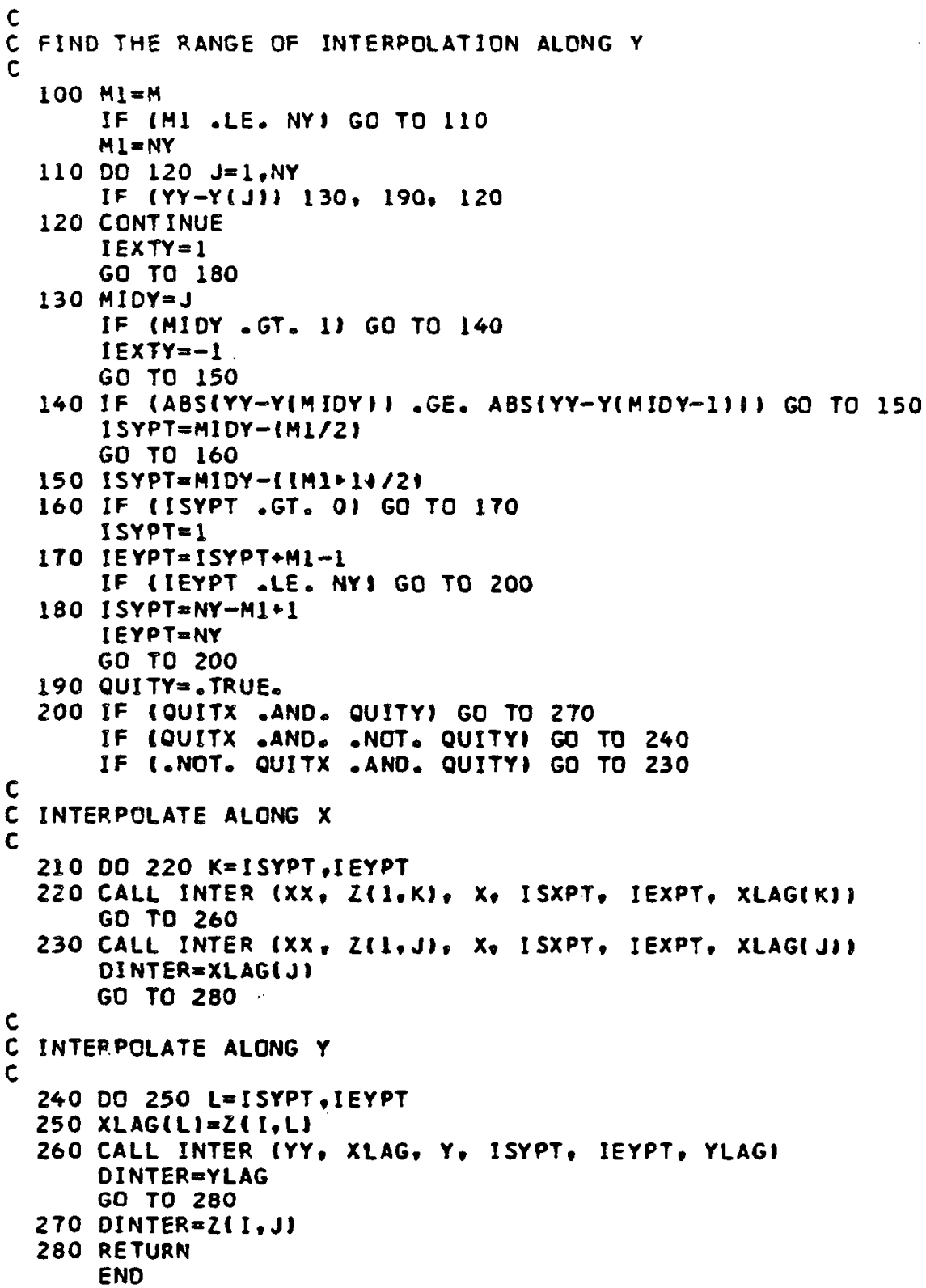


c

SUBROUTINE INTER (PT, FUN, ORD, ISPT, IEPT, ALAG)

C AN INTERPOLATION ROUTINE DESCRIBED IN UNION CARBIOE PUBLICATION CTC-39

C

DIMENSION FUN(1), ORDII)

SUM $=0.0$

$P R O D=1$.

DO $30 \mathrm{~J}=$ ISPT , I EPT

PROD $=P R O D *(P T-O R D(\mathrm{~J}))$

$D E N O M=1$.

DO $20 \quad K=$ ISPT, IEPT

IF IK.NE. JI GO TO 10

$D=P T$

60 ro 20

$10 D=O R D(J)$

20 DENOM $=D E N O M *(D-O R D(K))$

30 SUM $=$ SUM FUNA J / /OENOM

ALAG $=$ SUM* PROD

RETURN

END 
FUNCTION DCGAM(Z)

C CALCULATES gamma funct IONS OF a COMPLEX ARgument USING a SEP. IES EXPANSION; SEe EQ. 6.1.34 IN HANDBDOK OF MATHEMATICAL FUNCTIONS. PG. 256

COMPLEX 16 Z, SUM, DCGAM DOUBLE PRECISI ON C (26)

DATA C/ 1.0, 5.7721566490153290-01, -6.5587807152025380-01,

$2-4.200263503409520-02$. $1.6653861138229150-01$.

3 -4.219773455554430-02, $-9.62197152787700-03,7.21894324666300-03$,

$4-1.16516759185910-03,-2.1524167411490-04,1.2805028238820-04$.

$5-2.013485478070-05,-1.25049348210-06,1.13302723200-06$

$5-2.013485478070-05,-1.25049348210-06,1.13302723200-06$,
$6-2.0563384170-07,6.11609500-09,5.00200750-09,-1.18127460-09$ 。

$71.0434270-10,7.78230-12,-3.69680-12,5.1000-13,-2.060-14$.

$8-5.40-15,1.40-15,1.0-161$

SUM $=0.000$

DO $11=1.26$

SUM $=S U M+C(I) *(Z * * 1)$

1 CONTINUE

DCGAM=1.000/ SUM

RETURN

END 
$c$

SUBROUTINE SORT (N, A, B)

C SORTS A ONE-OIMENSIONAL ARRAY IN DECREASING NUMERICAL ORDER

C N - NUMBER OF ELEMENTS IN ARRAY

c A - INPUT ARRAY

C B - SORTED ARRAY

DIMENSION A(1), B(1)

$N 1=N-1$

DO $1 \quad I=1 . N 1$

$N I=N-I$

DO $1 \mathrm{~K}=1, \mathrm{NI}$

IF (A(K). LE. $A(K+1))$ GO TO 1

$T=A(K)$

$A(K)=A(K+1)$

$A(K+1)=T$

1 CONTINUE

Do $2 I=1, N$

$N I=N-I \bullet I$

B(I)FAINI)

2 CONTINUE

RETURN

END 
SUBROUTINE DOSOUT

C WRITES DOSE-RATE FACTORS IN TABLES AND ON PUNCHEO CARDS

DIMENSION INUCL 21 . THALF131

DIMENSION DOSORG $(3.23)$. DOSORB $(3,4)$, DOSSKN(3), DOSEFF(3)

COMMON/ BLKL/ INUCL. THALF

C

COMMON/ BLKS/ DOSORG, DOSORB, DOSSKN, DOSEFF

C MAXIMUM NUMBER OF LINES OF OUTPUT PER PAGE FOR. EACH TYPE OF DOSE-RATE FACTOR TABLE

$C$

DATA MAXLOF/40\% MAXSKN/50\%, MAXSKT/50/, MAXEFF/50/

NUMBER OF LINES OF OUTPUT BETHEEN SPACES FOR SUMMARY TABLE OF ORGAN AND EFFECTIVE DOSE-RATE FACTORS

DATA MAXSKP/5/

c

LOGICAL FIRST/ .TRUE./

C TEST FOR FIRST TIME THROUGH SUBROUTINE

IF I.NOT. FIRSTI GO TO 1

C INITIALIZE LINE COUNTS HITHIN EACH TABLE AND RESET LOGICAL VARIABLE

c

LINORG $=0$
LINSKP $=0$

LINSKN=0

LINSKT $=0$

LINEFF $=0$

FIRST $=$.FALSE.

c

C WRITE DOSE-RATE FACTORS IN TABLES

C PHOTON DOSE-RATE FACTORS FOR ALL DRGANS EXCEPT SKIN, PHOTON PLUS ELECTRON DOSE-RATE FACTORS FOR SKIN. AND EFFECTIVE DOSE-RATE FACTORS FOR THREE EXPOSURE MODES

AIR IMMERSION

1 WRITE $(15.4)$ INUCL, THALF, (DOSORG $(1, \mathrm{~J}), \mathrm{J}=1,9)$

4 FORMAT IIH. 2A4, IX, 3A4, 9IIPE II. 211

WP.ITE (16.4i INUCL. THALF, (DOSORG I I,J), Je10.16), (DOSORG(1.J), $2 J=18,191$

WRITE $(17,4)$ INUCL, THALF, (DOSORG $(1, \mathrm{~J}), J=20,23)$, DOSEFFI1),

2 DOSORG $(1,17)$, DOSORB $(1,4)$, DOSSKN1 1$)$

$c$
$c$

WATER IMMERSION

HRITE $(20,4)$ INUCL. THALF, (DOSORG $(2,3), j=1,9)$

WRITE $(21,4)$ INUCL. THALF, (DOSORG $(2, \mathrm{~J}), J=10,16)$, IDOSORG $(2, \mathrm{~J})$, $2 \mathrm{~J}=18,191$ 
WRITE 122,4$)$ INUCL. THALF, IOOSORG $(2, \mathrm{~J} 1, \mathrm{~J}=20,231$, DOSEFFI 21 , 2 DOSORG $(2,17)$. DOSORBI 2, 4\%, DOSSKN 21

c

GROUNO SURFACE EXPOSURE

WRITE $(25,4)$ INUCL, THALF, (DOSORG $(3, J), J=1,9)$

HRITE $(26,4)$ INUCL. THALF, (DOSORG $(3, \mathrm{~J}), j=10,16)$, (DOSORG $(3, \mathrm{~J})$,

$2 J=18,191$

WRITE $(27,4)$ INUCL, THALF, IDOSORG $(3, \mathrm{~J}), J=20,23), \operatorname{DOSEFF}(3)$,

2 DOSORG (3.17), DOSORB (3.4), DOSSKN(3)

LINSKP $=$ LINSKP +1

IF (LINSKP . LT. MAXSKP) GO TO 3

WRITE $(15,15)$

15 FORMAT (IH)

WRITE $(16,15)$

WRITE $(17,15)$

WRITE $(20,15)$

WRITE 121.15

HRITE 122,151

WRITE 122.150

WRITE 126,15

WRITE 127,151

LINSKP $=0$

3 LINORG $=$ LI NORG +1

IF ILINORG -LT. MAXLOFI GO TO 5

CALL PRINT 161

LINORG $=0$

ELECTRON DOSE-RATE FACTORS FOR DIFFERENT DEPTHS IN SKIN FOR THREE EXPOSURE MODES

5 HRITE $(18,2)$ INUCL. THALF, (DOSORB $(1, \mathrm{~J}), \mathrm{J}=1,4)$

2 FORMAT IIH, 4X, 2A4, 5X, 3A4, 6X, IP510.2, 3(8X, (PE10.2))

WRITE $(23,2)$ INUCL. THALF, (DOSORB $(2, \mathrm{~J}), \mathrm{J}=1.41$

HRITE $(28,2)$ INUCL. THALF, (DOSORB $(3, \mathrm{~J}), \mathrm{J}=1,4)$

LINSKN $=$ LINSKN+I

IF (LINSKN - LT. MAXSKN) GO TO 11

CALL PRINT (7)

LINSKN=0

DOSE-RATE FACTORS FOR SKIN FOR PHDTONS. ELECTRONS. ANO PHOTONS PLUS ELECTRONS FOR THREE EXPOSURE MODES

11 HRITE $(19,2)$ INUCL. THALF, DOSORG 1,17$),$ DOSOR $8(1,4)$, DOSSKN(1) WRITE $(24,2)$ INUCL. THALF, DOSORG 2.17$)$, DOSORB(2,4), DOSSKN(2) WRITE $(29.2)$ INUCL. THALF, DOSORG 3,171 , DOSORB $(3,4)$, DOSSKN(3) LINSKT $=$ LINSKT+1

IF (LINSKT .LT. MAXSKTI GO TO 9

IF (LINSKT ILT

LINSKT $=0$

c

EFFECTIVE DOSE-RATE FACTORS FOR THREE EXPOSURE MODES

9 WRITE $(30.2)$ INUCL, THALF, (DOSEFF $(1) ; 1=1,3)$

LINEFF $=L$ INEFF $\bullet$ I

IF (LINEFF. LT. MAXEFF) GO TO 20

CALL PRINT (9)

$L$ INEFF $=0$ 
C WPITE DOSE-RATE FACTORS ON PUNCHED CARDS

RADI ONUCLIDE IDENT IFICATION

20 HRITE $(7.6)$ INUCL. THALF

6 FORMAT (2A4, 2X, 3A4)

PHOTON DOSE-RATE FACTORS FOR ALL ORGANS EXCEPT SKIN, EFFECTIVE DOSE-RATE FACTORS FOR PHOTONS, AND PHOTON PLUS ELECTRON DOSE-RATE FACTOPS FOR SKIN FOR THREE EXPOSURE MODES

DO $8 \quad 1=1.3$

HR.ITE (7, T) (DOSORG I $, J 1, J=1,16)$, (DOSORGI I,J), J=18,231,

2 DOSEFF(I), DOSSKN(I)

7 FORMAT $18(1 \mathrm{PE} 10.21)$

8 CONTINUE

C ELECTRON DOSE-RATE FACTORS FOR DIFFERENT DEPTHS IN SKIN FOR THREE EXPOSURE MODES

DO $10 \quad 1=1.3$

WRITE $(7,7) \quad$ COOSORB (I, J1, J=1,4)

10 CONTINUE

$c$

DOSE-RATE FACTORS FOR SKIN FOR PHOTONS. ELECTRONS, AND PHOTONS PLUS ELECTRONS FOR THREE EXPOSURE MODES

DO $12 I=1,3$

HRITE 17,71 DOSORG 1,171, DOSORB 11,41, DOSSKNI II

12 CONTINUE

c

EFFECTIVE DOSE-RATE FACTORS FOR THREE EXPOSURE MODES

WR ITE 17,7$)$ IDOSEFF(I), $[=1,3)$

RETURN

END 